\title{
Enantioselective Allenoate-Claisen Rearrangement using Chiral Phosphate Catalysts
}

\author{
Javier Miró,†,\# Tobias Gensch,†,\# Mario Ellwart, $\uparrow$ Seo-Jung Han, $\dagger, \S$ Hsin-Hui Lin, $\uparrow$ Matthew S. Sigman,,+ , F. \\ Dean Toste, ${ }^{*}, \dagger$ \\ + Department of Chemistry, University of California, Berkeley, California 94720, United States \\ + Department of Chemistry, University of Utah, 315 South 1400 East, Salt Lake City, Utah 84112, United States \\ $\S$ Chemical Kinomics Research Center and Division of Bio-Medical Science \& Technology, Korea Institute of \\ Science and Technolo-gy (KIST), Seoul, 02792, Republic of Korea
}

\section{SUPPORTING INFORMATION}

\section{TABLE OF CONTENTS}

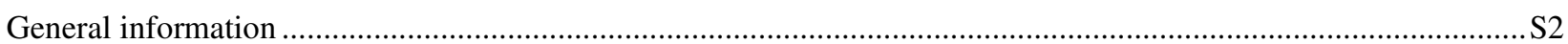

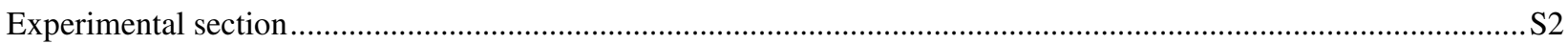

General procedure A: Enantioselective allenoate-Claisen rearrangement. .......................................................S2

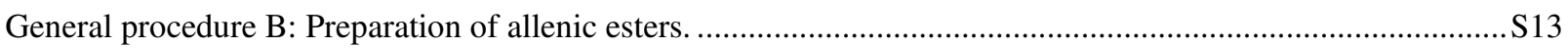

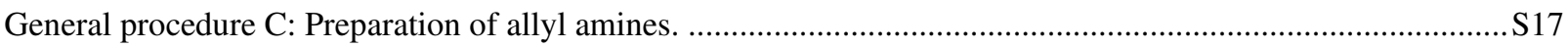

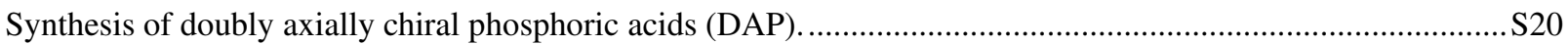

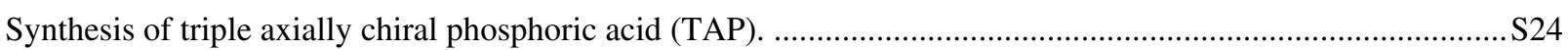

Summary data on the optimization of the enantioselective allenoate-Claisen rearrangement. ............................S29

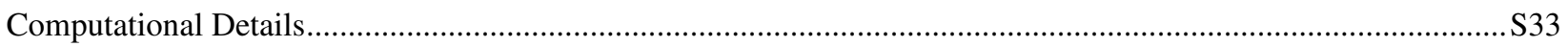

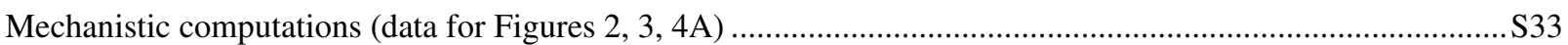

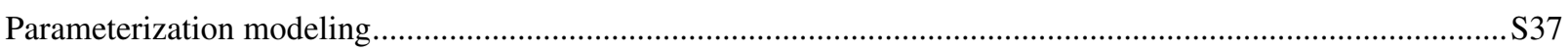

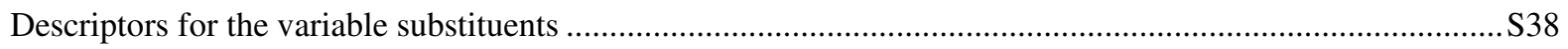

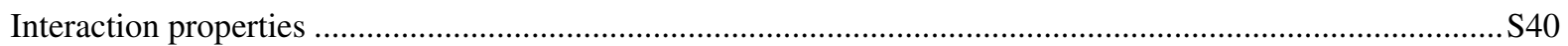

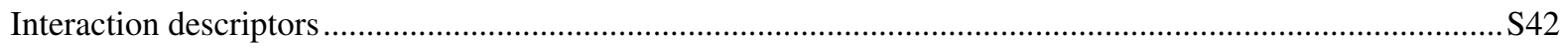

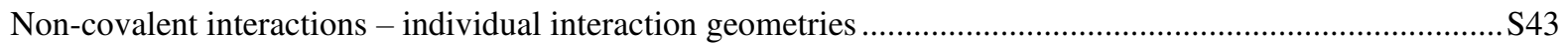

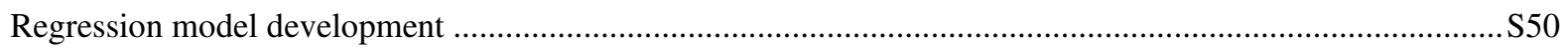

Additional discussion on confounding effects between descriptors for NCI and sterics ..................................S51

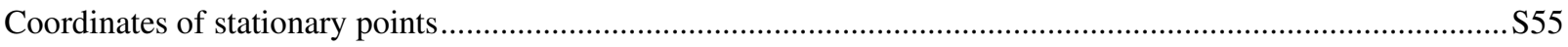

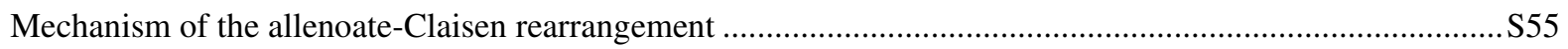

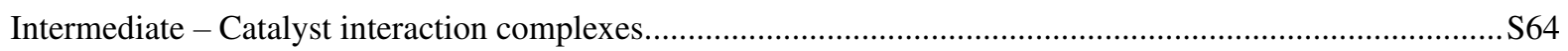

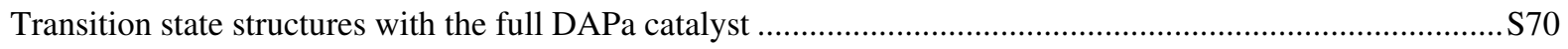

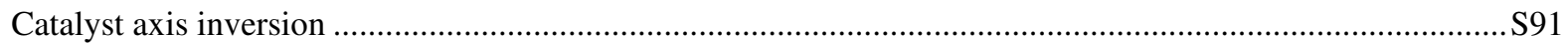

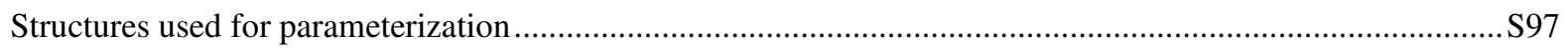

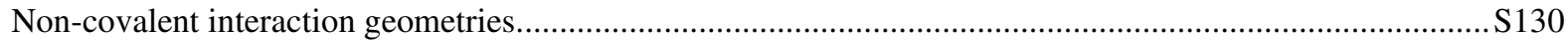

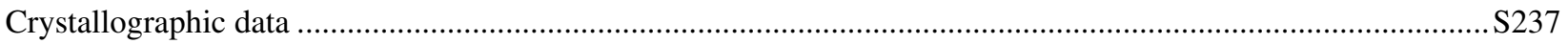

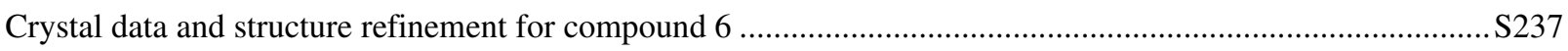

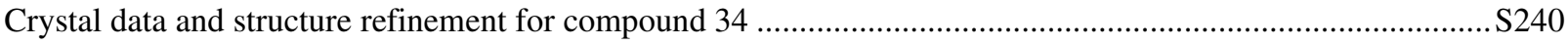

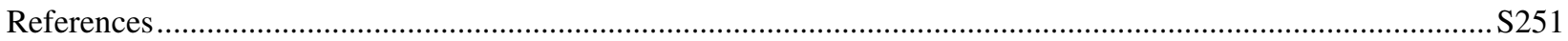

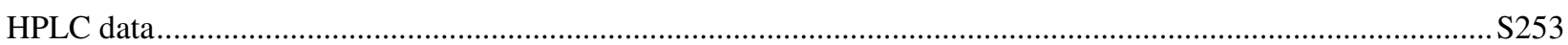

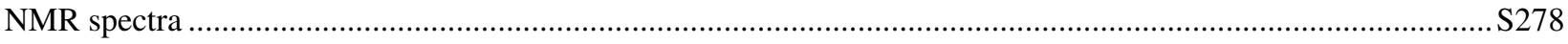




\section{General information}

Unless otherwise noted, all reagents were purchased from commercial suppliers and used without further purification. Chiral reactions were performed in 1-dram ( 0.5 " X 1.75 ") vials equipped with a screw cap and stirred using a magnetic Teflon stir bar (1/2" X 5/16"), placed on the surface of a magnetic stir plate. Cyclohexane was used as purchased from Fischer Scientific. Thin-layer chromatography (TLC) analysis of reaction mixtures was performed using Merck silica gel 60 F254 TLC plates, and visualized under UV or by staining with $\mathrm{KMnO}_{4}$. Column chromatography was performed on Merck Silica Gel $60 \AA$, 230 X 400 mesh. Nuclear magnetic resonance (NMR) spectra were recorded using Bruker AV-600, AV-500, DRX-500, AVQ-400, AVB-400 and AV-300 spectrometers. ${ }^{1} \mathrm{H},{ }^{13} \mathrm{C}$ and ${ }^{19} \mathrm{~F}$ chemical shifts are reported in ppm downfield of tetramethylsilane and referenced to residual solvent peak $\left(\mathrm{CHCl}_{3}, \delta \mathrm{H}=7.26 \mathrm{ppm}\right.$ and $\delta \mathrm{C}=77.23 \mathrm{ppm} ; \mathrm{CH}_{2} \mathrm{Cl}_{2}, \delta \mathrm{H}=5.32$ and $\left.\delta \mathrm{C}=53.8 \mathrm{ppm}\right)$. Multiplicities are reported using the following abbreviations: $\mathrm{s}=$ singlet, $\mathrm{d}=$ doublet, $\mathrm{t}=$ triplet, $\mathrm{q}=$ quartet, quin $=$ quintet, $\mathrm{h}=$ sextet, $\mathrm{m}=$ multiplet, $\mathrm{br}=$ broad resonance. Solvent abbreviations are reported as follows: EtOAc $=$ ethyl acetate, hex $=$ hexanes, $\mathrm{DCM}=$ dichloromethane, $\mathrm{Et}_{2} \mathrm{O}=$ diethyl ether, $\mathrm{MeOH}=$ methanol, $i \mathrm{PrOH}=$ isopropanol, $\mathrm{THF}=$ tetrahydrofuran, $\mathrm{DMF}=N, N$-dimethylformamide, $\mathrm{Et}_{3} \mathrm{~N}=$ triethylamine. Acid chlorides were distilled immediately prior to use. Mass spectral data were obtained from the Micro-Mass/Analytical Facility operated by the College of Chemistry, University of California, Berkeley or by usage of an Agilent Time of Flight (Q-TOF) mass spectrometer in ESI mode. Enantiomeric excesses were measured on a Shimadzu VP Series Chiral HPLC using Chiralpak ADH, ODH, OJH, IA, IB, or IC columns. The syntheses of the BINOL, VAPPOL, SPINOL, and BINAM-derived chiral phosphoric acids have been previously reported. Racemic products were synthesized utilizing conditions previously reported by MacMillan and Lambert. ${ }^{1}$

\section{Experimental section}

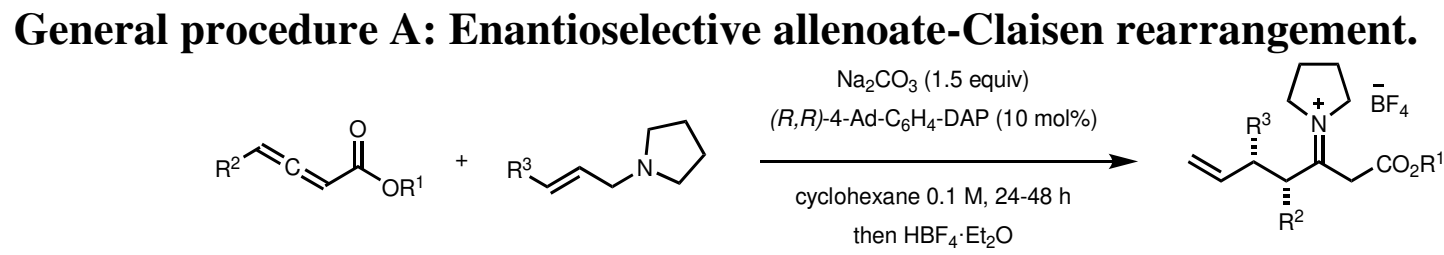

A 1 dram vial was charged with $\mathrm{Na}_{2} \mathrm{CO}_{3}$ (1.5 equiv), $(R, R)-4-\mathrm{Ad}_{-} \mathrm{C}_{6} \mathrm{H}_{4}-\mathrm{DAP}(10 \mathrm{~mol} \%)$, and $0.2 \mathrm{~mL}$ of cyclohexane. The vial was capped and the mixture was stirred for $30 \mathrm{~min}$. Then, allylamine ( 2.0 equiv) and allenoate ester $(0.06$ $\mathrm{mmol}$ ) were added with the rest of the solvent $(0.2 \mathrm{~mL})$. The vial was capped again and the reaction mixture was stirred vigorously monitoring the reaction progress by TLC analysis. Upon completion (24-72 h), the reaction was filtered through a plug of $\mathrm{SiO}_{2}$ eluting with $\mathrm{Et}_{2} \mathrm{O}$ to remove catalyst and remaining amine. To the filtrate, 1.0 equiv of $\mathrm{HBF}_{4} \cdot \mathrm{Et}_{2} \mathrm{O}$ was added. The mixture was sonicated for a few seconds, resulting in the precipitation of the tetrafluoroborate salt of the corresponding $\beta$-enamino ester as a white solid. Solvent was removed and the solid was washed with $\mathrm{Et}_{2} \mathrm{O}$, resulting in the pure iminium salts. Diastereomeric ratios could be determined either by ${ }^{1} \mathrm{H}$ NMR or ${ }^{19} \mathrm{~F} \mathrm{NMR.}{ }^{2}$ Isolated yields were assessed by dissolving the tetrafluoroborate iminium salts in DCM and washing with sat. aq. sol. $\mathrm{Na}_{2} \mathrm{CO}_{3}$. The combined organic layers were dried over anhydrous $\mathrm{Na}_{2} \mathrm{SO}_{4}$, filtered and concentrated under reduced pressure to afford the corresponding $\beta$-enamino esters. Generally, further derivatization to the corresponding hydrodeaminated $\alpha, \beta$-unsaturated esters was required to determine enantiomeric excesses.
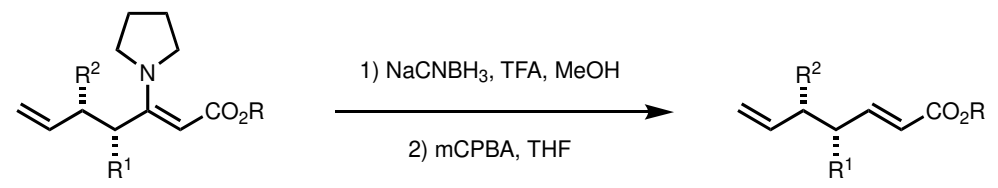

Over a solution of the $\beta$-amino- $\alpha, \beta$-unsaturated ester $(0.04 \mathrm{mmol})$ in $\mathrm{MeOH}(0.5 \mathrm{~mL}), \mathrm{NaCNBH}_{3}(15 \mathrm{mg}, 0.24 \mathrm{mmol})$ was added, followed by the addition of trifluoroacetic acid $(15 \mu \mathrm{L})$. After $30 \mathrm{~min}, 1 \mathrm{~mL}$ of $1 \mathrm{~N} \mathrm{NaOH}$ was added, and the solution was extracted with EtOAc, dried over $\mathrm{MgSO}_{4}$ and concentrated. The crude residue was taken up in THF 
$(0.5 \mathrm{~mL})$ and meta-chloroperbenzoic acid $(15 \mathrm{mg}, 0.09 \mathrm{mmol})$ was added. After $15 \mathrm{~min}$, the solution was diluted with EtOAc and successively washed with $\mathrm{H}_{2} \mathrm{O}, 20 \% \mathrm{Na}_{2} \mathrm{~S}_{2} \mathrm{O}_{3}$, and $1 \mathrm{~N} \mathrm{NaOH}$, dried over $\mathrm{MgSO}_{4}$ and concentrated. The crude residue was purified by preparative TLC to provide the $\alpha, \beta$-unsaturated ester product in sufficient amounts for analytical purposes.

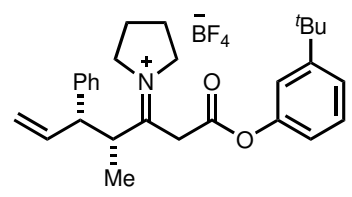

1-((4R,5S)-1-(3-(tert-butyl)phenoxy)-4-methyl-1-oxo-5-phenylhept-6-en-3-ylidene)pyrrolidin-1-ium

tetrafluoroborate (5). Following the general procedure A, 5 was isolated in $83 \%$ yield and $90 \%$ ee $(1: 0 \mathrm{dr}) .{ }^{1} \mathrm{H} \mathrm{NMR}$ $\left(400 \mathrm{MHz}, \mathrm{CDCl}_{3}\right) \delta$ 7.38-7.35 (m, 2H), 7.32-7.28 (m, 5H), 7.14-7.13 (m, 1H), $6.96(\mathrm{dt}, J=6.8,2.4 \mathrm{~Hz}, 1 \mathrm{H}), 6.05$ (ddd, $J=17.2,10.4,8.4 \mathrm{~Hz}, 1 \mathrm{H}), 5.17(\mathrm{~d}, J=10.0 \mathrm{~Hz}, 1 \mathrm{H}), 5.15(\mathrm{~d}, J=16.8 \mathrm{~Hz}, 1 \mathrm{H}), 4.32(\mathrm{~s}, 2 \mathrm{H}), 4.32-4.19(\mathrm{~m}, 3 \mathrm{H})$, 4.13-4.08 (m, 1H), 3.59-3.49 (m, 2H), 2.28-2.15 (m, 4H), $1.30(\mathrm{~s}, 9 \mathrm{H}), 1.16(\mathrm{~d}, J=6.0 \mathrm{~Hz}, 3 \mathrm{H}) .{ }^{13} \mathrm{C} \mathrm{NMR}(101 \mathrm{MHz}$, $\left.\mathrm{CDCl}_{3}\right) \delta 184.55,164.20,153.78,150.02,138.77,136.84,129.41,129.33,128.37,127.95,123.96,119.04,118.18$, $118.14,56.93,56.22,53.90,48.02,38.43,35.00,31.31,24.50,24.35,14.91 .{ }^{19} \mathrm{~F} \mathrm{NMR}\left(376 \mathrm{MHz} \mathrm{CDCl}_{3}\right) \delta-150.93$. HRMS (ESI) $\mathrm{m} / z$ [M+H] ${ }^{+}$calcd for $\mathrm{C}_{28} \mathrm{H}_{35} \mathrm{NO}_{2} 418.2741$, found 418.2755. Enantiomeric excess was determined by derivatization to the corresponding hydrodeaminated $\alpha, \beta$-unsaturated ester. ${ }^{1} \mathrm{H}$ NMR $\left(600 \mathrm{MHz}, \mathrm{CDCl}_{3}\right) \delta 7.34-7.30$ $(\mathrm{m}, 3 \mathrm{H}), 7.26-7.22(\mathrm{~m}, 2 \mathrm{H}), 7.20(\mathrm{~d}, J=7.1 \mathrm{~Hz}, 2 \mathrm{H}), 7.14(\mathrm{dd}, J=15.7,8.2 \mathrm{~Hz}, 1 \mathrm{H}), 7.11(\mathrm{t}, J=2.0 \mathrm{~Hz}, 1 \mathrm{H}), 6.95$ (ddd, $J=7.9,2.1,0.8 \mathrm{~Hz}, 1 \mathrm{H}), 6.01(\mathrm{dd}, J=15.6,0.6 \mathrm{~Hz}, 1 \mathrm{H}), 6.00(\mathrm{ddd}, J=17.0,10.2,8.6 \mathrm{~Hz}, 1 \mathrm{H}), 5.11(\mathrm{~d}, J=10.2$ $\mathrm{Hz}, 1 \mathrm{H}), 5.05(\mathrm{~d}, J=17.0 \mathrm{~Hz}, 1 \mathrm{H}), 3.22(\mathrm{t}, J=8.6 \mathrm{~Hz}, 1 \mathrm{H}), 2.79(\mathrm{~h}, J=7.2 \mathrm{~Hz}, 1 \mathrm{H}), 1.32(\mathrm{~s}, 9 \mathrm{H}), 0.99(\mathrm{~d}, J=6.7 \mathrm{~Hz}$, $3 \mathrm{H}) .{ }^{13} \mathrm{C}$ NMR $\left(151 \mathrm{MHz}, \mathrm{CDCl}_{3}\right) \delta 165.20,154.70,153.14,150.80,142.43,139.60,128.98,128.76,128.16,126.76$, 122.86, 120.60, 118.78, 118.77, 116.59, 55.96, 41.92, 34.94, 31.41, 18.22. HRMS (ESI) $\mathrm{m} / z$ [M+Na] ${ }^{+} \mathrm{calcd}$ for

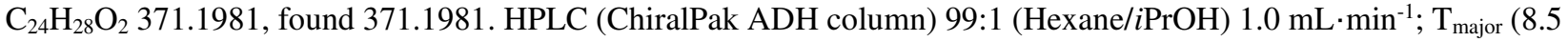
$\min ), \mathrm{T}_{\text {minor }}(7.6 \mathrm{~min}) ; 95.1: 4.9 \mathrm{er}$.

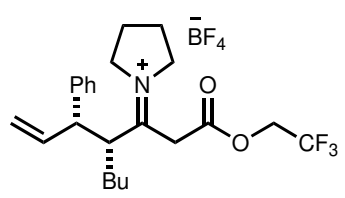

1-((R)-1-oxo-4-((S)-1-phenylallyl)-1-(2,2,2-trifluoroethoxy)octan-3-ylidene)pyrrolidin-1-ium tetrafluo-roborate (8). Following the general procedure A, 8 was isolated in $82 \%$ yield and $95 \%$ ee $(96: 4 \mathrm{dr}) .{ }^{1} \mathrm{H} \mathrm{NMR}\left(500 \mathrm{MHz}, \mathrm{CDCl}_{3}\right)$ $\delta 7.36(\mathrm{t}, J=8.0 \mathrm{~Hz}, 2 \mathrm{H}), 7.29-7.26(\mathrm{~m}, 3 \mathrm{H}), 5.98(\mathrm{dt}, J=16.5,10.0 \mathrm{~Hz}, 1 \mathrm{H}), 5.16(\mathrm{~d}, J=10.0 \mathrm{~Hz}, 1 \mathrm{H}), 5.14(\mathrm{~d}, J=$ $17.0 \mathrm{~Hz}, 1 \mathrm{H}), 4.73-4.67(\mathrm{~m}, 1 \mathrm{H}), 4.60-4.53(\mathrm{~m}, 1 \mathrm{H}), 4.27-4.23(\mathrm{~m}, 1 \mathrm{H}), 4.17-4.06(\mathrm{~m}, 3 \mathrm{H}), 4.16(\mathrm{~s}, 2 \mathrm{H}), 3.52-3.47(\mathrm{~m}$, $1 \mathrm{H}), 3.37(\mathrm{td}, J=9.5,4.0 \mathrm{~Hz}, 1 \mathrm{H}), 2.23-2.18(\mathrm{~m}, 4 \mathrm{H}), 1.52-1.42(\mathrm{~m}, 2 \mathrm{H}), 1.14-1.04(\mathrm{~m}, 4 \mathrm{H}), 0.72(\mathrm{t}, J=6.5 \mathrm{~Hz}$, $3 \mathrm{H}) .{ }^{13} \mathrm{C}$ NMR $\left(126 \mathrm{MHz}, \mathrm{CDCl}_{3}\right) \delta 184.03,163.73,139.19,136.13,129.49,128.15,127.98,122.67\left(\mathrm{q},{ }^{1} J_{\mathrm{CF}}=278.5\right.$ $\mathrm{Hz}), 119.17,61.82\left(\mathrm{q},{ }^{2} J_{\mathrm{CF}}=36.5 \mathrm{~Hz}\right), 57.22,56.78,54.79,53.74,29.75,29.65,24.43,24.40,22.49,13.49 .{ }^{19} \mathrm{~F} \mathrm{NMR}$ $\left(376 \mathrm{MHz}, \mathrm{CDCl}_{3}\right) \delta-72.74,-150.52$. HRMS (ESI) $\mathrm{m} / z[\mathrm{M}+\mathrm{H}]^{+}$calcd for $\mathrm{C}_{23} \mathrm{H}_{30} \mathrm{~F}_{3} \mathrm{NO}_{2}$ 410.2302, found 410.2322 . Enantiomeric excess was determined by derivatization to the corresponding hydrodeaminated $\alpha, \beta$-unsaturated ester. ${ }^{1} \mathrm{H}$ NMR $\left(600 \mathrm{MHz}, \mathrm{CDCl}_{3}\right) \delta 7.31(\mathrm{t}, J=7.6 \mathrm{~Hz}, 2 \mathrm{H}), 7.22(\mathrm{t}, J=7.4 \mathrm{~Hz}, 1 \mathrm{H}), 7.15(\mathrm{~d}, J=7.1 \mathrm{~Hz}, 2 \mathrm{H}), 6.89(\mathrm{dd}, J=$ $15.7,9.8 \mathrm{~Hz}, 1 \mathrm{H}), 5.90(\mathrm{ddd}, J=17.0,10.1,8.9 \mathrm{~Hz}, 1 \mathrm{H}), 5.82(\mathrm{~d}, J=15.7 \mathrm{~Hz}, 1 \mathrm{H}), 5.04(\mathrm{~d}, J=10.2 \mathrm{~Hz}, 1 \mathrm{H}), 4.99(\mathrm{~d}$, $J=17.0 \mathrm{~Hz}, 1 \mathrm{H}), 4.52(\mathrm{qd}, J=8.5,3.7 \mathrm{~Hz}, 2 \mathrm{H}), 3.27(\mathrm{t}, J=8.6 \mathrm{~Hz}, 1 \mathrm{H}), 2.54(\mathrm{qd}, J=9.4,3.8 \mathrm{~Hz}, 1 \mathrm{H}), 1.38-1.33(\mathrm{~m}$, $1 \mathrm{H}), 1.25-1.07(\mathrm{~m}, 5 \mathrm{H}), 0.79(\mathrm{t}, J=6.9 \mathrm{~Hz}, 2 \mathrm{H}) .{ }^{13} \mathrm{C}$ NMR $\left(151 \mathrm{MHz}, \mathrm{CDCl}_{3}\right) \delta 164.63,154.73,142.42,139.48$, $128.76,128.11,126.74,123.25\left(\mathrm{q},{ }^{1} J_{\mathrm{CF}}=277.8 \mathrm{~Hz}\right), 120.57,116.40,60.40\left(\mathrm{q},{ }^{2} J_{\mathrm{CF}}=36.2 \mathrm{~Hz}\right), 54.74,48.16,31.82$, 29.44, 22.61, 14.01. ${ }^{19} \mathrm{~F} \mathrm{NMR}\left(376 \mathrm{MHz}, \mathrm{CDCl}_{3}\right) \delta$-72.92. HRMS (EI) $m / z[\mathrm{M}]^{+}$calcd for $\mathrm{C}_{19} \mathrm{H}_{23} \mathrm{~F}_{3} \mathrm{O}_{2} 340.1650$, found 340.1651. HPLC (ChiralPak IA column) 99.9:0.1 (Hexane/iPrOH) 0.5 mL $\cdot \mathrm{min}^{-1} ; \mathrm{T}_{\text {major }}(10.7 \mathrm{~min}), \mathrm{T}_{\operatorname{minor}}(10.3 \mathrm{~min})$; 97.4:2.6 er. 


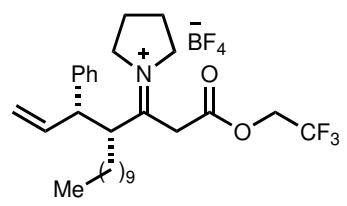

1-((R)-1-oxo-4-((S)-1-phenylallyl)-1-(2,2,2-trifluoroethoxy)tetradecan-3-ylidene)pyrrolidin-1-ium

tetrafluoroborate (10). Following the general procedure A, 10 was isolated in $78 \%$ yield and $94 \%$ ee $(91: 9 \mathrm{dr}) .{ }^{1} \mathrm{H} \mathrm{NMR}$ $\left(600 \mathrm{MHz} \mathrm{CDCl}_{3}\right) \delta 7.38-7.36(\mathrm{~m}, 2 \mathrm{H}), 7.30-7.26(\mathrm{~m}, 3 \mathrm{H}), 5.97(\mathrm{dt}, J=16.8,9.6 \mathrm{~Hz}, 1 \mathrm{H}), 5.19(\mathrm{~d}, J=10.2 \mathrm{~Hz}, 1 \mathrm{H})$, $5.15(\mathrm{~d}, J=16.8 \mathrm{~Hz}, 1 \mathrm{H}), 4.76-4.70(\mathrm{~m}, 1 \mathrm{H}), 4.58-4.52(\mathrm{~m}, 1 \mathrm{H}), 4.29-4.26(\mathrm{~m}, 1 \mathrm{H}), 4.12(\mathrm{q}, J=17.4 \mathrm{~Hz}, 2 \mathrm{H}), 4.05$ (br s, 2H), 3.50 (t, $J=9.3 \mathrm{~Hz}, 1 \mathrm{H}), 3.34-3.30(\mathrm{~m}, 1 \mathrm{H}), 2.24-2.18(\mathrm{~m}, 4 \mathrm{H}), 1.56-1.52(\mathrm{~m}, 1 \mathrm{H}), 1.46-1.43(\mathrm{~m}, 1 \mathrm{H}), 1.25-$ $1.07(\mathrm{~m}, 17 \mathrm{H}), 0.86(\mathrm{t}, J=7.2 \mathrm{~Hz}, 3 \mathrm{H}) .{ }^{13} \mathrm{C} \mathrm{NMR}\left(151 \mathrm{MHz}, \mathrm{CDCl}_{3}\right) \delta 184.00,163.84,139.24,135.86,129.54,128.15$, $128.04,122.66\left(\mathrm{q},{ }^{1} J_{\mathrm{CF}}=277.7 \mathrm{~Hz}\right), 119.47,61.85\left(\mathrm{q},{ }^{2} J_{\mathrm{CF}}=37.0 \mathrm{~Hz}\right), 57.29,56.70,54.91,53.76,37.99,31.98,30.04$, 29.84, 29.56, 29.53, 29.42, 29.36, 29.10, 27.75, 24.44, 24.39, 22.77, 14.21. ${ }^{19} \mathrm{~F} \mathrm{NMR}\left(376 \mathrm{MHz} \mathrm{CDCl}_{3}\right) \delta-72.72,-$ 150.82. HRMS (ESI) $\mathrm{m} / z$ [M+H] ${ }^{+}$calcd for $\mathrm{C}_{2} \mathrm{H}_{42} \mathrm{~F}_{3} \mathrm{NO}_{2}$ 494.3241, found 494.3250. Enantiomeric excess was determined by derivatization to the corresponding hydrodeaminated $\alpha, \beta$-unsaturated ester. ${ }^{1} \mathrm{H} \mathrm{NMR}(600 \mathrm{MHz}$, $\left.\mathrm{CDCl}_{3}\right) \delta 7.31(\mathrm{t}, J=7.6 \mathrm{~Hz}, 2 \mathrm{H}), 7.22(\mathrm{t}, J=7.4 \mathrm{~Hz}, 1 \mathrm{H}), 7.15(\mathrm{~d}, J=7.2 \mathrm{~Hz}, 2 \mathrm{H}), 6.89(\mathrm{dd}, J=15.7,9.8 \mathrm{~Hz}, 1 \mathrm{H})$, $5.91(\mathrm{ddd}, J=17.0,10.0,8.9 \mathrm{~Hz}, 1 \mathrm{H}), 5.82(\mathrm{~d}, J=15.7 \mathrm{~Hz}, 1 \mathrm{H}), 5.04(\mathrm{~d}, J=10.2 \mathrm{~Hz}, 1 \mathrm{H}), 4.99(\mathrm{~d}, J=17.0 \mathrm{~Hz}, 1 \mathrm{H})$, $4.55-4.49(\mathrm{~m}, 2 \mathrm{H}), 3.27(\mathrm{t}, J=8.6 \mathrm{~Hz}, 1 \mathrm{H}), 2.54(\mathrm{qd}, J=9.3,3.7 \mathrm{~Hz}, 1 \mathrm{H}), 1.38-1.11(\mathrm{~m}, 18 \mathrm{H}), 0.88(\mathrm{t}, J=7.1 \mathrm{~Hz}$, $3 \mathrm{H}) .{ }^{13} \mathrm{C} \mathrm{NMR}\left(151 \mathrm{MHz}, \mathrm{CDCl}_{3}\right) \delta 164.62,154.73,142.44,139.48,128.76,128.12,126.74,123.26\left(\mathrm{q},{ }^{1} J_{\mathrm{CF}}=277.8\right.$ $\mathrm{Hz}), 120.56,116.39,60.40\left(\mathrm{q},{ }^{2} J_{\mathrm{CF}}=36.2 \mathrm{~Hz}\right), 54.73,48.17,32.11,32.04,29.70,29.69,29.54,29.43,27.24,22.81$, 14.23. ${ }^{19} \mathrm{~F}$ NMR $\left(376 \mathrm{MHz}, \mathrm{CDCl}_{3}\right) \delta$-72.93. HRMS (EI) $m / z[\mathrm{M}]^{+}$calcd for $\mathrm{C}_{25} \mathrm{H}_{35} \mathrm{~F}_{3} \mathrm{O}_{2}$ 424.2589, found 424.2582. HPLC (ChiralPak IA column) 99.9:0.1 (Hexane/iPrOH) $0.5 \mathrm{~mL} \cdot \mathrm{min}^{-1}$; $_{\text {major }}(12.6 \mathrm{~min}), \mathrm{T}_{\operatorname{minor}}(11.3 \mathrm{~min}) ; 96.9: 3.1 \mathrm{er}$.

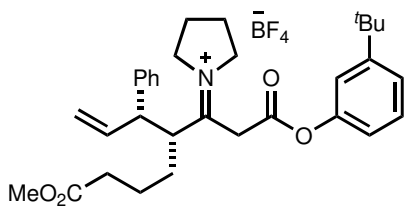

1-((R)-1-(3-(tert-butyl)phenoxy)-8-methoxy-1,8-dioxo-4-((S)-1-phenylallyl)octan-3-ylidene)pyrrolidin-1-ium tetrafluoroborate (11). Following the general procedure A, 11 was isolated in $88 \%$ yield and $72 \%$ ee $(96: 4 \mathrm{dr}) .{ }^{1} \mathrm{H}$ NMR $\left(600 \mathrm{MHz}, \mathrm{CDCl}_{3}\right) \delta$ 7.38-7.36 (m, 2H), 7.33-7.27 (m, 5H), $7.17(\mathrm{t}, J=1.8 \mathrm{~Hz}, 1 \mathrm{H}), 7.00(\mathrm{dt}, J=7.2,1.8 \mathrm{~Hz}$, $1 \mathrm{H}), 6.04(\mathrm{dt}, J=16.8,9.6 \mathrm{~Hz}, 1 \mathrm{H}), 5.23(\mathrm{~d}, J=10.2 \mathrm{~Hz}, 1 \mathrm{H}), 5.22(\mathrm{~d}, J=17.4 \mathrm{~Hz}, 1 \mathrm{H}), 4.37(\mathrm{~d}, J=3.0 \mathrm{~Hz}, 2 \mathrm{H}), 4.33$ $(\mathrm{t}, J=6.6 \mathrm{~Hz}, 1 \mathrm{H}), 4.26-4.22(\mathrm{~m}, 1 \mathrm{H}), 4.20-4.16(\mathrm{~m}, 1 \mathrm{H}), 3.98-3.95(\mathrm{~m}, 1 \mathrm{H}), 3.64(\mathrm{t}, J=9.0 \mathrm{~Hz}, 1 \mathrm{H}), 3.55(\mathrm{~s}, 3 \mathrm{H})$, 3.47-3.42 (m, 1H), 2.23-2.12 (m, 6H), 1.76-1.70 (m, 1H), 1.64-1.46 (m, 3H), 1.30 (s, 9H). ${ }^{13} \mathrm{C} \mathrm{NMR}\left(151 \mathrm{MHz}^{\mathrm{C}} \mathrm{CDCl} 3\right)$ $\delta 184.14,173.62,164.17,153.75,150.06,139.11,135.81,129.55,129.31,128.25,128.04,123.95,119.70,118.28$, $118.24,57.18,56.78,54.57,53.44,51.79,35.00,33.37,31.30,29.53,24.49,24.42,22.70 .{ }^{19} \mathrm{~F} \mathrm{NMR}\left(376 \mathrm{MHz} \mathrm{CDCl}_{3}\right)$ $\delta$-150.66. HRMS (ESI) $\mathrm{m} / z[\mathrm{M}+\mathrm{H}]^{+}$calcd for $\mathrm{C}_{32} \mathrm{H}_{41} \mathrm{NO}_{4}$ 504.3109, found 504.3091. Enantiomeric excess was determined by derivatization to the corresponding hydrodeaminated $\alpha, \beta$-unsaturated ester. ${ }^{1} \mathrm{H} \mathrm{NMR}(400 \mathrm{MHz}$, $\left.\mathrm{CDCl}_{3}\right) \delta 7.35-7.30(\mathrm{~m}, 2 \mathrm{H}), 7.28-7.21(\mathrm{~m}, 3 \mathrm{H}), 7.18(\mathrm{~d}, J=8.0 \mathrm{~Hz}, 2 \mathrm{H}), 7.12(\mathrm{t}, J=2.0 \mathrm{~Hz}, 1 \mathrm{H}), 6.97-6.91(\mathrm{~m}, 2 \mathrm{H})$, $5.99(\mathrm{~d}, J=15.6 \mathrm{~Hz}, 1 \mathrm{H}), 5.96(\mathrm{ddd}, J=16.8,10.0,8.8 \mathrm{~Hz}, 1 \mathrm{H}), 5.08(\mathrm{~d}, J=10.4 \mathrm{~Hz}, 1 \mathrm{H}), 5.02(\mathrm{~d}, J=17.6 \mathrm{~Hz}, 1 \mathrm{H})$, $3.62(\mathrm{~s}, 3 \mathrm{H}), 3.30(\mathrm{t}, J=8.8 \mathrm{~Hz}, 1 \mathrm{H}), 2.65-2.57(\mathrm{~m}, 1 \mathrm{H}), 2.24-2.19(\mathrm{~m}, 2 \mathrm{H}), 1.71-1.24(\mathrm{~m}, 4 \mathrm{H}), 1.32$ (s, 9H). HPLC (ChiralPak IB column) 99:1 (Hexane $/ \mathrm{iPrOH}) 1.0 \mathrm{~mL} \cdot \mathrm{min}^{-1} ; \mathrm{T}_{\text {major }}(15.7 \mathrm{~min}), \mathrm{T}_{\text {minor }}(16.7 \mathrm{~min}) ; 85.8: 14.2 \mathrm{er}$.

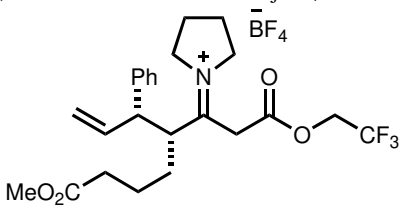

1-((R)-8-methoxy-1,8-dioxo-4-( $(S)$-1-phenylallyl)-1-(2,2,2-trifluoroethoxy)octan-3-ylidene)pyrrolidin-1-ium tetrafluoroborate (12). Following the general procedure A, 12 was isolated in $84 \%$ yield and $90 \%$ ee $(97: 3 \mathrm{dr}) .{ }^{1} \mathrm{H}$ NMR (400 MHz, $\left.\mathrm{CDCl}_{3}\right) \delta$ 7.38-7.34 (m, 2H), 7.29-7.26 (m, 3H), $5.97(\mathrm{dt}, J=16.8,9.6 \mathrm{~Hz}, 1 \mathrm{H}), 5.17(\mathrm{~d}, J=10.4 \mathrm{~Hz}$, $1 \mathrm{H}), 5.15(\mathrm{~d}, J=16.8 \mathrm{~Hz}, 1 \mathrm{H}), 4.71-4.61(\mathrm{~m}, 2 \mathrm{H}), 4.24-4.12(\mathrm{~m}, 5 \mathrm{H}), 4.07-4.01(\mathrm{~m}, 1 \mathrm{H}), 3.57(\mathrm{~s}, 3 \mathrm{H}), 3.52(\mathrm{t}, J=9.2$ $\mathrm{Hz}, 1 \mathrm{H}), 3.43(\mathrm{dt}, J=8.4,3.2 \mathrm{~Hz}, 1 \mathrm{H}), 2.20-2.14(\mathrm{~m}, 6 \mathrm{H}), 1.64-1.38(\mathrm{~m}, 4 \mathrm{H}) .{ }^{13} \mathrm{C}$ NMR $\left(151 \mathrm{MHz}^{\mathrm{C}} \mathrm{CDCl}\right)_{3} \delta 183.69$, 
$173.73,163.66,139.00,135.95,129.54,128.17,128.06,122.73\left(\mathrm{q},{ }^{1} J_{\mathrm{CF}}=277.8 \mathrm{~Hz}\right), 119.38,61.98\left(\mathrm{q},{ }^{2} J_{\mathrm{CF}}=36.2 \mathrm{~Hz}\right)$, $57.27,56.93,54.58,53.61,51.79,37.98,33.20,29.38,24.43,24.40,22.67 .{ }^{19} \mathrm{~F} \mathrm{NMR}\left(376 \mathrm{MHz}, \mathrm{CDCl}_{3}\right) \delta-72.66$, 150.74. HRMS (ESI) $\mathrm{m} / z[\mathrm{M}+\mathrm{H}]^{+}$calcd for $\mathrm{C}_{24} \mathrm{H}_{30} \mathrm{~F}_{3} \mathrm{NO}_{4} 454.2200$, found 454.2184. Enantiomeric excess was determined by derivatization to the corresponding hydrodeaminated $\alpha, \beta$-unsaturated ester. ${ }^{1} \mathrm{H}$ NMR $(400 \mathrm{MHz}$, $\left.\mathrm{CDCl}_{3}\right) \delta 7.31(\mathrm{t}, J=7.6 \mathrm{~Hz}, 2 \mathrm{H}), 7.22(\mathrm{t}, J=7.2 \mathrm{~Hz}, 1 \mathrm{H}), 7.14(\mathrm{~d}, J=7.2 \mathrm{~Hz}, 2 \mathrm{H}), 6.87(\mathrm{dd}, J=15.6,9.6 \mathrm{~Hz}, 1 \mathrm{H})$, 5.89 (ddd, $J=16.4,9.6,8.8 \mathrm{~Hz}, 1 \mathrm{H}), 5.84(\mathrm{~d}, J=16.0 \mathrm{~Hz}, 1 \mathrm{H}), 4.52(\mathrm{q}, J=8.4 \mathrm{~Hz}, 2 \mathrm{H}), 3.60(\mathrm{~s}, 3 \mathrm{H}), 3.26(\mathrm{t}, J=8.4$ $\mathrm{Hz} 1 \mathrm{H}), 2.60-2.52(\mathrm{~m}, 1 \mathrm{H}), 2.21-2.16(\mathrm{~m}, 2 \mathrm{H}), 0.90-0.79(\mathrm{~m}, 4 \mathrm{H}) .{ }^{19} \mathrm{~F}$ NMR $\left(376 \mathrm{MHz}, \mathrm{CDCl}_{3}\right) \delta-72.89$. HPLC (ChiralPak OJH column) 99:1 (Hexane/ $i \mathrm{PrOH}) 1.0 \mathrm{~mL} \cdot \mathrm{min}^{-1}$; $\mathrm{T}_{\text {major }}(19.6 \mathrm{~min}), \mathrm{T}_{\text {minor }}(26.5 \mathrm{~min}) ;$ 95.2:4.8 er.

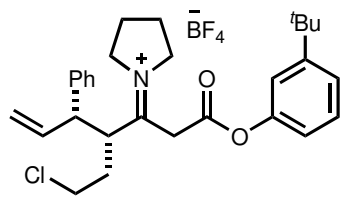

1-((4R,5S)-1-(3-(tert-butyl)phenoxy)-4-(2-chloroethyl)-1-oxo-5-phenylhept-6-en-3-ylidene)pyrrolidin-1-ium

tetrafluoroborate (13). Following the general procedure A, 13 was isolated in $70 \%$ yield and $80 \%$ ee $(96: 4 \mathrm{dr}) .{ }^{1} \mathrm{H}$ $\operatorname{NMR}\left(600 \mathrm{MHz}, \mathrm{CDCl}_{3}\right) \delta 7.38-7.31(\mathrm{~m}, 4 \mathrm{H}), 7.30(\mathrm{~m}, 3 \mathrm{H}), 7.18(\mathrm{~s}, 1 \mathrm{H}), 7.00(\mathrm{~d}, J=7.2 \mathrm{~Hz}, 1 \mathrm{H}), 5.99(\mathrm{dt}, J=16.8$, $9.0 \mathrm{~Hz}, 1 \mathrm{H}), 5.21(\mathrm{~d}, J=18.0 \mathrm{~Hz}, 1 \mathrm{H}), 5.20(\mathrm{~d}, J=9.6 \mathrm{~Hz}, 1 \mathrm{H}), 4.57(\mathrm{~d}, J=17.4 \mathrm{~Hz}, 1 \mathrm{H}), 4.45-4.37(\mathrm{~m}, 2 \mathrm{H}), 4.42(\mathrm{~d}$, $J=17.4 \mathrm{~Hz}, 1 \mathrm{H}), 4.24-4.21(\mathrm{~m}, 1 \mathrm{H}), 4.17-4.14(\mathrm{~m}, 1 \mathrm{H}), 3.80-3.76(\mathrm{~m}, 2 \mathrm{H}), 3.40(\mathrm{td}, J=12.0,4.2 \mathrm{~Hz}, 1 \mathrm{H}), 3.34(\mathrm{dt}, J$ $=11.4,5.4 \mathrm{~Hz}, 1 \mathrm{H}), 2.27-2.21(\mathrm{~m}, 5 \mathrm{H}), 2.04-1.99(\mathrm{~m}, 1 \mathrm{H}), 1.29(\mathrm{~s}, 9 \mathrm{H}) .{ }^{13} \mathrm{C} \mathrm{NMR}\left(151 \mathrm{MHz}, \mathrm{CDCl}_{3}\right) \delta 184.22,164.56$, 153.78, 150.09, 138.54, 135.96, 129.58, 129.31, 128.41, 128.17, 123.96, 119.83, 118.39, 118.31, 57.35, 56.86, 52.72, 51.27, 42.32, 35.01, 32.43, 31.31, 24.54, 24.51. ${ }^{19} \mathrm{~F}$ NMR (376 MHz, $\left.\mathrm{CDCl}_{3}\right) \delta-149.96$. HRMS (ESI) $\mathrm{m} / z$ [M+H] ${ }^{+}$ calcd for $\mathrm{C}_{29} \mathrm{H}_{36} \mathrm{ClNO}_{2} 466.2508$, found 466.2491 . Enantiomeric excess was determined by derivatization following the general procedure developed by Deng. ${ }^{3}$ In our system, further decarboxylation of the ester moiety was observed, resulting in the formation of 3-(2-chloroethyl)-1,1-difluoro-4-phenylhex-5-en-2-one. ${ }^{1} \mathrm{H} \mathrm{NMR}\left(400 \mathrm{MHz}, \mathrm{CDCl}_{3}\right) \delta$ $7.36(\mathrm{t}, J=7.4 \mathrm{~Hz}, 2 \mathrm{H}), 7.27(\mathrm{t}, J=7.2 \mathrm{~Hz}, 1 \mathrm{H}), 7.21(\mathrm{~d}, J=7.1 \mathrm{~Hz}, 2 \mathrm{H}), 5.98(\mathrm{dt}, J=17.0,9.7 \mathrm{~Hz}, 1 \mathrm{H}), 5.63(\mathrm{t}, J=$ $53.9 \mathrm{~Hz}, 1 \mathrm{H}), 5.07(\mathrm{~d}, J=10.0 \mathrm{~Hz}, 1 \mathrm{H}), 5.05(\mathrm{~d}, J=17.2 \mathrm{~Hz}, 1 \mathrm{H}), 3.73(\mathrm{tm}, J=10.0 \mathrm{~Hz}, 1 \mathrm{H}), 3.50(\mathrm{t}, J=9.7 \mathrm{~Hz}, 1 \mathrm{H})$, 3.37 (dt, $J=12.1,6.2 \mathrm{~Hz}, 1 \mathrm{H}$ ), 3.27 (ddd, $J=11.2,8.3,5.8 \mathrm{~Hz}, 1 \mathrm{H}$ ), 2.12 (ddt, $J=15.7,10.2,5.9 \mathrm{~Hz}, 1 \mathrm{H}$ ), 1.77 (dddd, $J=14.6,8.3,6.5,3.4 \mathrm{~Hz}, 1 \mathrm{H}) .{ }^{13} \mathrm{C} \mathrm{NMR}\left(151 \mathrm{MHz}, \mathrm{CDCl}_{3}\right) \delta 202.80\left(\mathrm{t},{ }^{2} J_{\mathrm{CF}}=24.2 \mathrm{~Hz}\right), 140.37,138.14,129.28$, $127.86,127.58,117.54,109.72\left(\mathrm{t},{ }^{1} J_{\mathrm{CF}}=252.2 \mathrm{~Hz}\right), 53.55,48.73,42.32,31.42 .{ }^{19} \mathrm{~F} \mathrm{NMR}\left(376 \mathrm{MHz}, \mathrm{CDCl}_{3}\right) \delta-127.49$, -127.64. HRMS (EI) $\mathrm{m} / z$ [M] ${ }^{+}$calcd for $\mathrm{C}_{14} \mathrm{H}_{15} \mathrm{ClF}_{2} \mathrm{O}$ 272.0779, found 272.0781. HPLC (ChiralPak OJH column) 99:1 (Hexane $/ \mathrm{iPrOH}) 1.0 \mathrm{~mL} \cdot \mathrm{min}^{-1} ; \mathrm{T}_{\text {major }}(8.5 \mathrm{~min}), \mathrm{T}_{\text {minor }}(8.9 \mathrm{~min}) ; 90.0: 10.0 \mathrm{er}$.

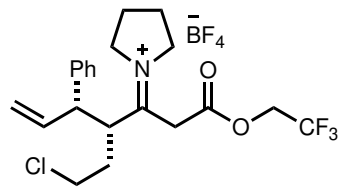

1-((4R,5S)-4-(2-chloroethyl)-1-oxo-5-phenyl-1-(2,2,2-trifluoroethoxy)hept-6-en-3-ylidene)pyrrolidin-1-ium tetrafluoroborate (14). Following the general procedure A, 14 was isolated in $84 \%$ yield and $86 \%$ ee $(97: 3 \mathrm{dr}) .{ }^{1} \mathrm{H}$ NMR (400 MHz, $\left.\mathrm{CDCl}_{3}\right) \delta 7.39-7.35(\mathrm{~m}, 2 \mathrm{H}), 7.31-7.24(\mathrm{~m}, 3 \mathrm{H}), 5.96(\mathrm{dt}, J=16.8,9.6 \mathrm{~Hz}, 1 \mathrm{H}), 5.19(\mathrm{~d}, J=10.0 \mathrm{~Hz}$, $1 \mathrm{H}), 5.17(\mathrm{~d}, J=17.2 \mathrm{~Hz}, 1 \mathrm{H}), 4.65(\mathrm{q}, J=8.4 \mathrm{~Hz}, 2 \mathrm{H}), 4.42-4.26(\mathrm{~m}, 3 \mathrm{H}), 4.19-4.09(\mathrm{~m}, 3 \mathrm{H}), 3.77(\mathrm{td}, J=10.0,3.6$ $\mathrm{Hz}, 1 \mathrm{H}), 3.66(\mathrm{t}, J=9.6 \mathrm{~Hz}, 2 \mathrm{H}), 3.41-3.34(\mathrm{~m}, 2 \mathrm{H}), 2.26-2.25(\mathrm{~m}, 4 \mathrm{H}), 2.18-2.09(\mathrm{~m}, 1 \mathrm{H}), 2.01-1.93(\mathrm{~m}, 1 \mathrm{H}) .{ }^{13} \mathrm{C}$ NMR $\left(126 \mathrm{MHz}, \mathrm{CDCl}_{3}\right) \delta 183.45,163.94,138.41,135.97,129.60,128.27,128.24,122.67\left(\mathrm{q},{ }^{1} J_{\mathrm{CF}}=278.5 \mathrm{~Hz}\right)$, $119.63,61.92\left(\mathrm{q},{ }^{2} J_{\mathrm{CF}}=36.5 \mathrm{~Hz}\right), 57.36,57.02,52.88,51.17,42.22,32.20,24.46 .{ }^{19} \mathrm{~F} \mathrm{NMR}\left(376 \mathrm{MHz}^{\mathrm{N}} \mathrm{CDCl}_{3}\right) \delta-$ 72.66, -150.53. HRMS (ESI) $\mathrm{m} / z[\mathrm{M}+\mathrm{H}]^{+}$calcd for $\mathrm{C}_{21} \mathrm{H}_{25} \mathrm{ClF}_{3} \mathrm{NO}_{2} 416.1599$, found 416.1603. Enantiomeric excess was determined by derivatization to 3-(2-chloroethyl)-1,1-difluoro-4-phenylhex-5-en-2-one. HPLC (ChiralPak OJH column) 99:1 (Hexane/iPrOH) $1.0 \mathrm{~mL} \cdot \mathrm{min}^{-1}$; $\mathrm{T}_{\text {major }}(8.3 \mathrm{~min}), \mathrm{T}_{\text {minor }}(8.8 \mathrm{~min})$; 92.8:7.2 er. 


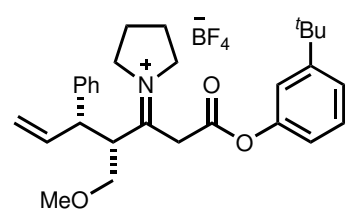

1-((4R,5S)-1-(3-(tert-butyl)phenoxy)-4-(methoxymethyl)-1-oxo-5-phenylhept-6-en-3-ylidene)pyrrole-din-1-ium tetrafluoroborate (15). Following the general procedure A, 15 was isolated in $98 \%$ yield and $86 \%$ ee $(98: 2 \mathrm{dr}) .{ }^{1} \mathrm{H}$

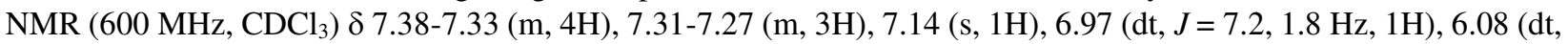
$J=16.8,10.2 \mathrm{~Hz}, 1 \mathrm{H}), 5.20(\mathrm{~d}, J=10.2 \mathrm{~Hz}, 1 \mathrm{H}), 5.19(\mathrm{~d}, J=16.8 \mathrm{~Hz}, 1 \mathrm{H}), 4.44-4.38(\mathrm{~m}, 1 \mathrm{H}), 4.41(\mathrm{~s}, 2 \mathrm{H}), 4.35-4.31$ $(\mathrm{m}, 1 \mathrm{H}), 4.29-4.23(\mathrm{~m}, 2 \mathrm{H}), 3.91(\mathrm{t}, J=10.2 \mathrm{~Hz}, 1 \mathrm{H}), 3.64-3.61(\mathrm{~m}, 1 \mathrm{H}), 3.44(\mathrm{dd}, J=10.2,3.0 \mathrm{~Hz}, 1 \mathrm{H}), 3.30(\mathrm{dd}, J$ $=10.2,6.0 \mathrm{~Hz}, 1 \mathrm{H}), 3.13(\mathrm{~s}, 3 \mathrm{H}), 2.31-2.18(\mathrm{~m}, 4 \mathrm{H}), 1.30(\mathrm{~s}, 9 \mathrm{H}) .{ }^{13} \mathrm{C} \mathrm{NMR}\left(151 \mathrm{MHz}, \mathrm{CDCl}_{3}\right) \delta 183.52,163.72$, $153.63,150.16,138.67,136.49,129.37,129.26,128.24,127.97,123.77,119.21,118.25,118.20,69.43,59.15,57.06$, 56.63, 53.52, 49.43, 39.67, 34.98, 31.32, 24.55, 24.45. ${ }^{19} \mathrm{~F} \mathrm{NMR}\left(376 \mathrm{MHz}, \mathrm{CDCl}_{3}\right) \delta-150.93$. HRMS (ESI) $m / z$ $[\mathrm{M}+\mathrm{H}]^{+}$calcd for $\mathrm{C}_{29} \mathrm{H}_{37} \mathrm{NO}_{3} 448.2846$, found 448.2828. Enantiomeric excess was directly determined over the $\beta$ enamino ester after basic washing. HPLC (ChiralPak IC column) 99:1 (Hexane/iPrOH) $1.0 \mathrm{~mL} \cdot \mathrm{min}^{-1} ; \mathrm{T}_{\text {major }}(17.8 \mathrm{~min})$, $\mathrm{T}_{\text {minor }}(22.6 \mathrm{~min}) ; 92.7: 7.3 \mathrm{er}$.

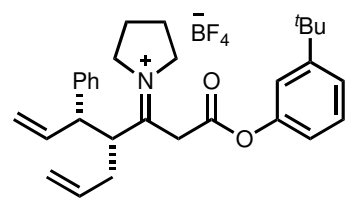

1-((4R,5S)-4-allyl-1-(3-(tert-butyl)phenoxy)-1-oxo-5-phenylhept-6-en-3-ylidene)pyrrolidin-1-ium

tetrafluoroborate (17). Following the general procedure A, 17 was isolated in $66 \%$ yield and $85 \%$ ee $(97: 3 \mathrm{dr}) .{ }^{1} \mathrm{H} \mathrm{NMR}$ $\left(400 \mathrm{MHz} \mathrm{CDCl}_{3}\right) \delta 7.40-7.27(\mathrm{~m}, 7 \mathrm{H}), 7.15(\mathrm{br} \mathrm{s}, 1 \mathrm{H}), 6.98(\mathrm{dt}, J=7.2,2.0 \mathrm{~Hz}, 1 \mathrm{H}), 6.06(\mathrm{dt}, J=16.8,10.0 \mathrm{~Hz}, 1 \mathrm{H})$, $5.71-5.61(\mathrm{~m}, 1 \mathrm{H}), 5.24(\mathrm{~d}, J=10.0 \mathrm{~Hz}, 1 \mathrm{H}), 5.23(\mathrm{~d}, J=18.4 \mathrm{~Hz}, 1 \mathrm{H}), 5.09(\mathrm{~d}, J=11.2 \mathrm{~Hz}, 1 \mathrm{H}), 5.09(\mathrm{~d}, J=15.6$ $\mathrm{Hz}, 1 \mathrm{H}), 4.42-4.32(\mathrm{~m}, 1 \mathrm{H}), 4.37(\mathrm{~s}, 2 \mathrm{H}), 4.30-4.23(\mathrm{~m}, 1 \mathrm{H}), 4.17-4.10(\mathrm{~m}, 1 \mathrm{H}), 3.93-3.86(\mathrm{~m}, 1 \mathrm{H}), 3.72(\mathrm{t}, J=9.2 \mathrm{~Hz}$, $1 \mathrm{H}), 3.55-3.45(\mathrm{~m}, 1 \mathrm{H}), 2.47-2.34(\mathrm{~m}, 2 \mathrm{H}), 2.23-2.10(\mathrm{~m}, 4 \mathrm{H}), 1.30(\mathrm{~s}, 9 \mathrm{H}) .{ }^{13} \mathrm{C} \mathrm{NMR}\left(101 \mathrm{MHz}, \mathrm{CDCl}_{3}\right) \delta 183.75$, $164.03,153.77,150.04,139.14,135.77,132.70,129.59,129.34,128.26,128.07,123.97,120.32,119.78,118.24$, $118.19,57.20,56.73,54.50,52.76,38.96,35.01,34.02,31.31,24.48,24.42 .{ }^{19} \mathrm{~F} \mathrm{NMR}\left(376 \mathrm{MHz} \mathrm{CDCl}_{3}\right) \delta-150.66$. HRMS (ESI) $\mathrm{m} / \mathrm{z}[\mathrm{M}+\mathrm{H}]^{+}$calcd for $\mathrm{C}_{30} \mathrm{H}_{37} \mathrm{NO}_{2} 444.2897$, found 444.2884. Enantiomeric excess was determined by derivatization to the corresponding hydrodeaminated $\alpha, \beta$-unsaturated ester. ${ }^{1} \mathrm{H}$ NMR $\left(600 \mathrm{MHz}, \mathrm{CDCl}_{3}\right) \delta 7.35-7.30$ $(\mathrm{m}, 3 \mathrm{H}), 7.26-7.23(\mathrm{~m}, 2 \mathrm{H}), 7.20(\mathrm{~d}, J=7.3 \mathrm{~Hz}, 2 \mathrm{H}), 7.12(\mathrm{t}, J=1.9 \mathrm{~Hz}, 1 \mathrm{H}), 7.01(\mathrm{dd}, J=15.7,9.4 \mathrm{~Hz}, 1 \mathrm{H}), 6.95$ $(\mathrm{dd}, J=7.6,1.7 \mathrm{~Hz}, 1 \mathrm{H}), 5.98(\mathrm{ddd}, J=18.0,10.2,9.0 \mathrm{~Hz}, 1 \mathrm{H}), 5.96(\mathrm{~d}, J=15.7 \mathrm{~Hz}, 1 \mathrm{H}), 5.70(\mathrm{ddt}, J=17.2,10.2$, $7.1 \mathrm{~Hz}, 1 \mathrm{H}), 5.11(\mathrm{~d}, J=9.6 \mathrm{~Hz}, 1 \mathrm{H}), 5.05(\mathrm{~d}, J=17.0 \mathrm{~Hz}, 1 \mathrm{H}), 5.03(\mathrm{~d}, J=9.8 \mathrm{~Hz}, 1 \mathrm{H}), 4.97(\mathrm{~d}, J=17.0 \mathrm{~Hz}, 1 \mathrm{H})$, $3.39(\mathrm{t}, J=8.5 \mathrm{~Hz}, 1 \mathrm{H}), 2.72(\mathrm{qd}, J=8.8,4.7 \mathrm{~Hz}, 1 \mathrm{H}), 2.20(\mathrm{dt}, J=11.9,5.8 \mathrm{~Hz}, 1 \mathrm{H}), 2.06(\mathrm{dt}, J=15.0,7.8 \mathrm{~Hz}, 1 \mathrm{H})$, 1.32 (s, 9H). ${ }^{13} \mathrm{C}$ NMR $\left(151 \mathrm{MHz}, \mathrm{CDCl}_{3}\right) \delta 164.85,153.14,152.47,150.79,142.28,139.09,135.52,128.96,128.83$, 128.18, 126.84, 122.86, 122.31, 118.77, 118.75, 117.34, 116.86, 53.86, 47.72, 36.72, 34.94, 31.41. HRMS (EI) m/z $[\mathrm{M}]^{+}$calcd for $\mathrm{C}_{26} \mathrm{H}_{30} \mathrm{O}_{2}$ 374.2246, found 374.2244. HPLC (ChiralPak OJH column) 99:1 (Hexane/iPrOH) 1.0 $\mathrm{mL} \cdot \min ^{-1} ; \mathrm{T}_{\text {major }}(8.5 \mathrm{~min}), \mathrm{T}_{\text {minor }}(7.5 \mathrm{~min})$; 92.5:7.5 er.

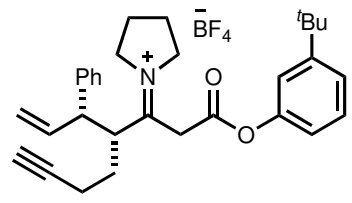

1-((R)-1-(3-(tert-butyl)phenoxy)-1-oxo-4-((S)-1-phenylallyl)oct-7-yn-3-ylidene)pyrrolidin-1-ium

tetrafluoroborate (18). Following the general procedure A, 18 was isolated in $87 \%$ yield and 83\% ee $(96: 4 \mathrm{dr}) .{ }^{1} \mathrm{H} \mathrm{NMR}$ $\left(600 \mathrm{MHz} \mathrm{CDCl}_{3}\right) \delta 7.38-7.31(\mathrm{~m}, 4 \mathrm{H}), 7.30-7.27(\mathrm{~m}, 3 \mathrm{H}), 7.17(\mathrm{~s}, 1 \mathrm{H}), 6.99(\mathrm{dt}, J=7.2,1.8 \mathrm{~Hz}, 1 \mathrm{H}), 6.00(\mathrm{dt}, J=$ 18.0, $9.0 \mathrm{~Hz}, 1 \mathrm{H}), 5.21(\mathrm{~d}, J=10.8 \mathrm{~Hz}, 1 \mathrm{H}), 5.20(\mathrm{~d}, J=15.0 \mathrm{~Hz}, 1 \mathrm{H}), 4.45(\mathrm{t}, J=17.4 \mathrm{~Hz}, 2 \mathrm{H}), 4.41(\mathrm{t}, J=17.4 \mathrm{~Hz}$, 2H), 4.23-4.19 (m, 1H), 4.17-4.12 (m, 1H), $3.76(\mathrm{td}, J=9.6,3.6 \mathrm{~Hz}, 1 \mathrm{H}), 3.71(\mathrm{t}, J=9.0 \mathrm{~Hz}, 1 \mathrm{H}), 2.26-2.16(\mathrm{~m}, 4 \mathrm{H})$, 
2.14-2.12 (m, 3H), 1.91-1.87 (m, 1H), 1.84-1.79 (m, 1H), $1.29(\mathrm{~s}, 9 \mathrm{H}) .{ }^{13} \mathrm{C}$ NMR $\left(151 \mathrm{MHz}, \mathrm{CDCl}_{3}\right) \delta 184.18,164.37$, $153.75,150.05,138.76,136.08,129.52,129.30,128.38,128.06,123.95,119.64,118.33,118.27,82.56,71.40,57.32$, $56.91,53.04,52.75,35.00,31.31,28.62,24.52,24.48,16.50 .{ }^{19} \mathrm{~F}$ NMR $\left(376 \mathrm{MHz}, \mathrm{CDCl}_{3}\right) \delta-150.27$. HRMS (ESI) $\mathrm{m} / z[\mathrm{M}+\mathrm{H}]^{+}$calcd for $\mathrm{C}_{31} \mathrm{H}_{37} \mathrm{NO}_{2} 456.2897$, found 456.2878. Enantiomeric excess was determined by derivatization to the corresponding hydrodeaminated $\alpha, \beta$-unsaturated ester. ${ }^{1} \mathrm{H}$ NMR $\left(400 \mathrm{MHz}, \mathrm{CDCl}_{3}\right) \delta$ 7.36-7.24 (m, $\left.5 \mathrm{H}\right), 7.20$ $(\mathrm{d}, J=7.2 \mathrm{~Hz}, 2 \mathrm{H}), 7.12(\mathrm{t}, J=1.9 \mathrm{~Hz}, 1 \mathrm{H}), 6.97-6.95(\mathrm{~m}, 1 \mathrm{H}), 6.91(\mathrm{dd}, J=14.8,8.9 \mathrm{~Hz}, 1 \mathrm{H}), 6.05(\mathrm{~d}, J=15.6 \mathrm{~Hz}$, $1 \mathrm{H}), 5.97(\mathrm{ddd}, J=16.8,10.0,8.4 \mathrm{~Hz}, 1 \mathrm{H}), 5.09(\mathrm{~d}, J=10.1 \mathrm{~Hz}, 1 \mathrm{H}), 5.04(\mathrm{~d}, J=16.7 \mathrm{~Hz}, 1 \mathrm{H}), 3.32(\mathrm{t}, J=8.4 \mathrm{~Hz}$, $1 \mathrm{H}), 2.89-2.81(\mathrm{~m}, 1 \mathrm{H}), 2.28-2.16(\mathrm{~m}, 1 \mathrm{H}), 2.13-2.03(\mathrm{~m}, 1 \mathrm{H}), 1.96(\mathrm{t}, J=2.6 \mathrm{~Hz}, 1 \mathrm{H}), 1.71-1.61(\mathrm{~m}, 1 \mathrm{H}), 1.50-1.42$ (m, 1H). HPLC (ChiralPak IC column) 99:1 (Hexane/iPrOH) $1.0 \mathrm{~mL} \cdot \mathrm{min}^{-1} ; \mathrm{T}_{\text {major }}(7.4 \mathrm{~min}), \mathrm{T}_{\text {minor }}(6.8 \mathrm{~min}) ; 91.7: 8.3$ er.

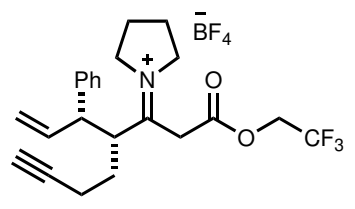

1-((R)-1-oxo-4-((S)-1-phenylallyl)-1-(2,2,2-trifluoroethoxy)oct-7-yn-3-ylidene)pyrrolidin-1-ium

tetrafluoroborate (19). Following the general procedure A, 19 was isolated in $91 \%$ yield and $95 \%$ ee $(98: 2 \mathrm{dr}) .{ }^{1} \mathrm{H} \mathrm{NMR}$ $\left(600 \mathrm{MHz} \mathrm{CDCl}_{3}\right) \delta 7.37-7.35(\mathrm{~m}, 2 \mathrm{H}), 7.30-7.26(\mathrm{~m}, 3 \mathrm{H}), 5.95(\mathrm{dt}, J=16.2,10.2 \mathrm{~Hz}, 1 \mathrm{H}), 5.17(\mathrm{~d}, J=9.6 \mathrm{~Hz}, 1 \mathrm{H})$, $5.15(\mathrm{~d}, J=16.8 \mathrm{~Hz}, 1 \mathrm{H}), 4.70-4.59(\mathrm{~m}, 2 \mathrm{H}), 4.45-4.41(\mathrm{~m}, 2 \mathrm{H}), 4.29-4.25(\mathrm{~m}, 2 \mathrm{H}), 4.19-4.10(\mathrm{~m}, 3 \mathrm{H}), 3.74(\mathrm{td}, J=$ 9.6, 4.2 Hz, 1H), $3.58(\mathrm{t}, J=9.6 \mathrm{~Hz}, 1 \mathrm{H}), 2.26-2.18(\mathrm{~m}, 4 \mathrm{H}), 2.13(\mathrm{t}, J=2.4 \mathrm{~Hz}, 1 \mathrm{H}), 2.10-2.08(\mathrm{~m}, 2 \mathrm{H}), 1.80-1.71$ $(\mathrm{m}, 2 \mathrm{H}) .{ }^{13} \mathrm{C} \mathrm{NMR}\left(151 \mathrm{MHz}, \mathrm{CDCl}_{3}\right) \delta 183.61,163.86,138.68,136.17,129.54,128.27,128.11,122.69\left(\mathrm{q},{ }^{1} J_{\mathrm{CF}}=\right.$ $277.8 \mathrm{~Hz}), 119.38,82.47,71.40,61.89\left(\mathrm{q},{ }^{2} J_{\mathrm{CF}}=36.2 \mathrm{~Hz}\right), 57.34,57.07,53.16,52.71,38.09,28.42,24.44,16.40 .{ }^{19} \mathrm{~F}$ NMR (376 MHz, $\mathrm{CDCl}_{3}$ ) $\delta-72.67,-150.51$. HRMS (ESI) $\mathrm{m} / z$ [M+H] ${ }^{+}$calcd for $\mathrm{C}_{23} \mathrm{H}_{26} \mathrm{~F}_{3} \mathrm{NO}_{2}$ 406.1989, found 406.1992. Enantiomeric excess was determined by derivatization to the corresponding hydrodeaminated $\alpha, \beta-$ unsaturated ester. ${ }^{1} \mathrm{H} \mathrm{NMR}\left(400 \mathrm{MHz}, \mathrm{CDCl}_{3}\right) \delta 7.32(\mathrm{t}, J=7.5 \mathrm{~Hz}, 2 \mathrm{H}), 7.25-7.21(\mathrm{~m}, 1 \mathrm{H}), 7.17(\mathrm{~d}, J=7.1 \mathrm{~Hz}, 2 \mathrm{H})$, $6.85(\mathrm{dd}, J=15.7,9.9 \mathrm{~Hz}, 1 \mathrm{H}), 5.91(\mathrm{ddd}, J=17.2,10.4,8.8 \mathrm{~Hz}, 1 \mathrm{H}), 5.91(\mathrm{~d}, J=15.7 \mathrm{~Hz}, 2 \mathrm{H}), 5.06(\mathrm{~d}, J=10.0 \mathrm{~Hz}$, $1 \mathrm{H}), 5.02(\mathrm{~d}, J=16.8 \mathrm{~Hz}, 1 \mathrm{H}), 4.52(\mathrm{q}, J=8.4 \mathrm{~Hz}, 2 \mathrm{H}), 3.29(\mathrm{t}, J=8.7 \mathrm{~Hz}, 1 \mathrm{H}), 2.80(\mathrm{qd}, J=10.0,3.5 \mathrm{~Hz}, 1 \mathrm{H}), 2.20-$ $2.12(\mathrm{~m}, 1 \mathrm{H}), 2.07-1.97(\mathrm{~m}, 1 \mathrm{H}), 1.94(\mathrm{t}, J=2.6 \mathrm{~Hz}, 1 \mathrm{H}), 1.67-1.58(\mathrm{~m}, 1 \mathrm{H}), 1.46-1.37(\mathrm{~m}, 1 \mathrm{H}) .{ }^{13} \mathrm{C} \mathrm{NMR}(151 \mathrm{MHz}$, $\left.\mathrm{CDCl}_{3}\right) \delta 164.39,153.02,141.82,139.13,128.88,128.12,126.94,123.20\left(\mathrm{q},{ }^{1} J_{\mathrm{CF}}=277.8 \mathrm{~Hz}\right), 121.50,116.71,83.34$, $69.35,60.45\left(\mathrm{q},{ }^{2} J_{\mathrm{CF}}=36.2 \mathrm{~Hz}\right), 54.47,46.65,30.55,16.44 .{ }^{19} \mathrm{~F} \mathrm{NMR}\left(376 \mathrm{MHz}, \mathrm{CDCl}_{3}\right) \delta-72.90 . \mathrm{HRMS}(\mathrm{EI}) \mathrm{m} / z$ $[\mathrm{M}]^{+}$calcd for $\mathrm{C}_{19} \mathrm{H}_{19} \mathrm{~F}_{3} \mathrm{O}_{2}$ 336.1337, found 336.1337. HPLC (ChiralPak IA column) 99.9:0.1 (Hexane/iPrOH) 0.5 $\mathrm{mL} \cdot \mathrm{min}^{-1} ; \mathrm{T}_{\text {major }}(17.0 \mathrm{~min}), \mathrm{T}_{\text {minor }}(16.1 \mathrm{~min}) ; 97.6: 2.4 \mathrm{er}$.

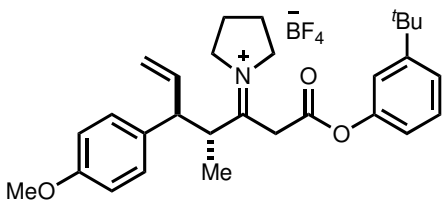

1-((4R,5S)-1-(3-(tert-butyl)phenoxy)-5-(4-methoxyphenyl)-4-methyl-1-oxohept-6-en-3-ylidene)pyro-ledin-1ium tetrafluoroborate (20). Following the general procedure A, 20 was isolated in 81\% yield and 71\% ee (95:5 dr). ${ }^{1} \mathrm{H}$ NMR $\left(600 \mathrm{MHz}, \mathrm{CDCl}_{3}\right) \delta$ 7.33-7.29 (m, 2H), 7.21-7.20 (m, 2H), $7.13(\mathrm{t}, J=2.1 \mathrm{~Hz}, 1 \mathrm{H}), 6.95(\mathrm{dt}, J=7.2,1.8 \mathrm{~Hz}$, $1 \mathrm{H}), 6.91-6.89$ (m, 2H), $6.02(\mathrm{ddd}, J=17.4,10.2,8.4 \mathrm{~Hz}, 1 \mathrm{H}), 5.15(\mathrm{~d}, J=10.2 \mathrm{~Hz}, 1 \mathrm{H}), 5.12(\mathrm{~d}, J=16.8 \mathrm{~Hz}, 1 \mathrm{H})$, 4.33-4.19 (m, 3H), 4.29 (s, 2H), 4.14-4.09 (m, 1H), 3.78 (s, 3H), 3.53-3.44 (m, 2H), 2.26-2.17 (m, 4H), 1.30 (s, 9H), $1.15(\mathrm{~d}, J=6.6 \mathrm{~Hz}, 3 \mathrm{H}) .{ }^{13} \mathrm{C}$ NMR $\left(151 \mathrm{MHz}, \mathrm{CDCl}_{3}\right) \delta 184.68,164.22,159.23,153.78,150.04,137.17,130.64$, 129.44, 129.32, 123.95, 118.59, 118.18, 118.14, 114.77, 56.89, 56.20, 55.45, 53.07, 48.17, 38.42, 35.00, 31.31, 24.49, 24.35, 14.84. ${ }^{19} \mathrm{~F}$ NMR $\left(376 \mathrm{MHz} \mathrm{CDCl}_{3}\right) \delta-150.83$. HRMS (ESI) $m / z[\mathrm{M}+\mathrm{H}]^{+}$calcd for $\mathrm{C}_{29} \mathrm{H}_{37} \mathrm{NO}_{3} 448.2846$, found 448.2841. Enantiomeric excess was determined by derivatization to the corresponding hydrodeaminated $\alpha, \beta$ unsaturated ester. ${ }^{1} \mathrm{H}$ NMR $\left(500 \mathrm{MHz}, \mathrm{CDCl}_{3}\right) \delta 7.31(\mathrm{t}, J=8.0 \mathrm{~Hz}, 1 \mathrm{H}), 7.27-7.25(\mathrm{~m}, 1 \mathrm{H}), 7.16-7.10(\mathrm{~m}, 4 \mathrm{H}), 6.95$ (ddd, $J=7.5,2.0,1.0 \mathrm{~Hz}, 1 \mathrm{H}), 6.00(\mathrm{dd}, J=16.0,1.0 \mathrm{~Hz}, 2 \mathrm{H}), 5.98(\mathrm{ddd}, J=17.5,10.5,8.5 \mathrm{~Hz}, 1 \mathrm{H}), 6.02-5.09$ (m, $1 \mathrm{H}), 5.09(\mathrm{~d}, J=10.0 \mathrm{~Hz}, 1 \mathrm{H}), 5.03(\mathrm{~d}, J=17.0 \mathrm{~Hz}, 1 \mathrm{H}), 3.80(\mathrm{~s}, 3 \mathrm{H}), 3.18(\mathrm{t}, J=8.5 \mathrm{~Hz}, 1 \mathrm{H}), 2.74(\mathrm{~h}, J=7.0 \mathrm{~Hz}$, $1 \mathrm{H}), 1.32(\mathrm{~s}, 9 \mathrm{H}), 0.98(\mathrm{~d}, J=6.5 \mathrm{~Hz}, 3 \mathrm{H}) .{ }^{13} \mathrm{C} \mathrm{NMR}\left(126 \mathrm{MHz}, \mathrm{CDCl}_{3}\right) \delta 165.23,158.35,154.89,153.10,150.74$, 
139.83, 134.43, 129.04, 128.97, 122.86, 120.46, 118.77, 116.26, 114.11, 55.39, 55.01, 42.05, 34.93, 31.40, 18.17. HRMS (ESI) $\mathrm{m} / \mathrm{z}[\mathrm{M}+\mathrm{H}]^{+}$calcd for $\mathrm{C}_{25} \mathrm{H}_{30} \mathrm{O}_{3}$ 379.2274, found 379.2274. HPLC (ChiralPak IB column) 99:1 (Hexane $/ \mathrm{PrOH}) 1.0 \mathrm{~mL} \cdot \mathrm{min}^{-1} ; \mathrm{T}_{\text {major }}(8.6 \mathrm{~min}), \mathrm{T}_{\text {minor }}(7.8 \mathrm{~min}) ; 85.5: 14.5 \mathrm{er}$.

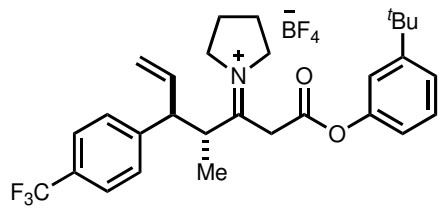

1-((4R,5S)-1-(3-(tert-butyl)phenoxy)-4-methyl-1-oxo-5-(4-(trifluoromethyl)phenyl)hept-6-en-3-ylide-

ne)pyrrolidin-1-ium tetrafluoroborate (21). Following the general procedure A, 21 was isolated in $79 \%$ yield and $72 \%$ ee $(99: 1 \mathrm{dr}) .{ }^{1} \mathrm{H}$ NMR $\left(600 \mathrm{MHz}, \mathrm{CD}_{3} \mathrm{CN}\right) \delta 7.75(\mathrm{~d}, J=7.8 \mathrm{~Hz}, 2 \mathrm{H}), 7.59(\mathrm{~d}, J=7.8 \mathrm{~Hz}, 2 \mathrm{H}), 7.39-7.37(\mathrm{~m}$, 2H), $7.22(\mathrm{~s}, 1 \mathrm{H}), 7.02-7.00(\mathrm{~m}, 1 \mathrm{H}), 6.04(\mathrm{dt}, J=16.8,10.2 \mathrm{~Hz}, 1 \mathrm{H}), 5.22(\mathrm{~d}, J=16.8 \mathrm{~Hz}, 1 \mathrm{H}), 5.14(\mathrm{~d}, J=10.2 \mathrm{~Hz}$, $1 \mathrm{H}), 4.39$ (d, $J=17.2 \mathrm{~Hz}, 1 \mathrm{H}), 4.28-4.20(\mathrm{~m}, 4 \mathrm{H}), 4.09-4.06(\mathrm{~m}, 1 \mathrm{H}), 3.82(\mathrm{t}, J=10.1 \mathrm{~Hz}, 1 \mathrm{H}), 3.59(\mathrm{dq}, J=13.2,6.6$ $\mathrm{Hz}, 1 \mathrm{H}), 2.24-2.17(\mathrm{~m}, 4 \mathrm{H}), 1.31(\mathrm{~s}, 9 \mathrm{H}), 1.06(\mathrm{~d}, J=6.6 \mathrm{~Hz}, 3 \mathrm{H}) .{ }^{13} \mathrm{C}$ NMR $\left(151 \mathrm{MHz}, \mathrm{CD}_{3} \mathrm{CN}\right) \delta 184.85,164.82$, $154.66,151.16,145.09,138.02,130.34,130.12\left(\mathrm{q},{ }^{2} J_{\mathrm{CF}}=33.2 \mathrm{~Hz}\right), 130.08,126.97\left(\mathrm{q},{ }^{3} J_{\mathrm{CF}}=4.5 \mathrm{~Hz}\right), 125.37\left(\mathrm{q},{ }^{1} J_{\mathrm{CF}}\right.$ $=271.8 \mathrm{~Hz}), 124.78,119.52,119.44,119.40,57.93,57.53,53.79,48.25,39.07,35.55,31.40,25.12,24.98,14.71 .{ }^{19} \mathrm{~F}$ NMR (376 MHz, $\mathrm{CD}_{3} \mathrm{CN}$ ) $\delta-62.21,-150.81$. HRMS (ESI) $m / z[\mathrm{M}+\mathrm{H}]^{+}$calcd for $\mathrm{C}_{29} \mathrm{H}_{34} \mathrm{~F}_{3} \mathrm{NO}_{2}$ 486.2615, found 486.2601. Enantiomeric excess was determined by derivatization to the corresponding hydrodeaminated $\alpha, \beta$ unsaturated ester. ${ }^{1} \mathrm{H}$ NMR $\left(500 \mathrm{MHz}, \mathrm{CDCl}_{3}\right) \delta 7.60(\mathrm{~d}, J=8.1 \mathrm{~Hz}, 2 \mathrm{H}), 7.34-7.31(\mathrm{~m}, 3 \mathrm{H}), 7.27(\mathrm{ddd}, J=8.0,1.5$, $1.5 \mathrm{~Hz}, 1 \mathrm{H}), 7.14-7.09(\mathrm{~m}, 2 \mathrm{H}), 6.95(\mathrm{ddd}, J=7.9,2.2,1.1 \mathrm{~Hz}, 1 \mathrm{H}), 6.03(\mathrm{dd}, J=15.7,1.0 \mathrm{~Hz}, 1 \mathrm{H}), 5.99$ (ddd, $J=$ 17.0, 10.2, 8.5 Hz, 1H), $5.16(\mathrm{~d}, J=10.2 \mathrm{~Hz}, 1 \mathrm{H}), 5.07(\mathrm{~d}, J=17.0 \mathrm{~Hz}, 1 \mathrm{H}), 3.30(\mathrm{t}, J=8.6 \mathrm{~Hz}, 1 \mathrm{H}), 2.81(\mathrm{~h}, J=7.7$ $\mathrm{Hz}, 1 \mathrm{H}), 1.32$ (s, 9H), $1.00(\mathrm{~d}, J=6.7 \mathrm{~Hz}, 3 \mathrm{H}) .{ }^{13} \mathrm{C} \mathrm{NMR}\left(126 \mathrm{MHz}, \mathrm{CDCl}_{3}\right) \delta 165.07,153.77,153.16,150.68,146.50$, $138.62,129.09\left(\mathrm{q},{ }^{2} J_{\mathrm{CF}}=32.5 \mathrm{~Hz}\right), 129.00,128.50,125.73\left(\mathrm{q},{ }^{3} J_{\mathrm{CF}}=3.8 \mathrm{~Hz}\right), 124.31\left(\mathrm{~d},{ }^{1} J_{\mathrm{CF}}=271.8 \mathrm{~Hz}\right), 122.95$, 121.03, 118.73, 118.72, 117.50, 55.68, 41.76, 34.94, 31.40, 18.21. ${ }^{19} \mathrm{~F}$ NMR (376 MHz, CD 3 CN) $\delta-62.10$. HRMS (ESI) $\mathrm{m} / \mathrm{z}[\mathrm{M}+\mathrm{H}]^{+}$calcd for $\mathrm{C}_{25} \mathrm{H}_{27} \mathrm{~F}_{3} \mathrm{O}_{2}$ 417.2041, found 417.2055. HPLC (ChiralPak IB column) 99.5:0.5 (Hexane/iPrOH) $1.0 \mathrm{~mL} \cdot \mathrm{min}^{-1}$; $\mathrm{T}_{\text {major }}(13.2 \mathrm{~min}), \mathrm{T}_{\text {minor }}(14.1 \mathrm{~min})$; 85.8:14.2 er.

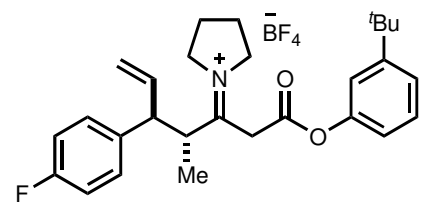

1-((4R,5S)-1-(3-(tert-butyl)phenoxy)-5-(4-fluorophenyl)-4-methyl-1-oxohept-6-en-3-ylidene)pyrrole-din-1-ium tetrafluoroborate (22). Following the general procedure A, 22 was isolated in $75 \%$ yield and $84 \%$ ee $(98: 2 \mathrm{dr}) .{ }^{1} \mathrm{H}$ NMR (600 MHz, $\left.\mathrm{CDCl}_{3}\right) \delta 7.33-7.27(\mathrm{~m}, 4 \mathrm{H}), 7.13(\mathrm{~s}, 1 \mathrm{H}), 7.05(\mathrm{t}, J=9.0 \mathrm{~Hz}, 2 \mathrm{H}), 6.95(\mathrm{dt}, J=7.2,2.4 \mathrm{~Hz}, 1 \mathrm{H})$, 6.01 (ddd, $J=17.4,10.2,8.4 \mathrm{~Hz}, 1 \mathrm{H}), 5.16(\mathrm{~d}, J=10.2 \mathrm{~Hz}, 1 \mathrm{H}), 5.13(\mathrm{~d}, J=17.4 \mathrm{~Hz}, 1 \mathrm{H}), 4.34-4.22(\mathrm{~m}, 3 \mathrm{H}), 4.30(\mathrm{~s}$, 2H), 4.19-4.15 (m, 1H), $3.58(\mathrm{t}, J=9.6 \mathrm{~Hz}, 1 \mathrm{H}), 3.51-3.46(\mathrm{~m}, 1 \mathrm{H}), 2.26-2.18(\mathrm{~m}, 4 \mathrm{H}), 1.30(\mathrm{~s}, 9 \mathrm{H}), 1.13(\mathrm{~d}, J=6.0$ $\mathrm{Hz}, 3 \mathrm{H}) .{ }^{13} \mathrm{C} \mathrm{NMR}\left(151 \mathrm{MHz}, \mathrm{CDCl}_{3}\right) \delta 184.48,164.20,162.28\left(\mathrm{~d},{ }^{1} J_{\mathrm{CF}}=247.0 \mathrm{~Hz}\right), 153.80,150.02,136.90,134.56$ $\left(\mathrm{d},{ }^{4} J_{\mathrm{CF}}=3.2 \mathrm{~Hz}\right), 130.07\left(\mathrm{~d},{ }^{3} J_{\mathrm{CF}}=7.9 \mathrm{~Hz}\right), 129.34,123.98,119.00,118.17,118.13,116.27\left(\mathrm{~d},{ }^{2} J_{\mathrm{CF}}=21.4 \mathrm{~Hz}\right), 56.94$, 56.31, 52.91, 47.96, 38.37, 35.00, 31.30, 24.48, 24.35, 14.79. ${ }^{19} \mathrm{~F}$ NMR (376 MHz, $\left.\mathrm{CDCl}_{3}\right) \delta$-115.74, -150.82. HRMS (ESI) $m / z[\mathrm{M}+\mathrm{H}]^{+}$calcd for $\mathrm{C}_{28} \mathrm{H}_{34} \mathrm{FNO}_{2}$ 436.2647, found 436.2635. Enantiomeric excess was determined by derivatization to the corresponding hydrodeaminated $\alpha, \beta$-unsaturated ester. ${ }^{1} \mathrm{H} \mathrm{NMR}\left(500 \mathrm{MHz}, \mathrm{CDCl}_{3}\right) \delta 7.35(\mathrm{t}, J=$ $7.9 \mathrm{~Hz}, 1 \mathrm{H}), 7.29(\mathrm{dt}, J=8.5,1.0 \mathrm{~Hz}, 1 \mathrm{H}), 7.20-7.17(\mathrm{~m}, 2 \mathrm{H}), 7.15-7.12(\mathrm{~m}, 2 \mathrm{H}), 7.06(\mathrm{t}, J=8.7 \mathrm{~Hz}, 2 \mathrm{H}), 6.98(\mathrm{ddd}$, $J=7.9,2.2,1.0 \mathrm{~Hz}, 1 \mathrm{H}), 6.04(\mathrm{dd}, J=16.0,1.0 \mathrm{~Hz}, 1 \mathrm{H}), 6.00(\mathrm{ddd}, J=17.0,10.5,8.5 \mathrm{~Hz}, 1 \mathrm{H}), 5.15(\mathrm{~d}, J=10.2 \mathrm{~Hz}$, $1 \mathrm{H}), 5.07(\mathrm{~d}, J=17.0 \mathrm{~Hz}, 1 \mathrm{H}), 3.25(\mathrm{t}, J=8.5 \mathrm{~Hz}, 1 \mathrm{H}), 2.77(\mathrm{~h}, J=7.3 \mathrm{~Hz}, 1 \mathrm{H}), 1.35(\mathrm{~s}, 9 \mathrm{H}), 1.01(\mathrm{~d}, J=6.7 \mathrm{~Hz}, 3 \mathrm{H})$. ${ }^{13} \mathrm{C} \mathrm{NMR}\left(126 \mathrm{MHz} \mathrm{CDCl}_{3}\right) \delta 165.15,161.69\left(\mathrm{~d},{ }^{1} J_{\mathrm{CF}}=244.4 \mathrm{~Hz}\right), 154.34,153.13,150.70,139.32,138.04\left(\mathrm{~d},{ }^{4} J_{\mathrm{CF}}=\right.$ $3.8 \mathrm{~Hz}), 129.52\left(\mathrm{~d},{ }^{3} J_{\mathrm{CF}}=7.6 \mathrm{~Hz}\right), 128.98,122.91,120.74,118.75,118.73,116.80,115.57\left(\mathrm{~d},{ }^{2} J_{\mathrm{CF}}=21.4 \mathrm{~Hz}\right), 55.03$, 42.00, 34.93, 31.40, 18.17. ${ }^{19} \mathrm{~F}$ NMR $\left(376 \mathrm{MHz} \mathrm{CDCl}_{3}\right) \delta-115.61$. HRMS (ESI) $\mathrm{m} / z[\mathrm{M}+\mathrm{H}]^{+}$calcd for $\mathrm{C}_{24} \mathrm{H}_{27} \mathrm{FO}_{2}$ 367.2073, found 367.2064. HPLC (ChiralPak ADH column) 99:1 (Hexane/iPrOH) $1.0 \mathrm{~mL} \cdot \mathrm{min}^{-1} ; \mathrm{T}_{\text {major }}(10.1 \mathrm{~min}$ ), $\mathrm{T}_{\text {minor }}(7.9 \mathrm{~min})$; 92.1:7.9 er. 


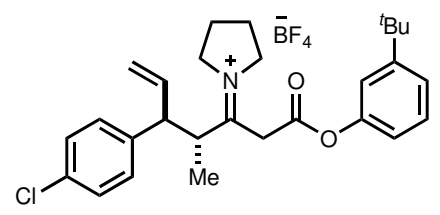

1-((4R,5S)-1-(3-(tert-butyl)phenoxy)-5-(4-chlorophenyl)-4-methyl-1-oxohept-6-en-3-ylidene)pyrrole-din-1-ium tetrafluoroborate (23). Following the general procedure A, 23 was isolated in $74 \%$ yield and $86 \%$ ee $(94: 6 \mathrm{dr}) .{ }^{1} \mathrm{H}$ NMR (600 MHz, CD 3 CN) $\delta 7.44(\mathrm{~d}, J=8.4 \mathrm{~Hz}, 2 \mathrm{H}), 7.39-7.37(\mathrm{~m}, 4 \mathrm{H}), 7.21(\mathrm{~s}, 1 \mathrm{H}), 7.01-6.99(\mathrm{~m}, 1 \mathrm{H}), 6.01(\mathrm{dt}, J=$ $16.8,10.2 \mathrm{~Hz}, 1 \mathrm{H}), 5.19(\mathrm{~d}, J=17.4 \mathrm{~Hz}, 1 \mathrm{H}), 5.11(\mathrm{~d}, J=10.2 \mathrm{~Hz}, 1 \mathrm{H}), 4.37(\mathrm{~d}, J=16.8 \mathrm{~Hz}, 1 \mathrm{H}), 4.28-4.14(\mathrm{~m}, 3 \mathrm{H})$, $4.19(\mathrm{~d}, J=17.4 \mathrm{~Hz}, 1 \mathrm{H}), 4.08-4.05(\mathrm{~m}, 1 \mathrm{H}), 3.71(\mathrm{t}, J=10.8 \mathrm{~Hz}, 1 \mathrm{H}), 3.54-3.49(\mathrm{~m}, 1 \mathrm{H}), 2.23-2.15(\mathrm{~m}, 4 \mathrm{H}), 1.31(\mathrm{~s}$, $9 \mathrm{H}), 1.06(\mathrm{~d}, J=6.6 \mathrm{~Hz}, 3 \mathrm{H}) .{ }^{13} \mathrm{C}$ NMR $\left(151 \mathrm{MHz}, \mathrm{CD}_{3} \mathrm{CN}\right) \delta 185.02,164.83,154.65,151.16,139.36,138.37,133.87$, 130.98, 130.33, 130.09, 124.77, 119.44, 119.40, 119.03, 57.85, 57.46, 53.45, 48.44, 39.06, 35.54, 31.40, 25.11, 24.97, 14.69. ${ }^{19} \mathrm{~F}$ NMR (376 MHz, $\mathrm{CD}_{3} \mathrm{CN}$ ) $\delta$-150.81. HRMS (ESI) $\mathrm{m} / z$ [M+H] ${ }^{+}$calcd for $\mathrm{C}_{28} \mathrm{H}_{34} \mathrm{ClNO}_{2} 452.2351$, found 452.2335. Enantiomeric excess was determined by derivatization to the corresponding hydrodeaminated $\alpha, \beta$ unsaturated ester. ${ }^{1} \mathrm{H}$ NMR $\left(400 \mathrm{MHz}, \mathrm{CDCl}_{3}\right) \delta$ 7.34-7.30 (m, 3H), $7.26(\mathrm{dt}, J=8.0,1.2 \mathrm{~Hz}, 1 \mathrm{H}), 7.14-7.08(\mathrm{~m}, 4 \mathrm{H})$, $6.95(\mathrm{ddd}, J=7.8,2.2,1.1 \mathrm{~Hz}, 1 \mathrm{H}), 6.01(\mathrm{dd}, J=16.0,0.8 \mathrm{~Hz}, 1 \mathrm{H}), 5.96(\mathrm{ddd}, J=17.2,10.4,8.4 \mathrm{~Hz}, 1 \mathrm{H}), 5.12(\mathrm{~d}, J$ $=10.2 \mathrm{~Hz}, 1 \mathrm{H}), 5.04(\mathrm{~d}, J=17.0 \mathrm{~Hz}, 1 \mathrm{H}), 3.21(\mathrm{t}, J=8.5 \mathrm{~Hz}, 1 \mathrm{H}), 2.75(\mathrm{~h}, J=7.2 \mathrm{~Hz}, 1 \mathrm{H}), 1.32(\mathrm{~s}, 9 \mathrm{H}), 0.99(\mathrm{~d}, J=$ $6.7 \mathrm{~Hz}, 9 \mathrm{H}) .{ }^{13} \mathrm{C} \mathrm{NMR}\left(101 \mathrm{MHz}, \mathrm{CDCl}_{3}\right) \delta 165.09,154.10,153.15,150.72,140.87,139.02,132.49,129.50,128.99$, $128.90,122.90,120.87,118.74,117.05,55.19,41.85,34.94,31.41,18.17$. HRMS (ESI) $m / z[M+H]^{+}$calcd for $\mathrm{C}_{24} \mathrm{H}_{27} \mathrm{ClO}_{2}$ 383.1778, found 383.1754. HPLC (ChiralPak ADH column) 99:1 (Hexane/iPrOH) $1.0 \mathrm{~mL} \cdot \mathrm{min}^{-1} ; \mathrm{T}_{\mathrm{major}}$ (10.1 min), $\mathrm{T}_{\text {minor }}(9.1 \mathrm{~min}) ; 92.8: 7.2 \mathrm{er}$.

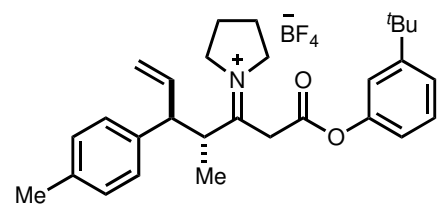

1-((4R,5S)-1-(3-(tert-butyl)phenoxy)-4-methyl-1-oxo-5-(p-tolyl)hept-6-en-3-ylidene)pyrrolidin-1-ium

tetrafluoroborate (24). Following the general procedure A, $\mathbf{2 4}$ was isolated in $89 \%$ yield and $86 \%$ ee $(97: 3 \mathrm{dr}) .{ }^{1} \mathrm{H}$ NMR (600 MHz, $\left.\mathrm{CDCl}_{3}\right) \delta$ 7.33-7.29 (m, 2H), $7.17(\mathrm{~s}, 4 \mathrm{H}), 7.13(\mathrm{~s}, 1 \mathrm{H}), 6.96$ (dt, J= 7.4, 1.8 Hz, 1H), $6.02(\mathrm{ddd}, J=$ $18.1,10.1,8.1 \mathrm{~Hz}, 1 \mathrm{H}), 5.15(\mathrm{~d}, J=9.9 \mathrm{~Hz}, 1 \mathrm{H}), 5.12(\mathrm{~d}, J=16.8 \mathrm{~Hz}, 1 \mathrm{H}), 4.34-4.27(\mathrm{~m}, 2 \mathrm{H}), 4.30(\mathrm{~s}, 2 \mathrm{H}), 4.23(\mathrm{dt}$, $J=13.2,7.2 \mathrm{~Hz}, 1 \mathrm{H}), 4.12(\mathrm{dt}, J=13.5,5.5 \mathrm{~Hz}, 1 \mathrm{H}), 3.54-3.46(\mathrm{~m}, 2 \mathrm{H}), 2.32(\mathrm{~s}, 3 \mathrm{H}), 2.26-2.17(\mathrm{~m}, 4 \mathrm{H}), 1.30(\mathrm{~s}, 9 \mathrm{H})$, $1.15(\mathrm{~d}, J=6.3 \mathrm{~Hz}, 3 \mathrm{H}) .{ }^{13} \mathrm{C}$ NMR $\left(151 \mathrm{MHz}, \mathrm{CDCl}_{3}\right) \delta 184.63,164.18,153.77,150.05,137.68,137.09,135.69$, 130.06, 129.32, 128.22, 123.93, 118.73, 118.19, 118.15, 56.92, 56.21, 53.56, 48.04, 38.42, 35.00, 31.31, 24.48, 24.35, 21.16, 14.89. ${ }^{19} \mathrm{~F} \mathrm{NMR}\left(376 \mathrm{MHz}, \mathrm{CDCl}_{3}\right) \delta$-150.92. HRMS (ESI) $m / z[\mathrm{M}+\mathrm{H}]^{+}$calcd for $\mathrm{C}_{29} \mathrm{H}_{37} \mathrm{NO}_{2} 432.2897$, found 432.2918. Enantiomeric excess was directly determined over the $\beta$-enamino ester after basic washing. HPLC (ChiralPak ODH column) 99:1 (Hexane/iPrOH) 0.5 mL· $\mathrm{min}^{-1} ; \mathrm{T}_{\text {major }}(19.7 \mathrm{~min}), \mathrm{T}_{\text {minor }}(15.0 \mathrm{~min})$; 92.8:7.2 er.<smiles>C=CC(c1cccc(C)c1)[C@H](C)[C@H](CC(=O)Oc1cccc(C(F)(F)Br)c1)/C(C)=N\C1CCCC1</smiles>

1-((4R,5S)-1-(3-(tert-butyl)phenoxy)-4-methyl-1-oxo-5-(m-tolyl)hept-6-en-3-ylidene)pyrrolidin-1-ium tetrafluoroborate (25). Following the general procedure A, 25 was isolated in $86 \%$ yield and $65 \%$ ee $(97: 3 \mathrm{dr})$. ${ }^{1} \mathrm{H}$ NMR (400 MHz, $\left.\mathrm{CDCl}_{3}\right) \delta$ 7.35-7.29 (m, 2H), $7.25(\mathrm{t}, J=7.5 \mathrm{~Hz}, 1 \mathrm{H}), 7.13(\mathrm{~s}, 1 \mathrm{H}), 7.11-7.06(\mathrm{~m}, 3 \mathrm{H}), 6.96(\mathrm{dt}, J=$ 7.1, $2.2 \mathrm{~Hz}, 1 \mathrm{H}), 6.03$ (ddd, $J=17.1,10.1,8.0 \mathrm{~Hz}, 1 \mathrm{H}), 5.17(\mathrm{~d}, J=7.1 \mathrm{~Hz}, 1 \mathrm{H}), 5.16(\mathrm{~d}, J=13.6 \mathrm{~Hz}, 1 \mathrm{H}), 4.36-4.27$ $(\mathrm{m}, 2 \mathrm{H}), 4.31(\mathrm{~s}, 2 \mathrm{H}), 4.26-4.21(\mathrm{~m}, 1 \mathrm{H}), 4.12(\mathrm{dt}, J=13.6,6.4 \mathrm{~Hz}, 1 \mathrm{H}), 3.53-3.45(\mathrm{~m}, 2 \mathrm{H}), 2.36(\mathrm{~s}, 3 \mathrm{H}), 2.29-2.16$ $(\mathrm{m}, 4 \mathrm{H}), 1.31(\mathrm{~s}, 9 \mathrm{H}), 1.16(\mathrm{~d}, J=5.8 \mathrm{~Hz}, 3 \mathrm{H}) .{ }^{13} \mathrm{C} \mathrm{NMR}\left(151 \mathrm{MHz}, \mathrm{CDCl}_{3}\right) \delta 184.59,164.17,153.78,150.06,139.22$, 138.70 , 136.99, 129.33, 129.25, 129.01, 128.71, 125.34, 123.94, 118.87, 118.18, 118.15, 56.96, 56.24, 53.97, 48.00, 
38.42, 35.01, 31.31, 24.49, 24.35, 21.51, 14.97. ${ }^{19} \mathrm{~F}$ NMR (376 MHz, $\left.\mathrm{CDCl}_{3}\right) \delta-150.71$. HRMS (ESI) $m / z[\mathrm{M}+\mathrm{H}]^{+}$ calcd for $\mathrm{C}_{29} \mathrm{H}_{37} \mathrm{NO}_{2}$ 432.2897, found 432.2893. Enantiomeric excess was determined by derivatization to the corresponding hydrodeaminated $\alpha, \beta$-unsaturated ester. ${ }^{1} \mathrm{H}$ NMR $\left(600 \mathrm{MHz}, \mathrm{CDCl}_{3}\right) \delta 7.31(\mathrm{t}, J=7.9 \mathrm{~Hz}, 1 \mathrm{H}), 7.28$ $7.25(\mathrm{~m}, 1 \mathrm{H}), 7.22(\mathrm{t}, J=7.4 \mathrm{~Hz}, 1 \mathrm{H}), 7.14(\mathrm{dd}, J=15.8,8.3 \mathrm{~Hz}, 1 \mathrm{H}), 7.11(\mathrm{~s}, 1 \mathrm{H}), 7.04(\mathrm{~d}, J=7.5 \mathrm{~Hz}, 2 \mathrm{H}), 7.00(\mathrm{~s}$, $1 \mathrm{H}), 6.99(\mathrm{~d}, J=8.2 \mathrm{~Hz}, 1 \mathrm{H}), 6.95(\mathrm{~d}, J=7.9 \mathrm{~Hz}, 1 \mathrm{H}), 6.02-5.96(\mathrm{~m}, 2 \mathrm{H}), 5.09(\mathrm{~d}, J=10.2 \mathrm{~Hz}, 1 \mathrm{H}), 5.05(\mathrm{~d}, J=17.0$ $\mathrm{Hz}, 1 \mathrm{H}), 3.17(\mathrm{t}, J=8.7 \mathrm{~Hz}, 1 \mathrm{H}), 2.77(\mathrm{q}, J=7.2 \mathrm{~Hz}, 1 \mathrm{H}), 2.35(\mathrm{~s}, 3 \mathrm{H}), 1.32(\mathrm{~s}, 9 \mathrm{H}), 0.98(\mathrm{~d}, J=6.7 \mathrm{~Hz}, 3 \mathrm{H}){ }^{13} \mathrm{C} \mathrm{NMR}$ $\left(151 \mathrm{MHz}, \mathrm{CDCl}_{3}\right) \delta 165.23,154.86,153.14,150.82,142.41,139.77,138.33,128.98,128.90,128.64,127.51,125.13$, $122.85,120.52,118.78,116.42,56.00,41.88,34.94,31.41,21.65,18.26$. HRMS (EI) $m / z[M]^{+}$calcd for $\mathrm{C}_{25} \mathrm{H}_{30} \mathrm{O}_{2}$ 362.2246, found 362.2250. HPLC (ChiralPak OJH column) 99:1 (Hexane $/ \mathrm{PrOH}) 1.0 \mathrm{~mL} \cdot \mathrm{min}^{-1} ; \mathrm{T}_{\text {major }}(8.6 \mathrm{~min}), \mathrm{T}_{\text {minor }}$ (7.6 min); 82.4:17.6 er.

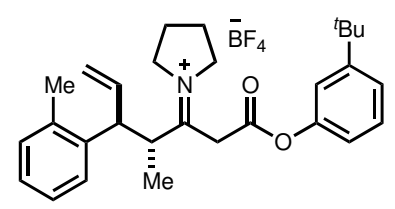

1-((4R,5S)-1-(3-(tert-butyl)phenoxy)-4-methyl-1-oxo-5-(o-tolyl)hept-6-en-3-ylidene)pyrrolidin-1-ium tetrafluoroborate (26). Following the general procedure A, 26 was isolated in $97 \%$ yield and $49 \%$ ee $(98: 2 \mathrm{dr}) .{ }^{1} \mathrm{H}$ NMR (400 MHz, $\left.\mathrm{CDCl}_{3}\right) \delta$ 7.35-7.30 (m, 2H), 7.26-7.25 (m, 2H), 7.22-7.16 (m, 2H), 7.15-7.11 (m, 1H), $6.97(\mathrm{dt}, J=$ 6.8, $2.4 \mathrm{~Hz}, 1 \mathrm{H}), 5.96(\mathrm{ddd}, J=17.2,10.4,8.4 \mathrm{~Hz}, 1 \mathrm{H}), 5.16(\mathrm{~d}, J=10.4 \mathrm{~Hz}, 1 \mathrm{H}), 5.09(\mathrm{~d}, J=16.8 \mathrm{~Hz}, 1 \mathrm{H}), 4.36(\mathrm{~s}$, $2 \mathrm{H}), 4.36-4.24(\mathrm{~m}, 3 \mathrm{H}), 4.08-4.01(\mathrm{~m}, 1 \mathrm{H}), 3.87(\mathrm{t}, J=9.2 \mathrm{~Hz}, 1 \mathrm{H}), 3.62-3.55(\mathrm{~m}, 1 \mathrm{H}), 2.38(\mathrm{~s}, 3 \mathrm{H}), 2.29-2.11(\mathrm{~m}$, $4 \mathrm{H}), 1.31(\mathrm{~s}, 9 \mathrm{H}), 1.18(\mathrm{~d}, J=6.4 \mathrm{~Hz}, 3 \mathrm{H}) .{ }^{13} \mathrm{C}$ NMR $\left(151 \mathrm{MHz}, \mathrm{CDCl}_{3}\right) \delta 184.47,164.25,153.83,150.05,136.78$, 136.60, 136.28, 131.34, 129.37, 127.52, 127.16, 126.95, 124.00, 119.05, 118.16, 118.12, 57.04, 56.27, 53.56, 47.67, 38.56, 35.01, 31.31, 24.51, 24.36, 20.02, 14.84. ${ }^{19} \mathrm{~F}$ NMR $\left(376 \mathrm{MHz}, \mathrm{CDCl}_{3}\right) \delta-150.98$. HRMS (ESI) $\left.\mathrm{m} / z . \mathrm{M}+\mathrm{H}\right]^{+}$ calcd for $\mathrm{C}_{29} \mathrm{H}_{37} \mathrm{NO}_{2}$ 432.2897, found 432.2889. Enantiomeric excess was determined by derivatization to the corresponding hydrodeaminated $\alpha, \beta$-unsaturated ester. ${ }^{1} \mathrm{H}$ NMR $\left(600 \mathrm{MHz}, \mathrm{CDCl}_{3}\right) \delta 7.32(\mathrm{t}, J=7.9 \mathrm{~Hz}, 1 \mathrm{H}), 7.27$ (s, 1H), 7.23-7.18 (m, 4H), 7.15-7.12 (m, 2H), $6.97(\mathrm{~d}, J=7.9 \mathrm{~Hz}, 1 \mathrm{H}), 6.05(\mathrm{~d}, J=15.7 \mathrm{~Hz}, 1 \mathrm{H}), 5.94(\mathrm{ddd}, J=17.2$, $10.1,8.5 \mathrm{~Hz}, 1 \mathrm{H}), 5.06(\mathrm{~d}, J=10.2 \mathrm{~Hz}, 1 \mathrm{H}), 5.00(\mathrm{~d}, J=17.0 \mathrm{~Hz}, 1 \mathrm{H}), 3.51(\mathrm{t}, J=8.9 \mathrm{~Hz}, 1 \mathrm{H}), 2.82(\mathrm{~h}, J=6.9 \mathrm{~Hz}$, $1 \mathrm{H}), 2.36(\mathrm{~s}, 3 \mathrm{H}), 1.33(\mathrm{~s}, 9 \mathrm{H}), 0.99(\mathrm{~d}, J=6.7 \mathrm{~Hz}, 3 \mathrm{H}){ }^{13} \mathrm{C}$ NMR $\left(151 \mathrm{MHz}, \mathrm{CDCl}_{3}\right) \delta 165.22,155.04,153.14,150.81$, 140.56, 139.76, 136.12, 130.80, 128.98, 126.93, 126.45, 126.33, 122.86, 120.58, 118.79, 118.77, 116.24, 50.77, 41.64, 34.94, 31.41, 20.05, 18.10. HRMS (ESI) $\mathrm{m} / z$ [M+Na ${ }^{+}$calcd for $\mathrm{C}_{25} \mathrm{H}_{30} \mathrm{O}_{2}$ 385.2159, found 385.2177. HPLC (ChiralPak IB column) 99.9:0.1 (Hexane $/ \mathrm{iPrOH}) 0.5 \mathrm{~mL} \cdot \mathrm{min}^{-1} ; \mathrm{T}_{\text {major }}(21.4 \mathrm{~min}), \mathrm{T}_{\text {minor }}(19.5 \mathrm{~min})$; 74.7:25.3 er.

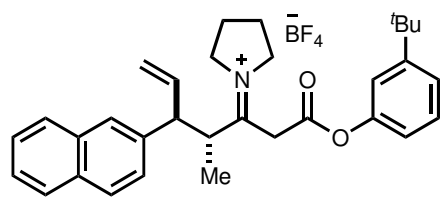

1-((4R,5S)-1-(3-(tert-butyl)phenoxy)-4-methyl-5-(naphthalen-2-yl)-1-oxohept-6-en-3-ylidene)pyrrole-din-1-ium tetrafluoroborate (27). Following the general procedure A, 27 was isolated in $83 \%$ yield and $87 \%$ ee $(93: 7 \mathrm{dr}) .{ }^{1} \mathrm{H}$ NMR (600 MHz, $\left.\mathrm{CDCl}_{3}\right) \delta$ 7.86-7.84 (m, 2H), 7.80-7.78 (m, 2H), 7.49-7.45 (m, 2H), $7.40(\mathrm{~d}, J=8.4 \mathrm{~Hz}, 1 \mathrm{H}), 7.33-$ $7.28(\mathrm{~m}, 2 \mathrm{H}), 7.15(\mathrm{br} \mathrm{s}, 1 \mathrm{H}), 6.97(\mathrm{dt}, J=7.8,1.8 \mathrm{~Hz}, 1 \mathrm{H}), 6.12(\mathrm{ddd}, J=18.0,9.6,8.4 \mathrm{~Hz}, 1 \mathrm{H}), 5.18(\mathrm{~d}, J=10.2 \mathrm{~Hz}$, $1 \mathrm{H}), 5.17(\mathrm{~d}, J=17.4 \mathrm{~Hz}, 1 \mathrm{H}), 4.35(\mathrm{~s}, 2 \mathrm{H}), 4.33-4.19(\mathrm{~m}, 4 \mathrm{H}), 3.72(\mathrm{t}, J=10.2 \mathrm{~Hz}, 1 \mathrm{H}), 3.66-3.62(\mathrm{~m}, 1 \mathrm{H}), 2.24-2.15$ $(\mathrm{m}, 4 \mathrm{H}), 1.30(\mathrm{~s}, 9 \mathrm{H}), 1.16(\mathrm{~d}, J=6.6 \mathrm{~Hz}, 3 \mathrm{H}) .{ }^{13} \mathrm{C}$ NMR $\left(151 \mathrm{MHz}, \mathrm{CDCl}_{3}\right) \delta 184.55,164.22,153.78,150.06,136.93$, $136.08,133.73,132.91,129.33,129.29,128.05,127.80,127.72,126.66,126.38,125.71,123.95,119.13,118.20$, $118.16,56.97,56.33,53.95,47.79,38.43,35.00,31.31,24.47,24.34,14.97 .{ }^{19} \mathrm{~F}$ NMR $\left(376 \mathrm{MHz}, \mathrm{CD}_{3} \mathrm{CN}\right) \delta-150.85$. HRMS (ESI) $\mathrm{m} / z$ [M+H] $]^{+}$calcd for $\mathrm{C}_{32} \mathrm{H}_{37} \mathrm{NO}_{2} 468.2897$, found 468.2879. Enantiomeric excess was determined by derivatization to the corresponding hydrodeaminated $\alpha, \beta$-unsaturated ester. ${ }^{1} \mathrm{H}$ NMR $\left(400 \mathrm{MHz}, \mathrm{CDCl}_{3}\right) \delta 7.84-7.80$ $(\mathrm{m}, 3 \mathrm{H}), 7.64(\mathrm{~s}, 1 \mathrm{H}), 7.50-7.43(\mathrm{~m}, 2 \mathrm{H}), 7.35(\mathrm{dd}, J=8.7,1.7 \mathrm{~Hz}, 1 \mathrm{H}), 7.32(\mathrm{t}, J=7.6 \mathrm{~Hz}, 1 \mathrm{H}), 7.26(\mathrm{dt}, J=7.6,1.6$ $\mathrm{Hz}, 1 \mathrm{H}), 7.19$ (dd, $J=15.7,8.2 \mathrm{~Hz}, 1 \mathrm{H}), 7.12(\mathrm{t}, J=2.0 \mathrm{~Hz}, 1 \mathrm{H}), 6.96$ (ddd, $J=7.8,2.2,1.1 \mathrm{~Hz}, 1 \mathrm{H}), 6.10$ (ddd, $J=$ $17.0,10.2,8.4 \mathrm{~Hz}, 1 \mathrm{H}), 6.05(\mathrm{dd}, J=15.7,0.9 \mathrm{~Hz}, 1 \mathrm{H}), 5.14(\mathrm{~d}, J=10.2 \mathrm{~Hz}, 1 \mathrm{H}), 5.09(\mathrm{~d}, J=17.0 \mathrm{~Hz}, 1 \mathrm{H}), 3.39(\mathrm{t}, J$ $=8.6 \mathrm{~Hz}, 1 \mathrm{H}), 2.91(\mathrm{~h}, J=7.6 \mathrm{~Hz}, 1 \mathrm{H}), 1.32(\mathrm{~s}, 9 \mathrm{H}), 1.01(\mathrm{~d}, J=6.7 \mathrm{~Hz}, 3 \mathrm{H}) \cdot{ }^{13} \mathrm{C} \mathrm{NMR}\left(101 \mathrm{MHz}, \mathrm{CDCl}_{3}\right) \delta 165.20$, 
$154.67,153.13,150.78,139.87,139.54,133.73,132.55,128.98,128.47,127.81,127.78,126.91,126.26,125.73$, 122.87, 120.70, 118.78, 116.86, 56.02, 41.83, 34.94, 31.42, 18.38. HRMS (ESI) $m / z[M+H]^{+}$calcd for $\mathrm{C}_{28} \mathrm{H}_{30} \mathrm{O}_{2}$ 399.2324, found 399.2360. HPLC (ChiralPak ADH column) 99:1 (Hexane/iPrOH) $1.0 \mathrm{~mL} \cdot \mathrm{min}^{-1}$; $\mathrm{T}_{\text {major }}(11.6 \mathrm{~min}$ ), $\mathrm{T}_{\text {minor }}(10.7 \mathrm{~min})$; 93.7:6.3 er.

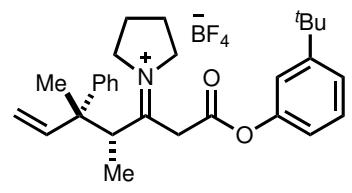

1-((4R,5S)-1-(3-(tert-butyl)phenoxy)-4,5-dimethyl-1-oxo-5-phenylhept-6-en-3-ylidene)pyrrolidin-1-ium tetrafluoroborate (28). Following the general procedure A, 28 was isolated in $96 \%$ yield and $49 \%$ ee $(1: 0 \mathrm{dr}) .{ }^{1} \mathrm{H}$ NMR (400 MHz, $\left.\mathrm{CDCl}_{3}\right) \delta$ 7.46-7.39 (m, 4H), 7.35-7.30 (m, 3H), 7.07 (br s, 1H), $6.92(\mathrm{dt}, J=7.2,2.4 \mathrm{~Hz}, 1 \mathrm{H}), 6.33$ $(\mathrm{dd}, J=17.2,10.8 \mathrm{~Hz}, 1 \mathrm{H}), 5.34(\mathrm{~d}, J=10.8 \mathrm{~Hz}, 1 \mathrm{H}), 5.14(\mathrm{~d}, J=18.8 \mathrm{~Hz}, 1 \mathrm{H}), 4.24-4.18(\mathrm{~m}, 1 \mathrm{H}), 4.14-4.01(\mathrm{~m}, 2 \mathrm{H})$, 4.06 (s, 2H), 3.50-3.37 (m, 2H), 2.26-2.06 (m, 3H), 1.92-1.81 (m, 1H), $1.65(\mathrm{~s}, 3 \mathrm{H}), 1.34(\mathrm{~d}, J=6.8 \mathrm{~Hz}, 3 \mathrm{H}), 1.32(\mathrm{~s}$, 9H). ${ }^{13} \mathrm{C} \mathrm{NMR}\left(101 \mathrm{MHz}, \mathrm{CDCl}_{3}\right) \delta 184.55,164.38,153.78,149.91,142.22,141.03,129.29,129.04,127.89,127.63$, 123.96, 118.07, 117.47, 56.86, 56.34, 53.05, 49.31, 39.55, 35.01, 31.33, 24.50, 24.17, 23.77, 12.72. ${ }^{19} \mathrm{~F} \mathrm{NMR}(376$ $\left.\mathrm{MHz}, \mathrm{CDCl}_{3}\right) \delta$-151.34. HRMS (ESI) $\mathrm{m} / z[\mathrm{M}+\mathrm{H}]^{+}$calcd for $\mathrm{C}_{29} \mathrm{H}_{37} \mathrm{NO}_{2}$ 432.2897, found 432.2893. Enantiomeric excess was determined by derivatization to the corresponding hydrodeaminated $\alpha, \beta$-unsaturated ester. ${ }^{1} \mathrm{H}$ NMR $(400$ $\left.\mathrm{MHz} \mathrm{CDCl}_{3}\right) \delta 7.36-7.28(\mathrm{~m}, 5 \mathrm{H}), 7.25-7.29(\mathrm{~m}, 2 \mathrm{H}), 7.14(\mathrm{dd}, J=15.7,7.4 \mathrm{~Hz}, 1 \mathrm{H}), 7.08(\mathrm{t}, J=1.9 \mathrm{~Hz}, 1 \mathrm{H}), 6.92$ $(\mathrm{d}, J=7.4 \mathrm{~Hz}, 1 \mathrm{H}), 6.16(\mathrm{dd}, J=17.5,10.9 \mathrm{~Hz}, 1 \mathrm{H}), 5.99(\mathrm{~d}, J=15.9 \mathrm{~Hz}, 1 \mathrm{H}), 5.22(\mathrm{~d}, J=10.8 \mathrm{~Hz}, 1 \mathrm{H}), 5.08(\mathrm{~d}, J=$ $17.4 \mathrm{~Hz}, 1 \mathrm{H}), 2.97$ (quin, $J=7.2 \mathrm{~Hz}, 1 \mathrm{H}), 1.42(\mathrm{~s}, 3 \mathrm{H}), 1.31(\mathrm{~s}, 9 \mathrm{H}), 0.96(\mathrm{~d}, J=6.8 \mathrm{~Hz}, 3 \mathrm{H})$. HRMS (ESI) $m / z$ [M+H] ${ }^{+}$ calcd for $\mathrm{C}_{25} \mathrm{H}_{30} \mathrm{O}_{2}$ 385.2159, found 385.2171. HPLC (ChiralPak OJH column) 99:1 (Hexane/ $\left.i \mathrm{PrOH}\right) 1.0 \mathrm{~mL} \cdot \mathrm{min}^{-1}$; $\mathrm{T}_{\text {major }}(8.4 \mathrm{~min}), \mathrm{T}_{\text {minor }}(7.6 \mathrm{~min}) ; 74.5: 25.5 \mathrm{er}$.

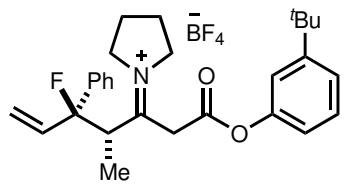

1-((4S,5R)-1-(3-(tert-butyl)phenoxy)-5-fluoro-4-methyl-1-oxo-5-phenylhept-6-en-3-ylidene)pyrrolidin-1-ium tetrafluoroborate (29). Following the general procedure A, 29 was isolated in $85 \%$ yield and $58 \%$ ee $(98: 2 \mathrm{dr}) .{ }^{1} \mathrm{H}$ NMR (600 MHz, $\left.\mathrm{CDCl}_{3}\right) \delta$ 7.46-7.43 (m, 4H), 7.37-7.31 (m, 3H), 7.14 (t, J=1.9 Hz, 1H), $6.97(\mathrm{~d}, J=7.6 \mathrm{~Hz}, 1 \mathrm{H})$, 6.28 (ddd, $J=19.8,17.1,11.0 \mathrm{~Hz}, 1 \mathrm{H}), 5.31$ (d, $J=10.8 \mathrm{~Hz}, 1 \mathrm{H}), 5.31$ (d, $J=16.8 \mathrm{~Hz}, 1 \mathrm{H}), 4.48-4.43$ (m, $1 \mathrm{H}), 4.40-$ $4.36(\mathrm{~m}, 1 \mathrm{H}), 4.33(\mathrm{~s}, 2 \mathrm{H}), 4.33-4.24(\mathrm{~m}, 2 \mathrm{H}), 3.85(\mathrm{dq}, J=28.4,6.3 \mathrm{~Hz}, 1 \mathrm{H}), 2.35-2.28(\mathrm{~m}, 2 \mathrm{H}), 2.24-2.16(\mathrm{~m}, 2 \mathrm{H})$, $1.32(\mathrm{~s}, 9 \mathrm{H}), 1.22(\mathrm{~d}, J=6.8 \mathrm{~Hz}, 3 \mathrm{H}) .{ }^{13} \mathrm{C} \mathrm{NMR}\left(151 \mathrm{MHz}, \mathrm{CDCl}_{3}\right) \delta 182.30,164.11,153.76,150.16,137.93\left(\mathrm{~d},{ }^{2} J_{\mathrm{CF}}\right.$ $=21.1 \mathrm{~Hz}), 136.15\left(\mathrm{~d},{ }^{2} J_{\mathrm{CF}}=22.7 \mathrm{~Hz}\right), 129.32,129.12,129.11,128.67,124.68\left(\mathrm{~d},{ }^{3} J_{\mathrm{CF}}=10.6 \mathrm{~Hz}\right), 123.90,118.46(\mathrm{~d}$, $\left.{ }^{3} J_{\mathrm{CF}}=12.1 \mathrm{~Hz}\right), 118.24\left(\mathrm{~d},{ }^{4} J_{\mathrm{CF}}=7.6 \mathrm{~Hz}\right), 99.58\left(\mathrm{~d},{ }^{1} J_{\mathrm{CF}}=187.2 \mathrm{~Hz}\right), 57.18,57.04,50.63\left(\mathrm{~d},{ }^{2} J_{\mathrm{CF}}=22.7 \mathrm{~Hz}\right), 39.44$ $\left(\mathrm{d},{ }^{4} J_{\mathrm{CF}}=6.0 \mathrm{~Hz}\right), 35.00,31.33,24.62,24.38,11.78\left(\mathrm{~d},{ }^{3} J_{\mathrm{CF}}=3.0 \mathrm{~Hz}\right) .{ }^{19} \mathrm{~F} \mathrm{NMR}\left(376 \mathrm{MHz}, \mathrm{CDCl}_{3}\right) \delta-151.12,-168.58$. HRMS (ESI) $\mathrm{m} / \mathrm{z}[\mathrm{M}+\mathrm{H}]^{+}$calcd for $\mathrm{C}_{28} \mathrm{H}_{34} \mathrm{FNO}_{2}$ 436.2647, found 436.2627. Enantiomeric excess was directly determined over the $\beta$-enamino ester after basic washing. HPLC (ChiralPak ADH column) 99:1 (Hexane/iPrOH) 1.0 $\mathrm{mL} \cdot \mathrm{min}^{-1} ; \mathrm{T}_{\text {major }}(6.9 \mathrm{~min}), \mathrm{T}_{\text {minor }}(5.9 \mathrm{~min}) ; 79.0: 21.0 \mathrm{er}$.

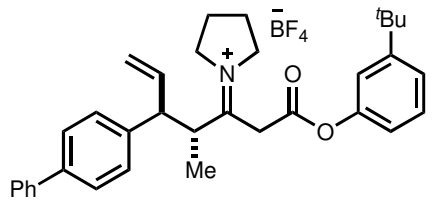

1-((4R,5S)-5-([1,1'-biphenyl]-4-yl)-1-(3-(tert-butyl)phenoxy)-4-methyl-1-oxohept-6-en-3-ylidene)pyro-ledin-1ium tetrafluoroborate (SI-1). Following the general procedure A, SI-1 was isolated in 94\% yield and 77\% ee (96:4 
dr). ${ }^{1} \mathrm{H}$ NMR $\left(600 \mathrm{MHz}, \mathrm{CDCl}_{3}\right) \delta 7.60(\mathrm{~d}, J=8.0 \mathrm{~Hz}, 2 \mathrm{H}), 7.56(\mathrm{~d}, J=7.5 \mathrm{~Hz}, 2 \mathrm{H}), 7.42(\mathrm{t}, J=7.6 \mathrm{~Hz}, 2 \mathrm{H}), 7.37-$ $7.29(\mathrm{~m}, 5 \mathrm{H}), 7.15(\mathrm{~s}, 1 \mathrm{H}), 6.97(\mathrm{~d}, J=7.4 \mathrm{~Hz}, 1 \mathrm{H}), 6.07(\mathrm{dt}, J=17.3,9.2 \mathrm{~Hz}, 1 \mathrm{H}), 5.19(\mathrm{~d}, J=9.6 \mathrm{~Hz}, 1 \mathrm{H}), 5.19(\mathrm{~d}$, $J=18.0 \mathrm{~Hz}, 1 \mathrm{H}), 4.37-4.34(\mathrm{~m}, 2 \mathrm{H}), 4.34(\mathrm{~s}, 2 \mathrm{H}), 4.25(\mathrm{dt}, J=13.8,6.0 \mathrm{~Hz}, 1 \mathrm{H}), 4.17(\mathrm{dt}, J=13.6,5.4 \mathrm{~Hz}, 1 \mathrm{H}), 3.61$ $(\mathrm{t}, J=9.1 \mathrm{~Hz}, 1 \mathrm{H}), 3.58-3.53(\mathrm{~m}, 1 \mathrm{H}), 2.25-2.18(\mathrm{~m}, 4 \mathrm{H}), 1.31(\mathrm{~s}, 9 \mathrm{H}), 1.20(\mathrm{~d}, J=6.5 \mathrm{~Hz}, 3 \mathrm{H}) .{ }^{13} \mathrm{C}$ NMR $(151 \mathrm{MHz}$, $\left.\mathrm{CDCl}_{3}\right) \delta 184.56,164.21,153.79,150.06,140.80,140.43,137.78,136.87,129.34,128.96,128.83,128.02,127.62$, 127.14, 123.96, 119.06, 118.19, 118.15, 56.97, 56.28, 53.57, 47.98, 38.44, 35.00, 31.31, 24.50, 24.36, 14.96. ${ }^{19} \mathrm{~F}$ NMR $\left(376 \mathrm{MHz}, \mathrm{CDCl}_{3}\right) \delta-150.68$. HRMS (ESI) $\mathrm{m} / z[\mathrm{M}+\mathrm{H}]^{+}$calcd for $\mathrm{C}_{34} \mathrm{H}_{39} \mathrm{NO}_{2} 494.3054$, found 494.3052. Enantiomeric excess was determined by derivatization to the corresponding hydrodeaminated $\alpha, \beta$-unsaturated ester. ${ }^{1} \mathrm{H}$ NMR (600 $\left.\mathrm{MHz}, \mathrm{CDCl}_{3}\right) \delta 7.60(\mathrm{~d}, J=8.0 \mathrm{~Hz}, 2 \mathrm{H}), 7.57(\mathrm{~d}, J=8.0 \mathrm{~Hz}, 2 \mathrm{H}), 7.44(\mathrm{t}, J=7.4 \mathrm{~Hz}, 2 \mathrm{H}), 7.35-7.26(\mathrm{~m}, 5 \mathrm{H}), 7.17$ $(\mathrm{dd}, J=15.7,8.2 \mathrm{~Hz}, 1 \mathrm{H}), 7.12(\mathrm{~s}, 1 \mathrm{H}), 6.96(\mathrm{~d}, J=7.9 \mathrm{~Hz}, 1 \mathrm{H}), 6.07-6.01(\mathrm{~m}, 2 \mathrm{H}), 5.14(\mathrm{~d}, J=10.3 \mathrm{~Hz}, 1 \mathrm{H}), 5.10$ $(\mathrm{d}, J=17.1 \mathrm{~Hz}, 1 \mathrm{H}), 3.27(\mathrm{t}, J=8.6 \mathrm{~Hz}, 1 \mathrm{H}), 2.83(\mathrm{q}, J=7.5 \mathrm{~Hz}, 1 \mathrm{H}), 1.33(\mathrm{~s}, 9 \mathrm{H}), 1.04(\mathrm{~d}, J=6.7 \mathrm{~Hz}, 3 \mathrm{H}){ }^{13} \mathrm{C} \mathrm{NMR}$ $\left(151 \mathrm{MHz}, \mathrm{CDCl}_{3}\right) \delta 165.20,154.61,153.14,150.80,141.51,140.98,139.69,139.50,128.98,128.90,128.55,128.06$, $127.49,127.33,127.17,122.87,120.68,118.78,116.72,55.64,41.91,34.94,31.41,18.27$. HRMS (EI) $\mathrm{m} / \mathrm{z}[\mathrm{M}]^{+}$calcd for $\mathrm{C}_{30} \mathrm{H}_{32} \mathrm{O}_{2}$ 424.2402, found 424.2402. HPLC (ChiralPak ADH column) 99:1 (Hexane/iPrOH) $1.0 \mathrm{~mL} \cdot \mathrm{min}^{-1} ; \mathrm{T}_{\text {major }}$ (13.0 min), $\mathrm{T}_{\text {minor }}(11.7 \mathrm{~min}) ;$ 88.3:11.7 er.

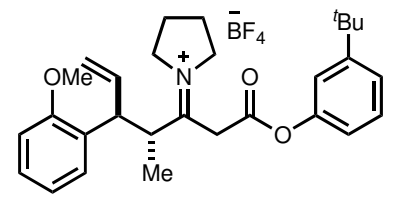

1-((4R,5S)-1-(3-(tert-butyl)phenoxy)-5-(2-methoxyphenyl)-4-methyl-1-oxohept-6-en-3-ylidene)pyrole-din-1ium tetrafluoroborate (SI-2). Following the general procedure A, SI-2 was isolated in $82 \%$ yield and $42 \%$ ee $(99: 1$ dr). ${ }^{1} \mathrm{H}$ NMR $\left(600 \mathrm{MHz}, \mathrm{CDCl}_{3}\right) \delta$ 7.34-7.26 (m, 3H), 7.22 (dd, $\left.J=7.5,1.4 \mathrm{~Hz}, 1 \mathrm{H}\right), 7.14,7.14(\mathrm{t}, J=1.8 \mathrm{~Hz}, 1 \mathrm{H})$, 6.98-6.96 (m, 2H), $6.92(\mathrm{~d}, J=8.3 \mathrm{~Hz}, 1 \mathrm{H}), 6.14(\mathrm{dt}, J=16.2,9.6 \mathrm{~Hz}, 1 \mathrm{H}), 5.16(\mathrm{~d}, J=16.2 \mathrm{~Hz}, 1 \mathrm{H}), 5.14(\mathrm{~d}, J=9.6$ $\mathrm{Hz}, 1 \mathrm{H}), 4.34-4.26(\mathrm{~m}, 4 \mathrm{H}), 4.25-4.20(\mathrm{~m}, 1 \mathrm{H}), 4.12-4.06(\mathrm{~m}, 1 \mathrm{H}), 3.90-3.83(\mathrm{~m}, 1 \mathrm{H}), 3.87(\mathrm{~s}, 3 \mathrm{H}), 3.80-3.75(\mathrm{~m}, 1 \mathrm{H})$, 2.30-2.13 (m, 4H), $1.31(\mathrm{~s}, 9 \mathrm{H}), 1.14(\mathrm{~d}, J=6.0 \mathrm{~Hz}, 3 \mathrm{H}) .{ }^{13} \mathrm{C}$ NMR $\left(151 \mathrm{MHz}, \mathrm{CDCl}_{3}\right) \delta 184.93,164.24,156.96$, 153.76, 150.07, 135.94, 129.31, 129.17, 126.70, 123.91, 121.50, 118.94, 118.21, 118.16, 111.41, 56.78, 56.12, 55.79, $46.15,38.34,35.00,31.31,24.51,24.39,14.78 .{ }^{19} \mathrm{~F}$ NMR $\left(376 \mathrm{MHz}, \mathrm{CDCl}_{3}\right) \delta-150.93$. HRMS (ESI) $\left.\mathrm{m} / z . \mathrm{M}+\mathrm{H}\right]^{+}$ calcd for $\mathrm{C}_{29} \mathrm{H}_{37} \mathrm{NO}_{3} 448.2846$, found 448.2861. Enantiomeric excess was determined by derivatization to the corresponding hydrodeaminated $\alpha, \beta$-unsaturated ester. ${ }^{1} \mathrm{H}$ NMR $\left(500 \mathrm{MHz}, \mathrm{CDCl}_{3}\right) \delta 7.31(\mathrm{t}, J=7.9 \mathrm{~Hz}, 1 \mathrm{H}), 7.26$ $7.14(\mathrm{~m}, 4 \mathrm{H}), 7.11(\mathrm{t}, J=2.0 \mathrm{~Hz}, 1 \mathrm{H}), 6.95-6.92(\mathrm{~m}, 2 \mathrm{H}), 6.89(\mathrm{~d}, J=8.2 \mathrm{~Hz}, 1 \mathrm{H}), 6.06(\mathrm{ddd}, J=17.0,10.2,8.8 \mathrm{~Hz}$, $1 \mathrm{H}), 5.98(\mathrm{dd}, J=15.7,0.9 \mathrm{~Hz}, 1 \mathrm{H}), 5.07(\mathrm{~d}, J=10.5 \mathrm{~Hz}, 1 \mathrm{H}), 5.06(\mathrm{~d}, J=17.0 \mathrm{~Hz}, 1 \mathrm{H}), 3.84(\mathrm{~s}, 3 \mathrm{H}), 3.71(\mathrm{t}, J=8.7$ $\mathrm{Hz}, 1 \mathrm{H}), 2.88(\mathrm{q}, J=7.9,7.5 \mathrm{~Hz}, 1 \mathrm{H}), 1.32(\mathrm{~s}, 9 \mathrm{H}), 0.98(\mathrm{~d}, J=6.7 \mathrm{~Hz}, 3 \mathrm{H}) .{ }^{13} \mathrm{C}$ NMR $\left(126 \mathrm{MHz}, \mathrm{CDCl}_{3}\right) \delta 165.35$, 157.11, 155.56, 153.08, 150.78, 141.98, 139.11, 130.72, 128.95, 128.82, 127.60, 122.82, 120.77, 120.10, 118.80, $116.46,110.96,55.55,48.92,40.67,34.93,31.41,17.89$. HRMS (ESI) $\mathrm{m} / z . \mathrm{M}+\mathrm{H}]^{+}$calcd for $\mathrm{C}_{25} \mathrm{H}_{30} \mathrm{O}_{3} 379.2274$, found 379.2288. HPLC (ChiralPak ADH column) 99:1 (Hexane/iPrOH) $1.0 \mathrm{~mL} \cdot \mathrm{min}^{-1} ; \mathrm{T}_{\text {major }}(8.5 \mathrm{~min}), \mathrm{T}_{\text {minor }}(7.7$ $\min ) ; 70.8: 29.2 \mathrm{er}$. 


\section{General procedure B: Preparation of allenic esters.}
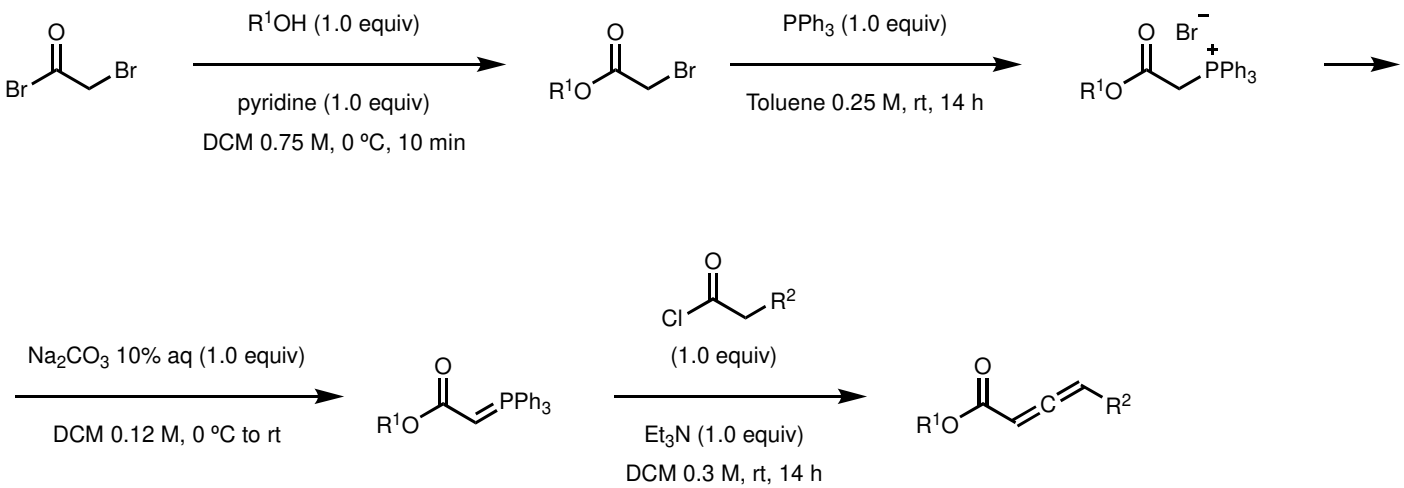

Step 1. Bromoacetyl bromide $(2.0 \mathrm{mmol})$ was dropwise added to a cooled solution of the corresponding alcohol (1.0 equiv, $2.0 \mathrm{mmol})$ and pyridine (1.0 equiv, $2.0 \mathrm{mmol})$ in $\mathrm{DCM}(2.7 \mathrm{~mL})$ at $0{ }^{\circ} \mathrm{C}$. This mixture was stirred at $0{ }^{\circ} \mathrm{C}$ for 20 minutes and then at room temperature for 30 minutes. The reaction mixture was quenched with $\mathrm{H}_{2} \mathrm{O}$ and extracted with DCM. Combined organic extracts were dried with anhydrous $\mathrm{Na}_{2} \mathrm{SO}_{4}$, filtered and concentrated under vacuum. Crude was directly employed in the second step. For trifluoroethanol derivatives, step 1 was carried out following the procedure reported by J. R. Morphy et al. ${ }^{4}$

Step 2. The crude was dissolved in toluene $(8.0 \mathrm{~mL})$ and $\mathrm{PPh}_{3}(1.0$ equiv, $2.0 \mathrm{mmol})$ was added. The reaction mixture was stirred at room temperature for 14 hours. The resulting solid was filtered and washed with toluene and hexanes, and directly used in step 3.

Step 3. Crude was dissolved in DCM $(16.0 \mathrm{~mL})$ and $\mathrm{Na}_{2} \mathrm{CO}_{3} 10 \%$ aq. (1.0 equiv, $2.0 \mathrm{mmol}$ ) was dropwise added at 0 ${ }^{\circ} \mathrm{C}$. This mixture was stirred for 30 minutes at $0{ }^{\circ} \mathrm{C}$. After this time, the mixture was warmed to room temperature, extracted with DCM and washed with brine. Collected organic fractions were dried with anhydrous $\mathrm{Na}_{2} \mathrm{SO}_{4}$, filtered, and concentrated under vacuum. The crude was directly employed in the final step.

Step 4. The corresponding acid chloride (1.0 equiv, $2.0 \mathrm{mmol}$ ) was dropwise added to a solution of the crude and $\mathrm{Et}_{3} \mathrm{~N}$ (1.0 equiv, $2.0 \mathrm{mmol})$ in DCM $(7.0 \mathrm{~mL})$. The reaction mixture was stirred at room temperature for 14 hours. After this time, the solution was filtered through a pad of $\mathrm{SiO}_{2}$ eluting with DCM. The filtrate was concentrated, and the residue was purified by silica gel chromatography to afford the corresponding allenic ester. Most of the allenoates were isolated with variable amounts of their 3-alkynyl isomers. For ${ }^{1} \mathrm{H}$ NMR, just signals corresponding to the allenic isomer are indicated. Control experiments showcased that isomerization is fast and ruled out a kinetic resolution.

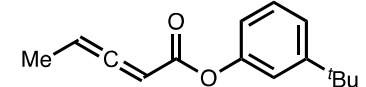

racemic

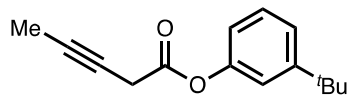

allenoate:alkyne (1.5:1)
$\mathrm{Na}_{2} \mathrm{CO}_{3}$ (1.5 equiv) $(R, R)-4-{ }^{\mathrm{t}} \mathrm{Bu}-\mathrm{C}_{6} \mathrm{H}_{4}$-DAP (10 mol\%)

cyclohexane $0.1 \mathrm{M}$ $\mathrm{rt}, 12 \mathrm{~h}$<smiles>CC=CC(=O)Oc1cccc(Br)c1</smiles><smiles></smiles>

allenoate:alkyne (1.5:1) 

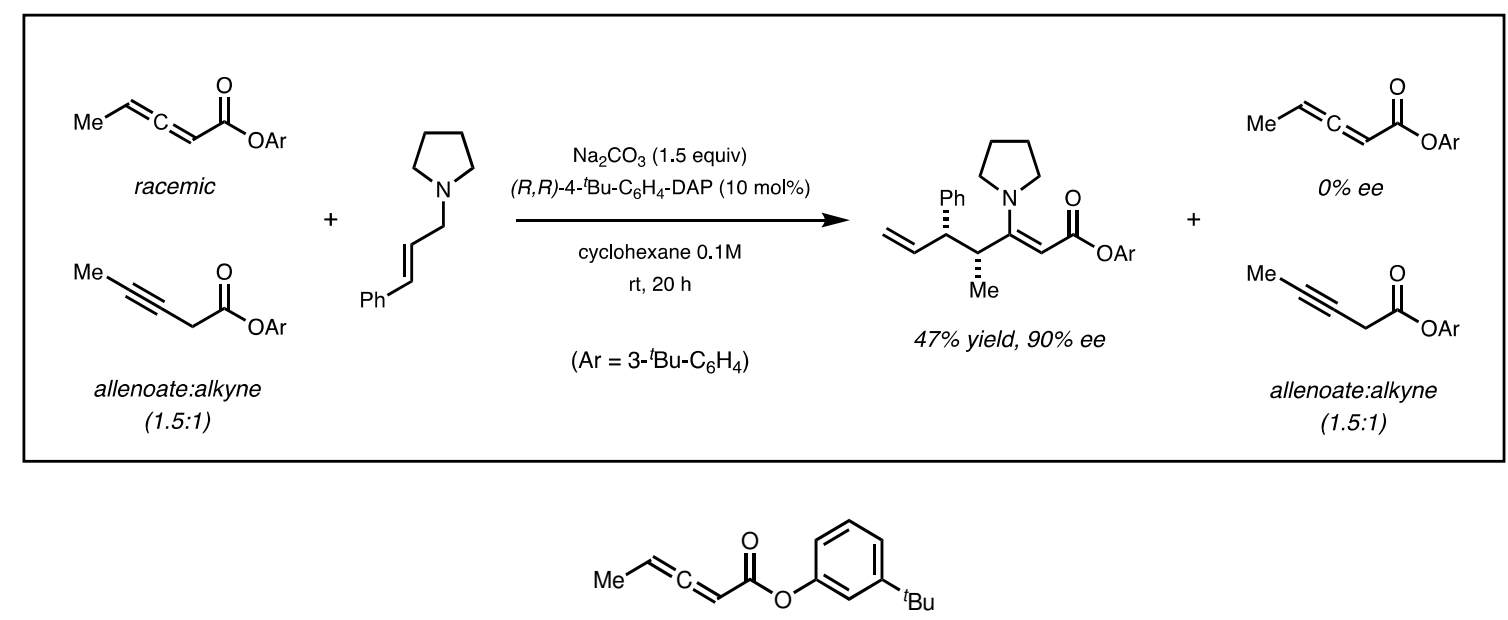

( \pm )-3-(tert-butyl)phenyl penta-2,3-dienoate (2). Following the general procedure B, 2 was isolated in $48 \%$ yield as a colorless oil. ${ }^{1} \mathrm{H}$ NMR $\left(600 \mathrm{MHz}, \mathrm{CDCl}_{3}\right) \delta 7.30(\mathrm{t}, J=8.4 \mathrm{~Hz}, 1 \mathrm{H}), 7.26(\mathrm{tdd}, J=7.8,1.8,1.2 \mathrm{~Hz}, 1 \mathrm{H}), 7.11(\mathrm{dt}, J=$ $5.4,1.8 \mathrm{~Hz}, 1 \mathrm{H}), 6.95-6.92(\mathrm{~m}, 1 \mathrm{H}), 5.75(\mathrm{dt}, J=6.3,3.2 \mathrm{~Hz}, 1 \mathrm{H}), 5.72-5.67(\mathrm{~m}, 1 \mathrm{H}), 1.85(\mathrm{dd}, J=7.2,3.0 \mathrm{~Hz}, 3 \mathrm{H})$, $1.31(\mathrm{~s}, 9 \mathrm{H}) .{ }^{13} \mathrm{C}$ NMR $\left(151 \mathrm{MHz}, \mathrm{CDCl}_{3}\right) \delta 214.03,153.25,153.12,150.90,129.05,128.97,123.17,122.92,118.76$, $118.73,118.53,118.46,90.87,87.57,80.01,70.20,34.93,31.39,31.37,26.42,12.90,12.89,3.80$. HRMS (ESI) $\mathrm{m} / \mathrm{z}$ $[\mathrm{M}+\mathrm{H}]^{+}$calcd for $\mathrm{C}_{15} \mathrm{H}_{18} \mathrm{O}_{2} 231.1380$, found 231.1359.

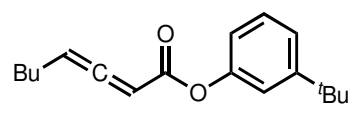

( \pm )-3-(tert-butyl)phenyl octa-2,3-dienoate (SI-3). Following the general procedure B, SI-3 was isolated in 86\% yield as a colorless oil. ${ }^{1} \mathrm{H}$ NMR $\left(600 \mathrm{MHz}, \mathrm{CDCl}_{3}\right) \delta 7.30(\mathrm{t}, J=8.4 \mathrm{~Hz}, 1 \mathrm{H}), 7.26(\mathrm{ddd}, J=7.8,1.7,1.1 \mathrm{~Hz}, 1 \mathrm{H}), 7.11(\mathrm{t}$, $J=2.1 \mathrm{~Hz}, 1 \mathrm{H}), 6.94(\mathrm{ddd}, J=7.9,2.3,1.0 \mathrm{~Hz}, 1 \mathrm{H}), 5.77(\mathrm{dt}, J=6.1,3.0 \mathrm{~Hz}, 1 \mathrm{H}), 5.72(\mathrm{dt}, J=7.2,6.0 \mathrm{~Hz}, 1 \mathrm{H}), 2.20$ (qd, $J=7.1,3.0 \mathrm{~Hz}, 2 \mathrm{H}), 1.52-1.47(\mathrm{~m}, 2 \mathrm{H}), 1.44-1.39(\mathrm{~m}, 2 \mathrm{H}), 1.31(\mathrm{~s}, 9 \mathrm{H}), 0.92(\mathrm{t}, J=7.3 \mathrm{~Hz}, 3 \mathrm{H}) .{ }^{13} \mathrm{C}$ NMR $(151$ $\left.\mathrm{MHz}, \mathrm{CDCl}_{3}\right) \delta 213.46,164.99,153.11,150.94,128.96,122.88,118.72,95.97,88.07,34.92,31.39,30.94,27.28$, 22.14, 13.93. HRMS (EI) $\mathrm{m} / z$ [M] ${ }^{+}$calcd for $\mathrm{C}_{18} \mathrm{H}_{24} \mathrm{O}_{2} 272.1776$, found 272.1776 .

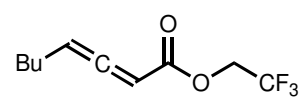

(士)-2,2,2-trifluoroethyl octa-2,3-dienoate (SI-4). Following the general procedure B, SI-4 was isolated in 43\% yield as a colorless oil. ${ }^{1} \mathrm{H} \mathrm{NMR}\left(400 \mathrm{MHz}, \mathrm{CDCl}_{3}\right) \delta 5.69(\mathrm{dt}, J=6.4,6.0 \mathrm{~Hz}, 1 \mathrm{H}), 5.63(\mathrm{dt}, J=6.1,3.0 \mathrm{~Hz}, 1 \mathrm{H}), 4.51(\mathrm{qq}$, $J=12.7,8.5 \mathrm{~Hz}, 2 \mathrm{H}), 2.15(\mathrm{tt}, J=7.2,3.5 \mathrm{~Hz}, 2 \mathrm{H}), 1.41(\mathrm{~m}, 4 \mathrm{H}), 0.91(\mathrm{t}, J=7.2 \mathrm{~Hz}, 3 \mathrm{H}) .{ }^{13} \mathrm{C} \mathrm{NMR}\left(101 \mathrm{MHz}, \mathrm{CDCl}_{3}\right)$ $\delta 213.79,164.73,123.14\left(\mathrm{q},{ }^{1} J_{\mathrm{CF}}=278.8 \mathrm{~Hz}\right), 96.28,86.85,60.67\left(\mathrm{q},{ }^{2} J_{\mathrm{CF}}=37.4 \mathrm{~Hz}\right), 30.85,27.15,22.10,13.87 .{ }^{19} \mathrm{~F}$ NMR (376 MHz, $\left.\mathrm{CDCl}_{3}\right) \delta-72.98,-73.02$. HRMS (EI) $\mathrm{m} / z$ [M] ${ }^{+}$calcd for $\mathrm{C}_{10} \mathrm{H}_{13} \mathrm{~F}_{3} \mathrm{O}_{2} 222.0868$, found 222.0866.

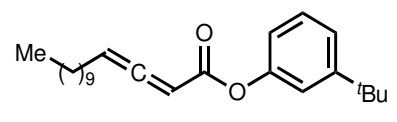

( \pm )-3-(tert-butyl)phenyl tetradeca-2,3-dienoate (SI-5). Following the general procedure B, SI-5 was isolated in 72\% yield as a colorless oil. ${ }^{1} \mathrm{H}$ NMR $\left(600 \mathrm{MHz}, \mathrm{CDCl}_{3}\right) \delta 7.30(\mathrm{t}, J=7.9 \mathrm{~Hz}, 1 \mathrm{H}), 7.25(\mathrm{dt}, J=7.9,1.4 \mathrm{~Hz}, 1 \mathrm{H}), 7.11(\mathrm{t}$, $J=2.0 \mathrm{~Hz}, 1 \mathrm{H}), 6.94(\mathrm{ddd}, J=7.9,2.2,1.0 \mathrm{~Hz}, 1 \mathrm{H}), 5.76(\mathrm{dt}, J=6.0,3.0 \mathrm{~Hz}, 1 \mathrm{H}), 5.72(\mathrm{dt}, J=7.2,6.0 \mathrm{~Hz}, 1 \mathrm{H}), 2.19$ $(\mathrm{qd}, J=7.2,3.0 \mathrm{~Hz}, 2 \mathrm{H}), 1.50(\mathrm{p}, J=7.6 \mathrm{~Hz}, 2 \mathrm{H}), 1.37(\mathrm{p}, J=6.6 \mathrm{~Hz}, 2 \mathrm{H}), 1.33-1.25(\mathrm{~m}, 12 \mathrm{H}), 1.31(\mathrm{~s}, 9 \mathrm{H}), 0.88(\mathrm{t}$, $J=7.0 \mathrm{~Hz}, 3 \mathrm{H}) .{ }^{13} \mathrm{C}$ NMR $\left(151 \mathrm{MHz}, \mathrm{CDCl}_{3}\right) \delta 213.45,165.00,153.10,150.94,128.95,122.88,118.73,96.03,88.08$, 
34.92, 32.04, 31.39, 29.75, 29.72, 29.52, 29.46, 29.11, 28.88, 27.61, 22.83, 14.26. HRMS (EI) $\mathrm{m} / z[\mathrm{M}]^{+}$calcd for $\mathrm{C}_{24} \mathrm{H}_{36} \mathrm{O}_{2} 356.2715$, found 356.2711 .

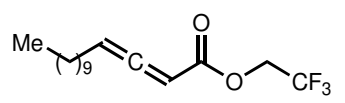

( \pm )-2,2,2-trifluoroethyl tetradeca-2,3-dienoate (SI-6). Following the general procedure B, SI-6 was isolated in $66 \%$ yield as a colorless oil. ${ }^{1} \mathrm{H}$ NMR $\left(600 \mathrm{MHz}, \mathrm{CDCl}_{3}\right) \delta 5.70(\mathrm{q}, J=7.0 \mathrm{~Hz}, 1 \mathrm{H}), 5.64(\mathrm{dt}, J=6.1,3.0 \mathrm{~Hz}, 1 \mathrm{H}), 4.51(\mathrm{~m}$, $2 \mathrm{H}), 2.15(\mathrm{qd}, J=7.2,3.0 \mathrm{~Hz}, 2 \mathrm{H}), 1.46(\mathrm{p}, J=7.3 \mathrm{~Hz}, 2 \mathrm{H}), 1.35-1.26(\mathrm{~m}, 14 \mathrm{H}), 0.88(\mathrm{t}, J=7.0 \mathrm{~Hz}, 3 \mathrm{H}) .{ }^{13} \mathrm{C}$ NMR $\left(151 \mathrm{MHz}, \mathrm{CDCl}_{3}\right) \delta 213.79,164.72,123.15\left(\mathrm{q},{ }^{1} J_{\mathrm{CF}}=277.8 \mathrm{~Hz}\right), 96.35,86.86,60.66\left(\mathrm{q},{ }^{2} J_{\mathrm{CF}}=36.2 \mathrm{~Hz}\right), 32.05,29.74$, 29.67, 29.47, 29.46, 29.07, 28.81, 27.48, 22.83, 14.25. $\left.{ }^{19} \mathrm{~F} \mathrm{NMR} \mathrm{(376} \mathrm{MHz,} \mathrm{CDCl}_{3}\right) \delta-72.97,-73.00$. HRMS (EI) $\mathrm{m} / z$ $[\mathrm{M}]^{+}$calcd for $\mathrm{C}_{16} \mathrm{H}_{25} \mathrm{~F}_{3} \mathrm{O}_{2} 306.1807$, found 306.1804.

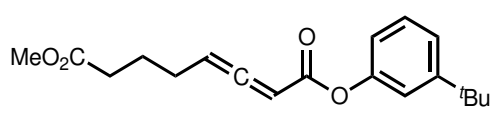

( \pm )-1-(3-(tert-butyl)phenyl) 8-methyl octa-2,3-dienedioate (SI-7). Following the general procedure B, SI-7 was isolated in $65 \%$ yield as a colorless oil. ${ }^{1} \mathrm{H}$ NMR $\left(600 \mathrm{MHz}, \mathrm{CDCl}_{3}\right) \delta 7.30(\mathrm{t}, J=7.9 \mathrm{~Hz}, 1 \mathrm{H}), 7.25(\mathrm{ddd}, J=7.8,1.7$, $1.1 \mathrm{~Hz}, 1 \mathrm{H}), 7.11(\mathrm{t}, J=2.0 \mathrm{~Hz}, 1 \mathrm{H}), 6.94(\mathrm{ddd}, J=7.9,2.3,1.0 \mathrm{~Hz}, 1 \mathrm{H}), 5.80(\mathrm{dt}, J=6.0,3.0 \mathrm{~Hz}, 1 \mathrm{H}), 5.71(\mathrm{dt}, J=$ 7.2, $6.0 \mathrm{~Hz}, 1 \mathrm{H}), 3.66(\mathrm{~s}, 3 \mathrm{H}), 2.43(\mathrm{t}, J=7.4 \mathrm{~Hz}, 2 \mathrm{H}), 2.25(\mathrm{qd}, J=7.2,3.0 \mathrm{~Hz}, 2 \mathrm{H}), 1.89-1.82(\mathrm{~m}, 2 \mathrm{H}), 1.31(\mathrm{~s}$, $12 \mathrm{H}){ }^{13} \mathrm{C}$ NMR $\left(101 \mathrm{MHz}, \mathrm{CDCl}_{3}\right) \delta 213.39,173.82,164.75,153.14,150.84,128.97,122.95,118.71,95.06$, 88.59, 51.72, 34.92, 33.12, 31.38, 26.94, 23.90. HRMS (EI) $\mathrm{m} / z$ [M] ${ }^{+}$calcd for $\mathrm{C}_{19} \mathrm{H}_{24} \mathrm{O}_{4} 316.1675$, found 316.1673 .

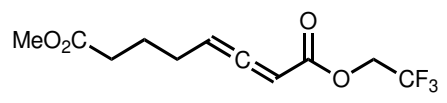

( \pm )-8-methyl 1-(2,2,2-trifluoroethyl) octa-2,3-dienedioate (SI-8). Following the general procedure B, SI-8 was isolated in $10 \%$ yield as a colorless oil. ${ }^{1} \mathrm{H}$ NMR $\left(600 \mathrm{MHz}, \mathrm{CDCl}_{3}\right) \delta 5.70-5.66(\mathrm{~m}, 2 \mathrm{H}), 4.56-4.46(\mathrm{~m}, 2 \mathrm{H}), 3.67(\mathrm{~s}$, $3 \mathrm{H}), 2.39(\mathrm{td}, J=7.4,2.4 \mathrm{~Hz}, 2 \mathrm{H}), 2.21(\mathrm{qd}, J=7.1,3.2 \mathrm{~Hz}, 2 \mathrm{H}), 1.84-1.78(\mathrm{~m}, 2 \mathrm{H}) .{ }^{13} \mathrm{C} \mathrm{NMR}\left(151 \mathrm{MHz}, \mathrm{CDCl}_{3}\right) \delta$ 213.72, 173.74, 164.45, $123.10\left(\mathrm{q},{ }^{1} J_{\mathrm{CF}}=277.8 \mathrm{~Hz}\right), 95.37,87.37,60.71\left(\mathrm{q},{ }^{2} J_{\mathrm{CF}}=36.2 \mathrm{~Hz}\right), 51.72,33.06,26.80,23.87$.

${ }^{19} \mathrm{~F}$ NMR $\left(376 \mathrm{MHz}, \mathrm{CDCl}_{3}\right) \delta$-73.01. HRMS (EI) $\mathrm{m} / z$ [M] ${ }^{+}$calcd for $\mathrm{C}_{11} \mathrm{H}_{13} \mathrm{~F}_{3} \mathrm{O}_{4} 266.0766$, found 266.0764 .

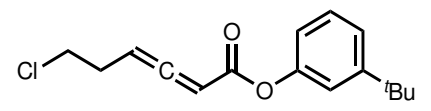

( \pm )-3-(tert-butyl)phenyl 6-chlorohexa-2,3-dienoate (SI-9). Following the general procedure B, SI-9 was isolated in $69 \%$ yield as a colorless oil. ${ }^{1} \mathrm{H}$ NMR $\left(400 \mathrm{MHz}, \mathrm{CDCl}_{3}\right) \delta 7.31(\mathrm{t}, J=7.8 \mathrm{~Hz}, 1 \mathrm{H}), 7.27-7.24(\mathrm{~m}, 1 \mathrm{H}), 7.11(\mathrm{t}, J=2.0$ $\mathrm{Hz}, 1 \mathrm{H}), 6.94(\mathrm{ddd}, J=7.8,2.3,1.2 \mathrm{~Hz}, 1 \mathrm{H}), 5.86(\mathrm{dt}, J=5.8,2.9 \mathrm{~Hz}, 1 \mathrm{H}), 5.80(\mathrm{dt}, J=6.8,6.4 \mathrm{~Hz}, 1 \mathrm{H}), 3.66(\mathrm{t}, J=$ $6.7 \mathrm{~Hz}, 2 \mathrm{H}), 2.67$ (qd, $J=6.6,2.6 \mathrm{~Hz}, 2 \mathrm{H}), 1.31(\mathrm{~s}, 9 \mathrm{H}) .{ }^{13} \mathrm{C} \mathrm{NMR}\left(101 \mathrm{MHz}, \mathrm{CDCl}_{3}\right) \delta 213.45,164.43,153.19,150.79$, 129.01, 123.04, 118.67, 118.65, 92.61, 89.15, 43.16, 34.94, 31.38, 30.93. HRMS (EI) $m / z[\mathrm{M}]^{+}$calcd for $\mathrm{C}_{16} \mathrm{H}_{19} \mathrm{ClO}_{2}$ 278.1074, found 278.1072 .

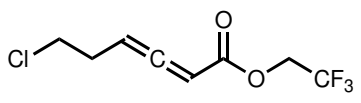

( \pm )-2,2,2-trifluoroethyl 6-chlorohexa-2,3-dienoate (SI-10). Following the general procedure B, SI-10 was isolated in $78 \%$ yield as a colorless liquid. ${ }^{1} \mathrm{H}$ NMR $\left(600 \mathrm{MHz}, \mathrm{CDCl}_{3}\right) \delta 5.78(\mathrm{dt}, J=6.6,6.6 \mathrm{~Hz}, 1 \mathrm{H}), 5.73(\mathrm{dt}, J=5.8,2.8$ $\mathrm{Hz}, 1 \mathrm{H}), 4.57-4.46(\mathrm{~m}, 2 \mathrm{H}), 3.61(\mathrm{t}, J=6.7 \mathrm{~Hz}, 2 \mathrm{H}), 2.65-2.61(\mathrm{~m}, 2 \mathrm{H}) .{ }^{13} \mathrm{C} \mathrm{NMR}\left(151 \mathrm{MHz}, \mathrm{CDCl}_{3}\right) \delta 213.77,164.14$, 
$123.06\left(\mathrm{q},{ }^{1} J_{\mathrm{CF}}=276.3 \mathrm{~Hz}\right), 92.93,87.91,60.79\left(\mathrm{q},{ }^{2} J_{\mathrm{CF}}=36.2 \mathrm{~Hz}\right), 42.97,30.79 .{ }^{19} \mathrm{~F} \mathrm{NMR}\left(376 \mathrm{MHz} \mathrm{CDCl}_{3}\right) \delta-$ 72.95, -72.99. HRMS (EI) $m / z[\mathrm{M}]^{+}$calcd for $\mathrm{C}_{8} \mathrm{H}_{8} \mathrm{ClF}_{3} \mathrm{O}_{2} 228.0165$, found 228.0164.

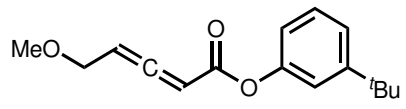

( \pm )-3-(tert-butyl)phenyl 5-methoxypenta-2,3-dienoate (SI-11). Following the general procedure B, SI-11 was isolated in $53 \%$ yield as a colorless oil. ${ }^{1} \mathrm{H} \mathrm{NMR}\left(600 \mathrm{MHz}, \mathrm{CDCl}_{3}\right) \delta 7.31(\mathrm{t}, J=7.9 \mathrm{~Hz}, 1 \mathrm{H}), 7.26(\mathrm{ddd}, J=8.4,1.8$, $1.2 \mathrm{~Hz}, 1 \mathrm{H}), 7.10(\mathrm{t}, J=2.0 \mathrm{~Hz}, 1 \mathrm{H}), 6.94(\mathrm{ddd}, J=7.9,2.4,1.1 \mathrm{~Hz}, 1 \mathrm{H}), 5.90(\mathrm{dt}, J=6.1,2.5 \mathrm{~Hz}, 1 \mathrm{H}), 5.79(\mathrm{dt}, J=$ 6.6, $6.6 \mathrm{~Hz}, 1 \mathrm{H}), 4.19-4.11(\mathrm{~m}, 2 \mathrm{H}), 3.41(\mathrm{~s}, 3 \mathrm{H}), 1.31(\mathrm{~s}, 9 \mathrm{H}) .{ }^{13} \mathrm{C} \mathrm{NMR}\left(151 \mathrm{MHz}, \mathrm{CDCl}_{3}\right) \delta 213.28,164.23,153.21$, $150.78,129.02,123.06,118.65,118.63,93.39,89.12,68.63,58.07,34.93,31.38$. HRMS (EI) $\mathrm{m} / z$ [M] ${ }^{+}$calcd for $\mathrm{C}_{16} \mathrm{H}_{20} \mathrm{O}_{3} 260.1412$, found 260.1412 .

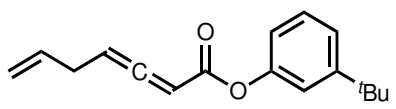

( \pm )-3-(tert-butyl)phenyl hepta-2,3,6-trienoate (SI-12). Following the general procedure B, SI-12 was isolated in 74\% yield as a colorless oil. ${ }^{1} \mathrm{H}$ NMR $\left(300 \mathrm{MHz} \mathrm{CDCl}_{3}\right) \delta 7.31(\mathrm{t}, J=7.8 \mathrm{~Hz}, 1 \mathrm{H}), 7.24(\mathrm{dt}, J=7.8,1.6 \mathrm{~Hz}, 1 \mathrm{H}), 7.11(\mathrm{t}$, $J=2.0 \mathrm{~Hz}, 1 \mathrm{H}), 6.94(\mathrm{ddd}, J=7.6,2.3,1.4 \mathrm{~Hz}, 1 \mathrm{H}), 5.95-5.71(\mathrm{~m}, 3 \mathrm{H}), 5.20(\mathrm{dq}, J=17.1,1.6 \mathrm{~Hz}, 1 \mathrm{H}), 5.12(\mathrm{dq}, J=$ 10.1, $1.4 \mathrm{~Hz}, 1 \mathrm{H}), 2.99-2.92(\mathrm{~m}, 2 \mathrm{H}), 1.31(\mathrm{~s}, 8 \mathrm{H}) .{ }^{13} \mathrm{C} \mathrm{NMR}\left(101 \mathrm{MHz}, \mathrm{CDCl}_{3}\right) \delta 213.60,164.81,153.13,150.85$, 134.76, 128.98, 122.95, 118.69, 116.84, 94.20, 88.55, 34.92, 31.69, 31.37. HRMS (EI) $m / z$ [M] ${ }^{+}$calcd for $\mathrm{C}_{17} \mathrm{H}_{20} \mathrm{O}_{2}$ 256.1463, found 256.1460 .

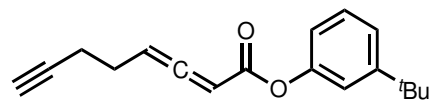

( \pm )-3-(tert-butyl)phenyl octa-2,3-dien-7-ynoate (SI-13). Following the general procedure B, SI-13 was isolated in $80 \%$ yield as a colorless oil. ${ }^{1} \mathrm{H}$ NMR $\left(600 \mathrm{MHz} \mathrm{CDCl}_{3}\right) \delta 7.30(\mathrm{t}, J=7.9 \mathrm{~Hz}, 1 \mathrm{H}), 7.27-7.24(\mathrm{~m}, 1 \mathrm{H}), 7.11-7.09(\mathrm{~m}$, $1 \mathrm{H}), 6.94(\mathrm{~d}, J=9.0 \mathrm{~Hz}, 1 \mathrm{H}), 5.84-5.80(\mathrm{~m}, 2 \mathrm{H}), 2.44-2.32(\mathrm{~m}, 4 \mathrm{H}), 2.01(\mathrm{~s}, 1 \mathrm{H}), 1.31(\mathrm{~s}, 9 \mathrm{H}) .{ }^{13} \mathrm{C} \mathrm{NMR}(151 \mathrm{MHz}$, $\left.\mathrm{CDCl}_{3}\right) \delta 213.17,164.61,153.16,150.86,128.99,122.97,118.71,118.68,94.47,89.04,82.96,69.63,34.93,31.39$, 26.85, 18.32. HRMS (ESI) $m / z[\mathrm{M}+\mathrm{H}]^{+}$calcd for $\mathrm{C}_{18} \mathrm{H}_{20} \mathrm{O}_{2} 269.1536$, found 269.1515 .

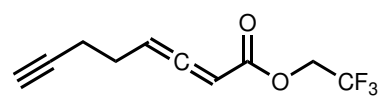

( \pm )-2,2,2-trifluoroethyl octa-2,3-dien-7-ynoate (SI-14). Following the general procedure B, SI-14 was isolated in $43 \%$ yield as a colorless oil. ${ }^{1} \mathrm{H}$ NMR $\left(600 \mathrm{MHz} \mathrm{CDCl}_{3}\right) \delta 5.80(\mathrm{dt}, J=6.3,6.3 \mathrm{~Hz}, 1 \mathrm{H}), 5.71(\mathrm{dt}, J=5.8,2.7 \mathrm{~Hz}$, $1 \mathrm{H}), 4.56-4.46(\mathrm{~m}, 2 \mathrm{H}), 2.42-2.34(\mathrm{~m}, 4 \mathrm{H}), 2.01(\mathrm{t}, J=2.3 \mathrm{~Hz}, 1 \mathrm{H}) .{ }^{13} \mathrm{C} \mathrm{NMR}\left(151 \mathrm{MHz}, \mathrm{CDCl}_{3}\right) \delta 213.50,164.34$, $123.09\left(\mathrm{q},{ }^{1} J_{\mathrm{CF}}=277.8 \mathrm{~Hz}\right), 94.77,87.81,82.76,69.65,60.73\left(\mathrm{q},{ }^{2} J_{\mathrm{CF}}=36.2 \mathrm{~Hz}\right), 26.70,18.21 .{ }^{19} \mathrm{~F} \mathrm{NMR}(376 \mathrm{MHz}$, $\left.\mathrm{CDCl}_{3}\right) \delta$-72.99. HRMS (EI) $\mathrm{m} / z[\mathrm{M}]^{+}$calcd for $\mathrm{C}_{10} \mathrm{H}_{9} \mathrm{~F}_{3} \mathrm{O}_{2} 218.0555$, found 218.0558. 


\section{General procedure C: Preparation of allyl amines.}

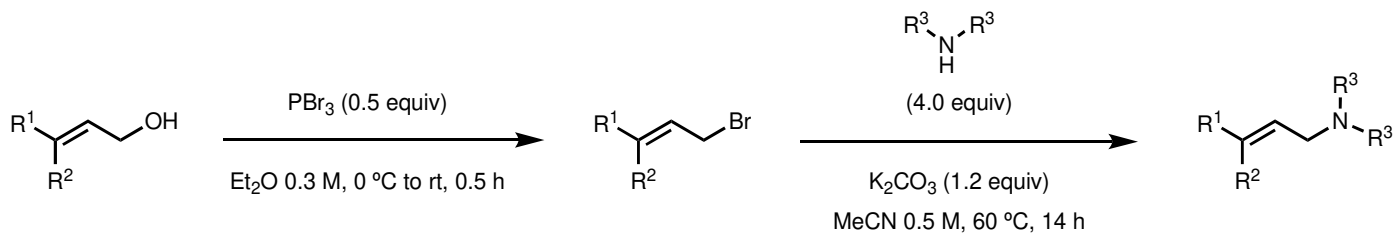

Bromination. To a solution of the appropriate allylic alcohol ${ }^{5}$ (1.0 equiv, $\left.2.0 \mathrm{mmol}\right)$ in $\mathrm{Et}_{2} \mathrm{O}(6.7 \mathrm{~mL}) \mathrm{PBr}_{3}(0.5$ equiv, $1.0 \mathrm{mmol}$ ) was added at $0{ }^{\circ} \mathrm{C}$, and the mixture was stirred for 10 minutes at this temperature. The reaction then proceeded at room temperature for 30 minutes. After this time, the reaction mixture was quenched with aq. sat. $\mathrm{NaHCO}_{3}$ and extracted with $\mathrm{Et}_{2} \mathrm{O}$. Collected organic phases were washed with aq. sat. $\mathrm{Na}_{2} \mathrm{~S}_{2} \mathrm{O}_{3}$ and brine, and dried over anhydrous $\mathrm{Na}_{2} \mathrm{SO}_{4}$. After filtration and removal of solvents, crude product was directly employed in the next step without further purification.

Allylic amination. A solution of the corresponding allylic bromide (1.0 equiv, $2.0 \mathrm{mmol}$ ), secondary amine (4.0 equiv, $8.0 \mathrm{mmol}$ ) and $\mathrm{K}_{2} \mathrm{CO}_{3}(1.2$ equiv, $2.4 \mathrm{mmol})$ in $\mathrm{MeCN}(4.0 \mathrm{~mL})$ was heated to $60^{\circ} \mathrm{C}$ for 14 hours. Then, solvent was removed under reduced pressure and the dissolved crude in DCM was washed with sat. aq. $\mathrm{NaHCO}_{3}$. Collected organic fractions were dried over anhydrous $\mathrm{Na}_{2} \mathrm{SO}_{4}$ and filtered. After removal of solvents, allylic amines were purified by column chromatography.

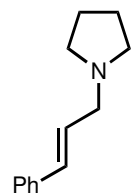

3

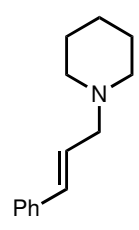

$\mathrm{SI}-15$

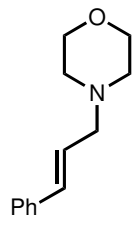

SI-16

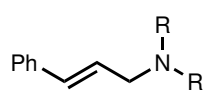

$\mathrm{SI}-17, \mathrm{R}=\mathrm{Me}$

$\mathrm{Sl}-18, \mathrm{R}=\mathrm{Et}$

$\mathrm{SI}-19, \mathrm{R}=\mathrm{Bn}$

1-cinnamylpyrrolidine (3), 1-cinnamylpiperidine (SI-15), 1-cinnamylmorpholine (SI-16), (E)- $N, N$-dimethyl-3phenylprop-2-en-1-amine (SI-17), (E)- $N, N$-diethyl-3-phenylprop-2-en-1-amine (SI-18) and (E)- $N, N$-dibenzyl-3phenylprop-2-en-1-amine (SI-19) were prepared following general procedure C. Spectroscopic data were in agreement with those previously reported in the literature. ${ }^{6}$

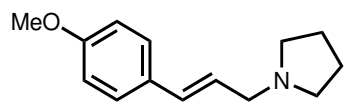

(E)-1-(3-(4-methoxyphenyl)allyl)pyrrolidine (SI-20). Following the general procedure C, SI-20 was isolated in 33\% yield as an orange solid. ${ }^{1} \mathrm{H}$ NMR $\left(400 \mathrm{MHz}, \mathrm{CDCl}_{3}\right) \delta 7.32(\mathrm{~d}, J=8.8 \mathrm{~Hz}, 2 \mathrm{H}), 6.84(\mathrm{~d}, J=8.8 \mathrm{~Hz}, 2 \mathrm{H}), 6.56(\mathrm{~d}, J=$ $15.8 \mathrm{~Hz}, 1 \mathrm{H}), 6.24(\mathrm{dt}, J=15.7,7.0 \mathrm{~Hz}, 1 \mathrm{H}), 3.79(\mathrm{~s}, 3 \mathrm{H}), 3.49(\mathrm{~d}, J=7.0 \mathrm{~Hz}, 2 \mathrm{H}), 2.99-2.79(\mathrm{~m}, 4 \mathrm{H}), 2.00-1.90(\mathrm{~m}$, $4 \mathrm{H}){ }^{13} \mathrm{C}$ NMR $\left(101 \mathrm{MHz}, \mathrm{CDCl}_{3}\right) \delta 159.75,135.01,128.89,128.00,120.55,114.15,57.92,55.42,53.41,23.56$. HRMS (ESI) $\mathrm{m} / z[\mathrm{M}+\mathrm{H}]^{+}$calcd for $\mathrm{C}_{14} \mathrm{H}_{19} \mathrm{NO} 218.1545$, found 218.1540 .

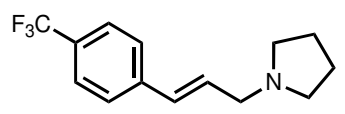

(E)-1-(3-(4-(trifluoromethyl)phenyl)allyl)pyrrolidine (SI-21). Following the general procedure C, SI-21 was isolated in $55 \%$ yield as a yellow oil. ${ }^{1} \mathrm{H}$ NMR $\left(600 \mathrm{MHz}, \mathrm{CDCl}_{3}\right) \delta 7.55(\mathrm{~d}, J=8.1 \mathrm{~Hz}, 2 \mathrm{H}), 7.46(\mathrm{~d}, J=8.0 \mathrm{~Hz}, 2 \mathrm{H})$, $6.58(\mathrm{~d}, J=15.9 \mathrm{~Hz}, 1 \mathrm{H}), 6.45(\mathrm{dt}, J=15.8,6.6 \mathrm{~Hz}, 1 \mathrm{H}), 3.32(\mathrm{~d}, J=6.6 \mathrm{~Hz}, 2 \mathrm{H}), 2.64-2.58(\mathrm{~m}, 4 \mathrm{H}), 1.85-1.83(\mathrm{~m}$, $4 \mathrm{H}) .{ }^{13} \mathrm{C}$ NMR $\left(151 \mathrm{MHz}, \mathrm{CDCl}_{3}\right) \delta 140.67,130.92,130.30,129.34\left(\mathrm{q},{ }^{2} J_{\mathrm{CF}}=32.3 \mathrm{~Hz}\right), 126.59,125.63\left(\mathrm{q},{ }^{3} J_{\mathrm{CF}}=3.9\right.$ 
$\mathrm{Hz}), 124.35\left(\mathrm{q},{ }^{1} J_{\mathrm{CF}}=271.8 \mathrm{~Hz}\right), 58.29,54.24,23.65 .{ }^{19} \mathrm{~F} \mathrm{NMR}\left(376 \mathrm{MHz}, \mathrm{CDCl}_{3}\right) \delta-61.68 . \mathrm{HRMS}(\mathrm{ESI}) \mathrm{m} / z[\mathrm{M}+\mathrm{H}]^{+}$ calcd for $\mathrm{C}_{14} \mathrm{H}_{16} \mathrm{~F}_{3} \mathrm{~N} 256.1313$, found 256.1323 .

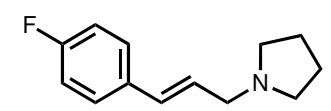

(E)-1-(3-(4-fluorophenyl)allyl)pyrrolidine (SI-22). Following the general procedure C, SI-22 was isolated in 62\% yield as a yellow oil. ${ }^{1} \mathrm{H}$ NMR $\left(600 \mathrm{MHz}, \mathrm{CDCl}_{3}\right) \delta 7.34-7.31(\mathrm{~m}, 2 \mathrm{H}), 7.00-6.96(\mathrm{~m}, 2 \mathrm{H}), 6.49(\mathrm{~d}, J=16.2 \mathrm{~Hz}, 1 \mathrm{H})$, $6.24(\mathrm{dt}, J=15.8,6.7 \mathrm{~Hz}, 1 \mathrm{H}), 3.24(\mathrm{dd}, J=6.7,1.4 \mathrm{~Hz}, 2 \mathrm{H}), 2.56-2.54(\mathrm{~m}, 4 \mathrm{H}), 1.81-1.79(\mathrm{~m}, 4 \mathrm{H}) .{ }^{13} \mathrm{C}$ NMR $(151$ $\left.\mathrm{MHz}, \mathrm{CDCl}_{3}\right) \delta 162.31\left(\mathrm{~d},{ }^{1} J_{\mathrm{CF}}=246.4 \mathrm{~Hz}\right), 133.51\left(\mathrm{~d},{ }^{4} J_{\mathrm{CF}}=3.0 \mathrm{~Hz}\right), 130.68,127.87\left(\mathrm{~d},{ }^{3} J_{\mathrm{CF}}=7.8 \mathrm{~Hz}\right), 127.81(\mathrm{~d}$, $\left.{ }^{5} J_{\mathrm{CF}}=2.3 \mathrm{~Hz}\right), 115.52\left(\mathrm{~d},{ }^{2} J_{\mathrm{CF}}=21.5 \mathrm{~Hz}\right), 58.48,54.25,23.64 .{ }^{19} \mathrm{~F}$ NMR $\left(376 \mathrm{MHz}, \mathrm{CDCl}_{3}\right) \delta-114.17 . \mathrm{HRMS}(\mathrm{ESI})$ $\mathrm{m} / z[\mathrm{M}+\mathrm{H}]^{+}$calcd for $\mathrm{C}_{13} \mathrm{H}_{16} \mathrm{FN} 206.1345$, found 206.1354 .

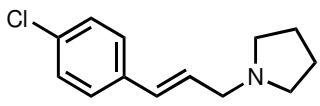

(E)-1-(3-(4-chlorophenyl)allyl)pyrrolidine (SI-23). Following the general procedure C, SI-23 was isolated in 70\% yield as a yellow oil. ${ }^{1} \mathrm{H}$ NMR $\left(400 \mathrm{MHz}, \mathrm{CDCl}_{3}\right) \delta 7.35-7.18(\mathrm{~m}, 4 \mathrm{H}), 6.47(\mathrm{dt}, J=15.7,1.4 \mathrm{~Hz}, 1 \mathrm{H}), 6.29(\mathrm{dt}, J=$ $15.8,6.6 \mathrm{~Hz}, 1 \mathrm{H}), 3.24(\mathrm{dd}, J=6.6,1.3 \mathrm{~Hz}, 2 \mathrm{H}), 2.60-2.44(\mathrm{~m}, 4 \mathrm{H}), 1.85-1.70(\mathrm{~m}, 4 \mathrm{H}) .{ }^{13} \mathrm{C} \mathrm{NMR}\left(101 \mathrm{MHz}, \mathrm{CDCl}_{3}\right)$ $\delta 135.78,133.00,130.61,128.77,128.72,127.59,58.42,54.26,23.60$. HRMS (ESI) $m / z[\mathrm{M}+\mathrm{H}]^{+}$calcd for $\mathrm{C}_{13} \mathrm{H}_{16} \mathrm{ClN}$ 222.1050 , found 222.1040 .

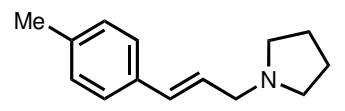

(E)-1-(3-(p-tolyl)allyl)pyrrolidine (SI-24). Following the general procedure C, SI-24 was isolated in 38\% yield as a yellow oil. ${ }^{1} \mathrm{H}$ NMR $\left(600 \mathrm{MHz}, \mathrm{CDCl}_{3}\right) \delta 7.27(\mathrm{~d}, J=8.1 \mathrm{~Hz}, 2 \mathrm{H}), 7.11(\mathrm{~d}, J=7.9 \mathrm{~Hz}, 2 \mathrm{H}), 6.50(\mathrm{~d}, J=15.8 \mathrm{~Hz}, 1 \mathrm{H})$, $6.28(\mathrm{dt}, J=15.8,6.7 \mathrm{~Hz}, 1 \mathrm{H}), 3.25(\mathrm{~d}, J=6.7 \mathrm{~Hz}, 2 \mathrm{H}), 2.56(\mathrm{t}, J=6.0 \mathrm{~Hz}, 4 \mathrm{H}), 2.33(\mathrm{~s}, 3 \mathrm{H}), 1.80(\mathrm{p}, J=3.1 \mathrm{~Hz}$, $4 \mathrm{H}) .{ }^{13} \mathrm{C}$ NMR $\left(151 \mathrm{MHz}, \mathrm{CDCl}_{3}\right) \delta 137.23,134.56,131.80,129.35,126.94,126.34,58.61,54.21,23.63,21.30$. HRMS (ESI) $\mathrm{m} / z[\mathrm{M}+\mathrm{H}]^{+}$calcd for $\mathrm{C}_{14} \mathrm{H}_{19} \mathrm{~N} 202.1590$, found 202.1578 .

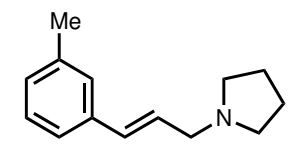

(E)-1-(3-( $\boldsymbol{m}$-tolyl)allyl)pyrrolidine (SI-25). Following the general procedure C, SI-25 was isolated in 35\% yield as a yellow oil. ${ }^{1} \mathrm{H}$ NMR $\left(400 \mathrm{MHz}, \mathrm{CDCl}_{3}\right) \delta$ 7.22-7.17 (m, 3H), 7.05-7.03 (m, 1H), $6.50(\mathrm{~d}, J=15.8 \mathrm{~Hz}, 1 \mathrm{H}), 6.32(\mathrm{dt}, J$ $=15.8,6.7 \mathrm{~Hz}, 1 \mathrm{H}), 3.26(\mathrm{dd}, J=6.7,1.2 \mathrm{~Hz}, 2 \mathrm{H}), 2.58-2.54(\mathrm{~m}, 4 \mathrm{H}), 2.34(\mathrm{~s}, 3 \mathrm{H}), 1.81(\mathrm{p}, J=3.6 \mathrm{~Hz}, 4 \mathrm{H}) .{ }^{13} \mathrm{C} \mathrm{NMR}$ $\left(101 \mathrm{MHz}, \mathrm{CDCl}_{3}\right) \delta 138.17,137.25,132.01,128.56,128.25,127.73,127.21,123.54,58.56,54.19,23.62,21.53$. HRMS (ESI) $m / z$ [M+H] $]^{+}$calcd for $\mathrm{C}_{14} \mathrm{H}_{19} \mathrm{~N} 202.1590$, found 202.1573 .

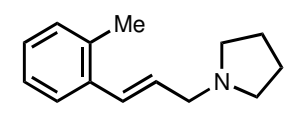

(E)-1-(3-(o-tolyl)allyl)pyrrolidine (SI-26). Following the general procedure C, SI-26 was isolated in $18 \%$ yield as a yellow oil. ${ }^{1} \mathrm{H}$ NMR $\left(400 \mathrm{MHz}, \mathrm{CDCl}_{3}\right) \delta$ 7.46-7.44 (m, 1H), 7.18-7.13 (m, 3H), $6.74(\mathrm{~d}, J=16.0 \mathrm{~Hz}, 1 \mathrm{H}), 6.21(\mathrm{dt}, J$ $=16.0,6.4 \mathrm{~Hz}, 1 \mathrm{H}), 3.29(\mathrm{dd}, J=6.8,1.6 \mathrm{~Hz}, 2 \mathrm{H}), 2.59-2.56(\mathrm{~m}, 4 \mathrm{H}), 2.35(\mathrm{~s}, 3 \mathrm{H}), 1.83-1.80(\mathrm{~m}, 4 \mathrm{H}) .{ }^{13} \mathrm{C} \mathrm{NMR}(101$ $\left.\mathrm{MHz}, \mathrm{CDCl}_{3}\right) \delta 136.42,135.34,130.32,129.82,129.28,127.37,126.20,125.87,58.86,54.17,23.61$, 19.98. HRMS (ESI) $m / z[\mathrm{M}+\mathrm{H}]^{+}$calcd for $\mathrm{C}_{14} \mathrm{H}_{19} \mathrm{~N} 202.1590$, found 202.1577 . 


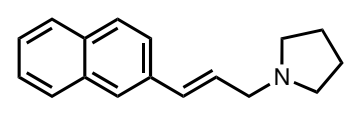

(E)-1-(3-(naphthalen-2-yl)allyl)pyrrolidine (SI-27). Following the general procedure C, SI-27 was isolated in 69\% yield as a white solid. ${ }^{1} \mathrm{H}$ NMR $\left(400 \mathrm{MHz}, \mathrm{CDCl}_{3}\right) \delta 7.83-7.76(\mathrm{~m}, 3 \mathrm{H}), 7.72(\mathrm{~d}, J=1.6 \mathrm{~Hz}, 1 \mathrm{H}), 7.62(\mathrm{dd}, J=8.6$, $1.7 \mathrm{~Hz}, 1 \mathrm{H}), 7.44(\mathrm{tt}, J=7.1,5.3 \mathrm{~Hz}, 2 \mathrm{H}), 6.70(\mathrm{~d}, J=15.8 \mathrm{~Hz}, 1 \mathrm{H}), 6.48(\mathrm{dt}, J=15.8,6.7 \mathrm{~Hz}, 1 \mathrm{H}), 3.32(\mathrm{dd}, J=6.7$, $1.4 \mathrm{~Hz}, 2 \mathrm{H}), 2.64-2.56(\mathrm{~m}, 4 \mathrm{H}), 1.89-1.78(\mathrm{~m}, 4 \mathrm{H}) .{ }^{13} \mathrm{C} \mathrm{NMR}\left(101 \mathrm{MHz}, \mathrm{CDCl}_{3}\right) \delta$ 134.75, 133.70, 132.97, 131.91, $128.44,128.23,127.99,127.73,126.26,126.15,125.78,123.67,58.61,54.25,23.61$. HRMS (ESI) $\mathrm{m} / z$ [M+H] ${ }^{+}$calcd for $\mathrm{C}_{17} \mathrm{H}_{19} \mathrm{~N} 238.1596$, found 238.1603.

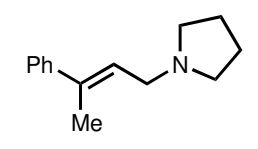

(E)-1-(3-phenylbut-2-en-1-yl)pyrrolidine (SI-28). Following the general procedure C, SI-28 was isolated in 44\% yield as a yellow oil. ${ }^{1} \mathrm{H}$ NMR $\left(400 \mathrm{MHz}, \mathrm{CDCl}_{3}\right) \delta$ 7.42-7.40 (m, 2H), 7.33-7.29 (m, 2H), $7.23(\mathrm{tt}, J=7.2,1.2 \mathrm{~Hz}$, $1 \mathrm{H}), 5.95(\mathrm{tq}, J=6.8,1.2 \mathrm{~Hz}, 1 \mathrm{H}), 3.31(\mathrm{dq}, J=6.8,0.8 \mathrm{~Hz}, 2 \mathrm{H}), 2.61-2.57(\mathrm{~m}, 4 \mathrm{H}), 2.07(\mathrm{~d}, J=1.2 \mathrm{~Hz}, 3 \mathrm{H}), 1.83-$ $1.80(\mathrm{~m}, 4 \mathrm{H}) .{ }^{13} \mathrm{C}$ NMR $\left(101 \mathrm{MHz}, \mathrm{CDCl}_{3}\right) \delta 143.63,136.48,128.33,126.98,125.87,125.70,54.31,54.27,23.64$, 16.30. HRMS (ESI) $\mathrm{m} / z,[\mathrm{M}+\mathrm{H}]^{+}$calcd for $\mathrm{C}_{14} \mathrm{H}_{19} \mathrm{~N} 202.1590$, found 202.1583 .

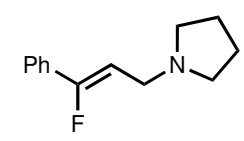

(Z)-1-(3-fluoro-3-phenylallyl)pyrrolidine (SI-29). Following the general procedure C, SI-29 was isolated in $41 \%$ yield as a yellow oil. ${ }^{1} \mathrm{H}$ NMR $\left(600 \mathrm{MHz}, \mathrm{CDCl}_{3}\right) \delta 7.53(\mathrm{dd}, J=7.8,1.2 \mathrm{~Hz}, 2 \mathrm{H}), 7.37-7.32(\mathrm{~m}, 3 \mathrm{H}), 5.66(\mathrm{dt}, J=$ $36.0,7.8 \mathrm{~Hz}, 1 \mathrm{H}), 3.49(\mathrm{dd}, J=7.8,1.8 \mathrm{~Hz}, 2 \mathrm{H}), 2.77-2.72(\mathrm{~m}, 4 \mathrm{H}), 1.89-1.87(\mathrm{~m}, 4 \mathrm{H}) .{ }^{13} \mathrm{C} \mathrm{NMR}\left(151 \mathrm{MHz}, \mathrm{CDCl}_{3}\right)$ $\delta 158.82\left(\mathrm{~d},{ }^{1} J_{\mathrm{CF}}=252.2 \mathrm{~Hz}\right), 131.85\left(\mathrm{~d},{ }^{2} J_{\mathrm{CF}}=28.7 \mathrm{~Hz}\right), 129.34,128.62\left(\mathrm{~d},{ }^{4} J_{\mathrm{CF}}=3.0 \mathrm{~Hz}\right), 124.47\left(\mathrm{~d},{ }^{3} J_{\mathrm{CF}}=6.0 \mathrm{~Hz}\right)$, $101.62\left(\mathrm{~d},{ }^{2} J_{\mathrm{CF}}=15.1 \mathrm{~Hz}\right), 53.83,49.30\left(\mathrm{~d},{ }^{3} J_{\mathrm{CF}}=4.5 \mathrm{~Hz}\right), 23.65 .{ }^{19} \mathrm{~F} \mathrm{NMR}\left(376 \mathrm{MHz}, \mathrm{CDCl}_{3}\right) \delta-116.25 . \mathrm{HRMS}$ (ESI) $\mathrm{m} / z[\mathrm{M}+\mathrm{H}]^{+}$calcd for $\mathrm{C}_{13} \mathrm{H}_{16} \mathrm{FN} 206.1340$, found 206.1336 .

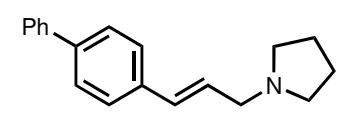

(E)-1-(3-([1,1'-biphenyl]-4-yl)allyl)pyrrolidine (SI-30). Following the general procedure C, SI-30 was isolated in $82 \%$ yield as a white solid. ${ }^{1} \mathrm{H}$ NMR $\left(600 \mathrm{MHz}, \mathrm{CDCl}_{3}\right) \delta 7.60(\mathrm{~d}, J=8.3 \mathrm{~Hz}, 2 \mathrm{H}), 7.55(\mathrm{~d}, J=8.2 \mathrm{~Hz}, 2 \mathrm{H}), 7.46-7.42$ (m, 4H), $7.34(\mathrm{t}, J=7.9 \mathrm{~Hz}, 1 \mathrm{H}), 6.58(\mathrm{~d}, J=15.8 \mathrm{~Hz}, 1 \mathrm{H}), 6.39(\mathrm{dt}, J=15.7,6.7 \mathrm{~Hz}, 1 \mathrm{H}), 3.29(\mathrm{~d}, J=6.7 \mathrm{~Hz}, 2 \mathrm{H})$, 2.58 (br s, 4H), 1.82 (br s, 4H). ${ }^{13} \mathrm{C} \mathrm{NMR}\left(151 \mathrm{MHz}, \mathrm{CDCl}_{3}\right.$ ) $\delta 140.91,140.26,136.37,131.50,128.91,128.12,127.38$, 127.06, 126.86, 58.61, 54.26, 23.65. HRMS (ESI) $\mathrm{m} / z[\mathrm{M}+\mathrm{H}]^{+}$calcd for $\mathrm{C}_{19} \mathrm{H}_{21} \mathrm{~N} 264.1747$, found 264.1748.

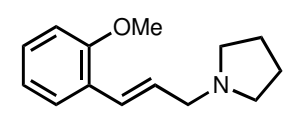

(E)-1-(3-(2-methoxyphenyl)allyl)pyrrolidine (SI-31). Following the general procedure C, SI-31 was isolated in 67\% yield as an orange oil. ${ }^{1} \mathrm{H}$ NMR $\left(600 \mathrm{MHz}, \mathrm{CDCl}_{3}\right) \delta 7.45(\mathrm{~d}, J=7.6 \mathrm{~Hz}, 1 \mathrm{H}), 7.20(\mathrm{t}, J=7.8 \mathrm{~Hz}, 1 \mathrm{H}), 6.91(\mathrm{t}, J=7.5$ $\mathrm{Hz}, 1 \mathrm{H}), 6.86(\mathrm{~s}, 1 \mathrm{H}), 6.84(\mathrm{~d}, J=9.4 \mathrm{~Hz}, 1 \mathrm{H}), 6.35(\mathrm{dt}, J=15.9,6.8 \mathrm{~Hz}, 1 \mathrm{H}), 3.84(\mathrm{~s}, 3 \mathrm{H}), 3.28(\mathrm{~d}, J=6.8 \mathrm{~Hz}, 2 \mathrm{H})$, 2.57-2.55 (m, 4H), 1.81-1.80 (m, 4H). $\left.{ }^{13} \mathrm{C} \mathrm{NMR} \mathrm{(151} \mathrm{MHz,} \mathrm{CDCl}_{3}\right) \delta 156.72,128.62,128.46,126.96,126.65,126.33$, $120.79,110.98,59.05,55.58,54.19,23.63$. HRMS (ESI) $\mathrm{m} / z[\mathrm{M}+\mathrm{H}]^{+}$calcd for $\mathrm{C}_{14} \mathrm{H}_{19} \mathrm{NO} 218.1545$, found 218.1543 . 


\section{Synthesis of doubly axially chiral phosphoric acids (DAP).}

DAP catalysts were synthesized following a similar route to the one previously developed in our group. ${ }^{7}$ Coupling of the two BINOL scaffolds could be efficiently accomplished by an homocoupling protocol as an alternative to the previously reported strategy based on a Suzuki cross-coupling reaction.
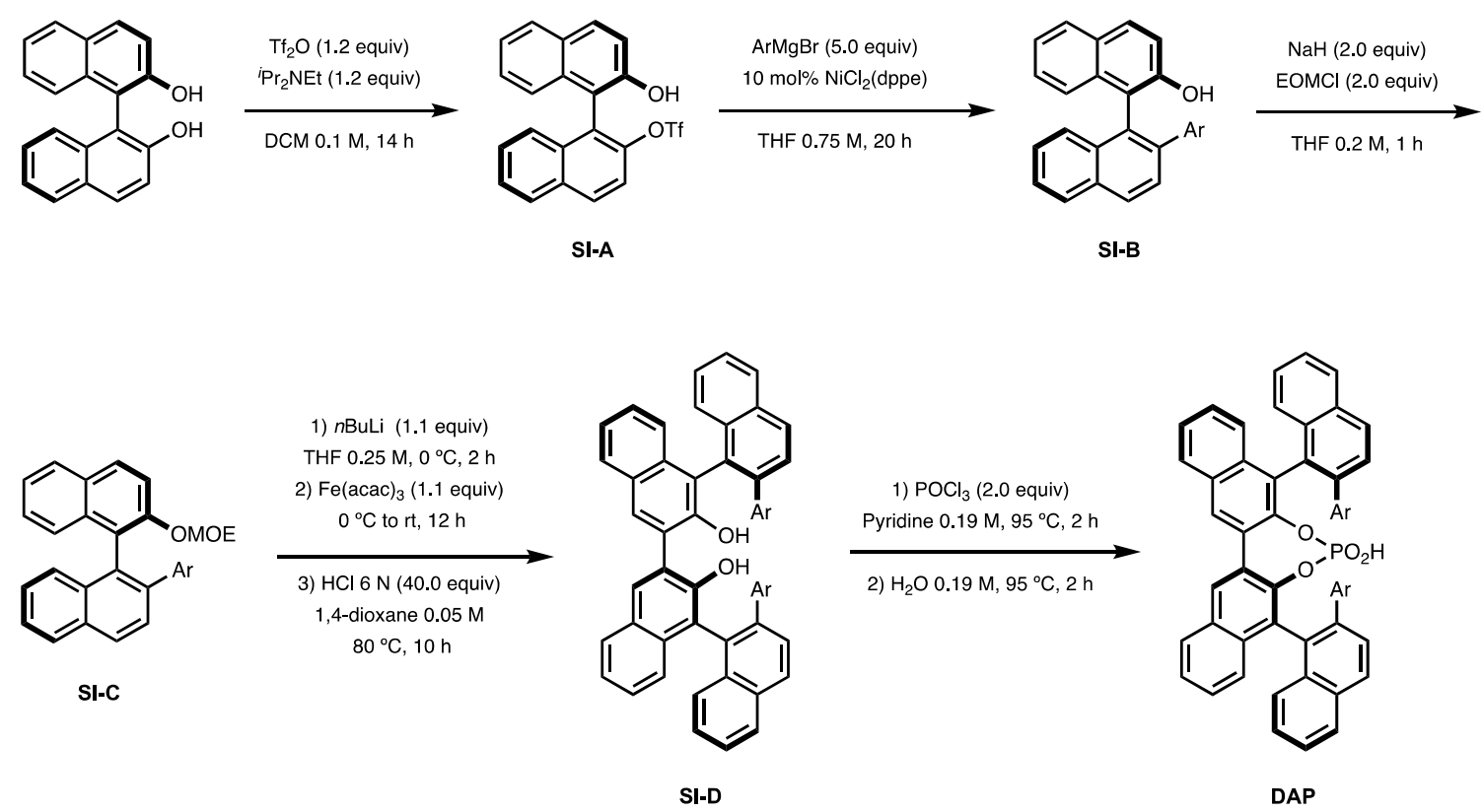

Homocoupling procedure. To a solution of monomer SI-C $(0.60 \mathrm{mmol})$ in THF $(2.4 \mathrm{~mL})$ was added $n \mathrm{BuLi}$ (1.1 equiv, $0.66 \mathrm{mmol}$ ) at $0^{\circ} \mathrm{C}$. After stirring for 2 hours at room temperature, Fe(acac) $)_{3}(1.1$ equiv, $0.66 \mathrm{mmol}$ ) was added. The resultant mixture was slowly warmed to room temperature and stirred for 12 hours. This mixture was quenched with $\mathrm{H}_{2} \mathrm{O}$, extracted with EtOAc, and washed with brine. Collected organic fractions were dried over $\mathrm{Na}_{2} \mathrm{SO}_{4}$ and filtered. After removal of solvents, crude product was purified by column chromatography.

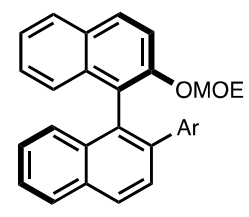

$\left(\mathrm{Ar}=4-{ }^{\mathrm{t}} \mathrm{Bu}-\mathrm{C}_{6} \mathrm{H}_{4}\right)$

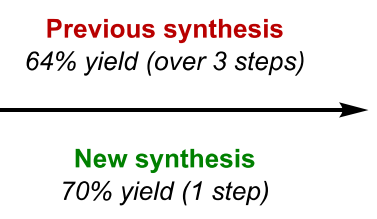

$70 \%$ yield (1 step)

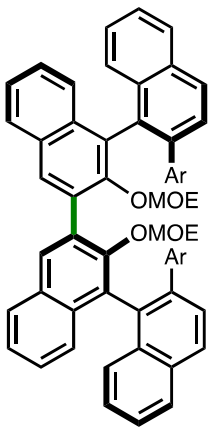

Spectroscopic data for DAPa, DAPf and DAPI were in agreement with those previously reported in the literature. ${ }^{\text {Fehler! }}$ Textmarke nicht definiert. 


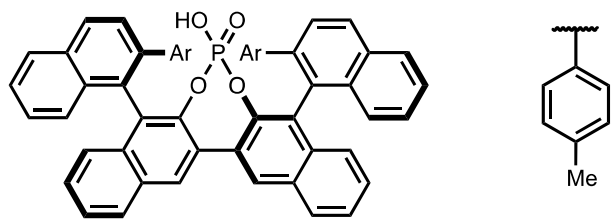

4-Me-C6H4-DAP (DAPb). ${ }^{1} \mathrm{H}$ NMR $\left(600 \mathrm{MHz}, \mathrm{CD}_{2} \mathrm{Cl}_{2}\right) \delta 8.03(\mathrm{~s}, 2 \mathrm{H}), 7.93(\mathrm{~d}, J=8.1 \mathrm{~Hz}, 2 \mathrm{H}), 7.78(\mathrm{~d}, J=8.2 \mathrm{~Hz}$, $4 \mathrm{H}), 7.47(\mathrm{t}, J=7.6 \mathrm{~Hz}, 2 \mathrm{H}), 7.39(\mathrm{t}, J=7.4 \mathrm{~Hz}, 2 \mathrm{H}), 7.32(\mathrm{~d}, J=8.3 \mathrm{~Hz}, 2 \mathrm{H}), 7.29(\mathrm{t}, J=7.5 \mathrm{~Hz}, 2 \mathrm{H}), 7.15(\mathrm{t}, J=7.7$ $\mathrm{Hz}, 2 \mathrm{H}), 7.09(\mathrm{~d}, J=8.4 \mathrm{~Hz}, 2 \mathrm{H}), 7.01(\mathrm{~d}, J=8.7 \mathrm{~Hz}, 2 \mathrm{H}), 6.89(\mathrm{~d}, J=7.9 \mathrm{~Hz}, 4 \mathrm{H}), 6.83(\mathrm{~d}, J=7.9 \mathrm{~Hz}, 4 \mathrm{H}), 2.21(\mathrm{~s}$, $6 \mathrm{H})$. HRMS (ESI) $\mathrm{m} / z$ [M-H] $]^{-}$calcd for $\mathrm{C}_{54} \mathrm{H}_{37} \mathrm{O}_{4} \mathrm{P} 779.2357$, found 779.2349.

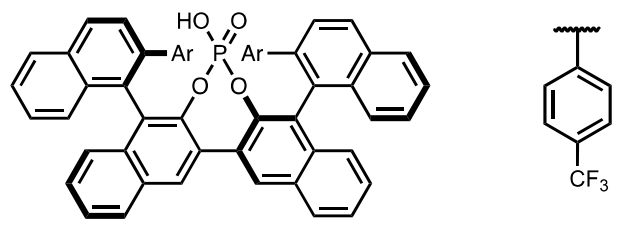

4-CF $3-\mathbf{C}_{6} \mathbf{H}_{4}-\mathbf{D A P}$ (DAPc). ${ }^{1} \mathrm{H}$ NMR $\left(600 \mathrm{MHz}, \mathrm{CD}_{2} \mathrm{Cl}_{2}\right) \delta 8.45$ (br s, $\left.1 \mathrm{H}\right), 7.98(\mathrm{~s}, 2 \mathrm{H}), 7.88(\mathrm{~d}, J=8.2 \mathrm{~Hz}, 2 \mathrm{H}), 7.80$ (br s, 4H), 7.47-7.44 (m, 4H), 7.34-7.21 (m, 10H), 7.09-7.02 (m, 8H). ${ }^{13} \mathrm{C}$ NMR $\left(151 \mathrm{MHz}, \mathrm{CD}_{2} \mathrm{Cl}_{2}\right) \delta 146.28,143.71$, $139.36,134.58,133.41,133.32,131.62,131.08,130.18,129.67,129.37,128.96,128.77,128.55,128.18,128.08$, 127.97, 127.93, 127.73, 126.79, 126.71, 126.64, $124.96\left(\mathrm{q},{ }^{1} J_{\mathrm{CF}}=271.8 \mathrm{~Hz}\right), 124.92\left(\mathrm{q},{ }^{3} J_{\mathrm{CF}}=3.0 \mathrm{~Hz}\right) .{ }^{31} \mathrm{P} \mathrm{NMR}(162$ $\left.\mathrm{MHz}, \mathrm{CD}_{2} \mathrm{Cl}_{2}\right) \delta-1.10 .{ }^{19} \mathrm{~F}$ NMR $\left(376 \mathrm{MHz}, \mathrm{CD}_{2} \mathrm{Cl}_{2}\right) \delta-61.82$. HRMS (ESI) $\mathrm{m} / z$ [M-H] ${ }^{-}$calcd for $\mathrm{C}_{54} \mathrm{H}_{31} \mathrm{~F}_{6} \mathrm{O}_{4} \mathrm{P}$ 887.1791 , found 887.1773 .

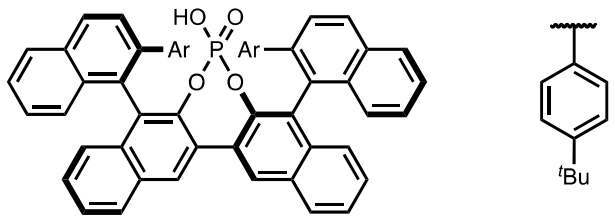

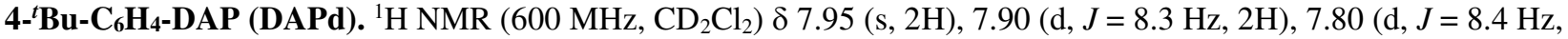
$4 \mathrm{H}), 7.46(\mathrm{t}, J=7.6 \mathrm{~Hz}, 2 \mathrm{H}), 7.42(\mathrm{t}, J=7.5 \mathrm{~Hz}, 2 \mathrm{H}), 7.35(\mathrm{~d}, J=8.5 \mathrm{~Hz}, 2 \mathrm{H}), 7.29(\mathrm{t}, J=8.1 \mathrm{~Hz}, 2 \mathrm{H}), 7.18(\mathrm{t}, J=7.7$ $\mathrm{Hz}, 2 \mathrm{H}), 7.12(\mathrm{~d}, J=8.5 \mathrm{~Hz}, 2 \mathrm{H}), 7.10(\mathrm{~d}, J=8.4 \mathrm{~Hz}, 4 \mathrm{H}), 7.03(\mathrm{~d}, J=8.5 \mathrm{~Hz}, 2 \mathrm{H}), 6.84(\mathrm{~d}, J=8.3 \mathrm{~Hz}, 4 \mathrm{H}), 1.23(\mathrm{~s}$, $18 \mathrm{H}) .{ }^{13} \mathrm{C}$ NMR $\left(151 \mathrm{MHz}, \mathrm{CD}_{2} \mathrm{Cl}_{2}\right) \delta 149.84,143.93,143.87,140.58,139.45,134.92,133.50,133.03,131.58,130.63$, $129.60,129.33,129.16,128.98,128.78,128.64,128.60,128.11,127.94,127.71,127.05,126.42,126.31,125.95$, 124.87, 34.81, 31.58. ${ }^{31} \mathrm{P}$ NMR (162 $\mathrm{MHz}, \mathrm{CD}_{2} \mathrm{Cl}_{2}$ ) $\delta$-1.77. HRMS (ESI) $\mathrm{m} / z$ [M-H] ${ }^{-}$calcd for $\mathrm{C}_{60} \mathrm{H}_{49} \mathrm{O}_{4} \mathrm{P} 863.3296$, found 863.3276 .
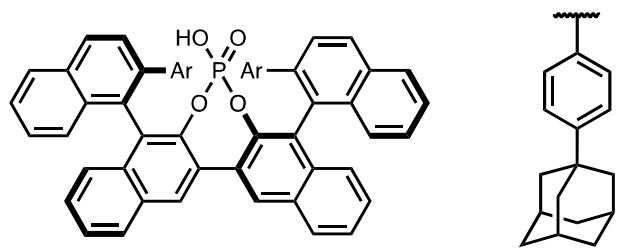

4-Ad-C6H4-DAP (DAPe). ${ }^{1} \mathrm{H}$ NMR $\left(600 \mathrm{MHz}, \mathrm{CD}_{3} \mathrm{Cl}\right) \delta 7.87(\mathrm{~s}, 2 \mathrm{H}), 7.87(\mathrm{~d}, J=9.1 \mathrm{~Hz}, 2 \mathrm{H}), 7.78(\mathrm{~d}, J=10.2 \mathrm{~Hz}$, $2 \mathrm{H}), 7.77(\mathrm{~d}, J=9.0 \mathrm{~Hz}, 2 \mathrm{H}), 7.43(\mathrm{t}, J=7.5 \mathrm{~Hz}, 2 \mathrm{H}), 7.40(\mathrm{t}, J=7.8 \mathrm{~Hz}, 2 \mathrm{H}), 7.37(\mathrm{~d}, J=8.4 \mathrm{~Hz}, 2 \mathrm{H}), 7.28(\mathrm{t}, J=$ $7.6 \mathrm{~Hz}, 2 \mathrm{H}), 7.21(\mathrm{~d}, J=8.5 \mathrm{~Hz}, 2 \mathrm{H}), 7.18(\mathrm{t}, J=7.6 \mathrm{~Hz}, 2 \mathrm{H}), 7.11(\mathrm{~d}, J=8.5 \mathrm{~Hz}, 2 \mathrm{H}), 7.00(\mathrm{~d}, J=8.2 \mathrm{~Hz}, 4 \mathrm{H}), 6.82$ $(\mathrm{d}, J=8.2 \mathrm{~Hz}, 4 \mathrm{H}), 6.28$ (br s, 1H), $2.05(\mathrm{~s}, 6 \mathrm{H}), 1.82-1.76(\mathrm{~m}, 18 \mathrm{H}), 1.71(\mathrm{~d}, J=11.6 \mathrm{~Hz}, 6 \mathrm{H}) .{ }^{13} \mathrm{C} \mathrm{NMR}(151 \mathrm{MHz}$, $\left.\mathrm{CD}_{3} \mathrm{Cl}\right) \delta 149.21,143.58,143.53,140.52,139.02,134.46,132.96,132.50,130.96,129.71,129.42,129.02,128.65$, $128.59,128.28,128.24,128.10,127.94,127.83,127.65,127.07,126.90,125.76,125.73,125.41,123.73,43.21,36.97$, 
35.97, 29.09. ${ }^{31} \mathrm{P}$ NMR $\left(162 \mathrm{MHz}, \mathrm{CD}_{3} \mathrm{Cl}\right) \delta$-2.02. HRMS (ESI) $\mathrm{m} / z$ [M-H] $]^{-}$calcd for $\mathrm{C}_{72} \mathrm{H}_{61} \mathrm{O}_{4} \mathrm{P} 1019.4235$, found 1019.4211.

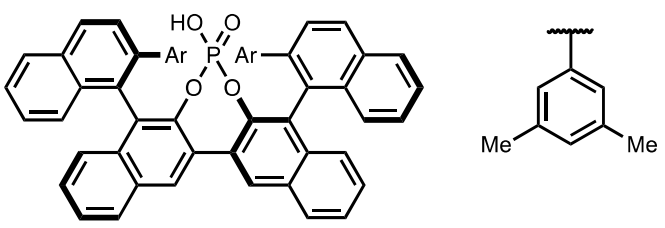

3,5-diMe-C $6 \mathbf{H}_{3}$-DAP (DAPg). ${ }^{1} \mathrm{H}$ NMR $\left(600 \mathrm{MHz}, \mathrm{CD}_{2} \mathrm{Cl}_{2}\right) \delta 7.97(\mathrm{~s}, 2 \mathrm{H}), 7.90(\mathrm{~d}, J=8.3 \mathrm{~Hz}, 2 \mathrm{H}), 7.74(\mathrm{~d}, J=8.0$ $\mathrm{Hz}, 2 \mathrm{H}), 7.70(\mathrm{~d}, J=8.4 \mathrm{~Hz}, 2 \mathrm{H}), 7.44(\mathrm{t}, J=7.5 \mathrm{~Hz}, 2 \mathrm{H}), 7.35-7.33(\mathrm{~m}, 4 \mathrm{H}), 7.27$ (t, $J=7.6 \mathrm{~Hz}, 2 \mathrm{H}), 7.16$ (t, $J=7.5$ $\mathrm{Hz}, 2 \mathrm{H}), 7.04(\mathrm{~d}, J=8.4 \mathrm{~Hz}, 4 \mathrm{H}), 6.72(\mathrm{~s}, 2 \mathrm{H}), 6.67$ (s, 4H), 6.21 (br s, 1H), 2.06 (s, 12H). ${ }^{13} \mathrm{C}$ NMR (151 MHz, $\left.\mathrm{CD}_{2} \mathrm{Cl}_{2}\right) \delta 144.42,144.37,142.18,140.66,137.26,134.76,133.42,133.08,131.52,130.43,129.72,129.12,129.06$, $128.85,128.81,128.69,128.57,128.30,128.08,127.97,127.61,127.23,126.98,126.38,126.34,125.93,21.45 .{ }^{31} \mathrm{P}$ NMR (162 MHz, $\left.\mathrm{CD}_{2} \mathrm{Cl}_{2}\right) \delta-2.46$. HRMS (ESI) $\mathrm{m} / z$ [M-H] calcd for $\mathrm{C}_{56} \mathrm{H}_{41} \mathrm{O}_{4} \mathrm{P}$ 807.2670, found 807.2662.

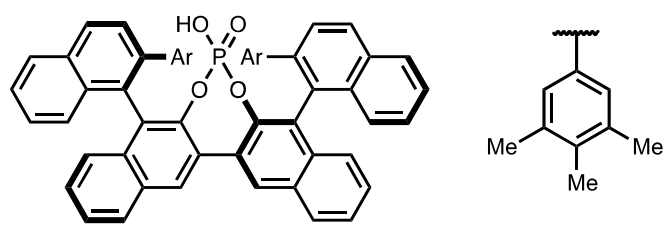

3,4,5-triMe-C $\mathbf{C}_{6} \mathbf{H}_{2}$-DAP (DAPi). ${ }^{1} \mathrm{H}$ NMR $\left(600 \mathrm{MHz}, \mathrm{CDCl}_{3}\right) \delta 7.73$ (br s, 4H), 7.64-7.61 (m, 4H), 7.34-7.21 (m, 8H), $7.13(\mathrm{~d}, J=7.8 \mathrm{~Hz}, 4 \mathrm{H}), 7.03(\mathrm{~d}, J=7.2 \mathrm{~Hz}, 2 \mathrm{H}), 6.84(\mathrm{br} \mathrm{s}, 1 \mathrm{H}), 6.70(\mathrm{~s}, 4 \mathrm{H}), 1.98(\mathrm{~s}, 12 \mathrm{H}), 1.90(\mathrm{~s}, 6 \mathrm{H}) .{ }^{13} \mathrm{C}$ NMR $\left(151 \mathrm{MHz}, \mathrm{CDCl}_{3}\right) \delta 143.87,143.82,140.25,138.92,135.16,134.36,133.06,133.00,132.44,130.75,129.55,129.24$, $128.74,128.63,128.59,128.48,128.12$, 128.07, 127.90, 127.78, 127.58, 126.89, 126.73, 125.75, 125.67, 125.24, 20.46, 14.98. ${ }^{31} \mathrm{P}$ NMR $\left(162 \mathrm{MHz}, \mathrm{CDCl}_{3}\right) \delta-2.53$. HRMS (ESI) $\mathrm{m} / z$ [M-H] ${ }^{-}$calcd for $\mathrm{C}_{58} \mathrm{H}_{45} \mathrm{O}_{4} \mathrm{P} 835.2963$, found 835.2964.

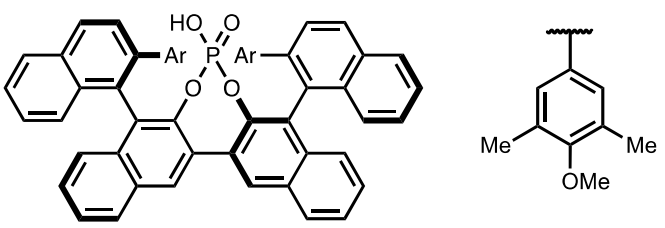

4-OMe-3,5-diMe-C6H2-DAP (DAPj). ${ }^{1} \mathrm{H}$ NMR (600 MHz, $\left.\mathrm{CD}_{2} \mathrm{Cl}_{2}\right) \delta 8.11(\mathrm{~s}, 2 \mathrm{H}), 7.96(\mathrm{~d}, J=8.2 \mathrm{~Hz}, 2 \mathrm{H}), 7.88$ (t, $J=7.6 \mathrm{~Hz}, 2 \mathrm{H}), 7.82(\mathrm{~d}, J=8.4 \mathrm{~Hz}, 2 \mathrm{H}), 7.66(\mathrm{~d}, J=7.9 \mathrm{~Hz}, 2 \mathrm{H}), 7.58(\mathrm{~d}, J=8.5 \mathrm{~Hz}, 2 \mathrm{H}), 7.53(\mathrm{~d}, J=8.2 \mathrm{~Hz}, 2 \mathrm{H})$, 7.42 (t, $J=7.5 \mathrm{~Hz}, 2 \mathrm{H}), 7.27$ (br s, 1H), 7.27-7.21 (m, 4H), $7.02(\mathrm{~d}, J=8.5 \mathrm{~Hz}, 2 \mathrm{H}), 6.87(\mathrm{~s}, 4 \mathrm{H}), 3.57(\mathrm{~s}, 6 \mathrm{H}), 1.98$ (s, 12H). ${ }^{13} \mathrm{C}$ NMR $\left(151 \mathrm{MHz}, \mathrm{CD}_{2} \mathrm{Cl}_{2}\right) \delta 156.20,146.88,146.82,143.74,142.15,140.17,137.55,134.84,134.03$, 132.76, 131.64, 131.50, 131.25, 130.37, 130.10, 130.00, 129.46, 128.85, 128.69, 128.37, 127.68, 127.65, 127.59, 127.07, 126.60, 125.98, 125.90, 125.59, 59.92, 16.20. $\left.{ }^{31} \mathrm{P} \mathrm{NMR} \mathrm{(162} \mathrm{MHz,} \mathrm{CD}_{2} \mathrm{Cl}_{2}\right) \delta-1.86$. HRMS (ESI) $m / z$ [M-H] $]^{-}$ calcd for $\mathrm{C}_{58} \mathrm{H}_{45} \mathrm{O}_{6} \mathrm{P} 867.2881$, found 867.2857.

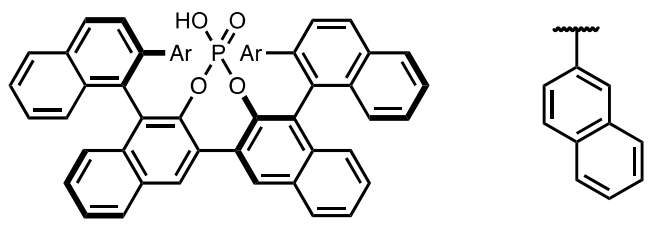


2-Naphthyl-DAP (DAPk). ${ }^{1} \mathrm{H}$ NMR $\left(600 \mathrm{MHz}, \mathrm{CD}_{2} \mathrm{Cl}_{2}\right) \delta 7.81(\mathrm{t}, J=7.2 \mathrm{~Hz}, 4 \mathrm{H}), 7.73(\mathrm{~s}, 2 \mathrm{H}), 7.71(\mathrm{~d}, J=8.2 \mathrm{~Hz}$, $2 \mathrm{H}), 7.66-7.65(\mathrm{~m}, 2 \mathrm{H}), 7.54(\mathrm{~d}, J=8.3 \mathrm{~Hz}, 2 \mathrm{H}), 7.51(\mathrm{~s}, 2 \mathrm{H}), 7.50-7.43(\mathrm{~m}, 6 \mathrm{H}), 7.38(\mathrm{t}, J=7.5 \mathrm{~Hz}, 2 \mathrm{H}), 7.33-7.28$ $(\mathrm{m}, 6 \mathrm{H}), 7.26(\mathrm{t}, J=7.5 \mathrm{~Hz}, 2 \mathrm{H}), 7.15(\mathrm{q}, J=8.2 \mathrm{~Hz}, 6 \mathrm{H}), 6.17(\mathrm{br} \mathrm{s}, 1 \mathrm{H}) .{ }^{13} \mathrm{C}$ NMR $\left(151 \mathrm{MHz}, \mathrm{CD}_{2} \mathrm{Cl}_{2}\right) \delta 144.19$, $144.13,140.55,140.01,134.65,133.51,133.33,133.23,132.57,131.47,130.82,130.17,129.24,128.82,128.79$, $128.54,128.50,128.40,128.16,128.12,127.89,127.82,127.75,127.48,126.84,126.53,126.42,126.25,126.17 .{ }^{31} \mathrm{P}$ NMR (162 MHz, $\left.\mathrm{CD}_{2} \mathrm{Cl}_{2}\right) \delta$-2.00. HRMS (ESI) $\mathrm{m} / z$ [M-H] calcd for $\mathrm{C}_{60} \mathrm{H}_{37} \mathrm{O}_{4} \mathrm{P}$ 851.2357, found 851.2334.

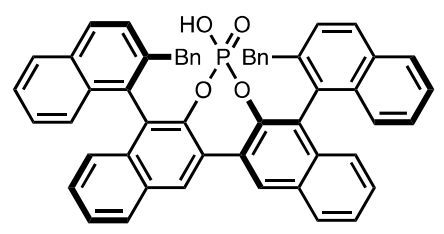

Bn-DAP (DAPm). ${ }^{1} \mathrm{H}$ NMR $\left(600 \mathrm{MHz}, \mathrm{CD}_{2} \mathrm{Cl}_{2}\right) \delta 8.50(\mathrm{~s}, 2 \mathrm{H}), 8.14(\mathrm{~d}, J=8.3 \mathrm{~Hz}, 2 \mathrm{H}), 7.71(\mathrm{~d}, J=8.1 \mathrm{~Hz}, 2 \mathrm{H})$, $7.65(\mathrm{~d}, J=8.3 \mathrm{~Hz}, 2 \mathrm{H}), 7.53(\mathrm{t}, J=7.5 \mathrm{~Hz}, 2 \mathrm{H}), 7.27-7.20(\mathrm{~m}, 6 \mathrm{H}), 7.17(\mathrm{t}, J=7.5 \mathrm{~Hz}, 2 \mathrm{H}), 7.12(\mathrm{~d}, J=8.5 \mathrm{~Hz}, 2 \mathrm{H})$, 6.99-6.98 (m, 6H), $6.95(\mathrm{~d}, J=8.5 \mathrm{~Hz}, 2 \mathrm{H}), 6.76(\mathrm{t}, J=3.3 \mathrm{~Hz}, 4 \mathrm{H}), 6.27(\mathrm{br} \mathrm{s}, 1 \mathrm{H}), 3.77(\mathrm{~d}, J=15.4 \mathrm{~Hz}, 2 \mathrm{H}), 3.62$ $(\mathrm{d}, J=15.4 \mathrm{~Hz}, 2 \mathrm{H}) .{ }^{13} \mathrm{C} \mathrm{NMR}\left(151 \mathrm{MHz}, \mathrm{CD}_{2} \mathrm{Cl}_{2}\right) \delta 144.20,144.14,141.10,139.08,134.36,133.48,132.49,132.24$, $131.27,130.58,129.68,128.81,128.77,128.52,128.45,128.28,128.25,128.05,127.63,127.44,127.00,126.79$, 126.23, 126.11, 125.60, 40.31. ${ }^{31} \mathrm{P}$ NMR $\left(162 \mathrm{MHz}, \mathrm{CD}_{2} \mathrm{Cl}_{2}\right) \delta-3.33$. HRMS (ESI) $m / z$ [M-H] $]^{-}$calcd for $\mathrm{C}_{54} \mathrm{H}_{37} \mathrm{O}_{4} \mathrm{P}$ 779.2357 , found 779.2345 . 


\section{Synthesis of triple axially chiral phosphoric acid (TAP).}
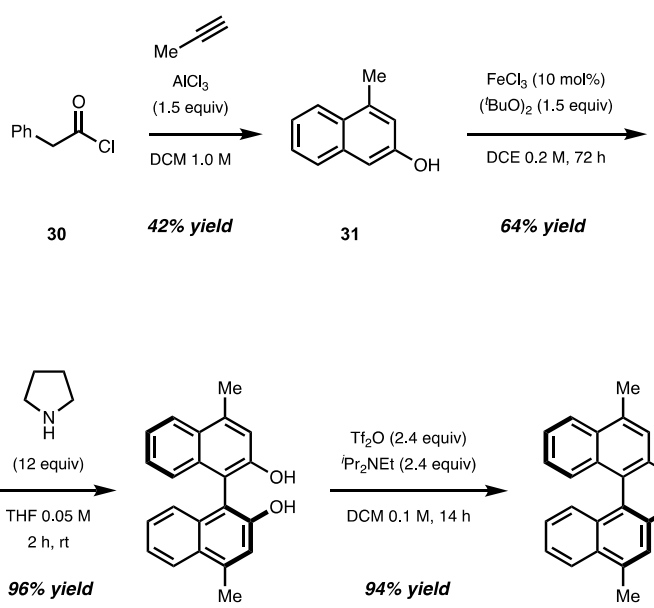

(S)-32

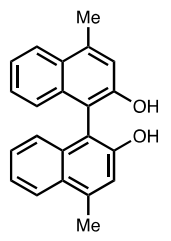

rac-32
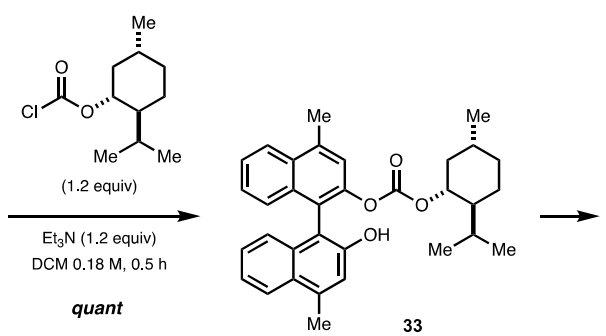

chromatographic separation

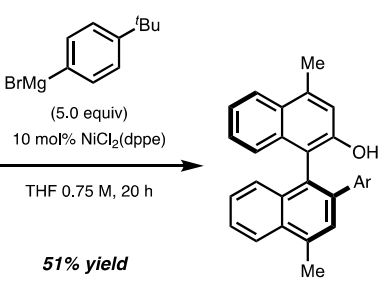<smiles>Cc1cc(O)c(-c2c(O)cc([N+](=O)[O-])c3ccccc23)c2ccccc12</smiles>

(S)-SI-F<smiles>CCOc1cc([N+](=O)[O-])c2ccccc2c1-c1c(O)cc([N+](=O)[O-])c2ccccc12</smiles>

(S)-SI-E
$\underset{\operatorname{THF} 0.05 \mathrm{M}, 4 \mathrm{~h}}{\stackrel{\operatorname{TBAF}(2.2 \text { equiv) }}{\longrightarrow}}$
$\mathbf{8 3 \%}$ yield<smiles>Cc1cc([Al])c2ccccc2c1-c1c(O)c(-c2c(O)c(-c3c([Al])cc([N+](=O)[O-])c4ccccc34)c3ccccc3c2C)c2ccccc2c1O</smiles>

$\left(R, S_{c}, R\right)-34$
1) $\mathrm{POCl}_{3}$ (2.0 equiv) Pyridine $0.19 \mathrm{M}, 95^{\circ} \mathrm{C}, 2 \mathrm{~h}$

2) $\mathrm{H}_{2} \mathrm{O} 0.19 \mathrm{M}, 95^{\circ} \mathrm{C}, 24 \mathrm{~h}$

$82 \%$ yield

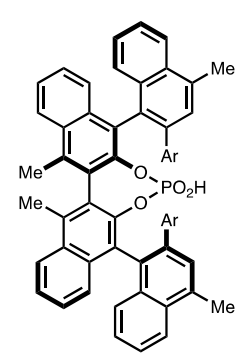

$\left(R, S_{c}, R\right)$-TAPa<smiles>O=[N+]([O-])c1cc(O)cc2ccccc12</smiles>

4-methylnaphthalen-2-ol (31). Compound 31 was prepared following a modified procedure of the methodology developed by $\mathrm{Oh}^{8}{ }^{8} \mathrm{~A}$ dry flask was charged with $\mathrm{AlCl}_{3}(1.5$ equiv, $30.0 \mathrm{mmol})$ and dry $\mathrm{DCM}(20.0 \mathrm{~mL})$ under nitrogen. To this flask was added phenyl acetyl chloride $30(1.0$ equiv, $20.0 \mathrm{mmol})$ at $0{ }^{\circ} \mathrm{C}$. Propyne gas was bubble into the reaction mixture for $5 \mathrm{~min}$. Then the flask was warmed to room temperature. After 4 hours, the reaction was carefully quenched with water at $0{ }^{\circ} \mathrm{C}$, extracted with DCM, and dried with $\mathrm{Na}_{2} \mathrm{SO}_{4}$. After removal of solvents, the crude mixture was purified by column chromatography, affording the title compound in $42 \%$ yield as a red solid. Spectroscopic data were in agreement with those previously reported in the literature. ${ }^{9}$<smiles>N#Cc1cc(O)c(-c2c(O)cc([N+](=O)[O-])c3ccccc23)c2ccccc12</smiles>

4,4'-dimethyl-[1,1'-binaphthalene]-2,2'-diol (rac-32). To a stirred solution of the 2-naphthol derivative SI-E (8.0 mmol) and anhydrous $\mathrm{FeCl}_{3}(0.08 \mathrm{mmol}, 10 \mathrm{~mol} \%)$ in 1,2-dichloroethane $(40.0 \mathrm{~mL})$, was added tert-butyl peroxide (12.0 mmol, 1.5 equiv). The reaction progress was monitored by TLC and, upon completion (72 hours), the crude 
material was isolated by filtration through a pad of $\mathrm{SiO}_{2}$ washing with 1:1 hexanes:ethyl acetate and evaporation of volatiles. The crude residue was purified by column chromatography, affording compound $\mathbf{3 2}$ in $64 \%$ yield as a white solid. ${ }^{1} \mathrm{H}$ NMR $\left(600 \mathrm{MHz}, \mathrm{CDCl}_{3}\right) \delta 8.05(\mathrm{~d}, J=8.4 \mathrm{~Hz}, 2 \mathrm{H}), 7.41(\mathrm{t}, J=7.5 \mathrm{~Hz}, 2 \mathrm{H}), 7.31(\mathrm{t}, J=7.6 \mathrm{~Hz}, 2 \mathrm{H}), 7.26$ $(\mathrm{s}, 2 \mathrm{H}), 7.19(\mathrm{~d}, J=8.4 \mathrm{~Hz}, 2 \mathrm{H}), 5.00(\mathrm{~s}, 2 \mathrm{H}), 2.80(\mathrm{~s}, 6 \mathrm{H}) \cdot{ }^{13} \mathrm{C} \mathrm{NMR}\left(151 \mathrm{MHz}, \mathrm{CDCl}_{3}\right) \delta 152.52,138.48,133.89$, 129.00, 127.28, 125.01, 124.72, 123.92, 118.50, 109.04, 19.76. HRMS (ESI) $\mathrm{m} / z$ [M-H] ${ }^{-}$calcd for $\mathrm{C}_{22} \mathrm{H}_{18} \mathrm{O}_{2} 313.1234$, found 313.1230 .

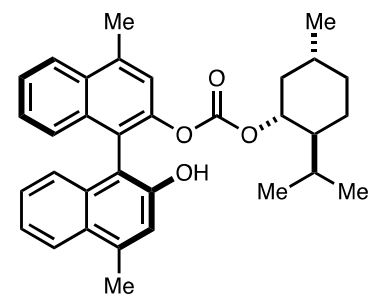

(S)-2'-hydroxy-4,4'-dimethyl-[1,1'-binaphthalen]-2-yl $((1 R, 2 S, 5 R)$-2-isopropyl-5-methylcyclohexyl) carbonate (33). Binaphthol $32(12.7 \mathrm{mmol})$ was dissolved in DCM $(70 \mathrm{~mL})$ and triethylamine $(14.0 \mathrm{mmol}, 1.2$ equiv) was added. Then, $(1 R)$-(-)-menthyl chloroformate $(14.0 \mathrm{mmol}, 1.2$ equiv) was added. The reaction mixture was stirred for $0.5 \mathrm{~h}$ and quenched with water. The aqueous layer was extracted with DCM, and the combined organic layers were washed with brine and dried over anhydrous $\mathrm{Na}_{2} \mathrm{SO}_{4}$, filtered and concentrated in vacuo. Diastereoisomers could be separated via automatic column chromatography eluting with hexanes and a 0 to $10 \%$ gradient of a 2:1ethyl acetate:methanol mixture. ${ }^{1} \mathrm{H}$ NMR $\left(600 \mathrm{MHz}, \mathrm{CDCl}_{3}\right) \delta 8.11(\mathrm{~d}, J=8.5 \mathrm{~Hz}, 1 \mathrm{H}), 7.98(\mathrm{~d}, J=8.3 \mathrm{~Hz}, 1 \mathrm{H}), 7.55(\mathrm{ddd}, J=8.3,5.5,2.5$ $\mathrm{Hz}, 1 \mathrm{H}), 7.36-7.34(\mathrm{~m}, 4 \mathrm{H}), 7.25(\mathrm{t}, J=7.2 \mathrm{~Hz}, 1 \mathrm{H}), 7.19(\mathrm{~s}, 1 \mathrm{H}), 7.08(\mathrm{~d}, J=8.4 \mathrm{~Hz}, 1 \mathrm{H}), 5.21(\mathrm{~s}, 1 \mathrm{H}), 4.37(\mathrm{td}, J=$ $10.9,4.5 \mathrm{~Hz}, 1 \mathrm{H}), 2.84(\mathrm{~s}, 3 \mathrm{H}), 2.77(\mathrm{~s}, 3 \mathrm{H}), 1.78(\mathrm{~d}, J=11.4 \mathrm{~Hz}, 1 \mathrm{H}), 1.61-1.54(\mathrm{~m}, 2 \mathrm{H}), 1.37-1.26(\mathrm{~m}, 2 \mathrm{H}), 1.20-$ $1.15(\mathrm{~m}, 1 \mathrm{H}), 0.95(\mathrm{qd}, J=12.9,3.1 \mathrm{~Hz}, 1 \mathrm{H}), 0.90-0.84(\mathrm{~m}, 1 \mathrm{H}), 0.85(\mathrm{~d}, J=6.6 \mathrm{~Hz}, 3 \mathrm{H}), 0.77(\mathrm{qd}, J=12.6,3.1 \mathrm{~Hz}$, $1 \mathrm{H}), 0.65(\mathrm{~d}, J=7.0 \mathrm{~Hz}, 3 \mathrm{H}), 0.56(\mathrm{~d}, J=6.9 \mathrm{~Hz}, 3 \mathrm{H}) .{ }^{13} \mathrm{C} \mathrm{NMR}\left(151 \mathrm{MHz}, \mathrm{CDCl}_{3}\right) \delta 154.03,151.54,147.80,138.13$, 137.06, 133.95, 133.87, 131.82, 128.58, 127.29, 126.68, 126.56, 126.27, 125.36, 124.59, 124.19, 123.40, 122.10, $121.67,119.22,112.07,79.65,46.83,40.43,34.07,31.37,26.20,23.69,22.03,20.42,19.71,19.66,16.50$. HRMS (ESI) $m / z$ [M-H] $]^{-}$calcd for $\mathrm{C}_{33} \mathrm{H}_{36} \mathrm{O}_{4}$ 495.2541, found 495.2534.

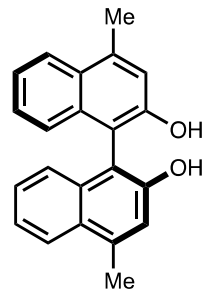

(S)-4,4'-dimethyl-[1,1'-binaphthalene]-2,2'-diol ((S)-32). Compound 33 (4.0 mmol) was dissolved in THF (80 mL) and pyrrolidine ( $48.0 \mathrm{mmol}, 12.0$ equiv) was added. The mixture was stirred at room temperature for 2 hours. After removal of solvents, the residue was purified by column chromatography, affording the title compound in $96 \%$ yield and $98 \%$ ee as a white solid. HPLC (ChiralPak ODH column) $90: 10$ (Hexane $/ \mathrm{PrOH}) 1.0 \mathrm{~mL} \cdot \mathrm{min}^{-1}$; $\mathrm{T}_{\text {major }}(7.8 \mathrm{~min}$ ), $\mathrm{T}_{\text {minor }}(9.4 \mathrm{~min}$ ); 99.0:1.0 er. Absolute configuration could be established by X-Ray analysis of derived compound $\mathbf{3 4}$ (see below). 


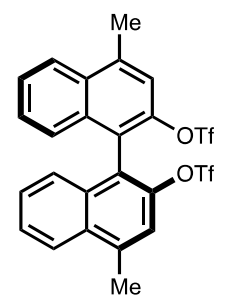

(S)-4,4'-dimethyl-[1,1'-binaphthalene]-2,2'-diyl bis(trifluoromethanesulfonate) ((S)-SI-E). To a flame-dried round bottom flask was added binaphthol $(S)$-32 $(3.0 \mathrm{mmol})$ followed by DCM $(30.0 \mathrm{~mL})$. The solution was cooled to $0{ }^{\circ} \mathrm{C}$ and $N, N$-diisopropylethylamine (7.2 mmol, 2.4 equiv) was added, followed by the slow addition of trifluoromethanesulfonic anhydride (7.2 mmol, 2.4 equiv). The reaction was warmed to room temperature and stirred overnight. The reaction was cooled to $0{ }^{\circ} \mathrm{C}$, quenched with $2 \mathrm{~N} \mathrm{HCl}$ and extracted with DCM. After washing with sat. aq. sol. $\mathrm{NaHCO}_{3}$ and brine, collected organic layers were dried with anhydrous $\mathrm{Na}_{2} \mathrm{SO}_{4}$. The crude was purified by column chromatography, affording compound (S)-SI-E in $94 \%$ yield as a white sticky solid. ${ }^{1} \mathrm{H} \mathrm{NMR}(600 \mathrm{MHz}$, $\left.\mathrm{CDCl}_{3}\right) \delta 8.13(\mathrm{~d}, J=8.5 \mathrm{~Hz}, 2 \mathrm{H}), 7.60(\mathrm{t}, J=7.6 \mathrm{~Hz}, 2 \mathrm{H}), 7.45(\mathrm{~s}, 2 \mathrm{H}), 7.39(\mathrm{t}, J=7.6 \mathrm{~Hz}, 2 \mathrm{H}), 7.26(\mathrm{~d}, J=8.4 \mathrm{~Hz}$, 2H), $2.87(\mathrm{~s}, 6 \mathrm{H}) .{ }^{13} \mathrm{C}$ NMR $\left(151 \mathrm{MHz}, \mathrm{CDCl}_{3}\right) \delta 145.19,139.63,133.52,131.87,127.69,127.17,124.68,121.68$, $119.91,118.28\left(\mathrm{q},{ }^{1} J_{\mathrm{CF}}=320.1 \mathrm{~Hz}\right), 19.98 .{ }^{19} \mathrm{~F} \mathrm{NMR}\left(376 \mathrm{MHz}, \mathrm{CDCl}_{3}\right) \delta-73.82 . \mathrm{HRMS}(\mathrm{ESI}) \mathrm{m} / z[\mathrm{M}+\mathrm{Na}]^{+} \mathrm{calcd}$ for $\mathrm{C}_{24} \mathrm{H}_{16} \mathrm{~F}_{6} \mathrm{O}_{6} \mathrm{~S}_{2} 601.0185$, found 601.0188 .

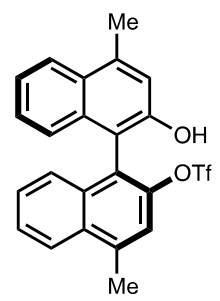

(S)-2'-hydroxy-4,4'-dimethyl-[1,1'-binaphthalen]-2-yl trifluoromethanesulfonate ((S)-SI-F). Over a solution of compound $(S)$-SI-E $(2.5 \mathrm{mmol})$ in THF $(50.0 \mathrm{~mL})$, tetrabutylammonium fluoride $(5.5 \mathrm{mmol}, 2.2$ equiv) was dropwise added. The mixture was stirred at room temperature for 4 hours and quenched with sat. aq. sol. $\mathrm{NH}_{4} \mathrm{Cl}$ and extracted with ethyl acetate. Collected organic layers were dried over anhydrous $\mathrm{Na}_{2} \mathrm{SO}_{4}$ and crude residue was purified by column chromatography after removal of solvents, yielding compound $(S)$-SI-F in $83 \%$ yield as a white solid. ${ }^{1} \mathrm{H}$ NMR $\left(600 \mathrm{MHz}, \mathrm{CDCl}_{3}\right) \delta 8.14(\mathrm{~d}, J=8.5 \mathrm{~Hz}, 1 \mathrm{H}), 8.02(\mathrm{~d}, J=8.4 \mathrm{~Hz}, 1 \mathrm{H}), 7.62(\mathrm{t}, J=7.2 \mathrm{~Hz}, 1 \mathrm{H}), 7.46-7.42(\mathrm{~m}, 3 \mathrm{H})$, $7.38(\mathrm{t}, J=7.5 \mathrm{~Hz}, 1 \mathrm{H}), 7.28(\mathrm{~d}, J=8.0 \mathrm{~Hz}, 1 \mathrm{H}), 7.20(\mathrm{~s}, 1 \mathrm{H}), 7.02(\mathrm{~d}, J=8.4 \mathrm{~Hz}, 1 \mathrm{H}), 4.78(\mathrm{br} \mathrm{s}, 1 \mathrm{H}), 2.87(\mathrm{~s}, 3 \mathrm{H})$, $2.79(\mathrm{~s}, 3 \mathrm{H}) .{ }^{13} \mathrm{C}$ NMR $\left(151 \mathrm{MHz}, \mathrm{CDCl}_{3}\right) \delta 151.51,146.02,139.38,138.37,133.71,133.62,132.46,128.68,128.16$, $127.50,127.33,126.81,124.99,124.87,124.52,123.66,123.20,120.41,118.65,118.39\left(\mathrm{q},{ }^{1} J_{\mathrm{CF}}=320.1 \mathrm{~Hz}\right), 110.37$, 19.88, 19.76. ${ }^{19} \mathrm{~F}$ NMR (376 MHz, $\left.\mathrm{CDCl}_{3}\right) \delta-73.60$. HRMS (ESI) $m / z$ [M-H] ${ }^{-}$calcd for $\mathrm{C}_{23} \mathrm{H}_{17} \mathrm{~F}_{3} \mathrm{O}_{4} \mathrm{~S} 445.0727$, found 445.0725 .

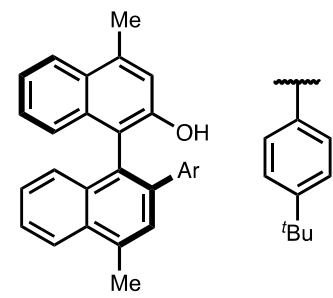

(R)-2'-(4-(tert-butyl)phenyl)-4,4'-dimethyl-[1,1'-binaphthalen]-2-ol ((R)-SI-G). Following the reported procedure, ${ }^{7}$ $(R)$-SI-G was isolated in $51 \%$ yield as a white solid. ${ }^{1} \mathrm{H}$ NMR $\left(600 \mathrm{MHz}, \mathrm{CD}_{2} \mathrm{Cl}_{2}\right) \delta 8.14(\mathrm{~d}, J=8.4 \mathrm{~Hz}, 1 \mathrm{H}), 7.98(\mathrm{~d}$, $J=8.4 \mathrm{~Hz}, 1 \mathrm{H}), 7.56(\mathrm{~s}, 1 \mathrm{H}), 7.54(\mathrm{t}, J=7.2 \mathrm{~Hz}, 1 \mathrm{H}), 7.33(\mathrm{t}, J=7.6 \mathrm{~Hz}, 1 \mathrm{H}), 7.29(\mathrm{t}, J=7.6 \mathrm{~Hz}, 1 \mathrm{H}), 7.23(\mathrm{t}, J=7.9$ $\mathrm{Hz}, 1 \mathrm{H}), 7.20(\mathrm{~d}, J=8.5 \mathrm{~Hz}, 1 \mathrm{H}), 7.15(\mathrm{~s}, 4 \mathrm{H}), 7.10(\mathrm{~d}, J=8.4 \mathrm{~Hz}, 1 \mathrm{H}), 7.03(\mathrm{~s}, 1 \mathrm{H}), 4.92(\mathrm{~s}, 1 \mathrm{H}), 2.85(\mathrm{~s}, 3 \mathrm{H}), 2.71$ $(\mathrm{s}, 3 \mathrm{H}), 1.22(\mathrm{~s}, 9 \mathrm{H}) .{ }^{13} \mathrm{C}$ NMR $\left(151 \mathrm{MHz}, \mathrm{CD}_{2} \mathrm{Cl}_{2}\right) \delta 151.30,150.34,141.62,138.95,137.22,136.38,135.31,134.12$, 
$133.08,130.38,128.89,128.72,127.26,127.11,126.96,126.88,126.56,126.15,125.28,124.96,124.91,118.43$, 116.76, 34.85, 31.53, 19.92, 19.79. HRMS (ESI) $\mathrm{m} / z$ [M-H] ${ }^{-}$calcd for $\mathrm{C}_{32} \mathrm{H}_{30} \mathrm{O} 429.2224$, found 429.2221 .

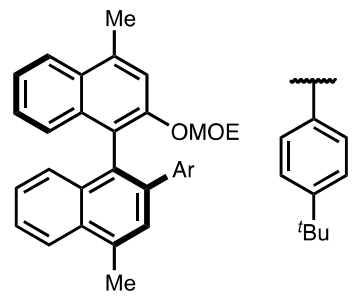

(R)-2-(4-(tert-butyl)phenyl)-2'-(ethoxymethoxy)-4,4'-dimethyl-1,1'-binaphthalene $((R)-S I-H)$. Following the reported procedure, ${ }^{7}(R)-\mathbf{S I}-\mathbf{H}$ was isolated in $91 \%$ yield as a white solid. ${ }^{1} \mathrm{H}$ NMR $\left(600 \mathrm{MHz}, \mathrm{CD}_{2} \mathrm{Cl}_{2}\right) \delta 8.11(\mathrm{~d}, J=$ $8.4 \mathrm{~Hz}, 1 \mathrm{H}), 7.99(\mathrm{~d}, J=8.4 \mathrm{~Hz}, 1 \mathrm{H}), 7.52(\mathrm{~s}, 1 \mathrm{H}), 7.48(\mathrm{t}, J=7.6 \mathrm{~Hz}, 1 \mathrm{H}), 7.41(\mathrm{~s}, 1 \mathrm{H}), 7.34(\mathrm{t}, J=7.6 \mathrm{~Hz}, 1 \mathrm{H}), 7.23$ $(\mathrm{t}, J=7.2 \mathrm{~Hz}, 1 \mathrm{H}), 7.20(\mathrm{t}, J=7.6 \mathrm{~Hz}, 1 \mathrm{H}), 7.16-7.11(\mathrm{~m}, 6 \mathrm{H}), 5.05(\mathrm{~d}, J=7.2 \mathrm{~Hz}, 1 \mathrm{H}), 4.91(\mathrm{~d}, J=7.2 \mathrm{~Hz}, 1 \mathrm{H}), 2.84$ $(\mathrm{s}, 3 \mathrm{H}), 2.76(\mathrm{~s}, 3 \mathrm{H}), 1.22(\mathrm{~s}, 9 \mathrm{H}), 1.03(\mathrm{t}, J=7.1 \mathrm{~Hz}, 3 \mathrm{H}) .{ }^{13} \mathrm{C}$ NMR $\left(151 \mathrm{MHz}, \mathrm{CD}_{2} \mathrm{Cl}_{2}\right) \delta 153.34,149.77,140.00$, 139.94, 136.73, 135.29, 134.65, 134.02, 132.53, 130.41, 130.06, 129.15, 129.03, 127.65, 126.71, 126.65, 126.31, $125.91,124.94,124.78,124.69,124.04,121.54,117.63,94.01,64.48,34.78,31.56,20.22,19.87,15.38$. HRMS (EI) $m / z[\mathrm{M}]^{+}$calcd for $\mathrm{C}_{35} \mathrm{H}_{36} \mathrm{O}_{2} 488.2715$, found 488.2720 .

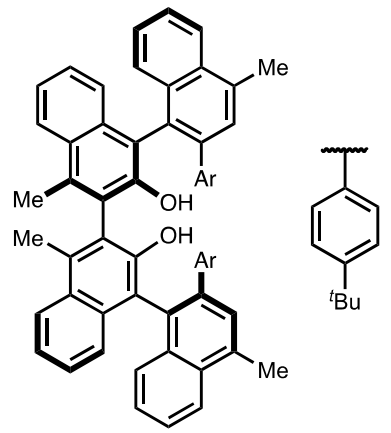

$\left(R, S_{c}, R\right)-34$. Following the homocoupling procedure described above, compound $\left(R, S_{c}, R\right)-34$ was isolated in $42 \%$ yield (over 2 steps after deprotection) as a single diastereoisomer. ${ }^{1} \mathrm{H} \mathrm{NMR}\left(600 \mathrm{MHz}, \mathrm{CD}_{2} \mathrm{Cl}_{2}\right) \delta 8.09(\mathrm{~d}, J=8.4 \mathrm{~Hz}, 2 \mathrm{H})$, $7.99(\mathrm{~d}, J=8.5 \mathrm{~Hz}, 2 \mathrm{H}), 7.54(\mathrm{~s}, 2 \mathrm{H}), 7.46(\mathrm{t}, J=8.2 \mathrm{~Hz}, 2 \mathrm{H}), 7.37(\mathrm{t}, J=7.6 \mathrm{~Hz}, 2 \mathrm{H}), 7.28(\mathrm{t}, J=7.3 \mathrm{~Hz}, 2 \mathrm{H}), 7.20-$ $7.12(\mathrm{~m}, 14 \mathrm{H}), 4.85(\mathrm{~s}, 2 \mathrm{H}), 2.82(\mathrm{~s}, 6 \mathrm{H}), 1.88(\mathrm{~s}, 6 \mathrm{H}), 1.29(\mathrm{~s}, 18 \mathrm{H}) .{ }^{13} \mathrm{C}$ NMR $\left(151 \mathrm{MHz}, \mathrm{CD}_{2} \mathrm{Cl}_{2}\right) \delta 150.26,149.15$, $141.26,139.24,136.00,135.62$, 135.38, 133.95, 132.91, 130.04, 129.07, 128.51, 128.06, 127.46, 127.04, 126.90, $126.37,126.31,125.25,125.22,125.17,124.86,123.80,117.24,34.90,31.69,19.90,15.72$. HRMS (ESI) $m / z$ [M+Na $]^{+}$ calcd for $\mathrm{C}_{64} \mathrm{H}_{58} \mathrm{O}_{2} 881.4329$, found 881.4319 . Configuration of the central axis could be established by $\mathrm{X}-\mathrm{Ray}$ analysis. 


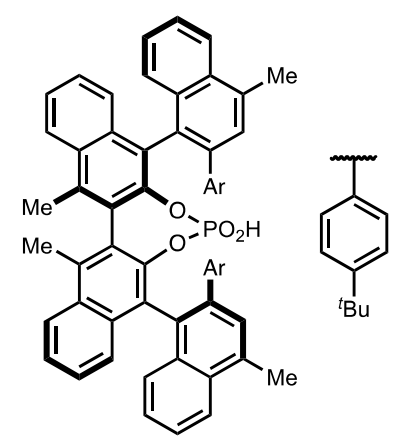

$\left(R, S_{c}, R\right)$-TAPa. Following the reported procedure, ${ }^{7}\left(R, S_{c}, R\right)$-TAPa was isolated in $82 \%$ yield as a white solid. ${ }^{10}{ }^{1} \mathrm{H}$ NMR $\left(400 \mathrm{MHz}, \mathrm{CDCl}_{3}\right) \delta 8.04(\mathrm{~d}, J=8.5 \mathrm{~Hz}, 2 \mathrm{H}), 7.91(\mathrm{~d}, J=8.4 \mathrm{~Hz}, 2 \mathrm{H}), 7.49(\mathrm{t}, J=7.1 \mathrm{~Hz}, 2 \mathrm{H}), 7.38(\mathrm{t}, J=7.4$ $\mathrm{Hz}, 2 \mathrm{H}), 7.31-7.28(\mathrm{~m}, 4 \mathrm{H}), 7.24(\mathrm{t}, J=3.8 \mathrm{~Hz}, 4 \mathrm{H}), 7.18(\mathrm{t}, J=7.6 \mathrm{~Hz}, 2 \mathrm{H}), 7.02(\mathrm{~d}, J=8.4 \mathrm{~Hz}, 4 \mathrm{H}), 6.88(\mathrm{~d}, J=8.3$ $\mathrm{Hz}, 4 \mathrm{H}), 3.81$ (br s, 1H), $2.63(\mathrm{~s}, 6 \mathrm{H}), 2.08(\mathrm{~s}, 6 \mathrm{H}), 1.22(\mathrm{~s}, 18 \mathrm{H}) \cdot{ }^{13} \mathrm{C}$ NMR $\left(151 \mathrm{MHz}, \mathrm{CDCl}_{3}\right) \delta 148.71,140.32$, $139.33,135.48,134.39,134.29,132.90,131.61,130.20,129.27,128.98,128.42,127.94,127.70,127.23,126.65$, $125.90,125.87,125.43,125.33,125.32,124.79,124.27,123.61,34.45,31.56,19.72,16.15 .{ }^{31} \mathrm{P}$ NMR (162 MHz, $\left.\mathrm{CDCl}_{3}\right) \delta$-4.37. HRMS (ESI) $\mathrm{m} / z$ [M-H] calcd for $\mathrm{C}_{64} \mathrm{H}_{57} \mathrm{O}_{4} \mathrm{P} 919.3922$, found 919.2905. 


\section{Summary data on the optimization of the enantioselective allenoate-Claisen} rearrangement.

\section{Catalyst screening}

$$
\text { (2.0 equiv) }
$$

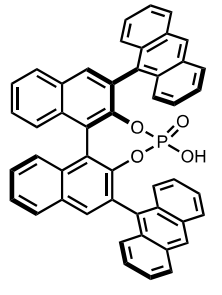

$65 \%$ yield, $8 \%$ ee

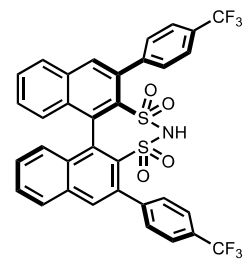

$82 \%$ yield, $<5 \%$ ee

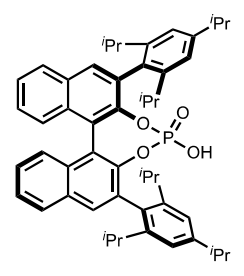

$72 \%$ yield, $14 \%$ ee

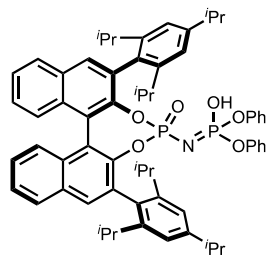

$72 \%$ yield, $<5 \%$ ee

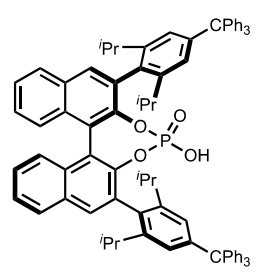

$64 \%$ yield, $6 \%$ ee

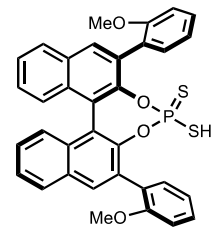

$59 \%$ yield, $<5 \%$ ee

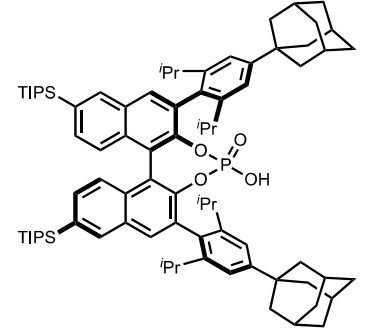

$57 \%$ yield, $14 \%$ ee

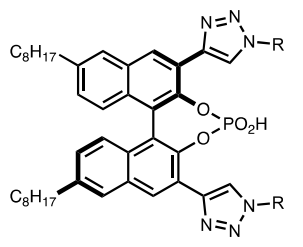

up to $87 \%$ yield, up to $18 \%$ ee ${ }^{*} 6$ catalysts screened
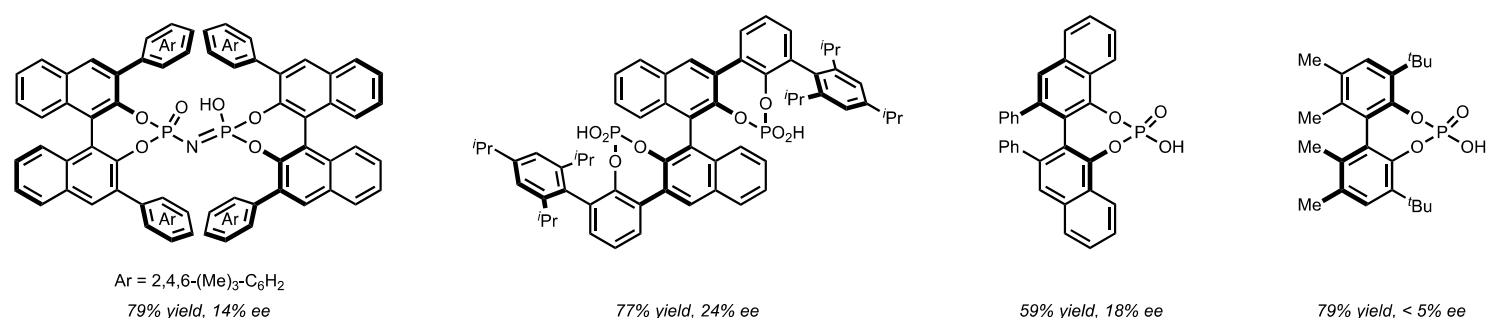

$77 \%$ yield, $24 \%$ ee

$59 \%$ yield, $18 \%$ ee

$79 \%$ yield, $<5 \%$ ee
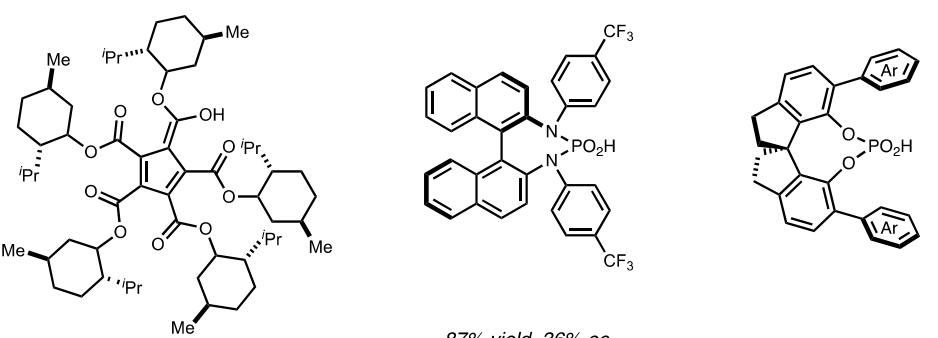

$87 \%$ yield, $36 \%$ ee *best among 9 catalysts

$<20 \%$ conversions
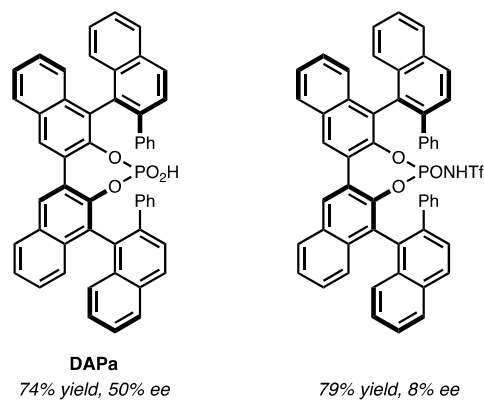

$79 \%$ yield, $8 \%$ ee 


\section{Ester substitution}

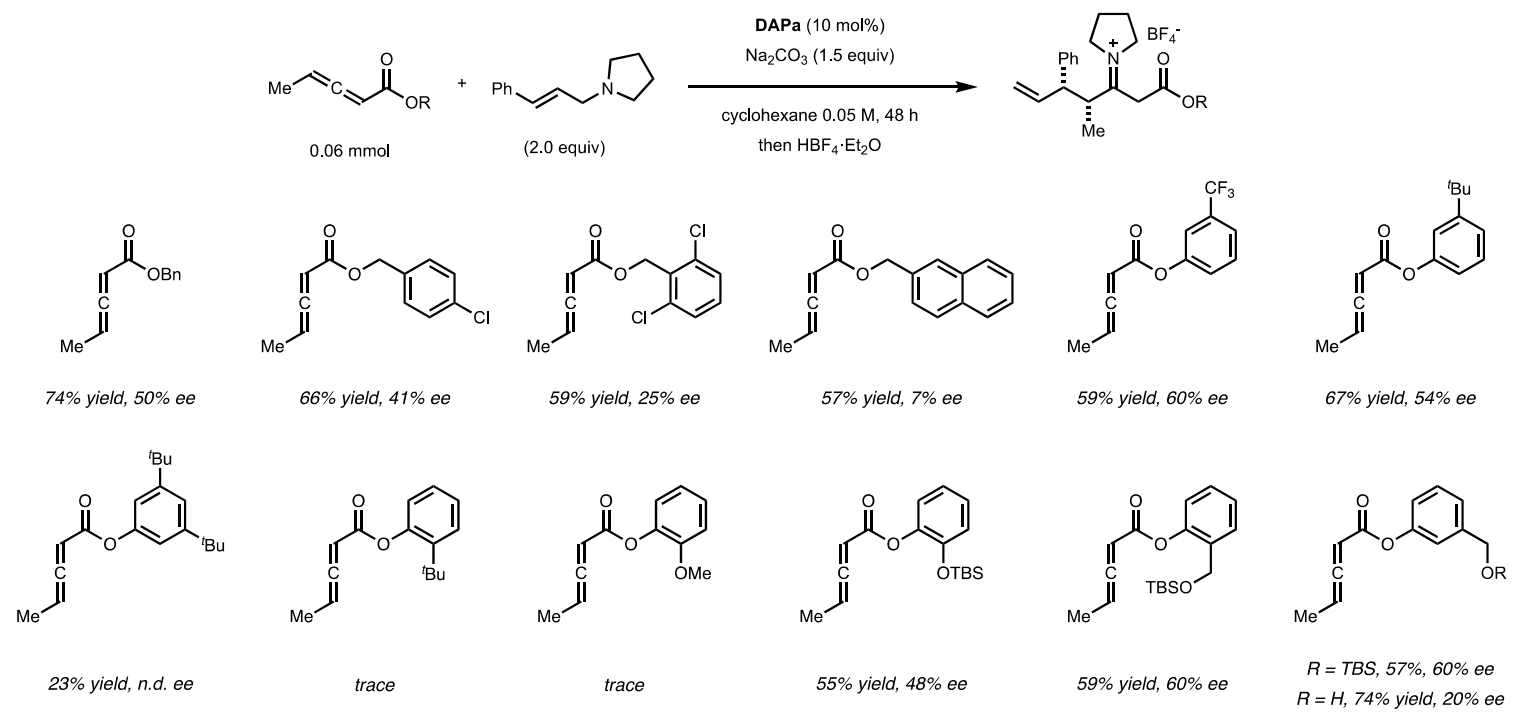

\section{Amine substitution}
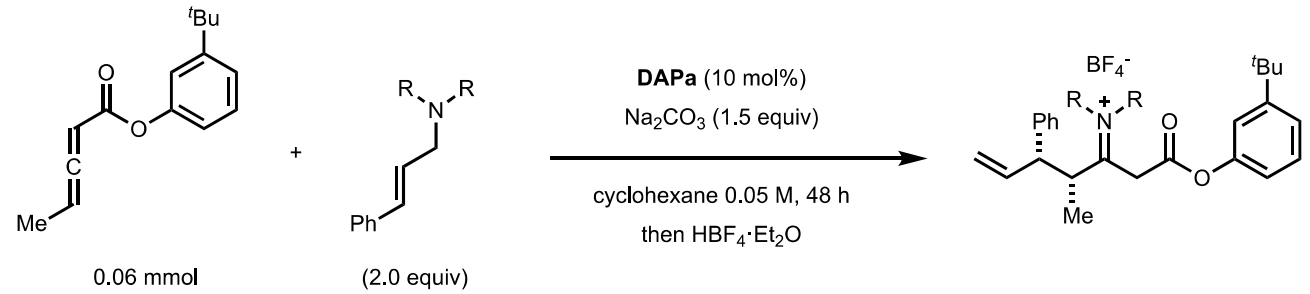<smiles>C(=C/c1ccccc1)\CN1CCCC1</smiles>

$67 \%$ yield, $54 \%$ ee<smiles>C(=C/c1ccccc1)\CN1CCCCC1</smiles>

$0 \%$ yield<smiles>C(=C/c1ccccc1)\CN1CCOCC1</smiles>

o\% yield<smiles>[2H]N([2H])C/C=C/[PH+](/C=C/CNP)c1ccccc1</smiles>

$\mathrm{R}=\mathrm{Me}, 74 \%$ yield, $12 \%$ ee

$\mathrm{R}=\mathrm{Et}, 0 \%$ yield

$\mathrm{R}=\mathrm{Bn}, 0 \%$ yield 


\section{DAP catalyst screening}
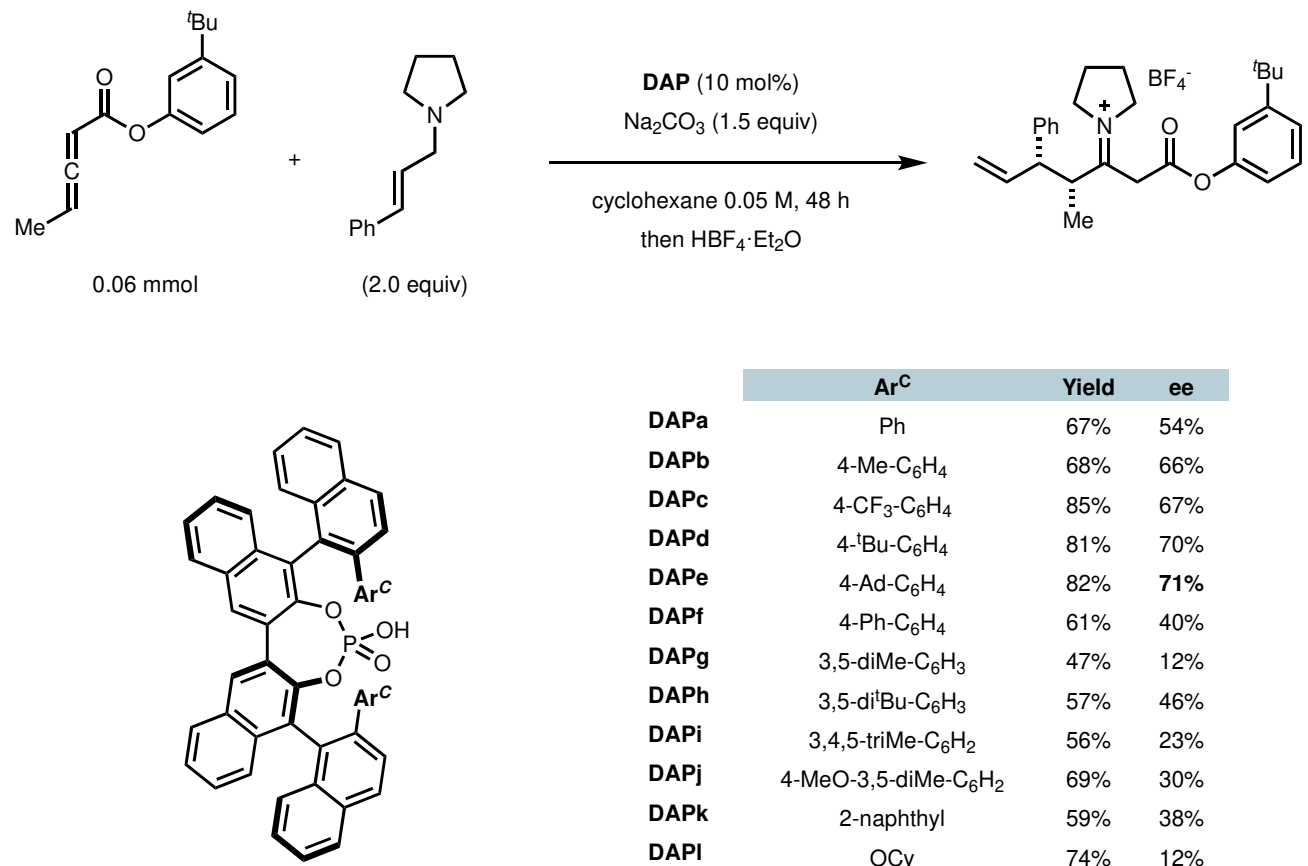

\begin{tabular}{|c|c|c|c|}
\hline & $\mathrm{Ar}^{\mathrm{C}}$ & Yield & ee \\
\hline DAPa & $\mathrm{Ph}$ & $67 \%$ & $54 \%$ \\
\hline $\mathrm{DAPb}$ & 4-Me- $\mathrm{C}_{6} \mathrm{H}_{4}$ & $68 \%$ & $66 \%$ \\
\hline DAPc & $4-\mathrm{CF}_{3}-\mathrm{C}_{6} \mathrm{H}_{4}$ & $85 \%$ & $67 \%$ \\
\hline DAPd & 4- ${ }^{\mathrm{t}} \mathrm{Bu}-\mathrm{C}_{6} \mathrm{H}_{4}$ & $81 \%$ & $70 \%$ \\
\hline DAPe & 4-Ad- $\mathrm{C}_{6} \mathrm{H}_{4}$ & $82 \%$ & $71 \%$ \\
\hline DAPf & 4-Ph- $\mathrm{C}_{6} \mathrm{H}_{4}$ & $61 \%$ & $40 \%$ \\
\hline DAPg & 3,5-diMe- $\mathrm{C}_{6} \mathrm{H}_{3}$ & $47 \%$ & $12 \%$ \\
\hline DAPh & 3,5-dit'Bu- $\mathrm{C}_{6} \mathrm{H}_{3}$ & $57 \%$ & $46 \%$ \\
\hline DAPi & 3,4,5-triMe- $\mathrm{C}_{6} \mathrm{H}_{2}$ & $56 \%$ & $23 \%$ \\
\hline DAPj & 4-MeO-3,5-diMe- $\mathrm{C}_{6} \mathrm{H}_{2}$ & $69 \%$ & $30 \%$ \\
\hline DAPk & 2-naphthyl & $59 \%$ & $38 \%$ \\
\hline DAPI & OCy & $74 \%$ & $12 \%$ \\
\hline DAPm & $\mathrm{Bn}$ & $54 \%$ & $21 \%$ \\
\hline
\end{tabular}

(from Table 1)

\section{Base screening}

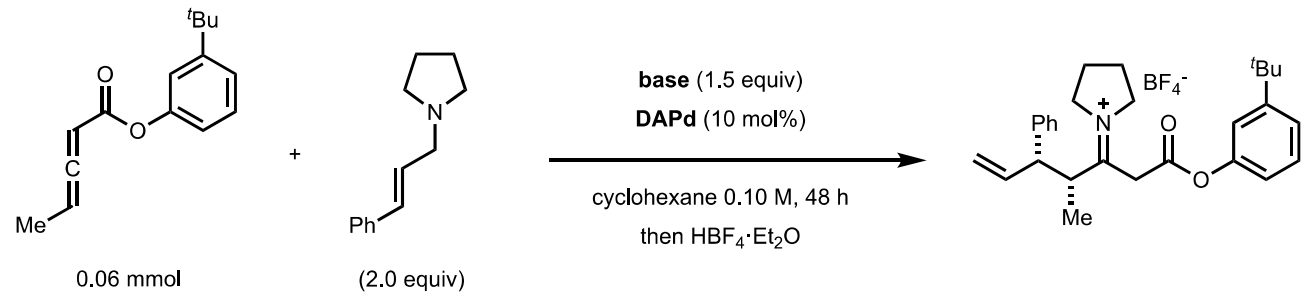

\begin{tabular}{ccccc} 
Entry & Base & Ionic radius $(\mathbf{p m})$ & Yield & ee \\
\hline 1 & $\mathrm{Li}_{2} \mathrm{CO}_{3}$ & 90 & $84 \%$ & $54 \%$ \\
2 & $\mathrm{Na}_{2} \mathrm{CO}_{3}$ & 116 & $81 \%$ & $70 \%$ \\
3 & $\mathrm{~K}_{2} \mathrm{CO}_{3}$ & 152 & $24 \%$ & $9 \%$ \\
4 & $\mathrm{Cs}_{2} \mathrm{CO}_{3}$ & 181 & $<5 \%$ & n.d. \\
5 & $\mathrm{MgCO}_{3}$ & 86 & $78 \%$ & $53 \%$ \\
6 & $\mathrm{CaCO}_{3}$ & 114 & $70 \%$ & $43 \%$ \\
7 & $\mathrm{Na}_{3} \mathrm{PO}_{4}$ & 116 & $69 \%$ & $67 \%$ \\
\hline
\end{tabular}

(from Table 1) 


\section{Concentration effect}

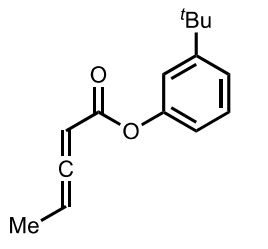

$0.06 \mathrm{mmol}$<smiles>C(=C/c1ccccc1)\CN1CCCC1</smiles>

(2.0 equiv)

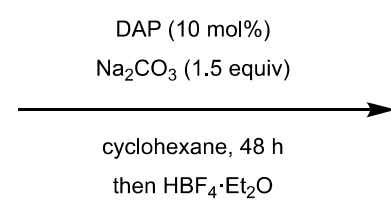

then $\mathrm{HBF}_{4} \cdot \mathrm{Et}_{2} \mathrm{O}$

$\begin{array}{ccc}\text { DAPa } & \text { DAPd } & \text { DAPe } \\ 42 \% \text { ee } & 70 \% \text { ee } & 71 \% \text { ee } \\ 47 \% \text { ee } & 78 \% \text { ee } & 90 \% \text { ee }\end{array}$

(from Table 1)

\section{Further optimization}
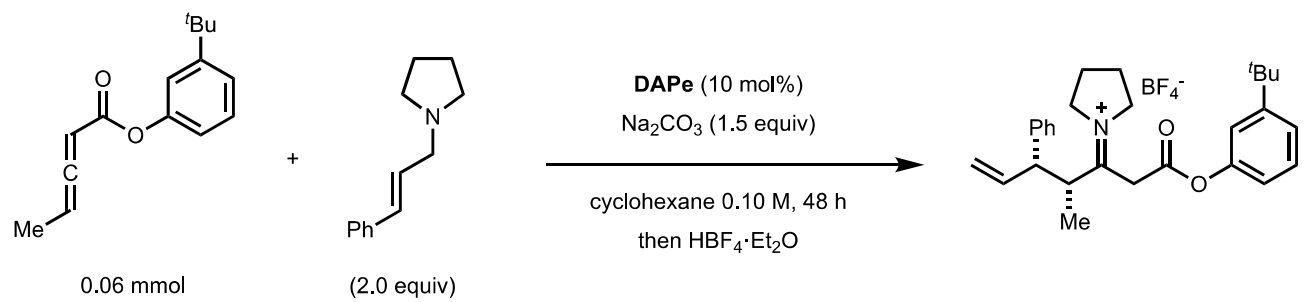

(2.0 equiv)

\begin{tabular}{cccc} 
Entry & Conditions & Yield & ee \\
\hline 1 & $0.05 \mathrm{M}$ & $70 \%$ & $71 \%$ \\
2 & as shown $0.10 \mathrm{M}$ & $83 \%$ & $90 \%$ \\
3 & $0.20 \mathrm{M}$ & $85 \%$ & $84 \%$ \\
4 & 1.5 equiv amine & $65 \%$ & $80 \%$ \\
5 & 1.0 equiv amine & $54 \%$ & $83 \%$ \\
6 & $10^{\circ} \mathrm{C}$ & $77 \%$ & $78 \%$ \\
7 & $35-38{ }^{\circ} \mathrm{C}$ & $86 \%$ & $84 \%$ \\
8 & w/o CPA & $14 \%$ & - \\
\end{tabular}

Table S1. Further optimization on the enantioselective allenoate-Claisen rearrangement. 


\section{Computational Details}

All DFT computations were performed using the Gaussian 16, Rev. A.03 suite ${ }^{11}$ using defaults unless noted otherwise. Optimized structures are visualized using CYLview. ${ }^{12}$ Non-covalent interactions were visualized with NCIPLOT. ${ }^{13}$

\section{Mechanistic computations (data for Figures 2, 3, 4A)}

The B97-D3 functional ${ }^{14}$ was used for geometry optimization and frequency analysis because of its accuracy in describing non-covalent interactions. ${ }^{15}$ Two regions of the system were treated with basis sets of varying augmentation, according to the following illustration: blue $=$ jun-cc-pVDZ, black $=c c-p V D Z .{ }^{16}$ Gas phase single point energies were obtained using $\omega \mathrm{B} 97 \mathrm{X}-\mathrm{D} / \mathrm{spAug}$-cc-pVTZ.${ }^{17}$ Corrections for the solvation energies were obtained from the difference between single points with the SMD continuum solvation mode ${ }^{18}$ for cyclohexane and a gas phase single point, both using B97-D3 with aug-cc-pVTZ and jun-cc-pVTZ basis sets for the blue and black regions, respectively. The "fine" integration grid setting was used in all computations and the density fitting approximation in all computations using B97-D3 to further increase computational efficiency.
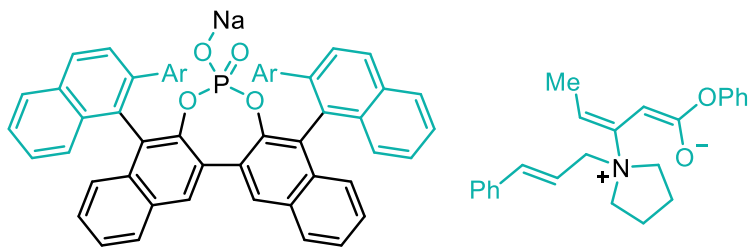

Conformers of higher energy were accounted for in all cases and are not included in the discussion. Stationary points were characterized as ground states or transition states by frequency analysis. Free energies were corrected using the approach described by Cramer and Truhlar ${ }^{19}$ and implemented by Paton and Funes-Ardoiz in the "Goodvibes" Python code. ${ }^{20}$ The reported free energies in $\mathrm{kcal} / \mathrm{mol}$ are the sum of the single point electronic energies, the solvation correction and the free energy corrections from the frequency analysis.

Additional consideration to the NCI in the transition states: One important feature in the transition states of DAPcatalyzed rearrangement is the stabilization of $\mathrm{Na}^{+}$via interactions with the arene of the allenoate ester. Experimentally, we found that trifluoroethyl allenoates also undergo the allenoate-Claisen rearrangement with high selectivity. While we did not compute the transition state with such a substrate, we demonstrate that trifluoroethyl allenoates can stabilize $\mathrm{Na}^{+}$via interaction with fluorine lone pairs via NCIPLOT visualizations of two model systems (Figure S1). The blue interaction isosurface between sodium (purple) and the arene (left: aryl ester enolate) or fluorine (right, trifluoroethyl ester enolate) indicates a strong, attractive interaction:
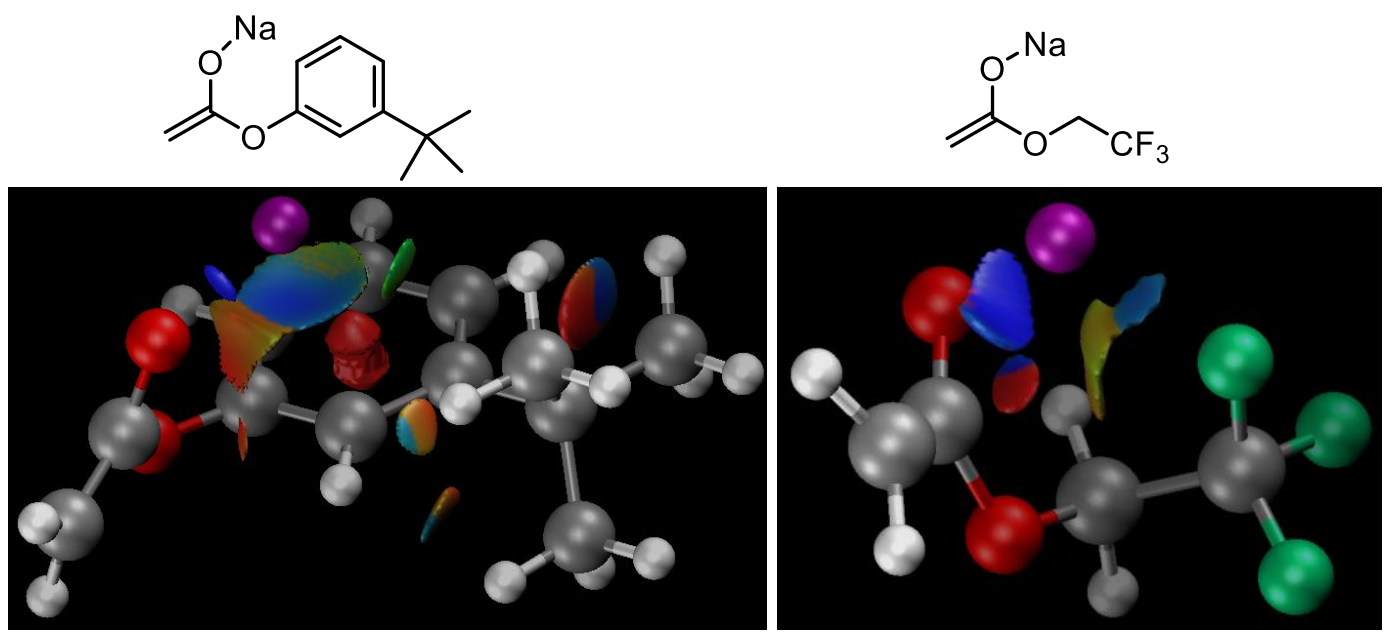

Figure S1. Visualization of the interactions stabilizing $\mathrm{Na}+$ in two model systems representing the two substrate classes employed in the synthetic part of the manuscript. 


\begin{tabular}{|c|c|c|c|c|c|c|c|c|}
\hline & NImag & $\begin{array}{c}E \text { B97-D3/ } \\
\text { (jun)-cc-pVDZ } \\
\text { in Hartree }\end{array}$ & $\begin{array}{c}G \text { B97-D3/ } \\
\text { (jun)-cc-pVDZ } \\
\text { in Hartree } \\
\text { qh-scaled: } \\
\text { Cramer,Truhlar }\end{array}$ & $\begin{array}{c}E \text { B97-D3/ } \\
\text { (aug/jun)-cc- } \\
\text { pVTZ (gas) } \\
\text { in Hartree }\end{array}$ & $\begin{array}{c}E \text { B97-D3/ } \\
\text { (aug/jun)-cc- } \\
\text { pVTZ (SMD: } \\
\text { cyclohexane) } \\
\text { in Hartree }\end{array}$ & $\begin{array}{c}E \omega B 97 X- \\
\text { D/spAug-cc- } \\
\text { pVTZ (gas) } \\
\text { in Hartree }\end{array}$ & $\begin{array}{c}G \text { corrected } \\
(\omega \mathrm{B} 97 \mathrm{X} \mathrm{TZ}, \\
\text { solv }) \\
\text { in Hartree }\end{array}$ & $\begin{array}{c}\Delta G \text { corrected } \\
(\omega \mathrm{B} 97 \mathrm{X} \mathrm{TZ}, \\
\text { solv }) \\
\text { in } \mathrm{kcal} / \mathrm{mol}\end{array}$ \\
\hline \multicolumn{9}{|l|}{ Uncatalyzed mechanism } \\
\hline Substrate_Amine & 0 & -560.031087 & -559.804600 & -560.172953 & -560.186594 & -560.347182 & -560.134336 & \\
\hline Substrate_Allenoate & 0 & -575.288582 & -575.151545 & -575.433348 & -575.445975 & -575.587682 & -575.463272 & \\
\hline Substrates_associated & 0 & -1135.341328 & -1134.951805 & -1135.626344 & -1135.647593 & -1135.956250 & -1135.587976 & 0.0 \\
\hline TS_Nucleophilic_addition & 1 & -1135.317426 & -1134.924622 & -1135.601334 & -1135.627232 & -1135.924398 & -1135.557492 & 19.1 \\
\hline Intermediate_1 & 0 & -1135.325200 & -1134.929796 & -1135.609374 & -1135.636052 & -1135.935588 & -1135.566862 & 13.2 \\
\hline Intermediate_2 & 0 & -1135.323261 & -1134.928032 & -1135.607470 & -1135.634208 & -1135.933428 & -1135.564937 & 14.5 \\
\hline TS_Rearrangement & 1 & -1135.317707 & -1134.923103 & -1135.600917 & -1135.625854 & -1135.914913 & -1135.545246 & 26.8 \\
\hline Product & 0 & -1135.380220 & -1134.984872 & -1135.662338 & -1135.684793 & -1135.999070 & -1135.626177 & -24.0 \\
\hline \multicolumn{9}{|l|}{$\mathrm{Na}^{+}$-catalyzed mechanism } \\
\hline $\mathrm{Na}+$ & 0 & -162.0570376 & -162.0714660 & -162.0622641 & -162.1192488 & -162.0869437 & -162.1583568 & \\
\hline Substrate_Allenoate_Na+ & 0 & -737.4146904 & -737.2784090 & -737.5650802 & -737.6119790 & -737.7442474 & -737.6548648 & \\
\hline Substrates_associated_Na+ & 0 & -1297.4918770 & -1297.1026300 & -1297.7831277 & -1297.8370086 & -1298.1340501 & -1297.7986840 & 0.0 \\
\hline TS_Nucleophilic_addition_Na+ & 1 & -1297.4803151 & -1297.0883670 & -1297.7696379 & -1297.8207252 & -1298.1159974 & -1297.7751366 & 14.8 \\
\hline Intermediate_1_Na+ & 0 & -1297.4759621 & -1297.0807180 & -1297.7652014 & -1297.8201240 & -1298.1189546 & -1297.7786331 & 12.6 \\
\hline Intermediate_2_Na+ & 0 & -1297.4889586 & -1297.0930910 & -1297.7784694 & -1297.8325282 & -1298.1325997 & -1297.7907909 & 5.0 \\
\hline TS_Rearrangement_Na+ & 1 & -1297.4672435 & -1297.0737430 & -1297.7556948 & -1297.8086109 & -1298.0967086 & -1297.7561242 & 26.7 \\
\hline Product_Na+ & 0 & -1297.5212639 & -1297.1261900 & -1297.8088670 & -1297.8623018 & -1298.1705927 & -1297.8289536 & -19.0 \\
\hline
\end{tabular}

Table S2. Electronic and free energies of stationary points relevant for the mechanism of the uncatalyzed and $\mathrm{Na}^{+}$-catalyzed reaction mechanism (Figure 2). 


\begin{tabular}{|c|c|c|c|c|c|c|c|c|c|c|}
\hline & NImag & $\begin{array}{c}E \text { B97-D3/ } \\
\text { (jun)-cc-pVDZ } \\
\text { in Hartree }\end{array}$ & $\begin{array}{c}G \text { B97-D3/ } \\
\text { (jun)-cc-pVDZ } \\
\text { in Hartree } \\
\text { qh-scaled: } \\
\text { Cramer,Truhlar } \\
\end{array}$ & $\begin{array}{c}E \text { B97-D3/ } \\
\text { (aug/jun)-cc- } \\
\text { pVTZ (gas) } \\
\text { in Hartree }\end{array}$ & $\begin{array}{c}E \text { B97-D3/ } \\
\text { (aug/jun)-cc- } \\
\text { pVTZ (SMD: } \\
\text { cyclohexane) } \\
\text { in Hartree } \\
\end{array}$ & $\begin{array}{c}E \omega B 97 X- \\
\text { D/spAug-cc- } \\
\text { pVTZ (gas) } \\
\text { in Hartree }\end{array}$ & $\begin{array}{l}G \text { corrected } \\
(\omega \mathrm{B} 97 \mathrm{X} \text { TZ, } \\
\text { solv })\end{array}$ & $\begin{array}{c}\Delta G \\
\text { in } \\
\mathrm{kcal} / \mathrm{mol}\end{array}$ & $\begin{array}{c}\Delta \Delta G^{\prime} \\
\text { in } \\
\mathrm{kcal} / \mathrm{mol}\end{array}$ & $\begin{array}{l}\text { Percentage } \\
\text { in } \\
\text { Boltzmann- } \\
\text { weighted } \\
\text { average } \\
\end{array}$ \\
\hline $\begin{array}{l}\text { R_DAPa_pre-major- } \\
\text { intermediate }\end{array}$ & 0 & -4018.317198 & -4017.233607 & -4019.256889 & -4019.199172 & -4020.234698 & -4019.208824 & 1.0 & & \\
\hline $\begin{array}{l}\text { S_DAPa_pre-major- } \\
\text { intermediate }\end{array}$ & 0 & -4018.322913 & -4017.238457 & -4019.263248 & -4019.205232 & -4020.236302 & -4019.209862 & 0.3 & & \\
\hline $\begin{array}{l}\text { R_DAPa_pre-minor- } \\
\text { intermediate }\end{array}$ & 0 & -4018.316588 & -4017.234092 & -4019.258212 & -4019.199719 & -4020.233886 & -4019.209883 & 0.3 & & \\
\hline $\begin{array}{l}\text { S_DAPa_pre-minor- } \\
\text { intermediate }\end{array}$ & 0 & -4018.324733 & -4017.240744 & -4019.263684 & -4019.206724 & -4020.237394 & -4019.210365 & 0.0 & & \\
\hline R_DAPa_major_TS_1 & 1 & -4018.311211 & -4017.229648 & -4019.249201 & -4019.192277 & -4020.212895 & -4019.188255 & 13.9 & 0.0 & $82 \%$ \\
\hline R_DAPa_major_TS_2 & 1 & -4018.300200 & -4017.219047 & -4019.243007 & -4019.184021 & -4020.205642 & -4019.183475 & 16.9 & 3.0 & $1 \%$ \\
\hline R_DAPa_major_TS_3 & 1 & -4018.302630 & -4017.221565 & -4019.243868 & -4019.185544 & -4020.200212 & -4019.177471 & 20.6 & 6.8 & $0 \%$ \\
\hline R_DAPa_major_TS_4 & 1 & -4018.295134 & -4017.214354 & -4019.237452 & -4019.179029 & -4020.197280 & -4019.174923 & 22.2 & 8.4 & $0 \%$ \\
\hline S_DAPa_major_TS_1 & 1 & -4018.307914 & -4017.226391 & -4019.248544 & -4019.190648 & -4020.209367 & -4019.185740 & 15.5 & 1.6 & $6 \%$ \\
\hline S_DAPa_major_TS_2 & 1 & -4018.307632 & -4017.226789 & -4019.247131 & -4019.189750 & -4020.202236 & -4019.178774 & 19.8 & 5.9 & $0 \%$ \\
\hline R_DAPa_minor_TS_1 & 1 & -4018.307141 & -4017.226116 & -4019.248239 & -4019.190202 & -4020.208543 & -4019.185555 & 15.6 & 1.7 & $5 \%$ \\
\hline R_DAPa_minor_TS_2 & 1 & -4018.304493 & -4017.223452 & -4019.246299 & -4019.187783 & -4020.207531 & -4019.185006 & 15.9 & 2.0 & $3 \%$ \\
\hline R_DAPa_minor_TS_3 & 1 & -4018.300412 & -4017.219149 & -4019.244500 & -4019.184066 & -4020.205761 & -4019.184932 & 16.0 & 2.1 & $2 \%$ \\
\hline R_DAPa_minor_TS_4 & 1 & -4018.296113 & -4017.215071 & -4019.237890 & -4019.178313 & -4020.195956 & -4019.174491 & 22.5 & 8.6 & $0 \%$ \\
\hline S_DAPa_minor_TS_1 & 1 & -4018.313006 & -4017.230688 & -4019.252001 & -4019.194594 & -4020.209367 & -4019.184455 & 16.3 & 2.4 & $1 \%$ \\
\hline S_DAPa_minor_TS_2 & 1 & -4018.301651 & -4017.220590 & -4019.242908 & -4019.184319 & -4020.201723 & -4019.179251 & 19.5 & 5.6 & $0 \%$ \\
\hline S_DAPa_minor_TS_3 & 1 & -4018.300638 & -4017.219480 & -4019.242731 & -4019.183466 & -4020.198398 & -4019.176505 & 21.2 & 7.4 & $0 \%$ \\
\hline S_DAPa_minor_TS_4 & 1 & -4018.301030 & -4017.219322 & -4019.240744 & -4019.184238 & -4020.199405 & -4019.174203 & 22.7 & 8.8 & $0 \%$ \\
\hline
\end{tabular}

Table S3. Electronic and free energies of stationary points relevant for the mechanism of the DAPa-catalyzed rearrangement (Figure 3). 


\begin{tabular}{|c|c|c|c|c|c|c|c|c|}
\hline & NImag & $\begin{array}{c}E \text { B97-D3/ } \\
\text { (jun)-cc-pVDZ } \\
\text { in Hartree }\end{array}$ & $\begin{array}{c}G \text { B97-D3/ } \\
\text { (jun)-cc-pVDZ } \\
\text { in Hartree } \\
\text { qh-scaled: } \\
\text { Cramer,Truhlar }\end{array}$ & $\begin{array}{c}E \text { B97-D3/ } \\
\text { (aug/jun)-cc- } \\
\text { pVTZ (gas) } \\
\text { in Hartree }\end{array}$ & $\begin{array}{l}E \text { B97-D3/ } \\
\text { (aug/jun)-cc- } \\
\text { pVTZ (SMD: } \\
\text { cyclohexane) } \\
\text { in Hartree }\end{array}$ & $\begin{array}{c}E \omega B 97 X- \\
\text { D/spAug-cc- } \\
\text { pVTZ (gas) } \\
\text { in Hartree }\end{array}$ & $\begin{array}{c}G \text { corrected } \\
(\omega \mathrm{B} 97 \mathrm{X} \mathrm{TZ}, \\
\text { solv }) \\
\text { in Hartree }\end{array}$ & $\begin{array}{c}\Delta G \text { corrected } \\
(\omega \mathrm{B} 97 \mathrm{X} \mathrm{TZ}, \\
\text { solv }) \\
\text { in } \mathrm{kcal} / \mathrm{mol}\end{array}$ \\
\hline \multicolumn{9}{|l|}{ Phosphoric acid } \\
\hline R_DAPa_H & 0 & -2642.570771 & -2641.953412 & -2643.149616 & -2643.194179 & -2643.808644 & -2643.235848 & 0.00 \\
\hline S_DAPa_H & 0 & -2642.568226 & -2641.950583 & -2643.147089 & -2643.191112 & -2643.804979 & -2643.231359 & 2.82 \\
\hline TS_inversion_DAPa_H & 1 & -2642.551328 & -2641.934275 & -2643.131398 & -2643.175819 & -2643.788735 & -2643.216104 & 12.39 \\
\hline \multicolumn{9}{|l|}{$\mathrm{Na}^{+}$-Phosphate } \\
\hline R_DAPa_Na & 0 & -2804.316477 & -2803.710850 & -2804.897802 & -2804.943731 & -2805.579885 & -2805.020188 & 0.00 \\
\hline S_DAPa_Na & 0 & -2804.323588 & -2803.717271 & -2804.902250 & -2804.947581 & -2805.580565 & -2805.019580 & 0.38 \\
\hline TS_inversion_DAPa_Na & 1 & -2804.303522 & -2803.697412 & -2804.886078 & -2804.932519 & -2805.567403 & -2805.007735 & 7.81 \\
\hline
\end{tabular}

Table S4. Electronic and free energies of stationary points relevant for the catalyst axis inversion (Figure 4A). 


\section{Parameterization modeling}

In the parameterization modeling, catalyst and allyl amine variations were considered. For both components, only structures where the variable group is an arene were considered for consistency. For ease of discussion, these groups are called $\mathbf{A} \mathbf{r}^{\mathbf{C}}$ and $\mathbf{A} \mathbf{r}^{\mathbf{A}}$ in the following:<smiles>Cc1ccc2ccccc2c1-c1c2c(cc3ccccc13)-c1cc3ccccc3c(-c3c([Te])ccc4ccccc34)c1OP(=O)(O)OP(=O)(O)O2</smiles><smiles>[Al]C(=[Te])C=CCN1CCCC1</smiles>

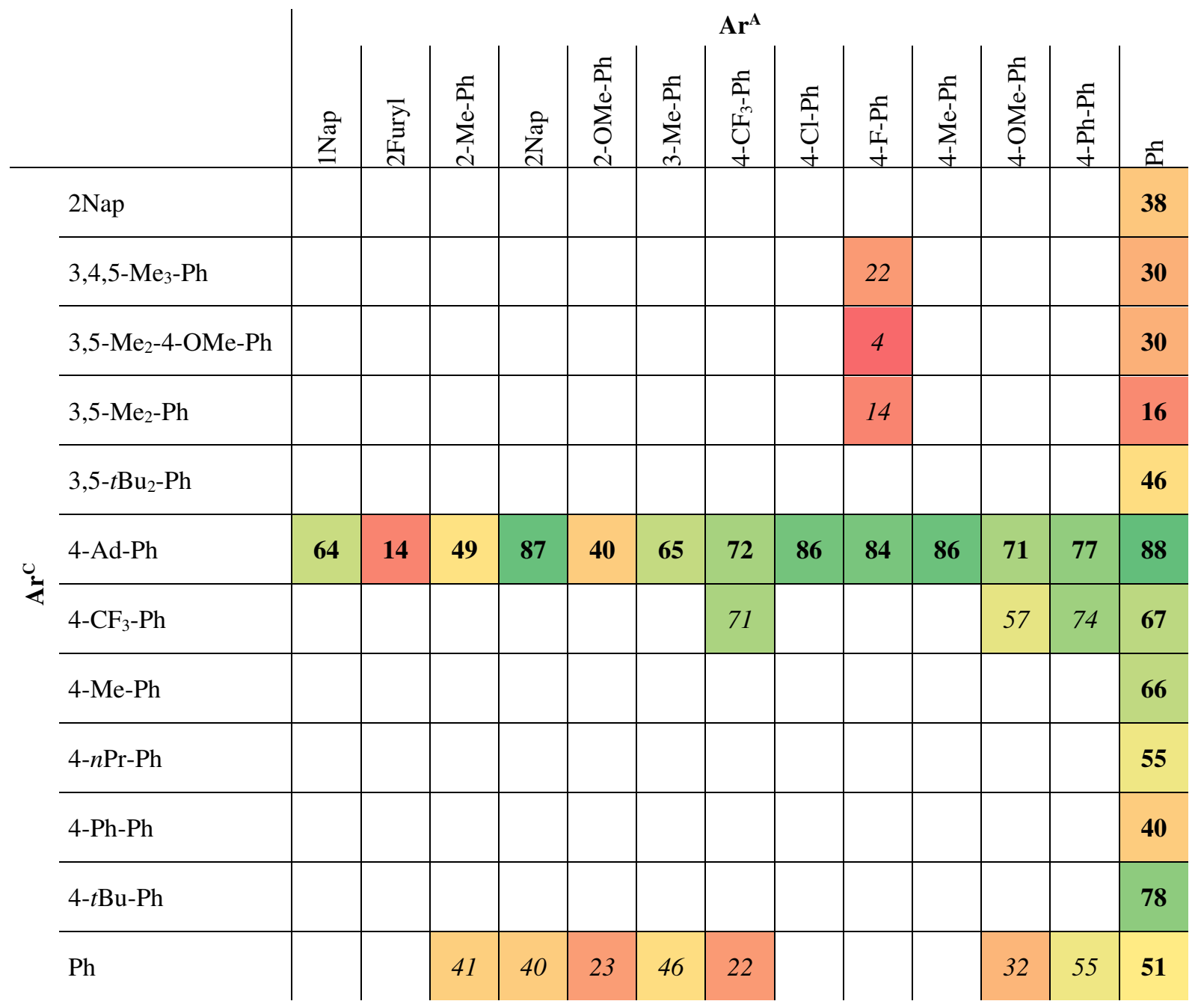

Table S5. Overview over the data used in modelling. Enantiomeric excess is given for all combinations of $\mathrm{Ar}^{\mathrm{C}}$ and $A r^{A}$ that have been employed together in the reaction. Numbers in boldface were used for model training and numbers in italics were used for model validation. 


\section{Descriptors for the variable substituents}

- Partial charges according to natural population analysis and natural bond orbital energies were obtained with the NBO 3.1 module $^{21}$ within Gaussian.

- Verloop's Sterimol parameters ${ }^{22}$ were obtained from optimized geometries using a modified version of Paton and Jackson's Python script ${ }^{23}$ using Bondi radii.

- The dispersion potential $P_{\text {int }}$ and related properties were obtained using a Python script from Pollice and Chen $^{24}$ based on promolecular densities from Multiwfn ${ }^{25}$ and dispersion parameters from DFT-D4. ${ }^{26}$

- All other descriptors were obtained from the Gaussian output directly.

\section{Descriptors for the catalyst variable substituent $\mathrm{Ar}^{\mathrm{C}}$}

The following properties were obtained from the corresponding benzene derivatives $\mathbf{A r}^{\mathrm{C}} \mathrm{H}$, using M06-2X-D3/6$31 \mathrm{G}(\mathrm{d}, \mathrm{p})^{27,28}$ for geometry optimization:

- Sterimol: $L, B_{1}, B_{5}$ with the 'capping' hydrogen atom as reference atom.

- $\quad P_{\text {int }}, \mathrm{d} P, P_{\min }, P_{\max }$ of the entire molecule

The partial charge of the carbonyl oxygen in the corresponding benzoic acid derivatives $\mathbf{A r}^{\mathbf{C}} \mathrm{COOH}$, according to reference (S29), was obtained using NBO at the M06-2X/def2-TZVP // B97-D3/jun-cc-pVDZ level, ${ }^{30}$ using the "fine" integration grid during optimization and the "ultrafine" integration grid for the single point.

\begin{tabular}{|c|c|c|c|c|c|c|c|c|}
\hline & $L$ & $B_{1}$ & $B_{5}$ & $P_{\text {int }}$ & $\mathrm{d} P$ & $P_{\min }$ & $P_{\max }$ & $q_{\mathrm{NBO}}(\mathrm{O})$ \\
\hline $2 \mathrm{Nap}$ & 8.58 & 1.70 & 4.44 & 17.36 & 4.53 & 10.65 & 28.94 & -0.593 \\
\hline 3,4,5--Me $-\mathrm{Ph}$ & 7.49 & 1.97 & 4.46 & 16.38 & 4.38 & 10.94 & 29.11 & -0.598 \\
\hline 3,5-- $\mathrm{Me}_{2}-4-\mathrm{OMe}-\mathrm{Ph}$ & 8.39 & 1.85 & 4.46 & 16.33 & 4.40 & 10.08 & 29.78 & -0.598 \\
\hline $3,5-\mathrm{Me}_{2}-\mathrm{Ph}$ & 6.43 & 1.89 & 4.44 & 15.76 & 4.13 & 10.38 & 28.11 & -0.595 \\
\hline $3,5-t \mathrm{Bu}_{2}-\mathrm{Ph}$ & 7.38 & 3.25 & 5.71 & 17.04 & 4.29 & 11.78 & 32.15 & -0.597 \\
\hline 4-Ad-Ph & 10.66 & 3.18 & 3.75 & 17.92 & 3.67 & 11.19 & 30.47 & -0.596 \\
\hline $4-\mathrm{CF}_{3}-\mathrm{Ph}$ & 7.77 & 2.18 & 3.24 & 16.65 & 3.79 & 10.23 & 27.24 & -0.582 \\
\hline 4-Me-Ph & 7.50 & 1.71 & 3.24 & 15.62 & 3.90 & 10.32 & 27.18 & -0.596 \\
\hline 4- $n \mathrm{Pr}-\mathrm{Ph}$ & 9.28 & 1.81 & 3.82 & 16.10 & 3.90 & 9.99 & 29.34 & -0.596 \\
\hline 4-Ph-Ph & 10.76 & 2.10 & 3.24 & 17.88 & 4.66 & 10.70 & 28.77 & -0.594 \\
\hline 4- $t \mathrm{Bu}-\mathrm{Ph}$ & 8.64 & 2.82 & 3.31 & 16.39 & 3.92 & 10.94 & 29.34 & -0.595 \\
\hline $\mathrm{Ph}$ & 6.45 & 1.70 & 3.24 & 15.46 & 3.61 & 10.26 & 26.21 & -0.592 \\
\hline
\end{tabular}

Table S6. Properties describing the catalyst as benzoic acid (first three) and as benzene (last 7) derivatives of $\mathrm{Ar}^{\mathrm{C}}$. 
Descriptors for the allyl amines and the variable substituent Ar ${ }^{A}$

A conformational search was performed for all allyl amines using the OPLS3 force field and low-frequency-mode conformational search in Schrödinger MacroModel ${ }^{31}$ with Python scripts based on work by Ermanis and Goodman. ${ }^{32}$ For each compound, five conformers were taken forward to DFT analysis. Properties were obtained at the M06-2X/def2-TZVP // B3LYP-D3/6-31G(d,p) level using the "fine" integration grid during optimization and the "ultrafine" integration grid for the single point.

Partial charge (NBO) for $\mathrm{N}, \mathrm{C} \alpha, \mathrm{C} \beta, \mathrm{C} \gamma$

Orbital energies and occupancies (NBO) for $\operatorname{LP}(N), \pi(C=C), \pi^{*}(C=C)$

Sterimol: $L, B_{1}, B_{5}$ of $\mathbf{A} \mathbf{r}^{\mathbf{A}}$ with $\mathrm{C} \gamma$ as reference atom

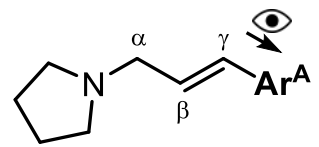

$P_{\text {int }}, \mathrm{d} P, P_{\min }, P_{\max }$ were obtained from the corresponding benzene derivative $\mathbf{A} \mathbf{r}^{\mathrm{A}} \mathrm{H}$, using M06-2X-D3/6-31G(d,p) for geometry optimization.

\begin{tabular}{|c|c|c|c|c|c|c|c|c|c|c|c|c|c|c|c|c|}
\hline & \multicolumn{4}{|c|}{$q_{\mathrm{NBO}}$} & \multicolumn{2}{|c|}{$\pi(\mathrm{C}=\mathrm{C})$} & \multicolumn{2}{|c|}{$\pi^{*}(\mathrm{C}=\mathrm{C})$} & \multicolumn{4}{|c|}{ Sterimol } & \multicolumn{4}{|c|}{ dispersion potential } \\
\hline & $\mathrm{C} \alpha$ & $C \beta$ & $\mathrm{C} \gamma$ & $\mathrm{N}$ & occ & $E$ & occ & E & $L$ & $B_{1}$ & $B_{5}$ & $B_{1} \times B_{5}$ & Pint & $\mathrm{dP}$ & Pmin & Pmax \\
\hline 1Nap & -0.247 & -0.154 & -0.212 & -0.427 & 1.928 & -0.328 & 0.067 & 0.099 & 6.95 & 1.70 & 5.72 & 9.74 & 17.36 & 4.53 & 10.65 & 28.94 \\
\hline 2Furyl & -0.247 & -0.157 & -0.248 & -0.430 & 1.918 & -0.323 & 0.100 & 0.087 & 6.12 & 1.70 & 3.16 & 5.37 & 14.73 & 3.33 & 9.28 & 22.99 \\
\hline 2-Me-Ph & -0.247 & -0.158 & -0.214 & -0.431 & 1.926 & -0.324 & 0.073 & 0.100 & 6.86 & 1.78 & 4.46 & 7.95 & 15.62 & 3.90 & 10.32 & 27.18 \\
\hline 2Nap & -0.245 & -0.157 & -0.202 & -0.428 & 1.917 & -0.322 & 0.081 & 0.091 & 8.96 & 1.70 & 4.56 & 7.75 & 17.36 & 4.53 & 10.65 & 28.94 \\
\hline 2-OMe-Ph & -0.245 & -0.160 & -0.203 & -0.428 & 1.922 & -0.317 & 0.073 & 0.101 & 6.86 & 1.75 & 5.45 & 9.54 & 15.92 & 4.11 & 9.34 & 27.14 \\
\hline 3-Me-Ph & -0.245 & -0.158 & -0.201 & -0.428 & 1.920 & -0.320 & 0.078 & 0.094 & 6.85 & 1.75 & 4.44 & 7.77 & 15.62 & 3.90 & 10.32 & 27.18 \\
\hline $4-\mathrm{CF}_{3}-\mathrm{Ph}$ & -0.248 & -0.139 & -0.214 & -0.428 & 1.914 & -0.333 & 0.073 & 0.080 & 8.21 & 2.03 & 3.34 & 6.76 & 16.65 & 3.79 & 10.23 & 27.24 \\
\hline 4-Cl-Ph & -0.246 & -0.152 & -0.207 & -0.429 & 1.919 & -0.327 & 0.078 & 0.086 & 8.18 & 1.72 & 3.34 & 5.75 & 17.26 & 3.98 & 10.38 & 27.23 \\
\hline 4-F-Ph & -0.245 & -0.159 & -0.203 & -0.429 & 1.923 & -0.325 & 0.081 & 0.089 & 7.48 & 1.70 & 3.33 & 5.66 & 15.81 & 3.62 & 10.16 & 26.07 \\
\hline 4-Me-Ph & -0.244 & -0.162 & -0.199 & -0.428 & 1.921 & -0.319 & 0.080 & 0.094 & 7.91 & 1.76 & 3.33 & 5.85 & 15.62 & 3.90 & 10.32 & 27.18 \\
\hline 4-OMe-Ph & -0.243 & -0.170 & -0.194 & -0.428 & 1.924 & -0.317 & 0.085 & 0.095 & 8.94 & 1.81 & 3.34 & 6.03 & 15.92 & 4.11 & 9.34 & 27.14 \\
\hline 4-Ph-Ph & -0.245 & -0.155 & -0.203 & -0.428 & 1.918 & -0.322 & 0.080 & 0.091 & 11.18 & 2.05 & 3.46 & 7.07 & 17.88 & 4.66 & 10.70 & 28.77 \\
\hline $\mathrm{Ph}$ & -0.245 & -0.157 & -0.204 & -0.429 & 1.920 & -0.321 & 0.078 & 0.092 & 6.86 & 1.70 & 3.34 & 5.68 & 15.46 & 3.61 & 10.26 & 26.21 \\
\hline
\end{tabular}

Table S7. Descriptors for the allyl amines. 


\section{Interaction properties}

Previously, we have reported the utility of equilibrium distances $D$ and energies $E$ of non-covalent interactions between two probe molecules representing reaction components as descriptors in multivariable regression analysis. ${ }^{33}$

In the updated procedure used herein, this process involves:

- Selecting representative probes by truncating the reactants/catalyst to the smallest meaningful fragments that are hypothesized to engage in NCI during the reaction. Here, these $\operatorname{are} \mathbf{A \mathbf { r } ^ { \mathbf { C } }}$ and $\mathbf{A} \mathbf{r}^{\mathbf{A}}$, the isolated arene residues of catalyst and allyl amine, respectively, capped with a $\mathrm{H}$, and benzene as a probe for a naphthyl portion of the catalyst. The structures of the probe molecules are fully optimized using M06-2X-D3/6-31G(d,p).

- $\quad$ Arranging the probe molecules into a defined interaction geometry. Here, the following orientations are aimed at representing the interactions in the transition states shown in Figure 3 (in the illustrations, $\mathbf{A r}^{\mathbf{C}}$ is blue, $\mathbf{A} \mathbf{r}^{\mathbf{A}}$ is yellow and the hydrogen atom that caps the arene where it would be connected to the rest of the molecule is grey):

- The edge-to-face complex between $\mathbf{A} \mathbf{r}^{\mathbf{A}}$ interacting through the $\pi$-face and $\mathbf{A r}^{\mathrm{C}}$ interacting with the edge, defined as the center of the hydrogens at the 2- and 3-positions. This center is oriented over the ring centroid of $\mathbf{A} \mathbf{r}^{\mathbf{A}}$ and $\mathbf{A} \mathbf{r}^{\mathbf{C}}$ then aligned along the ipso/para carbons of $\mathbf{A} \mathbf{r}^{\mathbf{A}}$ at a $90^{\circ}$ angle to the plane of $\mathbf{A} \mathbf{r}^{\mathrm{A}}$.

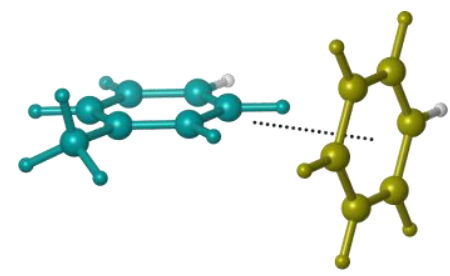

- The edge-to-face complex between benzene interacting through the $\pi$-face and $\mathbf{A} \mathbf{r}^{\mathrm{A}}$ interacting with the edge, defined as the center of the hydrogens at the 2- and 3positions. This center is oriented over the ring centroid of benzene and $\mathbf{A} \mathbf{r}^{\mathbf{A}}$ then aligned along the ipsolpara carbons of $\mathbf{A} \mathbf{r}^{\mathbf{A}}$ at a $90^{\circ}$ angle to the plane of $\mathbf{A} \mathbf{r}^{\mathbf{A}}$.

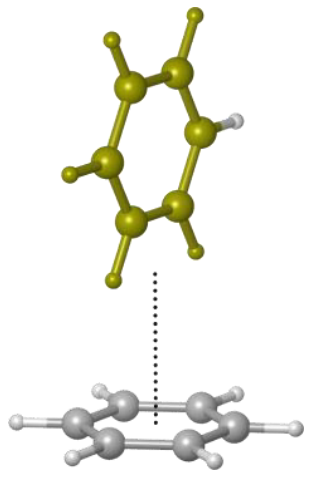

- The sandwich complex between $\mathbf{A} \mathbf{r}^{\mathbf{A}}$ and $\mathbf{A} \mathbf{r}^{\mathbf{C}}$ both interacting through the $\pi$-face. The probes are placed parallel to each other with the centroids on top of each other. The rings are oriented so that the ipso carbon of one is over an ortho carbon of the other.

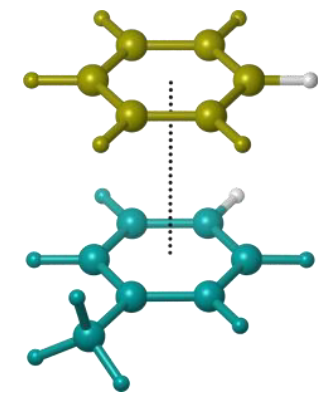

For each combination of probes there can be several variations depending on the symmetry of the probe molecules. In these cases, interaction distances and energies are determined separately for each variation. For example, the sandwich complex between $\mathbf{A} \mathbf{r}^{\mathbf{C}}=4-\mathrm{Ad}-\mathrm{Ph}$ and $\mathbf{A} \mathbf{r}^{\mathrm{A}}=4-\mathrm{OMe}-\mathrm{Ph}$ has four specific orientations (the orientation was rotated relative to the above illustration for clarity):

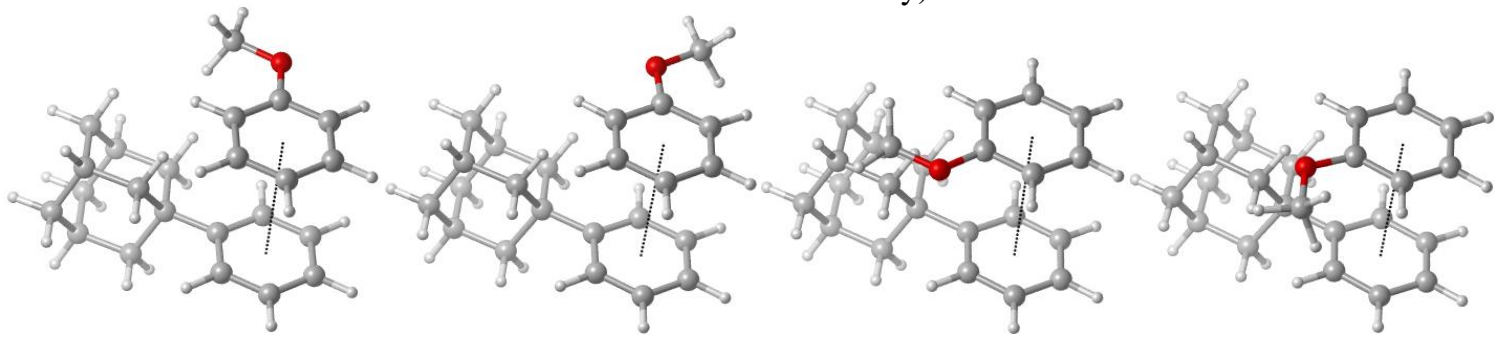

For each of these interaction geometries, the interaction energy is determined at a series of distances between both probes. In the sandwich complexes, the distance is scanned along the axis defined by both ring centroids. In the edge-to-face complexes, the axis is defined by the centroid of the $\pi$-component and the center of the hydrogens 
at the 2- and 3-positions. Only the distance between the probe molecules is changed, otherwise keeping the geometries of the probe molecules identical.

At each distance, the interaction energy $E$ is obtained by a single point computation at the sSAPT0/jun-cc-pVDZ

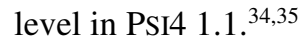

- $\quad$ In an iterative procedure, new scan points are generated by varying the distance $D$. A polynomial function is fitted for $E(D)$ and the minimum is determined by interpolation. This is used to generate the next scan point until 5 single points are present within \pm 20 pm of the interpolated minimum-energy distance, at which point a fourthdegree polynomial fit is a reasonably accurate approximation of the $E(D)$ function in that distance interval.
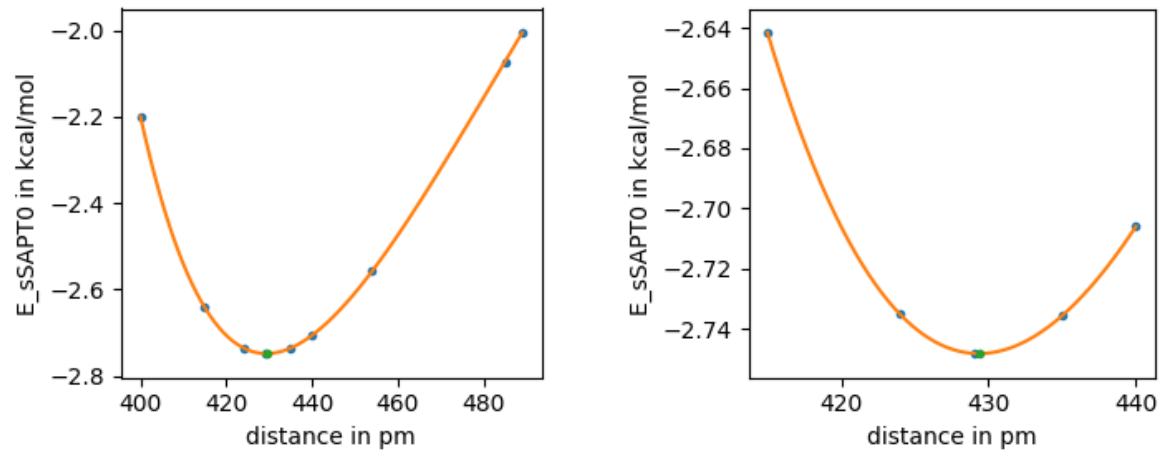

Example: sandwich complex between 4-Ad-PhH and 4-OMe-PhH (variation shown on the left above). Blue: distance scan single point energies. Orange: the polynomial fit interpolation. Green: the minimum of the interpolation function.

Left: All 9 scan points that were computed as part of the iterative procedure, right: the selection of 5 points within \pm 20 pm of the minimum that are used to determine the equilibrium distance and interaction energy by interpolation with a $4^{\text {th }}$-degree polynomial.

- We report the interpolated equilibrium distance as well as the energy components and the total interaction energy at that distance for each interaction geometry in tables S9-S11. Table S12 lists probe combinations that are equivalent and only listed once in Table S11, as well as any other combinations of $\mathbf{A} \mathbf{r}^{\mathbf{C}}$ and $\mathbf{A} \mathbf{r}^{\mathbf{A}}$ that is represented by these probes. In the "Coordinates of stationary points" section, we further depict $E(D)$ diagrams as explained in the example above. The coordinates of the interaction geometries are reported along with a graphical depiction at the equilibrium distance.

- In the regression modelling, the lowest ("min") and highest ("max") interaction energies / distances across all specific variations of each probe combination/orientation were used as features, along with the Boltzmannweighted average of the interaction energies / distances, weighted by the energies of interaction. These values are given in Table S8. 
Interaction descriptors

\begin{tabular}{|c|c|c|c|c|c|c|c|c|c|c|c|c|c|c|c|c|c|c|c|}
\hline \multirow[b]{2}{*}{$A \mathbf{r}^{\mathrm{C}}$} & \multirow[b]{2}{*}{$A r^{A}$} & \multicolumn{6}{|c|}{ Edge-to-face complex $A r_{\text {side }}^{C}-A r_{\pi}^{A}$} & \multicolumn{6}{|c|}{ Edge-to-face complex $A r_{\text {side }}^{A}-P h_{\pi}$} & \multicolumn{6}{|c|}{ Sandwich complex $A r_{\pi}^{C}-A r_{\pi}^{A}$} \\
\hline & & $D_{\text {Boltz }}$ & $E_{\text {Boltz }}$ & $D_{\min }$ & $E_{\min }$ & $D_{\max }$ & $E_{\max }$ & $D_{\text {Boltz }}$ & $E_{\text {Boltz }}$ & $D_{\min }$ & $E_{\min }$ & $D_{\max }$ & $E_{\max }$ & $D_{\text {Boltz }}$ & $E_{\text {Boltz }}$ & $D_{\min }$ & $E_{\min }$ & $D_{\max }$ & $E_{\max }$ \\
\hline \multicolumn{20}{|l|}{ Training set } \\
\hline 2Nap & $\mathrm{Ph}$ & 341.9 & -2.652 & 291.2 & -2.729 & 390.4 & -2.572 & 292.8 & -2.351 & 292.7 & -2.351 & 292.8 & -2.351 & 368.5 & -3.386 & 368.5 & -3.386 & 368.5 & -3.386 \\
\hline 3,4,5- $\mathrm{Me}_{3}-\mathrm{Ph}$ & $\mathrm{Ph}$ & 405.9 & -1.781 & 405.9 & -1.781 & 405.9 & -1.781 & 292.8 & -2.351 & 292.7 & -2.351 & 292.8 & -2.351 & 372.5 & -2.989 & 372.5 & -2.989 & 372.5 & -2.989 \\
\hline 3,5- $\mathrm{Me}_{2}-4-\mathrm{OMe}-\mathrm{Ph}$ & $\mathrm{Ph}$ & 403.3 & -1.763 & 403.3 & -1.763 & 403.3 & -1.763 & 292.8 & -2.351 & 292.7 & -2.351 & 292.8 & -2.351 & 377.6 & -2.993 & 369.5 & -3.149 & 422.6 & -2.140 \\
\hline $3,5-\mathrm{Me}_{2}-\mathrm{Ph}$ & $\mathrm{Ph}$ & 403.4 & -1.675 & 403.4 & -1.675 & 403.4 & -1.675 & 292.8 & -2.351 & 292.7 & -2.351 & 292.8 & -2.351 & 378.5 & -2.403 & 378.5 & -2.403 & 378.5 & -2.403 \\
\hline 3,5-tBu $2-\mathrm{Ph}$ & $\mathrm{Ph}$ & 520.1 & -1.748 & 509.9 & -1.879 & 539.5 & -1.498 & 292.8 & -2.351 & 292.7 & -2.351 & 292.8 & -2.351 & 448.6 & -2.301 & 448.6 & -2.301 & 448.6 & -2.301 \\
\hline 4-Ad-Ph & $1 \mathrm{Nap}$ & 276.3 & -4.183 & 275.9 & -4.272 & 276.8 & -4.054 & 371.2 & -2.587 & 289.8 & -2.592 & 532.1 & -2.585 & 436.4 & -3.497 & 412.7 & -3.762 & 467.1 & -3.241 \\
\hline 4-Ad-Ph & 2Furyl & 290.7 & -2.243 & 290.6 & -2.295 & 291.0 & -2.179 & 200.7 & -2.358 & 198.8 & -2.489 & 201.0 & -1.184 & 402.6 & -1.947 & 394.0 & -2.167 & 417.0 & -1.683 \\
\hline 4-Ad-Ph & 2-Me-Ph & 280.3 & -3.686 & 280.0 & -3.759 & 280.7 & -3.589 & 324.6 & -2.127 & 293.1 & -2.282 & 403.4 & -1.739 & 425.0 & -2.671 & 423.2 & -2.780 & 427.2 & -2.417 \\
\hline 4-Ad-Ph & 2Nap & 274.7 & -5.806 & 273.7 & -5.970 & 278.0 & -5.264 & 347.3 & -2.660 & 291.2 & -2.729 & 390.4 & -2.572 & 440.0 & -3.716 & 412.3 & -3.840 & 481.4 & -3.476 \\
\hline 4-Ad-Ph & 2-OMe-Ph & 281.6 & -3.560 & 280.7 & -3.729 & 282.8 & -3.343 & 332.6 & -2.194 & 290.5 & -2.323 & 501.2 & -0.475 & 429.1 & -2.632 & 422.5 & -2.783 & 458.9 & -2.350 \\
\hline 4-Ad-Ph & 3-Me-Ph & 279.3 & -3.844 & 279.0 & -3.930 & 279.7 & -3.722 & 324.6 & -2.127 & 293.1 & -2.282 & 403.4 & -1.739 & 438.0 & -2.312 & 425.6 & -2.455 & 534.4 & -1.229 \\
\hline 4-Ad-Ph & $4-\mathrm{CF}_{3}-\mathrm{Ph}$ & 322.7 & -3.044 & 318.5 & -3.201 & 333.8 & -2.640 & 285.1 & -3.261 & 285.1 & -3.269 & 285.1 & -3.257 & 425.7 & -3.493 & 419.0 & -3.577 & 464.9 & -2.522 \\
\hline 4-Ad-Ph & 4-Cl-Ph & 285.8 & -3.961 & 283.9 & -4.110 & 290.1 & -3.616 & 287.4 & -2.967 & 287.4 & -2.967 & 287.4 & -2.967 & 427.1 & -2.476 & 426.9 & -2.491 & 427.4 & -2.461 \\
\hline 4-Ad-Ph & 4-F-Ph & 284.6 & -3.231 & 283.6 & -3.340 & 286.3 & -3.056 & 289.3 & -2.788 & 289.3 & -2.788 & 289.3 & -2.788 & 411.2 & -3.271 & 403.5 & -3.350 & 421.8 & -3.163 \\
\hline 4-Ad-Ph & 4-Me-Ph & 292.7 & -3.702 & 288.7 & -3.831 & 300.3 & -3.465 & 292.2 & -2.392 & 292.2 & -2.392 & 292.2 & -2.392 & 424.3 & -2.744 & 423.2 & -2.780 & 425.3 & -2.703 \\
\hline 4-Ad-Ph & 4-OMe-Ph & 284.5 & -4.228 & 284.3 & -4.347 & 285.0 & -4.021 & 288.9 & -2.708 & 287.9 & -2.848 & 291.0 & -2.420 & 429.7 & -2.705 & 422.5 & -2.783 & 458.9 & -2.350 \\
\hline 4-Ad-Ph & 4-Ph-Ph & 340.7 & -4.271 & 333.0 & -4.425 & 359.7 & -3.885 & 286.4 & -3.030 & 286.4 & -3.030 & 286.5 & -3.030 & 415.8 & -4.634 & 410.4 & -4.872 & 502.3 & -2.694 \\
\hline 4-Ad-Ph & $\mathrm{Ph}$ & 284.8 & -3.224 & 284.4 & -3.291 & 285.2 & -3.139 & 292.8 & -2.351 & 292.7 & -2.351 & 292.8 & -2.351 & 431.7 & -2.032 & 431.7 & -2.032 & 431.7 & -2.032 \\
\hline $4-\mathrm{CF}_{3}-\mathrm{Ph}$ & $\mathrm{Ph}$ & 285.1 & -3.263 & 285.1 & -3.269 & 285.1 & -3.257 & 292.8 & -2.351 & 292.7 & -2.351 & 292.8 & -2.351 & 382.4 & -2.816 & 382.4 & -2.816 & 382.4 & -2.816 \\
\hline 4-Me-Ph & $\mathrm{Ph}$ & 292.2 & -2.392 & 292.2 & -2.392 & 292.2 & -2.392 & 292.8 & -2.351 & 292.7 & -2.351 & 292.8 & -2.351 & 384.4 & -1.947 & 384.4 & -1.947 & 384.4 & -1.947 \\
\hline 4-nPr-Ph & $\mathrm{Ph}$ & 289.7 & -2.647 & 287.5 & -2.849 & 291.9 & -2.424 & 292.8 & -2.351 & 292.7 & -2.351 & 292.8 & -2.351 & 399.4 & -2.019 & 380.9 & -2.255 & 581.2 & -0.769 \\
\hline 4-Ph-Ph & $\mathrm{Ph}$ & 286.4 & -3.030 & 286.4 & -3.030 & 286.4 & -3.030 & 292.8 & -2.351 & 292.7 & -2.351 & 292.8 & -2.351 & 376.8 & -3.147 & 376.8 & -3.147 & 376.8 & -3.147 \\
\hline 4-tBu-Ph & $\mathrm{Ph}$ & 286.5 & -2.981 & 286.4 & -3.017 & 286.7 & -2.941 & 292.8 & -2.351 & 292.7 & -2.351 & 292.8 & -2.351 & 438.1 & -1.634 & 438.1 & -1.634 & 438.1 & -1.634 \\
\hline $\mathrm{Ph}$ & $\mathrm{Ph}$ & 292.8 & -2.351 & 292.7 & -2.351 & 292.8 & -2.351 & 292.8 & -2.351 & 292.7 & -2.351 & 292.8 & -2.351 & 390.5 & -1.433 & 390.5 & -1.433 & 390.5 & -1.433 \\
\hline \multicolumn{20}{|l|}{ Validation set } \\
\hline 3,4,5-Me $3-\mathrm{Ph}$ & 4-F-Ph & 402.4 & -1.840 & 402.4 & -1.840 & 402.4 & -1.840 & 289.3 & -2.788 & 289.3 & -2.788 & 289.3 & -2.788 & 369.6 & -3.663 & 369.6 & -3.663 & 369.6 & -3.663 \\
\hline 3,5-Me2-4-OMe-Ph & 4-F-Ph & 400.2 & -1.727 & 400.2 & -1.727 & 400.2 & -1.727 & 289.3 & -2.788 & 289.3 & -2.788 & 289.3 & -2.788 & 373.3 & -3.606 & 366.7 & -3.761 & 418.8 & -2.519 \\
\hline 3,5-Me2-Ph & 4-F-Ph & 398.8 & -1.716 & 398.8 & -1.716 & 398.8 & -1.716 & 289.3 & -2.788 & 289.3 & -2.788 & 289.3 & -2.788 & 374.9 & -3.049 & 374.5 & -3.089 & 375.3 & -3.003 \\
\hline $4-\mathrm{CF}_{3}-\mathrm{Ph}$ & 4- $\mathrm{CF}_{3}-\mathrm{Ph}$ & 296.4 & -2.168 & 292.7 & -2.257 & 301.7 & -2.041 & 285.1 & -3.261 & 285.1 & -3.269 & 285.1 & -3.257 & 379.4 & -3.679 & 377.7 & -3.791 & 389.6 & -2.986 \\
\hline $4-\mathrm{CF}_{3}-\mathrm{Ph}$ & 4-OMe-Ph & 280.5 & -3.722 & 280.1 & -3.753 & 281.0 & -3.687 & 288.9 & -2.708 & 287.9 & -2.848 & 291.0 & -2.420 & 379.8 & -3.739 & 374.9 & -3.988 & 388.8 & -2.974 \\
\hline
\end{tabular}




\begin{tabular}{|c|c|c|c|c|c|c|c|c|c|c|c|c|c|c|c|c|c|c|c|}
\hline $4-\mathrm{CF}_{3}-\mathrm{Ph}$ & 4-Ph-Ph & 282.4 & -4.153 & 279.8 & -4.293 & 287.8 & -3.865 & 286.4 & -3.030 & 286.4 & -3.030 & 286.5 & -3.030 & 378.3 & -4.555 & 371.6 & -4.809 & 425.4 & -3.499 \\
\hline $\mathrm{Ph}$ & 2-Me-Ph & 287.7 & -2.723 & 287.7 & -2.723 & 287.7 & -2.723 & 324.6 & -2.127 & 293.1 & -2.282 & 403.4 & -1.739 & 384.4 & -1.947 & 384.4 & -1.947 & 384.4 & -1.947 \\
\hline $\mathrm{Ph}$ & 2Nap & 281.6 & -3.264 & 281.6 & -3.266 & 281.7 & -3.262 & 347.3 & -2.660 & 291.2 & -2.729 & 390.4 & -2.572 & 368.5 & -3.386 & 368.5 & -3.386 & 368.5 & -3.386 \\
\hline $\mathrm{Ph}$ & 2-OMe-Ph & 289.2 & -2.624 & 288.6 & -2.724 & 290.2 & -2.470 & 332.6 & -2.194 & 290.5 & -2.323 & 501.2 & -0.475 & 381.5 & -2.031 & 381.5 & -2.031 & 381.5 & -2.031 \\
\hline $\mathrm{Ph}$ & 3-Me-Ph & 287.7 & -2.723 & 287.7 & -2.723 & 287.7 & -2.723 & 324.6 & -2.127 & 293.1 & -2.282 & 403.4 & -1.739 & 384.4 & -1.947 & 384.4 & -1.947 & 384.4 & -1.947 \\
\hline $\mathrm{Ph}$ & $4-\mathrm{CF}_{3}-\mathrm{Ph}$ & 293.5 & -2.349 & 293.5 & -2.349 & 293.5 & -2.349 & 285.1 & -3.261 & 285.1 & -3.269 & 285.1 & -3.257 & 382.4 & -2.816 & 382.4 & -2.816 & 382.4 & -2.816 \\
\hline $\mathrm{Ph}$ & 4-OMe-Ph & 287.6 & -2.703 & 287.6 & -2.703 & 287.6 & -2.703 & 288.9 & -2.708 & 287.9 & -2.848 & 291.0 & -2.420 & 381.5 & -2.031 & 381.5 & -2.031 & 381.5 & -2.031 \\
\hline $\mathrm{Ph}$ & 4-Ph-Ph & 283.3 & -3.333 & 283.3 & -3.333 & 283.3 & -3.333 & 286.4 & -3.030 & 286.4 & -3.030 & 286.5 & -3.030 & 376.8 & -3.147 & 376.8 & -3.147 & 376.8 & -3.147 \\
\hline
\end{tabular}

Table S8. Parameters describing non-covalent interactions between $\mathrm{Ar}^{\mathrm{C}}$ and $\mathrm{Ar}^{\mathrm{A}}$.

\section{Non-covalent interactions - individual interaction geometries}

Interpolated minimum interaction energies as well as the interaction energy components as given by the PSI4 SAPT output.

The individual scan names contain first the name and interacting part (pi $=\pi$-face) of $\mathbf{A} \mathbf{r}^{\mathbf{C}}$ then name and interacting part (pi or side) of $\mathbf{A} \mathbf{r}^{\mathbf{A}}$. The final number indicates the dihedral angle between both moieties. Additional indices may be present to distinguish separate geometries for the same probe combination.

\begin{tabular}{|c|c|c|c|c|c|c|c|c|}
\hline Name - Edge-to-face complex $A r_{\text {side }}^{\mathrm{C}}-\mathbf{A} \mathbf{r}_{\pi}^{A}$ & $\mathrm{Ar}^{\mathrm{C}}$ & $A \mathbf{r}^{\mathrm{A}}$ & $\begin{array}{c}D_{\min } \\
\text { in } \mathbf{p m}\end{array}$ & \begin{tabular}{|c} 
Electrostatics \\
in $\mathrm{kcal} / \mathrm{mol}$
\end{tabular} & $\begin{array}{l}\text { Exchange } \\
\text { in } \mathbf{k c a l} / \mathbf{m o l}\end{array}$ & $\begin{array}{l}\text { Induction } \\
\text { in kcal/mol }\end{array}$ & $\begin{array}{l}\text { Dispersion } \\
\text { in kcal/mol }\end{array}$ & $\begin{array}{c}\text { Total } E_{\text {SSAPTO }} \\
\text { in kcal } / \mathrm{mol}\end{array}$ \\
\hline 1Nap_pi0_4AdPh_side1 & $4 \mathrm{AdPh}$ & 1Nap & 276.84 & -1.7648 & 4.5897 & -0.6127 & -6.2663 & -4.0541 \\
\hline 1Nap_piO_4AdPh_side2 & $4 \mathrm{AdPh}$ & 1Nap & 275.92 & -1.8033 & 4.7489 & -0.6435 & -6.5743 & -4.2721 \\
\hline 2Fur_pi0_4AdPh_side1 & $4 \mathrm{AdPh}$ & 2Furyl & 290.97 & -1.2245 & 2.7855 & -0.3649 & -3.3749 & -2.1788 \\
\hline 2Fur_pi0_4AdPh_side2 & $4 \mathrm{AdPh}$ & 2Furyl & 290.56 & -1.2859 & 2.8382 & -0.3788 & -3.4685 & -2.295 \\
\hline 2MePh_pi0_4AdPh_side_1 & $4 \mathrm{AdPh}$ & 2-Me-Ph & 280.72 & -1.9633 & 4.1728 & -0.5675 & -5.2314 & -3.5893 \\
\hline 2MePh_pi0_4AdPh_side_2 & $4 \mathrm{AdPh}$ & 2-Me-Ph & 279.95 & -2.0223 & 4.2655 & -0.5897 & -5.4129 & -3.7594 \\
\hline 2MePh_pi0_Ph_side & $\mathrm{Ph}$ & 2-Me-Ph & 287.7 & -1.7384 & 3.2247 & -0.4662 & -3.7413 & -2.7213 \\
\hline 2Nap_piO_4AdPh_side1 & $4 \mathrm{AdPh}$ & 2Nap & 277.95 & -2.5237 & 6.1712 & -0.7412 & -8.1702 & -5.2639 \\
\hline 2Nap_piO_4AdPh_side2 & $4 \mathrm{AdPh}$ & 2Nap & 273.66 & -3.0106 & 7.1742 & -0.9057 & -9.2279 & -5.97 \\
\hline 2Nap_pi_ph_side0 & $\mathrm{Ph}$ & 2Nap & 281.6 & -1.7902 & 3.667 & -0.5193 & -4.6238 & -3.2663 \\
\hline 2OMePh_pi0_1_4AdPh_side_1 & $4 \mathrm{AdPh}$ & 2-OMe-Ph & 282.76 & -1.672 & 3.954 & -0.5378 & -5.0867 & -3.3426 \\
\hline 2OMePh_pi0_1_4AdPh_side_2 & $4 \mathrm{AdPh}$ & 2-OMe-Ph & 282.09 & -1.7062 & 4.033 & -0.5588 & -5.2588 & -3.4909 \\
\hline 2OMePh_pi0_2_4AdPh_side_1 & $4 \mathrm{AdPh}$ & 2-OMe-Ph & 281.53 & -1.8922 & 4.0548 & -0.5558 & -5.1565 & -3.5496 \\
\hline 2OMePh_pi0_2_4AdPh_side_2 & $4 \mathrm{AdPh}$ & 2-OMe-Ph & 280.68 & -1.9576 & 4.1579 & -0.5796 & -5.3492 & -3.7285 \\
\hline 2OMePh_pi0_Ph_side_1 & $\mathrm{Ph}$ & 2-OMe-Ph & 290.23 & -1.4253 & 3.0186 & -0.4381 & -3.6252 & -2.47 \\
\hline 2OMePh_pi0_Ph_side_2 & $\mathrm{Ph}$ & 2-OMe-Ph & 288.6 & -1.7285 & 3.1272 & -0.4534 & -3.6695 & -2.7242 \\
\hline 3MePh_pi0_4AdPh_side_1 & $4 \mathrm{AdPh}$ & 3-Me-Ph & 279.72 & -1.9685 & 4.3237 & -0.5839 & -5.4928 & -3.7215 \\
\hline 3MePh_pi0_4AdPh_side_2 & $4 \mathrm{AdPh}$ & 3-Me-Ph & 278.97 & -2.0456 & 4.4681 & -0.6074 & -5.7454 & -3.9303 \\
\hline
\end{tabular}




\begin{tabular}{|c|c|c|c|c|c|c|c|c|}
\hline 3MePh_pi0_Ph_side & $\mathrm{Ph}$ & 3-Me-Ph & 287.71 & -1.7399 & 3.2247 & -0.4662 & -3.7412 & -2.7226 \\
\hline 4CF3Ph_pi0_4AdPh_side_1 & $4 \mathrm{AdPh}$ & 4CF3Ph & 333.77 & -1.03 & 2.3499 & -0.2592 & -3.7006 & -2.6399 \\
\hline 4CF3Ph_pi0_4AdPh_side_2 & $4 \mathrm{AdPh}$ & $4 \mathrm{CF} 3 \mathrm{Ph}$ & 318.47 & -1.7167 & 3.6675 & -0.4317 & -4.7195 & -3.2005 \\
\hline 4CF3Ph_pi_4CF3Ph_side_1 & 4CF3Ph & 4CF3Ph & 292.74 & -0.7204 & 2.7618 & -0.4157 & -3.8822 & -2.2565 \\
\hline 4CF3Ph_pi_4CF3Ph_side_2 & 4CF3Ph & 4CF3Ph & 301.66 & -0.4943 & 2.3359 & -0.3486 & -3.5335 & -2.0407 \\
\hline 4CF3Ph_pi_Ph_side0 & $\mathrm{Ph}$ & $4 \mathrm{CF} 3 \mathrm{Ph}$ & 293.52 & -1.1988 & 2.6936 & -0.3073 & -3.5369 & -2.3494 \\
\hline 4ClPh_pi0_4AdPh_side1 & $4 \mathrm{AdPh}$ & 4-Cl-Ph & 290.1 & -1.6674 & 3.9586 & -0.427 & -5.4802 & -3.616 \\
\hline 4ClPh_piO_4AdPh_side2 & $4 \mathrm{AdPh}$ & $4-\mathrm{Cl}-\mathrm{Ph}$ & 283.88 & -2.1199 & 4.7767 & -0.5588 & -6.2084 & -4.1103 \\
\hline 4FPh_pi_345Me3Ph_side & 345-Me3Ph & $4-\mathrm{F}-\mathrm{Ph}$ & 402.38 & -1.0945 & 2.1974 & -0.2147 & -2.728 & -1.8398 \\
\hline 4FPh_pi_35Me24OMePh_side & 35-Me2-4-OMePh & 4-F-Ph & 400.18 & -0.9922 & 2.1076 & -0.2171 & -2.6256 & -1.7273 \\
\hline 4FPh_pi_35Me2Ph_side & $35-\mathrm{Me} 2 \mathrm{Ph}$ & 4-F-Ph & 398.84 & -1.0251 & 2.1904 & -0.2266 & -2.6548 & -1.716 \\
\hline 4FPh_pi0_4AdPh_side1 & $4 \mathrm{AdPh}$ & 4-F-Ph & 286.32 & -1.434 & 3.3329 & -0.3935 & -4.5611 & -3.0557 \\
\hline 4FPh_pi0_4AdPh_side2 & $4 \mathrm{AdPh}$ & 4-F-Ph & 283.57 & -1.6767 & 3.6319 & -0.4405 & -4.8545 & -3.3398 \\
\hline 4MePh_pi0_4AdPh_side_1 & $4 \mathrm{AdPh}$ & $4 \mathrm{MePh}$ & 300.28 & -1.6351 & 3.942 & -0.5082 & -5.2632 & -3.4646 \\
\hline 4MePh_piO_4AdPh_side_2 & $4 \mathrm{AdPh}$ & $4 \mathrm{MePh}$ & 288.68 & -1.8597 & 5.0062 & -0.6799 & -6.2972 & -3.8306 \\
\hline 4OMePh_pi0_4AdPh_side_1 & $4 \mathrm{AdPh}$ & $4 \mathrm{OMePh}$ & 285.02 & -1.8272 & 4.567 & -0.6173 & -6.1436 & -4.0211 \\
\hline 4OMePh_pi0_4AdPh_side_2 & $4 \mathrm{AdPh}$ & $4 \mathrm{OMePh}$ & 284.26 & -2.0898 & 5.0017 & -0.678 & -6.5813 & -4.3474 \\
\hline 4OMePh_pi0_4CF3Ph_side_1 & $4 \mathrm{CF} 3 \mathrm{Ph}$ & $4 \mathrm{OMePh}$ & 280.98 & -2.3973 & 3.8105 & -0.6491 & -4.4508 & -3.6868 \\
\hline 4OMePh_pi0_4CF3Ph_side_2 & $4 \mathrm{CF} 3 \mathrm{Ph}$ & $4 \mathrm{OMePh}$ & 280.1 & -2.521 & 3.9075 & -0.6747 & -4.4653 & -3.7534 \\
\hline 4OMePh_pi0_Ph_side & $\mathrm{Ph}$ & $4 \mathrm{OMePh}$ & 287.63 & -1.6211 & 3.1389 & -0.4491 & -3.7718 & -2.7032 \\
\hline 4PhPh_pi0_4AdPh_side_1 & $4 \mathrm{AdPh}$ & 4-Ph-Ph & 333.02 & -2.2539 & 5.6988 & -0.6464 & -7.2239 & -4.4254 \\
\hline 4PhPh_pi0_4AdPh_side_2 & $4 \mathrm{AdPh}$ & 4-Ph-Ph & 359.71 & -2.1864 & 5.1547 & -0.646 & -6.2074 & -3.885 \\
\hline 4PhPh_pi0_4CF3Ph_side_1 & $4 \mathrm{CF} 3 \mathrm{Ph}$ & 4-Ph-Ph & 287.77 & -2.1007 & 4.2903 & -0.7037 & -5.3508 & -3.865 \\
\hline 4PhPh_pi0_4CF3Ph_side_2 & 4CF3Ph & 4-Ph-Ph & 279.8 & -2.4417 & 4.6333 & -0.7736 & -5.7109 & -4.293 \\
\hline 4PhPh_pi_Ph_side0 & $\mathrm{Ph}$ & 4-Ph-Ph & 283.26 & -1.8716 & 3.7542 & -0.5059 & -4.7097 & -3.333 \\
\hline Ph_pi_2Nap_side1 & 2Nap & $\mathrm{Ph}$ & 291.15 & -1.5286 & 2.9823 & -0.4281 & -3.5972 & -2.5715 \\
\hline Ph_pi_2Nap_side2 & 2Nap & $\mathrm{Ph}$ & 390.4 & -1.7573 & 3.1536 & -0.4943 & -3.6293 & -2.7273 \\
\hline Ph_pi0_345Me3Ph_side & 345-Me3Ph & $\mathrm{Ph}$ & 405.89 & -1.0783 & 2.2133 & -0.2683 & -2.6477 & -1.7809 \\
\hline $\mathrm{Ph} \_$pi0_35Me24OMePh_side & 35-Me2-4-OMePh & $\mathrm{Ph}$ & 403.26 & -1.093 & 2.1681 & -0.2733 & -2.5644 & -1.7626 \\
\hline Ph_pi0_35Me2Ph_side & 35-Me2Ph & $\mathrm{Ph}$ & 403.4 & -1.0501 & 2.1719 & -0.265 & -2.5315 & -1.6747 \\
\hline Ph_pi0_35tBu2Ph_side_1 & 35-tBu2Ph & $\mathrm{Ph}$ & 539.47 & -0.6627 & 1.961 & -0.2352 & -2.5606 & -1.4975 \\
\hline Ph_pi0_35tBu2Ph_side_2 & 35-tBu2Ph & $\mathrm{Ph}$ & 509.91 & -0.9194 & 2.553 & -0.2809 & -3.2316 & -1.8789 \\
\hline Ph_pi0_4AdPh_side1 & $4 \mathrm{AdPh}$ & $\mathrm{Ph}$ & 285.2 & -1.6612 & 3.6784 & -0.492 & -4.6641 & -3.1389 \\
\hline
\end{tabular}




\begin{tabular}{|c|c|c|c|c|c|c|c|c|}
\hline Ph_pi0_4AdPh_side2 & 4AdPh & $\mathrm{Ph}$ & 284.43 & -1.7086 & 3.7596 & -0.5112 & -4.8304 & -3.2906 \\
\hline Ph_piO_4CF3Ph_side & 4CF3Ph & $\mathrm{Ph}$ & 285.06 & -2.3402 & 3.4657 & -0.5786 & -3.8037 & -3.2567 \\
\hline Ph_pi_4MePh_side & $4 \mathrm{MePh}$ & $\mathrm{Ph}$ & 292.19 & -1.4133 & 2.8703 & -0.4011 & -3.4476 & -2.3916 \\
\hline Ph_pi0_4nPrPh1_side & $4 \mathrm{nPrPh}$ & $\mathrm{Ph}$ & 290.99 & -1.4429 & 2.9875 & -0.413 & -3.659 & -2.5274 \\
\hline Ph_pi0_4nPrPh2_side_1 & $4 \mathrm{nPrPh}$ & $\mathrm{Ph}$ & 287.47 & -1.591 & 3.3989 & -0.4581 & -4.1989 & -2.849 \\
\hline Ph_pi0_4nPrPh2_side_2 & $4 \mathrm{nPrPh}$ & $\mathrm{Ph}$ & 291.86 & -1.3966 & 2.907 & -0.4005 & -3.5337 & -2.4237 \\
\hline Ph_pi0_4nPrPh3_side_1 & $4 \mathrm{nPrPh}$ & $\mathrm{Ph}$ & 289.49 & -1.5018 & 3.1414 & -0.4335 & -3.8728 & -2.6666 \\
\hline Ph_pi0_4nPrPh3_side_2 & $4 \mathrm{nPrPh}$ & $\mathrm{Ph}$ & 290.39 & -1.4805 & 3.0477 & -0.4227 & -3.7343 & -2.5898 \\
\hline Ph_pi0_4PhPh_side & $4 \mathrm{PhPh}$ & $\mathrm{Ph}$ & 286.44 & -1.7927 & 3.4381 & -0.4904 & -4.1851 & -3.0301 \\
\hline Ph_pi0_4tBuPh_side_1 & $4 \mathrm{tBuPh}$ & $\mathrm{Ph}$ & 286.35 & -1.6842 & 3.5168 & -0.4904 & -4.3591 & -3.0169 \\
\hline Ph_pi0_4tBuPh_side_2 & $4 \mathrm{tBuPh}$ & $\mathrm{Ph}$ & 286.74 & -1.6544 & 3.4998 & -0.4731 & -4.3135 & -2.9412 \\
\hline Ph_pi_Ph_side & $\mathrm{Ph}$ & $\mathrm{Ph}$ & 292.74 & -1.4794 & 2.7958 & -0.3986 & -3.2685 & -2.3507 \\
\hline
\end{tabular}

Table S9. Names, equilibrium distances, energy contributions and final interaction energies of individual probe interactions in the Edge-to-face complex $\mathbf{A r}_{\text {side }}^{\mathrm{C}}-\mathrm{Ar}_{\pi}^{\mathrm{A}}$. 


\begin{tabular}{|c|c|c|c|c|c|c|c|}
\hline Name - Edge-to-face complex $\mathbf{A r}_{\text {side }}^{\mathrm{C}}-\mathbf{A r}_{\pi}^{\mathrm{A}}$ & $\mathbf{A} \mathbf{r}^{\mathrm{A}}$ & $\begin{array}{c}D_{\min } \\
\text { in pm }\end{array}$ & $\begin{array}{c}\text { Electrostatics } \\
\text { in } \mathrm{kcal} / \mathrm{mol}\end{array}$ & $\begin{array}{l}\text { Exchange } \\
\text { in } \mathrm{kcal} / \mathrm{mol}\end{array}$ & \begin{tabular}{|c} 
Induction \\
in kcal/mol
\end{tabular} & \begin{tabular}{|c|} 
Dispersion \\
in kcal/mol
\end{tabular} & $\begin{array}{c}\text { Total } E_{\text {SSAPTO }} \\
\text { in } \mathrm{kcal} / \mathrm{mol}\end{array}$ \\
\hline 1Nap_side1_Ph_pi_0 & $1 \mathrm{Nap}$ & 289.79 & -1.6493 & 3.0192 & -0.4567 & -3.4979 & -2.5847 \\
\hline 1Nap_side2_Ph_pi_0 & 1Nap & 532.13 & -1.6548 & 3.031 & -0.4591 & -3.5093 & -2.5922 \\
\hline 2Fur_side1_Ph_pi_0 & 2Furyl & 198.87 & -0.1 & 2.0206 & -0.3643 & -2.7404 & -1.1841 \\
\hline 2Fur_side2_Ph_pi_0 & 2Furyl & 200.93 & -1.72 & 2.9191 & -0.5101 & -3.1779 & -2.4888 \\
\hline 3MePh_side1_Ph_pi_0 & 2-Me-Ph, 3-Me-Ph & 293.13 & -1.3798 & 2.7742 & -0.3894 & -3.2869 & -2.2818 \\
\hline 3MePh_side2_Ph_pi_0 & 2-Me-Ph, 3-Me-Ph & 403.36 & -1.1372 & 2.1659 & -0.2781 & -2.4894 & -1.7387 \\
\hline 2Nap_side1_Ph_pi_0 & $2 \mathrm{Nap}$ & 291.15 & -1.5286 & 2.9823 & -0.4281 & -3.5972 & -2.5715 \\
\hline 2Nap_side2_Ph_pi_0 & 2Nap & 390.4 & -1.7573 & 3.1536 & -0.4943 & -3.6293 & -2.7273 \\
\hline 2OMePh_side1a_Ph_pi_0 & 2-OMe-Ph & 291.08 & -1.3781 & 2.7722 & -0.3953 & -3.2961 & -2.2973 \\
\hline 2OMePh_side1b_Ph_pi_0 & 2-OMe-Ph & 420.83 & 0.5586 & 1.0931 & -0.2018 & -1.9243 & -0.4745 \\
\hline 2OMePh_side2a_Ph_pi_0 & 2-OMe-Ph & 290.5 & -1.4236 & 2.8027 & -0.3995 & -3.303 & -2.3234 \\
\hline 2OMePh_side2b_Ph_pi_0 & 2-OMe-Ph & 501.15 & -1.3983 & 1.8969 & -0.2489 & -2.1161 & -1.8663 \\
\hline 4CF3Ph_side_Ph_pi_0 & 4CF3Ph & 285.08 & -2.3398 & 3.4647 & -0.5785 & -3.803 & -3.2567 \\
\hline 4ClPh_side_Ph_pi_0 & 4-Cl-Ph & 287.44 & -2.0644 & 3.2324 & -0.5186 & -3.6161 & -2.9667 \\
\hline 4FPh_side_Ph_pi_0 & 4-F-Ph & 289.32 & -1.956 & 3.0602 & -0.4821 & -3.4104 & -2.7884 \\
\hline 4MePh_side_Ph_pi_0 & $4 \mathrm{MePh}$ & 292.19 & -1.4133 & 2.8703 & -0.4011 & -3.4476 & -2.3916 \\
\hline 4OMePh_side1_Ph_pi_0 & $4 \mathrm{OMePh}$ & 287.9 & -1.6987 & 3.2694 & -0.4939 & -3.9245 & -2.8477 \\
\hline 4OMePh_side2_Ph_pi_0 & $4 \mathrm{OMePh}$ & 290.96 & -1.4902 & 2.9348 & -0.4149 & -3.45 & -2.4203 \\
\hline 4PhPh_side_Ph_pi_0 & 4-Ph-Ph & 286.45 & -1.7922 & 3.4381 & -0.4907 & -4.185 & -3.0299 \\
\hline Ph_side_Ph_pi_0 & $\mathrm{Ph}$ & 292.74 & -1.4794 & 2.7958 & -0.3986 & -3.2685 & -2.3507 \\
\hline
\end{tabular}

Table S10. Names, equilibrium distances, energy contributions and final interaction energies of individual probe interactions in the Edge-to-face complex $\mathrm{Ar}_{\text {side }}^{A}-\mathrm{Ph}_{\boldsymbol{\pi}}$.

\begin{tabular}{|c|c|c|c|c|c|c|c|c|}
\hline Name - Sandwich complex $A r_{\pi}^{C}-A r_{\pi}^{A}$ & $A \mathbf{r}^{\mathrm{C}}$ & $\mathbf{A r} \mathbf{r}^{\mathbf{A}}$ & $\begin{array}{c}D_{\min } \\
\text { in } \mathbf{p m} \\
\end{array}$ & \begin{tabular}{|c} 
Electrostatics \\
in kcal/mol
\end{tabular} & $\begin{array}{c}\text { Exchange } \\
\text { in kcal/mol }\end{array}$ & $\begin{array}{c}\text { Induction } \\
\text { in kcal/mol }\end{array}$ & $\begin{array}{l}\text { Dispersion } \\
\text { in kcal/mol }\end{array}$ & $\begin{array}{c}\text { Total } E_{\text {SSAPT0 }} \\
\text { in kcal } / \mathrm{mol}\end{array}$ \\
\hline 345Me3Ph_pi_4FPh_pi_300 & 3,4,5-Me3-Ph & 4-F-Ph & 369.63 & -1.1832 & 6.1208 & -0.4124 & -8.1881 & -3.6629 \\
\hline 345Me3Ph_pi_Ph_pi_0 & 3,4,5-Me3-Ph & $\mathrm{Ph}$ & 372.47 & -0.4097 & 5.8217 & -0.4432 & -7.958 & -2.989 \\
\hline 35Me24OMePh_pi_4FPh_pi_300 & 3,5-Me2-4-OMe-Ph & 4-F-Ph & 366.68 & -1.4779 & 6.3294 & -0.3709 & -8.2413 & -3.7607 \\
\hline 35Me24OMePh_pi_4FPh_pi_300_2 & 3,5-Me2-4-OMe-Ph & 4-F-Ph & 418.82 & -0.3975 & 3.8031 & -0.3485 & -5.5759 & -2.5188 \\
\hline 35Me24OMePh_pi_4FPh_pi_60 & 3,5-Me2-4-OMe-Ph & 4-F-Ph & 367.21 & -1.4699 & 6.2942 & -0.3643 & -8.1966 & -3.7366 \\
\hline 35Me24OMePh_pi_4FPh_pi_60_2 & 3,5-Me2-4-OMe-Ph & 4-F-Ph & 417.48 & -0.576 & 3.9573 & -0.357 & -5.6764 & -2.6521 \\
\hline 35Me2Ph_pi_4FPh_pi_300 & 3,5-Me2-Ph & 4-F-Ph & 375.29 & -0.9471 & 5.2574 & -0.3272 & -7.072 & -3.0889 \\
\hline 35Me2Ph_pi_4FPh_pi_60 & 3,5-Me2-Ph & 4-F-Ph & 374.54 & -0.7959 & 5.2416 & -0.3347 & -7.1137 & -3.0027 \\
\hline
\end{tabular}




\begin{tabular}{|c|c|c|c|c|c|c|c|c|}
\hline 35Me2Ph_pi_Ph_pi_0 & 3,5-Me2-Ph & $\mathrm{Ph}$ & 378.49 & -0.0947 & 4.9197 & -0.3687 & -6.8598 & -2.4034 \\
\hline 35Me4OMePh_pi1_Ph_pi0 & 3,5-Me2-4-OMe-Ph & $\mathrm{Ph}$ & 422.61 & 0.0075 & 3.5883 & -0.3624 & -5.3736 & -2.1402 \\
\hline 35Me4OMePh_pi2_Ph_pi0 & 3,5-Me2-4-OMe-Ph & $\mathrm{Ph}$ & 369.45 & -0.8048 & 6.1077 & -0.4103 & -8.0413 & -3.1486 \\
\hline 35tBuPh_pi_Ph_pi_60 & 3,5-tBu2-Ph & $\mathrm{Ph}$ & 448.6 & -0.0484 & 3.5383 & -0.4169 & -5.3739 & -2.3008 \\
\hline 4AdPh_pi_1Nap_pi_300 & 4-Ad-Ph & $1 \mathrm{Nap}$ & 467.08 & -1.1647 & 5.2915 & -0.6937 & -7.1955 & -3.7623 \\
\hline 4AdPh_pi_1Nap_pi_300_2 & 4-Ad-Ph & 1Nap & 414.76 & -0.4127 & 5.2598 & -0.6127 & -7.534 & -3.2997 \\
\hline 4AdPh_pi_1Nap_pi_60 & 4-Ad-Ph & $1 \mathrm{Nap}$ & 412.68 & -0.4504 & 5.4215 & -0.6267 & -7.7062 & -3.3618 \\
\hline 4AdPh_pi_1Nap_pi_60_2 & 4-Ad-Ph & 1Nap & 415.3 & -0.4215 & 5.3128 & -0.6044 & -7.5278 & -3.2409 \\
\hline 4AdPh_pi_2Furane_pi_300_N1 & 4-Ad-Ph & 2Furyl & 417 & 0.1052 & 2.8967 & -0.3253 & -4.3592 & -1.6826 \\
\hline 4AdPh_pi_2Furane_pi_300_N2 & 4-Ad-Ph & 2Furyl & 411.89 & 0.076 & 3.2806 & -0.3308 & -4.8276 & -1.8017 \\
\hline 4AdPh_pi_2Furane_pi_60_N1 & 4-Ad-Ph & 2Furyl & 398.56 & 0.1175 & 3.6394 & -0.3812 & -5.2819 & -1.9062 \\
\hline 4AdPh_pi_2Furane_pi_60_N2 & 4-Ad-Ph & 2Furyl & 393.96 & -0.1571 & 4.2788 & -0.3892 & -5.8997 & -2.1672 \\
\hline 4AdPh_pi_2MePh_pi_60_2 & 4-Ad-Ph & 2-Me-Ph & 427.21 & 0.0095 & 3.9331 & -0.4622 & -5.8975 & -2.4171 \\
\hline 4AdPh_pi_2Nap_pi1_300 & 4-Ad-Ph & 2Nap & 481.37 & -0.9146 & 4.7377 & -0.57 & -6.7292 & -3.4761 \\
\hline 4AdPh_pi_2Nap_pi1_60 & 4-Ad-Ph & $2 \mathrm{Nap}$ & 412.29 & -0.5455 & 5.7297 & -0.6573 & -8.2148 & -3.6878 \\
\hline 4AdPh_pi_2Nap_pi2_300 & 4-Ad-Ph & 2Nap & 414.06 & -0.5998 & 5.7867 & -0.667 & -8.3596 & -3.8398 \\
\hline 4AdPh_pi_2Nap_pi2_60 & 4-Ad-Ph & 2Nap & 469.24 & -1.172 & 5.2452 & -0.6847 & -7.1354 & -3.7469 \\
\hline 4AdPh_pi_2OMePh_pi_60 & 4-Ad-Ph & 2-OMe-Ph & 427.19 & -0.0257 & 3.9664 & -0.4635 & -5.862 & -2.3849 \\
\hline 4AdPh_pi_2OMePh_pi_60_2 & 4-Ad-Ph & 2-OMe-Ph & 426.87 & -0.041 & 3.9795 & -0.465 & -5.8781 & -2.4045 \\
\hline 4AdPh_pi_3MePh_pi_300_2 & 4-Ad-Ph & 3-Me-Ph & 425.6 & 0.0414 & 3.9789 & -0.4672 & -6.0079 & -2.4547 \\
\hline 4AdPh_pi_3MePh_pi_60_1 & 4-Ad-Ph & 3-Me-Ph & 425.84 & 0.0399 & 3.9671 & -0.4659 & -5.9864 & -2.4452 \\
\hline 4AdPh_pi_3MePh_pi_60_2_300_1 & 4-Ad-Ph & 3-Me-Ph & 534.4 & 0.3157 & 1.4616 & -0.2308 & -2.7759 & -1.2294 \\
\hline 4AdPh_pi_3MePh_pi2_60_2_300_1 & 4-Ad-Ph & 3-Me-Ph & 533.65 & 0.3216 & 1.4695 & -0.2317 & -2.7943 & -1.2348 \\
\hline 4AdPh_pi_4CF3Ph_pi_300 & 4-Ad-Ph & 4-CF3-Ph & 464.94 & -0.485 & 2.9298 & -0.333 & -4.6337 & -2.5219 \\
\hline 4AdPh_pi_4CF3Ph_pi_60 & 4-Ad-Ph & 4-CF3-Ph & 419.03 & -0.9825 & 4.8825 & -0.5233 & -6.927 & -3.5502 \\
\hline 4AdPh_pi_4CF3Ph_pi2_300 & 4-Ad-Ph & 4-CF3-Ph & 424.4 & -0.9227 & 4.6682 & -0.4795 & -6.785 & -3.519 \\
\hline 4AdPh_pi_4CF3Ph_pi2_60 & 4-Ad-Ph & 4-CF3-Ph & 426.69 & -1.0794 & 4.6679 & -0.5374 & -6.6283 & -3.5772 \\
\hline 4AdPh_pi_4ClPh_pi_270 & 4-Ad-Ph & 4-Cl-Ph & 403.5 & -0.9117 & 5.6599 & -0.4995 & -7.5985 & -3.3499 \\
\hline 4AdPh_pi_4ClPh_pi_300 & 4-Ad-Ph & 4-Cl-Ph & 421.8 & -0.5703 & 4.5493 & -0.4936 & -6.6482 & -3.1629 \\
\hline 4AdPh_pi_4FPh_pi_300 & 4-Ad-Ph & 4-F-Ph & 427.4 & -0.2797 & 3.665 & -0.4001 & -5.4461 & -2.461 \\
\hline 4AdPh_pi_4FPh_pi_60 & 4-Ad-Ph & 4-F-Ph & 426.86 & -0.3496 & 3.7327 & -0.4038 & -5.4702 & -2.4908 \\
\hline 4AdPh_pi_4MePh_pi_300 & 4-Ad-Ph & 4-Me-Ph & 425.34 & -0.0365 & 4.3542 & -0.5014 & -6.5966 & -2.7802 \\
\hline 4AdPh_pi_4MePh_pi_60 & 4-Ad-Ph & 4-Me-Ph & 423.18 & -0.0131 & 4.3462 & -0.5024 & -6.5337 & -2.703 \\
\hline
\end{tabular}




\begin{tabular}{|c|c|c|c|c|c|c|c|c|}
\hline 4AdPh_pi_4OMePh_pi1_300 & 4-Ad-Ph & 4-OMe-Ph & 422.97 & -0.0514 & 4.2307 & -0.5063 & -6.4316 & -2.7586 \\
\hline 4AdPh_pi_4OMePh_pi1_60 & 4-Ad-Ph & 4-OMe-Ph & 429.35 & -0.0236 & 4.6894 & -0.5849 & -6.8291 & -2.7482 \\
\hline 4AdPh_pi_4OMePh_pi2_300 & 4-Ad-Ph & 4-OMe-Ph & 458.88 & -0.0708 & 3.5833 & -0.4715 & -5.3912 & -2.3502 \\
\hline 4AdPh_pi_4OMePh_pi2_60 & 4-Ad-Ph & 4-OMe-Ph & 422.48 & -0.2218 & 4.3117 & -0.506 & -6.3668 & -2.783 \\
\hline 4AdPh_pi_4PhPh_pi1_300 & 4-Ad-Ph & 4-Ph-Ph & 410.38 & -0.9858 & 6.7171 & -0.7336 & -9.8698 & -4.8722 \\
\hline 4AdPh_pi_4PhPh_pi1_60 & 4-Ad-Ph & 4-Ph-Ph & 438.66 & -0.5406 & 5.7951 & -0.723 & -8.5771 & -4.0456 \\
\hline 4AdPh_pi_4PhPh_pi2_300 & 4-Ad-Ph & 4-Ph-Ph & 502.29 & -0.3304 & 3.2722 & -0.4139 & -5.2223 & -2.6944 \\
\hline 4AdPh_pi_4PhPh_pi2_60 & 4-Ad-Ph & 4-Ph-Ph & 411.79 & -0.8812 & 6.1561 & -0.6809 & -9.1531 & -4.5591 \\
\hline 4AdPh_pi_Ph_pi_60 & 4-Ad-Ph & $\mathrm{Ph}$ & 431.73 & 0.2009 & 3.3912 & -0.3927 & -5.2309 & -2.0315 \\
\hline 4CF3Ph_pi_4CF3Ph_pi_300 & 4-CF3-Ph & 4-CF3-Ph & 389.56 & -1.0371 & 4.7355 & -0.2526 & -6.432 & -2.9863 \\
\hline 4CF3Ph_pi_4CF3Ph_pi_60 & 4-CF3-Ph & 4-CF3-Ph & 377.65 & -1.8602 & 5.6153 & -0.2925 & -7.2536 & -3.7911 \\
\hline 4CF3Ph_pi_4CF3Ph_pi2_300 & 4-CF3-Ph & 4-CF3-Ph & 378.96 & -1.7357 & 5.5269 & -0.2812 & -7.2259 & -3.7158 \\
\hline 4CF3Ph_pi_4CF3Ph_pi2_60 & 4-CF3-Ph & 4-CF3-Ph & 378.98 & -1.7379 & 5.5257 & -0.2811 & -7.2236 & -3.7169 \\
\hline 4CF3Ph_pi_4OMePh_pi1_300 & 4-CF3-Ph & 4-OMe-Ph & 376.9 & -0.8547 & 5.3789 & -0.3241 & -7.1739 & -2.9737 \\
\hline 4CF3Ph_pi_4OMePh_pi1_60 & 4-CF3-Ph & 4-OMe-Ph & 374.9 & -2.0812 & 6.0371 & -0.385 & -7.5584 & -3.9875 \\
\hline 4CF3Ph_pi_4OMePh_pi2_300 & 4-CF3-Ph & 4-OMe-Ph & 388.84 & -2.1546 & 5.4822 & -0.5016 & -6.6112 & -3.7852 \\
\hline 4CF3Ph_pi_4OMePh_pi2_60 & 4-CF3-Ph & 4-OMe-Ph & 375.9 & -1.15 & 5.5806 & -0.3342 & -7.2422 & -3.1459 \\
\hline 4CF3Ph_pi_4PhPh_pi1_300 & 4-CF3-Ph & 4-Ph-Ph & 371.6 & -1.707 & 7.0108 & -0.4415 & -9.2638 & -4.4015 \\
\hline 4CF3Ph_pi_4PhPh_pi1_60 & 4-CF3-Ph & $4-\mathrm{Ph}-\mathrm{Ph}$ & 379.95 & -2.1626 & 6.5015 & -0.4487 & -8.6993 & -4.8092 \\
\hline 4CF3Ph_pi_4PhPh_pi2_300 & 4-CF3-Ph & 4-Ph-Ph & 425.35 & -1.7697 & 4.2407 & -0.5202 & -5.4503 & -3.4994 \\
\hline 4CF3Ph_pi_4PhPh_pi2_60 & 4-CF3-Ph & 4-Ph-Ph & 371.89 & -1.8186 & 6.9071 & -0.4288 & -9.1022 & -4.4425 \\
\hline 4CF3Ph_pi_Ph_pi_60 & 4-CF3-Ph & $\mathrm{Ph}$ & 382.37 & -1.1021 & 4.7056 & -0.269 & -6.1506 & -2.8161 \\
\hline 4MePh_pi_Ph_pi_60 & 4-Me-Ph & $\mathrm{Ph}$ & 384.36 & 0.1231 & 4.1463 & -0.3141 & -5.9019 & -1.9466 \\
\hline 4nPrPh_Ph_pi_1_pi_0 & 4-nPr-Ph & $\mathrm{Ph}$ & 446.17 & 0.1991 & 2.7959 & -0.2715 & -4.3094 & -1.5859 \\
\hline 4nPrPh_Ph_pi_2_pi_0 & 4-nPr-Ph & $\mathrm{Ph}$ & 380.85 & 0.0244 & 4.6009 & -0.3295 & -6.5507 & -2.2551 \\
\hline 4nPrPh_Ph_pi_3_pi1_0 & 4-nPr-Ph & $\mathrm{Ph}$ & 581.23 & 0.036 & 1.1992 & -0.1207 & -1.883 & -0.7685 \\
\hline 4nPrPh_Ph_pi_3_pi2_0 & 4-nPr-Ph & $\mathrm{Ph}$ & 382.7 & 0.0939 & 4.3084 & -0.3003 & -6.1289 & -2.0269 \\
\hline 4PhPh_pi_Ph_pi_60 & 4-Ph-Ph & $\mathrm{Ph}$ & 376.77 & -0.6962 & 5.6558 & -0.3806 & -7.7262 & -3.1473 \\
\hline 4tBuPh_pi_Ph_pi0 & 4-tBu-Ph & $\mathrm{Ph}$ & 438.11 & 0.2471 & 2.8868 & -0.3478 & -4.4201 & -1.6339 \\
\hline Nap_pi_Ph_pi0 & $2 \mathrm{Nap}$ & $\mathrm{Ph}$ & 368.47 & -1.0084 & 6.3339 & -0.4353 & -8.2765 & -3.3862 \\
\hline Ph_pi_4OMePh_pi_0 & $\mathrm{Ph}$ & 4-OMe-Ph & 381.49 & -0.0436 & 4.3948 & -0.3112 & -6.0704 & -2.0305 \\
\hline Ph_pi_Ph_piO & $\mathrm{Ph}$ & $\mathrm{Ph}$ & 390.51 & 0.3398 & 3.4124 & -0.2314 & -4.9539 & -1.4332 \\
\hline
\end{tabular}

Table S11. Names, equilibrium distances, energy contributions and final interaction energies of individual probe interactions in the sandwich complex. 


\begin{tabular}{|c|c|c|}
\hline name & ArC & ArA \\
\hline \multirow[t]{3}{*}{ 4MePh_pi_Ph_pi_60 } & 4-Me-Ph & $\mathrm{Ph}$ \\
\hline & $\mathrm{Ph}$ & 2-Me-Ph \\
\hline & $\mathrm{Ph}$ & 3-Me-Ph \\
\hline \multirow[t]{2}{*}{ Ph_pi_4OMePh_pi_0 } & $\mathrm{Ph}$ & 4-OMe-Ph \\
\hline & $\mathrm{Ph}$ & 2-OMe-Ph \\
\hline \multirow[t]{2}{*}{ 4AdPh_pi_4MePh_pi_300 } & 4-Ad-Ph & 4-Me-Ph \\
\hline & 4-Ad-Ph & 2-Me-Ph \\
\hline \multirow{2}{*}{ 4AdPh_pi_4MePh_pi_60 } & 4-Ad-Ph & 4-Me-Ph \\
\hline & 4-Ad-Ph & 2-Me-Ph \\
\hline \multirow{2}{*}{ 4AdPh_pi_4OMePh_pi1_300 } & 4-Ad-Ph & 4-OMe-Ph \\
\hline & 4-Ad-Ph & 2-OMe-Ph \\
\hline \multirow[t]{2}{*}{ 4AdPh_pi_4OMePh_pi1_60 } & 4-Ad-Ph & 4-OMe-Ph \\
\hline & 4-Ad-Ph & 2-OMe-Ph \\
\hline \multirow[t]{2}{*}{ 4AdPh_pi_4OMePh_pi2_300 } & 4-Ad-Ph & 4-OMe-Ph \\
\hline & 4-Ad-Ph & 2-OMe-Ph \\
\hline \multirow[t]{2}{*}{ 4AdPh_pi_4OMePh_pi2_60 } & 4-Ad-Ph & 4-OMe-Ph \\
\hline & 4-Ad-Ph & 2-OMe-Ph \\
\hline \multirow[t]{2}{*}{ Nap_pi_Ph_piO } & 2Nap & $\mathrm{Ph}$ \\
\hline & $\mathrm{Ph}$ & 2Nap \\
\hline \multirow[t]{2}{*}{ 4CF3Ph_pi_Ph_pi_60 } & 4-CF3-Ph & $\mathrm{Ph}$ \\
\hline & $\mathrm{Ph}$ & 4-CF3-Ph \\
\hline \multirow[t]{2}{*}{ 4PhPh_pi_Ph_pi_60 } & 4-Ph-Ph & $\mathrm{Ph}$ \\
\hline & $\mathrm{Ph}$ & 4-Ph-Ph \\
\hline
\end{tabular}

Table S12. Equivalent interaction geometries that represent several probe combinations. 


\section{Regression model development}

The observed enantioselectivity (cf. Table S5) of each catalyst-substrate pair was converted to relative free energies and used as target in the subsequent modelling. Multivariable model development was performed using Python with previously reported algorithms ${ }^{36}$ based on forward stepwise linear regression that keeps sets of candidates at each step based on leave-one-out internal validation. The final models were further internally validated by repeated $k$-fold $(k=$ 4,100 repeats) cross-validation. The training set for model development comprised of all catalyst variations with the standard substrate $\mathbf{A} \mathbf{r}^{\mathbf{A}}=\mathrm{Ph}$ as well as all substrate variations with the catalyst $\mathbf{A} \mathbf{r}^{\mathbf{C}}=4$ - $\mathrm{Ad}-\mathrm{Ph}(24$ training samples). The validation set comprised of all other combinations of $\mathbf{A} \mathbf{r}^{\mathbf{A}}$ and $\mathbf{A} \mathbf{r}^{\mathbf{C}}$ (13 samples).

\begin{tabular}{|c|c|c|c|c|c|c|}
\hline \multirow[b]{2}{*}{$A r^{C}$} & \multirow[b]{2}{*}{$A \mathbf{r}^{A}$} & \multirow[b]{2}{*}{$\begin{array}{c}\Delta \Delta G^{\ddagger} \\
\text { measured }\end{array}$} & \multicolumn{2}{|c|}{$\begin{array}{l}\text { Hypothesis-informed } \\
\text { model (Figure 5, top) }\end{array}$} & \multicolumn{2}{|c|}{$\begin{array}{c}\text { Full model } \\
\text { (Figure 5, bottom) }\end{array}$} \\
\hline & & & $\begin{array}{c}\Delta \Delta G^{\ddagger} \\
\text { predicted }\end{array}$ & $\begin{array}{l}\text { LOO } \\
\text { prediction }\end{array}$ & $\begin{array}{c}\Delta \Delta G^{\ddagger} \\
\text { predicted }\end{array}$ & $\begin{array}{l}\mathrm{LOO} \\
\text { prediction }\end{array}$ \\
\hline \multicolumn{7}{|l|}{ Training Set } \\
\hline 2Nap & $\mathrm{Ph}$ & 0.47 & 0.62 & 0.64 & 0.50 & 0.51 \\
\hline $3,4,5-\mathrm{Me}_{3}-\mathrm{Ph}$ & $\mathrm{Ph}$ & 0.37 & 0.26 & 0.23 & 0.25 & 0.23 \\
\hline 3,5-Me $-4-\mathrm{OMe}-\mathrm{Ph}$ & $\mathrm{Ph}$ & 0.37 & 0.24 & 0.21 & 0.22 & 0.19 \\
\hline $3,5-\mathrm{Me}_{2}-\mathrm{Ph}$ & $\mathrm{Ph}$ & 0.19 & 0.33 & 0.36 & 0.35 & 0.38 \\
\hline 3,5-tBu $-\mathrm{Ph}$ & $\mathrm{Ph}$ & 0.59 & 0.64 & 0.76 & 0.66 & 0.76 \\
\hline $4-\mathrm{CF}_{3}-\mathrm{Ph}$ & $\mathrm{Ph}$ & 0.96 & 0.77 & 0.75 & 1.07 & 1.09 \\
\hline 4-Me-Ph & $\mathrm{Ph}$ & 0.94 & 0.77 & 0.75 & 0.72 & 0.69 \\
\hline 4-nPr-Ph & $\mathrm{Ph}$ & 0.73 & 0.75 & 0.75 & 0.87 & 0.89 \\
\hline 4-Ph-Ph & $\mathrm{Ph}$ & 0.50 & 0.71 & 0.74 & 0.59 & 0.64 \\
\hline 4-tBu-Ph & $\mathrm{Ph}$ & 1.24 & 1.31 & 1.32 & 1.17 & 1.15 \\
\hline $\mathrm{Ph}$ & $\mathrm{Ph}$ & 0.67 & 0.82 & 0.84 & 0.86 & 0.93 \\
\hline 4-Ad-Ph & $1 \mathrm{Nap}$ & 0.90 & 1.19 & 1.22 & 0.77 & 0.69 \\
\hline 4-Ad-Ph & 2Furyl & 0.17 & 0.38 & 0.44 & 0.17 & 0.18 \\
\hline 4-Ad-Ph & 2-Me-Ph & 0.62 & 0.93 & 0.97 & 0.98 & 1.03 \\
\hline 4-Ad-Ph & $2 \mathrm{Nap}$ & 1.58 & 1.19 & 1.16 & 1.48 & 1.41 \\
\hline 4-Ad-Ph & 2-OMe-Ph & 0.50 & 0.40 & 0.29 & 0.47 & 0.44 \\
\hline 4-Ad-Ph & 3-Me-Ph & 0.92 & 0.96 & 0.96 & 0.93 & 0.93 \\
\hline 4-Ad-Ph & $4-\mathrm{CF}_{3}-\mathrm{Ph}$ & 1.08 & 1.39 & 1.45 & 1.10 & 1.10 \\
\hline 4-Ad-Ph & 4-Cl-Ph & 1.45 & 1.46 & 1.46 & 1.57 & 1.59 \\
\hline 4-Ad-Ph & 4-F-Ph & 1.41 & 1.16 & 1.14 & 1.15 & 1.11 \\
\hline 4-Ad-Ph & 4-Me-Ph & 1.53 & 1.17 & 1.14 & 1.36 & 1.34 \\
\hline 4-Ad-Ph & 4-OMe-Ph & 1.05 & 1.19 & 1.20 & 1.31 & 1.37 \\
\hline 4-Ad-Ph & 4-Ph-Ph & 1.21 & 1.16 & 1.15 & 1.17 & 1.17 \\
\hline 4-Ad-Ph & $\mathrm{Ph}$ & 1.63 & 1.25 & 1.21 & 1.35 & 1.29 \\
\hline \multicolumn{7}{|l|}{ Validation Set } \\
\hline $3,4,5-\mathrm{Me}_{3}-\mathrm{Ph}$ & 4-F-Ph & 0.27 & 0.43 & & 0.17 & \\
\hline 3,5-Me $-4-\mathrm{OMe}-\mathrm{Ph}$ & 4-F-Ph & 0.05 & 0.41 & & 0.11 & \\
\hline $3,5-\mathrm{Me}_{2}-\mathrm{Ph}$ & 4-F-Ph & 0.17 & 0.49 & & 0.25 & \\
\hline $4-\mathrm{CF}_{3}-\mathrm{Ph}$ & $4-\mathrm{CF}_{3}-\mathrm{Ph}$ & 1.05 & 1.08 & & 0.54 & \\
\hline $4-\mathrm{CF}_{3}-\mathrm{Ph}$ & 4-OMe-Ph & 0.77 & 0.75 & & 0.84 & \\
\hline $4-\mathrm{CF}_{3}-\mathrm{Ph}$ & 4-Ph-Ph & 1.13 & 0.97 & & 0.90 & \\
\hline
\end{tabular}




\begin{tabular}{|c|c|c|c|c|}
\hline $\mathrm{Ph}$ & 2-Me-Ph & 0.52 & 0.53 & 0.47 \\
\hline $\mathrm{Ph}$ & 2Nap & 0.50 & 0.74 & 0.37 \\
\hline $\mathrm{Ph}$ & 2-OMe-Ph & 0.28 & -0.03 & -0.04 \\
\hline $\mathrm{Ph}$ & 3-Me-Ph & 0.59 & 0.53 & 0.35 \\
\hline $\mathrm{Ph}$ & $4-\mathrm{CF}_{3}-\mathrm{Ph}$ & 0.27 & 1.12 & 0.67 \\
\hline $\mathrm{Ph}$ & 4-OMe-Ph & 0.39 & 0.78 & 0.58 \\
\hline $\mathrm{Ph}$ & 4-Ph-Ph & 0.73 & 1.01 & 0.67 \\
\hline
\end{tabular}

Table S13. Measured and predicted relative free energies of activation in kcal/mol of the models shown in Figure 5.

\section{Additional discussion on confounding effects between descriptors for NCI and sterics}

Two descriptors for NCI in the first model shown in the manuscript (Figure 5, top) have a correlation with a steric descriptor for the catalyst bulk: ${ }^{\mathrm{ETF}} D_{\min }\left(\mathrm{Ar}_{\text {side }}{ }-\mathrm{Ar}^{\mathrm{A}}\right)$ with $B_{5}\left(\mathrm{Ar}^{\mathrm{C}}\right)$ and ${ }^{\mathrm{S}} D^{\mathrm{min}}\left(\mathrm{Ar}^{\mathrm{C}}{ }^{-}-\mathrm{Ar}_{\pi}^{\mathrm{A}}\right)$ with $B_{1}\left(\mathrm{Ar}^{\mathrm{C}}\right)$, both with a correlation $R^{2}=0.72$. Physically, these correlations make sense because the interaction distance is impacted by the bulk of the interacting arene. Steric effects could influence the reaction outcome both directly and via modulation of $\mathrm{NCI}$ and the collinearity between relevant descriptors could complicate the interpretation of the model. Adding either of those steric descriptors $\left(B_{1}\left(\mathrm{Ar}^{\mathrm{C}}\right), B_{5}\left(\mathrm{Ar}^{\mathrm{C}}\right)\right)$ to the original model does not cause any of the NCI descriptors to become insignificant while the steric descriptors are highly insignificant ( $p$-value in the 4-term model $>0.9$ in both cases). Also, exchanging the NCI term with the respective steric descriptor results in poorer model statistics. This, together with the results from the transition state analysis, offers additional evidence for a causative relation of the NCI descriptors with the enantioselectivity.

In the following, ${ }^{\mathrm{ETF}} D_{\min }\left(\mathrm{Ar}^{\mathrm{C}}{ }_{\text {side }}-\mathrm{Ar}^{\mathrm{A}}\right)$ is the same as $\mathrm{ArC}$ _side_ArA_pi_0_D.min, ${ }^{\mathrm{S}} D^{\mathrm{min}}\left(\mathrm{Ar}_{\pi}^{\mathrm{C}}-\mathrm{Ar}^{\mathrm{A}}\right)$ the same as ArC_pi_ArA_pi_60_D.min and ${ }^{\mathrm{ETF}} E_{\max }\left(\mathrm{Ar}^{\mathrm{A}}{ }_{\text {side }}-\mathrm{Ph}_{\pi}\right)$ the same as ArA_side_Ph_pi_0_E.max. y $=\Delta \Delta G^{\ddagger}$

\section{Descriptor correlations}
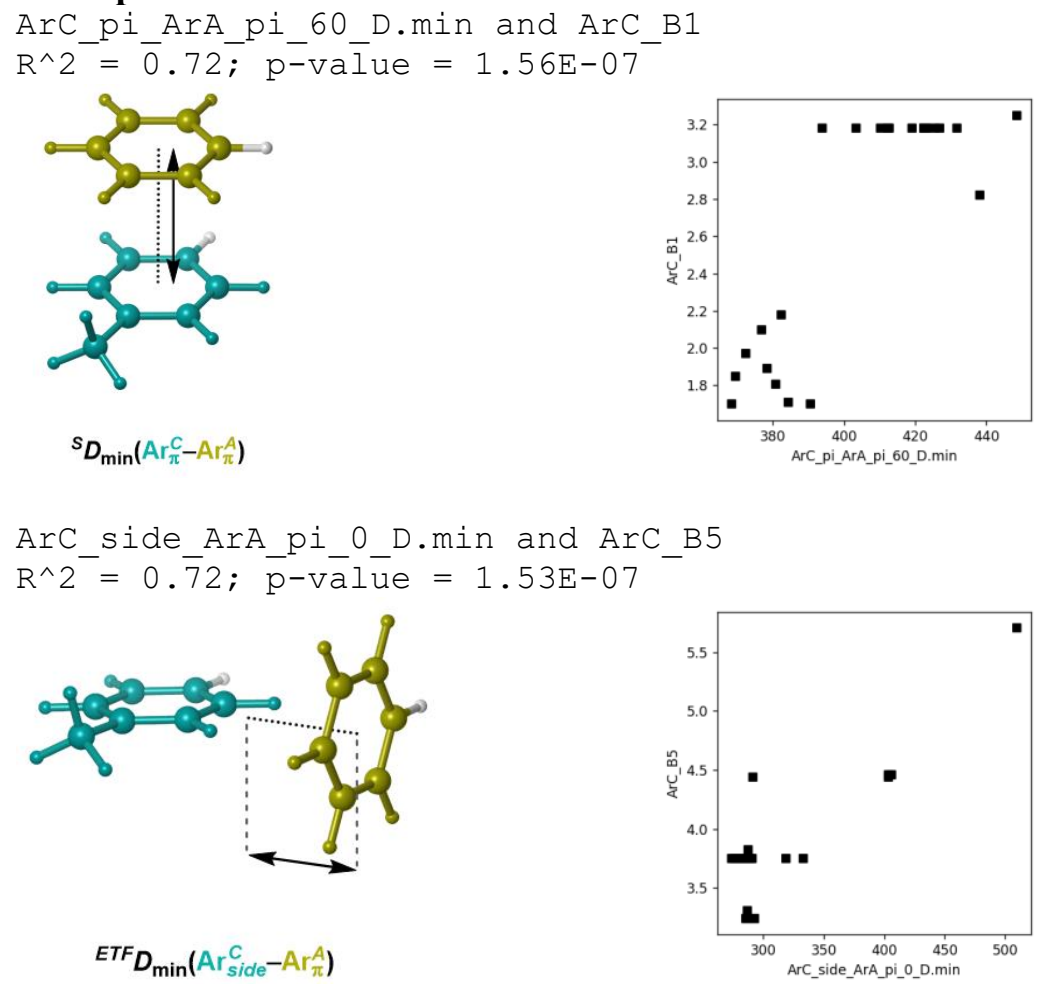


\section{Model as shown in the manuscript:}
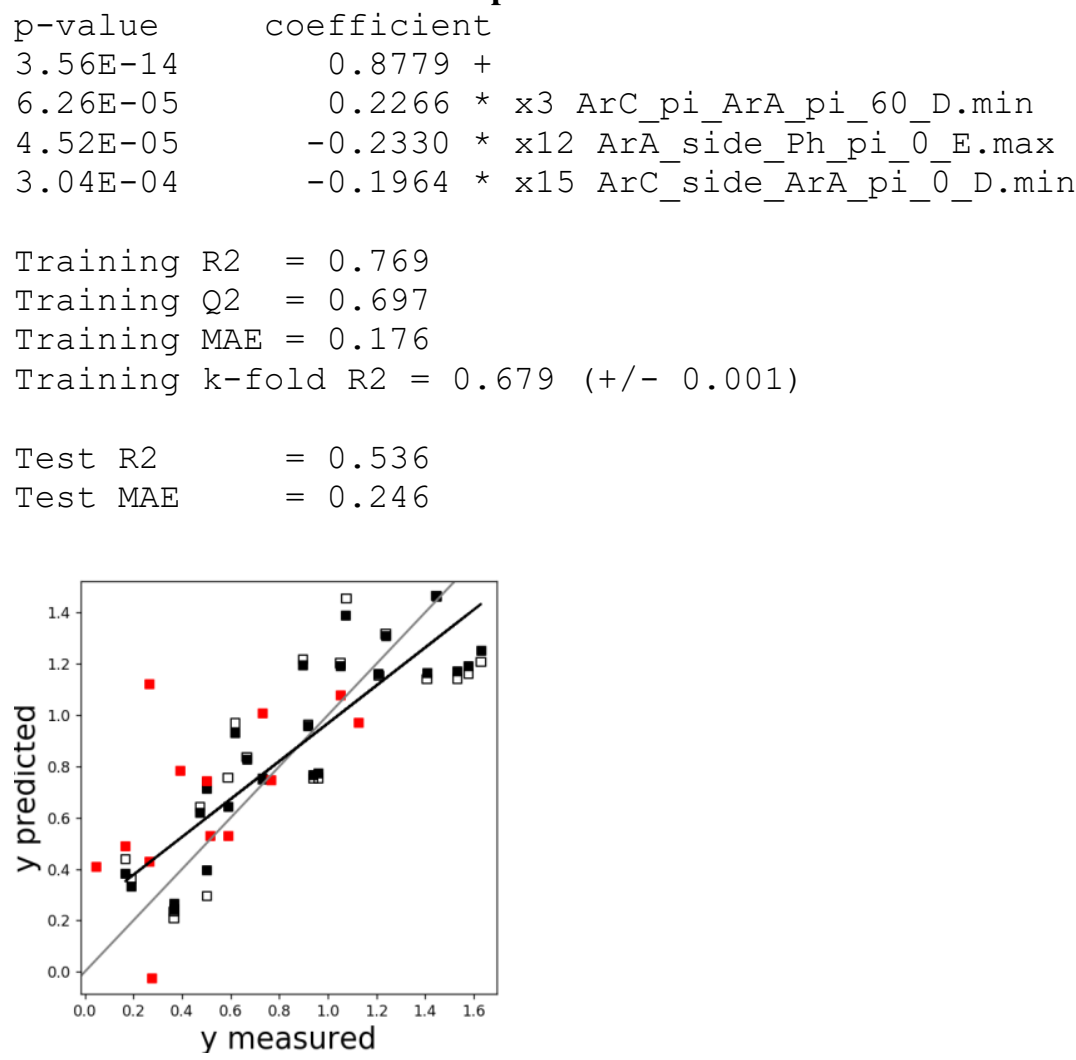

Addition of ArC_B1 to standard model:
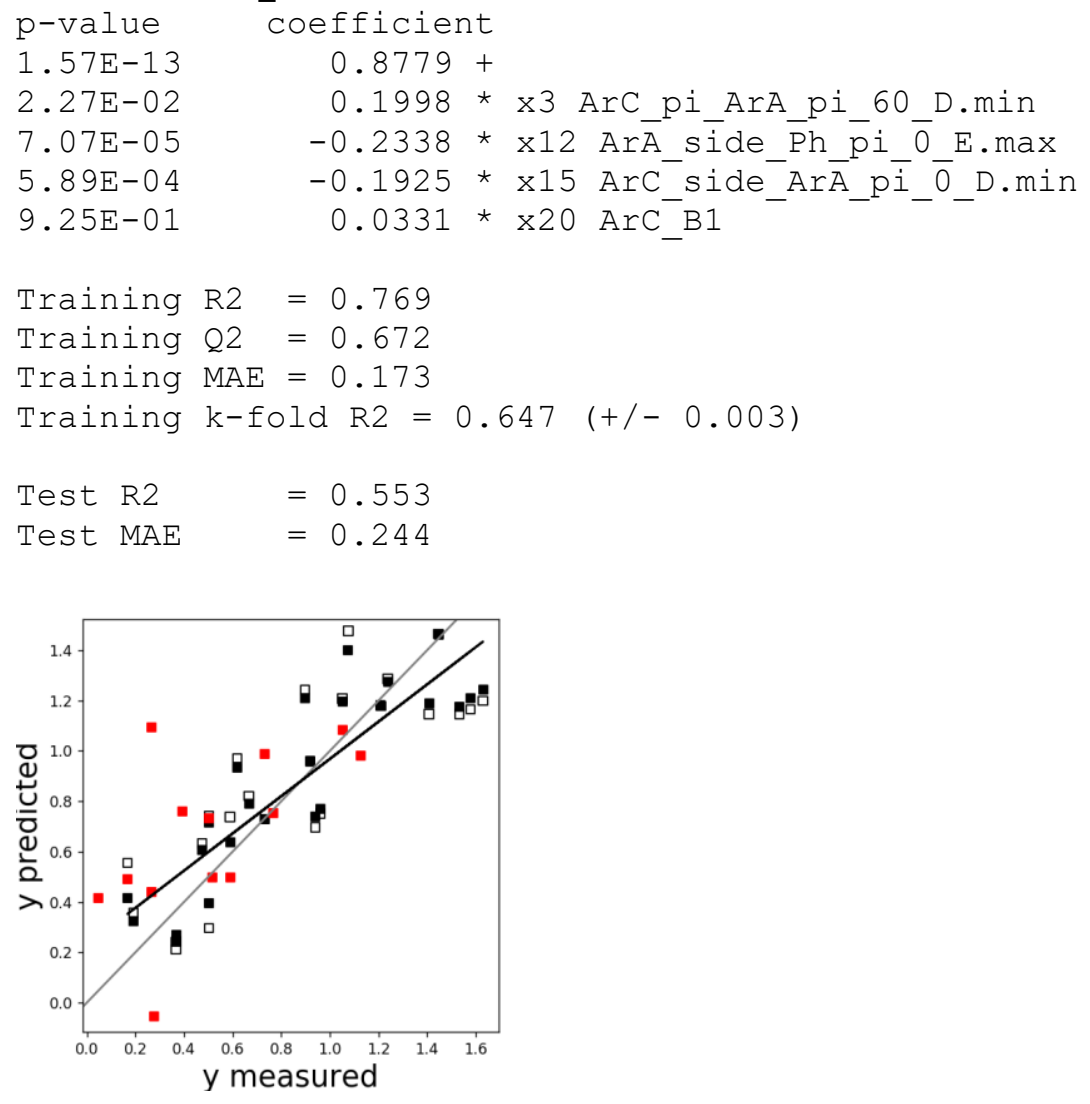


\section{Addition of ArC_B5 to standard model:}

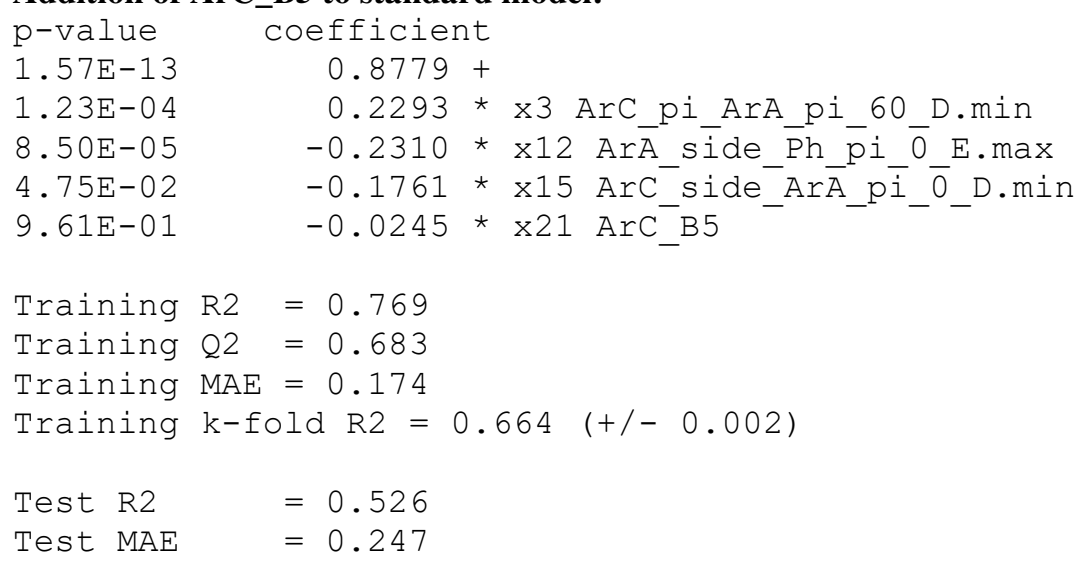

1.23E-04

$0.8779+$

$0.2293 * \times 3$ ArC_pi_ArA_pi_60_D.min

$8.50 \mathrm{E}-05$

$-0.2310 * x 12$ Ar $\overline{\mathrm{A}} \_\mathrm{s} \overline{\mathrm{i}} \mathrm{de} \_\overline{\mathrm{P}} \mathrm{p} \overline{\mathrm{p}}_{\mathrm{i}} \overline{0}_{-} \mathrm{E} \cdot \max$

4. $75 E-02$

$-0.1761 * x 15$ ArC_side_Ar $\bar{A} \_p \bar{i}-\overline{0}$ D.min

9. $61 \mathrm{E}-01$ $-0.0245 * \times 21 \mathrm{ArC}^{-} \mathrm{B} 5$

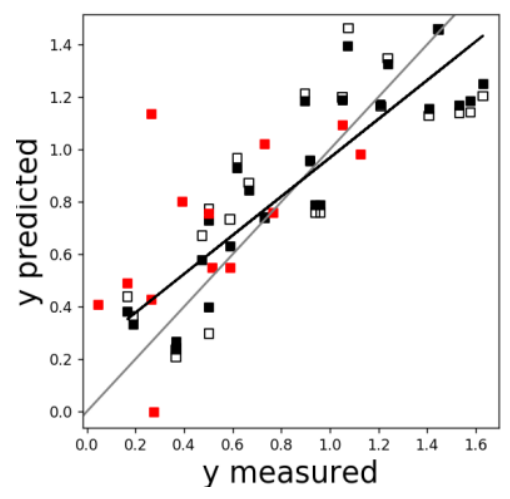

Exchange ArC_pi_ArA_pi_60_D.min with ArC_B1:
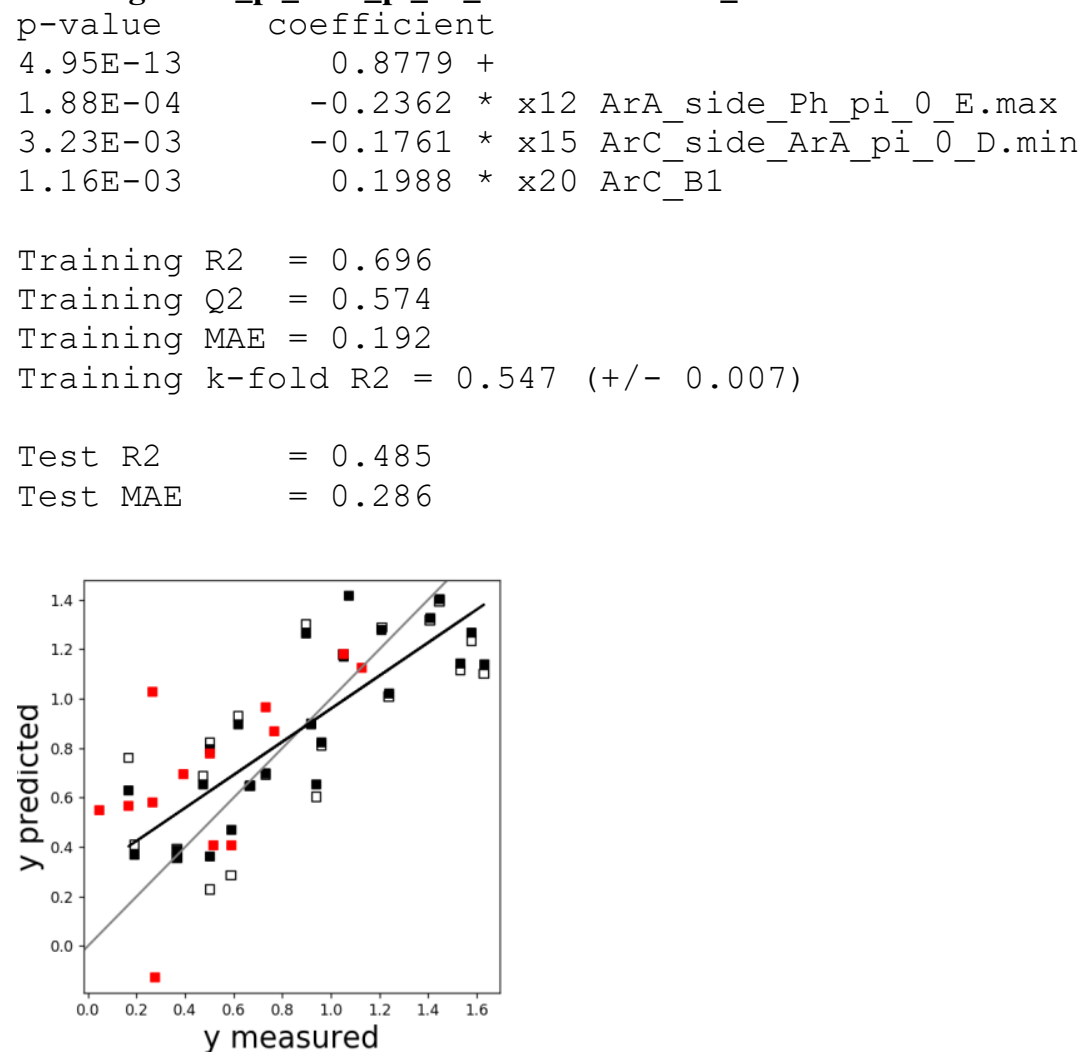
Exchange ArC_side_ArA_pi_0_D.min with ArC_B5:

p-value

2. $63 \mathrm{E}-13$

$7.46 \mathrm{E}-05$

$3.71 \mathrm{E}-04$

$2.85 \mathrm{E}-03$

coefficient

$0.8779+$

0.2475 * x3 ArC_pi_ArA_pi_60_D.min

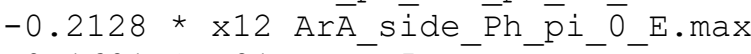

$-0.1691 * \times 21$ ArC B5

Training $\mathrm{R} 2=0.715$

Training $\mathrm{Q} 2=0.594$

Training $\mathrm{MAE}=0.189$

Training $\mathrm{k}$-fold $\mathrm{R} 2=0.575(+/-0.004)$

Test R2 $=0.434$

Test MAE $\quad=0.278$

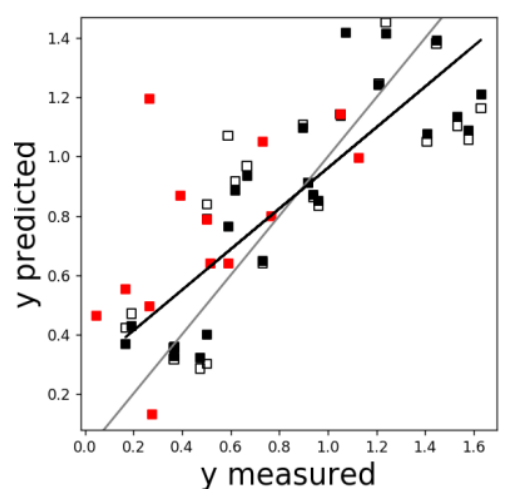




\section{Coordinates of stationary points}

\section{Mechanism of the allenoate-Claisen rearrangement}

\section{Uncatalyzed mechanism}

Substrate_Amine

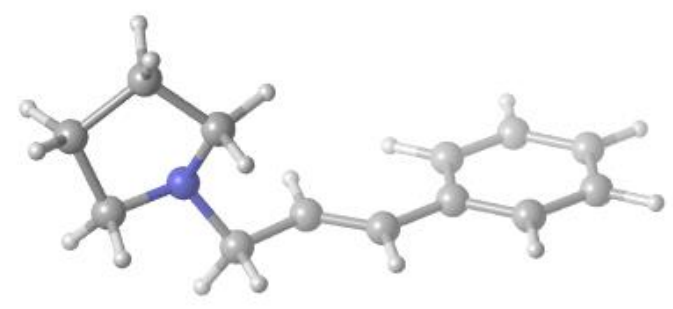

31

$\begin{array}{lrrr}\mathrm{C} & -3.95479 & -0.73790 & -0.36463 \\ \mathrm{C} & -2.51726 & 0.70327 & 0.73549 \\ \mathrm{C} & -3.84785 & 1.47519 & 0.67201 \\ \mathrm{C} & -4.82367 & 0.49583 & -0.04944 \\ \mathrm{H} & -4.05037 & -1.49907 & 0.45285 \\ \mathrm{H} & -4.21730 & -1.23564 & -1.31559 \\ \mathrm{H} & -2.44618 & 0.12023 & 1.69066 \\ \mathrm{H} & -1.62321 & 1.34641 & 0.67477 \\ \mathrm{H} & -4.19852 & 1.76043 & 1.67900 \\ \mathrm{H} & -3.72714 & 2.40321 & 0.08658 \\ \mathrm{H} & -5.69350 & 0.22662 & 0.57466 \\ \mathrm{H} & -5.20993 & 0.94654 & -0.98013 \\ \mathrm{~N} & -2.58942 & -0.20122 & -0.41982 \\ \mathrm{C} & -1.55740 & -1.23760 & -0.42126 \\ \mathrm{H} & -1.58000 & -1.84315 & 0.52122 \\ \mathrm{H} & -1.79240 & -1.93059 & -1.25488 \\ \mathrm{C} & -0.18513 & -0.66047 & -0.62824 \\ \mathrm{C} & 0.84930 & -0.84397 & 0.22168 \\ \mathrm{H} & -0.06505 & -0.05041 & -1.53420 \\ \mathrm{H} & 0.66633 & -1.44049 & 1.12856 \\ \mathrm{C} & 2.21964 & -0.32665 & 0.08706 \\ \mathrm{C} & 2.66702 & 0.39853 & -1.04372 \\ \mathrm{C} & 3.14440 & -0.55629 & 1.13260 \\ \mathrm{C} & 3.97874 & 0.87885 & -1.11662 \\ \mathrm{H} & 1.98056 & 0.58602 & -1.87634 \\ \mathrm{C} & 4.45931 & -0.07619 & 1.06057 \\ \mathrm{H} & 2.81828 & -1.11961 & 2.01577 \\ \mathrm{C} & 4.88374 & 0.64503 & -0.06557 \\ \mathrm{H} & 4.30187 & 1.43844 & -2.00234 \\ \mathrm{H} & 5.15482 & -0.26687 & 1.88629 \\ \mathrm{H} & 5.91135 & 1.02079 & -0.12776\end{array}$

Substrate_Allenoate

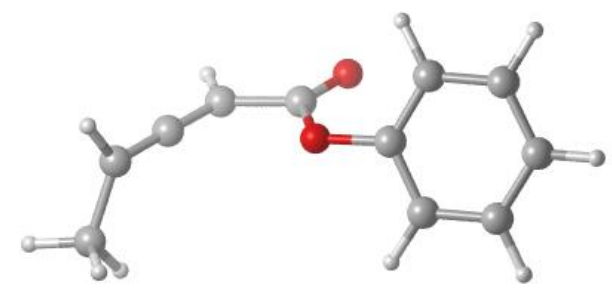

23

$\begin{array}{lrrr}\mathrm{C} & -3.70832 & -0.56472 & 0.75204 \\ \mathrm{H} & -3.91249 & -0.52692 & 1.83424 \\ \mathrm{C} & -2.96329 & 0.38729 & 0.23599 \\ \mathrm{C} & -2.20532 & 1.33647 & -0.29510 \\ \mathrm{H} & -2.64148 & 2.26266 & -0.69085 \\ \mathrm{C} & -4.29084 & -1.72124 & -0.02748 \\ \mathrm{H} & -4.03374 & -1.65657 & -1.09751 \\ \mathrm{H} & -5.39297 & -1.74050 & 0.07204 \\ \mathrm{H} & -3.90809 & -2.68111 & 0.36952 \\ \mathrm{C} & -0.72682 & 1.26456 & -0.40815 \\ \mathrm{O} & -0.03286 & 2.14792 & -0.87704 \\ \mathrm{O} & -0.23746 & 0.06409 & 0.07764 \\ \mathrm{C} & 1.15291 & -0.13430 & 0.07301 \\ \mathrm{C} & 1.99730 & 0.65773 & 0.86148 \\ \mathrm{C} & 1.64889 & -1.20259 & -0.68283 \\ \mathrm{C} & 3.36926 & 0.36778 & 0.88696 \\ \mathrm{H} & 1.58375 & 1.48830 & 1.44032 \\ \mathrm{C} & 3.02316 & -1.48603 & -0.64516 \\ \mathrm{H} & 0.95797 & -1.79862 & -1.28754 \\ \mathrm{C} & 3.88549 & -0.70193 & 0.13705 \\ \mathrm{H} & 4.03802 & 0.98386 & 1.49860 \\ \mathrm{H} & 4.95822 & -0.92346 & 0.16273 \\ \mathrm{H} & 3.41885 & -2.32186 & -1.23324\end{array}$

Substrates_associated

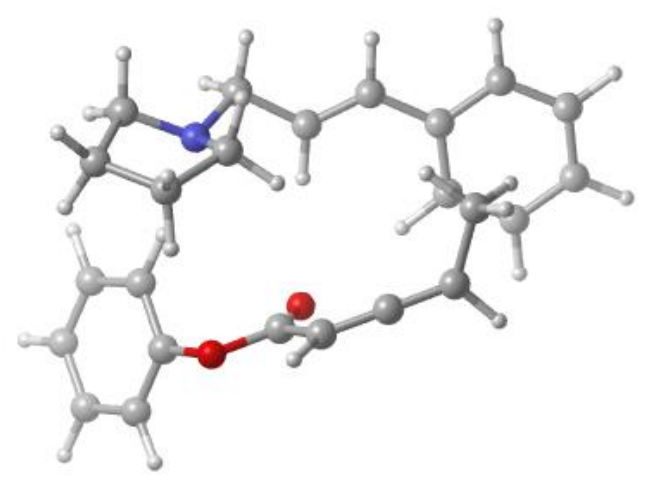

54

C $\quad-0.04260 \quad 2.39413 \quad-1.33020$ 


\begin{tabular}{|c|c|c|c|}
\hline $\mathrm{C}$ & 0.95268 & 1.26854 & -1.29703 \\
\hline $\mathrm{C}$ & 2.29091 & 1.44484 & -1.23375 \\
\hline $\mathrm{C}$ & 2.53321 & -1.22545 & 2.38542 \\
\hline $\mathrm{C}$ & 1.22192 & -1.26463 & 2.32599 \\
\hline $\mathrm{H}$ & 0.48117 & 3.38091 & -1.26022 \\
\hline $\mathrm{H}$ & -0.58217 & 2.38356 & -2.29981 \\
\hline $\mathrm{H}$ & 3.06335 & -2.18014 & 2.53323 \\
\hline $\mathrm{H}$ & 2.67728 & 2.47568 & -1.21583 \\
\hline $\mathrm{C}$ & 3.37658 & 0.02227 & 2.27546 \\
\hline $\mathrm{H}$ & 2.76566 & 0.90242 & 2.01936 \\
\hline $\mathrm{H}$ & 3.89638 & 0.21348 & 3.23401 \\
\hline $\mathrm{H}$ & 4.14607 & -0.10791 & 1.49481 \\
\hline $\mathrm{H}$ & 0.52058 & 0.26054 & -1.30990 \\
\hline $\mathrm{C}$ & -0.52612 & 2.32950 & 1.08776 \\
\hline $\mathrm{C}$ & -2.13313 & 3.22616 & -0.32789 \\
\hline $\mathrm{C}$ & -1.79931 & 2.39482 & 1.95196 \\
\hline $\mathrm{H}$ & 0.11926 & 1.46510 & 1.31442 \\
\hline $\mathrm{H}$ & 0.09070 & 3.25510 & 1.21854 \\
\hline $\mathrm{C}$ & -2.87465 & 3.01490 & 1.00809 \\
\hline $\mathrm{H}$ & -1.72735 & 4.26744 & -0.39353 \\
\hline $\mathrm{H}$ & -2.76850 & 3.05866 & -1.21715 \\
\hline $\mathrm{H}$ & -1.63796 & 2.99372 & 2.86462 \\
\hline $\mathrm{H}$ & -2.10133 & 1.38119 & 2.26516 \\
\hline $\mathrm{H}$ & -3.28310 & 3.96432 & 1.39471 \\
\hline $\mathrm{H}$ & -3.71956 & 2.31781 & 0.87426 \\
\hline $\mathrm{N}$ & -1.04931 & 2.23906 & -0.28087 \\
\hline $\mathrm{C}$ & -0.10300 & -1.28711 & 2.26820 \\
\hline $\mathrm{C}$ & -0.83379 & -1.47121 & 0.99051 \\
\hline $\mathrm{H}$ & -0.71066 & -1.16043 & 3.17384 \\
\hline $\mathrm{O}$ & -2.19414 & -1.36478 & 1.23985 \\
\hline $\mathrm{O}$ & -0.35920 & -1.68815 & -0.10926 \\
\hline $\mathrm{C}$ & -3.07220 & -1.41504 & 0.13950 \\
\hline $\mathrm{C}$ & -3.05641 & -0.39696 & -0.82107 \\
\hline $\mathrm{C}$ & -4.00030 & -2.46046 & 0.09745 \\
\hline $\mathrm{C}$ & -4.00548 & -0.43887 & -1.85326 \\
\hline $\mathrm{H}$ & -2.31949 & 0.41231 & -0.74225 \\
\hline $\mathrm{C}$ & -4.94648 & -2.48556 & -0.93994 \\
\hline $\mathrm{H}$ & -3.97705 & -3.23612 & 0.86969 \\
\hline $\mathrm{C}$ & -4.95020 & -1.47729 & -1.91687 \\
\hline $\mathrm{H}$ & -4.00622 & 0.35058 & -2.61411 \\
\hline $\mathrm{H}$ & -5.68058 & -3.29814 & -0.98249 \\
\hline $\mathrm{H}$ & -5.68893 & -1.50051 & -2.72597 \\
\hline $\mathrm{C}$ & 3.31704 & 0.39238 & -1.17873 \\
\hline $\mathrm{C}$ & 2.99990 & -0.98315 & -1.06810 \\
\hline $\mathrm{C}$ & 4.68233 & 0.76198 & -1.19868 \\
\hline $\mathrm{C}$ & 4.01397 & -1.94280 & -0.97971 \\
\hline $\mathrm{H}$ & 1.95551 & -1.30355 & -1.01673 \\
\hline $\mathrm{C}$ & 5.69781 & -0.20074 & -1.10988 \\
\hline $\mathrm{H}$ & 4.94571 & 1.82416 & -1.27888 \\
\hline $\mathrm{C}$ & 5.36774 & -1.56074 & -0.99855 \\
\hline $\mathrm{H}$ & 3.74463 & -3.00145 & -0.88581 \\
\hline $\mathrm{H}$ & 6.74850 & 0.11193 & -1.12562 \\
\hline $\mathrm{H}$ & 6.15744 & -2.31764 & -0.92711 \\
\hline
\end{tabular}

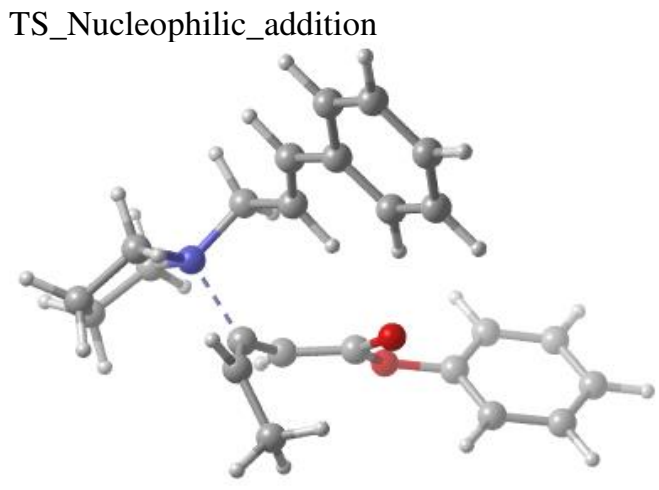

54

$\begin{array}{lrrr}\mathrm{C} & 1.36324 & 1.19016 & -1.63199 \\ \mathrm{C} & 1.54129 & -0.17061 & -1.03112 \\ \mathrm{C} & 2.64868 & -0.92747 & -1.19463 \\ \mathrm{C} & 0.95151 & 1.42382 & 1.84046 \\ \mathrm{C} & 0.32226 & 1.87072 & 0.74609 \\ \mathrm{H} & 2.07865 & 1.38588 & -2.45410 \\ \mathrm{H} & 0.33463 & 1.30488 & -2.01458 \\ \mathrm{H} & 2.02303 & 1.19818 & 1.83936 \\ \mathrm{H} & 3.46666 & -0.54331 & -1.82451 \\ \mathrm{C} & 0.20455 & 1.09625 & 3.10570 \\ \mathrm{H} & -0.79585 & 1.56006 & 3.10445 \\ \mathrm{H} & 0.06097 & 0.00041 & 3.18545 \\ \mathrm{H} & 0.76486 & 1.42666 & 4.00111 \\ \mathrm{H} & 0.70178 & -0.51693 & -0.41778 \\ \mathrm{C} & 2.94086 & 2.47411 & -0.16245 \\ \mathrm{C} & 1.15245 & 3.62175 & -1.12600 \\ \mathrm{C} & 2.93332 & 3.84396 & 0.53740 \\ \mathrm{H} & 3.27001 & 1.62981 & 0.45711 \\ \mathrm{H} & 3.58089 & 2.48942 & -1.06717 \\ \mathrm{C} & 1.71152 & 4.59073 & -0.07440 \\ \mathrm{H} & 1.63133 & 3.76202 & -2.11525 \\ \mathrm{H} & 0.06100 & 3.65887 & -1.25898 \\ \mathrm{H} & 3.88459 & 4.37352 & 0.36245 \\ \mathrm{H} & 2.80804 & 3.72473 & 1.62536 \\ \mathrm{H} & 1.98415 & 5.55747 & -0.52900 \\ \mathrm{H} & 0.95132 & 4.78301 & 0.70053 \\ \mathrm{~N} & 1.53355 & 2.27706 & -0.61050 \\ \mathrm{C} & -0.99697 & 1.92379 & 0.29799 \\ \mathrm{C} & -1.75445 & 0.70891 & 0.23699 \\ \mathrm{H} & -1.48979 & 2.85022 & -0.01229 \\ \mathrm{O} & -3.07294 & 0.99008 & -0.23379 \\ \mathrm{O} & -1.40880 & -0.44303 & 0.51318 \\ \mathrm{C} & -4.01370 & -0.02943 & -0.23097 \\ \mathrm{C} & -4.73706 & -0.23779 & -1.41583 \\ \mathrm{C} & -4.30696 & -0.77577 & 0.92328 \\ \mathrm{C} & -5.76424 & -1.19347 & -1.44585 \\ \mathrm{H} & -4.48637 & 0.35696 & -2.30062 \\ \mathrm{C} & -5.33029 & -1.73346 & 0.87959 \\ \mathrm{H} & -3.73369 & -0.60706 & 1.83826 \\ \mathrm{C} & -6.06309 & -1.94762 & -0.30002 \\ \mathrm{H} & -6.32948 & -1.35100 & -2.37213 \\ \mathrm{H} & -5.55762 & -2.31727 & 1.77950\end{array}$




$\begin{array}{lccc}\mathrm{H} & -6.86235 & -2.69713 & -0.32494 \\ \mathrm{C} & 2.88549 & -2.24896 & -0.59390 \\ \mathrm{C} & 1.95193 & -2.86846 & 0.27325 \\ \mathrm{C} & 4.09240 & -2.92957 & -0.87482 \\ \mathrm{C} & 2.22432 & -4.12115 & 0.83235 \\ \mathrm{H} & 1.00747 & -2.36732 & 0.50977 \\ \mathrm{C} & 4.36407 & -4.18514 & -0.31303 \\ \mathrm{H} & 4.82535 & -2.46299 & -1.54513 \\ \mathrm{C} & 3.43004 & -4.78650 & 0.54397 \\ \mathrm{H} & 1.48863 & -4.58501 & 1.49948 \\ \mathrm{H} & 5.30635 & -4.69465 & -0.54568 \\ \mathrm{H} & 3.63748 & -5.76807 & 0.98536\end{array}$

Intermediate_1

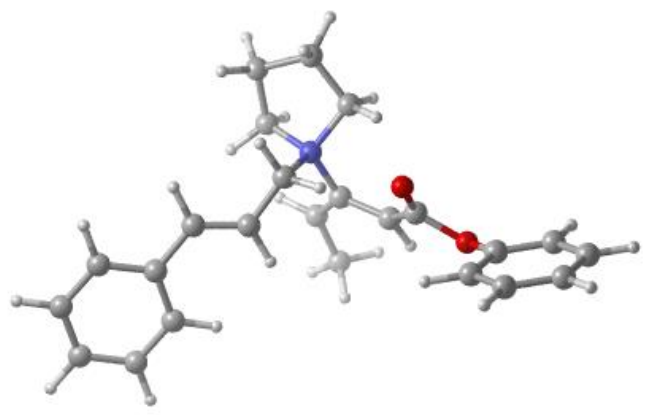

54

$\begin{array}{lrrr}\text { C } & 0.73302 & 0.83276 & -1.04234 \\ \mathrm{C} & 1.74118 & -0.11883 & -0.49596 \\ \mathrm{C} & 2.98060 & -0.26084 & -1.02355 \\ \mathrm{C} & 0.91587 & 1.39452 & 2.31161 \\ \mathrm{C} & 0.00540 & 1.33986 & 1.28460 \\ \mathrm{H} & 1.08748 & 1.31360 & -1.96650 \\ \mathrm{H} & -0.24949 & 0.36417 & -1.22871 \\ \mathrm{H} & 1.86425 & 1.91776 & 2.19566 \\ \mathrm{H} & 3.25265 & 0.37311 & -1.88183 \\ \mathrm{C} & 0.67364 & 0.71450 & 3.63166 \\ \mathrm{H} & 0.43504 & -0.36212 & 3.50255 \\ \mathrm{H} & 1.56186 & 0.79005 & 4.28245 \\ \mathrm{H} & -0.18161 & 1.15395 & 4.18668 \\ \mathrm{H} & 1.41043 & -0.73268 & 0.34778 \\ \mathrm{C} & -0.66574 & 2.90248 & -0.57742 \\ \mathrm{C} & 1.61181 & 2.91004 & 0.04327 \\ \mathrm{C} & 0.03596 & 3.77420 & -1.62492 \\ \mathrm{H} & -1.49192 & 2.28438 & -0.95331 \\ \mathrm{H} & -1.00162 & 3.48151 & 0.29718 \\ \mathrm{C} & 1.53813 & 3.76769 & -1.22677 \\ \mathrm{H} & 1.46197 & 3.51861 & 0.94613 \\ \mathrm{H} & 2.53181 & 2.31944 & 0.14571 \\ \mathrm{H} & -0.39454 & 4.78915 & -1.62259 \\ \mathrm{H} & -0.10830 & 3.36548 & -2.63935 \\ \mathrm{H} & 1.92515 & 4.77809 & -1.01453 \\ \mathrm{H} & 2.16517 & 3.34707 & -2.03135 \\ \mathrm{~N} & 0.41911 & 1.97112 & -0.03421 \\ \mathrm{C} & -1.26563 & 0.68821 & 1.36863 \\ \mathrm{C} & -2.20283 & 0.29412 & 0.38917 \\ \mathrm{H} & -1.53749 & 0.39571 & 2.38439\end{array}$

$\begin{array}{lrrr} & & & \\ \mathrm{O} & -3.33876 & -0.29015 & 1.03445 \\ \mathrm{O} & -2.19831 & 0.39663 & -0.85837 \\ \mathrm{C} & -4.27162 & -0.97226 & 0.27452 \\ \mathrm{C} & -5.62489 & -0.70508 & 0.54125 \\ \mathrm{C} & -3.91559 & -1.96090 & -0.66065 \\ \mathrm{C} & -6.62459 & -1.43053 & -0.12406 \\ \mathrm{H} & -5.87505 & 0.06907 & 1.27445 \\ \mathrm{C} & -4.92361 & -2.67350 & -1.32576 \\ \mathrm{H} & -2.86104 & -2.16286 & -0.86326 \\ \mathrm{C} & -6.27969 & -2.41507 & -1.06387 \\ \mathrm{H} & -7.67892 & -1.21986 & 0.09139 \\ \mathrm{H} & -4.64372 & -3.44272 & -2.05565 \\ \mathrm{H} & -7.06086 & -2.97821 & -1.58715 \\ \mathrm{C} & 4.02871 & -1.19048 & -0.58339 \\ \mathrm{C} & 3.88696 & -2.02587 & 0.55114 \\ \mathrm{C} & 5.24047 & -1.25422 & -1.31024 \\ \mathrm{C} & 4.91614 & -2.89163 & 0.93455 \\ \mathrm{H} & 2.96509 & -1.99376 & 1.14074 \\ \mathrm{C} & 6.27180 & -2.12204 & -0.92637 \\ \mathrm{H} & 5.36828 & -0.61334 & -2.19156 \\ \mathrm{C} & 6.11356 & -2.94598 & 0.19832 \\ \mathrm{H} & 4.78630 & -3.52989 & 1.81611 \\ \mathrm{H} & 7.20052 & -2.15581 & -1.50735 \\ \mathrm{H} & 6.91697 & -3.62645 & 0.50228\end{array}$

Intermediate_2

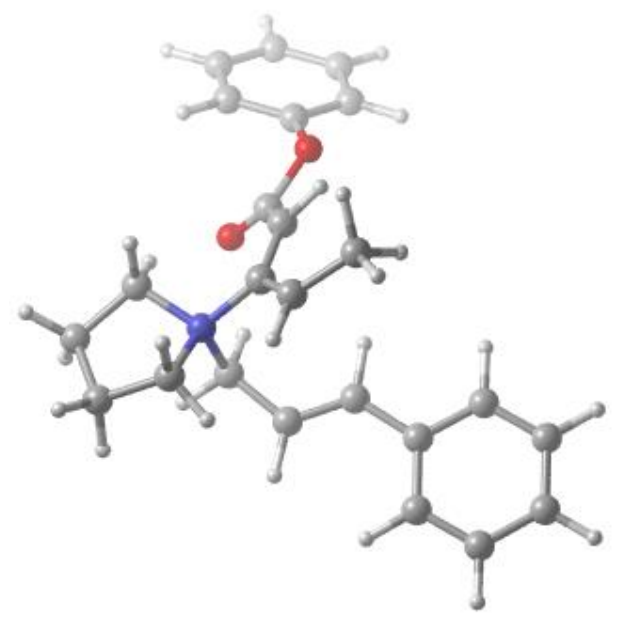

54

$\begin{array}{lrcc}\mathrm{N} & 0.54044 & 2.16163 & 0.10658 \\ \mathrm{O} & -2.03147 & 0.52406 & -0.79939 \\ \mathrm{O} & -2.64199 & -0.97429 & 0.85958 \\ \mathrm{C} & -1.78562 & 0.05060 & 0.33233 \\ \mathrm{C} & -0.75068 & 0.30152 & 1.25686 \\ \mathrm{C} & 0.41308 & 1.13716 & 1.21440 \\ \mathrm{C} & -3.85891 & -1.21826 & 0.25363 \\ \mathrm{C} & 1.45450 & 1.04270 & 2.10341 \\ \mathrm{C} & -4.20689 & -2.56384 & 0.04590 \\ \mathrm{C} & -4.77144 & -0.19529 & -0.06371 \\ \mathrm{C} & 1.49128 & 0.00103 & 3.18713 \\ \mathrm{C} & 1.63007 & 3.20024 & 0.30814\end{array}$




$\begin{array}{cccc}\mathrm{C} & -0.70761 & 3.04135 & 0.01351 \\ \mathrm{C} & -5.46913 & -2.88807 & -0.47316 \\ \mathrm{C} & -6.02694 & -0.53085 & -0.59031 \\ \mathrm{C} & 1.26978 & 4.35230 & -0.63627 \\ \mathrm{C} & -0.27679 & 4.28531 & -0.77613 \\ \mathrm{C} & -6.38419 & -1.87378 & -0.79811 \\ \mathrm{C} & 0.77939 & 1.42068 & -1.25718 \\ \mathrm{C} & 2.05915 & 0.65458 & -1.26967 \\ \mathrm{C} & 2.14183 & -0.59026 & -0.74232 \\ \mathrm{C} & 3.33455 & -1.43507 & -0.62272 \\ \mathrm{C} & 4.61352 & -1.04836 & -1.09024 \\ \mathrm{C} & 3.20709 & -2.69716 & 0.00328 \\ \mathrm{C} & 5.71728 & -1.89337 & -0.93872 \\ \mathrm{C} & 4.31323 & -3.54357 & 0.15803 \\ \mathrm{C} & 5.57352 & -3.14528 & -0.31285 \\ \mathrm{H} & -0.80918 & -0.31061 & 2.15829 \\ \mathrm{H} & 2.32146 & 1.69762 & 2.02444 \\ \mathrm{H} & -3.47901 & -3.34143 & 0.30103 \\ \mathrm{H} & -4.49445 & 0.84879 & 0.10093 \\ \mathrm{H} & 0.67977 & 0.13126 & 3.93320 \\ \mathrm{H} & 2.45030 & 0.03348 & 3.73215 \\ \mathrm{H} & 1.37054 & -1.02261 & 2.77441 \\ \mathrm{H} & 2.61106 & 2.74222 & 0.12580 \\ \mathrm{H} & 1.56573 & 3.51243 & 1.36015 \\ \mathrm{H} & -0.96608 & 3.27339 & 1.05844 \\ \mathrm{H} & -1.51017 & 2.44316 & -0.44077 \\ \mathrm{H} & -5.73535 & -3.94045 & -0.62855 \\ \mathrm{H} & -6.73590 & 0.26864 & -0.83747 \\ \mathrm{H} & 1.61704 & 5.30746 & -0.20888 \\ \mathrm{H} & 1.76684 & 4.23895 & -1.61479 \\ \mathrm{H} & -0.77016 & 5.18167 & -0.36535 \\ \mathrm{H} & -0.57700 & 4.20941 & -1.83486 \\ \mathrm{H} & -7.36851 & -2.12700 & -1.20826 \\ \mathrm{H} & -0.11766 & 0.79526 & -1.37495 \\ \mathrm{H} & 0.76023 & 2.20412 & -2.02865 \\ \mathrm{H} & 2.94645 & 1.14736 & -1.68649 \\ \mathrm{H} & 1.21827 & -1.02432 & -0.33459 \\ \mathrm{H} & 4.74602 & -0.07766 & -1.57991 \\ \mathrm{H} & 2.22299 & -3.00874 & 0.37304 \\ \mathrm{H} & 6.69896 & -1.57677 & -1.30975 \\ \mathrm{H} & 4.19068 & -4.51666 & 0.64699 \\ \mathrm{H} & 6.44117 & -3.80431 & -0.19477 \\ & & & \end{array}$

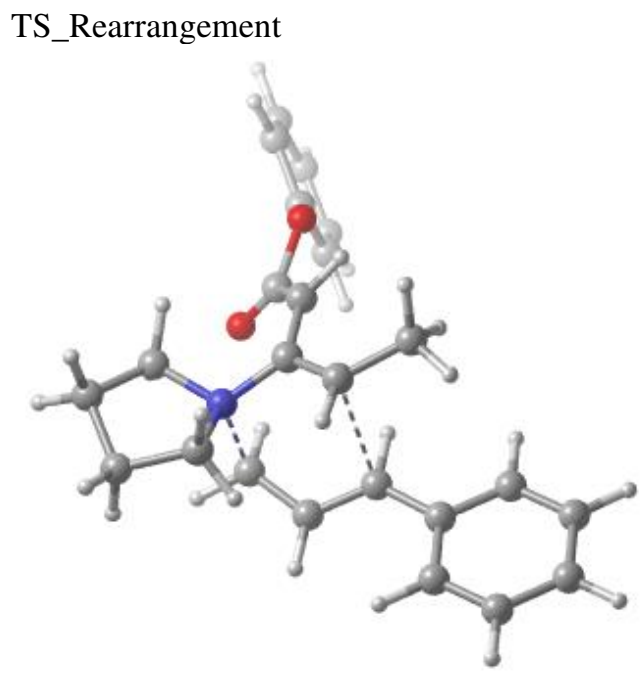

$\begin{array}{lrrr}54 & & & \\ & & & \\ \mathrm{C} & -0.75870 & 1.26899 & -1.61628 \\ \mathrm{C} & -1.98686 & 0.52729 & -1.60864 \\ \mathrm{C} & -2.08272 & -0.63019 & -0.86386 \\ \mathrm{C} & -1.64834 & 0.49218 & 1.57618 \\ \mathrm{C} & -0.46847 & 0.96110 & 1.01105 \\ \mathrm{H} & 0.19387 & 0.72819 & -1.55779 \\ \mathrm{H} & -0.70975 & 2.15175 & -2.26618 \\ \mathrm{H} & -2.56999 & 1.06961 & 1.48707 \\ \mathrm{H} & -1.14457 & -1.09744 & -0.53781 \\ \mathrm{C} & -1.70690 & -0.71228 & 2.46568 \\ \mathrm{H} & -1.32750 & -0.50061 & 3.48803 \\ \mathrm{H} & -2.74546 & -1.07292 & 2.56515 \\ \mathrm{H} & -1.08886 & -1.54136 & 2.07040 \\ \mathrm{H} & -2.88893 & 0.99083 & -2.02621 \\ \mathrm{C} & -1.80063 & 2.95158 & 0.21615 \\ \mathrm{C} & 0.61000 & 3.01118 & 0.01949 \\ \mathrm{C} & -1.41802 & 4.25190 & -0.48279 \\ \mathrm{H} & -2.67661 & 2.44114 & -0.20985 \\ \mathrm{H} & -1.97529 & 3.12695 & 1.29121 \\ \mathrm{C} & 0.04276 & 4.43967 & -0.04430 \\ \mathrm{H} & 1.21470 & 2.81641 & 0.91862 \\ \mathrm{H} & 1.22638 & 2.73407 & -0.84612 \\ \mathrm{H} & -2.07626 & 5.08237 & -0.17638 \\ \mathrm{H} & -1.49879 & 4.14993 & -1.58022 \\ \mathrm{H} & 0.07385 & 4.91121 & 0.95481 \\ \mathrm{H} & 0.62144 & 5.07774 & -0.73293 \\ \mathrm{~N} & -0.58499 & 2.07580 & 0.07067 \\ \mathrm{C} & 0.78753 & 0.31377 & 1.22119 \\ \mathrm{C} & 1.93213 & 0.24844 & 0.38565 \\ \mathrm{H} & 0.84871 & -0.29108 & 2.12762 \\ \mathrm{O} & 2.13559 & 0.67623 & -0.76371 \\ \mathrm{O} & 2.98059 & -0.43844 & 1.08119 \\ \mathrm{C} & 4.06673 & -0.91004 & 0.36541 \\ \mathrm{C} & 5.33667 & -0.70713 & 0.93043 \\ \mathrm{C} & 3.93032 & -1.64518 & -0.82602 \\ \mathrm{C} & 6.47231 & -1.24674 & 0.30787 \\ \mathrm{H} & 5.41542 & -0.13100 & 1.85849\end{array}$




$\begin{array}{lrrr}\mathrm{C} & 5.07338 & -2.17255 & -1.44359 \\ \mathrm{H} & 2.93954 & -1.79509 & -1.26196 \\ \mathrm{C} & 6.34746 & -1.97880 & -0.88367 \\ \mathrm{H} & 7.46041 & -1.08888 & 0.75634 \\ \mathrm{H} & 4.96481 & -2.74435 & -2.37309 \\ \mathrm{H} & 7.23525 & -2.39613 & -1.37235 \\ \mathrm{C} & -3.29782 & -1.38943 & -0.57436 \\ \mathrm{C} & -3.18223 & -2.70037 & -0.05183 \\ \mathrm{C} & -4.59790 & -0.86025 & -0.76634 \\ \mathrm{C} & -4.31861 & -3.45945 & 0.25423 \\ \mathrm{H} & -2.18302 & -3.12056 & 0.11009 \\ \mathrm{C} & -5.73256 & -1.61803 & -0.46047 \\ \mathrm{H} & -4.71853 & 0.16045 & -1.14477 \\ \mathrm{C} & -5.59953 & -2.92228 & 0.05024 \\ \mathrm{H} & -4.20480 & -4.47293 & 0.65541 \\ \mathrm{H} & -6.72984 & -1.18947 & -0.61309 \\ \mathrm{H} & -6.49078 & -3.51208 & 0.29254\end{array}$

Product

$\begin{array}{lrrr}\mathrm{H} & 1.34414 & 5.30888 & 0.57480 \\ \mathrm{H} & 0.25764 & 4.38625 & 1.65674 \\ \mathrm{H} & -0.10834 & 4.79782 & -1.38145 \\ \mathrm{H} & -1.29546 & 5.19056 & -0.10119 \\ \mathrm{~N} & 0.27248 & 2.25940 & -0.20544 \\ \mathrm{C} & -0.63722 & 0.08152 & -0.80409 \\ \mathrm{C} & -1.97161 & 0.12647 & -0.25964 \\ \mathrm{H} & -0.40030 & -0.84195 & -1.33651 \\ \mathrm{O} & -2.42971 & 0.82346 & 0.64237 \\ \mathrm{O} & -2.75590 & -0.87569 & -0.88943 \\ \mathrm{C} & -4.01541 & -1.16442 & -0.37178 \\ \mathrm{C} & -5.09252 & -1.15734 & -1.26998 \\ \mathrm{C} & -4.20215 & -1.53631 & 0.96932 \\ \mathrm{C} & -6.36900 & -1.53298 & -0.82405 \\ \mathrm{H} & -4.91477 & -0.86256 & -2.30948 \\ \mathrm{C} & -5.48356 & -1.90280 & 1.40551 \\ \mathrm{H} & -3.35373 & -1.53408 & 1.65864 \\ \mathrm{C} & -6.57012 & -1.90383 & 0.51486 \\ \mathrm{H} & -7.20956 & -1.53193 & -1.52780 \\ \mathrm{H} & -5.63216 & -2.19216 & 2.45247 \\ \mathrm{H} & -7.56791 & -2.19370 & 0.86314 \\ \mathrm{C} & 3.53579 & -1.25053 & 0.18519 \\ \mathrm{C} & 4.70003 & -0.45694 & 0.22742 \\ \mathrm{C} & 3.67623 & -2.63103 & -0.04536 \\ \mathrm{C} & 5.96776 & -1.02654 & 0.04147 \\ \mathrm{H} & 4.61904 & 0.62255 & 0.40313 \\ \mathrm{C} & 4.94298 & -3.20755 & -0.23050 \\ \mathrm{H} & 2.77963 & -3.26166 & -0.07958 \\ \mathrm{C} & 6.09394 & -2.40639 & -0.18888 \\ \mathrm{H} & 6.86087 & -0.39175 & 0.07598 \\ \mathrm{H} & 5.02968 & -4.28572 & -0.40833 \\ \mathrm{H} & 7.08395 & -2.85343 & -0.33422 \\ & & & \end{array}$

\section{Na+-catalyzed mechanism}

Substrate_Allenoate_Na+

$\begin{array}{lrrr}\mathrm{C} & 0.89391 & -0.10723 & 2.50371 \\ \mathrm{C} & 2.00620 & -0.03319 & 1.75425 \\ \mathrm{C} & 2.15649 & -0.63623 & 0.37167 \\ \mathrm{C} & 1.84048 & 0.42299 & -0.74414 \\ \mathrm{C} & 0.41708 & 0.95687 & -0.56286 \\ \mathrm{H} & -0.00337 & -0.62921 & 2.15069 \\ \mathrm{H} & 0.83884 & 0.37044 & 3.48922 \\ \mathrm{H} & 2.55174 & 1.25330 & -0.61967 \\ \mathrm{H} & 1.40390 & -1.43633 & 0.26160 \\ \mathrm{C} & 2.06334 & -0.15034 & -2.15706 \\ \mathrm{H} & 1.76171 & 0.58208 & -2.92610 \\ \mathrm{H} & 3.12853 & -0.39669 & -2.30308 \\ \mathrm{H} & 1.48247 & -1.07459 & -2.32051 \\ \mathrm{H} & 2.87965 & 0.51331 & 2.13960 \\ \mathrm{C} & 1.36951 & 3.13063 & 0.26869 \\ \mathrm{C} & -0.96671 & 3.03764 & -0.40683 \\ \mathrm{C} & 0.66192 & 4.44360 & 0.62894 \\ \mathrm{H} & 1.88929 & 2.66951 & 1.12462 \\ \mathrm{H} & 2.10923 & 3.30272 & -0.53966 \\ \mathrm{C} & -0.48954 & 4.49424 & -0.38746 \\ \mathrm{H} & -1.43352 & 2.72191 & -1.35738 \\ \mathrm{H} & -1.69062 & 2.82647 & 0.39847\end{array}$

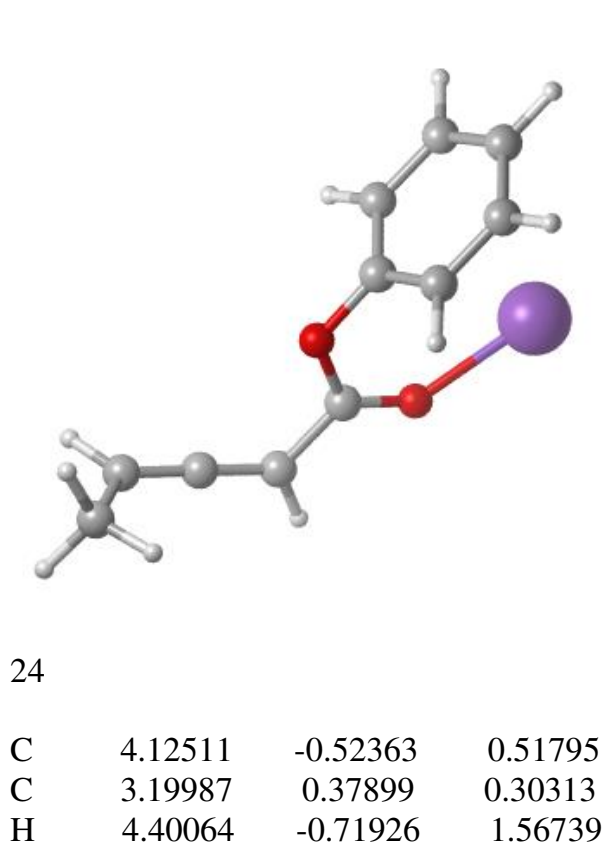




$\begin{array}{lrrr}\mathrm{C} & 4.84013 & -1.31446 & -0.55263 \\ \mathrm{H} & 5.92911 & -1.13584 & -0.48439 \\ \mathrm{H} & 4.67224 & -2.39595 & -0.39454 \\ \mathrm{H} & 4.49556 & -1.04097 & -1.56241 \\ \mathrm{C} & 2.27407 & 1.31033 & 0.07797 \\ \mathrm{C} & 0.84338 & 1.02359 & 0.00657 \\ \mathrm{H} & 2.53924 & 2.36564 & -0.06407 \\ \mathrm{O} & 0.52659 & -0.30378 & 0.17878 \\ \mathrm{O} & -0.02646 & 1.88470 & -0.19151 \\ \mathrm{C} & -3.58338 & -1.15628 & 0.02334 \\ \mathrm{C} & -2.97526 & -0.84097 & 1.25360 \\ \mathrm{C} & -1.59574 & -0.56724 & 1.31407 \\ \mathrm{C} & -0.84228 & -0.61457 & 0.13059 \\ \mathrm{C} & -1.42549 & -0.94895 & -1.10158 \\ \mathrm{C} & -2.80610 & -1.22090 & -1.14890 \\ \mathrm{H} & -4.65474 & -1.37999 & -0.01682 \\ \mathrm{H} & -3.57101 & -0.82110 & 2.17280 \\ \mathrm{H} & -1.10286 & -0.32325 & 2.26097 \\ \mathrm{H} & -0.80283 & -0.99643 & -2.00108 \\ \mathrm{H} & -3.27006 & -1.49706 & -2.10221 \\ \mathrm{Na} & -2.26929 & 2.01286 & -0.38349\end{array}$

Substrates_associated_Na+

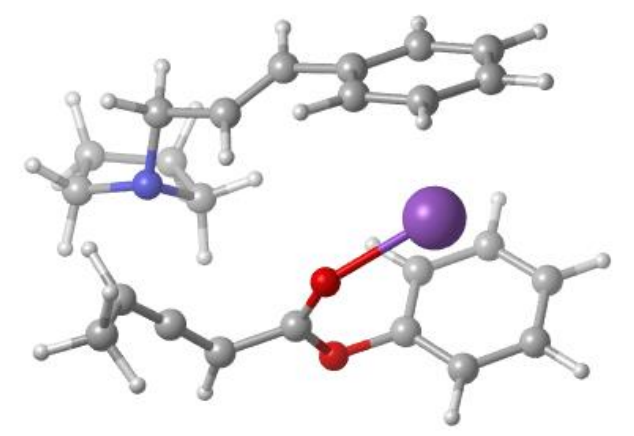

55

$\begin{array}{lrrr}\mathrm{C} & -2.69407 & 1.68517 & -0.90813 \\ \mathrm{C} & -1.34223 & 1.98549 & -0.32774 \\ \mathrm{C} & -0.30193 & 2.48586 & -1.03122 \\ \mathrm{C} & -3.07177 & 0.02660 & 2.69965 \\ \mathrm{C} & -2.36143 & -0.92156 & 2.13802 \\ \mathrm{H} & -3.45306 & 2.28990 & -0.37358 \\ \mathrm{H} & -2.73119 & 2.00010 & -1.97602 \\ \mathrm{H} & -0.42685 & 2.66863 & -2.10841 \\ \mathrm{C} & -3.15040 & 0.28610 & 4.18614 \\ \mathrm{H} & -4.19612 & 0.18385 & 4.53110 \\ \mathrm{H} & -2.83240 & 1.32218 & 4.40743 \\ \mathrm{H} & -2.51837 & -0.41129 & 4.75874 \\ \mathrm{H} & -1.21495 & 1.71535 & 0.72925 \\ \mathrm{C} & -4.44097 & -0.04507 & -1.04419 \\ \mathrm{C} & -2.27109 & -0.63445 & -1.57003 \\ \mathrm{C} & -4.47685 & -0.27206 & -2.58030 \\ \mathrm{H} & -4.71784 & -0.97597 & -0.51317 \\ \mathrm{H} & -5.11670 & 0.75400 & -0.69527 \\ \mathrm{C} & -3.01942 & -0.68772 & -2.93254\end{array}$

\begin{tabular}{lrrr}
$\mathrm{H}$ & -1.22564 & -0.29077 & -1.64658 \\
$\mathrm{H}$ & -2.26228 & -1.63677 & -1.10088 \\
$\mathrm{H}$ & -4.76040 & 0.65533 & -3.10802 \\
$\mathrm{H}$ & -5.21648 & -1.04199 & -2.85810 \\
$\mathrm{H}$ & -2.57271 & 0.01515 & -3.65789 \\
$\mathrm{H}$ & -2.96362 & -1.69423 & -3.38190 \\
$\mathrm{~N}$ & -3.03929 & 0.26718 & -0.68956 \\
$\mathrm{C}$ & -1.63802 & -1.91158 & 1.61875 \\
$\mathrm{C}$ & -0.26977 & -1.68270 & 1.16619 \\
$\mathrm{H}$ & -2.06170 & -2.90947 & 1.45230 \\
$\mathrm{O}$ & 0.23073 & -2.80401 & 0.52419 \\
$\mathrm{O}$ & 0.39773 & -0.65167 & 1.29259 \\
$\mathrm{C}$ & 1.51946 & -2.66868 & -0.01510 \\
$\mathrm{C}$ & 2.63534 & -2.96923 & 0.78082 \\
$\mathrm{C}$ & 1.65888 & -2.21654 & -1.33660 \\
$\mathrm{C}$ & 3.92309 & -2.79404 & 0.24112 \\
$\mathrm{H}$ & 2.48901 & -3.33365 & 1.80279 \\
$\mathrm{C}$ & 2.95163 & -2.04584 & -1.86491 \\
$\mathrm{H}$ & 0.76562 & -2.00298 & -1.93192 \\
$\mathrm{C}$ & 4.08327 & -2.32354 & -1.07576 \\
$\mathrm{H}$ & 4.80137 & -3.03655 & 0.84940 \\
$\mathrm{H}$ & 3.07241 & -1.70283 & -2.89826 \\
$\mathrm{H}$ & 5.08749 & -2.19674 & -1.49382 \\
$\mathrm{C}$ & 1.03991 & 2.75075 & -0.48289 \\
$\mathrm{C}$ & 1.25800 & 3.03771 & 0.89160 \\
$\mathrm{C}$ & 2.17466 & 2.67883 & -1.33414 \\
$\mathrm{C}$ & 2.55857 & 3.20119 & 1.39797 \\
$\mathrm{H}$ & 0.39858 & 3.14898 & 1.56092 \\
$\mathrm{C}$ & 3.47673 & 2.83917 & -0.82692 \\
$\mathrm{H}$ & 2.02807 & 2.49065 & -2.40474 \\
$\mathrm{C}$ & 3.67723 & 3.09127 & 0.54490 \\
$\mathrm{H}$ & 2.70093 & 3.43914 & 2.45863 \\
$\mathrm{H}$ & 4.33536 & 2.78469 & -1.50607 \\
$\mathrm{H}$ & 4.68905 & 3.24024 & 0.93806 \\
$\mathrm{Na}$ & 2.39037 & 0.20554 & 0.58414 \\
$\mathrm{H}$ & -3.65726 & 0.66838 & 2.02489 \\
& & & \\
\hline
\end{tabular}

TS_Nucleophilic_addition_Na+

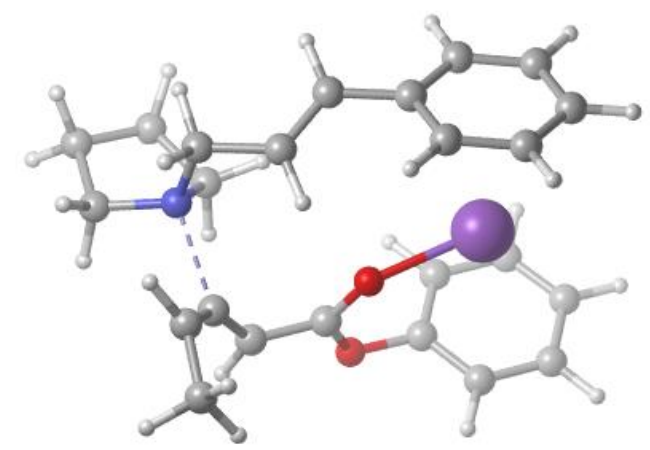

55

$\begin{array}{llll}\mathrm{C} & -2.93819 & 1.52702 & -0.08030 \\ \mathrm{C} & -1.53580 & 1.87230 & 0.31930 \\ \mathrm{C} & -0.70697 & 2.64649 & -0.41318\end{array}$




\begin{tabular}{|c|c|c|c|}
\hline $\mathrm{C}$ & -3.08208 & -0.62865 & 2.28696 \\
\hline $\mathrm{C}$ & -2.40823 & -1.09620 & 1.24240 \\
\hline $\mathrm{H}$ & -3.63579 & 1.76297 & 0.74296 \\
\hline $\mathrm{H}$ & -3.25786 & 2.12373 & -0.95741 \\
\hline $\mathrm{H}$ & -1.05279 & 3.05151 & -1.37555 \\
\hline C & -2.88530 & -1.15444 & 3.68818 \\
\hline $\mathrm{H}$ & -3.83212 & -1.57849 & 4.07399 \\
\hline $\mathrm{H}$ & -2.59929 & -0.33240 & 4.37228 \\
\hline $\mathrm{H}$ & -2.10847 & -1.93483 & 3.72210 \\
\hline $\mathrm{H}$ & -1.17317 & 1.39208 & 1.23464 \\
\hline $\mathrm{C}$ & -4.48923 & -0.33038 & -0.65521 \\
\hline $\mathrm{C}$ & -2.34990 & -0.34348 & -1.58812 \\
\hline $\mathrm{C}$ & -4.72013 & -0.02585 & -2.15143 \\
\hline $\mathrm{H}$ & -4.55772 & -1.41331 & -0.45276 \\
\hline $\mathrm{H}$ & -5.17914 & 0.18958 & 0.02749 \\
\hline $\mathrm{C}$ & -3.29400 & -0.04453 & -2.77429 \\
\hline $\mathrm{H}$ & -1.37870 & 0.17035 & -1.63422 \\
\hline $\mathrm{H}$ & -2.15238 & -1.42593 & -1.48893 \\
\hline $\mathrm{H}$ & -5.19815 & 0.95945 & -2.28608 \\
\hline $\mathrm{H}$ & -5.39040 & -0.77449 & -2.60494 \\
\hline $\mathrm{H}$ & -3.05311 & 0.93040 & -3.23279 \\
\hline $\mathrm{H}$ & -3.18764 & -0.80727 & -3.56349 \\
\hline $\mathrm{N}$ & -3.08011 & 0.06774 & -0.36533 \\
\hline $\mathrm{C}$ & -1.52958 & -2.06131 & 0.79491 \\
\hline $\mathrm{C}$ & -0.16710 & -1.75382 & 0.53286 \\
\hline $\mathrm{H}$ & -1.88401 & -3.06884 & 0.54832 \\
\hline $\mathrm{O}$ & 0.53307 & -2.85480 & -0.00312 \\
\hline $\mathrm{O}$ & 0.42519 & -0.66757 & 0.71342 \\
\hline C & 1.87322 & -2.61905 & -0.29769 \\
\hline $\mathrm{C}$ & 2.85061 & -2.81608 & 0.69267 \\
\hline $\mathrm{C}$ & 2.22573 & -2.16231 & -1.57969 \\
\hline $\mathrm{C}$ & 4.19646 & -2.52993 & 0.39892 \\
\hline $\mathrm{H}$ & 2.54756 & -3.18397 & 1.67835 \\
\hline $\mathrm{C}$ & 3.57427 & -1.87860 & -1.86452 \\
\hline $\mathrm{H}$ & 1.44369 & -2.03200 & -2.33508 \\
\hline $\mathrm{C}$ & 4.55972 & -2.05039 & -0.87394 \\
\hline $\mathrm{H}$ & 4.96368 & -2.69081 & 1.16454 \\
\hline $\mathrm{H}$ & 3.85613 & -1.53312 & -2.86557 \\
\hline $\mathrm{H}$ & 5.60979 & -1.83767 & -1.10149 \\
\hline $\mathrm{C}$ & 0.69469 & 2.92997 & -0.05390 \\
\hline $\mathrm{C}$ & 1.13420 & 2.98050 & 1.29449 \\
\hline C & 1.65916 & 3.10883 & -1.07827 \\
\hline $\mathrm{C}$ & 2.49278 & 3.16397 & 1.60279 \\
\hline $\mathrm{H}$ & 0.40113 & 2.89425 & 2.10325 \\
\hline $\mathrm{C}$ & 3.01985 & 3.28359 & -0.77070 \\
\hline $\mathrm{H}$ & 1.33588 & 3.10094 & -2.12620 \\
\hline $\mathrm{C}$ & 3.44556 & 3.30418 & 0.57197 \\
\hline $\mathrm{H}$ & 2.80801 & 3.22109 & 2.65121 \\
\hline $\mathrm{H}$ & 3.74676 & 3.42205 & -1.57916 \\
\hline $\mathrm{H}$ & 4.50185 & 3.46624 & 0.81406 \\
\hline $\mathrm{Na}$ & 2.40259 & 0.32131 & 0.33744 \\
\hline $\mathrm{H}$ & -3.82590 & 0.16795 & 2.16827 \\
\hline
\end{tabular}

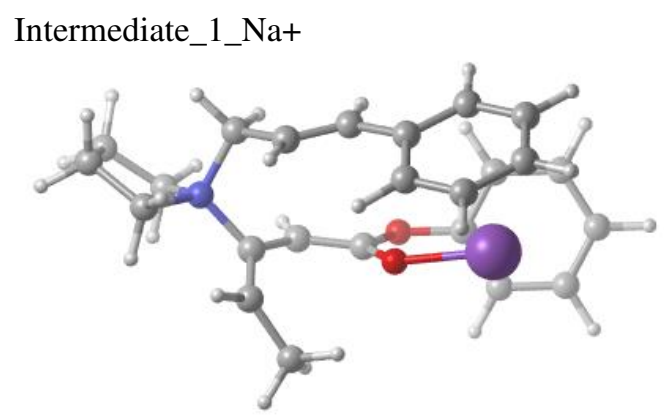

55

$\begin{array}{lrrr}\mathrm{C} & -2.71204 & 0.64916 & -1.25466 \\ \mathrm{C} & -1.67128 & 1.55756 & -0.68241 \\ \mathrm{C} & -0.39218 & 1.51049 & -1.10986 \\ \mathrm{C} & -2.25585 & -0.34303 & 2.05214 \\ \mathrm{C} & -2.26745 & -0.77726 & 0.76435 \\ \mathrm{H} & -3.52749 & 1.20087 & -1.75106 \\ \mathrm{H} & -2.25502 & -0.04644 & -1.97202 \\ \mathrm{H} & -2.90452 & 0.48001 & 2.36773 \\ \mathrm{H} & -0.14731 & 0.80334 & -1.91410 \\ \mathrm{C} & -1.35287 & -0.91096 & 3.10450 \\ \mathrm{H} & -1.05287 & -1.94271 & 2.85198 \\ \mathrm{H} & -0.41742 & -0.32410 & 3.19406 \\ \mathrm{H} & -1.85341 & -0.90699 & 4.08950 \\ \mathrm{H} & -1.97872 & 2.25835 & 0.10254 \\ \mathrm{C} & -4.14924 & -1.37082 & -0.81505 \\ \mathrm{C} & -4.49079 & 0.54620 & 0.51280 \\ \mathrm{C} & -5.43386 & -0.73531 & -1.35785 \\ \mathrm{H} & -3.51815 & -1.85466 & -1.57102 \\ \mathrm{H} & -4.34309 & -2.08522 & 0.00113 \\ \mathrm{C} & -5.67796 & 0.51139 & -0.46263 \\ \mathrm{H} & -4.71454 & -0.00221 & 1.43933 \\ \mathrm{H} & -4.13637 & 1.55350 & 0.77140 \\ \mathrm{H} & -6.26309 & -1.45906 & -1.31136 \\ \mathrm{H} & -5.31515 & -0.44726 & -2.41578 \\ \mathrm{H} & -6.62083 & 0.44418 & 0.10360 \\ \mathrm{H} & -5.73246 & 1.43234 & -1.06655 \\ \mathrm{~N} & -3.37082 & -0.22690 & -0.16681 \\ \mathrm{C} & -1.32304 & -1.62832 & 0.09396 \\ \mathrm{C} & 0.05400 & -1.46887 & 0.26800 \\ \mathrm{H} & -1.64258 & -2.39620 & -0.61442 \\ \mathrm{O} & 0.80408 & -2.41938 & -0.51465 \\ \mathrm{O} & 0.69065 & -0.62265 & 0.94967 \\ \mathrm{C} & 2.17014 & -2.21236 & -0.52694 \\ \mathrm{C} & 2.74382 & -1.44628 & -1.55885 \\ \mathrm{C} & 2.97815 & -2.73824 & 0.49940 \\ \mathrm{C} & 4.12582 & -1.18548 & -1.55120 \\ \mathrm{H} & 2.09624 & -1.05779 & -2.35166 \\ \mathrm{C} & 4.35957 & -2.47307 & 0.50160 \\ \mathrm{H} & 2.51206 & -3.33917 & 1.28694 \\ \mathrm{C} & 4.93601 & -1.68727 & -0.51558 \\ \mathrm{H} & 4.57157 & -0.59379 & -2.35902 \\ \mathrm{H} & 4.98979 & -2.88943 & 1.29594 \\ \mathrm{H} & 6.01364 & -1.49073 & -0.51515 \\ \mathrm{C} & 0.75155 & 2.26485 & -0.58163\end{array}$




$\begin{array}{lccr}\mathrm{C} & 0.73180 & 2.88416 & 0.69364 \\ \mathrm{C} & 1.95961 & 2.29664 & -1.32207 \\ \mathrm{C} & 1.87878 & 3.50632 & 1.20754 \\ \mathrm{H} & -0.17630 & 2.84058 & 1.30281 \\ \mathrm{C} & 3.10985 & 2.91964 & -0.80884 \\ \mathrm{H} & 1.99483 & 1.82075 & -2.30887 \\ \mathrm{C} & 3.07666 & 3.52364 & 0.46210 \\ \mathrm{H} & 1.84267 & 3.98033 & 2.19510 \\ \mathrm{H} & 4.03024 & 2.93900 & -1.40323 \\ \mathrm{H} & 3.96868 & 4.01768 & 0.86311 \\ \mathrm{Na} & 2.66566 & 0.34843 & 1.06381\end{array}$

Intermediate_2_Na+

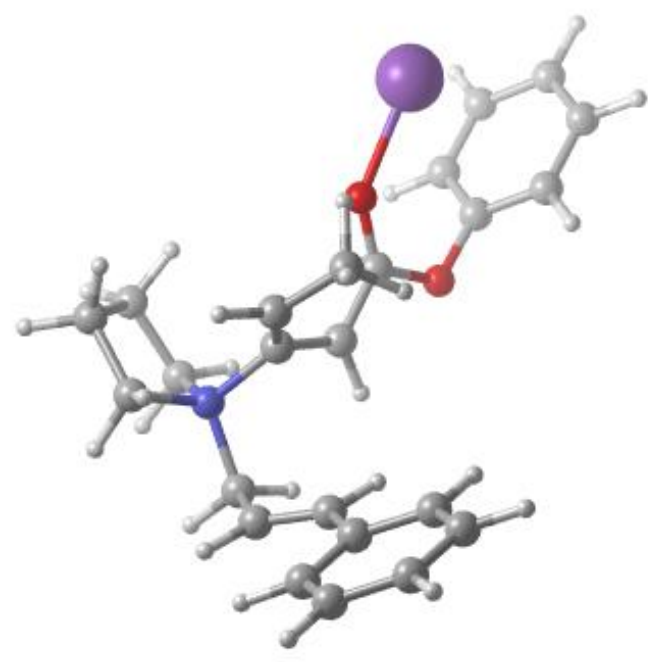

55

$\begin{array}{lrrr}\mathrm{C} & 2.05010 & 1.83509 & 1.62365 \\ \mathrm{C} & 3.20123 & 0.99925 & 1.17528 \\ \mathrm{C} & 3.10299 & -0.34503 & 1.03644 \\ \mathrm{C} & 0.86853 & 0.57124 & -1.36145 \\ \mathrm{C} & 0.45292 & 1.04903 & -0.16268 \\ \mathrm{H} & 1.37659 & 1.29656 & 2.30492 \\ \mathrm{H} & 2.37382 & 2.76862 & 2.11259 \\ \mathrm{H} & 1.56091 & 1.14151 & -1.98534 \\ \mathrm{H} & 2.14857 & -0.81612 & 1.31197 \\ \mathrm{C} & 0.43226 & -0.75090 & -1.91351 \\ \mathrm{H} & 0.13951 & -1.43794 & -1.09964 \\ \mathrm{H} & -0.45122 & -0.64684 & -2.57497 \\ \mathrm{H} & 1.24530 & -1.20941 & -2.50419 \\ \mathrm{H} & 4.12150 & 1.52726 & 0.89972 \\ \mathrm{C} & 1.97041 & 3.10078 & -0.55727 \\ \mathrm{C} & 0.11472 & 3.31082 & 0.85941 \\ \mathrm{C} & 0.93985 & 3.92134 & -1.35326 \\ \mathrm{H} & 2.61689 & 3.75033 & 0.05547 \\ \mathrm{H} & 2.61777 & 2.43780 & -1.14238 \\ \mathrm{C} & -0.33978 & 3.91577 & -0.46848 \\ \mathrm{H} & -0.67245 & 2.79835 & 1.42705 \\ \mathrm{H} & 0.63214 & 4.04367 & 1.50108 \\ \mathrm{H} & 0.74330 & 3.47377 & -2.33988\end{array}$

$\begin{array}{lccc}\mathrm{H} & 1.32625 & 4.93993 & -1.52078 \\ \mathrm{H} & -1.11746 & 3.27404 & -0.91431 \\ \mathrm{H} & -0.76414 & 4.92147 & -0.31934 \\ \mathrm{~N} & 1.15788 & 2.28827 & 0.43275 \\ \mathrm{C} & -0.56354 & 0.49626 & 0.69708 \\ \mathrm{C} & -1.80347 & 0.07371 & 0.22650 \\ \mathrm{H} & -0.40217 & 0.40346 & 1.77491 \\ \mathrm{O} & -2.30834 & 0.14375 & -0.93151 \\ \mathrm{O} & -2.58955 & -0.51976 & 1.28366 \\ \mathrm{C} & -3.85504 & -0.93787 & 0.93099 \\ \mathrm{C} & -4.06374 & -2.28029 & 0.55608 \\ \mathrm{C} & -4.92960 & -0.02579 & 0.92985 \\ \mathrm{C} & -5.34539 & -2.70141 & 0.15367 \\ \mathrm{H} & -3.21729 & -2.97498 & 0.58153 \\ \mathrm{C} & -6.20728 & -0.45381 & 0.52466 \\ \mathrm{H} & -4.74866 & 1.00872 & 1.24013 \\ \mathrm{C} & -6.41709 & -1.78801 & 0.12419 \\ \mathrm{H} & -5.50922 & -3.74808 & -0.12769 \\ \mathrm{H} & -7.04430 & 0.25393 & 0.53247 \\ \mathrm{H} & -7.41534 & -2.12072 & -0.18028 \\ \mathrm{C} & 4.11641 & -1.26839 & 0.52011 \\ \mathrm{C} & 3.75032 & -2.61800 & 0.30537 \\ \mathrm{C} & 5.43932 & -0.87306 & 0.20697 \\ \mathrm{C} & 4.66769 & -3.54096 & -0.21355 \\ \mathrm{H} & 2.72978 & -2.93872 & 0.54726 \\ \mathrm{C} & 6.35598 & -1.79516 & -0.30657 \\ \mathrm{H} & 5.76011 & 0.16021 & 0.37717 \\ \mathrm{C} & 5.97397 & -3.13205 & -0.52201 \\ \mathrm{H} & 4.36493 & -4.58160 & -0.37350 \\ \mathrm{H} & 7.37784 & -1.47452 & -0.53749 \\ \mathrm{H} & 6.69621 & -3.85107 & -0.92387 \\ \mathrm{Na} & -4.08016 & -0.54407 & -2.00345 \\ & & & \end{array}$

TS_Rearrangement_Na+

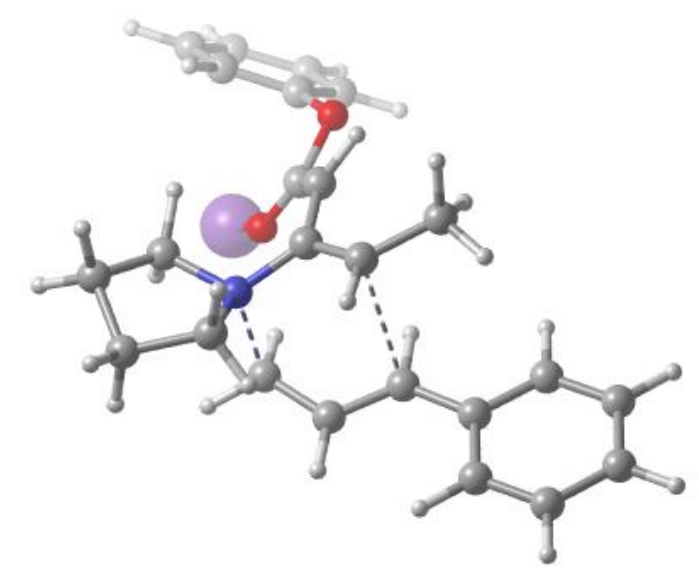

55

$\begin{array}{lrrr}\mathrm{C} & -0.93180 & 1.30795 & -1.55828 \\ \mathrm{C} & -2.22636 & 0.70097 & -1.54191 \\ \mathrm{C} & -2.41844 & -0.51031 & -0.91117 \\ \mathrm{C} & -1.87587 & 0.58117 & 1.64687 \\ \mathrm{C} & -0.65943 & 0.91117 & 1.09358\end{array}$




$\begin{array}{lrrc}\mathrm{H} & -0.04763 & 0.66162 & -1.52520 \\ \mathrm{H} & -0.80417 & 2.21500 & -2.16174 \\ \mathrm{H} & -2.72514 & 1.25836 & 1.53307 \\ \mathrm{H} & -1.51894 & -1.06420 & -0.61106 \\ \mathrm{C} & -2.11824 & -0.62756 & 2.49903 \\ \mathrm{H} & -1.49438 & -1.48457 & 2.19081 \\ \mathrm{H} & -1.89398 & -0.42738 & 3.56692 \\ \mathrm{H} & -3.17836 & -0.93029 & 2.44272 \\ \mathrm{H} & -3.08530 & 1.28059 & -1.89872 \\ \mathrm{C} & -1.68579 & 3.09462 & 0.36589 \\ \mathrm{C} & 0.70985 & 2.79287 & 0.12760 \\ \mathrm{C} & -1.10044 & 4.35296 & -0.26551 \\ \mathrm{H} & -2.62377 & 2.74329 & -0.08693 \\ \mathrm{H} & -1.84586 & 3.24286 & 1.44734 \\ \mathrm{C} & 0.36425 & 4.28897 & 0.19088 \\ \mathrm{H} & 1.33114 & 2.44367 & 0.96553 \\ \mathrm{H} & 1.21993 & 2.50525 & -0.80280 \\ \mathrm{H} & -1.62589 & 5.25798 & 0.08066 \\ \mathrm{H} & -1.18113 & 4.32162 & -1.36740 \\ \mathrm{H} & 0.44940 & 4.65721 & 1.22882 \\ \mathrm{H} & 1.04180 & 4.89237 & -0.43556 \\ \mathrm{~N} & -0.61101 & 2.04842 & 0.19101 \\ \mathrm{C} & 0.52436 & 0.10515 & 1.32648 \\ \mathrm{C} & 1.57965 & -0.21885 & 0.48697 \\ \mathrm{H} & 0.53505 & -0.42333 & 2.28171 \\ \mathrm{O} & 2.45485 & -1.18206 & 1.11773 \\ \mathrm{O} & 1.86619 & 0.13819 & -0.70457 \\ \mathrm{C} & 6.36594 & -1.42143 & -0.37597 \\ \mathrm{C} & 5.51185 & -2.53885 & -0.42180 \\ \mathrm{C} & 4.20139 & -2.45619 & 0.08676 \\ \mathrm{C} & 3.74586 & -1.24677 & 0.65110 \\ \mathrm{C} & 4.60971 & -0.13118 & 0.73049 \\ \mathrm{C} & 5.91284 & -0.22312 & 0.21093 \\ \mathrm{H} & 7.38597 & -1.49104 & -0.76848 \\ \mathrm{H} & 5.86677 & -3.48325 & -0.85001 \\ \mathrm{H} & 3.52213 & -3.31514 & 0.05806 \\ \mathrm{H} & 4.24785 & 0.79199 & 1.19559 \\ \mathrm{H} & 6.58456 & 0.64045 & 0.28003 \\ \mathrm{C} & -3.68098 & -1.19078 & -0.65670 \\ \mathrm{C} & -3.64932 & -2.50018 & -0.11606 \\ \mathrm{C} & -4.94320 & -0.59383 & -0.90284 \\ \mathrm{C} & -4.83306 & -3.19348 & 0.16151 \\ \mathrm{H} & -2.67967 & -2.97051 & 0.08414 \\ \mathrm{C} & -6.12407 & -1.28706 & -0.62621 \\ \mathrm{H} & -5.00121 & 0.42184 & -1.30761 \\ \mathrm{C} & -6.07415 & -2.58874 & -0.09285 \\ \mathrm{H} & -4.78942 & -4.20577 & 0.57746 \\ \mathrm{H} & -7.09219 & -0.81328 & -0.82148 \\ & 3.53517 & -0.36649 & -2.06512\end{array}$

Product_Na+

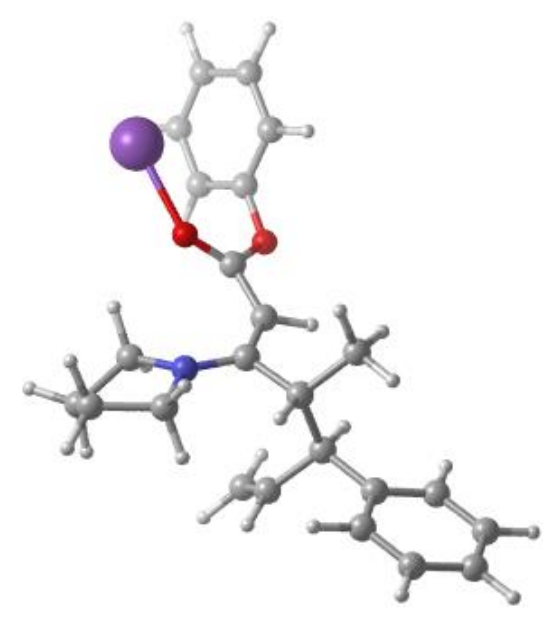

55

$\begin{array}{lrrr}\mathrm{C} & 1.95494 & 0.70252 & 2.68935 \\ \mathrm{C} & 2.70915 & 0.64611 & 1.57940 \\ \mathrm{C} & 2.65510 & -0.47062 & 0.55956 \\ \mathrm{C} & 1.91573 & -0.02624 & -0.76036 \\ \mathrm{C} & 0.55280 & 0.55052 & -0.39420 \\ \mathrm{H} & 1.23357 & -0.08838 & 2.93232 \\ \mathrm{H} & 2.04650 & 1.53109 & 3.40163 \\ \mathrm{H} & 2.52453 & 0.76908 & -1.21364 \\ \mathrm{H} & 2.06906 & -1.30090 & 0.99223 \\ \mathrm{C} & 1.82698 & -1.17503 & -1.77771 \\ \mathrm{H} & 1.32378 & -2.06135 & -1.35377 \\ \mathrm{H} & 1.26584 & -0.86082 & -2.67514 \\ \mathrm{H} & 2.83988 & -1.48059 & -2.08669 \\ \mathrm{H} & 3.43472 & 1.44842 & 1.38193 \\ \mathrm{C} & 1.40595 & 2.85191 & -0.92445 \\ \mathrm{C} & -0.61046 & 2.60003 & 0.41853 \\ \mathrm{C} & 0.77116 & 4.22254 & -0.66279 \\ \mathrm{H} & 2.35547 & 2.75786 & -0.36788 \\ \mathrm{H} & 1.59955 & 2.65180 & -1.99126 \\ \mathrm{C} & 0.00053 & 3.98689 & 0.64510 \\ \mathrm{H} & -1.56291 & 2.65587 & -0.13653 \\ \mathrm{H} & -0.79041 & 2.02275 & 1.34210 \\ \mathrm{H} & 0.07281 & 4.48525 & -1.47902 \\ \mathrm{H} & 1.53017 & 5.01848 & -0.59327 \\ \mathrm{H} & -0.76692 & 4.75239 & 0.84640 \\ \mathrm{H} & 0.69846 & 3.96364 & 1.50199 \\ \mathrm{~N} & 0.40736 & 1.88909 & -0.38555 \\ \mathrm{C} & -0.44161 & -0.35371 & 0.01673 \\ \mathrm{C} & -1.84710 & -0.21341 & -0.03481 \\ \mathrm{H} & -0.10477 & -1.35876 & 0.27830 \\ \mathrm{O} & -2.47928 & -1.33370 & 0.56324 \\ \mathrm{O} & -2.55347 & 0.67911 & -0.56676 \\ \mathrm{C} & -6.63773 & -1.54217 & 0.04036 \\ \mathrm{C} & -5.77628 & -2.11487 & -0.91572 \\ \mathrm{C} & -4.38086 & -2.05924 & -0.73835 \\ \mathrm{C} & -3.85487 & -1.42318 & 0.40197\end{array}$




$\begin{array}{lrrr}\mathrm{C} & -4.70610 & -0.86999 & 1.37616 \\ \mathrm{C} & -6.10018 & -0.93217 & 1.19030 \\ \mathrm{H} & -7.72316 & -1.59771 & -0.09538 \\ \mathrm{H} & -6.19078 & -2.61957 & -1.79578 \\ \mathrm{H} & -3.69480 & -2.49969 & -1.46967 \\ \mathrm{H} & -4.26866 & -0.39997 & 2.26321 \\ \mathrm{H} & -6.76749 & -0.51367 & 1.95219 \\ \mathrm{C} & 4.04839 & -0.99765 & 0.24109 \\ \mathrm{C} & 4.38116 & -2.33490 & 0.52231 \\ \mathrm{C} & 5.02500 & -0.16768 & -0.34428 \\ \mathrm{C} & 5.65824 & -2.83553 & 0.22430 \\ \mathrm{H} & 3.63223 & -2.99189 & 0.98102 \\ \mathrm{C} & 6.30164 & -0.66349 & -0.64430 \\ \mathrm{H} & 4.79319 & 0.88001 & -0.57231 \\ \mathrm{C} & 6.62205 & -2.00132 & -0.36139 \\ \mathrm{H} & 5.89970 & -3.87995 & 0.45100 \\ \mathrm{H} & 7.04936 & -0.00402 & -1.09894 \\ \mathrm{H} & 7.61923 & -2.39000 & -0.59513 \\ \mathrm{Na} & -4.68630 & 0.98616 & -1.10489\end{array}$

\section{Intermediate - Catalyst interaction complexes}

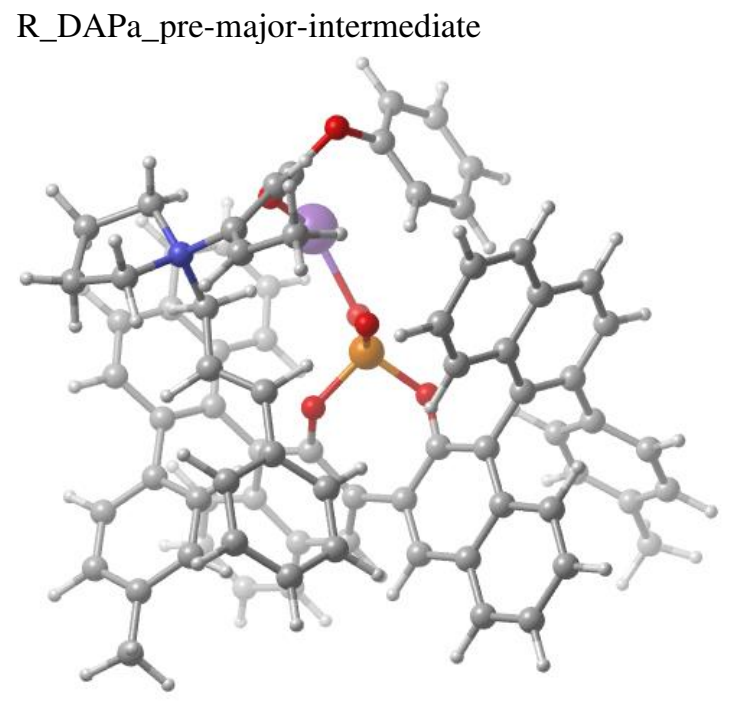

$\begin{array}{lccc}150 & & & \\ \mathrm{P} & 0.36979 & -0.68161 & 0.94846 \\ \mathrm{Na} & -1.52444 & -2.60520 & 2.70510 \\ \mathrm{~N} & -4.12287 & -2.38753 & -1.61393 \\ \mathrm{O} & 1.94970 & -0.07329 & 1.07932 \\ \mathrm{O} & -0.43028 & 0.75813 & 0.46797 \\ \mathrm{O} & 0.00123 & -1.03649 & 2.39276 \\ \mathrm{O} & 0.17991 & -1.62647 & -0.22375 \\ \mathrm{O} & -2.62124 & -3.50277 & 0.97315 \\ \mathrm{O} & -1.62863 & -5.53312 & 0.51622 \\ \mathrm{C} & -0.75937 & 6.37166 & 3.57859 \\ \mathrm{C} & 0.27690 & 5.78911 & 2.86677 \\ \mathrm{C} & 0.12555 & 4.50190 & 2.27215 \\ \mathrm{C} & -1.12538 & 3.80248 & 2.42938\end{array}$

\begin{tabular}{|c|c|c|c|}
\hline$C$ & -2.17276 & 4.42863 & 3.16588 \\
\hline $\mathrm{C}$ & -1.99381 & 5.68427 & 3.72749 \\
\hline $\mathrm{C}$ & 1.16605 & 3.90093 & 1.51208 \\
\hline $\mathrm{C}$ & -1.27307 & 2.50686 & 1.83808 \\
\hline $\mathrm{C}$ & -0.21214 & 1.94631 & 1.13280 \\
\hline $\mathrm{C}$ & 1.02281 & 2.64629 & 0.92504 \\
\hline $\mathrm{C}$ & -2.59191 & 1.79922 & 1.89289 \\
\hline $\mathrm{C}$ & -2.84817 & 0.84040 & 2.93064 \\
\hline $\mathrm{C}$ & -3.59925 & 2.12747 & 0.97371 \\
\hline $\mathrm{C}$ & -1.88289 & 0.54608 & 3.94019 \\
\hline $\mathrm{C}$ & -4.12385 & 0.16645 & 2.98343 \\
\hline $\mathrm{C}$ & -4.87515 & 1.49103 & 1.07907 \\
\hline C & -2.14664 & -0.39106 & 4.93012 \\
\hline $\mathrm{C}$ & -4.35236 & -0.81997 & 3.98873 \\
\hline $\mathrm{C}$ & -5.12841 & 0.53046 & 2.04145 \\
\hline $\mathrm{C}$ & -3.38350 & -1.09697 & 4.94559 \\
\hline C & 2.06953 & 2.10116 & 0.02311 \\
\hline $\mathrm{C}$ & 2.69724 & 2.92552 & -0.90744 \\
\hline $\mathrm{C}$ & 2.46791 & 0.72527 & 0.07575 \\
\hline $\mathrm{C}$ & 3.70091 & 2.43659 & -1.78687 \\
\hline C & 3.44773 & 0.19602 & -0.75867 \\
\hline $\mathrm{C}$ & 4.32531 & 3.27873 & -2.75441 \\
\hline $\mathrm{C}$ & 4.08216 & 1.04775 & -1.71942 \\
\hline C & 5.28370 & 2.77765 & -3.62124 \\
\hline $\mathrm{C}$ & 5.07576 & 0.56428 & -2.61985 \\
\hline C & 5.66045 & 1.40879 & -3.55148 \\
\hline $\mathrm{C}$ & 3.85364 & -1.23801 & -0.61360 \\
\hline C & 3.21087 & -2.24372 & -1.40946 \\
\hline $\mathrm{C}$ & 4.87840 & -1.58832 & 0.27262 \\
\hline $\mathrm{C}$ & 2.22890 & -1.91907 & -2.38941 \\
\hline $\mathrm{C}$ & 3.57300 & -3.62740 & -1.23130 \\
\hline C & 5.24477 & -2.96076 & 0.41563 \\
\hline $\mathrm{C}$ & 1.61695 & -2.90560 & -3.14294 \\
\hline C & 2.90549 & -4.62405 & -2.00248 \\
\hline $\mathrm{C}$ & 4.59646 & -3.95456 & -0.29657 \\
\hline C & 1.94609 & -4.27286 & -2.93933 \\
\hline $\mathrm{C}$ & 5.63209 & -0.55228 & 1.03789 \\
\hline $\mathrm{C}$ & 5.01536 & 0.23891 & 2.02878 \\
\hline $\mathrm{C}$ & 6.99916 & -0.34419 & 0.77211 \\
\hline $\mathrm{C}$ & 5.74487 & 1.21874 & 2.71302 \\
\hline $\mathrm{C}$ & 7.72377 & 0.64175 & 1.45940 \\
\hline $\mathrm{C}$ & 7.10893 & 1.44508 & 2.43743 \\
\hline $\mathrm{C}$ & -3.39514 & 3.15258 & -0.08617 \\
\hline $\mathrm{C}$ & -2.32127 & 3.06822 & -0.99673 \\
\hline $\mathrm{C}$ & -4.28947 & 4.23460 & -0.21084 \\
\hline $\mathrm{C}$ & -2.14380 & 4.03667 & -1.98828 \\
\hline $\mathrm{C}$ & -4.10496 & 5.20691 & -1.20588 \\
\hline $\mathrm{C}$ & -3.02987 & 5.12621 & -2.11090 \\
\hline $\mathrm{C}$ & 7.87417 & 2.52793 & 3.16411 \\
\hline $\mathrm{C}$ & -2.82065 & 6.16447 & -3.18897 \\
\hline $\mathrm{C}$ & 1.51246 & -4.82879 & 3.20958 \\
\hline $\mathrm{C}$ & -2.31243 & -4.34633 & 0.07647 \\
\hline $\mathrm{C}$ & 0.43852 & -5.66023 & 3.57298 \\
\hline $\mathrm{C}$ & 1.53390 & -4.23648 & 1.93418 \\
\hline $\mathrm{C}$ & -2.54764 & -4.36449 & -1.29474 \\
\hline $\mathrm{C}$ & -0.61849 & -5.89156 & 2.67283 \\
\hline $\mathrm{C}$ & 0.49438 & -4.47267 & 1.02272 \\
\hline
\end{tabular}




$\begin{array}{lrrrrrrr}\mathrm{C} & -0.58471 & -5.29552 & 1.39925 & \mathrm{H} & -1.47022 & -6.52620 & 2.94062 \\ \mathrm{C} & -3.14882 & -3.39430 & -2.17924 & \mathrm{H} & 0.49085 & -3.97648 & 0.04904 \\ \mathrm{C} & -2.87089 & -3.31283 & -3.51409 & \mathrm{H} & -3.38702 & -2.58953 & -4.14663 \\ \mathrm{C} & -5.11627 & -1.84082 & -2.62396 & \mathrm{H} & -4.60840 & -1.16036 & -3.31731 \\ \mathrm{C} & -5.06398 & -3.01426 & -0.58459 & \mathrm{H} & -5.49818 & -2.71165 & -3.17795 \\ \mathrm{C} & -1.85376 & -4.19092 & -4.18869 & \mathrm{H} & -5.31654 & -4.00560 & -0.98940 \\ \mathrm{C} & -6.20016 & -1.18467 & -1.77187 & \mathrm{H} & -4.50261 & -3.13901 & 0.34999 \\ \mathrm{C} & -6.28439 & -2.07985 & -0.50790 & \mathrm{H} & -2.24263 & -5.21223 & -4.38621 \\ \mathrm{C} & -3.30869 & -1.20523 & -0.96096 & \mathrm{H} & -1.55024 & -3.76514 & -5.16119 \\ \mathrm{C} & -2.85510 & -0.19760 & -1.95852 & \mathrm{H} & -0.95003 & -4.31006 & -3.56528 \\ \mathrm{C} & -1.57771 & -0.15075 & -2.39878 & \mathrm{H} & -7.14914 & -1.12893 & -2.32926 \\ \mathrm{C} & -1.05321 & 0.85363 & -3.33471 & \mathrm{H} & -5.91101 & -0.15371 & -1.50607 \\ \mathrm{C} & -1.83684 & 1.39305 & -4.38006 & \mathrm{H} & -7.21141 & -2.67651 & -0.48767 \\ \mathrm{C} & 0.27228 & 1.31955 & -3.17801 & \mathrm{H} & -6.26341 & -1.47031 & 0.40864 \\ \mathrm{C} & -1.31304 & 2.36680 & -5.23947 & \mathrm{H} & -2.47131 & -1.69190 & -0.44842 \\ \mathrm{C} & 0.79561 & 2.29911 & -4.03234 & \mathrm{H} & -3.97752 & -0.76413 & -0.20714 \\ \mathrm{C} & 0.00478 & 2.82448 & -5.06771 & \mathrm{H} & -3.57144 & 0.57153 & -2.27336 \\ \mathrm{H} & 2.10839 & 4.44597 & 1.38592 & \mathrm{H} & -0.85410 & -0.85184 & -1.96366 \\ \mathrm{H} & -0.63011 & 7.36285 & 4.02761 & \mathrm{H} & -2.85951 & 1.02830 & -4.52918 \\ \mathrm{H} & 1.23228 & 6.31369 & 2.74512 & \mathrm{H} & 0.87809 & 0.93399 & -2.35383 \\ \mathrm{H} & -3.12407 & 3.90021 & 3.28362 & \mathrm{H} & -1.93277 & 2.76794 & -6.05004 \\ \mathrm{H} & -2.81009 & 6.14925 & 4.29189 & \mathrm{H} & 1.82329 & 2.64715 & -3.88748 \\ \mathrm{H} & -0.91839 & 1.05685 & 3.90968 & \mathrm{H} & 0.41631 & 3.58318 & -5.74349\end{array}$

R_DAPa_pre-minor-intermediate

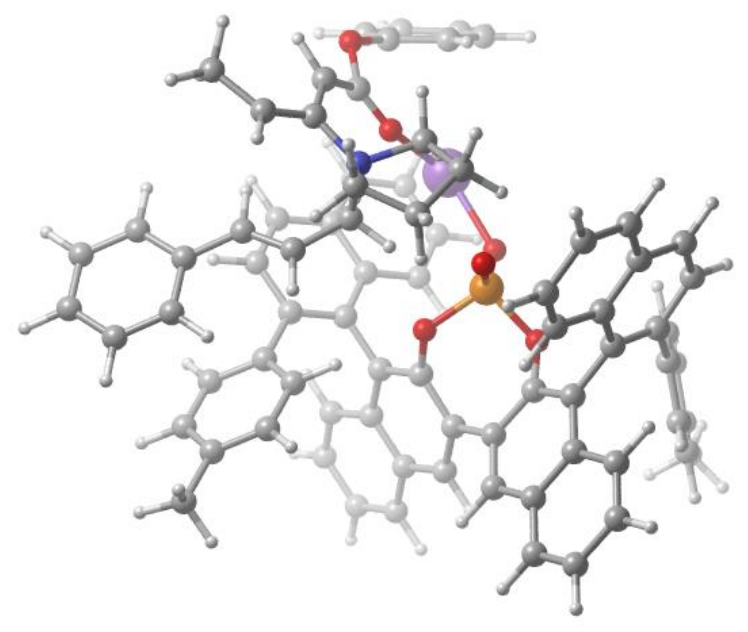

150

$\begin{array}{lccc}\mathrm{P} & 1.17029 & -0.85788 & 0.39100 \\ \mathrm{Na} & -1.24176 & -2.49567 & 2.12160 \\ \mathrm{~N} & -2.26546 & -2.20427 & -2.68243 \\ \mathrm{O} & 2.74617 & -0.35448 & 0.73664 \\ \mathrm{O} & 0.42640 & 0.69123 & 0.24371 \\ \mathrm{O} & 1.03806 & -1.47429 & -0.99209 \\ \mathrm{O} & 0.70115 & -1.55193 & 1.67490 \\ \mathrm{O} & -2.59110 & -2.87300 & 0.42533 \\ \mathrm{O} & -4.50052 & -4.13266 & 0.79288 \\ \mathrm{C} & 1.48516 & 5.23923 & 3.39145 \\ \mathrm{C} & 6.35640 & -0.11228 & 3.51953 \\ \mathrm{C} & 6.86179 & 0.27725 & 2.26259 \\ \mathrm{C} & 6.48876 & 2.94643 & -3.32318\end{array}$




\begin{tabular}{|c|c|c|c|c|c|c|c|}
\hline C & 5.49313 & 3.36443 & -2.45541 & $\mathrm{C}$ & -1.30229 & -3.33487 & -2.24316 \\
\hline $\mathrm{C}$ & -0.78944 & 5.15792 & 4.24234 & $\mathrm{C}$ & -4.30554 & -4.63976 & 4.42254 \\
\hline $\mathrm{C}$ & 1.24535 & 4.07377 & 2.60573 & $\mathrm{C}$ & -2.31104 & -5.58191 & 3.41168 \\
\hline $\mathrm{C}$ & 6.46885 & -0.37703 & 1.08905 & $\mathrm{C}$ & -0.31074 & -2.05910 & -4.07918 \\
\hline $\mathrm{C}$ & 6.78868 & 1.56261 & -3.44461 & $\mathrm{C}$ & -0.10416 & -3.31148 & -3.20977 \\
\hline $\mathrm{C}$ & 5.45660 & -1.19432 & 3.56016 & $\mathrm{C}$ & -3.07127 & -5.29532 & 4.55975 \\
\hline $\mathrm{C}$ & 2.24265 & 3.52224 & 1.75765 & $\mathrm{C}$ & -1.98972 & -0.92916 & -1.79962 \\
\hline $\mathrm{C}$ & 4.75632 & 2.42420 & -1.67556 & $\mathrm{C}$ & -2.69025 & 0.28945 & -2.29096 \\
\hline $\mathrm{C}$ & 3.74643 & 2.83964 & -0.76536 & $\mathrm{C}$ & -3.97372 & 0.55546 & -1.95561 \\
\hline $\mathrm{C}$ & 6.08361 & 0.62159 & -2.70878 & $\mathrm{C}$ & -4.77318 & 1.72399 & -2.33221 \\
\hline $\mathrm{C}$ & 5.04604 & 1.01844 & -1.81403 & $\mathrm{C}$ & -4.25377 & 2.81273 & -3.06960 \\
\hline $\mathrm{C}$ & -1.05356 & 4.02054 & 3.49359 & $\mathrm{C}$ & -6.12291 & 1.78558 & -1.91244 \\
\hline $\mathrm{C}$ & 3.04641 & 1.92660 & 0.01954 & $\mathrm{C}$ & -5.05148 & 3.92139 & -3.36482 \\
\hline $\mathrm{C}$ & -0.04971 & 3.44515 & 2.66025 & $\mathrm{C}$ & -6.92499 & 2.89498 & -2.21099 \\
\hline $\mathrm{C}$ & 5.55639 & -1.45125 & 1.13293 & $\mathrm{C}$ & -6.39017 & 3.97037 & -2.93775 \\
\hline $\mathrm{C}$ & 2.01437 & 2.38911 & 0.98140 & $\mathrm{C}$ & 6.73701 & 0.63906 & 4.77498 \\
\hline $\mathrm{C}$ & 5.06394 & -1.85576 & 2.38885 & $\mathrm{C}$ & -1.94992 & 6.43279 & -2.74628 \\
\hline $\mathrm{C}$ & 4.30437 & 0.07009 & -1.03418 & $\mathrm{H}$ & 0.68617 & 6.67125 & 4.79117 \\
\hline $\mathrm{C}$ & 3.35718 & 0.53789 & -0.12529 & $\mathrm{H}$ & 2.47517 & 5.70871 & 3.34387 \\
\hline $\mathrm{C}$ & 0.73216 & 1.74664 & 1.08107 & $\mathrm{H}$ & 7.57470 & 1.10929 & 2.20108 \\
\hline $\mathrm{C}$ & -0.28400 & 2.26564 & 1.88072 & $\mathrm{H}$ & 7.05092 & 3.68004 & -3.91202 \\
\hline $\mathrm{C}$ & 5.09465 & -2.12756 & -0.10883 & $\mathrm{H}$ & 5.26059 & 4.43077 & -2.34670 \\
\hline $\mathrm{C}$ & 4.52856 & -1.40156 & -1.16489 & $\mathrm{H}$ & 3.22503 & 4.00590 & 1.71513 \\
\hline $\mathrm{C}$ & -0.89234 & 0.18955 & 3.83601 & $\mathrm{H}$ & 3.50593 & 3.90629 & -0.69343 \\
\hline $\mathrm{C}$ & -2.13348 & 5.30998 & -1.75182 & $\mathrm{H}$ & -1.57415 & 5.58473 & 4.87729 \\
\hline $\mathrm{C}$ & 5.20474 & -3.54705 & -0.21060 & $\mathrm{H}$ & 7.58449 & 1.23611 & -4.12363 \\
\hline $\mathrm{C}$ & -1.27727 & 4.18973 & -1.76522 & $\mathrm{H}$ & 6.87631 & -0.05525 & 0.12551 \\
\hline $\mathrm{C}$ & -3.16623 & 5.33522 & -0.79816 & $\mathrm{H}$ & 5.04129 & -1.51435 & 4.52396 \\
\hline $\mathrm{C}$ & -1.16304 & -0.79097 & 4.78273 & $\mathrm{H}$ & 6.31718 & -0.44302 & -2.80596 \\
\hline $\mathrm{C}$ & -1.63482 & 1.62249 & 1.91854 & $\mathrm{H}$ & -2.04039 & 3.54951 & 3.53431 \\
\hline $\mathrm{C}$ & -1.44159 & 3.13662 & -0.85941 & $\mathrm{H}$ & 4.32897 & -2.66636 & 2.44038 \\
\hline $\mathrm{C}$ & -3.33696 & 4.28232 & 0.11117 & $\mathrm{H}$ & 0.10585 & 0.62931 & 3.76875 \\
\hline $\mathrm{C}$ & 4.08165 & -2.08044 & -2.35021 & $\mathrm{H}$ & -0.37621 & -1.10658 & 5.47717 \\
\hline $\mathrm{C}$ & -2.47221 & 3.17098 & 0.10382 & $\mathrm{H}$ & 5.64957 & -4.09710 & 0.62621 \\
\hline $\mathrm{C}$ & -1.89606 & 0.61770 & 2.91178 & $\mathrm{H}$ & -0.47068 & 4.13549 & -2.50797 \\
\hline $\mathrm{C}$ & 3.47903 & -1.39096 & -3.44478 & $\mathrm{H}$ & -3.85374 & 6.18900 & -0.76844 \\
\hline $\mathrm{C}$ & -2.66877 & 2.06989 & 1.08523 & $\mathrm{H}$ & 3.32697 & -0.31146 & -3.37066 \\
\hline $\mathrm{C}$ & 4.79047 & -4.22053 & -1.34465 & $\mathrm{H}$ & -0.78812 & 2.26435 & -0.91500 \\
\hline $\mathrm{C}$ & -2.44885 & -1.40254 & 4.84270 & $\mathrm{H}$ & -4.14421 & 4.33135 & 0.85075 \\
\hline $\mathrm{C}$ & 4.22979 & -3.51158 & -2.44562 & $\mathrm{H}$ & -2.64816 & -2.19399 & 5.57353 \\
\hline $\mathrm{C}$ & -3.21078 & 0.02438 & 3.00071 & $\mathrm{H}$ & 4.89778 & -5.30992 & -1.41347 \\
\hline $\mathrm{C}$ & 3.08798 & -2.06750 & -4.58891 & $\mathrm{H}$ & 2.64084 & -1.51164 & -5.42096 \\
\hline $\mathrm{C}$ & -3.44973 & -0.99821 & 3.96784 & $\mathrm{H}$ & -4.76337 & 1.85526 & 0.55036 \\
\hline $\mathrm{C}$ & -3.97093 & 1.49304 & 1.21176 & $\mathrm{H}$ & -4.44029 & -1.46352 & 4.00732 \\
\hline $\mathrm{C}$ & 3.80360 & -4.18075 & -3.63106 & $\mathrm{H}$ & 3.92350 & -5.26954 & -3.69050 \\
\hline $\mathrm{C}$ & -4.23201 & 0.48644 & 2.12093 & $\mathrm{H}$ & -5.23005 & 0.03664 & 2.18153 \\
\hline $\mathrm{C}$ & 3.25181 & -3.47539 & -4.68878 & $\mathrm{H}$ & 2.93531 & -4.00106 & -5.59737 \\
\hline $\mathrm{C}$ & 0.49018 & 5.77355 & 4.19401 & $\mathrm{H}$ & -5.12492 & -3.80199 & -1.48204 \\
\hline $\mathrm{C}$ & -3.63346 & -3.36985 & -0.07980 & $\mathrm{H}$ & -4.19442 & -1.74819 & -4.45095 \\
\hline $\mathrm{C}$ & -4.16276 & -3.29972 & -1.36522 & $\mathrm{H}$ & -5.71526 & -3.71472 & 3.03545 \\
\hline $\mathrm{C}$ & -3.71822 & -2.59292 & -2.54519 & $\mathrm{H}$ & -2.19448 & -5.45201 & 1.23948 \\
\hline $\mathrm{C}$ & -3.99453 & -4.51528 & 2.01255 & $\mathrm{H}$ & -6.35614 & -3.48983 & -3.56357 \\
\hline $\mathrm{C}$ & -4.57116 & -2.22805 & -3.54754 & $\mathrm{H}$ & -6.57503 & -1.86554 & -4.26378 \\
\hline $\mathrm{C}$ & -4.76412 & -4.24329 & 3.15736 & $\mathrm{H}$ & -6.45946 & -2.07949 & -2.48929 \\
\hline $\mathrm{C}$ & -2.77005 & -5.20765 & 2.13782 & $\mathrm{H}$ & -2.19774 & -0.92090 & -4.40749 \\
\hline $\mathrm{C}$ & -6.05989 & -2.42453 & -3.46370 & $\mathrm{H}$ & -2.28201 & -2.68233 & -4.74782 \\
\hline $\mathrm{C}$ & -1.82458 & -1.91015 & -4.11020 & $\mathrm{H}$ & -1.88880 & -4.26289 & -2.28656 \\
\hline
\end{tabular}




$\begin{array}{lrrr}\mathrm{H} & -1.02450 & -3.13143 & -1.20496 \\ \mathrm{H} & -4.91249 & -4.42116 & 5.30903 \\ \mathrm{H} & -1.35883 & -6.11853 & 3.50401 \\ \mathrm{H} & 0.08912 & -2.17472 & -5.09909 \\ \mathrm{H} & 0.17958 & -1.18446 & -3.62259 \\ \mathrm{H} & -0.10545 & -4.21797 & -3.83978 \\ \mathrm{H} & 0.84466 & -3.26316 & -2.65822 \\ \mathrm{H} & -2.70920 & -5.59403 & 5.54966 \\ \mathrm{H} & -2.31768 & -1.23305 & -0.79878 \\ \mathrm{H} & -0.89733 & -0.82285 & -1.77808 \\ \mathrm{H} & -2.12523 & 0.98108 & -2.92573 \\ \mathrm{H} & -4.48645 & -0.17124 & -1.31196 \\ \mathrm{H} & -3.20652 & 2.80970 & -3.38275 \\ \mathrm{H} & -6.53914 & 0.94593 & -1.34221 \\ \mathrm{H} & -4.62270 & 4.76365 & -3.91895 \\ \mathrm{H} & -7.96769 & 2.92181 & -1.87418 \\ \mathrm{H} & -7.01049 & 4.84417 & -3.16778 \\ \mathrm{H} & 6.54725 & 0.03830 & 5.68177 \\ \mathrm{H} & 7.80415 & 0.92716 & 4.76899 \\ \mathrm{H} & 6.14821 & 1.57370 & 4.86794 \\ \mathrm{H} & -2.73048 & 7.20505 & -2.63548 \\ \mathrm{H} & -0.96638 & 6.92457 & -2.62044 \\ \mathrm{H} & -1.98934 & 6.05645 & -3.78681\end{array}$

S_DAPa_pre-major-intermediate

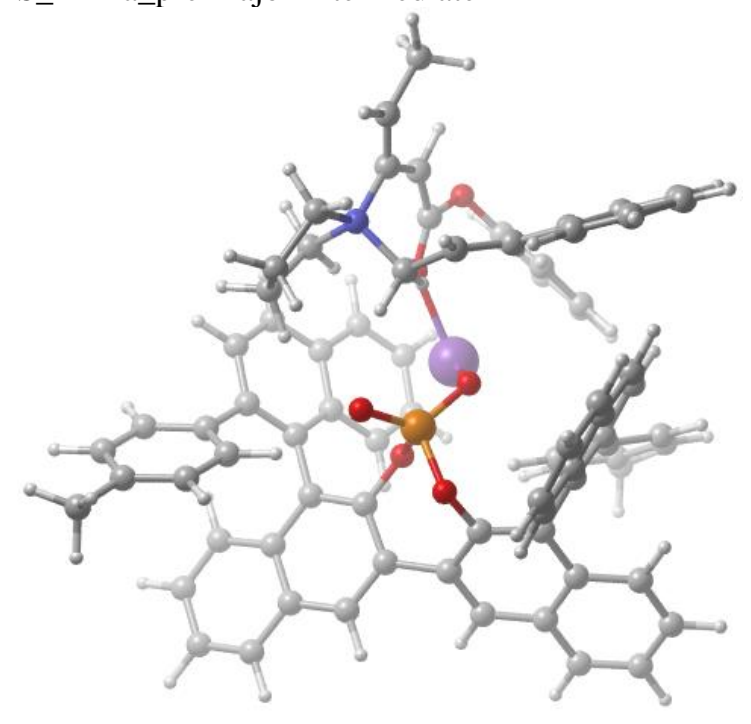

150

$\begin{array}{lccc}\mathrm{P} & -0.44455 & 0.19490 & -1.13311 \\ \mathrm{Na} & 0.30132 & -0.29467 & 1.83175 \\ \mathrm{~N} & 0.44298 & -4.47031 & -0.72025 \\ \mathrm{O} & -0.38544 & 1.58430 & -2.09197 \\ \mathrm{O} & -1.39754 & 0.84083 & 0.17501 \\ \mathrm{O} & 0.87337 & -0.06164 & -0.39959 \\ \mathrm{O} & -1.20359 & -0.85191 & -1.93076 \\ \mathrm{O} & 0.51734 & -2.54210 & 1.87029 \\ \mathrm{O} & 1.96173 & -3.39393 & 3.45544 \\ \mathrm{C} & -7.57154 & 2.59987 & -0.30666 \\ \mathrm{C} & -6.41651 & 3.18613 & -0.79762\end{array}$

\begin{tabular}{|c|c|c|c|}
\hline & -5.13693 & 2.60766 & -0.54776 \\
\hline & -5.05729 & 1.38139 & 0.20753 \\
\hline & -6.26171 & 0.81982 & 0.72170 \\
\hline & -7.48921 & 1.41255 & 0.46867 \\
\hline & -3.93914 & 3.23602 & -0.9878 \\
\hline & -3.77367 & 0.77322 & 0.4122 \\
\hline & -2.63433 & 1.41277 & -0.077 \\
\hline & -2.68564 & 2.68581 & -0.7390 \\
\hline & -3.61703 & -0.50175 & 1.1779 \\
\hline & -2.91654 & -0.46791 & 2.4379 \\
\hline & -4.12141 & -1.71910 & 0.6942 \\
\hline 7 & -2.45131 & 0.75335 & $3.0227^{\circ}$ \\
\hline & -2.64295 & -1.69884 & 3.1394 \\
\hline C & -3.90656 & -2.91392 & 1.4508 \\
\hline & -1.69914 & 0.74918 & 4.1912 \\
\hline$\theta$ & -1.84922 & -1.67138 & 4.32437 \\
\hline $\mathrm{C}$ & -3.16485 & -2.91561 & 2.61560 \\
\hline C & -1.37349 & -0.47543 & 4.8386 \\
\hline C & -1.43225 & 3.45268 & -0.9742 \\
\hline $\mathrm{C}$ & -1.33417 & 4.76926 & -0.5289 \\
\hline & -0.25584 & 2.83341 & -1.505 \\
\hline & -0.10745 & 5.48649 & -0.5742 \\
\hline & 0.99678 & 3.44363 & -1.448 \\
\hline & -0.01179 & 6.83694 & -0.125 \\
\hline & 1.08433 & 4.81061 & -1.02082 \\
\hline & 1.20469 & 7.49974 & -0.1188 \\
\hline & 2.31981 & 5.52043 & -1.0026 \\
\hline & 2.37841 & 6.83485 & -0.5653 \\
\hline & 2.23295 & 2.63326 & -1.6710 \\
\hline & 2.58468 & 2.17666 & -2.9834 \\
\hline & 3.03796 & 2.30142 & -0.5669 \\
\hline & 1.81416 & 2.51681 & -4.13554 \\
\hline C & 3.75565 & 1.35475 & -3.16153 \\
\hline & 4.20123 & 1.49930 & -0.7 \\
\hline & 2.16704 & 2.05206 & -5.3928 \\
\hline & 4.08920 & 0.88653 & -4.4662 \\
\hline & 4.54936 & 1.03336 & -2.0256 \\
\hline & 3.31016 & 1.22374 & -5.5621 \\
\hline C & 2.74136 & 2.75878 & 0.82102 \\
\hline$C$ & 1.45291 & 2.67755 & 1.39727 \\
\hline & 3.77245 & 3.29984 & 1.6181 \\
\hline & 1.21366 & 3.12101 & $2.7012 \mathrm{~S}$ \\
\hline & 3.52706 & 3.74447 & 2.9268 \\
\hline & 2.24288 & 3.66753 & 3.4944 \\
\hline & -4.88626 & -1.81953 & -0.578 \\
\hline & -4.40268 & -1.27007 & -1.783 \\
\hline & -6.12949 & -2.48503 & -0.5972 \\
\hline & -5.15512 & -1.37045 & -2.9595 \\
\hline & -6.88014 & -2.57409 & -1.777 \\
\hline & -6.41048 & -2.01171 & -2.979 \\
\hline & 1.33105 & -3.46459 & 2.1522 \\
\hline & 1.75456 & -4.59909 & 1.462 \\
\hline & 1.45736 & -5.15103 & 0.1636 \\
\hline & 2.11784 & -6.23430 & -0.3472 \\
\hline & 3.20196 & -6.96257 & 0.4007 \\
\hline & 0.06256 & -5.21324 & -1.992 \\
\hline & -0.9 & $-43520^{\circ}$ & \\
\hline
\end{tabular}




$\begin{array}{lrrrrrrr}\mathrm{C} & -1.24619 & -4.56404 & -2.45579 & \mathrm{H} & -0.85224 & -3.58261 & 0.74606 \\ \mathrm{C} & -1.92077 & -4.04922 & -1.15593 & \mathrm{H} & -1.85881 & -5.30756 & -2.99156 \\ \mathrm{C} & 3.38430 & -2.54435 & -1.07557 & \mathrm{H} & -1.05827 & -3.72985 & -3.15088 \\ \mathrm{C} & 2.32433 & -3.07691 & -1.72192 & \mathrm{H} & -2.88076 & -4.55036 & -0.95743 \\ \mathrm{C} & 0.97025 & -3.06264 & -1.09976 & \mathrm{H} & -2.11241 & -2.96956 & -1.22571 \\ \mathrm{C} & 2.26572 & -2.13457 & 3.91849 & \mathrm{H} & 3.21985 & -2.19402 & -0.04997 \\ \mathrm{C} & 2.89147 & -1.16701 & 3.10147 & \mathrm{H} & 2.42947 & -3.45500 & -2.74642 \\ \mathrm{C} & 1.97787 & -1.83342 & 5.26087 & \mathrm{H} & 0.96527 & -2.49085 & -0.16929 \\ \mathrm{C} & 3.18266 & 0.10482 & 3.61943 & \mathrm{H} & 0.21617 & -2.61395 & -1.76167 \\ \mathrm{C} & 2.30180 & -0.56914 & 5.77790 & \mathrm{H} & 3.13349 & -1.41260 & 2.06234 \\ \mathrm{C} & 2.88888 & 0.40995 & 4.96000 & \mathrm{H} & 1.49344 & -2.59615 & 5.87940 \\ \mathrm{C} & 4.74615 & -2.36222 & -1.58590 & \mathrm{H} & 3.63917 & 0.86182 & 2.97292 \\ \mathrm{C} & 5.07628 & -2.46664 & -2.95710 & \mathrm{H} & 2.07579 & -0.34357 & 6.82715 \\ \mathrm{C} & 5.77497 & -2.02540 & -0.67607 & \mathrm{H} & 3.12159 & 1.40218 & 5.35806 \\ \mathrm{C} & 6.38677 & -2.24940 & -3.39669 & \mathrm{H} & 4.29149 & -2.68136 & -3.68944 \\ \mathrm{C} & 7.08752 & -1.80763 & -1.11444 & \mathrm{H} & 5.53118 & -1.92792 & 0.38857 \\ \mathrm{C} & 7.39981 & -1.91772 & -2.47908 & \mathrm{H} & 6.61953 & -2.32259 & -4.46550 \\ \mathrm{C} & 1.95796 & 4.16883 & 4.89037 & \mathrm{H} & 7.86722 & -1.54356 & -0.39086 \\ \mathrm{C} & -7.23365 & -2.06713 & -4.24605 & \mathrm{H} & 8.42294 & -1.73845 & -2.82841 \\ \mathrm{H} & -4.00793 & 4.19233 & -1.51878 & \mathrm{H} & 1.33844 & 3.44876 & 5.45665 \\ \mathrm{H} & -8.54829 & 3.05521 & -0.50520 & \mathrm{H} & 2.88808 & 4.34281 & 5.45884 \\ \mathrm{H} & -6.46701 & 4.11296 & -1.38153 & \mathrm{H} & 1.40030 & 5.12536 & 4.86270 \\ \mathrm{H} & -6.20502 & -0.09344 & 1.31976 & \mathrm{H} & -6.59852 & -2.23895 & -5.13406 \\ \mathrm{H} & -8.40309 & 0.96047 & 0.87037 & \mathrm{H} & -7.99301 & -2.86771 & -4.20412 \\ \mathrm{H} & -2.69037 & 1.70022 & 2.52977 & \mathrm{H} & -7.76848 & -1.11054 & -4.41019\end{array}$

S_DAPa_pre-minor-intermediate

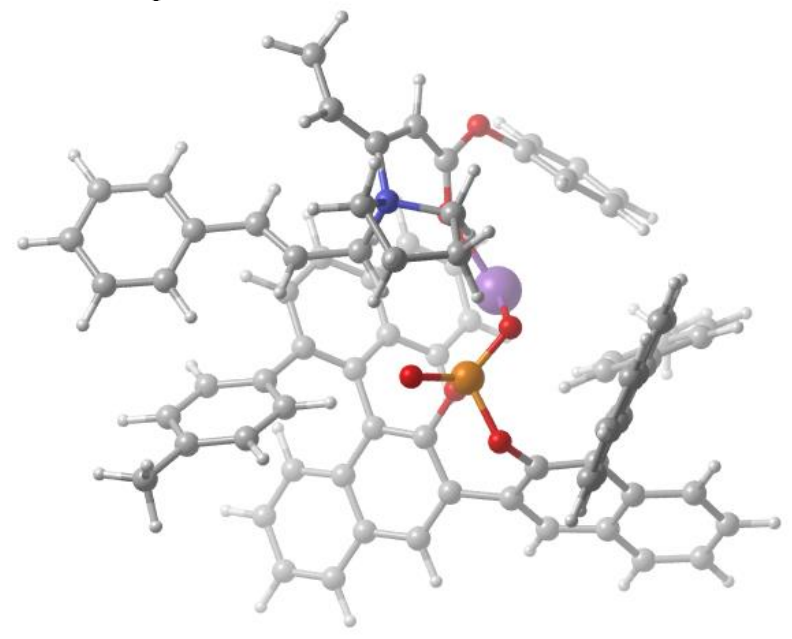

150

$\begin{array}{lrcc}\mathrm{P} & 0.64236 & 0.51724 & -1.28323 \\ \mathrm{Na} & 0.91403 & -1.42595 & 1.13246 \\ \mathrm{~N} & -2.36039 & -2.90205 & -1.98072 \\ \mathrm{O} & 1.74043 & 1.65424 & -1.87165 \\ \mathrm{O} & 0.59921 & 1.03718 & 0.37726 \\ \mathrm{O} & 1.25924 & -0.87409 & -1.10936 \\ \mathrm{O} & -0.66177 & 0.78320 & -2.01594 \\ \mathrm{O} & -0.74962 & -2.86091 & 0.76835 \\ \mathrm{O} & -0.62532 & -4.99386 & 1.64563 \\ \mathrm{C} & -1.88668 & 6.62649 & 2.39262 \\ \mathrm{C} & -0.74784 & 6.34773 & 1.65541\end{array}$




\begin{tabular}{|c|c|c|c|c|c|c|c|}
\hline $\mathrm{C}$ & -0.38447 & 5.00341 & 1.34695 & $\mathrm{C}$ & 1.70508 & -4.37484 & 1.26577 \\
\hline $\mathrm{C}$ & -1.23577 & 3.92513 & 1.78806 & $\mathrm{C}$ & 0.85796 & -4.82631 & 3.50720 \\
\hline $\mathrm{C}$ & -2.37841 & 4.24570 & 2.57715 & $\mathrm{C}$ & -4.90236 & -5.54854 & -0.71879 \\
\hline $\mathrm{C}$ & -2.69898 & 5.56297 & 2.86786 & $\mathrm{C}$ & -2.94114 & -2.74951 & -3.37832 \\
\hline $\mathrm{C}$ & 0.82686 & 4.70174 & 0.66593 & $\mathrm{C}$ & -0.85888 & -3.08300 & -2.27114 \\
\hline $\mathrm{C}$ & -0.89987 & 2.57652 & 1.42282 & $\mathrm{C}$ & 2.97109 & -4.09691 & 1.80528 \\
\hline $\mathrm{C}$ & 0.26786 & 2.34706 & 0.69363 & $\mathrm{C}$ & 2.13590 & -4.57748 & 4.03255 \\
\hline $\mathrm{C}$ & 1.19435 & 3.39446 & 0.36460 & $\mathrm{C}$ & -1.94426 & -1.84903 & -4.09743 \\
\hline $\mathrm{C}$ & -1.71993 & 1.41555 & 1.89151 & $\mathrm{C}$ & -0.57341 & -2.35718 & -3.60064 \\
\hline $\mathrm{C}$ & -1.13043 & 0.49293 & 2.83284 & $\mathrm{C}$ & 3.19225 & -4.19638 & 3.19009 \\
\hline $\mathrm{C}$ & -3.04765 & 1.22657 & 1.47767 & $\mathrm{C}$ & -3.95690 & -1.01363 & -1.31351 \\
\hline $\mathrm{C}$ & 0.18286 & 0.67643 & 3.37300 & $\mathrm{C}$ & -2.58105 & -1.57074 & -1.17988 \\
\hline $\mathrm{C}$ & -1.87220 & -0.67233 & 3.25258 & $\mathrm{C}$ & -4.99979 & -1.43170 & -0.56145 \\
\hline $\mathrm{C}$ & -3.79536 & 0.12219 & 1.99244 & $\mathrm{C}$ & -6.35697 & -0.87388 & -0.57021 \\
\hline $\mathrm{C}$ & 0.75436 & -0.27331 & 4.21186 & $\mathrm{C}$ & -6.65966 & 0.37289 & -1.16723 \\
\hline $\mathrm{C}$ & -1.24655 & -1.64104 & 4.09145 & $\mathrm{C}$ & -7.39937 & -1.57994 & 0.07459 \\
\hline $\mathrm{C}$ & -3.22018 & -0.82034 & 2.81976 & $\mathrm{C}$ & -7.96178 & 0.88109 & -1.13452 \\
\hline $\mathrm{C}$ & 0.04564 & -1.45721 & 4.55660 & $\mathrm{C}$ & -8.70563 & -1.07237 & 0.10262 \\
\hline $\mathrm{C}$ & 2.57066 & 3.05728 & -0.09299 & $\mathrm{C}$ & -8.99244 & 0.16084 & -0.50467 \\
\hline $\mathrm{C}$ & 3.67248 & 3.58812 & 0.57526 & $\mathrm{H}$ & 1.50083 & 5.52260 & 0.39557 \\
\hline $\mathrm{C}$ & 2.81156 & 2.07020 & -1.10004 & $\mathrm{H}$ & -2.15497 & 7.66371 & 2.62263 \\
\hline $\mathrm{C}$ & 4.99889 & 3.15838 & 0.29412 & $\mathrm{H}$ & -0.09889 & 7.15868 & 1.30373 \\
\hline $\mathrm{C}$ & 4.07430 & 1.52150 & -1.32225 & $\mathrm{H}$ & -3.00663 & 3.43494 & 2.95368 \\
\hline $\mathrm{C}$ & 6.12457 & 3.70790 & 0.97616 & $\mathrm{H}$ & -3.58687 & 5.78368 & 3.47100 \\
\hline $\mathrm{C}$ & 5.20659 & 2.09063 & -0.65179 & $\mathrm{H}$ & 0.74223 & 1.57980 & 3.11297 \\
\hline $\mathrm{C}$ & 7.40293 & 3.22968 & 0.73890 & $\mathrm{H}$ & -4.83411 & 0.00926 & 1.67175 \\
\hline $\mathrm{C}$ & 6.53338 & 1.61993 & -0.87197 & $\mathrm{H}$ & 1.75855 & -0.10785 & 4.61790 \\
\hline $\mathrm{C}$ & 7.60684 & 2.17829 & -0.19516 & $\mathrm{H}$ & -1.80827 & -2.54458 & 4.35421 \\
\hline $\mathrm{C}$ & 4.19010 & 0.25665 & -2.10934 & $\mathrm{H}$ & -3.79176 & -1.69568 & 3.14922 \\
\hline $\mathrm{C}$ & 3.97538 & 0.25964 & -3.52716 & $\mathrm{H}$ & 0.52236 & -2.21502 & 5.18564 \\
\hline $\mathrm{C}$ & 4.45004 & -0.94875 & -1.43456 & $\mathrm{H}$ & 3.51215 & 4.31996 & 1.37502 \\
\hline $\mathrm{C}$ & 3.75335 & 1.46665 & -4.25520 & $\mathrm{H}$ & 5.95719 & 4.51737 & 1.69685 \\
\hline $\mathrm{C}$ & 3.98054 & -0.98607 & -4.25226 & $\mathrm{H}$ & 8.25938 & 3.66072 & 1.26951 \\
\hline $\mathrm{C}$ & 4.47395 & -2.16762 & -2.17903 & $\mathrm{H}$ & 6.69026 & 0.80964 & -1.59036 \\
\hline $\mathrm{C}$ & 3.52535 & 1.44497 & -5.62211 & $\mathrm{H}$ & 8.62060 & 1.80648 & -0.38210 \\
\hline $\mathrm{C}$ & 3.73484 & -0.97662 & -5.65668 & $\mathrm{H}$ & 3.75966 & 2.41737 & -3.71430 \\
\hline $\mathrm{C}$ & 4.23151 & -2.19183 & -3.53892 & $\mathrm{H}$ & 4.64333 & -3.10635 & -1.64054 \\
\hline $\mathrm{C}$ & 3.50750 & 0.21281 & -6.33098 & $\mathrm{H}$ & 3.35243 & 2.38385 & -6.16032 \\
\hline $\mathrm{C}$ & 4.69894 & -1.02904 & 0.03323 & $\mathrm{H}$ & 3.73288 & -1.93201 & -6.19583 \\
\hline $\mathrm{C}$ & 3.89872 & -0.35748 & 0.98618 & $\mathrm{H}$ & 4.22437 & -3.14408 & -4.08302 \\
\hline $\mathrm{C}$ & 5.76536 & -1.81228 & 0.52297 & $\mathrm{H}$ & 3.31933 & 0.20724 & -7.41084 \\
\hline $\mathrm{C}$ & 4.15782 & -0.46849 & 2.35515 & $\mathrm{H}$ & 3.06717 & 0.26387 & 0.65309 \\
\hline $\mathrm{C}$ & 6.02163 & -1.91971 & 1.89888 & $\mathrm{H}$ & 6.42173 & -2.32903 & -0.18634 \\
\hline $\mathrm{C}$ & 5.22354 & -1.25119 & 2.84355 & $\mathrm{H}$ & 3.51672 & 0.07172 & 3.06135 \\
\hline $\mathrm{C}$ & -3.72937 & 2.13320 & 0.51479 & $\mathrm{H}$ & 6.86465 & -2.53268 & 2.24124 \\
\hline $\mathrm{C}$ & -3.15947 & 2.44641 & -0.73704 & $\mathrm{H}$ & -2.20042 & 2.01025 & -1.03010 \\
\hline $\mathrm{C}$ & -4.98931 & 2.67762 & 0.83350 & $\mathrm{H}$ & -5.44921 & 2.44523 & 1.80086 \\
\hline $\mathrm{C}$ & -3.83783 & 3.28277 & -1.63137 & $\mathrm{H}$ & -3.38077 & 3.49933 & -2.60518 \\
\hline $\mathrm{C}$ & -5.65298 & 3.52838 & -0.06005 & $\mathrm{H}$ & -6.62986 & 3.94363 & 0.21593 \\
\hline $\mathrm{C}$ & -5.09176 & 3.84452 & -1.31067 & $\mathrm{H}$ & 4.57630 & -1.64727 & 4.87574 \\
\hline $\mathrm{C}$ & 5.48938 & -1.34870 & 4.32690 & $\mathrm{H}$ & 6.27920 & -2.08595 & 4.55172 \\
\hline $\mathrm{C}$ & -5.80088 & 4.75872 & -2.28343 & $\mathrm{H}$ & 5.81167 & -0.37320 & 4.73921 \\
\hline $\mathrm{C}$ & -1.17465 & -4.04422 & 0.70490 & $\mathrm{H}$ & -5.86412 & 4.30379 & -3.28992 \\
\hline $\mathrm{C}$ & -2.13415 & -4.63080 & -0.11820 & $\mathrm{H}$ & -6.82658 & 4.99162 & -1.94828 \\
\hline $\mathrm{C}$ & -2.93180 & -4.05263 & -1.17881 & $\mathrm{H}$ & -5.25859 & 5.71736 & -2.39744 \\
\hline $\mathrm{C}$ & 0.63671 & -4.71842 & 2.12405 & $\mathrm{H}$ & -2.36166 & -5.67527 & 0.10699 \\
\hline $\mathrm{C}$ & -4.17218 & -4.50712 & -1.52124 & $\mathrm{H}$ & -4.68475 & -4.12109 & -2.40358 \\
\hline
\end{tabular}




$\begin{array}{cccc}\mathrm{H} & 1.53416 & -4.31540 & 0.18563 \\ \mathrm{H} & 0.01729 & -5.09467 & 4.15539 \\ \mathrm{H} & -4.53594 & -6.57690 & -0.92313 \\ \mathrm{H} & -5.98215 & -5.53869 & -0.95148 \\ \mathrm{H} & -4.77543 & -5.38187 & 0.36860 \\ \mathrm{H} & -3.96156 & -2.35029 & -3.30870 \\ \mathrm{H} & -2.96507 & -3.75966 & -3.81663 \\ \mathrm{H} & -0.70003 & -4.16915 & -2.32639 \\ \mathrm{H} & -0.29806 & -2.67438 & -1.42521 \\ \mathrm{H} & 3.79022 & -3.79801 & 1.14207 \\ \mathrm{H} & 2.30041 & -4.66708 & 5.11319 \\ \mathrm{H} & -2.06653 & -1.93024 & -5.19010 \\ \mathrm{H} & -2.09049 & -0.79243 & -3.81909 \\ \mathrm{H} & -0.12918 & -3.06417 & -4.32194 \\ \mathrm{H} & 0.13708 & -1.53244 & -3.45111 \\ \mathrm{H} & 4.18347 & -3.98337 & 3.60160 \\ \mathrm{H} & -4.08928 & -0.19878 & -2.03424 \\ \mathrm{H} & -2.31884 & -1.82952 & -0.14816 \\ \mathrm{H} & -1.83585 & -0.85722 & -1.55648 \\ \mathrm{H} & -4.82789 & -2.25792 & 0.13995 \\ \mathrm{H} & -5.86369 & 0.96748 & -1.62489 \\ \mathrm{H} & -7.17410 & -2.54135 & 0.55271 \\ \mathrm{H} & -8.16979 & 1.85722 & -1.58731 \\ \mathrm{H} & -9.50024 & -1.63699 & 0.60381 \\ \mathrm{H} & -10.01096 & 0.56497 & -0.47795\end{array}$

\section{Transition state structures with the full DAPa catalyst}
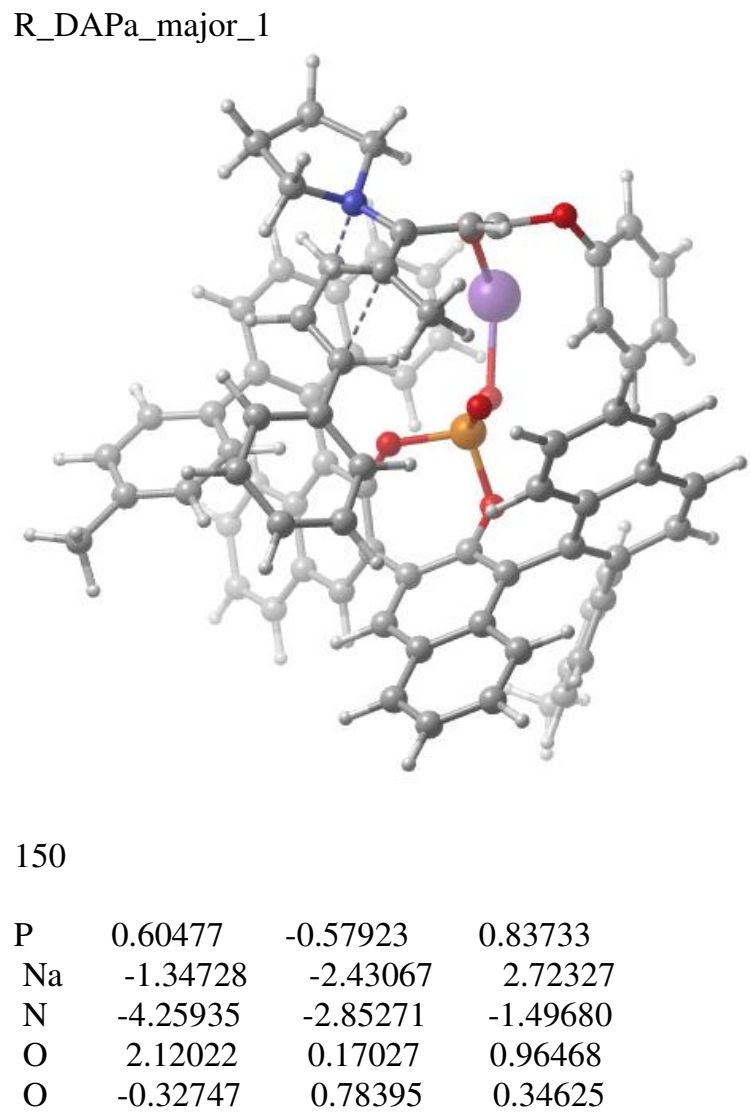

\begin{tabular}{|c|c|c|c|}
\hline $\mathrm{O}$ & 0.24507 & -0.95706 & 2.27662 \\
\hline $\mathrm{O}$ & 0.52663 & -1.53901 & -0.33910 \\
\hline $\mathrm{O}$ & -2.47660 & -3.53512 & 1.11491 \\
\hline $\mathrm{O}$ & -1.26686 & -5.44536 & 0.64065 \\
\hline $\mathrm{C}$ & -0.68928 & 6.67560 & 2.89837 \\
\hline $\mathrm{C}$ & 0.32150 & 6.06056 & 2.17700 \\
\hline $\mathrm{C}$ & 0.18685 & 4.71418 & 1.72815 \\
\hline $\mathrm{C}$ & -1.01789 & 3.98799 & 2.04206 \\
\hline $\mathrm{C}$ & -2.03993 & 4.64913 & 2.78381 \\
\hline $\mathrm{C}$ & -1.87908 & 5.96166 & 3.20272 \\
\hline $\mathrm{C}$ & 1.19775 & 4.07639 & 0.95810 \\
\hline $\mathrm{C}$ & -1.15108 & 2.63387 & 1.59230 \\
\hline $\mathrm{C}$ & -0.11536 & 2.03799 & 0.87691 \\
\hline $\mathrm{C}$ & 1.07061 & 2.76346 & 0.51675 \\
\hline $\mathrm{C}$ & -2.44898 & 1.91172 & 1.77995 \\
\hline $\mathrm{C}$ & -2.62387 & 0.99798 & 2.87259 \\
\hline $\mathrm{C}$ & -3.52171 & 2.19313 & 0.92176 \\
\hline $\mathrm{C}$ & -1.58716 & 0.75196 & 3.82260 \\
\hline $\mathrm{C}$ & -3.88835 & 0.32105 & 3.04286 \\
\hline $\mathrm{C}$ & -4.78355 & 1.56270 & 1.14620 \\
\hline $\mathrm{C}$ & -1.77361 & -0.14119 & 4.86941 \\
\hline $\mathrm{C}$ & -4.03651 & -0.62270 & 4.10293 \\
\hline $\mathrm{C}$ & -4.96040 & 0.63919 & 2.16007 \\
\hline $\mathrm{C}$ & -3.00100 & -0.85169 & 5.00190 \\
\hline $\mathrm{C}$ & 2.08864 & 2.16415 & -0.38321 \\
\hline $\mathrm{C}$ & 2.58823 & 2.86993 & -1.47301 \\
\hline $\mathrm{C}$ & 2.57963 & 0.84108 & -0.15436 \\
\hline $\mathrm{C}$ & 3.55930 & 2.30410 & -2.34367 \\
\hline C & 3.53562 & 0.23773 & -0.96864 \\
\hline $\mathrm{C}$ & 4.04741 & 3.01603 & -3.47933 \\
\hline $\mathrm{C}$ & 4.04881 & 0.97072 & -2.08942 \\
\hline $\mathrm{C}$ & 4.98891 & 2.45157 & -4.32527 \\
\hline C & 5.03455 & 0.42785 & -2.96568 \\
\hline $\mathrm{C}$ & 5.49050 & 1.14845 & -4.05971 \\
\hline $\mathrm{C}$ & 4.02590 & -1.13128 & -0.61494 \\
\hline $\mathrm{C}$ & 3.63697 & -2.26503 & -1.40608 \\
\hline $\mathrm{C}$ & 4.78147 & -1.31351 & 0.55227 \\
\hline $\mathrm{C}$ & 2.85839 & -2.13545 & -2.59333 \\
\hline $\mathrm{C}$ & 4.01279 & -3.59008 & -0.97939 \\
\hline $\mathrm{C}$ & 5.15420 & -2.63223 & 0.95094 \\
\hline $\mathrm{C}$ & 2.47191 & -3.24660 & -3.32342 \\
\hline C & 3.58773 & -4.71652 & -1.74402 \\
\hline C & 4.78120 & -3.73875 & 0.21000 \\
\hline $\mathrm{C}$ & 2.83147 & -4.55250 & -2.89355 \\
\hline $\mathrm{C}$ & 5.14621 & -0.16152 & 1.42066 \\
\hline $\mathrm{C}$ & 4.76903 & -0.15740 & 2.77925 \\
\hline $\mathrm{C}$ & 5.82156 & 0.96620 & 0.91448 \\
\hline $\mathrm{C}$ & 5.03900 & 0.94717 & 3.59562 \\
\hline $\mathrm{C}$ & 6.09119 & 2.06892 & 1.73613 \\
\hline $\mathrm{C}$ & 5.69615 & 2.08569 & 3.08754 \\
\hline $\mathrm{C}$ & -3.38753 & 3.16196 & -0.20030 \\
\hline C & -2.37847 & 3.02211 & -1.17647 \\
\hline $\mathrm{C}$ & -4.27269 & 4.25233 & -0.30992 \\
\hline $\mathrm{C}$ & -2.25038 & 3.95263 & -2.21152 \\
\hline $\mathrm{C}$ & -4.13789 & 5.18533 & -1.34945 \\
\hline $\mathrm{C}$ & -3.12264 & 5.05544 & -2.31557 \\
\hline $\mathrm{C}$ & 5.93396 & 3.29632 & 3.96109 \\
\hline
\end{tabular}




\begin{tabular}{|c|c|c|c|}
\hline $\mathrm{C}$ & -2.95769 & 6.06186 & -3.43121 \\
\hline $\mathrm{C}$ & 1.65163 & -4.54382 & 3.51646 \\
\hline $\mathrm{C}$ & -2.05305 & -4.32338 & 0.21170 \\
\hline $\mathrm{C}$ & 0.57445 & -5.38667 & 3.84334 \\
\hline $\mathrm{C}$ & 1.74955 & -4.00807 & 2.22027 \\
\hline $\mathrm{C}$ & -2.24371 & -4.31595 & -1.16494 \\
\hline $\mathrm{C}$ & -0.40965 & -5.68570 & 2.88302 \\
\hline $\mathrm{C}$ & 0.78411 & -4.31331 & 1.24944 \\
\hline $\mathrm{C}$ & -0.29854 & -5.14592 & 1.58902 \\
\hline $\mathrm{C}$ & -3.00562 & -3.39533 & -1.98148 \\
\hline $\mathrm{C}$ & -2.53677 & -2.94319 & -3.19686 \\
\hline $\mathrm{C}$ & -5.25779 & -2.43012 & -2.52589 \\
\hline $\mathrm{C}$ & -5.02547 & -3.70933 & -0.53708 \\
\hline $\mathrm{C}$ & -1.16332 & -3.26335 & -3.70804 \\
\hline $\mathrm{C}$ & -6.56224 & -2.21129 & -1.74797 \\
\hline $\mathrm{C}$ & -6.43723 & -3.10202 & -0.48137 \\
\hline $\mathrm{C}$ & -3.78837 & -1.22209 & -0.55000 \\
\hline $\mathrm{C}$ & -3.27829 & -0.32387 & -1.53376 \\
\hline $\mathrm{C}$ & -1.99250 & -0.48893 & -1.99750 \\
\hline $\mathrm{C}$ & -1.35520 & 0.25324 & -3.08071 \\
\hline $\mathrm{C}$ & -2.08392 & 0.94346 & -4.07835 \\
\hline $\mathrm{C}$ & 0.05971 & 0.28835 & -3.12361 \\
\hline $\mathrm{C}$ & -1.41724 & 1.65740 & -5.07929 \\
\hline $\mathrm{C}$ & 0.72516 & 1.01276 & -4.12017 \\
\hline $\mathrm{C}$ & -0.01062 & 1.69963 & -5.09994 \\
\hline $\mathrm{H}$ & 2.10494 & 4.63754 & 0.70683 \\
\hline $\mathrm{H}$ & -0.57422 & 7.71224 & 3.23452 \\
\hline $\mathrm{H}$ & 1.24245 & 6.60420 & 1.93376 \\
\hline $\mathrm{H}$ & -2.95770 & 4.10201 & 3.02075 \\
\hline $\mathrm{H}$ & -2.67577 & 6.45163 & 3.77401 \\
\hline $\mathrm{H}$ & -0.63068 & 1.26484 & 3.70094 \\
\hline $\mathrm{H}$ & -5.61122 & 1.80112 & 0.46832 \\
\hline $\mathrm{H}$ & -0.96303 & -0.31241 & 5.58684 \\
\hline $\mathrm{H}$ & -4.99340 & -1.14754 & 4.21220 \\
\hline $\mathrm{H}$ & -5.92875 & 0.14261 & 2.29866 \\
\hline $\mathrm{H}$ & -3.13606 & -1.56180 & 5.82689 \\
\hline $\mathrm{H}$ & 2.20125 & 3.87190 & -1.68915 \\
\hline $\mathrm{H}$ & 3.66109 & 4.02467 & -3.66936 \\
\hline $\mathrm{H}$ & 5.35417 & 3.01011 & -5.19445 \\
\hline $\mathrm{H}$ & 5.41962 & -0.57651 & -2.76570 \\
\hline $\mathrm{H}$ & 6.24348 & 0.70961 & -4.72393 \\
\hline $\mathrm{H}$ & 2.56326 & -1.13916 & -2.92374 \\
\hline $\mathrm{H}$ & 5.74989 & -2.75376 & 1.86263 \\
\hline $\mathrm{H}$ & 1.87388 & -3.11704 & -4.23169 \\
\hline $\mathrm{H}$ & 3.86631 & -5.71939 & -1.39871 \\
\hline $\mathrm{H}$ & 5.06560 & -4.74633 & 0.53580 \\
\hline $\mathrm{H}$ & 2.50675 & -5.42650 & -3.46974 \\
\hline $\mathrm{H}$ & 4.21393 & -1.01139 & 3.18259 \\
\hline $\mathrm{H}$ & 6.13633 & 0.98067 & -0.13364 \\
\hline $\mathrm{H}$ & 4.71352 & 0.93451 & 4.64350 \\
\hline $\mathrm{H}$ & 6.61710 & 2.93572 & 1.31667 \\
\hline $\mathrm{H}$ & -1.70589 & 2.16536 & -1.13886 \\
\hline $\mathrm{H}$ & -5.05640 & 4.38948 & 0.44471 \\
\hline $\mathrm{H}$ & -1.46325 & 3.80718 & -2.96051 \\
\hline $\mathrm{H}$ & -4.82957 & 6.03521 & -1.40334 \\
\hline $\mathrm{H}$ & 6.68432 & 3.97519 & 3.51934 \\
\hline $\mathrm{H}$ & 4.99881 & 3.87552 & 4.09556 \\
\hline
\end{tabular}

$\begin{array}{rrrr}\mathrm{H} & 6.28372 & 3.00726 & 4.96932 \\ \mathrm{H} & -2.95285 & 5.56544 & -4.42021 \\ \mathrm{H} & -3.76825 & 6.81082 & -3.42829 \\ \mathrm{H} & -1.99633 & 6.60338 & -3.33889 \\ \mathrm{H} & 2.40819 & -4.29974 & 4.27098 \\ \mathrm{H} & 0.49266 & -5.80948 & 4.85206 \\ \mathrm{H} & 2.57207 & -3.33641 & 1.95775 \\ \mathrm{H} & -1.61370 & -5.01351 & -1.72036 \\ \mathrm{H} & -1.26451 & -6.32831 & 3.11909 \\ \mathrm{H} & 0.83661 & -3.86466 & 0.25356 \\ \mathrm{H} & -3.18744 & -2.37251 & -3.86310 \\ \mathrm{H} & -4.89988 & -1.52735 & -3.04029 \\ \mathrm{H} & -5.34656 & -3.24733 & -3.26354 \\ \mathrm{H} & -5.02210 & -4.73419 & -0.94843 \\ \mathrm{H} & -4.49891 & -3.72031 & 0.42707 \\ \mathrm{H} & -1.10938 & -4.26196 & -4.19010 \\ \mathrm{H} & -0.85153 & -2.51792 & -4.46079 \\ \mathrm{H} & -0.42516 & -3.25751 & -2.88704 \\ \mathrm{H} & -7.43176 & -2.47708 & -2.37133 \\ \mathrm{H} & -6.67458 & -1.14836 & -1.47272 \\ \mathrm{H} & -7.19780 & -3.89981 & -0.45819 \\ \mathrm{H} & -6.57256 & -2.50129 & 0.43467 \\ \mathrm{H} & -3.08387 & -1.73147 & 0.11561 \\ \mathrm{H} & -4.74830 & -0.98504 & -0.08326 \\ \mathrm{H} & -3.95220 & 0.41122 & -1.98881 \\ \mathrm{H} & -1.32432 & -1.14965 & -1.43414 \\ \mathrm{H} & -3.17890 & 0.91465 & -4.07084 \\ \mathrm{H} & 0.61217 & -0.24560 & -2.34533 \\ \mathrm{H} & -1.99341 & 2.18654 & -5.84733 \\ \mathrm{H} & 1.81920 & 1.04876 & -4.13121 \\ \mathrm{H} & 0.51026 & 2.26248 & -5.88339\end{array}$

R_DAPa_major_2

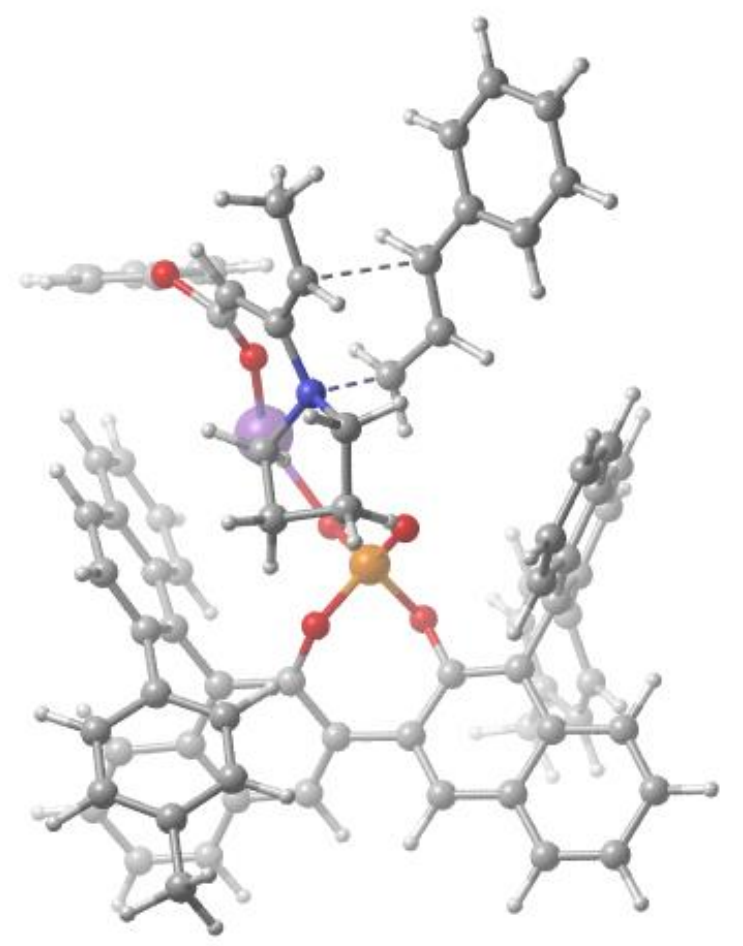


150

\begin{tabular}{|c|c|c|c|}
\hline $\mathrm{P}$ & -0.70907 & 0.29768 & 0.77459 \\
\hline $\mathrm{Na}$ & 1.45666 & -1.95563 & 2.18797 \\
\hline $\mathrm{N}$ & 3.40028 & -0.20508 & -1.63889 \\
\hline $\mathrm{O}$ & -2.00438 & 1.19137 & 1.39209 \\
\hline $\mathrm{O}$ & -1.55919 & -0.56868 & -0.44042 \\
\hline $\mathrm{O}$ & 0.27316 & 1.15802 & 0.00092 \\
\hline $\mathrm{O}$ & -0.29198 & -0.64317 & 1.91194 \\
\hline $\mathrm{O}$ & 3.33100 & -1.84439 & 1.06748 \\
\hline $\mathrm{O}$ & 4.97081 & -3.47875 & 1.02454 \\
\hline $\mathrm{C}$ & -6.56384 & -3.04917 & 0.20190 \\
\hline $\mathrm{C}$ & -4.59397 & 2.95165 & 4.84051 \\
\hline $\mathrm{C}$ & -4.81233 & 3.81213 & 3.74839 \\
\hline $\mathrm{C}$ & -5.40626 & 5.32046 & -2.18560 \\
\hline $\mathrm{C}$ & -5.54998 & 3.95807 & -1.97864 \\
\hline $\mathrm{C}$ & -5.50864 & -5.21495 & -0.12909 \\
\hline $\mathrm{C}$ & -5.30276 & -2.39789 & 0.06434 \\
\hline $\mathrm{C}$ & -3.75906 & 4.20420 & 2.91049 \\
\hline $\mathrm{C}$ & -4.32328 & 6.01883 & -1.58675 \\
\hline $\mathrm{C}$ & -3.27732 & 2.50477 & 5.07374 \\
\hline $\mathrm{C}$ & -5.17804 & -0.98211 & 0.11785 \\
\hline $\mathrm{C}$ & -4.62418 & 3.23702 & -1.16739 \\
\hline $\mathrm{C}$ & -4.74970 & 1.83613 & -0.95663 \\
\hline $\mathrm{C}$ & -3.40109 & 5.34612 & -0.79844 \\
\hline $\mathrm{C}$ & -3.51962 & 3.94399 & -0.56786 \\
\hline $\mathrm{C}$ & -4.26550 & -4.61431 & -0.26212 \\
\hline $\mathrm{C}$ & -3.85553 & 1.13505 & -0.15166 \\
\hline $\mathrm{C}$ & -4.12578 & -3.19942 & -0.16329 \\
\hline $\mathrm{C}$ & -2.44709 & 3.74676 & 3.14002 \\
\hline $\mathrm{C}$ & -3.95005 & -0.34179 & -0.02524 \\
\hline $\mathrm{C}$ & -2.22197 & 2.89605 & 4.24247 \\
\hline $\mathrm{C}$ & -2.59059 & 3.22564 & 0.25600 \\
\hline $\mathrm{C}$ & -2.80216 & 1.86806 & 0.48274 \\
\hline $\mathrm{C}$ & -2.78209 & -1.16118 & -0.19011 \\
\hline $\mathrm{C}$ & -2.85875 & -2.54711 & -0.28430 \\
\hline $\mathrm{C}$ & -1.32271 & 4.11405 & 2.23814 \\
\hline $\mathrm{C}$ & -1.39683 & 3.89630 & 0.85700 \\
\hline $\mathrm{C}$ & -1.25765 & -3.72680 & 1.86665 \\
\hline $\mathrm{C}$ & -3.71718 & -2.44825 & -5.28200 \\
\hline $\mathrm{C}$ & -0.12918 & 4.66154 & 2.79956 \\
\hline $\mathrm{C}$ & -3.23312 & -1.46163 & -4.39875 \\
\hline $\mathrm{C}$ & -3.34549 & -3.78357 & -5.04043 \\
\hline $\mathrm{C}$ & -0.50417 & -4.27095 & 2.90002 \\
\hline $\mathrm{C}$ & -1.61264 & -3.31989 & -0.57900 \\
\hline $\mathrm{C}$ & -2.42041 & -1.79282 & -3.30864 \\
\hline $\mathrm{C}$ & -2.52601 & -4.12145 & -3.95315 \\
\hline $\mathrm{C}$ & -0.28178 & 4.22560 & 0.01169 \\
\hline $\mathrm{C}$ & -2.05941 & -3.13442 & -3.06227 \\
\hline $\mathrm{C}$ & -0.83768 & -3.85180 & 0.50604 \\
\hline $\mathrm{C}$ & -0.28545 & 3.96688 & -1.39158 \\
\hline $\mathrm{C}$ & -1.21564 & -3.54628 & -1.90276 \\
\hline $\mathrm{C}$ & 0.94991 & 5.00039 & 2.00468 \\
\hline $\mathrm{C}$ & 0.72303 & -4.94347 & 2.62472 \\
\hline $\mathrm{C}$ & 0.90540 & 4.79736 & 0.59546 \\
\hline $\mathrm{C}$ & 0.39266 & -4.55434 & 0.22684 \\
\hline
\end{tabular}

\begin{tabular}{|c|c|c|c|}
\hline $\mathrm{C}$ & 0.80593 & 4.28797 & -2.18293 \\
\hline $\mathrm{C}$ & 1.16045 & -5.07330 & 1.31248 \\
\hline $\mathrm{C}$ & 0.00409 & -4.24641 & -2.15870 \\
\hline $\mathrm{C}$ & 2.01148 & 5.12166 & -0.24502 \\
\hline $\mathrm{C}$ & 0.79966 & -4.71435 & -1.12946 \\
\hline $\mathrm{C}$ & 1.96390 & 4.87986 & -1.60935 \\
\hline $\mathrm{C}$ & -6.66780 & -4.42783 & 0.10789 \\
\hline $\mathrm{C}$ & 4.34916 & -2.29702 & 0.48351 \\
\hline $\mathrm{C}$ & 5.02086 & -1.86620 & -0.66237 \\
\hline $\mathrm{C}$ & 4.73036 & -0.76933 & -1.55760 \\
\hline $\mathrm{C}$ & 4.61171 & -3.91408 & 2.28002 \\
\hline $\mathrm{C}$ & 5.73754 & -0.14155 & -2.26772 \\
\hline $\mathrm{C}$ & 4.49266 & -5.30537 & 2.45336 \\
\hline $\mathrm{C}$ & 4.43501 & -3.05368 & 3.38463 \\
\hline $\mathrm{C}$ & 7.18339 & -0.53401 & -2.18844 \\
\hline $\mathrm{C}$ & 3.09732 & 0.66212 & -2.83695 \\
\hline $\mathrm{C}$ & 2.23599 & -1.17612 & -1.51228 \\
\hline $\mathrm{C}$ & 4.17673 & -5.83401 & 3.71282 \\
\hline $\mathrm{C}$ & 4.10228 & -3.59466 & 4.63706 \\
\hline $\mathrm{C}$ & 1.57800 & 0.63367 & -2.95566 \\
\hline $\mathrm{C}$ & 1.26296 & -0.83468 & -2.64419 \\
\hline $\mathrm{C}$ & 3.96408 & -4.98220 & 4.80934 \\
\hline $\mathrm{C}$ & 3.33100 & 1.02503 & -0.10810 \\
\hline $\mathrm{C}$ & 4.27157 & 2.06033 & -0.40031 \\
\hline $\mathrm{C}$ & 5.62595 & 1.83575 & -0.29365 \\
\hline $\mathrm{C}$ & 6.69522 & 2.73777 & -0.71625 \\
\hline $\mathrm{C}$ & 6.45608 & 3.89419 & -1.50003 \\
\hline $\mathrm{C}$ & 8.03134 & 2.44296 & -0.35230 \\
\hline $\mathrm{C}$ & 7.51129 & 4.72344 & -1.89187 \\
\hline $\mathrm{C}$ & 9.08844 & 3.27301 & -0.74630 \\
\hline $\mathrm{C}$ & 8.83285 & 4.41782 & -1.51754 \\
\hline $\mathrm{C}$ & -4.60459 & -2.07210 & -6.44631 \\
\hline $\mathrm{C}$ & -5.72915 & 2.50563 & 5.73383 \\
\hline $\mathrm{H}$ & -7.64405 & -4.91444 & 0.21279 \\
\hline $\mathrm{H}$ & -7.45499 & -2.43477 & 0.37822 \\
\hline $\mathrm{H}$ & -5.82514 & 4.18305 & 3.54777 \\
\hline $\mathrm{H}$ & -6.12716 & 5.86263 & -2.80793 \\
\hline $\mathrm{H}$ & -6.38441 & 3.41037 & -2.43325 \\
\hline $\mathrm{H}$ & -6.08063 & -0.37858 & 0.26691 \\
\hline $\mathrm{H}$ & -5.54854 & 1.29232 & -1.47321 \\
\hline $\mathrm{H}$ & -5.59824 & -6.30441 & -0.20683 \\
\hline $\mathrm{H}$ & -4.21573 & 7.09736 & -1.74852 \\
\hline $\mathrm{H}$ & -3.95709 & 4.87597 & 2.06979 \\
\hline $\mathrm{H}$ & -3.07955 & 1.82429 & 5.91180 \\
\hline $\mathrm{H}$ & -2.56702 & 5.88592 & -0.33964 \\
\hline $\mathrm{H}$ & -3.37222 & -5.22028 & -0.44497 \\
\hline $\mathrm{H}$ & -1.21447 & 2.50281 & 4.41722 \\
\hline $\mathrm{H}$ & -2.18038 & -3.18256 & 2.08391 \\
\hline $\mathrm{H}$ & -0.85059 & -4.17201 & 3.93542 \\
\hline $\mathrm{H}$ & -0.09006 & 4.82834 & 3.88185 \\
\hline $\mathrm{H}$ & -3.49805 & -0.40983 & -4.56623 \\
\hline $\mathrm{H}$ & -3.70904 & -4.57467 & -5.70789 \\
\hline $\mathrm{H}$ & -1.16139 & 3.49092 & -1.83904 \\
\hline $\mathrm{H}$ & -2.05224 & -1.00211 & -2.65245 \\
\hline $\mathrm{H}$ & -2.26540 & -5.17130 & -3.77401 \\
\hline $\mathrm{H}$ & 1.32834 & -5.34900 & 3.44280 \\
\hline $\mathrm{H}$ & 1.85429 & 5.43149 & 2.45099 \\
\hline
\end{tabular}




$\begin{array}{cccc}\mathrm{H} & 0.77843 & 4.07710 & -3.25831 \\ \mathrm{H} & 0.31224 & -4.38778 & -3.20061 \\ \mathrm{H} & 2.10591 & -5.58063 & 1.09582 \\ \mathrm{H} & 2.90618 & 5.56201 & 0.21226 \\ \mathrm{H} & 1.74500 & -5.22641 & -1.34400 \\ \mathrm{H} & 2.81630 & 5.14312 & -2.24751 \\ \mathrm{H} & 5.94360 & -2.40750 & -0.87816 \\ \mathrm{H} & 5.48772 & 0.63245 & -2.99443 \\ \mathrm{H} & 4.65576 & -5.95763 & 1.58897 \\ \mathrm{H} & 4.57628 & -1.97670 & 3.26442 \\ \mathrm{H} & 7.39893 & -1.46045 & -2.76116 \\ \mathrm{H} & 7.82571 & 0.26348 & -2.59976 \\ \mathrm{H} & 7.50090 & -0.72852 & -1.14632 \\ \mathrm{H} & 3.53047 & 1.66198 & -2.69100 \\ \mathrm{H} & 3.56448 & 0.19422 & -3.72091 \\ \mathrm{H} & 2.63569 & -2.19841 & -1.55561 \\ \mathrm{H} & 1.77387 & -1.02183 & -0.53267 \\ \mathrm{H} & 4.08855 & -6.91994 & 3.83444 \\ \mathrm{H} & 3.96827 & -2.92074 & 5.49204 \\ \mathrm{H} & 1.25293 & 0.94805 & -3.96182 \\ \mathrm{H} & 1.10503 & 1.29020 & -2.20838 \\ \mathrm{H} & 1.45159 & -1.47183 & -3.52772 \\ \mathrm{H} & 0.22512 & -0.99032 & -2.32037 \\ \mathrm{H} & 3.70833 & -5.39545 & 5.79121 \\ \mathrm{H} & 2.25860 & 1.25440 & -0.11880 \\ \mathrm{H} & 3.61729 & 0.25052 & 0.60919 \\ \mathrm{H} & 3.89270 & 2.98415 & -0.84961 \\ \mathrm{H} & 5.94942 & 0.92696 & 0.22979 \\ \mathrm{H} & 5.43505 & 4.13777 & -1.81167 \\ \mathrm{H} & 8.23195 & 1.54862 & 0.24889 \\ \mathrm{H} & 7.30671 & 5.61294 & -2.49881 \\ \mathrm{H} & 10.11474 & 3.02602 & -0.45183 \\ \mathrm{H} & 9.65750 & 5.06839 & -1.83002 \\ \mathrm{H} & -4.08315 & -1.38665 & -7.14181 \\ \mathrm{H} & -4.92105 & -2.96019 & -7.01990 \\ \mathrm{H} & -5.51599 & -1.54838 & -6.10013 \\ \mathrm{H} & -5.86928 & 1.40850 & 5.68212 \\ \mathrm{H} & -5.53086 & 2.75650 & 6.79340 \\ \mathrm{H} & -6.68277 & 2.98095 & 5.44565\end{array}$

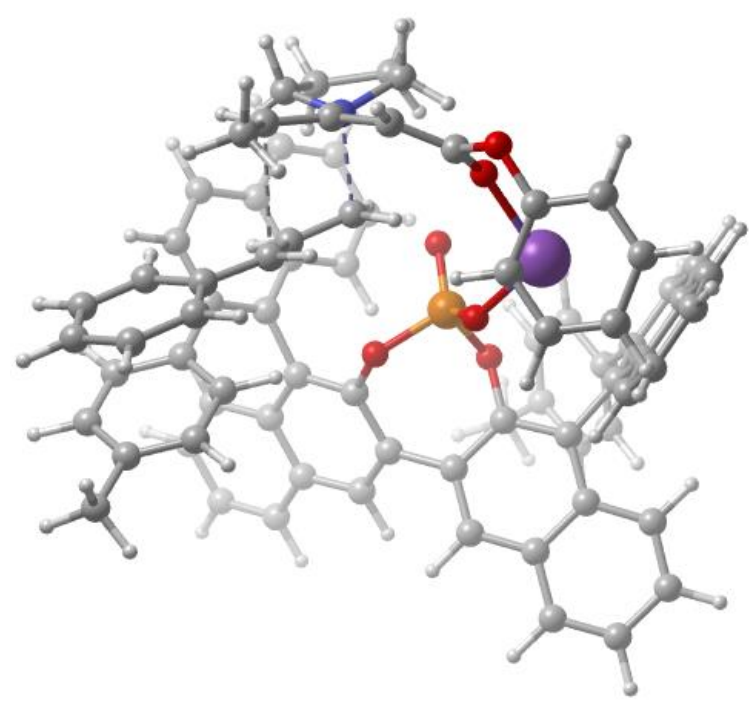

150

$\begin{array}{lrrr}\mathrm{P} & 0.91327 & 0.04460 & -0.71906 \\ \mathrm{Na} & 0.83280 & 3.50040 & -0.50312 \\ \mathrm{~N} & -3.43105 & 2.15356 & -2.48645 \\ \mathrm{O} & 2.46869 & -0.61173 & -0.53515 \\ \mathrm{O} & 0.06244 & -1.06655 & 0.27442 \\ \mathrm{O} & 0.54163 & -0.10021 & -2.18404 \\ \mathrm{O} & 0.81116 & 1.37433 & 0.04351 \\ \mathrm{O} & -1.13598 & 4.10330 & -1.31574 \\ \mathrm{O} & -2.10363 & 6.08468 & -0.66231 \\ \mathrm{C} & 1.36782 & -6.36502 & 1.65139 \\ \mathrm{C} & 5.58833 & -3.36604 & -1.86170 \\ \mathrm{C} & 6.22012 & -2.67305 & -0.81015 \\ \mathrm{C} & 5.98825 & 0.19155 & 4.78504 \\ \mathrm{C} & 4.93881 & -0.66962 & 4.50869 \\ \mathrm{C} & -0.57803 & -7.14460 & 0.42574 \\ \mathrm{C} & 1.08033 & -5.02069 & 1.27212 \\ \mathrm{C} & 6.04460 & -1.29363 & -0.64244 \\ \mathrm{C} & 6.43243 & 1.11028 & 3.79587 \\ \mathrm{C} & 4.80299 & -2.62150 & -2.76380 \\ \mathrm{C} & 1.92073 & -3.94168 & 1.65192 \\ \mathrm{C} & 4.28531 & -0.65025 & 3.24049 \\ \mathrm{C} & 3.23776 & -1.55883 & 2.92674 \\ \mathrm{C} & 5.81208 & 1.16325 & 2.55630 \\ \mathrm{C} & 4.71860 & 0.30163 & 2.24658 \\ \mathrm{C} & -0.88232 & -5.84845 & 0.03667 \\ \mathrm{C} & 2.61836 & -1.56410 & 1.67886 \\ \mathrm{C} & -0.07059 & -4.75035 & 0.44953 \\ \mathrm{C} & 5.23430 & -0.55902 & -1.53060 \\ \mathrm{C} & 1.65197 & -2.62301 & 1.29227 \\ \mathrm{C} & 4.62971 & -1.24140 & -2.60597 \\ \mathrm{C} & 4.04797 & 0.33763 & 0.98146 \\ \mathrm{C} & 3.03448 & -0.58459 & 0.72346 \\ \mathrm{C} & 0.47507 & -2.36302 & 0.50614 \\ \mathrm{C} & -0.34848 & -3.39680 & 0.06362 \\ \mathrm{C} & 4.93415 & 0.88021 & -1.30627 \\ \mathrm{C} & 4.39930 & 1.32259 & -0.08673\end{array}$




\begin{tabular}{|c|c|c|c|}
\hline $\mathrm{C}$ & 0.19511 & -3.15853 & -2.77158 \\
\hline $\mathrm{C}$ & -4.05091 & -3.02484 & 3.67798 \\
\hline C & 5.11620 & 1.80949 & -2.37428 \\
\hline $\mathrm{C}$ & -3.05849 & -2.14825 & 3.19761 \\
\hline $\mathrm{C}$ & -4.58562 & -3.96710 & 2.77906 \\
\hline C & 0.47577 & -3.12158 & -4.12711 \\
\hline $\mathrm{C}$ & -1.46925 & -3.14866 & -0.89655 \\
\hline $\mathrm{C}$ & -2.62751 & -2.19261 & 1.86767 \\
\hline $\mathrm{C}$ & -4.16623 & -4.01157 & 1.44243 \\
\hline $\mathrm{C}$ & 4.04864 & 2.70681 & 0.08395 \\
\hline $\mathrm{C}$ & -3.18452 & -3.12093 & 0.96227 \\
\hline $\mathrm{C}$ & -1.15053 & -3.11739 & -2.29862 \\
\hline $\mathrm{C}$ & 3.46475 & 3.20380 & 1.29225 \\
\hline $\mathrm{C}$ & -2.80439 & -3.11535 & -0.47533 \\
\hline $\mathrm{C}$ & 4.77369 & 3.14192 & -2.23881 \\
\hline $\mathrm{C}$ & -0.57384 & -3.04853 & -5.08117 \\
\hline $\mathrm{C}$ & 4.22827 & 3.62820 & -1.01486 \\
\hline $\mathrm{C}$ & -2.21509 & -3.06129 & -3.26961 \\
\hline $\mathrm{C}$ & 3.08765 & 4.53468 & 1.41453 \\
\hline $\mathrm{C}$ & -1.89337 & -3.02350 & -4.65805 \\
\hline $\mathrm{C}$ & -3.84554 & -3.07530 & -1.45611 \\
\hline $\mathrm{C}$ & 3.81682 & 4.98899 & -0.86157 \\
\hline $\mathrm{C}$ & -3.56303 & -3.04839 & -2.80932 \\
\hline $\mathrm{C}$ & 3.25489 & 5.43914 & 0.32652 \\
\hline $\mathrm{C}$ & 0.55487 & -7.40890 & 1.24130 \\
\hline $\mathrm{C}$ & -2.22038 & 4.71859 & -1.11408 \\
\hline $\mathrm{C}$ & -3.55200 & 4.33755 & -1.25767 \\
\hline $\mathrm{C}$ & -4.13839 & 3.07459 & -1.65949 \\
\hline $\mathrm{C}$ & -1.20459 & 6.31317 & 0.35251 \\
\hline $\mathrm{C}$ & -5.35217 & 2.66142 & -1.13079 \\
\hline $\mathrm{C}$ & -0.50950 & 7.53611 & 0.34800 \\
\hline $\mathrm{C}$ & -1.01699 & 5.40121 & 1.41380 \\
\hline $\mathrm{C}$ & -6.16565 & 3.46308 & -0.15856 \\
\hline $\mathrm{C}$ & -4.18830 & 1.04969 & -3.14665 \\
\hline $\mathrm{C}$ & -2.52961 & 2.69839 & -3.54606 \\
\hline $\mathrm{C}$ & 0.35524 & 7.85150 & 1.40688 \\
\hline $\mathrm{C}$ & -0.14276 & 5.72606 & 2.46196 \\
\hline $\mathrm{C}$ & -3.17208 & 0.38830 & -4.08629 \\
\hline $\mathrm{C}$ & -2.10689 & 1.47897 & -4.37930 \\
\hline $\mathrm{C}$ & 0.54636 & 6.95031 & 2.46728 \\
\hline $\mathrm{C}$ & -2.22924 & 1.14172 & -1.11381 \\
\hline C & -3.16732 & 0.46728 & -0.30451 \\
\hline $\mathrm{C}$ & -3.89902 & 1.15130 & 0.64958 \\
\hline $\mathrm{C}$ & -4.95273 & 0.59215 & 1.48704 \\
\hline $\mathrm{C}$ & -5.57938 & -0.64299 & 1.19351 \\
\hline $\mathrm{C}$ & -5.35485 & 1.28910 & 2.65179 \\
\hline $\mathrm{C}$ & -6.54882 & -1.17292 & 2.04711 \\
\hline $\mathrm{C}$ & -6.32774 & 0.75790 & 3.50787 \\
\hline $\mathrm{C}$ & -6.92558 & -0.47718 & 3.21016 \\
\hline $\mathrm{C}$ & -4.55306 & -2.92101 & 5.09842 \\
\hline $\mathrm{C}$ & 5.70747 & -4.86721 & -1.99041 \\
\hline $\mathrm{H}$ & 0.78423 & -8.43784 & 1.54055 \\
\hline $\mathrm{H}$ & 2.24832 & -6.55587 & 2.27686 \\
\hline $\mathrm{H}$ & 6.85266 & -3.22436 & -0.10332 \\
\hline $\mathrm{H}$ & 6.48358 & 0.16081 & 5.76204 \\
\hline $\mathrm{H}$ & 4.59885 & -1.39131 & 5.26127 \\
\hline $\mathrm{H}$ & 2.83226 & -4.16167 & 2.21855 \\
\hline
\end{tabular}

\begin{tabular}{|c|c|c|c|}
\hline $\mathrm{H}$ & 2.91882 & -2.27845 & 3.68905 \\
\hline $\mathrm{H}$ & -1.21625 & -7.97299 & 0.09766 \\
\hline $\mathrm{H}$ & 7.27256 & 1.77967 & 4.01284 \\
\hline $\mathrm{H}$ & 6.53400 & -0.78053 & 0.19167 \\
\hline $\mathrm{H}$ & 4.29508 & -3.13643 & -3.58885 \\
\hline $\mathrm{H}$ & 6.15527 & 1.87194 & 1.79600 \\
\hline $\mathrm{H}$ & -1.75479 & -5.65501 & -0.59404 \\
\hline $\mathrm{H}$ & 3.96164 & -0.69621 & -3.28120 \\
\hline $\mathrm{H}$ & 1.01297 & -3.19697 & -2.04871 \\
\hline $\mathrm{H}$ & 1.51835 & -3.13118 & -4.46348 \\
\hline $\mathrm{H}$ & 5.53747 & 1.44303 & -3.31684 \\
\hline $\mathrm{H}$ & -2.62713 & -1.39953 & 3.87343 \\
\hline $\mathrm{H}$ & -5.34573 & -4.67662 & 3.12960 \\
\hline $\mathrm{H}$ & 3.29923 & 2.51285 & 2.12201 \\
\hline $\mathrm{H}$ & -1.88277 & -1.47652 & 1.51320 \\
\hline $\mathrm{H}$ & -4.59330 & -4.75976 & 0.76416 \\
\hline $\mathrm{H}$ & -0.33823 & -3.01409 & -6.15119 \\
\hline $\mathrm{H}$ & 4.91514 & 3.83995 & -3.07261 \\
\hline $\mathrm{H}$ & 2.63953 & 4.89251 & 2.34647 \\
\hline $\mathrm{H}$ & -4.88684 & -3.05779 & -1.11471 \\
\hline $\mathrm{H}$ & -2.71205 & -2.97210 & -5.38636 \\
\hline $\mathrm{H}$ & 3.95035 & 5.67545 & -1.70674 \\
\hline $\mathrm{H}$ & -4.37564 & -3.01257 & -3.54535 \\
\hline $\mathrm{H}$ & 2.93031 & 6.48001 & 0.43173 \\
\hline $\mathrm{H}$ & -4.26795 & 5.07959 & -0.90111 \\
\hline $\mathrm{H}$ & -5.81626 & 1.74824 & -1.50658 \\
\hline $\mathrm{H}$ & -0.66644 & 8.22833 & -0.48568 \\
\hline $\mathrm{H}$ & -1.57502 & 4.45862 & 1.42450 \\
\hline $\mathrm{H}$ & -6.73128 & 4.27753 & -0.65745 \\
\hline $\mathrm{H}$ & -6.90062 & 2.81813 & 0.35348 \\
\hline $\mathrm{H}$ & -5.53377 & 3.94156 & 0.61249 \\
\hline $\mathrm{H}$ & -4.58739 & 0.35780 & -2.39154 \\
\hline $\mathrm{H}$ & -5.03523 & 1.49911 & -3.69886 \\
\hline $\mathrm{H}$ & -3.12541 & 3.42595 & -4.13042 \\
\hline $\mathrm{H}$ & -1.69021 & 3.22517 & -3.07551 \\
\hline $\mathrm{H}$ & 0.88669 & 8.81054 & 1.39944 \\
\hline $\mathrm{H}$ & -0.00761 & 5.01374 & 3.28450 \\
\hline $\mathrm{H}$ & -3.67321 & 0.02015 & -4.99685 \\
\hline $\mathrm{H}$ & -2.70614 & -0.48074 & -3.60016 \\
\hline $\mathrm{H}$ & -2.05866 & 1.74259 & -5.44877 \\
\hline $\mathrm{H}$ & -1.10774 & 1.12738 & -4.07406 \\
\hline $\mathrm{H}$ & 1.22534 & 7.19953 & 3.29022 \\
\hline $\mathrm{H}$ & -1.60168 & 0.57337 & -1.80480 \\
\hline $\mathrm{H}$ & -1.80048 & 2.08567 & -0.77677 \\
\hline $\mathrm{H}$ & -3.39380 & -0.57803 & -0.53024 \\
\hline $\mathrm{H}$ & -3.57490 & 2.16856 & 0.90355 \\
\hline $\mathrm{H}$ & -5.29613 & -1.19155 & 0.29256 \\
\hline $\mathrm{H}$ & -4.88239 & 2.25003 & 2.88676 \\
\hline $\mathrm{H}$ & -7.00681 & -2.13911 & 1.81210 \\
\hline $\mathrm{H}$ & -6.61962 & 1.30733 & 4.41008 \\
\hline $\mathrm{H}$ & -7.68494 & -0.89692 & 3.87978 \\
\hline $\mathrm{H}$ & -5.07677 & -3.84009 & 5.41439 \\
\hline $\mathrm{H}$ & -3.72716 & -2.73270 & 5.80811 \\
\hline $\mathrm{H}$ & -5.26609 & -2.07847 & 5.19541 \\
\hline $\mathrm{H}$ & 4.87823 & -5.37218 & -1.45568 \\
\hline $\mathrm{H}$ & 5.65935 & -5.19026 & -3.04576 \\
\hline $\mathrm{H}$ & 6.65295 & -5.23947 & -1.55773 \\
\hline
\end{tabular}




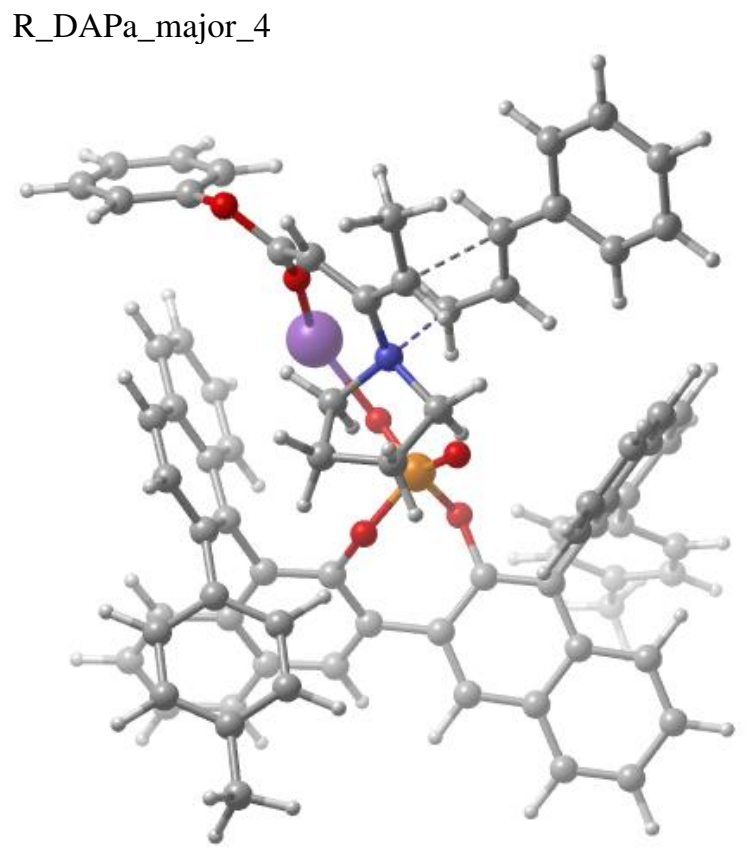

$\begin{array}{lccc}150 & & & \\ & & & \\ \mathrm{P} & 0.76055 & 0.04799 & -0.75460 \\ \mathrm{Na} & -2.08753 & -1.06132 & -2.21435 \\ \mathrm{~N} & -3.10744 & 1.12807 & 1.12035 \\ \mathrm{O} & 2.35758 & 0.33282 & -1.22288 \\ \mathrm{O} & 1.03703 & -1.16317 & 0.42126 \\ \mathrm{O} & 0.06669 & -0.54454 & -1.98836 \\ \mathrm{O} & 0.16915 & 1.20378 & 0.03493 \\ \mathrm{O} & -4.04079 & -0.56600 & -1.34953 \\ \mathrm{O} & -6.25929 & -1.15654 & -1.06608 \\ \mathrm{C} & 3.96190 & -6.86027 & -0.14804 \\ \mathrm{C} & 4.48113 & -5.57601 & -0.12235 \\ \mathrm{C} & 3.62433 & -4.44148 & -0.00948 \\ \mathrm{C} & 2.19978 & -4.64720 & 0.06798 \\ \mathrm{C} & 1.69609 & -5.98057 & 0.04226 \\ \mathrm{C} & 2.55791 & -7.06225 & -0.06253 \\ \mathrm{C} & 4.13744 & -3.11717 & 0.04453 \\ \mathrm{C} & 1.33958 & -3.50648 & 0.16305 \\ \mathrm{C} & 1.89022 & -2.22857 & 0.19778 \\ \mathrm{C} & 3.30854 & -2.00462 & 0.16974 \\ \mathrm{C} & -0.14540 & -3.68455 & 0.22009 \\ \mathrm{C} & -0.86906 & -3.80938 & -1.01623 \\ \mathrm{C} & -0.82249 & -3.79094 & 1.44169 \\ \mathrm{C} & -0.21215 & -3.74808 & -2.28502 \\ \mathrm{C} & -2.30237 & -3.99679 & -0.99312 \\ \mathrm{C} & -2.24215 & -3.97201 & 1.44277 \\ \mathrm{C} & -0.93100 & -3.86047 & -3.46794 \\ \mathrm{C} & -3.01617 & -4.09632 & -2.22715 \\ \mathrm{C} & -2.96540 & -4.05499 & 0.26766 \\ \mathrm{C} & -2.34516 & -4.03176 & -3.44189 \\ \mathrm{C} & 3.88073 & -0.65042 & 0.38283 \\ \mathrm{C} & 4.96968 & -0.47626 & 1.23464 \\ \mathrm{C} & 3.32878 & 0.51391 & -0.24440 \\ \mathrm{C} & 5.53155 & 0.80482 & 1.48502\end{array}$

\begin{tabular}{|c|c|c|c|}
\hline C & 3.79885 & 1.79741 & 0.01436 \\
\hline $\mathrm{C}$ & 6.65865 & 0.97576 & 2.34226 \\
\hline $\mathrm{C}$ & 4.93114 & 1.96586 & 0.87779 \\
\hline $\mathrm{C}$ & 7.17907 & 2.23546 & 2.58962 \\
\hline $\mathrm{C}$ & 5.48496 & 3.24905 & 1.15846 \\
\hline $\mathrm{C}$ & 6.58556 & 3.38001 & 1.99201 \\
\hline $\mathrm{C}$ & 3.11026 & 2.99992 & -0.55165 \\
\hline $\mathrm{C}$ & 2.10129 & 3.64279 & 0.24534 \\
\hline $\mathrm{C}$ & 3.49282 & 3.54785 & -1.78032 \\
\hline $\mathrm{C}$ & 1.74889 & 3.17359 & 1.54612 \\
\hline $\mathrm{C}$ & 1.43839 & 4.81775 & -0.26040 \\
\hline $\mathrm{C}$ & 2.82856 & 4.71793 & -2.26163 \\
\hline $\mathrm{C}$ & 0.79455 & 3.82678 & 2.30794 \\
\hline $\mathrm{C}$ & 0.44041 & 5.45426 & 0.53709 \\
\hline $\mathrm{C}$ & 1.81957 & 5.32449 & -1.53588 \\
\hline $\mathrm{C}$ & 0.12773 & 4.97430 & 1.79981 \\
\hline $\mathrm{C}$ & 4.62922 & 2.99212 & -2.56973 \\
\hline $\mathrm{C}$ & 4.63226 & 1.67378 & -3.06350 \\
\hline $\mathrm{C}$ & 5.74979 & 3.80781 & -2.83689 \\
\hline $\mathrm{C}$ & 5.73009 & 1.18732 & -3.78864 \\
\hline $\mathrm{C}$ & 6.84435 & 3.31398 & -3.55703 \\
\hline $\mathrm{C}$ & 6.85626 & 1.99129 & -4.04491 \\
\hline $\mathrm{C}$ & -0.11043 & -3.78419 & 2.75147 \\
\hline $\mathrm{C}$ & 0.70651 & -2.71461 & 3.16854 \\
\hline $\mathrm{C}$ & -0.26742 & -4.88153 & 3.62590 \\
\hline $\mathrm{C}$ & 1.35525 & -2.75466 & 4.41122 \\
\hline $\mathrm{C}$ & 0.38704 & -4.91663 & 4.86298 \\
\hline $\mathrm{C}$ & 1.21506 & -3.85390 & 5.27863 \\
\hline $\mathrm{C}$ & -5.09972 & -0.40467 & -0.68704 \\
\hline $\mathrm{C}$ & -5.38702 & 0.39481 & 0.42705 \\
\hline $\mathrm{C}$ & -4.53552 & 1.26367 & 1.20195 \\
\hline $\mathrm{C}$ & -6.18727 & -2.01399 & -2.14469 \\
\hline $\mathrm{C}$ & -5.05966 & 2.31895 & 1.93287 \\
\hline $\mathrm{C}$ & -6.66464 & -3.32145 & -1.94581 \\
\hline $\mathrm{C}$ & -5.73894 & -1.61229 & -3.41743 \\
\hline $\mathrm{C}$ & -6.52099 & 2.64349 & 2.00539 \\
\hline $\mathrm{C}$ & -2.68730 & 2.12647 & -0.63137 \\
\hline $\mathrm{C}$ & -2.58747 & -0.27792 & 1.22161 \\
\hline C & -2.23481 & 1.83951 & 2.11328 \\
\hline $\mathrm{C}$ & -6.68541 & -4.23015 & -3.01459 \\
\hline $\mathrm{C}$ & -5.74948 & -2.53426 & -4.47470 \\
\hline $\mathrm{C}$ & -2.95034 & 3.47174 & -0.27209 \\
\hline $\mathrm{C}$ & -2.42463 & -0.50596 & 2.73618 \\
\hline $\mathrm{C}$ & -2.16365 & 0.90573 & 3.33114 \\
\hline $\mathrm{C}$ & -6.21698 & -3.84506 & -4.28139 \\
\hline C & -4.24921 & 3.93621 & -0.16503 \\
\hline $\mathrm{C}$ & -4.66098 & 5.23331 & 0.36395 \\
\hline $\mathrm{C}$ & -6.00472 & 5.65251 & 0.20675 \\
\hline $\mathrm{C}$ & -3.76965 & 6.09233 & 1.05529 \\
\hline $\mathrm{C}$ & -6.44151 & 6.88357 & 0.71165 \\
\hline $\mathrm{C}$ & -4.20675 & 7.32076 & 1.55926 \\
\hline $\mathrm{C}$ & -5.54438 & 7.72394 & 1.39028 \\
\hline $\mathrm{C}$ & 8.04473 & 1.46390 & -4.81645 \\
\hline $\mathrm{C}$ & 1.93262 & -3.90829 & 6.60777 \\
\hline $\mathrm{H}$ & 5.22207 & -2.97084 & -0.00863 \\
\hline $\mathrm{H}$ & 4.63193 & -7.72334 & -0.23167 \\
\hline $\mathrm{H}$ & 5.56370 & -5.41167 & -0.18290 \\
\hline
\end{tabular}




\begin{tabular}{|c|c|c|c|}
\hline $\mathrm{H}$ & 0.61482 & -6.13686 & 0.10772 \\
\hline $\mathrm{H}$ & 2.15349 & -8.08063 & -0.08018 \\
\hline $\mathrm{H}$ & 0.86802 & -3.58559 & -2.31193 \\
\hline $\mathrm{H}$ & -2.75749 & -4.02196 & 2.40819 \\
\hline $\mathrm{H}$ & -0.40589 & -3.80518 & -4.42819 \\
\hline $\mathrm{H}$ & -4.10334 & -4.21499 & -2.20227 \\
\hline $\mathrm{H}$ & -4.05569 & -4.16461 & 0.28827 \\
\hline $\mathrm{H}$ & -2.90879 & -4.11184 & -4.37802 \\
\hline $\mathrm{H}$ & 5.39189 & -1.34651 & 1.74938 \\
\hline $\mathrm{H}$ & 7.10860 & 0.08687 & 2.80079 \\
\hline $\mathrm{H}$ & 8.04818 & 2.35286 & 3.24680 \\
\hline $\mathrm{H}$ & 5.02884 & 4.13124 & 0.69926 \\
\hline $\mathrm{H}$ & 7.00100 & 4.37418 & 2.19183 \\
\hline $\mathrm{H}$ & 2.23766 & 2.27655 & 1.93348 \\
\hline $\mathrm{H}$ & 3.12900 & 5.12336 & -3.23433 \\
\hline $\mathrm{H}$ & 0.54392 & 3.45005 & 3.30629 \\
\hline $\mathrm{H}$ & -0.06268 & 6.34359 & 0.13739 \\
\hline $\mathrm{H}$ & 1.31045 & 6.21284 & -1.92921 \\
\hline $\mathrm{H}$ & -0.62419 & 5.48332 & 2.41494 \\
\hline $\mathrm{H}$ & 3.76282 & 1.03373 & -2.89497 \\
\hline $\mathrm{H}$ & 5.76778 & 4.83480 & -2.45294 \\
\hline $\mathrm{H}$ & 5.70730 & 0.15714 & -4.16563 \\
\hline $\mathrm{H}$ & 7.70925 & 3.96484 & -3.73879 \\
\hline $\mathrm{H}$ & 0.81736 & -1.83616 & 2.52961 \\
\hline $\mathrm{H}$ & -0.89355 & -5.72695 & 3.31702 \\
\hline $\mathrm{H}$ & 1.98303 & -1.90683 & 4.71258 \\
\hline $\mathrm{H}$ & 0.25769 & -5.78853 & 5.51668 \\
\hline $\mathrm{H}$ & -6.43417 & 0.37525 & 0.73332 \\
\hline $\mathrm{H}$ & -4.41007 & 2.88181 & 2.60438 \\
\hline $\mathrm{H}$ & -7.01580 & -3.60705 & -0.94856 \\
\hline $\mathrm{H}$ & -5.39805 & -0.58576 & -3.57262 \\
\hline $\mathrm{H}$ & -7.06477 & 1.96888 & 2.70014 \\
\hline $\mathrm{H}$ & -6.67033 & 3.67568 & 2.36712 \\
\hline $\mathrm{H}$ & -7.01559 & 2.54802 & 1.02086 \\
\hline $\mathrm{H}$ & -1.64479 & 1.79743 & -0.71740 \\
\hline $\mathrm{H}$ & -3.44244 & 1.57284 & -1.19463 \\
\hline $\mathrm{H}$ & -3.27144 & -0.98262 & 0.74752 \\
\hline $\mathrm{H}$ & -1.61321 & -0.29460 & 0.71472 \\
\hline $\mathrm{H}$ & -1.25135 & 1.91321 & 1.62763 \\
\hline $\mathrm{H}$ & -2.59458 & 2.85374 & 2.31408 \\
\hline $\mathrm{H}$ & -7.06110 & -5.24706 & -2.85216 \\
\hline $\mathrm{H}$ & -5.39829 & -2.21909 & -5.46467 \\
\hline $\mathrm{H}$ & -2.11137 & 4.07327 & 0.09506 \\
\hline $\mathrm{H}$ & -1.60545 & -1.21300 & 2.93649 \\
\hline $\mathrm{H}$ & -3.35326 & -0.93724 & 3.14753 \\
\hline $\mathrm{H}$ & -1.17293 & 0.98282 & 3.81030 \\
\hline $\mathrm{H}$ & -2.92610 & 1.16553 & 4.08490 \\
\hline $\mathrm{H}$ & -6.22553 & -4.55727 & -5.11396 \\
\hline $\mathrm{H}$ & -5.04561 & 3.32372 & -0.60624 \\
\hline $\mathrm{H}$ & -6.70665 & 4.99760 & -0.32218 \\
\hline $\mathrm{H}$ & -2.72955 & 5.78734 & 1.20399 \\
\hline $\mathrm{H}$ & -7.48533 & 7.18883 & 0.57586 \\
\hline $\mathrm{H}$ & -3.50289 & 7.97070 & 2.09201 \\
\hline $\mathrm{H}$ & -5.88368 & 8.68685 & 1.78840 \\
\hline $\mathrm{H}$ & 7.89156 & 0.41668 & -5.12965 \\
\hline $\mathrm{H}$ & 8.23266 & 2.06585 & -5.72628 \\
\hline $\mathrm{H}$ & 8.96770 & 1.50343 & -4.20649 \\
\hline
\end{tabular}

$\begin{array}{llll}\mathrm{H} & 2.69672 & -4.70976 & 6.61415 \\ \mathrm{H} & 2.44303 & -2.95584 & 6.83176 \\ \mathrm{H} & 1.23126 & -4.12390 & 7.43575\end{array}$

S_DAPa_major_1

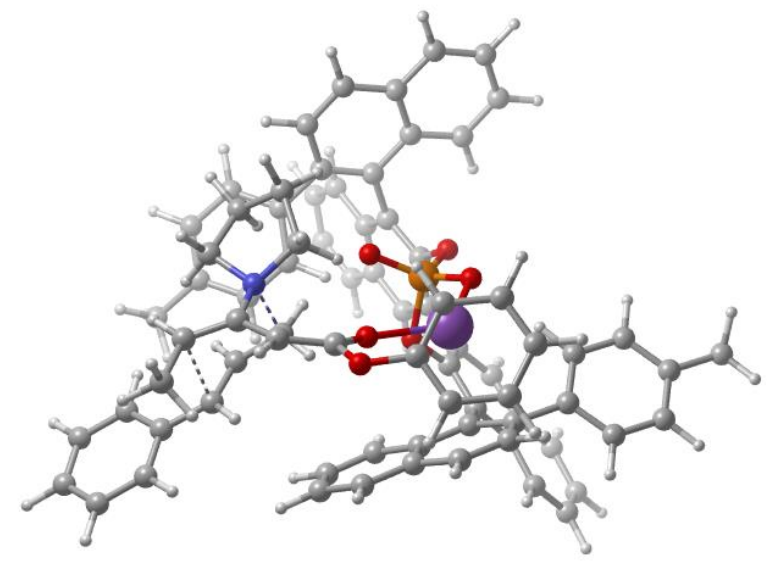

150

$\begin{array}{lccc}\mathrm{P} & 0.87693 & 0.98373 & 0.94143 \\ \mathrm{Na} & 1.71891 & -1.96134 & 1.71296 \\ \mathrm{~N} & -3.18458 & -1.89299 & 1.77119 \\ \mathrm{O} & 0.88824 & 0.52329 & -0.71919 \\ \mathrm{O} & 1.34287 & 2.60166 & 0.76483 \\ \mathrm{O} & 2.03267 & 0.27304 & 1.66227 \\ \mathrm{O} & -0.56857 & 0.84317 & 1.38921 \\ \mathrm{O} & -0.21719 & -3.04854 & 1.69987 \\ \mathrm{O} & -0.23636 & -5.22949 & 2.48291 \\ \mathrm{C} & -1.94728 & 6.90567 & -2.73200 \\ \mathrm{C} & -0.98739 & 5.97623 & -3.09684 \\ \mathrm{C} & -0.41867 & 5.09363 & -2.13122 \\ \mathrm{C} & -0.86215 & 5.16897 & -0.76282 \\ \mathrm{C} & -1.84356 & 6.14360 & -0.41858 \\ \mathrm{C} & -2.37213 & 6.99228 & -1.37895 \\ \mathrm{C} & 0.54769 & 4.11659 & -2.49153 \\ \mathrm{C} & -0.31893 & 4.25602 & 0.20366 \\ \mathrm{C} & 0.69338 & 3.37985 & -0.18670 \\ \mathrm{C} & 1.11508 & 3.26300 & -1.54889 \\ \mathrm{C} & -0.91137 & 4.16768 & 1.57064 \\ \mathrm{C} & -0.15065 & 4.57695 & 2.71622 \\ \mathrm{C} & -2.22420 & 3.68901 & 1.72269 \\ \mathrm{C} & 1.16427 & 5.11961 & 2.59925 \\ \mathrm{C} & -0.72423 & 4.45596 & 4.03315 \\ \mathrm{C} & -2.77850 & 3.60378 & 3.03658 \\ \mathrm{C} & 1.88434 & 5.49711 & 3.72146 \\ \mathrm{C} & 0.04424 & 4.84601 & 5.16895 \\ \mathrm{C} & -2.05194 & 3.95847 & 4.15710 \\ \mathrm{C} & 1.32521 & 5.35340 & 5.02038 \\ \mathrm{C} & 2.09023 & 2.22620 & -1.96935 \\ \mathrm{C} & 3.12053 & 2.54260 & -2.84836 \\ \mathrm{C} & 1.95243 & 0.86259 & -1.53994 \\ \mathrm{C} & 3.99435 & 1.55281 & -3.37153 \\ \mathrm{C} & 2.80807 & -0.14183 & -1.99875 \\ \mathrm{C} & 5.02965 & 1.88336 & -4.29477\end{array}$




\begin{tabular}{|c|c|c|c|c|c|c|c|}
\hline $\mathrm{C}$ & 3.81844 & 0.18124 & -2.96944 & $\mathrm{H}$ & 1.60359 & 5.23045 & 1.60419 \\
\hline $\mathrm{C}$ & 5.84842 & 0.90124 & -4.82710 & $\mathrm{H}$ & -3.79375 & 3.21126 & 3.15110 \\
\hline $\mathrm{C}$ & 4.66197 & -0.80719 & -3.55564 & $\mathrm{H}$ & 2.89473 & 5.90608 & 3.60733 \\
\hline $\mathrm{C}$ & 5.65117 & -0.45679 & -4.46165 & $\mathrm{H}$ & -0.40133 & 4.74040 & 6.16576 \\
\hline $\mathrm{C}$ & 2.70643 & -1.54002 & -1.47785 & $\mathrm{H}$ & -2.49195 & 3.85868 & 5.15678 \\
\hline $\mathrm{C}$ & 1.56897 & -2.35622 & -1.81603 & $\mathrm{H}$ & 1.90688 & 5.64909 & 5.90111 \\
\hline $\mathrm{C}$ & 3.76072 & -2.08232 & -0.71458 & $\mathrm{H}$ & 3.25943 & 3.58610 & -3.15291 \\
\hline $\mathrm{C}$ & 0.50895 & -1.87939 & -2.64607 & $\mathrm{H}$ & 5.15898 & 2.93386 & -4.58168 \\
\hline $\mathrm{C}$ & 1.49559 & -3.71443 & -1.33305 & $\mathrm{H}$ & 6.63950 & 1.16672 & -5.53740 \\
\hline $\mathrm{C}$ & 3.67036 & -3.43816 & -0.25880 & $\mathrm{H}$ & 4.51631 & -1.85603 & -3.28360 \\
\hline $\mathrm{C}$ & -0.56272 & -2.69349 & -2.97376 & $\mathrm{H}$ & 6.28650 & -1.23560 & -4.89841 \\
\hline $\mathrm{C}$ & 0.36776 & -4.51973 & -1.66855 & $\mathrm{H}$ & 0.55619 & -0.85640 & -3.02731 \\
\hline $\mathrm{C}$ & 2.56799 & -4.22531 & -0.54762 & $\mathrm{H}$ & 4.48604 & -3.84080 & 0.35247 \\
\hline $\mathrm{C}$ & -0.63808 & -4.02422 & -2.48042 & $\mathrm{H}$ & -1.36538 & -2.30741 & -3.61090 \\
\hline $\mathrm{C}$ & 4.99212 & -1.30649 & -0.39054 & $\mathrm{H}$ & 0.31822 & -5.54215 & -1.27636 \\
\hline $\mathrm{C}$ & 4.93855 & -0.02973 & 0.20811 & $\mathrm{H}$ & 2.50783 & -5.24991 & -0.16892 \\
\hline $\mathrm{C}$ & 6.25622 & -1.84620 & -0.70427 & $\mathrm{H}$ & -1.49016 & -4.65703 & -2.75078 \\
\hline $\mathrm{C}$ & 6.11526 & 0.68463 & 0.45706 & $\mathrm{H}$ & 3.97373 & 0.38744 & 0.50767 \\
\hline $\mathrm{C}$ & 7.42963 & -1.12011 & -0.45793 & $\mathrm{H}$ & 6.31973 & -2.83229 & -1.17946 \\
\hline $\mathrm{C}$ & 7.38072 & 0.16268 & 0.11876 & $\mathrm{H}$ & 6.04672 & 1.67264 & 0.92918 \\
\hline $\mathrm{C}$ & -3.07983 & 3.27018 & 0.57563 & $\mathrm{H}$ & 8.39974 & -1.55478 & -0.72924 \\
\hline $\mathrm{C}$ & -2.61336 & 2.42976 & -0.45669 & $\mathrm{H}$ & -1.59499 & 2.04790 & -0.42128 \\
\hline $\mathrm{C}$ & -4.42874 & 3.68664 & 0.51821 & $\mathrm{H}$ & -4.81696 & 4.35827 & 1.29265 \\
\hline $\mathrm{C}$ & -3.45877 & 2.03323 & -1.50024 & $\mathrm{H}$ & -3.05889 & 1.38129 & -2.28671 \\
\hline $\mathrm{C}$ & -5.27356 & 3.27776 & -0.52121 & $\mathrm{H}$ & -6.31464 & 3.62516 & -0.53683 \\
\hline $\mathrm{C}$ & -4.80475 & 2.44077 & -1.55394 & $\mathrm{H}$ & -2.62104 & -5.24811 & 2.26279 \\
\hline $\mathrm{C}$ & -0.92082 & -4.03403 & 2.03379 & $\mathrm{H}$ & -5.39716 & -3.09224 & 0.99790 \\
\hline $\mathrm{C}$ & -2.30244 & -4.23342 & 2.01593 & $\mathrm{H}$ & 0.65506 & -3.43458 & 4.28238 \\
\hline $\mathrm{C}$ & -3.36561 & -3.32428 & 1.65863 & $\mathrm{H}$ & 1.71563 & -6.75196 & 1.72998 \\
\hline $\mathrm{C}$ & 1.05654 & -5.08421 & 2.92719 & $\mathrm{H}$ & -5.08460 & -5.80670 & 1.72518 \\
\hline $\mathrm{C}$ & -4.55905 & -3.77843 & 1.12651 & $\mathrm{H}$ & -5.67613 & -5.31659 & 0.11647 \\
\hline $\mathrm{C}$ & 1.42114 & -4.10189 & 3.87416 & $\mathrm{H}$ & -3.94524 & -5.71192 & 0.36542 \\
\hline $\mathrm{C}$ & 2.02416 & -5.98305 & 2.44581 & $\mathrm{H}$ & -4.89520 & -1.01140 & 0.82427 \\
\hline $\mathrm{C}$ & -4.82818 & -5.22113 & 0.81718 & $\mathrm{H}$ & -5.13776 & -1.53995 & 2.52096 \\
\hline $\mathrm{C}$ & -4.43350 & -1.05675 & 1.82047 & $\mathrm{H}$ & -2.21548 & -2.20902 & 3.63421 \\
\hline $\mathrm{C}$ & -2.29727 & -1.39418 & 2.89776 & $\mathrm{H}$ & -1.30543 & -1.18916 & 2.48449 \\
\hline $\mathrm{C}$ & 2.76125 & -3.99246 & 4.28178 & $\mathrm{H}$ & 3.04083 & -3.22239 & 5.01101 \\
\hline $\mathrm{C}$ & 3.35630 & -5.87600 & 2.87291 & $\mathrm{H}$ & 4.10476 & -6.57717 & 2.48559 \\
\hline $\mathrm{C}$ & -3.96245 & 0.28589 & 2.35816 & $\mathrm{H}$ & -4.80880 & 0.88118 & 2.73823 \\
\hline $\mathrm{C}$ & -2.96040 & -0.12116 & 3.44805 & $\mathrm{H}$ & -3.46808 & 0.87264 & 1.57013 \\
\hline $\mathrm{C}$ & 3.73709 & -4.87013 & 3.77600 & $\mathrm{H}$ & -3.49118 & -0.33709 & 4.39350 \\
\hline $\mathrm{C}$ & -3.32447 & -1.56397 & -0.91427 & $\mathrm{H}$ & -2.21035 & 0.66263 & 3.63282 \\
\hline $\mathrm{C}$ & -2.31326 & -1.39830 & 0.07992 & $\mathrm{H}$ & 4.78082 & -4.77735 & 4.09495 \\
\hline $\mathrm{C}$ & -3.68456 & -2.82106 & -1.34390 & $\mathrm{H}$ & -3.90874 & -0.68589 & -1.20829 \\
\hline $\mathrm{C}$ & -4.79901 & -3.15320 & -2.22756 & $\mathrm{H}$ & -1.53212 & -2.15913 & 0.16381 \\
\hline $\mathrm{C}$ & -5.78680 & -2.20896 & -2.60342 & $\mathrm{H}$ & -1.96845 & -0.39411 & 0.34528 \\
\hline $\mathrm{C}$ & -4.91433 & -4.47350 & -2.72592 & $\mathrm{H}$ & -3.02265 & -3.65151 & -1.07787 \\
\hline $\mathrm{C}$ & -6.83620 & -2.57109 & -3.45323 & $\mathrm{H}$ & -5.73430 & -1.18497 & -2.22038 \\
\hline $\mathrm{C}$ & -5.96632 & -4.83632 & -3.57654 & $\mathrm{H}$ & -4.16360 & -5.21743 & -2.43536 \\
\hline $\mathrm{C}$ & -6.93141 & -3.88580 & -3.94563 & $\mathrm{H}$ & -7.59000 & -1.82588 & -3.73256 \\
\hline $\mathrm{C}$ & -5.71558 & 2.00806 & -2.68066 & $\mathrm{H}$ & -6.03491 & -5.86356 & -3.95219 \\
\hline $\mathrm{C}$ & 8.63669 & 0.96918 & 0.35475 & $\mathrm{H}$ & -7.75656 & -4.16646 & -4.60983 \\
\hline $\mathrm{H}$ & 0.83163 & 4.01502 & -3.54498 & $\mathrm{H}$ & -6.63245 & 1.52136 & -2.29538 \\
\hline $\mathrm{H}$ & -2.37648 & 7.57880 & -3.48276 & $\mathrm{H}$ & -6.04386 & 2.87392 & -3.28718 \\
\hline $\mathrm{H}$ & -0.64788 & 5.90377 & -4.13710 & $\mathrm{H}$ & -5.21065 & 1.29773 & -3.35803 \\
\hline $\mathrm{H}$ & -2.17301 & 6.21344 & 0.62192 & $\mathrm{H}$ & 8.65452 & 1.39860 & 1.37370 \\
\hline $\mathrm{H}$ & -3.12240 & 7.73757 & -1.09135 & $\mathrm{H}$ & 9.54529 & 0.35603 & 0.22446 \\
\hline
\end{tabular}


$\begin{array}{llll}\mathrm{H} & 8.70007 & 1.81775 & -0.35455\end{array}$

S_DAPa_major_2

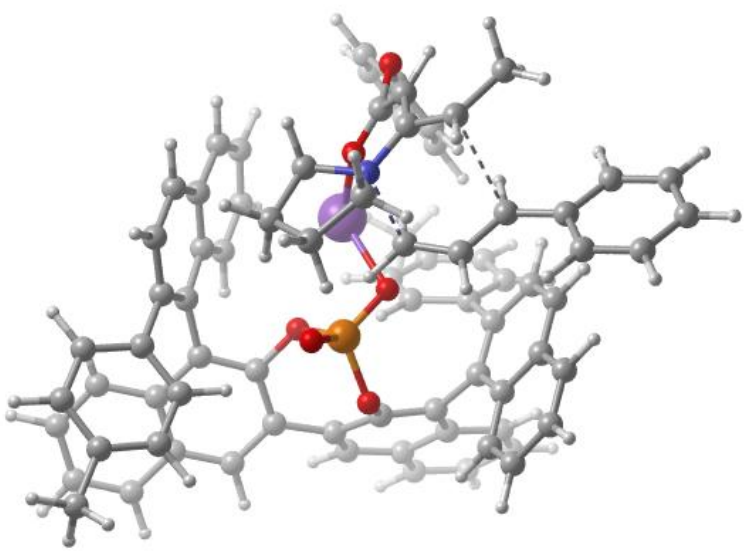

150

$\begin{array}{lccc}\mathrm{P} & -0.62576 & -0.16124 & -0.92840 \\ \mathrm{Na} & 0.22918 & 0.19198 & 2.06531 \\ \mathrm{~N} & 2.42638 & -3.95802 & 0.37939 \\ \mathrm{O} & -0.93561 & 0.88783 & -2.21854 \\ \mathrm{O} & -1.82425 & 0.45673 & 0.15588 \\ \mathrm{O} & 0.64523 & 0.23215 & -0.17205 \\ \mathrm{O} & -0.93687 & -1.56755 & -1.40872 \\ \mathrm{O} & 1.57397 & -1.58563 & 2.40604 \\ \mathrm{O} & 3.12406 & -1.45577 & 4.10749 \\ \mathrm{C} & -8.16352 & -0.01128 & -0.80813 \\ \mathrm{C} & -7.21664 & 0.82738 & -1.37312 \\ \mathrm{C} & -5.84380 & 0.74422 & -0.99527 \\ \mathrm{C} & -5.44534 & -0.24356 & -0.02187 \\ \mathrm{C} & -6.44741 & -1.07316 & 0.55813 \\ \mathrm{C} & -7.77433 & -0.96236 & 0.17268 \\ \mathrm{C} & -4.86849 & 1.62484 & -1.54223 \\ \mathrm{C} & -4.05808 & -0.35960 & 0.32037 \\ \mathrm{C} & -3.14573 & 0.52969 & -0.24783 \\ \mathrm{C} & -3.52856 & 1.55817 & -1.17267 \\ \mathrm{C} & -3.53048 & -1.41318 & 1.24151 \\ \mathrm{C} & -2.89217 & -1.01483 & 2.47001 \\ \mathrm{C} & -3.56946 & -2.77078 & 0.88636 \\ \mathrm{C} & -2.89777 & 0.34103 & 2.92664 \\ \mathrm{C} & -2.19192 & -1.99481 & 3.26519 \\ \mathrm{C} & -2.93384 & -3.73336 & 1.73022 \\ \mathrm{C} & -2.19939 & 0.72034 & 4.06650 \\ \mathrm{C} & -1.46643 & -1.57124 & 4.41976 \\ \mathrm{C} & -2.23450 & -3.36028 & 2.86180 \\ \mathrm{C} & -1.45834 & -0.24067 & 4.81243 \\ \mathrm{C} & -2.53481 & 2.58507 & -1.59185 \\ \mathrm{C} & -2.83971 & 3.93994 & -1.48734 \\ \mathrm{C} & -1.19602 & 2.22301 & -1.95480 \\ \mathrm{C} & -1.85989 & 4.94660 & -1.70939 \\ \mathrm{C} & -0.16400 & 3.16065 & -2.02813\end{array}$

\begin{tabular}{|c|c|c|c|}
\hline $\mathrm{C}$ & -2.17885 & 6.33374 & -1.61750 \\
\hline $\mathrm{C}$ & -0.49493 & 4.55608 & -1.95946 \\
\hline $\mathrm{C}$ & -1.19904 & 7.30108 & -1.77091 \\
\hline $\mathrm{C}$ & 0.48844 & 5.57574 & -2.11326 \\
\hline $\mathrm{C}$ & 0.14471 & 6.91599 & -2.02519 \\
\hline $\mathrm{C}$ & 1.26001 & 2.70623 & -1.96525 \\
\hline $\mathrm{C}$ & 1.86403 & 1.97353 & -3.04020 \\
\hline $\mathrm{C}$ & 1.98465 & 2.94511 & -0.78437 \\
\hline $\mathrm{C}$ & 1.18127 & 1.73113 & -4.26932 \\
\hline $\mathrm{C}$ & 3.19969 & 1.45165 & -2.88678 \\
\hline $\mathrm{C}$ & 3.31980 & 2.45390 & -0.67145 \\
\hline $\mathrm{C}$ & 1.77090 & 0.98759 & -5.27968 \\
\hline $\mathrm{C}$ & 3.77829 & 0.68638 & -3.94115 \\
\hline $\mathrm{C}$ & 3.90695 & 1.71565 & -1.68138 \\
\hline $\mathrm{C}$ & 3.07750 & 0.45162 & -5.11361 \\
\hline $\mathrm{C}$ & 1.41339 & 3.66290 & 0.39148 \\
\hline $\mathrm{C}$ & 0.17380 & 3.30581 & 0.96732 \\
\hline $\mathrm{C}$ & 2.13209 & 4.71713 & 0.99244 \\
\hline $\mathrm{C}$ & -0.31514 & 3.97218 & 2.09610 \\
\hline $\mathrm{C}$ & 1.63396 & 5.38651 & 2.12045 \\
\hline $\mathrm{C}$ & 0.40151 & 5.02677 & 2.69637 \\
\hline $\mathrm{C}$ & -4.25311 & -3.25100 & -0.34645 \\
\hline $\mathrm{C}$ & -3.94410 & -2.73120 & -1.62024 \\
\hline $\mathrm{C}$ & -5.25557 & -4.23666 & -0.24803 \\
\hline $\mathrm{C}$ & -4.64832 & -3.16421 & -2.74885 \\
\hline $\mathrm{C}$ & -5.96016 & -4.66031 & -1.38363 \\
\hline $\mathrm{C}$ & -5.67805 & -4.12264 & -2.65331 \\
\hline $\mathrm{C}$ & 2.67607 & -2.00045 & 2.84664 \\
\hline $\mathrm{C}$ & 3.59483 & -2.93022 & 2.34974 \\
\hline $\mathrm{C}$ & 3.61584 & -3.68341 & 1.11750 \\
\hline $\mathrm{C}$ & 4.82817 & -4.03187 & 0.53147 \\
\hline $\mathrm{C}$ & 6.17060 & -3.73378 & 1.12976 \\
\hline $\mathrm{C}$ & 2.46232 & -4.98994 & -0.69574 \\
\hline $\mathrm{C}$ & 1.16583 & -4.22318 & 1.13729 \\
\hline $\mathrm{C}$ & 0.99931 & -5.14742 & -1.13602 \\
\hline $\mathrm{C}$ & 0.15177 & -4.69437 & 0.08370 \\
\hline $\mathrm{C}$ & 4.49894 & -1.76483 & -0.99199 \\
\hline $\mathrm{C}$ & 3.26488 & -2.09684 & -1.52163 \\
\hline $\mathrm{C}$ & 2.13452 & -2.17232 & -0.68103 \\
\hline $\mathrm{C}$ & 2.71541 & -0.16759 & 4.38301 \\
\hline $\mathrm{C}$ & 2.87151 & 0.87500 & 3.44393 \\
\hline $\mathrm{C}$ & 2.16901 & 0.10283 & 5.64829 \\
\hline $\mathrm{C}$ & 2.43658 & 2.17070 & 3.76275 \\
\hline $\mathrm{C}$ & 1.76304 & 1.40910 & 5.96752 \\
\hline $\mathrm{C}$ & 1.87664 & 2.44270 & 5.02399 \\
\hline $\mathrm{C}$ & 5.76685 & -1.70098 & -1.70806 \\
\hline $\mathrm{C}$ & 5.95080 & -2.25961 & -2.99742 \\
\hline $\mathrm{C}$ & 6.86200 & -1.03313 & -1.10762 \\
\hline $\mathrm{C}$ & 7.17521 & -2.13870 & -3.66107 \\
\hline $\mathrm{C}$ & 8.08807 & -0.91086 & -1.77314 \\
\hline $\mathrm{C}$ & 8.24949 & -1.46154 & -3.05449 \\
\hline $\mathrm{C}$ & -0.15054 & 5.75785 & 3.89775 \\
\hline $\mathrm{C}$ & -6.46656 & -4.53557 & -3.87475 \\
\hline $\mathrm{H}$ & -5.18760 & 2.38153 & -2.26816 \\
\hline $\mathrm{H}$ & -9.21472 & 0.06305 & -1.10870 \\
\hline $\mathrm{H}$ & -7.50972 & 1.57527 & -2.11973 \\
\hline $\mathrm{H}$ & -6.15168 & -1.80635 & 1.31355 \\
\hline
\end{tabular}




\begin{tabular}{|c|c|c|c|}
\hline $\mathrm{H}$ & -8.52838 & -1.61453 & 0.62758 \\
\hline $\mathrm{H}$ & -3.45665 & 1.08626 & 2.35311 \\
\hline $\mathrm{H}$ & -2.96251 & -4.78465 & 1.42451 \\
\hline $\mathrm{H}$ & -2.21487 & 1.76628 & 4.39446 \\
\hline $\mathrm{H}$ & -0.90103 & -2.31878 & 4.98790 \\
\hline $\mathrm{H}$ & -1.69914 & -4.10806 & 3.45850 \\
\hline $\mathrm{H}$ & -0.88214 & 0.07355 & 5.68794 \\
\hline $\mathrm{H}$ & -3.84663 & 4.24072 & -1.17645 \\
\hline $\mathrm{H}$ & -3.21867 & 6.62019 & -1.41902 \\
\hline $\mathrm{H}$ & -1.45747 & 8.36362 & -1.69883 \\
\hline $\mathrm{H}$ & 1.52384 & 5.28077 & -2.30834 \\
\hline $\mathrm{H}$ & 0.91538 & 7.68445 & -2.15378 \\
\hline $\mathrm{H}$ & 0.17256 & 2.13324 & -4.40030 \\
\hline $\mathrm{H}$ & 3.86430 & 2.63125 & 0.26285 \\
\hline $\mathrm{H}$ & 1.22426 & 0.80764 & -6.21251 \\
\hline $\mathrm{H}$ & 4.79029 & 0.28792 & -3.80581 \\
\hline $\mathrm{H}$ & 4.92129 & 1.31831 & -1.56336 \\
\hline $\mathrm{H}$ & 3.53138 & -0.13941 & -5.91791 \\
\hline $\mathrm{H}$ & -0.41157 & 2.49810 & 0.52765 \\
\hline $\mathrm{H}$ & 3.08918 & 5.02828 & 0.55837 \\
\hline $\mathrm{H}$ & -1.27832 & 3.66232 & 2.52088 \\
\hline $\mathrm{H}$ & 2.21674 & 6.20549 & 2.56027 \\
\hline $\mathrm{H}$ & -3.12598 & -2.01438 & -1.72329 \\
\hline $\mathrm{H}$ & -5.51104 & -4.65145 & 0.73466 \\
\hline $\mathrm{H}$ & -4.38776 & -2.74828 & -3.73028 \\
\hline $\mathrm{H}$ & -6.75026 & -5.41437 & -1.27878 \\
\hline $\mathrm{H}$ & 4.51164 & -2.99800 & 2.93669 \\
\hline $\mathrm{H}$ & 4.83141 & -4.66402 & -0.35776 \\
\hline $\mathrm{H}$ & 6.41809 & -4.42017 & 1.96675 \\
\hline $\mathrm{H}$ & 6.96446 & -3.83980 & 0.37055 \\
\hline $\mathrm{H}$ & 6.22399 & -2.70756 & 1.53871 \\
\hline $\mathrm{H}$ & 3.12649 & -4.66501 & -1.50928 \\
\hline $\mathrm{H}$ & 2.86459 & -5.92631 & -0.26425 \\
\hline $\mathrm{H}$ & 1.39906 & -5.00802 & 1.88315 \\
\hline $\mathrm{H}$ & 0.86056 & -3.31316 & 1.66793 \\
\hline $\mathrm{H}$ & 0.79886 & -6.19022 & -1.43288 \\
\hline $\mathrm{H}$ & 0.78575 & -4.51060 & -2.01050 \\
\hline $\mathrm{H}$ & -0.46650 & -5.51277 & 0.48724 \\
\hline $\mathrm{H}$ & -0.52642 & -3.87300 & -0.19656 \\
\hline $\mathrm{H}$ & 4.50995 & -1.36903 & 0.03096 \\
\hline $\mathrm{H}$ & 3.17546 & -2.41077 & -2.56773 \\
\hline $\mathrm{H}$ & 2.11876 & -1.61945 & 0.25647 \\
\hline $\mathrm{H}$ & 1.14909 & -2.37477 & -1.10663 \\
\hline $\mathrm{H}$ & 3.31532 & 0.66106 & 2.46523 \\
\hline $\mathrm{H}$ & 2.05923 & -0.71985 & 6.36256 \\
\hline $\mathrm{H}$ & 2.53752 & 2.97197 & 3.02428 \\
\hline $\mathrm{H}$ & 1.33782 & 1.61375 & 6.95749 \\
\hline $\mathrm{H}$ & 1.53821 & 3.45593 & 5.26392 \\
\hline $\mathrm{H}$ & 5.12608 & -2.79381 & -3.47983 \\
\hline $\mathrm{H}$ & 6.73466 & -0.59806 & -0.10978 \\
\hline $\mathrm{H}$ & 7.29775 & -2.57529 & -4.65888 \\
\hline $\mathrm{H}$ & 8.91977 & -0.38389 & -1.29216 \\
\hline $\mathrm{H}$ & 9.20718 & -1.36803 & -3.57870 \\
\hline $\mathrm{H}$ & -0.70381 & 5.07409 & 4.56698 \\
\hline $\mathrm{H}$ & 0.65128 & 6.24024 & 4.48411 \\
\hline $\mathrm{H}$ & -0.85761 & 6.55277 & 3.58843 \\
\hline $\mathrm{H}$ & -7.20206 & -3.75409 & -4.15096 \\
\hline
\end{tabular}

$\begin{array}{llll}\mathrm{H} & -5.80804 & -4.68381 & -4.75028 \\ \mathrm{H} & -7.02567 & -5.47187 & -3.70194\end{array}$

R_DAPa_minor_1

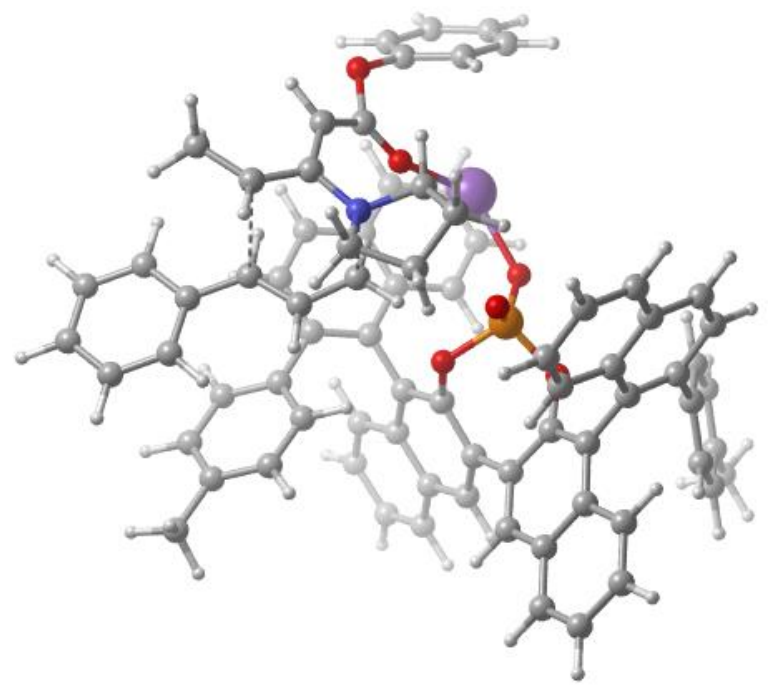

150

$\begin{array}{lccc}\mathrm{P} & 1.27776 & -0.88656 & 0.29585 \\ \mathrm{Na} & -1.04028 & -2.74385 & 1.89361 \\ \mathrm{~N} & -2.31451 & -2.07745 & -2.78532 \\ \mathrm{O} & 2.82984 & -0.32935 & 0.66306 \\ \mathrm{O} & 0.45063 & 0.63266 & 0.29354 \\ \mathrm{O} & 1.14434 & -1.39537 & -1.13022 \\ \mathrm{O} & 0.86552 & -1.70592 & 1.52487 \\ \mathrm{O} & -2.54746 & -2.86931 & 0.28945 \\ \mathrm{O} & -4.43429 & -4.17485 & 0.60457 \\ \mathrm{C} & 1.37804 & 5.06706 & 3.63729 \\ \mathrm{C} & 6.50236 & -0.04952 & 3.39187 \\ \mathrm{C} & 6.95374 & 0.43677 & 2.14919 \\ \mathrm{C} & 6.32544 & 3.35964 & -3.28044 \\ \mathrm{C} & 5.33523 & 3.68407 & -2.36760 \\ \mathrm{C} & -0.86974 & 4.83898 & 4.53117 \\ \mathrm{C} & 1.17292 & 3.93078 & 2.80065 \\ \mathrm{C} & 6.56814 & -0.17229 & 0.94806 \\ \mathrm{C} & 6.68289 & 1.99965 & -3.48653 \\ \mathrm{C} & 5.66507 & -1.18245 & 3.39001 \\ \mathrm{C} & 2.17464 & 3.46643 & 1.90716 \\ \mathrm{C} & 4.66110 & 2.67008 & -1.62397 \\ \mathrm{C} & 3.65475 & 2.98769 & -0.67143 \\ \mathrm{C} & 6.04031 & 0.98893 & -2.78691 \\ \mathrm{C} & 5.01136 & 1.28910 & -1.84629 \\ \mathrm{C} & -1.09949 & 3.72766 & 3.73369 \\ \mathrm{C} & 3.01279 & 2.00133 & 0.07448 \\ \mathrm{C} & -0.09051 & 3.24038 & 2.85147 \\ \mathrm{C} & 5.71881 & -1.29678 & 0.95008 \\ \mathrm{C} & 1.98159 & 2.36236 & 1.08057 \\ \mathrm{C} & 5.28047 & -1.79772 & 2.19224 \\ \mathrm{C} & 4.33386 & 0.26684 & -1.10305 \\ & & & \\ & & & \end{array}$




\begin{tabular}{|c|c|c|c|c|c|c|c|}
\hline $\mathrm{C}$ & 3.38461 & 0.63937 & -0.15426 & $\mathrm{H}$ & 2.34447 & 5.58348 & 3.59164 \\
\hline $\mathrm{C}$ & 0.73263 & 1.65488 & 1.17591 & $\mathrm{H}$ & 7.61797 & 1.30970 & 2.11987 \\
\hline $\mathrm{C}$ & -0.28794 & 2.08911 & 2.02198 & $\mathrm{H}$ & 6.83858 & 4.14928 & -3.84090 \\
\hline $\mathrm{C}$ & 5.26226 & -1.93054 & -0.31593 & $\mathrm{H}$ & 5.05803 & 4.73124 & -2.19544 \\
\hline $\mathrm{C}$ & 4.62597 & -1.18325 & -1.31556 & $\mathrm{H}$ & 3.13262 & 3.99692 & 1.86778 \\
\hline $\mathrm{C}$ & -0.66515 & -0.16436 & 3.81952 & $\mathrm{H}$ & 3.36834 & 4.03668 & -0.53576 \\
\hline $\mathrm{C}$ & -2.68783 & 5.26156 & -1.31099 & $\mathrm{H}$ & -1.65789 & 5.19758 & 5.20296 \\
\hline $\mathrm{C}$ & 5.44440 & -3.33466 & -0.49706 & $\mathrm{H}$ & 7.47281 & 1.74740 & -4.20302 \\
\hline $\mathrm{C}$ & -1.65683 & 4.30177 & -1.37264 & $\mathrm{H}$ & 6.93337 & 0.22578 & -0.00376 \\
\hline $\mathrm{C}$ & -3.70157 & 5.07195 & -0.35598 & $\mathrm{H}$ & 5.29311 & -1.57999 & 4.34281 \\
\hline $\mathrm{C}$ & -0.82179 & -1.23064 & 4.69618 & $\mathrm{H}$ & 6.31567 & -0.05759 & -2.94884 \\
\hline $\mathrm{C}$ & -1.60003 & 1.37020 & 2.07082 & $\mathrm{H}$ & -2.06270 & 3.20985 & 3.77230 \\
\hline $\mathrm{C}$ & -1.63916 & 3.19372 & -0.51915 & $\mathrm{H}$ & 4.59324 & -2.65055 & 2.21260 \\
\hline $\mathrm{C}$ & -3.69095 & 3.96162 & 0.49855 & $\mathrm{H}$ & 0.30147 & 0.33782 & 3.73511 \\
\hline $\mathrm{C}$ & 4.16910 & -1.82429 & -2.51767 & $\mathrm{H}$ & 0.02348 & -1.55144 & 5.31520 \\
\hline $\mathrm{C}$ & -2.65974 & 3.00160 & 0.43641 & $\mathrm{H}$ & 5.94671 & -3.90228 & 0.29423 \\
\hline $\mathrm{C}$ & -1.74415 & 0.27306 & 2.98950 & $\mathrm{H}$ & -0.85550 & 4.41616 & -2.11411 \\
\hline $\mathrm{C}$ & 3.48512 & -1.11422 & -3.54923 & $\mathrm{H}$ & -4.51910 & 5.79914 & -0.28293 \\
\hline $\mathrm{C}$ & -2.70617 & 1.81527 & 1.33254 & $\mathrm{H}$ & 3.28390 & -0.04877 & -3.41439 \\
\hline C & 5.02477 & -3.97080 & -1.65063 & $\mathrm{H}$ & -0.84983 & 2.44859 & -0.62176 \\
\hline $\mathrm{C}$ & -2.06403 & -1.92314 & 4.77810 & $\mathrm{H}$ & -4.49178 & 3.84617 & 1.23742 \\
\hline C & 4.38324 & -3.23938 & -2.69097 & $\mathrm{H}$ & -2.17481 & -2.77726 & 5.45567 \\
\hline $\mathrm{C}$ & -3.01293 & -0.40910 & 3.10075 & $\mathrm{H}$ & 5.18574 & -5.04806 & -1.77973 \\
\hline $\mathrm{C}$ & 3.07070 & -1.75502 & -4.70536 & $\mathrm{H}$ & 2.55487 & -1.18493 & -5.48635 \\
\hline $\mathrm{C}$ & -3.13643 & -1.51542 & 3.99401 & $\mathrm{H}$ & -4.80764 & 1.48837 & 0.88301 \\
\hline $\mathrm{C}$ & -3.95781 & 1.13504 & 1.47414 & $\mathrm{H}$ & -4.09327 & -2.04459 & 4.04648 \\
\hline $\mathrm{C}$ & 3.93492 & -3.87232 & -3.88816 & $\mathrm{H}$ & 4.10490 & -4.94949 & -4.00754 \\
\hline $\mathrm{C}$ & -4.10654 & 0.04679 & 2.31021 & $\mathrm{H}$ & -5.06750 & -0.47623 & 2.37862 \\
\hline $\mathrm{C}$ & 3.29663 & -3.14674 & -4.88174 & $\mathrm{H}$ & 2.96010 & -3.64457 & -5.79876 \\
\hline $\mathrm{C}$ & 0.37898 & 5.51541 & 4.48593 & $\mathrm{H}$ & -5.17699 & -3.55038 & -1.59812 \\
\hline $\mathrm{C}$ & -3.60465 & -3.32142 & -0.21959 & $\mathrm{H}$ & -4.24599 & -1.08472 & -4.26385 \\
\hline $\mathrm{C}$ & -4.18572 & -3.11160 & -1.46880 & $\mathrm{H}$ & -5.52021 & -4.08037 & 2.95137 \\
\hline $\mathrm{C}$ & -3.71999 & -2.28744 & -2.56414 & $\mathrm{H}$ & -2.10960 & -5.52685 & 0.73336 \\
\hline $\mathrm{C}$ & -3.86037 & -4.71669 & 1.73013 & $\mathrm{H}$ & -6.56103 & -2.53304 & -3.64074 \\
\hline $\mathrm{C}$ & -4.60968 & -1.60417 & -3.37515 & $\mathrm{H}$ & -6.56435 & -0.75125 & -3.66663 \\
\hline $\mathrm{C}$ & -4.56195 & -4.61027 & 2.94372 & $\mathrm{H}$ & -6.37641 & -1.63339 & -2.11886 \\
\hline $\mathrm{C}$ & -2.63124 & -5.41077 & 1.68874 & $\mathrm{H}$ & -2.15091 & -0.54317 & -4.27168 \\
\hline $\mathrm{C}$ & -6.09753 & -1.63074 & -3.18873 & $\mathrm{H}$ & -2.43422 & -2.19608 & -4.90345 \\
\hline $\mathrm{C}$ & -1.88962 & -1.60403 & -4.14512 & $\mathrm{H}$ & -1.95049 & -4.10386 & -2.26000 \\
\hline $\mathrm{C}$ & -1.35986 & -3.18272 & -2.38494 & $\mathrm{H}$ & -0.91293 & -2.91825 & -1.42015 \\
\hline $\mathrm{C}$ & -4.02955 & -5.17161 & 4.11417 & $\mathrm{H}$ & -4.58381 & -5.08098 & 5.05578 \\
\hline $\mathrm{C}$ & -2.09885 & -5.95317 & 2.87013 & $\mathrm{H}$ & -1.14369 & -6.49118 & 2.83220 \\
\hline $\mathrm{C}$ & -0.39446 & -1.89912 & -4.19602 & $\mathrm{H}$ & -0.02506 & -1.91961 & -5.23416 \\
\hline $\mathrm{C}$ & -0.30107 & -3.26185 & -3.49495 & $\mathrm{H}$ & 0.18638 & -1.14661 & -3.63843 \\
\hline $\mathrm{C}$ & -2.78976 & -5.83040 & 4.08907 & $\mathrm{H}$ & -0.54332 & -4.07249 & -4.20684 \\
\hline $\mathrm{C}$ & -1.85938 & -0.50003 & -1.59178 & $\mathrm{H}$ & 0.70113 & -3.44396 & -3.07873 \\
\hline C & -2.63832 & 0.58122 & -2.07471 & $\mathrm{H}$ & -2.37080 & -6.25811 & 5.00649 \\
\hline $\mathrm{C}$ & -3.98717 & 0.64484 & -1.79986 & $\mathrm{H}$ & -2.19782 & -1.03176 & -0.69901 \\
\hline $\mathrm{C}$ & -4.91159 & 1.69760 & -2.20018 & $\mathrm{H}$ & -0.77670 & -0.50769 & -1.73996 \\
\hline $\mathrm{C}$ & -4.52953 & 2.78310 & -3.02549 & $\mathrm{H}$ & -2.17568 & 1.31069 & -2.74727 \\
\hline C & -6.23633 & 1.67209 & -1.69902 & $\mathrm{H}$ & -4.38453 & -0.09569 & -1.09711 \\
\hline $\mathrm{C}$ & -5.43269 & 3.80619 & -3.32265 & $\mathrm{H}$ & -3.50868 & 2.83912 & -3.41283 \\
\hline $\mathrm{C}$ & -7.14203 & 2.69634 & -1.99887 & $\mathrm{H}$ & -6.54456 & 0.83802 & -1.05832 \\
\hline $\mathrm{C}$ & -6.74184 & 3.77061 & -2.81005 & $\mathrm{H}$ & -5.11295 & 4.64711 & -3.94796 \\
\hline $\mathrm{C}$ & 6.87016 & 0.64541 & 4.68308 & $\mathrm{H}$ & -8.16071 & 2.66028 & -1.59640 \\
\hline $\mathrm{C}$ & -2.70172 & 6.44308 & -2.25245 & $\mathrm{H}$ & -7.44562 & 4.57802 & -3.04220 \\
\hline $\mathrm{H}$ & 0.54782 & 6.39161 & 5.12205 & $\mathrm{H}$ & 6.95321 & -0.07016 & 5.52093 \\
\hline
\end{tabular}




$\begin{array}{cccc}\mathrm{H} & 7.82988 & 1.18511 & 4.59492 \\ \mathrm{H} & 6.09821 & 1.38970 & 4.96405 \\ \mathrm{H} & -1.81997 & 7.09333 & -2.09496 \\ \mathrm{H} & -2.67278 & 6.11335 & -3.30890 \\ \mathrm{H} & -3.60629 & 7.05970 & -2.11428\end{array}$

R_DAPa_minor_2

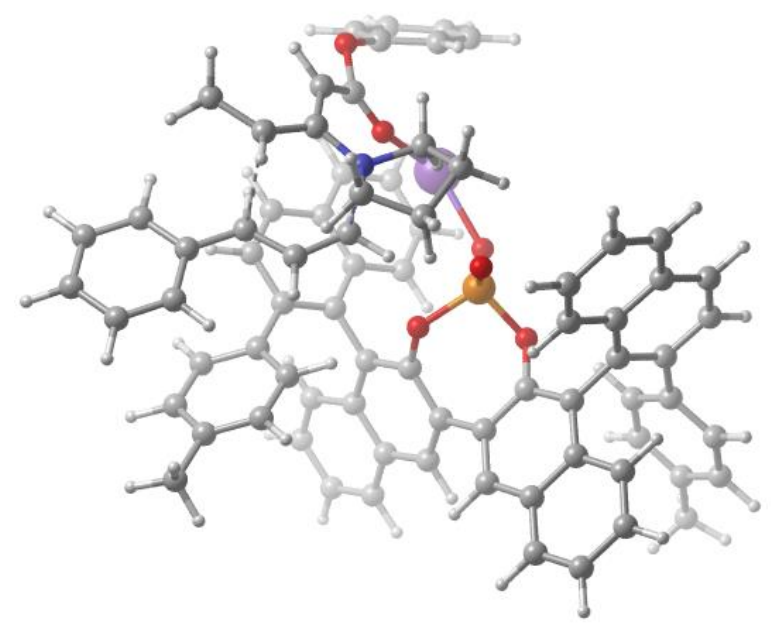

\begin{tabular}{|c|c|c|c|}
\hline C & 3.71748 & 2.72404 & -0.88032 \\
\hline $\mathrm{C}$ & 3.32060 & 0.42556 & -0.22767 \\
\hline $\mathrm{C}$ & 4.77346 & 2.31191 & -1.73681 \\
\hline $\mathrm{C}$ & 4.35431 & -0.02970 & -1.03944 \\
\hline C & 5.51993 & 3.24697 & -2.51322 \\
\hline $\mathrm{C}$ & 5.11042 & 0.91271 & -1.81045 \\
\hline $\mathrm{C}$ & 6.56231 & 2.82671 & -3.32306 \\
\hline $\mathrm{C}$ & 6.18937 & 0.51208 & -2.65196 \\
\hline $\mathrm{C}$ & 6.89861 & 1.44753 & -3.39033 \\
\hline C & 4.71568 & -1.48093 & -1.07806 \\
\hline $\mathrm{C}$ & 4.16983 & -2.31783 & -2.10762 \\
\hline C & 5.65923 & -1.99128 & -0.17928 \\
\hline $\mathrm{C}$ & 3.28089 & -1.81125 & -3.10152 \\
\hline C & 4.56199 & -3.70363 & -2.17671 \\
\hline $\mathrm{C}$ & 6.03885 & -3.36484 & -0.26507 \\
\hline C & 2.80706 & -2.62903 & -4.11426 \\
\hline $\mathrm{C}$ & 4.02924 & -4.52686 & -3.21247 \\
\hline C & 5.49638 & -4.20151 & -1.22379 \\
\hline $\mathrm{C}$ & 3.17355 & -4.00099 & -4.16784 \\
\hline C & 6.33339 & -1.11553 & 0.82311 \\
\hline $\mathrm{C}$ & 5.62071 & -0.48106 & 1.86125 \\
\hline C & 7.72276 & -0.90043 & 0.73899 \\
\hline $\mathrm{C}$ & 6.27994 & 0.35666 & 2.76900 \\
\hline $\mathrm{C}$ & 8.37659 & -0.05647 & 1.64963 \\
\hline $\mathrm{C}$ & 7.66690 & 0.59230 & 2.67713 \\
\hline $\mathrm{C}$ & -2.61819 & 3.12778 & 0.17717 \\
\hline $\mathrm{C}$ & -1.61785 & 3.14253 & -0.81842 \\
\hline $\mathrm{C}$ & -3.56206 & 4.17490 & 0.17988 \\
\hline $\mathrm{C}$ & -1.57220 & 4.16675 & -1.77012 \\
\hline $\mathrm{C}$ & -3.50839 & 5.20121 & -0.77315 \\
\hline $\mathrm{C}$ & -2.51624 & 5.21438 & -1.76930 \\
\hline $\mathrm{C}$ & -3.91375 & -3.29371 & 0.07511 \\
\hline C & -4.52475 & -3.14259 & -1.16930 \\
\hline $\mathrm{C}$ & -4.07185 & -2.39674 & -2.32396 \\
\hline C & -4.16144 & -4.50080 & 2.14614 \\
\hline $\mathrm{C}$ & -4.96752 & -1.74360 & -3.15350 \\
\hline C & -4.85922 & -4.24455 & 3.33878 \\
\hline $\mathrm{C}$ & -2.94180 & -5.21092 & 2.18409 \\
\hline $\mathrm{C}$ & -6.44952 & -1.72272 & -2.92460 \\
\hline $\mathrm{C}$ & -4.33193 & -4.67322 & 4.56646 \\
\hline $\mathrm{C}$ & -2.41265 & -5.61704 & 3.42029 \\
\hline $\mathrm{C}$ & -3.10011 & -5.34539 & 4.61677 \\
\hline $\mathrm{C}$ & -2.14855 & -0.60698 & -1.49519 \\
\hline $\mathrm{C}$ & -2.27131 & -1.84388 & -3.98701 \\
\hline $\mathrm{C}$ & -1.72949 & -3.33828 & -2.15941 \\
\hline $\mathrm{C}$ & -2.89595 & 0.46913 & -2.03446 \\
\hline $\mathrm{C}$ & -0.78316 & -2.16832 & -4.05758 \\
\hline $\mathrm{C}$ & -0.69312 & -3.49160 & -3.28341 \\
\hline $\mathrm{C}$ & -4.23944 & 0.59954 & -1.75598 \\
\hline $\mathrm{C}$ & -5.12456 & 1.65850 & -2.22478 \\
\hline $\mathrm{C}$ & -6.45986 & 1.69643 & -1.75333 \\
\hline $\mathrm{C}$ & -4.69158 & 2.69070 & -3.09234 \\
\hline $\mathrm{C}$ & -7.33017 & 2.72580 & -2.13159 \\
\hline $\mathrm{C}$ & -5.55841 & 3.72025 & -3.46562 \\
\hline $\mathrm{C}$ & -6.88144 & 3.74400 & -2.98863 \\
\hline $\mathrm{C}$ & 8.35805 & 1.52666 & 3.64427 \\
\hline $\mathrm{C}$ & -2.46488 & 6.29917 & -2.81955 \\
\hline
\end{tabular}




\begin{tabular}{|c|c|c|c|}
\hline $\mathrm{H}$ & 3.23157 & 3.90413 & 1.55581 \\
\hline $\mathrm{H}$ & 0.81268 & 6.62755 & 4.67390 \\
\hline $\mathrm{H}$ & 2.54656 & 5.63299 & 3.18175 \\
\hline $\mathrm{H}$ & -1.98747 & 3.53147 & 3.52022 \\
\hline $\mathrm{H}$ & -1.45701 & 5.56923 & 4.83420 \\
\hline $\mathrm{H}$ & 0.21130 & 0.56617 & 3.66051 \\
\hline $\mathrm{H}$ & -4.85785 & 1.80952 & 0.77829 \\
\hline $\mathrm{H}$ & -0.15803 & -1.17854 & 5.38389 \\
\hline $\mathrm{H}$ & -4.30904 & -1.52805 & 4.18084 \\
\hline $\mathrm{H}$ & -5.21363 & -0.02154 & 2.41735 \\
\hline $\mathrm{H}$ & -2.41952 & -2.26511 & 5.62575 \\
\hline $\mathrm{H}$ & 3.46552 & 3.78918 & -0.82839 \\
\hline $\mathrm{H}$ & 5.25571 & 4.30965 & -2.45090 \\
\hline $\mathrm{H}$ & 7.13129 & 3.55553 & -3.91144 \\
\hline $\mathrm{H}$ & 6.44926 & -0.54946 & -2.70557 \\
\hline $\mathrm{H}$ & 7.72552 & 1.12092 & -4.03108 \\
\hline $\mathrm{H}$ & 2.97088 & -0.76474 & -3.04806 \\
\hline $\mathrm{H}$ & 6.76989 & -3.74897 & 0.45534 \\
\hline $\mathrm{H}$ & 2.13596 & -2.21722 & -4.87543 \\
\hline $\mathrm{H}$ & 4.32438 & -5.58274 & -3.24908 \\
\hline $\mathrm{H}$ & 5.79202 & -5.25676 & -1.27036 \\
\hline $\mathrm{H}$ & 2.78131 & -4.64039 & -4.96759 \\
\hline $\mathrm{H}$ & 4.54660 & -0.65794 & 1.95944 \\
\hline $\mathrm{H}$ & 8.29243 & -1.38268 & -0.06417 \\
\hline $\mathrm{H}$ & 5.70262 & 0.83842 & 3.56867 \\
\hline $\mathrm{H}$ & 9.45771 & 0.10545 & 1.55481 \\
\hline $\mathrm{H}$ & -0.90025 & 2.32290 & -0.87173 \\
\hline $\mathrm{H}$ & -4.34218 & 4.19439 & 0.94916 \\
\hline $\mathrm{H}$ & -0.79047 & 4.14560 & -2.54052 \\
\hline $\mathrm{H}$ & -4.25694 & 6.00214 & -0.74441 \\
\hline $\mathrm{H}$ & -5.52800 & -3.56633 & -1.24640 \\
\hline $\mathrm{H}$ & -4.61727 & -1.28904 & -4.08204 \\
\hline $\mathrm{H}$ & -5.80959 & -3.70332 & 3.28484 \\
\hline $\mathrm{H}$ & -2.42516 & -5.44506 & 1.24774 \\
\hline $\mathrm{H}$ & -6.69633 & -1.63873 & -1.84994 \\
\hline $\mathrm{H}$ & -6.91266 & -0.87182 & -3.45476 \\
\hline $\mathrm{H}$ & -6.94440 & -2.64624 & -3.29270 \\
\hline $\mathrm{H}$ & -4.88300 & -4.46714 & 5.49157 \\
\hline $\mathrm{H}$ & -1.46416 & -6.16740 & 3.44510 \\
\hline $\mathrm{H}$ & -2.68445 & -5.66932 & 5.57723 \\
\hline $\mathrm{H}$ & -2.49559 & -1.08070 & -0.57367 \\
\hline $\mathrm{H}$ & -1.06817 & -0.65926 & -1.65218 \\
\hline $\mathrm{H}$ & -2.51755 & -0.78698 & -4.16540 \\
\hline $\mathrm{H}$ & -2.33519 & -4.24093 & -1.98202 \\
\hline $\mathrm{H}$ & -1.25971 & -3.04034 & -1.21545 \\
\hline $\mathrm{H}$ & -2.41615 & 1.13715 & -2.75646 \\
\hline $\mathrm{H}$ & -0.44409 & -2.25193 & -5.10357 \\
\hline $\mathrm{H}$ & -0.17867 & -1.39361 & -3.55766 \\
\hline $\mathrm{H}$ & -0.95880 & -4.33715 & -3.94442 \\
\hline $\mathrm{H}$ & 0.31397 & -3.66283 & -2.87429 \\
\hline $\mathrm{H}$ & -4.66266 & -0.07997 & -1.00835 \\
\hline $\mathrm{H}$ & -6.80481 & 0.90703 & -1.07569 \\
\hline $\mathrm{H}$ & -3.65856 & 2.70341 & -3.44973 \\
\hline $\mathrm{H}$ & -8.35888 & 2.73801 & -1.75393 \\
\hline $\mathrm{H}$ & -5.19905 & 4.51995 & -4.12273 \\
\hline $\mathrm{H}$ & -7.55742 & 4.55549 & -3.28127 \\
\hline $\mathrm{H}$ & -2.84406 & -2.46692 & -4.69833 \\
\hline
\end{tabular}

$\begin{array}{cccc}\mathrm{H} & 9.45671 & 1.45790 & 3.56082 \\ \mathrm{H} & 8.07317 & 2.57988 & 3.45202 \\ \mathrm{H} & 8.07928 & 1.30263 & 4.69094 \\ \mathrm{H} & -2.58387 & 5.87609 & -3.83610 \\ \mathrm{H} & -3.26168 & 7.04784 & -2.67010 \\ \mathrm{H} & -1.49314 & 6.82820 & -2.80456\end{array}$

R_DAPa_minor_3

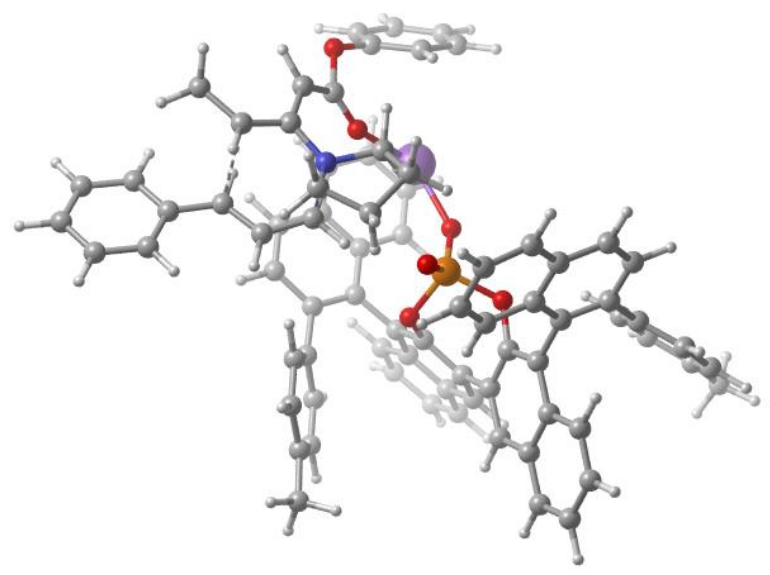

150

$\begin{array}{lccc}\mathrm{P} & 0.78967 & -0.29952 & 0.46368 \\ \mathrm{Na} & -1.65828 & 0.44964 & 2.89680 \\ \mathrm{~N} & -3.27988 & -2.94228 & -0.13147 \\ \mathrm{O} & 0.66392 & 0.94226 & -0.70566 \\ \mathrm{O} & 2.46791 & -0.24728 & 0.73429 \\ \mathrm{O} & 0.34464 & -1.56280 & -0.25321 \\ \mathrm{O} & 0.20025 & 0.16493 & 1.80131 \\ \mathrm{O} & -3.34681 & -0.77001 & 2.18869 \\ \mathrm{O} & -5.14163 & -1.17675 & 3.59078 \\ \mathrm{C} & 6.37662 & 0.21391 & -3.28650 \\ \mathrm{C} & 0.30104 & 2.33653 & -5.10578 \\ \mathrm{C} & 0.40082 & 3.52847 & -4.36119 \\ \mathrm{C} & 2.33244 & 7.10154 & 0.14287 \\ \mathrm{C} & 3.10207 & 5.97253 & -0.08729 \\ \mathrm{C} & 7.06284 & -2.07961 & -2.86658 \\ \mathrm{C} & 5.36049 & 0.10463 & -2.29174 \\ \mathrm{C} & -0.27242 & 3.68337 & -3.14319 \\ \mathrm{C} & 0.91962 & 6.98802 & 0.24158 \\ \mathrm{C} & -0.52304 & 1.31270 & -4.59930 \\ \mathrm{C} & 4.50268 & 1.19224 & -1.97379 \\ \mathrm{C} & 2.49901 & 4.68722 & -0.22362 \\ \mathrm{C} & 3.27039 & 3.52469 & -0.49652 \\ \mathrm{C} & 0.30227 & 5.75126 & 0.12146 \\ \mathrm{C} & 1.06785 & 4.56814 & -0.09962 \\ \mathrm{C} & 6.08305 & -2.21943 & -1.89485 \\ \mathrm{C} & 2.68617 & 2.26968 & -0.64350 \\ \mathrm{C} & 5.20607 & -1.13990 & -1.58170 \\ \mathrm{C} & -1.07551 & 2.64609 & -2.62742 \\ \mathrm{C} & 3.51206 & 1.08894 & -1.00082 \\ \mathrm{C} & -1.20153 & 1.46462 & -3.38412 \\ \mathrm{C} & 0.46311 & 3.27474 & -0.22740\end{array}$




\begin{tabular}{|c|c|c|c|c|c|c|c|}
\hline $\mathrm{C}$ & 1.26559 & 2.16308 & -0.48590 & $\mathrm{H}$ & 6.48684 & 1.16520 & -3.82127 \\
\hline $\mathrm{C}$ & 3.35817 & -0.16504 & -0.32206 & $\mathrm{H}$ & 1.02054 & 4.35088 & -4.73968 \\
\hline $\mathrm{C}$ & 4.18332 & -1.25339 & -0.58380 & $\mathrm{H}$ & 2.80910 & 8.08319 & 0.24215 \\
\hline $\mathrm{C}$ & -1.74466 & 2.75870 & -1.30351 & $\mathrm{H}$ & 4.19230 & 6.05088 & -0.17686 \\
\hline $\mathrm{C}$ & -1.01861 & 3.09401 & -0.15110 & $\mathrm{H}$ & 4.62061 & 2.13533 & -2.51953 \\
\hline $\mathrm{C}$ & 2.53392 & -3.48153 & -1.53030 & $\mathrm{H}$ & 4.35842 & 3.62126 & -0.58314 \\
\hline $\mathrm{C}$ & 7.58602 & 0.36991 & 2.70354 & $\mathrm{H}$ & 7.72696 & -2.92117 & -3.09420 \\
\hline $\mathrm{C}$ & -3.13887 & 2.46794 & -1.19750 & $\mathrm{H}$ & 0.31223 & 7.88421 & 0.41179 \\
\hline $\mathrm{C}$ & 6.19678 & 0.61080 & 2.69196 & $\mathrm{H}$ & -0.17408 & 4.62003 & -2.58666 \\
\hline $\mathrm{C}$ & 8.03607 & -0.89932 & 2.29556 & $\mathrm{H}$ & -0.61836 & 0.37180 & -5.15593 \\
\hline $\mathrm{C}$ & 1.81726 & -4.55459 & -2.03343 & $\mathrm{H}$ & -0.78669 & 5.67014 & 0.19586 \\
\hline $\mathrm{C}$ & 4.05599 & -2.51867 & 0.20474 & $\mathrm{H}$ & 5.96911 & -3.16404 & -1.35443 \\
\hline $\mathrm{C}$ & 5.28947 & -0.37052 & 2.27571 & $\mathrm{H}$ & -1.80462 & 0.64253 & -2.98978 \\
\hline $\mathrm{C}$ & 7.13175 & -1.88882 & 1.88043 & $\mathrm{H}$ & 2.55559 & -2.53075 & -2.06851 \\
\hline $\mathrm{C}$ & -1.68438 & 3.17786 & 1.11924 & $\mathrm{H}$ & 1.28236 & -4.45241 & -2.98439 \\
\hline $\mathrm{C}$ & 5.74695 & -1.63583 & 1.85281 & $\mathrm{H}$ & -3.68549 & 2.19245 & -2.10481 \\
\hline $\mathrm{C}$ & 3.25011 & -3.59171 & -0.30161 & $\mathrm{H}$ & 5.81625 & 1.58849 & 3.01486 \\
\hline $\mathrm{C}$ & -0.98232 & 3.49209 & 2.32646 & $\mathrm{H}$ & 9.11169 & -1.11593 & 2.29334 \\
\hline $\mathrm{C}$ & 4.81195 & -2.69300 & 1.36945 & $\mathrm{H}$ & 0.09239 & 3.68527 & 2.27641 \\
\hline $\mathrm{C}$ & -3.79793 & 2.52812 & 0.01583 & $\mathrm{H}$ & 4.21622 & -0.16266 & 2.28251 \\
\hline $\mathrm{C}$ & 1.75481 & -5.78076 & -1.31770 & $\mathrm{H}$ & 7.50501 & -2.86433 & 1.54696 \\
\hline C & -3.09876 & 2.89008 & 1.20355 & $\mathrm{H}$ & 1.17848 & -6.62135 & -1.72239 \\
\hline $\mathrm{C}$ & 3.19696 & -4.83989 & 0.41856 & $\mathrm{H}$ & -4.86760 & 2.29639 & 0.07916 \\
\hline $\mathrm{C}$ & -1.64262 & 3.53541 & 3.54883 & $\mathrm{H}$ & -1.08540 & 3.78130 & 4.46039 \\
\hline $\mathrm{C}$ & 2.42319 & -5.91497 & -0.11087 & $\mathrm{H}$ & 5.33250 & -4.04523 & 2.99018 \\
\hline $\mathrm{C}$ & 4.74239 & -3.93303 & 2.07352 & $\mathrm{H}$ & 2.38254 & -6.85884 & 0.44677 \\
\hline $\mathrm{C}$ & -3.74450 & 2.93325 & 2.47701 & $\mathrm{H}$ & -4.81099 & 2.68666 & 2.53382 \\
\hline $\mathrm{C}$ & 3.94921 & -4.97227 & 1.62097 & $\mathrm{H}$ & 3.90425 & -5.91809 & 2.17465 \\
\hline $\mathrm{C}$ & -3.03710 & 3.25200 & 3.62823 & $\mathrm{H}$ & -3.54271 & 3.26462 & 4.59866 \\
\hline $\mathrm{C}$ & 7.21267 & -0.85396 & -3.56987 & $\mathrm{H}$ & -6.01378 & -2.70862 & 1.94299 \\
\hline $\mathrm{C}$ & -4.39965 & -1.42951 & 2.37832 & $\mathrm{H}$ & -5.33706 & -3.63448 & -1.62060 \\
\hline $\mathrm{C}$ & -5.04251 & -2.37082 & 1.57639 & $\mathrm{H}$ & -6.03213 & 0.90812 & 4.84664 \\
\hline $\mathrm{C}$ & -4.66788 & -2.85117 & 0.26581 & $\mathrm{H}$ & -2.79117 & -1.93924 & 4.64892 \\
\hline $\mathrm{C}$ & -4.46964 & -0.56185 & 4.62232 & $\mathrm{H}$ & -7.52594 & -3.85502 & 0.12482 \\
\hline $\mathrm{C}$ & -5.62795 & -3.16096 & -0.68118 & $\mathrm{H}$ & -7.64226 & -2.95827 & -1.41172 \\
\hline $\mathrm{C}$ & -5.07378 & 0.54875 & 5.23548 & $\mathrm{H}$ & -7.32973 & -2.08877 & 0.12394 \\
\hline $\mathrm{C}$ & -3.23764 & -1.05091 & 5.10804 & $\mathrm{H}$ & -3.27565 & -3.43887 & -2.21994 \\
\hline $\mathrm{C}$ & -7.10123 & -3.00555 & -0.45045 & $\mathrm{H}$ & -3.54430 & -4.81297 & -1.10265 \\
\hline $\mathrm{C}$ & -2.96643 & -3.88574 & -1.26402 & $\mathrm{H}$ & -2.80906 & -3.57641 & 1.84307 \\
\hline $\mathrm{C}$ & -2.25745 & -3.22517 & 0.95746 & $\mathrm{H}$ & -1.75478 & -2.28420 & 1.20623 \\
\hline $\mathrm{C}$ & -4.44490 & 1.17637 & 6.32159 & $\mathrm{H}$ & -4.92555 & 2.03933 & 6.79746 \\
\hline $\mathrm{C}$ & -2.60621 & -0.40080 & 6.18111 & $\mathrm{H}$ & -1.64901 & -0.78430 & 6.55474 \\
\hline $\mathrm{C}$ & -1.47245 & -4.14811 & -1.13954 & $\mathrm{H}$ & -1.18286 & -5.06342 & -1.67962 \\
\hline $\mathrm{C}$ & -1.27838 & -4.25846 & 0.37869 & $\mathrm{H}$ & -0.87623 & -3.31050 & -1.53414 \\
\hline $\mathrm{C}$ & -3.20324 & 0.71739 & 6.79031 & $\mathrm{H}$ & -1.53770 & -5.27608 & 0.72438 \\
\hline $\mathrm{C}$ & -2.83016 & -1.16407 & -0.85504 & $\mathrm{H}$ & -0.24320 & -4.03362 & 0.67504 \\
\hline $\mathrm{C}$ & -3.68601 & -0.98874 & -1.98269 & $\mathrm{H}$ & -2.70940 & 1.21685 & 7.63094 \\
\hline $\mathrm{C}$ & -5.02144 & -0.70099 & -1.80372 & $\mathrm{H}$ & -3.08684 & -0.64861 & 0.07538 \\
\hline $\mathrm{C}$ & -6.05606 & -0.62313 & -2.83077 & $\mathrm{H}$ & -1.75280 & -1.30703 & -1.00042 \\
\hline $\mathrm{C}$ & -5.84704 & -1.03903 & -4.16904 & $\mathrm{H}$ & -3.30771 & -1.24344 & -2.97939 \\
\hline $\mathrm{C}$ & -7.33303 & -0.12269 & -2.47886 & $\mathrm{H}$ & -5.33543 & -0.40458 & -0.79494 \\
\hline $\mathrm{C}$ & -6.87286 & -0.94853 & -5.11442 & $\mathrm{H}$ & -4.87404 & -1.44160 & -4.46917 \\
\hline $\mathrm{C}$ & -8.36101 & -0.03323 & -3.42563 & $\mathrm{H}$ & -7.51006 & 0.19770 & -1.44570 \\
\hline $\mathrm{C}$ & -8.13499 & -0.44545 & -4.74831 & $\mathrm{H}$ & -6.69228 & -1.27510 & -6.14488 \\
\hline $\mathrm{C}$ & 8.55322 & 1.44990 & 3.13275 & $\mathrm{H}$ & -9.34152 & 0.35776 & -3.13141 \\
\hline $\mathrm{C}$ & 1.08565 & 2.14464 & -6.38302 & $\mathrm{H}$ & -8.93709 & -0.37864 & -5.49191 \\
\hline $\mathrm{H}$ & 7.99149 & -0.75615 & -4.33469 & $\mathrm{H}$ & 8.30200 & 1.83943 & 4.13765 \\
\hline
\end{tabular}




$\begin{array}{llll} & & & \\ \mathrm{H} & 9.59160 & 1.07658 & 3.16257 \\ \mathrm{H} & 8.52451 & 2.31058 & 2.43646 \\ \mathrm{H} & 1.28661 & 3.10705 & -6.88625 \\ \mathrm{H} & 2.06560 & 1.67129 & -6.17302 \\ \mathrm{H} & 0.55181 & 1.48829 & -7.09353\end{array}$

R_DAPa_minor_4

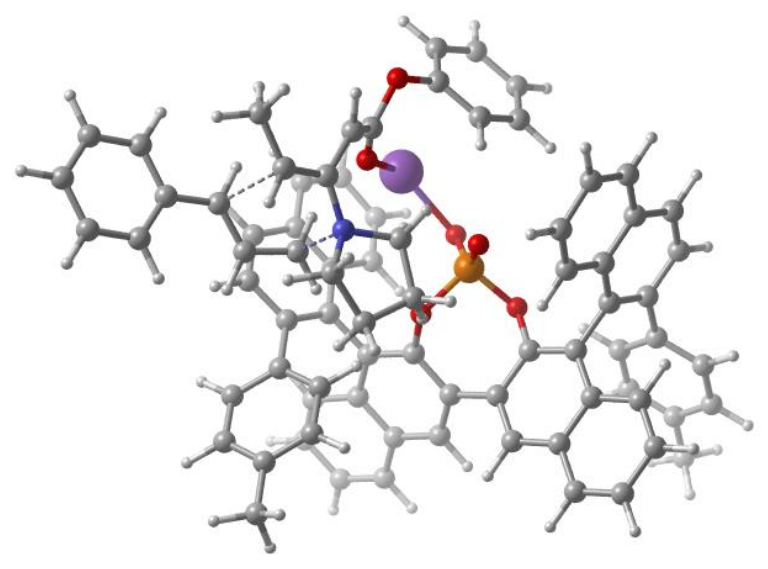

\begin{tabular}{|c|c|c|c|}
\hline$C$ & 4.01279 & -0.45075 & -1.29894 \\
\hline $\mathrm{C}$ & 4.77593 & 2.02199 & -4.04888 \\
\hline $\mathrm{C}$ & 4.54608 & 0.10543 & -2.50624 \\
\hline $\mathrm{C}$ & 5.57027 & 1.25752 & -4.88819 \\
\hline $\mathrm{C}$ & 5.36729 & -0.65448 & -3.38921 \\
\hline $\mathrm{C}$ & 5.86740 & -0.09153 & -4.55385 \\
\hline $\mathrm{C}$ & 4.34672 & -1.85046 & -0.88858 \\
\hline $\mathrm{C}$ & 3.51923 & -2.94093 & -1.31690 \\
\hline $\mathrm{C}$ & 5.48661 & -2.08975 & -0.11337 \\
\hline $\mathrm{C}$ & 2.40609 & -2.75280 & -2.18805 \\
\hline $\mathrm{C}$ & 3.82463 & -4.27711 & -0.86884 \\
\hline $\mathrm{C}$ & 5.79380 & -3.42275 & 0.29274 \\
\hline $\mathrm{C}$ & 1.61748 & -3.82332 & -2.57565 \\
\hline $\mathrm{C}$ & 2.97534 & -5.35341 & -1.26253 \\
\hline $\mathrm{C}$ & 4.97452 & -4.48315 & -0.05316 \\
\hline $\mathrm{C}$ & 1.89099 & -5.13294 & -2.09739 \\
\hline $\mathrm{C}$ & 6.41601 & -0.98072 & 0.25281 \\
\hline $\mathrm{C}$ & 6.03132 & 0.05918 & 1.11995 \\
\hline $\mathrm{C}$ & 7.71484 & -0.94904 & -0.29538 \\
\hline $\mathrm{C}$ & 6.91569 & 1.10834 & 1.41090 \\
\hline $\mathrm{C}$ & 8.59217 & 0.10308 & -0.00331 \\
\hline $\mathrm{C}$ & 8.20636 & 1.15536 & 0.85163 \\
\hline $\mathrm{C}$ & -2.40403 & 3.45101 & -0.06722 \\
\hline $\mathrm{C}$ & -1.56118 & 3.09216 & -1.14028 \\
\hline $\mathrm{C}$ & -3.24750 & 4.56850 & -0.23566 \\
\hline $\mathrm{C}$ & -1.55434 & 3.83411 & -2.32787 \\
\hline $\mathrm{C}$ & -3.23365 & 5.31047 & -1.42466 \\
\hline $\mathrm{C}$ & -2.38364 & 4.96119 & -2.49242 \\
\hline $\mathrm{C}$ & -2.97493 & -3.31921 & 0.59235 \\
\hline $\mathrm{C}$ & -3.72303 & -3.48340 & -0.57613 \\
\hline $\mathrm{C}$ & -4.08272 & -2.50235 & -1.57307 \\
\hline $\mathrm{C}$ & -1.55224 & -4.66199 & 1.95055 \\
\hline $\mathrm{C}$ & -5.32729 & -2.50906 & -2.18682 \\
\hline $\mathrm{C}$ & -1.57824 & -5.01405 & 3.31231 \\
\hline $\mathrm{C}$ & -0.32584 & -4.41320 & 1.30167 \\
\hline $\mathrm{C}$ & -6.40800 & -3.48567 & -1.83872 \\
\hline $\mathrm{C}$ & -3.58664 & 0.04253 & -0.62152 \\
\hline $\mathrm{C}$ & -3.32805 & -0.82921 & -3.28068 \\
\hline $\mathrm{C}$ & -1.70339 & -1.73259 & -1.76688 \\
\hline $\mathrm{C}$ & -0.36840 & -5.12324 & 4.02306 \\
\hline $\mathrm{C}$ & 0.87221 & -4.50831 & 2.02456 \\
\hline $\mathrm{C}$ & -4.90904 & 0.42046 & -0.95990 \\
\hline $\mathrm{C}$ & -2.07415 & 0.02592 & -3.45464 \\
\hline $\mathrm{C}$ & -0.96887 & -0.74456 & -2.69386 \\
\hline $\mathrm{C}$ & 0.85707 & -4.86463 & 3.38482 \\
\hline $\mathrm{C}$ & -5.97397 & -0.39569 & -0.61687 \\
\hline $\mathrm{C}$ & -7.36778 & -0.22892 & -1.01895 \\
\hline $\mathrm{C}$ & -8.36812 & -0.99229 & -0.36883 \\
\hline $\mathrm{C}$ & -7.76916 & 0.65420 & -2.05220 \\
\hline $\mathrm{C}$ & -9.71699 & -0.87080 & -0.72512 \\
\hline $\mathrm{C}$ & -9.11598 & 0.77521 & -2.40766 \\
\hline $\mathrm{C}$ & -10.09750 & 0.01449 & -1.74608 \\
\hline $\mathrm{C}$ & 9.15615 & 2.29316 & 1.15130 \\
\hline $\mathrm{C}$ & -2.33627 & 5.78711 & -3.75717 \\
\hline $\mathrm{H}$ & 3.43357 & 4.23651 & 0.02454 \\
\hline $\mathrm{H}$ & 1.57720 & 7.91706 & 2.47926 \\
\hline $\mathrm{H}$ & 3.02781 & 6.42256 & 1.10766 \\
\hline
\end{tabular}




\begin{tabular}{|c|c|c|c|}
\hline $\mathrm{H}$ & -1.32468 & 4.73154 & 2.92091 \\
\hline $\mathrm{H}$ & -0.60222 & 7.06289 & 3.38293 \\
\hline $\mathrm{H}$ & 0.72849 & 1.77514 & 3.67029 \\
\hline $\mathrm{H}$ & -4.60652 & 2.45668 & 1.09633 \\
\hline $\mathrm{H}$ & 0.54229 & 0.43829 & 5.74756 \\
\hline $\mathrm{H}$ & -3.71119 & -0.08105 & 5.06937 \\
\hline $\mathrm{H}$ & -4.79769 & 1.06755 & 3.14245 \\
\hline $\mathrm{H}$ & -1.67083 & -0.53426 & 6.43735 \\
\hline $\mathrm{H}$ & 3.19921 & 3.28053 & -2.23182 \\
\hline $\mathrm{H}$ & 4.54448 & 3.06476 & -4.29821 \\
\hline $\mathrm{H}$ & 5.97247 & 1.69103 & -5.81093 \\
\hline $\mathrm{H}$ & 5.59439 & -1.69317 & 3.12974 \\
\hline $\mathrm{H}$ & 6.49648 & -0.69073 & -5.22182 \\
\hline $\mathrm{H}$ & 2.17130 & -1.74264 & -2.53088 \\
\hline $\mathrm{H}$ & 6.68629 & -3.58836 & 0.90685 \\
\hline $\mathrm{H}$ & 0.76256 & -3.65876 & -3.24141 \\
\hline $\mathrm{H}$ & 3.20011 & -6.36254 & -0.89646 \\
\hline $\mathrm{H}$ & 5.20808 & -5.49897 & 0.28846 \\
\hline $\mathrm{H}$ & 1.24596 & -5.96845 & -2.39329 \\
\hline $\mathrm{H}$ & 5.03656 & & 1.57330 \\
\hline $\mathrm{H}$ & 8.02709 & -1.748 & -0.97721 \\
\hline $\mathrm{H}$ & 6.59240 & 1.90881 & 2.08826 \\
\hline $\mathrm{H}$ & 9.59300 & 0.11262 & -0.45390 \\
\hline $\mathrm{H}$ & -0.91871 & 2.21403 & -1.05061 \\
\hline $\mathrm{H}$ & -3.90616 & 4.87282 & 0.58596 \\
\hline $\mathrm{H}$ & -0.88949 & 3.52739 & -3.14511 \\
\hline $\mathrm{H}$ & -3.89111 & & -1.52178 \\
\hline $\mathrm{H}$ & -4.14029 & 37 & -0.72545 \\
\hline $\mathrm{H}$ & -5.51344 & -1.87274 & -3.05444 \\
\hline $\mathrm{H}$ & -2.54393 & -5.19785 & 3.79568 \\
\hline $\mathrm{H}$ & -0.30663 & -4.10133 & 0.25472 \\
\hline $\mathrm{H}$ & -6.27019 & -4.45845 & -2.35621 \\
\hline $\mathrm{H}$ & -7.39647 & -3.09440 & -2.13748 \\
\hline $\mathrm{H}$ & -6.42496 & -3.70200 & -0.75478 \\
\hline $\mathrm{H}$ & -3.40358 & -0.61700 & 0.23247 \\
\hline $\mathrm{H}$ & -2.76265 & 0.718 & -0.86072 \\
\hline $\mathrm{H}$ & -4.26462 & -0.25457 & -3.32571 \\
\hline $\mathrm{H}$ & -3.37678 & -1.64168 & -4.02970 \\
\hline $\mathrm{H}$ & -1.53599 & -2.78796 & -2.04196 \\
\hline $\mathrm{H}$ & -1.41801 & -1.62166 & -0.71630 \\
\hline $\mathrm{H}$ & -0.38880 & -5.40253 & 5.08328 \\
\hline $\mathrm{H}$ & 1.81477 & -4.28332 & 1.51557 \\
\hline $\mathrm{H}$ & -5.05629 & 1.27364 & -1.63298 \\
\hline $\mathrm{H}$ & -1.84277 & 0.16774 & -4.52365 \\
\hline $\mathrm{H}$ & -2.23277 & 1.02775 & -3.02228 \\
\hline $\mathrm{H}$ & -0.31736 & -1.29645 & -3.39067 \\
\hline $\mathrm{H}$ & -0.32027 & -0.07590 & -2.10890 \\
\hline $\mathrm{H}$ & 1.79564 & -4.92985 & 3.94606 \\
\hline $\mathrm{H}$ & -5.78592 & -1.17639 & 0.13045 \\
\hline $\mathrm{H}$ & -8.07230 & -1.68609 & 0.42650 \\
\hline $\mathrm{H}$ & -7.01745 & 1.24350 & -2.58789 \\
\hline $\mathrm{H}$ & -10.47378 & -1.47032 & -0.20669 \\
\hline $\mathrm{H}$ & -9.40593 & 1.46243 & -3.21075 \\
\hline $\mathrm{H}$ & -11.15160 & 0.10949 & -2.03002 \\
\hline $\mathrm{H}$ & 9.44143 & 2.83087 & 0.22651 \\
\hline $\mathrm{H}$ & 8.70484 & 3.02590 & 1.84254 \\
\hline $\mathrm{H}$ & 10.09260 & 1.92472 & 1.61252 \\
\hline
\end{tabular}

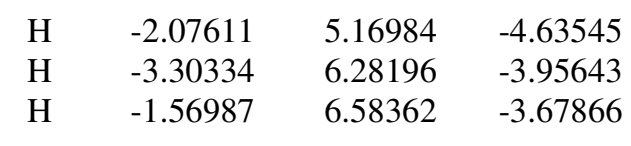

S_DAPa_minor_1

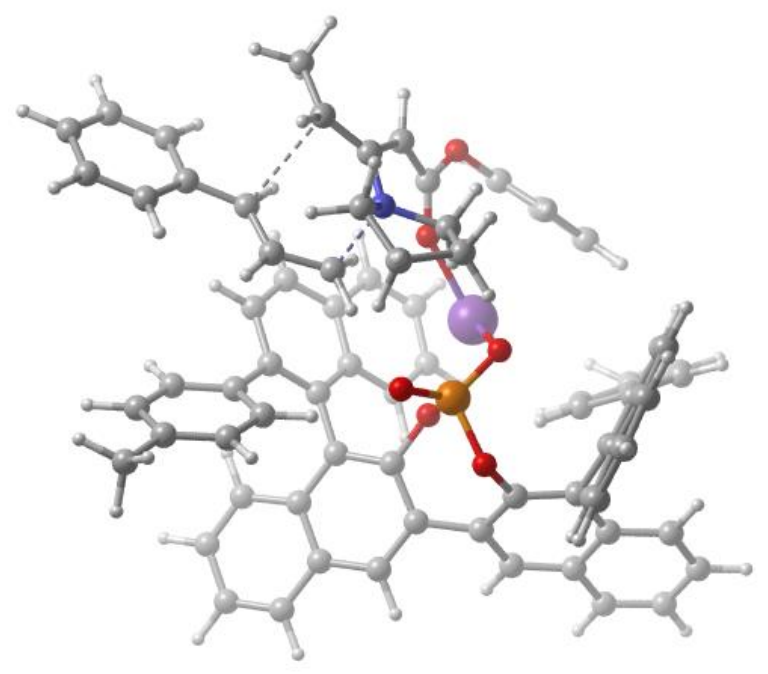

150

$\begin{array}{lccc}\mathrm{P} & -0.80859 & 0.57535 & 1.31244 \\ \mathrm{Na} & -0.75451 & -1.42204 & -1.05282 \\ \mathrm{~N} & 2.68592 & -2.36362 & 2.16232 \\ \mathrm{O} & -2.05102 & 1.60085 & 1.81240 \\ \mathrm{O} & -0.75295 & 1.03774 & -0.37329 \\ \mathrm{O} & -1.27122 & -0.87603 & 1.16259 \\ \mathrm{O} & 0.42877 & 1.01048 & 2.07656 \\ \mathrm{O} & 1.11537 & -2.57262 & -0.60206 \\ \mathrm{O} & 1.33448 & -4.72378 & -1.41347 \\ \mathrm{C} & 1.07761 & 6.83736 & -2.49337 \\ \mathrm{C} & -0.04683 & 6.43523 & -1.79183 \\ \mathrm{C} & -0.25284 & 5.06353 & -1.46017 \\ \mathrm{C} & 0.74140 & 4.08984 & -1.84155 \\ \mathrm{C} & 1.86732 & 4.53275 & -2.59538 \\ \mathrm{C} & 2.03429 & 5.87325 & -2.90818 \\ \mathrm{C} & -1.44295 & 4.62820 & -0.81455 \\ \mathrm{C} & 0.56114 & 2.71859 & -1.45073 \\ \mathrm{C} & -0.58596 & 2.36509 & -0.73805 \\ \mathrm{C} & -1.65005 & 3.29467 & -0.47908 \\ \mathrm{C} & 1.51631 & 1.65577 & -1.89650 \\ \mathrm{C} & 1.04174 & 0.67097 & -2.84041 \\ \mathrm{C} & 2.85360 & 1.61858 & -1.46958 \\ \mathrm{C} & -0.28683 & 0.69034 & -3.37396 \\ \mathrm{C} & 1.91786 & -0.39491 & -3.26600 \\ \mathrm{C} & 3.72958 & 0.61945 & -2.00044 \\ \mathrm{C} & -0.74441 & -0.32731 & -4.20261 \\ \mathrm{C} & 1.41028 & -1.43628 & -4.09660 \\ \mathrm{C} & 3.27611 & -0.37432 & -2.84132 \\ \mathrm{C} & 0.10018 & -1.41765 & -4.54773 \\ \mathrm{C} & -2.98842 & 2.79495 & -0.05927 \\ \mathrm{C} & -4.11904 & 3.13777 & -0.79823\end{array}$




\begin{tabular}{|c|c|c|c|c|c|c|c|}
\hline $\mathrm{C}$ & -3.13940 & 1.83566 & 0.99033 & $\mathrm{H}$ & 1.22482 & 7.89463 & -2.74096 \\
\hline $\mathrm{C}$ & -5.38795 & 2.54727 & -0.54352 & $\mathrm{H}$ & -0.80433 & 7.16668 & -1.48572 \\
\hline $\mathrm{C}$ & -4.33004 & 1.14180 & 1.20465 & $\mathrm{H}$ & 2.60743 & 3.79876 & -2.92306 \\
\hline $\mathrm{C}$ & -6.54223 & 2.89421 & -1.30624 & $\mathrm{H}$ & 2.91254 & 6.19003 & -3.48198 \\
\hline $\mathrm{C}$ & -5.49664 & 1.51915 & 0.46114 & $\mathrm{H}$ & -0.95008 & 1.52136 & -3.11783 \\
\hline $\mathrm{C}$ & -7.75434 & 2.25870 & -1.09114 & $\mathrm{H}$ & 4.77129 & 0.61698 & -1.67061 \\
\hline $\mathrm{C}$ & -6.75847 & 0.88830 & 0.66139 & $\mathrm{H}$ & -1.76404 & -0.28622 & -4.60173 \\
\hline $\mathrm{C}$ & -7.86189 & 1.24992 & -0.09595 & $\mathrm{H}$ & 2.07871 & -2.26261 & -4.36406 \\
\hline $\mathrm{C}$ & -4.31757 & -0.07778 & 2.06796 & $\mathrm{H}$ & 3.95204 & -1.16808 & -3.17993 \\
\hline $\mathrm{C}$ & -4.15693 & 0.04228 & 3.48792 & $\mathrm{H}$ & -0.28539 & -2.23382 & -5.16628 \\
\hline $\mathrm{C}$ & -4.40182 & -1.34694 & 1.46898 & $\mathrm{H}$ & -4.02046 & 3.84245 & -1.63183 \\
\hline $\mathrm{C}$ & -4.11202 & 1.31149 & 4.13884 & $\mathrm{H}$ & -6.45023 & 3.67357 & -2.07230 \\
\hline $\mathrm{C}$ & -4.03481 & -1.14514 & 4.29565 & $\mathrm{H}$ & -8.63299 & 2.53339 & -1.68557 \\
\hline $\mathrm{C}$ & -4.29866 & -2.50882 & 2.29386 & $\mathrm{H}$ & -6.84032 & 0.11069 & 1.42665 \\
\hline $\mathrm{C}$ & -3.93311 & 1.40555 & 5.50989 & $\mathrm{H}$ & -8.82473 & 0.75526 & 0.07475 \\
\hline $\mathrm{C}$ & -3.84358 & -1.01586 & 5.70259 & $\mathrm{H}$ & -4.21447 & 2.21811 & 3.53555 \\
\hline $\mathrm{C}$ & -4.10564 & -2.41582 & 3.65852 & $\mathrm{H}$ & -4.32751 & -3.49381 & 1.81549 \\
\hline $\mathrm{C}$ & -3.78974 & 0.23316 & 6.30097 & $\mathrm{H}$ & -3.89612 & 2.39109 & 5.98790 \\
\hline $\mathrm{C}$ & -4.59677 & -1.55200 & 0.00538 & $\mathrm{H}$ & -3.74240 & -1.92723 & 6.30465 \\
\hline $\mathrm{C}$ & -3.88760 & -0.82601 & -0.97901 & $\mathrm{H}$ & -3.99758 & -3.32288 & 4.26531 \\
\hline $\mathrm{C}$ & -5.52631 & -2.51304 & -0.44754 & $\mathrm{H}$ & -3.64154 & 0.32023 & 7.38356 \\
\hline $\mathrm{C}$ & -4.10765 & -1.04951 & -2.34153 & $\mathrm{H}$ & -3.16125 & -0.07175 & -0.67486 \\
\hline $\mathrm{C}$ & -5.74111 & -2.73542 & -1.81637 & $\mathrm{H}$ & -6.11176 & -3.08042 & 0.28475 \\
\hline $\mathrm{C}$ & -5.03932 & -2.00599 & -2.79222 & $\mathrm{H}$ & -3.54283 & -0.46084 & -3.07358 \\
\hline $\mathrm{C}$ & 3.41079 & 2.56382 & -0.46699 & $\mathrm{H}$ & -6.47717 & -3.48697 & -2.12831 \\
\hline $\mathrm{C}$ & 2.72137 & 2.86707 & 0.72552 & $\mathrm{H}$ & 1.76108 & 2.39608 & 0.94636 \\
\hline $\mathrm{C}$ & 4.68129 & 3.14509 & -0.66569 & $\mathrm{H}$ & 5.23800 & 2.93181 & -1.58552 \\
\hline $\mathrm{C}$ & 3.28375 & 3.72936 & 1.67370 & $\mathrm{H}$ & 2.72817 & 3.93122 & 2.59792 \\
\hline $\mathrm{C}$ & 5.23520 & 4.01180 & 0.28503 & $\mathrm{H}$ & 6.22098 & 4.45608 & 0.09819 \\
\hline $\mathrm{C}$ & 4.54546 & 4.32415 & 1.47304 & $\mathrm{H}$ & -4.33163 & -2.21429 & -4.83370 \\
\hline $\mathrm{C}$ & -5.28188 & -2.21120 & -4.26869 & $\mathrm{H}$ & -5.80322 & -3.16372 & -4.46759 \\
\hline $\mathrm{C}$ & 5.12461 & 5.28620 & 2.48442 & $\mathrm{H}$ & -5.90628 & -1.39682 & -4.68552 \\
\hline $\mathrm{C}$ & 1.71774 & -3.66991 & -0.50247 & $\mathrm{H}$ & 4.82658 & 5.01904 & 3.51417 \\
\hline $\mathrm{C}$ & 2.76557 & -4.07315 & 0.33044 & $\mathrm{H}$ & 6.22799 & 5.31068 & 2.43827 \\
\hline $\mathrm{C}$ & 3.40952 & -3.35789 & 1.40857 & $\mathrm{H}$ & 4.76456 & 6.31778 & 2.29862 \\
\hline $\mathrm{C}$ & 0.06149 & -4.65241 & -1.93621 & $\mathrm{H}$ & 3.18472 & -5.05564 & 0.10316 \\
\hline $\mathrm{C}$ & 4.74570 & -3.55058 & 1.71257 & $\mathrm{H}$ & 5.16990 & -3.10533 & 2.61424 \\
\hline $\mathrm{C}$ & -1.07211 & -4.45030 & -1.11778 & $\mathrm{H}$ & -0.94633 & -4.33422 & -0.03602 \\
\hline $\mathrm{C}$ & -0.09691 & -4.83243 & -3.32016 & $\mathrm{H}$ & 0.79421 & -4.98972 & -3.93650 \\
\hline $\mathrm{C}$ & 5.64462 & -4.46384 & 0.93397 & $\mathrm{H}$ & 5.50804 & -5.52925 & 1.21623 \\
\hline $\mathrm{C}$ & 3.23642 & -1.96427 & 3.50015 & $\mathrm{H}$ & 6.70514 & -4.21262 & 1.11168 \\
\hline $\mathrm{C}$ & 1.20432 & -2.60051 & 2.36042 & $\mathrm{H}$ & 5.44737 & -4.39887 & -0.15262 \\
\hline $\mathrm{C}$ & -2.34799 & -4.38308 & -1.69995 & $\mathrm{H}$ & 4.14120 & -1.35374 & 3.36648 \\
\hline $\mathrm{C}$ & -1.38102 & -4.79927 & -3.88688 & $\mathrm{H}$ & 3.50446 & -2.88418 & 4.05170 \\
\hline $\mathrm{C}$ & 2.07472 & -1.24348 & 4.17677 & $\mathrm{H}$ & 1.02096 & -3.67629 & 2.20827 \\
\hline $\mathrm{C}$ & 0.87019 & -2.11203 & 3.77956 & $\mathrm{H}$ & 0.64611 & -2.05140 & 1.59630 \\
\hline $\mathrm{C}$ & -2.50829 & -4.55745 & -3.08586 & $\mathrm{H}$ & -3.22305 & -4.19194 & -1.06885 \\
\hline $\mathrm{C}$ & 4.26999 & -0.38469 & 1.11117 & $\mathrm{H}$ & -1.49646 & -4.94673 & -4.96747 \\
\hline $\mathrm{C}$ & 2.88388 & -0.70303 & 1.05593 & $\mathrm{H}$ & 2.22446 & -1.17931 & 5.26774 \\
\hline $\mathrm{C}$ & 5.18114 & -1.12225 & 0.38642 & $\mathrm{H}$ & 1.95634 & -0.22100 & 3.78202 \\
\hline $\mathrm{C}$ & 6.63281 & -0.97268 & 0.38600 & $\mathrm{H}$ & 0.77443 & -2.96873 & 4.47171 \\
\hline $\mathrm{C}$ & 7.31993 & -0.10883 & 1.27507 & $\mathrm{H}$ & -0.07746 & -1.55201 & 3.78678 \\
\hline $\mathrm{C}$ & 7.39715 & -1.71107 & -0.55057 & $\mathrm{H}$ & -3.50668 & -4.50955 & -3.53055 \\
\hline $\mathrm{C}$ & 8.71162 & 0.01195 & 1.22160 & $\mathrm{H}$ & 4.60535 & 0.38193 & 1.81803 \\
\hline $\mathrm{C}$ & 8.79155 & -1.59192 & -0.60187 & $\mathrm{H}$ & 2.48911 & -1.19463 & 0.16249 \\
\hline $\mathrm{C}$ & 9.45542 & -0.72888 & 0.28443 & $\mathrm{H}$ & 2.16221 & -0.05888 & 1.56880 \\
\hline $\mathrm{H}$ & -2.22789 & 5.36182 & -0.59817 & $\mathrm{H}$ & 4.77844 & -1.81817 & -0.35849 \\
\hline
\end{tabular}




$\begin{array}{cccc}\mathrm{H} & 6.75746 & 0.47497 & 2.01058 \\ \mathrm{H} & 6.87883 & -2.38390 & -1.24341 \\ \mathrm{H} & 9.22462 & 0.68837 & 1.91492 \\ \mathrm{H} & 9.36256 & -2.17270 & -1.33517 \\ \mathrm{H} & 10.54638 & -0.63206 & 0.24733\end{array}$

S_DAPa_minor_2

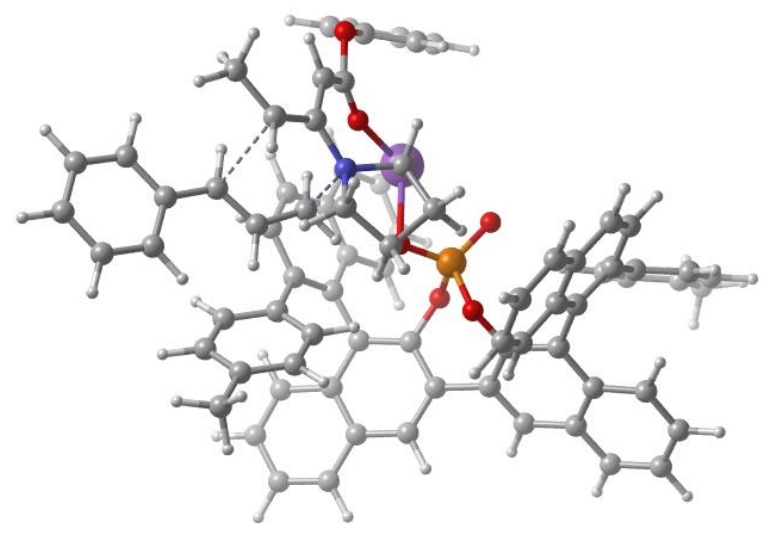

150

$\begin{array}{lccc}\mathrm{P} & 1.28625 & -0.41990 & 0.46803 \\ \mathrm{Na} & -0.60311 & -2.40214 & 1.44524 \\ \mathrm{~N} & -2.85972 & -1.38798 & -2.42116 \\ \mathrm{O} & 1.96799 & 0.62598 & -0.67570 \\ \mathrm{O} & 1.69299 & 0.44088 & 1.87701 \\ \mathrm{O} & 1.94734 & -1.78786 & 0.61747 \\ \mathrm{O} & -0.22856 & -0.35799 & 0.23369 \\ \mathrm{O} & -2.39070 & -2.94397 & 0.27743 \\ \mathrm{O} & -3.53021 & -4.94061 & 0.26421 \\ \mathrm{C} & -0.35341 & 6.39759 & 3.19712 \\ \mathrm{C} & 0.72263 & 5.94281 & 2.45300 \\ \mathrm{C} & 0.94640 & 4.54688 & 2.26356 \\ \mathrm{C} & 0.02464 & 3.60356 & 2.84597 \\ \mathrm{C} & -1.06557 & 4.10441 & 3.61468 \\ \mathrm{C} & -1.24931 & 5.46739 & 3.78901 \\ \mathrm{C} & 2.03693 & 4.06803 & 1.48651 \\ \mathrm{C} & 0.22583 & 2.19824 & 2.62581 \\ \mathrm{C} & 1.37709 & 1.78581 & 1.94878 \\ \mathrm{C} & 2.27629 & 2.70634 & 1.32351 \\ \mathrm{C} & -0.83728 & 1.20286 & 2.96269 \\ \mathrm{C} & -0.57542 & 0.12112 & 3.87345 \\ \mathrm{C} & -2.08075 & 1.27981 & 2.30774 \\ \mathrm{C} & 0.65111 & 0.01470 & 4.59673 \\ \mathrm{C} & -1.56065 & -0.92256 & 4.04999 \\ \mathrm{C} & -3.06760 & 0.27758 & 2.55791 \\ \mathrm{C} & 0.92221 & -1.08708 & 5.39284 \\ \mathrm{C} & -1.24498 & -2.05447 & 4.86339 \\ \mathrm{C} & -2.81322 & -0.80477 & 3.37969 \\ \mathrm{C} & -0.02283 & -2.14298 & 5.51266 \\ \mathrm{C} & 3.40780 & 2.21626 & 0.49305 \\ \mathrm{C} & 4.66964 & 2.79350 & 0.60545 \\ \mathrm{C} & 3.23235 & 1.17583 & -0.48141 \\ \mathrm{C} & 5.75285 & 2.40930 & -0.23018\end{array}$

\begin{tabular}{|c|c|c|c|}
\hline$C$ & 4.26222 & 0.75405 & -1.31950 \\
\hline C & 7.03894 & 3.01432 & -0.11089 \\
\hline $\mathrm{C}$ & 5.54479 & 1.39176 & -1.22911 \\
\hline $\mathrm{C}$ & 8.07741 & 2.64793 & -0.95090 \\
\hline $\mathrm{C}$ & 6.62777 & 1.05387 & -2.09144 \\
\hline $\mathrm{C}$ & 7.86422 & 1.66552 & -1.95469 \\
\hline $\mathrm{C}$ & 4.02193 & -0.34738 & -2.30247 \\
\hline $\mathrm{C}$ & 3.19392 & -0.10182 & -3.45171 \\
\hline $\mathrm{C}$ & 4.63763 & -1.59639 & -2.13173 \\
\hline C & 2.60179 & 1.17236 & -3.70649 \\
\hline $\mathrm{C}$ & 2.97073 & -1.15879 & -4.40700 \\
\hline $\mathrm{C}$ & 4.40146 & -2.62879 & -3.09038 \\
\hline $\mathrm{C}$ & 1.82356 & 1.38934 & -4.83271 \\
\hline $\mathrm{C}$ & 2.15827 & -0.90636 & -5.55158 \\
\hline $\mathrm{C}$ & 3.58395 & -2.42493 & -4.18604 \\
\hline $\mathrm{C}$ & 1.59409 & 0.34126 & -5.76477 \\
\hline $\mathrm{C}$ & 5.58288 & -1.86077 & -1.01164 \\
\hline $\mathrm{C}$ & 5.22783 & -1.64870 & 0.33651 \\
\hline $\mathrm{C}$ & 6.88911 & -2.30614 & -1.29694 \\
\hline $\mathrm{C}$ & 6.16649 & -1.84498 & 1.35510 \\
\hline $\mathrm{C}$ & 7.82543 & -2.49626 & -0.27069 \\
\hline $\mathrm{C}$ & 7.48509 & -2.25758 & 1.07351 \\
\hline $\mathrm{C}$ & -2.42062 & 2.32874 & 1.30413 \\
\hline $\mathrm{C}$ & -1.57828 & 2.62209 & 0.20986 \\
\hline $\mathrm{C}$ & -3.65450 & 3.00360 & 1.38692 \\
\hline $\mathrm{C}$ & -1.96704 & 3.55059 & -0.76081 \\
\hline $\mathrm{C}$ & -4.03718 & 3.93771 & 0.41432 \\
\hline $\mathrm{C}$ & -3.20227 & 4.22780 & -0.68002 \\
\hline $\mathrm{C}$ & -3.23414 & -3.65955 & -0.31900 \\
\hline $\mathrm{C}$ & -3.99326 & -3.41361 & -1.46892 \\
\hline $\mathrm{C}$ & -4.00394 & -2.25705 & -2.33537 \\
\hline $\mathrm{C}$ & -2.79391 & -5.23631 & 1.40016 \\
\hline C & -5.14120 & -1.88576 & -3.03475 \\
\hline $\mathrm{C}$ & -3.43574 & -5.23724 & 2.64773 \\
\hline $\mathrm{C}$ & -1.42297 & -5.54321 & 1.30779 \\
\hline $\mathrm{C}$ & -6.44909 & -2.61293 & -2.93896 \\
\hline $\mathrm{C}$ & -3.09160 & -0.21128 & -0.78252 \\
\hline $\mathrm{C}$ & -2.79732 & -0.40150 & -3.54542 \\
\hline C & -1.49016 & -2.02102 & -2.33060 \\
\hline $\mathrm{C}$ & -2.70216 & -5.53521 & 3.80801 \\
\hline $\mathrm{C}$ & -0.69291 & -5.82479 & 2.47381 \\
\hline $\mathrm{C}$ & -4.20337 & 0.61829 & -1.07909 \\
\hline $\mathrm{C}$ & -1.35662 & 0.09773 & -3.51512 \\
\hline $\mathrm{C}$ & -0.56378 & -1.18773 & -3.23203 \\
\hline $\mathrm{C}$ & -1.32883 & -5.81657 & 3.72830 \\
\hline $\mathrm{C}$ & -5.48944 & 0.12255 & -1.02706 \\
\hline $\mathrm{C}$ & -6.70388 & 0.83123 & -1.41802 \\
\hline $\mathrm{C}$ & -6.68202 & 2.12366 & -2.00179 \\
\hline $\mathrm{C}$ & -7.96211 & 0.21524 & -1.20838 \\
\hline $\mathrm{C}$ & -7.87078 & 2.76722 & -2.35744 \\
\hline $\mathrm{C}$ & -9.15219 & 0.85932 & -1.56859 \\
\hline $\mathrm{C}$ & -9.11203 & 2.13868 & -2.14584 \\
\hline $\mathrm{C}$ & 8.49673 & -2.42304 & 2.18431 \\
\hline $\mathrm{C}$ & -3.60420 & 5.23610 & -1.73163 \\
\hline $\mathrm{H}$ & 2.69136 & 4.79287 & 0.98933 \\
\hline $\mathrm{H}$ & -0.51221 & 7.47280 & 3.33672 \\
\hline $\mathrm{H}$ & 1.42497 & 6.65181 & 1.99851 \\
\hline
\end{tabular}




\begin{tabular}{|c|c|c|c|}
\hline $\mathrm{H}$ & -1.75852 & 3.39171 & 4.07134 \\
\hline $\mathrm{H}$ & -2.09186 & 5.82960 & 4.38889 \\
\hline $\mathrm{H}$ & 1.39026 & 0.81408 & 4.49841 \\
\hline $\mathrm{H}$ & -4.02177 & 0.34488 & 2.02674 \\
\hline $\mathrm{H}$ & 1.87786 & -1.15069 & 5.92498 \\
\hline $\mathrm{H}$ & -1.98815 & -2.85439 & 4.95995 \\
\hline $\mathrm{H}$ & -3.56276 & -1.59486 & 3.50533 \\
\hline $\mathrm{H}$ & 0.21126 & -3.01967 & 6.12730 \\
\hline $\mathrm{H}$ & 4.83718 & 3.56450 & 1.36598 \\
\hline $\mathrm{H}$ & 7.18828 & 3.78082 & 0.65899 \\
\hline $\mathrm{H}$ & 9.06141 & 3.11943 & -0.84924 \\
\hline $\mathrm{H}$ & 6.46846 & 0.29915 & -2.86611 \\
\hline $\mathrm{H}$ & 8.68415 & & -2.62659 \\
\hline $\mathrm{H}$ & & & 9736 \\
\hline $\mathrm{H}$ & 4.87053 & -3.60542 & 2782 \\
\hline $\mathrm{H}$ & 1.38341 & 2.37811 & -5.00706 \\
\hline $\mathrm{H}$ & 1.99430 & -1.72110 & -6.26763 \\
\hline $\mathrm{H}$ & 3.40399 & -3.23526 & -4.90291 \\
\hline $\mathrm{H}$ & 0.97636 & 0.52342 & -6.65202 \\
\hline $\mathrm{H}$ & 4.20126 & -1.37165 & 0.58569 \\
\hline $\mathrm{H}$ & 7.18609 & $-2.4^{7}$ & -2.33928 \\
\hline $\mathrm{H}$ & 5.86360 & & 9681 \\
\hline $\mathrm{H}$ & 8.84187 & -2.82505 & 2119 \\
\hline $\mathrm{H}$ & -0.63789 & 2.08131 & 0.09740 \\
\hline $\mathrm{H}$ & -4.32452 & 2.79891 & 2.22960 \\
\hline $\mathrm{H}$ & -1.29809 & 3.74564 & -1.60871 \\
\hline $\mathrm{H}$ & -5.00561 & 4.44462 & 0.50533 \\
\hline $\mathrm{H}$ & -4.73862 & -4.17741 & -1.69863 \\
\hline $\mathrm{H}$ & -5.088 & 208 & -3.77076 \\
\hline $\mathrm{H}$ & -4.50214 & -4.99280 & 69424 \\
\hline $\mathrm{H}$ & -0.94288 & -5.55209 & 0.32339 \\
\hline $\mathrm{H}$ & -6.45797 & -3.54823 & -3.53783 \\
\hline $\mathrm{H}$ & -7.27350 & -1.97974 & -3.31133 \\
\hline $\mathrm{H}$ & -6.67885 & -2.90628 & -1.89682 \\
\hline $\mathrm{H}$ & -3.22200 & -1.07577 & -0.13034 \\
\hline $\mathrm{H}$ & -2.08657 & 0.20969 & -0.74621 \\
\hline $\mathrm{H}$ & -3.55595 & 0.38106 & -3.40216 \\
\hline $\mathrm{H}$ & -3.00773 & 46 & -4.49082 \\
\hline $\mathrm{H}$ & -1.58991 & -3.07326 & -2.64379 \\
\hline $\mathrm{H}$ & -1.17501 & -1.99763 & -1.28426 \\
\hline $\mathrm{H}$ & -3.20691 & -5.53630 & 4.78137 \\
\hline $\mathrm{H}$ & 0.37614 & -6.05725 & 2.39999 \\
\hline $\mathrm{H}$ & -4.00862 & 1.62415 & -1.46432 \\
\hline $\mathrm{H}$ & -1.07019 & 0.57401 & -4.46591 \\
\hline $\mathrm{H}$ & & & -2.70882 \\
\hline $\mathrm{H}$ & -0.35708 & -1.71629 & -4.17841 \\
\hline $\mathrm{H}$ & 0.40034 & -0.98605 & -2.74291 \\
\hline $\mathrm{H}$ & -0.75633 & -6.03491 & 4.63653 \\
\hline $\mathrm{H}$ & -5.63490 & -0.86407 & -0.57049 \\
\hline $\mathrm{H}$ & -5.72588 & 2.62917 & -2.16921 \\
\hline $\mathrm{H}$ & -7.99502 & -0.78166 & -0.75433 \\
\hline $\mathrm{H}$ & -7.83359 & 3.76762 & -2.80423 \\
\hline $\mathrm{H}$ & & 0.36320 & -1.39765 \\
\hline $\mathrm{H}$ & -10.04122 & 2.64613 & -2.42875 \\
\hline $\mathrm{H}$ & 8.08803 & -3.02798 & 3.01536 \\
\hline $\mathrm{H}$ & 9.41866 & -2.91315 & 1.82566 \\
\hline $\mathrm{H}$ & 8.78227 & -1.44078 & 2.60959 \\
\hline
\end{tabular}

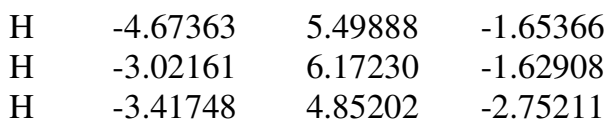

S_DAPa_minor_3

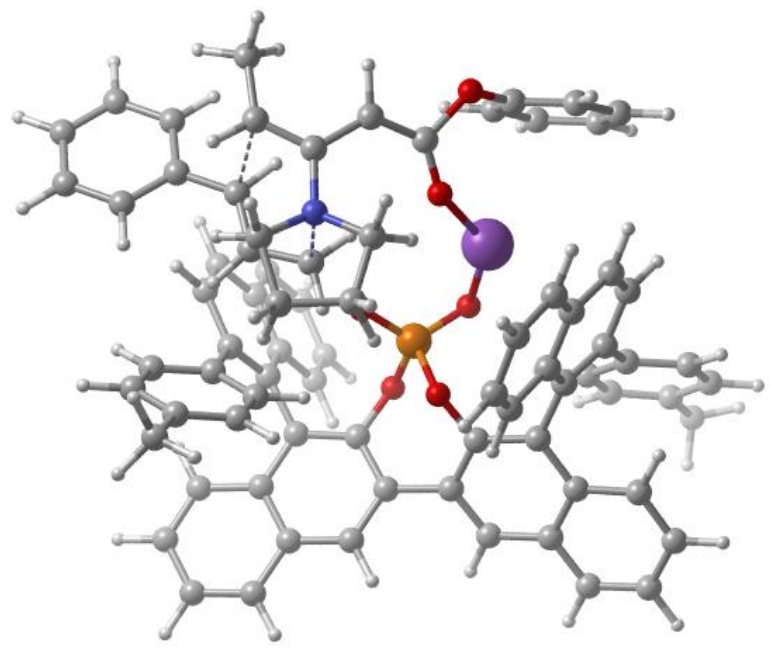

150

$\begin{array}{lccc}\mathrm{P} & 0.74193 & 0.52445 & 0.74392 \\ \mathrm{Na} & 1.20800 & -2.40914 & 0.83670 \\ \mathrm{~N} & -3.07854 & -3.15498 & -1.90226 \\ \mathrm{O} & 0.92362 & 2.14676 & 1.18976 \\ \mathrm{O} & 1.42659 & 0.62296 & -0.83824 \\ \mathrm{O} & -0.70323 & 0.11245 & 0.52513 \\ \mathrm{O} & 1.67883 & -0.30240 & 1.63540 \\ \mathrm{O} & -0.45902 & -3.65082 & -0.04390 \\ \mathrm{O} & -0.69591 & -5.72717 & 0.94443 \\ \mathrm{C} & 7.44275 & 2.53669 & -2.12456 \\ \mathrm{C} & 6.30019 & 3.26068 & -1.82528 \\ \mathrm{C} & 5.06633 & 2.60172 & -1.54819 \\ \mathrm{C} & 5.02002 & 1.16134 & -1.56482 \\ \mathrm{C} & 6.20793 & 0.44767 & -1.89718 \\ \mathrm{C} & 7.39049 & 1.11807 & -2.16912 \\ \mathrm{C} & 3.88187 & 3.33135 & -1.25409 \\ \mathrm{C} & 3.78843 & 0.49718 & -1.24479 \\ \mathrm{C} & 2.64280 & 1.25999 & -1.00887 \\ \mathrm{C} & 2.67137 & 2.69527 & -1.00064 \\ \mathrm{C} & 3.73250 & -0.99139 & -1.11130 \\ \mathrm{C} & 2.99337 & -1.77125 & -2.06889 \\ \mathrm{C} & 4.40716 & -1.63223 & -0.05470 \\ \mathrm{C} & 2.37795 & -1.17989 & -3.21455 \\ \mathrm{C} & 2.88423 & -3.20158 & -1.89990 \\ \mathrm{C} & 4.28984 & -3.05154 & 0.08980 \\ \mathrm{C} & 1.69094 & -1.95426 & -4.13542 \\ \mathrm{C} & 2.13888 & -3.96519 & -2.84700 \\ \mathrm{C} & 3.53684 & -3.81324 & -0.78919 \\ \mathrm{C} & 1.55830 & -3.35769 & -3.94727 \\ \mathrm{C} & 1.42375 & 3.46976 & -0.77386 \\ \mathrm{C} & 1.07300 & 4.51932 & -1.61817 \\ \mathrm{C} & 0.52236 & 3.12335 & 0.28442 \\ \mathrm{C} & -0.14092 & 5.24033 & -1.45189\end{array}$




\begin{tabular}{|c|c|c|c|c|c|c|c|}
\hline $\mathrm{C}$ & -0.71547 & 3.74564 & 0.44518 & $\mathrm{H}$ & 6.17385 & -0.64463 & -1.93552 \\
\hline $\mathrm{C}$ & -0.49510 & 6.31781 & -2.31704 & $\mathrm{H}$ & 8.29223 & 0.54799 & -2.41970 \\
\hline $\mathrm{C}$ & -1.05370 & 4.85066 & -0.40653 & $\mathrm{H}$ & 2.47076 & -0.10053 & -3.36229 \\
\hline $\mathrm{C}$ & -1.69442 & 6.99255 & -2.15900 & $\mathrm{H}$ & 4.78816 & -3.53001 & 0.93978 \\
\hline $\mathrm{C}$ & -2.27440 & 5.57262 & -0.26417 & $\mathrm{H}$ & 1.24491 & -1.48106 & -5.01775 \\
\hline $\mathrm{C}$ & -2.58735 & 6.61817 & -1.11908 & $\mathrm{H}$ & 2.04326 & -5.04595 & -2.68987 \\
\hline $\mathrm{C}$ & -1.69930 & 3.20893 & 1.43303 & $\mathrm{H}$ & 3.43346 & -4.89345 & -0.63944 \\
\hline $\mathrm{C}$ & -1.44544 & 3.35189 & 2.83899 & $\mathrm{H}$ & 0.99873 & -3.95594 & -4.67482 \\
\hline $\mathrm{C}$ & -2.86365 & 2.56155 & 0.98756 & $\mathrm{H}$ & 1.73382 & 4.77871 & -2.45310 \\
\hline $\mathrm{C}$ & -0.31081 & 4.06100 & 3.33597 & $\mathrm{H}$ & 0.20366 & 6.60158 & -3.11332 \\
\hline $\mathrm{C}$ & -2.36338 & 2.78000 & 3.79219 & $\mathrm{H}$ & -1.95497 & 7.81820 & -2.83092 \\
\hline $\mathrm{C}$ & -3.76508 & 2.01496 & 1.95252 & $\mathrm{H}$ & -2.96286 & 5.28820 & 0.53671 \\
\hline $\mathrm{C}$ & -0.08179 & 4.18162 & 4.69753 & $\mathrm{H}$ & -3.53041 & 7.16142 & -0.99025 \\
\hline $\mathrm{C}$ & -2.09539 & 2.91247 & 5.18616 & $\mathrm{H}$ & 0.38586 & 4.50883 & 2.62180 \\
\hline $\mathrm{C}$ & -3.51875 & 2.10427 & 3.30827 & $\mathrm{H}$ & -4.65020 & 1.48003 & 1.59899 \\
\hline $\mathrm{C}$ & -0.97668 & 3.59685 & 5.63433 & $\mathrm{H}$ & 0.79857 & 4.72797 & 5.05505 \\
\hline $\mathrm{C}$ & -3.22569 & 2.41438 & -0.44939 & $\mathrm{H}$ & -2.79752 & 2.46444 & 5.90010 \\
\hline $\mathrm{C}$ & -2.30564 & 2.00118 & -1.43473 & $\mathrm{H}$ & -4.21371 & 1.65163 & 4.02591 \\
\hline $\mathrm{C}$ & -4.55846 & 2.64953 & -0.85766 & $\mathrm{H}$ & -0.78158 & 3.69175 & 6.70882 \\
\hline $\mathrm{C}$ & -2.70680 & 1.82432 & -2.76461 & $\mathrm{H}$ & -1.28283 & 1.76222 & -1.14940 \\
\hline $\mathrm{C}$ & -4.95704 & 2.46090 & -2.18684 & $\mathrm{H}$ & -5.29293 & 2.99257 & -0.12046 \\
\hline $\mathrm{C}$ & -4.03814 & 2.03923 & -3.16929 & $\mathrm{H}$ & -1.96392 & 1.49808 & -3.50381 \\
\hline $\mathrm{C}$ & 5.28630 & -0.89390 & 0.89508 & $\mathrm{H}$ & -5.99989 & 2.65519 & -2.46970 \\
\hline $\mathrm{C}$ & 4.84589 & 0.23517 & 1.61600 & $\mathrm{H}$ & 3.79789 & 0.53385 & 1.55298 \\
\hline $\mathrm{C}$ & 6.62369 & -1.31085 & 1.05481 & $\mathrm{H}$ & 6.98809 & -2.18184 & 0.49674 \\
\hline $\mathrm{C}$ & 5.73211 & 0.93776 & 2.43897 & $\mathrm{H}$ & 5.36458 & 1.81124 & 2.99193 \\
\hline $\mathrm{C}$ & 7.50617 & -0.59826 & 1.87850 & $\mathrm{H}$ & 8.54730 & -0.93211 & 1.97029 \\
\hline $\mathrm{C}$ & 7.08006 & 0.54749 & 2.57539 & $\mathrm{H}$ & -3.62874 & 1.52169 & -5.24039 \\
\hline $\mathrm{C}$ & -4.47413 & 1.83073 & -4.60168 & $\mathrm{H}$ & -5.25679 & 1.05085 & -4.67552 \\
\hline $\mathrm{C}$ & 8.03596 & 1.35206 & 3.42554 & $\mathrm{H}$ & -4.90128 & 2.75677 & -5.03086 \\
\hline $\mathrm{C}$ & -1.24165 & -4.60364 & 0.22043 & $\mathrm{H}$ & 7.56912 & 1.65698 & 4.38010 \\
\hline $\mathrm{C}$ & -2.59079 & -4.80382 & -0.07605 & $\mathrm{H}$ & 8.95353 & 0.78336 & 3.65706 \\
\hline $\mathrm{C}$ & -3.51607 & -3.98242 & -0.82468 & $\mathrm{H}$ & 8.34169 & 2.28031 & 2.90323 \\
\hline $\mathrm{C}$ & 0.41207 & -5.46122 & 1.72377 & $\mathrm{H}$ & -3.03999 & -5.66516 & 0.42229 \\
\hline $\mathrm{C}$ & -4.85272 & -3.90271 & -0.45601 & $\mathrm{H}$ & -5.56953 & -3.40451 & -1.11074 \\
\hline $\mathrm{C}$ & 1.60844 & -6.14804 & 1.45974 & $\mathrm{H}$ & 1.63491 & -6.85971 & 0.62807 \\
\hline $\mathrm{C}$ & 0.34623 & -4.53898 & 2.78885 & $\mathrm{H}$ & -0.59830 & -4.02136 & 2.99159 \\
\hline $\mathrm{C}$ & -5.42770 & -4.60920 & 0.73487 & $\mathrm{H}$ & -5.62195 & -5.68382 & 0.53363 \\
\hline $\mathrm{C}$ & -4.09307 & -2.68856 & -2.88692 & $\mathrm{H}$ & -6.38901 & -4.15241 & 1.02872 \\
\hline $\mathrm{C}$ & -1.89783 & -3.59133 & -2.70148 & $\mathrm{H}$ & -4.74272 & -4.56897 & 1.60237 \\
\hline $\mathrm{C}$ & 2.74357 & -5.89990 & 2.24881 & $\mathrm{H}$ & -4.81927 & -2.02254 & -2.40057 \\
\hline $\mathrm{C}$ & 1.49309 & -4.28078 & 3.55832 & $\mathrm{H}$ & -4.63034 & -3.57437 & -3.27654 \\
\hline $\mathrm{C}$ & -3.27806 & -1.98785 & -3.97537 & $\mathrm{H}$ & -2.04357 & -4.65824 & -2.95583 \\
\hline $\mathrm{C}$ & -1.89191 & -2.68331 & -3.94767 & $\mathrm{H}$ & -0.99032 & -3.50344 & -2.08934 \\
\hline $\mathrm{C}$ & 2.69718 & -4.95657 & 3.28799 & $\mathrm{H}$ & 3.67658 & -6.43489 & 2.03574 \\
\hline $\mathrm{C}$ & -3.69921 & -0.87867 & -0.47814 & $\mathrm{H}$ & 1.44442 & -3.54775 & 4.37184 \\
\hline $\mathrm{C}$ & -2.46709 & -1.39446 & -0.92343 & $\mathrm{H}$ & -3.77996 & -2.06751 & -4.95375 \\
\hline $\mathrm{C}$ & -4.32507 & -1.42618 & 0.62602 & $\mathrm{H}$ & -3.18367 & -0.91479 & -3.74117 \\
\hline $\mathrm{C}$ & -5.63938 & -1.05045 & 1.13427 & $\mathrm{H}$ & -1.71184 & -3.28113 & -4.85657 \\
\hline $\mathrm{C}$ & -6.54036 & -0.24538 & 0.39274 & $\mathrm{H}$ & -1.08047 & -1.94154 & -3.88314 \\
\hline $\mathrm{C}$ & -6.03175 & -1.47894 & 2.42557 & $\mathrm{H}$ & 3.59023 & -4.74864 & 3.88710 \\
\hline $\mathrm{C}$ & -7.77581 & 0.12521 & 0.93125 & $\mathrm{H}$ & -4.20421 & -0.12391 & -1.08673 \\
\hline $\mathrm{C}$ & -7.26993 & -1.10707 & 2.96394 & $\mathrm{H}$ & -1.83483 & -1.97652 & -0.25497 \\
\hline $\mathrm{C}$ & -8.14692 & -0.30137 & 2.21977 & $\mathrm{H}$ & -1.94063 & -0.90397 & -1.74444 \\
\hline $\mathrm{H}$ & 3.92839 & 4.42589 & -1.22177 & $\mathrm{H}$ & -3.73612 & -2.09654 & 1.26223 \\
\hline $\mathrm{H}$ & 8.38491 & 3.05507 & -2.33552 & $\mathrm{H}$ & -6.26267 & 0.10010 & -0.60758 \\
\hline $\mathrm{H}$ & 6.32521 & 4.35677 & -1.80052 & $\mathrm{H}$ & -5.34321 & -2.09972 & 3.01015 \\
\hline
\end{tabular}




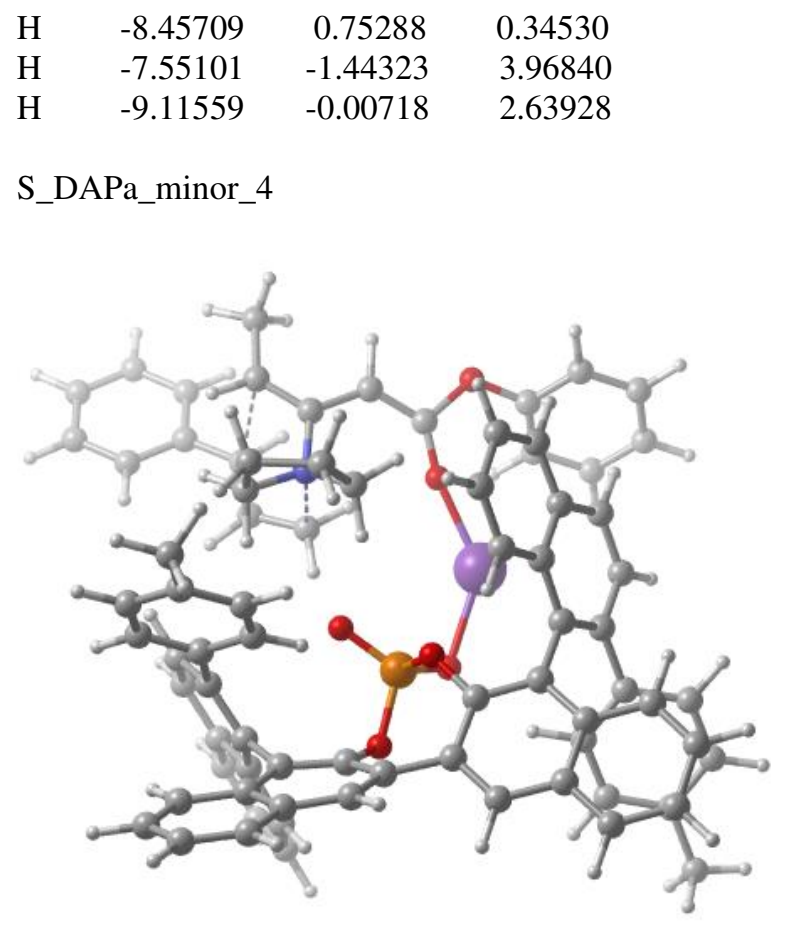

\begin{tabular}{|c|c|c|c|}
\hline $\mathrm{C}$ & 3.45758 & 2.21518 & -0.45753 \\
\hline $\mathrm{C}$ & 4.81937 & 2.47988 & -0.55829 \\
\hline $\mathrm{C}$ & 3.01798 & 0.86001 & -0.63770 \\
\hline $\mathrm{C}$ & 5.76101 & 1.46388 & -0.87678 \\
\hline $\mathrm{C}$ & 3.90654 & -0.18468 & -0.89969 \\
\hline $\mathrm{C}$ & 7.15149 & 1.75182 & -1.00918 \\
\hline $\mathrm{C}$ & 5.30076 & 0.11304 & -1.07701 \\
\hline $\mathrm{C}$ & 8.05548 & 0.75842 & -1.34762 \\
\hline $\mathrm{C}$ & 6.25379 & -0.88003 & -1.44570 \\
\hline $\mathrm{C}$ & 7.59760 & -0.56592 & -1.57839 \\
\hline $\mathrm{C}$ & 3.41580 & -1.59759 & -0.92930 \\
\hline $\mathrm{C}$ & 2.50575 & -2.03484 & -1.95678 \\
\hline $\mathrm{C}$ & 3.84256 & -2.50371 & 0.06105 \\
\hline $\mathrm{C}$ & 2.08432 & -1.17612 & -3.01769 \\
\hline $\mathrm{C}$ & 1.99087 & -3.38477 & -1.93097 \\
\hline $\mathrm{C}$ & 3.35864 & -3.85105 & 0.03541 \\
\hline $\mathrm{C}$ & 1.17491 & -1.60901 & -3.96888 \\
\hline $\mathrm{C}$ & 1.03551 & -3.79128 & -2.91048 \\
\hline $\mathrm{C}$ & 2.44742 & -4.27933 & -0.91719 \\
\hline $\mathrm{C}$ & 0.63089 & -2.92105 & -3.90892 \\
\hline $\mathrm{C}$ & 4.79520 & -2.11604 & 1.13974 \\
\hline $\mathrm{C}$ & 4.55407 & -1.01051 & 1.98241 \\
\hline $\mathrm{C}$ & 5.97833 & -2.85727 & 1.32846 \\
\hline $\mathrm{C}$ & 5.48739 & -0.64904 & 2.95937 \\
\hline $\mathrm{C}$ & 6.91165 & -2.48429 & 2.30613 \\
\hline $\mathrm{C}$ & 6.68841 & -1.36766 & 3.13258 \\
\hline $\mathrm{C}$ & -2.15262 & 3.59708 & -0.83641 \\
\hline $\mathrm{C}$ & -1.18675 & 3.01809 & -1.68892 \\
\hline C & -3.18880 & 4.34512 & -1.43224 \\
\hline $\mathrm{C}$ & -1.24366 & 3.21340 & -3.07151 \\
\hline $\mathrm{C}$ & -3.24491 & 4.53101 & -2.82184 \\
\hline $\mathrm{C}$ & -2.26767 & 3.97727 & -3.66840 \\
\hline C & -2.11588 & -4.08543 & -0.51246 \\
\hline $\mathrm{C}$ & -3.32015 & -3.70111 & -1.11532 \\
\hline $\mathrm{C}$ & -3.92823 & -2.39760 & -1.23504 \\
\hline $\mathrm{C}$ & -0.80941 & -6.09306 & -0.09251 \\
\hline C & -5.29747 & -2.25838 & -1.39985 \\
\hline $\mathrm{C}$ & -0.09175 & -7.00996 & -0.88178 \\
\hline $\mathrm{C}$ & -0.43827 & -5.88305 & 1.25156 \\
\hline $\mathrm{C}$ & -6.25090 & -3.41058 & -1.50532 \\
\hline C & -3.04398 & -1.10717 & 1.01010 \\
\hline C & -1.81179 & -1.19474 & -1.75646 \\
\hline C & -3.69791 & 0.12680 & -1.39186 \\
\hline C & 1.00359 & -7.69832 & -0.33950 \\
\hline C & 0.67218 & -6.56217 & 1.77635 \\
\hline $\mathrm{C}$ & -4.38324 & -0.78843 & 1.35189 \\
\hline $\mathrm{C}$ & -2.10773 & -0.49489 & -3.10415 \\
\hline $\mathrm{C}$ & -3.43285 & 0.28435 & -2.88988 \\
\hline $\mathrm{C}$ & 1.40292 & -7.46582 & 0.98677 \\
\hline $\mathrm{C}$ & -5.37680 & -1.74927 & 1.30388 \\
\hline $\mathrm{C}$ & -6.81236 & -1.52018 & 1.44629 \\
\hline $\mathrm{C}$ & -7.67554 & -2.62721 & 1.63401 \\
\hline $\mathrm{C}$ & -7.39070 & -0.22766 & 1.38067 \\
\hline $\mathrm{C}$ & -9.05884 & -2.45197 & 1.76375 \\
\hline $\mathrm{C}$ & -8.77184 & -0.05307 & 1.50998 \\
\hline $\mathrm{C}$ & -9.61378 & -1.16364 & 1.70373 \\
\hline $\mathrm{C}$ & 7.70397 & -0.93388 & 4.16413 \\
\hline
\end{tabular}




$\begin{array}{lrrrrrrc}\mathrm{C} & -2.30166 & 4.18726 & -5.16428 & \mathrm{H} & -10.69611 & -1.02347 & 1.80247 \\ \mathrm{H} & 3.35746 & 4.60904 & -1.71522 & \mathrm{H} & 7.23042 & -0.76355 & 5.14901 \\ \mathrm{H} & 0.72822 & 8.59647 & -2.12664 & \mathrm{H} & 8.50159 & -1.68601 & 4.29296 \\ \mathrm{H} & 2.51000 & 6.85870 & -2.28626 & \mathrm{H} & 8.18506 & 0.01883 & 3.86773 \\ \mathrm{H} & -1.43773 & 6.14251 & 0.70914 & \mathrm{H} & -3.21276 & 4.72428 & -5.47978 \\ \mathrm{H} & -1.24114 & 8.23432 & -0.61335 & \mathrm{H} & -1.42760 & 4.77655 & -5.50275 \\ \mathrm{H} & 1.05431 & 4.20410 & 3.18058 & \mathrm{H} & -2.26925 & 3.22273 & -5.70596\end{array}$

\section{Catalyst axis inversion}

R_DAPa_H: $\mathbf{R}=\mathbf{H},\left(\boldsymbol{R}_{\mathrm{a}}, \boldsymbol{R}_{\mathrm{a}}, \boldsymbol{R}_{\mathrm{a}}\right)$

H $\quad-1.13386$

$\mathrm{H} \quad 5.17969$

H 7.49086

H $\quad 9.12118$

$\mathrm{H} \quad 5.90772$

$\mathrm{H} \quad 8.31176$

H 2.48916

$\mathrm{H} \quad 3.69833$

$\mathrm{H} \quad 0.86466$

$\mathrm{H} \quad 0.62760$

$\mathrm{H} \quad 2.06277$

H $\quad-0.10697$

$\mathrm{H} \quad 3.61771$

$\mathrm{H} \quad 6.18665$

$\mathrm{H} \quad 5.27545$

H 7.83509

$\mathrm{H} \quad-0.40690$

$\mathrm{H} \quad-3.94536$

$\mathrm{H} \quad-0.47550$

H $\quad-4.05635$

H -3.90536

$\mathrm{H} \quad-5.71684$

H $\quad-0.40773$

H -1.02805

H $\quad-6.21730$

$\mathrm{H} \quad-7.28781$

H $\quad-6.02506$

H $\quad-2.70806$

H $\quad-2.25948$

H $\quad-1.42824$

H $\quad-1.12859$

H $\quad-3.09835$

H $\quad-4.74412$

$\begin{array}{ll}\mathrm{H} & 1.55447\end{array}$

$\mathrm{H} \quad 0.95870$

H $\quad-4.64417$

$\mathrm{H} \quad-1.27362$

H $\quad-2.21015$

H $\quad-3.34834$

$\mathrm{H} \quad-4.25273$

$\mathrm{H} \quad 2.26610$

$\begin{array}{ll}2.83835 & 0.70027\end{array}$

$3.92787 \quad 5.64218$

$2.59927 \quad 5.37252$

$2.50925 \quad 3.16344$

$3.10436 \quad 6.75003$

$\begin{array}{ll}3.50152 & -0.39230\end{array}$

$2.78146 \quad-0.84405$

$\begin{array}{ll}0.99299 & -1.44788\end{array}$

$\begin{array}{ll}-1.90103 & -1.62872\end{array}$

$\begin{array}{ll}-1.34684 & -1.86300\end{array}$

$\begin{array}{ll}-0.16209 & -3.06759\end{array}$

$\begin{array}{ll}-4.54289 & 0.81470\end{array}$

$-0.93029 \quad-4.77122$

$\begin{array}{ll}-4.80598 & -2.85128\end{array}$

$\begin{array}{ll}-5.30352 & -0.89371\end{array}$

$\begin{array}{ll}-3.24166 & -4.65274\end{array}$

$\begin{array}{ll}-0.45623 & 1.88670\end{array}$

$\begin{array}{ll}-3.71582 & 0.67895\end{array}$

$0.21192 \quad 3.60566$

$-3.06549 \quad 2.42036$

$2.38787-1.26466$

$4.81809 \quad-0.79504$

$\begin{array}{ll}2.75199 & -3.70509\end{array}$

$5.13086-3.25266$

$\begin{array}{ll}-4.52949 & -1.51708\end{array}$

$\begin{array}{ll}-1.27135 & -1.59975\end{array}$

$\begin{array}{ll}-7.17211 & -1.91763\end{array}$

$\begin{array}{ll}-5.21057 & 1.87922\end{array}$

$\begin{array}{ll}-3.89083 & -2.50600\end{array}$

$-3.07134 \quad-1.33830$

$\begin{array}{ll}-4.20273 & -0.76710\end{array}$

$-2.14176 \quad 1.10842$

$\begin{array}{ll}-0.34357 & 1.06795\end{array}$

$\begin{array}{ll}-2.20924 & -1.87178\end{array}$

$\begin{array}{ll}-0.59723 & -1.13884\end{array}$

$\begin{array}{ll}0.85283 & -0.82213\end{array}$

$\begin{array}{ll}0.22508 & -1.08177\end{array}$

$\begin{array}{ll}-8.41172 & -0.96338\end{array}$

$-6.39281 \quad 2.82131$

$0.26914 \quad 1.46924$

$0.17591-3.36406$

$\begin{array}{ll}-1.23837 & -3.91240\end{array}$

$1.34984-3.15681$

$\begin{array}{ll}-0.15974 & -3.47961\end{array}$

$\begin{array}{ll}-7.99404 & 1.40625\end{array}$

$\begin{array}{ll}-2.79931 & 1.25837\end{array}$

$\mathrm{H} \quad-5.06166$

H $\quad-7.24483$

$\begin{array}{ll}-3.63389 & 1.68149\end{array}$

$\mathrm{H} \quad-6.75171$

0.64601

1.21487

H $\quad-9.70696$

$-3.32328$

1.91176

H $\quad-9.19945$

0.95457

1.45427

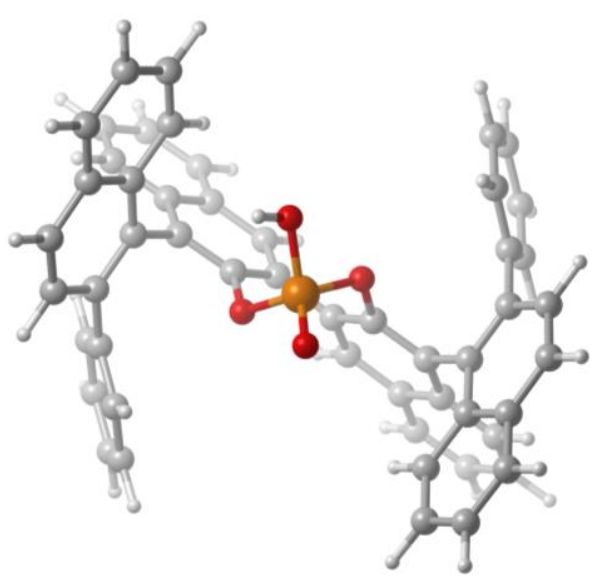

90

$\begin{array}{llll}\mathrm{C} & 4.69962 & 3.92831 & -2.00969 \\ \mathrm{C} & 3.33674 & 3.90919 & -1.76273 \\ \mathrm{C} & 2.72149 & 2.78510 & -1.13594 \\ \mathrm{C} & 3.53866 & 1.65438 & -0.77277 \\ \mathrm{C} & 4.93892 & 1.70692 & -1.03379 \\ \mathrm{C} & 5.50629 & 2.81886 & -1.63815 \\ \mathrm{H} & 0.70976 & 3.61088 & -1.15362 \\ \mathrm{H} & 5.15992 & 4.79963 & -2.48866 \\ \mathrm{H} & 2.70832 & 4.76351 & -2.04087 \\ \mathrm{C} & 1.32792 & 2.75713 & -0.85546 \\ \mathrm{C} & 2.92400 & 0.52351 & -0.14131 \\ \mathrm{H} & 5.56077 & 0.85280 & -0.74872 \\ \mathrm{H} & 6.58459 & 2.84219 & -1.83107 \\ \mathrm{C} & 1.55827 & 0.55813 & 0.11088 \\ \mathrm{C} & 0.72559 & 1.66725 & -0.23252 \\ \mathrm{C} & 3.71829 & -0.66984 & 0.28375 \\ \mathrm{C} & 4.15813 & -1.61733 & -0.70232 \\ \mathrm{C} & 3.95379 & -0.90439 & 1.64479 \\ \mathrm{C} & 3.90238 & -1.44150 & -2.09666 \\ \mathrm{C} & 4.85443 & -2.80808 & -0.27794 \\ \mathrm{C} & 4.63491 & -2.09342 & 2.04456 \\ \mathrm{C} & 4.32609 & -2.38114 & -3.02394 \\ \mathrm{H} & 3.35428 & -0.55553 & -2.42752 \\ \mathrm{C} & 5.28087 & -3.75255 & -1.25839 \\ \mathrm{C} & 5.07823 & -3.01490 & 1.11390 \\ \mathrm{H} & 4.81364 & -2.25807 & 3.11273 \\ \mathrm{C} & 5.02534 & -3.54596 & -2.60508 \\ \mathrm{H} & 4.11585 & -2.22642 & -4.08825\end{array}$




\begin{tabular}{|c|c|c|c|}
\hline $\mathrm{H}$ & 5.81269 & -4.65152 & -0.92406 \\
\hline $\mathrm{H}$ & 5.60760 & -3.91886 & 1.43796 \\
\hline $\mathrm{H}$ & 5.35673 & -4.28041 & -3.34795 \\
\hline $\mathrm{C}$ & -0.72175 & 1.67425 & 0.09496 \\
\hline $\mathrm{C}$ & -1.31885 & 2.79986 & 0.65606 \\
\hline $\mathrm{C}$ & -1.55501 & 0.54094 & -0.16150 \\
\hline $\mathrm{C}$ & -2.70645 & 2.83906 & 0.96191 \\
\hline $\mathrm{H}$ & -0.69930 & 3.67270 & 0.88894 \\
\hline $\mathrm{C}$ & -2.91415 & 0.51406 & 0.12051 \\
\hline $\mathrm{C}$ & -3.31544 & 3.99841 & 1.52763 \\
\hline $\mathrm{C}$ & -3.52156 & 1.68155 & 0.69282 \\
\hline $\mathrm{C}$ & -4.67212 & 4.02447 & 1.80537 \\
\hline $\mathrm{H}$ & -2.68729 & 4.87301 & 1.73435 \\
\hline $\mathrm{C}$ & -4.91545 & 1.74143 & 0.98427 \\
\hline $\mathrm{C}$ & -5.47745 & 2.88703 & 1.52770 \\
\hline $\mathrm{H}$ & -5.12839 & 4.92218 & 2.23723 \\
\hline $\mathrm{H}$ & -5.53571 & 0.86515 & 0.77376 \\
\hline $\mathrm{H}$ & -6.55078 & 2.91487 & 1.74595 \\
\hline $\mathrm{C}$ & -3.71900 & -0.70219 & -0.20567 \\
\hline C & -4.13537 & -1.57475 & 0.85492 \\
\hline $\mathrm{C}$ & -4.00970 & & 945 \\
\hline C & -3.81489 & $-1.31 \mathrm{c}$ & 2.22172 \\
\hline $\mathrm{C}$ & -4.88206 & -2.76587 & 0.53523 \\
\hline $\mathrm{C}$ & -4.73115 & -2.21023 & -1.83646 \\
\hline $\mathrm{C}$ & -4.23252 & -2.17997 & 3.22423 \\
\hline $\mathrm{H}$ & -3.22245 & -0.43456 & 2.47183 \\
\hline $\mathrm{C}$ & -5.30013 & -3.62879 & 1.59036 \\
\hline $\mathrm{C}$ & -5.16407 & -3.05477 & -0.83064 \\
\hline $\mathrm{H}$ & -4.95571 & -2.4 & 8422 \\
\hline $\mathrm{C}$ & -4.98706 & -3.34232 & 2.90963 \\
\hline $\mathrm{H}$ & -3.97088 & -1.96795 & 4.26707 \\
\hline $\mathrm{H}$ & -5.86967 & -4.53100 & 1.33640 \\
\hline $\mathrm{H}$ & -5.73009 & -3.96169 & -1.07494 \\
\hline $\mathrm{H}$ & -5.31079 & -4.01591 & 3.71134 \\
\hline $\mathrm{O}$ & -0.99646 & -0.56642 & -0.82209 \\
\hline $\mathrm{O}$ & 0.99527 & -0.51355 & 0.81832 \\
\hline $\mathrm{P}$ & 0.002 & -1.582 & 0.01820 \\
\hline $\mathrm{O}$ & 0.82858 & -2.11973 & -1.29727 \\
\hline $\mathrm{O}$ & -0.55192 & -2.60372 & 0.96297 \\
\hline $\mathrm{C}$ & -3.54325 & -0.14917 & -2.65804 \\
\hline $\mathrm{C}$ & -2.74236 & -0.69740 & -3.68139 \\
\hline $\mathrm{C}$ & -3.86089 & 1.22427 & -2.70976 \\
\hline $\mathrm{C}$ & -2.25576 & 0.10973 & -4.71962 \\
\hline $\mathrm{H}$ & -2.47042 & -1.75782 & -3.63692 \\
\hline C & -3.37722 & 2.03096 & -3.74925 \\
\hline $\mathrm{H}$ & -4.49389 & 1.66019 & -1.93096 \\
\hline C & -2.56821 & 1.47807 & -4.75522 \\
\hline $\mathrm{H}$ & -1.61948 & -0.32950 & -5.49679 \\
\hline $\mathrm{H}$ & -3.63573 & 3.09607 & -3.77382 \\
\hline $\mathrm{H}$ & -2.18412 & 2.11027 & -5.56420 \\
\hline C & 3.46194 & 0.03738 & 2.68844 \\
\hline $\mathrm{C}$ & 2.60751 & -0.43286 & 3.70709 \\
\hline C & 3.80534 & 1.40529 & 2.66753 \\
\hline $\mathrm{C}$ & 2.09560 & 0.44595 & 4.67215 \\
\hline $\mathrm{H}$ & 2.31055 & -1.48736 & 3.71334 \\
\hline $\mathrm{C}$ & 3.29693 & 2.28295 & 3.63515 \\
\hline $\mathrm{H}$ & 4.47742 & 1.78106 & 1.88995 \\
\hline
\end{tabular}

$\begin{array}{cccc}\mathrm{C} & 2.43633 & 1.80750 & 4.63793 \\ \mathrm{H} & 1.41720 & 0.06750 & 5.44541 \\ \mathrm{H} & 3.57548 & 3.34277 & 3.60533 \\ \mathrm{H} & 2.03274 & 2.49533 & 5.38994 \\ \mathrm{H} & 1.38878 & -2.87508 & -1.03643\end{array}$

S_DAPa_H R $=\mathbf{H},\left(R_{\mathrm{a}}, S_{\mathrm{a}}, R_{\mathrm{a}}\right)$

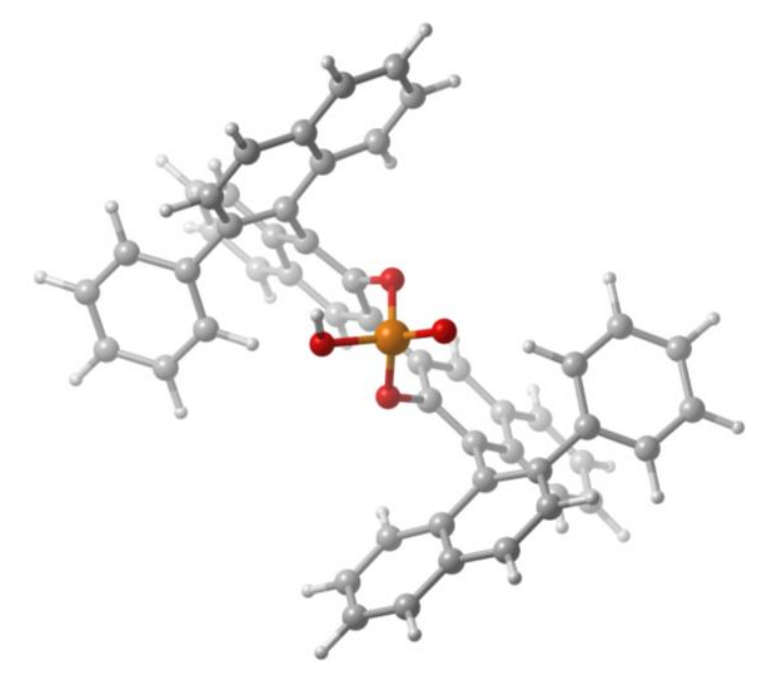

90

$\begin{array}{lrrr}\text { C } & -5.02693 & 0.11005 & 4.17736 \\ \mathrm{C} & -3.65000 & 0.25433 & 4.15848 \\ \mathrm{C} & -2.90709 & 0.01574 & 2.96434 \\ \mathrm{C} & -3.61016 & -0.35607 & 1.76273 \\ \mathrm{C} & -5.02454 & -0.51362 & 1.82079 \\ \mathrm{C} & -5.71612 & -0.28734 & 3.00038 \\ \mathrm{H} & -0.96621 & 0.43541 & 3.84674 \\ \mathrm{H} & -5.58724 & 0.29398 & 5.10084 \\ \mathrm{H} & -3.10711 & 0.54818 & 5.06455 \\ \mathrm{C} & -1.49182 & 0.13205 & 2.93482 \\ \mathrm{C} & -2.86831 & -0.55357 & 0.54850 \\ \mathrm{H} & -5.55755 & -0.82011 & 0.91695 \\ \mathrm{H} & -6.80406 & -0.41454 & 3.02470 \\ \mathrm{C} & -1.48118 & -0.46603 & 0.59754 \\ \mathrm{C} & -0.75415 & -0.12490 & 1.78116 \\ \mathrm{C} & -3.56845 & -0.81834 & -0.74523 \\ \mathrm{C} & -3.46360 & -2.11106 & -1.36335 \\ \mathrm{C} & -4.34766 & 0.18812 & -1.33598 \\ \mathrm{C} & -2.75019 & -3.19022 & -0.76087 \\ \mathrm{C} & -4.11991 & -2.34698 & -2.62529 \\ \mathrm{C} & -5.00382 & -0.07458 & -2.57650 \\ \mathrm{C} & -2.66288 & -4.42627 & -1.38235 \\ \mathrm{H} & -2.26910 & -3.03232 & 0.20872 \\ \mathrm{C} & -4.00291 & -3.62630 & -3.24420 \\ \mathrm{C} & -4.88156 & -1.29607 & -3.21132 \\ \mathrm{H} & -5.58950 & 0.72945 & -3.03519 \\ \mathrm{C} & -3.28637 & -4.64713 & -2.64014 \\ \mathrm{H} & -2.10817 & -5.23961 & -0.90060 \\ \mathrm{H} & -4.49863 & -3.78935 & -4.20887 \\ \mathrm{H} & -5.37348 & -1.47048 & -4.17572 \\ & & & \end{array}$




\begin{tabular}{|c|c|c|c|}
\hline $\mathrm{H}$ & -3.20500 & -5.62628 & -3.12581 \\
\hline C & 0.72998 & -0.08498 & 1.80770 \\
\hline C & 1.43308 & -0.66099 & 2.86441 \\
\hline $\mathrm{C}$ & 1.49233 & 0.51909 & 0.76077 \\
\hline $\mathrm{C}$ & 2.85186 & -0.63158 & 2.93004 \\
\hline $\mathrm{H}$ & 0.87725 & -1.16610 & 3.66180 \\
\hline C & 2.88419 & 0.50631 & 0.72699 \\
\hline $\mathrm{C}$ & 3.56099 & -1.20302 & 4.02795 \\
\hline $\mathrm{C}$ & 3.59520 & -0.03269 & 1.85117 \\
\hline $\mathrm{C}$ & 4.94466 & -1.17311 & 4.07107 \\
\hline $\mathrm{H}$ & 2.98736 & -1.66562 & 4.83971 \\
\hline $\mathrm{C}$ & 5.01678 & -0.00442 & 1.93340 \\
\hline C & 5.67590 & -0.56113 & 3.01794 \\
\hline $\mathrm{H}$ & 5.47872 & -1.61547 & 4.91936 \\
\hline $\mathrm{H}$ & 5.58204 & 0.46714 & 1.12481 \\
\hline $\mathrm{H}$ & 6.77012 & -0.52945 & 3.06310 \\
\hline $\mathrm{C}$ & 3.60418 & 0.95420 & -0.50297 \\
\hline $\mathrm{C}$ & 3.59038 & 2.33849 & -0.88721 \\
\hline $\mathrm{C}$ & 4.27586 & 0.00530 & -1.29364 \\
\hline C & 2.99563 & 3.34738 & -0.07160 \\
\hline $\mathrm{C}$ & 4.21108 & 2.73883 & -2.12553 \\
\hline C & 4.89669 & 0.42936 & -2.50930 \\
\hline $\mathrm{C}$ & 2.99082 & 4.67515 & -0.46793 \\
\hline $\mathrm{H}$ & 2.53876 & 3.05841 & 0.87915 \\
\hline C & 4.17886 & 4.11139 & -2.51138 \\
\hline $\mathrm{C}$ & 4.84966 & 1.74786 & -2.92453 \\
\hline $\mathrm{H}$ & 5.39506 & -0.32449 & -3.12867 \\
\hline $\mathrm{C}$ & 3.57904 & 5.06280 & -1.70235 \\
\hline $\mathrm{H}$ & 2.52726 & 5.43279 & 0.17392 \\
\hline $\mathrm{H}$ & 4.64530 & 4.40114 & -3.46068 \\
\hline $\mathrm{H}$ & 5.31238 & 2.04634 & -3.87295 \\
\hline $\mathrm{H}$ & 3.56120 & 6.11481 & -2.00873 \\
\hline $\mathrm{O}$ & 0.81215 & 1.21492 & -0.25232 \\
\hline $\mathrm{O}$ & -0.75472 & -0.80343 & -0.56455 \\
\hline $\mathrm{P}$ & -0.03556 & 0.40795 & -1.42933 \\
\hline $\mathrm{O}$ & 1.02713 & -0.55488 & -2.22946 \\
\hline $\mathrm{O}$ & -0.84877 & 1.36442 & -2.24756 \\
\hline C & 4.41425 & -1.42652 & -0.89570 \\
\hline $\mathrm{C}$ & 3.31148 & -2.23310 & -0.54537 \\
\hline $\mathrm{C}$ & 5.70573 & -1.99512 & -0.84543 \\
\hline $\mathrm{C}$ & 3.49921 & -3.56161 & -0.14160 \\
\hline $\mathrm{H}$ & 2.30237 & -1.82481 & -0.60521 \\
\hline $\mathrm{C}$ & 5.89371 & -3.32297 & -0.43710 \\
\hline $\mathrm{H}$ & 6.57395 & -1.37610 & -1.09973 \\
\hline $\mathrm{C}$ & 4.78947 & -4.11124 & -0.07847 \\
\hline $\mathrm{H}$ & 2.62753 & -4.17157 & 0.12202 \\
\hline $\mathrm{H}$ & 6.90679 & -3.73920 & -0.39045 \\
\hline $\mathrm{H}$ & 4.93343 & -5.14858 & 0.24495 \\
\hline $\mathrm{C}$ & -4.55656 & 1.51315 & -0.68518 \\
\hline $\mathrm{C}$ & -3.48010 & 2.34254 & -0.31107 \\
\hline C & -5.87145 & 1.94403 & -0.40780 \\
\hline C & -3.71388 & 3.55692 & 0.34821 \\
\hline $\mathrm{H}$ & -2.46125 & 2.04817 & -0.56854 \\
\hline C & -6.10489 & 3.15718 & 0.25518 \\
\hline $\mathrm{H}$ & -6.71591 & 1.30354 & -0.68841 \\
\hline $\mathrm{C}$ & -5.02459 & 3.96510 & 0.64325 \\
\hline $\mathrm{H}$ & -2.86397 & 4.19089 & 0.62634 \\
\hline
\end{tabular}

$\begin{array}{rrrr}\mathrm{H} & -7.13280 & 3.46735 & 0.47695 \\ \mathrm{H} & -5.20413 & 4.91188 & 1.16584 \\ \mathrm{H} & 1.46022 & -0.02947 & -2.92860\end{array}$

TS_inversion _DAPa_H

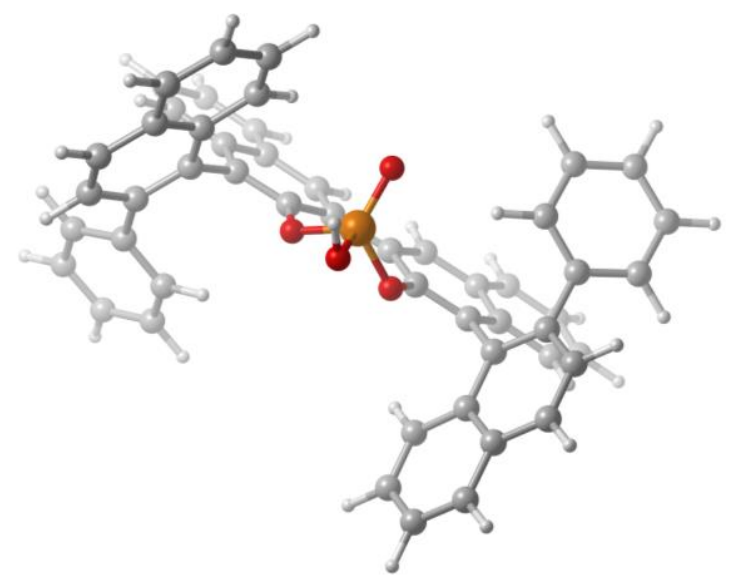

90

$\begin{array}{lrrr}\text { C } & -4.68677 & 4.42090 & 0.11660 \\ \mathrm{C} & -3.34454 & 4.22168 & -0.15499 \\ \mathrm{C} & -2.75983 & 2.92597 & -0.01673 \\ \mathrm{C} & -3.58397 & 1.82675 & 0.39319 \\ \mathrm{C} & -4.96033 & 2.06153 & 0.67739 \\ \mathrm{C} & -5.49724 & 3.33209 & 0.54267 \\ \mathrm{H} & -0.82994 & 3.58798 & -0.57874 \\ \mathrm{H} & -5.12819 & 5.41791 & 0.00958 \\ \mathrm{H} & -2.70960 & 5.05624 & -0.47582 \\ \mathrm{C} & -1.38583 & 2.70514 & -0.26549 \\ \mathrm{C} & -2.97822 & 0.53810 & 0.46406 \\ \mathrm{H} & -5.58530 & 1.22473 & 1.00131 \\ \mathrm{H} & -6.55671 & 3.50056 & 0.76553 \\ \mathrm{C} & -1.61170 & 0.38131 & 0.22236 \\ \mathrm{C} & -0.72451 & 1.47240 & -0.12726 \\ \mathrm{C} & -3.82966 & -0.65677 & 0.76164 \\ \mathrm{C} & -3.79059 & -1.25468 & 2.06596 \\ \mathrm{C} & -4.71662 & -1.13622 & -0.21146 \\ \mathrm{C} & -2.93141 & -0.77336 & 3.09871 \\ \mathrm{C} & -4.65674 & -2.36921 & 2.35931 \\ \mathrm{C} & -5.57851 & -2.23034 & 0.10468 \\ \mathrm{C} & -2.91053 & -1.37352 & 4.34833 \\ \mathrm{H} & -2.28110 & 0.08166 & 2.89070 \\ \mathrm{C} & -4.60918 & -2.96665 & 3.65316 \\ \mathrm{C} & -5.54529 & -2.83476 & 1.34767 \\ \mathrm{H} & -6.25877 & -2.59909 & -0.67100 \\ \mathrm{C} & -3.75242 & -2.48332 & 4.62957 \\ \mathrm{H} & -2.23940 & -0.99036 & 5.12554 \\ \mathrm{H} & -5.26816 & -3.81805 & 3.86259 \\ \mathrm{H} & -6.20292 & -3.68412 & 1.56849 \\ \mathrm{H} & -3.72455 & -2.95212 & 5.61987 \\ \mathrm{C} & 0.78042 & 1.45418 & -0.34854 \\ \mathrm{C} & 1.43364 & 2.66896 & -0.61630 \\ \mathrm{C} & 1.67492 & 0.32260 & -0.29452\end{array}$




\begin{tabular}{|c|c|c|c|}
\hline C & 2.82312 & 2.80678 & -0.84776 \\
\hline $\mathrm{H}$ & 0.86892 & 3.59989 & -0.65114 \\
\hline C & 3.05330 & 0.39677 & -0.49470 \\
\hline C & 3.40750 & 4.08007 & -1.12479 \\
\hline C & 3.66661 & 1.64717 & -0.80142 \\
\hline C & 4.76665 & 4.19683 & -1.35849 \\
\hline $\mathrm{H}$ & 2.75867 & 4.96361 & -1.15258 \\
\hline C & 5.06026 & 1.79362 & -1.05734 \\
\hline C & 5.59651 & 3.04201 & -1.33082 \\
\hline $\mathrm{H}$ & 5.20649 & 5.17740 & -1.57152 \\
\hline $\mathrm{H}$ & 5.69866 & 0.90603 & -1.03627 \\
\hline $\mathrm{H}$ & 6.66968 & 3.14121 & -1.52843 \\
\hline $\mathrm{C}$ & 3.89647 & -0.82801 & -0.31984 \\
\hline C & 3.88157 & -1.86539 & -1.31237 \\
\hline C & 4.72543 & -0.93907 & 0.80441 \\
\hline C & 3.05955 & -1.79252 & -2.47596 \\
\hline C & 4.71356 & -3.02975 & -1.13156 \\
\hline C & 5.57011 & -2.08184 & 0.94635 \\
\hline $\mathrm{C}$ & 3.02960 & -2.83047 & -3.39557 \\
\hline $\mathrm{H}$ & 2.43349 & -0.91069 & -2.63468 \\
\hline $\mathrm{C}$ & 4.66692 & -4.07559 & -2.09968 \\
\hline C & 5.56025 & -3.10084 & 0.01232 \\
\hline $\mathrm{H}$ & 6.20857 & -2.15226 & 1.83395 \\
\hline $\mathrm{C}$ & 3.83673 & -3.98496 & -3.20680 \\
\hline $\mathrm{H}$ & 2.37597 & -2.75648 & -4.27180 \\
\hline $\mathrm{H}$ & 5.30175 & -4.95750 & -1.95032 \\
\hline $\mathrm{H}$ & 6.19720 & -3.98320 & 0.14794 \\
\hline $\mathrm{H}$ & 3.80676 & -4.79850 & -3.94089 \\
\hline $\mathrm{O}$ & 1.25953 & -0.96276 & 0.07036 \\
\hline $\mathrm{O}$ & -1.22208 & -0.96059 & 0.35738 \\
\hline$P$ & -0.08646 & -1.60238 & -0.63615 \\
\hline $\mathrm{O}$ & -0.06385 & -3.12211 & -0.04405 \\
\hline $\mathrm{O}$ & -0.24727 & -1.41120 & -2.11912 \\
\hline $\mathrm{C}$ & 4.75044 & 0.11261 & 1.85977 \\
\hline C & 3.57116 & 0.50197 & 2.52897 \\
\hline C & 5.96392 & 0.74257 & 2.20450 \\
\hline C & 3.60272 & 1.51164 & 3.50046 \\
\hline $\mathrm{H}$ & 2.62889 & -0.00022 & 2.29136 \\
\hline C & 5.99573 & 1.75457 & 3.17515 \\
\hline $\mathrm{H}$ & 6.88453 & 0.45242 & 1.68497 \\
\hline C & 4.81348 & 2.14641 & 3.82248 \\
\hline $\mathrm{H}$ & 2.67706 & 1.80153 & 4.01111 \\
\hline $\mathrm{H}$ & 6.94544 & 2.24427 & 3.42000 \\
\hline $\mathrm{H}$ & 4.83617 & 2.94008 & 4.57811 \\
\hline C & -4.81065 & -0.50590 & -1.55832 \\
\hline $\mathrm{C}$ & -3.67973 & -0.37294 & -2.39025 \\
\hline $\mathrm{C}$ & -6.05100 & -0.01433 & -2.01691 \\
\hline $\mathrm{C}$ & -3.78380 & 0.25598 & -3.63848 \\
\hline $\mathrm{H}$ & -2.71500 & -0.77658 & -2.07485 \\
\hline $\mathrm{C}$ & -6.15456 & 0.61738 & -3.26477 \\
\hline $\mathrm{H}$ & -6.93411 & -0.10464 & -1.37354 \\
\hline C & -5.01884 & 0.75933 & -4.07794 \\
\hline $\mathrm{H}$ & -2.89234 & 0.34582 & -4.26964 \\
\hline $\mathrm{H}$ & -7.12333 & 1.00700 & -3.59889 \\
\hline $\mathrm{H}$ & -5.09781 & 1.25561 & -5.05222 \\
\hline $\mathrm{H}$ & 0.24677 & -3.71249 & -0.75680 \\
\hline
\end{tabular}

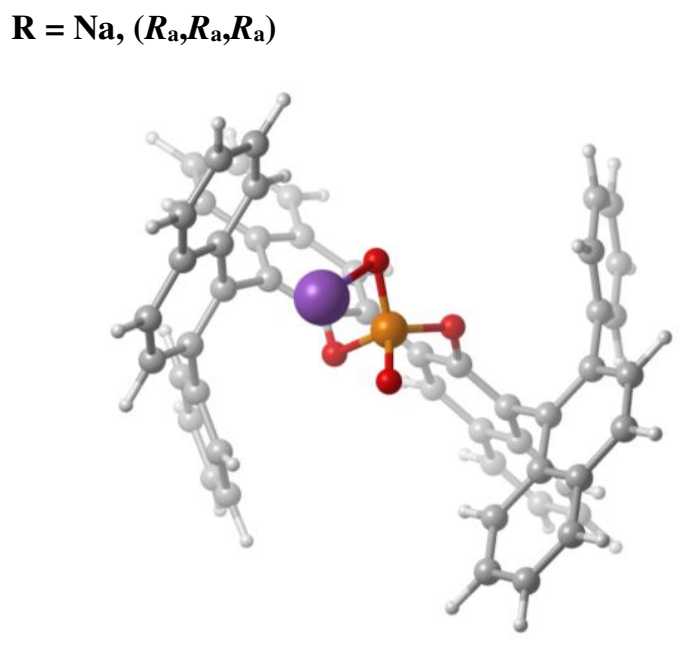

\begin{tabular}{lrrr}
90 & & & \\
O 1 & & & \\
$\mathrm{C}$ & -4.58475 & 4.12443 & 1.77010 \\
$\mathrm{C}$ & -3.25758 & 4.10538 & 1.37296 \\
$\mathrm{C}$ & -2.67376 & 2.92495 & 0.82529 \\
$\mathrm{C}$ & -3.48040 & 1.73836 & 0.69790 \\
$\mathrm{C}$ & -4.84424 & 1.79099 & 1.11076 \\
$\mathrm{C}$ & -5.38343 & 2.95692 & 1.63430 \\
$\mathrm{H}$ & -0.69525 & 3.77868 & 0.54649 \\
$\mathrm{H}$ & -5.02222 & 5.03857 & 2.18704 \\
$\mathrm{H}$ & -2.63382 & 5.00223 & 1.46994 \\
$\mathrm{C}$ & -1.31548 & 2.88594 & 0.40871 \\
$\mathrm{C}$ & -2.89688 & 0.55358 & 0.14109 \\
$\mathrm{H}$ & -5.46016 & 0.89221 & 1.00937 \\
$\mathrm{H}$ & -6.43365 & 2.97753 & 1.94656 \\
$\mathrm{C}$ & -1.56486 & 0.57168 & -0.26222 \\
$\mathrm{C}$ & -0.73912 & 1.73841 & -0.12907 \\
$\mathrm{C}$ & -3.70156 & -0.69171 & -0.04837 \\
$\mathrm{C}$ & -3.98470 & -1.52808 & 1.08278 \\
$\mathrm{C}$ & -4.11964 & -1.06987 & -1.32987 \\
$\mathrm{C}$ & -3.53864 & -1.20262 & 2.39877 \\
$\mathrm{C}$ & -4.72642 & -2.74966 & 0.89159 \\
$\mathrm{C}$ & -4.83049 & -2.29511 & -1.50325 \\
$\mathrm{C}$ & -3.83314 & -2.02479 & 3.47500 \\
$\mathrm{H}$ & -2.95024 & -0.29286 & 2.54824 \\
$\mathrm{C}$ & -5.01548 & -3.57202 & 2.02008 \\
$\mathrm{C}$ & -5.13492 & -3.10660 & -0.42537 \\
$\mathrm{H}$ & -5.15229 & -2.57401 & -2.51298 \\
$\mathrm{C}$ & -4.58299 & -3.21768 & 3.28833 \\
$\mathrm{H}$ & -3.47881 & -1.75717 & 4.47713 \\
$\mathrm{H}$ & -5.58496 & -4.49655 & 1.86386 \\
$\mathrm{H}$ & -5.69702 & -4.03686 & -0.57320 \\
$\mathrm{H}$ & -4.81057 & -3.85920 & 4.14765 \\
$\mathrm{C}$ & 0.70007 & 1.73839 & -0.49769 \\
$\mathrm{C}$ & 1.29668 & 2.79444 & -1.18105 \\
$\mathrm{C}$ & 1.54106 & 0.66823 & -0.06253 \\
$\mathrm{C}$ & 2.69719 & 2.82553 & -1.43491 \\
$\mathrm{H}$ & 0.67467 & 3.62001 & -1.54464 \\
$\mathrm{C}$ & 2.91932 & 0.66229 & -0.25707 \\
$\mathrm{C}$ & 3.30816 & 3.90494 & -2.13912 \\
& & & \\
\hline
\end{tabular}




\begin{tabular}{|c|c|c|c|}
\hline $\mathrm{C}$ & 3.53076 & 1.74837 & -0.95649 \\
\hline $\mathrm{C}$ & 4.67661 & 3.93354 & -2.35649 \\
\hline $\mathrm{H}$ & 2.67073 & 4.71918 & -2.50413 \\
\hline $\mathrm{C}$ & 4.93598 & 1.81023 & -1.18824 \\
\hline C & 5.49662 & 2.87777 & -1.87419 \\
\hline $\mathrm{H}$ & 5.13033 & 4.77116 & -2.89794 \\
\hline $\mathrm{H}$ & 5.56740 & 0.99635 & -0.81606 \\
\hline $\mathrm{H}$ & 6.57855 & 2.90757 & -2.04590 \\
\hline $\mathrm{C}$ & 3.69214 & -0.47065 & 0.34268 \\
\hline $\mathrm{C}$ & 4.11552 & -1.57454 & -0.47256 \\
\hline $\mathrm{C}$ & 3.83910 & -0.52957 & 1.73541 \\
\hline $\mathrm{C}$ & 3.94120 & -1.57728 & -1.89023 \\
\hline $\mathrm{C}$ & 4.65693 & -2.75839 & 0.15881 \\
\hline $\mathrm{C}$ & 4.40365 & -1.69287 & 2.33958 \\
\hline $\mathrm{C}$ & 4.27801 & -2.68673 & -2.65052 \\
\hline $\mathrm{H}$ & 3.49860 & -0.69862 & -2.36440 \\
\hline $\mathrm{C}$ & 4.98721 & -3.88990 & -0.65315 \\
\hline $\mathrm{C}$ & 4.79751 & -2.77969 & 1.57887 \\
\hline $\mathrm{H}$ & 4.51420 & -1.71373 & 3.42940 \\
\hline $\mathrm{C}$ & 4.79906 & -3.85648 & -2.03010 \\
\hline $\mathrm{H}$ & 4.12102 & -2.67037 & -3.73431 \\
\hline $\mathrm{H}$ & 5.40936 & -4.78022 & -0.16949 \\
\hline $\mathrm{H}$ & 5.22304 & -3.66956 & 2.05901 \\
\hline $\mathrm{H}$ & 5.06278 & -4.72748 & -2.64158 \\
\hline $\mathrm{O}$ & 0.96840 & -0.37778 & 0.63882 \\
\hline $\mathrm{O}$ & -1.07896 & -0.54770 & -0.92248 \\
\hline $\mathrm{P}$ & 0.13020 & -1.53264 & -0.29737 \\
\hline $\mathrm{O}$ & -0.29681 & -2.61924 & 0.69747 \\
\hline $\mathrm{O}$ & 0.98048 & -1.99320 & -1.49705 \\
\hline C & 3.32935 & 0.56808 & 2.60345 \\
\hline $\mathrm{C}$ & 2.34443 & 0.28802 & 3.57226 \\
\hline C & 3.77759 & 1.89593 & 2.44929 \\
\hline $\mathrm{C}$ & 1.80996 & 1.31562 & 4.36263 \\
\hline $\mathrm{H}$ & 1.96284 & -0.73426 & 3.66728 \\
\hline $\mathrm{C}$ & 3.24837 & 2.92134 & 3.24478 \\
\hline $\mathrm{H}$ & 4.54174 & 2.12400 & 1.69983 \\
\hline $\mathrm{C}$ & 2.25934 & 2.63554 & 4.20029 \\
\hline $\mathrm{H}$ & 1.02831 & 1.08632 & 5.09595 \\
\hline $\mathrm{H}$ & 3.60631 & 3.94898 & 3.11326 \\
\hline $\mathrm{H}$ & 1.83780 & 3.43977 & 4.81431 \\
\hline $\mathrm{C}$ & -3.80318 & -0.23842 & -2.52468 \\
\hline $\mathrm{C}$ & -3.08479 & -0.80019 & -3.60005 \\
\hline $\mathrm{C}$ & -4.19103 & 1.11527 & -2.60288 \\
\hline $\mathrm{C}$ & -2.75098 & -0.02576 & -4.72021 \\
\hline $\mathrm{H}$ & -2.75159 & -1.84163 & -3.53267 \\
\hline $\mathrm{C}$ & -3.86090 & 1.88914 & -3.72430 \\
\hline $\mathrm{H}$ & -4.75711 & 1.56160 & -1.77991 \\
\hline $\mathrm{C}$ & -3.13655 & 1.32266 & -4.78574 \\
\hline $\mathrm{H}$ & -2.17444 & -0.47343 & -5.53812 \\
\hline $\mathrm{H}$ & -4.17119 & 2.93978 & -3.76810 \\
\hline $\mathrm{H}$ & -2.87187 & 1.92976 & -5.65937 \\
\hline $\mathrm{Na}$ & 1.69127 & -3.81083 & -0.29983 \\
\hline
\end{tabular}

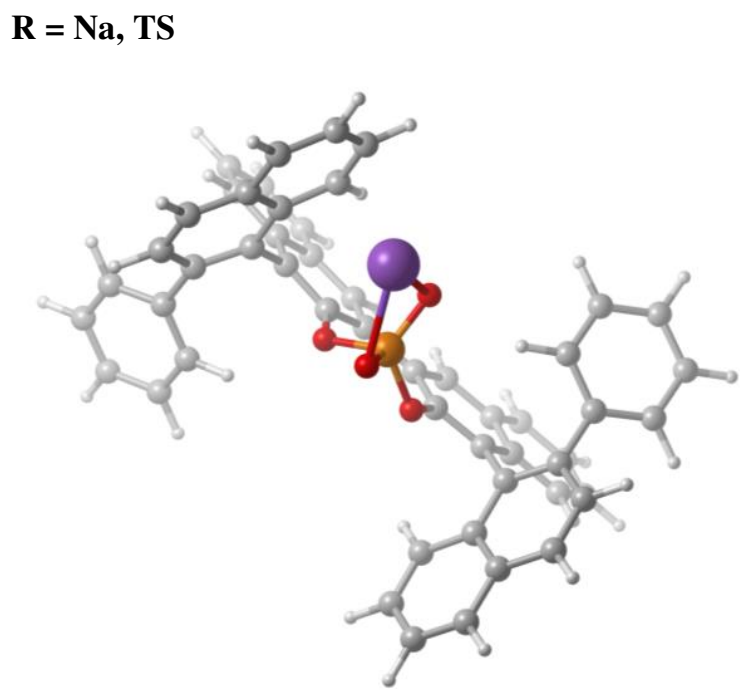

\begin{tabular}{lrrr}
90 & & & \\
O 1 & & & \\
$\mathrm{C}$ & -4.80212 & 4.39726 & -0.83701 \\
$\mathrm{C}$ & -3.43464 & 4.21356 & -0.94504 \\
$\mathrm{C}$ & -2.82875 & 2.99286 & -0.51820 \\
$\mathrm{C}$ & -3.65472 & 1.95065 & 0.01725 \\
$\mathrm{C}$ & -5.05947 & 2.16890 & 0.12493 \\
$\mathrm{C}$ & -5.61814 & 3.36658 & -0.29249 \\
$\mathrm{H}$ & -0.87732 & 3.61332 & -1.04835 \\
$\mathrm{H}$ & -5.25997 & 5.33679 & -1.16621 \\
$\mathrm{H}$ & -2.79580 & 5.00356 & -1.35834 \\
$\mathrm{C}$ & -1.43447 & 2.78354 & -0.61458 \\
$\mathrm{C}$ & -3.01942 & 0.73347 & 0.39543 \\
$\mathrm{H}$ & -5.68847 & 1.37713 & 0.54160 \\
$\mathrm{H}$ & -6.69918 & 3.52233 & -0.20273 \\
$\mathrm{C}$ & -1.63001 & 0.58225 & 0.30835 \\
$\mathrm{C}$ & -0.75630 & 1.62263 & -0.20532 \\
$\mathrm{C}$ & -3.86345 & -0.41610 & 0.85226 \\
$\mathrm{C}$ & -3.95830 & -0.72467 & 2.25059 \\
$\mathrm{C}$ & -4.61836 & -1.13663 & -0.08250 \\
$\mathrm{C}$ & -3.23943 & 0.01048 & 3.23957 \\
$\mathrm{C}$ & -4.81715 & -1.79825 & 2.68416 \\
$\mathrm{C}$ & -5.47441 & -2.18790 & 0.36792 \\
$\mathrm{C}$ & -3.34723 & -0.30707 & 4.58465 \\
$\mathrm{H}$ & -2.59317 & 0.83313 & 2.91948 \\
$\mathrm{C}$ & -4.90275 & -2.10354 & 4.07428 \\
$\mathrm{C}$ & -5.56579 & -2.51634 & 1.70772 \\
$\mathrm{H}$ & -6.04888 & -2.74912 & -0.37772 \\
$\mathrm{C}$ & -4.18235 & -1.37607 & 5.00855 \\
$\mathrm{H}$ & -2.78225 & 0.26797 & 5.32732 \\
$\mathrm{H}$ & -5.55430 & -2.92687 & 4.39189 \\
$\mathrm{H}$ & -6.21649 & -3.33662 & 2.03442 \\
$\mathrm{H}$ & -4.25615 & -1.62150 & 6.07432 \\
$\mathrm{C}$ & 0.75124 & 1.60162 & -0.38491 \\
$\mathrm{C}$ & 1.39513 & 2.73817 & -0.90287 \\
$\mathrm{C}$ & 1.64920 & 0.50923 & -0.09354 \\
$\mathrm{C}$ & 2.78241 & 2.82909 & -1.17817 \\
$\mathrm{H}$ & 0.82554 & 3.64090 & -1.12376 \\
$\mathrm{C}$ & 3.01141 & 0.52651 & -0.40529 \\
& & & \\
\hline
\end{tabular}




\begin{tabular}{|c|c|c|c|}
\hline $\mathrm{C}$ & 3.36592 & 4.02395 & -1.69785 \\
\hline $\mathrm{C}$ & 3.62410 & 1.68781 & -0.95164 \\
\hline $\mathrm{C}$ & 4.71860 & 4.08315 & -1.98900 \\
\hline $\mathrm{H}$ & 2.72170 & 4.89559 & -1.86559 \\
\hline $\mathrm{C}$ & 5.01010 & 1.77025 & -1.27074 \\
\hline $\mathrm{C}$ & 5.54503 & 2.94494 & -1.77736 \\
\hline $\mathrm{H}$ & 5.15588 & 5.00559 & -2.38706 \\
\hline $\mathrm{H}$ & 5.64529 & 0.89474 & -1.10393 \\
\hline $\mathrm{H}$ & 6.61324 & 2.99874 & -2.01600 \\
\hline $\mathrm{C}$ & 3.80492 & -0.71692 & -0.15183 \\
\hline $\mathrm{C}$ & 3.73527 & -1.78842 & -1.10770 \\
\hline $\mathrm{C}$ & 4.60727 & -0.84804 & 0.98605 \\
\hline $\mathrm{C}$ & 3.01466 & -1.66042 & -2.33596 \\
\hline $\mathrm{C}$ & 4.42189 & -3.03078 & -0.83913 \\
\hline $\mathrm{C}$ & 5.31879 & -2.06668 & 1.21096 \\
\hline $\mathrm{C}$ & 2.94869 & -2.71285 & -3.24338 \\
\hline $\mathrm{H}$ & 2.50054 & -0.72028 & -2.55049 \\
\hline $\mathrm{C}$ & 4.30372 & -4.10678 & -1.77062 \\
\hline $\mathrm{C}$ & 5.20971 & -3.13775 & 0.34377 \\
\hline $\mathrm{H}$ & 5.93034 & -2.15235 & 2.11590 \\
\hline $\mathrm{C}$ & 3.58256 & -3.95688 & -2.95154 \\
\hline $\mathrm{H}$ & 2.40313 & -2.58346 & -4.18561 \\
\hline $\mathrm{H}$ & 4.81945 & -5.05005 & -1.55231 \\
\hline $\mathrm{H}$ & 5.73332 & -4.07799 & 0.55323 \\
\hline $\mathrm{H}$ & 3.53075 & -4.78124 & -3.67341 \\
\hline $\mathrm{O}$ & 1.27454 & -0.61991 & 0.60351 \\
\hline $\mathrm{O}$ & -1.22679 & -0.65745 & 0.78766 \\
\hline $\mathrm{P}$ & -0.02269 & -1.56107 & 0.08390 \\
\hline $\mathrm{O}$ & 0.10731 & -2.91558 & 0.78616 \\
\hline $\mathrm{O}$ & -0.09050 & -1.56595 & -1.45657 \\
\hline $\mathrm{C}$ & 4.74498 & 0.26304 & 1.96877 \\
\hline $\mathrm{C}$ & 3.62004 & 0.80119 & 2.62709 \\
\hline $\mathrm{C}$ & 6.01995 & 0.79701 & 2.24709 \\
\hline $\mathrm{C}$ & 3.76955 & 1.86361 & 3.52936 \\
\hline $\mathrm{H}$ & 2.63273 & 0.36985 & 2.44219 \\
\hline $\mathrm{C}$ & 6.16716 & 1.86343 & 3.14632 \\
\hline $\mathrm{H}$ & 6.89764 & 0.38777 & 1.73276 \\
\hline $\mathrm{C}$ & 5.04045 & 2.40276 & 3.78675 \\
\hline $\mathrm{H}$ & 2.88683 & 2.27016 & 4.03617 \\
\hline $\mathrm{H}$ & 7.16289 & 2.27846 & 3.34101 \\
\hline $\mathrm{H}$ & 5.15328 & 3.23833 & 4.48719 \\
\hline $\mathrm{C}$ & -4.58350 & -0.80736 & -1.53558 \\
\hline $\mathrm{C}$ & -3.37204 & -0.78170 & -2.25757 \\
\hline $\mathrm{C}$ & -5.78295 & -0.50166 & -2.21297 \\
\hline $\mathrm{C}$ & -3.36255 & -0.44035 & -3.61729 \\
\hline $\mathrm{H}$ & -2.43069 & -1.04278 & -1.76844 \\
\hline $\mathrm{C}$ & -5.77220 & -0.15889 & -3.57306 \\
\hline $\mathrm{H}$ & -6.72810 & -0.50683 & -1.65736 \\
\hline $\mathrm{C}$ & -4.55936 & -0.12203 & -4.27956 \\
\hline $\mathrm{H}$ & -2.40872 & -0.42445 & -4.15760 \\
\hline $\mathrm{H}$ & -6.71240 & 0.09050 & -4.07911 \\
\hline $\mathrm{H}$ & -4.54852 & 0.15279 & -5.34096 \\
\hline $\mathrm{Na}$ & 0.87945 & -3.76910 & -1.27652 \\
\hline
\end{tabular}

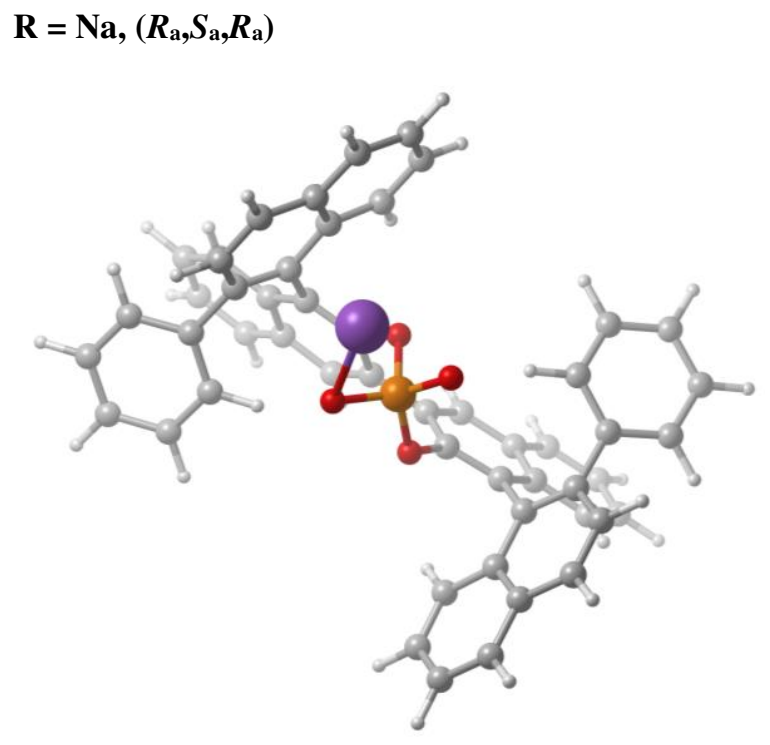

\begin{tabular}{lrrr}
90 & & & \\
O 1 & & & \\
$\mathrm{C}$ & -5.00795 & -1.75392 & 3.94018 \\
$\mathrm{C}$ & -3.62356 & -1.71938 & 3.93086 \\
$\mathrm{C}$ & -2.90765 & -1.43537 & 2.73048 \\
$\mathrm{C}$ & -3.64128 & -1.16471 & 1.52114 \\
$\mathrm{C}$ & -5.06470 & -1.22060 & 1.56184 \\
$\mathrm{C}$ & -5.73156 & -1.50965 & 2.74229 \\
$\mathrm{H}$ & -0.93750 & -1.55896 & 3.63364 \\
$\mathrm{H}$ & -5.54761 & -1.97480 & 4.86800 \\
$\mathrm{H}$ & -3.05421 & -1.91408 & 4.84767 \\
$\mathrm{C}$ & -1.48856 & -1.39953 & 2.70007 \\
$\mathrm{C}$ & -2.91997 & -0.85152 & 0.32017 \\
$\mathrm{H}$ & -5.62519 & -1.03445 & 0.64157 \\
$\mathrm{H}$ & -6.82662 & -1.54964 & 2.75137 \\
$\mathrm{C}$ & -1.52799 & -0.88724 & 0.33381 \\
$\mathrm{C}$ & -0.77682 & -1.14249 & 1.53030 \\
$\mathrm{C}$ & -3.65888 & -0.45807 & -0.91852 \\
$\mathrm{C}$ & -3.74385 & -1.36479 & -2.02804 \\
$\mathrm{C}$ & -4.31473 & 0.78040 & -0.96556 \\
$\mathrm{C}$ & -3.14007 & -2.65746 & -2.00233 \\
$\mathrm{C}$ & -4.48248 & -0.97767 & -3.20439 \\
$\mathrm{C}$ & -5.05437 & 1.13746 & -2.13292 \\
$\mathrm{C}$ & -3.23916 & -3.51444 & -3.08739 \\
$\mathrm{H}$ & -2.59011 & -2.96663 & -1.10899 \\
$\mathrm{C}$ & -4.55964 & -1.87893 & -4.30698 \\
$\mathrm{C}$ & -5.12787 & 0.29186 & -3.22435 \\
$\mathrm{H}$ & -5.54878 & 2.11497 & -2.15712 \\
$\mathrm{C}$ & -3.94950 & -3.12225 & -4.25436 \\
$\mathrm{H}$ & -2.76448 & -4.50159 & -3.04671 \\
$\mathrm{H}$ & -5.11824 & -1.57000 & -5.19911 \\
$\mathrm{H}$ & -5.68644 & 0.58976 & -4.12001 \\
$\mathrm{H}$ & -4.01691 & -3.80627 & -5.10829 \\
$\mathrm{C}$ & 0.70845 & -1.10794 & 1.56857 \\
$\mathrm{C}$ & 1.44598 & -2.05553 & 2.27326 \\
$\mathrm{C}$ & 1.42515 & -0.05549 & 0.91728 \\
$\mathrm{C}$ & 2.86063 & -1.96834 & 2.39067 \\
$\mathrm{H}$ & 0.92655 & -2.89298 & 2.75221 \\
& & & \\
\hline
\end{tabular}




\begin{tabular}{|c|c|c|c|}
\hline $\mathrm{C}$ & 2.81881 & 0.03523 & 0.93094 \\
\hline $\mathrm{C}$ & 3.60419 & -2.92214 & 3.14680 \\
\hline $\mathrm{C}$ & 3.56367 & -0.89850 & 1.72681 \\
\hline $\mathrm{C}$ & 4.98037 & -2.82058 & 3.26551 \\
\hline $\mathrm{H}$ & 3.06086 & -3.73747 & 3.63900 \\
\hline $\mathrm{C}$ & 4.97653 & -0.81294 & 1.88423 \\
\hline $\mathrm{C}$ & 5.66871 & -1.75001 & 2.63538 \\
\hline $\mathrm{H}$ & 5.53951 & -3.55929 & 3.85052 \\
\hline $\mathrm{H}$ & 5.51211 & 0.01002 & 1.40248 \\
\hline $\mathrm{H}$ & 6.75585 & -1.66411 & 2.74254 \\
\hline $\mathrm{C}$ & 3.49250 & 1.02569 & 0.03548 \\
\hline $\mathrm{C}$ & 3.27793 & 2.43845 & 0.21955 \\
\hline $\mathrm{C}$ & 4.27724 & 0.57231 & -1.04162 \\
\hline $\mathrm{C}$ & 2.54886 & 2.95685 & 1.33282 \\
\hline $\mathrm{C}$ & 3.80119 & 3.37890 & -0.74702 \\
\hline $\mathrm{C}$ & 4.82043 & 1.52117 & -1.96144 \\
\hline $\mathrm{C}$ & 2.31034 & 4.31502 & 1.46628 \\
\hline $\mathrm{H}$ & 2.17102 & 2.25745 & 2.08284 \\
\hline $\mathrm{C}$ & 3.52897 & 4.77320 & -0.58654 \\
\hline $\mathrm{C}$ & 4.57436 & 2.88022 & -1.84003 \\
\hline $\mathrm{H}$ & 5.41748 & 1.14497 & -2.79969 \\
\hline $\mathrm{C}$ & 2.79141 & 5.23341 & 0.49333 \\
\hline $\mathrm{H}$ & 1.74141 & 4.68482 & 2.32641 \\
\hline $\mathrm{H}$ & 3.93107 & 5.47615 & -1.32706 \\
\hline $\mathrm{H}$ & 4.99280 & 3.58879 & -2.56667 \\
\hline $\mathrm{H}$ & 2.59022 & 6.30463 & 0.60663 \\
\hline $\mathrm{O}$ & 0.69104 & 0.93082 & 0.28311 \\
\hline $\mathrm{O}$ & -0.86107 & -0.75595 & -0.88565 \\
\hline $\mathrm{P}$ & 0.05201 & 0.59837 & -1.26184 \\
\hline $\mathrm{O}$ & 1.17688 & 0.16131 & -2.21676 \\
\hline $\mathrm{O}$ & -0.70509 & 1.88000 & -1.63326 \\
\hline $\mathrm{C}$ & 4.57329 & -0.87080 & -1.27263 \\
\hline $\mathrm{C}$ & 3.54752 & -1.82980 & -1.39679 \\
\hline $\mathrm{C}$ & 5.91725 & -1.29201 & -1.35420 \\
\hline $\mathrm{C}$ & 3.86690 & -3.18272 & -1.57096 \\
\hline $\mathrm{H}$ & 2.50666 & -1.49920 & -1.38789 \\
\hline C & 6.23353 & -2.64735 & -1.52502 \\
\hline $\mathrm{H}$ & 6.72100 & -0.55344 & -1.24778 \\
\hline $\mathrm{C}$ & 5.20705 & -3.59906 & -1.62647 \\
\hline $\mathrm{H}$ & 3.05886 & -3.91689 & -1.66819 \\
\hline $\mathrm{H}$ & 7.28324 & -2.96044 & -1.56767 \\
\hline $\mathrm{H}$ & 5.45091 & -4.66026 & -1.75333 \\
\hline $\mathrm{C}$ & -4.29860 & 1.72155 & 0.19097 \\
\hline $\mathrm{C}$ & -3.09130 & 2.21439 & 0.72705 \\
\hline $\mathrm{C}$ & -5.51672 & 2.12464 & 0.77733 \\
\hline $\mathrm{C}$ & -3.10586 & 3.06959 & 1.83736 \\
\hline $\mathrm{H}$ & -2.14520 & 1.95795 & 0.24634 \\
\hline $\mathrm{C}$ & -5.52992 & 2.97951 & 1.88914 \\
\hline $\mathrm{H}$ & -6.46003 & 1.74018 & 0.37179 \\
\hline $\mathrm{C}$ & -4.32197 & 3.45009 & 2.42796 \\
\hline $\mathrm{H}$ & -2.15682 & 3.44565 & 2.23786 \\
\hline $\mathrm{H}$ & -6.48553 & 3.27005 & 2.34151 \\
\hline $\mathrm{H}$ & -4.32961 & 4.11375 & 3.30071 \\
\hline $\mathrm{Na}$ & 1.49653 & 2.43131 & -2.67621 \\
\hline
\end{tabular}

\section{Structures used for parameterization}

Catalyst $\mathrm{ArC}$ as benzoic acid derivatives

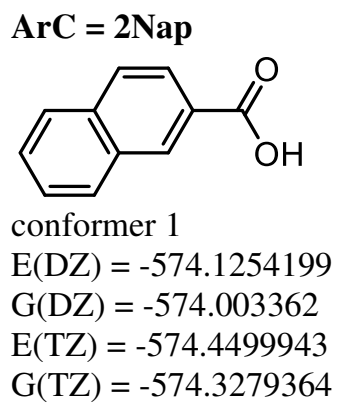

$\begin{array}{lrrr}21 & & & \\ 01 & & & \\ \mathrm{C} & 1.44980 & 0.13148 & -0.00001 \\ \mathrm{C} & 0.48467 & -0.87715 & -0.00001 \\ \mathrm{C} & 1.04597 & 1.50515 & 0.00000 \\ \mathrm{C} & -0.90372 & -0.55930 & 0.00000 \\ \mathrm{H} & 0.78783 & -1.92955 & -0.00001 \\ \mathrm{C} & -0.29694 & 1.83962 & 0.00001 \\ \mathrm{H} & 1.82311 & 2.27667 & 0.00000 \\ \mathrm{C} & -1.90841 & -1.57573 & 0.00000 \\ \mathrm{C} & -1.30791 & 0.82867 & 0.00001 \\ \mathrm{H} & -0.60417 & 2.89308 & 0.00001 \\ \mathrm{C} & -3.25549 & -1.23967 & 0.00001 \\ \mathrm{H} & -1.59448 & -2.62733 & -0.00000 \\ \mathrm{C} & -2.70024 & 1.14027 & 0.00002 \\ \mathrm{C} & -3.65337 & 0.12901 & 0.00002 \\ \mathrm{H} & -4.01902 & -2.02706 & 0.00001 \\ \mathrm{H} & -3.00628 & 2.19421 & 0.00002 \\ \mathrm{H} & -4.72072 & 0.38244 & 0.00002 \\ \mathrm{C} & 2.91281 & -0.16164 & -0.00001 \\ \mathrm{O} & 3.79648 & 0.68522 & -0.00002 \\ \mathrm{O} & 3.19816 & -1.50532 & -0.00002 \\ \mathrm{H} & 4.17361 & -1.56601 & -0.00003\end{array}$

conformer 2

$$
\begin{aligned}
& \mathrm{E}(\mathrm{DZ})=-574.1252421 \\
& \mathrm{G}(\mathrm{DZ})=-574.003104 \\
& \mathrm{E}(\mathrm{TZ})=-574.4497381 \\
& \mathrm{G}(\mathrm{TZ})=-574.3276
\end{aligned}
$$

$\begin{array}{lccc}21 & & & \\ 01 & & & \\ \mathrm{C} & -1.44470 & 0.06884 & -0.00001 \\ \mathrm{C} & -0.45513 & -0.91516 & -0.00000 \\ \mathrm{C} & -1.07354 & 1.45249 & -0.00000 \\ \mathrm{C} & 0.92438 & -0.56858 & 0.00000 \\ \mathrm{H} & -0.75186 & -1.97041 & -0.00000 \\ \mathrm{C} & 0.26352 & 1.81550 & 0.00000 \\ \mathrm{H} & -1.85451 & 2.21882 & -0.00001 \\ \mathrm{C} & 1.95185 & -1.56244 & 0.00001 \\ \mathrm{C} & 1.29696 & 0.82870 & 0.00001 \\ \mathrm{H} & 0.54653 & 2.87571 & 0.00000\end{array}$




$\begin{array}{lrrr}\mathrm{C} & 3.29056 & -1.19539 & 0.00001 \\ \mathrm{H} & 1.66141 & -2.62059 & 0.00001 \\ \mathrm{C} & 2.68204 & 1.17203 & 0.00001 \\ \mathrm{C} & 3.65729 & 0.18232 & 0.00001 \\ \mathrm{H} & 4.07201 & -1.96499 & 0.00002 \\ \mathrm{H} & 2.96452 & 2.23253 & 0.00001 \\ \mathrm{H} & 4.71870 & 0.45969 & 0.00002 \\ \mathrm{C} & -2.87046 & -0.37520 & -0.00001 \\ \mathrm{O} & -3.24914 & -1.53888 & -0.00001 \\ \mathrm{O} & -3.75683 & 0.67425 & -0.00001 \\ \mathrm{H} & -4.64567 & 0.26756 & -0.00002\end{array}$

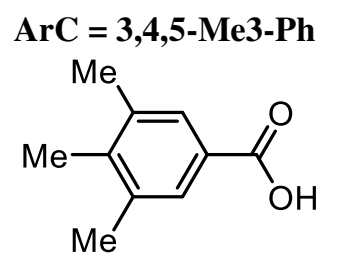

$$
\begin{aligned}
& E(D Z)=-538.4598736 \\
& G(D Z)=-538.30616 \\
& E(T Z)=-538.7468582 \\
& G(T Z)=-538.5931446
\end{aligned}
$$

$\begin{array}{lrrr}24 & & & \\ 01 & & & \\ \mathrm{C} & -0.36767 & -1.24064 & 0.00001 \\ \mathrm{C} & -0.43674 & 1.18901 & -0.00001 \\ \mathrm{C} & 1.03485 & -1.21268 & 0.00001 \\ \mathrm{H} & -0.90074 & -2.19809 & 0.00002 \\ \mathrm{C} & 0.96886 & 1.24549 & -0.00002 \\ \mathrm{H} & -1.00964 & 2.12205 & -0.00003 \\ \mathrm{C} & 1.71520 & 0.03664 & -0.00000 \\ \mathrm{C} & 1.81564 & -2.50990 & 0.00003 \\ \mathrm{H} & 2.47560 & -2.58285 & -0.88813 \\ \mathrm{H} & 2.47565 & -2.58279 & 0.88815 \\ \mathrm{H} & 1.13744 & -3.38173 & 0.00008 \\ \mathrm{C} & 3.22972 & 0.03938 & -0.00000 \\ \mathrm{H} & 3.62293 & -0.49880 & 0.88644 \\ \mathrm{H} & 3.62294 & -0.49896 & -0.88634 \\ \mathrm{H} & 3.65570 & 1.05513 & -0.00009 \\ \mathrm{C} & 1.65184 & 2.60089 & -0.00003 \\ \mathrm{H} & 2.29897 & 2.73037 & 0.89049 \\ \mathrm{H} & 2.29900 & 2.73034 & -0.89053 \\ \mathrm{H} & 0.90501 & 3.41521 & -0.00006 \\ \mathrm{C} & -1.11602 & -0.04626 & 0.00000 \\ \mathrm{C} & -2.60278 & -0.14712 & -0.00001 \\ \mathrm{O} & -3.24060 & -1.19252 & -0.00010 \\ \mathrm{O} & -3.22872 & 1.07769 & 0.00010 \\ \mathrm{H} & -4.18579 & 0.88003 & 0.00007\end{array}$

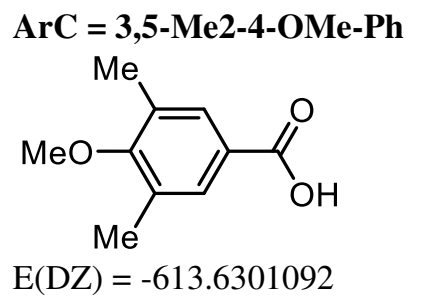

$$
\begin{aligned}
& G(D Z)=-613.47433 \\
& E(T Z)=-613.9592786 \\
& G(T Z)=-613.8034994
\end{aligned}
$$

25

01

$\begin{array}{llll}\text { C } & -1.41568 & -0.03823 & -0.00554\end{array}$

$\begin{array}{llll}\text { C } & -0.72970 & 1.19421 & -0.06674\end{array}$

$\begin{array}{llll}\text { C } & -0.68493 & -1.24373 & -0.06577\end{array}$

$\begin{array}{llll}\text { C } & 0.67044 & 1.23712 & -0.17749\end{array}$

$\begin{array}{llll}\mathrm{H} & -1.29186 & 2.13320 & -0.03288\end{array}$

$\begin{array}{llll}\text { C } & 0.71434 & -1.24050 & -0.17692\end{array}$

$\begin{array}{llll}\mathrm{H} & -1.22988 & -2.19379 & -0.03052\end{array}$

$\begin{array}{llll}\text { C } & 1.37460 & 0.01079 & -0.22270\end{array}$

$\begin{array}{llll}\text { O } & 2.75509 & 0.03791 & -0.37856\end{array}$

$\begin{array}{llll}\text { C } & 1.41174 & 2.55338 & -0.26543\end{array}$

$\begin{array}{llll}\mathrm{H} & 2.05385 & 2.71753 & 0.62265\end{array}$

$\begin{array}{llll}\mathrm{H} & 2.07971 & 2.57066 & -1.14742\end{array}$

$\begin{array}{llll}\mathrm{H} & 0.70198 & 3.39711 & -0.33457\end{array}$

$\begin{array}{llll}\text { C } & 1.49917 & -2.53123 & -0.26467\end{array}$

$\begin{array}{llll}\mathrm{H} & 2.19580 & -2.51042 & -1.12394\end{array}$

$\begin{array}{llll}\mathrm{H} & 2.11579 & -2.69335 & 0.64191\end{array}$

$\begin{array}{llll}\mathrm{H} & 0.81845 & -3.39449 & -0.37190\end{array}$

$\begin{array}{llll}\mathrm{C} & 3.46548 & 0.03447 & 0.87254\end{array}$

$\begin{array}{llll}\mathrm{H} & 3.23987 & -0.88232 & 1.45676\end{array}$

$\begin{array}{llll}\mathrm{H} & 4.54064 & 0.06004 & 0.62086\end{array}$

$\begin{array}{llll}\mathrm{H} & 3.20258 & 0.92491 & 1.48166\end{array}$

$\begin{array}{llll}\text { C } & -2.89892 & -0.12465 & 0.11204\end{array}$

$\begin{array}{llll}\mathrm{O} & -3.54407 & -1.16381 & 0.16958\end{array}$

$\begin{array}{llll}\text { O } & -3.51093 & 1.10622 & 0.15307\end{array}$

$\begin{array}{llll}\mathrm{H} & -4.46705 & 0.91871 & 0.22874\end{array}$

$\mathrm{ArC}=3,5-\mathrm{Me} 2-\mathrm{Ph}$

$\mathrm{Me}$

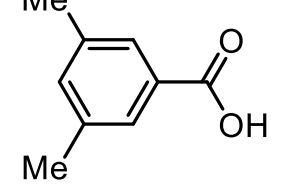

$\mathrm{E}(\mathrm{DZ})=-499.1684323$

$\mathrm{G}(\mathrm{DZ})=-499.043276$

$E(T Z)=-499.4366407$

$G(T Z)=-499.3114844$

$\begin{array}{lrrr}21 & & & \\ 01 & & & \\ \mathrm{H} & -0.55012 & -2.20093 & -0.00513 \\ \mathrm{C} & -0.01861 & -1.24279 & -0.00291 \\ \mathrm{C} & 1.38388 & -1.20407 & -0.00064 \\ \mathrm{C} & -0.11289 & 1.19926 & 0.00333 \\ \mathrm{C} & 2.02242 & 0.05692 & 0.00286 \\ \mathrm{C} & 1.29534 & 1.26191 & 0.00385 \\ \mathrm{H} & -0.69890 & 2.12451 & 0.00649 \\ \mathrm{H} & 3.12063 & 0.09973 & 0.00574 \\ \mathrm{C} & 1.99918 & 2.60365 & -0.00469 \\ \mathrm{H} & 1.81413 & 3.14148 & -0.95669 \\ \mathrm{H} & 1.62909 & 3.25291 & 0.81309 \\ \mathrm{H} & 3.09211 & 2.48650 & 0.11340\end{array}$




$\begin{array}{lrrr}\mathrm{C} & 2.20508 & -2.47695 & 0.00154 \\ \mathrm{H} & 1.55767 & -3.37220 & -0.02520 \\ \mathrm{H} & 2.88312 & -2.51394 & -0.87455 \\ \mathrm{H} & 2.84025 & -2.53779 & 0.90819 \\ \mathrm{C} & -0.77212 & -0.04664 & -0.00041 \\ \mathrm{C} & -2.26122 & -0.16207 & -0.00061 \\ \mathrm{O} & -2.88690 & -1.21394 & -0.00492 \\ \mathrm{O} & -2.89817 & 1.05570 & 0.00452 \\ \mathrm{H} & -3.85376 & 0.85022 & 0.00396\end{array}$

$\begin{array}{cccc}\mathrm{H} & -2.68518 & -1.32791 & 2.18242 \\ \mathrm{H} & -4.18569 & -1.71949 & -1.27374 \\ \mathrm{H} & -3.55810 & -0.04062 & -1.29272 \\ \mathrm{H} & -2.68496 & -1.32781 & -2.18243 \\ \mathrm{C} & -0.24318 & 3.16445 & -0.00001 \\ \mathrm{O} & 0.70801 & 3.93520 & -0.00003 \\ \mathrm{O} & -1.53964 & 3.62324 & 0.00002 \\ \mathrm{H} & -1.46969 & 4.59802 & 0.00001\end{array}$

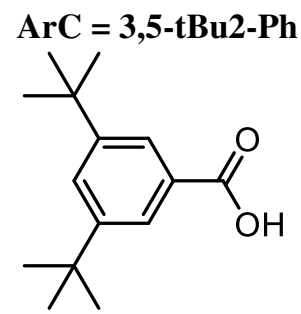

conformer 2

$$
\begin{aligned}
& E(D Z)=-734.8998081 \\
& G(D Z)=-734.61529 \\
& E(T Z)=-735.2818632 \\
& G(T Z)=-734.9973451
\end{aligned}
$$

conformer 1

$$
\begin{aligned}
& \mathrm{E}(\mathrm{DZ})=-734.8997729 \\
& \mathrm{G}(\mathrm{DZ})=-734.615431 \\
& \mathrm{E}(\mathrm{TZ})=-735.2819057 \\
& \mathrm{G}(\mathrm{TZ})=-734.9975638
\end{aligned}
$$

$\begin{array}{lrrr}39 & & & \\ \text { O } 1 & & & \\ \mathrm{C} & -0.01238 & 1.68138 & -0.00002 \\ \mathrm{C} & 1.22409 & 0.99397 & -0.00002 \\ \mathrm{C} & -1.22012 & 0.96160 & -0.00005 \\ \mathrm{C} & 1.25544 & -0.40925 & -0.00003 \\ \mathrm{H} & 2.14899 & 1.57392 & 0.00000 \\ \mathrm{C} & -1.22342 & -0.44767 & -0.00007 \\ \mathrm{H} & -2.15843 & 1.52478 & -0.00007 \\ \mathrm{C} & 0.02173 & -1.10343 & -0.00005 \\ \mathrm{H} & 0.04913 & -2.19654 & -0.00001 \\ \mathrm{C} & 2.56851 & -1.21486 & 0.00000 \\ \mathrm{C} & 2.61821 & -2.11159 & 1.26445 \\ \mathrm{C} & 2.61826 & -2.11159 & -1.26445 \\ \mathrm{C} & 3.81655 & -0.30726 & 0.00002 \\ \mathrm{H} & 2.58635 & -1.49372 & 2.18250 \\ \mathrm{H} & 1.76562 & -2.81586 & 1.29347 \\ \mathrm{H} & 3.55407 & -2.70454 & 1.27201 \\ \mathrm{H} & 2.58649 & -1.49372 & -2.18250 \\ \mathrm{H} & 3.55410 & -2.70457 & -1.27194 \\ \mathrm{H} & 1.76564 & -2.81583 & -1.29352 \\ \mathrm{H} & 4.72813 & -0.93400 & 0.00003 \\ \mathrm{H} & 3.84437 & 0.34100 & -0.89660 \\ \mathrm{H} & 3.84435 & 0.34099 & 0.89666 \\ \mathrm{C} & -2.56817 & -1.19991 & -0.00001 \\ \mathrm{C} & -2.38880 & -2.73234 & -0.00068 \\ \mathrm{C} & -3.37287 & -0.80295 & 1.26478 \\ \mathrm{C} & -3.37360 & -0.80195 & -1.26400 \\ \mathrm{H} & -1.83966 & -3.07530 & -0.89871 \\ \mathrm{H} & -1.83836 & -3.07594 & 0.89632 \\ \mathrm{H} & -3.38196 & -3.21963 & -0.00012 \\ \mathrm{H} & -3.56292 & 0.28606 & 1.29626 \\ \mathrm{H} & -4.35058 & -1.32410 & 1.26948 \\ \mathrm{H} & -2.82104 & -1.08363 & 2.18273 \\ \mathrm{H} & -4.35121 & -1.32328 & -1.26867 \\ \mathrm{H} & -3.56391 & 0.28705 & -1.29436 \\ \mathrm{H} & -2.82221 & -1.08166 & -2.18250 \\ \mathrm{C} & -0.09295 & 3.17247 & 0.00000 \\ \mathrm{O} & -1.12923 & 3.82454 & 0.00002 \\ \mathrm{O} & 1.13976 & 3.78140 & -0.00000 \\ \mathrm{H} & 0.95592 & 4.74131 & 0.00002\end{array}$




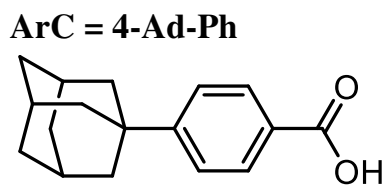

$\mathrm{E}(\mathrm{DZ})=-809.8625299$

$\mathrm{G}(\mathrm{DZ})=-809.575281$

$E(T Z)=-810.3110037$

$G(T Z)=-810.0237548$

39

01

$\begin{array}{llll}\text { C } & -3.33917 & 0.03677 & 0.00001\end{array}$

$\begin{array}{llll}\text { C } & -2.59960 & -1.16859 & 0.00022\end{array}$

$\begin{array}{llll}\text { C } & -2.64093 & 1.26184 & -0.00022\end{array}$

$\begin{array}{llll}\text { C } & -1.20100 & -1.13375 & 0.00021\end{array}$

$\begin{array}{llll}\mathrm{H} & -3.12391 & -2.12945 & 0.00040\end{array}$

$\begin{array}{llll}\mathrm{C} & -1.23901 & 1.28357 & -0.00024\end{array}$

$\begin{array}{llll}\mathrm{H} & -3.21384 & 2.19567 & -0.00039\end{array}$

$\begin{array}{llll}\text { C } & -0.48591 & 0.08820 & -0.00002\end{array}$

$\begin{array}{llll}\mathrm{H} & -0.64893 & -2.08043 & 0.00039\end{array}$

$\begin{array}{llll}\mathrm{H} & -0.73213 & 2.25312 & -0.00043\end{array}$

$\begin{array}{llll}\text { C } & 1.04432 & 0.06739 & -0.00003\end{array}$

$\begin{array}{llll}\text { C } & 1.56083 & -0.68699 & -1.26684\end{array}$

$\begin{array}{llll}\text { C } & 1.66918 & 1.48912 & -0.00032\end{array}$

$\begin{array}{llll}\text { C } & 1.56083 & -0.68646 & 1.26711\end{array}$

$\begin{array}{llll}\mathrm{H} & 1.20449 & -0.15597 & -2.17207\end{array}$

$\begin{array}{llll}\mathrm{H} & 1.13354 & -1.70773 & -1.29188\end{array}$

$\begin{array}{llll}\text { C } & 3.10513 & -0.76737 & -1.26470\end{array}$

$\begin{array}{llll}\mathrm{H} & 1.32314 & 2.04567 & 0.89313\end{array}$

$\begin{array}{llll}\mathrm{H} & 1.32314 & 2.04529 & -0.89401\end{array}$

$\begin{array}{llll}\text { C } & 3.21361 & 1.41543 & -0.00031\end{array}$

$\begin{array}{llll}\mathrm{H} & 1.13354 & -1.70719 & 1.29257\end{array}$

$\begin{array}{llll}\mathrm{H} & 1.20448 & -0.15506 & 2.17211\end{array}$

$\begin{array}{llll}\text { C } & 3.10512 & -0.76683 & 1.26500\end{array}$

$\begin{array}{llll}\mathrm{H} & 3.43995 & -1.30928 & -2.17070\end{array}$

$\begin{array}{llll}\text { C } & 3.69358 & 0.66298 & -1.26355\end{array}$

$\begin{array}{llll}\text { C } & 3.57897 & -1.52236 & 0.00031\end{array}$

$\begin{array}{llll}\mathrm{H} & 3.62299 & 2.44426 & -0.00053\end{array}$

$\begin{array}{llll}\text { C } & 3.69358 & 0.66352 & 1.26325\end{array}$

$\begin{array}{llll}\mathrm{H} & 3.43995 & -1.30836 & 2.17123\end{array}$

$\begin{array}{llll}\mathrm{H} & 3.36759 & 1.20570 & -2.17390\end{array}$

$\begin{array}{llll}\mathrm{H} & 4.80115 & 0.61701 & -1.27809\end{array}$

$\begin{array}{llll}\mathrm{H} & 3.16988 & -2.55336 & 0.00053\end{array}$

$\begin{array}{llll}\mathrm{H} & 4.68467 & -1.60208 & 0.00033\end{array}$

$\begin{array}{llll}\mathrm{H} & 4.80115 & 0.61756 & 1.27782\end{array}$

$\begin{array}{llll}\mathrm{H} & 3.36758 & 1.20662 & 2.17337\end{array}$

$\begin{array}{llll}\text { C } & -4.82918 & 0.07157 & 0.00001\end{array}$

$\begin{array}{llll}\mathrm{O} & -5.51204 & 1.08765 & -0.00015\end{array}$

$\begin{array}{llll}\mathrm{O} & -5.39764 & -1.18034 & 0.00022\end{array}$

$\begin{array}{llll}\mathrm{H} & -6.36313 & -1.02870 & 0.00021\end{array}$

\section{$\mathrm{ArC}=4-\mathrm{CF} 3-\mathrm{Ph}$}<smiles>O=C(O)c1ccc(C(F)(F)F)cc1</smiles>

$\mathrm{E}(\mathrm{DZ})=-757.4962102$

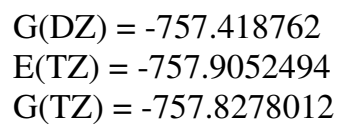

$\begin{array}{lccc}18 & & & \\ 01 & & & \\ \text { C } & -1.73978 & 0.03609 & -0.00350 \\ \text { C } & -1.03194 & -1.18755 & -0.00698 \\ \text { C } & -1.02812 & 1.25416 & -0.00707 \\ \text { C } & 0.36827 & -1.18756 & -0.01375 \\ \text { H } & -1.57897 & -2.13494 & -0.00513 \\ \text { C } & 0.37326 & 1.25602 & -0.01384 \\ \text { H } & -1.58882 & 2.19470 & -0.00496 \\ \text { C } & 1.07020 & 0.03409 & -0.01860 \\ \text { H } & 0.91942 & -2.13395 & -0.01874 \\ \text { H } & 0.92384 & 2.20162 & -0.01759 \\ \text { C } & 2.58543 & -0.00507 & -0.00046 \\ \text { F } & 3.05540 & -0.49603 & 1.19059 \\ \text { F } & 3.08185 & -0.82377 & -0.97925 \\ \text { F } & 3.15535 & 1.21984 & -0.17699 \\ \text { C } & -3.23634 & 0.09982 & 0.00318 \\ \text { O } & -3.88926 & 1.13251 & 0.00691 \\ \text { O } & -3.82177 & -1.13851 & 0.00486 \\ \text { H } & -4.78656 & -0.97976 & 0.00932\end{array}$

$\mathrm{ArC}=4-\mathrm{Me}-\mathrm{Ph}$

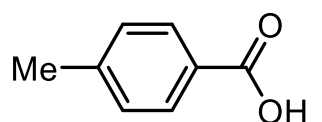

$\mathrm{E}(\mathrm{DZ})=-459.8775954$

$\mathrm{G}(\mathrm{DZ})=-459.774637$

$E(T Z)=-460.1268942$

$G(T Z)=-460.0239358$

$\begin{array}{lrcc}18 & & & \\ 01 & & & \\ \mathrm{C} & -0.71110 & 0.02961 & -0.00050 \\ \mathrm{C} & -0.01685 & -1.20067 & -0.00357 \\ \mathrm{C} & 0.02640 & 1.23577 & -0.00333 \\ \mathrm{C} & 1.38580 & -1.21650 & -0.00780 \\ \mathrm{H} & -0.57629 & -2.14152 & -0.00501 \\ \mathrm{C} & 1.42520 & 1.20806 & -0.00731 \\ \mathrm{H} & -0.51844 & 2.18624 & -0.00453 \\ \mathrm{C} & 2.12966 & -0.01758 & -0.00767 \\ \mathrm{H} & 1.91654 & -2.17721 & -0.01365 \\ \mathrm{H} & 1.98632 & 2.15162 & -0.01254 \\ \mathrm{C} & 3.64223 & -0.03586 & 0.01099 \\ \mathrm{H} & 4.02458 & 0.24949 & 1.01286 \\ \mathrm{H} & 4.03761 & -1.04026 & -0.22783 \\ \mathrm{H} & 4.05982 & 0.68866 & -0.71485 \\ \mathrm{C} & -2.19960 & 0.11514 & 0.00233 \\ \mathrm{O} & -2.84607 & 1.15454 & 0.00371 \\ \mathrm{O} & -2.81021 & -1.11608 & 0.00388 \\ \mathrm{H} & -3.77024 & -0.93258 & 0.00604\end{array}$


$\operatorname{ArC}=4-\mathrm{Ph}-\mathrm{Ph}$

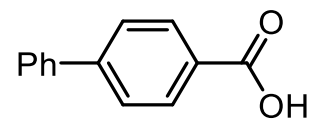

$\mathrm{E}(\mathrm{DZ})=-651.4881055$

$\mathrm{G}(\mathrm{DZ})=-651.336434$

$E(T Z)=-651.8575921$

$G(T Z)=-651.7059206$

$\begin{array}{lrrr}25 & & & \\ 01 & & & \\ \text { C } & 2.39800 & 0.03001 & -0.00716 \\ \text { C } & 1.69052 & -1.15894 & 0.28331 \\ \text { C } & 1.67161 & 1.20730 & -0.29562 \\ \text { C } & 0.29067 & -1.16416 & 0.28394 \\ \text { H } & 2.24039 & -2.07574 & 0.51782 \\ \text { C } & 0.27342 & 1.19531 & -0.29276 \\ \text { H } & 2.22399 & 2.12389 & -0.52944 \\ \text { C } & -0.44745 & 0.01001 & -0.00328 \\ \text { H } & -0.24349 & -2.08758 & 0.53494 \\ \text { H } & -0.27446 & 2.1114 & -0.54200 \\ \text { C } & -1.93288 & -0.00060 & -0.00068 \\ \text { C } & -2.65233 & -1.14582 & -0.41940 \\ \text { C } & -2.66646 & 1.13456 & 0.42089 \\ \text { C } & -4.05584 & -1.15517 & -0.41829 \\ \text { H } & -2.10397 & -2.02672 & -0.77332 \\ \text { C } & -4.06997 & 1.12432 & 0.42546 \\ \text { H } & -2.12893 & 2.02316 & 0.77211 \\ \text { C } & -4.77137 & -0.02036 & 0.00507 \\ \text { H } & -4.59322 & -2.04946 & -0.75733 \\ \text { H } & -4.61834 & 2.01117 & 0.76643 \\ \text { H } & -5.86824 & -0.02795 & 0.00720 \\ \text { C } & 3.88736 & 0.09884 & -0.02365 \\ \text { O } & 4.54343 & 1.10137 & -0.27390 \\ \text { O } & 4.48502 & -1.10142 & 0.27662 \\ \text { H } & 5.44691 & -0.93324 & 0.23477\end{array}$

$\mathrm{ArC}=4-\mathrm{nPr}-\mathrm{Ph}$

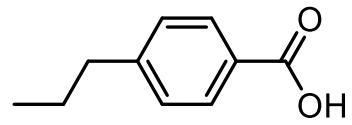

conformer 1

$\mathrm{E}(\mathrm{DZ})=-538.4536384$

$\mathrm{G}(\mathrm{DZ})=-538.298575$

$E(T Z)=-538.7408485$

$\mathrm{G}(\mathrm{TZ})=-538.5857851$

$\begin{array}{lccc}24 & & & \\ 01 & & & \\ \text { C } & -1.65839 & 0.03097 & -0.04162 \\ \text { C } & -0.96384 & -1.19476 & -0.15907 \\ \text { C } & -0.93961 & 1.24309 & -0.14654 \\ \text { C } & 0.42065 & -1.19880 & -0.37833 \\ \text { H } & -1.51125 & -2.13957 & -0.08238 \\ \text { C } & 0.44306 & 1.22621 & -0.36584 \\ \text { H } & -1.48541 & 2.18884 & -0.05768 \\ \text { H } & 0.95219 & -2.15469 & -0.47233\end{array}$

$\begin{array}{lrrr}\mathrm{H} & 0.99223 & 2.17297 & -0.45042 \\ \mathrm{C} & -3.13017 & 0.10363 & 0.18617 \\ \mathrm{O} & -3.77651 & 1.13712 & 0.29824 \\ \mathrm{O} & -3.72561 & -1.13280 & 0.26298 \\ \mathrm{H} & -4.67545 & -0.95692 & 0.41184 \\ \mathrm{C} & 1.14592 & 0.00721 & -0.48659 \\ \mathrm{C} & 2.64706 & -0.00600 & -0.66893 \\ \mathrm{H} & 2.94684 & -0.89930 & -1.25278 \\ \mathrm{H} & 2.96280 & 0.88506 & -1.24776 \\ \mathrm{C} & 3.39783 & -0.01636 & 0.68421 \\ \mathrm{H} & 3.07412 & -0.90336 & 1.26624 \\ \mathrm{H} & 3.08961 & 0.87283 & 1.27123 \\ \mathrm{C} & 4.92350 & -0.02909 & 0.50378 \\ \mathrm{H} & 5.44370 & -0.03565 & 1.48122 \\ \mathrm{H} & 5.24814 & -0.92553 & -0.06210 \\ \mathrm{H} & 5.26335 & 0.86414 & -0.05821\end{array}$

conformer 2

$$
\begin{aligned}
& E(D Z)=-538.4537698 \\
& G(D Z)=-538.298262 \\
& E(T Z)=-538.7410994 \\
& G(T Z)=-538.5855916
\end{aligned}
$$

$\begin{array}{lrrr}24 & & & \\ 01 & & & \\ \mathrm{C} & 1.49009 & -0.00707 & -0.09232 \\ \mathrm{C} & 0.83716 & 1.24384 & 0.00556 \\ \mathrm{C} & 0.74430 & -1.14522 & -0.47074 \\ \mathrm{C} & -0.53288 & 1.34330 & -0.26928 \\ \mathrm{H} & 1.40708 & 2.13326 & 0.29247 \\ \mathrm{C} & -0.62514 & -1.03269 & -0.74299 \\ \mathrm{H} & 1.25710 & -2.11031 & -0.54720 \\ \mathrm{H} & -1.03050 & 2.31891 & -0.19363 \\ \mathrm{H} & -1.19687 & -1.92184 & -1.03733 \\ \mathrm{C} & 2.94487 & -0.17975 & 0.18507 \\ \mathrm{O} & 3.55574 & -1.23813 & 0.11478 \\ \mathrm{O} & 3.56848 & 0.99238 & 0.54079 \\ \mathrm{H} & 4.50379 & 0.75448 & 0.69554 \\ \mathrm{C} & -1.28695 & 0.21018 & -0.64617 \\ \mathrm{C} & -2.77662 & 0.32978 & -0.88747 \\ \mathrm{H} & -2.98026 & 1.24172 & -1.48304 \\ \mathrm{H} & -3.12830 & -0.53782 & -1.48035 \\ \mathrm{C} & -3.58861 & 0.40484 & 0.43172 \\ \mathrm{H} & -4.65132 & 0.59261 & 0.17615 \\ \mathrm{H} & -3.24055 & 1.28167 & 1.01578 \\ \mathrm{C} & -3.47332 & -0.86674 & 1.28683 \\ \mathrm{H} & -4.07273 & -0.77823 & 2.21340 \\ \mathrm{H} & -3.83732 & -1.75285 & 0.72779 \\ \mathrm{H} & -2.42132 & -1.05844 & 1.57461\end{array}$

conformer 3

$$
\begin{aligned}
& \mathrm{E}(\mathrm{DZ})=-538.4537922 \\
& \mathrm{G}(\mathrm{DZ})=-538.29824 \\
& \mathrm{E}(\mathrm{TZ})=-538.741126 \\
& \mathrm{G}(\mathrm{TZ})=-538.5855738
\end{aligned}
$$




$\begin{array}{lrrr}24 & & & \\ 01 & & & \\ \text { C } & 1.49081 & 0.05547 & -0.07972 \\ \text { C } & 0.80594 & 1.28741 & 0.03159 \\ \text { C } & 0.77589 & -1.09644 & -0.47771 \\ \text { C } & -0.56311 & 1.36026 & -0.24768 \\ \text { H } & 1.36844 & 2.17713 & 0.33526 \\ \mathrm{C} & -0.59630 & -1.01039 & -0.75476 \\ \mathrm{H} & 1.29605 & -2.05508 & -0.57008 \\ \mathrm{H} & -1.08434 & 2.32254 & -0.16111 \\ \mathrm{H} & -1.14520 & -1.90881 & -1.06410 \\ \mathrm{C} & 2.94958 & 0.03247 & 0.22740 \\ \mathrm{O} & 3.61263 & 1.00168 & 0.57295 \\ \mathrm{O} & 3.51114 & -1.21401 & 0.08615 \\ \mathrm{H} & 4.45573 & -1.10248 & 0.31102 \\ \mathrm{C} & -1.28805 & 0.21390 & -0.64437 \\ \mathrm{C} & -2.77891 & 0.30122 & -0.89239 \\ \mathrm{H} & -2.99895 & 1.20843 & -1.48948 \\ \mathrm{H} & -3.10932 & -0.57381 & -1.48656 \\ \mathrm{C} & -3.59830 & 0.35993 & 0.42288 \\ \mathrm{H} & -4.66369 & 0.52435 & 0.16246 \\ \mathrm{H} & -3.27219 & 1.24446 & 1.00790 \\ \mathrm{C} & -3.45888 & -0.90829 & 1.27936 \\ \mathrm{H} & -4.06345 & -0.83233 & 2.20369 \\ \mathrm{H} & -3.80142 & -1.80248 & 0.71955 \\ \mathrm{H} & -2.40395 & -1.07665 & 1.57111 \\ & & & \\ \text { conformer } 4 & & \\ \mathrm{E}(\mathrm{DZ})=-538.4519399 & \\ \mathrm{G}(\mathrm{DZ})=-538.296997 & & \\ \mathrm{E}(\mathrm{TZ})=-538.7395126 & \\ \mathrm{G}(\mathrm{TZ})=-538.5845697 & \\ & & & \end{array}$

$\begin{array}{lrrr}24 & & & \\ 01 & & & \\ \mathrm{C} & -1.68931 & -0.02169 & -0.00002 \\ \mathrm{C} & -1.14511 & 1.28452 & 0.00004 \\ \mathrm{C} & -0.81321 & -1.12733 & -0.00015 \\ \mathrm{C} & 0.24238 & 1.46527 & -0.00003 \\ \mathrm{H} & -1.81274 & 2.15195 & 0.00015 \\ \mathrm{C} & 0.57592 & -0.93460 & -0.00022 \\ \mathrm{H} & -1.23837 & -2.13703 & -0.00020 \\ \mathrm{H} & 0.65409 & 2.48329 & 0.00004 \\ \mathrm{H} & 1.23400 & -1.80983 & -0.00034 \\ \mathrm{C} & -3.15670 & -0.28412 & 0.00005 \\ \mathrm{O} & -3.67571 & -1.39278 & 0.00001 \\ \mathrm{O} & -3.90983 & 0.86598 & 0.00017 \\ \mathrm{H} & -4.84088 & 0.56868 & 0.00021 \\ \mathrm{C} & 1.12955 & 0.36362 & -0.00016 \\ \mathrm{C} & 2.62758 & 0.62327 & -0.00029 \\ \mathrm{H} & 2.87065 & 1.25265 & -0.88303 \\ \mathrm{H} & 2.87064 & 1.25374 & 0.88166 \\ \mathrm{C} & 3.54369 & -0.61005 & 0.00044 \\ \mathrm{H} & 3.32131 & -1.23614 & -0.88750 \\ \mathrm{H} & 3.32143 & -1.23497 & 0.88924 \\ \mathrm{C} & 5.02932 & -0.21668 & 0.00008 \\ \mathrm{H} & 5.68331 & -1.10957 & 0.00070\end{array}$

$\begin{array}{llll}\mathrm{H} & 5.27801 & 0.38847 & -0.89493 \\ \mathrm{H} & 5.27810 & 0.38979 & 0.89416\end{array}$

conformer 5

$\mathrm{E}(\mathrm{DZ})=-538.4519613$

$G(D Z)=-538.296947$

$E(T Z)=-538.7395559$

$G(T Z)=-538.5845416$

$\begin{array}{lrrr}24 & & & \\ 01 & & & \\ \mathrm{C} & 1.69319 & 0.03928 & 0.00001 \\ \mathrm{C} & 0.83781 & -1.08320 & 0.00009 \\ \mathrm{C} & 1.12488 & 1.33457 & -0.00004 \\ \mathrm{C} & -0.55583 & -0.91199 & 0.00014 \\ \mathrm{H} & 1.26376 & -2.09163 & 0.00012 \\ \mathrm{C} & -0.26358 & 1.49342 & 0.00001 \\ \mathrm{H} & 1.79230 & 2.20332 & -0.00011 \\ \mathrm{H} & -1.19878 & -1.79847 & 0.00022 \\ \mathrm{H} & -0.69294 & 2.50413 & -0.00004 \\ \mathrm{C} & 3.17913 & -0.07838 & -0.00004 \\ \mathrm{O} & 3.96126 & 0.86342 & -0.00012 \\ \mathrm{O} & 3.61753 & -1.38113 & 0.00003 \\ \mathrm{H} & 4.59342 & -1.32809 & -0.00001 \\ \mathrm{C} & -1.13172 & 0.37567 & 0.00010 \\ \mathrm{C} & -2.63380 & 0.60959 & 0.00020 \\ \mathrm{H} & -2.88701 & 1.23521 & 0.88278 \\ \mathrm{H} & -2.88703 & 1.23604 & -0.88177 \\ \mathrm{C} & -3.52902 & -0.63870 & -0.00035 \\ \mathrm{H} & -3.29614 & -1.26088 & 0.88770 \\ \mathrm{H} & -3.29634 & -1.25994 & -0.88912 \\ \mathrm{C} & -5.02123 & -0.27091 & 0.00000 \\ \mathrm{H} & -5.65969 & -1.17499 & -0.00054 \\ \mathrm{H} & -5.28039 & 0.32988 & 0.89493 \\ \mathrm{H} & -5.28052 & 0.33105 & -0.89411\end{array}$

$\mathrm{ArC}=4-\mathrm{tBu}-\mathrm{Ph}$

$E(D Z)=-577.7425558$

$\mathrm{G}(\mathrm{DZ})=-577.560734$

$E(T Z)=-578.0490062$

$G(T Z)=-577.8671844$

$\begin{array}{lrrr}27 & & & \\ 01 & & & \\ \text { C } & 1.78792 & 0.04218 & 0.00000 \\ \text { C } & 1.07029 & -1.17621 & 0.00023 \\ \text { C } & 1.06796 & 1.25448 & -0.00023 \\ \text { C } & -0.32880 & -1.16640 & 0.00023 \\ \text { H } & 1.61152 & -2.12763 & 0.00041 \\ \text { C } & -0.33437 & 1.25092 & -0.00023 \\ \text { H } & 1.62400 & 2.19847 & -0.00040 \\ \text { C } & -1.06525 & 0.04241 & -0.00001 \\ \text { H } & -0.86334 & -2.12316 & 0.00041 \\ \text { H } & -0.85878 & 2.21122 & -0.00042 \\ \text { C } & -2.60323 & -0.00645 & -0.00001 \\ \text { C } & -3.08817 & -0.76240 & -1.26486 \\ \text { C } & -3.08818 & -0.76181 & 1.26519\end{array}$




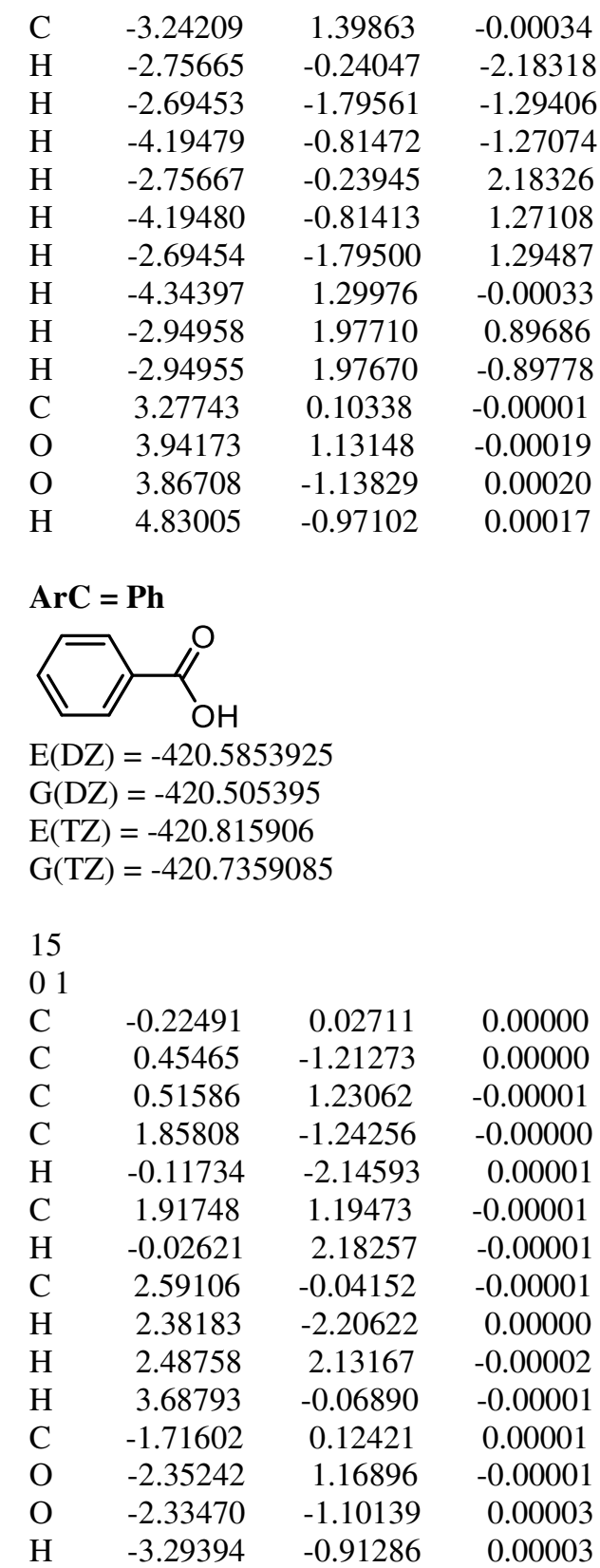

\section{Amines}

\section{$\operatorname{ArA}=1 \mathrm{Nap}$}<smiles>C(=C/c1cccc2ccccc12)\CN1CCCC1</smiles>

conformer 1

$$
\begin{aligned}
& \mathrm{E}(\mathrm{DZ})=-714.090376 \\
& \mathrm{G}(\mathrm{DZ})=-713.815607 \\
& \mathrm{E}(\mathrm{TZ})=-713.92681 \\
& \mathrm{G}(\mathrm{TZ})=-713.652041
\end{aligned}
$$

37

\begin{tabular}{lrrr}
0 & & & \\
$\mathrm{C}$ & 4.84090 & -0.92080 & -0.52932 \\
$\mathrm{C}$ & 3.53120 & 0.31495 & 0.92351 \\
$\mathrm{C}$ & 4.95349 & 0.86105 & 1.13603 \\
$\mathrm{C}$ & 5.83366 & 0.03430 & 0.15535 \\
$\mathrm{H}$ & 4.80533 & -1.88718 & 0.01299 \\
$\mathrm{H}$ & 5.09104 & -1.13743 & -1.57312 \\
$\mathrm{H}$ & 2.74881 & 1.07096 & 1.02892 \\
$\mathrm{H}$ & 3.31366 & -0.48822 & 1.65629 \\
$\mathrm{H}$ & 4.99812 & 1.92432 & 0.88649 \\
$\mathrm{H}$ & 5.27081 & 0.75499 & 2.17658 \\
$\mathrm{H}$ & 6.30375 & 0.68999 & -0.58210 \\
$\mathrm{H}$ & 6.63108 & -0.51337 & 0.66421 \\
$\mathrm{~N}$ & 3.56523 & -0.21275 & -0.44087 \\
$\mathrm{C}$ & 2.41149 & -1.02744 & -0.79471 \\
$\mathrm{H}$ & 2.61069 & -1.46620 & -1.78229 \\
$\mathrm{H}$ & 2.27178 & -1.87184 & -0.08962 \\
$\mathrm{C}$ & 1.15815 & -0.20449 & -0.85878 \\
$\mathrm{C}$ & 0.06107 & -0.44313 & -0.12938 \\
$\mathrm{H}$ & 1.18232 & 0.63398 & -1.55249 \\
$\mathrm{H}$ & 0.06915 & -1.30428 & 0.53661 \\
$\mathrm{C}$ & -1.16222 & 0.37589 & -0.14220 \\
$\mathrm{C}$ & -2.45370 & -0.22871 & 0.02970 \\
$\mathrm{C}$ & -1.08194 & 1.75121 & -0.29503 \\
$\mathrm{C}$ & -2.63346 & -1.63169 & 0.16335 \\
$\mathrm{C}$ & -3.61925 & 0.60640 & 0.04798 \\
$\mathrm{C}$ & -2.23316 & 2.56586 & -0.29503 \\
$\mathrm{H}$ & -0.10432 & 2.21293 & -0.38814 \\
$\mathrm{C}$ & -3.88572 & -2.17782 & 0.33336 \\
$\mathrm{H}$ & -1.77020 & -2.28514 & 0.11428 \\
$\mathrm{C}$ & -4.89617 & 0.01147 & 0.22549 \\
$\mathrm{C}$ & -3.47757 & 2.00777 & -0.12075 \\
$\mathrm{H}$ & -2.12644 & 3.63955 & -0.41553 \\
$\mathrm{C}$ & -5.03072 & -1.34916 & 0.37011 \\
$\mathrm{H}$ & -3.99707 & -3.25316 & 0.43370 \\
$\mathrm{H}$ & -5.77003 & 0.65698 & 0.24127 \\
$\mathrm{H}$ & -4.36830 & 2.62939 & -0.10522 \\
$\mathrm{H}$ & -6.01314 & -1.79097 & 0.50517 \\
& & & \\
\hline
\end{tabular}

conformer 2

$\mathrm{E}(\mathrm{DZ})=-714.090646$

$\mathrm{G}(\mathrm{DZ})=-713.815472$

$\mathrm{E}(\mathrm{TZ})=-713.9270937$

$G(T Z)=-713.6519197$

$\begin{array}{lccc}37 & & & \\ 01 & & & \\ \text { C } & 4.85682 & -0.27069 & -0.54854 \\ \text { C } & 3.17544 & 0.91412 & 0.51008 \\ \text { C } & 4.45111 & 1.29862 & 1.27817 \\ \text { C } & 5.58344 & 0.50755 & 0.56235 \\ \text { H } & 5.29182 & -1.25535 & -0.74945 \\ \text { H } & 4.88147 & 0.30443 & -1.49619 \\ \text { H } & 2.97693 & 1.64788 & -0.29762 \\ \text { H } & 2.28045 & 0.86819 & 1.13575 \\ \text { H } & 4.61805 & 2.37872 & 1.26272\end{array}$




$\begin{array}{lrrr}\mathrm{H} & 4.37416 & 0.99313 & 2.32481 \\ \mathrm{H} & 6.35852 & 1.16020 & 0.15230 \\ \mathrm{H} & 6.06859 & -0.18116 & 1.25875 \\ \mathrm{~N} & 3.49232 & -0.40330 & -0.04195 \\ \mathrm{C} & 2.53917 & -0.87970 & -1.03313 \\ \mathrm{H} & 2.43376 & -0.17028 & -1.87930 \\ \mathrm{H} & 2.93992 & -1.81363 & -1.45220 \\ \mathrm{C} & 1.19421 & -1.14501 & -0.42395 \\ \mathrm{C} & 0.05437 & -0.58211 & -0.84446 \\ \mathrm{H} & 1.18682 & -1.82728 & 0.42447 \\ \mathrm{H} & 0.11062 & 0.13770 & -1.65935 \\ \mathrm{C} & -1.28327 & -0.86633 & -0.30108 \\ \mathrm{C} & -2.27173 & 0.17186 & -0.20966 \\ \mathrm{C} & -1.61655 & -2.14696 & 0.11056 \\ \mathrm{C} & -2.00522 & 1.51901 & -0.57331 \\ \mathrm{C} & -3.57715 & -0.14835 & 0.28924 \\ \mathrm{C} & -2.89601 & -2.45206 & 0.62027 \\ \mathrm{H} & -0.88139 & -2.93844 & 0.00786 \\ \mathrm{C} & -2.97784 & 2.48899 & -0.47996 \\ \mathrm{H} & -1.01276 & 1.79059 & -0.91415 \\ \mathrm{C} & -4.55794 & 0.87492 & 0.37134 \\ \mathrm{C} & -3.86096 & -1.47605 & 0.70104 \\ \mathrm{H} & -3.11826 & -3.46863 & 0.92991 \\ \mathrm{C} & -4.27063 & 2.16492 & -0.00812 \\ \mathrm{H} & -2.75002 & 3.51172 & -0.76441 \\ \mathrm{H} & -5.54457 & 0.61730 & 0.74661 \\ \mathrm{H} & -4.85366 & -1.70609 & 1.07750 \\ \mathrm{H} & -5.03012 & 2.93761 & 0.06051\end{array}$

$\begin{array}{lrrr}\mathrm{C} & 1.47509 & -1.18914 & 0.16707 \\ \mathrm{C} & 2.03160 & 0.12899 & 0.01830 \\ \mathrm{C} & 2.33578 & -2.25889 & 0.36437 \\ \mathrm{C} & 1.24185 & 1.30896 & -0.04835 \\ \mathrm{C} & 3.45949 & 0.27596 & -0.01585 \\ \mathrm{C} & 3.73670 & -2.10535 & 0.34464 \\ \mathrm{H} & 1.91338 & -3.25004 & 0.50446 \\ \mathrm{C} & 1.81963 & 2.55002 & -0.19420 \\ \mathrm{H} & 0.16695 & 1.22861 & 0.04266 \\ \mathrm{C} & 4.02211 & 1.56916 & -0.18270 \\ \mathrm{C} & 4.28939 & -0.86411 & 0.13792 \\ \mathrm{H} & 4.37272 & -2.97440 & 0.48164 \\ \mathrm{C} & 3.22398 & 2.68384 & -0.28075 \\ \mathrm{H} & 1.19130 & 3.43462 & -0.23325 \\ \mathrm{H} & 5.10421 & 1.66026 & -0.21966 \\ \mathrm{H} & 5.36735 & -0.73370 & 0.10559 \\ \mathrm{H} & 3.66804 & 3.66671 & -0.40496\end{array}$

conformer 4

$$
\begin{aligned}
& E(D Z)=-714.087414 \\
& G(D Z)=-713.812774 \\
& E(T Z)=-713.9242155 \\
& G(T Z)=-713.6495755
\end{aligned}
$$

conformer 3

$$
\begin{aligned}
& \mathrm{E}(\mathrm{DZ})=-714.089016 \\
& \mathrm{G}(\mathrm{DZ})=-713.814012 \\
& \mathrm{E}(\mathrm{TZ})=-713.925108 \\
& \mathrm{G}(\mathrm{TZ})=-713.650104
\end{aligned}
$$

$\begin{array}{lrrr}37 & & & \\ \text { O 1 } & & & \\ \text { C } & -3.11695 & 0.31994 & 0.91713 \\ \text { C } & -4.62577 & -0.49845 & -0.63341 \\ \text { C } & -5.33458 & 0.72351 & -0.02357 \\ \text { C } & -4.31379 & 1.28097 & 1.00961 \\ \text { H } & -3.21451 & -0.49176 & 1.66607 \\ \text { H } & -2.15202 & 0.80455 & 1.08831 \\ \text { H } & -4.85065 & -0.65058 & -1.69419 \\ \mathrm{H} & -4.92293 & -1.42020 & -0.09401 \\ \mathrm{H} & -5.54631 & 1.46944 & -0.79389 \\ \mathrm{H} & -6.28704 & 0.44553 & 0.43504 \\ \mathrm{H} & -4.01075 & 2.29545 & 0.73789 \\ \mathrm{H} & -4.71953 & 1.31990 & 2.02376 \\ \mathrm{~N} & -3.20564 & -0.20624 & -0.44516 \\ \mathrm{C} & -2.33153 & -1.34096 & -0.70871 \\ \mathrm{H} & -2.50191 & -2.16763 & 0.01045 \\ \mathrm{H} & -2.58825 & -1.72697 & -1.70496 \\ \mathrm{C} & -0.88643 & -0.93657 & -0.69003 \\ \mathrm{C} & 0.03213 & -1.48343 & 0.11707 \\ \mathrm{H} & -0.61162 & -0.15794 & -1.39654 \\ \mathrm{H} & -0.29116 & -2.29477 & 0.77042\end{array}$

$\begin{array}{lrrr}37 & & & \\ \text { O 1 } & & & \\ \mathrm{C} & 3.77758 & -0.67380 & 0.86869 \\ \mathrm{C} & 4.64332 & 0.71926 & -0.76074 \\ \mathrm{C} & 5.50508 & 1.01274 & 0.47669 \\ \mathrm{C} & 4.95156 & 0.04526 & 1.56018 \\ \mathrm{H} & 2.94162 & -0.87408 & 1.54446 \\ \mathrm{H} & 4.11416 & -1.64605 & 0.45384 \\ \mathrm{H} & 5.12301 & -0.06174 & -1.38577 \\ \mathrm{H} & 4.47906 & 1.59520 & -1.39710 \\ \mathrm{H} & 6.56805 & 0.85762 & 0.27467 \\ \mathrm{H} & 5.37670 & 2.05161 & 0.79114 \\ \mathrm{H} & 5.70317 & -0.66786 & 1.90838 \\ \mathrm{H} & 4.60162 & 0.60392 & 2.43177 \\ \mathrm{~N} & 3.38021 & 0.24805 & -0.19588 \\ \mathrm{C} & 2.48784 & -0.32731 & -1.19790 \\ \mathrm{H} & 2.30888 & 0.43147 & -1.96809 \\ \mathrm{H} & 2.97724 & -1.18827 & -1.70432 \\ \mathrm{C} & 1.17491 & -0.78242 & -0.62921 \\ \mathrm{C} & -0.00560 & -0.23137 & -0.93741 \\ \mathrm{H} & 1.20568 & -1.61285 & 0.07381 \\ \mathrm{H} & -0.01129 & 0.62408 & -1.61064 \\ \mathrm{C} & -1.31140 & -0.69756 & -0.44325 \\ \mathrm{C} & -2.36602 & 0.23700 & -0.16461 \\ \mathrm{C} & -1.54952 & -2.05058 & -0.25970 \\ \mathrm{C} & -2.20022 & 1.64203 & -0.29324 \\ \mathrm{C} & -3.63544 & -0.25589 & 0.28421 \\ \mathrm{C} & -2.79394 & -2.52857 & 0.20136 \\ \mathrm{H} & -0.76609 & -2.75941 & -0.50737 \\ \mathrm{C} & -3.23468 & 2.50978 & -0.02425 \\ \mathrm{H} & -1.23658 & 2.03820 & -0.59175 \\ \mathrm{C} & -4.68157 & 0.66575 & 0.55241 \\ \mathrm{C} & -3.81995 & -1.65135 & 0.46190\end{array}$




$\begin{array}{cccc}\mathrm{H} & -2.94056 & -3.59659 & 0.33014 \\ \mathrm{C} & -4.49141 & 2.01875 & 0.39822 \\ \mathrm{H} & -3.08355 & 3.57968 & -0.13020 \\ \mathrm{H} & -5.63958 & 0.27798 & 0.88781 \\ \mathrm{H} & -4.78619 & -2.01208 & 0.80301 \\ \mathrm{H} & -5.29994 & 2.71241 & 0.60724\end{array}$

conformer 5

$\mathrm{E}(\mathrm{DZ})=-714.087251$
$\mathrm{G}(\mathrm{DZ})=-713.812733$
$\mathrm{E}(\mathrm{TZ})=-713.9240701$
$\mathrm{G}(\mathrm{TZ})=-713.6495521$

37

01

$\begin{array}{lrrr}\mathrm{C} & -4.66920 & -1.26021 & -0.20225 \\ \mathrm{C} & -3.96708 & 0.82605 & 0.50395 \\ \mathrm{C} & -5.31304 & 1.09042 & -0.19672 \\ \mathrm{C} & -5.76628 & -0.30791 & -0.70299 \\ \mathrm{H} & -4.48692 & -2.10790 & -0.87104 \\ \mathrm{H} & -4.94292 & -1.67168 & 0.79081 \\ \mathrm{H} & -4.12137 & 0.69405 & 1.59459 \\ \mathrm{H} & -3.24792 & 1.63895 & 0.36885 \\ \mathrm{H} & -6.03479 & 1.54262 & 0.48834 \\ \mathrm{H} & -5.18197 & 1.77850 & -1.03560 \\ \mathrm{H} & -6.75166 & -0.59416 & -0.32640 \\ \mathrm{H} & -5.81302 & -0.32198 & -1.79483 \\ \mathrm{~N} & -3.48512 & -0.40718 & -0.11763 \\ \mathrm{C} & -2.37207 & -1.04578 & 0.57990 \\ \mathrm{H} & -2.66726 & -1.31393 & 1.61749 \\ \mathrm{H} & -2.14372 & -1.98381 & 0.06125 \\ \mathrm{C} & -1.13908 & -0.18952 & 0.62765 \\ \mathrm{C} & -0.00600 & -0.47342 & -0.02621 \\ \mathrm{H} & -1.18259 & 0.70067 & 1.25248 \\ \mathrm{H} & 0.03154 & -1.38994 & -0.61267 \\ \mathrm{C} & 1.20338 & 0.36623 & -0.02855 \\ \mathrm{C} & 2.51057 & -0.22816 & -0.05146 \\ \mathrm{C} & 1.09531 & 1.74784 & -0.03353 \\ \mathrm{C} & 2.71538 & -1.63348 & -0.01726 \\ \mathrm{C} & 3.66418 & 0.62247 & -0.08853 \\ \mathrm{C} & 2.23391 & 2.58031 & -0.05096 \\ \mathrm{H} & 0.10735 & 2.19594 & -0.05714 \\ \mathrm{C} & 3.98257 & -2.17082 & -0.04659 \\ \mathrm{H} & 1.85806 & -2.29334 & 0.04898 \\ \mathrm{C} & 4.95706 & 0.03635 & -0.11851 \\ \mathrm{C} & 3.49378 & 2.03088 & -0.08444 \\ \mathrm{H} & 2.10638 & 3.65845 & -0.05712 \\ \mathrm{C} & 5.11723 & -1.32913 & -0.10268 \\ \mathrm{H} & 4.11330 & -3.24831 & -0.02025 \\ \mathrm{H} & 5.82230 & 0.69281 & -0.15063 \\ \mathrm{H} & 4.37570 & 2.66454 & -0.11267 \\ \mathrm{H} & 6.11173 & -1.76387 & -0.12584\end{array}$

ArA = 2-Me-Ph<smiles>Cc1ccccc1/C=C/CN1CCCC1</smiles>

conformer 1

$$
\begin{aligned}
& E(D Z)=-599.752528 \\
& G(D Z)=-599.495751 \\
& E(T Z)=-599.6035154 \\
& G(T Z)=-599.3467384
\end{aligned}
$$

\begin{tabular}{lccc}
34 & & & \\
O 1 & & & \\
$\mathrm{C}$ & -4.12247 & 0.66061 & -0.57961 \\
$\mathrm{C}$ & -2.72510 & -0.44819 & 0.89327 \\
$\mathrm{C}$ & -4.08323 & -1.14675 & 1.06418 \\
$\mathrm{C}$ & -5.03242 & -0.37909 & 0.10015 \\
$\mathrm{H}$ & -4.37763 & 0.84654 & -1.62816 \\
$\mathrm{H}$ & -4.18551 & 1.62943 & -0.04464 \\
$\mathrm{H}$ & -1.86789 & -1.11518 & 1.01556 \\
$\mathrm{H}$ & -2.61595 & 0.36961 & 1.63427 \\
$\mathrm{H}$ & -4.00974 & -2.19702 & 0.77024 \\
$\mathrm{H}$ & -4.42343 & -1.11846 & 2.10253 \\
$\mathrm{H}$ & -5.46138 & -1.05976 & -0.63967 \\
$\mathrm{H}$ & -5.86301 & 0.10090 & 0.62415 \\
$\mathrm{~N}$ & -2.78539 & 0.07965 & -0.46986 \\
$\mathrm{C}$ & -1.71192 & 1.00540 & -0.80247 \\
$\mathrm{H}$ & -1.93114 & 1.41537 & -1.79842 \\
$\mathrm{H}$ & -1.67684 & 1.86482 & -0.10230 \\
$\mathrm{C}$ & -0.37869 & 0.31706 & -0.82757 \\
$\mathrm{C}$ & 0.67171 & 0.68640 & -0.08294 \\
$\mathrm{H}$ & -0.30395 & -0.53066 & -1.50610 \\
$\mathrm{H}$ & 0.55335 & 1.55798 & 0.55831 \\
$\mathrm{C}$ & 1.98878 & 0.03028 & -0.05994 \\
$\mathrm{C}$ & 2.11566 & -1.33518 & -0.36310 \\
$\mathrm{C}$ & 3.14853 & 0.76493 & 0.28064 \\
$\mathrm{C}$ & 3.35470 & -1.96725 & -0.35620 \\
$\mathrm{H}$ & 1.22159 & -1.90911 & -0.58499 \\
$\mathrm{C}$ & 4.38526 & 0.11316 & 0.28624 \\
$\mathrm{C}$ & 4.49873 & -1.23943 & -0.03063 \\
$\mathrm{H}$ & 3.42495 & -3.02481 & -0.59179 \\
$\mathrm{H}$ & 5.27573 & 0.68142 & 0.54150 \\
$\mathrm{H}$ & 5.47152 & -1.72133 & -0.01596 \\
$\mathrm{C}$ & 3.07430 & 2.23490 & 0.61673 \\
$\mathrm{H}$ & 2.48120 & 2.41620 & 1.52119 \\
$\mathrm{H}$ & 4.07258 & 2.64357 & 0.79120 \\
$\mathrm{H}$ & 2.61036 & 2.81179 & -0.19034 \\
& & & \\
\hline
\end{tabular}

conformer 2

$$
\begin{aligned}
& E(D Z)=-599.752565 \\
& G(D Z)=-599.495087 \\
& E(T Z)=-599.6033056 \\
& G(T Z)=-599.3458276 \\
& 34 \\
& 01
\end{aligned}
$$




$\begin{array}{lrrr}\mathrm{C} & -3.82754 & -0.71116 & -0.61280 \\ \mathrm{C} & -4.43797 & 0.70181 & -0.59490 \\ \mathrm{C} & -3.59027 & 1.47192 & 0.45780 \\ \mathrm{H} & -1.62023 & 0.84445 & 1.25252 \\ \mathrm{H} & -2.99742 & -0.09010 & 1.86728 \\ \mathrm{H} & -3.83244 & -1.17658 & -1.60369 \\ \mathrm{H} & -4.38901 & -1.37708 & 0.07229 \\ \mathrm{H} & -4.34764 & 1.17170 & -1.57758 \\ \mathrm{H} & -5.50053 & 0.67485 & -0.34025 \\ \mathrm{H} & -3.06134 & 2.30502 & -0.01272 \\ \mathrm{H} & -4.19691 & 1.88359 & 1.26844 \\ \mathrm{~N} & -2.45696 & -0.50257 & -0.14913 \\ \mathrm{C} & -1.74010 & -1.72599 & 0.16211 \\ \mathrm{H} & -2.12670 & -2.19626 & 1.08988 \\ \mathrm{H} & -1.94065 & -2.45179 & -0.63983 \\ \mathrm{C} & -0.25025 & -1.53836 & 0.28273 \\ \mathrm{C} & 0.41471 & -0.47708 & -0.19144 \\ \mathrm{H} & 0.28417 & -2.33954 & 0.79035 \\ \mathrm{H} & -0.17936 & 0.30379 & -0.65790 \\ \mathrm{C} & 1.86835 & -0.26696 & -0.12179 \\ \mathrm{C} & 2.75753 & -1.35286 & -0.07237 \\ \mathrm{C} & 2.39526 & 1.04620 & -0.11179 \\ \mathrm{C} & 4.13319 & -1.16177 & 0.00801 \\ \mathrm{H} & 2.35790 & -2.36092 & -0.12149 \\ \mathrm{C} & 3.78000 & 1.22069 & -0.03486 \\ \mathrm{C} & 4.64997 & 0.13323 & 0.02803 \\ \mathrm{H} & 4.79866 & -2.01915 & 0.04173 \\ \mathrm{H} & 4.18112 & 2.23067 & -0.02094 \\ \mathrm{H} & 5.72184 & 0.29692 & 0.08418 \\ \mathrm{C} & 1.48808 & 2.25211 & -0.16097 \\ \mathrm{H} & 0.90460 & 2.28574 & -1.08853 \\ \mathrm{H} & 0.76978 & 2.25090 & 0.66616 \\ \mathrm{H} & 2.06786 & 3.17648 & -0.10112\end{array}$

$\begin{array}{lrrr}\mathrm{H} & 2.17238 & -2.37238 & -0.49249 \\ \mathrm{C} & 0.28232 & -1.37234 & -0.65090 \\ \mathrm{C} & -0.39555 & -0.27161 & -0.30066 \\ \mathrm{H} & -0.24385 & -2.31069 & -0.81843 \\ \mathrm{H} & 0.17834 & 0.64634 & -0.20591 \\ \mathrm{C} & -1.84317 & -0.19311 & -0.05579 \\ \mathrm{C} & -2.56704 & -1.31755 & 0.37380 \\ \mathrm{C} & -2.53017 & 1.03026 & -0.23902 \\ \mathrm{C} & -3.93902 & -1.25672 & 0.59575 \\ \mathrm{H} & -2.03451 & -2.24534 & 0.55781 \\ \mathrm{C} & -3.90794 & 1.07503 & -0.00744 \\ \mathrm{C} & -4.61638 & -0.05317 & 0.40333 \\ \mathrm{H} & -4.47377 & -2.14057 & 0.93032 \\ \mathrm{H} & -4.43391 & 2.01445 & -0.15601 \\ \mathrm{H} & -5.68592 & 0.01048 & 0.57915 \\ \mathrm{C} & -1.80447 & 2.27249 & -0.69615 \\ \mathrm{H} & -1.04870 & 2.59255 & 0.03098 \\ \mathrm{H} & -1.28239 & 2.10934 & -1.64488 \\ \mathrm{H} & -2.50307 & 3.10159 & -0.83308\end{array}$

conformer 3

$$
\begin{aligned}
& E(D Z)=-599.752538 \\
& G(D Z)=-599.494781 \\
& E(T Z)=-599.6031985 \\
& G(T Z)=-599.3454415
\end{aligned}
$$

$\begin{array}{lccc}34 & & & \\ 01 & & & \\ \mathrm{C} & 3.84542 & -0.12204 & -0.82666 \\ \mathrm{C} & 2.62280 & -0.29955 & 1.12713 \\ \mathrm{C} & 3.62180 & 0.83476 & 1.40867 \\ \mathrm{C} & 4.44732 & 0.95257 & 0.09545 \\ \mathrm{H} & 3.84208 & 0.16148 & -1.88410 \\ \mathrm{H} & 4.41717 & -1.06695 & -0.73491 \\ \mathrm{H} & 1.65621 & -0.16553 & 1.61728 \\ \mathrm{H} & 3.03695 & -1.27020 & 1.46604 \\ \mathrm{H} & 3.09299 & 1.76928 & 1.61371 \\ \mathrm{H} & 4.24487 & 0.61272 & 2.27880 \\ \mathrm{H} & 4.32567 & 1.94537 & -0.34531 \\ \mathrm{H} & 5.51670 & 0.79177 & 0.25407 \\ \mathrm{~N} & 2.47907 & -0.27595 & -0.33011 \\ \mathrm{C} & 1.76947 & -1.41435 & -0.88339 \\ \mathrm{H} & 1.95707 & -1.44010 & -1.96695\end{array}$

conformer 4

$$
\begin{aligned}
& E(D Z)=-599.750918 \\
& G(D Z)=-599.493681 \\
& E(T Z)=-599.6014817 \\
& G(T Z)=-599.3442447
\end{aligned}
$$

$\begin{array}{lrrr}34 & & & \\ \text { O 1 } & & & \\ \mathrm{C} & 2.65546 & 0.27368 & 0.97033 \\ \mathrm{C} & 4.07792 & -0.67851 & -0.58507 \\ \mathrm{C} & 4.95542 & 0.34880 & 0.15154 \\ \mathrm{C} & 3.99433 & 0.99801 & 1.18852 \\ \mathrm{H} & 2.57224 & -0.59731 & 1.65152 \\ \mathrm{H} & 1.78010 & 0.90538 & 1.14269 \\ \mathrm{H} & 4.18265 & -1.67664 & -0.11398 \\ \mathrm{H} & 4.32839 & -0.78746 & -1.64552 \\ \mathrm{H} & 5.82037 & -0.12726 & 0.62069 \\ \mathrm{H} & 5.33298 & 1.10185 & -0.54495 \\ \mathrm{H} & 4.34755 & 0.88743 & 2.21697 \\ \mathrm{H} & 3.88642 & 2.06782 & 0.99165 \\ \mathrm{~N} & 2.72277 & -0.15429 & -0.42695 \\ \mathrm{C} & 1.67835 & -1.09036 & -0.81839 \\ \mathrm{H} & 1.90688 & -1.42475 & -1.84031 \\ \mathrm{H} & 1.67518 & -1.99603 & -0.17838 \\ \mathrm{C} & 0.32338 & -0.44351 & -0.79804 \\ \mathrm{C} & -0.71072 & -0.92400 & -0.09177 \\ \mathrm{H} & 0.23642 & 0.44484 & -1.41206 \\ \mathrm{H} & -0.52498 & -1.83977 & 0.47133 \\ \mathrm{C} & -2.09752 & -0.44580 & 0.01593 \\ \mathrm{C} & -3.04428 & -1.39127 & 0.45487 \\ \mathrm{C} & -2.53995 & 0.87398 & -0.25380 \\ \mathrm{C} & -4.39100 & -1.08007 & 0.59706 \\ \mathrm{H} & -2.70185 & -2.39773 & 0.67988 \\ \mathrm{C} & -3.89863 & 1.17021 & -0.09472 \\ \mathrm{C} & -4.82463 & 0.21415 & 0.31585 \\ \mathrm{H} & -5.09285 & -1.83819 & 0.93053 \\ \mathrm{H} & -4.23322 & 2.18487 & -0.29387\end{array}$




$\begin{array}{lrrr}\mathrm{H} & -5.87077 & 0.48264 & 0.42630 \\ \mathrm{C} & -1.61116 & 1.98379 & -0.67600 \\ \mathrm{H} & -2.10028 & 2.95441 & -0.56056 \\ \mathrm{H} & -0.69140 & 1.98939 & -0.08340 \\ \mathrm{H} & -1.31509 & 1.89197 & -1.72767\end{array}$

$G(D Z)=-674.698949$
$E(T Z)=-674.8193827$
$G(T Z)=-674.5590337$

35

01

conformer 5

$E(D Z)=-599.749554$

$\mathrm{G}(\mathrm{DZ})=-599.492581$

$\mathrm{E}(\mathrm{TZ})=-599.6009377$

$G(T Z)=-599.3439647$

$\begin{array}{lrrr}34 & & & \\ 01 & & & \\ \mathrm{C} & 2.99712 & -1.06314 & 0.50192 \\ \mathrm{C} & 3.99424 & 0.69604 & -0.61711 \\ \mathrm{C} & 4.92666 & 0.43840 & 0.57636 \\ \mathrm{C} & 4.27760 & -0.77058 & 1.30709 \\ \mathrm{H} & 3.18751 & -1.86253 & -0.24343 \\ \mathrm{H} & 2.16227 & -1.38767 & 1.12917 \\ \mathrm{H} & 4.34227 & 0.12908 & -1.50486 \\ \mathrm{H} & 3.93175 & 1.75100 & -0.90424 \\ \mathrm{H} & 5.95089 & 0.23634 & 0.25245 \\ \mathrm{H} & 4.95404 & 1.31165 & 1.23308 \\ \mathrm{H} & 4.93464 & -1.64360 & 1.33772 \\ \mathrm{H} & 4.03215 & -0.50909 & 2.33944 \\ \mathrm{~N} & 2.69655 & 0.21103 & -0.15111 \\ \mathrm{C} & 1.70347 & 0.13050 & -1.21878 \\ \mathrm{H} & 1.60433 & 1.12726 & -1.66348 \\ \mathrm{H} & 2.05797 & -0.54681 & -2.02656 \\ \mathrm{C} & 0.35910 & -0.34126 & -0.74486 \\ \mathrm{C} & -0.73638 & 0.42882 & -0.72447 \\ \mathrm{H} & 0.29557 & -1.37230 & -0.40197 \\ \mathrm{H} & -0.63028 & 1.46887 & -1.02759 \\ \mathrm{C} & -2.08150 & 0.00018 & -0.30995 \\ \mathrm{C} & -2.48902 & -1.33462 & -0.46535 \\ \mathrm{C} & -2.98913 & 0.93204 & 0.24575 \\ \mathrm{C} & -3.75248 & -1.75807 & -0.06523 \\ \mathrm{H} & -1.80796 & -2.04033 & -0.93041 \\ \mathrm{C} & -4.25592 & 0.49057 & 0.63819 \\ \mathrm{C} & -4.64304 & -0.84059 & 0.49068 \\ \mathrm{H} & -4.04446 & -2.79520 & -0.19937 \\ \mathrm{H} & -4.94926 & 1.20650 & 1.07151 \\ \mathrm{H} & -5.63457 & -1.15558 & 0.80126 \\ \mathrm{C} & -2.60354 & 2.37849 & 0.44001 \\ \mathrm{H} & -1.69594 & 2.47645 & 1.04487 \\ \mathrm{H} & -2.40617 & 2.87884 & -0.51578 \\ \mathrm{H} & -3.40371 & 2.92935 & 0.94029 \\ & & & \end{array}$

ArA = 2-OMe-Ph<smiles>COc1ccccc1/C=C/CN1CCCC1</smiles>

conformer 1

$E(D Z)=-674.959298$

$\begin{array}{lrrr}\mathrm{C} & -4.38755 & -0.22709 & -0.74016 \\ \mathrm{C} & -2.84453 & 0.62303 & 0.75810 \\ \mathrm{C} & -4.17700 & 0.66086 & 1.52486 \\ \mathrm{C} & -5.21730 & 0.09721 & 0.51469 \\ \mathrm{H} & -4.41101 & 0.62901 & -1.44448 \\ \mathrm{H} & -4.74251 & -1.10948 & -1.28301 \\ \mathrm{H} & -1.97683 & 0.41937 & 1.39040 \\ \mathrm{H} & -2.66400 & 1.59310 & 0.25218 \\ \mathrm{H} & -4.12542 & 0.02680 & 2.41382 \\ \mathrm{H} & -4.41978 & 1.67346 & 1.85746 \\ \mathrm{H} & -5.68381 & -0.80853 & 0.91070 \\ \mathrm{H} & -6.01709 & 0.80876 & 0.29327 \\ \mathrm{~N} & -3.04147 & -0.45003 & -0.21690 \\ \mathrm{C} & -2.01172 & -0.51649 & -1.24486 \\ \mathrm{H} & -2.32995 & -1.27157 & -1.97785 \\ \mathrm{H} & -1.91608 & 0.44187 & -1.79428 \\ \mathrm{C} & -0.68215 & -0.90595 & -0.66960 \\ \mathrm{C} & 0.42971 & -0.16794 & -0.78801 \\ \mathrm{H} & -0.67479 & -1.83906 & -0.10887 \\ \mathrm{H} & 0.36460 & 0.79047 & -1.29604 \\ \mathrm{C} & 1.75585 & -0.52013 & -0.26652 \\ \mathrm{C} & 2.71880 & 0.49633 & -0.05713 \\ \mathrm{C} & 2.12599 & -1.83861 & 0.02830 \\ \mathrm{C} & 3.98715 & 0.18785 & 0.44128 \\ \mathrm{C} & 3.38617 & -2.15328 & 0.53185 \\ \mathrm{H} & 1.41155 & -2.63259 & -0.16313 \\ \mathrm{C} & 4.31499 & -1.13623 & 0.73797 \\ \mathrm{H} & 4.71951 & 0.96812 & 0.60517 \\ \mathrm{H} & 3.64334 & -3.18500 & 0.74830 \\ \mathrm{H} & 5.30358 & -1.36527 & 1.12389 \\ \mathrm{O} & 2.31546 & 1.76659 & -0.36247 \\ \mathrm{C} & 3.24561 & 2.82348 & -0.20425 \\ \mathrm{H} & 2.72711 & 3.73012 & -0.51846 \\ \mathrm{H} & 3.55999 & 2.93155 & 0.84146 \\ \mathrm{H} & 4.13412 & 2.67817 & -0.83157 \\ & & & \\ & & & \end{array}$

conformer 2

$$
\begin{aligned}
& E(D Z)=-674.959171 \\
& G(D Z)=-674.698845 \\
& E(T Z)=-674.8191211 \\
& G(T Z)=-674.5587951
\end{aligned}
$$




$\begin{array}{cccc}\mathrm{H} & -2.16297 & -0.75791 & 1.30341 \\ \mathrm{H} & -2.76553 & 0.90620 & 1.41393 \\ \mathrm{H} & -4.38362 & -1.66743 & 1.54437 \\ \mathrm{H} & -4.64873 & -0.15396 & 2.40800 \\ \mathrm{H} & -5.79979 & -0.99566 & -0.16732 \\ \mathrm{H} & -6.10757 & 0.54210 & 0.63801 \\ \mathrm{~N} & -3.08358 & -0.04739 & -0.46363 \\ \mathrm{C} & -1.97356 & 0.63249 & -1.11830 \\ \mathrm{H} & -2.22747 & 0.71905 & -2.18422 \\ \mathrm{H} & -1.84043 & 1.66409 & -0.73462 \\ \mathrm{C} & -0.69232 & -0.13414 & -0.96888 \\ \mathrm{C} & 0.41503 & 0.36456 & -0.40313 \\ \mathrm{H} & -0.71622 & -1.15265 & -1.35187 \\ \mathrm{H} & 0.39899 & 1.39428 & -0.05708 \\ \mathrm{C} & 1.68684 & -0.34340 & -0.21139 \\ \mathrm{C} & 2.86042 & 0.39347 & 0.07977 \\ \mathrm{C} & 1.79656 & -1.73845 & -0.28358 \\ \mathrm{C} & 4.08276 & -0.25542 & 0.27466 \\ \mathrm{C} & 3.01201 & -2.39167 & -0.09608 \\ \mathrm{H} & 0.90117 & -2.32124 & -0.47349 \\ \mathrm{C} & 4.15515 & -1.64599 & 0.18244 \\ \mathrm{H} & 4.97876 & 0.31139 & 0.49384 \\ \mathrm{H} & 3.06258 & -3.47391 & -0.15631 \\ \mathrm{H} & 5.10948 & -2.14022 & 0.33627 \\ \mathrm{O} & 2.70873 & 1.75069 & 0.14653 \\ \mathrm{C} & 3.83930 & 2.53864 & 0.47488 \\ \mathrm{H} & 4.63173 & 2.44219 & -0.27801 \\ \mathrm{H} & 3.48979 & 3.57156 & 0.49743 \\ \mathrm{H} & 4.24620 & 2.27198 & 1.45848 \\ & & & \end{array}$

$\begin{array}{rrrr}\mathrm{H} & -0.41286 & -1.56007 & -0.04358 \\ \mathrm{C} & -1.51734 & 0.30327 & -0.19146 \\ \mathrm{C} & -2.77314 & -0.29344 & 0.07340 \\ \mathrm{C} & -1.44880 & 1.70243 & -0.19825 \\ \mathrm{C} & -3.90408 & 0.49425 & 0.30454 \\ \mathrm{C} & -2.57285 & 2.49447 & 0.02397 \\ \mathrm{H} & -0.48396 & 2.17251 & -0.35885 \\ \mathrm{C} & -3.80042 & 1.88595 & 0.27452 \\ \mathrm{H} & -4.86443 & 0.03521 & 0.50269 \\ \mathrm{H} & -2.48681 & 3.57611 & 0.01535 \\ \mathrm{H} & -4.68493 & 2.48864 & 0.45642 \\ \mathrm{O} & -2.79084 & -1.66033 & 0.07471 \\ \mathrm{C} & -4.01289 & -2.31567 & 0.36593 \\ \mathrm{H} & -3.79734 & -3.38421 & 0.33049 \\ \mathrm{H} & -4.78477 & -2.07793 & -0.37676 \\ \mathrm{H} & -4.38442 & -2.05345 & 1.36456\end{array}$

conformer 3

$E(D Z)=-674.957381$
$G(D Z)=-674.697038$
$E(T Z)=-674.8165943$
$G(T Z)=-674.5562513$

$\begin{array}{lrrr}35 & & & \\ 01 & & & \\ \mathrm{C} & 2.97023 & -0.66248 & 1.01616 \\ \mathrm{C} & 3.75680 & 0.60574 & -0.76419 \\ \mathrm{C} & 4.84117 & 0.75859 & 0.31336 \\ \mathrm{C} & 4.26689 & -0.00870 & 1.53856 \\ \mathrm{H} & 2.09136 & -0.06426 & 1.31449 \\ \mathrm{H} & 2.81452 & -1.67986 & 1.39210 \\ \mathrm{H} & 4.15375 & 0.59440 & -1.78534 \\ \mathrm{H} & 3.03841 & 1.44453 & -0.69395 \\ \mathrm{H} & 5.77106 & 0.29296 & -0.02336 \\ \mathrm{H} & 5.05531 & 1.80800 & 0.53277 \\ \mathrm{H} & 4.97575 & -0.76470 & 1.88556 \\ \mathrm{H} & 4.05964 & 0.65538 & 2.38198 \\ \mathrm{~N} & 3.15479 & -0.68327 & -0.43448 \\ \mathrm{C} & 1.98200 & -1.07526 & -1.21807 \\ \mathrm{H} & 2.29476 & -1.11399 & -2.26992 \\ \mathrm{H} & 1.71211 & -2.09694 & -0.92453 \\ \mathrm{C} & 0.77506 & -0.18189 & -1.07245 \\ \mathrm{C} & -0.34115 & -0.54400 & -0.42433 \\ \mathrm{H} & 0.84714 & 0.81380 & -1.50653\end{array}$

conformer 4

$$
\begin{aligned}
& E(D Z)=-674.956037 \\
& G(D Z)=-674.695875 \\
& E(T Z)=-674.8163683 \\
& G(T Z)=-674.5562063
\end{aligned}
$$

$\begin{array}{lccc}35 & & & \\ \text { O 1 } & & & \\ \mathrm{C} & 4.19556 & -1.16991 & 0.04602 \\ \mathrm{C} & 3.44797 & 1.00917 & 0.22244 \\ \mathrm{C} & 4.74891 & 1.11611 & -0.59440 \\ \mathrm{C} & 5.23700 & -0.35335 & -0.73579 \\ \mathrm{H} & 4.52412 & -1.30290 & 1.09735 \\ \mathrm{H} & 4.01743 & -2.16665 & -0.37123 \\ \mathrm{H} & 2.69197 & 1.74039 & -0.07740 \\ \mathrm{H} & 3.65802 & 1.16762 & 1.30042 \\ \mathrm{H} & 4.55088 & 1.55085 & -1.57737 \\ \mathrm{H} & 5.48119 & 1.75545 & -0.09448 \\ \mathrm{H} & 5.24632 & -0.65341 & -1.78673 \\ \mathrm{H} & 6.24537 & -0.50299 & -0.34104 \\ \mathrm{~N} & 2.98598 & -0.35407 & -0.03523 \\ \mathrm{C} & 1.92444 & -0.81747 & 0.85589 \\ \mathrm{H} & 1.69754 & -1.85723 & 0.59467 \\ \mathrm{H} & 2.27938 & -0.81892 & 1.90944 \\ \mathrm{C} & 0.67144 & 0.00502 & 0.76137 \\ \mathrm{C} & -0.47088 & -0.44415 & 0.22515 \\ \mathrm{H} & 0.71809 & 1.01014 & 1.17635 \\ \mathrm{H} & -0.50774 & -1.46843 & -0.13559 \\ \mathrm{C} & -1.71847 & 0.31781 & 0.09016 \\ \mathrm{C} & -2.94353 & -0.36936 & -0.08639 \\ \mathrm{C} & -1.75432 & 1.71809 & 0.10998 \\ \mathrm{C} & -4.14410 & 0.33285 & -0.22134 \\ \mathrm{C} & -2.94773 & 2.42503 & -0.01631 \\ \mathrm{H} & -0.81863 & 2.25946 & 0.20493 \\ \mathrm{C} & -4.14234 & 1.72803 & -0.18114 \\ \mathrm{H} & -5.07986 & -0.19553 & -0.35279 \\ \mathrm{H} & -2.94187 & 3.50997 & 0.00041 \\ \mathrm{H} & -5.08049 & 2.26397 & -0.28759 \\ \mathrm{O} & -2.86056 & -1.73375 & -0.10438 \\ \mathrm{C} & -4.04741 & -2.47715 & -0.31866\end{array}$




$\begin{array}{llll}\mathrm{H} & -4.50905 & -2.23311 & -1.28379 \\ \mathrm{H} & -3.74961 & -3.52637 & -0.31688 \\ \mathrm{H} & -4.77956 & -2.30828 & 0.48102\end{array}$

conformer 5

$E(D Z)=-674.955994$
$G(D Z)=-674.695548$
$E(T Z)=-674.8163957$
$G(T Z)=-674.5559497$

35

01

$\begin{array}{lll}\text { C } & -3.38214 & -1.01951\end{array}$

$\begin{array}{lll}\text { C } & -4.20068 & 0.87636\end{array}$

$\begin{array}{llll}\text { C } & -5.17129 & 0.64191 & 0.59127\end{array}$

$\begin{array}{llll}\text { C } & -4.63326 & -0.64572 & 1.27634\end{array}$

$\begin{array}{llll}\mathrm{H} & -3.64281 & -1.76049 & -0.32381\end{array}$

$\begin{array}{llll}\mathrm{H} & -2.58595 & -1.45195 & 1.07221\end{array}$

$\begin{array}{llll}\mathrm{H} & -4.03841 & 1.93505 & -0.80564\end{array}$

$\begin{array}{llll}\mathrm{H} & -4.58775 & 0.39319 & -1.49817\end{array}$

$\begin{array}{llll}\mathrm{H} & -5.14383 & 1.48511 & 1.28619\end{array}$

$\begin{array}{llll}\mathrm{H} & -6.20193 & 0.53499 & 0.24300\end{array}$

$\begin{array}{llll}\mathrm{H} & -4.36598 & -0.44371 & 2.31662\end{array}$

$\begin{array}{llll}\mathrm{H} & -5.36490 & -1.45788 & 1.27740\end{array}$

$\begin{array}{llll}\mathrm{N} & -2.95889 & 0.25054 & -0.12827\end{array}$

$\begin{array}{llll}\text { C } & -1.96503 & 0.13077 & -1.19200\end{array}$

$\begin{array}{llll}\mathrm{H} & -2.37215 & -0.47367 & -2.03251\end{array}$

$\begin{array}{llll}\mathrm{H} & -1.77222 & 1.13374 & -1.58883\end{array}$

$\begin{array}{llll}\text { C } & -0.67274 & -0.48095 & -0.73429\end{array}$

$\begin{array}{llll}\text { C } & 0.49355 & 0.17754 & -0.71203\end{array}$

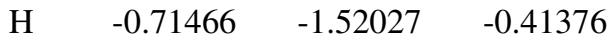

$\begin{array}{llll}\mathrm{H} & 0.50280 & 1.22839 & -0.98814\end{array}$

$\begin{array}{llll}\text { C } & 1.79205 & -0.38576 & -0.32212\end{array}$

$\begin{array}{llll}\text { C } & 2.83987 & 0.47924 & 0.07574\end{array}$

$\begin{array}{llll}\text { C } & 2.05513 & -1.76153 & -0.34014\end{array}$

$\begin{array}{llll}\text { C } & 4.08471 & -0.03183 & 0.45234\end{array}$

$\begin{array}{llll}\text { C } & 3.29126 & -2.27913 & 0.04012\end{array}$

$\begin{array}{llll}\mathrm{H} & 1.27504 & -2.43434 & -0.68128\end{array}$

$\begin{array}{llll}\text { C } & 4.30463 & -1.41016 & 0.43708\end{array}$

$\begin{array}{llll}\mathrm{H} & 4.88235 & 0.63214 & 0.76071\end{array}$

$\begin{array}{llll}\mathrm{H} & 3.46430 & -3.35000 & 0.01297\end{array}$

$\begin{array}{llll}\mathrm{H} & 5.27583 & -1.79672 & 0.73044\end{array}$

$\begin{array}{llll}\mathrm{O} & 2.54112 & 1.81299 & 0.07120\end{array}$

$\begin{array}{llll}\text { C } & 3.55592 & 2.73057 & 0.43902\end{array}$

$\begin{array}{llll}\mathrm{H} & 3.11126 & 3.72247 & 0.35017\end{array}$

$\begin{array}{llll}\mathrm{H} & 3.88541 & 2.57391 & 1.47384\end{array}$

$\begin{array}{llll}\mathrm{H} & 4.42493 & 2.66150 & -0.22750\end{array}$

ArA = 2Furyl

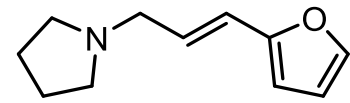

conformer 1

$\mathrm{E}(\mathrm{DZ})=-558.195269$

$\mathrm{G}(\mathrm{DZ})=-557.993268$

$\mathrm{E}(\mathrm{TZ})=-558.0908995$
$\mathrm{G}(\mathrm{TZ})=-557.8888985$

$\begin{array}{lccc}28 & & & \\ 0 \text { 1 } & & & \\ \text { C } & -2.18379 & 0.81799 & 0.58871 \\ \text { C } & -3.57051 & -0.83828 & -0.23876 \\ \text { C } & -4.45214 & 0.42223 & -0.22984 \\ \text { C } & -3.52133 & 1.53423 & 0.33352 \\ \text { H } & -2.13166 & 0.46444 & 1.63856 \\ \text { H } & -1.30718 & 1.44645 & 0.41240 \\ \text { H } & -3.78649 & -1.51731 & -1.07023 \\ \text { H } & -3.70968 & -1.40817 & 0.70221 \\ \text { H } & -4.77394 & 0.67075 & -1.24441 \\ \text { H } & -5.35133 & 0.27894 & 0.37492 \\ \text { H } & -3.39441 & 2.33483 & -0.39977 \\ \text { H } & -3.91324 & 1.98660 & 1.24818 \\ \text { N } & -2.21271 & -0.30828 & -0.34462 \\ \text { C } & -1.17279 & -1.30075 & -0.11413 \\ \text { H } & -1.21239 & -1.70594 & 0.91786 \\ \text { H } & -1.36949 & -2.14324 & -0.79239 \\ \text { C } & 0.19290 & -0.74778 & -0.39078 \\ \text { C } & 1.19393 & -0.74399 & 0.50231 \\ \text { H } & 0.34942 & -0.32451 & -1.37997 \\ \text { H } & 1.02934 & -1.15278 & 1.49733 \\ \text { C } & 2.52425 & -0.23353 & 0.27564 \\ \text { C } & 3.63021 & -0.15389 & 1.08392 \\ \text { O } & 2.82516 & 0.29547 & -0.95515 \\ \text { C } & 4.66630 & 0.45717 & 0.30998 \\ \text { H } & 3.68989 & -0.49233 & 2.10805 \\ \text { C } & 4.12420 & 0.70789 & -0.91415 \\ \text { H } & 5.67597 & 0.67867 & 0.62299 \\ \text { H } & 4.50547 & 1.15016 & -1.82083\end{array}$

conformer 2

$\mathrm{E}(\mathrm{DZ})=-558.195327$

$\mathrm{G}(\mathrm{DZ})=-557.992828$

$\mathrm{E}(\mathrm{TZ})=-558.0908098$

$G(T Z)=-557.8883108$

$\begin{array}{lrrr}28 & & & \\ 01 & & & \\ \mathrm{C} & 2.20318 & 0.42203 & 1.02555 \\ \mathrm{C} & 3.37506 & -0.61804 & -0.67477 \\ \mathrm{C} & 4.07813 & 0.73928 & -0.50845 \\ \mathrm{C} & 3.30176 & 1.43100 & 0.64861 \\ \mathrm{H} & 1.26605 & 0.88876 & 1.33702 \\ \mathrm{H} & 2.54452 & -0.22529 & 1.85779 \\ \mathrm{H} & 3.88113 & -1.38799 & -0.05876 \\ \mathrm{H} & 3.35942 & -0.97870 & -1.70832 \\ \mathrm{H} & 5.14214 & 0.61805 & -0.28985 \\ \mathrm{H} & 3.99498 & 1.32499 & -1.42754 \\ \mathrm{H} & 3.94152 & 1.66143 & 1.50436 \\ \mathrm{H} & 2.86126 & 2.37119 & 0.30671 \\ \mathrm{~N} & 2.01718 & -0.36056 & -0.19846 \\ \mathrm{C} & 1.20901 & -1.55499 & -0.03220 \\ \mathrm{H} & 1.37023 & -2.19945 & -0.90918 \\ \mathrm{H} & 1.54502 & -2.15473 & 0.83926\end{array}$




$\begin{array}{lrrr}\mathrm{C} & -0.26313 & -1.27441 & 0.09706 \\ \mathrm{C} & -0.84275 & -0.12254 & -0.27188 \\ \mathrm{H} & -0.87502 & -2.07992 & 0.49591 \\ \mathrm{H} & -0.22063 & 0.66926 & -0.68010 \\ \mathrm{C} & -2.25078 & 0.17436 & -0.18071 \\ \mathrm{C} & -2.97858 & 1.28929 & -0.51281 \\ \mathrm{O} & -3.09796 & -0.78392 & 0.32094 \\ \mathrm{C} & -4.34478 & 1.00224 & -0.20028 \\ \mathrm{H} & -2.57901 & 2.20155 & -0.93112 \\ \mathrm{C} & -4.35879 & -0.26400 & 0.30093 \\ \mathrm{H} & -5.19869 & 1.65060 & -0.33103 \\ \mathrm{H} & -5.13957 & -0.90985 & 0.67017\end{array}$

conformer 3

$E(D Z)=-558.193639$
$G(D Z)=-557.992285$
$E(T Z)=-558.0890416$
$G(T Z)=-557.8876876$

$\begin{array}{lccc}28 & & & \\ \text { O } 1 & & & \\ \mathrm{C} & -2.20359 & 0.89136 & -0.45868 \\ \mathrm{C} & -3.60658 & -0.86711 & 0.08022 \\ \mathrm{C} & -4.50611 & 0.37851 & 0.17188 \\ \mathrm{C} & -3.55620 & 1.56241 & -0.16899 \\ \mathrm{H} & -1.34045 & 1.49529 & -0.16733 \\ \mathrm{H} & -2.10538 & 0.67458 & -1.54167 \\ \mathrm{H} & -3.68664 & -1.32123 & -0.92781 \\ \mathrm{H} & -3.85738 & -1.64257 & 0.81156 \\ \mathrm{H} & -5.35420 & 0.30924 & -0.51423 \\ \mathrm{H} & -4.90944 & 0.48877 & 1.18176 \\ \mathrm{H} & -3.90438 & 2.14995 & -1.02234 \\ \mathrm{H} & -3.47060 & 2.24031 & 0.68417 \\ \mathrm{~N} & -2.26310 & -0.34457 & 0.32254 \\ \mathrm{C} & -1.20013 & -1.29331 & 0.02329 \\ \mathrm{H} & -1.41852 & -2.21492 & 0.58160 \\ \mathrm{H} & -1.18188 & -1.56805 & -1.05097 \\ \mathrm{C} & 0.14286 & -0.76739 & 0.43268 \\ \mathrm{C} & 1.18867 & -0.65515 & -0.39959 \\ \mathrm{H} & 0.23167 & -0.46000 & 1.47321 \\ \mathrm{H} & 1.07842 & -0.94693 & -1.44205 \\ \mathrm{C} & 2.50143 & -0.16676 & -0.04234 \\ \mathrm{C} & 3.09336 & 0.27501 & 1.11503 \\ \mathrm{O} & 3.41608 & -0.10334 & -1.06743 \\ \mathrm{C} & 4.43698 & 0.62893 & 0.78348 \\ \mathrm{H} & 2.62325 & 0.33720 & 2.08525 \\ \mathrm{C} & 4.57874 & 0.37964 & -0.54939 \\ \mathrm{H} & 5.19508 & 1.01535 & 1.44883 \\ \mathrm{H} & 5.39699 & 0.48490 & -1.24384\end{array}$

conformer 4

$\mathrm{E}(\mathrm{DZ})=-558.1923$

$\mathrm{G}(\mathrm{DZ})=-557.990883$

$E(T Z)=-558.0882891$

$\mathrm{G}(\mathrm{TZ})=-557.8868721$

28

$\begin{array}{lrrr}\text { O } 1 & & & \\ \mathrm{C} & 2.42197 & 1.06660 & 0.27995 \\ \mathrm{C} & 3.47268 & -0.97782 & 0.03280 \\ \mathrm{C} & 4.42409 & 0.01576 & -0.65186 \\ \mathrm{C} & 3.73506 & 1.39528 & -0.45509 \\ \mathrm{H} & 1.58837 & 1.70484 & -0.02571 \\ \mathrm{H} & 2.55397 & 1.18916 & 1.37461 \\ \mathrm{H} & 3.76893 & -1.12432 & 1.09177 \\ \mathrm{H} & 3.45342 & -1.96403 & -0.44297 \\ \mathrm{H} & 5.42665 & -0.02134 & -0.21789 \\ \mathrm{H} & 4.51603 & -0.21696 & -1.71580 \\ \mathrm{H} & 4.34882 & 2.09212 & 0.12153 \\ \mathrm{H} & 3.52856 & 1.86277 & -1.42111 \\ \mathrm{~N} & 2.16624 & -0.33056 & -0.07054 \\ \mathrm{C} & 1.14206 & -0.98450 & 0.74028 \\ \mathrm{H} & 1.08391 & -2.03323 & 0.42693 \\ \mathrm{H} & 1.44003 & -0.98598 & 1.81160 \\ \mathrm{C} & -0.21043 & -0.34776 & 0.61224 \\ \mathrm{C} & -1.26333 & -0.94877 & 0.03858 \\ \mathrm{H} & -0.33491 & 0.65175 & 1.02142 \\ \mathrm{H} & -1.15475 & -1.94926 & -0.37515 \\ \mathrm{C} & -2.58488 & -0.38544 & -0.09443 \\ \mathrm{C} & -3.74253 & -0.87260 & -0.64684 \\ \mathrm{O} & -2.81465 & 0.87619 & 0.39684 \\ \mathrm{C} & -4.73716 & 0.14284 & -0.48789 \\ \mathrm{H} & -3.86260 & -1.84081 & -1.11059 \\ \mathrm{C} & -4.12132 & 1.17723 & 0.14926 \\ \mathrm{H} & -5.76905 & 0.10657 & -0.80466 \\ \mathrm{H} & -4.45114 & 2.14825 & 0.48300\end{array}$

conformer 5

$$
\begin{aligned}
& E(D Z)=-558.193476 \\
& G(D Z)=-557.991819 \\
& E(T Z)=-558.0883328 \\
& G(T Z)=-557.8866758
\end{aligned}
$$

$\begin{array}{lrrr}28 & & & \\ 01 & & & \\ \mathrm{C} & -2.29606 & -0.38272 & 1.08844 \\ \mathrm{C} & -2.77375 & 0.63079 & -0.94691 \\ \mathrm{C} & -3.88581 & 1.12637 & -0.00872 \\ \mathrm{C} & -3.51999 & 0.51177 & 1.37288 \\ \mathrm{H} & -2.30768 & -1.32547 & 1.64697 \\ \mathrm{H} & -1.36379 & 0.14614 & 1.35438 \\ \mathrm{H} & -1.94442 & 1.36237 & -0.96364 \\ \mathrm{H} & -3.10744 & 0.48359 & -1.97996 \\ \mathrm{H} & -3.94183 & 2.21784 & 0.01547 \\ \mathrm{H} & -4.85592 & 0.75192 & -0.34567 \\ \mathrm{H} & -3.28396 & 1.27662 & 2.11749 \\ \mathrm{H} & -4.35322 & -0.07783 & 1.76343 \\ \mathrm{~N} & -2.38901 & -0.64479 & -0.34715 \\ \mathrm{C} & -1.23968 & -1.32526 & -0.94358 \\ \mathrm{H} & -1.48128 & -1.50181 & -2.00026 \\ \mathrm{H} & -1.15012 & -2.30965 & -0.46747 \\ \mathrm{C} & 0.07726 & -0.59878 & -0.83700 \\ \mathrm{C} & 1.09778 & -1.01929 & -0.07313\end{array}$




$\begin{array}{lrrr}\mathrm{H} & 0.18731 & 0.32996 & -1.39164 \\ \mathrm{H} & 1.00185 & -1.94615 & 0.49030 \\ \mathrm{C} & 2.36719 & -0.35229 & 0.08536 \\ \mathrm{C} & 3.48747 & -0.66351 & 0.81415 \\ \mathrm{O} & 2.57759 & 0.82790 & -0.58446 \\ \mathrm{C} & 4.43718 & 0.38012 & 0.58098 \\ \mathrm{H} & 3.61175 & -1.53520 & 1.44001 \\ \mathrm{C} & 3.83431 & 1.25459 & -0.27184 \\ \mathrm{H} & 5.43215 & 0.46646 & 0.99210 \\ \mathrm{H} & 4.14354 & 2.18079 & -0.72963\end{array}$

$\begin{array}{rrrr}\mathrm{H} & 6.27587 & 1.94335 & 0.57374 \\ \mathrm{H} & 5.10050 & -2.19377 & 0.52152 \\ \mathrm{H} & 6.84551 & -0.46640 & 0.82084\end{array}$

conformer 2

$$
\begin{aligned}
& \mathrm{E}(\mathrm{DZ})=-714.092554 \\
& \mathrm{G}(\mathrm{DZ})=-713.818432 \\
& \mathrm{E}(\mathrm{TZ})=-713.9287441 \\
& \mathrm{G}(\mathrm{TZ})=-713.6546221
\end{aligned}
$$

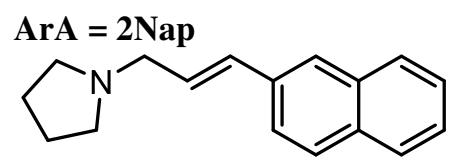

conformer 1

$$
\begin{aligned}
& \mathrm{E}(\mathrm{DZ})=-714.093605 \\
& \mathrm{G}(\mathrm{DZ})=-713.820031 \\
& \mathrm{E}(\mathrm{TZ})=-713.9299799 \\
& \mathrm{G}(\mathrm{TZ})=-713.6564059
\end{aligned}
$$

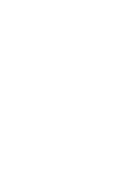

\begin{tabular}{lccc}
37 & & & \\
$\mathrm{O} 1$ & & & \\
$\mathrm{C}$ & -3.74271 & 0.15856 & 1.03358 \\
$\mathrm{C}$ & -5.19099 & -0.25885 & -0.72143 \\
$\mathrm{C}$ & -5.94585 & -0.76355 & 0.52080 \\
$\mathrm{C}$ & -4.96758 & -0.49109 & 1.69921 \\
$\mathrm{H}$ & -2.79444 & -0.09318 & 1.51540 \\
$\mathrm{H}$ & -3.83777 & 1.26334 & 1.04761 \\
$\mathrm{H}$ & -5.47722 & 0.78942 & -0.94156 \\
$\mathrm{H}$ & -5.38618 & -0.84984 & -1.62240 \\
$\mathrm{H}$ & -6.90388 & -0.25149 & 0.64226 \\
$\mathrm{H}$ & -6.15355 & -1.83322 & 0.43516 \\
$\mathrm{H}$ & -5.40004 & 0.15952 & 2.46367 \\
$\mathrm{H}$ & -4.68600 & -1.42814 & 2.18642 \\
$\mathrm{~N}$ & -3.78444 & -0.35620 & -0.33546 \\
$\mathrm{C}$ & -2.87532 & 0.31256 & -1.25432 \\
$\mathrm{H}$ & -3.08295 & -0.07770 & -2.26104 \\
$\mathrm{H}$ & -3.05662 & 1.40600 & -1.29318 \\
$\mathrm{C}$ & -1.44015 & 0.04893 & -0.90704 \\
$\mathrm{C}$ & -0.52989 & 1.00996 & -0.70161 \\
$\mathrm{H}$ & -1.17076 & -1.00175 & -0.81608 \\
$\mathrm{H}$ & -0.86265 & 2.04648 & -0.75738 \\
$\mathrm{C}$ & 0.89288 & 0.82987 & -0.38571 \\
$\mathrm{C}$ & 1.55466 & -0.38088 & -0.51749 \\
$\mathrm{C}$ & 1.63324 & 1.95683 & 0.07596 \\
$\mathrm{C}$ & 2.92296 & -0.52095 & -0.18720 \\
$\mathrm{H}$ & 1.02664 & -1.25123 & -0.89570 \\
$\mathrm{C}$ & 2.96259 & 1.85606 & 0.40329 \\
$\mathrm{H}$ & 1.12194 & 2.91065 & 0.17255 \\
$\mathrm{C}$ & 3.60698 & -1.76044 & -0.31629 \\
$\mathrm{C}$ & 3.64919 & 0.61993 & 0.28645 \\
$\mathrm{H}$ & 3.50816 & 2.72685 & 0.75631 \\
$\mathrm{C}$ & 4.93863 & -1.86788 & 0.00961 \\
$\mathrm{H}$ & 3.05576 & -2.62458 & -0.67683 \\
$\mathrm{C}$ & 5.02211 & 0.47592 & 0.61299 \\
$\mathrm{C}$ & 5.65424 & -0.73900 & 0.47933 \\
$\mathrm{H}$ & 5.44840 & -2.82104 & -0.09220 \\
$\mathrm{H}$ & 5.56705 & 1.34485 & 0.97171 \\
$\mathrm{H}$ & 6.70525 & -0.83743 & 0.73283 \\
& & & \\
\hline
\end{tabular}

conformer 3

$$
\begin{aligned}
& \mathrm{E}(\mathrm{DZ})=-714.089237 \\
& \mathrm{G}(\mathrm{DZ})=-713.816162 \\
& \mathrm{E}(\mathrm{TZ})=-713.9257824 \\
& \mathrm{G}(\mathrm{TZ})=-713.6527074
\end{aligned}
$$




$\begin{array}{lrrr}37 & & & \\ \text { O } 1 & & & \\ \mathrm{C} & -3.79713 & 1.22145 & 0.28063 \\ \mathrm{C} & -5.15091 & -0.62510 & -0.03497 \\ \mathrm{C} & -5.90261 & 0.51491 & -0.74064 \\ \mathrm{C} & -5.00478 & 1.76239 & -0.50658 \\ \mathrm{H} & -3.95723 & 1.35640 & 1.37009 \\ \mathrm{H} & -2.85900 & 1.71949 & 0.01991 \\ \mathrm{H} & -5.27065 & -1.59729 & -0.52449 \\ \mathrm{H} & -5.50947 & -0.73126 & 1.00946 \\ \mathrm{H} & -5.99592 & 0.30625 & -1.80939 \\ \mathrm{H} & -6.91172 & 0.64036 & -0.33969 \\ \mathrm{H} & -4.68234 & 2.18745 & -1.46036 \\ \mathrm{H} & -5.51932 & 2.55197 & 0.04713 \\ \mathrm{~N} & -3.75408 & -0.19602 & -0.07792 \\ \mathrm{C} & -2.88270 & -1.01130 & 0.76458 \\ \mathrm{H} & -3.21445 & -0.96320 & 1.82467 \\ \mathrm{H} & -2.98677 & -2.05470 & 0.44611 \\ \mathrm{C} & -1.43891 & -0.60704 & 0.68392 \\ \mathrm{C} & -0.48098 & -1.36918 & 0.14094 \\ \mathrm{H} & -1.18025 & 0.35782 & 1.11563 \\ \mathrm{H} & -0.76396 & -2.34710 & -0.24873 \\ \mathrm{C} & 0.94667 & -1.04219 & 0.02678 \\ \mathrm{C} & 1.44352 & 0.24298 & 0.17474 \\ \mathrm{C} & 1.86641 & -2.09297 & -0.25731 \\ \mathrm{C} & 2.82638 & 0.52304 & 0.07413 \\ \mathrm{H} & 0.76418 & 1.07065 & 0.35644 \\ \mathrm{C} & 3.21462 & -1.85592 & -0.36198 \\ \mathrm{H} & 1.48160 & -3.10096 & -0.38493 \\ \mathrm{C} & 3.34300 & 1.83830 & 0.22783 \\ \mathrm{C} & 3.73901 & -0.54785 & -0.19729 \\ \mathrm{H} & 3.90000 & -2.67168 & -0.57493 \\ \mathrm{C} & 4.69234 & 2.08227 & 0.12406 \\ \mathrm{H} & 2.64970 & 2.64978 & 0.43138 \\ \mathrm{C} & 5.12492 & -0.26250 & -0.29931 \\ \mathrm{C} & 5.59325 & 1.02168 & -0.14154 \\ \mathrm{H} & 5.07363 & 3.09154 & 0.24532 \\ \mathrm{H} & 5.81187 & -1.07893 & -0.50486 \\ \mathrm{H} & 6.65614 & 1.22778 & -0.22112 \\ & & & \\ & & & \\ & & \end{array}$

$\begin{array}{lrrr}\mathrm{H} & -4.46802 & 0.59415 & -1.75478 \\ \mathrm{H} & -2.90483 & -1.31048 & 1.05878 \\ \mathrm{H} & -1.94516 & 0.11717 & 1.49224 \\ \mathrm{H} & -4.56785 & -0.22798 & 2.36922 \\ \mathrm{H} & -3.92150 & 1.38036 & 2.04935 \\ \mathrm{H} & -5.33670 & 1.64253 & 0.22902 \\ \mathrm{H} & -6.03014 & 0.04254 & 0.48866 \\ \mathrm{~N} & -2.88683 & 0.24321 & -0.39755 \\ \mathrm{C} & -1.91443 & -0.42042 & -1.25399 \\ \mathrm{H} & -2.02996 & -1.52323 & -1.23313 \\ \mathrm{H} & -2.11651 & -0.10063 & -2.28619 \\ \mathrm{C} & -0.50874 & -0.04882 & -0.88469 \\ \mathrm{C} & 0.44488 & -0.93552 & -0.57117 \\ \mathrm{H} & -0.30429 & 1.01987 & -0.87099 \\ \mathrm{H} & 0.17485 & -1.99168 & -0.56524 \\ \mathrm{C} & 1.84082 & -0.65196 & -0.21358 \\ \mathrm{C} & 2.40648 & 0.63268 & -0.30513 \\ \mathrm{C} & 2.65299 & -1.70127 & 0.24044 \\ \mathrm{C} & 3.73007 & 0.88062 & 0.05279 \\ \mathrm{H} & 1.80259 & 1.45668 & -0.67288 \\ \mathrm{C} & 3.97925 & -1.46933 & 0.60106 \\ \mathrm{H} & 2.23689 & -2.70228 & 0.31325 \\ \mathrm{C} & 4.51592 & -0.18858 & 0.51055 \\ \mathrm{H} & 4.59550 & -2.29183 & 0.95200 \\ \mathrm{H} & 5.55133 & -0.01169 & 0.78948 \\ \mathrm{C} & 4.31435 & 2.26872 & -0.04623 \\ \mathrm{H} & 3.60599 & 2.96880 & -0.49663 \\ \mathrm{H} & 4.58425 & 2.65650 & 0.94281 \\ \mathrm{H} & 5.22620 & 2.27428 & -0.65325\end{array}$

conformer 2

$$
\begin{aligned}
& E(D Z)=-599.753871 \\
& G(D Z)=-599.498596 \\
& E(T Z)=-599.6043704 \\
& G(T Z)=-599.3490954
\end{aligned}
$$

$\operatorname{ArA}=3-\mathrm{Me}-\mathrm{Ph}$

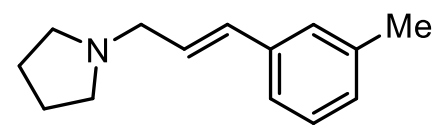

conformer 1

$$
\begin{aligned}
& E(D Z)=-599.753922 \\
& G(D Z)=-599.499992 \\
& E(T Z)=-599.6043999 \\
& G(T Z)=-599.3504699
\end{aligned}
$$

$\begin{array}{lrrr}34 & & & \\ 01 & & & \\ \text { C } & -4.27398 & 0.05825 & -0.81974 \\ \text { C } & -2.86408 & -0.20415 & 0.99525 \\ \text { C } & -4.14055 & 0.41170 & 1.59271 \\ \text { C } & -5.08972 & 0.58750 & 0.37272 \\ \text { H } & -4.50434 & -1.01208 & -0.99478\end{array}$

$\begin{array}{lrrc}34 & & & \\ 01 & & & \\ \mathrm{C} & 2.83835 & 0.79119 & 0.69039 \\ \mathrm{C} & 4.36227 & -0.20261 & -0.73718 \\ \mathrm{C} & 5.19497 & 0.18060 & 0.49910 \\ \mathrm{C} & 4.16642 & 0.83724 & 1.46408 \\ \mathrm{H} & 1.96060 & 0.65764 & 1.32767 \\ \mathrm{H} & 2.69316 & 1.73101 & 0.11983 \\ \mathrm{H} & 4.69328 & -1.12930 & -1.21754 \\ \mathrm{H} & 4.41395 & 0.60369 & -1.49644 \\ \mathrm{H} & 5.63522 & -0.70935 & 0.95617 \\ \mathrm{H} & 6.01463 & 0.85439 & 0.23636 \\ \mathrm{H} & 4.43761 & 1.86083 & 1.73519 \\ \mathrm{H} & 4.08979 & 0.26144 & 2.39005 \\ \mathrm{~N} & 3.00715 & -0.35005 & -0.20962 \\ \mathrm{C} & 1.98229 & -0.45608 & -1.23827 \\ \mathrm{H} & 1.92189 & 0.46252 & -1.85667 \\ \mathrm{H} & 2.27901 & -1.27232 & -1.91229 \\ \mathrm{C} & 0.63712 & -0.76040 & -0.64790 \\ \mathrm{C} & -0.45593 & -0.01247 & -0.84716 \\ \mathrm{H} & 0.60122 & -1.64271 & -0.01193\end{array}$




$\begin{array}{lrrr}\mathrm{H} & -0.35496 & 0.88244 & -1.46142 \\ \mathrm{C} & -1.80274 & -0.24769 & -0.31080 \\ \mathrm{C} & -2.77756 & 0.74975 & -0.46903 \\ \mathrm{C} & -2.16643 & -1.42981 & 0.35676 \\ \mathrm{C} & -4.07611 & 0.60738 & 0.02721 \\ \mathrm{H} & -2.50855 & 1.66485 & -0.99195 \\ \mathrm{C} & -3.45537 & -1.58728 & 0.85307 \\ \mathrm{H} & -1.44430 & -2.23085 & 0.47398 \\ \mathrm{C} & -4.40653 & -0.57823 & 0.69218 \\ \mathrm{H} & -3.72715 & -2.50759 & 1.36176 \\ \mathrm{H} & -5.41289 & -0.71477 & 1.07877 \\ \mathrm{C} & -5.08783 & 1.71626 & -0.12809 \\ \mathrm{H} & -4.87019 & 2.33598 & -1.00256 \\ \mathrm{H} & -6.10154 & 1.31937 & -0.23601 \\ \mathrm{H} & -5.08831 & 2.37599 & 0.74823\end{array}$

$$
\begin{aligned}
& H \quad 5.97469 \quad-1.16196 \quad 1.15219 \\
& \text { conformer } 4 \\
& E(D Z)=-599.753928 \\
& G(D Z)=-599.498485 \\
& E(T Z)=-599.604398 \\
& G(T Z)=-599.348955
\end{aligned}
$$

\begin{tabular}{|c|c|c|c|}
\hline \multicolumn{4}{|c|}{$E(D Z)=-599.75061$} \\
\hline \multicolumn{4}{|c|}{$G(D Z)=-599.496639$} \\
\hline \multicolumn{4}{|c|}{$E(T Z)=-599.6014254$} \\
\hline \multicolumn{4}{|c|}{$G(T Z)=-599.3474544$} \\
\hline \multicolumn{4}{|c|}{34} \\
\hline 01 & & & \\
\hline $\mathrm{C}$ & 4.18468 & -1.09494 & 0.13046 \\
\hline $\mathrm{C}$ & 3.30190 & 1.03852 & 0.02963 \\
\hline $\mathrm{C}$ & 4.57492 & 1.10994 & -0.83347 \\
\hline & 5.15430 & -0.33207 & -0.78632 \\
\hline
\end{tabular}

conformer 3

$$
\begin{aligned}
& E(D Z)=-599.75382 \\
& G(D Z)=-599.498543 \\
& E(T Z)=-599.6043247 \\
& G(T Z)=-599.3490477
\end{aligned}
$$

$\begin{array}{lrrr}34 & & & \\ 01 & & & \\ \mathrm{C} & -2.87866 & -0.30068 & 0.99851 \\ \mathrm{C} & -4.35891 & -0.24762 & -0.77792 \\ \mathrm{C} & -5.22487 & 0.09528 & 0.44720 \\ \mathrm{C} & -4.22626 & 0.07042 & 1.63977 \\ \mathrm{H} & -2.01669 & 0.16371 & 1.48431 \\ \mathrm{H} & -2.72500 & -1.39841 & 1.03247 \\ \mathrm{H} & -4.66946 & 0.26953 & -1.69186 \\ \mathrm{H} & -4.39974 & -1.33683 & -0.98092 \\ \mathrm{H} & -5.66725 & 1.08887 & 0.33865 \\ \mathrm{H} & -6.04441 & -0.61759 & 0.56984 \\ \mathrm{H} & -4.51308 & -0.64563 & 2.41421 \\ \mathrm{H} & -4.16742 & 1.05583 & 2.10915 \\ \mathrm{~N} & -3.01589 & 0.16960 & -0.38034 \\ \mathrm{C} & -1.96542 & -0.29043 & -1.27701 \\ \mathrm{H} & -1.89611 & -1.39713 & -1.29871 \\ \mathrm{H} & -2.23991 & 0.02943 & -2.29246 \\ \mathrm{C} & -0.63278 & 0.29478 & -0.91308 \\ \mathrm{C} & 0.46421 & -0.43406 & -0.66969 \\ \mathrm{H} & -0.61021 & 1.38071 & -0.84730 \\ \mathrm{H} & 0.37650 & -1.51887 & -0.73238 \\ \mathrm{C} & 1.80312 & 0.05683 & -0.31880 \\ \mathrm{C} & 2.82449 & -0.87700 & -0.08709 \\ \mathrm{C} & 2.11330 & 1.42251 & -0.19411 \\ \mathrm{C} & 4.12300 & -0.49119 & 0.25832 \\ \mathrm{H} & 2.59415 & -1.93630 & -0.17772 \\ \mathrm{C} & 3.39820 & 1.82114 & 0.15380 \\ \mathrm{H} & 1.34753 & 2.17152 & -0.36548 \\ \mathrm{C} & 4.39922 & 0.87404 & 0.38062 \\ \mathrm{H} & 3.62498 & 2.87863 & 0.25309 \\ \mathrm{H} & 5.39984 & 1.19702 & 0.65475 \\ \mathrm{C} & 5.20313 & -1.52430 & 0.46692 \\ \mathrm{H} & 4.79429 & -2.45310 & 0.87544 \\ \mathrm{H} & 5.69787 & -1.77496 & -0.47943 \\ & & & \\ & & & \\ & & & \\ & & & \\ & & & \\ & & & \end{array}$

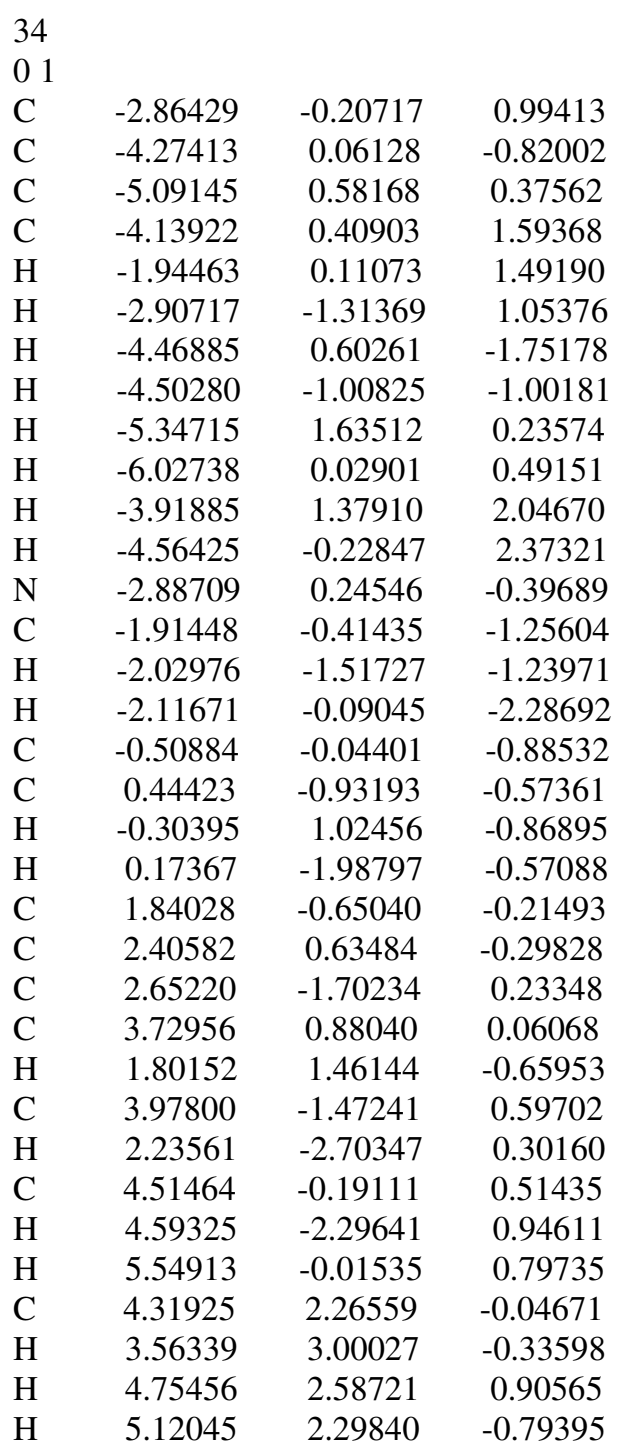

conformer 5 


$\begin{array}{lrrr}\mathrm{H} & 4.06231 & -2.14854 & -0.14193 \\ \mathrm{H} & 4.54365 & -1.06170 & 1.17969 \\ \mathrm{H} & 2.49514 & 1.67431 & -0.34545 \\ \mathrm{H} & 3.52426 & 1.35685 & 1.06901 \\ \mathrm{H} & 4.32743 & 1.39054 & -1.86027 \\ \mathrm{H} & 5.27478 & 1.85637 & -0.44900 \\ \mathrm{H} & 5.16155 & -0.77394 & -1.78594 \\ \mathrm{H} & 6.17801 & -0.36299 & -0.40437 \\ \mathrm{~N} & 2.92306 & -0.37324 & -0.02402 \\ \mathrm{C} & 1.91546 & -0.77173 & 0.95570 \\ \mathrm{H} & 1.76018 & -1.85228 & 0.85924 \\ \mathrm{H} & 2.28798 & -0.59135 & 1.98783 \\ \mathrm{C} & 0.60479 & -0.06076 & 0.77800 \\ \mathrm{C} & -0.52678 & -0.67148 & 0.40438 \\ \mathrm{H} & 0.60446 & 1.00723 & 0.98512 \\ \mathrm{H} & -0.48731 & -1.74444 & 0.21614 \\ \mathrm{C} & -1.84975 & -0.06198 & 0.21551 \\ \mathrm{C} & -2.93485 & -0.89184 & -0.10385 \\ \mathrm{C} & -2.08557 & 1.31888 & 0.33825 \\ \mathrm{C} & -4.22517 & -0.38986 & -0.29896 \\ \mathrm{H} & -2.76353 & -1.96176 & -0.20024 \\ \mathrm{C} & -3.36287 & 1.83219 & 0.15064 \\ \mathrm{H} & -1.26839 & 1.99081 & 0.57817 \\ \mathrm{C} & -4.42914 & 0.98661 & -0.16406 \\ \mathrm{H} & -3.53391 & 2.90000 & 0.25112 \\ \mathrm{H} & -5.42454 & 1.39828 & -0.30645 \\ \mathrm{C} & -5.36036 & -1.31159 & -0.67205 \\ \mathrm{H} & -5.26553 & -2.28301 & -0.17765 \\ \mathrm{H} & -6.32840 & -0.88344 & -0.39731 \\ \mathrm{H} & -5.37885 & -1.49763 & -1.75282\end{array}$

$\begin{array}{lrrc}\mathrm{H} & -3.55047 & 1.75246 & -1.42818 \\ \mathrm{H} & -3.36054 & 1.88587 & 0.32840 \\ \mathrm{C} & -1.85025 & 0.72390 & -0.66001 \\ \mathrm{C} & -0.85096 & 1.13276 & 0.13253 \\ \mathrm{H} & -1.67486 & 0.00468 & -1.45758 \\ \mathrm{H} & -1.08889 & 1.82901 & 0.93627 \\ \mathrm{C} & 0.56028 & 0.73766 & 0.05324 \\ \mathrm{C} & 1.07931 & -0.05824 & -0.98470 \\ \mathrm{C} & 1.44449 & 1.17294 & 1.05415 \\ \mathrm{C} & 2.42081 & -0.41215 & -1.01068 \\ \mathrm{H} & 0.42911 & -0.39442 & -1.78482 \\ \mathrm{C} & 2.79028 & 0.82241 & 1.03363 \\ \mathrm{H} & 1.06574 & 1.79537 & 1.85959 \\ \mathrm{C} & 3.28287 & 0.02676 & -0.00042 \\ \mathrm{H} & 2.80908 & -1.01904 & -1.82112 \\ \mathrm{H} & 3.45988 & 1.17024 & 1.81186 \\ \mathrm{C} & 4.71915 & -0.40775 & -0.00426 \\ \mathrm{~F} & 5.51322 & 0.46105 & 0.66068 \\ \mathrm{~F} & 5.20624 & -0.52438 & -1.26085 \\ \mathrm{~F} & 4.87916 & -1.61622 & 0.58731\end{array}$

$\mathrm{ArA}=$ 4-CF3-Ph

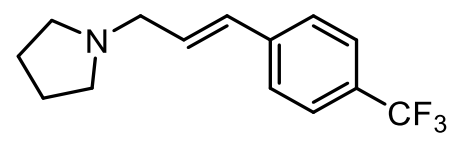

conformer 1

$\mathrm{E}(\mathrm{DZ})=-897.4712$
$\mathrm{G}(\mathrm{DZ})=-897.242651$
$\mathrm{E}(\mathrm{TZ})=-897.3873132$
$\mathrm{G}(\mathrm{TZ})=-897.1587642$

34

01

$\begin{array}{lrrr}\mathrm{C} & -4.05015 & -0.70660 & 0.81948 \\ \mathrm{C} & -5.60051 & 0.44731 & -0.45407 \\ \mathrm{C} & -6.33680 & -0.83538 & -0.02935 \\ \mathrm{C} & -5.29019 & -1.61582 & 0.81679 \\ \mathrm{H} & -3.10374 & -1.25363 & 0.83237 \\ \mathrm{H} & -4.06083 & -0.04266 & 1.70731 \\ \mathrm{H} & -5.81559 & 1.26575 & 0.26178 \\ \mathrm{H} & -5.87802 & 0.80113 & -1.45243 \\ \mathrm{H} & -7.24569 & -0.60670 & 0.53302 \\ \mathrm{H} & -6.63100 & -1.41732 & -0.90646 \\ \mathrm{H} & -5.63861 & -1.82147 & 1.83205 \\ \mathrm{H} & -5.05926 & -2.57570 & 0.34787 \\ \mathrm{~N} & -4.18969 & 0.06491 & -0.41648 \\ \mathrm{C} & -3.27099 & 1.18687 & -0.52779\end{array}$

conformer 2

$\mathrm{E}(\mathrm{DZ})=-897.471205$

$\mathrm{G}(\mathrm{DZ})=-897.242432$

$E(T Z)=-897.3873098$

$G(T Z)=-897.1585368$

$\begin{array}{lrrr}34 & & & \\ 01 & & & \\ \mathrm{C} & 5.60032 & -0.46444 & -0.47149 \\ \mathrm{C} & 4.06776 & 0.69733 & 0.81642 \\ \mathrm{C} & 5.31535 & 1.59638 & 0.80869 \\ \mathrm{C} & 6.34844 & 0.81260 & -0.05087 \\ \mathrm{H} & 5.81534 & -1.28537 & 0.24155 \\ \mathrm{H} & 5.86766 & -0.81858 & -1.47250 \\ \mathrm{H} & 3.12598 & 1.25204 & 0.83697 \\ \mathrm{H} & 4.07869 & 0.03080 & 1.70234 \\ \mathrm{H} & 5.08881 & 2.56129 & 0.34802 \\ \mathrm{H} & 5.67386 & 1.79243 & 1.82234 \\ \mathrm{H} & 6.63597 & 1.39542 & -0.92961 \\ \mathrm{H} & 7.26224 & 0.57734 & 0.50070 \\ \mathrm{~N} & 4.19261 & -0.07212 & -0.42240 \\ \mathrm{C} & 3.26483 & -1.18710 & -0.52894 \\ \mathrm{H} & 3.53454 & -1.75425 & -1.43128 \\ \mathrm{H} & 3.35417 & -1.88721 & 0.32636 \\ \mathrm{C} & 1.84689 & -0.71336 & -0.65253 \\ \mathrm{C} & 0.85049 & -1.10881 & 0.15038 \\ \mathrm{H} & 1.67095 & 0.00389 & -1.45179 \\ \mathrm{H} & 1.08889 & -1.79960 & 0.95868 \\ \mathrm{C} & -0.55794 & -0.70243 & 0.07702 \\ \mathrm{C} & -1.08372 & 0.05779 & -0.98355 \\ \mathrm{C} & -1.43037 & -1.08382 & 1.11013 \\ \mathrm{C} & -2.42133 & 0.42777 & -1.00286 \\ \mathrm{H} & -0.44129 & 0.35622 & -1.80466 \\ \mathrm{C} & -2.77153 & -0.71687 & 1.09657 \\ \mathrm{H} & -1.04417 & -1.67123 & 1.93802 \\ \mathrm{C} & -3.27119 & 0.04311 & 0.03874\end{array}$




$\begin{array}{rrrr}\mathrm{H} & -2.81245 & 1.01830 & -1.82374 \\ \mathrm{H} & -3.42924 & -1.01170 & 1.90639 \\ \mathrm{C} & -4.73052 & 0.38806 & -0.01708 \\ \mathrm{~F} & -5.44683 & -0.56273 & -0.66435 \\ \mathrm{~F} & -4.94926 & 1.55164 & -0.67053 \\ \mathrm{~F} & -5.26869 & 0.50753 & 1.21766\end{array}$

conformer 3

$E(D Z)=-897.471212$
$G(D Z)=-897.242244$
$E(T Z)=-897.3873275$
$G(T Z)=-897.1583595$

$\begin{array}{lrrr}34 & & & \\ 01 & & & \\ \text { C } & -4.07080 & 0.67624 & 0.83557 \\ \text { C } & -5.60086 & -0.45452 & -0.48267 \\ \text { C } & -6.35533 & 0.80369 & -0.01815 \\ \text { C } & -5.32083 & 1.57132 & 0.85381 \\ \text { H } & -4.07947 & -0.01627 & 1.70135 \\ \text { H } & -3.13058 & 1.23282 & 0.87186 \\ \text { H } & -5.86976 & -0.77824 & -1.49353 \\ \text { H } & -5.80810 & -1.29897 & 0.20461 \\ \text { H } & -7.26094 & 0.54373 & 0.53587 \\ \text { H } & -6.65825 & 1.40904 & -0.87626 \\ \text { H } & -5.09857 & 2.54661 & 0.41327 \\ \text { H } & -5.67513 & 1.74431 & 1.87312 \\ \text { N } & -4.19492 & -0.05617 & -0.42579 \\ \text { C } & -3.26358 & -1.16451 & -0.56646 \\ \text { H } & -3.35419 & -1.89320 & 0.26464 \\ \text { H } & -3.52929 & -1.70192 & -1.48809 \\ \text { C } & -1.84661 & -0.68327 & -0.66949 \\ \text { C } & -0.84795 & -1.11717 & 0.11030 \\ \text { H } & -1.67242 & 0.07035 & -1.43505 \\ \text { H } & -1.08319 & -1.84717 & 0.88439 \\ \text { C } & 0.56018 & -0.70776 & 0.05201 \\ \text { C } & 1.07931 & 0.11676 & -0.96298 \\ \text { C } & 1.43952 & -1.15359 & 1.05258 \\ \text { C } & 2.41679 & 0.48692 & -0.96832 \\ \text { H } & 0.43139 & 0.46657 & -1.75923 \\ \text { C } & 2.78111 & -0.78698 & 1.05267 \\ \text { H } & 1.05887 & -1.79151 & 1.84491 \\ \text { C } & 3.27391 & 0.03751 & 0.04127 \\ \text { H } & 2.80210 & 1.12758 & -1.75369 \\ \text { H } & 3.44382 & -1.13346 & 1.83739 \\ \text { C } & 4.73171 & 0.38981 & -0.00295 \\ \text { F } & 5.29046 & 0.38609 & 1.22838 \\ \text { F } & 4.93637 & 1.61469 & -0.53920 \\ \text { F } & 5.43859 & -0.48675 & -0.75674\end{array}$

conformer 4

$\mathrm{E}(\mathrm{DZ})=-897.467914$

$\mathrm{G}(\mathrm{DZ})=-897.239704$

$E(T Z)=-897.3844082$

$\mathrm{G}(\mathrm{TZ})=-897.1561982$

$\begin{array}{lrrr}34 & & & \\ \text { O } 1 & & & \\ \text { C } & -4.30470 & 1.20811 & -0.16409 \\ \text { C } & -5.51777 & -0.75080 & 0.02787 \\ \text { C } & -6.33375 & 0.27431 & 0.83088 \\ \text { C } & -5.53390 & 1.59861 & 0.67723 \\ \text { H } & -3.40082 & 1.75236 & 0.12355 \\ \text { H } & -4.49331 & 1.41293 & -1.23790 \\ \text { H } & -5.88793 & -0.80193 & -1.01642 \\ \text { H } & -5.55841 & -1.76368 & 0.44190 \\ \mathrm{H} & -7.35797 & 0.35430 & 0.45788 \\ \mathrm{H} & -6.38718 & -0.02012 & 1.88200 \\ \mathrm{H} & -6.11468 & 2.38561 & 0.18953 \\ \mathrm{H} & -5.22576 & 1.97665 & 1.65513 \\ \mathrm{~N} & -4.15398 & -0.22622 & 0.08378 \\ \mathrm{C} & -3.24466 & -0.91157 & -0.82956 \\ \mathrm{H} & -3.26330 & -1.98024 & -0.58822 \\ \mathrm{H} & -3.59857 & -0.81288 & -1.87911 \\ \mathrm{C} & -1.83365 & -0.40511 & -0.74641 \\ \mathrm{C} & -0.80659 & -1.13638 & -0.29543 \\ \mathrm{H} & -1.66752 & 0.61636 & -1.08201 \\ \mathrm{H} & -1.01241 & -2.14681 & 0.05652 \\ \mathrm{C} & 0.59742 & -0.71913 & -0.19707 \\ \mathrm{C} & 1.08755 & 0.46896 & -0.76952 \\ \mathrm{C} & 1.50210 & -1.53608 & 0.50093 \\ \mathrm{C} & 2.42092 & 0.82966 & -0.63538 \\ \mathrm{H} & 0.42053 & 1.11301 & -1.33206 \\ \mathrm{C} & 2.83966 & -1.18082 & 0.63851 \\ \mathrm{H} & 1.14425 & -2.45837 & 0.94898 \\ \mathrm{C} & 3.30287 & 0.00671 & 0.07221 \\ \mathrm{H} & 2.78382 & 1.75166 & -1.07585 \\ \mathrm{H} & 3.52248 & -1.81757 & 1.18928 \\ \mathrm{C} & 4.75568 & 0.37182 & 0.16234 \\ \mathrm{~F} & 4.93526 & 1.71166 & 0.21080 \\ \mathrm{~F} & 5.45411 & -0.07509 & -0.90931 \\ \mathrm{~F} & 5.34260 & -0.15740 & 1.25910\end{array}$

conformer 5

$$
\begin{aligned}
& E(D Z)=-897.467934 \\
& G(D Z)=-897.239374 \\
& E(T Z)=-897.3843472 \\
& G(T Z)=-897.1557872
\end{aligned}
$$

$\begin{array}{lrrr}34 & & & \\ 01 & & & \\ \mathrm{C} & 5.51383 & 0.76402 & 0.12252 \\ \mathrm{C} & 4.33387 & -1.18138 & -0.29085 \\ \mathrm{C} & 5.56960 & -1.64260 & 0.50426 \\ \mathrm{C} & 6.34182 & -0.32957 & 0.81476 \\ \mathrm{H} & 5.88854 & 0.93476 & -0.90730 \\ \mathrm{H} & 5.53531 & 1.72609 & 0.64495 \\ \mathrm{H} & 3.43951 & -1.76928 & -0.06590 \\ \mathrm{H} & 4.52488 & -1.26505 & -1.38032 \\ \mathrm{H} & 5.26886 & -2.13955 & 1.42985 \\ \mathrm{H} & 6.16733 & -2.35451 & -0.07060 \\ \mathrm{H} & 6.37816 & -0.15392 & 1.89284 \\ \mathrm{H} & 7.37134 & -0.34850 & 0.44830\end{array}$




\begin{tabular}{|c|c|c|c|c|c|c|c|}
\hline $\mathrm{N}$ & 4.15864 & 0.21460 & 0.11178 & $\mathrm{C}$ & 2.19021 & -1.01004 & 1.05760 \\
\hline $\mathrm{C}$ & 3.24289 & 0.98091 & -0.72776 & $\mathrm{C}$ & 3.12024 & 0.61467 & -1.00183 \\
\hline $\mathrm{H}$ & 3.25523 & 2.02051 & -0.38202 & $\mathrm{H}$ & 1.13195 & 0.53588 & -1.77464 \\
\hline $\mathrm{H}$ & 3.59537 & 0.98834 & -1.78246 & $\mathrm{C}$ & 3.52623 & -0.61838 & 1.04640 \\
\hline $\mathrm{C}$ & 1.83514 & 0.46023 & -0.69106 & $\mathrm{H}$ & 1.83172 & -1.64411 & 1.86352 \\
\hline $\mathrm{C}$ & 0.79901 & 1.15418 & -0.20324 & $\mathrm{C}$ & 3.98151 & 0.19540 & 0.01292 \\
\hline $\mathrm{H}$ & 1.67862 & -0.53309 & -1.10613 & $\mathrm{H}$ & 3.49302 & 1.24332 & -1.80229 \\
\hline $\mathrm{H}$ & 0.99298 & 2.14877 & 0.19723 & $\mathrm{H}$ & 4.20655 & -0.93881 & 1.82694 \\
\hline $\mathrm{C}$ & -0.60477 & 0.72887 & -0.14469 & $\mathrm{Cl}$ & 5.66481 & 0.69826 & -0.01995 \\
\hline $\mathrm{C}$ & -1.04331 & -0.54165 & -0.56103 & & & & \\
\hline $\mathrm{C}$ & -1.56535 & 1.62724 & 0.34861 & \multicolumn{4}{|c|}{ conformer 2} \\
\hline $\mathrm{C}$ & -2.38318 & -0.89494 & -0.48758 & \multicolumn{4}{|c|}{$E(D Z)=-1020.026246$} \\
\hline $\mathrm{H}$ & -0.32926 & -1.26179 & -0.94512 & \multicolumn{4}{|c|}{$G(D Z)=-1019.806786$} \\
\hline $\mathrm{C}$ & -2.90986 & 1.27974 & 0.42488 & \multirow{2}{*}{\multicolumn{4}{|c|}{$\begin{array}{l}E(T Z)=-1019.8974696 \\
G(T Z)=-1019.6780096\end{array}$}} \\
\hline $\mathrm{H}$ & -1.24846 & 2.61397 & 0.67328 & & & & \\
\hline $\mathrm{C}$ & -3.32349 & 0.01576 & 0.00481 & \\
\hline $\mathrm{H}$ & -2.70785 & -1.87521 & -0.81829 & \multicolumn{4}{|l|}{31} \\
\hline $\mathrm{H}$ & -3.63888 & 1.98798 & 0.80182 & \multicolumn{4}{|c|}{01} \\
\hline $\mathrm{C}$ & -4.76134 & -0.39250 & 0.13832 & $\mathrm{C}$ & -4.61765 & 0.31443 & -0.71244 \\
\hline $\mathrm{F}$ & -5.00021 & -1.01068 & 1.32004 & $\mathrm{C}$ & -3.33123 & -0.35580 & 1.09019 \\
\hline $\mathrm{F}$ & -5.13150 & -1.25743 & -0.83348 & $\mathrm{C}$ & -4.29583 & -1.52973 & 0.85551 \\
\hline \multirow[t]{2}{*}{$\mathrm{F}$} & \multirow{2}{*}{-5.59621} & \multirow{2}{*}{0.66905} & \multirow[t]{2}{*}{0.07740} & $\mathrm{C}$ & -5.16467 & -1.07772 & -0.35296 \\
\hline & & & & $\mathrm{H}$ & -5.20761 & 1.09994 & -0.19986 \\
\hline \multicolumn{4}{|c|}{$\mathrm{ArA}=4-\mathrm{Cl}-\mathrm{Ph}$} & $\mathrm{H}$ & -4.64211 & 0.52972 & -1.78553 \\
\hline & & & & $\mathrm{H}$ & -3.75147 & 0.34570 & 1.83810 \\
\hline & & & & $\mathrm{H}$ & -2.34534 & -0.66314 & 1.44599 \\
\hline & & & & $\mathrm{H}$ & -3.74027 & -2.43821 & 0.60864 \\
\hline \multicolumn{4}{|c|}{ conformer 1} & $\mathrm{H}$ & -4.89213 & -1.74203 & 1.74650 \\
\hline \multicolumn{4}{|c|}{$E(D Z)=-1020.026442$} & $\mathrm{H}$ & -5.04002 & -1.76615 & -1.19263 \\
\hline \multicolumn{4}{|c|}{$G(D Z)=-1019.807891$} & $\mathrm{H}$ & -6.23052 & -1.03965 & -0.11433 \\
\hline \multicolumn{4}{|c|}{$E(T Z)=-1019.8979768$} & $\mathrm{~N}$ & -3.23879 & 0.28058 & -0.22578 \\
\hline \multirow{2}{*}{\multicolumn{4}{|c|}{$G(T Z)=-1019.6794258$}} & $\mathrm{C}$ & -2.57755 & 1.57168 & -0.22362 \\
\hline & & & & $\mathrm{H}$ & -2.80763 & 2.07597 & -1.17380 \\
\hline 31 & & & & $\mathrm{H}$ & -2.98388 & 2.23548 & 0.56793 \\
\hline 01 & & & & $\mathrm{C}$ & -1.08129 & 1.48581 & -0.07520 \\
\hline $\mathrm{C}$ & -3.35978 & 0.67497 & 0.81919 & $\mathrm{C}$ & -0.36889 & 0.37200 & -0.28769 \\
\hline $\mathrm{C}$ & -4.87211 & -0.52354 & -0.45798 & $\mathrm{H}$ & -0.58957 & 2.41703 & 0.20017 \\
\hline $\mathrm{C}$ & -5.65005 & 0.73248 & -0.02774 & $\mathrm{H}$ & -0.92025 & -0.51607 & -0.58946 \\
\hline $\mathrm{C}$ & -4.62985 & 1.54196 & 0.82333 & $\mathrm{C}$ & 1.08473 & 0.21470 & -0.17302 \\
\hline $\mathrm{H}$ & -3.34681 & 0.00667 & 1.70381 & $\mathrm{C}$ & 1.93618 & 1.21632 & 0.32706 \\
\hline $\mathrm{H}$ & -2.43209 & 1.25300 & 0.83351 & $\mathrm{C}$ & 1.66941 & -0.99774 & -0.57447 \\
\hline $\mathrm{H}$ & -5.05946 & -1.35139 & 0.25499 & $\mathrm{C}$ & 3.30956 & 1.02265 & 0.41236 \\
\hline $\mathrm{H}$ & -5.13905 & -0.88254 & -1.45740 & $\mathrm{H}$ & 1.52130 & 2.16016 & 0.66467 \\
\hline $\mathrm{H}$ & -5.96164 & 1.30902 & -0.90247 & $\mathrm{C}$ & 3.04323 & -1.20950 & -0.49627 \\
\hline $\mathrm{H}$ & -6.55191 & 0.47203 & 0.53219 & $\mathrm{H}$ & 1.03241 & -1.78878 & -0.95959 \\
\hline $\mathrm{H}$ & -4.43193 & 2.51249 & 0.36121 & $\mathrm{C}$ & 3.85544 & -0.19257 & -0.00267 \\
\hline $\mathrm{H}$ & -4.98440 & 1.72851 & 1.84020 & $\mathrm{H}$ & 3.95584 & 1.80125 & 0.80105 \\
\hline $\mathrm{N}$ & -3.47502 & -0.09458 & -0.42022 & $\mathrm{H}$ & 3.48007 & -2.14971 & -0.81240 \\
\hline $\mathrm{C}$ & -2.51882 & -1.18476 & -0.53812 & $\mathrm{Cl}$ & 5.59201 & -0.44310 & 0.10673 \\
\hline
\end{tabular}

conformer 3

$\mathrm{E}(\mathrm{DZ})=-1020.023153$

$\mathrm{G}(\mathrm{DZ})=-1019.805128$

$\mathrm{E}(\mathrm{TZ})=-1019.8950871$

$\mathrm{G}(\mathrm{TZ})=-1019.6770621$ 


$\begin{array}{lrrr}31 & & & \\ 01 & & & \\ \mathrm{C} & 4.77934 & 0.80309 & 0.02525 \\ \mathrm{C} & 3.60393 & -1.18561 & 0.12458 \\ \mathrm{C} & 4.85574 & -1.51873 & -0.70928 \\ \mathrm{C} & 5.62433 & -0.17122 & -0.80945 \\ \mathrm{H} & 4.80402 & 1.83296 & -0.34609 \\ \mathrm{H} & 5.13727 & 0.81841 & 1.07509 \\ \mathrm{H} & 2.71572 & -1.73498 & -0.19999 \\ \mathrm{H} & 3.77811 & -1.42975 & 1.19264 \\ \mathrm{H} & 5.44890 & -2.30536 & -0.23601 \\ \mathrm{H} & 4.57300 & -1.87486 & -1.70299 \\ \mathrm{H} & 6.64858 & -0.24026 & -0.43424 \\ \mathrm{H} & 5.67494 & 0.16321 & -1.84872 \\ \mathrm{~N} & 3.42772 & 0.25401 & -0.06875 \\ \mathrm{C} & 2.49321 & 0.88481 & 0.85892 \\ \mathrm{H} & 2.84459 & 0.76340 & 1.90691 \\ \mathrm{H} & 2.48455 & 1.96062 & 0.65060 \\ \mathrm{C} & 1.09648 & 0.34389 & 0.75225 \\ \mathrm{C} & 0.05808 & 1.05380 & 0.29307 \\ \mathrm{H} & 0.95068 & -0.68396 & 1.07812 \\ \mathrm{H} & 0.24618 & 2.07115 & -0.04922 \\ \mathrm{C} & -1.33570 & 0.60867 & 0.17601 \\ \mathrm{C} & -1.81115 & -0.58615 & 0.74486 \\ \mathrm{C} & -2.24992 & 1.40564 & -0.53169 \\ \mathrm{C} & -3.13663 & -0.97780 & 0.60083 \\ \mathrm{H} & -1.14056 & -1.21558 & 1.32004 \\ \mathrm{C} & -3.58125 & 1.02891 & -0.68623 \\ \mathrm{H} & -1.90848 & 2.33643 & -0.97544 \\ \mathrm{C} & -4.01458 & -0.16574 & -0.11798 \\ \mathrm{H} & -3.49339 & -1.89968 & 1.04555 \\ \mathrm{H} & -4.27513 & 1.65191 & -1.23853 \\ \mathrm{Cl} & -5.69261 & -0.65489 & -0.29901\end{array}$

$\begin{array}{lrrr}\mathrm{H} & -2.83183 & 0.95596 & 1.81477 \\ \mathrm{C} & -1.09509 & 0.40003 & 0.69679 \\ \mathrm{C} & -0.04738 & 1.06589 & 0.19472 \\ \mathrm{H} & -0.96095 & -0.59972 & 1.10458 \\ \mathrm{H} & -0.21999 & 2.06941 & -0.19354 \\ \mathrm{C} & 1.34252 & 0.60276 & 0.10619 \\ \mathrm{C} & 1.74895 & -0.69318 & 0.47111 \\ \mathrm{C} & 2.32517 & 1.48611 & -0.36898 \\ \mathrm{C} & 3.07708 & -1.08897 & 0.37209 \\ \mathrm{H} & 1.01814 & -1.40870 & 0.83241 \\ \mathrm{C} & 3.66064 & 1.10701 & -0.47464 \\ \mathrm{H} & 2.03647 & 2.49185 & -0.66068 \\ \mathrm{C} & 4.02714 & -0.18261 & -0.10051 \\ \mathrm{H} & 3.37895 & -2.09099 & 0.65459 \\ \mathrm{H} & 4.40820 & 1.80032 & -0.84227 \\ \mathrm{Cl} & 5.70763 & -0.67947 & -0.22783\end{array}$

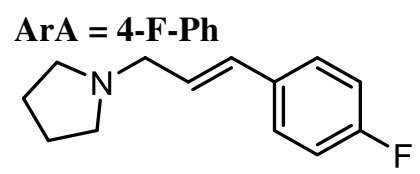

conformer 1

$E(D Z)=-659.660037$
$G(D Z)=-659.438948$
$E(T Z)=-659.5475442$
$G(T Z)=-659.3264552$

conformer 4

$\mathrm{E}(\mathrm{DZ})=-1020.023158$

$\mathrm{G}(\mathrm{DZ})=-1019.805026$

$\mathrm{E}(\mathrm{TZ})=-1019.8950257$

$\mathrm{G}(\mathrm{TZ})=-1019.6768937$

$\begin{array}{lrrr}31 & & & \\ 01 & & & \\ \text { C } & -4.77051 & 0.83368 & -0.07630 \\ \text { C } & -3.64507 & -1.15461 & 0.27696 \\ \text { C } & -4.89930 & -1.55938 & -0.51991 \\ \text { C } & -5.63643 & -0.21723 & -0.78834 \\ \text { H } & -5.13141 & 0.98896 & 0.96098 \\ \text { H } & -4.76830 & 1.80911 & -0.57390 \\ \text { H } & -3.83124 & -1.25979 & 1.36555 \\ \text { H } & -2.76937 & -1.76197 & 0.03111 \\ \text { H } & -4.61951 & -2.03895 & -1.46117 \\ \text { H } & -5.51258 & -2.26989 & 0.04019 \\ \text { H } & -5.67824 & -0.01229 & -1.86106 \\ \text { H } & -6.66253 & -0.21649 & -0.41181 \\ \text { N } & -3.43219 & 0.24498 & -0.09213 \\ \text { C } & -2.48616 & 0.96214 & 0.75785 \\ \text { H } & -2.46931 & 2.00942 & 0.43607\end{array}$

$\begin{array}{lrrr}31 & & & \\ \text { O 1 } & & & \\ \mathrm{C} & 2.92460 & 0.71149 & 0.75641 \\ \mathrm{C} & 4.41097 & -0.63810 & -0.39281 \\ \mathrm{C} & 5.22131 & 0.63001 & -0.07278 \\ \mathrm{C} & 4.22116 & 1.53723 & 0.69977 \\ \mathrm{H} & 2.01495 & 1.31560 & 0.70906 \\ \mathrm{H} & 2.88253 & 0.12440 & 1.69618 \\ \mathrm{H} & 4.56933 & -1.40108 & 0.39581 \\ \mathrm{H} & 4.67494 & -1.09550 & -1.35205 \\ \mathrm{H} & 6.11602 & 0.39747 & 0.51050 \\ \mathrm{H} & 5.54748 & 1.11848 & -0.99454 \\ \mathrm{H} & 4.57505 & 1.79593 & 1.70103 \\ \mathrm{H} & 4.05630 & 2.47230 & 0.15831 \\ \mathrm{~N} & 3.02716 & -0.16766 & -0.40873 \\ \mathrm{C} & 2.03997 & -1.23677 & -0.43963 \\ \mathrm{H} & 2.29405 & -1.89206 & -1.28490 \\ \mathrm{H} & 2.08028 & -1.86415 & 0.47414 \\ \mathrm{C} & 0.65118 & -0.70029 & -0.62530 \\ \mathrm{C} & -0.36762 & -0.95526 & 0.20577 \\ \mathrm{H} & 0.51843 & -0.05922 & -1.49469 \\ \mathrm{H} & -0.16733 & -1.57689 & 1.07847 \\ \mathrm{C} & -1.75234 & -0.48175 & 0.09111 \\ \mathrm{C} & -2.23155 & 0.23107 & -1.02337 \\ \mathrm{C} & -2.65140 & -0.74553 & 1.13799 \\ \mathrm{C} & -3.54809 & 0.67200 & -1.08640 \\ \mathrm{H} & -1.56892 & 0.43869 & -1.85656 \\ \mathrm{C} & -3.97439 & -0.31282 & 1.09205 \\ \mathrm{H} & -2.30355 & -1.29795 & 2.00617 \\ \mathrm{C} & -4.40211 & 0.39339 & -0.02373\end{array}$




$\begin{array}{lllr}\mathrm{H} & -3.92335 & 1.22046 & -1.94311 \\ \mathrm{H} & -4.66902 & -0.51416 & 1.89967 \\ \mathrm{~F} & -5.68194 & 0.81813 & -0.08410\end{array}$

conformer 2

$$
\begin{aligned}
& E(D Z)=-659.659683 \\
& G(D Z)=-659.438017 \\
& E(T Z)=-659.5469262 \\
& G(T Z)=-659.3252602
\end{aligned}
$$

$\begin{array}{lccc}31 & & & \\ 01 & & & \\ \mathrm{C} & -4.16911 & 0.36421 & -0.72446 \\ \mathrm{C} & -2.90021 & -0.31855 & 1.08521 \\ \mathrm{C} & -3.88970 & -1.47322 & 0.85921 \\ \mathrm{C} & -4.74885 & -1.01142 & -0.35238 \\ \mathrm{H} & -4.74130 & 1.16798 & -0.21990 \\ \mathrm{H} & -4.18795 & 0.57003 & -1.79955 \\ \mathrm{H} & -1.92181 & -0.64380 & 1.44550 \\ \mathrm{H} & -3.30658 & 0.39855 & 1.82609 \\ \mathrm{H} & -3.35353 & -2.39500 & 0.61868 \\ \mathrm{H} & -4.49023 & -1.66664 & 1.75171 \\ \mathrm{H} & -4.64203 & -1.71016 & -1.18601 \\ \mathrm{H} & -5.81320 & -0.94566 & -0.11292 \\ \mathrm{~N} & -2.79194 & 0.30354 & -0.23614 \\ \mathrm{C} & -2.10187 & 1.57984 & -0.24469 \\ \mathrm{H} & -2.31627 & 2.07736 & -1.20214 \\ \mathrm{H} & -2.49925 & 2.26103 & 0.53669 \\ \mathrm{C} & -0.60863 & 1.46264 & -0.08457 \\ \mathrm{C} & 0.08187 & 0.33321 & -0.28587 \\ \mathrm{H} & -0.09940 & 2.38469 & 0.18990 \\ \mathrm{H} & -0.48657 & -0.54434 & -0.58731 \\ \mathrm{C} & 1.53109 & 0.14261 & -0.15873 \\ \mathrm{C} & 2.40013 & 1.12689 & 0.34737 \\ \mathrm{C} & 2.09075 & -1.08447 & -0.55360 \\ \mathrm{C} & 3.76851 & 0.90273 & 0.44449 \\ \mathrm{H} & 2.00132 & 2.07953 & 0.67948 \\ \mathrm{C} & 3.45922 & -1.32751 & -0.46387 \\ \mathrm{H} & 1.43744 & -1.86029 & -0.94218 \\ \mathrm{C} & 4.27940 & -0.32481 & 0.03451 \\ \mathrm{H} & 4.44177 & 1.65712 & 0.83601 \\ \mathrm{H} & 3.89216 & -2.27301 & -0.77046 \\ \mathrm{~F} & 5.60815 & -0.54612 & 0.13040 \\ & & & \\ & & & \end{array}$

$\begin{array}{lccc}\mathrm{H} & 4.27903 & -1.90893 & -0.43795 \\ \mathrm{H} & 2.36451 & 1.73990 & -0.07408 \\ \mathrm{H} & 3.39552 & 1.29241 & 1.30336 \\ \mathrm{H} & 4.24343 & 1.88778 & -1.54991 \\ \mathrm{H} & 5.12289 & 2.17598 & -0.05063 \\ \mathrm{H} & 6.22284 & 0.07243 & -0.37829 \\ \mathrm{H} & 5.24418 & -0.18943 & -1.82229 \\ \mathrm{~N} & 2.97773 & -0.28592 & -0.06861 \\ \mathrm{C} & 2.00388 & -0.92831 & 0.81002 \\ \mathrm{H} & 1.95566 & -1.98946 & 0.54075 \\ \mathrm{H} & 2.34565 & -0.88020 & 1.86710 \\ \mathrm{C} & 0.63127 & -0.32690 & 0.71369 \\ \mathrm{C} & -0.42832 & -0.97531 & 0.21376 \\ \mathrm{H} & 0.52176 & 0.68741 & 1.09195 \\ \mathrm{H} & -0.27677 & -1.99225 & -0.14791 \\ \mathrm{C} & -1.80479 & -0.47966 & 0.09204 \\ \mathrm{C} & -2.18994 & 0.82440 & 0.45396 \\ \mathrm{C} & -2.79492 & -1.33984 & -0.41142 \\ \mathrm{C} & -3.50586 & 1.25157 & 0.32261 \\ \mathrm{H} & -1.45154 & 1.51838 & 0.84081 \\ \mathrm{C} & -4.11868 & -0.92992 & -0.55013 \\ \mathrm{H} & -2.51979 & -2.35040 & -0.69928 \\ \mathrm{C} & -4.45380 & 0.36469 & -0.17859 \\ \mathrm{H} & -3.80775 & 2.25554 & 0.59916 \\ \mathrm{H} & -4.88367 & -1.59288 & -0.93814 \\ \mathrm{~F} & -5.73240 & 0.77741 & -0.30732\end{array}$

$\mathrm{ArA}=4-\mathrm{Me}-\mathrm{Ph}$<smiles>Cc1ccc(/C=C/CN2CCCC2)cc1</smiles>

conformer 1

$$
\begin{aligned}
& E(D Z)=-599.753973 \\
& G(D Z)=-599.49931 \\
& E(T Z)=-599.6043868 \\
& G(T Z)=-599.3497238
\end{aligned}
$$

$\begin{array}{lrrr}34 & & & \\ 01 & & & \\ \mathrm{C} & -2.94608 & 0.74130 & 0.73012 \\ \mathrm{C} & -4.44329 & -0.63234 & -0.37464 \\ \mathrm{C} & -5.24622 & 0.64850 & -0.08717 \\ \mathrm{C} & -4.23682 & 1.57397 & 0.65085 \\ \mathrm{H} & -2.90669 & 0.18164 & 1.68669 \\ \mathrm{H} & -2.03215 & 1.33697 & 0.66386 \\ \mathrm{H} & -4.71341 & -1.11519 & -1.31966 \\ \mathrm{H} & -4.60302 & -1.37217 & 0.43561 \\ \mathrm{H} & -6.13744 & 0.43718 & 0.50947 \\ \mathrm{H} & -5.57767 & 1.11063 & -1.02060 \\ \mathrm{H} & -4.06710 & 2.48880 & 0.07720 \\ \mathrm{H} & -4.58511 & 1.86917 & 1.64400 \\ \mathrm{~N} & -3.05711 & -0.17055 & -0.40860 \\ \mathrm{C} & -2.07629 & -1.24666 & -0.41038 \\ \mathrm{H} & -2.12160 & -1.84763 & 0.52079 \\ \mathrm{H} & -2.33599 & -1.92347 & -1.23685 \\ \mathrm{C} & -0.68357 & -0.72575 & -0.60971 \\ \mathrm{C} & 0.33070 & -0.95870 & 0.23379\end{array}$




$\begin{array}{rrrr}\mathrm{H} & -0.54239 & -0.11501 & -1.49927 \\ \mathrm{H} & 0.12261 & -1.54904 & 1.12647 \\ \mathrm{C} & 1.71830 & -0.49785 & 0.10891 \\ \mathrm{C} & 2.21077 & 0.17095 & -1.02627 \\ \mathrm{C} & 2.61142 & -0.72204 & 1.16758 \\ \mathrm{C} & 3.53012 & 0.59944 & -1.08672 \\ \mathrm{H} & 1.55491 & 0.35382 & -1.87132 \\ \mathrm{C} & 3.93410 & -0.29093 & 1.10310 \\ \mathrm{H} & 2.25723 & -1.23623 & 2.05720 \\ \mathrm{C} & 4.41979 & 0.37660 & -0.02498 \\ \mathrm{H} & 3.88303 & 1.11570 & -1.97597 \\ \mathrm{H} & 4.59959 & -0.47533 & 1.94234 \\ \mathrm{C} & 5.85827 & 0.81944 & -0.11574 \\ \mathrm{H} & 6.32086 & 0.87851 & 0.87330 \\ \mathrm{H} & 5.94476 & 1.80186 & -0.59055 \\ \mathrm{H} & 6.45210 & 0.11810 & -0.71491\end{array}$

$\begin{array}{llll}\mathrm{H} & -6.31229 & 0.61523 & -1.03600 \\ \mathrm{H} & -5.91078 & 1.93312 & 0.06295\end{array}$

conformer 2

$$
\begin{aligned}
& E(D Z)=-599.753987 \\
& G(D Z)=-599.49911 \\
& E(T Z)=-599.6043873 \\
& G(T Z)=-599.3495103
\end{aligned}
$$

\begin{tabular}{lrrr}
34 & & & \\
0 & & & \\
$\mathrm{C}$ & 2.94386 & 0.70924 & \multicolumn{1}{c}{0.76201} \\
$\mathrm{C}$ & 4.44408 & -0.61433 & -0.39836 \\
$\mathrm{C}$ & 5.24383 & 0.65586 & -0.06114 \\
$\mathrm{C}$ & 4.23592 & 1.54353 & 0.72400 \\
$\mathrm{H}$ & 2.03069 & 1.30815 & 0.71830 \\
$\mathrm{H}$ & 2.90060 & 0.10922 & 1.69354 \\
$\mathrm{H}$ & 4.60520 & -1.38498 & 0.38242 \\
$\mathrm{H}$ & 4.71518 & -1.05911 & -1.36158 \\
$\mathrm{H}$ & 6.14115 & 0.42343 & 0.51827 \\
$\mathrm{H}$ & 5.56468 & 1.15975 & -0.97650 \\
$\mathrm{H}$ & 4.58520 & 1.78597 & 1.73099 \\
$\mathrm{H}$ & 4.06785 & 2.48746 & 0.19909 \\
$\mathrm{~N}$ & 3.05741 & -0.15340 & -0.41431 \\
$\mathrm{C}$ & 2.07723 & -1.22889 & -0.46315 \\
$\mathrm{H}$ & 2.33811 & -1.86959 & -1.31754 \\
$\mathrm{H}$ & 2.12144 & -1.86913 & 0.44150 \\
$\mathrm{C}$ & 0.68450 & -0.70031 & -0.64178 \\
$\mathrm{C}$ & -0.33006 & -0.96752 & 0.19107 \\
$\mathrm{H}$ & 0.54392 & -0.05484 & -1.50658 \\
$\mathrm{H}$ & -0.12248 & -1.59575 & 1.05761 \\
$\mathrm{C}$ & -1.71768 & -0.50192 & 0.08630 \\
$\mathrm{C}$ & -2.20441 & 0.23705 & -1.00772 \\
$\mathrm{C}$ & -2.61854 & -0.79984 & 1.11931 \\
$\mathrm{C}$ & -3.52550 & 0.66057 & -1.05256 \\
$\mathrm{H}$ & -1.54332 & 0.47467 & -1.83485 \\
$\mathrm{C}$ & -3.94394 & -0.37363 & 1.07032 \\
$\mathrm{H}$ & -2.27093 & -1.37451 & 1.97382 \\
$\mathrm{C}$ & -4.42258 & 0.36645 & -0.01398 \\
$\mathrm{H}$ & -3.87511 & 1.22769 & -1.91168 \\
$\mathrm{H}$ & -4.61723 & -0.62111 & 1.88670 \\
$\mathrm{C}$ & -5.85104 & 0.84614 & -0.06988 \\
$\mathrm{H}$ & -6.45792 & 0.38359 & 0.71295
\end{tabular}

conformer 3

$\mathrm{E}(\mathrm{DZ})=-599.750741$
$\mathrm{G}(\mathrm{DZ})=-599.496605$
$\mathrm{E}(\mathrm{TZ})=-599.601457$
$\mathrm{G}(\mathrm{TZ})=-599.347321$

$\begin{array}{lccc}34 & & & \\ \mathrm{O} 1 & & & \\ \mathrm{C} & 4.33753 & -0.88599 & -0.00673 \\ \mathrm{C} & 3.23930 & 1.13420 & 0.22513 \\ \mathrm{C} & 4.51172 & 1.47733 & -0.57258 \\ \mathrm{C} & 5.22948 & 0.11147 & -0.76188 \\ \mathrm{H} & 4.68249 & -0.99002 & 1.04256 \\ \mathrm{H} & 4.32707 & -1.88698 & -0.45069 \\ \mathrm{H} & 2.37622 & 1.74008 & -0.06476 \\ \mathrm{H} & 3.41193 & 1.29366 & 1.30953 \\ \mathrm{H} & 4.25362 & 1.91352 & -1.54084 \\ \mathrm{H} & 5.12937 & 2.20509 & -0.03992 \\ \mathrm{H} & 5.27970 & -0.14997 & -1.82195 \\ \mathrm{H} & 6.25124 & 0.11386 & -0.37349 \\ \mathrm{~N} & 3.00958 & -0.27917 & -0.07336 \\ \mathrm{C} & 2.03956 & -0.93660 & 0.79894 \\ \mathrm{H} & 1.99825 & -1.99524 & 0.51870 \\ \mathrm{H} & 2.38267 & -0.89716 & 1.85606 \\ \mathrm{C} & 0.66319 & -0.34303 & 0.70982 \\ \mathrm{C} & -0.39326 & -0.99222 & 0.20353 \\ \mathrm{H} & 0.54667 & 0.66610 & 1.09955 \\ \mathrm{H} & -0.23619 & -2.00472 & -0.16880 \\ \mathrm{C} & -1.77213 & -0.50292 & 0.08932 \\ \mathrm{C} & -2.16568 & 0.79651 & 0.45815 \\ \mathrm{C} & -2.76108 & -1.35949 & -0.41656 \\ \mathrm{C} & -3.48494 & 1.20806 & 0.32804 \\ \mathrm{H} & -1.42998 & 1.49473 & 0.84413 \\ \mathrm{C} & -4.08415 & -0.94315 & -0.54546 \\ \mathrm{H} & -2.48454 & -2.36724 & -0.71508 \\ \mathrm{C} & -4.47276 & 0.34664 & -0.17362 \\ \mathrm{H} & -3.75909 & 2.21939 & 0.61788 \\ \mathrm{H} & -4.82601 & -1.63088 & -0.94269 \\ \mathrm{C} & -5.90616 & 0.80004 & -0.28858 \\ \mathrm{H} & -5.97486 & 1.79920 & -0.73112 \\ \mathrm{H} & -6.38665 & 0.85048 & 0.69613 \\ \mathrm{H} & -6.49271 & 0.11515 & -0.90673\end{array}$

$\mathrm{ArA}=4-\mathrm{OMe}-\mathrm{Ph}$<smiles>COc1ccc(/C=C/CN2CCCC2)cc1</smiles>

conformer 1

$$
\begin{aligned}
& E(D Z)=-674.960098 \\
& G(D Z)=-674.700466 \\
& E(T Z)=-674.8200065 \\
& G(T Z)=-674.5603745
\end{aligned}
$$




\begin{tabular}{lrrr} 
O 1 & & & \\
$\mathrm{C}$ & -4.90312 & -0.62624 & -0.24973 \\
$\mathrm{C}$ & -3.36080 & 0.80878 & 0.70381 \\
$\mathrm{C}$ & -4.64527 & 1.64827 & 0.60015 \\
$\mathrm{C}$ & -5.68569 & 0.68129 & -0.03441 \\
$\mathrm{H}$ & -5.20651 & -1.17500 & -1.14755 \\
$\mathrm{H}$ & -5.04451 & -1.30217 & 0.61785 \\
$\mathrm{H}$ & -3.29875 & 0.32148 & 1.69815 \\
$\mathrm{H}$ & -2.44342 & 1.38681 & 0.56669 \\
$\mathrm{H}$ & -4.96000 & 2.02230 & 1.57785 \\
$\mathrm{H}$ & -4.48477 & 2.51472 & -0.04666 \\
$\mathrm{H}$ & -6.55898 & 0.52465 & 0.60419 \\
$\mathrm{H}$ & -6.04328 & 1.07599 & -0.98894 \\
$\mathrm{~N}$ & -3.51472 & -0.18397 & -0.35972 \\
$\mathrm{C}$ & -2.54383 & -1.26816 & -0.31163 \\
$\mathrm{H}$ & -2.56821 & -1.79964 & 0.66170 \\
$\mathrm{H}$ & -2.83521 & -2.00003 & -1.07863 \\
$\mathrm{C}$ & -1.15244 & -0.77965 & -0.58762 \\
$\mathrm{C}$ & -0.11509 & -0.96735 & 0.23946 \\
$\mathrm{H}$ & -1.03188 & -0.23653 & -1.52294 \\
$\mathrm{H}$ & -0.30256 & -1.49734 & 1.17381 \\
$\mathrm{C}$ & 1.27292 & -0.53687 & 0.04505 \\
$\mathrm{C}$ & 1.72739 & 0.10919 & -1.12260 \\
$\mathrm{C}$ & 2.21159 & -0.76626 & 1.05900 \\
$\mathrm{C}$ & 3.04590 & 0.50651 & -1.25879 \\
$\mathrm{H}$ & 1.03638 & 0.29932 & -1.93720 \\
$\mathrm{C}$ & 3.54511 & -0.37364 & 0.93904 \\
$\mathrm{H}$ & 1.89157 & -1.26437 & 1.97035 \\
$\mathrm{C}$ & 3.96831 & 0.26920 & -0.22736 \\
$\mathrm{H}$ & 3.39483 & 1.00303 & -2.15786 \\
$\mathrm{H}$ & 4.23405 & -0.57231 & 1.75054 \\
$\mathrm{O}$ & 5.24176 & 0.69887 & -0.46155 \\
$\mathrm{C}$ & 6.21101 & 0.47901 & 0.54959 \\
$\mathrm{H}$ & 5.94424 & 0.99104 & 1.48291 \\
$\mathrm{H}$ & 6.34443 & -0.59076 & 0.75506 \\
$\mathrm{H}$ & 7.14558 & 0.89133 & 0.16750 \\
& & & \\
\hline
\end{tabular}

conformer 2

$\begin{array}{lrrc}\mathrm{N} & 3.46583 & -0.06839 & -0.45951 \\ \mathrm{C} & 2.52877 & 0.96090 & -0.88777 \\ \mathrm{H} & 2.62839 & 1.88527 & -0.28297 \\ \mathrm{H} & 2.79026 & 1.22949 & -1.92132 \\ \mathrm{C} & 1.11034 & 0.47447 & -0.83927 \\ \mathrm{C} & 0.13448 & 1.08009 & -0.14924 \\ \mathrm{H} & 0.91560 & -0.43500 & -1.40447 \\ \mathrm{H} & 0.39690 & 1.97360 & 0.41788 \\ \mathrm{C} & -1.27447 & 0.68435 & -0.05288 \\ \mathrm{C} & -1.82221 & -0.40433 & -0.74765 \\ \mathrm{C} & -2.13986 & 1.42098 & 0.77733 \\ \mathrm{C} & -3.16423 & -0.75357 & -0.62263 \\ \mathrm{H} & -1.19301 & -0.99524 & -1.40529 \\ \mathrm{C} & -3.47919 & 1.08878 & 0.91330 \\ \mathrm{H} & -1.74578 & 2.27125 & 1.32747 \\ \mathrm{C} & -4.00315 & -0.00502 & 0.21328 \\ \mathrm{H} & -3.54501 & -1.60183 & -1.17800 \\ \mathrm{H} & -4.14141 & 1.65944 & 1.55526 \\ \mathrm{O} & -5.32968 & -0.25845 & 0.40766 \\ \mathrm{C} & -5.90241 & -1.35879 & -0.27893 \\ \mathrm{H} & -5.42334 & -2.30482 & 0.00353 \\ \mathrm{H} & -6.95221 & -1.38538 & 0.01541 \\ \mathrm{H} & -5.83502 & -1.23561 & -1.36736\end{array}$

conformer 3

$$
\begin{aligned}
& E(D Z)=-674.956882 \\
& G(D Z)=-674.697755 \\
& E(T Z)=-674.8171236 \\
& G(T Z)=-674.5579966
\end{aligned}
$$

$\begin{array}{lrrr}35 & & & \\ 01 & & & \\ \mathrm{C} & -3.69096 & 1.12728 & 0.17485 \\ \mathrm{C} & -4.78140 & -0.89704 & -0.05141 \\ \mathrm{C} & -5.65599 & 0.08798 & -0.84316 \\ \mathrm{C} & -4.93880 & 1.45589 & -0.66596 \\ \mathrm{H} & -3.89643 & 1.30405 & 1.25090 \\ \mathrm{H} & -2.81944 & 1.72863 & -0.09858 \\ \mathrm{H} & -4.75967 & -1.90479 & -0.47952 \\ \mathrm{H} & -5.15201 & -0.98550 & 0.99057 \\ \mathrm{H} & -5.68753 & -0.19335 & -1.89888 \\ \mathrm{H} & -6.68486 & 0.10001 & -0.47407 \\ \mathrm{H} & -4.65106 & 1.86743 & -1.63668 \\ \mathrm{H} & -5.56849 & 2.19844 & -0.16895 \\ \mathrm{~N} & -3.45252 & -0.29034 & -0.09420 \\ \mathrm{C} & -2.50460 & -0.93193 & 0.81419 \\ \mathrm{H} & -2.88011 & -0.88236 & 1.85974 \\ \mathrm{H} & -2.45018 & -1.99357 & 0.54739 \\ \mathrm{C} & -1.12898 & -0.33231 & 0.75905 \\ \mathrm{C} & -0.05959 & -0.97406 & 0.26974 \\ \mathrm{H} & -1.02521 & 0.67501 & 1.15711 \\ \mathrm{H} & -0.20647 & -1.98488 & -0.11155 \\ \mathrm{C} & 1.31852 & -0.48123 & 0.18332 \\ \mathrm{C} & 1.70395 & 0.81825 & 0.57075 \\ \mathrm{C} & 2.31997 & -1.32772 & -0.30892 \\ \mathrm{C} & 3.01825 & 1.23953 & 0.47184 \\ \mathrm{H} & 0.96054 & 1.51069 & 0.95180\end{array}$




$\begin{array}{cccc}\mathrm{C} & 3.65025 & -0.92076 & -0.41554 \\ \mathrm{H} & 2.05362 & -2.33500 & -0.61779 \\ \mathrm{C} & 4.00557 & 0.37226 & -0.02236 \\ \mathrm{H} & 3.31322 & 2.24016 & 0.76896 \\ \mathrm{H} & 4.38927 & -1.61163 & -0.80180 \\ \mathrm{O} & 5.26860 & 0.88414 & -0.07988 \\ \mathrm{C} & 6.29993 & 0.04662 & -0.57545 \\ \mathrm{H} & 6.42473 & -0.84991 & 0.04496 \\ \mathrm{H} & 6.10761 & -0.26023 & -1.61139 \\ \mathrm{H} & 7.21457 & 0.63949 & -0.53938\end{array}$

conformer 4

$$
\begin{aligned}
& E(D Z)=-674.956637 \\
& G(D Z)=-674.697441 \\
& E(T Z)=-674.8167993 \\
& G(T Z)=-674.5576033
\end{aligned}
$$

$\begin{array}{lrrr}35 & & & \\ \text { O } & & & \\ \mathrm{C} & -4.79687 & -0.74137 & -0.00474 \\ \mathrm{C} & -3.57123 & 1.20257 & 0.23095 \\ \mathrm{C} & -4.81226 & 1.62818 & -0.57522 \\ \mathrm{C} & -5.62206 & 0.31367 & -0.75878 \\ \mathrm{H} & -4.85103 & -1.74011 & -0.45067 \\ \mathrm{H} & -5.14923 & -0.82513 & 1.04401 \\ \mathrm{H} & -3.74144 & 1.37158 & 1.31440 \\ \mathrm{H} & -2.66817 & 1.75069 & -0.05157 \\ \mathrm{H} & -5.38053 & 2.40151 & -0.05180 \\ \mathrm{H} & -4.51905 & 2.03705 & -1.54542 \\ \mathrm{H} & -6.63947 & 0.38622 & -0.36569 \\ \mathrm{H} & -5.69516 & 0.05470 & -1.81811 \\ \mathrm{~N} & -3.43244 & -0.22221 & -0.06865 \\ \mathrm{C} & -2.50671 & -0.94070 & 0.80441 \\ \mathrm{H} & -2.85287 & -0.88671 & 1.85979 \\ \mathrm{H} & -2.52718 & -1.99846 & 0.51818 \\ \mathrm{C} & -1.09745 & -0.42873 & 0.72332 \\ \mathrm{C} & -0.08232 & -1.12851 & 0.19955 \\ \mathrm{H} & -0.92098 & 0.56414 & 1.13220 \\ \mathrm{H} & -0.30022 & -2.12462 & -0.18656 \\ \mathrm{C} & 1.32130 & -0.71961 & 0.08127 \\ \mathrm{C} & 1.78814 & 0.55664 & 0.42915 \\ \mathrm{C} & 2.26602 & -1.63988 & -0.40999 \\ \mathrm{C} & 3.13001 & 0.90619 & 0.30374 \\ \mathrm{H} & 1.09262 & 1.30220 & 0.80074 \\ \mathrm{C} & 3.60623 & -1.30982 & -0.54184 \\ \mathrm{H} & 1.93484 & -2.63567 & -0.69192 \\ \mathrm{C} & 4.05031 & -0.03088 & -0.18371 \\ \mathrm{H} & 3.44608 & 1.90374 & 0.58261 \\ \mathrm{H} & 4.33016 & -2.02330 & -0.92014 \\ \mathrm{O} & 5.38405 & 0.20540 & -0.34676 \\ \mathrm{C} & 5.87917 & 1.48688 & 0.00417 \\ \mathrm{H} & 6.95060 & 1.46242 & -0.19789 \\ \mathrm{H} & 5.41564 & 2.27839 & -0.59831 \\ \mathrm{H} & 5.71643 & 1.70579 & 1.06716\end{array}$

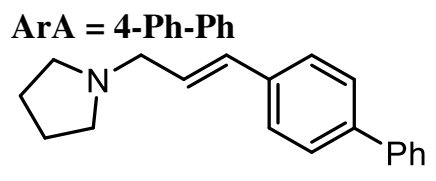

conformer 1

$$
\begin{aligned}
& E(D Z)=-791.518405 \\
& G(D Z)=-791.214483 \\
& E(T Z)=-791.3377065 \\
& G(T Z)=-791.0337845
\end{aligned}
$$

$\begin{array}{lrrr}41 & & & \\ \text { O } 1 & & & \\ \mathrm{C} & -4.61566 & 0.92270 & 0.65126 \\ \mathrm{C} & -6.21113 & -0.50006 & -0.23314 \\ \mathrm{C} & -6.89950 & 0.12593 & 0.99238 \\ \mathrm{C} & -5.82364 & 1.07728 & 1.59046 \\ \mathrm{H} & -4.64506 & 1.69478 & -0.14412 \\ \mathrm{H} & -3.65037 & 1.01184 & 1.15620 \\ \mathrm{H} & -6.45247 & 0.08226 & -1.14521 \\ \mathrm{H} & -6.50588 & -1.53842 & -0.41784 \\ \mathrm{H} & -7.81628 & 0.65095 & 0.71188 \\ \mathrm{H} & -7.17170 & -0.64702 & 1.71566 \\ \mathrm{H} & -6.16156 & 2.11552 & 1.64119 \\ \mathrm{H} & -5.56135 & 0.76741 & 2.60531 \\ \mathrm{~N} & -4.78807 & -0.41771 & 0.09019 \\ \mathrm{C} & -3.90999 & -0.71357 & -1.03235 \\ \mathrm{H} & -4.21517 & -1.68895 & -1.43763 \\ \mathrm{H} & -4.02552 & 0.02326 & -1.85314 \\ \mathrm{C} & -2.47273 & -0.77956 & -0.60770 \\ \mathrm{C} & -1.49373 & -0.05023 & -1.15980 \\ \mathrm{H} & -2.26536 & -1.46872 & 0.20852 \\ \mathrm{H} & -1.76451 & 0.64281 & -1.95649 \\ \mathrm{C} & -0.06869 & -0.06477 & -0.81350 \\ \mathrm{C} & 0.80102 & 0.82938 & -1.45877 \\ \mathrm{C} & 0.48656 & -0.93368 & 0.14306 \\ \mathrm{C} & 2.15906 & 0.86141 & -1.16366 \\ \mathrm{H} & 0.40071 & 1.50423 & -2.21063 \\ \mathrm{C} & 1.84152 & -0.90106 & 0.43948 \\ \mathrm{H} & -0.15071 & -1.63940 & 0.66568 \\ \mathrm{C} & 2.70984 & -0.00330 & -0.20644 \\ \mathrm{H} & 2.80784 & 1.54590 & -1.70082 \\ \mathrm{H} & 2.23606 & -1.56500 & 1.20213 \\ \mathrm{C} & 4.15503 & 0.02514 & 0.11117 \\ \mathrm{C} & 4.86531 & 1.23650 & 0.12860 \\ \mathrm{C} & 4.85319 & -1.15809 & 0.40225 \\ \mathrm{C} & 6.22601 & 1.26373 & 0.42469 \\ \mathrm{H} & 4.33770 & 2.16487 & -0.06580 \\ \mathrm{C} & 6.21346 & -1.13102 & 0.70058 \\ \mathrm{H} & 4.32854 & -2.10757 & 0.36685 \\ \mathrm{C} & 6.90664 & 0.08002 & 0.71268 \\ \mathrm{H} & 6.75385 & 2.21259 & 0.44027 \\ \mathrm{H} & 6.73530 & -2.05894 & 0.91467 \\ \mathrm{H} & 7.96697 & 0.10114 & 0.94453\end{array}$

conformer 2

$\mathrm{E}(\mathrm{DZ})=-791.518406$
$\mathrm{G}(\mathrm{DZ})=-791.214132$ 
$\mathrm{E}(\mathrm{TZ})=-791.337707$

$\mathrm{G}(\mathrm{TZ})=-791.033433$

41

0

C $\quad 4.62271$

0.64236

0.91913

C $\quad 6.21107$

$-0.26667$

C $\quad 6.90675$

0.96285

$-0.49579$

C $\quad 5.83619$

1.57670

0.11370

$\mathrm{H} \quad 4.64888$

$-0.14423$

1.06117

$\mathrm{H} \quad 3.66024$

1.15351

1.70024

1.00390

$-1.17276$

0.09737

$\begin{array}{ll}\mathrm{H} & 6.44857 \\ \mathrm{H} & 6.50306\end{array}$

$-0.46572$

0.68477

$-1.53228$

0.63912

$-0.66851$

H 7.17942

1.67592

2.09809

H $\quad 6.17666$

1.63665

2.58961

0.06552

4.79006

$-1.05511$

0.74113

$-0.41515$

$-0.69844$

$-1.67049$

0.04614

$-1.86926$

$-0.76571$

C 2.47059

$-0.62201$

$-1.17063$

0.19545

$-1.97613$

$-0.81487$

$-1.51797$

0.20774

$-1.21823$

$-2.30659$

0.50816

0.76863

$-0.19747$

$-1.76246$

1.28477

0.12733

$-0.88384$

1.45533

$-0.57793$

$-1.91894$

1.76144

2.25393

0.74629

$-1.37642$

2.79606

0.98485

$-0.04093$

$-1.45528$

0.64492

$-0.05672$

0.77170

$-0.86189$

0.79901

1.41149

$-0.83447$

$-1.52413$

$-0.00406$

1.47156

$-1.48658$

0.02109

0.16116

$-0.09350$

0.18744

0.22292

$-0.06927$

$-0.17460$

0.07182

0.28847

$-0.15124$

0.09123

conformer 3

$\mathrm{E}(\mathrm{DZ})=-791.515157$

$\mathrm{G}(\mathrm{DZ})=-791.211503$

$\mathrm{E}(\mathrm{TZ})=-791.3347881$

$\mathrm{G}(\mathrm{TZ})=-791.0311341$

41

01

$\begin{array}{rrrr}\mathrm{C} & 6.14526 & 0.61070 & -0.06817 \\ \mathrm{C} & 4.84922 & -1.28823 & 0.16970 \\ \mathrm{C} & 6.04598 & -1.52323 & 1.10868\end{array}$

$\begin{array}{lrrr}\mathrm{C} & 6.91537 & -0.24393 & 0.95198 \\ \mathrm{H} & 6.22852 & 1.68777 & 0.11137 \\ \mathrm{H} & 6.52105 & 0.41392 & -1.09304 \\ \mathrm{H} & 3.91690 & -1.71504 & 0.54969 \\ \mathrm{H} & 5.04612 & -1.74078 & -0.82407 \\ \mathrm{H} & 5.70532 & -1.63047 & 2.14155 \\ \mathrm{H} & 6.58770 & -2.43522 & 0.84490 \\ \mathrm{H} & 6.99982 & 0.28127 & 1.90673 \\ \mathrm{H} & 7.92892 & -0.46372 & 0.60667 \\ \mathrm{~N} & 4.75976 & 0.16915 & 0.08286 \\ \mathrm{C} & 3.88798 & 0.66690 & -0.97820 \\ \mathrm{H} & 3.93867 & 1.76162 & -0.96967 \\ \mathrm{H} & 4.26109 & 0.33607 & -1.97222 \\ \mathrm{C} & 2.45902 & 0.23116 & -0.82728 \\ \mathrm{C} & 1.45312 & 1.06944 & -0.54444 \\ \mathrm{H} & 2.26080 & -0.82886 & -0.97076 \\ \mathrm{H} & 1.69455 & 2.12203 & -0.39681 \\ \mathrm{C} & 0.03191 & 0.73818 & -0.39764 \\ \mathrm{C} & -0.86679 & 1.74316 & -0.00434 \\ \mathrm{C} & -0.49286 & -0.54535 & -0.63272 \\ \mathrm{C} & -2.22305 & 1.48278 & 0.15393 \\ \mathrm{H} & -0.49031 & 2.74711 & 0.17256 \\ \mathrm{C} & -1.84621 & -0.80636 & -0.47436 \\ \mathrm{H} & 0.16636 & -1.35098 & -0.93907 \\ \mathrm{C} & -2.74334 & 0.20092 & -0.07733 \\ \mathrm{H} & -2.89397 & 2.28891 & 0.43320 \\ \mathrm{H} & -2.21744 & -1.81324 & -0.63721 \\ \mathrm{C} & -4.18673 & -0.08206 & 0.08628 \\ \mathrm{C} & -4.93131 & 0.53517 & 1.10445 \\ \mathrm{C} & -4.84865 & -0.97375 & -0.77336 \\ \mathrm{C} & -6.29047 & 0.27165 & 1.25668 \\ \mathrm{H} & -4.43156 & 1.20551 & 1.79645 \\ \mathrm{C} & -6.20736 & -1.23918 & -0.62009 \\ \mathrm{H} & -4.29689 & -1.43959 & -1.58367 \\ & -6.93497 & -0.61715 & 0.39528 \\ \mathrm{H} & -6.84539 & 0.75392 & 2.05584 \\ -6.70113 & -1.92586 & -1.30115 \\ \mathrm{H} & -7.99414 & -0.82331 & 0.51432\end{array}$

conformer 4

$\mathrm{E}(\mathrm{DZ})=-791.515196$

$\mathrm{G}(\mathrm{DZ})=-791.210279$

$\mathrm{E}(\mathrm{TZ})=-791.3348078$

$\mathrm{G}(\mathrm{TZ})=-791.0298908$

41

01

$\begin{array}{llll}\text { C } & 6.13873 & -0.62008 & -0.12366\end{array}$

$\begin{array}{llll}\mathrm{C} & 4.85181 & 1.26857 & 0.22211\end{array}$

$\begin{array}{llll}\text { C } & 6.05532 & 1.76889 & -0.59849\end{array}$

$\begin{array}{llll}\text { C } & 6.90160 & 0.49150 & -0.86114\end{array}$

$\mathrm{H} \quad 6.21420 \quad-1.59897 \quad-0.60874$

$\begin{array}{llll}\mathrm{H} & 6.52579 & -0.72955 & 0.91026\end{array}$

$\mathrm{H} \quad 3.92320 \quad 1.79405 \quad-0.01731$

$\begin{array}{llll}\mathrm{H} & 5.04432 & 1.40389 & 1.30632\end{array}$

$\begin{array}{llll}\mathrm{H} & 5.72209 & 2.20956 & -1.54139\end{array}$

$\begin{array}{llll}\mathrm{H} & 6.61311 & 2.53714 & -0.05679\end{array}$ 


\begin{tabular}{|c|c|c|c|c|c|c|c|}
\hline $\mathrm{H}$ & 6.94633 & 0.27651 & -1.93181 & $\mathrm{C}$ & -1.54940 & -1.27502 & -0.29904 \\
\hline $\mathrm{H}$ & 7.92905 & 0.58377 & -0.49973 & $\mathrm{H}$ & -1.56434 & -1.79538 & 0.68018 \\
\hline $\mathrm{N}$ & 4.75528 & -0.14879 & -0.12742 & $\mathrm{H}$ & -1.78190 & -2.03075 & -1.06290 \\
\hline $\mathrm{C}$ & 3.88462 & -0.93411 & 0.74335 & $\mathrm{C}$ & -0.18219 & -0.71311 & -0.55550 \\
\hline $\mathrm{H}$ & 3.93604 & -1.97930 & 0.41797 & $\mathrm{C}$ & 0.84822 & -0.83115 & 0.29168 \\
\hline $\mathrm{H}$ & 4.25744 & -0.90526 & 1.79072 & $\mathrm{H}$ & -0.07641 & -0.17350 & -1.49459 \\
\hline $\mathrm{C}$ & 2.45533 & -0.47463 & 0.72392 & $\mathrm{H}$ & 0.67430 & -1.35151 & 1.23363 \\
\hline $\mathrm{C}$ & 1.44846 & -1.19974 & 0.21913 & $\mathrm{C}$ & 2.21398 & -0.32191 & 0.11177 \\
\hline $\mathrm{H}$ & 2.25682 & 0.50362 & 1.15690 & $\mathrm{C}$ & 2.65914 & 0.28148 & -1.07803 \\
\hline $\mathrm{H}$ & 1.68875 & -2.16606 & -0.22440 & $\mathrm{C}$ & 3.12533 & -0.43573 & 1.17441 \\
\hline $\mathrm{C}$ & 0.02783 & -0.83734 & 0.17644 & $\mathrm{C}$ & 3.96041 & 0.75871 & -1.19177 \\
\hline $\mathrm{C}$ & -0.86315 & -1.64386 & -0.55010 & $\mathrm{H}$ & 1.98360 & 0.37109 & -1.92236 \\
\hline $\mathrm{C}$ & -0.50331 & 0.28692 & 0.83359 & $\mathrm{C}$ & 4.42944 & 0.04163 & 1.06184 \\
\hline $\mathrm{C}$ & -2.21676 & -1.33824 & -0.63199 & $\mathrm{H}$ & 2.79937 & -0.90273 & 2.10000 \\
\hline $\mathrm{H}$ & -0.47934 & -2.51678 & -1.07123 & $\mathrm{C}$ & 4.85292 & 0.64313 & -0.12253 \\
\hline $\mathrm{C}$ & -1.85447 & 0.59182 & 0.75375 & $\mathrm{H}$ & 4.28316 & 1.22067 & -2.12009 \\
\hline $\mathrm{H}$ & 0.14504 & 0.91910 & 1.43154 & $\mathrm{H}$ & 5.11434 & -0.05721 & 1.89870 \\
\hline $\mathrm{C}$ & -2.74289 & -0.21185 & 0.01764 & $\mathrm{H}$ & 5.86852 & 1.01532 & -0.21549 \\
\hline $\mathrm{H}$ & -2.87190 & -1.96550 & -1.22813 & & & & \\
\hline $\mathrm{H}$ & -2.23991 & 1.44920 & 1.29641 & \multicolumn{4}{|c|}{ conformer 2} \\
\hline $\mathrm{C}$ & -4.18342 & 0.11778 & -0.06229 & \multicolumn{4}{|c|}{$E(D Z)=-560.427944$} \\
\hline $\mathrm{C}$ & -5.15551 & -0.89542 & -0.08543 & \multicolumn{4}{|c|}{$\mathrm{G}(\mathrm{DZ})=-560.197785$} \\
\hline $\mathrm{C}$ & -4.61494 & 1.45309 & -0.11639 & \multicolumn{4}{|c|}{$E(T Z)=-560.2937728$} \\
\hline $\mathrm{C}$ & -6.51113 & -0.58465 & -0.16103 & \multicolumn{4}{|c|}{$G(T Z)=-560.0636138$} \\
\hline $\mathrm{H}$ & -4.84392 & -1.93278 & -0.01618 & \multirow{2}{*}{\multicolumn{4}{|c|}{31}} \\
\hline $\mathrm{C}$ & -5.97056 & 1.76439 & -0.18951 & & & & \\
\hline $\mathrm{H}$ & -3.87817 & 2.25002 & -0.12762 & \multicolumn{4}{|c|}{01} \\
\hline $\mathrm{C}$ & -6.92529 & 0.74693 & -0.21268 & $\mathrm{C}$ & -2.51076 & 0.72881 & -0.71769 \\
\hline $\mathrm{H}$ & -7.24613 & -1.38394 & -0.16953 & $\mathrm{C}$ & -3.94048 & -0.75066 & 0.33920 \\
\hline $\mathrm{H}$ & -6.28153 & 2.80369 & -0.23818 & $\mathrm{C}$ & -4.80381 & 0.49906 & 0.08872 \\
\hline \multirow[t]{2}{*}{$\mathrm{H}$} & \multirow[t]{2}{*}{-7.98188} & \multirow[t]{2}{*}{0.98938} & \multirow[t]{2}{*}{-0.27041} & $\mathrm{C}$ & -3.83437 & 1.50180 & -0.60054 \\
\hline & & & & $\mathrm{H}$ & -2.45424 & 0.20567 & -1.69386 \\
\hline \multicolumn{4}{|c|}{$\mathbf{A r A}=\mathbf{P h}$} & $\mathrm{H}$ & -1.62231 & 1.35995 & -0.63426 \\
\hline & & & & $\mathrm{H}$ & -4.06541 & -1.47287 & -0.49260 \\
\hline & & & & $\mathrm{H}$ & -4.18703 & -1.27390 & 1.26906 \\
\hline & & & & $\mathrm{H}$ & -5.67503 & 0.26530 & -0.52868 \\
\hline \multicolumn{4}{|c|}{ conformer 1} & $\mathrm{H}$ & -5.17131 & 0.90839 & 1.03320 \\
\hline \multicolumn{4}{|c|}{$E(D Z)=-560.427924$} & $\mathrm{H}$ & -4.19501 & 1.83290 & -1.57780 \\
\hline \multicolumn{4}{|c|}{$\mathrm{G}(\mathrm{DZ})=-560.198195$} & $\mathrm{H}$ & -3.70157 & 2.39171 & 0.02005 \\
\hline \multicolumn{4}{|c|}{$E(T Z)=-560.2937606$} & $\mathrm{~N}$ & -2.57716 & -0.22609 & 0.38868 \\
\hline \multirow{2}{*}{\multicolumn{4}{|c|}{$G(T Z)=-560.0640316$}} & $\mathrm{C}$ & -1.54703 & -1.25375 & 0.35688 \\
\hline & & & & $\mathrm{H}$ & -1.77473 & -1.96936 & 1.15989 \\
\hline 31 & & & & $\mathrm{H}$ & -1.56148 & -1.82576 & -0.59322 \\
\hline 01 & & & & $\mathrm{C}$ & -0.18151 & -0.67378 & 0.58004 \\
\hline $\mathrm{C}$ & -3.94058 & -0.76345 & -0.30026 & $\mathrm{C}$ & 0.85420 & -0.85166 & -0.25035 \\
\hline $\mathrm{C}$ & -2.50077 & 0.76285 & 0.67585 & $\mathrm{H}$ & -0.07992 & -0.06948 & 1.47929 \\
\hline $\mathrm{C}$ & -3.82659 & 1.52919 & 0.53515 & $\mathrm{H}$ & 0.68643 & -1.43798 & -1.15392 \\
\hline $\mathrm{C}$ & -4.79846 & 0.50023 & -0.11016 & $\mathrm{C}$ & 2.21846 & -0.33061 & -0.09593 \\
\hline $\mathrm{H}$ & -4.06364 & -1.44243 & 0.56737 & $\mathrm{C}$ & 2.66064 & 0.33264 & 1.06289 \\
\hline $\mathrm{H}$ & -4.19317 & -1.33168 & -1.20164 & $\mathrm{C}$ & 3.13203 & -0.49604 & -1.14988 \\
\hline $\mathrm{H}$ & -2.43468 & 0.28812 & 1.67569 & $\mathrm{C}$ & 3.96004 & 0.81940 & 1.15390 \\
\hline $\mathrm{H}$ & -1.61390 & 1.38997 & 0.55367 & $\mathrm{H}$ & 1.98413 & 0.46067 & 1.90154 \\
\hline $\mathrm{H}$ & -4.18213 & 1.89628 & 1.50139 & $\mathrm{C}$ & 4.43449 & -0.00972 & -1.05974 \\
\hline $\mathrm{H}$ & -3.69876 & 2.39604 & -0.11840 & $\mathrm{H}$ & 2.80873 & -1.01011 & -2.05113 \\
\hline $\mathrm{H}$ & -5.67337 & 0.29900 & 0.51350 & $\mathrm{C}$ & 4.85454 & 0.65236 & 0.09318 \\
\hline $\mathrm{H}$ & -5.16004 & 0.86716 & -1.07419 & $\mathrm{H}$ & 4.28044 & 1.32773 & 2.05851 \\
\hline $\mathrm{N}$ & -2.57567 & -0.24567 & -0.38214 & $\mathrm{H}$ & 5.12079 & -0.14869 & -1.88970 \\
\hline
\end{tabular}




\begin{tabular}{|c|c|c|c|}
\hline & 5.86900 & 1.03134 & 0.16882 \\
\hline \multicolumn{4}{|c|}{ conformer 3} \\
\hline \multicolumn{4}{|c|}{$E(D Z)=-560.427538$} \\
\hline \multicolumn{4}{|c|}{$G(D Z)=-560.196407$} \\
\hline \multicolumn{4}{|c|}{$E(T Z)=-560.2931039$} \\
\hline \multicolumn{4}{|c|}{$G(T Z)=-560.0619729$} \\
\hline \multicolumn{4}{|c|}{31} \\
\hline 01 & & & \\
\hline $\mathrm{C}$ & 3.71502 & 0.43317 & -0.73841 \\
\hline $\mathrm{C}$ & 2.47621 & -0.28409 & 1.07830 \\
\hline $\mathrm{C}$ & 3.49836 & -1.40906 & 0.85025 \\
\hline $\mathrm{C}$ & 4.34509 & -0.91863 & -0.35866 \\
\hline $\mathrm{H}$ & 4.26045 & 1.26015 & -0.24171 \\
\hline $\mathrm{H}$ & 3.72320 & 0.63196 & -1.81498 \\
\hline $\mathrm{H}$ & 2.86545 & 0.44766 & 1.81421 \\
\hline $\mathrm{H}$ & 1.51015 & -0.63723 & 1.44537 \\
\hline $\mathrm{H}$ & 4.10294 & -1.58945 & 1.74279 \\
\hline $\mathrm{H}$ & 2.98797 & -2.34416 & 0.60507 \\
\hline $\mathrm{H}$ & 5.40468 & -0.81037 & -0.11339 \\
\hline $\mathrm{H}$ & 4.27100 & -1.62414 & -1.19016 \\
\hline $\mathrm{N}$ & 2.34246 & 0.32859 & -0.24487 \\
\hline $\mathrm{C}$ & 1.61088 & 1.58154 & -0.25617 \\
\hline $\mathrm{H}$ & 1.99144 & 2.28005 & 0.51840 \\
\hline $\mathrm{H}$ & 1.80291 & 2.07988 & -1.21799 \\
\hline $\mathrm{C}$ & 0.12343 & 1.41842 & -0.08528 \\
\hline $\mathrm{C}$ & -0.53477 & 0.26760 & -0.27347 \\
\hline $\mathrm{H}$ & -0.41232 & 2.32620 & 0.18588 \\
\hline $\mathrm{H}$ & 0.05616 & -0.59588 & -0.57241 \\
\hline $\mathrm{C}$ & -1.97780 & 0.03766 & -0.13508 \\
\hline $\mathrm{C}$ & -2.87018 & 1.00234 & 0.36680 \\
\hline $\mathrm{C}$ & -2.50308 & -1.20868 & -0.51528 \\
\hline $\mathrm{C}$ & -4.23017 & 0.73171 & 0.47141 \\
\hline $\mathrm{H}$ & -2.49452 & 1.96897 & 0.68675 \\
\hline $\mathrm{C}$ & -3.86534 & -1.48155 & -0.41123 \\
\hline $\mathrm{H}$ & -1.82751 & -1.96767 & -0.90051 \\
\hline $\mathrm{C}$ & -4.73679 & -0.51126 & 0.08195 \\
\hline $\mathrm{H}$ & -4.90003 & 1.49151 & 0.86327 \\
\hline $\mathrm{H}$ & -4.24555 & -2.45256 & -0.71439 \\
\hline $\mathrm{H}$ & -5.79873 & -0.72003 & 0.16709 \\
\hline
\end{tabular}

$\begin{array}{lrrr}\mathrm{H} & 1.98105 & 1.72330 & 0.21452 \\ \mathrm{H} & 4.75099 & 2.06410 & 0.19427 \\ \mathrm{H} & 3.87742 & 1.67537 & 1.67444 \\ \mathrm{H} & 5.77814 & -0.09739 & 0.31727 \\ \mathrm{H} & 4.81450 & -0.44680 & 1.75282 \\ \mathrm{~N} & 2.51918 & -0.31558 & 0.03423 \\ \mathrm{C} & 1.51087 & -0.84769 & -0.87860 \\ \mathrm{H} & 1.83960 & -0.72171 & -1.93352 \\ \mathrm{H} & 1.42923 & -1.92563 & -0.69913 \\ \mathrm{C} & 0.16157 & -0.21088 & -0.70947 \\ \mathrm{C} & -0.91453 & -0.86459 & -0.25353 \\ \mathrm{H} & 0.08365 & 0.83681 & -0.99235 \\ \mathrm{H} & -0.79247 & -1.91130 & 0.02519 \\ \mathrm{C} & -2.27222 & -0.33486 & -0.07243 \\ \mathrm{C} & -2.64176 & 0.97567 & -0.42496 \\ \mathrm{C} & -3.25709 & -1.17071 & 0.47899 \\ \mathrm{C} & -3.94156 & 1.42813 & -0.22707 \\ \mathrm{H} & -1.90709 & 1.64487 & -0.86074 \\ \mathrm{C} & -4.55999 & -0.71913 & 0.67762 \\ \mathrm{H} & -2.99021 & -2.18699 & 0.75616 \\ \mathrm{C} & -4.90864 & 0.58408 & 0.32571 \\ \mathrm{H} & -4.20453 & 2.44390 & -0.50714 \\ \mathrm{H} & -5.30214 & -1.38547 & 1.10695 \\ \mathrm{H} & -5.92271 & 0.94046 & 0.47776\end{array}$

Catalyst $\mathrm{ArC}$ and Amine ArA as benzene derivatives, $\mathrm{NCI}$ probes<smiles>Cc1cccc(C)c1C(C)(C)c1ccccc1</smiles>

conformer 4

$\begin{array}{lrrr}E(D Z)=-560.424719 & \\ G(D Z)=-560.19486 & & \\ E(T Z)=-560.2908269 & & \\ G(T Z)=-560.0609679 & & \\ & & & \\ 31 & & & \\ 01 & & \\ C & 3.81648 & -0.97463 & -0.10476 \\ C & 2.81174 & 1.10693 & -0.14047 \\ C & 4.10605 & 1.31888 & 0.66700 \\ C & 4.76254 & -0.08927 & 0.72131 \\ H & 4.14510 & -1.00207 & -1.16403 \\ H & 3.76363 & -2.00914 & 0.25040 \\ H & 2.98084 & 1.35506 & -1.20855\end{array}$

21

$\begin{array}{lrrr}\mathrm{H} & -0.09913 & 3.22921 & -0.00024 \\ \mathrm{C} & -0.06562 & 2.14440 & 0.00007 \\ \mathrm{C} & 1.15551 & 1.48125 & -0.00026 \\ \mathrm{C} & -1.24337 & 1.40862 & 0.00020 \\ \mathrm{C} & 1.20854 & 0.08789 & 0.00021 \\ \mathrm{H} & 2.08308 & 2.04725 & -0.00105 \\ \mathrm{C} & -1.22102 & 0.01201 & 0.00049 \\ \mathrm{H} & -2.20177 & 1.92060 & -0.00021 \\ \mathrm{C} & 0.01493 & -0.65515 & 0.00115 \\ \mathrm{C} & 2.54155 & -0.61578 & -0.00032 \\ \mathrm{H} & 2.66158 & -1.24896 & 0.88548 \\ \mathrm{H} & 2.65121 & -1.26769 & -0.87370 \\ \mathrm{H} & 3.36233 & 0.10404 & -0.01246 \\ \mathrm{C} & 0.09753 & -2.16216 & 0.00014 \\ \mathrm{H} & 0.61576 & -2.52718 & -0.89362 \\ \mathrm{H} & 0.66505 & -2.52588 & 0.86357 \\ \mathrm{H} & -0.88489 & -2.63242 & 0.02790 \\ \mathrm{C} & -2.52730 & -0.74608 & -0.00056 \\ \mathrm{H} & -2.62015 & -1.39166 & -0.87963 \\ \mathrm{H} & -2.62622 & -1.38379 & 0.88366\end{array}$




\begin{tabular}{|c|c|c|c|}
\hline $\mathrm{H}$ & -3.37138 & -0.05352 & -0.00637 \\
\hline \multicolumn{4}{|c|}{ 13_Me2_2OMe_Ph } \\
\hline & & & \\
\hline \multicolumn{4}{|c|}{22} \\
\hline $\mathrm{H}$ & -3.40880 & -0.65678 & 0.43590 \\
\hline $\mathrm{C}$ & -2.35896 & -0.44917 & 0.25637 \\
\hline $\mathrm{C}$ & -1.91867 & 0.86686 & 0.14260 \\
\hline $\mathrm{C}$ & -1.45548 & -1.49670 & 0.12420 \\
\hline $\mathrm{C}$ & -0.57483 & 1.15135 & -0.09230 \\
\hline $\mathrm{H}$ & -2.62493 & 1.68771 & 0.23155 \\
\hline $\mathrm{C}$ & -0.10050 & -1.25207 & -0.11071 \\
\hline $\mathrm{H}$ & -1.79945 & -2.52518 & 0.19512 \\
\hline $\mathrm{C}$ & 0.31653 & 0.07852 & -0.20898 \\
\hline $\mathrm{O}$ & 1.64166 & 0.36347 & -0.47280 \\
\hline $\mathrm{C}$ & -0.06878 & 2.56111 & -0.24374 \\
\hline $\mathrm{H}$ & 0.62938 & 2.82543 & 0.55697 \\
\hline $\mathrm{H}$ & 0.47100 & 2.67928 & -1.18655 \\
\hline $\mathrm{H}$ & -0.89599 & 3.27328 & -0.21890 \\
\hline $\mathrm{C}$ & 0.88508 & -2.38008 & -0.26957 \\
\hline $\mathrm{H}$ & 1.72232 & -2.07355 & -0.89979 \\
\hline $\mathrm{H}$ & 1.29069 & -2.69638 & 0.69790 \\
\hline $\mathrm{H}$ & 0.40380 & -3.25073 & -0.72106 \\
\hline $\mathrm{C}$ & 2.45163 & 0.37038 & 0.69030 \\
\hline $\mathrm{H}$ & 2.48233 & -0.61970 & 1.16104 \\
\hline $\mathrm{H}$ & 3.45769 & 0.65134 & 0.37589 \\
\hline $\mathrm{H}$ & 2.08260 & 1.09628 & 1.42524 \\
\hline \multicolumn{4}{|c|}{$\mathrm{AdPh}$} \\
\hline & & & \\
\hline & & & \\
\hline
\end{tabular}

36

$\begin{array}{lccc}\mathrm{H} & 0.18656 & -0.17257 & -2.15310 \\ \mathrm{H} & 0.15140 & -1.70789 & -1.28687 \\ \mathrm{C} & 2.08825 & -0.73704 & -1.25552 \\ \mathrm{H} & 0.26243 & 2.01609 & 0.88436 \\ \mathrm{H} & 0.26248 & 2.01602 & -0.88463 \\ \mathrm{C} & 2.15092 & 1.42916 & -0.00005 \\ \mathrm{H} & 0.15130 & -1.70780 & 1.28686 \\ \mathrm{H} & 0.18634 & -0.17242 & 2.15300 \\ \mathrm{C} & 2.08813 & -0.73692 & 1.25565 \\ \mathrm{H} & 2.43175 & -1.26658 & -2.15181 \\ \mathrm{C} & 2.64492 & 0.69347 & -1.25391 \\ \mathrm{C} & 2.57498 & -1.47673 & 0.00012 \\ \mathrm{H} & 2.53481 & 2.45592 & -0.00008 \\ \mathrm{C} & 2.64480 & 0.69360 & 1.25394 \\ \mathrm{H} & 2.43156 & -1.26637 & 2.15202 \\ \mathrm{H} & 2.31647 & 1.22530 & -2.15571 \\ \mathrm{H} & 3.74182 & 0.67188 & -1.26912 \\ \mathrm{H} & 2.19444 & -2.50609 & 0.00015 \\ \mathrm{H} & 3.67065 & -1.53490 & 0.00016 \\ \mathrm{H} & 3.74169 & 0.67200 & 1.26928 \\ \mathrm{H} & 2.31626 & 1.22552 & 2.15567\end{array}$

Benzene<smiles>c1ccccc1</smiles>

12

$\begin{array}{rrrc}\mathrm{H} & 2.47838 & -0.03654 & 0.00002 \\ \mathrm{C} & 1.39307 & -0.02055 & -0.00000 \\ \mathrm{C} & 0.71405 & 1.19580 & -0.00010 \\ \mathrm{C} & 0.67849 & -1.21632 & 0.00007 \\ \mathrm{C} & -0.67861 & 1.21638 & 0.00007 \\ \mathrm{H} & 1.27070 & 2.12791 & 0.00001 \\ \mathrm{C} & -0.71421 & -1.19584 & -0.00005 \\ \mathrm{H} & 1.20740 & -2.16444 & 0.00009 \\ \mathrm{C} & -1.39267 & 0.02052 & -0.00003 \\ \mathrm{H} & -1.20783 & 2.16438 & 0.00016 \\ \mathrm{H} & -1.27108 & -2.12785 & -0.00008 \\ \mathrm{H} & -2.47826 & 0.03656 & 0.00006\end{array}$

CF3Ph<smiles>FC(F)(F)c1ccccc1</smiles>

15

$\begin{array}{llll}\mathrm{H} & -3.90791 & -0.03241 & 0.00031 \\ \mathrm{C} & -2.82289 & -0.01641 & 0.00015 \\ \mathrm{C} & -2.11071 & -1.21537 & 0.00003 \\ \mathrm{C} & -2.14678 & 1.19942 & 0.00002 \\ \mathrm{C} & -0.72203 & -1.19681 & -0.00022 \\ \mathrm{H} & -2.63918 & -2.16268 & 0.00008 \\ \mathrm{C} & -0.75416 & 1.22399 & -0.00021\end{array}$




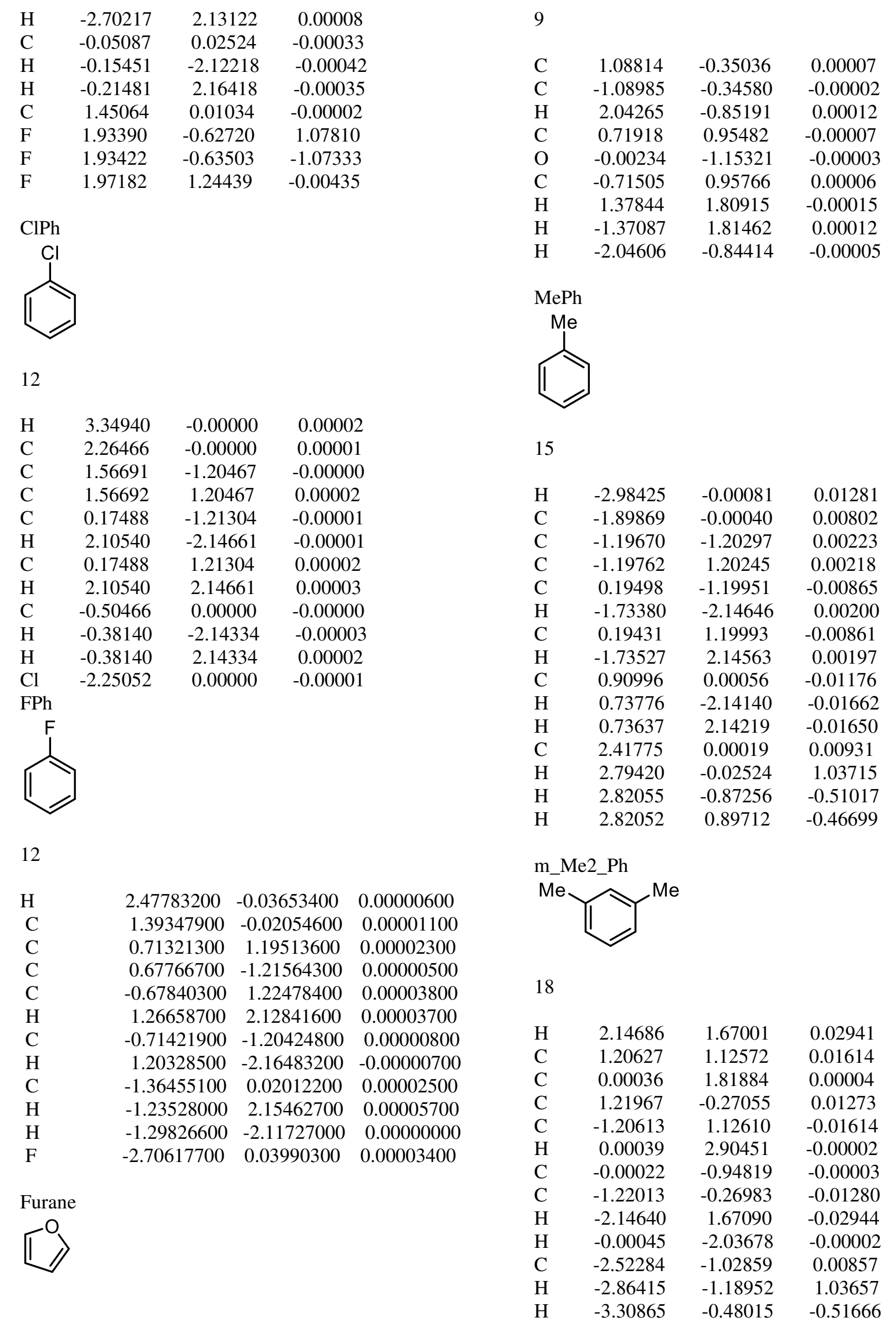




$\begin{array}{lrrr}\mathrm{H} & -2.41825 & -2.00957 & -0.46115 \\ \mathrm{C} & 2.52281 & -1.02876 & -0.00854 \\ \mathrm{H} & 3.29813 & -0.49843 & 0.55025 \\ \mathrm{H} & 2.40936 & -2.02497 & 0.42580 \\ \mathrm{H} & 2.88446 & -1.15445 & -1.03458 \\ \mathrm{~m} & & \end{array}$

36

$\begin{array}{lrrr}\mathrm{H} & -0.00000 & 3.47934 & -0.00000 \\ \mathrm{C} & -0.00000 & 2.39362 & -0.00000 \\ \mathrm{C} & 1.20556 & 1.70248 & 0.00000 \\ \mathrm{C} & -1.20556 & 1.70248 & -0.00000 \\ \mathrm{C} & 1.22577 & 0.30476 & -0.00000 \\ \mathrm{H} & 2.13751 & 2.26082 & 0.00001 \\ \mathrm{C} & -1.22576 & 0.30475 & 0.00000 \\ \mathrm{H} & -2.13751 & 2.26081 & -0.00001 \\ \mathrm{C} & 0.00000 & -0.36880 & -0.00000 \\ \mathrm{H} & 0.00001 & -1.45108 & -0.00000 \\ \mathrm{C} & 2.57004 & -0.43108 & -0.00000 \\ \mathrm{C} & 3.36627 & -0.03512 & -1.25505 \\ \mathrm{C} & 3.36633 & -0.03499 & 1.25497 \\ \mathrm{C} & 2.40165 & -1.95399 & 0.00008 \\ \mathrm{H} & 2.81725 & -0.30503 & -2.16218 \\ \mathrm{H} & 3.55983 & 1.04061 & -1.28596 \\ \mathrm{H} & 4.33186 & -0.55179 & -1.26932 \\ \mathrm{H} & 2.81733 & -0.30476 & 2.16215 \\ \mathrm{H} & 4.33189 & -0.55170 & 1.26927 \\ \mathrm{H} & 3.55994 & 1.04073 & 1.28573 \\ \mathrm{H} & 3.38680 & -2.42991 & 0.00007 \\ \mathrm{H} & 1.86321 & -2.29991 & 0.88799 \\ \mathrm{H} & 1.86315 & -2.29999 & -0.88776 \\ \mathrm{C} & -2.57003 & -0.43108 & 0.00000 \\ \mathrm{C} & -3.36625 & -0.03515 & 1.25507 \\ \mathrm{C} & -3.36635 & -0.03496 & -1.25494 \\ \mathrm{C} & -2.40166 & -1.95399 & -0.00012 \\ \mathrm{H} & -2.81721 & -0.30507 & 2.16218 \\ \mathrm{H} & -3.55979 & 1.04059 & 1.28601 \\ \mathrm{H} & -4.33183 & -0.55180 & 1.26934 \\ \mathrm{H} & -2.81737 & -0.30470 & -2.16214 \\ \mathrm{H} & -4.33192 & -0.55165 & -1.26924 \\ \mathrm{H} & -3.55994 & 1.04077 & -1.28568 \\ \mathrm{H} & -1.86323 & -2.29989 & -0.88804 \\ \mathrm{H} & & -2.30003 & 0.88771\end{array}$

Naphthalene<smiles>c1ccc2ccccc2c1</smiles>

18

$\begin{array}{lrrr}\mathrm{H} & 3.36934 & 1.24391 & 0.00000 \\ \mathrm{C} & 2.42573 & 0.70834 & 0.00000 \\ \mathrm{C} & 1.24228 & 1.39942 & -0.00000 \\ \mathrm{C} & 2.42607 & -0.70827 & 0.00000 \\ \mathrm{C} & -0.00003 & 0.71147 & -0.00000 \\ \mathrm{H} & 1.23624 & 2.48603 & -0.00001 \\ \mathrm{C} & 1.24254 & -1.39925 & -0.00000 \\ \mathrm{H} & 3.36993 & -1.24372 & 0.00001 \\ \mathrm{C} & -1.24247 & 1.39929 & -0.00000 \\ \mathrm{C} & 0.00008 & -0.71139 & -0.00000 \\ \mathrm{H} & 1.23655 & -2.48583 & -0.00001 \\ \mathrm{C} & -2.42598 & 0.70824 & 0.00000 \\ \mathrm{H} & -1.23649 & 2.48589 & 0.00000 \\ \mathrm{C} & -1.24230 & -1.39937 & -0.00000 \\ \mathrm{C} & -2.42591 & -0.70848 & 0.00000 \\ \mathrm{H} & -3.36978 & 1.24376 & 0.00000 \\ \mathrm{H} & -1.23613 & -2.48596 & 0.00000 \\ \mathrm{H} & -3.36966 & -1.24409 & 0.00000\end{array}$

nPrPh_1<smiles>CCCc1ccccc1</smiles>

21

$\begin{array}{lrrr}\mathrm{H} & 3.88929 & -0.00080 & -0.57642 \\ \mathrm{C} & 2.83712 & -0.00046 & -0.31076 \\ \mathrm{C} & 2.15805 & -1.20364 & -0.13721 \\ \mathrm{C} & 2.15869 & 1.20315 & -0.13780 \\ \mathrm{C} & 0.80965 & -1.20009 & 0.20761 \\ \mathrm{H} & 2.68009 & -2.14653 & -0.26697 \\ \mathrm{C} & 0.81029 & 1.20049 & 0.20706 \\ \mathrm{H} & 2.68123 & 2.14571 & -0.26804 \\ \mathrm{C} & 0.11825 & 0.00042 & 0.38605 \\ \mathrm{H} & 0.28249 & -2.14130 & 0.34495 \\ \mathrm{H} & 0.28365 & 2.14205 & 0.34396 \\ \mathrm{C} & -1.35637 & 0.00083 & 0.70346 \\ \mathrm{H} & -1.60680 & -0.87865 & 1.30903 \\ \mathrm{H} & -1.60677 & 0.88169 & 1.30702 \\ \mathrm{C} & -2.22055 & -0.00060 & -0.56419 \\ \mathrm{H} & -1.96131 & -0.87835 & -1.16784 \\ \mathrm{H} & -1.96111 & 0.87566 & -1.16992 \\ \mathrm{C} & -3.71311 & -0.00006 & -0.24876 \\ \mathrm{H} & -4.31543 & -0.00116 & -1.16061 \\ \mathrm{H} & -3.98881 & -0.88250 & 0.33714 \\ \mathrm{H} & -3.98864 & 0.88389 & 0.33491\end{array}$

nPrPh_2<smiles>CCCc1ccccc1</smiles> 
21

$\begin{array}{lrrc}\mathrm{H} & -3.93745 & 0.57090 & -0.00017 \\ \mathrm{C} & -2.87845 & 0.33404 & -0.00010 \\ \mathrm{C} & -2.45507 & -0.99442 & -0.00009 \\ \mathrm{C} & -1.93186 & 1.35110 & -0.00001 \\ \mathrm{C} & -1.09841 & -1.29169 & 0.00001 \\ \mathrm{H} & -3.18437 & -1.79855 & -0.00015 \\ \mathrm{C} & -0.57043 & 1.04730 & 0.00008 \\ \mathrm{H} & -2.24911 & 2.38931 & -0.00002 \\ \mathrm{C} & -0.13354 & -0.27750 & 0.00009 \\ \mathrm{H} & -0.77273 & -2.32946 & 0.00002 \\ \mathrm{H} & 0.15155 & 1.85746 & 0.00014 \\ \mathrm{C} & 1.33274 & -0.65971 & 0.00018 \\ \mathrm{H} & 1.52752 & -1.29533 & 0.87453 \\ \mathrm{H} & 1.52756 & -1.29559 & -0.87397 \\ \mathrm{C} & 2.32873 & 0.49622 & 0.00006 \\ \mathrm{H} & 2.15615 & 1.12878 & 0.87891 \\ \mathrm{H} & 2.15584 & 1.12887 & -0.87867 \\ \mathrm{C} & 3.77220 & 0.00074 & -0.00021 \\ \mathrm{H} & 4.48043 & 0.83275 & -0.00022 \\ \mathrm{H} & 3.97475 & -0.61293 & 0.88300 \\ \mathrm{H} & 3.97449 & -0.61275 & -0.88359\end{array}$

nPrPh_3<smiles>CCCc1ccccc1</smiles>

21

$\begin{array}{lrrr}\mathrm{H} & 3.59877 & 0.34523 & -0.76402 \\ \mathrm{C} & 2.58660 & 0.19246 & -0.40375 \\ \mathrm{C} & 2.00148 & -1.07007 & -0.47239 \\ \mathrm{C} & 1.86579 & 1.25518 & 0.13244 \\ \mathrm{C} & 0.70488 & -1.26379 & -0.00752 \\ \mathrm{H} & 2.55810 & -1.90555 & -0.88540 \\ \mathrm{C} & 0.56818 & 1.05483 & 0.59740 \\ \mathrm{H} & 2.31383 & 2.24218 & 0.19116 \\ \mathrm{C} & -0.02989 & -0.20528 & 0.53485 \\ \mathrm{H} & 0.25284 & -2.25179 & -0.05916 \\ \mathrm{H} & 0.00766 & 1.88715 & 1.01605 \\ \mathrm{C} & -1.45156 & -0.42259 & 0.99435 \\ \mathrm{H} & -1.51090 & -1.35447 & 1.56777 \\ \mathrm{H} & -1.74519 & 0.38794 & 1.67194 \\ \mathrm{C} & -2.44729 & -0.48530 & -0.17493 \\ \mathrm{H} & -3.43959 & -0.72950 & 0.22038 \\ \mathrm{H} & -2.16227 & -1.30938 & -0.84002 \\ \mathrm{C} & -2.51267 & 0.81615 & -0.96881 \\ \mathrm{H} & -3.24893 & 0.75243 & -1.77392 \\ \mathrm{H} & -2.79495 & 1.65281 & -0.32066 \\ \mathrm{H} & -1.54249 & 1.05329 & -1.41408\end{array}$

$\mathrm{OMePh}$<smiles>COc1ccccc1</smiles>

16

$\begin{array}{lrrr}\mathrm{H} & 3.32913 & 0.57409 & -0.00007 \\ \mathrm{C} & 2.27126 & 0.33580 & -0.00002 \\ \mathrm{C} & 1.84658 & -0.99446 & -0.00004 \\ \mathrm{C} & 1.32276 & 1.34926 & 0.00001 \\ \mathrm{C} & 0.49560 & -1.30222 & 0.00003 \\ \mathrm{H} & 2.57590 & -1.79834 & 0.00000 \\ \mathrm{C} & -0.04219 & 1.05706 & 0.00002 \\ \mathrm{H} & 1.63757 & 2.38812 & 0.00002 \\ \mathrm{C} & -0.45511 & -0.27550 & 0.00000 \\ \mathrm{O} & -1.75488 & -0.67674 & 0.00002 \\ \mathrm{C} & -2.74446 & 0.32709 & -0.00003 \\ \mathrm{H} & -2.67347 & 0.95961 & -0.89318 \\ \mathrm{H} & -3.70355 & -0.18986 & 0.00010 \\ \mathrm{H} & -2.67325 & 0.95987 & 0.89289 \\ \mathrm{H} & 0.14270 & -2.32773 & 0.00011 \\ \mathrm{H} & -0.76250 & 1.86600 & 0.00018\end{array}$

$\mathrm{PhPh}$<smiles>Pc1ccccc1</smiles>

22

$\begin{array}{lrrr}\mathrm{H} & -4.63400 & 0.00001 & 0.00000 \\ \mathrm{C} & -3.54879 & 0.00000 & 0.00000 \\ \mathrm{C} & -2.84819 & 1.13510 & 0.39965 \\ \mathrm{C} & -2.84820 & -1.13509 & -0.39965 \\ \mathrm{C} & -1.45748 & 1.13442 & 0.40026 \\ \mathrm{H} & -3.38613 & 2.02152 & 0.72051 \\ \mathrm{C} & -1.45749 & -1.13442 & -0.40026 \\ \mathrm{H} & -3.38615 & -2.02151 & -0.72052 \\ \mathrm{C} & -0.74204 & -0.00001 & 0.00000 \\ \mathrm{H} & -0.91537 & 2.01280 & 0.73813 \\ \mathrm{H} & -0.91539 & -2.01281 & -0.73815 \\ \mathrm{C} & 0.74205 & -0.00000 & -0.00000 \\ \mathrm{C} & 1.45749 & -1.13443 & 0.40026 \\ \mathrm{C} & 1.45748 & 1.13443 & -0.40026 \\ \mathrm{C} & 2.84819 & -1.13509 & 0.39965 \\ \mathrm{H} & 0.91540 & -2.01281 & 0.73815 \\ \mathrm{C} & 2.84818 & 1.13509 & -0.39965 \\ \mathrm{H} & 0.91539 & 2.01281 & -0.73814 \\ \mathrm{C} & 3.54880 & 0.00001 & -0.00000 \\ \mathrm{H} & 3.38613 & -2.02151 & 0.72052 \\ \mathrm{H} & 3.38612 & 2.02153 & -0.72051 \\ \mathrm{H} & 4.63396 & 0.00001 & 0.00000\end{array}$




$\begin{array}{lrrr}\text { tBuPh } & & \\ \end{array}$




\section{Non-covalent interaction geometries}

Edge-to-face complex $\mathbf{A r}_{\text {side }}^{\mathrm{C}}-\mathbf{A r}_{\pi}^{\mathrm{A}}$

1Nap_piO_4AdPh_side1
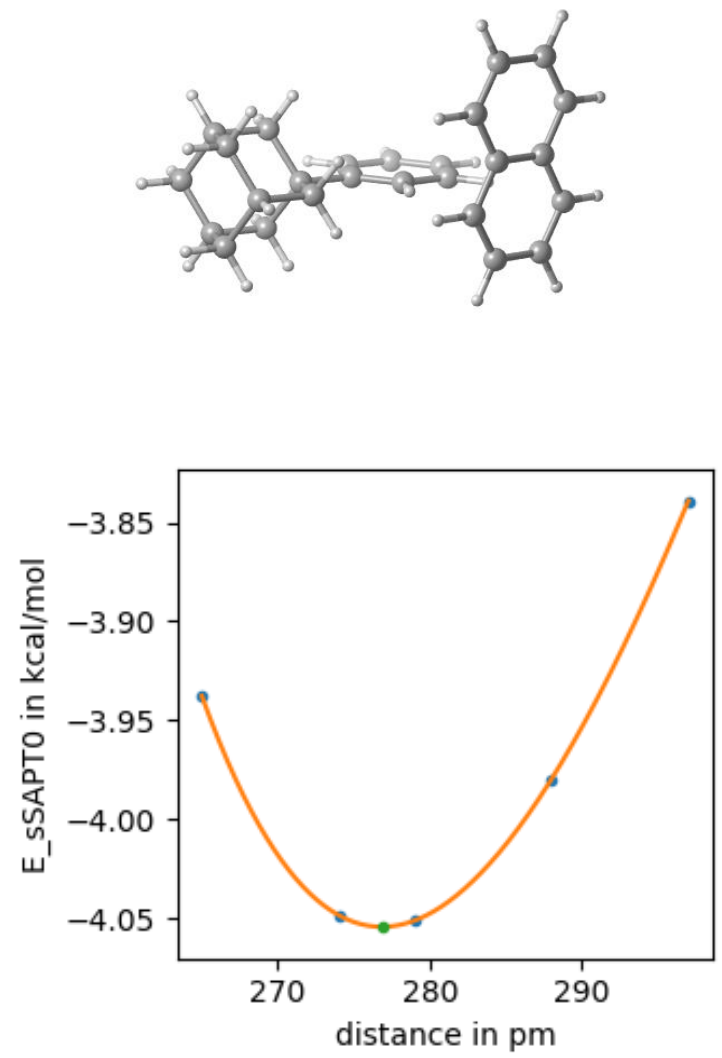

54

1Nap_pi0_4AdPh_side1-276

H $\quad-4.87787100 \quad-1.61916550 \quad-1.45943376$

$\begin{array}{llll}\text { C } & -3.99786918 & -1.59519376 & -0.82491427\end{array}$

$\begin{array}{llll}\text { C } & -3.38949090 & -2.78086647 & -0.41845613\end{array}$

$\begin{array}{llll}\text { C } & -3.46380978 & -0.38260933 & -0.40775501\end{array}$

$\begin{array}{llll}\text { C } & -2.26307715 & -2.74484269 & 0.39387826\end{array}$

H $\quad-3.79363954 \quad-3.73734728 \quad-0.73519497$

$\begin{array}{llll}\text { C } & -2.33295028 & -0.35209607 & 0.40763031\end{array}$

$\begin{array}{llll}\mathrm{H} & -3.92578411 & 0.55036108 & -0.71554004\end{array}$

C $-1.70973869-1.53086760 \quad 0.82498350$

$\begin{array}{llll}\mathrm{H} & -1.80527517 & -3.68203710 & 0.69851648\end{array}$

$\begin{array}{llll}\mathrm{H} & -1.94124754 & 0.61065561 & 0.71555150\end{array}$

$\begin{array}{llll}\text { C } & -0.47124831 & -1.54104542 & 1.71676248\end{array}$

$\begin{array}{llll}\text { C } & 0.69668588 & -2.25164418 & 0.99100847\end{array}$

$\begin{array}{llll}\text { C } & -0.00117287 & -0.12200067 & 2.09288817\end{array}$

$\begin{array}{llll}\text { C } & -0.77103042 & -2.30556437 & 3.02877768\end{array}$

$\begin{array}{llll}\mathrm{H} & 0.91977266 & -1.71458653 & 0.06009237\end{array}$

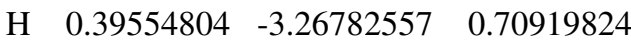

$\begin{array}{llll}\text { C } & 1.94247617 & -2.31162629 & 1.88718472\end{array}$

$\begin{array}{llll}\mathrm{H} & -0.80816213 & 0.40786696 & 2.61593008\end{array}$

$\begin{array}{lrrr}\mathrm{H} & 0.22545428 & 0.44584009 & 1.18087872 \\ \mathrm{C} & 1.24508838 & -0.17341921 & 2.98915476 \\ \mathrm{H} & -1.10820652 & -3.32307457 & 2.79702616 \\ \mathrm{H} & -1.59629528 & -1.80702388 & 3.55337204 \\ \mathrm{C} & 0.47520603 & -2.36552385 & 3.92433289 \\ \mathrm{H} & 2.74846962 & -2.82419922 & 1.34926781 \\ \mathrm{C} & 2.38357017 & -0.88546866 & 2.24465119 \\ \mathrm{C} & 1.60887984 & -3.08160645 & 3.17423035 \\ \mathrm{H} & 1.54962030 & 0.85035271 & 3.23558194 \\ \mathrm{C} & 0.91822282 & -0.93929567 & 4.27912818 \\ \mathrm{H} & 0.23373243 & -2.91657824 & 4.84070690 \\ \mathrm{H} & 2.64031581 & -0.33203666 & 1.33265751 \\ \mathrm{H} & 3.28276308 & -0.91452372 & 2.87271069 \\ \mathrm{H} & 1.30703180 & -4.10798370 & 2.92966655 \\ \mathrm{H} & 2.49840579 & -3.14757637 & 3.81317108 \\ \mathrm{H} & 1.79957245 & -0.96900735 & 4.93196178 \\ \mathrm{H} & 0.12116859 & -0.42457675 & 4.83022263 \\ \mathrm{C} & -1.76746590 & 3.40230206 & -0.59745338 \\ \mathrm{H} & -0.79191697 & 3.44483448 & -1.07045651 \\ \mathrm{C} & -1.86999762 & 3.39849915 & 0.76915182 \\ \mathrm{C} & -2.93252847 & 3.35081517 & -1.40167577 \\ \mathrm{C} & -3.14024923 & 3.34312621 & 1.40161293 \\ \mathrm{H} & -0.97960943 & 3.43785607 & 1.39078031 \\ \mathrm{C} & -4.17194539 & 3.29677187 & -0.81932325 \\ \mathrm{H} & -2.83782181 & 3.35441208 & -2.48269426 \\ \mathrm{C} & -3.27890580 & 3.33775802 & 2.81494892 \\ \mathrm{C} & -4.31057594 & 3.29140784 & 0.59404171 \\ \mathrm{H} & -5.06911998 & 3.25713043 & -1.43103359 \\ \mathrm{C} & -4.51837326 & 3.28370646 & 3.39723603 \\ \mathrm{H} & -2.3817653 & 3.37740311 & 3.42666947 \\ \mathrm{C} & -5.58089322 & 3.23602904 & 1.22654729 \\ \mathrm{C} & -5.68367565 & 3.23220915 & 2.59318217 \\ \mathrm{H} & -4.61298339 & 3.28010478 & 4.47823825 \\ \mathrm{H} & -6.47118855 & 3.19667915 & 0.60481900 \\ \mathrm{H} & -6.65933307 & 3.18966902 & 3.06627433\end{array}$

1Nap_pi0_4AdPh_side2

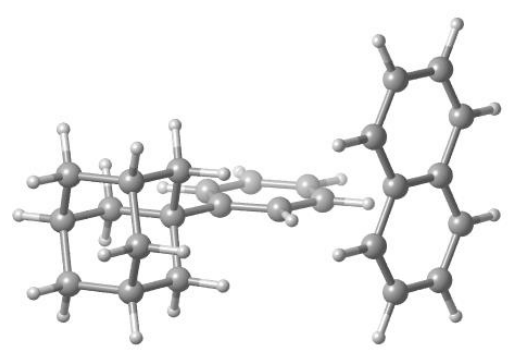




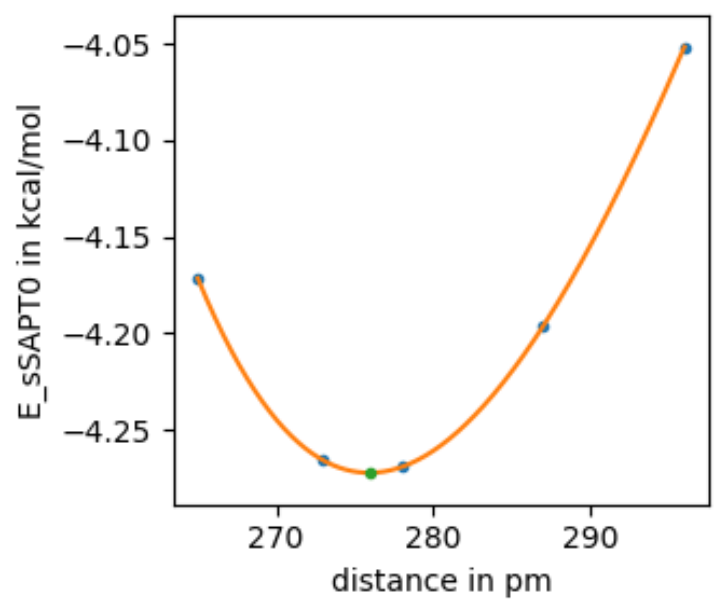

54

1Nap_pi0_4AdPh_side2-275

$\begin{array}{lrrc}\text { H } & -4.89876045 & 0.64420611 & -1.53031600 \\ \mathrm{C} & -4.04189092 & 0.67064166 & -0.86498254 \\ \mathrm{C} & -3.45203466 & -0.51353653 & -0.42801144 \\ \mathrm{C} & -3.51934862 & 1.88492925 & -0.43830061 \\ \mathrm{C} & -2.35521925 & -0.47435815 & 0.42372433 \\ \mathrm{H} & -3.84754906 & -1.47131305 & -0.75165884 \\ \mathrm{C} & -2.41821603 & 1.91860855 & 0.41668489 \\ \mathrm{H} & -3.96718905 & 2.81677034 & -0.76950570 \\ \mathrm{C} & -1.81390362 & 0.74137440 & 0.86502066 \\ \mathrm{H} & -1.91144913 & -1.41043586 & 0.75166802 \\ \mathrm{H} & -2.03480573 & 2.88262209 & 0.73102823 \\ \mathrm{C} & -0.60805962 & 0.73465604 & 1.80050283 \\ \mathrm{C} & 0.58284737 & 0.01520558 & 1.12236470 \\ \mathrm{C} & -0.14736425 & 2.15525729 & 2.18231605 \\ \mathrm{C} & -0.95680473 & -0.01883746 & 3.10678258 \\ \mathrm{H} & 0.84065178 & 0.54441160 & 0.19592998 \\ \mathrm{H} & 0.28884866 & -1.00228582 & 0.83775688 \\ \mathrm{C} & 1.79567728 & -0.04130165 & 2.06288250 \\ \mathrm{H} & -0.97087769 & 2.69140690 & 2.67214605 \\ \mathrm{H} & 0.11339782 & 2.71538198 & 1.27466652 \\ \mathrm{C} & 1.06595930 & 2.10731274 & 3.12287550 \\ \mathrm{H} & -1.28860886 & -1.03716944 & 2.87092352 \\ \mathrm{H} & -1.79873332 & 0.48604900 & 3.59775089 \\ \mathrm{C} & 0.25649327 & -0.07532812 & 4.04669551 \\ \mathrm{H} & 2.61877924 & -0.56026961 & 1.55801969 \\ \mathrm{C} & 2.22809737 & 1.38634841 & 2.42495981 \\ \mathrm{C} & 1.41401389 & -0.80035544 & 3.34304374 \\ \mathrm{H} & 1.36463569 & 3.13211084 & 3.37218703 \\ \mathrm{C} & 0.69093045 & 1.35236643 & 4.40617154 \\ \mathrm{H} & -0.01921010 & -0.61859184 & 4.95804832 \\ \mathrm{H} & 2.51891396 & 1.93198107 & 1.51851153 \\ \mathrm{H} & 3.10421390 & 1.35965544 & 3.08492808 \\ \mathrm{H} & 1.11794519 & -1.82775182 & 3.09571009 \\ \mathrm{H} & 2.27996844 & -0.86385079 & 4.01382297 \\ \mathrm{H} & 1.54832915 & 1.32525849 & 5.09026531 \\ \mathrm{H} & -0.12370166 & 1.87355750 & 4.92450587 \\ \mathrm{C} & -2.66108014 & -4.20394363 & -1.39489994 \\ \mathrm{H} & -2.52800186 & -4.20719621 & -2.47170831\end{array}$

$\begin{array}{llll}\text { C } & -3.92133139 & -4.23235131 & -0.85722273\end{array}$

$\begin{array}{llll}\text { C } & -1.52432588 & -4.17017144 & -0.55024348\end{array}$

$\begin{array}{llll}\text { C } & -4.11029145 & -4.22847755 & 0.55021873\end{array}$

H $\quad-4.79671829-4.25831939-1.50048434$

C $-1.67580709-4.16563605 \quad 0.81182932$

H $\quad-0.53182177 \quad-4.14788197 \quad-0.98844665$

$\begin{array}{llll}\text { C } & -5.40311915 & -4.25739748 & 1.13718827\end{array}$

$\begin{array}{llll}\text { C } & -2.96867058 & -4.19455998 & 1.39878343\end{array}$

H $-0.80761812-4.13979801 \quad 1.46471792$

C $\quad-5.55452223 \quad-4.25285396 \quad 2.49928808$

$\mathrm{H} \quad-6.27132250 \quad-4.28323895 \quad 0.48428895$

$\begin{array}{llll}\text { C } & -3.15765158 & -4.19068328 & 2.80630156\end{array}$

$\begin{array}{llll}\text { C } & -4.41785014 & -4.21908211 & 3.34422610\end{array}$

$\begin{array}{llll}\mathrm{H} & -6.54704217 & -4.27513542 & 2.93739487\end{array}$

$\begin{array}{llll}\mathrm{H} & -2.28220013 & -4.16471731 & 3.44944291\end{array}$

H $\quad-4.55097772 \quad-4.21582697 \quad 4.42116607$

2Fur_pi0_4AdPh_side1
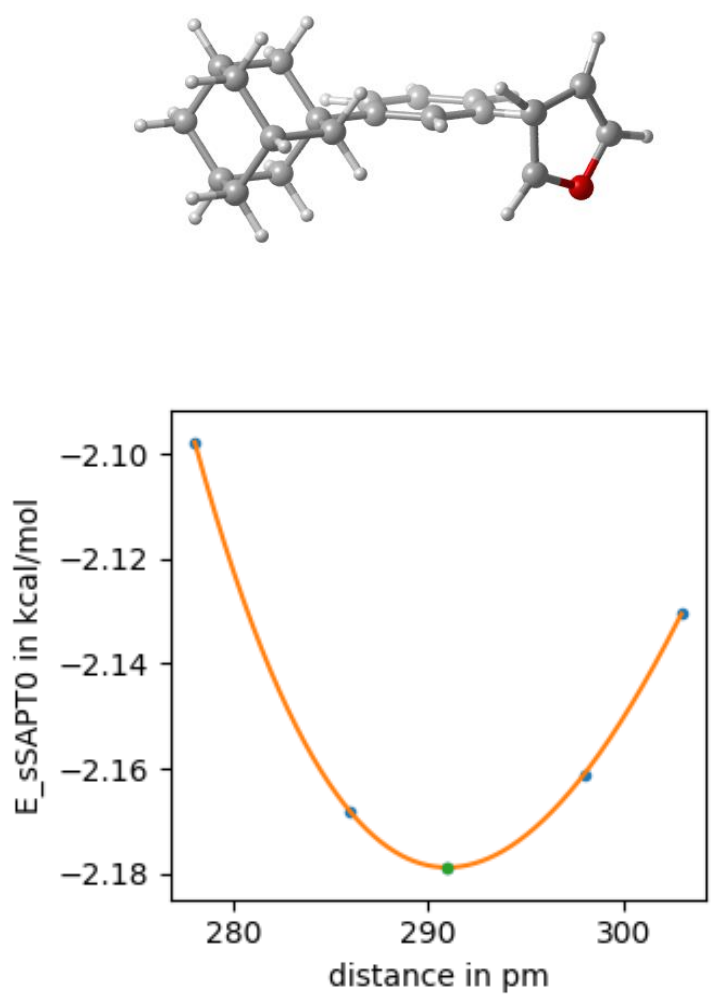

45

2Fur_pi0_4AdPh_side1-290

H $\quad-5.40420277-0.06983710 \quad-0.11780099$

$\begin{array}{llll}\text { C } & -4.32070148 & -0.03830540 & -0.06658052\end{array}$

$\begin{array}{llll}\text { C } & -3.58197593 & -1.21913575 & -0.03377221\end{array}$

$\begin{array}{llll}\text { C } & -3.65284733 & 1.17924947 & -0.03291661\end{array}$

$\begin{array}{llll}\text { C } & -2.19503530 & -1.17343346 & 0.03180068\end{array}$

$\begin{array}{llll}\mathrm{H} & -4.08771491 & -2.17939061 & -0.05934245\end{array}$

$\begin{array}{llll}\text { C } & -2.26048337 & 1.21947754 & 0.03289969\end{array}$ 


$\begin{array}{lrrc}\mathrm{H} & -4.21352817 & 2.10855295 & -0.05777047 \\ \mathrm{C} & -1.50342452 & 0.04567840 & 0.06660682 \\ \mathrm{H} & -1.63954243 & -2.10699869 & 0.05637912 \\ \mathrm{H} & -1.77002283 & 2.18589805 & 0.05774247 \\ \mathrm{C} & 0.02106058 & 0.04612521 & 0.13855632 \\ \mathrm{C} & 0.60838832 & -0.68607024 & -1.09216530 \\ \mathrm{C} & 0.61166538 & 1.46964759 & 0.16854764 \\ \mathrm{C} & 0.48999384 & -0.68980885 & 1.41693228 \\ \mathrm{H} & 0.28439937 & -0.16935582 & -2.00476641 \\ \mathrm{H} & 0.20839669 & -1.70592468 & -1.14344119 \\ \mathrm{C} & 2.14168957 & -0.73537139 & -1.01950252 \\ \mathrm{H} & 0.21738455 & 2.01486646 & 1.03621303 \\ \mathrm{H} & 0.30077073 & 2.01750031 & -0.73076661 \\ \mathrm{C} & 2.14535914 & 1.42890709 & 0.24085554 \\ \mathrm{H} & 0.08710793 & -1.70975947 & 1.42728438 \\ \mathrm{H} & 0.08143052 & -0.17576850 & 2.29650177 \\ \mathrm{C} & 2.02333016 & -0.73910266 & 1.48883087 \\ \mathrm{H} & 2.52691869 & -1.26359510 & -1.89943961 \\ \mathrm{C} & 2.69778395 & 0.69505850 & -0.98948338 \\ \mathrm{C} & 2.56869565 & -1.47706806 & 0.25650464 \\ \mathrm{H} & 2.52885130 & 2.45561235 & 0.26047817 \\ \mathrm{C} & 2.57957877 & 0.69133201 & 1.51556163 \\ \mathrm{H} & 2.32406096 & -1.26999479 & 2.39955931 \\ \mathrm{H} & 2.41222810 & 1.22832453 & -1.90491933 \\ \mathrm{H} & 3.79416549 & 0.67332862 & -0.95306743 \\ \mathrm{H} & 2.18848495 & -2.50635645 & 0.23703239 \\ \mathrm{H} & 3.66313167 & -1.53542563 & 0.30805949 \\ \mathrm{H} & 3.67452131 & 0.66955558 & 1.58248162 \\ \mathrm{H} & 2.20901636 & 1.22191460 & 2.40162128 \\ \mathrm{C} & -2.21360175 & 5.08794987 & 0.72737254 \\ \mathrm{C} & -4.24511682 & 5.01228148 & -0.05420009 \\ \mathrm{C} & -2.09252710 & 5.09225857 & -0.62352827 \\ \mathrm{O} & -3.51789115 & 5.03936619 & 1.08816015 \\ \mathrm{C} & -3.43035967 & 5.04262152 & -1.13805660 \\ \mathrm{H} & -1.50145053 & 5.11452058 & 1.53656612 \\ \mathrm{H} & -5.31547030 & 4.97245450 & 0.07004407 \\ \mathrm{H} & -1.17240803 & 5.12637824 & -1.18630463 \\ \mathrm{H} & -3.73699907 & 5.10992883 & -2.17049095\end{array}$

2Fur_pi0_4AdPh_side2

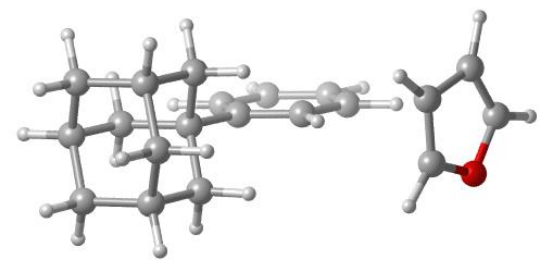

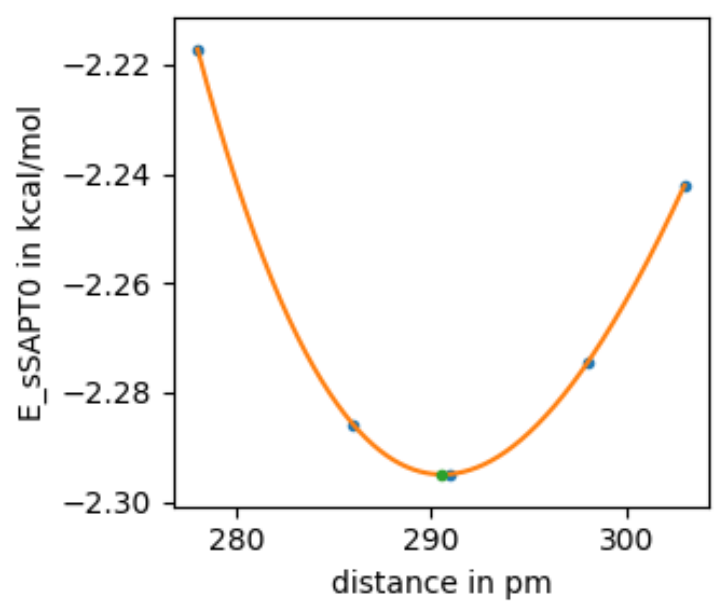

45

2Fur_pi0_4AdPh_side2-290

H $\quad-5.40628059 \quad-0.06992282-0.05920527$

$\begin{array}{llll}\text { C } & -4.32187596 & -0.03835385 & -0.03345982\end{array}$

$\begin{array}{llll}\text { C } & -3.58257763 & -1.21916048 & -0.01655335\end{array}$

$\begin{array}{llll}\text { C } & -3.65342237 & 1.17922564 & -0.01696008\end{array}$

$\begin{array}{llll}\text { C } & -2.19448052 & -1.17341048 & 0.01640362\end{array}$

H $-4.08877216 \quad-2.17943406 \quad-0.02907917$

C $-2.25989768 \quad 1.21950161 \quad 0.01611965$

H $\quad-4.21453701 \quad 2.10851115-0.02978505$

$\begin{array}{llll}\text { C } & -1.50225004 & 0.04572685 & 0.03348597\end{array}$

$\begin{array}{llll}\mathrm{H} & -1.63855884 & -2.10695794 & 0.02908017\end{array}$

$\begin{array}{llll}\mathrm{H} & -1.76899448 & 2.18594029 & 0.02826919\end{array}$

$\begin{array}{llll}\text { C } & 0.02350354 & 0.04622598 & 0.06964574\end{array}$

$\begin{array}{llll}\text { C } & 0.58179684 & -0.68748324 & -1.17362379\end{array}$

$\begin{array}{llll}\text { C } & 0.61464176 & 1.46977016 & 0.08403522\end{array}$

$\begin{array}{llll}\text { C } & 0.52230649 & -0.68815962 & 1.33756364\end{array}$

H $\quad 0.23648173-0.17187446 \quad-2.07900132$

H $\quad 0.18071772 \quad-1.70739016 \quad-1.21425667$

C $\quad 2.11638113 \quad-0.73673137 \quad-1.13689711$

$\begin{array}{llll}\mathrm{H} & 0.24082476 & 2.01605577 & 0.96004726\end{array}$

$\mathrm{H} \quad 0.28272893 \quad 2.01653311 \quad-0.80840433$

$\begin{array}{llll}\text { C } & 2.14961010 & 1.42908224 & 0.12038746\end{array}$

$\begin{array}{llll}\mathrm{H} & 0.11977989 & -1.70808751 & 1.35860978\end{array}$

$\begin{array}{llll}\mathrm{H} & 0.13449041 & -0.17303766 & 2.22584964\end{array}$

$\begin{array}{llll}\text { C } & 2.05690792 & -0.73740134 & 1.37352551\end{array}$

H $\quad 2.48086089-1.26603658-2.02498582$

C $\quad 2.67301905 \quad 0.69372116 \quad-1.12167852$

$\begin{array}{llll}\text { C } & 2.57321291 & -1.47688150 & 0.12964341\end{array}$

$\begin{array}{llll}\mathrm{H} & 2.53345156 & 2.45580176 & 0.12975458\end{array}$

C $\quad 2.61362288 \quad 0.69305194 \quad 1.38545300$

H $\quad 2.37892742 \quad-1.26718956 \quad 2.27759389$

$\mathrm{H} \quad 2.36605987 \quad 1.22587718 \quad-2.03081206$

H $\quad 3.769953320 .67201023-1.11097076$

$\mathrm{H} \quad 2.19265554 \quad-2.50618404 \quad 0.12035278$

H $\quad 3.66855759 \quad-1.53520158 \quad 0.15557622$

$\begin{array}{llll}\mathrm{H} & 3.70983425 & 0.67133170 & 1.42669021\end{array}$

$\begin{array}{llll}\mathrm{H} & 2.26394943 & 1.22472316 & 2.27931557\end{array}$

$\begin{array}{llll}\text { C } & -3.94462707 & -5.07397115 & -0.02722475\end{array}$

$\begin{array}{llll}\text { C } & -1.89447482 & -5.02764053 & 0.70650830\end{array}$ 


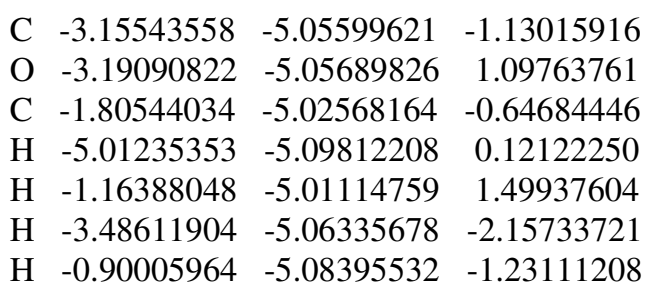

2MePh_pi0_4AdPh_side_1

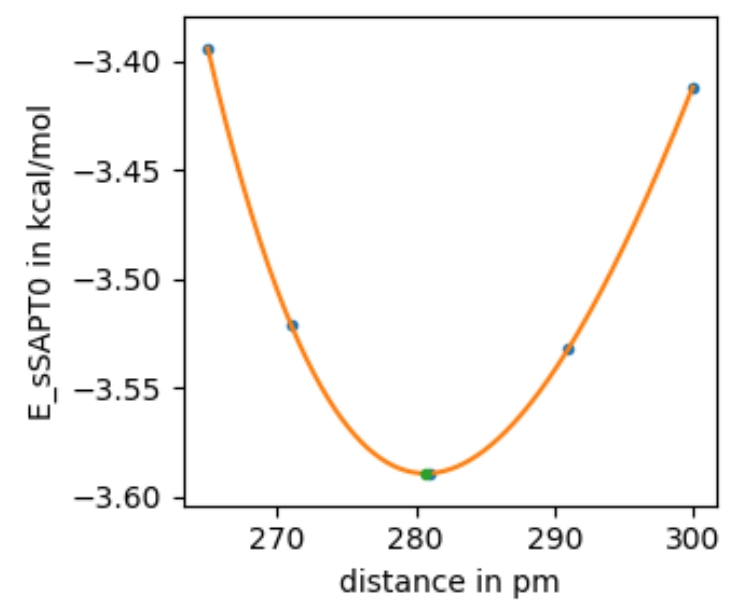

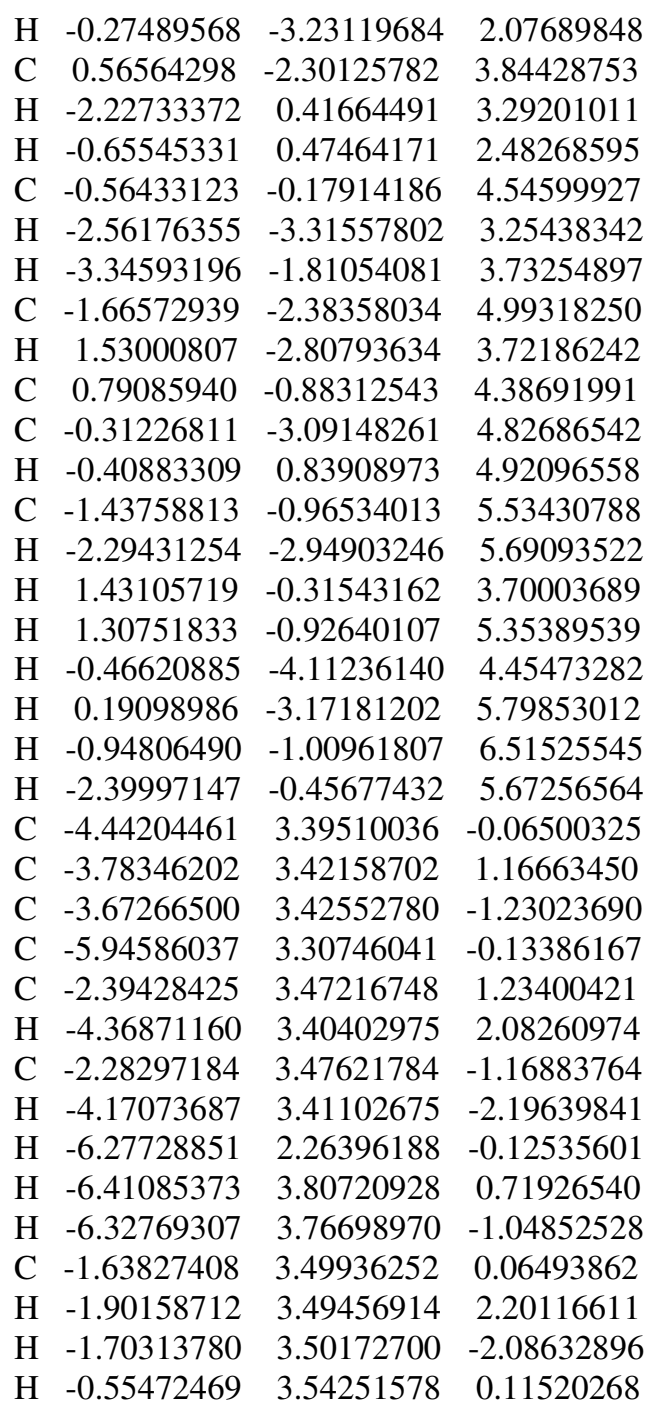

2MePh_pi0_4AdPh_side_2

51
2MePh_pi0_4AdPh_side_1-280
$\mathrm{H} \quad-3.99785357 \quad-1.52490840 \quad-2.21939728$
C $\quad-3.50144292 \quad-1.51519664-1.25447503$
$\begin{array}{llll}\text { C } & -3.13877813 & -2.71000375 & -0.63636309\end{array}$
$\begin{array}{llll}\text { C } & -3.21958060 & -0.31198763 & -0.62009028\end{array}$
$\begin{array}{llll}\text { C } & -2.50345172 & -2.69223613 & 0.59896581\end{array}$
H $-3.35144150 \quad-3.65936609-1.11802748$
$\begin{array}{llll}\text { C } & -2.58165338 & -0.29979912 & 0.61988060\end{array}$

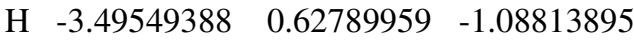
$\begin{array}{llll}\text { C } & -2.21073794 & -1.48794975 & 1.25454468\end{array}$
$\begin{array}{llll}\mathrm{H} & -2.22981268 & -3.63627680 & 1.06224688\end{array}$
$\begin{array}{llll}\mathrm{H} & -2.37610847 & 0.65603214 & 1.08814888\end{array}$
$\begin{array}{llll}\text { C } & -1.51136916 & -1.51816941 & 2.61071644\end{array}$
$\begin{array}{llll}\text { C } & -0.13843911 & -2.22113771 & 2.48172657\end{array}$
$\begin{array}{llll}\text { C } & -1.26875739 & -0.10758061 & 3.18299980\end{array}$
$\begin{array}{llll}\text { C } & -2.37049066 & -2.30349153 & 3.63097221\end{array}$
H $\quad 0.48040919 \quad-1.66936067 \quad 1.76244060$

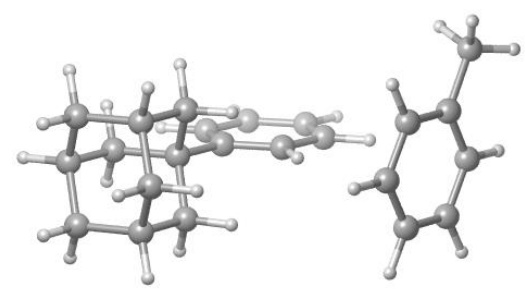




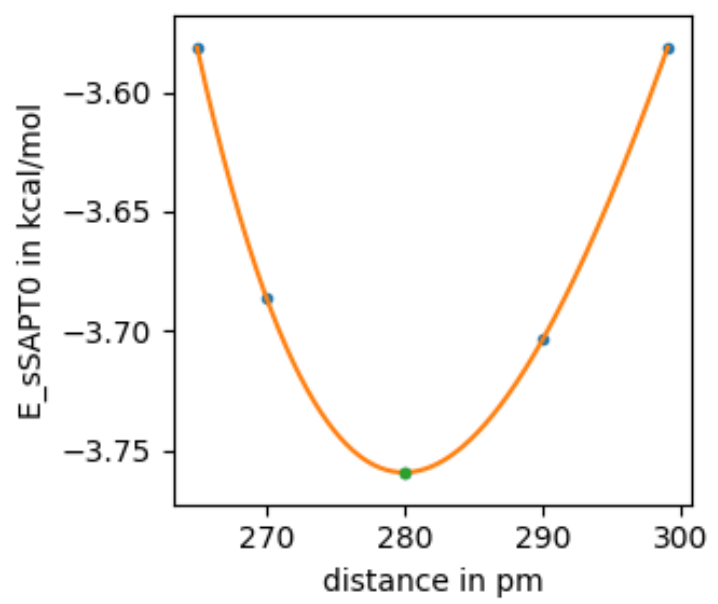

51

2MePh_pi0_4AdPh_side_2-279

$\mathrm{H} \quad-1.82908652 \quad 1.01096450 \quad 2.23783076$

$\begin{array}{llll}\text { C } & -2.30971701 & 1.00725611 & 1.26491072\end{array}$

$\begin{array}{llll}\text { C } & -2.59829089 & -0.19671971 & 0.62591192\end{array}$

$\begin{array}{llll}\text { C } & -2.64496312 & 2.20221128 & 0.64097604\end{array}$

$\begin{array}{llll}\text { C } & -3.21369913 & -0.19613061 & -0.61958603\end{array}$

H $-2.34318744 \quad-1.13983293 \quad 1.09917174$

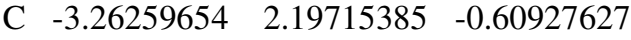

$\mathrm{H} \quad-2.42702580 \quad 3.14905735 \quad 1.12530190$

$\begin{array}{llll}\text { C } & -3.55949615 & 0.99960861 & -1.26487884\end{array}$

H $\quad-3.42922142 \quad-1.14706714-1.09915910$

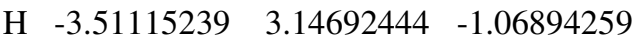

$\begin{array}{llll}\text { C } & -4.23434922 & 0.95050401 & -2.63286782\end{array}$

$\begin{array}{llll}\text { C } & -5.56982296 & 0.17409265 & -2.53573364\end{array}$

$\begin{array}{llll}\text { C } & -4.54244075 & 2.35377870 & -3.19146358\end{array}$

$\begin{array}{llll}\text { C } & -3.31834542 & 0.22553735 & -3.64830014\end{array}$

$\mathrm{H} \quad-6.228653220 .68262391 \quad-1.82009262$

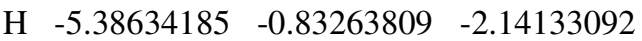

$\begin{array}{llll}\text { C } & -6.24662374 & 0.07499784 & -3.91081332\end{array}$

$\mathrm{H} \quad-3.61148954 \quad 2.92948546 \quad-3.27781045$

H $\quad-5.19705019 \quad 2.89325731 \quad-2.49431689$

$\begin{array}{llll}\text { C } & -5.22003348 & 2.26321758 & -4.56688045\end{array}$

H $\quad-3.07957041 \quad-0.77993463 \quad-3.28123599$

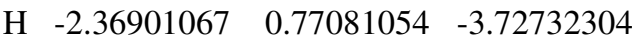

$\begin{array}{llll}\text { C } & -3.99583166 & 0.12643309 & -5.02304022\end{array}$

$\begin{array}{llll}\mathrm{H} & -7.18450442 & -0.48367439 & -3.81093238\end{array}$

$\begin{array}{llll}\text { C } & -6.53822547 & 1.48633026 & -4.43937839\end{array}$

$\begin{array}{llll}\text { C } & -5.31220491 & -0.65437218 & -4.88832198\end{array}$

H $\quad-5.42344221 \quad 3.27669993 \quad-4.93164531$

$\begin{array}{llll}\text { C } & -4.29038369 & 1.53769800 & -5.55014636\end{array}$

$\mathrm{H} \quad-3.32690043 \quad-0.39552482 \quad-5.71716031$

H $\quad-7.21869773 \quad 2.01005629 \quad-3.75629251$

$\mathrm{H} \quad-7.03622012 \quad 1.42866796-5.41535338$

H $\quad-5.11016469-1.67054242 \quad-4.52644945$

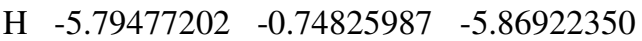

$\begin{array}{llll}\mathrm{H} & -4.76100640 & 1.48066002 & -6.53964754\end{array}$

$\mathrm{H} \quad-3.35432731 \quad 2.09836143 \quad-5.66586616$

$\begin{array}{llll}\text { C } & -3.60488645 & -3.95982780 & 1.16654496\end{array}$

$\begin{array}{llll}\text { C } & -4.20298588 & -3.97528475 & -0.09575542\end{array}$ $\begin{array}{llll}\text { C } & -2.21050041 & -3.93022358 & 1.24037899\end{array}$

$\begin{array}{llll}\text { C } & -4.44557008 & -3.94708341 & 2.41833276\end{array}$

$\begin{array}{llll}\text { C } & -3.43076639 & -3.95679094 & -1.25344579\end{array}$

H $\quad-5.28721339-4.00392020-0.16969266$

$\begin{array}{llll}\text { C } & -1.43317130 & -3.91170505 & 0.08582153\end{array}$

$\mathrm{H} \quad-1.73003221 \quad-3.92342784 \quad 2.21549850$

H $\quad-4.69556764 \quad-2.92199740 \quad 2.71074685$

$\begin{array}{llll}\mathrm{H} & -5.38486602 & -4.48506213 & 2.26968686\end{array}$

$\begin{array}{llll}\mathrm{H} & -3.91639387 & -4.40869087 & 3.25527005\end{array}$

C $-2.04137574-3.92456540 \quad-1.16649502$

$\mathrm{H} \quad-3.91487481-3.97176568 \quad-2.22507804$

$\begin{array}{llll}\mathrm{H} & -0.35058322 & -3.89125631 & 0.16477364\end{array}$

H $\quad-1.43704887 \quad-3.91379451 \quad-2.06823831$

2MePh_pi0_Ph_side
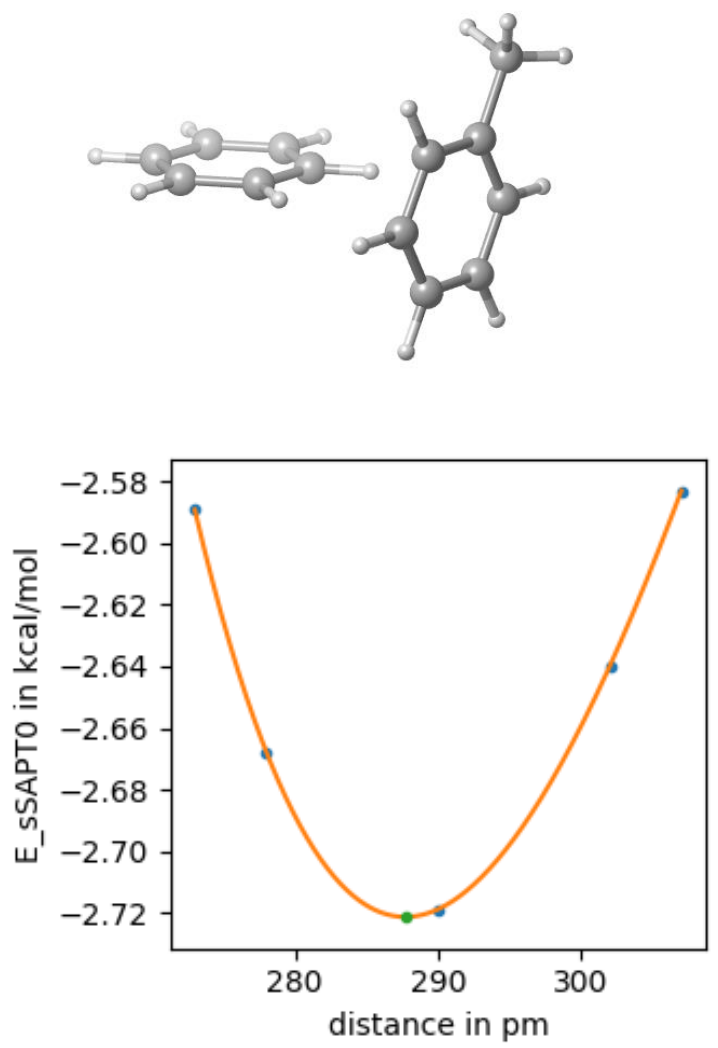

27

2MePh_pi0_Ph_side-287

$\begin{array}{llll}\mathrm{H} & 2.45716611 & -0.07215285 & 0.31745281\end{array}$

$\begin{array}{llll}\text { C } & 1.38113275 & -0.04058081 & 0.17844178\end{array}$

$\begin{array}{llll}\text { C } & 0.69026887 & 1.15587907 & 0.35662439\end{array}$

$\begin{array}{llll}\text { C } & 0.69031604 & -1.19646273 & -0.17823171\end{array}$

$\begin{array}{llll}\text { C } & -0.69050972 & 1.19641639 & 0.17845575\end{array}$

$\begin{array}{llll}\mathrm{H} & 1.22837374 & 2.05685976 & 0.63476174\end{array}$

C $\quad-0.69044928-1.15595928 \quad-0.35671763$

H $\quad 1.22847405 \quad-2.12906436-0.31723038$

$\begin{array}{llll}\text { C } & -1.38077634 & 0.04048312 & -0.17833987\end{array}$

$\begin{array}{llll}\mathrm{H} & -1.22897904 & 2.12887648 & 0.31748857\end{array}$ 


$\begin{array}{lrrr}\mathrm{H} & -1.22879046 & -2.05686224 & -0.63478353 \\ \mathrm{H} & -2.45710151 & 0.07208070 & -0.31726990 \\ \mathrm{C} & -4.12617441 & 2.88801338 & 1.35686775 \\ \mathrm{H} & -3.98423245 & 3.13097267 & 2.40533864 \\ \mathrm{C} & -4.81589157 & 1.73498503 & 0.99105199 \\ \mathrm{C} & -3.62471575 & 3.72959239 & 0.36759593 \\ \mathrm{C} & -5.00048299 & 1.42836838 & -0.35387335 \\ \mathrm{H} & -5.21499529 & 1.07442954 & 1.75462065 \\ \mathrm{C} & -3.81222693 & 3.41791005 & -0.97601119 \\ \mathrm{H} & -3.08950136 & 4.63341676 & 0.64199637 \\ \mathrm{C} & -4.49982574 & 2.26310173 & -1.35583475 \\ \mathrm{H} & -5.54274909 & 0.52862832 & -0.63360770 \\ \mathrm{H} & -3.42135281 & 4.08047437 & -1.74411266 \\ \mathrm{C} & -4.67361456 & 1.91068424 & -2.81167933 \\ \mathrm{H} & -3.85070616 & 1.27896020 & -3.16175448 \\ \mathrm{H} & -5.60345347 & 1.36144739 & -2.97760714 \\ \mathrm{H} & -4.69004455 & 2.80664989 & -3.43661817\end{array}$

2Nap_pi0_4AdPh_side1
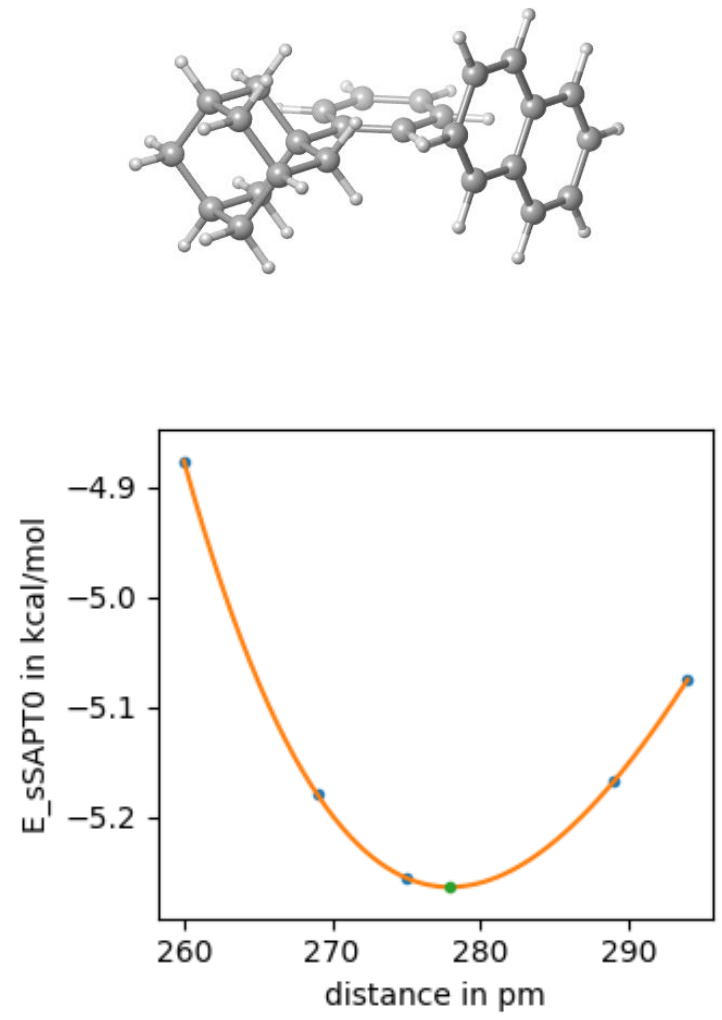

54

2Nap_pi0_4AdPh_side1-277

H $\quad-2.67006689-1.53707944-2.48906283$

C $-2.74993318-1.54879555-1.40689975$

$\begin{array}{llll}\text { C } & -2.75641495 & -2.75732873 & -0.71367708\end{array}$

$\begin{array}{llll}\text { C } & -2.84695055 & -0.35967453 & -0.69543569\end{array}$

$\begin{array}{llll}\text { C } & -2.85885972 & -2.76699386 & 0.67174865\end{array}$

H $\quad-2.68141086-3.69599466 \quad-1.25385836$

$\begin{array}{lrrr}\mathrm{C} & -2.94956730 & -0.37502186 & 0.69519789 \\ \mathrm{H} & -2.84333950 & 0.59060632 & -1.22035373 \\ \mathrm{C} & -2.95768040 & -1.57726602 & 1.40697163 \\ \mathrm{H} & -2.86190142 & -3.72132242 & 1.19133331 \\ \mathrm{H} & -3.02369215 & 0.57041037 & 1.22036519 \\ \mathrm{C} & -3.06825952 & -1.63760218 & 2.92794617 \\ \mathrm{C} & -1.82580777 & -2.34541334 & 3.52044660 \\ \mathrm{C} & -3.16750376 & -0.23972470 & 3.56998870 \\ \mathrm{C} & -4.32912365 & -2.43787116 & 3.33480443 \\ \mathrm{H} & -0.92565131 & -1.78317009 & 3.24043894 \\ \mathrm{H} & -1.72553539 & -3.34666990 & 3.08437738 \\ \mathrm{C} & -1.93542567 & -2.45578948 & 5.04836159 \\ \mathrm{H} & -4.04504792 & 0.28750769 & 3.17311786 \\ \mathrm{H} & -2.28214019 & 0.35261984 & 3.30386185 \\ \mathrm{C} & -3.27710365 & -0.34155410 & 5.09862320 \\ \mathrm{H} & -4.29033745 & -3.44140352 & 2.89418903 \\ \mathrm{H} & -5.21700774 & -1.94167102 & 2.92218932 \\ \mathrm{C} & -4.43797934 & -2.54821290 & 4.86277520 \\ \mathrm{H} & -1.04604122 & -2.96524977 & 5.43715014 \\ \mathrm{C} & -2.03408537 & -1.04969988 & 5.65618566 \\ \mathrm{C} & -3.19263967 & -3.26012673 & 5.41318614 \\ \mathrm{H} & -3.34559857 & 0.66834870 & 5.51931282 \\ \mathrm{C} & -4.53335820 & -1.14200222 & 5.47084163 \\ \mathrm{H} & -5.33513409 & -3.12365764 & 5.11907283 \\ \mathrm{H} & -1.13270371 & -0.47228810 & 5.41526137 \\ \mathrm{H} & -2.09745805 & -1.11454287 & 6.74963437 \\ \mathrm{H} & -3.12427971 & -4.27273967 & 4.99567457 \\ \mathrm{H} & -3.26968892 & -3.36203388 & 6.50292558 \\ \mathrm{H} & -4.62716461 & -1.20797037 & 6.56203374 \\ \mathrm{H} & -5.42932097 & -0.63097295 & 5.09662794 \\ \mathrm{C} & -1.76788007 & 3.41343996 & -0.59745352 \\ \mathrm{H} & -0.79233114 & 3.45597238 & -1.07045665 \\ \mathrm{C} & -1.87041179 & 3.40963705 & 0.76915168 \\ \mathrm{C} & -2.93294264 & 3.36195307 & -1.40167591 \\ \mathrm{C} & -3.14066340 & 3.35426411 & 1.40161279 \\ \mathrm{H} & -0.98002360 & 3.44899397 & 1.39078017 \\ \mathrm{C} & -4.17235956 & 3.30790977 & -0.81932339 \\ \mathrm{H} & -2.83823598 & 3.36554998 & -2.48269440 \\ \mathrm{C} & -3.27931997 & 3.34889592 & 2.81494878 \\ \mathrm{C} & -4.31099011 & 3.30254574 & 0.59404157 \\ \mathrm{H} & -5.06953415 & 3.26826833 & -1.43103373 \\ \mathrm{C} & -4.51878743 & 3.29484436 & 3.39723589 \\ \mathrm{H} & -2.38213070 & 3.38854101 & 3.42666933 \\ \mathrm{C} & -5.58130739 & 3.24716694 & 1.22654715 \\ \mathrm{C} & -5.68408982 & 3.24334705 & 2.59318203 \\ \mathrm{H} & -4.61339756 & 3.29124268 & 4.47823811 \\ \mathrm{H} & -6.47160272 & 3.20781705 & 0.60481886 \\ \mathrm{H} & -6.65974724 & 3.20080692 & 3.06627419\end{array}$

2Nap_pi0_4AdPh_side2 

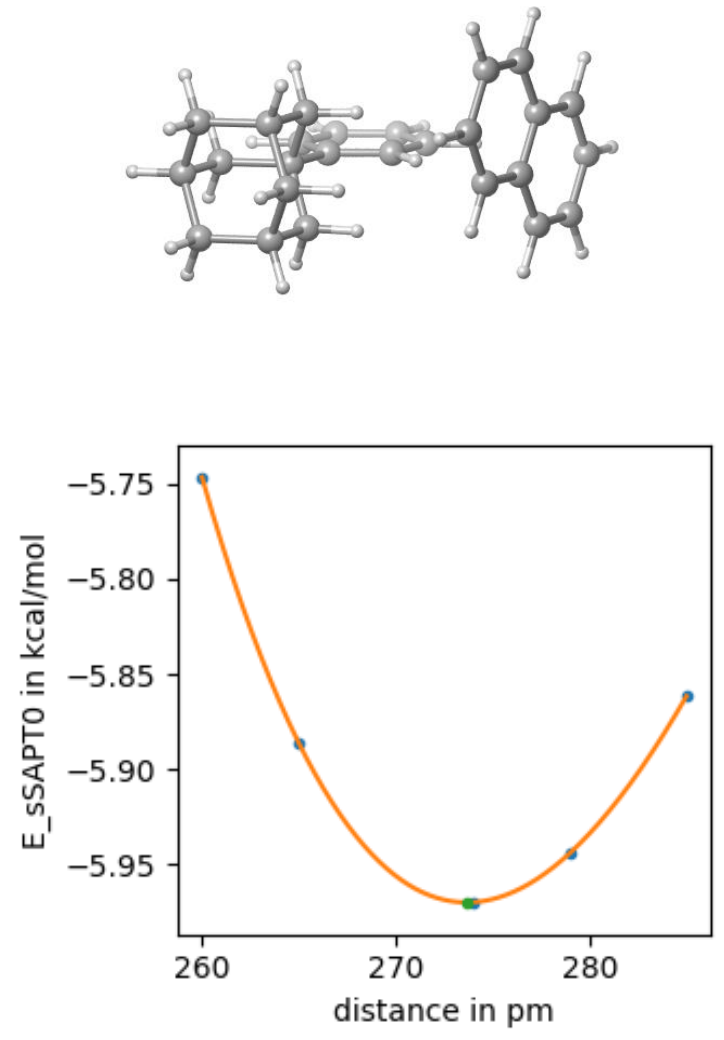

54

2Nap_pi0_4AdPh_side2-273

$\begin{array}{rrrc}\mathrm{H} & -2.65000301 & 0.69490910 & -2.48044268 \\ \mathrm{C} & -2.77081227 & 0.69930081 & -1.40202779 \\ \mathrm{C} & -2.82307295 & -0.49935526 & -0.69375907 \\ \mathrm{C} & -2.87526460 & 1.89945148 & -0.71041348 \\ \mathrm{C} & -2.97784760 & -0.48839662 & 0.68679065 \\ \mathrm{H} & -2.74300698 & -1.44640882 & -1.21834857 \\ \mathrm{C} & -3.03049986 & 1.90480332 & 0.67540788 \\ \mathrm{H} & -2.83641672 & 2.84226599 & -1.24723499 \\ \mathrm{C} & -3.08498808 & 0.71271511 & 1.40206836 \\ \mathrm{H} & -3.01599121 & -1.43534010 & 1.21835775 \\ \mathrm{H} & -3.10901105 & 2.85840186 & 1.18494013 \\ \mathrm{C} & -3.25382649 & 0.67500164 & 2.91841365 \\ \mathrm{C} & -2.04627273 & -0.04408125 & 3.56685290 \\ \mathrm{C} & -3.35449345 & 2.08294579 & 3.53783994 \\ \mathrm{C} & -4.54197602 & -0.09966496 & 3.28760856 \\ \mathrm{H} & -1.12717871 & 0.50002940 & 3.31384458 \\ \mathrm{H} & -1.94590391 & -1.05268099 & 3.14807102 \\ \mathrm{C} & -2.21522555 & -0.13174368 & 5.09082581 \\ \mathrm{H} & -4.20776075 & 2.61843000 & 3.10108395 \\ \mathrm{H} & -2.45021437 & 2.65757446 & 3.29774528 \\ \mathrm{C} & -3.52331654 & 2.00383782 & 5.06242036 \\ \mathrm{H} & -4.50290699 & -1.10963462 & 2.86198152 \\ \mathrm{H} & -5.40548483 & 0.40474048 & 2.83513575 \\ \mathrm{C} & -4.71016882 & -0.18730424 & 4.81166568 \\ \mathrm{H} & -1.34953433 & -0.64975692 & 5.51972210\end{array}$

$\begin{array}{lrrr}\mathrm{C} & -2.31388428 & 1.28393209 & 5.67597568 \\ \mathrm{C} & -3.49820858 & -0.91111186 & 5.41821271 \\ \mathrm{H} & -3.59121411 & 3.02037074 & 5.46692713 \\ \mathrm{C} & -4.80555666 & 1.22844427 & 5.39718059 \\ \mathrm{H} & -5.62558378 & -0.74498607 & 5.04127008 \\ \mathrm{H} & -1.39479391 & 1.84372493 & 5.46186678 \\ \mathrm{H} & -2.41950272 & 1.23510374 & 6.76698958 \\ \mathrm{H} & -3.43060978 & -1.93030860 & 5.01691187 \\ \mathrm{H} & -3.61795433 & -0.99683191 & 6.50548763 \\ \mathrm{H} & -4.94151630 & 1.17893907 & 6.48479995 \\ \mathrm{H} & -5.67834493 & 1.74832966 & 4.98257728 \\ \mathrm{C} & -2.66158774 & -4.18142835 & -1.39489981 \\ \mathrm{H} & -2.52850946 & -4.18468093 & -2.47170818 \\ \mathrm{C} & -3.92183899 & -4.20983603 & -0.85722260 \\ \mathrm{C} & -1.52483348 & -4.14765616 & -0.55024335 \\ \mathrm{C} & -4.11079905 & -4.20596227 & 0.55021886 \\ \mathrm{H} & -4.79722589 & -4.23580411 & -1.50048421 \\ \mathrm{C} & -1.67631469 & -4.14312077 & 0.81182945 \\ \mathrm{H} & -0.53232937 & -4.12536669 & -0.98844652 \\ \mathrm{C} & -5.40362675 & -4.23488220 & 1.13718840 \\ \mathrm{C} & -2.96917818 & -4.17204470 & 1.39878356 \\ \mathrm{H} & -0.80812572 & -4.11728273 & 1.46471805 \\ \mathrm{C} & -5.55502983 & -4.23033868 & 2.49928821 \\ \mathrm{H} & -6.27183010 & -4.26072367 & 0.48428908 \\ \mathrm{C} & -3.15815918 & -4.16816800 & 2.80630169 \\ \mathrm{C} & -4.41835774 & -4.19656683 & 3.34422623 \\ \mathrm{H} & -6.54754977 & -4.25262014 & 2.93739500 \\ \mathrm{H} & -2.28270773 & -4.14220203 & 3.44944304 \\ \mathrm{H} & -4.55148532 & -4.19331169 & 4.42116620\end{array}$

2nap_pi_ph_side0

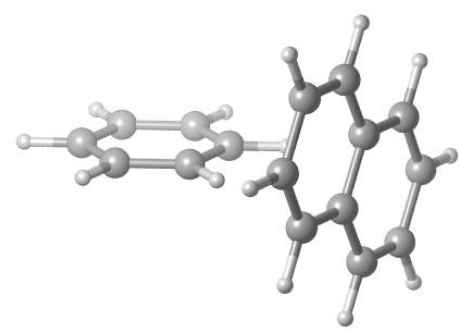




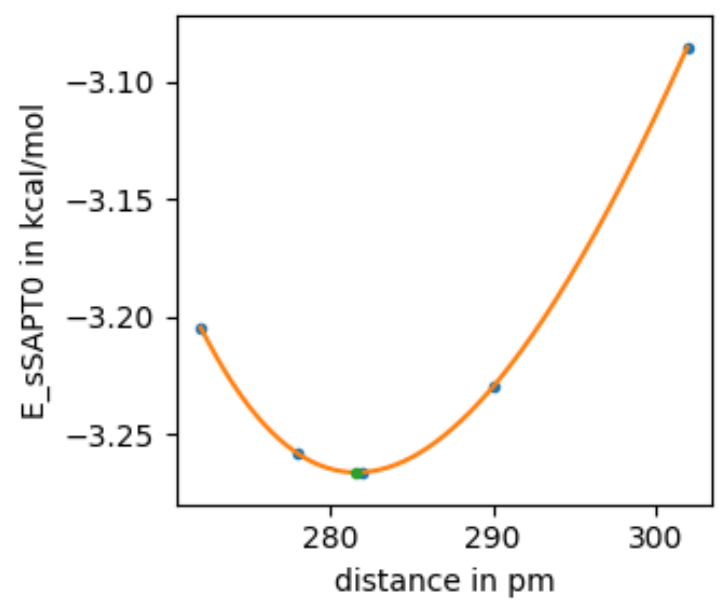

30

2nap_pi_ph_side0-281
$\begin{array}{llll}\mathrm{H} & 3.36933800 & 1.24391000 & 0.00000300\end{array}$
$\begin{array}{llll}\text { C } & 2.42572600 & 0.70834200 & 0.00000200\end{array}$
$\begin{array}{llll}\text { C } & 1.24227900 & 1.39942100 & -0.00000100\end{array}$
$\begin{array}{llll}\text { C } & 2.42607300 & -0.70827200 & 0.00000200\end{array}$
$\begin{array}{lrrr}\text { C } & -0.00003000 & 0.71146700 & -0.00000200\end{array}$
$\begin{array}{llll}\mathrm{H} & 1.23624300 & 2.48603300 & -0.00000600\end{array}$
$\begin{array}{llll}\text { C } & 1.24253700 & -1.39925100 & -0.00000200\end{array}$
$\begin{array}{llll}\mathrm{H} & 3.36993500 & -1.24372300 & 0.00001200\end{array}$
$\begin{array}{llll}\text { C } & -1.24247400 & 1.39929400 & 0.00000000\end{array}$
$\begin{array}{llll}\text { C } & 0.00008400 & -0.71138600 & -0.00000300\end{array}$
$\mathrm{H} \quad 1.23655200 \quad-2.48582700 \quad-0.00000500$
$\begin{array}{llll}\text { C } & -2.42598500 & 0.70823600 & 0.00000200\end{array}$
$\mathrm{H} \quad-1.23648900 \quad 2.48588800 \quad 0.00000000$
C $\quad-1.24229900 \quad-1.39936900-0.00000100$
$\begin{array}{llll}\text { C } & -2.42591300 & -0.70848000 & 0.00000200\end{array}$
$\begin{array}{llll}\mathrm{H} & -3.36977900 & 1.24375700 & 0.00000100\end{array}$
$\mathrm{H}-1.23612800 \quad-2.48596100 \quad 0.00000100$
$\begin{array}{llll}\mathrm{H} & -3.36966000 & -1.24408700 & 0.00000400\end{array}$
$\begin{array}{lrrr}\text { C } & 1.80215963 & 0.33074560 & 3.76009718\end{array}$
$\begin{array}{llll}\text { C } & 0.59883575 & -0.37061089 & 3.75212425\end{array}$
$\begin{array}{llll}\mathrm{H} & 2.27410264 & 0.60928146 & 2.82284123\end{array}$
H $\quad 0.13300272 \quad-0.63855836 \quad 2.80873696$
$\begin{array}{llll}\text { C } & 2.39977419 & 0.67442773 & 4.97033529\end{array}$
$\begin{array}{llll}\text { C } & -0.00700721 & -0.72806274 & 4.95421700\end{array}$
$\begin{array}{llll}\text { C } & 1.79412874 & 0.31723048 & 6.17290001\end{array}$
$\begin{array}{llll}\mathrm{H} & 3.33772431 & 1.22113926 & 4.97662171\end{array}$
$\begin{array}{llll}\text { C } & 0.59054470 & -0.38406864 & 6.16442788\end{array}$
$\mathrm{H} \quad-0.94502794 \quad-1.27471848 \quad 4.94768791$
$\begin{array}{llll}\mathrm{H} & 2.25981804 & 0.58522362 & 7.11602342\end{array}$
$\begin{array}{llll}\mathrm{H} & 0.11832225 & -0.66264512 & 7.10148795\end{array}$

2OMePh_pi0_1_4AdPh_side_1
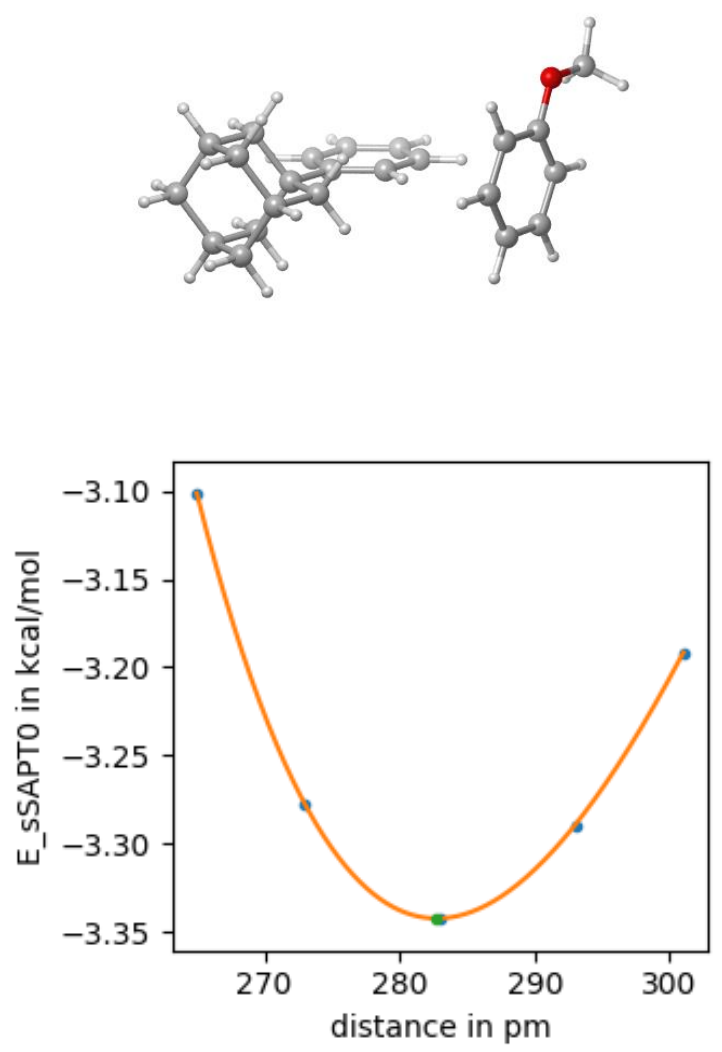

52

2OMePh_pi0_1_4AdPh_side_1-282

$\begin{array}{llll}\mathrm{H} & -2.19727671 & 0.04944670 & 2.39104047\end{array}$

$\begin{array}{llll}\text { C } & -2.50802409 & 0.02911837 & 1.35151382\end{array}$

$\begin{array}{llll}\text { C } & -2.66243637 & -1.18493285 & 0.68563949\end{array}$

$\begin{array}{llll}\text { C } & -2.75682968 & 1.21257745 & 0.66805010\end{array}$

$\begin{array}{llll}\text { C } & -3.06046651 & -1.20562377 & -0.64520111\end{array}$

H $\quad-2.47220823 \quad-2.11930074 \quad 1.20456632$

C $-3.15614899 \quad 1.18616265-0.66779189$

$\begin{array}{llll}\mathrm{H} & -2.64122399 & 2.16703587 & 1.17226248\end{array}$

C $-3.31611020-0.02174568 \quad-1.35149401$

$\mathrm{H} \quad-3.17440949-2.16408911 \quad-1.14428191$

$\begin{array}{llll}\mathrm{H} & -3.34232701 & 2.12741513 & -1.17229048\end{array}$

$\begin{array}{llll}\text { C } & -3.75127089 & -0.09418923 & -2.81252750\end{array}$

$\begin{array}{llll}\text { C } & -5.06905887 & -0.89721399 & -2.93221808\end{array}$

$\begin{array}{llll}\text { C } & -3.98823571 & 1.29855115 & -3.42917277\end{array}$

$\begin{array}{llll}\text { C } & -2.66445787 & -0.80717359 & -3.65290430\end{array}$

$\mathrm{H} \quad-5.84803381 \quad-0.39739968 \quad-2.34214426$

$\mathrm{H} \quad-4.93500572 \quad-1.89720348 \quad-2.50236183$

C $\quad-5.50399615-1.01972512 \quad-4.40005450$

$\begin{array}{llll}\mathrm{H} & -3.06715145 & 1.89267284 & -3.36446269\end{array}$

$\begin{array}{llll}\mathrm{H} & -4.76054468 & 1.82926432 & -2.85694328\end{array}$

C $\quad-4.42406030 \quad 1.18455307 \quad-4.89756174$

$\mathrm{H} \quad-2.47134766-1.80495655 \quad-3.24076219$

$\begin{array}{llll}\mathrm{H} & -1.72589777 & -0.24304947 & -3.57758582\end{array}$

$\begin{array}{llll}\text { C } & -3.10012749 & -0.92970591 & -5.12052052\end{array}$ 


$\begin{array}{lrrr}\mathrm{H} & -6.43450299 & -1.59686514 & -4.45275619 \\ \mathrm{C} & -5.72989041 & 0.38161814 & -4.98441795 \\ \mathrm{C} & -4.40529847 & -1.73647052 & -5.19991814 \\ \mathrm{H} & -4.58284848 & 2.19108786 & -5.30160261 \\ \mathrm{C} & -3.32917287 & 0.47151927 & -5.70393856 \\ \mathrm{H} & -2.31454351 & -1.44258699 & -5.68755011 \\ \mathrm{H} & -6.52503619 & 0.89595315 & -4.43021542 \\ \mathrm{H} & -6.05621032 & 0.30697031 & -6.02930733 \\ \mathrm{H} & -4.24732346 & -2.74574074 & -4.79893002 \\ \mathrm{H} & -4.71489392 & -1.84705200 & -6.24671139 \\ \mathrm{H} & -3.62625920 & 0.39796498 & -6.75758990 \\ \mathrm{H} & -2.39784977 & 1.05050285 & -5.66717730 \\ \mathrm{C} & -4.05177169 & 4.93679967 & 1.01658597 \\ \mathrm{C} & -2.69566691 & 4.98729458 & 1.34051836 \\ \mathrm{C} & -4.44997157 & 4.92248353 & -0.32475462 \\ \mathrm{C} & -1.74925282 & 5.02304757 & 0.31524769 \\ \mathrm{C} & -3.49712915 & 4.95847033 & -1.33031763 \\ \mathrm{C} & -2.13730518 & 5.00911079 & -1.01757918 \\ \mathrm{O} & -5.05865570 & 4.89882825 & 1.93034503 \\ \mathrm{H} & -2.36701753 & 4.99914377 & 2.37252716 \\ \mathrm{H} & -5.51123965 & 4.88295026 & -0.54424008 \\ \mathrm{H} & -0.69579637 & 5.06227178 & 0.57398825 \\ \mathrm{H} & -3.81696714 & 4.94695630 & -2.36746371 \\ \mathrm{H} & -1.39340435 & 5.03720910 & -1.80601438 \\ \mathrm{C} & -4.69976450 & 4.91156428 & 3.29385047 \\ \mathrm{H} & -5.63268973 & 4.87646446 & 3.85531230 \\ \mathrm{H} & -4.08659006 & 4.04067811 & 3.55441403 \\ \mathrm{H} & -4.15322166 & 5.82549357 & 3.55527719\end{array}$

2OMePh_pi0_1_4AdPh_side_2

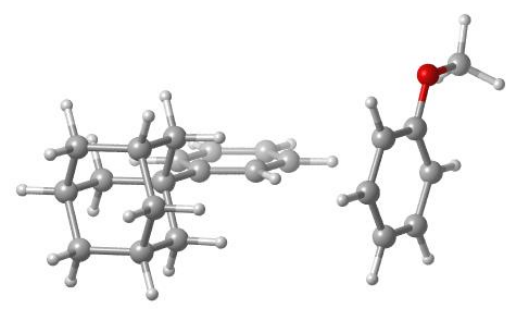

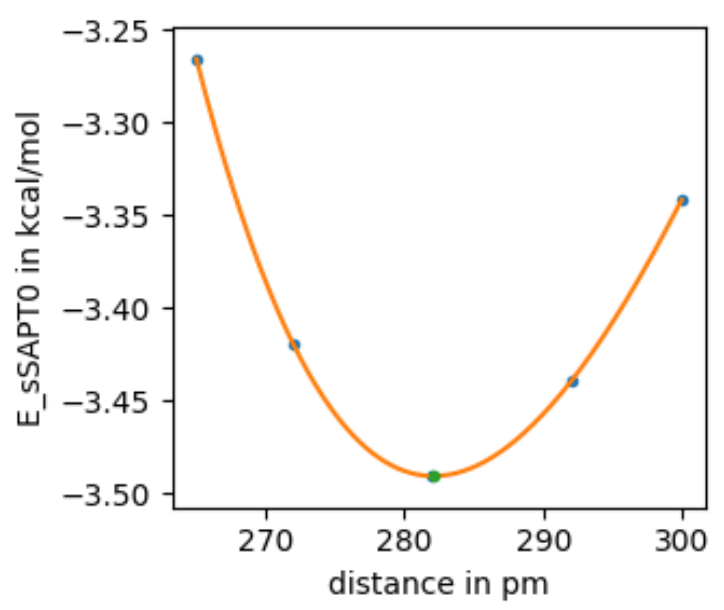

52

2OMePh_pi0_1_4AdPh_side_2-282

$\begin{array}{llll}\mathrm{H} & -5.42051699 & 0.93000875 & 0.21187192\end{array}$

$\begin{array}{llll}\text { C } & -4.33972205 & 0.96149702 & 0.11976528\end{array}$

$\begin{array}{llll}\text { C } & -3.60279399 & -0.21936259 & 0.05926939\end{array}$

$\begin{array}{llll}\text { C } & -3.67358540 & 2.17902472 & 0.06068457\end{array}$

$\begin{array}{llll}\text { C } & -2.21931806 & -0.17371590 & -0.05864382\end{array}$

$\begin{array}{llll}\mathrm{H} & -4.10723227 & -1.17959691 & 0.10407147\end{array}$

C $-2.28469986 \quad 2.21919698 \quad-0.05768193$

$\begin{array}{llll}\mathrm{H} & -4.23290473 & 3.10835036 & 0.10652574\end{array}$

$\begin{array}{llll}\text { C } & -1.52948182 & 1.04536791 & -0.11972818\end{array}$

H $\quad-1.66517658-1.10730316 \quad-0.10405883$

$\begin{array}{lllll}\mathrm{H} & -1.79550434 & 3.18559749 & -0.10121454\end{array}$

$\begin{array}{llll}\text { C } & -0.00880815 & 1.04575348 & -0.24927813\end{array}$

C $\quad 0.41143462 \quad 0.30893226 \quad-1.54397124$

$\begin{array}{llll}\text { C } & 0.58021288 & 2.46925032 & -0.30257406\end{array}$

$\begin{array}{llll}\text { C } & 0.62467819 & 0.31440462 & 0.95884699\end{array}$

$\begin{array}{llll}\mathrm{H} & -0.03011628 & 0.82237119 & -2.40780958\end{array}$

H $\quad 0.00845084 \quad-0.71101787 \quad-1.53836611$

$\begin{array}{llll}\text { C } & 1.94095505 & 0.25957094 & -1.67379383\end{array}$

$\begin{array}{llll}\mathrm{H} & 0.30354746 & 3.01772598 & 0.60747809\end{array}$

$\begin{array}{llll}\mathrm{H} & 0.15338438 & 3.01387320 & -1.15508069\end{array}$

C $\quad 2.11007333 \quad 2.42844822 \quad-0.43283146$

H $\quad 0.22694565 \quad-0.70541546 \quad 1.02592411$

$\begin{array}{llll}\mathrm{H} & 0.33543177 & 0.83174859 & 1.88269529\end{array}$

$\begin{array}{llll}\text { C } & 2.15413270 & 0.26504783 & 0.82826223\end{array}$

$\begin{array}{llll}\mathrm{H} & 2.20702613 & -0.27195267 & -2.59488008\end{array}$

$\begin{array}{llll}\text { C } & 2.49576834 & 1.68998270 & -1.72254027\end{array}$

$\begin{array}{llll}\text { C } & 2.53256921 & -0.47754025 & -0.46247134\end{array}$

H $\quad 2.49252892 \quad 3.45513675 \quad-0.46766472$

$\begin{array}{llll}\text { C } & 2.70866558 & 1.69545229 & 0.77623577\end{array}$

$\begin{array}{llll}\mathrm{H} & 2.57238533 & -0.26257062 & 1.69336064\end{array}$

H $\quad 2.09193744 \quad 2.21995457 \quad-2.59431329$

$\mathrm{H} \quad 3.58739500 \quad 1.66815280 \quad-1.83082461$

H $\quad 2.15338697 \quad-1.50681204 \quad-0.42791194$

H $\quad 3.62427198 \quad-0.53594174 \quad-0.55535857$

$\begin{array}{llll}\mathrm{H} & 3.80288508 & 1.67368783 & 0.69837909\end{array}$

$\begin{array}{llll}\mathrm{H} & 2.45793942 & 2.22935406 & 1.70145000\end{array}$

$\begin{array}{llll}\text { C } & -3.41726861 & -3.97651577 & 1.26211417\end{array}$

$\begin{array}{llll}\text { C } & -4.21661474 & -3.99459303 & 0.11877649\end{array}$ 


$\begin{array}{lrrr}\mathrm{C} & -2.02365438 & -3.94560444 & 1.14026774 \\ \mathrm{C} & -3.61081440 & -3.98150061 & -1.13859872 \\ \mathrm{C} & -1.43854194 & -3.93297295 & -0.11585509 \\ \mathrm{C} & -2.22884459 & -3.95085367 & -1.26676475 \\ \mathrm{O} & -3.89715989 & -3.98680129 & 2.53483229 \\ \mathrm{H} & -5.29702579 & -4.01856621 & 0.19178498 \\ \mathrm{H} & -1.42973321 & -3.93215363 & 2.04751821 \\ \mathrm{H} & -4.23837072 & -3.99569077 & -2.02416487 \\ \mathrm{H} & -0.35659008 & -3.90897254 & -0.19895168 \\ \mathrm{H} & -1.76889899 & -3.94092411 & -2.24868097 \\ \mathrm{C} & -5.29694825 & -4.01773347 & 2.70140936 \\ \mathrm{H} & -5.47639443 & -4.02137016 & 3.77593564 \\ \mathrm{H} & -5.77240786 & -3.13514942 & 2.25722183 \\ \mathrm{H} & -5.73287957 & -4.92077087 & 2.25777990\end{array}$

2OMePh_pi0_2_4AdPh_side_1

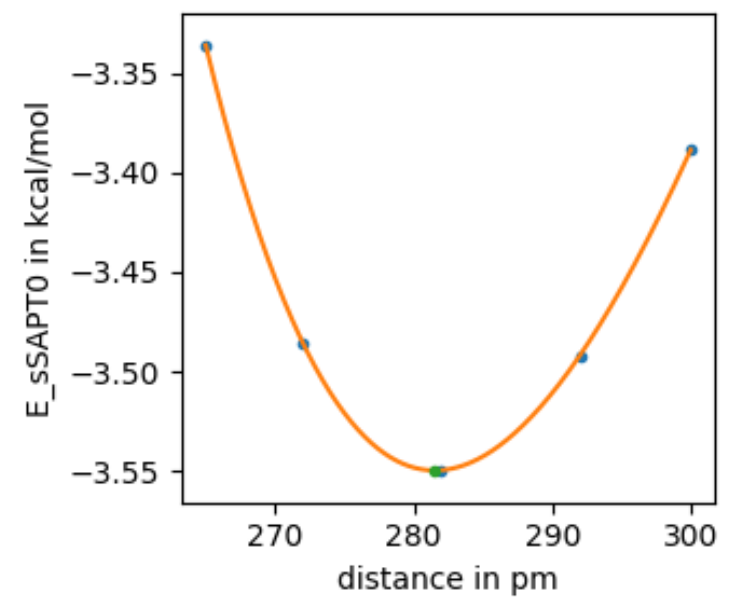

52

2OMePh_pi0_2_4AdPh_side_1-281

$\mathrm{H} \quad-5.33821776 \quad-0.06738924-0.58202427$

$\begin{array}{llll}\text { C } & -4.28340412 & -0.03692177 & -0.32897735\end{array}$

$\begin{array}{llll}\text { C } & -3.56305192 & -1.21843372 & -0.16688368\end{array}$

$\begin{array}{llll}\text { C } & -3.63441162 & 1.17993338 & -0.16262064\end{array}$

$\begin{array}{llll}\text { C } & -2.21283803 & -1.17409389 & 0.15707502\end{array}$

$\mathrm{H} \quad-4.05446802 \quad-2.17815724 \quad-0.29320127$

C $\quad-2.27891186 \quad 1.21879390 \quad 0.16255277$

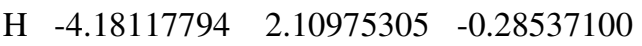

$\begin{array}{llll}\text { C } & -1.54072206 & 0.04429476 & 0.32900485\end{array}$

$\begin{array}{crrr}\mathrm{H} & -1.67111397 & -2.10816990 & 0.27855555 \\ \mathrm{H} & -1.80237306 & 2.18469795 & 0.28534300 \\ \mathrm{C} & -0.05654957 & 0.04324609 & 0.68462078 \\ \mathrm{C} & 0.75093701 & -0.68076098 & -0.41969754 \\ \mathrm{C} & 0.51710584 & 1.46613970 & 0.83436293 \\ \mathrm{C} & 0.16567001 & -0.70186146 & 2.02296887 \\ \mathrm{H} & 0.60288676 & -0.15750465 & -1.37315826 \\ \mathrm{H} & 0.36824847 & -1.69997301 & -0.55191675 \\ \mathrm{C} & 2.24366968 & -0.73156712 & -0.06207889 \\ \mathrm{H} & -0.03275595 & 2.00557209 & 1.61681352 \\ \mathrm{H} & 0.37941530 & 2.02043267 & -0.10338161 \\ \mathrm{C} & 2.01028482 & 1.42389621 & 1.19176583 \\ \mathrm{H} & -0.23138028 & -1.72159613 & 1.95074852 \\ \mathrm{H} & -0.40042702 & -0.19368025 & 2.81422734 \\ \mathrm{C} & 1.65857996 & -0.75265497 & 2.37984343 \\ \mathrm{H} & 2.78692174 & -1.25391356 & -0.85815831 \\ \mathrm{C} & 2.78341308 & 0.69825618 & 0.08128845 \\ \mathrm{C} & 2.42514746 & -1.48239330 & 1.26606578 \\ \mathrm{H} & 2.38267627 & 2.45018968 & 1.28985413 \\ \mathrm{C} & 2.19908949 & 0.67719585 & 2.52000927 \\ \mathrm{H} & 1.78414126 & -1.29006047 & 3.32701893 \\ \mathrm{H} & 2.67363762 & 1.23805832 & -0.86765874 \\ \mathrm{H} & 3.85368206 & 0.67555785 & 0.32182930 \\ \mathrm{H} & 2.05595323 & -2.51127302 & 1.16872056 \\ \mathrm{H} & 3.49069976 & -1.54182238 & 1.52086019 \\ \mathrm{H} & 3.26224351 & 0.65423990 & 2.79024660 \\ \mathrm{H} & 1.66909979 & 1.20184898 & 3.32486088 \\ \mathrm{C} & -4.05131576 & 4.92453894 & 1.01658613 \\ \mathrm{C} & -2.69521098 & 4.97503385 & 1.34051852 \\ \mathrm{C} & -4.44951564 & 4.91022280 & -0.32475446 \\ \mathrm{C} & -1.74879689 & 5.01078684 & 0.31524785 \\ \mathrm{C} & -3.49667322 & 4.94620960 & -1.33031747 \\ \mathrm{C} & -2.13684925 & 4.99685006 & -1.01757902 \\ \mathrm{O} & -5.05819977 & 4.88656752 & 1.93034519 \\ \mathrm{H} & -2.36656160 & 4.98688304 & 2.37252732 \\ \mathrm{H} & -5.51078372 & 4.87068953 & -0.54423992 \\ \mathrm{H} & -0.69534044 & 5.05001105 & 0.57398841 \\ \mathrm{H} & -3.81651121 & 4.93469557 & -2.36746355 \\ \mathrm{H} & -1.39294842 & 5.02494837 & -1.80601422 \\ \mathrm{C} & -4.69930857 & 4.89930355 & 3.29385063 \\ \mathrm{H} & -5.63223380 & 4.86420373 & 3.85531246 \\ \mathrm{H} & -4.08613413 & 4.02841738 & 3.55441419 \\ \mathrm{H} & -4.15276573 & 5.81323284 & 3.55527735\end{array}$


2OMePh_pi0_2_4AdPh_side_2
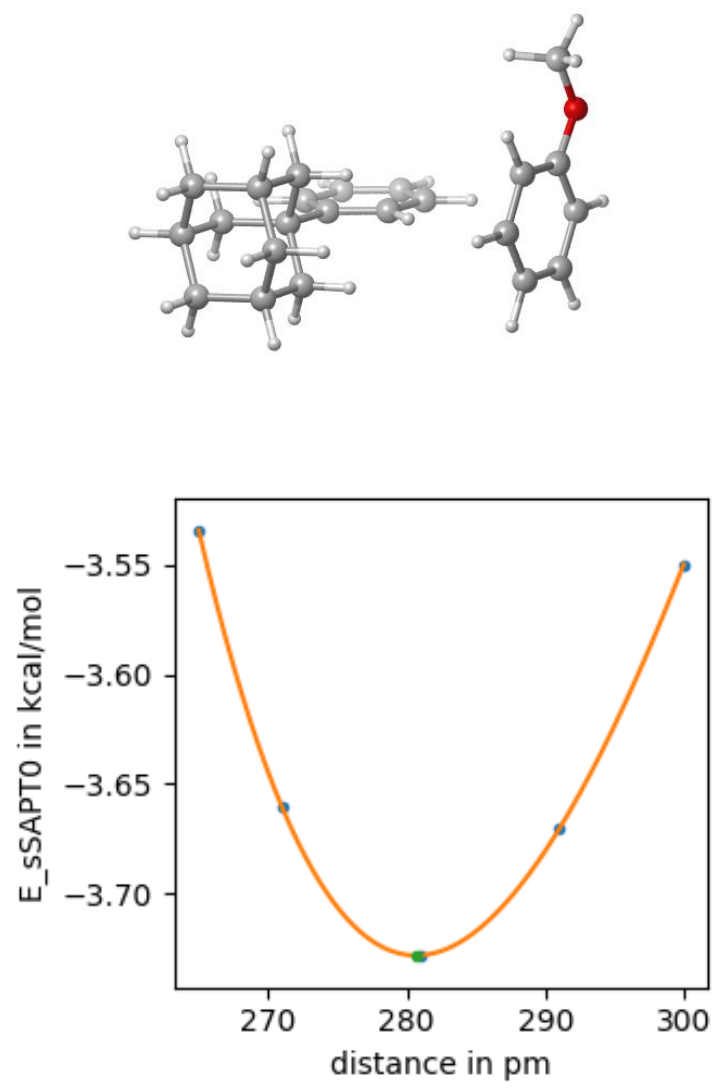

52

2OMePh_pi0_2_4AdPh_side_2-280
H $\quad-1.50208275 \quad 1.01833782 \quad 2.04395812$
$\begin{array}{llll}\text { C } & -2.12488269 & 1.01142377 & 1.15532701\end{array}$
C $-2.50683030 \quad-0.19465745 \quad 0.57168651$
C $\quad-2.55130300 \quad 2.20432313 \quad 0.58545060$
C $\quad-3.30423884-0.19817211 \quad-0.56590748$
$\begin{array}{llll}\mathrm{H} & -2.18257027 & -1.13621131 & 1.00394443\end{array}$
C $\quad-3.35163141 \quad 2.19514628 \quad-0.55648534$
H $-2.26259343 \quad 3.15276498 \quad 1.02781982$
C $\quad-3.74433129 \quad 0.99544093 \quad-1.15529464$
$\mathrm{H} \quad-3.58983858 \quad-1.15068875 \quad-1.00393179$
H $-3.66735726 \quad 3.14340231 \quad-0.97632458$
C $\quad-4.61908330 \quad 0.94182898 \quad-2.40476110$
$\begin{array}{llll}\text { C } & -5.92411467 & 0.16610276 & -2.10329517\end{array}$
C $\quad-5.00879645 \quad 2.34326326 \quad-2.91489354$
C $-3.86771303 \quad 0.21315144 \quad-3.54503044$
$\mathrm{H} \quad-6.46676255 \quad 0.67725278 \quad-1.29765111$
H $\quad-5.68260463 \quad-0.83931957 \quad-1.73788520$
C $\quad-6.80185547 \quad 0.06247713 \quad-3.35930313$
$\mathrm{H} \quad-4.10190538 \quad 2.91842842 \quad-3.14358073$
H $\quad-5.55008909 \quad 2.88529604 \quad-2.12827612$
C $\quad-5.88737347 \quad 2.24817032 \quad-4.17114374$
$\mathrm{H} \quad-3.57570225 \quad-0.79112014 \quad-3.21504288$
H $\quad-2.94153143 \quad 0.75790349 \quad-3.76916611$
C $\quad-4.74607977 \quad 0.10951771 \quad-4.80059863$

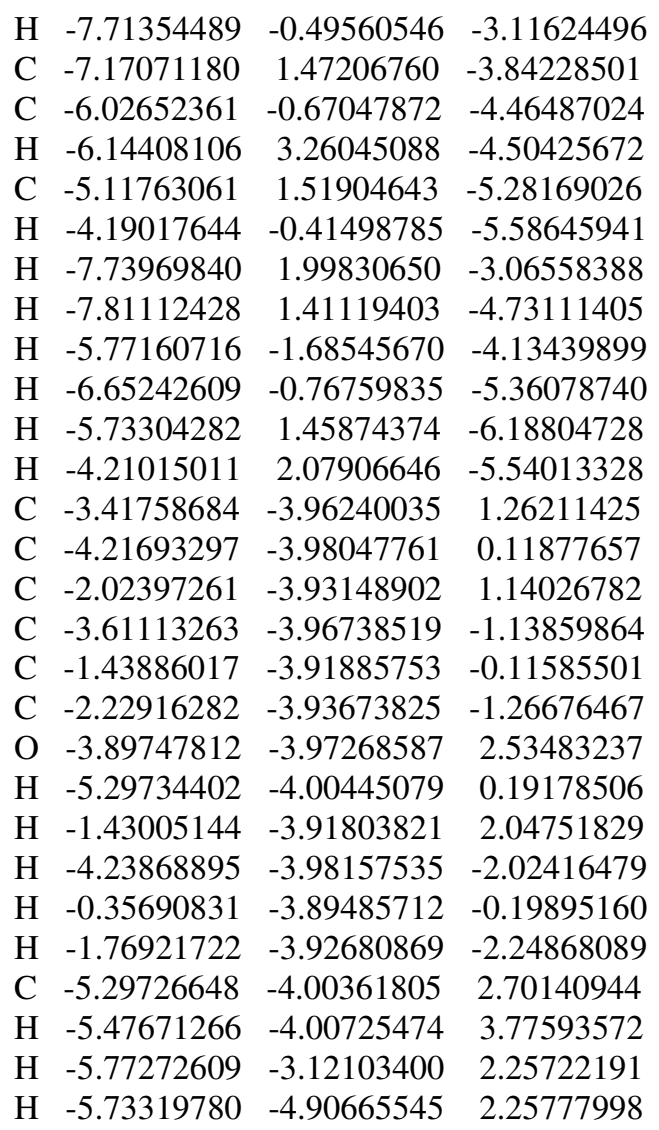

2OMePh_pi0_Ph_side_1

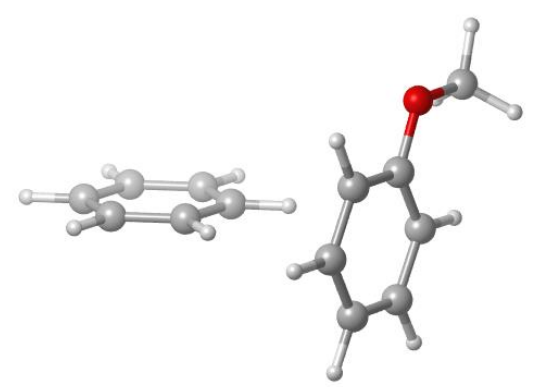




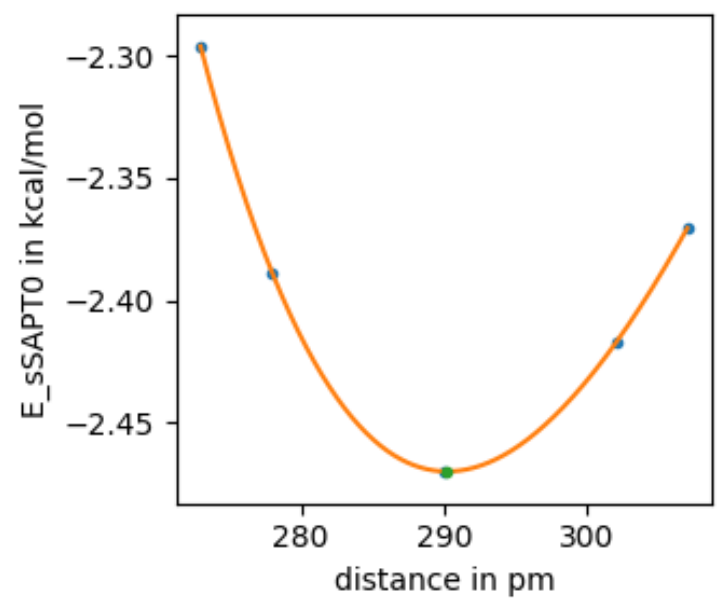

2OMePh_pi0_Ph_side_2

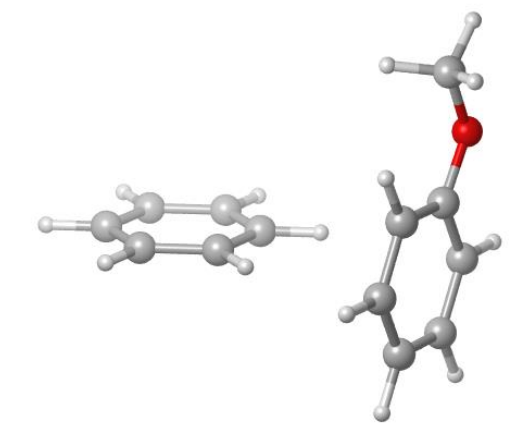

\begin{tabular}{lrrr}
\multicolumn{4}{l}{ 28 } \\
2OMePh_pi0_Ph_side_1-290 & \\
H & 2.44193634 & -0.09768052 & 0.41356424 \\
C & 1.37256924 & -0.05493471 & 0.23246914 \\
C & 0.67317385 & 1.12722486 & 0.46463938 \\
C & 0.69884353 & -1.18216915 & -0.23222742 \\
C & -0.69905938 & 1.18208569 & 0.23246893 \\
H & 1.19795207 & 2.00586780 & 0.82696224 \\
C & -0.67336319 & -1.12732004 & -0.46471592 \\
H & 1.24365651 & -2.10361591 & -0.41332181 \\
C & -1.37223032 & 0.05480772 & -0.23233006 \\
H & -1.24418460 & 2.10338933 & 0.41357455 \\
H & -1.19838060 & -2.00589006 & -0.82697198 \\
H & -2.44189239 & 0.09757380 & -0.41335479 \\
C & -4.46601628 & 2.36779361 & -1.37084628 \\
C & -5.01065306 & 1.45570739 & -0.46642846 \\
C & -3.79059396 & 3.49960809 & -0.90099166 \\
C & -4.87313566 & 1.68671212 & 0.90320056 \\
C & -3.66317682 & 3.71369165 & 0.46220085 \\
C & -4.20431128 & 2.80748159 & 1.37622921 \\
O & -4.53909043 & 2.24475209 & -2.72352282 \\
H & -5.53706260 & 0.57361114 & -0.80992836 \\
H & -3.37843875 & 4.18980700 & -1.62885169 \\
H & -5.29916207 & 0.97325766 & 1.60158435 \\
H & -3.13731400 & 4.59488442 & 0.81585108 \\
H & -4.10343984 & 2.97696057 & 2.44248895 \\
C & -5.21168815 & 1.11744826 & -3.23821745 \\
H & -5.16140113 & 1.20113916 & -4.32324644 \\
H & -4.72836822 & 0.18487298 & -2.92353635 \\
H & -6.26206924 & 1.10016949 & -2.92401882
\end{tabular}

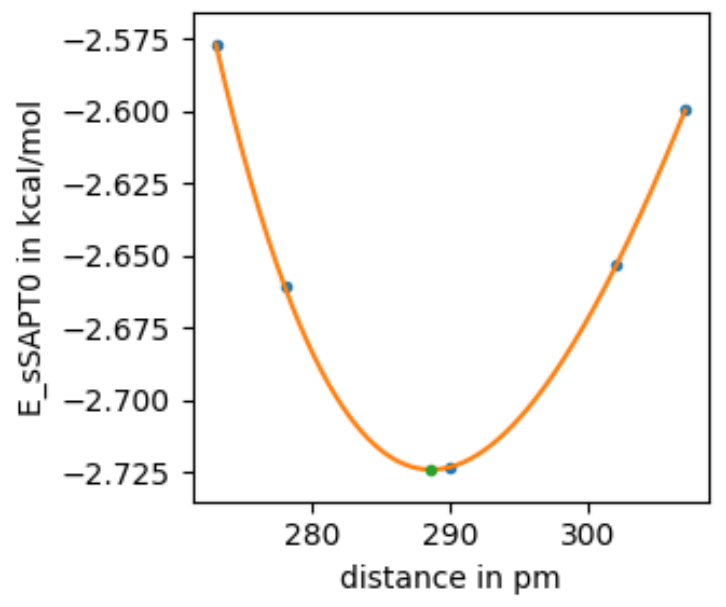

28

2OMePh_pi0_Ph_side_2-288

$\begin{array}{llll}\mathrm{H} & 2.46789903 & -0.05403252 & -0.22440329\end{array}$

$\begin{array}{llll}\text { C } & 1.38717839 & -0.03037416 & -0.12615956\end{array}$

$\begin{array}{llll}\text { C } & 0.70222189 & 1.17606077 & -0.25228463\end{array}$

$\begin{array}{llll}\text { C } & 0.68443536 & -1.20639307 & 0.12610710\end{array}$

$\begin{array}{llll}\text { C } & -0.68452609 & 1.20651917 & -0.12603414\end{array}$

$\begin{array}{llll}\mathrm{H} & 1.24965844 & 2.09279703 & -0.44873131\end{array}$

$\begin{array}{llll}\text { C } & -0.70236769 & -1.17608300 & 0.25208017\end{array}$

$\begin{array}{llll}\mathrm{H} & 1.21797321 & -2.14679603 & 0.22439317\end{array}$

$\begin{array}{llll}\text { C } & -1.38675742 & 0.03038463 & 0.12602049\end{array}$

$\begin{array}{llll}\mathrm{H} & -1.21835396 & 2.14681617 & -0.22416932\end{array}$

$\begin{array}{llll}\mathrm{H} & -1.25002224 & -2.09271088 & 0.44861470\end{array}$

$\begin{array}{llll}\mathrm{H} & -2.46774588 & 0.05410870 & 0.22439199\end{array}$

$\begin{array}{llll}\text { C } & -4.08377359 & 2.97613714 & -1.31618013\end{array}$

$\begin{array}{llll}\text { C } & -4.78719745 & 1.79835021 & -1.06218229\end{array}$

$\begin{array}{llll}\text { C } & -3.61851485 & 3.75588241 & -0.25152324\end{array}$

$\begin{array}{llll}\text { C } & -5.01833978 & 1.41207023 & 0.25899858\end{array}$

$\begin{array}{llll}\text { C } & -3.85676571 & 3.35769318 & 1.05424955\end{array}$

$\begin{array}{llll}\text { C } & -4.55961237 & 2.18088392 & 1.31998916\end{array}$

$\begin{array}{llll}\text { O } & -3.80110857 & 3.44872902 & -2.55995272\end{array}$

$\mathrm{H} \quad-5.15561547 \quad 1.18092910 \quad-1.87227631$

$\begin{array}{llll}\mathrm{H} & -3.07508530 & 4.66575543 & -0.48139728\end{array}$

$\begin{array}{llll}\mathrm{H} & -5.56625270 & 0.49466362 & 0.44981850\end{array}$

$\begin{array}{llll}\mathrm{H} & -3.49141842 & 3.96998503 & 1.87261435\end{array}$

H $\quad-4.74491970 \quad 1.87118619 \quad 2.34251374$ 


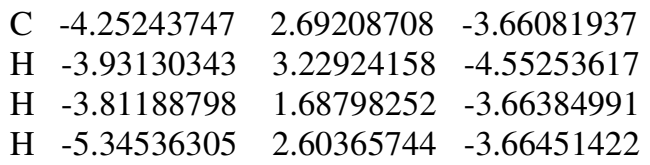

3MePh_pi0_4AdPh_side_1
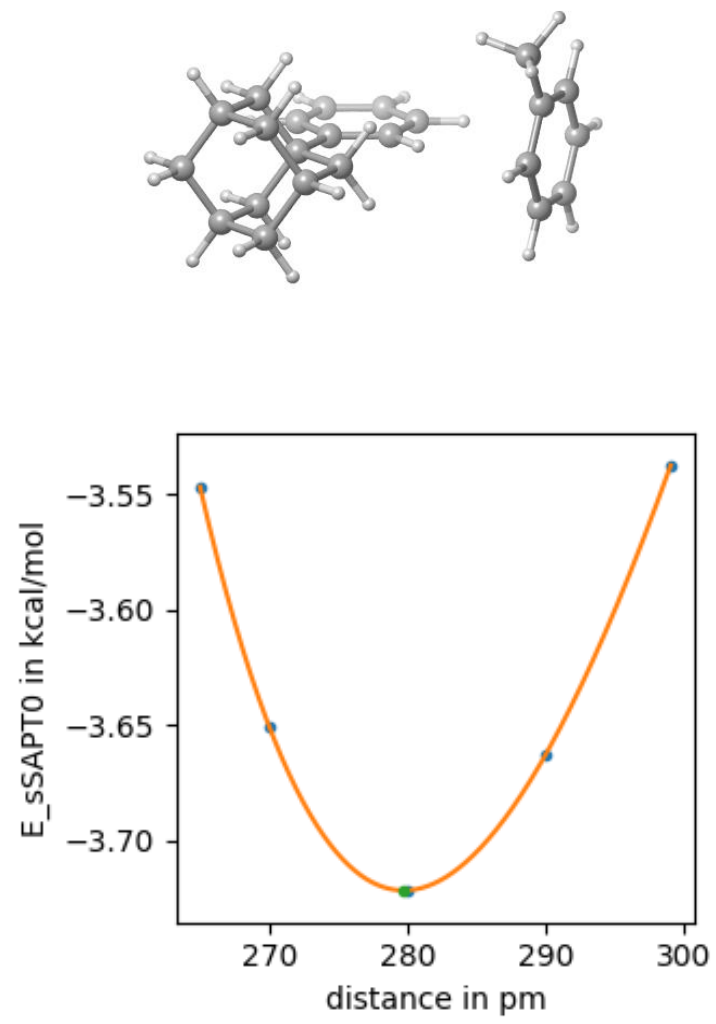

51

3MePh_pi0_4AdPh_side_1-279

H $\quad-2.73214281 \quad-1.47784534 \quad-2.49277851$

$\begin{array}{llll}\text { C } & -2.78601447 & -1.48859478 & -1.40900035\end{array}$

$\begin{array}{llll}\text { C } & -2.77584469 & -2.69650876 & -0.71474351\end{array}$

$\begin{array}{llll}\text { C } & -2.86594138 & -0.29883823 & -0.69647434\end{array}$

$\begin{array}{llll}\text { C } & -2.84501014 & -2.70493633 & 0.67274990\end{array}$

H $\quad-2.71381660 \quad-3.63565721 \quad-1.25573134$

$\begin{array}{llll}\text { C } & -2.93515373 & -0.31294337 & 0.69623459\end{array}$

H $\quad-2.87493912 \quad 0.65097374 \quad-1.22217572$

$\begin{array}{llll}\text { C } & -2.92616966 & -1.51455173 & 1.40907069\end{array}$

$\mathrm{H} \quad-2.83557120 \quad-3.65880078 \quad 1.19310937$

$\begin{array}{llll}\mathrm{H} & -2.99666323 & 0.63295799 & 1.22218565\end{array}$

$\begin{array}{llll}\text { C } & -3.00021348 & -1.57352927 & 2.93231425\end{array}$

$\begin{array}{llll}\text { C } & -1.74389242 & -2.28082506 & 3.49545708\end{array}$

$\begin{array}{llll}\text { C } & -3.08403488 & -0.17507827 & 3.57530588\end{array}$

$\begin{array}{llll}\text { C } & -4.25094165 & -2.37342095 & 3.37002963\end{array}$

H $\quad-0.85072117 \quad-1.71884184 \quad 3.19342497$

$\begin{array}{llll}\mathrm{H} & -1.65410401 & -3.28247149 & 3.05800070\end{array}$

$\begin{array}{llll}\text { C } & -1.81680820 & -2.38983637 & 5.02566066\end{array}$

H $\quad-3.97085653 \quad 0.35180940 \quad 3.19913972$

$\begin{array}{rrrr}\mathrm{H} & -2.20531945 & 0.41701879 & 3.28747875 \\ \mathrm{C} & -3.15691548 & -0.27554220 & 5.10622070 \\ \mathrm{H} & -4.22273079 & -3.37734656 & 2.92950607 \\ \mathrm{H} & -5.14847778 & -1.87757946 & 2.97839959 \\ \mathrm{C} & -4.32309410 & -2.48239782 & 4.90027066 \\ \mathrm{H} & -0.91834383 & -2.89895928 & 5.39344684 \\ \mathrm{C} & -1.90086656 & -0.98320378 & 5.63442234 \\ \mathrm{C} & -3.06489605 & -3.19383387 & 5.42127049 \\ \mathrm{H} & -3.21530478 & 0.73473639 & 5.52753156 \\ \mathrm{C} & -4.40386675 & -1.07564398 & 5.50919579 \\ \mathrm{H} & -5.21383339 & -3.05760372 & 5.17853931 \\ \mathrm{H} & -1.00553264 & -0.40601718 & 5.37141940 \\ \mathrm{H} & -1.93797794 & -1.04707022 & 6.72913446 \\ \mathrm{H} & -3.00656537 & -4.20681976 & 5.00314244 \\ \mathrm{H} & -3.11576870 & -3.29476759 & 6.51263572 \\ \mathrm{H} & -4.47145724 & -1.14063724 & 6.60238336 \\ \mathrm{H} & -5.30855776 & -0.56493901 & 5.15613674 \\ \mathrm{C} & -4.28530475 & 3.39089048 & 0.64709713 \\ \mathrm{C} & -3.09842275 & 3.43701615 & 1.38231658 \\ \mathrm{C} & -4.20340170 & 3.39575066 & -0.74714408 \\ \mathrm{C} & -5.62081179 & 3.30951886 & 1.34257084 \\ \mathrm{C} & -1.86268745 & 3.48188183 & 0.74367954 \\ \mathrm{H} & -3.14591845 & 3.43945785 & 2.46839861 \\ \mathrm{C} & -2.97021214 & 3.44061210 & -1.39120727 \\ \mathrm{H} & -5.11842777 & 3.36574961 & -1.33330204 \\ \mathrm{H} & -5.89816292 & 2.26803356 & 1.53542012 \\ \mathrm{H} & -5.59830690 & 3.82739713 & 2.30438640 \\ \mathrm{H} & -6.41180944 & 3.75383687 & 0.73390249 \\ \mathrm{C} & -1.79425836 & 3.48350580 & -0.64711883 \\ \mathrm{H} & -0.95185111 & 3.51982759 & 1.33324865 \\ \mathrm{H} & -2.92820251 & 3.44611970 & -2.47603601 \\ \mathrm{H} & -0.83155580 & 3.52215834 & -1.14729652\end{array}$

3MePh_pi0_4AdPh_side_2

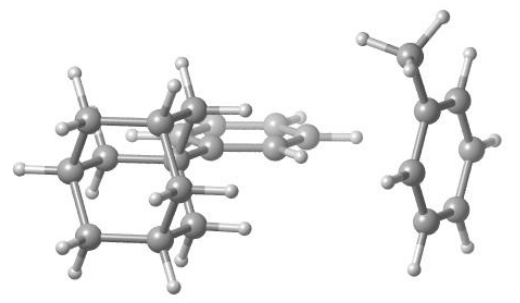




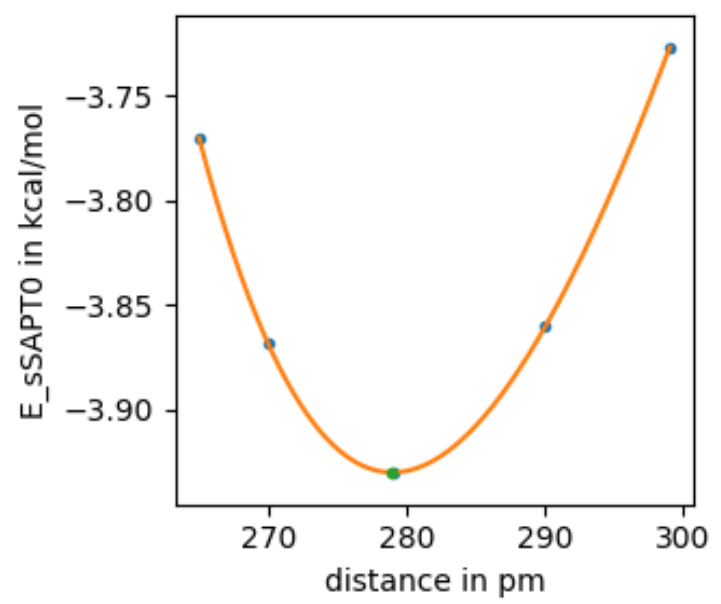

51

3MePh_pi0_4AdPh_side_2-278

$\begin{array}{lll}\mathrm{H} & -0.44364793 & 1.04221087\end{array}$

$\begin{array}{llll}\text { C } & -1.52661693 & 1.02491769 & 0.08696070\end{array}$

$\begin{array}{llll}\text { C } & -2.21079559 & -0.18798037 & 0.04302908\end{array}$

$\begin{array}{llll}\text { C } & -2.24813747 & 2.21116105 & 0.04409948\end{array}$

$\begin{array}{llll}\text { C } & -3.59729553 & -0.20478202 & -0.04257713\end{array}$

$\begin{array}{llll}\mathrm{H} & -1.66269362 & -1.12448547 & 0.07554969\end{array}$

$\begin{array}{llll}\text { C } & -3.63980431 & 2.18864653 & -0.04183060\end{array}$

$\begin{array}{llll}\mathrm{H} & -1.73034743 & 3.16476982 & 0.07740819\end{array}$

$\begin{array}{llll}\text { C } & -4.34259979 & 0.98194696 & -0.08692344\end{array}$

H $\quad-4.10971524 \quad-1.16241460-0.07553704$

$\begin{array}{llll}\text { H } & -4.17293349 & 3.13199901 & -0.07340056\end{array}$

$\begin{array}{llll}\text { C } & -5.86435733 & 0.91374176 & -0.18091171\end{array}$

$\begin{array}{llll}\text { C } & -6.43577011 & 0.15454935 & 1.04091236\end{array}$

$\begin{array}{llll}\text { C } & -6.51805546 & 2.30922184 & -0.21890619\end{array}$

$\begin{array}{llll}\text { C } & -6.28113369 & 0.15872961 & -1.46621189\end{array}$

$\begin{array}{llll}\mathrm{H} & -6.14861254 & 0.68440640 & 1.95829748\end{array}$

$\begin{array}{llll}\mathrm{H} & -5.99098041 & -0.84628832 & 1.09758427\end{array}$

$\begin{array}{llll}\text { C } & -7.96408042 & 0.03625010 & 0.94606469\end{array}$

$\begin{array}{llll}\mathrm{H} & -6.13628099 & 2.87255211 & -1.08057404\end{array}$

H $\quad-6.24518984 \quad 2.86960886 \quad 0.68501579$

$\begin{array}{llll}\text { C } & -8.04714898 & 2.19945647 & -0.31340148\end{array}$

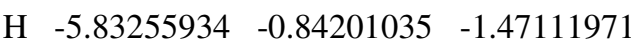

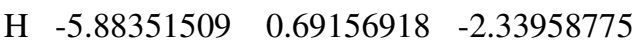

$\begin{array}{llll}\text { C } & -7.80949039 & 0.04043532 & -1.56029595\end{array}$

$\begin{array}{llll}\mathrm{H} & -8.33775674 & -0.50970684 & 1.82014024\end{array}$

$\begin{array}{llll}\text { C } & -8.58361790 & 1.44018646 & 0.90854407\end{array}$

$\begin{array}{llll}\text { C } & -8.33875096 & -0.72263112 & -0.33626274\end{array}$

H $\quad-8.47622337 \quad 3.20784929-0.33818225$

$\begin{array}{llll}\text { C } & -8.42922960 & 1.44436612 & -1.59453076\end{array}$

H $\quad-8.07280363 \quad-0.50253844 \quad-2.47547756$

$\begin{array}{llll}\mathrm{H} & -8.33563757 & 1.98484275 & 1.82821414\end{array}$

$\begin{array}{llll}\mathrm{H} & -9.67726471 & 1.36909002 & 0.85626835\end{array}$

H $\quad-7.91283570 \quad-1.73375221 \quad-0.31168076$

H $\quad-9.42858438-0.83021473 \quad-0.40366140$

$\begin{array}{llll}\mathrm{H} & -9.52099674 & 1.37331938 & -1.67728654\end{array}$

H $\quad-8.07022155 \quad 1.99202464 \quad-2.47493944$

$\begin{array}{llll}\text { C } & -3.60510669 & -3.95005869 & 1.16654501\end{array}$

$\begin{array}{llll}\text { C } & -4.20320612 & -3.96551564 & -0.09575537\end{array}$ $\begin{array}{lrrr}\text { C } & -2.21072065 & -3.92045447 & 1.24037904 \\ \text { C } & -4.44579032 & -3.93731430 & 2.41833281 \\ \text { C } & -3.43098663 & -3.94702183 & -1.25344574 \\ \text { H } & -5.28743363 & -3.99415109 & -0.16969261 \\ \text { C } & -1.43339154 & -3.90193594 & 0.08582158 \\ \text { H } & -1.73025245 & -3.91365873 & 2.21549855 \\ \text { H } & -4.69578788 & -2.91222829 & 2.71074690 \\ \text { H } & -5.38508626 & -4.47529302 & 2.26968691 \\ \text { H } & -3.91661411 & -4.39892176 & 3.25527010 \\ \text { C } & -2.04159598 & -3.91479629 & -1.16649497 \\ \text { H } & -3.91509505 & -3.96199657 & -2.22507799 \\ \text { H } & -0.35080346 & -3.88148720 & 0.16477369 \\ \text { H } & -1.43726911 & -3.90402540 & -2.06823826\end{array}$

3MePh_pi0_Ph_side
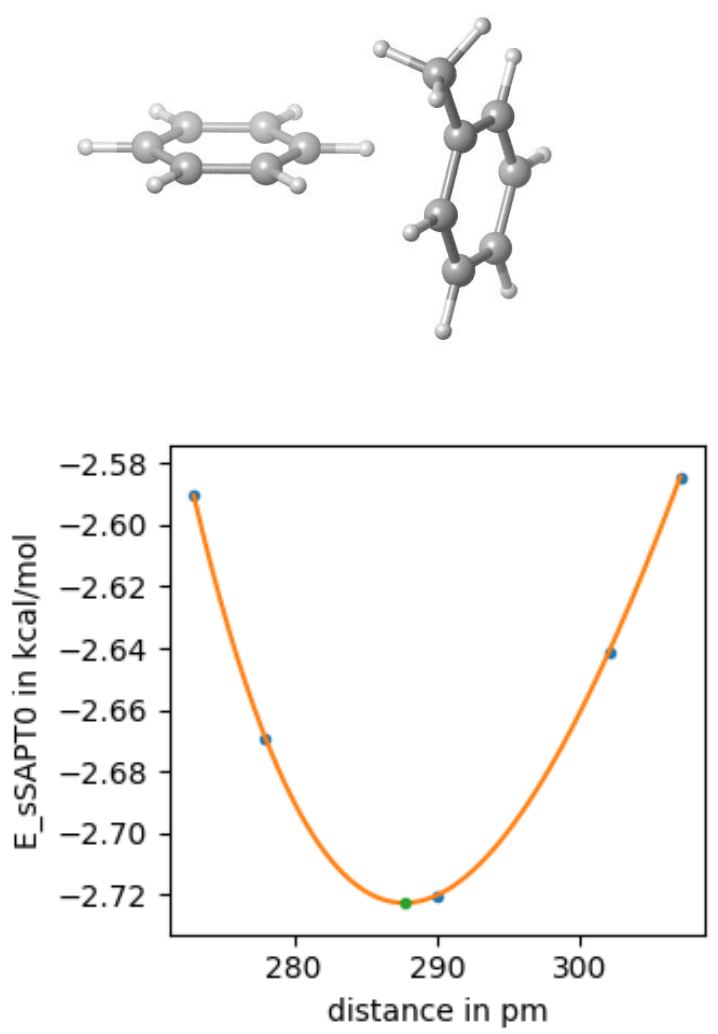

27

3MePh_pi0_Ph_side-287

H $\quad 2.45605103 \quad-0.07384341 \quad-0.32588572$

C $\quad 1.38052037 \quad-0.04150695-0.18320852$

$\begin{array}{llll}\text { C } & 0.68888772 & 1.15376481 & -0.36631467\end{array}$

$\begin{array}{llll}\text { C } & 0.69111726 & -1.19522029 & 0.18309137\end{array}$

$\begin{array}{llll}\text { C } & -0.69119276 & 1.19537189 & -0.18305642\end{array}$

$\begin{array}{llll}\mathrm{H} & 1.22593447 & 2.05312840 & -0.65163794\end{array}$

$\begin{array}{llll}\text { C } & -0.68902994 & -1.15378105 & 0.36608624\end{array}$

$\begin{array}{llll}\mathrm{H} & 1.22985827 & -2.12692304 & 0.32580994\end{array}$

$\begin{array}{llll}\text { C } & -1.38009359 & 0.04152717 & 0.18301851\end{array}$

H $\quad-1.23021688 \quad 2.12698028 \quad-0.32560453$ 


$\begin{array}{lrrr}\mathrm{H} & -1.22629089 & -2.05302987 & 0.65149861 \\ \mathrm{H} & -2.45588644 & 0.07393877 & 0.32582981 \\ \mathrm{C} & -4.49990087 & 2.26228456 & 1.35666302 \\ \mathrm{H} & -4.64684011 & 2.02153012 & 2.40495407 \\ \mathrm{C} & -5.00289961 & 1.42207598 & 0.36668974 \\ \mathrm{C} & -3.81177996 & 3.41624668 & 0.99164820 \\ \mathrm{C} & -4.81713820 & 1.73559186 & -0.97648279 \\ \mathrm{H} & -5.54500125 & 0.52221366 & 0.64060222 \\ \mathrm{C} & -3.62887376 & 3.72480580 & -0.35331388 \\ \mathrm{H} & -3.41956165 & 4.08049858 & 1.75557407 \\ \mathrm{C} & -4.12612360 & 2.88889594 & -1.35563000 \\ \mathrm{H} & -5.21502953 & 1.07773262 & -1.74507872 \\ \mathrm{H} & -3.09366430 & 4.62891655 & -0.63239906 \\ \mathrm{C} & -3.89872247 & 3.20824636 & -2.81171657 \\ \mathrm{H} & -2.97756931 & 2.74103074 & -3.17502435 \\ \mathrm{H} & -4.72046888 & 2.84012804 & -3.43059886 \\ \mathrm{H} & -3.80701118 & 4.28516017 & -2.97114877\end{array}$

4CF3Ph_pi0_4AdPh_side_1
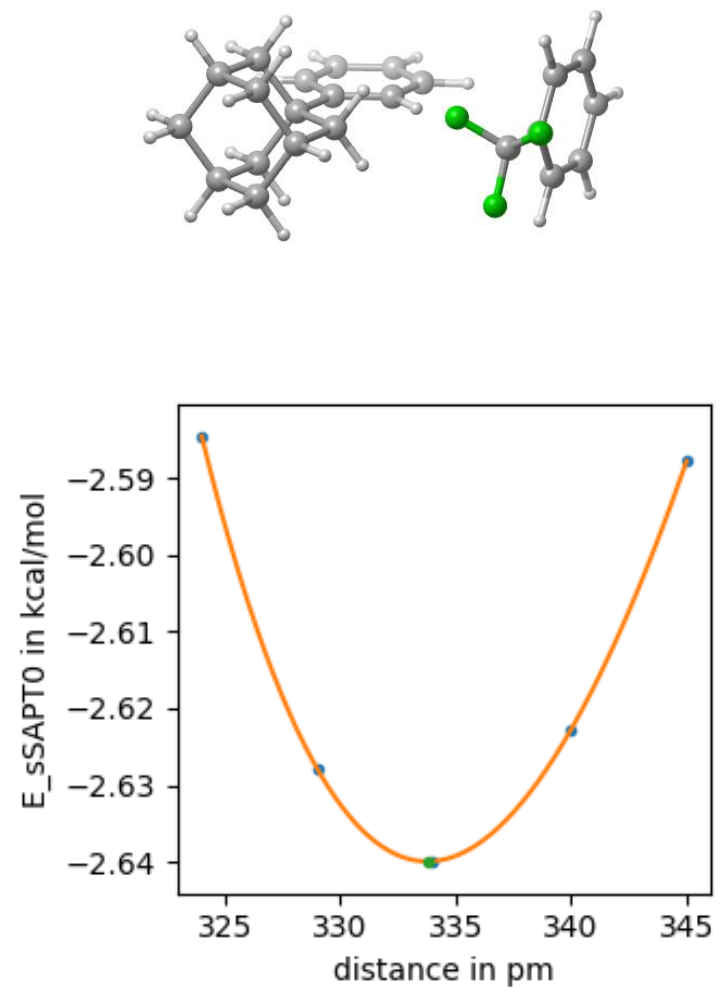

51

4CF3Ph_pi0_4AdPh_side_1-333

H $\quad-1.56705843-0.89034021 \quad-2.12396171$

C $-2.13624957 \quad-0.92025426-1.20053363$

$\begin{array}{llll}\text { C } & -2.45618298 & -2.14044468 & -0.60898475\end{array}$

$\begin{array}{llll}\text { C } & -2.55497814 & 0.25690235 & -0.59343316\end{array}$

$\begin{array}{llll}\text { C } & -3.18507995 & -2.17340751 & 0.57322313\end{array}$

H $\quad-2.13691973-3.07002664-1.06992240$

$\begin{array}{lrrr}\mathrm{C} & -3.28640226 & 0.21816972 & 0.59322013 \\ \mathrm{H} & -2.31401845 & 1.21601017 & -1.04135759 \\ \mathrm{C} & -3.61635298 & -0.99604355 & 1.20058933 \\ \mathrm{H} & -3.42306125 & -3.13647346 & 1.01660711 \\ \mathrm{H} & -3.59799927 & 1.15477038 & 1.04135381 \\ \mathrm{C} & -4.41468000 & -1.08195700 & 2.49848171 \\ \mathrm{C} & -3.58171576 & -1.80498907 & 3.58436151 \\ \mathrm{C} & -4.80428930 & 0.30512184 & 3.04652037 \\ \mathrm{C} & -5.71789332 & -1.88380882 & 2.26521984 \\ \mathrm{H} & -2.65610276 & -1.24179347 & 3.76001652 \\ \mathrm{H} & -3.28770607 & -2.79904036 & 3.22638674 \\ \mathrm{C} & -4.38217634 & -1.94105759 & 4.88799927 \\ \mathrm{H} & -5.40267926 & 0.84273040 & 2.29921140 \\ \mathrm{H} & -3.89832533 & 0.89823806 & 3.22819760 \\ \mathrm{C} & -5.60510099 & 0.17758633 & 4.35095133 \\ \mathrm{H} & -5.47635841 & -2.87980117 & 1.87485639 \\ \mathrm{H} & -6.31808123 & -1.37691493 & 1.49864498 \\ \mathrm{C} & -6.51770316 & -2.01984709 & 3.56925871 \\ \mathrm{H} & -3.76962879 & -2.46080780 & 5.63389220 \\ \mathrm{C} & -4.75554318 & -0.54518433 & 5.40607923 \\ \mathrm{C} & -5.66277567 & -2.74627243 & 4.61904421 \\ \mathrm{H} & -5.86385731 & 1.18041333 & 4.71005878 \\ \mathrm{C} & -6.88826991 & -0.62387061 & 4.08906669 \\ \mathrm{H} & -7.42967776 & -2.59584861 & 3.37371757 \\ \mathrm{H} & -3.84690440 & 0.03250300 & 5.61724426 \\ \mathrm{H} & -5.31506874 & -0.62847911 & 6.34620898 \\ \mathrm{H} & -5.40559133 & -3.75186301 & 4.26264870 \\ \mathrm{H} & -6.23257793 & -2.86650502 & 5.54894916 \\ \mathrm{H} & -7.47376589 & -0.70812474 & 5.01315941 \\ \mathrm{H} & -7.51337510 & -0.10277383 & 3.35310628 \\ \mathrm{C} & -3.80956598 & 4.49368057 & 1.17831171 \\ \mathrm{C} & -4.47724126 & 4.46989336 & -0.04541934 \\ \mathrm{C} & -2.42169722 & 4.54475335 & 1.23146158 \\ \mathrm{C} & -4.63033678 & 4.46320687 & 2.43535513 \\ \mathrm{C} & -3.74540488 & 4.49762742 & -1.22542564 \\ \mathrm{H} & -5.56185266 & 4.42986422 & -0.06571228 \\ \mathrm{C} & -1.69279374 & 4.57236604 & 0.04489277 \\ \mathrm{H} & -1.92038847 & 4.56252969 & 2.19231186 \\ \mathrm{~F} & -5.46682248 & 5.51129520 & 2.49965799 \\ \mathrm{~F} & -5.39461927 & 3.36106978 & 2.49461530 \\ \mathrm{~F} & -3.87166503 & 4.48616495 & 3.53917696 \\ \mathrm{C} & -2.35257149 & 4.54888333 & -1.17965117 \\ \mathrm{H} & -4.25855939 & 4.47931919 & -2.18094189 \\ \mathrm{H} & -0.60930155 & 4.61219996 & 0.07887255 \\ \mathrm{H} & -1.78202108 & 4.57047252 & -2.10243532\end{array}$

$\begin{array}{llll}\text { C } & -3.28640226 & 0.21816972 & 0.59322013\end{array}$

$\begin{array}{llll}\mathrm{H} & -2.31401845 & 1.21601017 & -1.04135759\end{array}$

$\begin{array}{llll}\text { C } & -3.61635298 & -0.99604355 & 1.20058933\end{array}$

$\begin{array}{llll}\mathrm{H} & -3.42306125 & -3.13647346 & 1.01660711\end{array}$

$\mathrm{H} \quad-3.59799927 \quad 1.15477038 \quad 1.04135381$

$\begin{array}{llll}\text { C } & -4.41468000 & -1.08195700 & 2.49848171\end{array}$

$\begin{array}{llll}\text { C } & -3.58171576 & -1.80498907 & 3.58436151\end{array}$

$\begin{array}{llll}\text { C } & -4.80428930 & 0.30512184 & 3.04652037\end{array}$

$\begin{array}{llll}\text { C } & -5.71789332 & -1.88380882 & 2.26521984\end{array}$

$\mathrm{H} \quad-2.65610276-1.24179347 \quad 3.76001652$

H $\quad-3.28770607 \quad-2.79904036 \quad 3.22638674$

$\begin{array}{llll}\text { C } & -4.38217634 & -1.94105759 & 4.88799927\end{array}$

$\begin{array}{llll}\mathrm{H} & -5.40267926 & 0.84273040 & 2.29921140\end{array}$

$\begin{array}{llll}\mathrm{H} & -3.89832533 & 0.89823806 & 3.22819760\end{array}$

$\begin{array}{llll}\text { C } & -5.60510099 & 0.17758633 & 4.35095133\end{array}$

$\begin{array}{llll}\mathrm{H} & -5.47635841 & -2.87980117 & 1.87485639\end{array}$

$\begin{array}{llll}\mathrm{H} & -6.31808123 & -1.37691493 & 1.49864498\end{array}$

$\begin{array}{llll}\text { C } & -6.51770316 & -2.01984709 & 3.56925871\end{array}$

$\begin{array}{llll}\mathrm{H} & -3.76962879 & -2.46080780 & 5.63389220\end{array}$

$\begin{array}{llll}\text { C } & -4.75554318 & -0.54518433 & 5.40607923\end{array}$

$\begin{array}{llll}\text { C } & -5.66277567 & -2.74627243 & 4.61904421\end{array}$

$\begin{array}{llll}\mathrm{H} & -5.86385731 & 1.18041333 & 4.71005878\end{array}$

$\begin{array}{llll}\text { C } & -6.88826991 & -0.62387061 & 4.08906669\end{array}$

$\mathrm{H} \quad-7.42967776-2.59584861 \quad 3.37371757$

$\mathrm{H} \quad-3.84690440 \quad 0.03250300 \quad 5.61724426$

$\begin{array}{llll}\mathrm{H} & -5.31506874 & -0.62847911 & 6.34620898\end{array}$

H $\quad-5.40559133 \quad-3.75186301 \quad 4.26264870$

$\mathrm{H} \quad-6.23257793 \quad-2.86650502 \quad 5.54894916$

$\mathrm{H} \quad-7.47376589-0.70812474 \quad 5.01315941$

$\begin{array}{llll}\mathrm{H} & -7.51337510 & -0.10277383 & 3.35310628\end{array}$

$\begin{array}{llll}\text { C } & -3.80956598 & 4.49368057 & 1.17831171\end{array}$

$\begin{array}{llll}\text { C } & -4.47724126 & 4.46989336 & -0.04541934\end{array}$

$\begin{array}{llll}\text { C } & -2.42169722 & 4.54475335 & 1.23146158\end{array}$

$\begin{array}{llll}\text { C } & -4.63033678 & 4.46320687 & 2.43535513\end{array}$

$\begin{array}{llll}\text { C } & -3.74540488 & 4.49762742 & -1.22542564\end{array}$

H $\quad-5.56185266 \quad 4.42986422-0.06571228$

$\begin{array}{llll}\text { C } & -1.69279374 & 4.57236604 & 0.04489277\end{array}$

$\begin{array}{llll}\mathrm{H} & -1.92038847 & 4.56252969 & 2.19231186\end{array}$

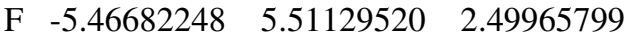

$\begin{array}{llll}\text { F } & -5.39461927 & 3.36106978 & 2.49461530\end{array}$

$\begin{array}{llll}\text { F } & -3.87166503 & 4.48616495 & 3.53917696\end{array}$

C $-2.35257149 \quad 4.54888333-1.17965117$

$\begin{array}{llll}\text { H } & -4.25855939 & 4.47931919 & -2.18094189\end{array}$

$\begin{array}{llll}\mathrm{H} & -1.78202108 & 4.57047252 & -2.10243532\end{array}$ 
4CF3Ph_pi0_4AdPh_side_2
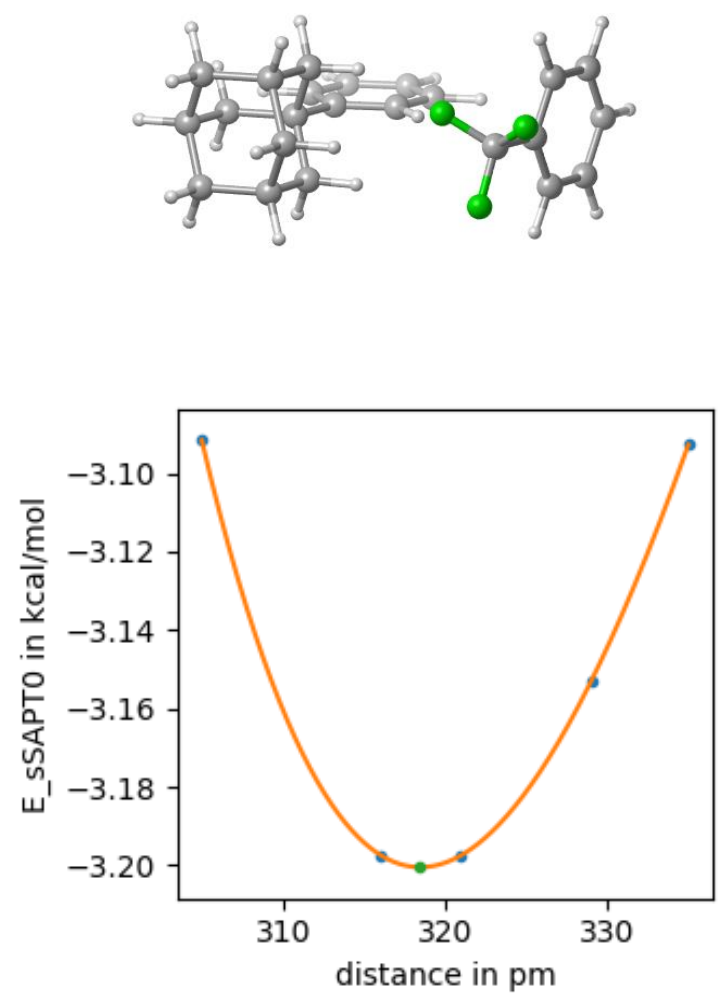

51

4CF3Ph_pi0_4AdPh_side_2-318

H $\quad-1.46085122 \quad 1.01929099 \quad-2.01438639$

C $\quad-2.10157630 \quad 1.01196256 \quad-1.13859504$

C $-2.49530400 \quad-0.19439099 \quad-0.56340659$

C $\quad-2.53946267 \quad 2.20459684 \quad-0.57691782$

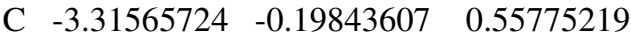

H $-2.16232981 \quad-1.13574339-0.98943357$

C $\quad-3.36281845 \quad 2.19488764 \quad 0.54852364$

H $\quad-2.24180644 \quad 3.15324550 \quad-1.01286755$

C $\quad-3.76763778 \quad 0.99490214 \quad 1.13863787$

H $\quad-3.61007904 \quad-1.15115667 \quad 0.98944621$

H $\quad-3.68698082 \quad 3.14294862 \quad 0.96233088$

C $\quad-4.66752863 \quad 0.94070900 \quad 2.37009689$

$\begin{array}{llll}\text { C } & -3.93969058 & 0.21080815 & 3.52475539\end{array}$

$\begin{array}{llll}\text { C } & -5.06687789 & 2.34192014 & 2.87334335\end{array}$

C $\quad \begin{array}{llll}-5.96653129 & 0.16580179 & 2.04168660\end{array}$

H $\quad-3.01800106 \quad 0.75497442 \quad 3.76801432$

H $\quad-3.64149113 \quad-0.79333212 \quad 3.19994004$

C $\quad-4.84332222 \quad 0.10658430 \quad 4.76221668$

H $\quad-5.59190765 \quad 2.88480645 \quad 2.07636002$

H $\quad-4.16455252 \quad 2.91650193 \quad 3.12078809$

C $\quad \begin{array}{llll}-5.97072431 & 2.24624299 & 4.11149210\end{array}$

$\mathrm{H} \quad-5.71812214 \quad-0.83944875 \quad 1.68045574$

H $\quad-6.49254624 \quad 0.67781802 \quad 1.22562802$

C $\quad-6.86954530 \quad 0.06159788 \quad 3.27959888$
H $\quad-4.30365749 \quad-0.41878123 \quad 5.55874921$

C $\quad \begin{array}{llll}-5.22391952 & 1.51590450 & 5.23680498\end{array}$

C $-6.11705264 \quad-0.67257127 \quad 4.40004293$

$\mathrm{H} \quad-6.23367548 \quad 3.25837894 \quad 4.44014464$

C $\quad \begin{array}{llll}-7.24748573 & 1.47097698 & 3.75613009\end{array}$

H $\quad-7.77637212 \quad-0.49588747 \quad 3.01770778$

$\mathrm{H} \quad-4.32160931 \quad 2.07531618 \quad 5.51399200$

H $\quad-5.85756415 \quad 1.45517314 \quad 6.13048109$

H $\quad-5.85594585 \quad-1.68740587 \quad 4.07399397$

H $-6.76098913 \quad-0.77010783 \quad 5.28304130$

H $\quad-7.90577159 \quad 1.40969734 \quad 4.63177625$

H $\quad-7.80041715 \quad 1.99807560 \quad 2.96849441$

C $\quad-3.63075718 \quad-4.34579348 \quad 1.12013423$

C $\quad-2.24582623 \quad-4.31562741 \quad 1.27802679$

C $\quad-4.20303248-4.35815116 \quad-0.14634171$

$\begin{array}{llll}\text { C } & -4.48557581 & -4.36510376 & 2.35450198\end{array}$

C $-1.42882975 \quad-4.29773672 \quad 0.15509972$

H $-1.81827081 \quad-4.30629729 \quad 2.27577693$

C $-3.37885326 \quad-4.34008631 \quad-1.26902179$

H $\quad-5.28180989-4.38159426 \quad-0.24905217$

F $\quad-4.22068729 \quad-5.43785797 \quad 3.11697480$

F $-4.26156446 \quad-3.28680956 \quad 3.12225597$

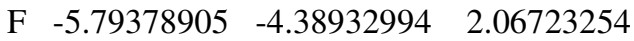

C $\quad-1.99614576-4.30998115 \quad-1.11875661$

H $-0.35052861 \quad-4.27421301 \quad 0.27080339$

H $\quad-3.81866353 \quad-4.34953904 \quad-2.26057322$

H $\quad-1.35657911 \quad-4.29594149 \quad-1.99527349$

4CF3Ph_pi_4CF3Ph_side1_0_1

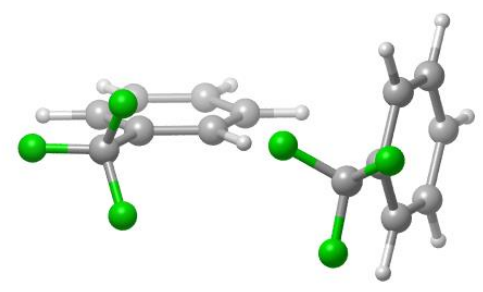




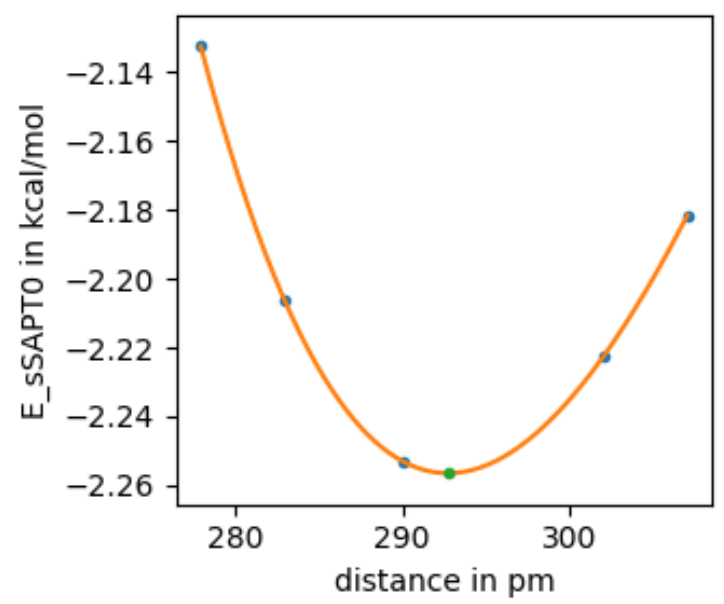

30

4CF3Ph_pi_4CF3Ph_side1_0_1-292

$\begin{array}{lrrc}\text { H } & 1.01746462 & 1.37935383 & -0.15653724 \\ \mathrm{C} & -0.06532867 & 1.36001626 & -0.08791347 \\ \mathrm{C} & -0.73667652 & 0.13850367 & -0.04425158 \\ \mathrm{C} & -0.77930395 & 2.55318582 & -0.04373564 \\ \mathrm{C} & -2.12243854 & 0.11184121 & 0.04361938 \\ \mathrm{H} & -0.17870355 & -0.79109368 & -0.07873300 \\ \mathrm{C} & -2.16920066 & 2.53239960 & 0.04437434 \\ \mathrm{H} & -0.25566022 & 3.50257352 & -0.07774891 \\ \mathrm{C} & -2.83168769 & 1.31138937 & 0.08747866 \\ \mathrm{H} & -2.65838950 & -0.83152102 & 0.07855247 \\ \mathrm{H} & -2.73778956 & 3.45452950 & 0.07962775 \\ \mathrm{C} & -4.32894319 & 1.24760114 & 0.18186378 \\ \mathrm{~F} & -4.85823923 & 0.59369112 & -0.86435787 \\ \mathrm{~F} & -4.72258355 & 0.58779770 & 1.28279628 \\ \mathrm{~F} & -4.88870366 & 2.46403372 & 0.22049674 \\ \mathrm{C} & 0.01238730 & -3.71677996 & -0.08801277 \\ \mathrm{H} & 1.09513292 & -3.69951155 & -0.15792841 \\ \mathrm{C} & -0.60258721 & -3.72742129 & 1.16354643 \\ \mathrm{C} & -0.75773563 & -3.72819241 & -1.24652320 \\ \mathrm{C} & -1.98820254 & -3.74947721 & 1.25493699 \\ \mathrm{H} & -0.00087195 & -3.71845647 & 2.06606094 \\ \mathrm{C} & -2.14776126 & -3.75033207 & -1.16080937 \\ \mathrm{H} & -0.27790820 & -3.71985284 & -2.21935040 \\ \mathrm{C} & -2.75389171 & -3.76082780 & 0.08983397 \\ \mathrm{H} & -2.48063907 & -3.75786827 & 2.22232103 \\ \mathrm{H} & -2.75976026 & -3.75937361 & -2.05536803 \\ \mathrm{C} & -4.24931277 & -3.78524236 & 0.22351744 \\ \mathrm{~F} & -4.66323302 & -4.87062263 & 0.89653456 \\ \mathrm{~F} & -4.69746569 & -2.71947209 & 0.90589444 \\ \mathrm{~F} & -4.86651855 & -3.78996219 & -0.96541791\end{array}$

4CF3Ph_pi_4CF3Ph_side1_0_2
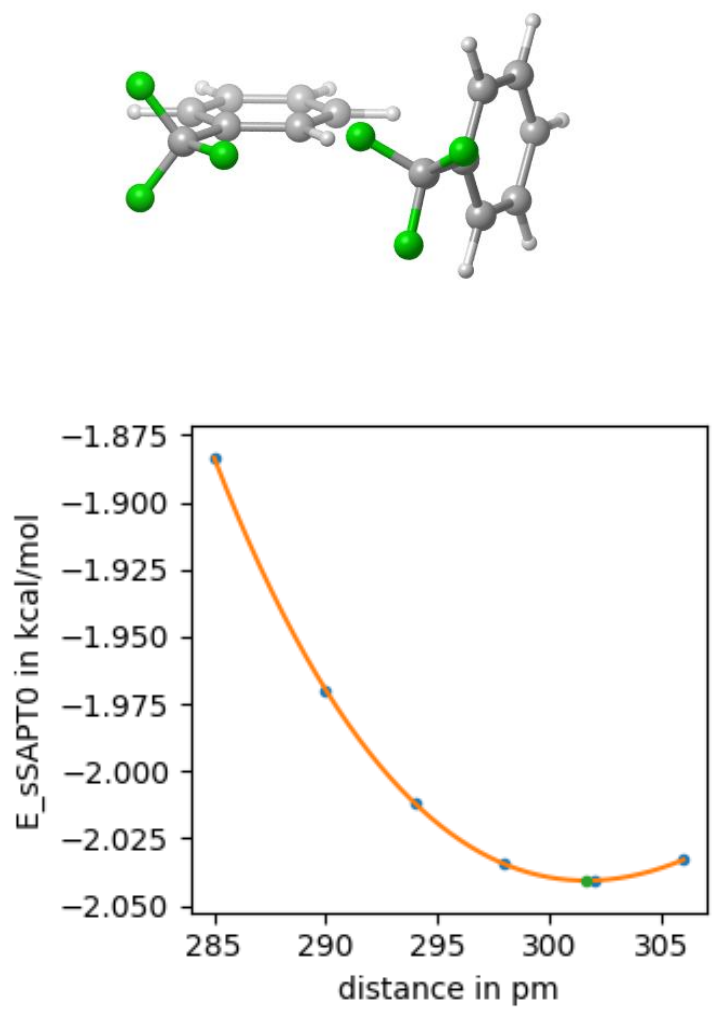

30

4CF3Ph_pi_4CF3Ph_side1_0_2-301

H $\quad 1.05974963 \quad-1.44169514 \quad 0.19470329$

C $-0.02196194-1.45441506 \quad 0.10948156$

$\begin{array}{llll}\text { C } & -0.69997597 & -2.67180169 & 0.05494629\end{array}$

$\begin{array}{llll}\text { C } & -0.72791453 & -0.25690486 & 0.05501823\end{array}$

$\begin{array}{llll}\text { C } & -2.08437147 & -2.68999490 & -0.05407957\end{array}$

$\begin{array}{llll}\mathrm{H} & -0.14826191 & -3.60479138 & 0.09755931\end{array}$

$\begin{array}{llll}\text { C } & -2.11640201 & -0.26919665 & -0.05434207\end{array}$

$\begin{array}{llll}\mathrm{H} & -0.19909731 & 0.68926464 & 0.09758939\end{array}$

C $-2.78556260-1.48613508 \quad-0.10817519$

$\begin{array}{llll}\mathrm{H} & -2.62546632 & -3.63006403 & -0.09746650\end{array}$

$\begin{array}{llll}\mathrm{H} & -2.67877863 & 0.65639048 & -0.09770928\end{array}$

$\begin{array}{llll}\text { C } & -4.28147070 & -1.54077113 & -0.22660388\end{array}$

F $-4.66136779 \quad-2.18986548 \quad-1.33866822$

$\begin{array}{llll}\text { F } & -4.83075310 & -2.19971778 & 0.80607411\end{array}$

$\begin{array}{llll}\text { F } & -4.83383880 & -0.32094697 & -0.26469598\end{array}$

$\begin{array}{llll}\text { C } & -0.09715900 & 3.70853497 & 0.10861106\end{array}$

$\begin{array}{llll}\mathrm{H} & 0.98443168 & 3.72325837 & 0.19503608\end{array}$

C $\quad-0.69301721 \quad 3.70111041-1.15218441$

$\begin{array}{llll}\text { C } & -0.88486464 & 3.69708827 & 1.25523862\end{array}$

$\begin{array}{llll}\text { C } & -2.07712402 & 3.68222583 & -1.26470217\end{array}$

$\begin{array}{llll}\mathrm{H} & -0.07760327 & 3.71000578 & -2.04541484\end{array}$

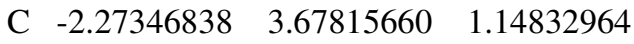

$\begin{array}{llll}\mathrm{H} & -0.41989516 & 3.70284476 & 2.23527277\end{array}$

$\begin{array}{llll}\text { C } & -2.86049708 & 3.67084772 & -0.11141514\end{array}$ 


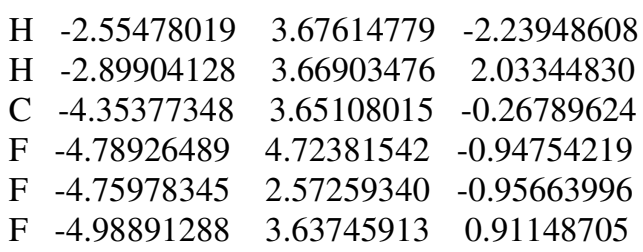

4CF3Ph_pi_Ph_side0
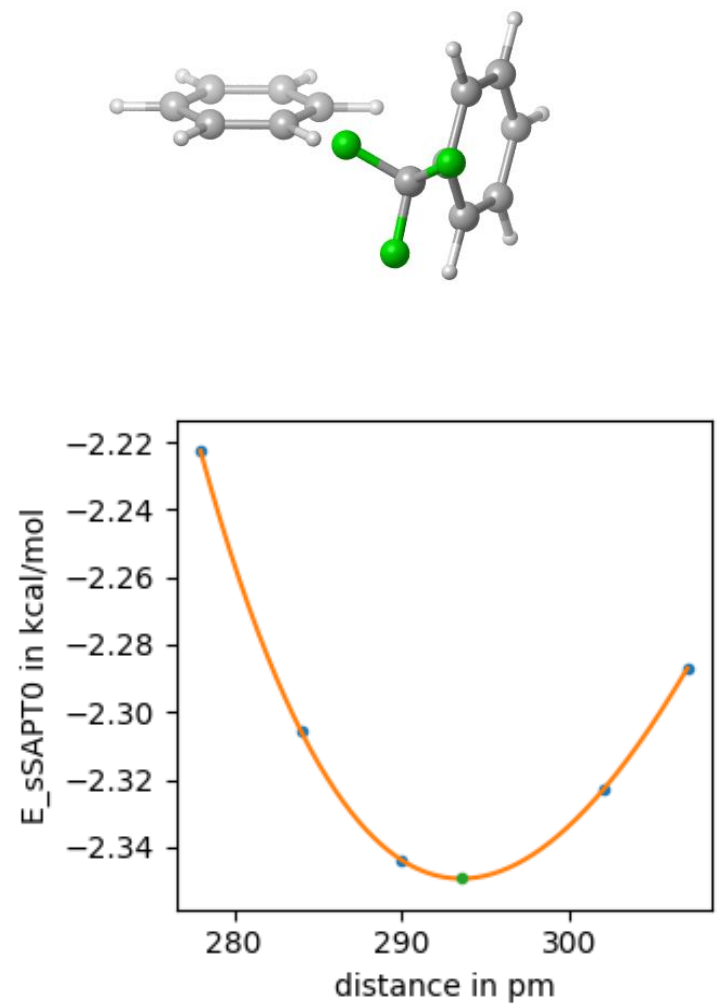

27

4CF3Ph_pi_Ph_side0-293

H $\quad 1.80975569-1.15577473-1.23836864$

C $\quad 1.01722848 \quad-0.64967605 \quad-0.69621239$

$\begin{array}{llll}\text { C } & -0.03755645 & -0.06233992 & -1.39121110\end{array}$

$\begin{array}{llll}\text { C } & 1.05435013 & -0.58714968 & 0.69490914\end{array}$

$\begin{array}{llll}\text { C } & -1.05444685 & 0.58726597 & -0.69559413\end{array}$

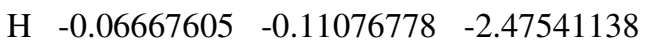

$\begin{array}{llll}\text { C } & 0.03733312 & 0.06218797 & 1.39064447\end{array}$

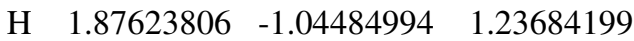

$\begin{array}{llll}\text { C } & -1.01699405 & 0.64937438 & 0.69533818\end{array}$

$\mathrm{H} \quad-1.87644110 \quad 1.04516782-1.23726975$

H $\quad 0.06628770 \quad 0.11081296 \quad 2.47487472$

$\begin{array}{llll}\mathrm{H} & -1.80968803 & 1.15570798 & 1.23759124\end{array}$

$\begin{array}{llll}\text { C } & -4.32666339 & 2.66780260 & 1.38485577\end{array}$

$\begin{array}{llll}\mathrm{H} & -4.29852271 & 2.71537423 & 2.46858521\end{array}$

$\begin{array}{llll}\text { C } & -3.72608924 & 3.67468341 & 0.62973390\end{array}$

$\begin{array}{llll}\text { C } & -4.96136622 & 1.60312541 & 0.75320650\end{array}$

$\begin{array}{llll}\text { C } & -3.76108780 & 3.61541850 & -0.75736066\end{array}$

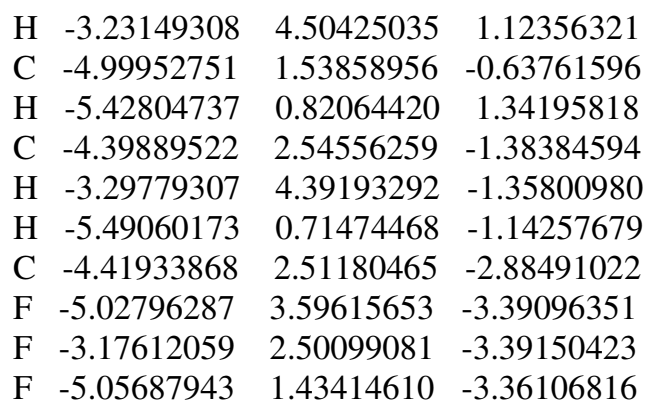

4ClPh_pi0_4AdPh_side1
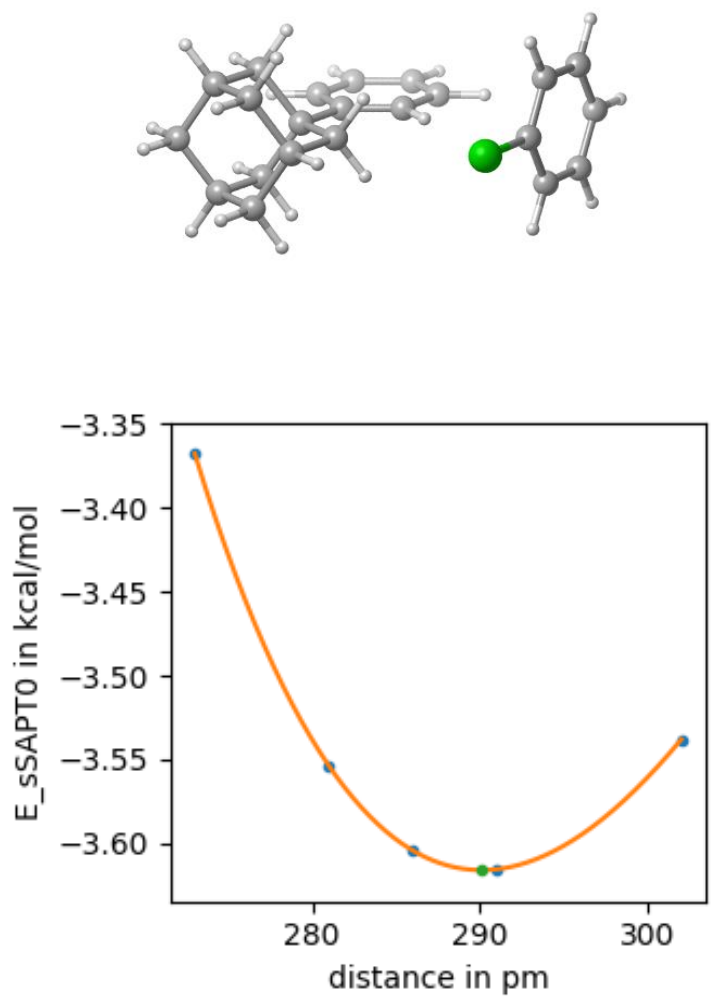

48

4ClPh_pi0_4AdPh_side1-290

H $\quad-1.55274689 \quad 0.07335761 \quad-2.09221723$

$\begin{array}{llll}\text { C } & -2.14371011 & 0.04263377 & -1.18259569\end{array}$

$\begin{array}{llll}\text { C } & -2.47759037 & -1.17807539 & -0.59989057\end{array}$

$\begin{array}{llll}\text { C } & -2.57675290 & 1.21925798 & -0.58457253\end{array}$

$\begin{array}{llll}\text { C } & -3.23436078 & -1.21207493 & 0.56464169\end{array}$

$\mathrm{H} \quad-2.14745912-2.10725312-1.05393600$

$\begin{array}{llll}\text { C } & -3.33615528 & 1.17948474 & 0.58433876\end{array}$

$\begin{array}{llll}\mathrm{H} & -2.32523258 & 2.17875860 & -1.02580012\end{array}$

$\begin{array}{llll}\text { C } & -3.68042586 & -0.03526114 & 1.18262708\end{array}$

$\begin{array}{llll}\mathrm{H} & -3.48279568 & -2.17552969 & 1.00139736\end{array}$

$\begin{array}{llll}\mathrm{H} & -3.65831842 & 2.11569240 & 1.02577212\end{array}$

$\begin{array}{llll}\text { C } & -4.50935385 & -0.12231276 & 2.46111530\end{array}$

$\begin{array}{llll}\text { C } & -3.70240828 & -0.84631212 & 3.56583405\end{array}$ 


$\begin{array}{lrrr}\mathrm{C} & -4.91188505 & 1.26428546 & 3.00097037 \\ \mathrm{C} & -5.80665051 & -0.92394497 & 2.19625761 \\ \mathrm{H} & -2.78123458 & -0.28328131 & 3.76392173 \\ \mathrm{H} & -3.39996830 & -1.84004985 & 3.21406566 \\ \mathrm{C} & -4.53360516 & -0.98352384 & 4.84997107 \\ \mathrm{H} & -5.49236216 & 1.80255996 & 2.24013457 \\ \mathrm{H} & -4.01049820 & 1.85723176 & 3.20463830 \\ \mathrm{C} & -5.74345187 & 1.13560606 & 4.28589956 \\ \mathrm{H} & -5.55590121 & -1.91959461 & 1.81086162 \\ \mathrm{H} & -6.38846713 & -0.41636809 & 1.41609154 \\ \mathrm{C} & -6.63720637 & -1.06112679 & 3.48081110 \\ \mathrm{H} & -3.93894102 & -1.50393888 & 5.60974326 \\ \mathrm{C} & -4.91918700 & 0.41189510 & 5.36026945 \\ \mathrm{C} & -5.80744639 & -1.78848777 & 4.54996441 \\ \mathrm{H} & -6.01067542 & 2.13811813 & 4.63964538 \\ \mathrm{C} & -7.02002995 & 0.33439381 & 3.99290362 \\ \mathrm{H} & -7.54427304 & -1.63694609 & 3.26315554 \\ \mathrm{H} & -4.01582631 & 0.98938644 & 5.59346675 \\ \mathrm{H} & -5.50088348 & 0.32777570 & 6.28677036 \\ \mathrm{H} & -5.54185877 & -2.79376579 & 4.19888998 \\ \mathrm{H} & -6.39917341 & -1.90953582 & 5.46596671 \\ \mathrm{H} & -7.62730860 & 0.24932949 & 4.90275435 \\ \mathrm{H} & -7.62748412 & 0.85614678 & 3.24276406 \\ \mathrm{C} & -2.34177561 & 5.07435327 & -1.16583927 \\ \mathrm{C} & -3.73110021 & 5.02268903 & -1.23782307 \\ \mathrm{C} & -1.71284344 & 5.09775534 & 0.07592117 \\ \mathrm{C} & -4.49662170 & 4.99423896 & -0.07549711 \\ \mathrm{C} & -2.46435102 & 5.06982469 & 1.24737037 \\ \mathrm{C} & -3.85076303 & 5.01826951 & 1.15556486 \\ \mathrm{H} & -1.75070450 & 5.09632080 & -2.07513437 \\ \mathrm{H} & -4.22672573 & 5.00424751 & -2.20282589 \\ \mathrm{H} & -0.63038179 & 5.13800796 & 0.13814384 \\ \mathrm{H} & -5.57903009 & 4.95398964 & -0.11646014 \\ \mathrm{H} & -1.98816837 & 5.08754311 & 2.22094217 \\ \mathrm{Cl} & -4.80207664 & 4.98291469 & 2.61905164\end{array}$

4ClPh_pi0_4AdPh_side2

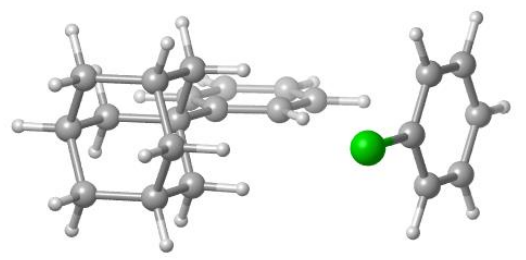

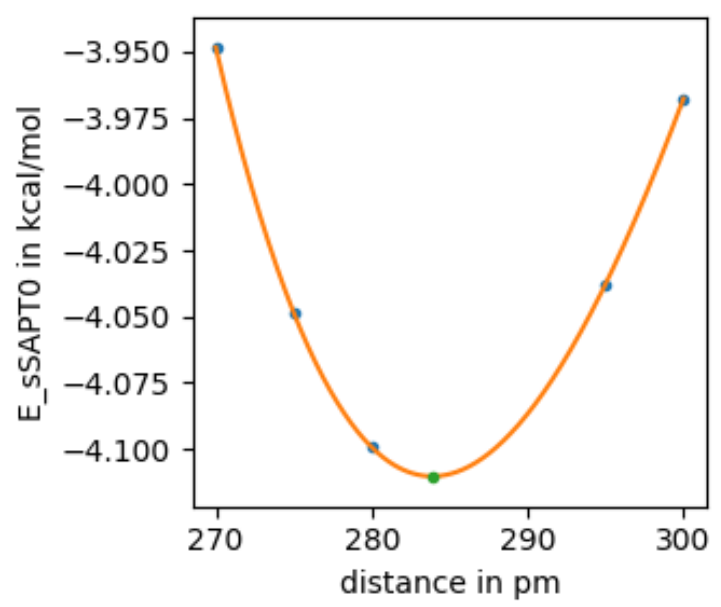

48

4ClPh_pi0_4AdPh_side2-283

$\mathrm{H} \quad-1.52920985 \quad 0.83605401 \quad-2.06545636$

$\begin{array}{llll}\text { C } & -2.13843504 & 0.82943560 & -1.16746210\end{array}$

$\begin{array}{llll}\text { C } & -2.51147447 & -0.37645162 & -0.57769119\end{array}$

$\begin{array}{llll}\text { C } & -2.55611963 & 2.02252524 & -0.59154631\end{array}$

$\begin{array}{llll}\text { C } & -3.29150259 & -0.37958775 & 0.57189095\end{array}$

$\mathrm{H}-2.19382314 \quad-1.31814941-1.01451845$

$\begin{array}{llll}\text { C } & -3.33899626 & 2.01372847 & 0.56242695\end{array}$

$\begin{array}{llll}\mathrm{H} & -2.27414357 & 2.97082046 & -1.03854894\end{array}$

$\begin{array}{llll}\text { C } & -3.72259047 & 0.81422139 & 1.16750294\end{array}$

$\begin{array}{llll}\mathrm{H} & -3.57039732 & -1.33195837 & 1.01452898\end{array}$

$\begin{array}{llll}\mathrm{H} & -3.64827528 & 2.96212492 & 0.98672394\end{array}$

$\begin{array}{llll}\text { C } & -4.57818902 & 0.76102663 & 2.43017994\end{array}$

$\begin{array}{llll}\text { C } & -3.80980213 & 0.03204008 & 3.55885171\end{array}$

$\begin{array}{llll}\text { C } & -4.95943844 & 2.16264574 & 2.94616658\end{array}$

$\begin{array}{llll}\text { C } & -5.88802195 & -0.01412503 & 2.14871943\end{array}$

$\begin{array}{llll}\mathrm{H} & -2.88006448 & 0.57638799 & 3.76879614\end{array}$

H $\quad-3.52330909-0.97236405 \quad 3.22445720$

$\begin{array}{llll}\text { C } & -4.66892527 & -0.07118052 & 4.82770005\end{array}$

$\begin{array}{llll}\mathrm{H} & -5.51244262 & 2.70490127 & 2.16789314\end{array}$

$\begin{array}{llll}\mathrm{H} & -4.04890455 & 2.73741278 & 3.16095737\end{array}$

$\begin{array}{llll}\text { C } & -5.81875194 & 2.06797239 & 4.21570274\end{array}$

$\begin{array}{llll}\mathrm{H} & -5.65258077 & -1.01966788 & 1.77970112\end{array}$

$\begin{array}{llll}\mathrm{H} & -6.44268753 & 0.49724518 & 1.35144246\end{array}$

C $-6.74651183-0.11732533 \quad 3.41799654$

$\begin{array}{llll}\mathrm{H} & -4.10131160 & -0.59591584 & 5.60498932\end{array}$

$\begin{array}{llll}\text { C } & -5.03245221 & 1.33852446 & 5.31437431\end{array}$

$\begin{array}{llll}\text { C } & -5.95470064 & -0.85060791 & 4.51160346\end{array}$

$\begin{array}{llll}\text { H } & -6.06988279 & 3.08037477 & 4.55267409\end{array}$

$\begin{array}{llll}\text { C } & -7.10731460 & 1.29244005 & 3.90651809\end{array}$

$\mathrm{H} \quad-7.66205752 \quad-0.67500744 \quad 3.18891463$

$\begin{array}{llll}\mathrm{H} & -4.12087723 & 1.89814520 & 5.55890130\end{array}$

$\mathrm{H} \quad-5.63396562 \quad 1.27851735 \quad 6.23003340$

$\mathrm{H} \quad-5.70532052-1.86570684 \quad 4.17730169$

$\mathrm{H} \quad-6.56687801 \quad-0.94742860 \quad 5.41698644$

$\begin{array}{llll}\mathrm{H} & -7.73409386 & 1.23187059 & 4.80503396\end{array}$

$\begin{array}{llll}\mathrm{H} & -7.68787072 & 1.81891578 & 3.13858972\end{array}$

C $-2.03755333-4.14556836 \quad-1.15088682$

C $-1.43252031 \quad-4.13193524 \quad 0.10283680$ 


\begin{abstract}
$\begin{array}{llll}\text { C } & -3.42582903 & -4.17686504 & -1.24979801\end{array}$
$\begin{array}{llll}\text { C } & -2.20690763 & -4.14940212 & 1.25949291\end{array}$

$\begin{array}{llll}\text { C } & -4.21405811 & -4.19464210 & -0.10253351\end{array}$

$\begin{array}{llll}\text { C } & -3.59188658 & -4.18062355 & 1.14082103\end{array}$

$\begin{array}{llll}\mathrm{H} & -1.42872027 & -4.13183671 & -2.04854987\end{array}$

H $-0.35099436 \quad-4.10755398 \quad 0.18603756$

H $-3.90288407 \quad-4.18761401 \quad-2.22423021$

H $-1.74946627 \quad-4.13909642 \quad 2.24211547$

H $\quad-5.29594259 \quad-4.21903136 \quad-0.16447758$

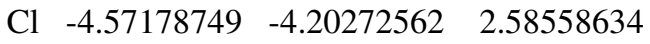

4FPh_pi0_4AdPh_side1
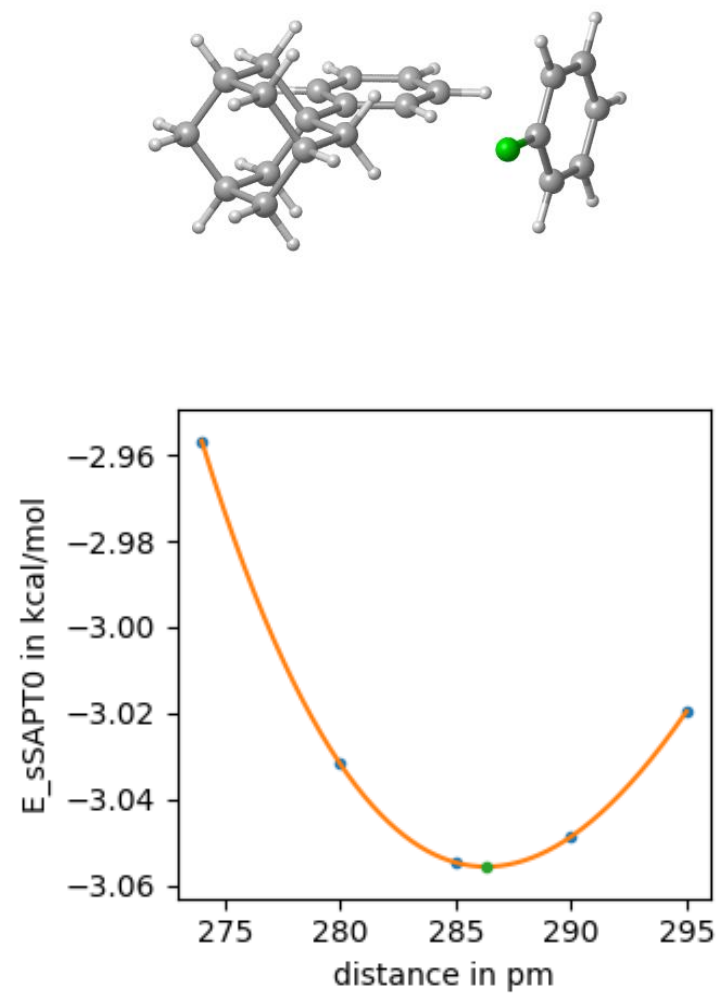

48

4FPh_pi0_4AdPh_side1-286

H $\quad-1.57672208 \quad 0.07246588 \quad-2.10762925$

$\begin{array}{llll}\text { C } & -2.15726180 & 0.04212973 & -1.19130716\end{array}$

$\begin{array}{llll}\text { C } & -2.48446493 & -1.17833108 & -0.60431000\end{array}$

$\begin{array}{llll}\text { C } & -2.58345159 & 1.21900883 & -0.58887863\end{array}$

$\begin{array}{llll}\text { C } & -3.22789079 & -1.21183429 & 0.56880054\end{array}$

H $\quad-2.15953679-2.10770233 \quad-1.06170035$

$\begin{array}{llll}\text { C } & -3.32945923 & 1.17973379 & 0.58864316\end{array}$

$\begin{array}{llll}\mathrm{H} & -2.33698722 & 2.17832139 & -1.03335630\end{array}$

C $-3.66687410 \quad-0.03475710 \quad 1.19133859$

$\begin{array}{llll}\mathrm{H} & -3.47132097 & -2.17510290 & 1.00877304\end{array}$

$\mathrm{H} \quad-3.64656378 \quad 2.11612961 \quad 1.03332830$

$\begin{array}{llll}\text { C } & -4.48115172 & -0.12126381 & 2.47924398\end{array}$

$\begin{array}{llll}\text { C } & -3.66164480 & -0.84479618 & 3.57498532\end{array}$
$\begin{array}{llll}\text { C } & -4.87749646 & 1.26556451 & 3.02307074\end{array}$
$\begin{array}{llll}\text { C } & -5.78138581 & -0.92300506 & 2.22955479\end{array}$
$\begin{array}{llll}\mathrm{H} & -2.73827090 & -0.28168369 & 3.76230425\end{array}$
H $\quad-3.36323831 \quad-1.83868393 \quad 3.22020889$
C $-4.47812655 \quad-0.98146059 \quad 4.86858633$
$\begin{array}{llll}\mathrm{H} & -5.46662322 & 1.80351744 & 2.26868323\end{array}$
$\begin{array}{llll}\mathrm{H} & -3.97384448 & 1.85859491 & 3.21618275\end{array}$
$\begin{array}{llll}\text { C } & -5.69433910 & 1.13743276 & 4.31746448\end{array}$
$\begin{array}{llll}\mathrm{H} & -5.53505054 & -1.91881887 & 1.84174391\end{array}$
$\begin{array}{llll}\mathrm{H} & -6.37207261 & -0.41575794 & 1.45586660\end{array}$
C $-6.59722182-1.05963940 \quad 3.52356493$
$\begin{array}{llll}\mathrm{H} & -3.87482586 & -1.50155455 & 5.62174076\end{array}$
$\begin{array}{llll}\text { C } & -4.85786050 & 0.41417585 & 5.38266108\end{array}$
C $-5.75530802-1.78654854 \quad 4.58348411$
$\begin{array}{llll}\mathrm{H} & -5.95750890 & 2.14009560 & 4.67381243\end{array}$
$\begin{array}{llll}\text { C } & -6.97417720 & 0.33609947 & 4.03940216\end{array}$
$\begin{array}{llll}\mathrm{H} & -7.50671297 & -1.63554871 & 3.31652376\end{array}$
$\begin{array}{llll}\mathrm{H} & -3.95189765 & 0.99176382 & 5.60528456\end{array}$
H $\quad-5.42894126 \quad 0.33045130 \quad 6.31577832$
$\begin{array}{llll}\mathrm{H} & -5.49374354 & -2.79197620 & 4.22982723\end{array}$
$\mathrm{H} \quad-6.33653845 \quad-1.90720618 \quad 5.50623360$
$\begin{array}{llll}\mathrm{H} & -7.57102856 & 0.25142298 & 4.95616276\end{array}$
$\begin{array}{llll}\mathrm{H} & -7.59015712 & 0.85753548 & 3.29602519\end{array}$
C $-2.35457369 \quad 5.03532169-1.17305244$
$\begin{array}{llll}\text { C } & -3.74556159 & 4.98399218 & -1.22928848\end{array}$
$\begin{array}{llll}\text { C } & -1.71100275 & 5.05967082 & 0.06220755\end{array}$
$\begin{array}{llll}\text { C } & -4.49836461 & 4.95681786 & -0.05880855\end{array}$
$\begin{array}{llll}\text { C } & -2.44840131 & 5.03305762 & 1.24246667\end{array}$
$\begin{array}{llll}\text { C } & -3.83113870 & 4.98202254 & 1.15617454\end{array}$
$\mathrm{H} \quad-1.77404519 \quad 5.05627737 \quad-2.08881691$
$\begin{array}{lllll}\mathrm{H} & -4.25108059 & 4.96488065 & -2.18914244\end{array}$
$\begin{array}{llll}\mathrm{H} & -0.62782558 & 5.09964140 & 0.11082457\end{array}$
$\begin{array}{llll}\mathrm{H} & -5.58140856 & 4.91687747 & -0.07066453\end{array}$
$\begin{array}{llll}\mathrm{H} & -1.97617147 & 5.05095485 & 2.21786337\end{array}$
$\begin{array}{llll}\text { F } & -4.54940292 & 4.95609775 & 2.28921295\end{array}$

4FPh_pi0_4AdPh_side2

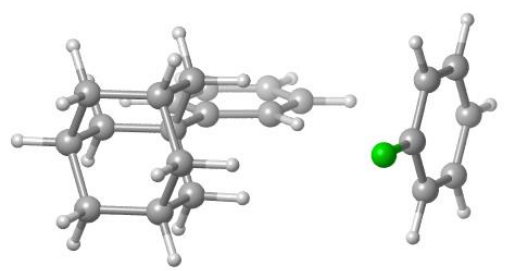




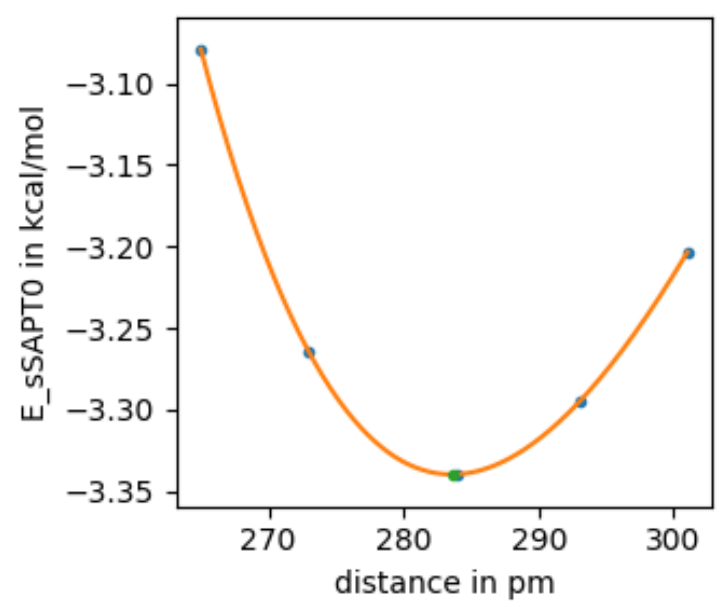

48

4FPh_pi0_4AdPh_side2-283

$\begin{array}{llll}\mathrm{H} & -1.52757436 & 0.01753301 & -2.07676841 \\ \mathrm{C} & -2.12949149 & 0.01107933 & -1.17385815\end{array}$

$\begin{array}{llll}\text { C } & -2.12949149 & 0.01107933 & -1.17385815\end{array}$

C $-2.49773120-1.19469971-0.58085851$

$\begin{array}{llll}\text { C } & -2.54248922 & 1.20427461 & -0.59478977\end{array}$

C $\quad-3.26840379-1.19762496 \quad 0.57501692$

$\begin{array}{llll}\mathrm{H} & -2.18363481 & -2.13647763 & -1.02007708\end{array}$

$\begin{array}{llll}\text { C } & -3.31597459 & 1.19568952 & 0.56550078\end{array}$

$\mathrm{H} \quad-2.26415100 \quad 2.15248783-1.04423966$

C $-3.69464452-0.00370656 \quad 1.17388950$

$\begin{array}{llll}\mathrm{H} & -3.54369619 & -2.14991437 & 1.02007808\end{array}$

$\begin{array}{llll}\mathrm{H} & -3.62180065 & 2.14416381 & 0.99212033\end{array}$

$\begin{array}{llll}\text { C } & -4.53996715 & -0.05666969 & 2.44347853\end{array}$

$\begin{array}{llll}\text { C } & -3.76244487 & -0.78545025 & 3.56601038\end{array}$

$\begin{array}{llll}\text { C } & -4.91701746 & 1.34504407 & 2.96228587\end{array}$

$\begin{array}{llll}\text { C } & -5.85204062 & -0.83187192 & 2.17279953\end{array}$

$\begin{array}{llll}\mathrm{H} & -2.83103438 & -0.24106459 & 3.76830268\end{array}$

$\begin{array}{llll}\mathrm{H} & -3.47867437 & -1.78991575 & 3.22948553\end{array}$

C $-4.61124185 \quad-0.88843809 \quad 4.84180831$

$\begin{array}{llll}\mathrm{H} & -5.47632020 & 1.88715758 & 2.18842696\end{array}$

$\begin{array}{llll}\mathrm{H} & -4.00477079 & 1.91984977 & 3.16957467\end{array}$

$\begin{array}{llll}\text { C } & -5.76599921 & 1.25060361 & 4.23877158\end{array}$

$\begin{array}{llll}\mathrm{H} & -5.61960130 & -1.83748244 & 1.80206655\end{array}$

$\begin{array}{llll}\mathrm{H} & -6.41315892 & -0.32064721 & 1.37995685\end{array}$

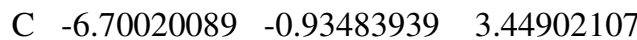

$\begin{array}{llll}\mathrm{H} & -4.03733810 & -1.41303158 & 5.61456131\end{array}$

$\begin{array}{llll}\text { C } & -4.97080815 & 0.52135616 & 5.33115893\end{array}$

$\begin{array}{llll}\text { C } & -5.89953965 & -1.66792241 & 4.53629991\end{array}$

$\begin{array}{llll}\mathrm{H} & -6.01438779 & 2.26306780 & 4.57758473\end{array}$

C $\quad-7.05702812 \quad 0.47501561 \quad 3.94019680$

$\begin{array}{llll}\mathrm{H} & -7.61757518 & -1.49256277 & 3.22747918\end{array}$

$\begin{array}{llll}\mathrm{H} & -4.05727909 & 1.08102100 & 5.56817727\end{array}$

$\mathrm{H} \quad-5.56487036 \quad 0.46151701 \quad 6.25168062$

$\mathrm{H} \quad-5.65288009 \quad-2.68308266 \quad 4.20017095$

H $\quad-6.50434883-1.76457701 \quad 5.44663910$

$\begin{array}{llll}\mathrm{H} & -7.67649447 & 0.41461098 & 4.84378099\end{array}$

$\begin{array}{llll}\mathrm{H} & -7.64379793 & 1.00135122 & 3.17690911\end{array}$

C $-2.02946611 \quad-4.95998198-1.15583815$

C $-1.41376856 \quad-4.94650463 \quad 0.09371355$
C $-3.41940432-4.99171882-1.24363450$

$\begin{array}{llll}\text { C } & -2.17801611 & -4.96454529 & 1.25693078\end{array}$

$\begin{array}{llll}\text { C } & -4.19883812 & -5.01008997 & -0.09054216\end{array}$

C $\quad-3.55902073-4.99605674 \quad 1.13927505$

$\mathrm{H}-1.42810220 \quad-4.94579924-2.05818916$

$\begin{array}{llll}\mathrm{H} & -0.33151762 & -4.92180491 & 0.16690623\end{array}$

$\mathrm{H} \quad-3.90326434 \quad-5.00231289 \quad-2.21471703$

H $\quad-1.72780437 \quad-4.95473466 \quad 2.24279747$

H $\quad-5.28178786 \quad-5.03482902 \quad-0.12698257$

$\begin{array}{llll}\text { F } & -4.30306410 & -5.01360729 & 2.25571719\end{array}$

4FPh_pi_345Me3Ph_side
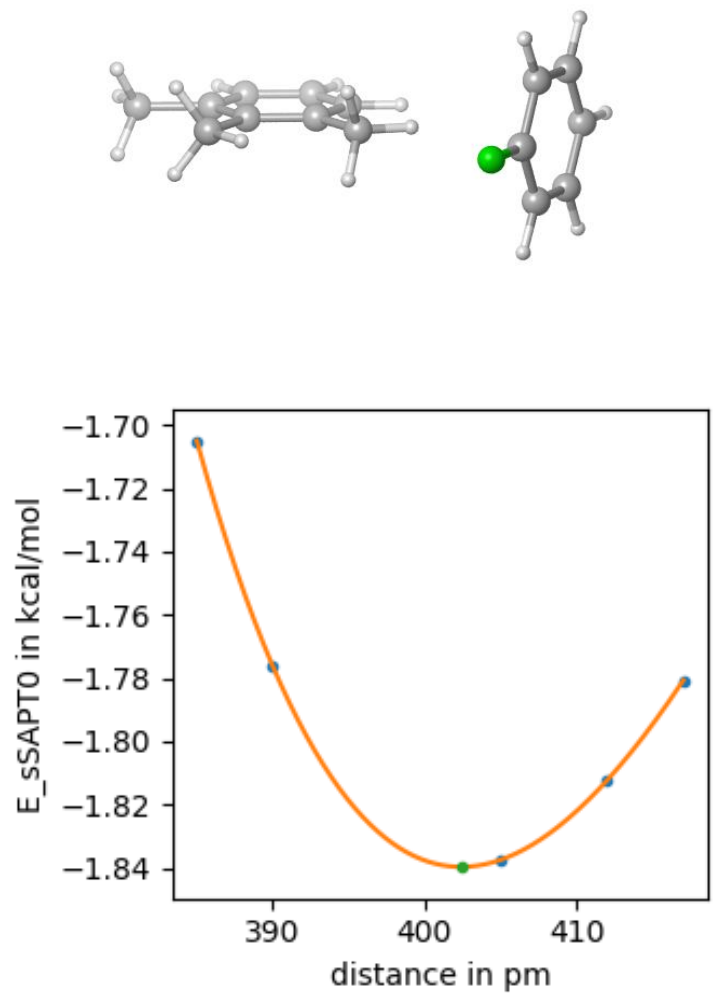

33

4FPh_pi_345Me3Ph_side-402

$\begin{array}{lllll}\mathrm{H} & 1.43033577 & -2.02108841 & 0.67995934\end{array}$

$\begin{array}{llll}\text { C } & 0.80506010 & -1.13502560 & 0.68022588\end{array}$

$\begin{array}{llll}\text { C } & 1.38751308 & 0.13043580 & 0.68095390\end{array}$

$\begin{array}{llll}\text { C } & -0.58241935 & -1.25970228 & 0.67984059\end{array}$

$\begin{array}{llll}\text { C } & 0.59243978 & 1.27294725 & 0.68130873\end{array}$

$\begin{array}{llll}\mathrm{H} & 2.46772101 & 0.23234300 & 0.68126542\end{array}$

C $-1.39240833-0.12771542 \quad 0.68017513$

$\begin{array}{llll}\mathrm{H} & -1.04044299 & -2.24329166 & 0.67927083\end{array}$

C $\quad-0.78531367 \quad 1.11865898 \quad 0.68090511$

$\begin{array}{llll}\mathrm{H} & 1.01642536 & 2.27042201 & 0.68188397\end{array}$

$\begin{array}{llll}\mathrm{H} & -2.47429127 & -0.19290188 & 0.67988608\end{array}$

$\begin{array}{llll}\text { F } & -1.55894215 & 2.21494839 & 0.68123770\end{array}$

$\begin{array}{llll}\text { C } & 0.41215688 & -0.57434670 & -4.28348032\end{array}$ 


$\begin{array}{rrrr}\mathrm{C} & -0.39309055 & 0.56675056 & -4.26134112 \\ \mathrm{H} & 0.72448777 & -1.01761092 & -3.34188257 \\ \mathrm{C} & -0.80576870 & 1.15198016 & -2.93157975 \\ \mathrm{C} & 0.81522790 & -1.14407195 & -5.48407542 \\ \mathrm{C} & -0.79995037 & 1.14395341 & -5.47541670 \\ \mathrm{H} & -0.45769503 & 2.18348691 & -2.81757492 \\ \mathrm{H} & -1.89396254 & 1.16055420 & -2.81303404 \\ \mathrm{H} & -0.38702545 & 0.56736246 & -2.10998163 \\ \mathrm{H} & 1.44106067 & -2.03080061 & -5.48513152 \\ \mathrm{C} & 0.41175331 & -0.56996754 & -6.68345963 \\ \mathrm{C} & -0.39231873 & 0.56918777 & -6.69208129 \\ \mathrm{C} & -1.66881043 & 2.37755984 & -5.50997470 \\ \mathrm{H} & 0.72199716 & -1.00726404 & -7.62858274 \\ \mathrm{C} & -0.82096391 & 1.17945935 & -8.00199997 \\ \mathrm{H} & -1.15795922 & 3.20455478 & -6.01566584 \\ \mathrm{H} & -2.59401463 & 2.19196647 & -6.06624317 \\ \mathrm{H} & -1.94496393 & 2.71966562 & -4.51308821 \\ \mathrm{H} & -1.91174171 & 1.18921042 & -8.10244438 \\ \mathrm{H} & -0.48478455 & 2.21820859 & -8.09021292 \\ \mathrm{H} & -0.41093220 & 0.62025353 & -8.84528922\end{array}$

4FPh_pi_35Me24OMePh_side
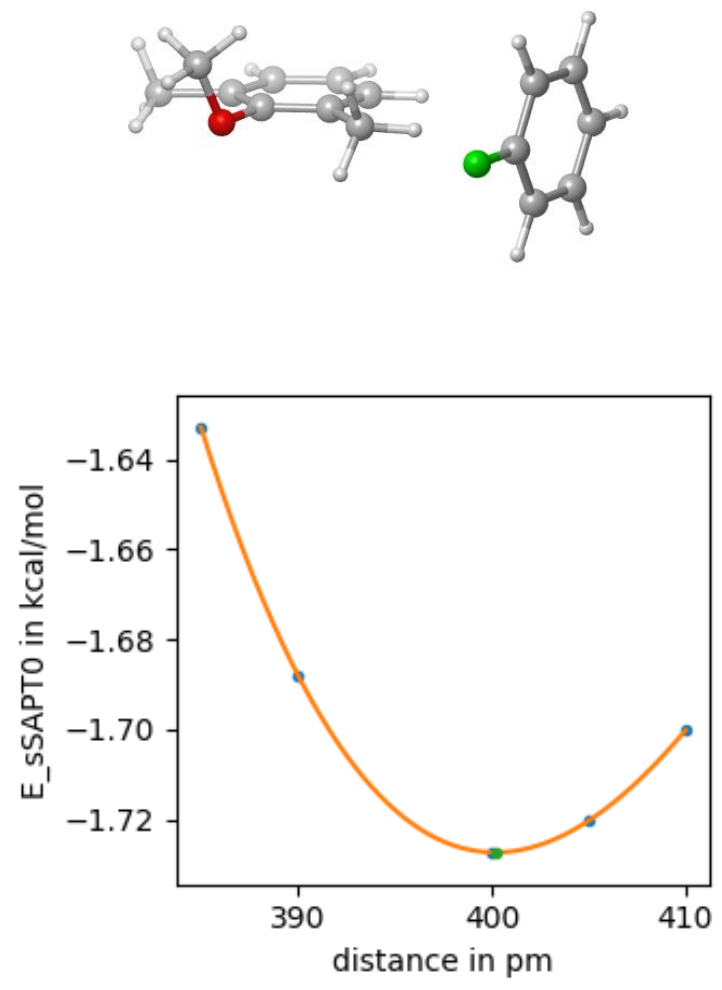

34

4FPh_pi_35Me24OMePh_side-400

H $\quad 0.32570696 \quad-2.45264196 \quad 0.73573242$

C $\quad 0.18471230 \quad-1.37737583 \quad 0.73627381$

$\begin{array}{llll}\text { C } & 1.28923753 & -0.52845444 & 0.73729725\end{array}$ $\begin{array}{llll}\text { C } & -1.10133929 & -0.84191821 & 0.73594698\end{array}$

$\begin{array}{llll}\text { C } & 1.11732418 & 0.85282011 & 0.73800574\end{array}$

$\begin{array}{llll}\mathrm{H} & 2.29273330 & -0.94104446 & 0.73756453\end{array}$

$\begin{array}{llll}\text { C } & -1.29135281 & 0.53698385 & 0.73663339\end{array}$

$\begin{array}{llll}\mathrm{H} & -1.96453751 & -1.49927826 & 0.73514795\end{array}$

$\begin{array}{llll}\text { C } & -0.17390198 & 1.35754232 & 0.73765212\end{array}$

$\begin{array}{llll}\mathrm{H} & 1.95685922 & 1.53831282 & 0.73881615\end{array}$

$\begin{array}{llll}\mathrm{H} & -2.27923933 & 0.98285404 & 0.73639836\end{array}$

$\begin{array}{llll}\text { F } & -0.34834926 & 2.68792546 & 0.73832478\end{array}$

C $\quad 0.10013752 \quad-0.69042039-4.23120546$

C $\quad-0.08629432 \quad 0.69030050-4.20137649$

$\begin{array}{llll}\mathrm{H} & 0.16831119 & -1.23486487 & -3.29342724\end{array}$

C $\quad-0.21318206 \quad 1.45404371 \quad-2.91024038$

$\begin{array}{llll}\text { C } & 0.19213850 & -1.37087673 & -5.44250994\end{array}$

$\begin{array}{llll}\text { C } & -0.17691772 & 1.37076652 & -5.42115226\end{array}$

H $\quad 0.61268926 \quad 2.16047372-2.77862501$

$\mathrm{H} \quad-1.13597982 \quad 2.03924315 \quad-2.89592975$

H $\quad-0.213834620 .77291696 \quad-2.05693281$

$\begin{array}{llll}\mathrm{H} & 0.33388362 & -2.44667109 & -5.45143317\end{array}$

C $\quad 0.08657973-0.67327098-6.63969662$

$\begin{array}{llll}\text { C } & -0.09966960 & 0.71099334 & -6.65110770\end{array}$

$\begin{array}{llll}\text { O } & -0.39290934 & 2.73420825 & -5.38745943\end{array}$

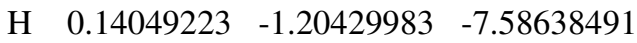

$\begin{array}{llll}\text { C } & -0.22942015 & 1.47524017 & -7.94265205\end{array}$

$\begin{array}{lllll}\text { C } & 0.79779568 & 3.48993061 & -5.52888583\end{array}$

$\begin{array}{llll}\mathrm{H} & -0.82852519 & 2.37647167 & -7.79788169\end{array}$

H $\quad 0.75009164 \quad 1.78082170 \quad-8.32701067$

$\begin{array}{llll}\mathrm{H} & -0.70162429 & 0.85704271 & -8.70969925\end{array}$

H $\quad 1.26455960 \quad 3.31987558 \quad-6.50664548$

$\mathrm{H} \quad 0.52008049 \quad 4.54108810 \quad-5.43999957$

H $\quad 1.52287742 \quad 3.23628039 \quad-4.74582649$

4FPh_pi_35Me2Ph_side
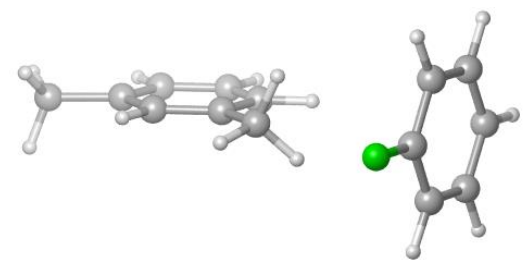


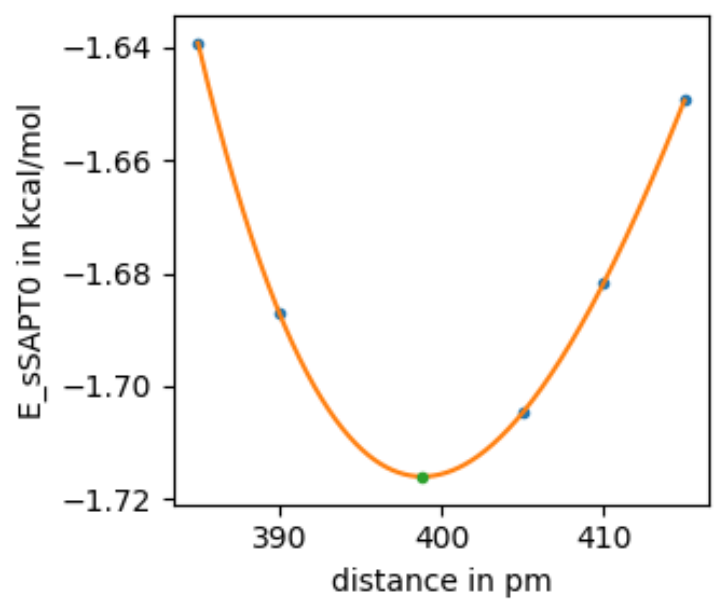

30

4FPh_pi_35Me2Ph_side-398

$\begin{array}{lrrr}\mathrm{H} & 0.04628355 & 2.47314262 & 0.76063106 \\ \mathrm{C} & 0.02778926 & 1.38882964 & 0.76123881 \\ \mathrm{C} & -1.18946164 & 0.71137507 & 0.76094363 \\ \mathrm{C} & 1.22122838 & 0.67025774 & 0.76231602 \\ \mathrm{C} & -1.22232610 & -0.68016847 & 0.76172699 \\ \mathrm{H} & -2.12145996 & 1.26690436 & 0.76011490 \\ \mathrm{C} & 1.20661626 & -0.72159798 & 0.76309791 \\ \mathrm{H} & 2.17162959 & 1.19368064 & 0.76254698 \\ \mathrm{C} & -0.01925344 & -1.36909856 & 0.76278590 \\ \mathrm{H} & -2.15345362 & -1.23489498 & 0.76153166 \\ \mathrm{H} & 2.11828573 & -1.30775336 & 0.76393844 \\ \mathrm{~F} & -0.04213537 & -2.71067504 & 0.76354066 \\ \mathrm{C} & 0.01892250 & 0.69886102 & -4.18544186 \\ \mathrm{C} & -0.00512688 & -0.69898105 & -4.16318671 \\ \mathrm{H} & 0.02767097 & 1.24863186 & -3.24783844 \\ \mathrm{C} & -0.04377408 & -1.44223063 & -2.85189691 \\ \mathrm{C} & 0.02892067 & 1.38329698 & -5.39429437 \\ \mathrm{C} & -0.01375134 & -1.38341204 & -5.37709990 \\ \mathrm{H} & -1.03355665 & -1.36913206 & -2.38975155 \\ \mathrm{H} & 0.18222021 & -2.50194054 & -2.98961593 \\ \mathrm{H} & 0.67876320 & -1.02897260 & -2.14284052 \\ \mathrm{H} & 0.04489746 & 2.46882464 & -5.40173943 \\ \mathrm{C} & 0.01780302 & 0.68200197 & -6.59799528 \\ \mathrm{C} & -0.00075512 & -0.71199223 & -6.60300873 \\ \mathrm{H} & -0.03147550 & -2.47186169 & -5.37098228 \\ \mathrm{H} & 0.02485360 & 1.22108845 & -7.54144520 \\ \mathrm{C} & 0.01971295 & -1.48620098 & -7.89662130 \\ \mathrm{H} & -0.63573714 & -2.35959555 & -7.84573914 \\ \mathrm{H} & -0.30397387 & -0.86506581 & -8.73489291 \\ \mathrm{H} & 1.02913698 & -1.84672113 & -8.12033911\end{array}$

4MePh_pi0_4AdPh_side_1
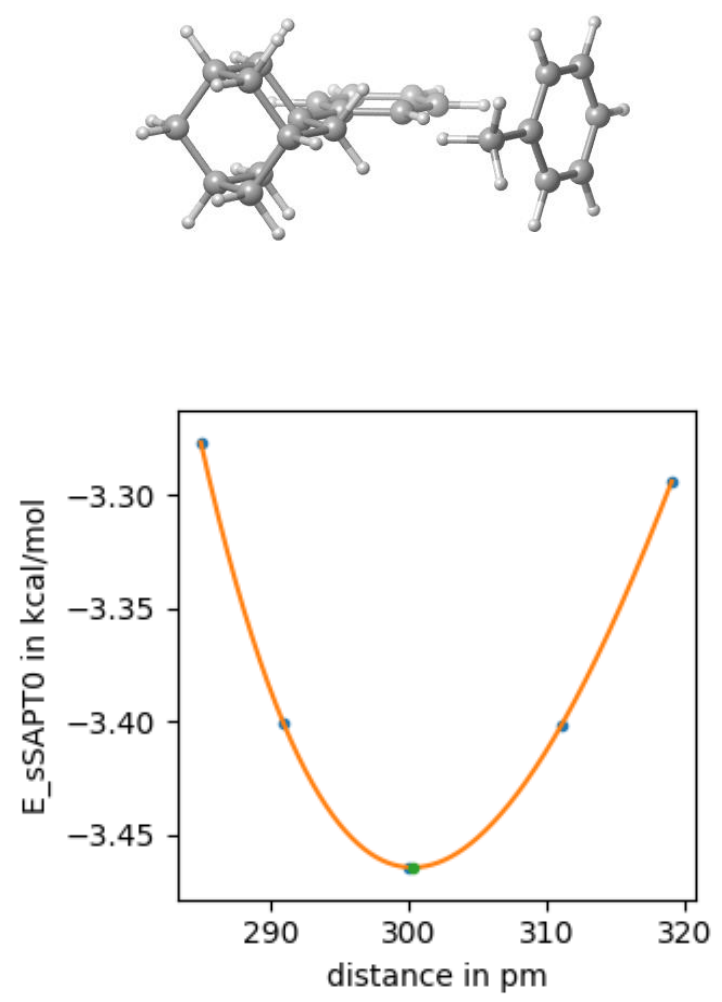

51

4MePh_pi0_4AdPh_side_1-300

H $\quad-1.50883247 \quad-1.43235058-2.09999916$

C $-2.09455250 \quad-1.46287940 \quad-1.18698610$

$\begin{array}{llll}\text { C } & -2.42507406 & -2.68346364 & -0.60210854\end{array}$

$\begin{array}{llll}\text { C } & -2.52414812 & -0.28612698 & -0.58673313\end{array}$

$\begin{array}{llll}\text { C } & -3.17513195 & -2.71721352 & 0.56676568\end{array}$

$\begin{array}{llll}\mathrm{H} & -2.09756006 & -3.61273871 & -1.05784702\end{array}$

C $-3.27681273-0.32564962 \quad 0.58653641$

$\begin{array}{llll}\mathrm{H} & -2.27517103 & 0.67327904 & -1.02960577\end{array}$

C $-3.61763478-1.54026723 \quad 1.18705542$

$\begin{array}{llll}\mathrm{H} & -3.42104939 & -3.68057464 & 1.00514956\end{array}$

$\begin{array}{llll}\mathrm{H} & -3.59643132 & 0.61065269 & 1.02961569\end{array}$

$\begin{array}{llll}\text { C } & -4.43919340 & -1.62704475 & 2.47031021\end{array}$

$\begin{array}{llll}\text { C } & -3.62590493 & -2.35080831 & 3.57052289\end{array}$

$\begin{array}{llll}\text { C } & -4.83861270 & -0.24033079 & 3.01217559\end{array}$

$\begin{array}{llll}\text { C } & -5.73799197 & -2.42873272 & 2.21309224\end{array}$

$\mathrm{H} \quad-2.70360711 \quad-1.78773576 \quad 3.76318698$

$\begin{array}{llll}\mathrm{H} & -3.32549327 & -3.34462148 & 3.21723288\end{array}$

$\begin{array}{llll}\text { C } & -4.44969987 & -2.48774472 & 4.85945016\end{array}$

$\begin{array}{llll}\mathrm{H} & -5.42345786 & 0.29778132 & 2.25457693\end{array}$

$\begin{array}{llll}\mathrm{H} & -3.93606931 & 0.35265845 & 3.21052727\end{array}$

C $-5.66277302-0.36873472 \quad 4.30189532$

$\begin{array}{llll}\mathrm{H} & -5.48946360 & -3.42446496 & 1.82647299\end{array}$

$\begin{array}{llll}\mathrm{H} & -6.32428782 & -1.92132236 & 1.43617791\end{array}$

$\begin{array}{llll}\text { C } & -6.56114350 & -2.56563915 & 3.50243228\end{array}$ 


$\begin{array}{lrrr}\mathrm{H} & -3.85067403 & -3.00799761 & 5.61589978 \\ \mathrm{C} & -4.83234015 & -1.09221637 & 5.37165993 \\ \mathrm{C} & -5.72524563 & -3.29277194 & 4.56694957 \\ \mathrm{H} & -5.92795746 & 0.63385319 & 4.65695824 \\ \mathrm{C} & -6.94101524 & -1.17000875 & 4.01642027 \\ \mathrm{H} & -7.46944710 & -3.14150437 & 3.29012220 \\ \mathrm{H} & -3.92765306 & -0.51467578 & 5.59953245 \\ \mathrm{H} & -5.40869645 & -1.17613715 & 6.30151020 \\ \mathrm{H} & -5.46168172 & -4.29812523 & 4.21456801 \\ \mathrm{H} & -6.31169269 & -3.41362360 & 5.48636701 \\ \mathrm{H} & -7.54304910 & -1.25487800 & 4.92976802 \\ \mathrm{H} & -7.55277547 & -0.64841587 & 3.26967642 \\ \mathrm{C} & -3.80585122 & 3.61450542 & 1.18160791 \\ \mathrm{C} & -2.41075279 & 3.66836511 & 1.22812246 \\ \mathrm{C} & -4.42827523 & 3.59317642 & -0.06850157 \\ \mathrm{C} & -4.61812904 & 3.55259773 & 2.45056664 \\ \mathrm{C} & -1.65658100 & 3.69531677 & 0.05875919 \\ \mathrm{H} & -1.91178444 & 3.69112601 & 2.19370441 \\ \mathrm{C} & -3.67901072 & 3.62003605 & -1.24130491 \\ \mathrm{H} & -5.51347258 & 3.55685413 & -0.12123994 \\ \mathrm{H} & -4.75769665 & 2.51623768 & 2.77504292 \\ \mathrm{H} & -4.12277570 & 4.08805739 & 3.26401705 \\ \mathrm{H} & -5.60925824 & 3.98947955 & 2.30784276 \\ \mathrm{C} & -2.28896573 & 3.67088229 & -1.18160277 \\ \mathrm{H} & -0.57336084 & 3.73966606 & 0.11639309 \\ \mathrm{H} & -4.18215413 & 3.60527323 & -2.20321827 \\ \mathrm{H} & -1.70276061 & 3.69552926 & -2.09496143\end{array}$

4MePh_pi0_4AdPh_side_2

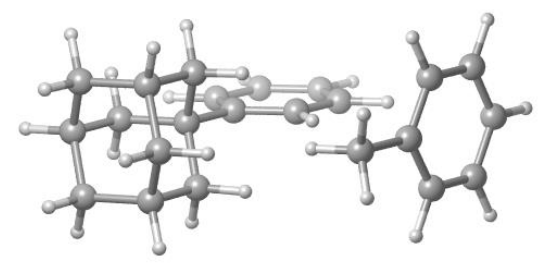

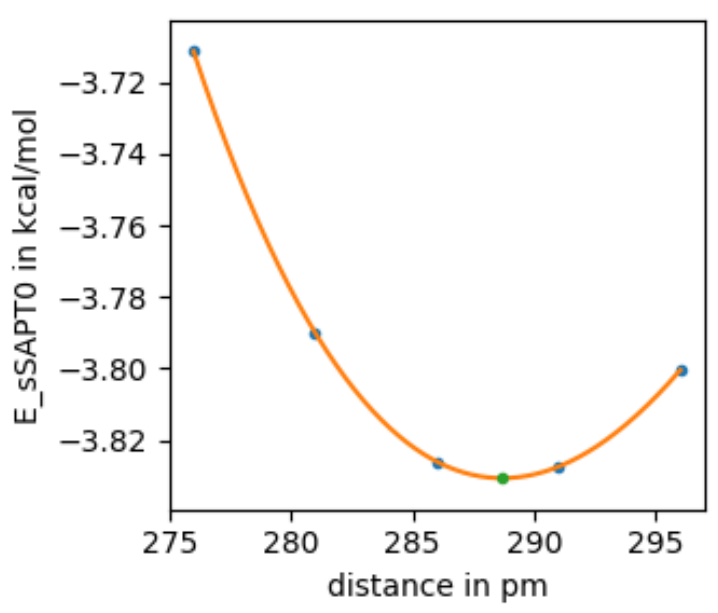

51

4MePh_pi0_4AdPh_side_2-288

$\mathrm{H} \quad-1.54480041 \quad 1.01739873 \quad-2.07321011$

$\begin{array}{llll}\text { C } & -2.14902739 & 1.01089299 & -1.17184434\end{array}$

$\begin{array}{llll}\text { C } & -2.51878414 & -0.19492024 & -0.57985909\end{array}$

$\begin{array}{llll}\text { C } & -2.56350649 & 2.20405488 & -0.59376643\end{array}$

$\begin{array}{llll}\text { C } & -3.29241372 & -0.19791215 & 0.57403914\end{array}$

$\begin{array}{llll}\mathrm{H} & -2.20356414 & -1.13667284 & -1.01832637\end{array}$

$\begin{array}{llll}\text { C } & -3.33996014 & 2.19540288 & 0.56453938\end{array}$

$\begin{array}{llll}\mathrm{H} & -2.28401846 & 3.15229402 & -1.04244745\end{array}$

$\begin{array}{llll}\text { C } & -3.72018648 & 0.99597172 & 1.17188732\end{array}$

$\begin{array}{llll}\mathrm{H} & -3.56884471 & -1.15022723 & 1.01833901\end{array}$

$\begin{array}{llll}\mathrm{H} & -3.64687757 & 3.14385257 & 0.99042924\end{array}$

$\begin{array}{llll}\text { C } & -4.56875701 & 0.94293537 & 2.43930476\end{array}$

$\begin{array}{llll}\text { C } & -3.79411137 & 0.21408995 & 3.56378163\end{array}$

$\begin{array}{llll}\text { C } & -4.94713452 & 2.34461922 & 2.95722591\end{array}$

$\begin{array}{llll}\text { C } & -5.88013309 & 0.16774889 & 2.16522245\end{array}$

$\begin{array}{llll}\mathrm{H} & -2.86322193 & 0.75846386 & 3.76848949\end{array}$

$\begin{array}{llll}\mathrm{H} & -3.50948010 & -0.79035615 & 3.22792647\end{array}$

$\begin{array}{llll}\text { C } & -4.64617214 & 0.11102854 & 4.83739617\end{array}$

$\begin{array}{llll}\mathrm{H} & -5.50445406 & 2.88677744 & 2.18196878\end{array}$

$\begin{array}{llll}\mathrm{H} & -4.03542163 & 2.91941287 & 3.16688293\end{array}$

C $\quad-5.79938182 \quad 2.25010515 \quad 4.23152824$

$\begin{array}{llll}\mathrm{H} & -5.64674524 & -0.83784025 & 1.79502778\end{array}$

$\begin{array}{llll}\mathrm{H} & -6.43921957 & 0.67901941 & 1.37097519\end{array}$

$\begin{array}{llll}\text { C } & -6.73155822 & 0.06470782 & 3.43926222\end{array}$

$\begin{array}{llll}\mathrm{H} & -4.07424880 & -0.41360960 & 5.61158578\end{array}$

$\begin{array}{llll}\text { C } & -5.00699028 & 1.52079458 & 5.32590593\end{array}$

$\begin{array}{llll}\text { C } & -5.93368343 & -0.66843802 & 4.52854520\end{array}$

$\begin{array}{lllll}\mathrm{H} & -6.04863714 & 3.26254981 & 4.56976274\end{array}$

$\begin{array}{llll}\text { C } & -7.08964197 & 1.47453450 & 3.92960411\end{array}$

$\begin{array}{llll}\mathrm{H} & -7.64836223 & -0.49300268 & 3.21534001\end{array}$

$\begin{array}{llll}\mathrm{H} & -4.09407111 & 2.08044565 & 5.56529480\end{array}$

$\mathrm{H} \quad-5.60340745 \quad 1.46090235 \quad 6.24490010$

$\mathrm{H} \quad-5.68616400 \quad-1.68357889 \quad 4.19299030$

$\begin{array}{llll}\mathrm{H} & -6.54082147 & -0.76514512 & 5.43732727\end{array}$

$\begin{array}{llll}\mathrm{H} & -7.71141984 & 1.41407777 & 4.83159575\end{array}$

$\begin{array}{llll}\mathrm{H} & -7.67445556 & 2.00091422 & 3.16484693\end{array}$

$\begin{array}{llll}\text { C } & -3.60291819 & -4.04713242 & 1.16654445\end{array}$

$\begin{array}{llll}\text { C } & -4.20101762 & -4.06258937 & -0.09575593\end{array}$ 


$\begin{array}{cccc}\text { C } & -2.20853215 & -4.01752820 & 1.24037848 \\ \text { C } & -4.44360182 & -4.03438803 & 2.41833225 \\ \text { C } & -3.42879813 & -4.04409556 & -1.25344630 \\ \text { H } & -5.28524513 & -4.09122482 & -0.16969317 \\ \text { C } & -1.43120304 & -3.99900967 & 0.08582102 \\ \text { H } & -1.72806395 & -4.01073246 & 2.21549799 \\ \text { H } & -4.69359938 & -3.00930202 & 2.71074634 \\ \text { H } & -5.38289776 & -4.57236675 & 2.26968635 \\ \text { H } & -3.91442561 & -4.49599549 & 3.25526954 \\ \text { C } & -2.03940748 & -4.01187002 & -1.16649553 \\ \text { H } & -3.91290655 & -4.05907030 & -2.22507855 \\ \text { H } & -0.34861496 & -3.97856093 & 0.16477313 \\ \text { H } & -1.43508061 & -4.00109913 & -2.06823882\end{array}$

4OMePh_pi0_4AdPh_side_1
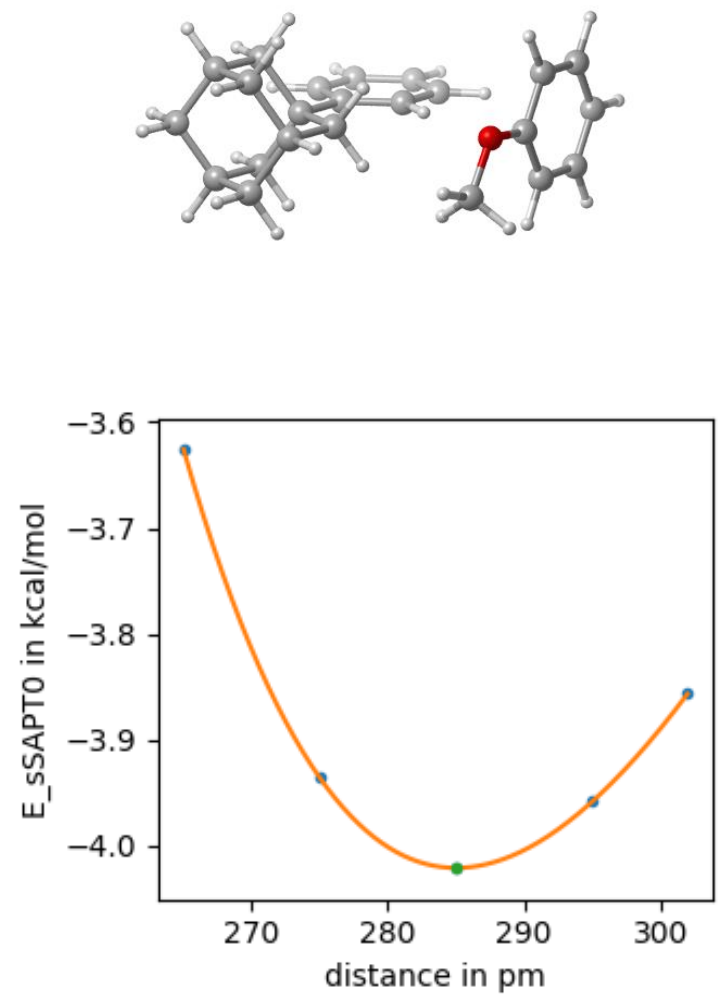

52

4OMePh_pi0_4AdPh_side_1-285

$\begin{array}{llll}\mathrm{H} & -1.20091542 & 0.08644425 & -1.81524029\end{array}$

C $\quad-1.94484149 \quad 0.05003084-1.02603760$

$\begin{array}{llll}\text { C } & -2.37670781 & -1.17432298 & -0.52046729\end{array}$

$\begin{array}{llll}\text { C } & -2.47845091 & 1.22291440 & -0.50718557\end{array}$

$\begin{array}{llll}\text { C } & -3.32930715 & -1.21560653 & 0.48990045\end{array}$

H $\quad-1.97022210 \quad-2.10066065 \quad-0.91440008$

$\begin{array}{llll}\text { C } & -3.43441865 & 1.17582976 & 0.50698217\end{array}$

H $\quad-2.15273528 \quad 2.18517476-0.89000406$

$\begin{array}{llll}\text { C } & -3.87929538 & -0.04265824 & 1.02606827\end{array}$

$\begin{array}{llll}\mathrm{H} & -3.65118579 & -2.18179308 & 0.86884394\end{array}$

$\begin{array}{lrrr}\mathrm{H} & -3.83081572 & 2.10927624 & 0.88997606 \\ \mathrm{C} & -4.92321591 & -0.13770666 & 2.13531463 \\ \mathrm{C} & -4.32615129 & -0.86950910 & 3.36157675 \\ \mathrm{C} & -5.41653994 & 1.24551445 & 2.60378046 \\ \mathrm{C} & -6.15185388 & -0.93678867 & 1.63766104 \\ \mathrm{H} & -3.45551630 & -0.30835553 & 3.72476375 \\ \mathrm{H} & -3.96514253 & -1.86106822 & 3.06289837 \\ \mathrm{C} & -5.37328315 & -1.01475267 & 4.47564245 \\ \mathrm{H} & -5.85209516 & 1.78917688 & 1.75508321 \\ \mathrm{H} & -4.56638830 & 1.83655756 & 2.96912200 \\ \mathrm{C} & -6.46418241 & 1.10879795 & 3.71861616 \\ \mathrm{H} & -5.83569633 & -1.93000550 & 1.29665043 \\ \mathrm{H} & -6.58525567 & -0.42369398 & 0.76950167 \\ \mathrm{C} & -7.19842944 & -1.08200551 & 2.75225121 \\ \mathrm{H} & -4.92358980 & -1.54055743 & 5.32594609 \\ \mathrm{C} & -5.84465599 & 0.37747520 & 4.91811390 \\ \mathrm{C} & -6.57256226 & -1.81694682 & 3.94757375 \\ \mathrm{H} & -6.79089966 & 2.10909711 & 4.02558915 \\ \mathrm{C} & -7.66740893 & 0.31031046 & 3.19698129 \\ \mathrm{H} & -8.05168119 & -1.65582570 & 2.37224649 \\ \mathrm{H} & -4.99782785 & 0.95286636 & 5.31275914 \\ \mathrm{H} & -6.58244749 & 0.28754977 & 5.72522476 \\ \mathrm{H} & -6.24793016 & -2.82002864 & 3.64291853 \\ \mathrm{H} & -7.31832543 & -1.94372436 & 4.74231802 \\ \mathrm{H} & -8.42739634 & 0.21956598 & 3.98313410 \\ \mathrm{H} & -8.13140808 & 0.83739675 & 2.35387933 \\ \mathrm{C} & -4.05261085 & 4.95936627 & 1.01658569 \\ \mathrm{C} & -2.69650607 & 5.00986118 & 1.34051808 \\ \mathrm{C} & -4.45081073 & 4.94505013 & -0.32475490 \\ \mathrm{C} & -1.75009198 & 5.04561417 & 0.31524741 \\ \mathrm{C} & -3.49796831 & 4.98103693 & -1.33031791 \\ \mathrm{C} & -2.13814434 & 5.03167739 & -1.01757946 \\ \mathrm{O} & -5.05949486 & 4.92139485 & 1.93034475 \\ \mathrm{H} & -2.36785669 & 5.02171037 & 2.37252688 \\ \mathrm{H} & -5.51207881 & 4.90551686 & -0.54424036 \\ \mathrm{H} & -0.69663553 & 5.08483838 & 0.57398797 \\ \mathrm{H} & -3.81780630 & 4.96952290 & -2.36746399 \\ \mathrm{H} & -1.39424351 & 5.05977570 & -1.80601466 \\ \mathrm{C} & -4.70060366 & 4.93413088 & 3.29385019 \\ \mathrm{H} & -5.63352889 & 4.89903106 & 3.85531202 \\ \mathrm{H} & -4.08742922 & 4.06324471 & 3.55441375 \\ \mathrm{H} & -4.15406082 & 5.84806017 & 3.55527691\end{array}$

4OMePh_pi0_4AdPh_side_2 


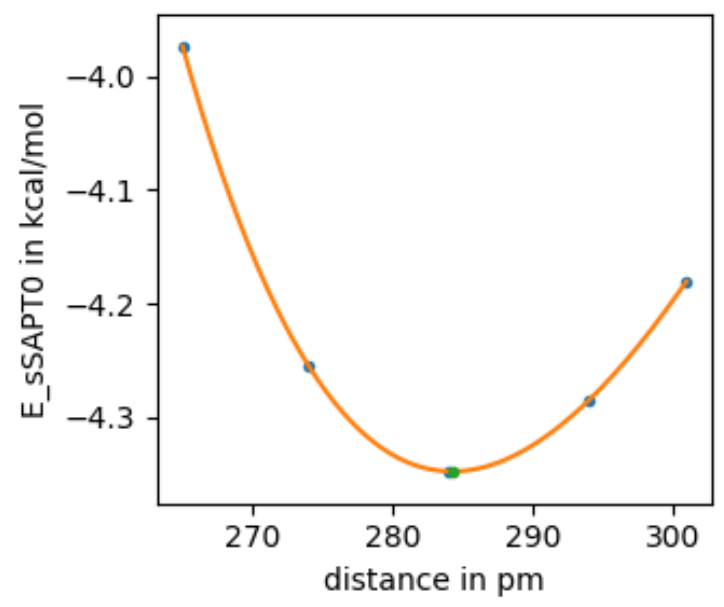

52

4OMePh_pi0_4AdPh_side_2-284

$\begin{array}{lrrc}\mathrm{H} & -1.87492337 & 1.00995731 & -2.25986224 \\ \mathrm{C} & -2.33562470 & 1.00668683 & -1.27734690 \\ \mathrm{C} & -2.61111769 & -0.19700157 & -0.63206402 \\ \mathrm{C} & -2.65805706 & 2.20192358 & -0.64722883 \\ \mathrm{C} & -3.20101059 & -0.19585180 & 0.62571890 \\ \mathrm{H} & -2.36571449 & -1.14032793 & -1.1000523 \\ \mathrm{C} & -3.25007224 & 2.19742908 & 0.61535792 \\ \mathrm{H} & -2.45001418 & 3.14855225 & -1.13630756 \\ \mathrm{C} & -3.53358832 & 1.00017790 & 1.27739036 \\ \mathrm{H} & -3.40669436 & -1.14657214 & 1.11001787 \\ \mathrm{H} & -3.48917584 & 3.14740738 & 1.07958630 \\ \mathrm{C} & -4.18034683 & 0.95169067 & 2.65890553 \\ \mathrm{C} & -3.24410174 & 0.22648919 & 3.65553369 \\ \mathrm{C} & -4.47626203 & 2.35523333 & 3.22338021 \\ \mathrm{C} & -5.51790653 & 0.17591269 & 2.58929935 \\ \mathrm{H} & -2.29308669 & 0.77131767 & 3.71499569 \\ \mathrm{H} & -3.01337288 & -0.77917191 & 3.28386777 \\ \mathrm{C} & -3.89335833 & 0.12799927 & 5.04387399 \\ \mathrm{H} & -5.14473640 & 2.89488507 & 2.53965349 \\ \mathrm{H} & -3.54346038 & 2.93050324 & 3.29053544 \\ \mathrm{C} & -5.12560104 & 2.26529308 & 4.61239943 \\ \mathrm{H} & -5.34303154 & -0.83099544 & 2.19145735 \\ \mathrm{H} & -6.19099625 & 0.68461251 & 1.88717495 \\ \mathrm{C} & -6.16647041 & 0.07744440 & 3.97796361 \\ \mathrm{H} & -3.21061500 & -0.39413993 & 5.72427332 \\ \mathrm{C} & -4.17637453 & 1.53951906 & 5.57658919 \\ \mathrm{C} & -5.21259476 & -0.65218370 & 4.93629992 \\ \mathrm{H} & -5.32100388 & 3.27895165 & 4.98103071 \\ \mathrm{C} & -6.44650624 & 1.48903038 & 4.51207534 \\ \mathrm{H} & -7.10646994 & -0.48078988 & 3.89742084 \\ \mathrm{H} & -3.23787243 & 2.09974773 & 5.67300192 \\ \mathrm{H} & -4.62667168 & 1.48292059 & 6.57552858 \\ \mathrm{H} & -5.01850112 & -1.66852886 & 4.57058949 \\ \mathrm{H} & -5.67502649 & -0.74562893 & 5.92689499 \\ \mathrm{H} & -6.92444700 & 1.43181592 & 5.49805242 \\ \mathrm{H} & -7.14056256 & 2.01294671 & 3.84294635 \\ \mathrm{C} & -3.41677960 & -3.99820625 & 1.26211405 \\ \mathrm{C} & -4.21612573 & -4.01628351 & 0.11877637\end{array}$

$\begin{array}{llll}\text { C } & -2.02316537 & -3.96729492 & 1.14026762\end{array}$

$\begin{array}{llll}\text { C } & -3.61032539 & -4.00319109 & -1.13859884\end{array}$

C $-1.43805293 \quad-3.95466343 \quad-0.11585521$

$\begin{array}{llll}\text { C } & -2.22835558 & -3.97254415 & -1.26676487\end{array}$

$\begin{array}{llll}\text { O } & -3.89667088 & -4.00849177 & 2.53483217\end{array}$

$\begin{array}{llll}\mathrm{H} & -5.29653678 & -4.04025669 & 0.19178486\end{array}$

$\begin{array}{llll}\mathrm{H} & -1.42924420 & -3.95384411 & 2.04751809\end{array}$

H $-4.23788171-4.01738125 \quad-2.02416499$

$\mathrm{H} \quad-0.35610107 \quad-3.93066302-0.19895180$

H -1.76840998 -3.96261459 -2.24868109

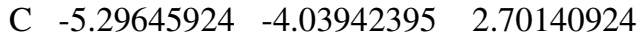

H $\quad-5.47590542 \quad-4.04306064 \quad 3.77593552$

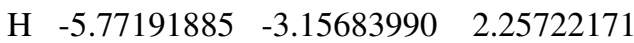

$\begin{array}{llll}\mathrm{H} & -5.73239056 & -4.94246135 & 2.25777978\end{array}$

4OMePh_pi0_4CF3Ph_side_1
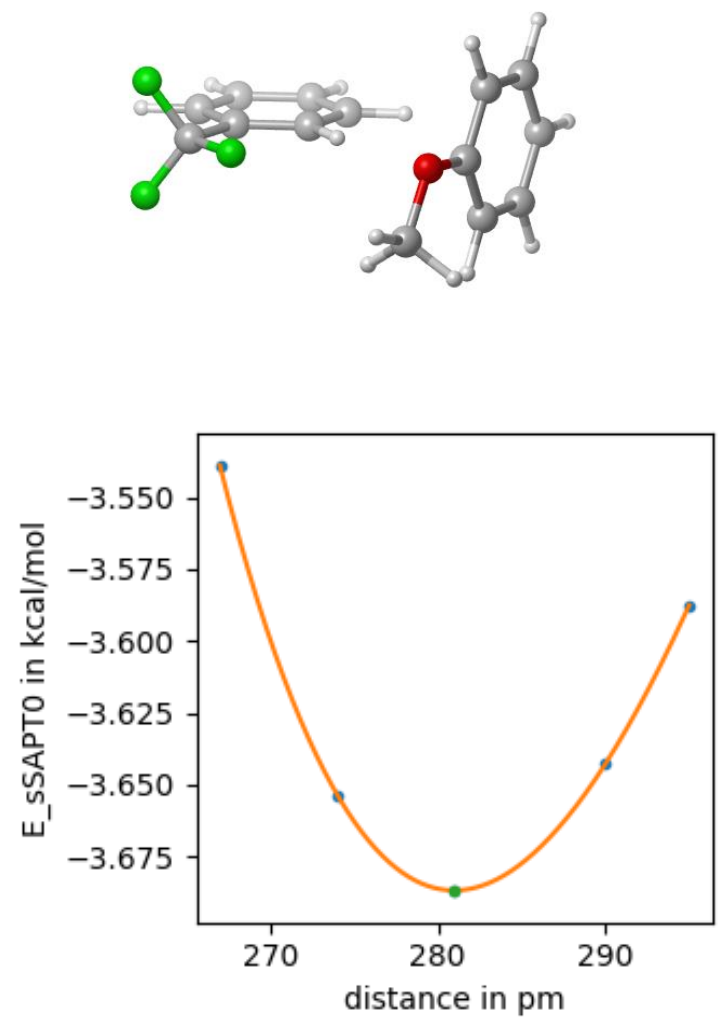

31

4OMePh_pi0_4CF3Ph_side_1-280

H $\quad-2.65599297 \quad-0.65268927 \quad-2.14870733$

C $-2.11546924 \quad-0.64385584-1.20781386$

$\begin{array}{llll}\text { C } & -1.75256867 & -1.84741268 & -0.60410744\end{array}$

$\begin{array}{llll}\text { C } & -1.78670708 & 0.56740179 & -0.60770023\end{array}$

C $-1.06072116-1.83802051 \quad 0.60006338$

H $\quad-2.00952121-2.79114648-1.07316600$

C $-1.09294788 \quad 0.58277515 \quad 0.59997114$

$\begin{array}{llll}\mathrm{H} & -2.06965253 & 1.50278606 & -1.07854503\end{array}$

$\begin{array}{llll}\text { C } & -0.73447460 & -0.62050994 & 1.19597024\end{array}$ 


$\begin{array}{lrrc}\text { H } & -0.77164484 & -2.76705984 & 1.08143313 \\ \text { H } & -0.83044591 & 1.51932178 & 1.07833588 \\ \text { C } & 0.01331242 & -0.64533575 & 2.49787260 \\ \text { F } & -0.67649405 & -1.29843049 & 3.44653831 \\ \text { F } & 1.18916160 & -1.28147853 & 2.37520750 \\ \text { F } & 0.26842976 & 0.58521637 & 2.96175744 \\ \text { C } & -0.78983892 & 4.32936889 & 1.20814556 \\ \text { C } & -0.08801886 & 4.33867072 & 0.00238266 \\ \text { C } & -2.18898322 & 4.31133305 & 1.20243301 \\ \text { C } & -0.79618927 & 4.32979502 & -1.20035330 \\ \text { C } & -2.87642979 & 4.30272651 & -0.00077264 \\ \text { C } & -2.18425193 & 4.31190758 & -1.21333049 \\ \text { O } & -0.20589009 & 4.33663116 & 2.43662587 \\ \text { H } & 0.99489876 & 4.35263748 & -0.01456858 \\ \text { H } & -2.70564921 & 4.30446863 & 2.15586670 \\ \text { H } & -0.24419894 & 4.33710952 & -2.13497026 \\ \text { H } & -3.96172044 & 4.28873535 & 0.00625324 \\ \text { H } & -2.72418410 & 4.30514826 & -2.15366850 \\ \text { C } & 1.20311973 & 4.35468145 & 2.48640225 \\ \text { H } & 1.47116126 & 4.35787366 & 3.54232101 \\ \text { H } & 1.63192031 & 3.46718753 & 2.00598200 \\ \text { H } & 1.60902343 & 5.25309972 & 2.00639541\end{array}$

4OMePh_pi0_4CF3Ph_side_2
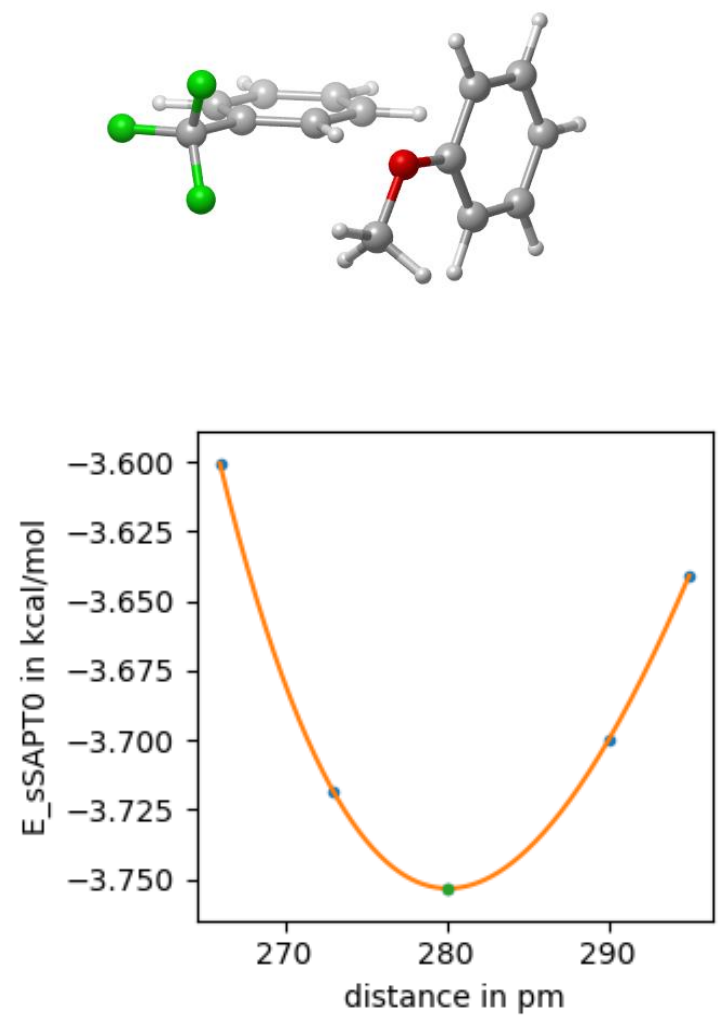

31

4OMePh_pi0_4CF3Ph_side_2-280

H $\quad-3.00181927 \quad 0.68507050 \quad-1.92426132$

$\begin{array}{lrrr}\text { C } & -2.31886113 & 0.69446450 & -1.08105057 \\ \text { C } & -1.86332438 & -0.50871191 & -0.54294265 \\ \text { C } & -1.90049521 & 1.90605905 & -0.54033383 \\ \text { C } & -0.98918566 & -0.49860221 & 0.53619952 \\ \text { H } & -2.19031645 & -1.45270904 & -0.96557682 \\ \text { C } & -1.02392075 & 1.92215190 & 0.54196304 \\ \text { H } & -2.25568696 & 2.84114672 & -0.96003198 \\ \text { C } & -0.57397847 & 0.71924264 & 1.07316386 \\ \text { H } & -0.62627831 & -1.42733858 & 0.96531844 \\ \text { H } & -0.68996432 & 2.85896766 & 0.97290277 \\ \text { C } & 0.37093930 & 0.69519236 & 2.23991297 \\ \text { F } & -0.15881857 & 0.04114185 & 3.28581300 \\ \text { F } & 1.51319041 & 0.06051268 & 1.93203354 \\ \text { F } & 0.69495465 & 1.92600591 & 2.65781797 \\ \text { C } & -0.48663711 & -4.22584599 & 1.08555989 \\ \text { C } & -1.86407754 & -4.24837448 & 1.30613280 \\ \text { C } & 0.01187355 & -4.21814529 & -0.22187672 \\ \text { C } & -2.73094953 & -4.26300689 & 0.21229702 \\ \text { C } & -0.86289930 & -4.23290223 & -1.29654662 \\ \text { C } & -2.24320569 & -4.25548408 & -1.08741667 \\ \text { O } & 0.44898146 & -4.21011786 & 2.07276979 \\ \text { H } & -2.26985360 & -4.25466755 & 2.31037340 \\ \text { H } & 1.08727140 & -4.20054173 & -0.36057155 \\ \text { H } & -3.80151601 & -4.28051515 & 0.39072507 \\ \text { H } & -0.46552682 & -4.22675333 & -2.30657544 \\ \text { H } & -2.92590160 & -4.26700353 & -1.92979522 \\ \text { C } & -0.01198116 & -4.21708954 & 3.40527759 \\ \text { H } & 0.87643495 & -4.20223384 & 4.03560844 \\ \text { H } & -0.62485655 & -3.33390058 & 3.62133319 \\ \text { H } & -0.59555466 & -5.11971903 & 3.62212254\end{array}$

4OMePh_piO_Ph_side

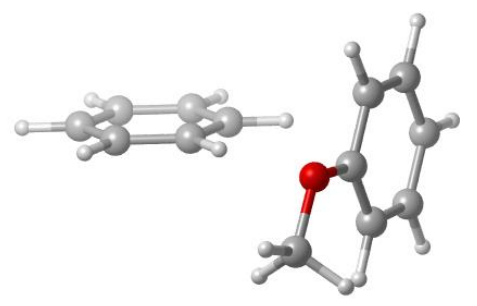




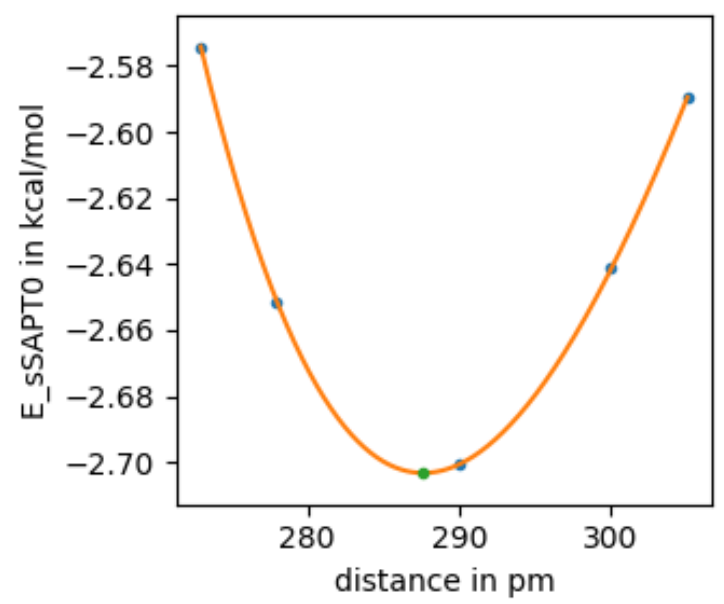

\begin{tabular}{lrrr}
\multicolumn{4}{l}{ 48 } \\
4OMePh_pi0_Ph_side-287 \\
H & 2.25553476 & -0.40994362 & 0.94239980 \\
C & 1.26777245 & -0.23049206 & 0.52973603 \\
C & 0.46381383 & 0.77650128 & 1.05904986 \\
C & 0.80339013 & -1.00703074 & -0.52943509 \\
C & -0.80375799 & 1.00669275 & 0.52970079 \\
H & 0.82540123 & 1.38176410 & 1.88463748 \\
C & -0.46407387 & -0.77671485 & -1.05905782 \\
H & 1.42975046 & -1.79186790 & -0.94221090 \\
C & -1.26757625 & 0.23012605 & -0.52943547 \\
H & -1.43041401 & 1.79141455 & 0.94234480 \\
H & -0.82591345 & -1.38192650 & -1.88461761 \\
H & -2.25564238 & 0.40958308 & -0.94210523 \\
C & -4.77768155 & 1.79516263 & -1.05857551 \\
C & -5.01734125 & 1.39362919 & 0.25589633 \\
C & -4.07244787 & 2.97709257 & -1.31086455 \\
C & -4.54712943 & 2.18179750 & 1.30749010 \\
C & -3.61263115 & 3.74784308 & -0.25498912 \\
C & -3.84679485 & 3.35552540 & 1.06438392 \\
O & -5.18775226 & 1.10777170 & -2.15837030 \\
H & -5.56116689 & 0.48221713 & 0.47217095 \\
H & -3.90222173 & 3.26227971 & -2.34320075 \\
H & -4.73692214 & 1.86381346 & 2.32784326 \\
H & -3.06667693 & 4.66283151 & -0.46194583 \\
H & -3.48609000 & 3.96014573 & 1.88908647 \\
C & -5.90202480 & -0.08951313 & -1.94757193 \\
H & -6.13666677 & -0.48294478 & -2.93599267 \\
H & -5.29995627 & -0.82331550 & -1.39860329 \\
H & -6.83380688 & 0.09173043 & -1.39870703
\end{tabular}
4PhPh_pi0_4AdPh_side_1
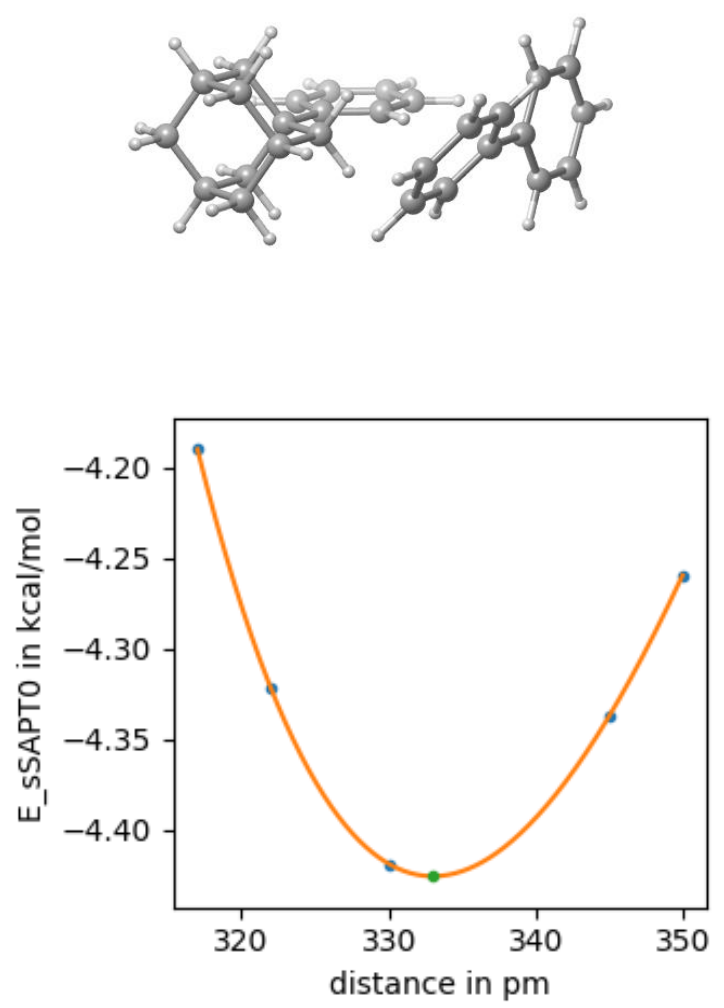

58

4PhPh_pi0_4AdPh_side_1-333

H $-1.32316546 \quad-1.91948614-1.98248152$

$\begin{array}{llll}\text { C } & -1.98163028 & -1.95272068 & -1.12055787\end{array}$

$\begin{array}{llll}\text { C } & -2.35875112 & -3.17503816 & -0.56840573\end{array}$

$\begin{array}{llll}\text { C } & -2.45905254 & -0.77774715 & -0.55389441\end{array}$

C $-3.20193966-3.21225201 \quad 0.53506200$

H $\quad-1.99492502 \quad-4.10296262 \quad-0.99864046$

$\begin{array}{llll}\text { C } & -3.30519802 & -0.82074678 & 0.55372301\end{array}$

$\begin{array}{llll}\mathrm{H} & -2.17479028 & 0.18297129 & -0.97198618\end{array}$

C $-3.69386551 \quad-2.03714400 \quad 1.12063932$

$\begin{array}{llll}\mathrm{H} & -3.48278471 & -4.17691225 & 0.94891843\end{array}$

$\begin{array}{llll}\mathrm{H} & -3.66012014 & 0.11424242 & 0.97200852\end{array}$

$\begin{array}{llll}\text { C } & -4.61766823 & -2.12772445 & 2.33209112\end{array}$

$\begin{array}{llll}\text { C } & -3.89704816 & -2.85493319 & 3.49295886\end{array}$

$\begin{array}{llll}\text { C } & -5.06026385 & -0.74261642 & 2.84367135\end{array}$

C $\quad-5.89096160-2.92846534 \quad 1.96701520$

$\mathrm{H} \quad-2.99368030-2.29256361 \quad 3.76216209$

H $\quad-3.56860888 \quad-3.84770385 \quad 3.16241434$

$\begin{array}{llll}\text { C } & -4.82353757 & -2.99568929 & 4.70972847\end{array}$

$\begin{array}{llll}\mathrm{H} & -5.58121996 & -0.20212909 & 2.04239586\end{array}$

$\begin{array}{llll}\mathrm{H} & -4.17705335 & -0.15034514 & 3.11701757\end{array}$

C $\quad-5.98718334-0.87484244 \quad 4.06122682$

$\begin{array}{llll}\mathrm{H} & -5.61150399 & -3.92304722 & 1.59900185\end{array}$

$\begin{array}{llll}\mathrm{H} & -6.41177904 & -2.41862067 & 1.14627677\end{array}$

$\begin{array}{llll}\text { C } & -6.81684354 & -3.06919279 & 3.18424894\end{array}$ 


$\begin{array}{lrrr}\text { H } & -4.28835657 & -3.51831574 & 5.51107355 \\ \text { C } & -5.24698481 & -1.60167875 & 5.19315256 \\ \text { C } & -6.07076847 & -3.79966493 & 4.31138107 \\ \text { H } & -6.28065973 & 0.12669317 & 4.39644769 \\ \text { C } & -7.23767705 & -1.67508596 & 3.66967213 \\ \text { H } & -7.70465485 & -3.64429694 & 2.89657194 \\ \text { H } & -4.36405158 & -1.02494614 & 5.49604888 \\ \text { H } & -5.89748839 & -1.68835739 & 6.07246065 \\ \text { H } & -5.77912626 & -4.80397386 & 3.97869152 \\ \text { H } & -6.73047060 & -3.92324130 & 5.17935325 \\ \text { H } & -7.91242152 & -1.76265967 & 4.53042901 \\ \text { H } & -7.78634414 & -1.15114748 & 2.87696223 \\ \text { C } & -2.18924903 & 3.50812901 & -1.11469001 \\ \text { H } & -1.53046692 & 3.53261465 & -1.97660939 \\ \text { C } & -1.65940118 & 3.52784787 & 0.17289451 \\ \text { C } & -3.56978727 & 3.45678990 & -1.28926962 \\ \text { C } & -2.50406408 & 3.49712614 & 1.27727373 \\ \text { H } & -0.58490767 & 3.57625133 & 0.31833638 \\ \text { C } & -4.41371936 & 3.42474734 & -0.18436684 \\ \text { H } & -3.99116937 & 3.43266069 & -2.28921363 \\ \text { C } & -3.89321050 & 3.44479387 & 1.11469525 \\ \text { H } & -2.08752308 & 3.53948684 & 2.27939749 \\ \text { H } & -5.48854237 & 3.35791548 & -0.32522596 \\ \text { C } & -4.79415676 & 3.41130431 & 2.29345386 \\ \text { C } & -4.45682167 & 2.66871032 & 3.43084385 \\ \text { C } & -6.00012501 & 4.12161107 & 2.29254955 \\ \text { C } & -5.30052656 & 2.63760426 & 4.53594563 \\ \text { H } & -3.53659389 & 2.09189680 & 3.43525334 \\ \text { C } & -6.84501123 & 4.08994815 & 3.39673260 \\ \text { H } & -6.26207320 & 4.72289410 & 1.42687604 \\ \text { C } & -6.49811412 & 3.34796544 & 4.52284216 \\ \text { H } & -5.02610070 & 2.04989032 & 5.40618466 \\ \text { H } & -7.77254881 & 4.65338561 & 3.38099668 \\ \text { H } & -7.15689342 & 3.32347763 & 5.38476363\end{array}$

4PhPh_pi0_4AdPh_side_2

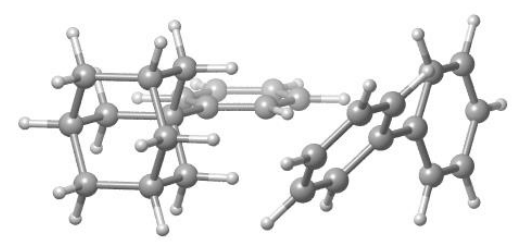

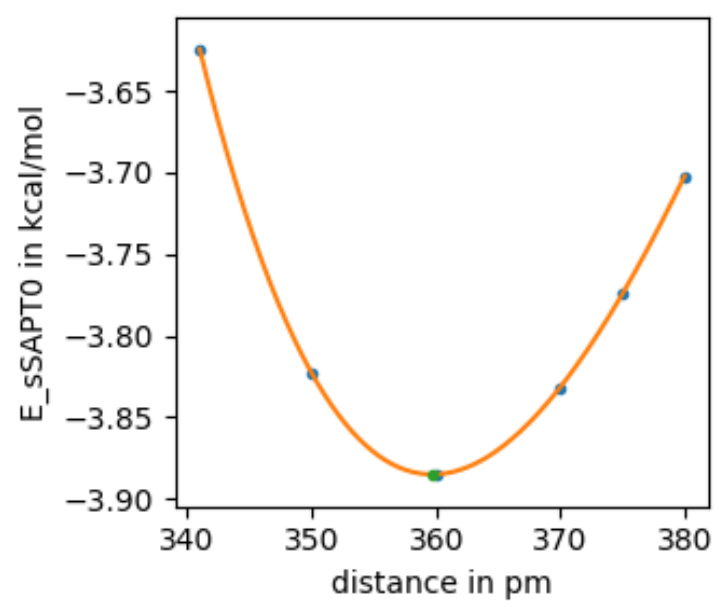

58

4PhPh_pi0_4AdPh_side_2-359

$\mathrm{H} \quad-1.53872900 \quad 1.01753558-2.06912626$

$\begin{array}{llll}\text { C } & -2.14559561 & 1.01097034 & -1.16953600\end{array}$

$\begin{array}{llll}\text { C } & -2.51708600 & -0.19488197 & -0.57871687\end{array}$

$\begin{array}{llll}\text { C } & -2.56176758 & 2.20409407 & -0.59259671\end{array}$

$\begin{array}{llll}\text { C } & -3.29409475 & -0.19795004 & 0.57290841\end{array}$

H $\quad-2.20058198 \quad-1.13660562-1.01632048$

$\begin{array}{llll}\text { C } & -3.34161331 & 2.19536562 & 0.56342748\end{array}$

$\begin{array}{llll}\mathrm{H} & -2.28096558 & 3.15236283 & -1.04039385\end{array}$

$\begin{array}{llll}\text { C } & -3.72361827 & 0.99589436 & 1.16957897\end{array}$

$\begin{array}{llll}\mathrm{H} & -3.57182687 & -1.15029445 & 1.01633312\end{array}$

$\begin{array}{llll}\mathrm{H} & -3.64977793 & 3.14378719 & 0.98847851\end{array}$

$\begin{array}{llll}\text { C } & -4.57590041 & 0.94277435 & 2.43449999\end{array}$

$\begin{array}{llll}\text { C } & -3.80455429 & 0.21385453 & 3.56119463\end{array}$

$\begin{array}{llll}\text { C } & -4.95579462 & 2.34442402 & 2.95140238\end{array}$

$\begin{array}{llll}\text { C } & -5.88646736 & 0.16760613 & 2.15652351\end{array}$

$\begin{array}{llll}\mathrm{H} & -2.87426896 & 0.75821480 & 3.76866635\end{array}$

$\begin{array}{llll}\mathrm{H} & -3.51893965 & -0.79056940 & 3.22610889\end{array}$

$\begin{array}{llll}\text { C } & -4.66034481 & 0.11070905 & 4.83229918\end{array}$

$\begin{array}{llll}\mathrm{H} & -5.51083925 & 2.88663353 & 2.17455071\end{array}$

$\begin{array}{llll}\mathrm{H} & -4.04470027 & 2.91920370 & 3.16376905\end{array}$

$\begin{array}{llll}\text { C } & -5.81177369 & 2.24982583 & 4.22319474\end{array}$

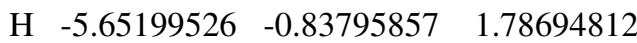

$\begin{array}{llll}\mathrm{H} & -6.44322326 & 0.67892921 & 1.36067457\end{array}$

$\begin{array}{llll}\text { C } & -6.74162349 & 0.06448097 & 3.42805516\end{array}$

$\begin{array}{llll}\mathrm{H} & -4.09069331 & -0.41398032 & 5.60812731\end{array}$

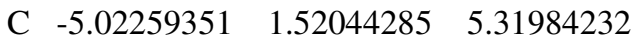

$\begin{array}{llll}\text { C } & -5.94694516 & -0.66873695 & 4.51962387\end{array}$

$\begin{array}{llll}\mathrm{H} & -6.06201951 & 3.26224816 & 4.56076405\end{array}$

$\begin{array}{llll}\text { C } & -7.10114319 & 1.47427528 & 3.91743844\end{array}$

$\begin{array}{llll}\mathrm{H} & -7.65776712 & -0.49321463 & 3.20140958\end{array}$

$\begin{array}{llll}\mathrm{H} & -4.11038004 & 2.08007799 & 5.56194324\end{array}$

$\begin{array}{llll}\mathrm{H} & -5.62170202 & 1.46048995 & 6.23708027\end{array}$

$\begin{array}{llll}\mathrm{H} & -5.69844307 & -1.68385567 & 4.18472889\end{array}$

$\mathrm{H} \quad-6.55674455 \quad-0.76550404 \quad 5.42661590$

$\begin{array}{llll}\mathrm{H} & -7.72556244 & 1.41375901 & 4.81759956\end{array}$

$\begin{array}{llll}\mathrm{H} & -7.68371253 & 2.00070561 & 3.15100503\end{array}$

C $-2.02054022-4.72196318-1.16337306$

H $-1.41385067 \quad-4.70827948-2.06295018$ 


$\begin{array}{lllc}\text { C } & -3.40961426 & -4.75327865 & -1.25553668 \\ \text { C } & -1.41489079 & -4.70831587 & 0.09042200 \\ \text { C } & -4.18677994 & -4.77147985 & -0.10239535 \\ \text { H } & -3.88997298 & -4.77254452 & -2.22863322 \\ \text { C } & -2.19280001 & -4.72518640 & 1.24308452 \\ \text { H } & -0.33306289 & -4.67548342 & 0.17168423 \\ \text { C } & -3.58976507 & -4.75735436 & 1.16341346 \\ \text { H } & -5.26893321 & -4.82272309 & -0.17901956 \\ \text { H } & -1.71687536 & -4.68761236 & 2.21860296 \\ \text { C } & -4.41947284 & -4.77606502 & 2.39367054 \\ \text { C } & -5.61289244 & -4.04814807 & 2.46283242 \\ \text { C } & -4.02600684 & -5.52202285 & 3.51064294 \\ \text { C } & -6.39105279 & -4.06595191 & 3.61531017 \\ \text { H } & -5.91702710 & -3.44423360 & 1.61292545 \\ \text { C } & -4.80292536 & -5.53928601 & 4.66396642 \\ \text { H } & -3.11564337 & -6.11226656 & 3.46165824 \\ \text { C } & -5.98870192 & -4.81145242 & 4.72045427 \\ \text { H } & -7.30958151 & -3.48899154 & 3.65345339 \\ \text { H } & -4.48586666 & -6.12981076 & 5.51765819 \\ \text { H } & -6.59539438 & -4.82513391 & 5.62002946\end{array}$

4PhPh_pi0_4CF3Ph_side_1
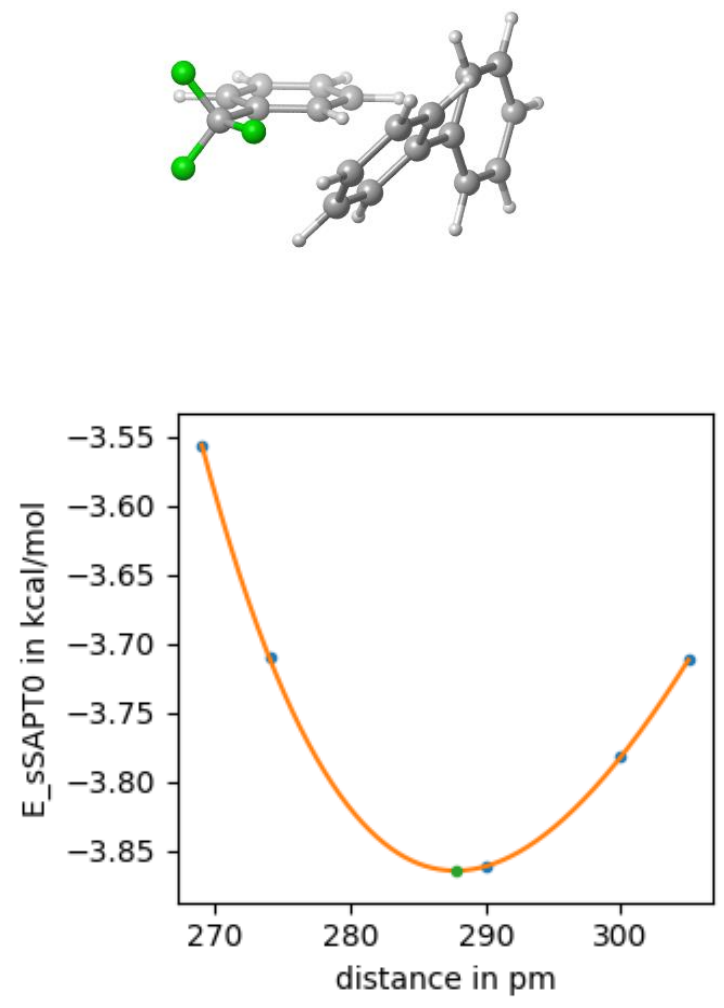

$\begin{array}{lrrc}\mathrm{C} & -1.81961850 & 0.54191446 & -0.58734627 \\ \mathrm{C} & -1.02756473 & -1.86263432 & 0.57995871 \\ \mathrm{H} & -2.06790748 & -2.81697063 & -1.03722908 \\ \mathrm{C} & -1.05978938 & 0.55816137 & 0.57987437 \\ \mathrm{H} & -2.12832049 & 1.47695818 & -1.04242099 \\ \mathrm{C} & -0.66871343 & -0.64469266 & 1.15590806 \\ \mathrm{H} & -0.71215631 & -2.79132550 & 1.04519657 \\ \mathrm{H} & -0.77111413 & 1.49505405 & 1.04221452 \\ \mathrm{C} & 0.15029966 & -0.66857675 & 2.41423974 \\ \mathrm{~F} & -0.48568512 & -1.32095623 & 3.40027053 \\ \mathrm{~F} & 1.31752148 & -1.30483723 & 2.22685917 \\ \mathrm{~F} & 0.43079146 & 0.56231085 & 2.86231629 \\ \mathrm{C} & -2.25349261 & 4.35323473 & -1.17625603 \\ \mathrm{H} & -2.84552993 & 4.34534018 & -2.08561180 \\ \mathrm{C} & -0.86286924 & 4.37166139 & -1.24574420 \\ \mathrm{C} & -2.87961191 & 4.34500062 & 0.06749088 \\ \mathrm{C} & -0.10447960 & 4.38244655 & -0.08007636 \\ \mathrm{H} & -0.36654471 & 4.38662989 & -2.21087136 \\ \mathrm{C} & -2.12048340 & 4.35444835 & 1.23269167 \\ \mathrm{H} & -3.96287953 & 4.32220489 & 0.13108922 \\ \mathrm{C} & -0.72216625 & 4.37365204 & 1.17582335 \\ \mathrm{H} & 0.97920864 & 4.42364848 & -0.13902606 \\ \mathrm{H} & -2.61258414 & 4.32113087 & 2.20030684 \\ \mathrm{C} & 0.08750319 & 4.38444546 & 2.41945371 \\ \mathrm{C} & 1.27282581 & 3.64546246 & 2.50783468 \\ \mathrm{C} & -0.31718550 & 5.13383601 & 3.53010072 \\ \mathrm{C} & 2.03221709 & 3.65584115 & 3.67285512 \\ \mathrm{H} & 1.58516184 & 3.03889351 & 1.66280642 \\ \mathrm{C} & 0.44094536 & 5.14368544 & 4.69594636 \\ \mathrm{H} & -1.22110925 & 5.73251897 & 3.46646608 \\ \mathrm{C} & 1.61883379 & 4.40485895 & 4.77153036 \\ \mathrm{H} & 2.94460482 & 3.07036697 & 3.72578715 \\ \mathrm{H} & 0.11550152 & 5.73698465 & 5.54454384 \\ \mathrm{H} & 2.21087403 & 4.41275126 & 5.68088426\end{array}$

4PhPh_pi0_4CF3Ph_side_2

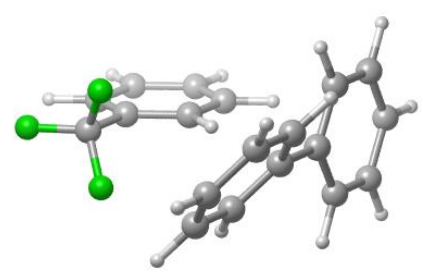

37

4PhPh_pi0_4CF3Ph_side_1-287

H $\quad-2.77321418 \quad-0.67929131 \quad-2.07674037$

C $-2.18121552 \quad-0.66977730 \quad-1.16736063$

$\begin{array}{llll}\text { C } & -1.78529061 & -1.87289750 & -0.58387748\end{array}$ 


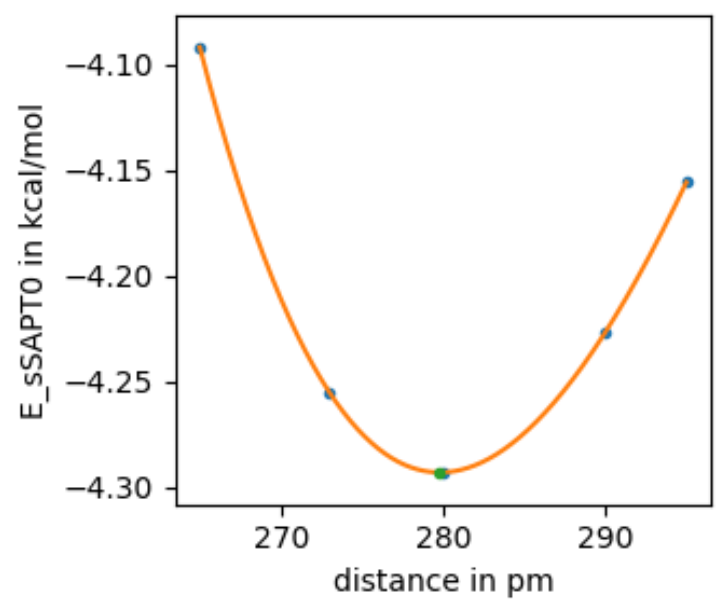

37

4PhPh_pi0_4CF3Ph_side_2-279

H $\quad-2.86476016 \quad 0.85953406-2.02959027$

$\begin{array}{llll}\text { C } & -2.24309086 & 0.86792615 & -1.14021896\end{array}$

$\begin{array}{llll}\text { C } & -1.82667194 & -0.33588973 & -0.57265405\end{array}$

$\begin{array}{llll}\text { C } & -1.86402173 & 2.07887830 & -0.56989672\end{array}$

$\begin{array}{llll}\text { C } & -1.03097092 & -0.32706228 & 0.56556847\end{array}$

H $\quad-2.12294905-1.27938476-1.01842423$

$\begin{array}{llll}\text { C } & -1.06611419 & 2.09368515 & 0.57165161\end{array}$

H $\quad-2.18870362 \quad 3.01446473 \quad-1.01256633$

C $\quad-0.65478770 \quad 0.89014463 \quad 1.13193170$

$\begin{array}{llll}\mathrm{H} & -0.69925862 & -1.25630860 & 1.01818780\end{array}$

H $\quad-0.76347674 \quad 3.02998893 \quad 1.02619042$

$\begin{array}{llll}\text { C } & 0.20532637 & 0.86470804 & 2.36250403\end{array}$

$\begin{array}{llll}\text { F } & -0.39698711 & 0.20947750 & 3.36760291\end{array}$

$\begin{array}{cccc}\text { F } & 1.36650019 & 0.23033166 & 2.13537494\end{array}$

$\begin{array}{llll}\text { F } & 0.49895797 & 2.09502487 & 2.80367691\end{array}$

C $\quad-2.16948406-4.07850829-1.15040242$

$\begin{array}{llll}\mathrm{H} & -2.79116870 & -4.08858403 & -2.03973089\end{array}$

$\begin{array}{llll}\text { C } & -2.75420909 & -4.08811914 & 0.11332452\end{array}$

C $-0.78197295 \quad-4.05588482-1.26573086$

$\begin{array}{llll}\text { C } & -1.95709349 & -4.07587249 & 1.25284610\end{array}$

H $\quad-3.83471469-4.11417809 \quad 0.21262771$

$\begin{array}{llll}\text { C } & 0.01441780 & -4.04230275 & -0.12571526\end{array}$

H $\quad-0.31780051 \quad-4.03981424-2.24670936$

C $\quad-0.56147649-4.05244484 \quad 1.14987736$

H $\quad-2.41690339-4.11027331 \quad 2.23617935$

H $\quad 1.09544051 \quad-3.99782974 \quad-0.22039559$

$\begin{array}{llll}\text { C } & 0.28873542 & -4.03866219 & 2.36612047\end{array}$

$\begin{array}{llll}\text { C } & -0.08138810 & -3.29003532 & 3.48926925\end{array}$

$\begin{array}{llll}\text { C } & 1.47857719 & -4.77400192 & 2.41559746\end{array}$

C $\quad 0.71474767 \quad-3.27738495 \quad 4.62947241$

H $\quad-0.98874425 \quad-2.69413049 \quad 3.45528840$

C $\quad 2.27594386 \quad-4.76081881 \quad 3.55493414$

$\begin{array}{llll}\mathrm{H} & 1.76472297 & -5.37997735 & 1.56092609\end{array}$

C $\quad 1.89673883 \quad-4.01259493 \quad 4.66640311$

$\mathrm{H} \quad 0.41566038 \quad-2.68471846 \quad 5.48814953$

H $\quad 3.19136463 \quad-5.34349470 \quad 3.57793330$

H $\quad 2.51842061 \quad-4.00251697 \quad 5.55573355$
4PhPh_pi_Ph_side0
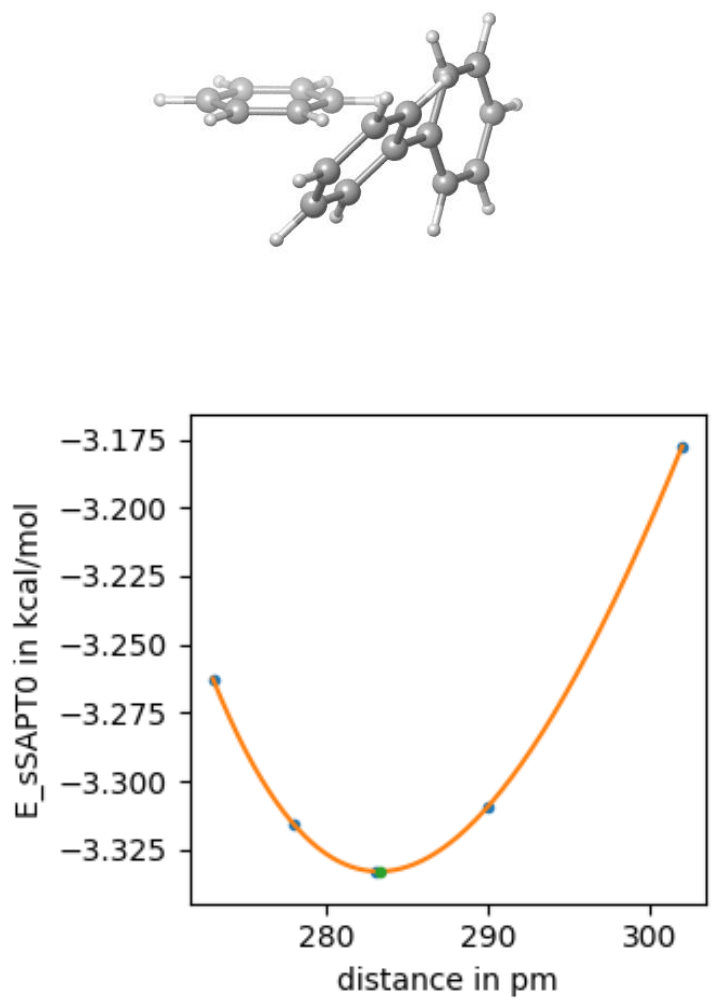

34

4PhPh_pi_Ph_side0-283

$\mathrm{H} \quad 1.89504003 \quad-1.01297204 \quad-1.23581368$

$\begin{array}{llll}\text { C } & 1.06517069 & -0.56940017 & -0.69476885\end{array}$

$\begin{array}{llll}\text { C } & 0.05828077 & 0.09813284 & -1.38840460\end{array}$

$\begin{array}{llll}\text { C } & 1.00645042 & -0.66735444 & 0.69356238\end{array}$

$\begin{array}{llll}\text { C } & -1.00652262 & 0.66751175 & -0.69418687\end{array}$

$\begin{array}{llll}\mathrm{H} & 0.10385687 & 0.17477773 & -2.47040822\end{array}$

C $\quad-0.05848636-0.09825509 \quad 1.38785786$

$\begin{array}{llll}\mathrm{H} & 1.79099197 & -1.18758861 & 1.23442415\end{array}$

$\begin{array}{llll}\text { C } & -1.06489789 & 0.56916273 & 0.69393151\end{array}$

$\begin{array}{llll}\mathrm{H} & -1.79118871 & 1.18791702 & -1.23477786\end{array}$

$\begin{array}{llll}\mathrm{H} & -0.10422992 & -0.17470694 & 2.46990251\end{array}$

$\begin{array}{llll}\mathrm{H} & -1.89494119 & 1.01295749 & 1.23508783\end{array}$

$\begin{array}{llll}\text { C } & -4.33376330 & 2.45375509 & 1.39912795\end{array}$

$\begin{array}{llll}\mathrm{H} & -4.37931836 & 2.37717748 & 2.48058948\end{array}$

$\begin{array}{llll}\text { C } & -4.91929832 & 1.47353117 & 0.60206378\end{array}$

$\begin{array}{llll}\text { C } & -3.68940166 & 3.53286258 & 0.79969099\end{array}$

$\begin{array}{llll}\text { C } & -4.86127305 & 1.57238459 & -0.78390655\end{array}$

$\begin{array}{llll}\mathrm{H} & -5.43084596 & 0.63331072 & 1.06073703\end{array}$

$\begin{array}{llll}\text { C } & -3.63065882 & 3.63029031 & -0.58635208\end{array}$

$\begin{array}{llll}\mathrm{H} & -3.22301584 & 4.29716523 & 1.41317191\end{array}$

C $-4.21593243 \quad 2.65182331-1.39810990$

$\mathrm{H} \quad-5.34226436 \quad 0.81951050-1.40146693$

$\begin{array}{llll}\mathrm{H} & -3.10414331 & 4.45968209 & -1.04943198\end{array}$

$\begin{array}{llll}\text { C } & -4.15362972 & 2.75654740 & -2.87711254\end{array}$ 


$\begin{array}{llll}\mathrm{C} & -3.95444271 & 1.61851979 & -3.66712169 \\ \mathrm{C} & -4.29275075 & 3.99554177 & -3.51306395 \\ \mathrm{C} & -3.89666420 & 1.71614155 & -5.05319063 \\ \mathrm{H} & -3.81889556 & 0.65446684 & -3.18568495 \\ \mathrm{C} & -4.23375939 & 4.09419470 & -4.89900878 \\ \mathrm{H} & -4.47381985 & 4.88307775 & -2.91386226 \\ \mathrm{C} & -4.03579807 & 2.95460935 & -5.67435079 \\ \mathrm{H} & -3.73370055 & 0.82382963 & -5.64922374 \\ \mathrm{H} & -4.35155942 & 5.06242311 & -5.37513086 \\ \mathrm{H} & -3.99024282 & 3.03118286 & -6.75581261\end{array}$

Ph_pi0_345Me3Ph_side
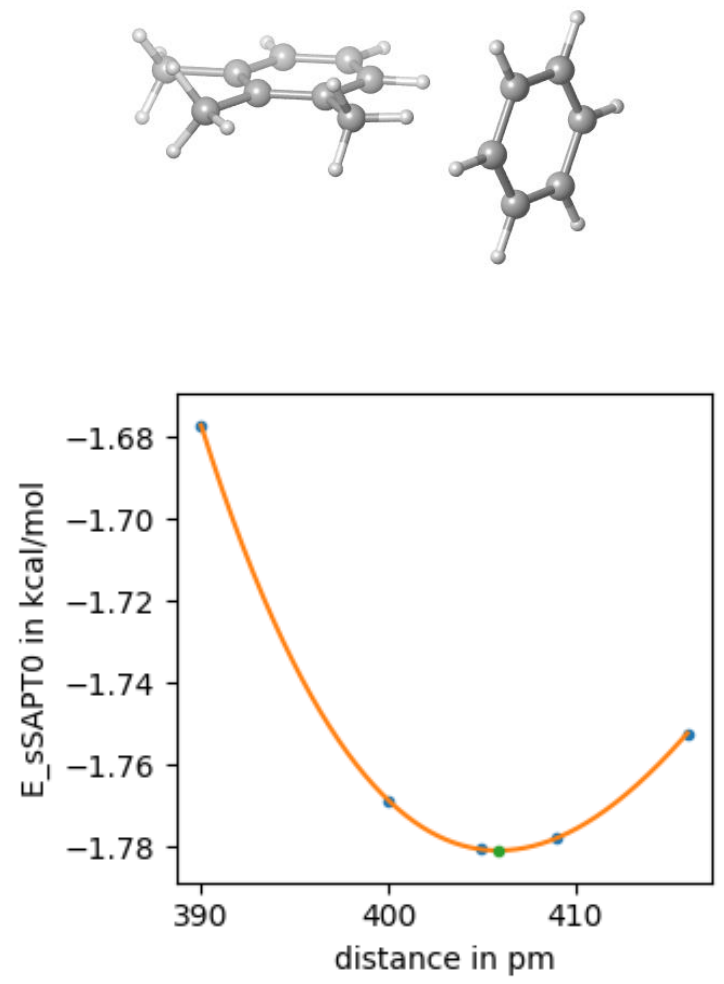

33

Ph_pi0_345Me3Ph_side-405

$\begin{array}{llll}\mathrm{H} & 2.47830959 & -0.04134128 & 0.00002015\end{array}$

$\begin{array}{llll}\text { C } & 1.39302564 & -0.02324781 & 0.00000008\end{array}$

$\begin{array}{llll}\text { C } & 0.71636748 & 1.19441056 & -0.00010067\end{array}$

$\begin{array}{llll}\text { C } & 0.67612530 & -1.21763495 & 0.00007275\end{array}$

$\begin{array}{llll}\text { C } & -0.67625398 & 1.21769798 & 0.00007325\end{array}$

$\begin{array}{llll}\mathrm{H} & 1.27482284 & 2.12543743 & 0.00000658\end{array}$

$\begin{array}{llll}\text { C } & -0.71652436 & -1.19445047 & -0.00005433\end{array}$

$\begin{array}{llll}\mathrm{H} & 1.20319885 & -2.16677876 & 0.00008956\end{array}$

$\begin{array}{llll}\text { C } & -1.39262449 & 0.02322482 & -0.00003308\end{array}$

$\begin{array}{llll}\mathrm{H} & -1.20362977 & 2.16671637 & 0.00016144\end{array}$

$\mathrm{H} \quad-1.27520554 \quad-2.12538191 \quad-0.00007858$

$\begin{array}{llll}\mathrm{H} & -2.47818735 & 0.04136582 & 0.00005785\end{array}$

$\begin{array}{llll}\text { C } & -0.34044594 & 0.61422569 & -4.99955630\end{array}$
C $\quad 0.33773900 \quad-0.60666156 \quad-4.97684006$

$\begin{array}{llll}\mathrm{H} & -0.60407344 & 1.08852115 & -4.05818260\end{array}$

C $\quad 0.68434845 \quad-1.23265924 \quad-3.64678328$

$\begin{array}{llll}\text { C } & -0.67925540 & 1.22378977 & -6.20043993\end{array}$

$\begin{array}{llll}\text { C } & 0.68116987 & -1.22428757 & -6.19062379\end{array}$

H $\quad 0.22755917 \quad-2.22086760 \quad-3.53300133$

H $\quad 1.76526250 \quad-1.35789927 \quad-3.52748453$

H $\quad 0.33016736-0.60642241 \quad-2.82548528$

$\begin{array}{llll}\mathrm{H} & -1.20636048 & 2.17253285 & -6.20194450\end{array}$

$\begin{array}{llll}\text { C } & -0.33886795 & 0.60960879 & -7.39953468\end{array}$

$\begin{array}{llll}\text { C } & 0.33837779 & -0.60922445 & -7.40758014\end{array}$

C $\quad 1.41271620 \quad-2.54397892 \quad-6.22455911$

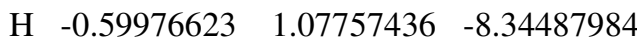

C $\quad 0.69998690 \quad-1.26207399 \quad-8.71719130$

$\mathrm{H} \quad 0.81645091 \quad-3.31145165 \quad-6.73058917$

H $\quad 2.35287157 \quad-2.45875033 \quad-6.78019053$

$\mathrm{H} \quad 1.64989604 \quad-2.91364150 \quad-5.22747583$

H $\quad 1.78349398 \quad-1.38878149 \quad-8.81688081$

$\mathrm{H} \quad 0.25438515 \quad-2.25877743 \quad-8.80561872$

$\begin{array}{llll}\mathrm{H} & 0.35288342 & -0.66218930 & -9.56077380\end{array}$

Ph_pi0_35Me24OMePh_side
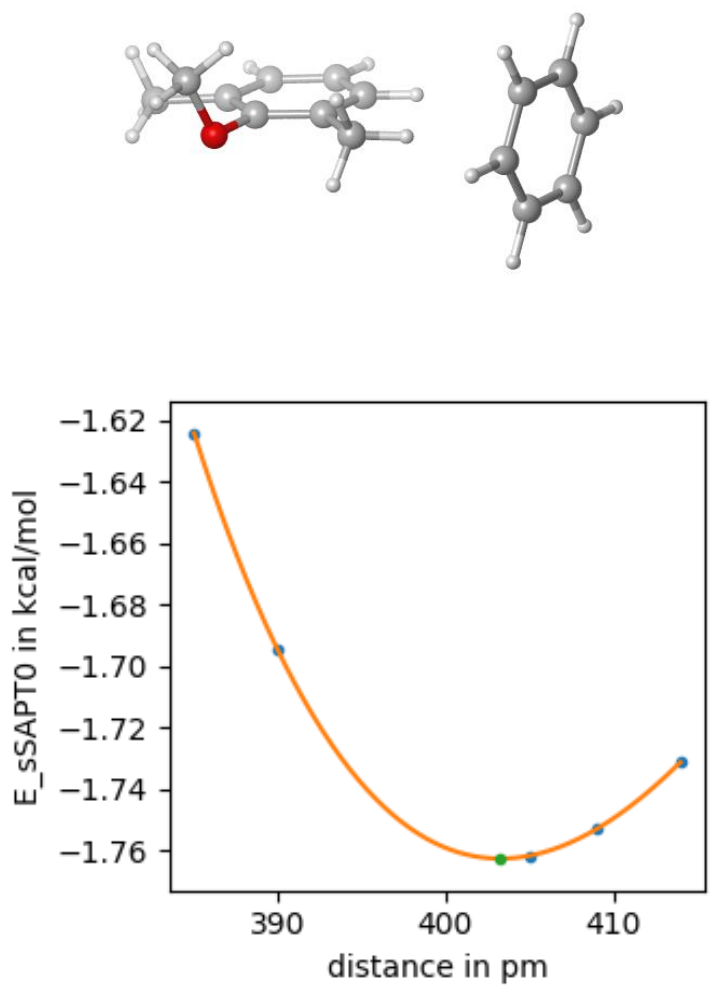

34

Ph_pi0_35Me24OMePh_side-403

$\begin{array}{llll}\mathrm{H} & 2.47830959 & -0.04134128 & 0.00002015\end{array}$

$\begin{array}{llll}\text { C } & 1.39302564 & -0.02324781 & 0.00000008\end{array}$

$\begin{array}{llll}\text { C } & 0.71636748 & 1.19441056 & -0.00010067\end{array}$ 

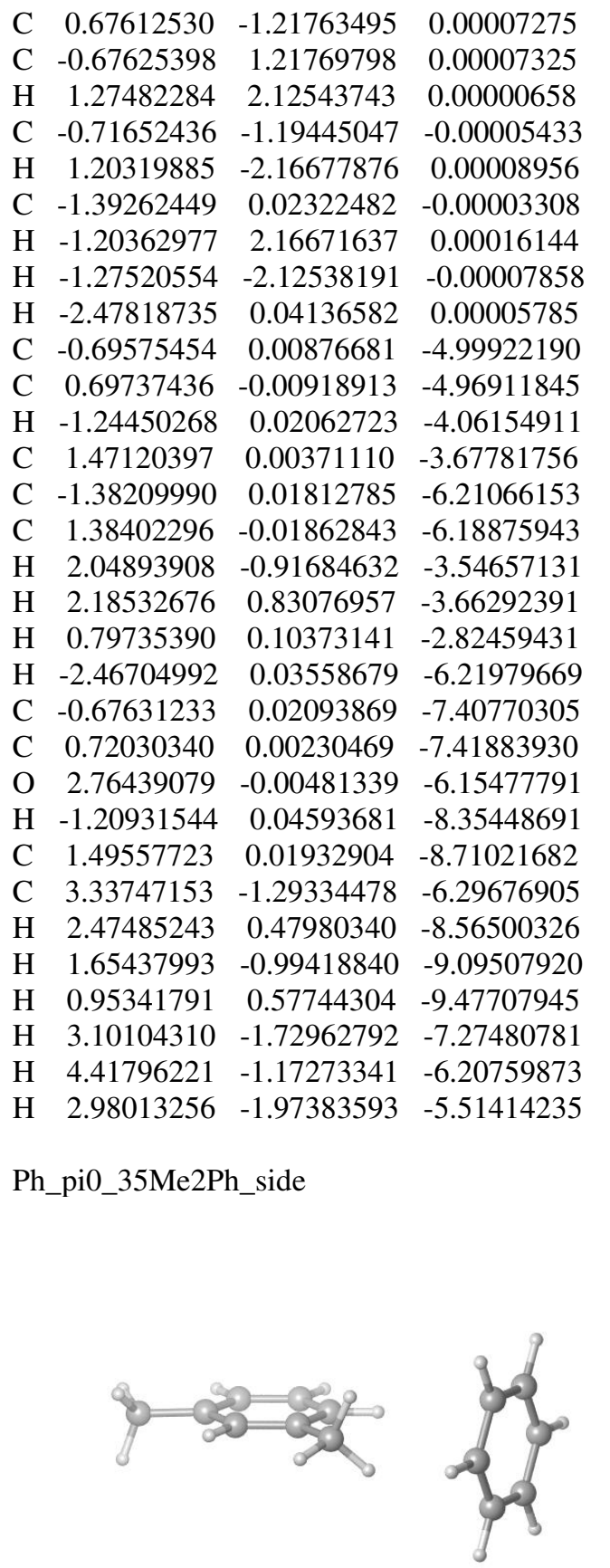

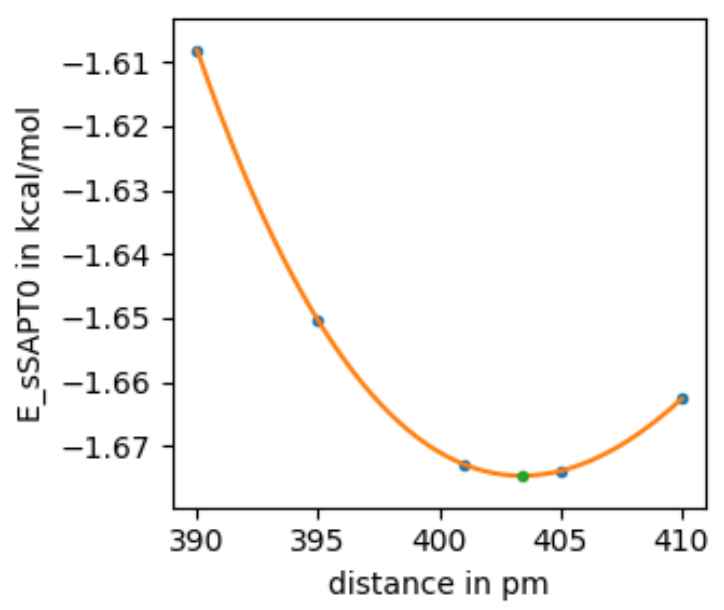

30

Ph_pi0_35Me2Ph_side-403

$\begin{array}{llll}\mathrm{H} & 2.47830959 & -0.04134128 & 0.00002015\end{array}$

$\begin{array}{llll}\text { C } & 1.39302564 & -0.02324781 & 0.00000008\end{array}$

$\begin{array}{llll}\text { C } & 0.71636748 & 1.19441056 & -0.00010067\end{array}$

$\begin{array}{llll}\text { C } & 0.67612530 & -1.21763495 & 0.00007275\end{array}$

$\begin{array}{llll}\text { C } & -0.67625398 & 1.21769798 & 0.00007325\end{array}$

$\begin{array}{llll}\mathrm{H} & 1.27482284 & 2.12543743 & 0.00000658\end{array}$

$\begin{array}{llll}\text { C } & -0.71652436 & -1.19445047 & -0.00005433\end{array}$

$\mathrm{H} \quad 1.20319885 \quad-2.16677876 \quad 0.00008956$

$\begin{array}{llll}\text { C } & -1.39262449 & 0.02322482 & -0.00003308\end{array}$

$\begin{array}{llll}\mathrm{H} & -1.20362977 & 2.16671637 & 0.00016144\end{array}$

$\begin{array}{llll}\mathrm{H} & -1.27520554 & -2.12538191 & -0.00007858\end{array}$

$\begin{array}{llll}\mathrm{H} & -2.47818735 & 0.04136582 & 0.00005785\end{array}$

$\begin{array}{llll}\text { C } & -0.69811410 & 0.01156555 & -4.99331385\end{array}$

$\begin{array}{llll}\text { C } & 0.69973342 & -0.01198772 & -4.97087153\end{array}$

$\begin{array}{llll}\mathrm{H} & -1.24800399 & 0.02068591 & -4.05578379\end{array}$

$\begin{array}{llll}\text { C } & 1.44283397 & -0.04959258 & -3.65946696\end{array}$

$\begin{array}{llll}\text { C } & -1.38240381 & 0.02060306 & -6.20225671\end{array}$

C $\quad 1.38431763 \quad-0.02110312-6.18469474$

H $\quad 1.37001995 \quad-1.03912327 \quad-3.19673766$

H $\quad 2.50248282 \quad 0.17668513 \quad-3.79719040$

$\mathrm{H} \quad 1.02923871 \quad 0.67322663 \quad-2.95089471$

$\begin{array}{llll}\mathrm{H} & -2.46793600 & 0.03620048 & -6.20984550\end{array}$

$\begin{array}{llll}\text { C } & -0.68095600 & 0.00900643 & -7.40586406\end{array}$

$\begin{array}{llll}\text { C } & 0.71304514 & -0.00907328 & -7.41069411\end{array}$

H $\quad 2.47277257 \quad-0.03844769 \quad-6.17843197$

$\begin{array}{llll}\mathrm{H} & -1.21992808 & 0.01530556 & -8.34938465\end{array}$

C $\quad 1.48740691 \quad 0.01088711 \quad-8.70422302$

H $\quad 2.36102144 \quad-0.64423070 \quad-8.65284018$

H $\quad 0.86648733 \quad-0.31351640 \quad-9.54237729$

$\begin{array}{llll}\mathrm{H} & 1.84760619 & 1.02030137 & -8.92850106\end{array}$ 
Ph_pi0_35tBu2Ph_side_1
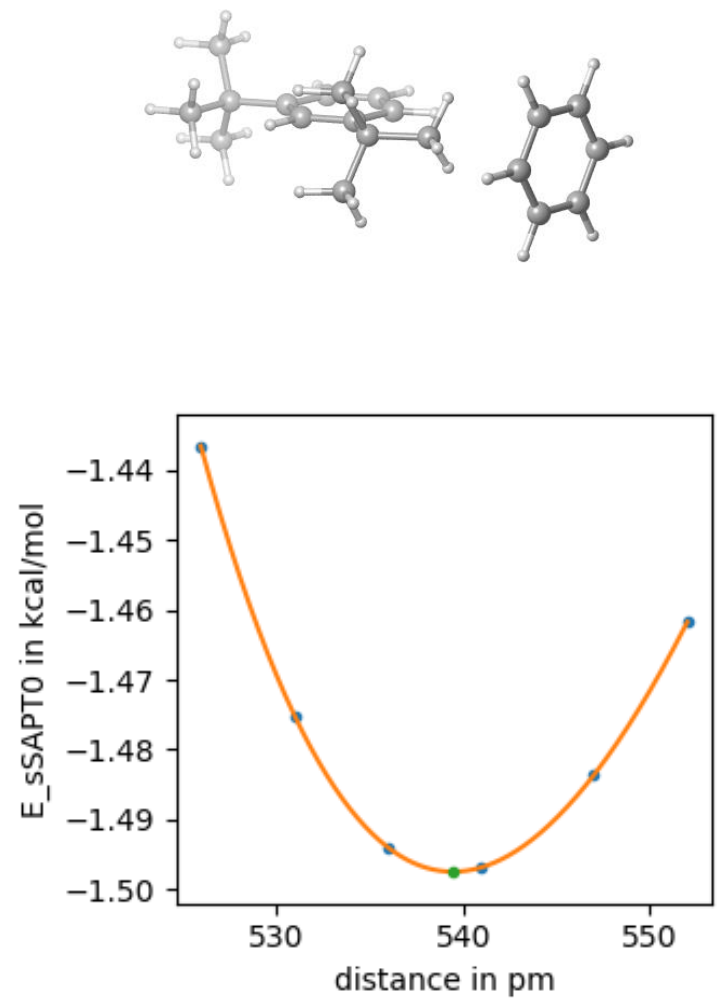

48

Ph_pi0_35tBu2Ph_side_1-539

H $2.47830959-0.04134128$

C $\quad 1.39302564 \quad-0.02324781 \quad 0.00000008$

C $\quad 0.71636748 \quad 1.19441056 \quad-0.00010067$

C $\quad 0.67612530 \quad-1.21763495 \quad 0.00007275$

$\begin{array}{llll}\text { C } & -0.67625398 & 1.21769798 & 0.00007325\end{array}$

H $\quad 1.27482284 \quad 2.12543743 \quad 0.00000658$

C $\quad-0.71652436-1.19445047 \quad-0.00005433$

H $\quad 1.20319885 \quad-2.16677876 \quad 0.00008956$

C $-1.39262449 \quad 0.02322482 \quad-0.00003308$

H $\quad-1.20362977 \quad 2.16671637 \quad 0.00016144$

H $\quad-1.27520554-2.12538191 \quad-0.00007858$

H $\quad-2.47818735 \quad 0.04136582 \quad 0.00005785$

C $\quad 0.35904855 \quad 0.59695841 \quad-6.34857402$

H $\quad 0.64964560 \quad 1.08184870 \quad-5.42368566$

C $-0.35709763 \quad-0.59746788 \quad-6.32993141$

C $-0.77593587-1.29574791 \quad-5.03220050$

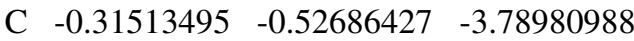

C $\quad 0.71342737 \quad 1.18772495 \quad-7.56196334$

C $\quad-0.70413690 \quad-1.17657159 \quad-7.56067523$

C $-2.30901881 \quad-1.41176646 \quad-4.98616861$

C $-0.15591698 \quad-2.70264251 \quad-4.98586427$

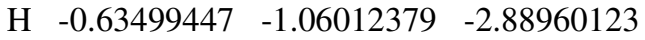

H $\quad 0.77493139-0.43528766 \quad-3.75523270$

H $\quad-0.74796417 \quad 0.47777862 \quad-3.75546744$

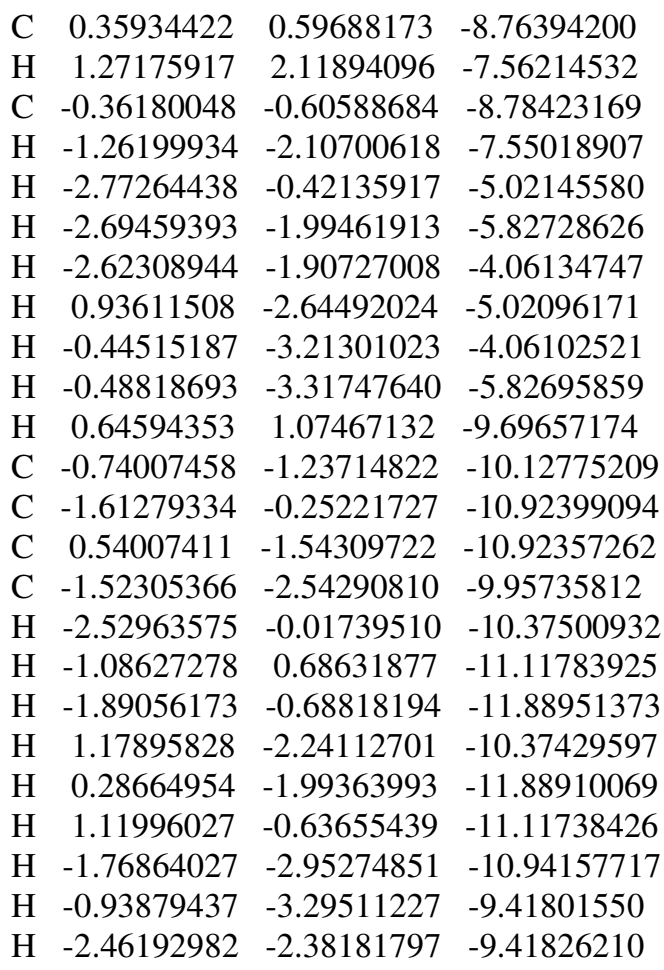

Ph_pi0_35tBu2Ph_side_2
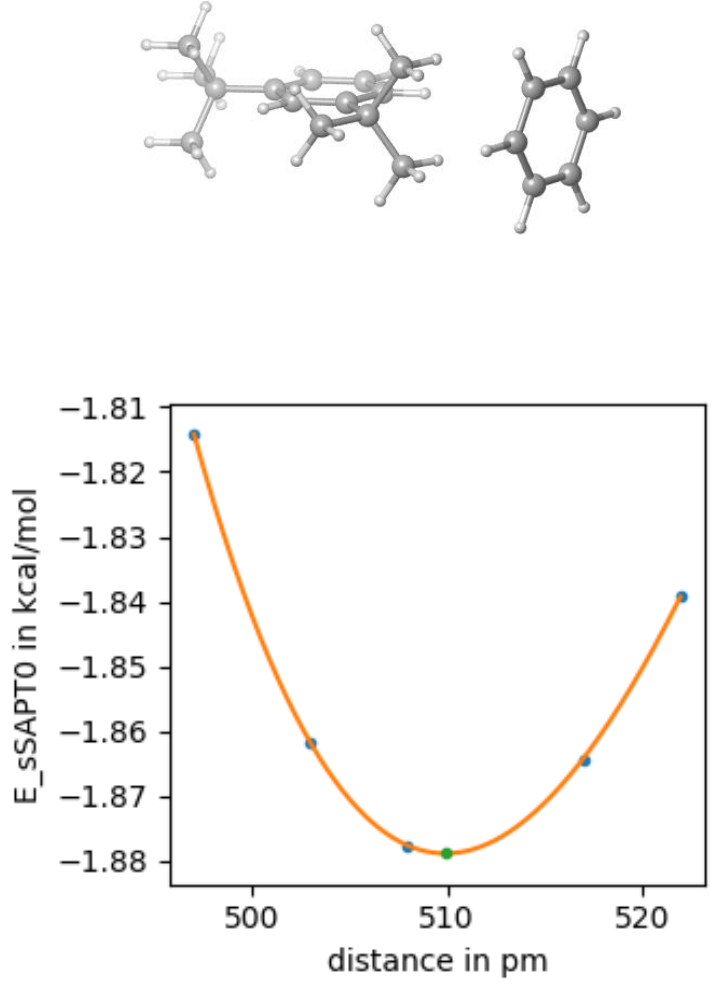


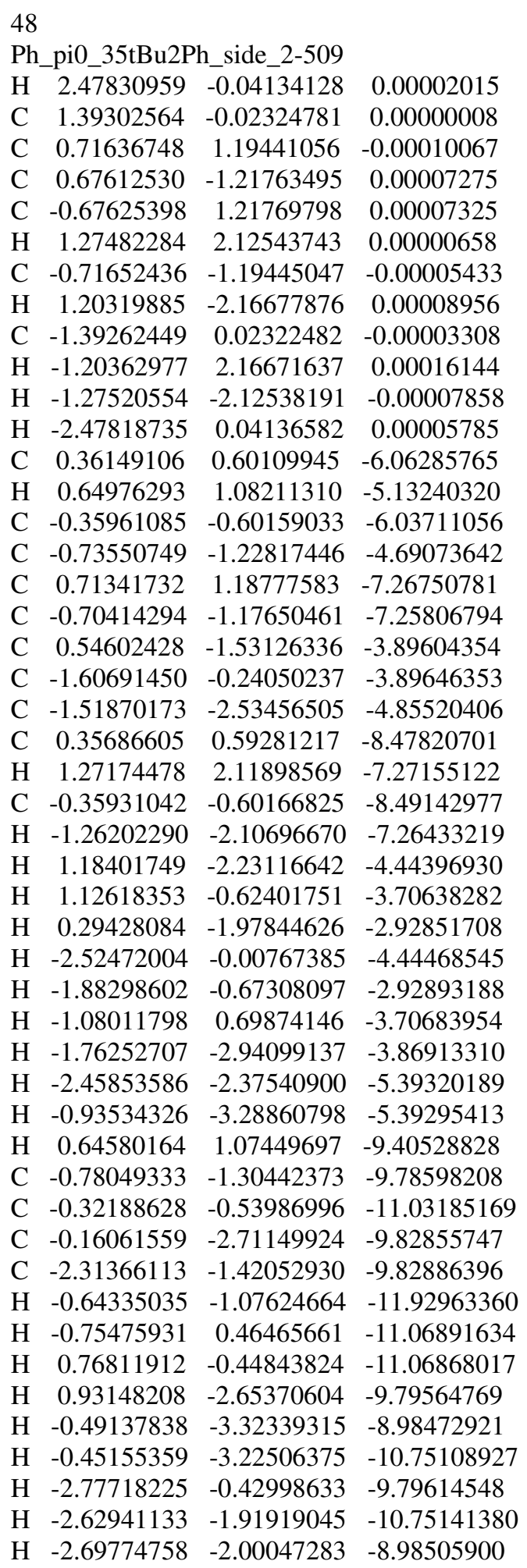

Ph_pi0_4AdPh_side1
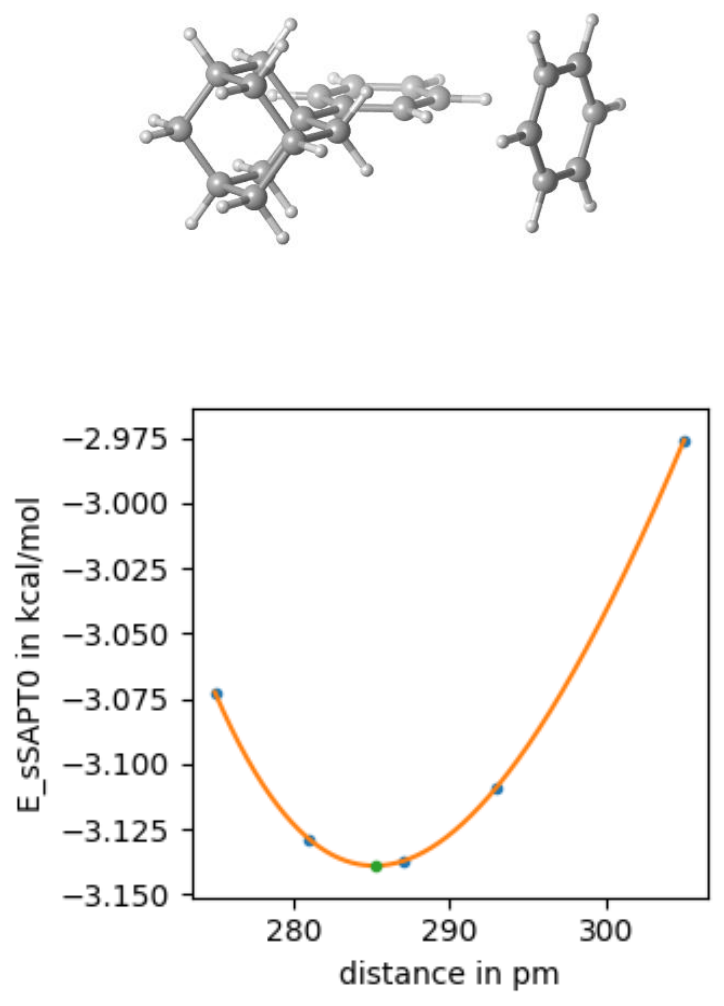

48

Ph_pi0 4AdPh_side1-285

$\begin{array}{llll}\text { H } & 2.47830959 & -0.04134128 & 0.00002015\end{array}$

$\begin{array}{llll}\text { C } & 1.39302564 & -0.02324781 & 0.00000008\end{array}$

C $\quad 0.71636748 \quad 1.19441056 \quad-0.00010067$

$\begin{array}{llll}\text { C } & 0.67612530 & -1.21763495 & 0.00007275\end{array}$

$\begin{array}{llll}\text { C } & -0.67625398 & 1.21769798 & 0.00007325\end{array}$

H $\quad 1.27482284 \quad 2.12543743 \quad 0.00000658$

C $\quad-0.71652436 \quad-1.19445047 \quad-0.00005433$

H $\quad 1.20319885 \quad-2.16677876 \quad 0.00008956$

C $\quad-1.39262449 \quad 0.02322482 \quad-0.00003308$

H $\quad-1.20362977 \quad 2.16671637 \quad 0.00016144$

H $-1.27520554 \quad-2.12538191 \quad-0.00007858$

H $-2.47818735 \quad 0.04136582 \quad 0.00005785$

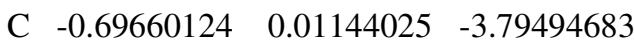

C $\quad 0.69765804 \quad-0.01178204 \quad-3.80631673$

H $\quad-1.22299134 \quad 0.02023516 \quad-2.84551448$

H $\quad 1.22409024 \quad-0.02052038 \quad-2.85872501$

$\begin{array}{lllll}\text { C } & -1.40978937 & 0.02328867 & -4.98693029\end{array}$

C $\quad 1.41141881 \quad-0.02371196 \quad-5.00735043$

H $-2.49477408 \quad 0.04136334 \quad-4.97830963$

C $-0.71462853 \quad 0.01168923 \quad-6.19431134$

C $0.67440889-0.01144906 \quad-6.20000993$

C $2.93636158-0.04907613 \quad-5.06336782$

H $\quad-1.25611553 \quad 0.02069525 \quad-7.13517577$

H $1.19545083 \quad-0.02013201 \quad-7.15350894$ 


$\begin{array}{lrrr}\mathrm{C} & 3.45829776 & 1.19815946 & -5.81684360 \\ \mathrm{C} & 3.57990234 & -0.05937462 & -3.66269701 \\ \mathrm{C} & 3.41657317 & -1.31338596 & -5.81613598 \\ \mathrm{H} & 3.12592482 & 2.10103802 & -5.28850473 \\ \mathrm{H} & 3.01944425 & 1.23601938 & -6.82118410 \\ \mathrm{C} & 4.99022659 & 1.17229409 & -5.92290133 \\ \mathrm{H} & 3.23277435 & -0.93804469 & -3.10322824 \\ \mathrm{H} & 3.26216738 & 0.83065909 & -3.10372590 \\ \mathrm{C} & 5.11252931 & -0.08486342 & -3.76021451 \\ \mathrm{H} & 2.97670836 & -1.33721376 & -6.82046396 \\ \mathrm{H} & 3.05438865 & -2.20442671 & -5.28729505 \\ \mathrm{C} & 4.94851395 & -1.33848643 & -5.92218769 \\ \mathrm{H} & 5.32856749 & 2.06297335 & -6.46505161 \\ \mathrm{C} & 5.59955328 & 1.16092197 & -4.51404951 \\ \mathrm{C} & 5.42827360 & -0.09093498 & -6.67987526 \\ \mathrm{H} & 5.53418874 & -0.09158075 & -2.74841750 \\ \mathrm{C} & 5.55789439 & -1.34656699 & -4.51333689 \\ \mathrm{H} & 5.25707346 & -2.24021976 & -6.46383313 \\ \mathrm{H} & 5.30609526 & 2.06782673 & -3.97059399 \\ \mathrm{H} & 6.69497920 & 1.15797669 & -4.57637262 \\ \mathrm{H} & 5.00980081 & -0.08427073 & -7.69437517 \\ \mathrm{H} & 6.52086286 & -0.10911380 & -6.77872851 \\ \mathrm{H} & 6.65281337 & -1.38004606 & -4.57565251 \\ \mathrm{H} & 5.23447770 & -2.24291537 & -3.96937245\end{array}$

Ph_pi0_4AdPh_side2
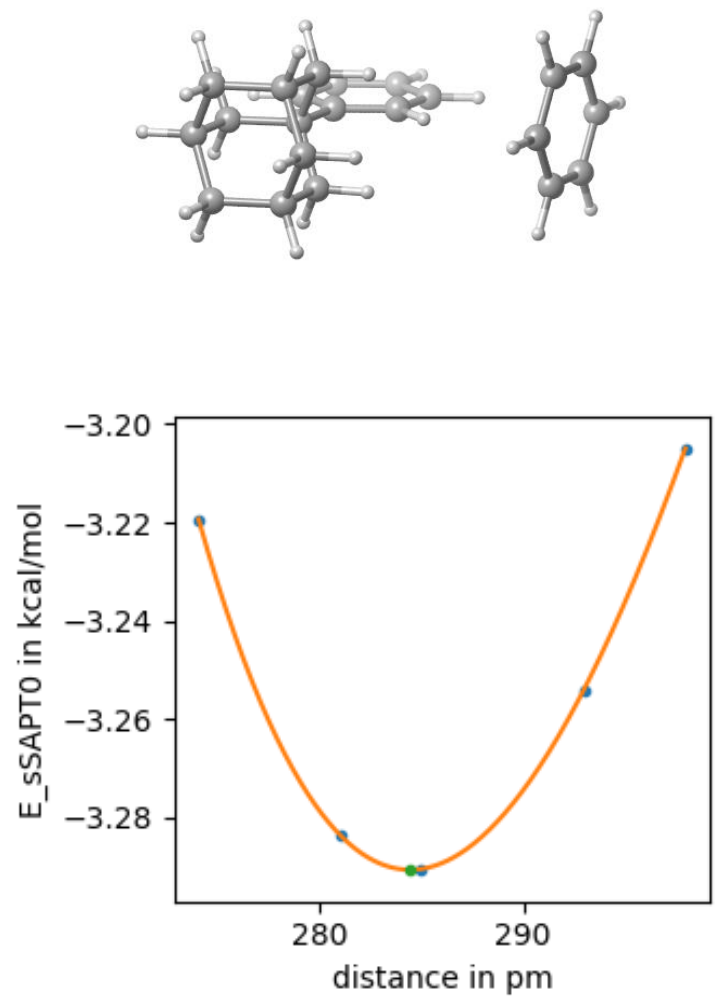

48

Ph_pi0_4AdPh_side2-284

$\begin{array}{llll}\text { H } & 2.47830959 & -0.04134128 & 0.00002015\end{array}$

$\begin{array}{llll}\text { C } & 1.39302564 & -0.02324781 & 0.00000008\end{array}$

$\begin{array}{llll}\text { C } & 0.71636748 & 1.19441056 & -0.00010067\end{array}$

$\begin{array}{llll}\text { C } & 0.67612530 & -1.21763495 & 0.00007275\end{array}$

$\begin{array}{llll}\text { C } & -0.67625398 & 1.21769798 & 0.00007325\end{array}$

$\begin{array}{llll}\mathrm{H} & 1.27482284 & 2.12543743 & 0.00000658\end{array}$

$\begin{array}{llll}\text { C } & -0.71652436 & -1.19445047 & -0.00005433\end{array}$

$\begin{array}{llll}\mathrm{H} & 1.20319885 & -2.16677876 & 0.00008956\end{array}$

$\begin{array}{llll}\text { C } & -1.39262449 & 0.02322482 & -0.00003308\end{array}$

$\begin{array}{llll}\mathrm{H} & -1.20362977 & 2.16671637 & 0.00016144\end{array}$

$\begin{array}{llll}\mathrm{H} & -1.27520554 & -2.12538191 & -0.00007858\end{array}$

$\begin{array}{llll}\mathrm{H} & -2.47818735 & 0.04136582 & 0.00005785\end{array}$

$\begin{array}{llll}\mathrm{H} & 0.63096679 & 1.05130567 & -2.83578931\end{array}$

$\begin{array}{llll}\text { C } & 0.35964386 & 0.59855558 & -3.78442853\end{array}$

$\begin{array}{llll}\text { C } & 0.72627056 & 1.20976932 & -4.98157295\end{array}$

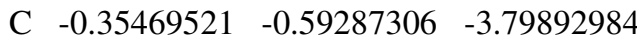

$\begin{array}{llll}\mathrm{H} & 1.28427531 & 2.14045309 & -4.97441493\end{array}$

$\begin{array}{llll}\text { C } & 0.36854355 & 0.61287728 & -6.18380154\end{array}$

C $\quad-0.72465216-1.21019766 \quad-5.00218079$

$\begin{array}{llll}\mathrm{H} & -0.62986977 & -1.05159039 & -2.85310858\end{array}$

C $-0.34852638-0.58309884 \quad-6.19270806$

$\begin{array}{llll}\mathrm{H} & 0.64641806 & 1.07614330 & -7.12547878\end{array}$

C $-1.50929316-2.51879439-4.96834494$

H $\quad-0.61207085-1.02284427 \quad-7.14785494$

C $\quad-2.84966408-2.31211945 \quad-4.22240192$

$\begin{array}{llll}\text { C } & -1.82995158 & -3.05315680 & -6.37822595\end{array}$

$\begin{array}{llll}\text { C } & -0.69537539 & -3.60387869 & -4.22277646\end{array}$

$\mathrm{H} \quad-3.43655471 \quad-1.54630902 \quad-4.74575381$

H $\quad-2.65754869-1.93203307 \quad-3.21178249$

C $-3.63795071-3.62753197 \quad-4.13863264$

$\mathrm{H} \quad-0.89632108-3.21586680 \quad-6.93268404$

$\mathrm{H} \quad-2.41343916 \quad-2.30618008-6.93242108$

C $-2.61886198-4.36885705-6.30300611$

$\mathrm{H} \quad-0.45035428 \quad-3.25553159-3.21216157$

H $\quad 0.25648058 \quad-3.76072438 \quad-4.74639237$

$\begin{array}{llll}\text { C } & -1.48431767 & -4.91889708 & -4.13901329\end{array}$

H $\quad-4.57700233 \quad-3.45080190 \quad-3.60136214$

C $-3.93955753-4.13331650-5.55619644$

$\begin{array}{llll}\text { C } & -2.80270350 & -4.67630451 & -3.38824564\end{array}$

$\mathrm{H}-2.82827501 \quad-4.71779838 \quad-7.32082786$

C $-1.78874735 \quad-5.42298788-5.55657650$

$\mathrm{H} \quad-0.88591778-5.66405253 \quad-3.60200987$

$\mathrm{H} \quad-4.55031342-3.39822525 \quad-6.09522878$

H $\quad-4.51633133 \quad-5.06553477 \quad-5.50980786$

H $-2.59494968-4.33012983-2.36776762$

H $\quad-3.36532288$ - $5.61461773-3.30529120$

$\mathrm{H} \quad-2.33933084-6.37091082-5.51019136$

H $\quad-0.85275469-5.61536012-6.09587859$ 
Ph_pi0_4CF3Ph_side
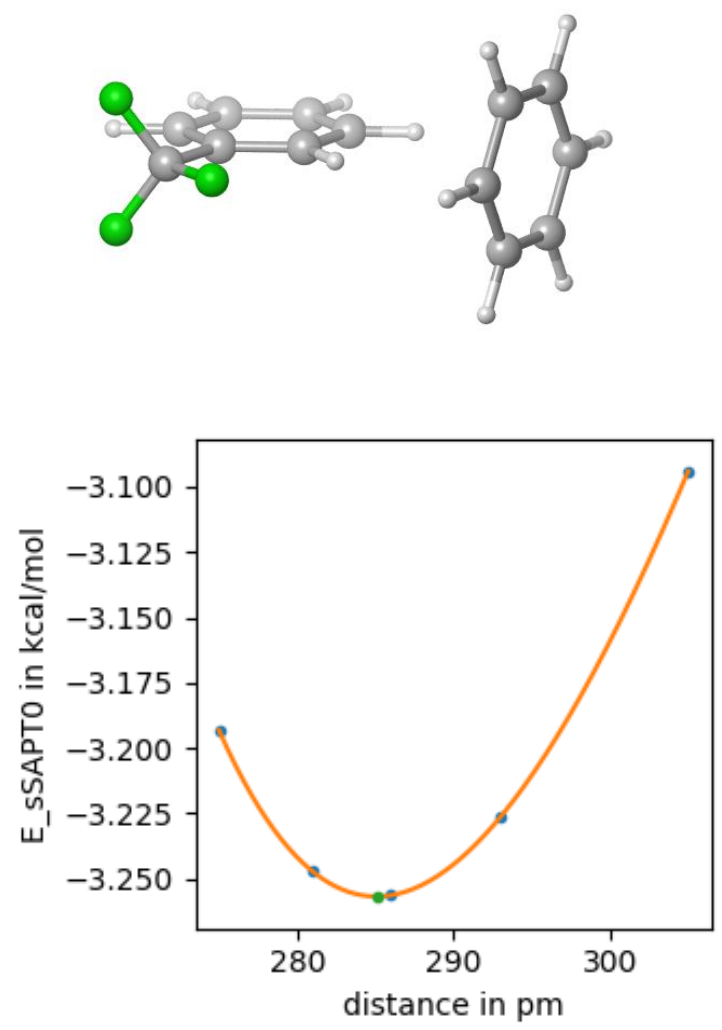

$\begin{array}{lrrr}27 & & & \\ \text { Ph_pi0_4CF3Ph_side-285 } & \\ \text { H } & 2.47830959 & -0.04134128 & 0.00002015 \\ \text { C } & 1.39302564 & -0.02324781 & 0.00000008 \\ \text { C } & 0.71636748 & 1.19441056 & -0.00010067 \\ \text { C } & 0.67612530 & -1.21763495 & 0.00007275 \\ \text { C } & -0.67625398 & 1.21769798 & 0.00007325 \\ \text { H } & 1.27482284 & 2.12543743 & 0.00000658 \\ \text { C } & -0.71652436 & -1.19445047 & -0.00005433 \\ \text { H } & 1.20319885 & -2.16677876 & 0.00008956 \\ \text { C } & -1.39262449 & 0.02322482 & -0.00003308 \\ \text { H } & -1.20362977 & 2.16671637 & 0.00016144 \\ \text { H } & -1.27520554 & -2.12538191 & -0.00007858 \\ \text { H } & -2.47818735 & 0.04136582 & 0.00005785 \\ \text { C } & -0.33964088 & 0.61246124 & -3.79002134 \\ \text { C } & 0.33671202 & -0.60512495 & -3.78378407 \\ \text { H } & -0.60343928 & 1.08715286 & -2.85099262 \\ \text { H } & 0.60453659 & -1.08743583 & -2.85077500 \\ \text { C } & -0.67555759 & 1.21752509 & -4.99684514 \\ \text { C } & 0.67069580 & -1.20599392 & -4.99168487 \\ \text { H } & -1.20247885 & 2.16614277 & -4.99856071 \\ \text { C } & -0.33726246 & 0.60888560 & -6.20507596 \\ \text { C } & 0.33715125 & -0.60517148 & -6.20479419 \\ \text { C } & 1.39917492 & -2.51857447 & -5.02635469 \\ \text { H } & -0.59976768 & 1.08181250 & -7.14534465 \\ \text { H } & 0.60701035 & -1.09047700 & -7.13755925\end{array}$
F $\quad 0.68719862 \quad-3.45702189 \quad-5.67035666$

F $\quad 2.56822344 \quad-2.41280107 \quad-5.67787843$

F $\quad 1.66368546 \quad-2.98636230 \quad-3.79926820$

Ph_pi0_4nPrPh1_side
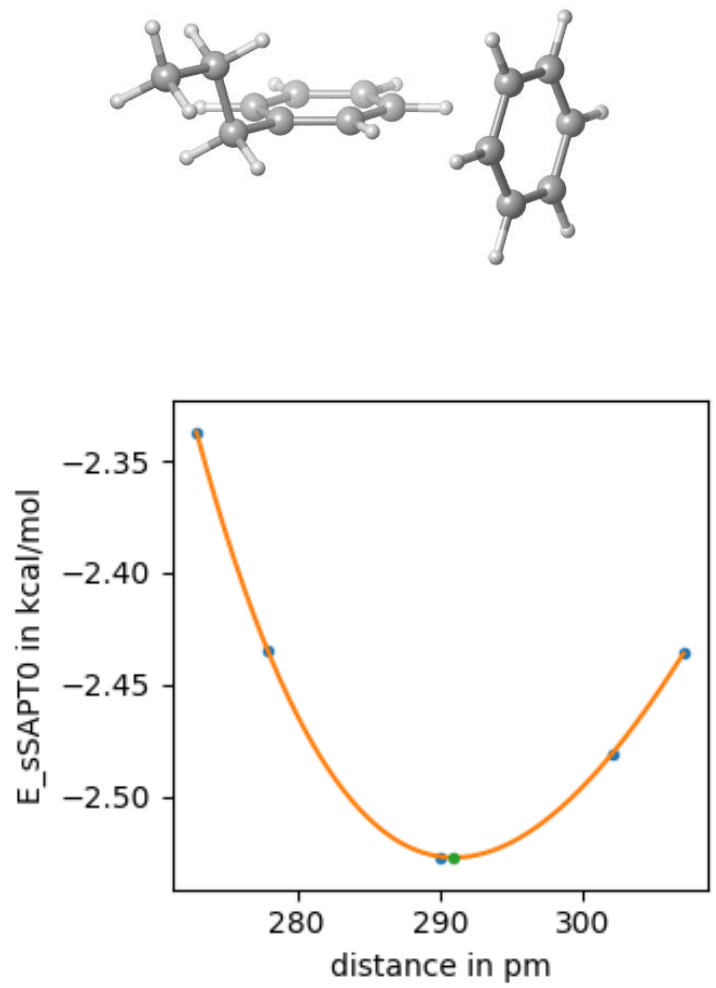

33

Ph_pi0_4nPrPh1_side-290

H $\quad 2.47830959 \quad-0.04134128 \quad 0.00002015$

C $\quad 1.39302564 \quad-0.02324781 \quad 0.00000008$

C $\quad 0.71636748 \quad 1.19441056 \quad-0.00010067$

C $\quad 0.67612530 \quad-1.21763495 \quad 0.00007275$

C $\quad-0.67625398 \quad 1.21769798 \quad 0.00007325$

H $\quad 1.27482284 \quad 2.12543743 \quad 0.00000658$

C $\quad-0.71652436-1.19445047 \quad-0.00005433$

H $\quad 1.20319885 \quad-2.16677876 \quad 0.00008956$

C $\quad-1.39262449 \quad 0.02322482 \quad-0.00003308$

H $\quad-1.20362977 \quad 2.16671637 \quad 0.00016144$

$\mathrm{H} \quad-1.27520554-2.12538191 \quad-0.00007858$

$\mathrm{H} \quad-2.47818735 \quad 0.04136582 \quad 0.00005785$

C $-0.35781265-0.60223200 \quad-3.84933237$

C $\quad 0.35776956 \quad 0.59151814 \quad-3.85488639$

H $-0.63604409-1.06101769-2.90565964$

H $\quad 0.63715715 \quad 1.06072875 \quad-2.91445505$

C $\quad-0.72010691 \quad-1.20424933 \quad-5.05148700$

$\begin{array}{llll}\text { C } & 0.72174477 & 1.20382417 & -5.05642622\end{array}$

H $\quad-1.28089766 \quad-2.13330873 \quad-5.04957310$

C $\quad-0.36151329-0.60494329-6.25612015$ 


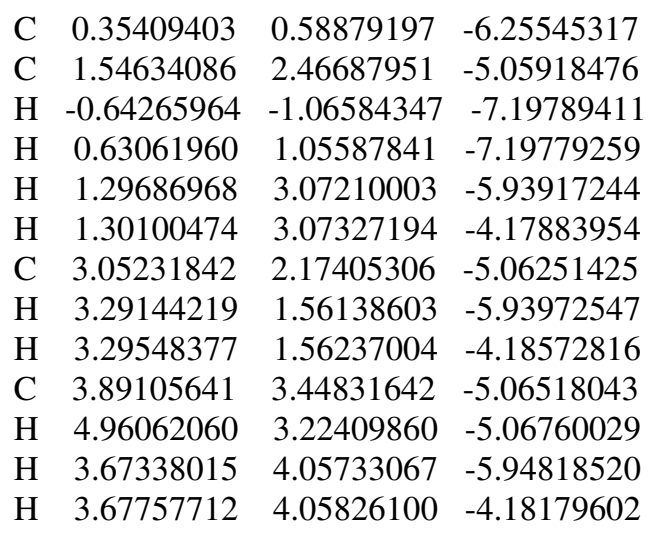

Ph_pi0_4nPrPh2_side_1
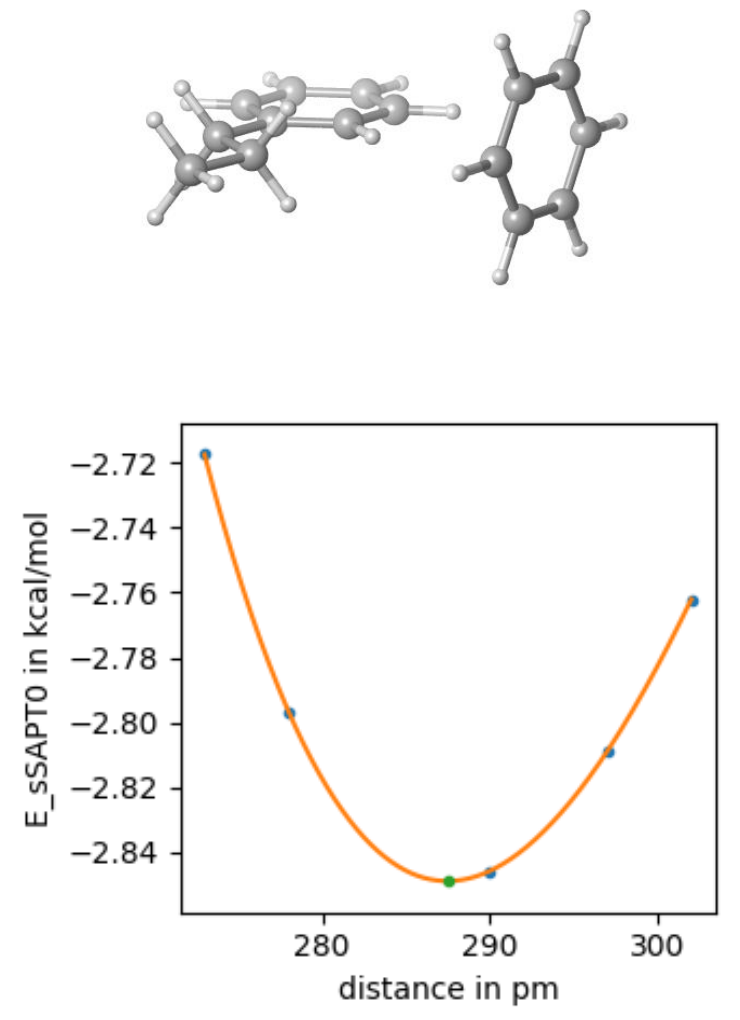

33

Ph_pi0_4nPrPh2_side_1-287

$\begin{array}{llll}\text { H } & 2.47830959 & -0.04134128 & 0.00002015\end{array}$

$\begin{array}{llll}\text { C } & 1.39302564 & -0.02324781 & 0.00000008\end{array}$

$\begin{array}{llll}\text { C } & 0.71636748 & 1.19441056 & -0.00010067\end{array}$

$\begin{array}{llll}\text { C } & 0.67612530 & -1.21763495 & 0.00007275\end{array}$

$\begin{array}{llll}\text { C } & -0.67625398 & 1.21769798 & 0.00007325\end{array}$

$\begin{array}{llll}\mathrm{H} & 1.27482284 & 2.12543743 & 0.00000658\end{array}$

$\begin{array}{lrrr}\text { C } & -0.71652436 & -1.19445047 & -0.00005433\end{array}$

$\begin{array}{llll}\mathrm{H} & 1.20319885 & -2.16677876 & 0.00008956\end{array}$

$\begin{array}{llll}\text { C } & -1.39262449 & 0.02322482 & -0.00003308\end{array}$

$\begin{array}{llll}\text { H } & -1.20362977 & 2.16671637 & 0.00016144\end{array}$

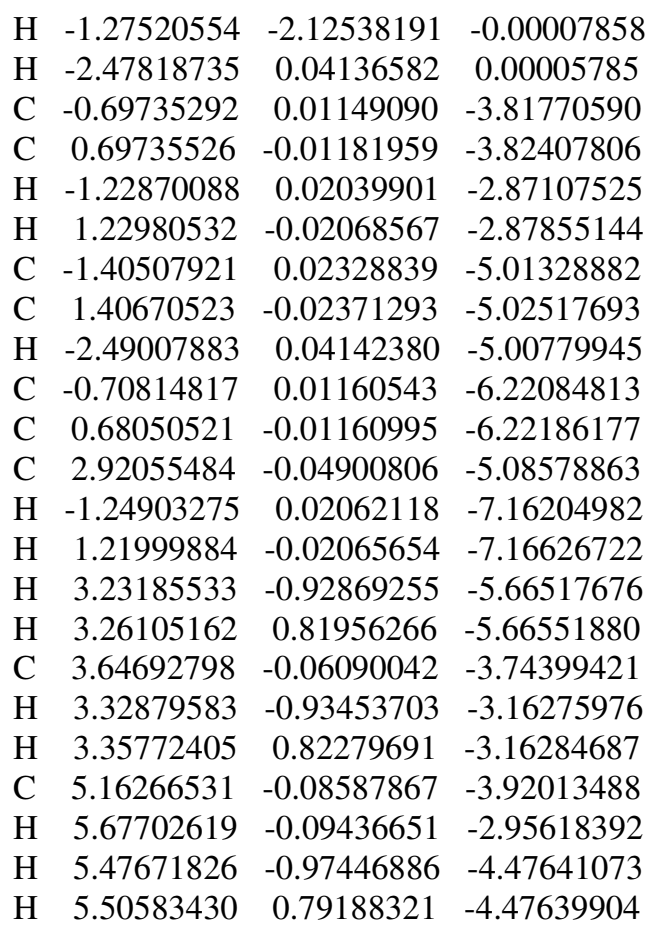

Ph_pi0_4nPrPh2_side_2

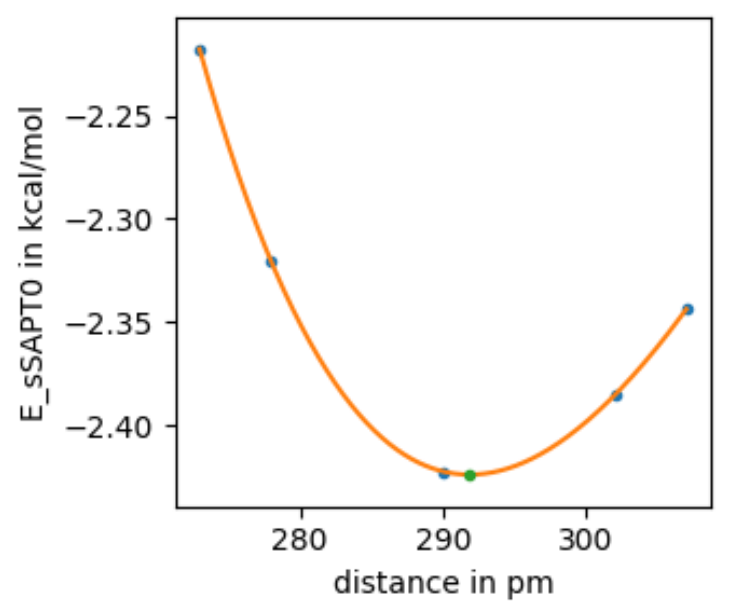

33

Ph_pi0_4nPrPh2_side_2-291 


$\begin{array}{lrrr}\mathrm{H} & 2.47830959 & -0.04134128 & 0.00002015 \\ \mathrm{C} & 1.39302564 & -0.02324781 & 0.00000008 \\ \mathrm{C} & 0.71636748 & 1.19441056 & -0.00010067 \\ \mathrm{C} & 0.67612530 & -1.21763495 & 0.00007275 \\ \mathrm{C} & -0.67625398 & 1.21769798 & 0.00007325 \\ \mathrm{H} & 1.27482284 & 2.12543743 & 0.00000658 \\ \mathrm{C} & -0.71652436 & -1.19445047 & -0.00005433 \\ \mathrm{H} & 1.20319885 & -2.16677876 & 0.00008956 \\ \mathrm{C} & -1.39262449 & 0.02322482 & -0.00003308 \\ \mathrm{H} & -1.20362977 & 2.16671637 & 0.00016144 \\ \mathrm{H} & -1.27520554 & -2.12538191 & -0.00007858 \\ \mathrm{H} & -2.47818735 & 0.04136582 & 0.00005785 \\ \mathrm{C} & 0.36108451 & 0.60043998 & -3.85707095 \\ \mathrm{C} & -0.35351905 & -0.59042509 & -3.86603380 \\ \mathrm{H} & 0.63584600 & 1.05854434 & -2.91200771 \\ \mathrm{H} & -0.63473082 & -1.05883384 & -2.92552858 \\ \mathrm{C} & 0.72431623 & 1.20546422 & -5.05959216 \\ \mathrm{C} & -0.72267574 & -1.20588963 & -5.06790496 \\ \mathrm{H} & 1.28267996 & 2.13595217 & -5.05728647 \\ \mathrm{C} & 0.36466473 & 0.60583360 & -6.26022872 \\ \mathrm{C} & -0.35308030 & -0.59025320 & -6.26387668 \\ \mathrm{C} & -1.50194438 & -2.50447392 & -5.01817072 \\ \mathrm{H} & 0.64169521 & 1.06726924 & -7.20301759 \\ \mathrm{H} & -0.62349221 & -1.04109354 & -7.21320415 \\ \mathrm{H} & -0.92214449 & -3.23738487 & -4.44100781 \\ \mathrm{H} & -2.42140758 & -2.33768549 & -4.44092793 \\ \mathrm{C} & -1.87081636 & -3.11909143 & -6.36514896 \\ \mathrm{H} & -0.95887389 & -3.30753094 & -6.94405653 \\ \mathrm{H} & -2.46569980 & -2.40280164 & -6.94422993 \\ \mathrm{C} & -2.65179402 & -4.41981298 & -6.19990232 \\ \mathrm{H} & -2.91289113 & -4.85498853 & -7.16752370 \\ \mathrm{H} & -2.06580253 & -5.15962879 & -5.64587059 \\ \mathrm{H} & -3.58038147 & -4.25028949 & -5.64614440\end{array}$

Ph_pi0_4nPrPh3_side_1

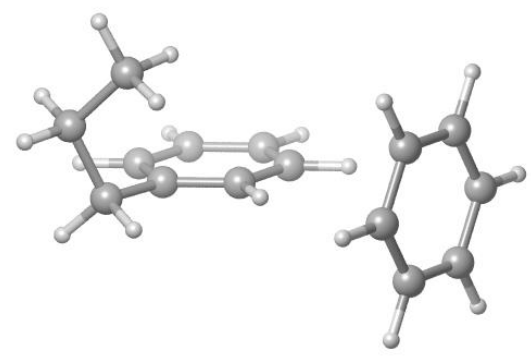

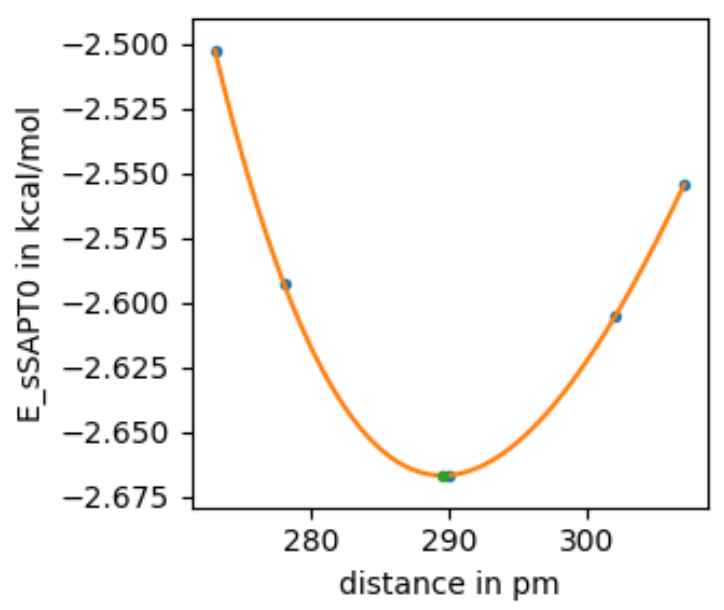

33

Ph_pi0_4nPrPh3_side_1-289

$\begin{array}{llll}\mathrm{H} & 2.47830959 & -0.04134128 & 0.00002015\end{array}$

$\begin{array}{llll}\text { C } & 1.39302564 & -0.02324781 & 0.00000008\end{array}$

$\begin{array}{llll}\text { C } & 0.71636748 & 1.19441056 & -0.00010067\end{array}$

$\begin{array}{llll}\text { C } & 0.67612530 & -1.21763495 & 0.00007275\end{array}$

$\begin{array}{llll}\text { C } & -0.67625398 & 1.21769798 & 0.00007325\end{array}$

$\begin{array}{llll}\mathrm{H} & 1.27482284 & 2.12543743 & 0.00000658\end{array}$

$\begin{array}{llll}\text { C } & -0.71652436 & -1.19445047 & -0.00005433\end{array}$

$\begin{array}{llll}\mathrm{H} & 1.20319885 & -2.16677876 & 0.00008956\end{array}$

$\begin{array}{llll}\text { C } & -1.39262449 & 0.02322482 & -0.00003308\end{array}$

$\begin{array}{llll}\mathrm{H} & -1.20362977 & 2.16671637 & 0.00016144\end{array}$

$\begin{array}{llll}\mathrm{H} & -1.27520554 & -2.12538191 & -0.00007858\end{array}$

$\begin{array}{llll}\mathrm{H} & -2.47818735 & 0.04136582 & 0.00005785\end{array}$

$\begin{array}{llll}\text { C } & -0.34079064 & 0.61258817 & -3.83441074\end{array}$

$\begin{array}{llll}\text { C } & 0.33635269 & -0.60460587 & -3.83997320\end{array}$

$\begin{array}{lllll}\mathrm{H} & -0.60032706 & 1.08155767 & -2.89045238\end{array}$

H $\quad 0.60146062-1.08188916 \quad-2.89970170$

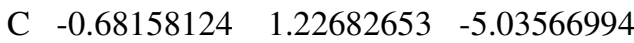

C $\quad 0.68321453 \quad-1.22725289 \quad-5.04058618$

$\mathrm{H} \quad-1.20656501 \quad 2.17656822 \quad-5.03411687$

$\begin{array}{llll}\text { C } & -0.34172420 & 0.61602667 & -6.24086076\end{array}$

$\begin{array}{llll}\text { C } & 0.33349261 & -0.60006933 & -6.24009691\end{array}$

$\begin{array}{llll}\text { C } & 1.37679938 & -2.56821638 & -5.05709465\end{array}$

$\begin{array}{llll}\mathrm{H} & -0.60008410 & 1.09024036 & -7.18256064\end{array}$

H $\quad 0.60061093 \quad-1.07196160 \quad-7.18302880$

$\mathrm{H} \quad 2.17254260 \quad-2.55778445 \quad-5.81036341$

H $\quad 1.85791006-2.74344493 \quad-4.08749066$

C $\quad 0.41349962 \quad-3.72791843 \quad-5.35670686$

H $\quad 0.99267172 \quad-4.65439787 \quad-5.43891587$

H $\quad-0.05098724 \quad-3.55957621 \quad-6.33579711$

C $-0.67049940 \quad-3.88804684 \quad-4.29482267$

H $\quad-1.32144203 \quad-4.73699764 \quad-4.51819913$

$\mathrm{H} \quad-0.22583252 \quad-4.05598273 \quad-3.30799040$

H $\quad-1.29255895 \quad-2.99100492 \quad-4.23077912$ 
Ph_pi0_4nPrPh3_side_2
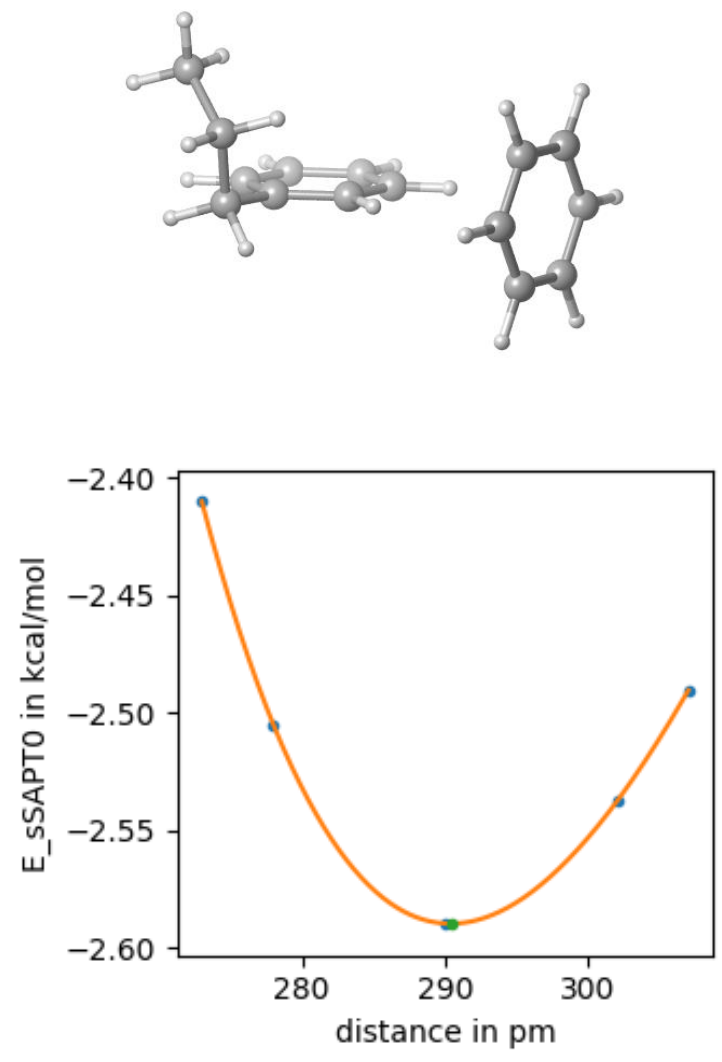

33

Ph_pi0_4nPrPh3_side_2-290

H $2.47830959 \quad-0.04134128 \quad 0.00002015$

$\begin{array}{llll}\text { C } & 1.39302564 & -0.02324781 & 0.00000008\end{array}$

C $\quad 0.71636748 \quad 1.19441056 \quad-0.00010067$

C $\quad 0.67612530 \quad-1.21763495 \quad 0.00007275$

$\begin{array}{llll}\text { C } & -0.67625398 & 1.21769798 & 0.00007325\end{array}$

H $\quad 1.27482284 \quad 2.12543743 \quad 0.00000658$

C $-0.71652436-1.19445047 \quad-0.00005433$

H $\quad 1.20319885 \quad-2.16677876 \quad 0.00008956$

C $\quad-1.39262449 \quad 0.02322482 \quad-0.00003308$

H $\quad-1.20362977 \quad 2.16671637 \quad 0.00016144$

H $-1.27520554-2.12538191 \quad-0.00007858$

$\begin{array}{llll}\mathrm{H} & -2.47818735 & 0.04136582 & 0.00005785\end{array}$

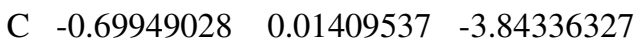

C $\quad 0.69127733 \quad-0.00906505 \quad-3.84943359$

$\mathrm{H} \quad-1.23587030 \quad 0.02051795 \quad-2.89960358$

H $\quad 1.23698190 \quad-0.02080651 \quad-2.90857031$

C $\quad-1.40299764 \quad 0.02412427 \quad-5.04587772$

C $\quad 1.40463386 \quad-0.02455075 \quad-5.05167038$

$\mathrm{H} \quad-2.48805820 \quad 0.04013978 \quad-5.04328694$

C $\quad-0.70527338 \quad 0.01042021 \quad-6.24980617$

C $\quad 0.68739431 \quad-0.01391113 \quad-6.24955526$

C $\quad 2.91427730 \quad-0.00509602 \quad-5.04100464$

H $\quad-1.24483373 \quad 0.01684679 \quad-7.19170970$

H $\quad 1.22966493 \quad-0.02483924 \quad-7.19190085$ $\begin{array}{llll}\text { H } & 3.28261263 & -0.71064166 & -4.28783510 \\ \text { H } & 3.29164951 & -0.34885241 & -6.01143042 \\ \text { C } & 3.48502327 & 1.39130997 & -4.74617913 \\ \text { H } & 4.57538370 & 1.31668544 & -4.66802474 \\ \text { H } & 3.12158948 & 1.72365994 & -3.76629943 \\ \text { C } & 3.11185453 & 2.42106962 & -5.80855061 \\ \text { H } & 3.55521367 & 3.39545852 & -5.58868757 \\ \text { H } & 3.46556870 & 2.10599987 & -6.79616712 \\ \text { H } & 2.02734288 & 2.54742404 & -5.86865797\end{array}$

Ph_pi0_4PhPh_side
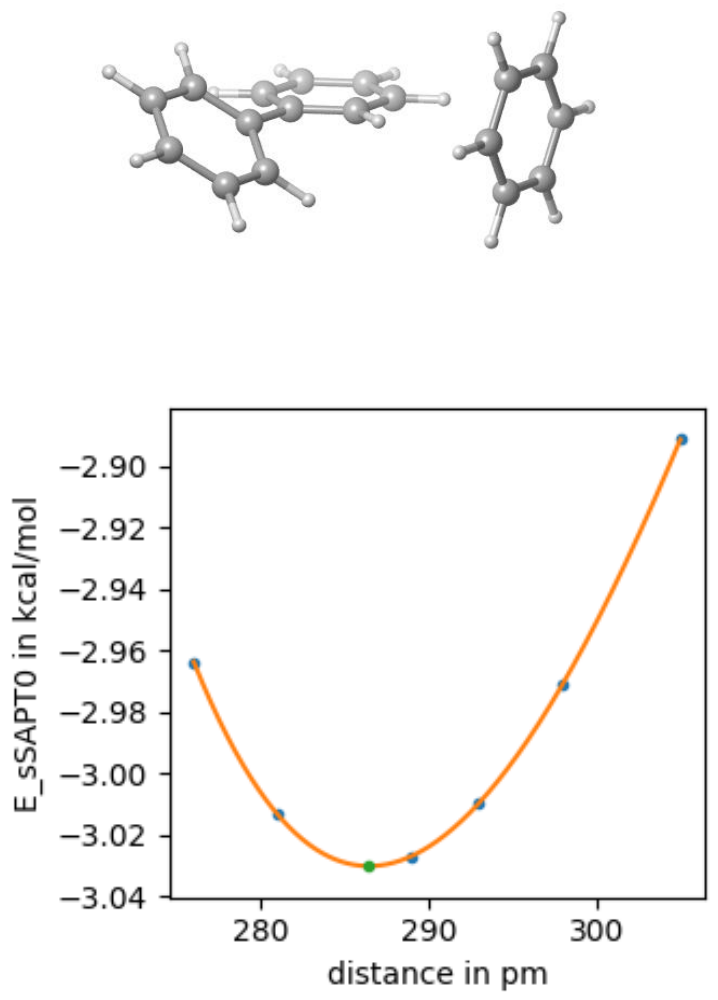

34

Ph_pi0_4PhPh_side-286

H $\quad 2.47830959 \quad-0.04134128 \quad 0.00002015$

C $\quad 1.39302564 \quad-0.02324781 \quad 0.00000008$

C $\quad 0.71636748 \quad 1.19441056 \quad-0.00010067$

$\begin{array}{llll}\text { C } & 0.67612530 & -1.21763495 & 0.00007275\end{array}$

C $\quad-0.67625398 \quad 1.21769798 \quad 0.00007325$

H $\quad 1.27482284 \quad 2.12543743 \quad 0.00000658$

C $\quad-0.71652436-1.19445047 \quad-0.00005433$

H $\quad 1.20319885 \quad-2.16677876 \quad 0.00008956$

$\begin{array}{llll}\text { C } & -1.39262449 & 0.02322482 & -0.00003308\end{array}$

H $\quad-1.20362977 \quad 2.16671637 \quad 0.00016144$

H $-1.27520554 \quad-2.12538191 \quad-0.00007858$

H $\quad-2.47818735 \quad 0.04136582 \quad 0.00005785$

C $\quad-0.33764342 \quad 0.60884875 \quad-3.80684842$

C $0.34647446 \quad-0.60195040 \quad-3.80593394$ 


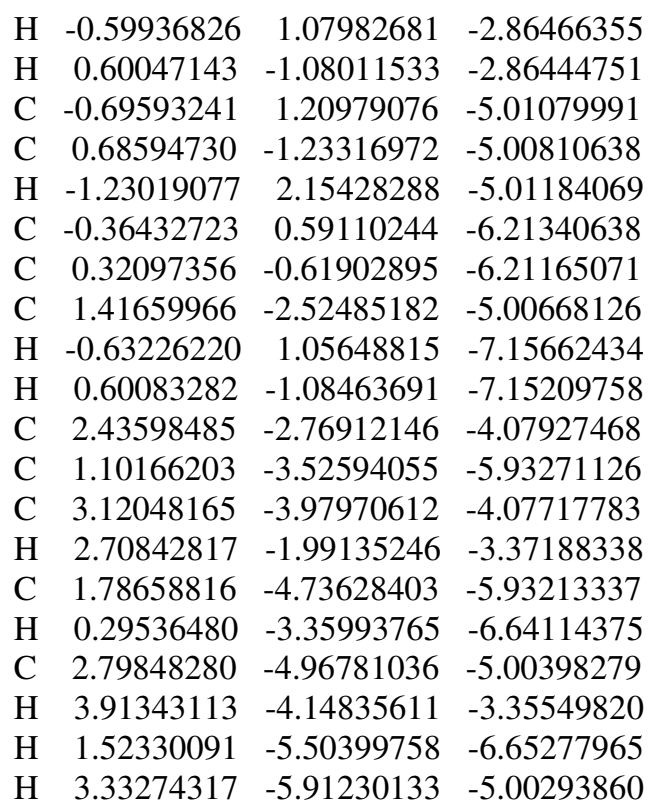

Ph_pi0_4tBuPh_side_1
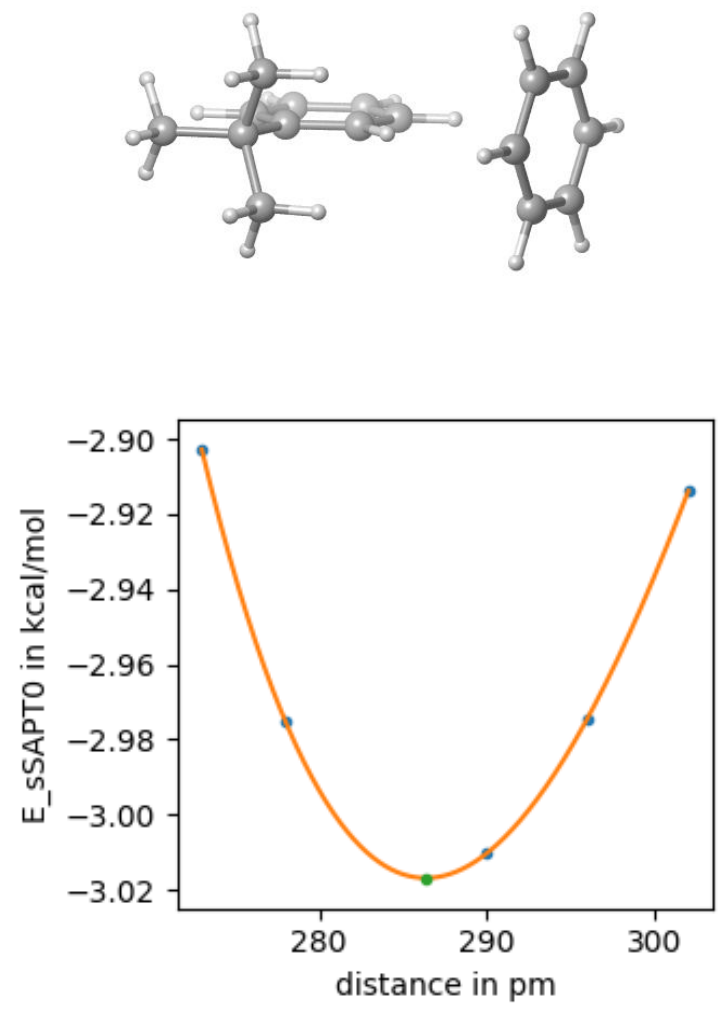

36

Ph_pi0_4tBuPh_side_1-286

$\begin{array}{llll}\text { H } & 2.47830959 & -0.04134128 & 0.00002015\end{array}$

$\begin{array}{llll}\text { C } & 1.39302564 & -0.02324781 & 0.00000008\end{array}$

$\begin{array}{llll}\text { C } & 0.71636748 & 1.19441056 & -0.00010067\end{array}$ $\begin{array}{llll}\text { C } & 0.67612530 & -1.21763495 & 0.00007275\end{array}$

$\begin{array}{llll}\text { C } & -0.67625398 & 1.21769798 & 0.00007325\end{array}$

$\begin{array}{llll}\mathrm{H} & 1.27482284 & 2.12543743 & 0.00000658\end{array}$

$\begin{array}{llll}\text { C } & -0.71652436 & -1.19445047 & -0.00005433\end{array}$

$\mathrm{H} \quad 1.20319885 \quad-2.16677876 \quad 0.00008956$

$\begin{array}{llll}\text { C } & -1.39262449 & 0.02322482 & -0.00003308\end{array}$

$\begin{array}{llll}\mathrm{H} & -1.20362977 & 2.16671637 & 0.00016144\end{array}$

H $\quad-1.27520554-2.12538191-0.00007858$

$\begin{array}{llll}\mathrm{H} & -2.47818735 & 0.04136582 & 0.00005785\end{array}$

$\begin{array}{llll}\text { C } & 0.35969628 & 0.59858086 & -3.80353156\end{array}$

$\begin{array}{llll}\text { C } & -0.35464847 & -0.59282150 & -3.81817614\end{array}$

$\mathrm{H} \quad 0.63104481 \quad 1.05143126-2.85497007$

H $\quad-0.62994309-1.05171722-2.87236142$

$\begin{array}{llll}\text { C } & 0.72639756 & 1.20980174 & -5.00076783\end{array}$

$\begin{array}{llll}\text { C } & -0.72431887 & -1.20972678 & -5.02137015\end{array}$

$\begin{array}{llll}\mathrm{H} & 1.28440389 & 2.14046273 & -4.99346549\end{array}$

C $\quad 0.36879936 \quad 0.61298890 \quad-6.20291316$

$\begin{array}{llll}\text { C } & -0.34839666 & -0.58317497 & -6.21160302\end{array}$

$\begin{array}{llll}\text { C } & -1.51166127 & -2.52299696 & -4.98625956\end{array}$

H $\quad 0.64666108 \quad 1.07610330 \quad-7.14465146$

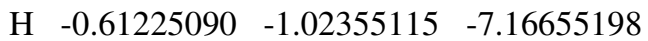

C $-0.68878293 \quad-3.59062102 \quad-4.24550755$

$\begin{array}{llll}\text { C } & -2.84174739 & -2.29996926 & -4.24680338\end{array}$

C $-1.82478418-3.04670612-6.39151180$

$\mathrm{H} \quad 0.26480040 \quad-3.76487737 \quad-4.75282636$

$\mathrm{H} \quad-0.47321750 \quad-3.29063274-3.21631769$

H $\quad-1.23989962-4.53642067-4.21208035$

H $\quad-3.44437191 \quad-1.54128977 \quad-4.75504373$

H $\quad-3.41624757 \quad-3.23175166-4.21342947$

$\mathrm{H} \quad-2.67969153 \quad-1.96796731 \quad-3.21762998$

$\mathrm{H} \quad-2.38609193 \quad-3.98277224-6.31606883$

H $\quad-2.43382707-2.33635235-6.95922232$

H $\quad-0.91074721 \quad-3.24959913 \quad-6.95825691$

Ph_pi0_4tBuPh_side_2

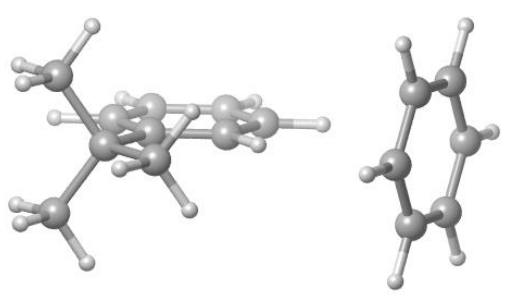




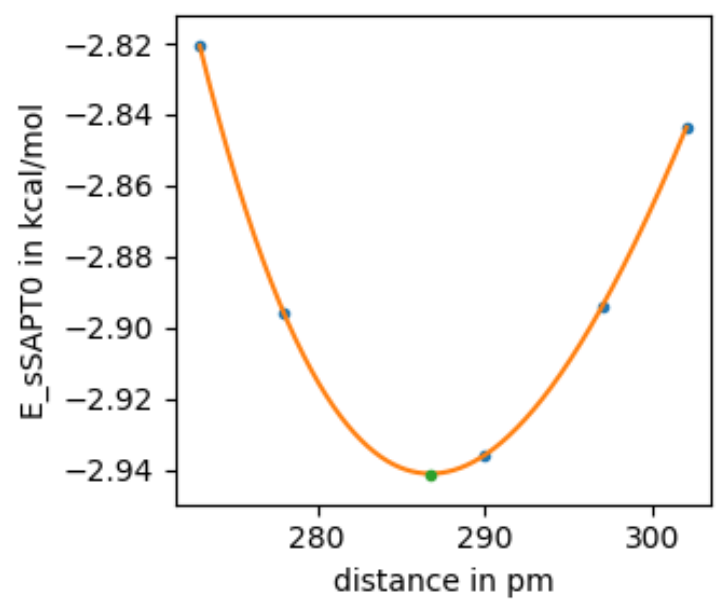

36

Ph_pi0_4tBuPh_side_2-286

$\begin{array}{llll}\text { H } & 2.47830959 & -0.04134128 & 0.00002015\end{array}$

$\begin{array}{llll}\text { C } & 1.39302564 & -0.02324781 & 0.00000008\end{array}$

$\begin{array}{llll}\text { C } & 0.71636748 & 1.19441056 & -0.00010067\end{array}$

$\begin{array}{llll}\text { C } & 0.67612530 & -1.21763495 & 0.00007275\end{array}$

$\begin{array}{llll}\text { C } & -0.67625398 & 1.21769798 & 0.00007325\end{array}$

$\begin{array}{llll}\mathrm{H} & 1.27482284 & 2.12543743 & 0.00000658\end{array}$

$\begin{array}{lrrr}\text { C } & -0.71652436 & -1.19445047 & -0.00005433\end{array}$

$\begin{array}{llll}\mathrm{H} & 1.20319885 & -2.16677876 & 0.00008956\end{array}$

$\begin{array}{llll}\text { C } & -1.39262449 & 0.02322482 & -0.00003308\end{array}$

$\begin{array}{llll}\mathrm{H} & -1.20362977 & 2.16671637 & 0.00016144\end{array}$

$\begin{array}{llll}\mathrm{H} & -1.27520554 & -2.12538191 & -0.00007858\end{array}$

$\begin{array}{llll}\mathrm{H} & -2.47818735 & 0.04136582 & 0.00005785\end{array}$

$\begin{array}{llll}\text { C } & 0.33966481 & -0.61036546 & -3.80993476\end{array}$

$\begin{array}{llll}\text { C } & -0.33759004 & 0.60882819 & -3.82203174\end{array}$

H $\quad 0.59497741 \quad-1.07022552 \quad-2.86025202$

$\begin{array}{llll}\mathrm{H} & -0.59387473 & 1.06993932 & -2.87483987\end{array}$

$\begin{array}{llll}\text { C } & 0.68631454 & -1.23409369 & -5.00157753\end{array}$

$\begin{array}{llll}\text { C } & -0.68363396 & 1.23205758 & -5.02302204\end{array}$

$\begin{array}{llll}\text { C } & 0.34903129 & -0.62658015 & -6.20930382\end{array}$

$\begin{array}{llll}\text { C } & -0.32557619 & 0.58783761 & -6.21536403\end{array}$

H $\quad 1.21324918 \quad-2.18267973-4.99270686$

C $\quad-1.42679019 \quad 2.57003466 \quad-5.08094903$

$\mathrm{H} \quad 0.61234516 \quad-1.10032270 \quad-7.14989205$

$\begin{array}{llll}\mathrm{H} & -0.57843224 & 1.04334440 & -7.16904913\end{array}$

$\begin{array}{llll}\text { C } & -0.56349424 & 3.60028809 & -5.82858624\end{array}$

$\begin{array}{llll}\text { C } & -2.75794885 & 2.38151484 & -5.82803856\end{array}$

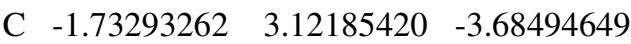

$\mathrm{H} \quad 0.39150446 \quad 3.74950152 \quad-5.31597364$

$\begin{array}{llll}\mathrm{H} & -0.35026712 & 3.28011381 & -6.85216614\end{array}$

H $-1.08273678 \quad 4.56325114 \quad-5.87831856$

$\mathrm{H} \quad-3.38914870 \quad 1.64974755 \quad-5.31504489$

$\mathrm{H} \quad-3.30102579 \quad 3.33124183 \quad-5.87772574$

H $-2.59926026 \quad 2.03111311-6.85162141$

$\mathrm{H} \quad-2.26236755 \quad 4.07488482 \quad-3.77664768$

H $\quad-2.36939339 \quad 2.43936034-3.11315040$

H $\quad-0.81695514 \quad 3.30175708 \quad-3.11358901$
Ph_pi_2Nap_side1
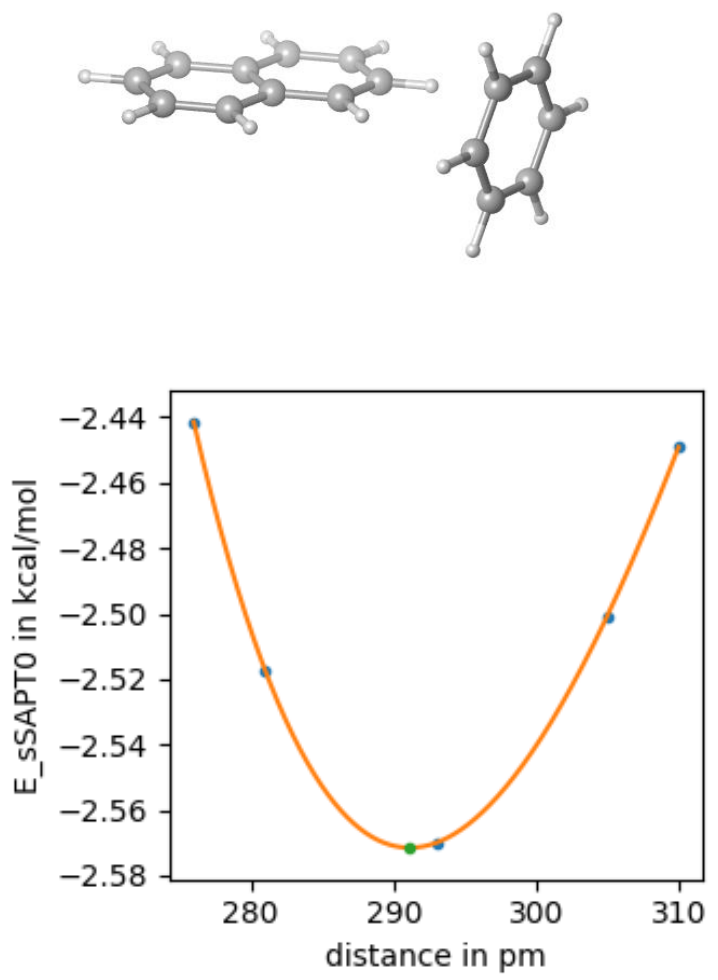

30

Ph_pi_2Nap_side1-291

$\begin{array}{llll}\mathrm{H} & 2.47830959 & -0.04134128 & 0.00002015\end{array}$

$\begin{array}{llll}\text { C } & 1.39302564 & -0.02324781 & 0.00000008\end{array}$

$\begin{array}{llll}\text { C } & 0.71636748 & 1.19441056 & -0.00010067\end{array}$

$\begin{array}{llll}\text { C } & 0.67612530 & -1.21763495 & 0.00007275\end{array}$

$\begin{array}{llll}\text { C } & -0.67625398 & 1.21769798 & 0.00007325\end{array}$

$\begin{array}{llll}\mathrm{H} & 1.27482284 & 2.12543743 & 0.00000658\end{array}$

$\begin{array}{llll}\text { C } & -0.71652436 & -1.19445047 & -0.00005433\end{array}$

$\begin{array}{llll}\mathrm{H} & 1.20319885 & -2.16677876 & 0.00008956\end{array}$

$\begin{array}{llll}\text { C } & -1.39262449 & 0.02322482 & -0.00003308\end{array}$

$\begin{array}{llll}\mathrm{H} & -1.20362977 & 2.16671637 & 0.00016144\end{array}$

$\begin{array}{llll}\mathrm{H} & -1.27520554 & -2.12538191 & -0.00007858\end{array}$

$\begin{array}{llll}\mathrm{H} & -2.47818735 & 0.04136582 & 0.00005785\end{array}$

$\begin{array}{llll}\mathrm{H} & -1.23358825 & 0.02047945 & -2.91181197\end{array}$

$\begin{array}{llll}\text { C } & -0.68728257 & 0.01132656 & -3.84938841\end{array}$

$\begin{array}{llll}\text { C } & -1.39950972 & 0.02319484 & -5.07388299\end{array}$

$\begin{array}{llll}\text { C } & 0.68300264 & -0.01157794 & -3.84758059\end{array}$

$\begin{array}{lllll}\mathrm{H} & -2.48429921 & 0.04132983 & -5.06206908\end{array}$

$\begin{array}{llll}\text { C } & -0.72444750 & 0.01187431 & -6.26648512\end{array}$

$\begin{array}{llll}\text { C } & 1.41067067 & -0.02378079 & -5.06708789\end{array}$

H $\quad 1.23469940 \quad-0.02076859 \quad-2.91150931$

$\begin{array}{llll}\text { C } & 0.69510407 & -0.01185785 & -6.29685736\end{array}$

$\begin{array}{lllll}\mathrm{H} & -1.26577444 & 0.02089941 & -7.20863467\end{array}$

$\begin{array}{llll}\text { C } & 2.83030072 & -0.04751726 & -5.09747229\end{array}$

$\begin{array}{llll}\text { C } & 1.42278338 & -0.02406389 & -7.51632729\end{array}$ 


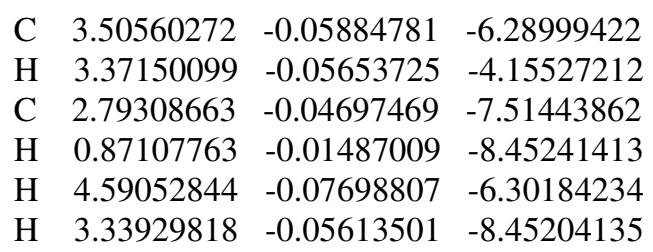

Ph_pi_2Nap_side2
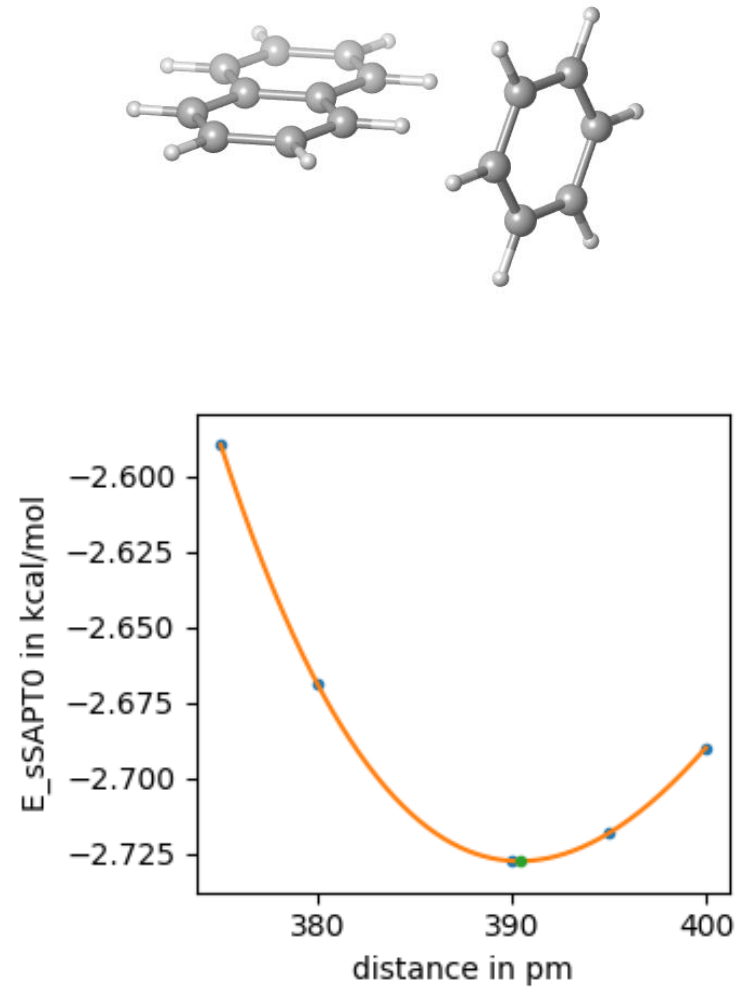

30

Ph_pi_2Nap_side2-390

$\begin{array}{llll}\text { H } & 2.47830959 & -0.04134128 & 0.00002015\end{array}$

$\begin{array}{llll}\text { C } & 1.39302564 & -0.02324781 & 0.00000008\end{array}$

$\begin{array}{llll}\text { C } & 0.71636748 & 1.19441056 & -0.00010067\end{array}$

$\begin{array}{llll}\text { C } & 0.67612530 & -1.21763495 & 0.00007275\end{array}$

$\begin{array}{llll}\text { C } & -0.67625398 & 1.21769798 & 0.00007325\end{array}$

$\begin{array}{llll}\mathrm{H} & 1.27482284 & 2.12543743 & 0.00000658\end{array}$

$\begin{array}{llll}\text { C } & -0.71652436 & -1.19445047 & -0.00005433\end{array}$

$\begin{array}{llll}\mathrm{H} & 1.20319885 & -2.16677876 & 0.00008956\end{array}$

$\begin{array}{llll}\text { C } & -1.39262449 & 0.02322482 & -0.00003308\end{array}$

$\begin{array}{llll}\mathrm{H} & -1.20362977 & 2.16671637 & 0.00016144\end{array}$

$\begin{array}{llll}\mathrm{H} & -1.27520554 & -2.12538191 & -0.00007858\end{array}$

$\begin{array}{llll}\mathrm{H} & -2.47818735 & 0.04136582 & 0.00005785\end{array}$

H $-1.27015725 \quad 0.02105988-3.93094403$

$\begin{array}{llll}\text { C } & -0.72820686 & 0.01197732 & -4.87273454\end{array}$

$\begin{array}{llll}\text { C } & -1.40247981 & 0.02321353 & -6.06578388\end{array}$

$\begin{array}{llll}\text { C } & 0.69132436 & -0.01174943 & -4.84142132\end{array}$

$\begin{array}{llll}\mathrm{H} & -2.48726127 & 0.04134570 & -6.07831687\end{array}$

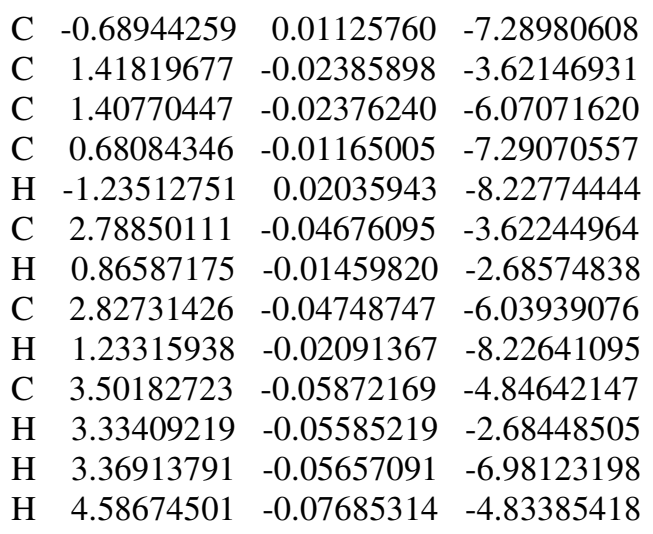

Ph_pi_4MePh_side
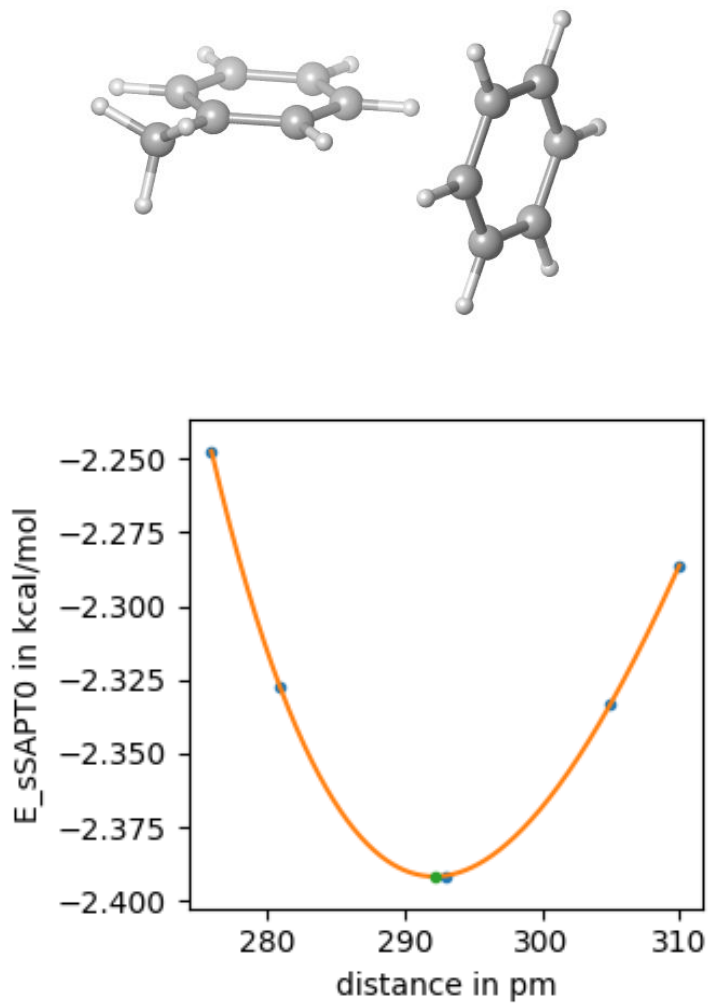

27

Ph_pi_4MePh_side-292

H $\quad 2.47830959 \quad-0.04134128 \quad 0.00002015$

$\begin{array}{llll}\text { C } & 1.39302564 & -0.02324781 & 0.00000008\end{array}$

$\begin{array}{llll}\text { C } & 0.71636748 & 1.19441056 & -0.00010067\end{array}$

$\begin{array}{llll}\text { C } & 0.67612530 & -1.21763495 & 0.00007275\end{array}$

$\begin{array}{llll}\text { C } & -0.67625398 & 1.21769798 & 0.00007325\end{array}$

$\begin{array}{llll}\mathrm{H} & 1.27482284 & 2.12543743 & 0.00000658\end{array}$

$\begin{array}{llll}\text { C } & -0.71652436 & -1.19445047 & -0.00005433\end{array}$

$\mathrm{H} \quad 1.20319885 \quad-2.16677876 \quad 0.00008956$

$\begin{array}{llll}\text { C } & -1.39262449 & 0.02322482 & -0.00003308\end{array}$

$\begin{array}{llll}\mathrm{H} & -1.20362977 & 2.16671637 & 0.00016144\end{array}$ 


$\begin{array}{rrrr}\mathrm{H} & -1.27520554 & -2.12538191 & -0.00007858 \\ \mathrm{H} & -2.47818735 & 0.04136582 & 0.00005785 \\ \mathrm{H} & 1.23620190 & -0.02072398 & -2.92191951 \\ \mathrm{C} & 0.69549613 & -0.01214982 & -3.86499944 \\ \mathrm{C} & -0.69603390 & 0.01142819 & -3.86455864 \\ \mathrm{C} & 1.41292036 & -0.02730990 & -5.06351383 \\ \mathrm{C} & -1.39542415 & 0.01807209 & -5.06864104 \\ \mathrm{H} & -1.23508564 & 0.02043424 & -2.92222295 \\ \mathrm{C} & 0.69983893 & -0.02316359 & -6.26440433 \\ \mathrm{C} & 2.92083880 & -0.01997295 & -5.06001852 \\ \mathrm{H} & -2.48089579 & 0.03275486 & -5.07056790 \\ \mathrm{C} & -0.69192437 & 0.00030006 & -6.26995157 \\ \mathrm{H} & 1.24378271 & -0.04033882 & -7.20545912 \\ \mathrm{H} & 3.30659021 & 1.00449729 & -5.03850803 \\ \mathrm{H} & 3.31705930 & -0.53907464 & -4.18404118 \\ \mathrm{H} & 3.32111991 & -0.50405650 & -5.95389271 \\ \mathrm{H} & -1.22757934 & 0.00063808 & -7.21426199\end{array}$

Ph_pi_Ph_side
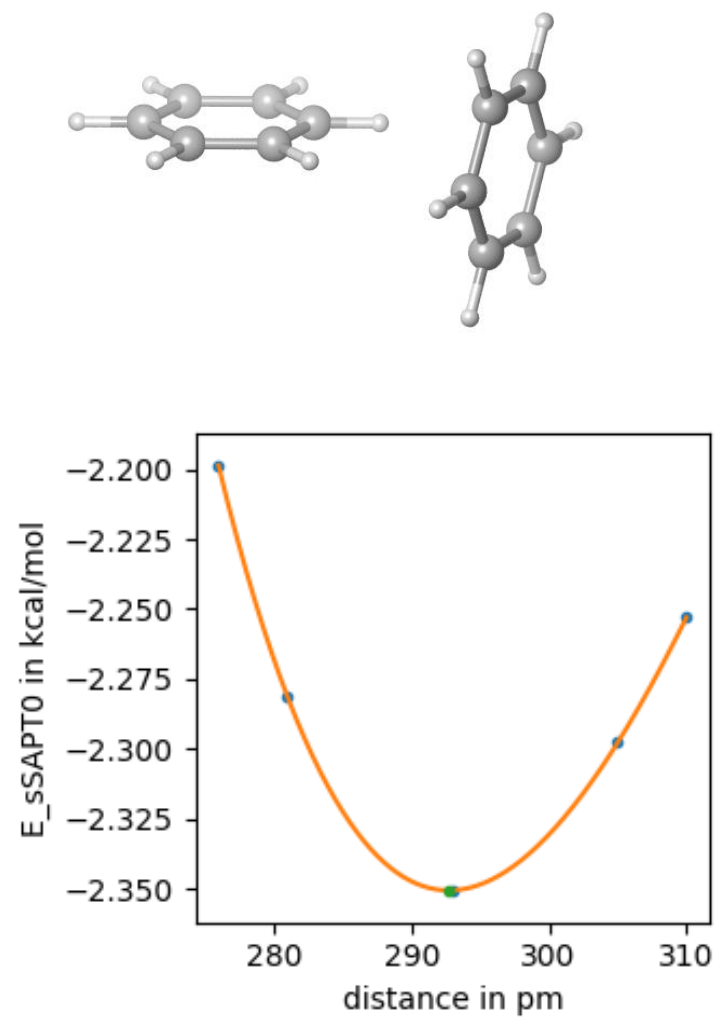

24

Ph_pi_Ph_side-292

$\mathrm{H} \quad 2.47838500 \quad-0.03653600 \quad 0.00002000$

$\begin{array}{llll}\text { C } & 1.39306800 & -0.02054700 & 0.00000000\end{array}$

$\begin{array}{llll}\text { C } & 0.71405000 & 1.19579700 & -0.00010100\end{array}$

$\begin{array}{llll}\text { C } & 0.67848500 & -1.21632200 & 0.00007300\end{array}$

$\begin{array}{llll}\text { C } & -0.67861400 & 1.21638400 & 0.00007300\end{array}$

H $\quad 1.27069900 \quad 2.12790500 \quad 0.00000600$
C $\quad-0.71420700 \quad-1.19583800 \quad-0.00005400$

$\begin{array}{llll}\mathrm{H} & 1.20739800 & -2.16444200 & 0.00009000\end{array}$

C $\quad-1.39266700 \quad 0.02052400 \quad-0.00003300$

$\begin{array}{llll}\mathrm{H} & -1.20782900 & 2.16437800 & 0.00016100\end{array}$

$\mathrm{H}-1.27108200 \quad-2.12785100 \quad-0.00007800$

$\begin{array}{llll}\mathrm{H} & -2.47826300 & 0.03656000 & 0.00005800\end{array}$

$\begin{array}{llll}\text { C } & -0.69580889 & 0.01016103 & -3.86777987\end{array}$

C $\quad 0.69685965-0.01050716-3.86772304$

$\mathrm{H} \quad-1.23841377 \quad 0.01815659 \quad-2.92741975$

H $\quad 1.23954223 \quad-0.01853243 \quad-2.92740019$

$\begin{array}{llll}\text { C } & -1.39198069 & 0.02039886 & -5.07408700\end{array}$

$\begin{array}{llll}\text { C } & 1.39336464 & -0.02067865 & -5.07380008\end{array}$

$\begin{array}{llll}\text { C } & -0.69577537 & 0.01035386 & -6.28063390\end{array}$

$\begin{array}{llll}\mathrm{H} & -2.47753267 & 0.03653361 & -5.07420292\end{array}$

C $\quad 0.69709450 \quad-0.01013835-6.28007841$

H $\quad 2.47895212-0.03673095 \quad-5.07344141$

$\mathrm{H} \quad-1.23835365 \quad 0.01848857 \quad-7.22069309$

H $\quad 1.23996390 \quad-0.01803281 \quad-7.22024382$

Edge-to-face complex $\mathbf{A r}_{\text {side }}^{A}-\mathbf{P h}_{\boldsymbol{\pi}}$

1Nap_side1_Ph_pi_0
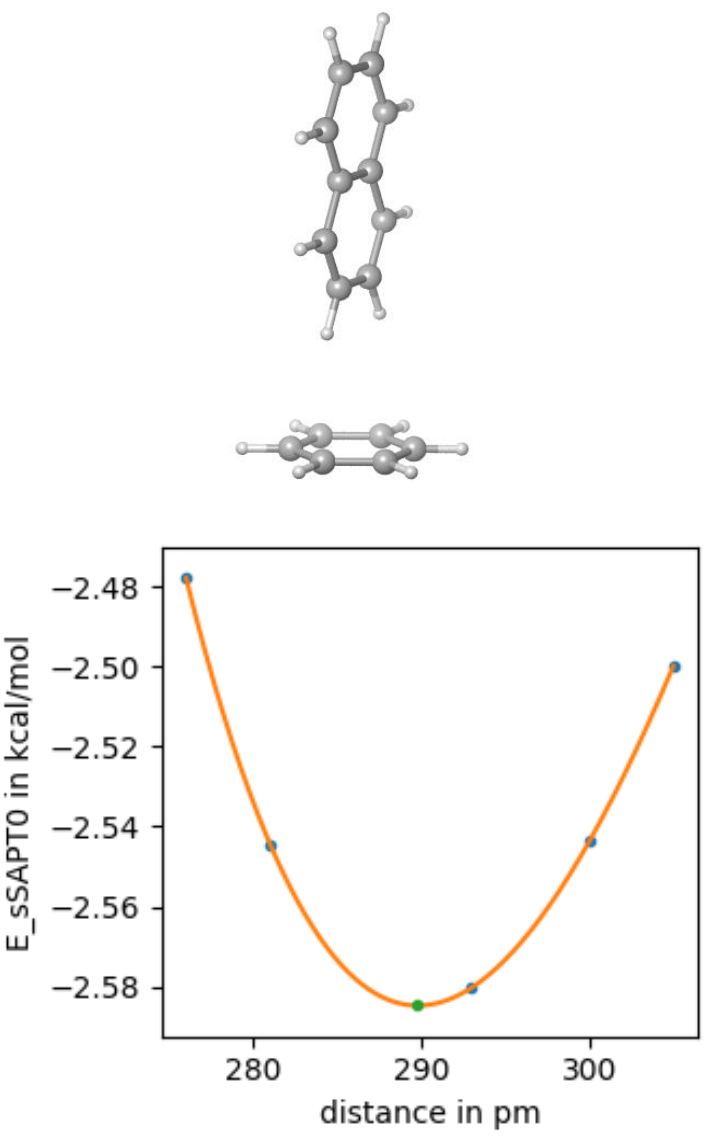

30

1Nap_side1_Ph_pi_0-289

H $\quad 2.47830959-0.04134128 \quad 0.00002015$

$\begin{array}{llll}\text { C } & 1.39302564 & -0.02324781 & 0.00000008\end{array}$

$\begin{array}{llll}\text { C } & 0.71636748 & 1.19441056 & -0.00010067\end{array}$ 


$\begin{array}{lrrr}\mathrm{C} & 0.67612530 & -1.21763495 & 0.00007275 \\ \mathrm{C} & -0.67625398 & 1.21769798 & 0.00007325 \\ \mathrm{H} & 1.27482284 & 2.12543743 & 0.00000658 \\ \mathrm{C} & -0.71652436 & -1.19445047 & -0.00005433 \\ \mathrm{H} & 1.20319885 & -2.16677876 & 0.00008956 \\ \mathrm{C} & -1.39262449 & 0.02322482 & -0.00003308 \\ \mathrm{H} & -1.20362977 & 2.16671637 & 0.00016144 \\ \mathrm{H} & -1.27520554 & -2.12538191 & -0.00007858 \\ \mathrm{H} & -2.47818735 & 0.04136582 & 0.00005785 \\ \mathrm{H} & 1.24419759 & -0.02085643 & -2.89793274 \\ \mathrm{C} & 0.70870996 & -0.01196179 & -3.84173205 \\ \mathrm{C} & -0.70770765 & 0.01162806 & -3.84191320 \\ \mathrm{C} & 1.39945397 & -0.02350408 & -5.02534893 \\ \mathrm{H} & -1.24309039 & 0.02057731 & -2.89823851 \\ \mathrm{C} & -1.39883000 & 0.02309918 & -5.02527929 \\ \mathrm{C} & 0.71153819 & -0.01209046 & -6.26772139 \\ \mathrm{H} & 2.48587864 & -0.04159526 & -5.03146114 \\ \mathrm{C} & -0.71111754 & 0.01160228 & -6.26766882 \\ \mathrm{H} & -2.48529195 & 0.04119855 & -5.03118808 \\ \mathrm{C} & 1.39927959 & -0.02359085 & -7.51018493 \\ \mathrm{C} & -1.39899537 & 0.02301227 & -7.51003228 \\ \mathrm{C} & 0.70834709 & -0.01212889 & -8.69371804 \\ \mathrm{H} & 2.48572161 & -0.04168688 & -7.50414114 \\ \mathrm{C} & -0.70817246 & 0.01146266 & -8.69362418 \\ \mathrm{H} & -2.48543799 & 0.04110674 & -7.50392008 \\ \mathrm{H} & 1.24376876 & -0.02108180 & -9.63752773 \\ \mathrm{H} & -1.24373023 & 0.02034947 & -9.63735548\end{array}$

1Nap_side2_Ph_pi_0
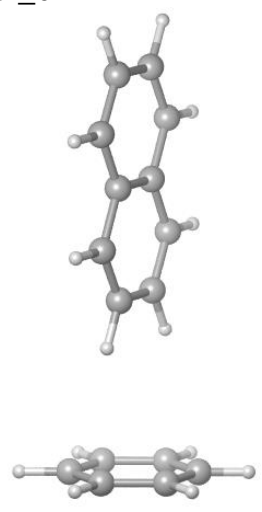

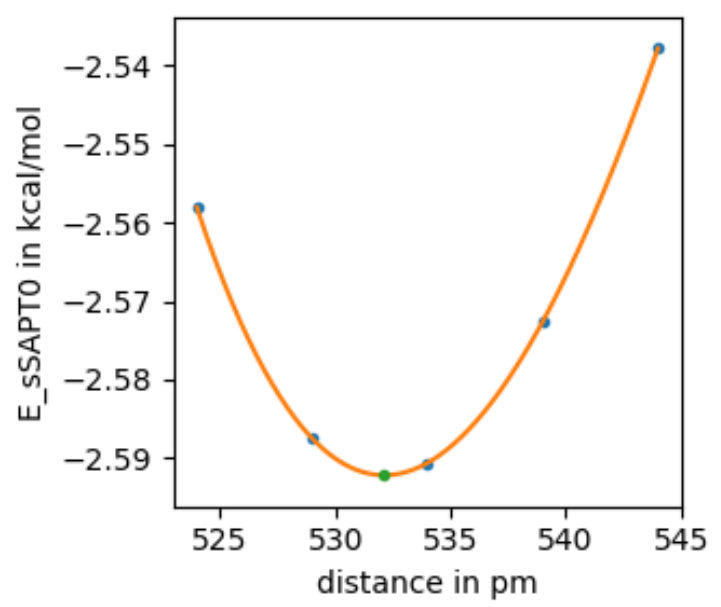

30

1Nap_side2_Ph_pi_0-532

$\begin{array}{llll}\mathrm{H} & 2.47830959 & -0.04134128 & 0.00002015\end{array}$

$\begin{array}{llll}\text { C } & 1.39302564 & -0.02324781 & 0.00000008\end{array}$

$\begin{array}{llll}\text { C } & 0.71636748 & 1.19441056 & -0.00010067\end{array}$

$\begin{array}{llll}\text { C } & 0.67612530 & -1.21763495 & 0.00007275\end{array}$

$\begin{array}{llll}\text { C } & -0.67625398 & 1.21769798 & 0.00007325\end{array}$

$\begin{array}{llll}\mathrm{H} & 1.27482284 & 2.12543743 & 0.00000658\end{array}$

$\begin{array}{llll}\text { C } & -0.71652436 & -1.19445047 & -0.00005433\end{array}$

$\begin{array}{llll}\mathrm{H} & 1.20319885 & -2.16677876 & 0.00008956\end{array}$

$\begin{array}{llll}\text { C } & -1.39262449 & 0.02322482 & -0.00003308\end{array}$

$\begin{array}{llll}\mathrm{H} & -1.20362977 & 2.16671637 & 0.00016144\end{array}$

$\begin{array}{llll}\mathrm{H} & -1.27520554 & -2.12538191 & -0.00007858\end{array}$

$\begin{array}{llll}\mathrm{H} & -2.47818735 & 0.04136582 & 0.00005785\end{array}$

C $\quad-0.68621364 \quad 0.01123166-6.25046946$

$\begin{array}{llll}\text { C } & 0.73613103 & -0.01253943 & -6.28015544\end{array}$

$\begin{array}{llll}\text { C } & -1.39985039 & 0.02312263 & -7.47824342\end{array}$

$\begin{array}{llll}\text { C } & -1.34793256 & 0.02232250 & -4.99394999\end{array}$

$\begin{array}{llll}\text { C } & 1.39782247 & -0.02363281 & -7.53660032\end{array}$

$\begin{array}{llll}\text { C } & 1.44972956 & -0.02443277 & -5.05238968\end{array}$

$\begin{array}{lllll}\text { C } & -0.73390431 & 0.01195676 & -8.67599155\end{array}$

$\begin{array}{lllll}\mathrm{H} & -2.48591101 & 0.04127594 & -7.44950270\end{array}$

$\begin{array}{llll}\text { C } & -0.63250513 & 0.01039598 & -3.82506545\end{array}$

$\begin{array}{llll}\mathrm{H} & -2.43426382 & 0.04047500 & -4.97736226\end{array}$

$\begin{array}{llll}\text { C } & 0.68220849 & -0.01170917 & -8.70531391\end{array}$

$\begin{array}{llll}\text { H } & 2.48417093 & -0.04178260 & -7.55332344\end{array}$

C $\quad 0.78370388 \quad-0.01327157 \quad-3.85466490$

$\begin{array}{llll}\mathrm{H} & 2.53580822 & -0.04258338 & -5.08113078\end{array}$

$\begin{array}{llll}\mathrm{H} & -1.28892902 & 0.02119626 & -9.60843213\end{array}$

$\begin{array}{llll}\mathrm{H} & -1.14815659 & 0.01903752 & -2.87030781\end{array}$

H $\quad 1.19782374-0.02035311-9.65993577$

H $\quad 1.33879699 \quad-0.02252121 \quad-2.92229376$ 
2Fur_side1_Ph_pi_0
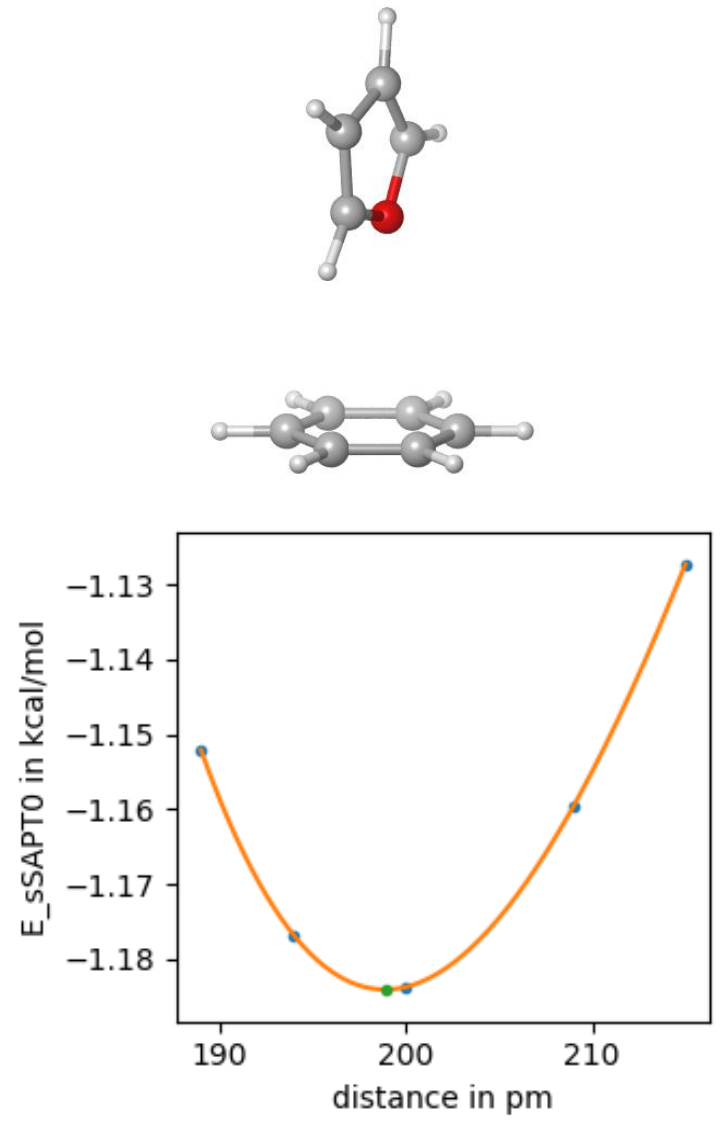

21

2Fur_side1_Ph_pi_0-198

H $\quad 2.47838500 \quad-0.03653600 \quad 0.00002000$

C $\quad 1.39306800 \quad-0.02054700 \quad 0.00000000$

$\begin{array}{llll}\text { C } & 0.71405000 & 1.19579700 & -0.00010100\end{array}$

C $\quad 0.67848500 \quad-1.21632200 \quad 0.00007300$

C $\quad-0.67861400 \quad 1.21638400 \quad 0.00007300$

H $\quad 1.27069900 \quad 2.12790500 \quad 0.00000600$

C $\quad-0.71420700 \quad-1.19583800 \quad-0.00005400$

H $\quad 1.20739800 \quad-2.16444200 \quad 0.00009000$

C $\quad-1.39266700 \quad 0.02052400 \quad-0.00003300$

H $\quad-1.20782900 \quad 2.16437800 \quad 0.00016100$

H $\quad-1.27108200 \quad-2.12785100 \quad-0.00007800$

H $\quad-2.47826300 \quad 0.03656000 \quad 0.00005800$

O $\quad 0.21860118 \quad-0.39088064 \quad-3.47253894$

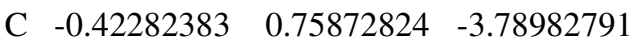

C $\quad 0.55461303 \quad-0.99288689 \quad-4.63841295$

C $\quad-0.50338724 \quad 0.90375486 \quad-5.13596588$

C $0.14012366 \quad-0.24984542 \quad-5.69462439$

$\mathrm{H} \quad-0.75689327 \quad 1.35721261 \quad-2.95743755$

H $\quad 1.07818942 \quad-1.93133759 \quad-4.54977094$

H $-0.96070796 \quad 1.72376637 \quad-5.66788634$

H $\quad 0.20281313 \quad-0.52348392 \quad-6.73657962$
2Fur_side2_Ph_pi_0
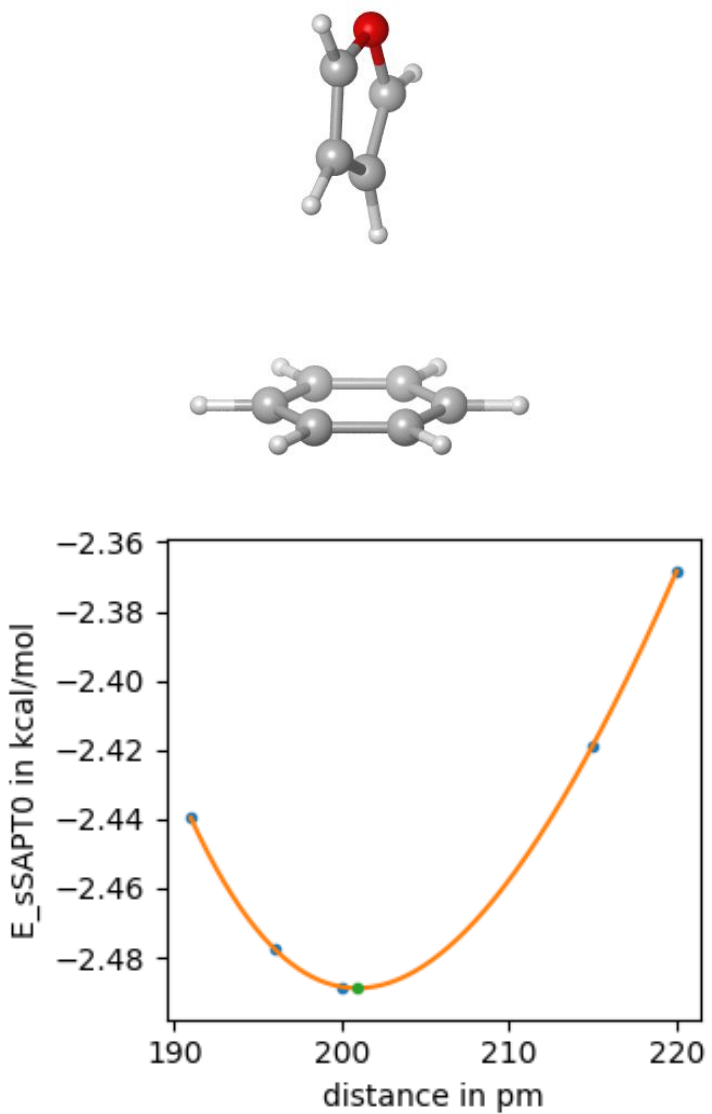

21

2Fur_side2_Ph_pi_0-200

$\mathrm{H} \quad 2.47838500 \quad-0.03653600 \quad 0.00002000$

$\begin{array}{llll}\text { C } & 1.39306800 & -0.02054700 & 0.00000000\end{array}$

C $\quad 0.71405000 \quad 1.19579700 \quad-0.00010100$

C $\quad 0.67848500 \quad-1.21632200 \quad 0.00007300$

C $-\begin{array}{llll}-0.67861400 & 1.21638400 & 0.00007300\end{array}$

H $\quad 1.27069900 \quad 2.12790500 \quad 0.00000600$

C $\quad-0.71420700 \quad-1.19583800 \quad-0.00005400$

H $\quad 1.20739800 \quad-2.16444200 \quad 0.00009000$

C $\quad-1.39266700 \quad 0.02052400 \quad-0.00003300$

$\mathrm{H} \quad-1.20782900 \quad 2.16437800 \quad 0.00016100$

H $\quad-1.27108200 \quad-2.12785100 \quad-0.00007800$

H $\quad-2.47826300 \quad 0.03656000 \quad 0.00005800$

C $\quad-0.10611138 \quad 0.27436719 \quad-3.64652127$

H $\quad-0.27780050 \quad 0.49861369 \quad-2.60502473$

C $\quad-0.41374974 \quad 1.06576159 \quad-4.70413567$

C $\quad 0.37314724 \quad-0.95755950 \quad-4.20299861$

C $\quad 0.31373764 \quad-0.80504323 \quad-5.54940863$

$\begin{array}{lllll}\mathrm{O} & -0.16312175 & 0.42143481 & -5.86887010\end{array}$

H $-0.80316828 \quad 2.06718936 \quad-4.79455136$

H $\quad 0.71319221 \quad-1.83179068 \quad-3.66952907$

H $\quad 0.56264107 \quad-1.44513468 \quad-6.38066650$ 
2Nap_side1_Ph_pi_0
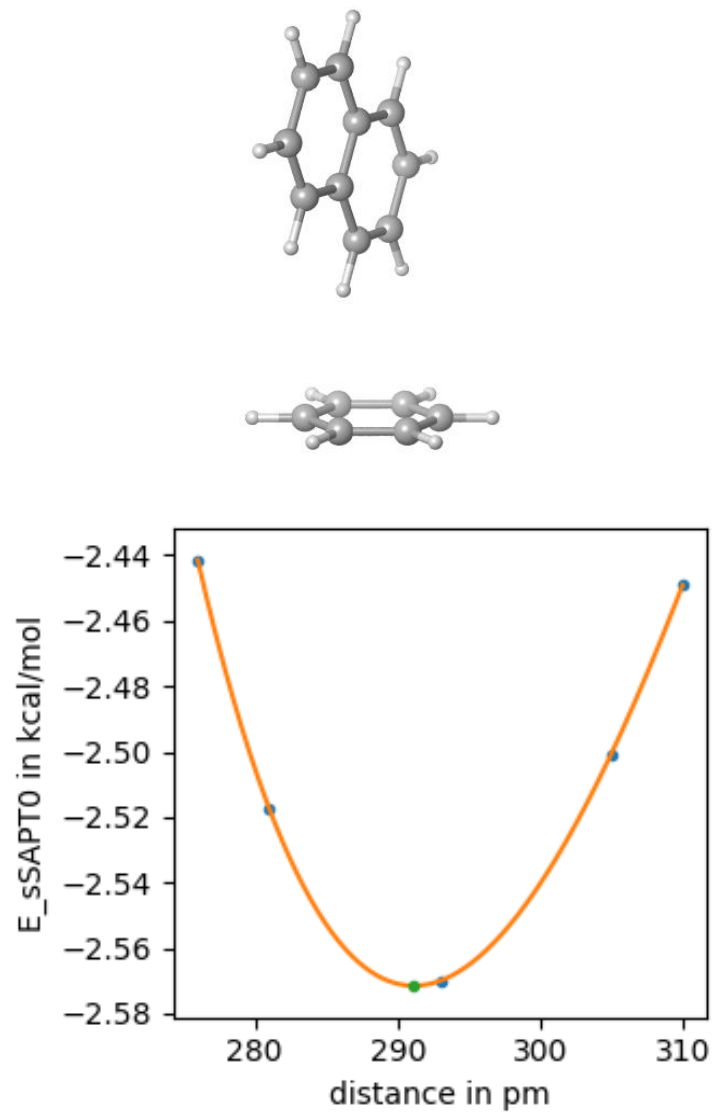

30

2Nap_side1_Ph_pi_0-291

H $2.47830959 \quad-0.04134128 \quad 0.00002015$

C $\quad 1.39302564 \quad-0.02324781 \quad 0.00000008$

C $\quad 0.71636748 \quad 1.19441056 \quad-0.00010067$

C $\quad 0.67612530 \quad-1.21763495 \quad 0.00007275$

C $\quad-0.67625398 \quad 1.21769798 \quad 0.00007325$

H $1.27482284 \quad 2.12543743 \quad 0.00000658$

C $\quad-0.71652436 \quad-1.19445047 \quad-0.00005433$

H $\quad 1.20319885 \quad-2.16677876 \quad 0.00008956$

C $\quad-1.39262449 \quad 0.02322482 \quad-0.00003308$

H $\quad-1.20362977 \quad 2.16671637 \quad 0.00016144$

H $\quad-1.27520554 \quad-2.12538191 \quad-0.00007858$

H $-2.47818735 \quad 0.04136582 \quad 0.00005785$

H $\quad-1.23358825 \quad 0.02047945 \quad-2.91181197$

C $\quad-0.68728257 \quad 0.01132656 \quad-3.84938841$

C $\quad-1.39950972 \quad 0.02319484 \quad-5.07388299$

C $0.68300264 \quad-0.01157794 \quad-3.84758059$

$\mathrm{H} \quad-2.48429921 \quad 0.04132983 \quad-5.06206908$

C $\quad-0.72444750 \quad 0.01187431 \quad-6.26648512$

C $\quad 1.41067067 \quad-0.02378079 \quad-5.06708789$

H $\quad 1.23469940 \quad-0.02076859 \quad-2.91150931$

C $0.69510407-0.01185785 \quad-6.29685736$

H $\quad-1.26577444 \quad 0.02089941 \quad-7.20863467$

C $\quad 2.83030072 \quad-0.04751726 \quad-5.09747229$

C $\quad 1.42278338 \quad-0.02406389 \quad-7.51632729$
$\begin{array}{llll}\text { C } & 3.50560272 & -0.05884781 & -6.28999422\end{array}$
H $\quad 3.37150099 \quad-0.05653725 \quad-4.15527212$
C $\quad 2.79308663 \quad-0.04697469-7.51443862$
H $\quad 0.87107763 \quad-0.01487009 \quad-8.45241413$
$\mathrm{H} \quad 4.59052844 \quad-0.07698807 \quad-6.30184234$
H $\quad 3.33929818 \quad-0.05613501 \quad-8.45204135$

2Nap_side2_Ph_pi_0
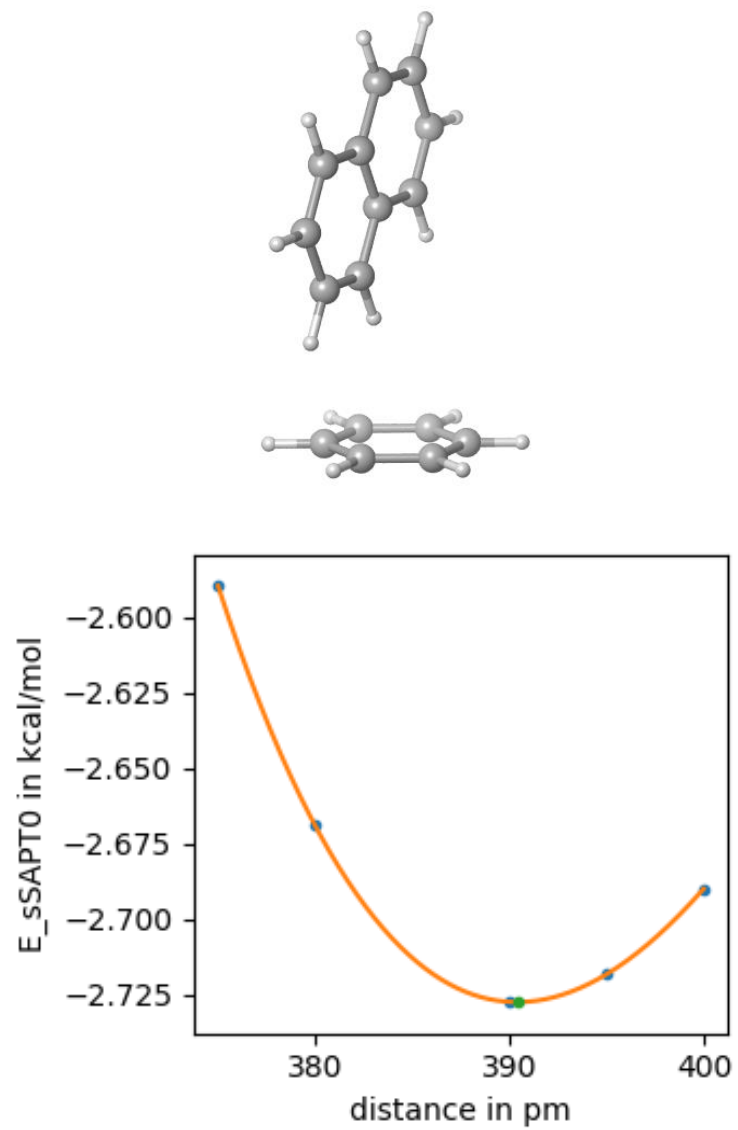

30

2Nap_side2_Ph_pi_0-390

H $\quad 2.47830959 \quad-0.04134128 \quad 0.00002015$

$\begin{array}{llll}\text { C } & 1.39302564 & -0.02324781 & 0.00000008\end{array}$

$\begin{array}{llll}\text { C } & 0.71636748 & 1.19441056 & -0.00010067\end{array}$

$\begin{array}{llll}\text { C } & 0.67612530 & -1.21763495 & 0.00007275\end{array}$

$\begin{array}{llll}\text { C } & -0.67625398 & 1.21769798 & 0.00007325\end{array}$

$\begin{array}{llll}\mathrm{H} & 1.27482284 & 2.12543743 & 0.00000658\end{array}$

$\begin{array}{llll}\text { C } & -0.71652436 & -1.19445047 & -0.00005433\end{array}$

$\begin{array}{llll}\mathrm{H} & 1.20319885 & -2.16677876 & 0.00008956\end{array}$

$\begin{array}{llll}\text { C } & -1.39262449 & 0.02322482 & -0.00003308\end{array}$

$\begin{array}{llll}\mathrm{H} & -1.20362977 & 2.16671637 & 0.00016144\end{array}$

$\begin{array}{llll}\mathrm{H} & -1.27520554 & -2.12538191 & -0.00007858\end{array}$

$\begin{array}{llll}\mathrm{H} & -2.47818735 & 0.04136582 & 0.00005785\end{array}$

$\begin{array}{llll}\mathrm{H} & -1.27015725 & 0.02105988 & -3.93094403\end{array}$

$\begin{array}{llll}\text { C } & -0.72820686 & 0.01197732 & -4.87273454\end{array}$

$\begin{array}{llll}\text { C } & -1.40247981 & 0.02321353 & -6.06578388\end{array}$

$\begin{array}{llll}\text { C } & 0.69132436 & -0.01174943 & -4.84142132\end{array}$

$\begin{array}{llll}\mathrm{H} & -2.48726127 & 0.04134570 & -6.07831687\end{array}$ 


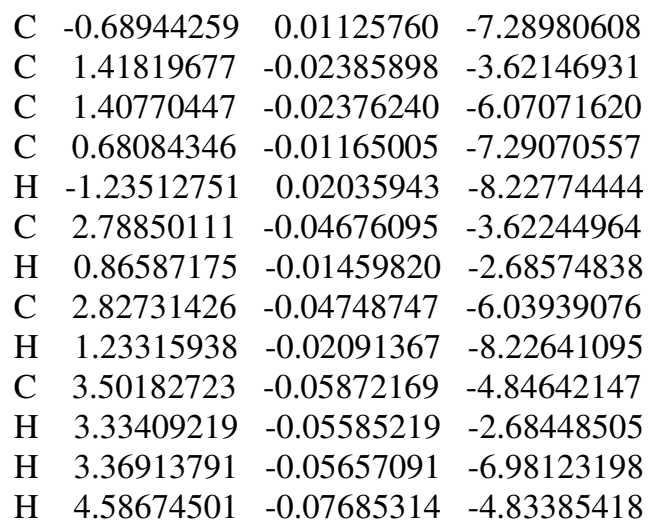

2OMePh_side1a_Ph_pi_0
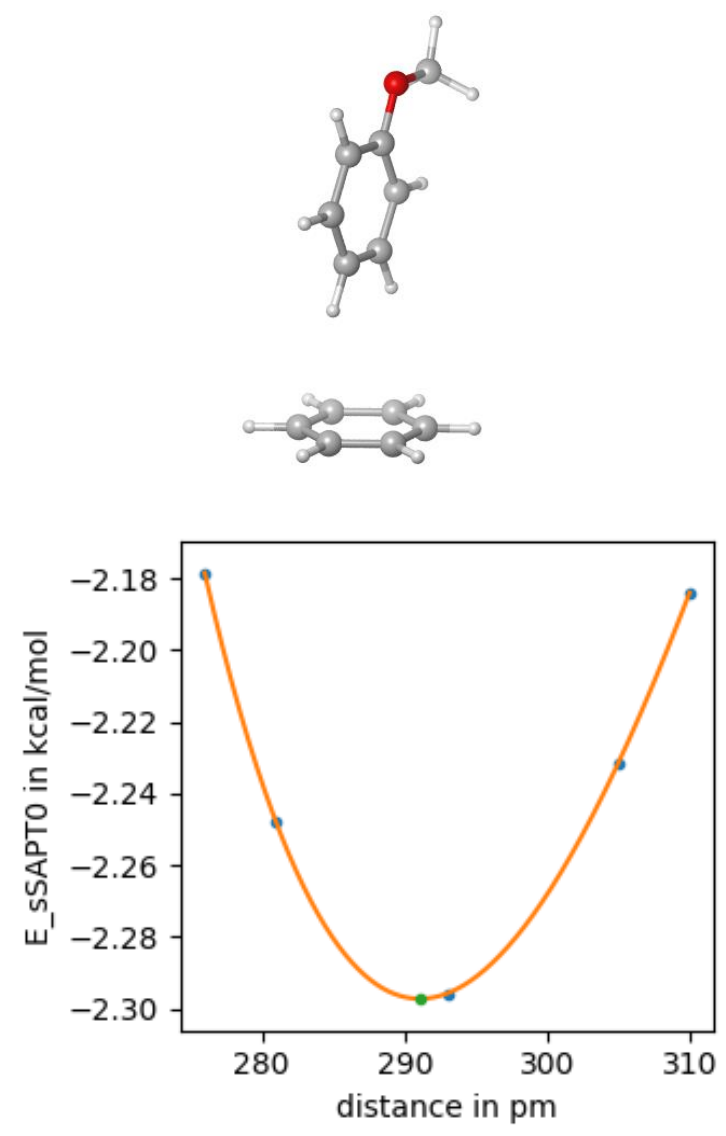

28

2OMePh_side1a_Ph_pi_0-291

H $\quad 2.47830959-0.04134128$

$\begin{array}{llll}\text { C } & 1.39302564 & -0.02324781 & 0.00000008\end{array}$

$\begin{array}{llll}\text { C } & 0.71636748 & 1.19441056 & -0.00010067\end{array}$

$\begin{array}{llll}\text { C } & 0.67612530 & -1.21763495 & 0.00007275\end{array}$

$\begin{array}{llll}\text { C } & -0.67625398 & 1.21769798 & 0.00007325\end{array}$

$\begin{array}{llll}\mathrm{H} & 1.27482284 & 2.12543743 & 0.00000658\end{array}$

$\begin{array}{llll}\text { C } & -0.71652436 & -1.19445047 & -0.00005433\end{array}$

$\begin{array}{llll}\mathrm{H} & 1.20319885 & -2.16677876 & 0.00008956\end{array}$

$\begin{array}{llll}\text { C } & -1.39262449 & 0.02322482 & -0.00003308\end{array}$

$\begin{array}{llll}\text { H } & -1.20362977 & 2.16671637 & 0.00016144\end{array}$

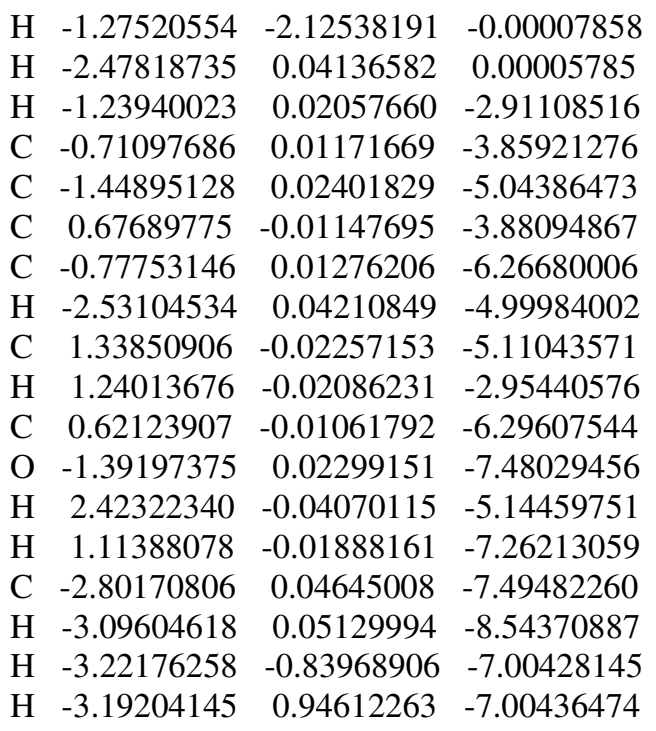

2OMePh_side1b_Ph_pi_0
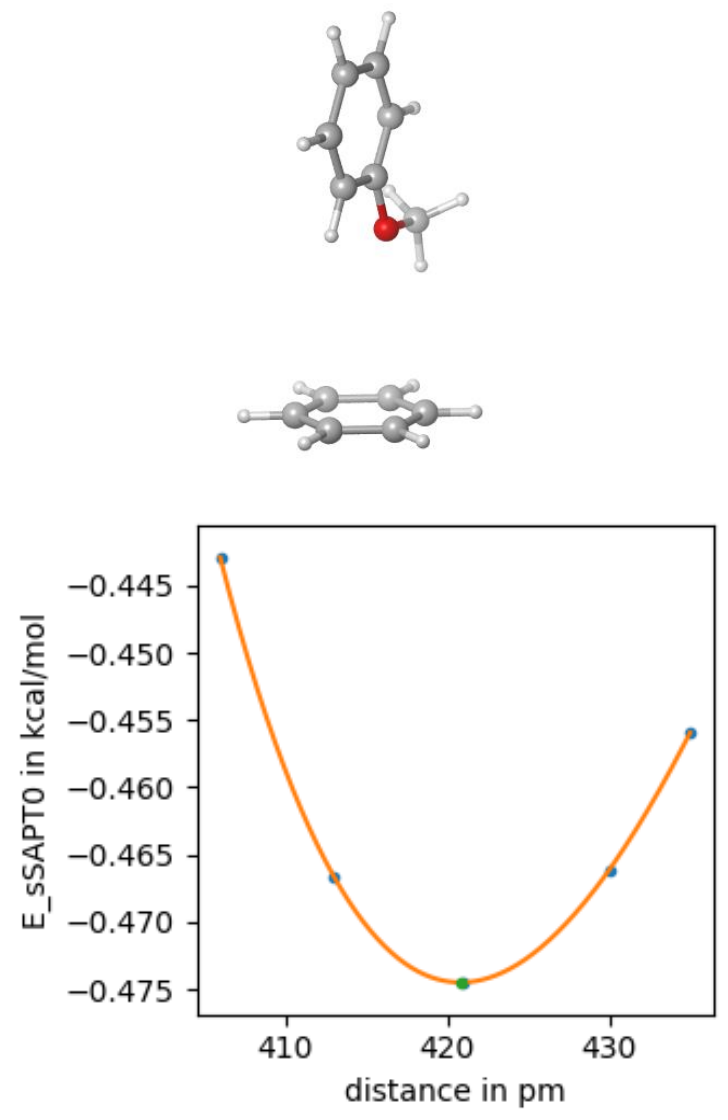

28

2OMePh_side1b_Ph_pi_0-420

H $\quad 2.47830959-0.04134128 \quad 0.00002015$

$\begin{array}{llll}\text { C } & 1.39302564 & -0.02324781 & 0.00000008\end{array}$

$\begin{array}{llll}\text { C } & 0.71636748 & 1.19441056 & -0.00010067\end{array}$

$\begin{array}{llll}\text { C } & 0.67612530 & -1.21763495 & 0.00007275\end{array}$

$\begin{array}{llll}\text { C } & -0.67625398 & 1.21769798 & 0.00007325\end{array}$ 


$\begin{array}{lrrc}\mathrm{H} & 1.27482284 & 2.12543743 & 0.00000658 \\ \mathrm{C} & -0.71652436 & -1.19445047 & -0.00005433 \\ \mathrm{H} & 1.20319885 & -2.16677876 & 0.00008956 \\ \mathrm{C} & -1.39262449 & 0.02322482 & -0.00003308 \\ \mathrm{H} & -1.20362977 & 2.16671637 & 0.00016144 \\ \mathrm{H} & -1.27520554 & -2.12538191 & -0.00007858 \\ \mathrm{H} & -2.47818735 & 0.04136582 & 0.00005785 \\ \mathrm{H} & -1.20968754 & 0.02004099 & -4.20864497 \\ \mathrm{C} & -0.64716948 & 0.01061139 & -5.13574324 \\ \mathrm{C} & 0.74995226 & -0.01273233 & -5.06179337 \\ \mathrm{C} & -1.27508255 & 0.02106260 & -6.37104751 \\ \mathrm{C} & 1.50969883 & -0.02546891 & -6.23188953 \\ \mathrm{O} & 1.27326389 & -0.02143962 & -3.80629269 \\ \mathrm{C} & -0.52463473 & 0.00848326 & -7.54840083 \\ \mathrm{H} & -2.35936302 & 0.03918097 & -6.41696570 \\ \mathrm{C} & 0.86106480 & -0.01467337 & -7.46773549 \\ \mathrm{H} & 2.59209339 & -0.04355202 & -6.19600063 \\ \mathrm{C} & 2.67808695 & -0.04501392 & -3.68785028 \\ \mathrm{H} & -1.01803647 & 0.01669718 & -8.51395522 \\ \mathrm{H} & 1.45794549 & -0.02467780 & -8.37431646 \\ \mathrm{H} & 2.89429932 & -0.04863000 & -2.62011435 \\ \mathrm{H} & 3.10335401 & -0.94530992 & -4.14712583 \\ \mathrm{H} & 3.13331963 & 0.84049769 & -4.14714557\end{array}$

2OMePh_side2a_Ph_pi_0
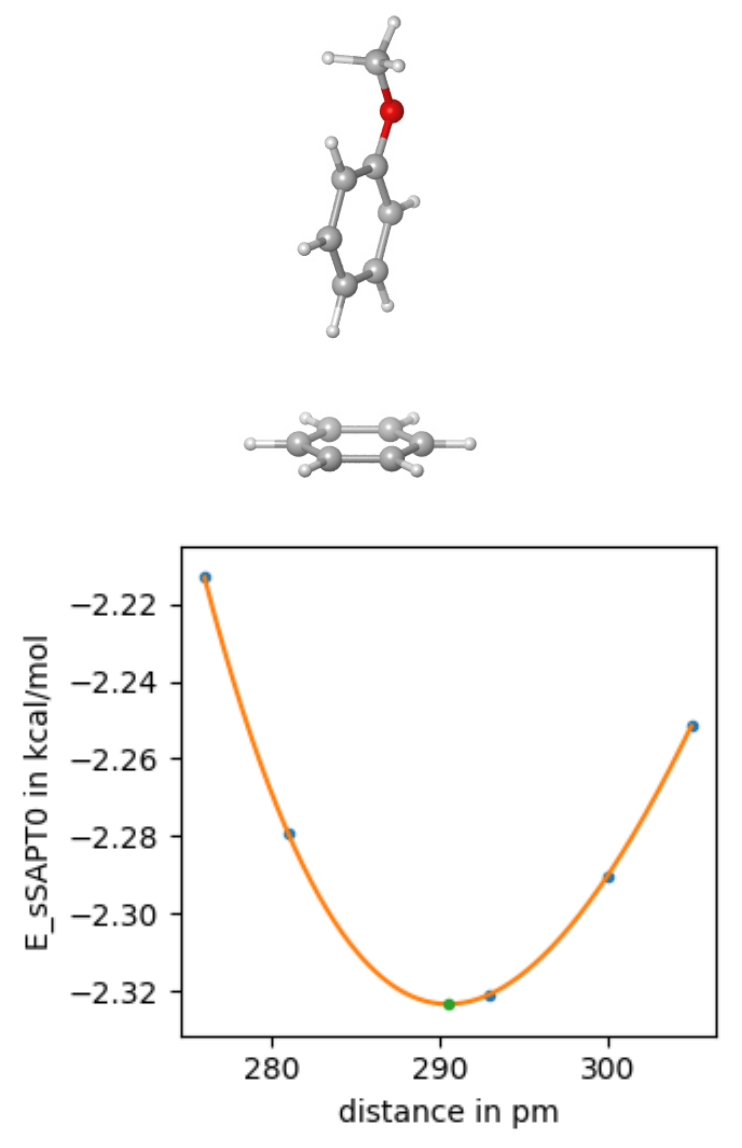

$\begin{array}{llll}\text { H } & 2.47830959 & -0.04134128 & 0.00002015\end{array}$

$\begin{array}{llll}\text { C } & 1.39302564 & -0.02324781 & 0.00000008\end{array}$

$\begin{array}{llll}\text { C } & 0.71636748 & 1.19441056 & -0.00010067\end{array}$

$\begin{array}{llll}\text { C } & 0.67612530 & -1.21763495 & 0.00007275\end{array}$

$\begin{array}{llll}\text { C } & -0.67625398 & 1.21769798 & 0.00007325\end{array}$

$\begin{array}{llll}\mathrm{H} & 1.27482284 & 2.12543743 & 0.00000658\end{array}$

$\begin{array}{llll}\text { C } & -0.71652436 & -1.19445047 & -0.00005433\end{array}$

$\begin{array}{llll}\mathrm{H} & 1.20319885 & -2.16677876 & 0.00008956\end{array}$

$\begin{array}{llll}\text { C } & -1.39262449 & 0.02322482 & -0.00003308\end{array}$

$\begin{array}{llll}\mathrm{H} & -1.20362977 & 2.16671637 & 0.00016144\end{array}$

$\mathrm{H}-1.27520554 \quad-2.12538191 \quad-0.00007858$

$\begin{array}{llll}\mathrm{H} & -2.47818735 & 0.04136582 & 0.00005785\end{array}$

$\begin{array}{llll}\mathrm{H} & -0.60386970 & 1.08793788 & -2.90524116\end{array}$

$\begin{array}{llll}\text { C } & -0.34614108 & 0.62415421 & -3.85090955\end{array}$

$\begin{array}{llll}\text { C } & -0.68619765 & 1.23654217 & -5.04947070\end{array}$

$\begin{array}{llll}\text { C } & 0.33175146 & -0.59617846 & -3.87812882\end{array}$

$\begin{array}{llll}\text { C } & -0.36092097 & 0.65121896 & -6.27411339\end{array}$

$\begin{array}{llll}\mathrm{H} & -1.21330227 & 2.18543111 & -5.04435268\end{array}$

C $\quad 0.66238014 \quad-1.19114357 \quad-5.08521701$

H $\quad 0.60479212 \quad-1.08787873 \quad-2.94980746$

C $\quad 0.31653976-0.56833430-6.28956984$

$\begin{array}{llll}\mathrm{H} & -0.63750622 & 1.14930723 & -7.19530916\end{array}$

$\mathrm{H} \quad 1.18853836 \quad-2.13832387 \quad-5.13031333$

$\begin{array}{lllll}\text { O } & 0.68346237 & -1.22865958 & -7.42074232\end{array}$

$\begin{array}{llll}\text { C } & 0.35345070 & -0.63455197 & -8.65615343\end{array}$

$\mathrm{H} \quad 0.72701124 \quad-1.30696588-9.42760202$

$\mathrm{H} \quad-0.73134100 \quad-0.52074858-8.76837983$

$\begin{array}{llll}\mathrm{H} & 0.82997770 & 0.34659835 & -8.76834006\end{array}$

2OMePh_side2b_Ph_pi_0
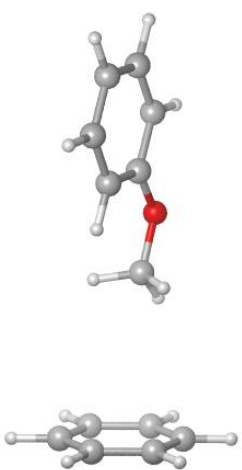

28

2OMePh_side2a_Ph_pi_0-290 


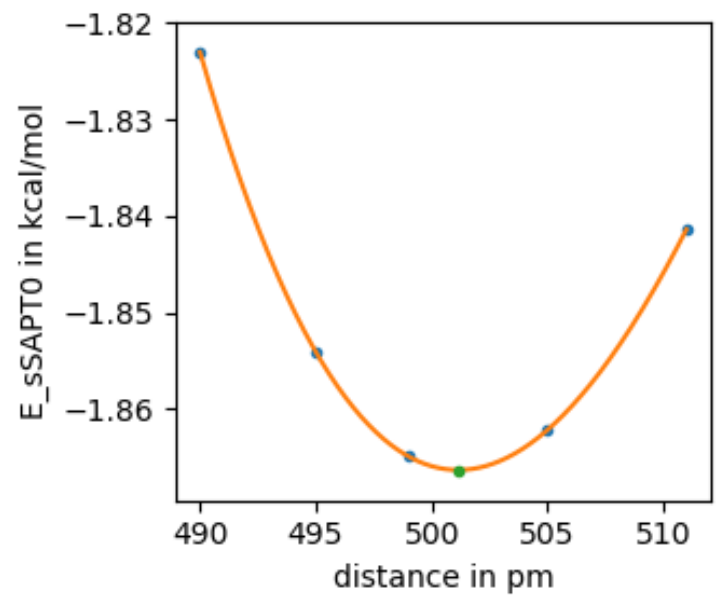

\begin{tabular}{lrrr}
\multicolumn{4}{l}{ 28 } \\
2OMePh_side2b_Ph_pi_0-501 \\
H & 2.47830959 & -0.04134128 & 0.00002015 \\
C & 1.39302564 & -0.02324781 & 0.00000008 \\
C & 0.71636748 & 1.19441056 & -0.00010067 \\
C & 0.67612530 & -1.21763495 & 0.00007275 \\
C & -0.67625398 & 1.21769798 & 0.00007325 \\
H & 1.27482284 & 2.12543743 & 0.00000658 \\
C & -0.71652436 & -1.19445047 & -0.00005433 \\
H & 1.20319885 & -2.16677876 & 0.00008956 \\
C & -1.39262449 & 0.02322482 & -0.00003308 \\
H & -1.20362977 & 2.16671637 & 0.00016144 \\
H & -1.27520554 & -2.12538191 & -0.00007858 \\
H & -2.47818735 & 0.04136582 & 0.00005785 \\
H & -1.23911360 & 0.02050855 & -5.01178667 \\
C & -0.72028355 & 0.01181347 & -5.96254046 \\
C & 0.67339037 & -0.01148651 & -6.02280832 \\
C & -1.45528045 & 0.02407016 & -7.14904255 \\
C & 1.31928638 & -0.02231602 & -7.26404309 \\
O & 1.48896636 & -0.02508618 & -4.93429387 \\
C & -0.82096978 & 0.01343049 & -8.38384743 \\
H & -2.53927027 & 0.04219152 & -7.09525253 \\
C & 0.57423198 & -0.00989263 & -8.43241815 \\
H & 2.40349521 & -0.04043867 & -7.27780771 \\
C & 0.87748117 & -0.01472773 & -3.66382516 \\
H & -1.40200608 & 0.02311746 & -9.29933145 \\
H & 1.08538161 & -0.01846358 & -9.38989060 \\
H & 1.68730940 & -0.02820662 & -2.93525297 \\
H & 0.27323889 & 0.88855098 & -3.51788741 \\
H & 0.24335881 & -0.89725806 & -3.51771277
\end{tabular}
3MePh_side1_Ph_pi_0
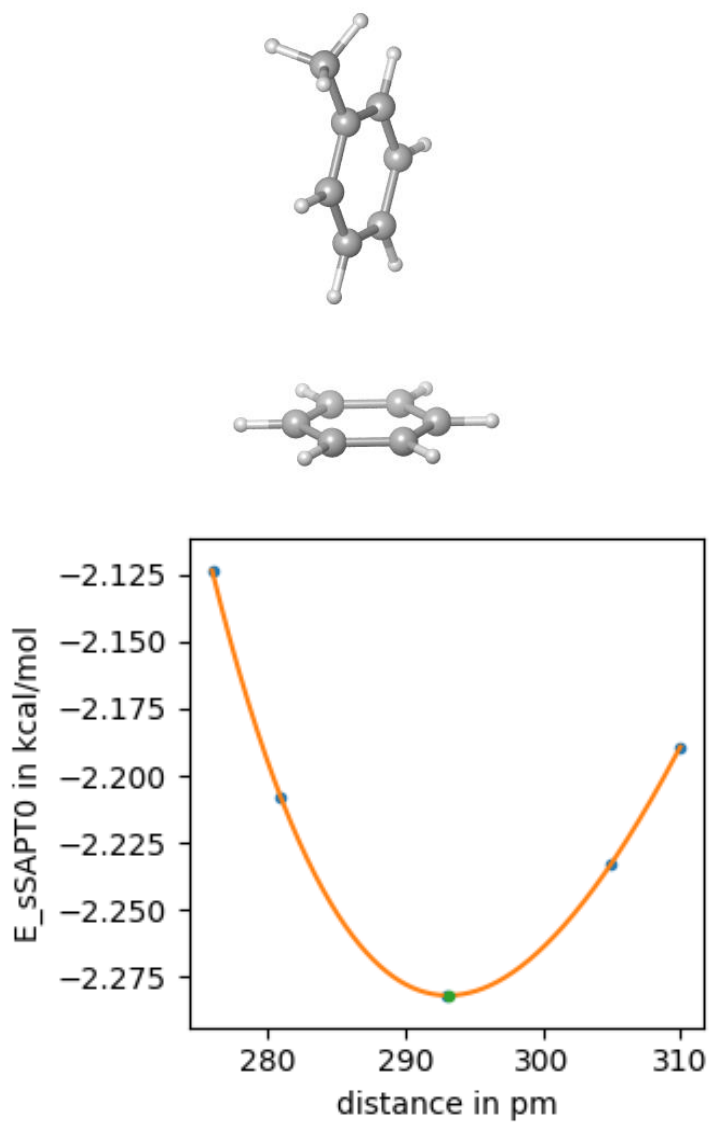

27

3MePh_side1_Ph_pi_0-293

$\begin{array}{llll}\mathrm{H} & 2.47830959 & -0.04134128 & 0.00002015\end{array}$

$\begin{array}{llll}\text { C } & 1.39302564 & -0.02324781 & 0.00000008\end{array}$

$\begin{array}{llll}\text { C } & 0.71636748 & 1.19441056 & -0.00010067\end{array}$

$\begin{array}{llll}\text { C } & 0.67612530 & -1.21763495 & 0.00007275\end{array}$

$\begin{array}{llll}\text { C } & -0.67625398 & 1.21769798 & 0.00007325\end{array}$

$\begin{array}{llll}\mathrm{H} & 1.27482284 & 2.12543743 & 0.00000658\end{array}$

$\begin{array}{llll}\text { C } & -0.71652436 & -1.19445047 & -0.00005433\end{array}$

H $\quad 1.20319885 \quad-2.16677876 \quad 0.00008956$

$\begin{array}{llll}\text { C } & -1.39262449 & 0.02322482 & -0.00003308\end{array}$

$\begin{array}{llll}\mathrm{H} & -1.20362977 & 2.16671637 & 0.00016144\end{array}$

$\mathrm{H} \quad-1.27520554-2.12538191 \quad-0.00007858$

$\begin{array}{llll}\mathrm{H} & -2.47818735 & 0.04136582 & 0.00005785\end{array}$

H $\quad-0.63836181-1.06488616 \quad-2.93145835$

$\begin{array}{llll}\text { C } & -0.35796555 & -0.59783629 & -3.87055195\end{array}$

$\begin{array}{llll}\text { C } & 0.35828571 & 0.59631125 & -3.86951033\end{array}$

$\begin{array}{llll}\text { C } & -0.72123455 & -1.19357697 & -5.07472828\end{array}$

$\begin{array}{lllll}\mathrm{H} & 0.63948075 & 1.06459714 & -2.93137088\end{array}$

$\begin{array}{llll}\text { C } & 0.70631012 & 1.18780160 & -5.08087029\end{array}$

C $\quad-0.37564764-0.61157550 \quad-6.29669722$

H $\quad-1.28337976 \quad-2.12406126 \quad-5.06946119$

$\begin{array}{llll}\text { C } & 0.34040615 & 0.58746509 & -6.28224316\end{array}$

H $\quad 1.26067692 \quad 2.12119258 \quad-5.09122721$

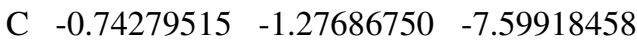

H $\quad 0.61199852 \quad 1.05559658 \quad-7.22502863$ 


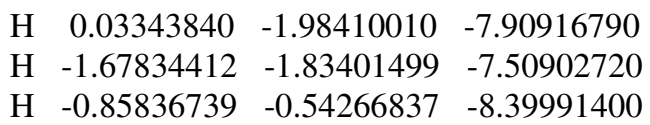

3MePh_side2_Ph_pi_0
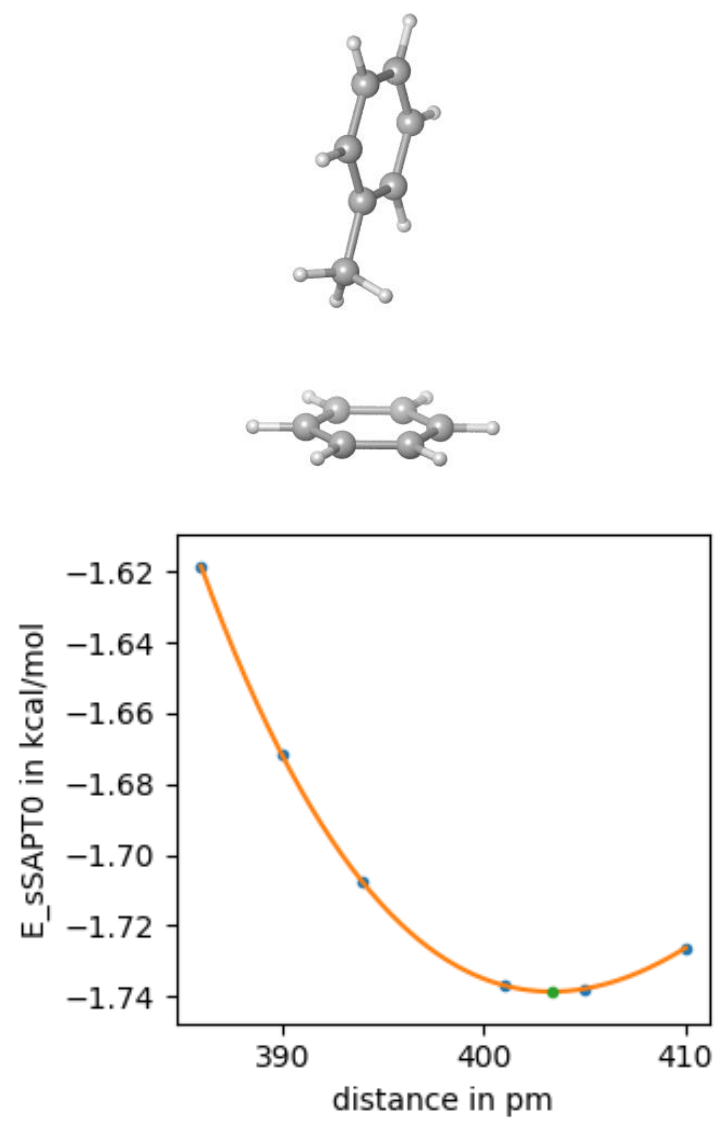

27

3MePh_side2_Ph_pi_0-403

$\begin{array}{llll}\text { H } & 2.47830959 & -0.04134128 & 0.00002015\end{array}$

$\begin{array}{llll}\text { C } & 1.39302564 & -0.02324781 & 0.00000008\end{array}$

$\begin{array}{llll}\text { C } & 0.71636748 & 1.19441056 & -0.00010067\end{array}$

$\begin{array}{llll}\text { C } & 0.67612530 & -1.21763495 & 0.00007275\end{array}$

$\begin{array}{llll}\text { C } & -0.67625398 & 1.21769798 & 0.00007325\end{array}$

$\begin{array}{llll}\mathrm{H} & 1.27482284 & 2.12543743 & 0.00000658\end{array}$

$\begin{array}{llll}\text { C } & -0.71652436 & -1.19445047 & -0.00005433\end{array}$

$\begin{array}{llll}\mathrm{H} & 1.20319885 & -2.16677876 & 0.00008956\end{array}$

$\begin{array}{llll}\text { C } & -1.39262449 & 0.02322482 & -0.00003308\end{array}$

$\begin{array}{llll}\mathrm{H} & -1.20362977 & 2.16671637 & 0.00016144\end{array}$

$\begin{array}{llll}\mathrm{H} & -1.27520554 & -2.12538191 & -0.00007858\end{array}$

$\begin{array}{llll}\mathrm{H} & -2.47818735 & 0.04136582 & 0.00005785\end{array}$

$\mathrm{H} \quad 0.58950860 \quad-1.06084790 \quad-4.03944063$

$\begin{array}{llll}\text { C } & 0.32838505 & -0.59029248 & -4.98397545\end{array}$

$\begin{array}{llll}\text { C } & 0.67306643 & -1.20742823 & -6.18311040\end{array}$

$\begin{array}{llll}\text { C } & -0.35299532 & 0.62883070 & -4.97398838\end{array}$

$\begin{array}{llll}\text { C } & 0.33820645 & -0.61284605 & -7.39666534\end{array}$

H $\quad 1.20051286-2.15624706-6.16926725$

$\begin{array}{llll}\text { C } & -0.68572476 & 1.21380017 & -6.19810681\end{array}$

$\begin{array}{llll}\text { C } & -0.69673321 & 1.31148309 & -3.67409836\end{array}$

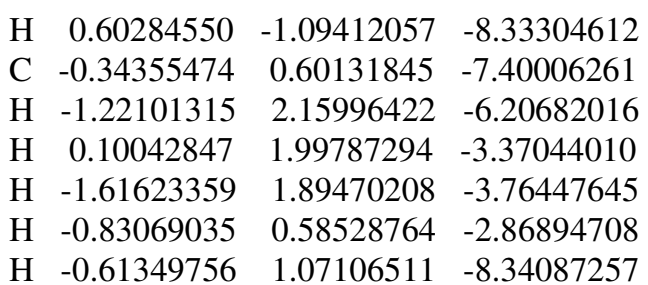

4CF3Ph_side_Ph_pi_0
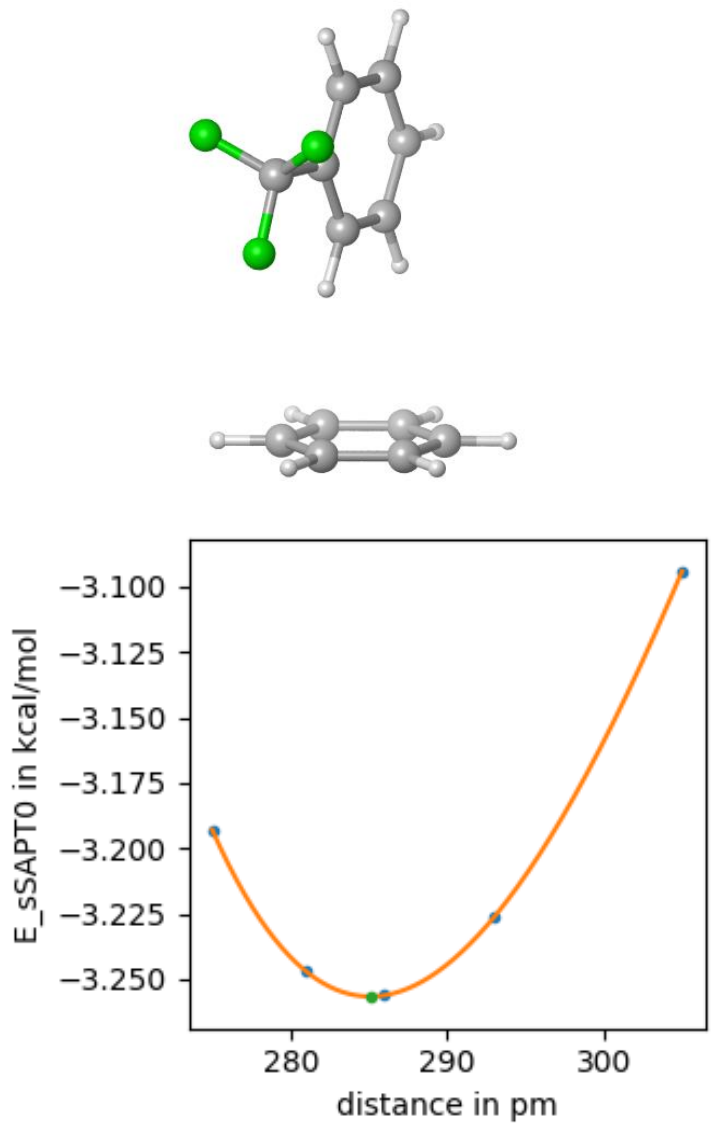

27

4CF3Ph_side_Ph_pi_0-285

$\begin{array}{llll}\mathrm{H} & 2.47830959 & -0.04134128 & 0.00002015\end{array}$

$\begin{array}{llll}\text { C } & 1.39302564 & -0.02324781 & 0.00000008\end{array}$

$\begin{array}{llll}\text { C } & 0.71636748 & 1.19441056 & -0.00010067\end{array}$

$\begin{array}{llll}\text { C } & 0.67612530 & -1.21763495 & 0.00007275\end{array}$

$\begin{array}{llll}\text { C } & -0.67625398 & 1.21769798 & 0.00007325\end{array}$

$\begin{array}{llll}\mathrm{H} & 1.27482284 & 2.12543743 & 0.00000658\end{array}$

$\begin{array}{llll}\text { C } & -0.71652436 & -1.19445047 & -0.00005433\end{array}$

H $\quad 1.20319885 \quad-2.16677876 \quad 0.00008956$

$\begin{array}{llll}\text { C } & -1.39262449 & 0.02322482 & -0.00003308\end{array}$

$\begin{array}{llll}\mathrm{H} & -1.20362977 & 2.16671637 & 0.00016144\end{array}$

$\begin{array}{llll}\mathrm{H} & -1.27520554 & -2.12538191 & -0.00007858\end{array}$

$\begin{array}{llll}\mathrm{H} & -2.47818735 & 0.04136582 & 0.00005785\end{array}$

$\begin{array}{llll}\mathrm{H} & -1.24306705 & 0.02063968 & -2.85110846\end{array}$

$\begin{array}{llll}\text { C } & -0.70004311 & 0.01154649 & -3.79011805\end{array}$

$\begin{array}{llll}\text { C } & -1.39196013 & 0.02313245 & -4.99696626\end{array}$

$\begin{array}{llll}\text { C } & 0.69258990 & -0.01173550 & -3.78383168\end{array}$ 


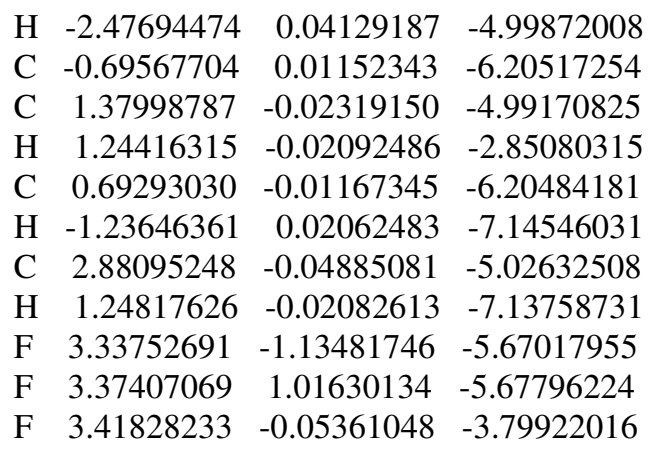

4CIPh_side_Ph_pi_0
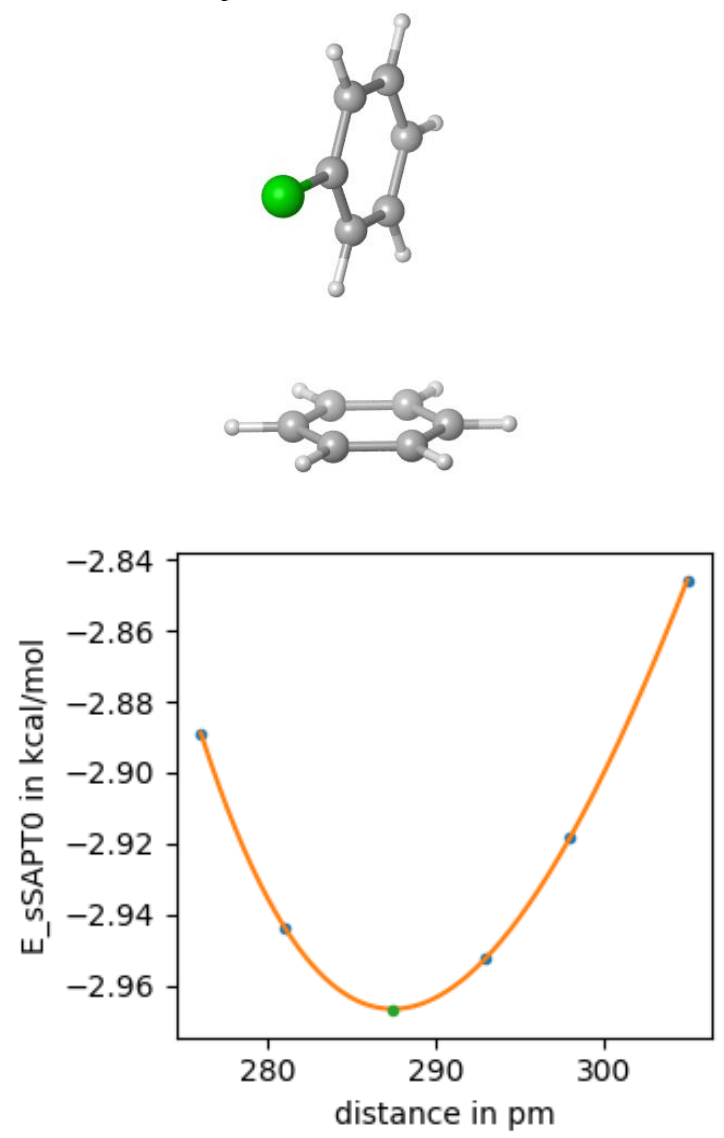

24

4ClPh_side_Ph_pi_0-287
H $2.47830959-0.04134128$
0.00002015
C $\quad 1.39302564-0.02324781$
0.00000008
C $\quad 0.71636748 \quad 1.19441056$
$-0.00010067$
C $\quad 0.67612530 \quad-1.21763495$
0.00007275
C $\quad-0.67625398 \quad 1.21769798$
0.00007325
$\mathrm{H} \quad 1.274822842 .12543743$
0.00000658
$\begin{array}{llll}\text { C } & -0.71652436 & -1.19445047 & -0.00005433\end{array}$
$\begin{array}{llll}\mathrm{H} & 1.20319885 & -2.16677876 & 0.00008956\end{array}$
$\begin{array}{llll}\text { C } & -1.39262449 & 0.02322482 & -0.00003308\end{array}$
$\begin{array}{llll}\mathrm{H} & -1.20362977 & 2.16671637 & 0.00016144\end{array}$
$\mathrm{H} \quad-1.27520554 \quad-2.12538191 \quad-0.00007858$
$\begin{array}{llll}\mathrm{H} & -2.47818735 & 0.04136582 & 0.00005785\end{array}$
$\begin{array}{llll}\text { C } & 0.70424510 & -0.01193158 & -3.81566911\end{array}$
$\begin{array}{llll}\text { C } & -0.68758658 & 0.01132862 & -3.80564342\end{array}$
H $\quad 1.24377859 \quad-0.02091977 \quad-2.87437320$
$\begin{array}{llll}\mathrm{H} & -1.24267537 & 0.02063242 & -2.87467844\end{array}$
C $1.40045062 \quad-0.02360222-5.02117066$
$\begin{array}{llll}\text { C } & -1.36847579 & 0.02267129 & -5.01786686\end{array}$
$\begin{array}{llll}\mathrm{H} & 2.48504040 & -0.04172809 & -5.02246476\end{array}$
$\begin{array}{llll}\text { C } & 0.70136894 & -0.01195628 & -6.22500740\end{array}$
$\begin{array}{llll}\text { C } & -0.69048167 & 0.01130555 & -6.23171169\end{array}$
$\begin{array}{lllll}\mathrm{Cl} & -3.11409476 & 0.05184574 & -5.01578405\end{array}$
$\mathrm{H} \quad 1.23865433 \quad-0.02096312 \quad-7.16758814$
$\begin{array}{llll}\mathrm{H} & -1.24779077 & 0.02059220 & -7.16134939\end{array}$

4FPh_side_Ph_pi_0
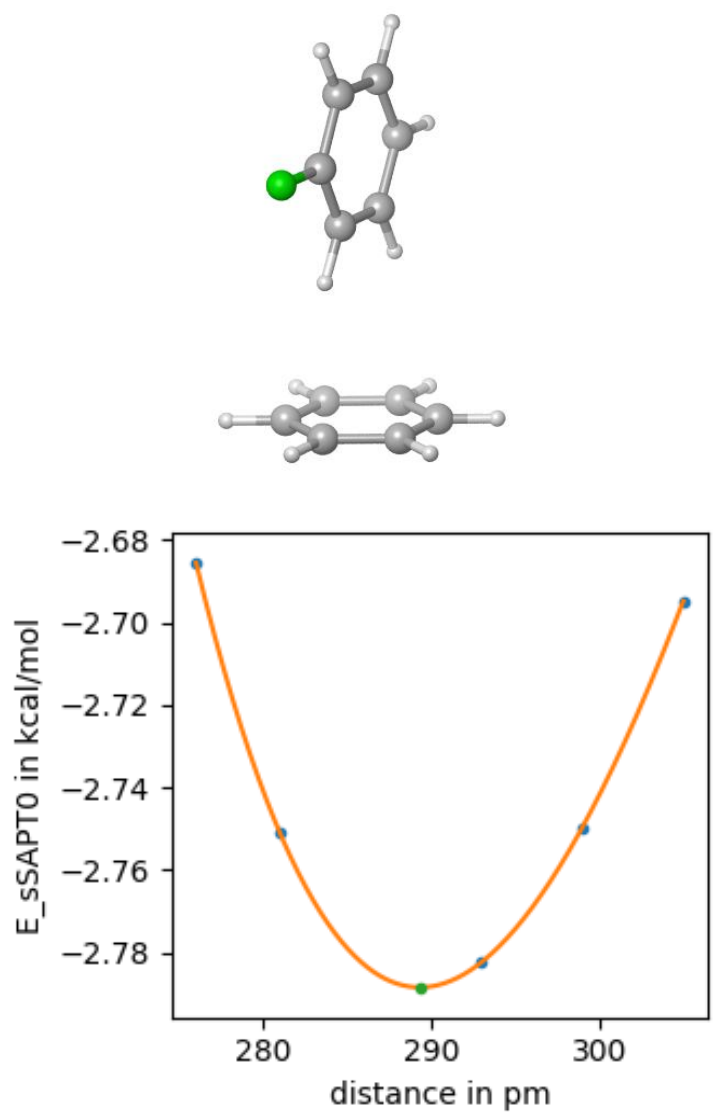

24

4FPh_side_Ph_pi_0-289

H $\quad 2.47830959-0.04134128 \quad 0.00002015$

$\begin{array}{llll}\text { C } & 1.39302564 & -0.02324781 & 0.00000008\end{array}$

$\begin{array}{llll}\text { C } & 0.71636748 & 1.19441056 & -0.00010067\end{array}$

$\begin{array}{llll}\text { C } & 0.67612530 & -1.21763495 & 0.00007275\end{array}$

$\begin{array}{llll}\text { C } & -0.67625398 & 1.21769798 & 0.00007325\end{array}$

$\begin{array}{llll}\mathrm{H} & 1.27482284 & 2.12543743 & 0.00000658\end{array}$

$\begin{array}{llll}\text { C } & -0.71652436 & -1.19445047 & -0.00005433\end{array}$

$\begin{array}{llll}\mathrm{H} & 1.20319885 & -2.16677876 & 0.00008956\end{array}$

$\begin{array}{llll}\text { C } & -1.39262449 & 0.02322482 & -0.00003308\end{array}$

$\begin{array}{llll}\mathrm{H} & -1.20362977 & 2.16671637 & 0.00016144\end{array}$

H $\quad-1.27520554 \quad-2.12538191 \quad-0.00007858$ 

$\begin{array}{llll}\mathrm{H} & -2.47818735 & 0.04136582 & 0.00005785\end{array}$
$\begin{array}{llll}\text { C } & -0.36333424 & -0.60677403 & -3.83236861\end{array}$
$\begin{array}{llll}\text { C } & 0.35282787 & 0.58669042 & -3.81725161\end{array}$

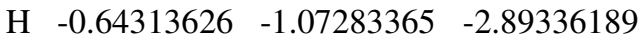
$\begin{array}{llll}\mathrm{H} & 0.64425292 & 1.07254618 & -2.89327346\end{array}$
$\begin{array}{llll}\text { C } & -0.71976277 & -1.20099525 & -5.04088191\end{array}$
$\begin{array}{llll}\text { C } & 0.69950970 & 1.16414918 & -5.02901178\end{array}$
H $\quad-1.27776740 \quad-2.13088024-5.04554768$
$\begin{array}{llll}\text { C } & -0.35777357 & -0.59801246 & -6.24338732\end{array}$
$\begin{array}{lllll}\text { C } & 0.35844126 & 0.59551297 & -6.24652514\end{array}$
F $\quad 1.38990380 \quad 2.31465962 \quad-5.02323932$

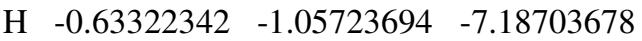
H $\quad 0.65412718 \quad 1.08805750 \quad-7.16559521$

4MePh_side_Ph_pi_0
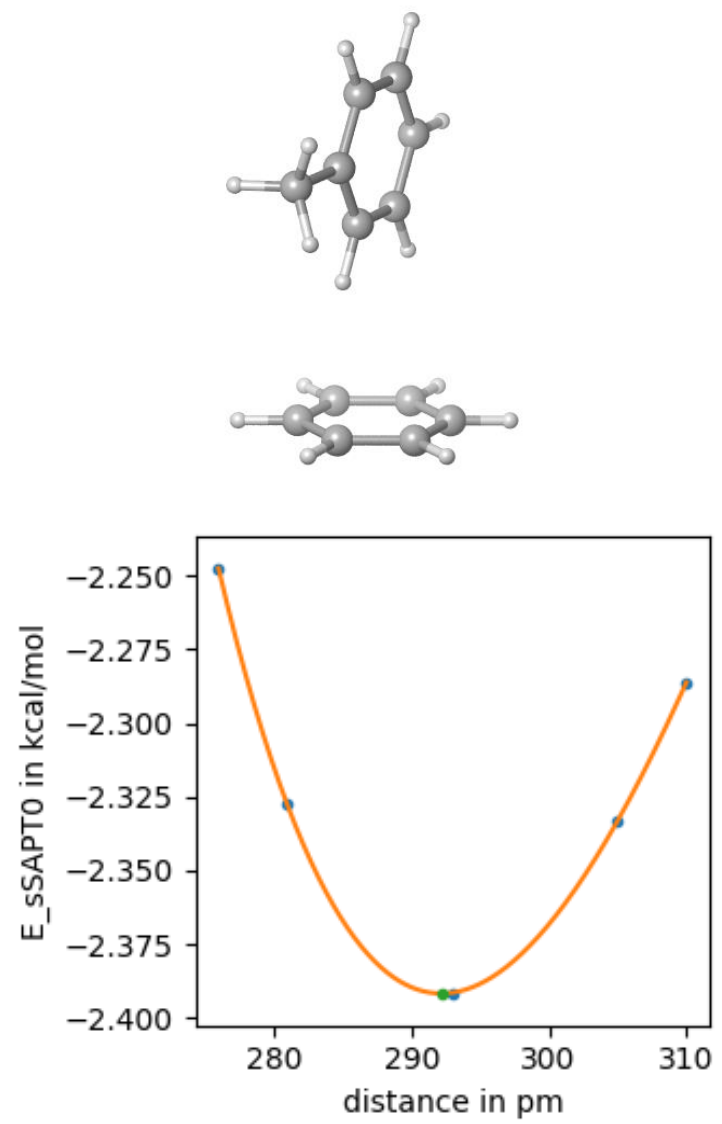

27

4MePh_side_Ph_pi_0-292

$\begin{array}{llll}\mathrm{H} & 2.47830959 & -0.04134128 & 0.00002015\end{array}$

$\begin{array}{llll}\text { C } & 1.39302564 & -0.02324781 & 0.00000008\end{array}$

$\begin{array}{llll}\text { C } & 0.71636748 & 1.19441056 & -0.00010067\end{array}$

$\begin{array}{llll}\text { C } & 0.67612530 & -1.21763495 & 0.00007275\end{array}$

$\begin{array}{llll}\text { C } & -0.67625398 & 1.21769798 & 0.00007325\end{array}$

$\begin{array}{llll}\mathrm{H} & 1.27482284 & 2.12543743 & 0.00000658\end{array}$

$\begin{array}{llll}\text { C } & -0.71652436 & -1.19445047 & -0.00005433\end{array}$

$\begin{array}{llll}\mathrm{H} & 1.20319885 & -2.16677876 & 0.00008956\end{array}$

$\begin{array}{llll}\text { C } & -1.39262449 & 0.02322482 & -0.00003308\end{array}$

$\begin{array}{llll}\text { H } & -1.20362977 & 2.16671637 & 0.00016144\end{array}$

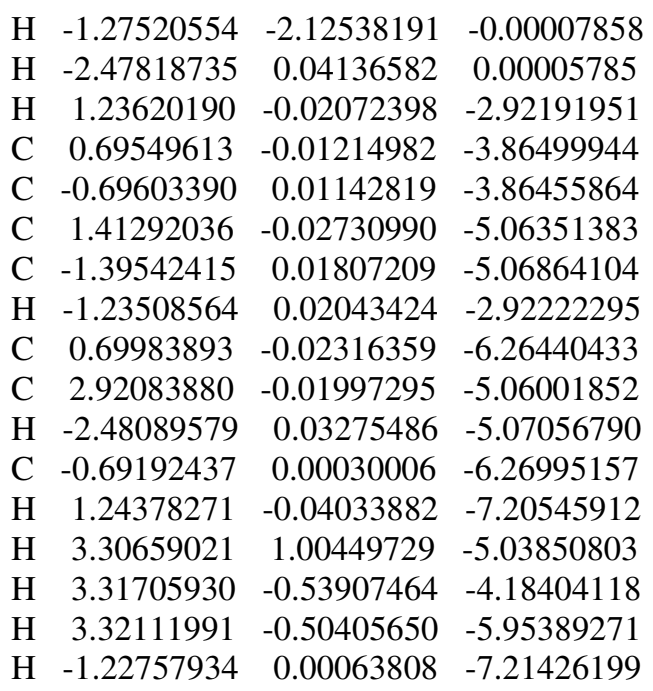

4OMePh_side1_Ph_pi_0
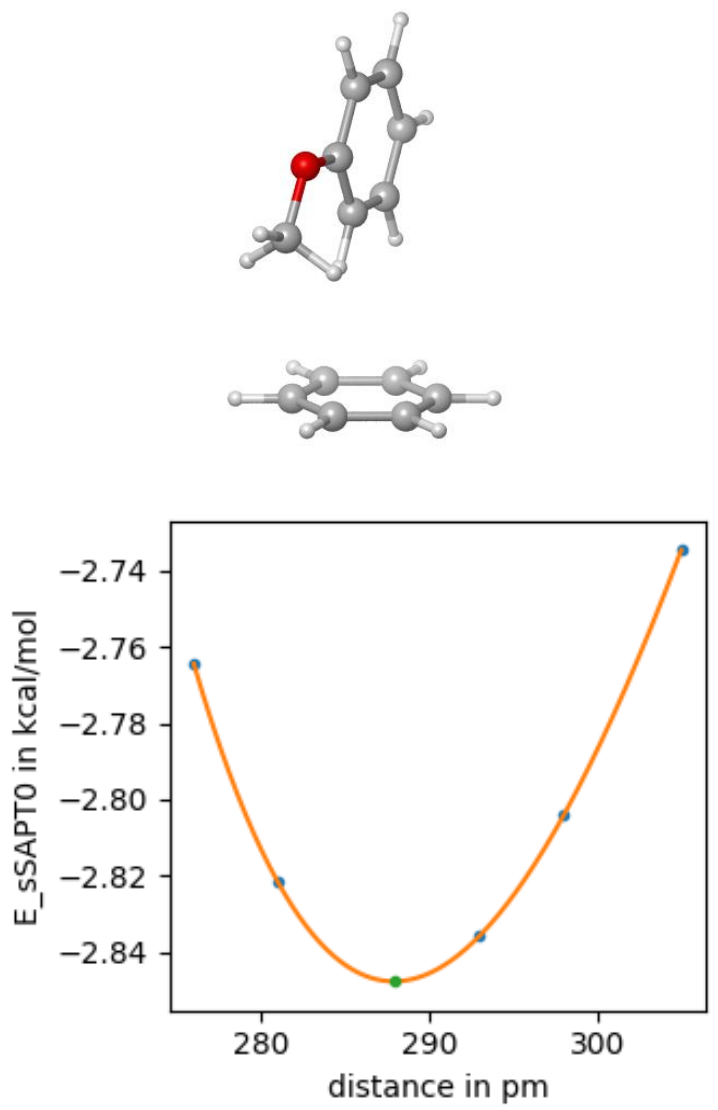

28

4OMePh_side1_Ph_pi_0-287

$\begin{array}{llll}\text { H } & 2.47830959 & -0.04134128 & 0.00002015\end{array}$

$\begin{array}{llll}\text { C } & 1.39302564 & -0.02324781 & 0.00000008\end{array}$

$\begin{array}{llll}\text { C } & 0.71636748 & 1.19441056 & -0.00010067\end{array}$

$\begin{array}{llll}\text { C } & 0.67612530 & -1.21763495 & 0.00007275\end{array}$

$\begin{array}{llll}\text { C } & -0.67625398 & 1.21769798 & 0.00007325\end{array}$

$\begin{array}{llll}\mathrm{H} & 1.27482284 & 2.12543743 & 0.00000658\end{array}$ 


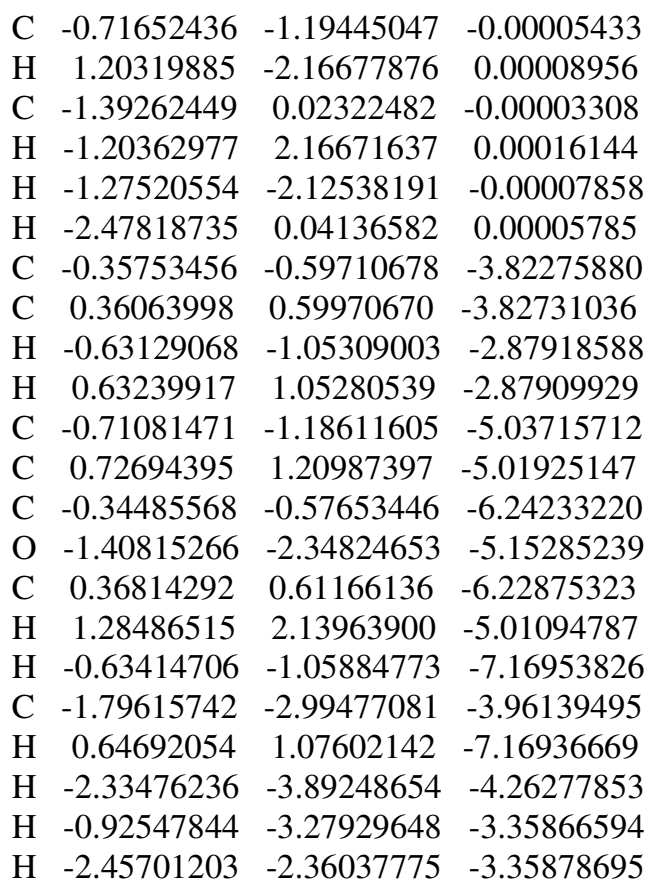

4OMePh_side2_Ph_pi_0
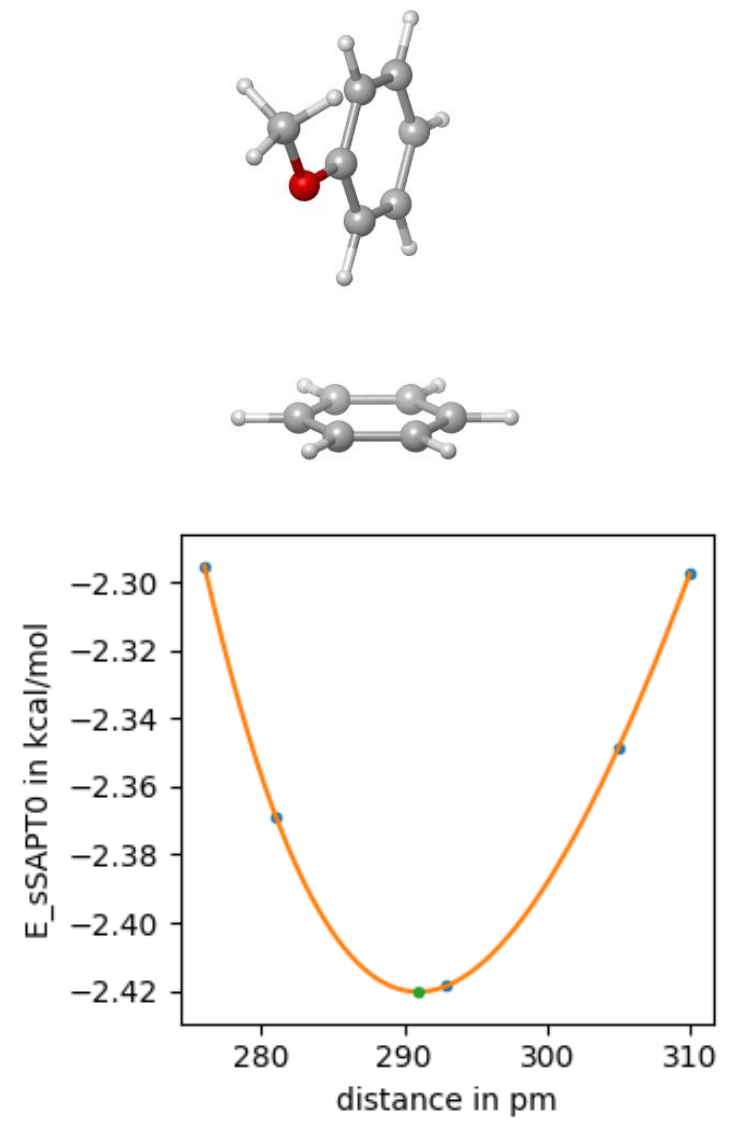

$\begin{array}{llll}\text { C } & 1.39302564 & -0.02324781 & 0.00000008\end{array}$

$\begin{array}{llll}\text { C } & 0.71636748 & 1.19441056 & -0.00010067\end{array}$

$\begin{array}{llll}\text { C } & 0.67612530 & -1.21763495 & 0.00007275\end{array}$

$\begin{array}{llll}\text { C } & -0.67625398 & 1.21769798 & 0.00007325\end{array}$

$\begin{array}{llll}\mathrm{H} & 1.27482284 & 2.12543743 & 0.00000658\end{array}$

$\begin{array}{llll}\text { C } & -0.71652436 & -1.19445047 & -0.00005433\end{array}$

$\begin{array}{llll}\mathrm{H} & 1.20319885 & -2.16677876 & 0.00008956\end{array}$

$\begin{array}{llll}\text { C } & -1.39262449 & 0.02322482 & -0.00003308\end{array}$

$\begin{array}{llll}\mathrm{H} & -1.20362977 & 2.16671637 & 0.00016144\end{array}$

$\begin{array}{llll}\mathrm{H} & -1.27520554 & -2.12538191 & -0.00007858\end{array}$

$\begin{array}{llll}\mathrm{H} & -2.47818735 & 0.04136582 & 0.00005785\end{array}$

$\begin{array}{llll}\text { C } & -0.31388792 & 0.56645787 & -3.83699419\end{array}$

$\begin{array}{llll}\text { C } & 0.35868284 & -0.64508276 & -3.85035648\end{array}$

$\begin{array}{llll}\mathrm{H} & -0.58700720 & 1.05827547 & -2.90987608\end{array}$

$\begin{array}{llll}\mathrm{H} & 0.62141615 & -1.11853722 & -2.90965834\end{array}$

$\begin{array}{llll}\text { C } & -0.65879267 & 1.18797898 & -5.04228043\end{array}$

$\begin{array}{llll}\text { C } & 0.69744103 & -1.25508544 & -5.05974916\end{array}$

C $-0.32524093 \quad 0.58736023 \quad-6.25657135$

$\begin{array}{llll}\text { O } & -1.31661614 & 2.37294512 & -4.92679708\end{array}$

$\begin{array}{llll}\text { C } & 0.35220784 & -0.63296689 & -6.25180154\end{array}$

H $\quad 1.22372678-2.20311770 \quad-5.06788321$

$\begin{array}{llll}\mathrm{H} & -0.58323702 & 1.05227338 & -7.20022742\end{array}$

C $-1.68231318 \quad 3.03213108-6.11837239$

H $\quad 0.60879446 \quad-1.09499662 \quad-7.19992998$

$\begin{array}{lllll}\mathrm{H} & -2.19044906 & 3.94749187 & -5.81715250\end{array}$

H $\quad-0.80238775 \quad 3.28714350-6.72088289$

$\begin{array}{llll}\mathrm{H} & -2.36400761 & 2.42033896 & -6.72113509\end{array}$

4PhPh_side_Ph_pi_0
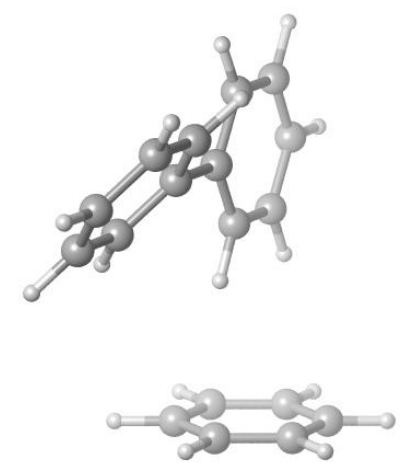

28

4OMePh_side2_Ph_pi_0-290

H $2.47830959 \quad-0.04134128 \quad 0.00002015$ 


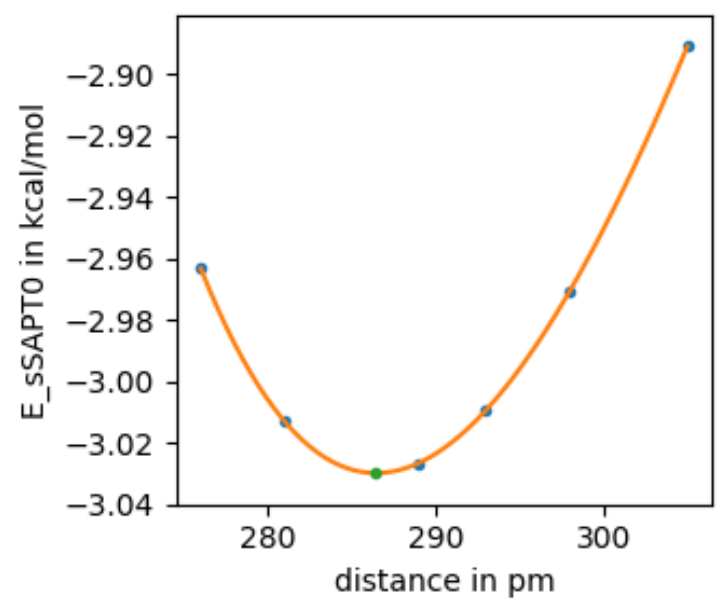

34

4PhPh_side_Ph_pi_0-286

$\begin{array}{llll}\mathrm{H} & 2.47830959 & -0.04134128 & 0.00002015\end{array}$

$\begin{array}{llll}\text { C } & 1.39302564 & -0.02324781 & 0.00000008\end{array}$

$\begin{array}{llll}\text { C } & 0.71636748 & 1.19441056 & -0.00010067\end{array}$

$\begin{array}{llll}\text { C } & 0.67612530 & -1.21763495 & 0.00007275\end{array}$

$\begin{array}{llll}\text { C } & -0.67625398 & 1.21769798 & 0.00007325\end{array}$

$\begin{array}{llll}\mathrm{H} & 1.27482284 & 2.12543743 & 0.00000658\end{array}$

$\begin{array}{llll}\text { C } & -0.71652436 & -1.19445047 & -0.00005433\end{array}$

$\begin{array}{llll}\mathrm{H} & 1.20319885 & -2.16677876 & 0.00008956\end{array}$

$\begin{array}{llll}\text { C } & -1.39262449 & 0.02322482 & -0.00003308\end{array}$

$\begin{array}{llll}\mathrm{H} & -1.20362977 & 2.16671637 & 0.00016144\end{array}$

$\mathrm{H} \quad-1.27520554-2.12538191 \quad-0.00007858$

$\begin{array}{llll}\mathrm{H} & -2.47818735 & 0.04136582 & 0.00005785\end{array}$

$\mathrm{H} \quad-1.23468655 \quad 0.02049922-2.86475350$

$\begin{array}{llll}\text { C } & -0.69591532 & 0.01146736 & -3.80691938\end{array}$

$\begin{array}{llll}\text { C } & -1.39545247 & 0.00161904 & -5.01089292\end{array}$

$\begin{array}{llll}\text { C } & 0.69472427 & -0.00169676 & -3.80595710\end{array}$

$\begin{array}{llll}\mathrm{H} & -2.48053420 & 0.01136166 & -5.01197094\end{array}$

$\begin{array}{llll}\text { C } & -0.69381378 & -0.02080825 & -6.21347335\end{array}$

C $\quad 1.41114903 \quad-0.02357834 \quad-5.00810308$

$\begin{array}{llll}\mathrm{H} & 1.23579131 & -0.02078543 & -2.86445036\end{array}$

$\begin{array}{llll}\text { C } & 0.69683917 & -0.03261396 & -6.21167004\end{array}$

H $\quad-1.23078565-0.02018073-7.15670924$

C $\quad 2.89510251 \quad-0.03689914 \quad-5.00662704$

H $\quad 1.24002993 \quad-0.02325642 \quad-7.15210002$

$\begin{array}{llll}\text { C } & 3.61643279 & 0.72377420 & -4.07928919\end{array}$

$\begin{array}{llll}\text { C } & 3.60450587 & -0.81041671 & -5.93253922\end{array}$

$\begin{array}{llll}\text { C } & 5.00707612 & 0.71104562 & -4.07714459\end{array}$

$\begin{array}{llll}\mathrm{H} & 3.07916575 & 1.34877587 & -3.37199189\end{array}$

C $\quad 4.99515512-0.82265308 \quad-5.93191364$

H $\quad 3.05751713 \quad-1.42568574 \quad-6.64091491$

C $\quad 5.70170405-0.06209258 \quad-5.00383226$

$\mathrm{H} \quad 5.54968087 \quad 1.31343395 \quad-3.35552033$

$\mathrm{H} \quad 5.52829450 \quad-1.43469857 \quad-6.65246763$

H $\quad 6.78678579 \quad-0.07183288 \quad-5.00275083$
$\mathrm{Ph} \_$side_Ph_pi_0
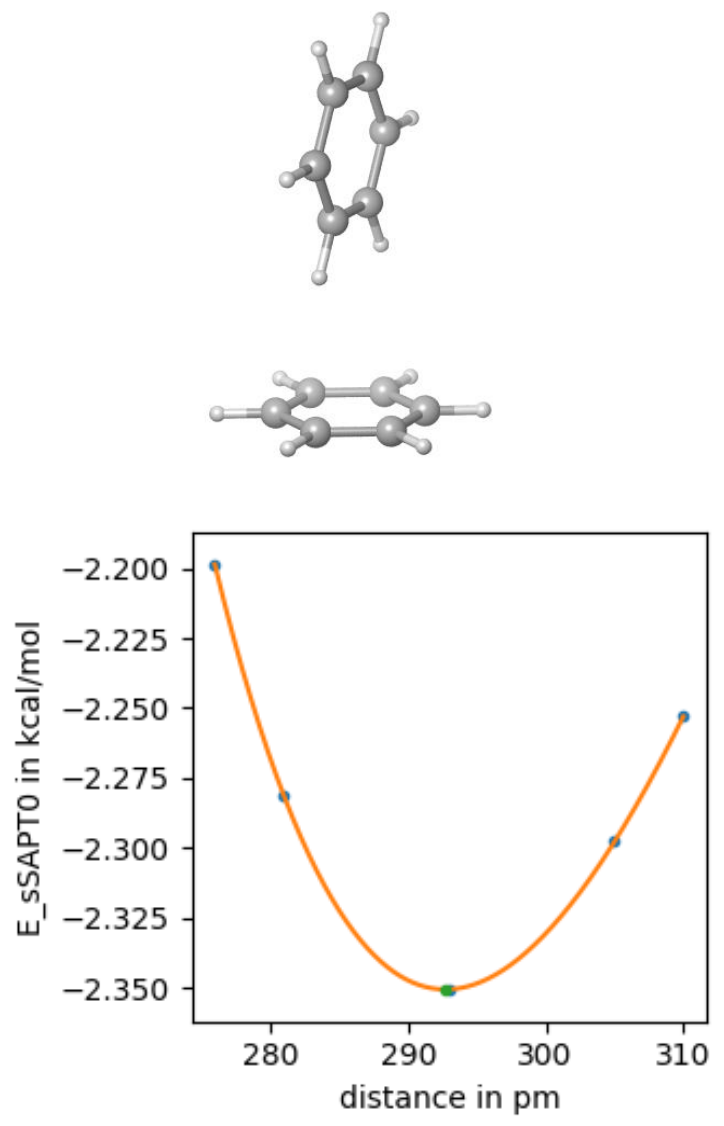

24

Ph_side_Ph_pi_0-292

H $\quad 2.47838500 \quad-0.03653600 \quad 0.00002000$

$\begin{array}{llll}\text { C } & 1.39306800 & -0.02054700 & 0.00000000\end{array}$

$\begin{array}{llll}\text { C } & 0.71405000 & 1.19579700 & -0.00010100\end{array}$

$\begin{array}{llll}\text { C } & 0.67848500 & -1.21632200 & 0.00007300\end{array}$

$\begin{array}{llll}\text { C } & -0.67861400 & 1.21638400 & 0.00007300\end{array}$

$\begin{array}{llll}\mathrm{H} & 1.27069900 & 2.12790500 & 0.00000600\end{array}$

C $-0.71420700-1.19583800-0.00005400$

H $\quad 1.20739800 \quad-2.16444200 \quad 0.00009000$

$\begin{array}{llll}\text { C } & -1.39266700 & 0.02052400 & -0.00003300\end{array}$

$\begin{array}{llll}\mathrm{H} & -1.20782900 & 2.16437800 & 0.00016100\end{array}$

$\mathrm{H} \quad-1.27108200 \quad-2.12785100-0.00007800$

$\begin{array}{llll}\mathrm{H} & -2.47826300 & 0.03656000 & 0.00005800\end{array}$

$\begin{array}{llll}\text { C } & -0.69580889 & 0.01016103 & -3.86777987\end{array}$

C $\quad 0.69685965 \quad-0.01050716 \quad-3.86772304$

$\begin{array}{llll}\mathrm{H} & -1.23841377 & 0.01815659 & -2.92741975\end{array}$

H $\quad 1.23954223 \quad-0.01853243 \quad-2.92740019$

$\begin{array}{llll}\text { C } & -1.39198069 & 0.02039886 & -5.07408700\end{array}$

$\begin{array}{lrrr}\text { C } & 1.39336464 & -0.02067865 & -5.07380008\end{array}$

$\begin{array}{llll}\text { C } & -0.69577537 & 0.01035386 & -6.28063390\end{array}$

$\begin{array}{lllll}\mathrm{H} & -2.47753267 & 0.03653361 & -5.07420292\end{array}$

C $\quad 0.69709450 \quad-0.01013835 \quad-6.28007841$

H $\quad 2.47895212 \quad-0.03673095 \quad-5.07344141$

$\begin{array}{llll}\mathrm{H} & -1.23835365 & 0.01848857 & -7.22069309\end{array}$

H $\quad 1.23996390 \quad-0.01803281 \quad-7.22024382$ 
Sandwich complex $\mathbf{A r}_{\pi}^{\mathrm{C}}-\mathrm{Ar}_{\pi}^{\mathrm{A}}$

345Me3Ph_pi_4FPh_pi_300
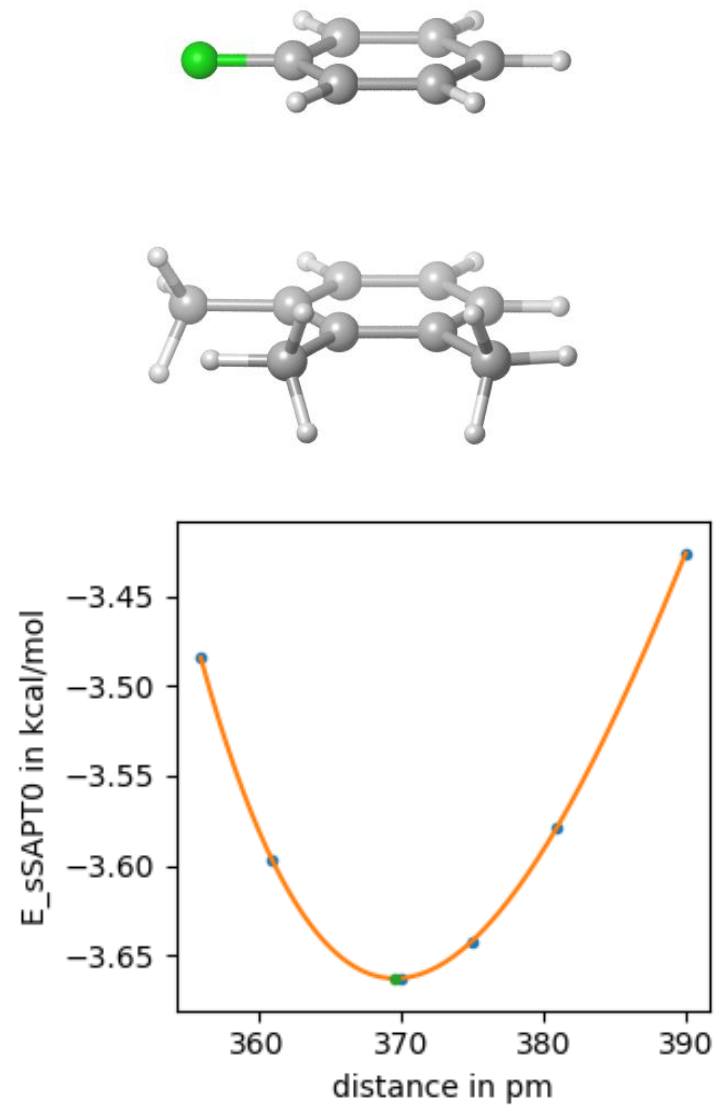

33

345Me3Ph_pi_4FPh_pi_300-369
$\begin{array}{llll}\mathrm{H} & -2.45976551 & -0.22046875 & 0.99720633\end{array}$
$\begin{array}{llll}\text { C } & -1.37960891 & -0.12383923 & 0.99843870\end{array}$
$\begin{array}{llll}\text { C } & -0.57685626 & -1.26236109 & 0.99928819\end{array}$
$\begin{array}{llll}\text { C } & -0.79168624 & 1.13908993 & 0.99917543\end{array}$
$\begin{array}{llll}\text { C } & 0.81032167 & -1.14743091 & 1.00087885\end{array}$
H $\quad-1.03038877 \quad-2.24802954 \quad 0.99872853$
$\begin{array}{llll}\text { C } & 0.59386600 & 1.27220257 & 1.00075338\end{array}$
$\begin{array}{llll}\mathrm{H} & -1.41297009 & 2.02860664 & 0.99851578\end{array}$
$\begin{array}{llll}\text { C } & 1.36774791 & 0.12193317 & 1.00157450\end{array}$
$\mathrm{H} \quad 1.46068803 \quad-2.01446210 \quad 1.00157976$
$\begin{array}{llll}\mathrm{H} & 1.08000819 & 2.24090557 & 1.00135486\end{array}$
$\begin{array}{llll}\text { F } & 2.70418183 & 0.24148924 & 1.00310207\end{array}$
$\begin{array}{llll}\text { C } & -0.79938608 & 1.14546675 & -2.69915268\end{array}$
$\begin{array}{llll}\text { C } & -1.38082414 & -0.11660997 & -2.69765503\end{array}$
$\begin{array}{llll}\text { C } & 0.58450864 & 1.26084665 & -2.69700952\end{array}$
$\begin{array}{llll}\text { C } & -0.58943041 & -1.26463798 & -2.69471168\end{array}$
$\begin{array}{llll}\text { C } & 1.40241651 & 0.12857753 & -2.69386527\end{array}$
$\begin{array}{llll}\text { C } & 0.81150402 & -1.14558876 & -2.69335446\end{array}$
$\mathrm{H} \quad-1.42176038 \quad 2.03462376 \quad-2.70150531$
H $\quad-2.46267463 \quad-0.21827477 \quad-2.69862327$
$\begin{array}{llll}\mathrm{H} & 1.04592004 & 2.24458409 & -2.69748709\end{array}$

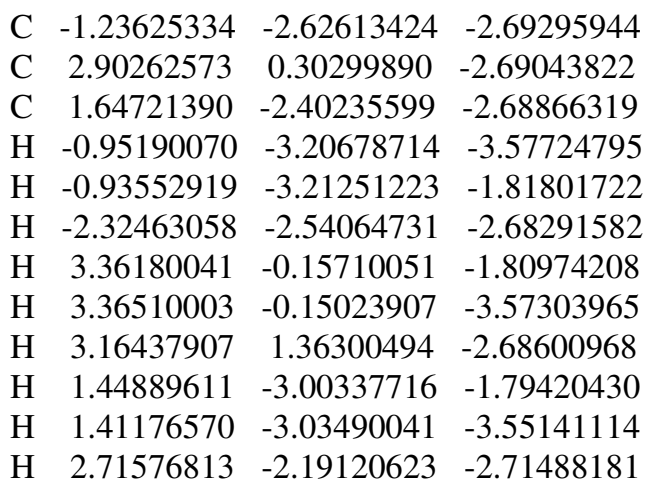

345Me3Ph_pi_Ph_pi_0
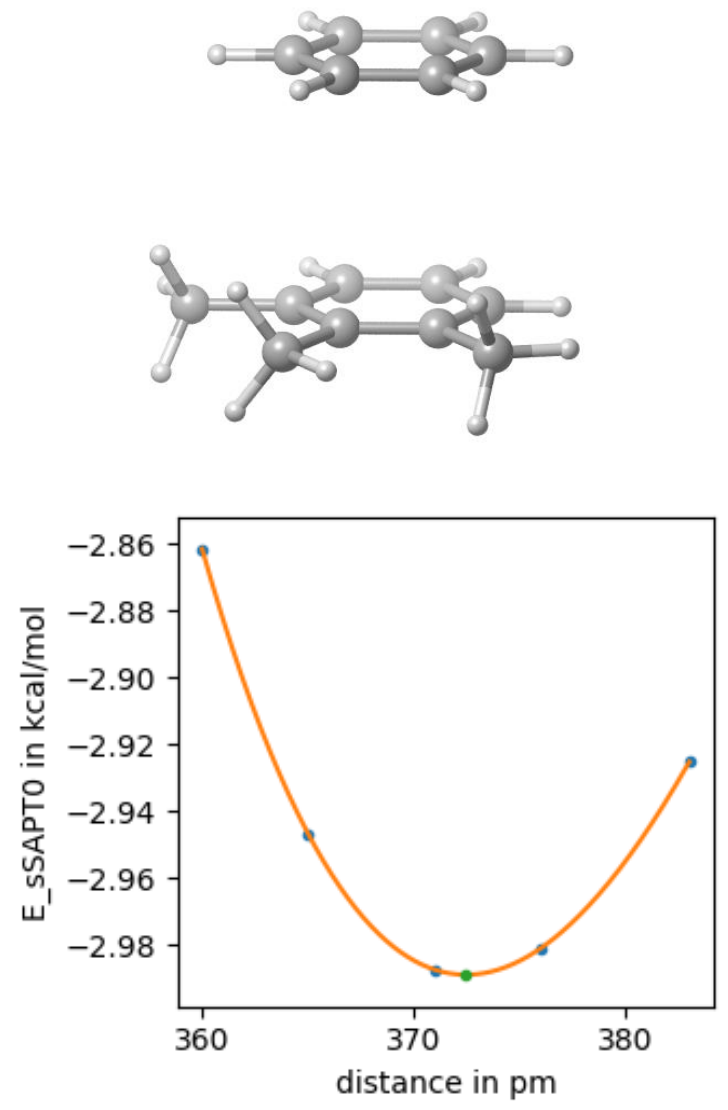

33

345Me3Ph_pi_Ph_pi_0-372

$\begin{array}{llll}\mathrm{H} & 2.47818743 & -0.04130912 & 1.00002014\end{array}$

$\begin{array}{llll}\text { C } & 1.39290347 & -0.02321566 & 1.00000007\end{array}$

$\begin{array}{llll}\text { C } & 0.71624531 & 1.19444271 & 0.99989932\end{array}$

$\begin{array}{llll}\text { C } & 0.67600314 & -1.21760279 & 1.00007275\end{array}$

$\begin{array}{llll}\text { C } & -0.67637615 & 1.21773013 & 1.00007324\end{array}$

$\begin{array}{llll}\mathrm{H} & 1.27470068 & 2.12546958 & 1.00000658\end{array}$

C $-0.71664652-1.194418320 .99994566$

$\begin{array}{llll}\mathrm{H} & 1.20307668 & -2.16674660 & 1.00008955\end{array}$

$\begin{array}{llll}\text { C } & -1.39274665 & 0.02325697 & 0.99996691\end{array}$

$\begin{array}{llll}\mathrm{H} & -1.20375194 & 2.16674853 & 1.00016143\end{array}$

H $\quad-1.27532771 \quad-2.12534975 \quad 0.99992141$ 


$\begin{array}{rrrr}\mathrm{H} & -2.47830951 & 0.04139798 & 1.00005785 \\ \mathrm{C} & -1.39962225 & 0.02318043 & -2.72741641 \\ \mathrm{C} & -0.72173948 & -1.18982462 & -2.72546547 \\ \mathrm{C} & -0.67816440 & 1.20976305 & -2.72600809 \\ \mathrm{C} & 0.67216408 & -1.22597369 & -2.72279773 \\ \mathrm{C} & 0.71861003 & 1.20432402 & -2.72315514 \\ \mathrm{C} & 1.40068728 & -0.02345741 & -2.72218467 \\ \mathrm{H} & -2.48476551 & 0.04355273 & -2.72955066 \\ \mathrm{H} & -1.27646471 & -2.12417837 & -2.72585788 \\ \mathrm{H} & -1.20171639 & 2.16188315 & -2.72684326 \\ \mathrm{C} & 1.39191831 & -2.55036178 & -2.72054628 \\ \mathrm{C} & 1.46081904 & 2.51968752 & -2.72053057 \\ \mathrm{C} & 2.90857903 & -0.08779988 & -2.71777915 \\ \mathrm{H} & 2.02849982 & -2.66278376 & -3.60491367 \\ \mathrm{H} & 2.04313610 & -2.65205616 & -1.84569103 \\ \mathrm{H} & 0.68207101 & -3.37980638 & -2.70994574 \\ \mathrm{H} & 2.10324483 & 2.62042008 & -1.84001902 \\ \mathrm{H} & 2.09926988 & 2.62626041 & -3.60331887 \\ \mathrm{H} & 0.75807499 & 3.35531942 & -2.71636469 \\ \mathrm{H} & 3.27784271 & -0.60150017 & -1.82314546 \\ \mathrm{H} & 3.28109499 & -0.65093576 & -3.58032895 \\ \mathrm{H} & 3.36696986 & 0.90024838 & -2.74457970\end{array}$

35Me24OMePh_pi_4FPh_pi_300
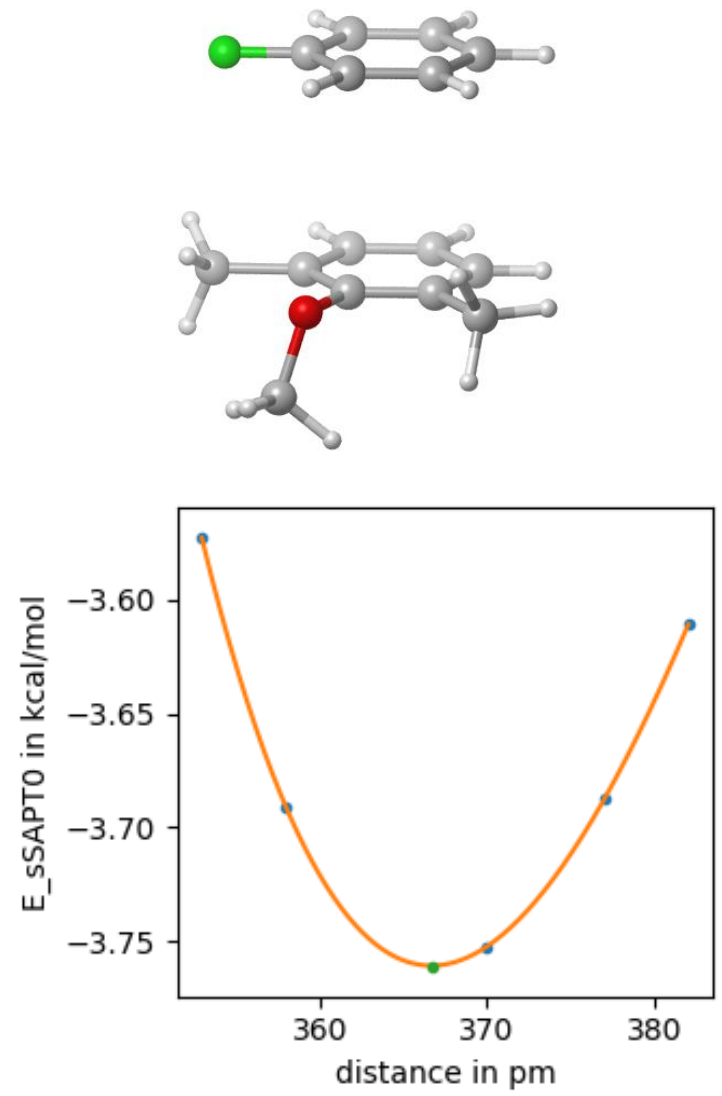

34

35Me24OMePh_pi_4FPh_pi_300-366

H $\quad-0.66998745 \quad-2.38005354 \quad 0.99821803$
C $\quad-0.37451178-1.33661187$
$\begin{array}{lrr}\text { C } & 0.97560900 & -0.99336697\end{array}$
0.99900685
C $-1.34421404-0.33645130$
1.00016879
C $1.36363081 \quad 0.34338736$
0.99886004
H $\quad 1.73432438-1.76898628$
1.00119470
C $-0.97375634 \quad 1.00527765$
H $-2.39694432 \quad-0.59911492$
1.00029678
C $\quad 0.37702740 \quad 1.31736030$
0.99986417
$\begin{array}{lll}\mathrm{H} & 2.40574384 & 0.64124568\end{array}$
0.99795412
H -1.70497971
1.80529793
1.00101447
$\begin{array}{ll}\text { F } & 0.74260717\end{array}$
2.60836845
1.00211941
C 1.34895828
$0.33145462-2.66605908$
$\begin{array}{llll}\text { C } & 0.98525113 & -1.02013020 & -2.66013267\end{array}$
$\begin{array}{llll}\text { C } & 0.40137893 & 1.35913089 & -2.66030074\end{array}$
$\begin{array}{llll}\text { O } & 2.69448790 & 0.64003233 & -2.63303203\end{array}$
C $\quad-0.37214565-1.33558029 \quad-2.66154928$
$\begin{array}{llll}\text { C } & 2.05415640 & -2.07983994 & -2.63024258\end{array}$
$\begin{array}{llll}\text { C } & -0.94920553 & 1.00286129 & -2.66088586\end{array}$
$\begin{array}{llll}\text { C } & 0.83281858 & 2.80218211 & -2.63889414\end{array}$
$\begin{array}{llll}\text { C } & 3.23336474 & 0.91767675 & -3.91421175\end{array}$
C $-1.33686608-0.33157748-2.66758286$
$\mathrm{H} \quad-0.67203014-2.37984368-2.65267775$
$\mathrm{H} \quad 2.65976073 \quad-2.06530107 \quad-3.54208765$
H $\quad 2.73763768-1.91651138-1.79334592$
H $\quad 1.61089761 \quad-3.07311069 \quad-2.53535975$
$\mathrm{H} \quad-1.70013265 \quad 1.78839045 \quad-2.64787415$
H $\quad 1.81076256 \quad 2.90435158 \quad-2.16424639$
H $\quad 0.90625339 \quad 3.21302549 \quad-3.65199335$
$\mathrm{H} \quad 0.10975034 \quad 3.41182328-2.09207038$
$\mathrm{H} \quad 2.76875921 \quad 1.80624733 \quad-4.35849234$
$\mathrm{H} \quad 4.30046130 \quad 1.09873691 \quad-3.77826792$
H $\quad 3.09066563 \quad 0.07007561 \quad-4.59573443$
$\begin{array}{llll}\mathrm{H} & -2.39049984 & -0.59110540 & -2.66514141\end{array}$

35Me24OMePh_pi_4FPh_pi_300_2
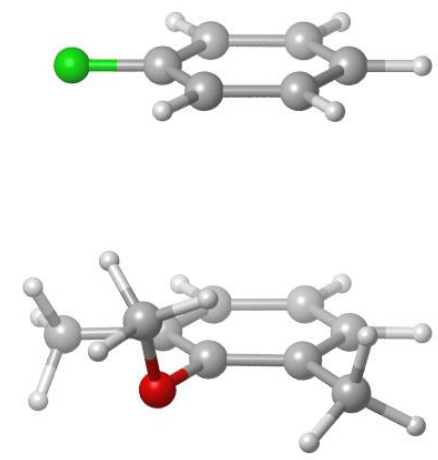


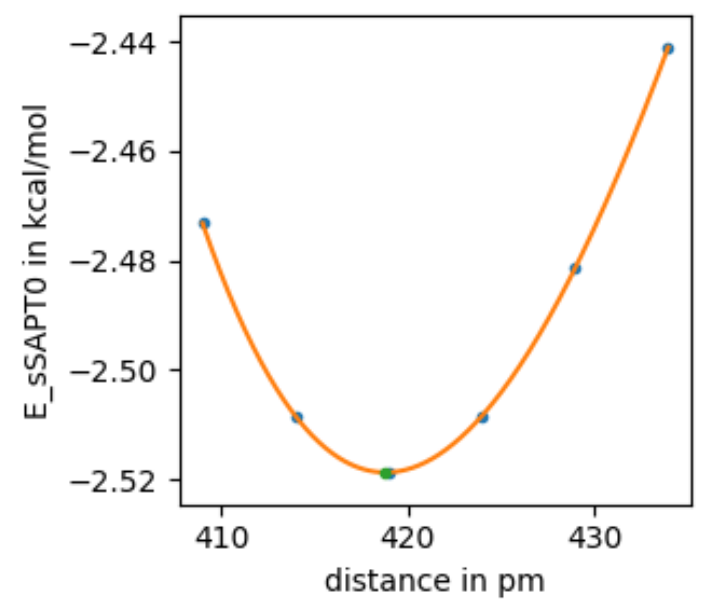

34

35Me24OMePh_pi_4FPh_pi_300_2-418

H $-0.32195001 \quad-2.45207176 \quad 0.99841525$

$\begin{array}{llll}\text { C } & -0.17906210 & -1.37705566 & 0.99911760\end{array}$

$\begin{array}{llll}\text { C } & 1.10793101 & -0.84386527 & 1.00024404\end{array}$

$\begin{array}{llll}\text { C } & -1.28208969 & -0.52618920 & 0.99889499\end{array}$

$\begin{array}{llll}\text { C } & 1.30037452 & 0.53469875 & 1.00115911\end{array}$

$\mathrm{H} \quad 1.96997016 \quad-1.50274525 \quad 1.00043076$

$\begin{array}{llll}\text { C } & -1.10774191 & 0.85478094 & 0.99978799\end{array}$

$\begin{array}{llll}\mathrm{H} & -2.28631036 & -0.93701047 & 0.99801637\end{array}$

C $\quad 0.18437324 \quad 1.35722577 \quad 1.00090530$

$\begin{array}{llll}\mathrm{H} & 2.28904371 & 0.97882985 & 1.00205372\end{array}$

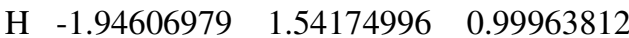

$\begin{array}{llll}\text { F } & 0.36116235 & 2.68729962 & 1.00177710\end{array}$

C $\quad 1.28733157 \quad 0.52184082-3.18746943$

$\begin{array}{llll}\text { C } & 0.19813009 & 1.40087101 & -3.19422029\end{array}$

$\begin{array}{llll}\text { C } & 1.12560573 & -0.86663398 & -3.19385725\end{array}$

$\begin{array}{llll}\text { O } & 2.55697750 & 1.06384190 & -3.21901249\end{array}$

$\begin{array}{llll}\text { C } & -1.08512278 & 0.85745693 & -3.19430213\end{array}$

$\begin{array}{llll}\text { C } & 0.43916075 & 2.88663389 & -3.22336706\end{array}$

$\begin{array}{llll}\text { C } & -0.17615003 & -1.37303089 & -3.19477721\end{array}$

C $\quad 2.32341480 \quad-1.77977645-3.21433932$

$\begin{array}{llll}\text { C } & 3.15476337 & 1.15589397 & -1.93718776\end{array}$

C $-1.27464841 \quad-0.52196130 \quad-3.18892353$

$\mathrm{H} \quad-1.94192364 \quad 1.52551153 \quad-3.20383003$

H $\quad 0.93651044 \quad 3.23082784 \quad-2.31089611$

H $\quad 1.08880951 \quad 3.15690228 \quad-4.05950722$

H $\quad-0.50349689 \quad 3.42913906-3.31903633$

$\begin{array}{llll}\mathrm{H} & -0.32128858 & -2.45000299 & -3.20829739\end{array}$

H $3.17483661 \quad-1.28694962 \quad-3.68794900$

H $2.62344393 \quad-2.06915169-2.20102819$

H $2.09788406 \quad-2.69794688 \quad-3.76170137$

H $3.30131019 \quad 0.16387565 \quad-1.49308766$

$\mathrm{H} \quad 4.12434098 \quad 1.63725659 \quad-2.07197588$

$\mathrm{H} \quad 2.54005412 \quad 1.75715008 \quad-1.25609488$

$\begin{array}{llll}\mathrm{H} & -2.27935685 & -0.93190364 & -3.19253300\end{array}$
35Me24OMePh_pi_4FPh_pi_60
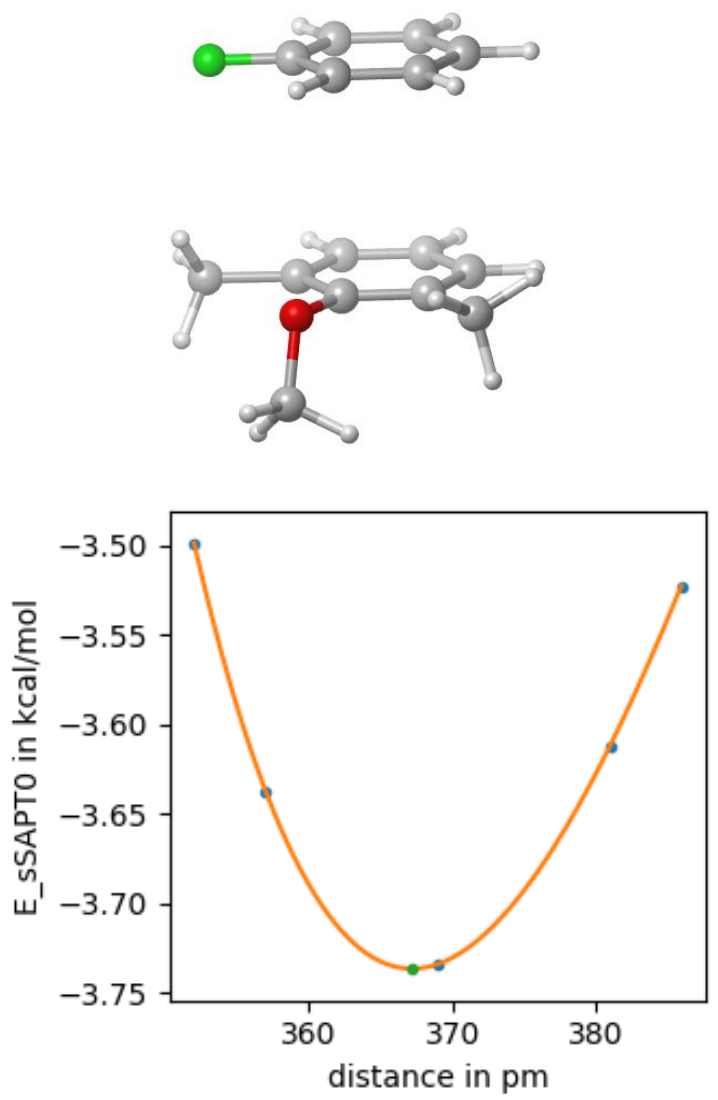

34

35Me24OMePh_pi_4FPh_pi_60-367

$\begin{array}{llll}\mathrm{H} & -1.71018682 & 1.78325222 & 0.99763421\end{array}$

$\begin{array}{llll}\text { C } & -0.95866271 & 1.00140348 & 0.99867899\end{array}$

$\begin{array}{llll}\text { C } & -1.34399825 & -0.33731271 & 0.99885454\end{array}$

$\begin{array}{llll}\text { C } & 0.39424118 & 1.33350792 & 0.99984812\end{array}$

$\begin{array}{llll}\text { C } & -0.38600978 & -1.34712971 & 1.00020025\end{array}$

$\begin{array}{llll}\mathrm{H} & -2.39655899 & -0.60065551 & 0.99795628\end{array}$

$\begin{array}{llll}\text { C } & 1.36539120 & 0.33634077 & 1.00118949\end{array}$

$\begin{array}{llll}\mathrm{H} & 0.69899334 & 2.37483406 & 0.99971375\end{array}$

C $\quad 0.95282171 \quad-0.98721505 \quad 1.00133764$

$\mathrm{H} \quad-0.65502165-2.39705951 \quad 1.00037963$

$\begin{array}{llll}\mathrm{H} & 2.42513518 & 0.56363333 & 1.00211514\end{array}$

$\begin{array}{llll}\text { F } & 1.88265223 & -1.95456458 & 1.00263312\end{array}$

$\begin{array}{llll}\text { C } & 1.34896124 & 0.33145463 & -2.67128128\end{array}$

$\begin{array}{llll}\text { C } & 0.98525409 & -1.02013019 & -2.66535487\end{array}$

$\begin{array}{llll}\text { C } & 0.40138189 & 1.35913090 & -2.66552294\end{array}$

$\begin{array}{lllll}\text { O } & 2.69449086 & 0.64003234 & -2.63825423\end{array}$

$\begin{array}{llll}\text { C } & -0.37214269 & -1.33558028 & -2.66677148\end{array}$

$\begin{array}{llll}\text { C } & 2.05415936 & -2.07983993 & -2.63546478\end{array}$

$\begin{array}{llll}\text { C } & -0.94920257 & 1.00286130 & -2.66610806\end{array}$

$\begin{array}{llll}\text { C } & 0.83282154 & 2.80218212 & -2.64411634\end{array}$

$\begin{array}{llll}\text { C } & 3.23336770 & 0.91767676 & -3.91943395\end{array}$

$\begin{array}{llll}\text { C } & -1.33686312 & -0.33157747 & -2.67280506\end{array}$

$\begin{array}{llll}\mathrm{H} & -0.67202718 & -2.37984367 & -2.65789995\end{array}$

H $\quad 2.65976369-2.06530106 \quad-3.54730985$ 


$\begin{array}{rrrr}\mathrm{H} & 2.73764064 & -1.91651137 & -1.79856812 \\ \mathrm{H} & 1.61090057 & -3.07311068 & -2.54058195 \\ \mathrm{H} & -1.70012969 & 1.78839046 & -2.65309635 \\ \mathrm{H} & 1.81076552 & 2.90435159 & -2.16946859 \\ \mathrm{H} & 0.90625635 & 3.21302550 & -3.65721555 \\ \mathrm{H} & 0.10975330 & 3.41182329 & -2.09729258 \\ \mathrm{H} & 2.76876217 & 1.80624734 & -4.36371454 \\ \mathrm{H} & 4.30046426 & 1.09873692 & -3.78349012 \\ \mathrm{H} & 3.09066859 & 0.07007562 & -4.60095663 \\ \mathrm{H} & -2.39049688 & -0.59110539 & -2.67036361\end{array}$

35Me24OMePh_pi_4FPh_pi_60_2
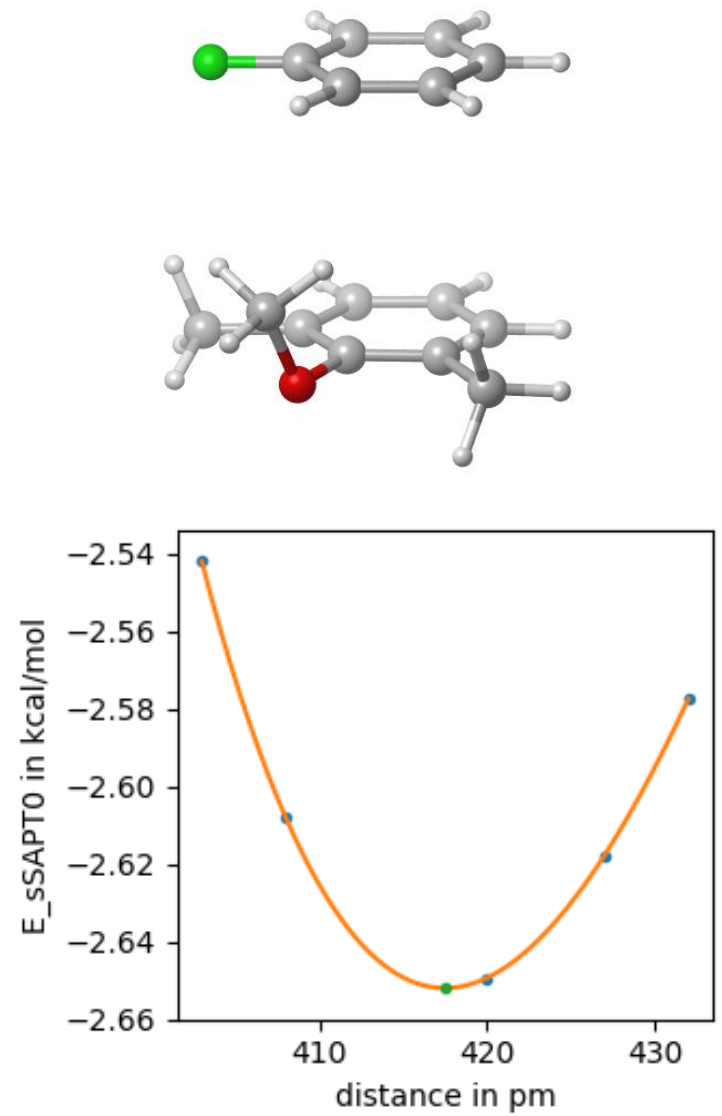

34

35Me24OMePh_pi_4FPh_pi_60_2-417

$\begin{array}{llll}\mathrm{H} & -1.94966523 & 1.51712543 & 0.99749804\end{array}$

$\begin{array}{llll}\text { C } & -1.09314823 & 0.85195293 & 0.99860252\end{array}$

$\begin{array}{llll}\text { C } & -1.28119611 & -0.52836676 & 0.99888988\end{array}$

$\begin{array}{llll}\text { C } & 0.19764387 & 1.37587862 & 0.99973671\end{array}$

$\begin{array}{llll}\text { C } & -0.18746873 & -1.38931536 & 1.00031276\end{array}$

H $\quad-2.28471706 \quad-0.94089470 \quad 0.99801920$

C $\quad 1.30256886 \quad 0.52934733 \quad 1.00115415$

$\begin{array}{llll}\mathrm{H} & 0.34888242 & 2.45029050 & 0.99951535\end{array}$

C $\quad 1.08538380 \quad-0.83990217 \quad 1.00141302$

H $\quad-0.30209935-2.46708145 \quad 1.00057963$

$\begin{array}{llll}\mathrm{H} & 2.31840188 & 0.90723957 & 1.00205512\end{array}$

$\begin{array}{llll}\text { F } & 2.14511774 & -1.66289288 & 1.00278235\end{array}$

C $\quad 1.28732395 \quad 0.52184080 \quad-3.17403983$ $\begin{array}{llll}\text { C } & 0.19812247 & 1.40087099 & -3.18079069\end{array}$

$\begin{array}{llll}\text { C } & 1.12559811 & -0.86663400 & -3.18042765\end{array}$

$\begin{array}{llll}\text { O } & 2.55696988 & 1.06384188 & -3.20558289\end{array}$

$\begin{array}{llll}\text { C } & -1.08513040 & 0.85745691 & -3.18087253\end{array}$

C $\quad 0.43915313 \quad 2.88663387 \quad-3.20993746$

$\begin{array}{llll}\text { C } & -0.17615765 & -1.37303091 & -3.18134761\end{array}$

$\begin{array}{llll}\text { C } & 2.32340718 & -1.77977647 & -3.20090972\end{array}$

$\begin{array}{llll}\text { C } & 3.15475575 & 1.15589395 & -1.92375816\end{array}$

C $-1.27465603-0.52196132 \quad-3.17549393$

$\mathrm{H} \quad-1.94193126 \quad 1.52551151 \quad-3.19040043$

$\mathrm{H} \quad 0.93650282 \quad 3.23082782-2.29746651$

H $\quad 1.08880189 \quad 3.15690226-4.04607762$

$\mathrm{H} \quad-0.50350451 \quad 3.42913904 \quad-3.30560673$

$\mathrm{H} \quad-0.32129620 \quad-2.45000301 \quad-3.19486779$

H $\quad 3.17482899-1.28694964 \quad-3.67451940$

H $\quad 2.62343631 \quad-2.06915171 \quad-2.18759859$

$\mathrm{H} \quad 2.09787644 \quad-2.69794690 \quad-3.74827177$

H $\quad 3.30130257 \quad 0.16387563 \quad-1.47965806$

H $\quad 4.12433336 \quad 1.63725657 \quad-2.05854628$

H $\quad 2.54004650 \quad 1.75715006-1.24266528$

H $\quad-2.27936447 \quad-0.93190366-3.17910340$

35Me2Ph_pi_4FPh_pi_300
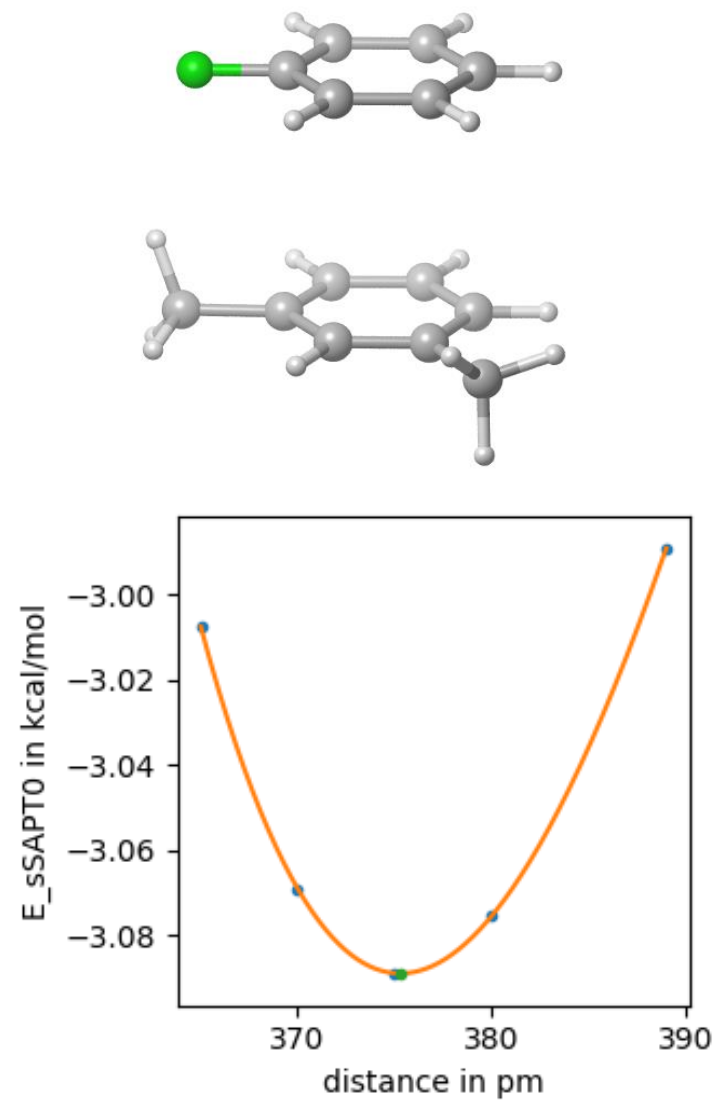

30

35Me2Ph_pi_4FPh_pi_300-375

H $\quad-0.74890824 \quad-2.35627789 \quad 0.99817332$

$\begin{array}{llll}\text { C } & -0.41883185 & -1.32325999 & 0.99898173\end{array}$ 


$\begin{array}{lrcl}\text { C } & 0.94197541 & -1.02518820 & 1.00014969 \\ \text { C } & -1.35467300 & -0.29134673 & 0.99885418 \\ \text { C } & 1.37431897 & 0.29789609 & 1.00120070 \\ \text { H } & 1.67442795 & -1.82565532 & 1.00026274 \\ \text { C } & -0.93971805 & 1.03729461 & 0.99988352 \\ \text { H } & -2.41557009 & -0.51879029 & 0.99794368 \\ \text { C } & 0.42071354 & 1.30419936 & 1.00103922 \\ \text { H } & 2.42577730 & 0.56086854 & 1.00213065 \\ \text { H } & -1.64388087 & 1.86123319 & 0.99980990 \\ \text { F } & 0.82910339 & 2.58231060 & 1.00204226 \\ \text { C } & -1.34721119 & -0.28775293 & -2.75048016 \\ \text { C } & -0.92080698 & 1.03438755 & -2.75144533 \\ \text { C } & -0.41729411 & -1.32509836 & -2.75216752 \\ \text { C } & 0.44397159 & 1.33838684 & -2.75136146 \\ \text { C } & 0.94912769 & -1.04859854 & -2.75752563 \\ \text { C } & 1.35939345 & 0.28762853 & -2.75525895 \\ \text { H } & -2.40900269 & -0.51418800 & -2.74784003 \\ \text { H } & -1.64858807 & 1.84170267 & -2.75015936 \\ \text { H } & -0.75488481 & -2.35795444 & -2.75057751 \\ \text { C } & 0.90777777 & 2.77280780 & -2.72462470 \\ \text { C } & 1.96806130 & -2.15937771 & -2.79210254 \\ \text { H } & 2.42434389 & 0.51335820 & -2.75620294 \\ \text { H } & 0.75966471 & 3.21348940 & -1.73343676 \\ \text { H } & 1.96949236 & 2.85117069 & -2.96885083 \\ \text { H } & 0.34772884 & 3.38181201 & -3.43951095 \\ \text { H } & 2.82417515 & -1.93140455 & -2.15169705 \\ \text { H } & 1.53449124 & -3.10484416 & -2.45834885 \\ \text { H } & 2.34917731 & -2.30858347 & -3.80770558\end{array}$

35Me2Ph_pi_4FPh_pi_60
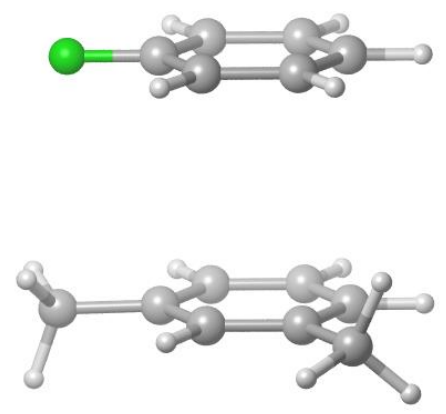

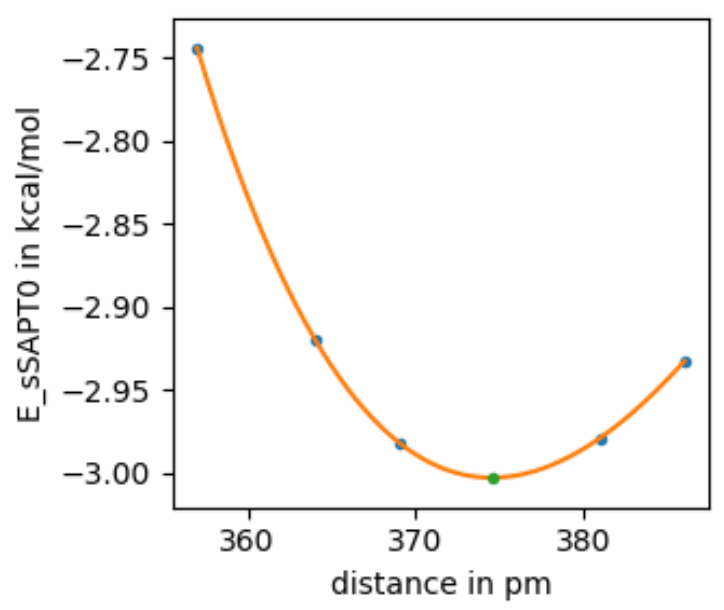

30

35Me2Ph_pi_4FPh_pi_60-374

$\begin{array}{llll}\mathrm{H} & -1.65122683 & 1.83810707 & 0.99766772\end{array}$

$\begin{array}{llll}\text { C } & -0.92555214 & 1.03220867 & 0.99869780\end{array}$

$\begin{array}{llll}\text { C } & -1.35426205 & -0.29325440 & 0.99884879\end{array}$

$\begin{array}{llll}\text { C } & 0.43744558 & 1.32009666 & 0.99987260\end{array}$

$\begin{array}{llll}\text { C } & -0.42965337 & -1.33372118 & 1.00017553\end{array}$

$\begin{array}{llll}\mathrm{H} & -2.41483743 & -0.52219412 & 0.99794603\end{array}$

C $\quad 1.37562061 \quad 0.29184458 \quad 1.00119523$

$\begin{array}{llll}\mathrm{H} & 0.77593400 & 2.35095048 & 0.99975734\end{array}$

$\begin{array}{llll}\text { C } & 0.92018470 & -1.01757961 & 1.00131909\end{array}$

$\mathrm{H} \quad-0.73270052-2.37433753 \quad 1.00033562$

$\begin{array}{llll}\mathrm{H} & 2.44220190 & 0.48451934 & 1.00212470\end{array}$

$\begin{array}{llll}\text { F } & 1.81803278 & -2.01468482 & 1.00259639\end{array}$

C $-1.34721543 \quad-0.28775294 \quad-2.74299576$

$\begin{array}{llll}\text { C } & -0.92081122 & 1.03438754 & -2.74396093\end{array}$

$\begin{array}{llll}\text { C } & -0.41729835 & -1.32509837 & -2.74468312\end{array}$

$\begin{array}{llll}\text { C } & 0.44396735 & 1.33838683 & -2.74387706\end{array}$

$\begin{array}{llll}\text { C } & 0.94912345 & -1.04859855 & -2.75004123\end{array}$

$\begin{array}{llll}\text { C } & 1.35938921 & 0.28762852 & -2.74777455\end{array}$

$\mathrm{H} \quad-2.40900693 \quad-0.51418801 \quad-2.74035563$

$\mathrm{H} \quad-1.64859231 \quad 1.84170266 \quad-2.74267496$

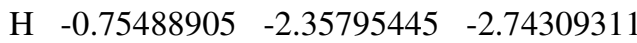

$\begin{array}{llll}\text { C } & 0.90777353 & 2.77280779 & -2.71714030\end{array}$

C $1.96805706-2.15937772-2.78461814$

$\mathrm{H} \quad 2.42433965 \quad 0.51335819 \quad-2.74871854$

H $\quad 0.75966047 \quad 3.21348939-1.72595236$

H $\quad 1.96948812 \quad 2.85117068 \quad-2.96136643$

$\mathrm{H} \quad 0.34772460 \quad 3.38181200 \quad-3.43202655$

H $\quad 2.82417091 \quad-1.93140456 \quad-2.14421265$

H $\quad 1.53448700 \quad-3.10484417 \quad-2.45086445$

H $\quad 2.34917307 \quad-2.30858348 \quad-3.80022118$ 
35Me2Ph_pi_Ph_pi_0
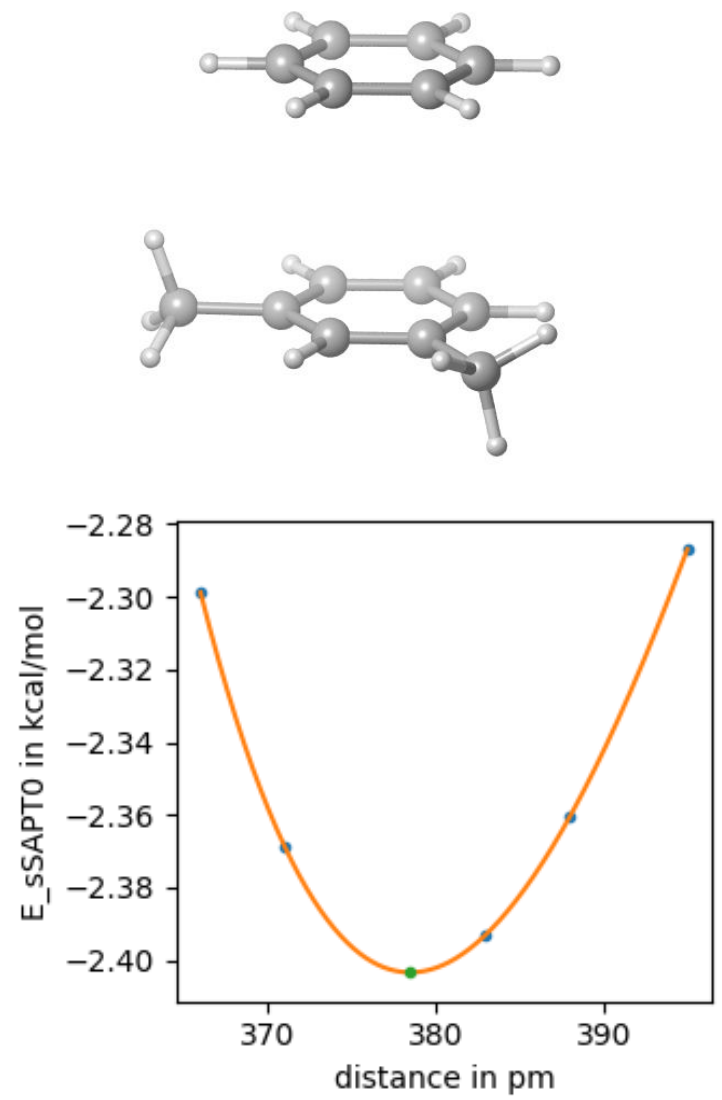

30

35Me2Ph_pi_Ph_pi_0-378

H $\quad 2.47830959 \quad-0.04134128 \quad 0.00002015$

C $\quad 1.39302564 \quad-0.02324781 \quad 0.00000008$

C $\quad 0.71636748 \quad 1.19441056 \quad-0.00010067$

C $\quad 0.67612530 \quad-1.21763495 \quad 0.00007275$

$\begin{array}{llll}\text { C } & -0.67625398 & 1.21769798 & 0.00007325\end{array}$

$\mathrm{H} \quad 1.27482284 \quad 2.12543743 \quad 0.00000658$

C $\quad-0.71652436 \quad-1.19445047 \quad-0.00005433$

H $\quad 1.20319885 \quad-2.16677876 \quad 0.00008956$

$\begin{array}{llll}\text { C } & -1.39262449 & 0.02322482 & -0.00003308\end{array}$

H $\quad-1.20362977 \quad 2.16671637 \quad 0.00016144$

H $\quad-1.27520554 \quad-2.12538191 \quad-0.00007858$

$\begin{array}{llll}\mathrm{H} & -2.47818735 & 0.04136582 & 0.00005785\end{array}$

C $\quad 0.71776816 \quad 1.19345093 \quad-3.78350411$

C $\quad-0.67309042 \quad 1.21389461 \quad-3.78565951$

C $\quad 1.40731684 \quad-0.02075222 \quad-3.78643222$

C $\quad-1.39163235 \quad 0.02249145 \quad-3.78725000$

C $\quad 0.66975537 \quad-1.20544926 \quad-3.78444544$

C $-0.72613453-1.20467266-3.78309672$

H $1.27590239 \quad 2.12597847 \quad-3.78162083$

H $\quad-1.20013369 \quad 2.16305221 \quad-3.78621506$

C $\quad 2.91438507 \quad-0.05113273 \quad-3.82277417$

$\mathrm{H} \quad-2.47822574 \quad 0.04224622 \quad-3.78966813$

H $\quad 1.19804976 \quad-2.15724953 \quad-3.78393273$

C $-1.49636342 \quad-2.50028189-3.74596705$

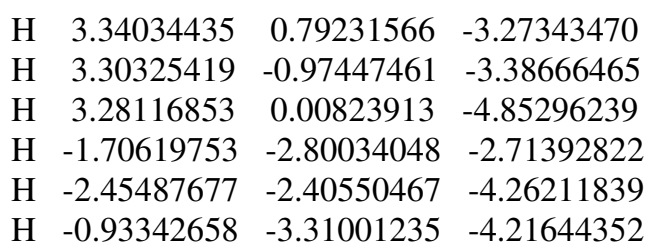

35Me4OMePh_pi1_Ph_pi0
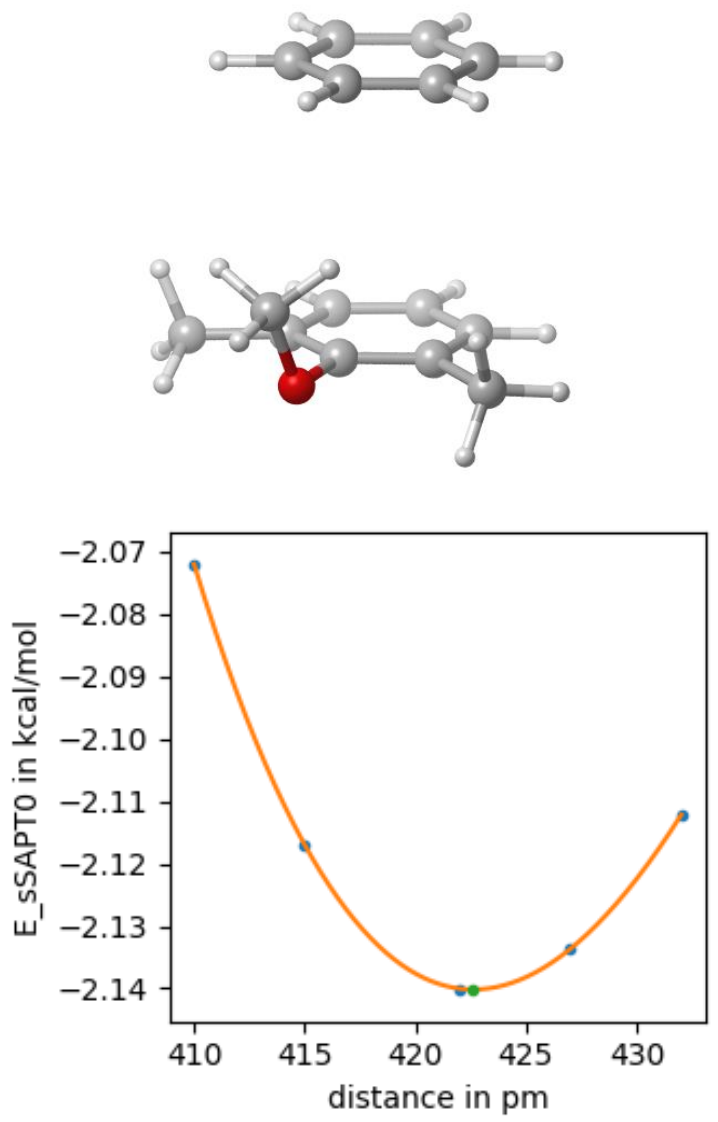

34

35Me4OMePh_pi1_Ph_pi0-422

H $\quad-3.57503868 \quad-0.70408434 \quad-0.54904609$

C $\quad-2.52519568-0.49647234-0.72857809$

C $\quad-2.08491168 \quad 0.81956266 \quad-0.84235609$

C $\quad-1.62171768 \quad-1.54399834 \quad-0.86074909$

C $-0.74106468 \quad 1.10405666 \quad-1.07725009$

H $-2.79116268 \quad 1.64041066 \quad-0.75339809$

C $\quad-0.26673368-1.29937034 \quad-1.09565809$

H $-1.96568368 \quad-2.57248034 \quad-0.78983309$

C $\quad 0.15029432 \quad 0.03122666 \quad-1.19393109$

O $\quad 1.47542332 \quad 0.31617066 \quad-1.45775109$

C $\quad-0.23502068 \quad 2.51381666 \quad-1.22868609$

H $\quad 0.46314232 \quad 2.77813566 \quad-0.42797809$

H $\quad 0.30476232 \quad 2.63197966 \quad-2.17149609$

H $\quad-1.06222968 \quad 3.22597966 \quad-1.20384609$

C $\quad 0.71884732 \quad-2.42737234 \quad-1.25451909$

H $\quad 1.55608532 \quad-2.12084534 \quad-1.88473909$

H $\quad 1.12446132 \quad-2.74367534 \quad-0.28705609$ 


$\begin{array}{lrrc}\mathrm{H} & 0.23757232 & -3.29802734 & -1.70600609 \\ \mathrm{C} & 2.28539332 & 0.32308366 & -0.29465309 \\ \mathrm{H} & 2.31609832 & -0.66699734 & 0.17608891 \\ \mathrm{H} & 3.29145732 & 0.60404766 & -0.60906209 \\ \mathrm{H} & 1.91636132 & 1.04898866 & 0.44028991 \\ \mathrm{C} & -0.04262939 & 1.19135213 & 3.09609209 \\ \mathrm{C} & -1.38974398 & 0.92503724 & 3.33043552 \\ \mathrm{C} & 0.86281002 & 0.14021869 & 2.97037541 \\ \mathrm{C} & -1.83111843 & -0.39151023 & 3.43919311 \\ \mathrm{C} & 0.42147361 & -1.17639513 & 3.07882293 \\ \mathrm{C} & -0.92546844 & -1.44217123 & 3.31324705 \\ \mathrm{H} & 0.30131277 & 2.21737173 & 3.01149781 \\ \mathrm{H} & -2.09561967 & 1.74405035 & 3.42862134 \\ \mathrm{H} & 1.91274103 & 0.34745341 & 2.78767580 \\ \mathrm{H} & -2.88100526 & -0.59906840 & 3.62200014 \\ \mathrm{H} & 1.12720549 & -1.99559124 & 2.98075032 \\ \mathrm{H} & -1.26952077 & -2.46843842 & 3.39797894\end{array}$

35Me4OMePh_pi2_Ph_pi0
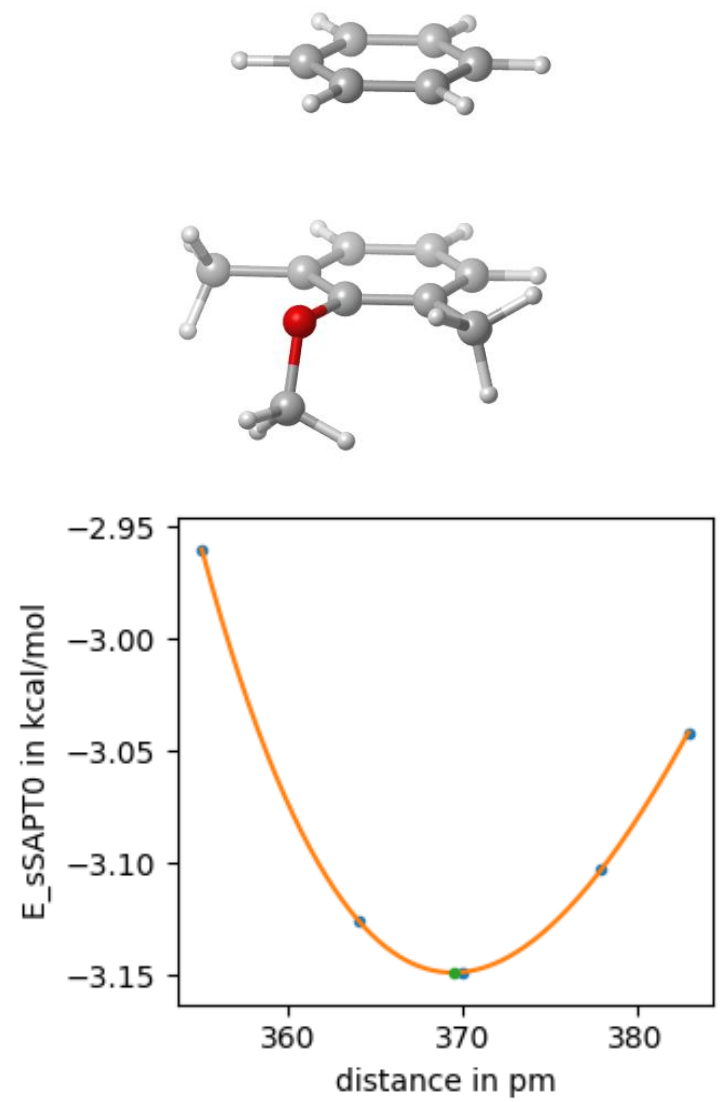

C $\quad-0.26673368-1.29937034-1.09565809$

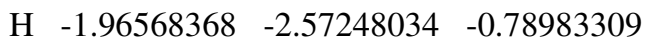

C $\quad 0.15029432 \quad 0.03122666-1.19393109$

$\begin{array}{lllll}\text { O } & 1.47542332 & 0.31617066 & -1.45775109\end{array}$

C $\quad-0.23502068 \quad 2.51381666-1.22868609$

H $\quad 0.46314232 \quad 2.77813566-0.42797809$

H $\quad 0.30476232 \quad 2.63197966-2.17149609$

H $\quad-1.06222968 \quad 3.22597966-1.20384609$

C $\quad 0.71884732 \quad-2.42737234-1.25451909$

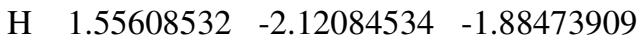

$\mathrm{H} \quad 1.12446132 \quad-2.74367534-0.28705609$

H $\quad 0.23757232 \quad-3.29802734 \quad-1.70600609$

$\begin{array}{llll}\text { C } & 2.28539332 & 0.32308366 & -0.29465309\end{array}$

H $\quad 2.31609832 \quad-0.66699734 \quad 0.17608891$

H $\quad 3.291457320 .60404766-0.60906209$

$\begin{array}{llll}\mathrm{H} & 1.91636132 & 1.04898866 & 0.44028991\end{array}$

C $\quad-0.89697144 \quad-1.37747448 \quad-4.72882982$

$\begin{array}{llll}\text { C } & -2.24432594 & -1.64260193 & -4.49451918\end{array}$

$\begin{array}{llll}\text { C } & -0.45557793 & -0.06069909 & -4.83737032\end{array}$

$\begin{array}{llll}\text { C } & -3.14973529 & -0.59168673 & -4.36902813\end{array}$

$\begin{array}{llll}\text { C } & -1.36087776 & 0.99031157 & -4.71159486\end{array}$

$\begin{array}{llll}\text { C } & -2.70790950 & 0.72474301 & -4.47745107\end{array}$

$\mathrm{H} \quad-0.19144380-2.19650240 \quad-4.82677181$

$\mathrm{H} \quad-2.58865592-2.66875188 \quad-4.41004077$

$\mathrm{H} \quad 0.59443623 \quad 0.14624790 \quad-5.01991812$

$\mathrm{H} \quad-4.19986528 \quad-0.79832440 \quad-4.18657530$

H $\quad-1.01677734 \quad 2.01657086 \quad-4.79612156$

$\begin{array}{llll}\mathrm{H} & -3.41366919 & 1.54395102 & -4.37958719\end{array}$

35tBuPh_pi_Ph_pi_60
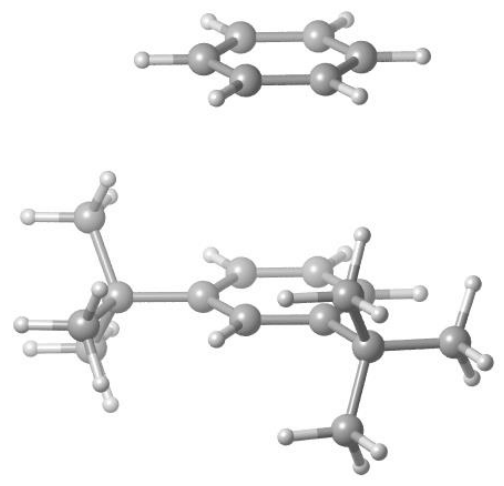

34

35Me4OMePh_pi2_Ph_pi0-369

H $\quad-3.57503868 \quad-0.70408434 \quad-0.54904609$

$\begin{array}{llll}\text { C } & -2.52519568 & -0.49647234 & -0.72857809\end{array}$

$\begin{array}{llll}\text { C } & -2.08491168 & 0.81956266 & -0.84235609\end{array}$

$\begin{array}{llll}\text { C } & -1.62171768 & -1.54399834 & -0.86074909\end{array}$

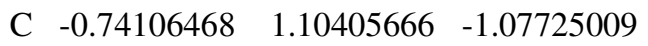

$\begin{array}{llll}\mathrm{H} & -2.79116268 & 1.64041066 & -0.75339809\end{array}$ 

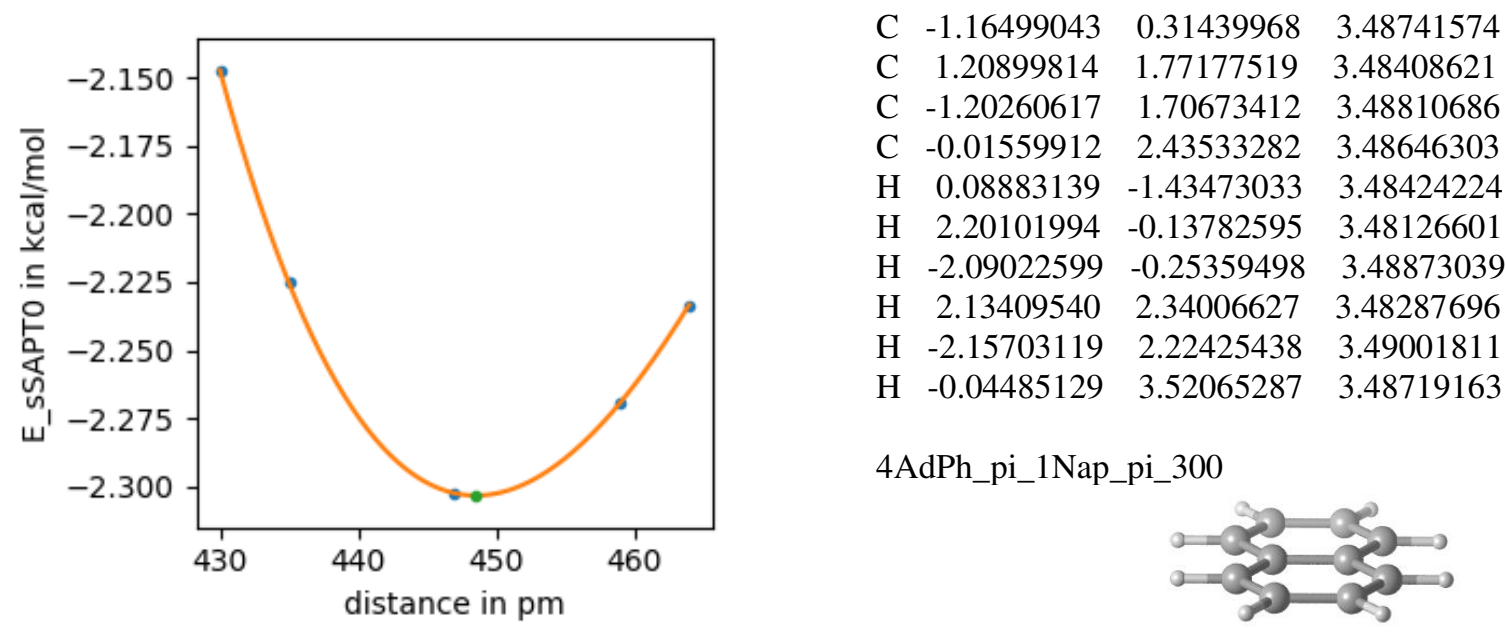

4AdPh_pi_1Nap_pi_300

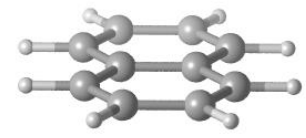

48

35tBuPh_pi_Ph_pi_60-448

$\begin{array}{llll}\text { C } & -0.02352049 & 2.42945833 & -0.99999536\end{array}$

$\begin{array}{llll}\text { C } & -1.21413849 & 1.72118433 & -0.99999036\end{array}$

C $\quad 1.20091151 \quad 1.76037733 \quad-0.99999336$

H $\quad-2.15570549 \quad 2.26309933 \quad-0.99999036$

$\begin{array}{llll}\mathrm{H} & 2.11648451 & 2.34064433 & -0.99999836\end{array}$

$\begin{array}{llll}\text { C } & 0.02259751 & -0.32685467 & -0.99998336\end{array}$

$\begin{array}{lllll}\mathrm{H} & 0.05072451 & -1.41139967 & -0.99997936\end{array}$

$\begin{array}{llll}\text { C } & 1.24219951 & 0.36819833 & -0.99998736\end{array}$

$\begin{array}{llll}\text { C } & 2.55300051 & -0.42485067 & -0.99999736\end{array}$

$\begin{array}{llll}\text { C } & 2.61361851 & -1.31183067 & 0.25519764\end{array}$

$\begin{array}{llll}\text { C } & 2.61361451 & -1.31178867 & -2.25522236\end{array}$

$\begin{array}{llll}\text { C } & 3.78064951 & 0.49162733 & -0.99998236\end{array}$

$\begin{array}{llll}\mathrm{H} & 2.56851051 & -0.70145967 & 1.16212264\end{array}$

H $\quad 1.78396951 \quad-2.02357567 \quad 0.28627664$

H $\quad 3.54785651 \quad-1.88318767 \quad 0.26965064$

H $\quad 2.56847351-0.70138967-3.16212536$

H $3.54786651 \quad-1.88312267 \quad-2.26971336$

H $\quad 1.78398151 \quad-2.02355167-2.28630636$

$\mathrm{H} \quad 4.69085151 \quad-0.11548367 \quad-0.99997636$

$\mathrm{H} \quad 3.80472051 \quad 1.13118033 \quad-1.88779936$

$\begin{array}{llll}\mathrm{H} & 3.80470351 & 1.13117233 & -0.11215836\end{array}$

C $-1.21161949 \quad 0.31864833-0.99998236$

$\begin{array}{llll}\text { C } & -2.54299449 & -0.43902567 & -0.99999636\end{array}$

$\begin{array}{llll}\text { C } & -3.34541349 & -0.05606167 & 0.25506764\end{array}$

$\begin{array}{llll}\text { C } & -3.34530149 & -0.05614167 & -2.25515336\end{array}$

$\begin{array}{llll}\text { C } & -2.34786349 & -1.95857267 & -0.99993936\end{array}$

$\begin{array}{llll}\mathrm{H} & -2.79200149 & -0.31715967 & 1.16208764\end{array}$

$\begin{array}{llll}\mathrm{H} & -3.55672549 & 1.01633433 & 0.28613264\end{array}$

$\begin{array}{llll}\mathrm{H} & -4.30240349 & -0.58843767 & 0.26923364\end{array}$

H $\quad-2.79181549-0.31731767 \quad-3.16210636$

$\begin{array}{llll}\mathrm{H} & -4.30230049 & -0.58850267 & -2.26936236\end{array}$

$\mathrm{H} \quad-3.55658449 \quad 1.01625733 \quad-2.28630936$

H $-3.32418249 \quad-2.45230367 \quad-0.99993836$

H $-1.80309549-2.29445267-1.88789936$

H $-1.80312549-2.29438867 \quad-0.11193536$

H $-0.04135949 \quad 3.51508233 \quad-1.00000136$

$\begin{array}{llll}\text { C } & 0.05954370 & -0.34969094 & 3.48485975\end{array}$

$\begin{array}{llll}\text { C } & 1.24650928 & 0.37946459 & 3.48309428\end{array}$
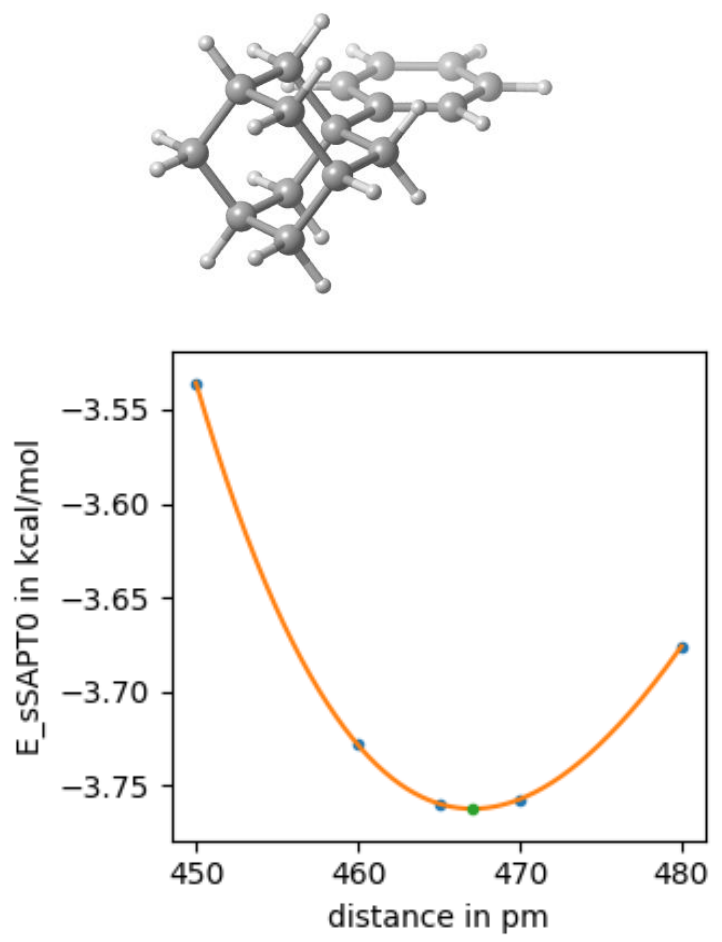

54

4AdPh_pi_1Nap_pi_300-467

H $\quad-5.40699838-0.07046942 \quad 1.99997493$

$\begin{array}{llll}\text { C } & -4.32228838 & -0.03889342 & 1.99999093\end{array}$

$\begin{array}{llll}\text { C } & -3.58278938 & -1.21969542 & 1.99999893\end{array}$

$\begin{array}{llll}\text { C } & -3.65363938 & 1.17869058 & 1.99998993\end{array}$

$\begin{array}{llll}\text { C } & -2.19430138 & -1.17393642 & 2.00001793\end{array}$

$\begin{array}{llll}\mathrm{H} & -4.08913238 & -2.17997242 & 1.99998893\end{array}$

C $\quad-2.25972238 \quad 1.21897558 \quad 2.00000593$

$\begin{array}{llll}\mathrm{H} & -4.21490638 & 2.10797258 & 1.99997293\end{array}$

$\begin{array}{llll}\text { C } & -1.50186838 & 0.04520558 & 2.00003493\end{array}$

$\begin{array}{llll}\mathrm{H} & -1.63822938 & -2.10748042 & 2.00001193\end{array}$

$\begin{array}{llll}\mathrm{H} & -1.76867538 & 2.18541758 & 1.99999893\end{array}$

$\begin{array}{llll}\text { C } & 0.02431362 & 0.04571458 & 2.00001693\end{array}$

$\begin{array}{llll}\text { C } & 0.55298362 & -0.68866242 & 0.74425493\end{array}$ 


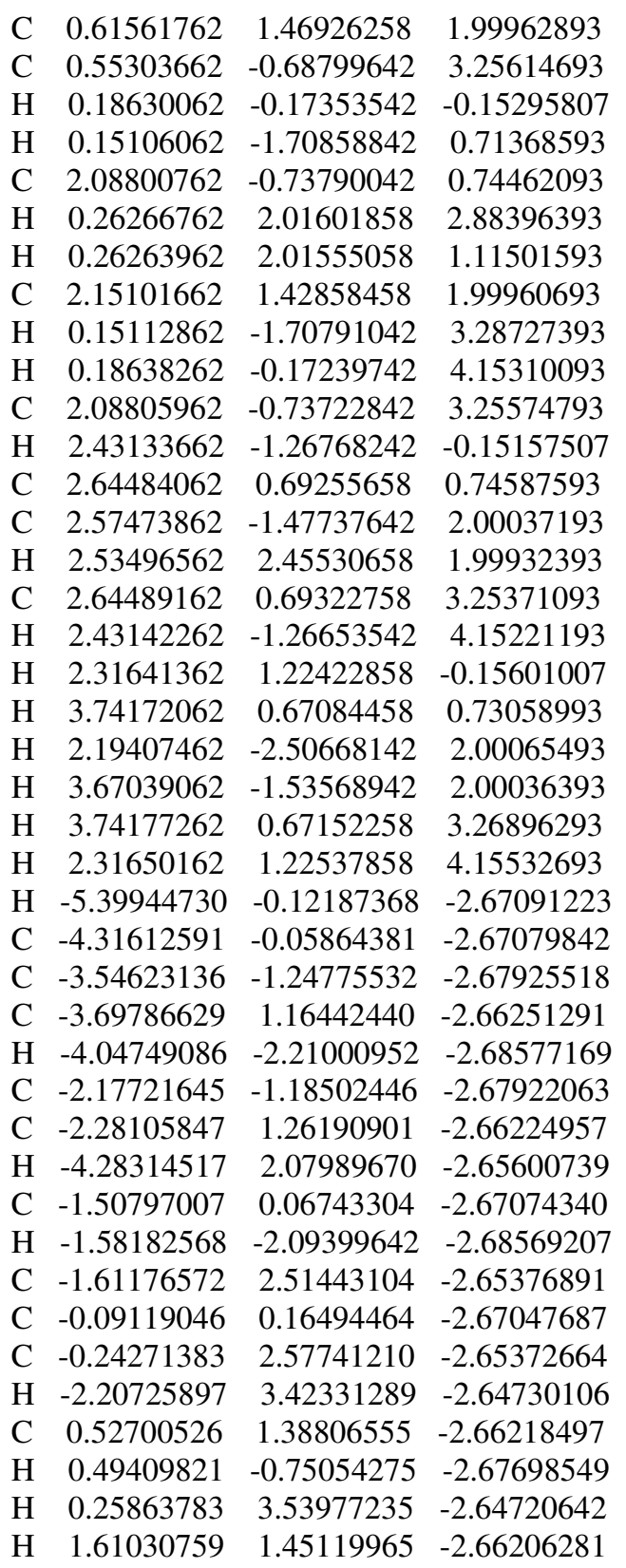

4AdPh_pi_1Nap_pi_300_2
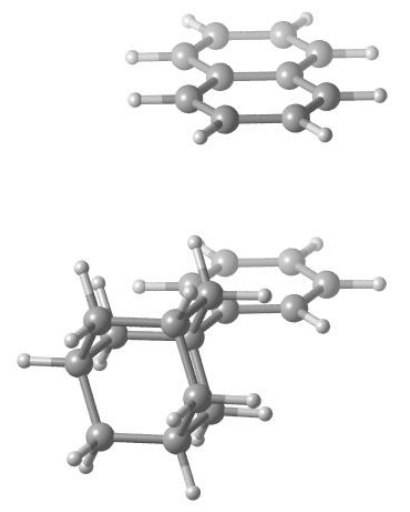

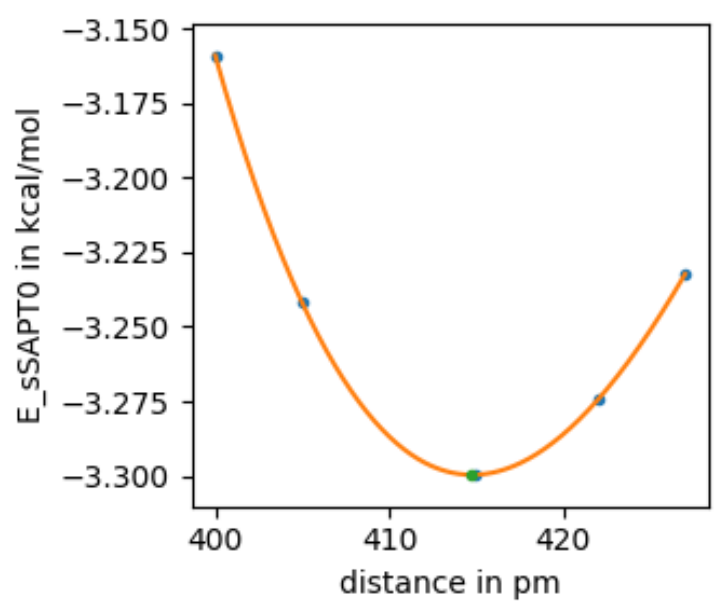

54

4AdPh_pi_1Nap_pi_300_2-414

H $\quad-5.40699838-0.07046942 \quad 1.99997493$

$\begin{array}{llll}\text { C } & -4.32228838 & -0.03889342 & 1.99999093\end{array}$

$\begin{array}{llll}\text { C } & -3.58278938 & -1.21969542 & 1.99999893\end{array}$

$\begin{array}{llll}\text { C } & -3.65363938 & 1.17869058 & 1.99998993\end{array}$

$\begin{array}{llll}\text { C } & -2.19430138 & -1.17393642 & 2.00001793\end{array}$

$\begin{array}{llll}\mathrm{H} & -4.08913238 & -2.17997242 & 1.99998893\end{array}$

$\begin{array}{llll}\text { C } & -2.25972238 & 1.21897558 & 2.00000593\end{array}$

$\begin{array}{llll}\mathrm{H} & -4.21490638 & 2.10797258 & 1.99997293\end{array}$

$\begin{array}{llll}\text { C } & -1.50186838 & 0.04520558 & 2.00003493\end{array}$

$\begin{array}{llll}\mathrm{H} & -1.63822938 & -2.10748042 & 2.00001193\end{array}$

$\begin{array}{llll}\mathrm{H} & -1.76867538 & 2.18541758 & 1.99999893\end{array}$

$\begin{array}{llll}\text { C } & 0.02431362 & 0.04571458 & 2.00001693\end{array}$

$\begin{array}{llll}\text { C } & 0.55298362 & -0.68866242 & 0.74425493\end{array}$

$\begin{array}{llll}\text { C } & 0.61561762 & 1.46926258 & 1.99962893\end{array}$

C $\quad 0.55303662 \quad-0.68799642 \quad 3.25614693$

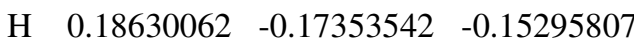

$\begin{array}{llll}\mathrm{H} & 0.15106062 & -1.70858842 & 0.71368593\end{array}$

$\begin{array}{llll}\text { C } & 2.08800762 & -0.73790042 & 0.74462093\end{array}$

$\begin{array}{llll}\mathrm{H} & 0.26266762 & 2.01601858 & 2.88396393\end{array}$

$\begin{array}{llll}\mathrm{H} & 0.26263962 & 2.01555058 & 1.11501593\end{array}$

C $\quad 2.15101662 \quad 1.42858458 \quad 1.99960693$

H $\quad 0.15112862 \quad-1.70791042 \quad 3.28727393$

H $\quad 0.18638262-0.17239742 \quad 4.15310093$

C $\quad 2.08805962 \quad-0.73722842 \quad 3.25574793$

H $\quad 2.43133662 \quad-1.26768242 \quad-0.15157507$

$\begin{array}{llll}\text { C } & 2.64484062 & 0.69255658 & 0.74587593\end{array}$

C $\quad 2.57473862 \quad-1.47737642 \quad 2.00037193$

$\begin{array}{llll}\mathrm{H} & 2.53496562 & 2.45530658 & 1.99932393\end{array}$

$\begin{array}{llll}\text { C } & 2.64489162 & 0.69322758 & 3.25371093\end{array}$

H $\quad 2.43142262 \quad-1.26653542 \quad 4.15221193$

H $\quad 2.31641362 \quad 1.22422858-0.15601007$

$\begin{array}{llll}\mathrm{H} & 3.74172062 & 0.67084458 & 0.73058993\end{array}$

$\mathrm{H} \quad 2.19407462 \quad-2.50668142 \quad 2.00065493$

H $\quad 3.67039062 \quad-1.53568942 \quad 2.00036393$

H $\quad 3.74177262 \quad 0.67152258 \quad 3.26896293$

$\mathrm{H} \quad 2.31650162 \quad 1.22537858 \quad 4.15532693$

H $\quad-1.66494181 \quad-2.15162716-2.14821980$

C $-2.21541635-1.21644753 \quad-2.14788730$ 


$\begin{array}{rrrr}\mathrm{C} & -1.50847226 & 0.01113721 & -2.15570361 \\ \mathrm{C} & -3.58584903 & -1.22423393 & -2.13994352 \\ \mathrm{H} & -0.42350798 & 0.00405643 & -2.16195461 \\ \mathrm{C} & -2.18883515 & 1.20077716 & -2.15538596 \\ \mathrm{C} & -4.31894157 & -0.00791869 & -2.13939998 \\ \mathrm{H} & -4.13350792 & -2.16269906 & -2.13392939 \\ \mathrm{C} & -3.60868078 & 1.22495544 & -2.14725054 \\ \mathrm{H} & -1.65157380 & 2.14527520 & -2.16136628 \\ \mathrm{C} & -5.73886577 & 0.01627140 & -2.13126116 \\ \mathrm{C} & -4.34178446 & 2.44123325 & -2.14670384 \\ \mathrm{C} & -6.41946818 & 1.20583013 & -2.13093587 \\ \mathrm{H} & -6.27600018 & -0.92827673 & -2.12528441 \\ \mathrm{C} & -5.71223489 & 2.43336595 & -2.13875370 \\ \mathrm{H} & -3.79411662 & 3.37971398 & -2.15272105 \\ \mathrm{H} & -7.50456888 & 1.21294453 & -2.12468119 \\ \mathrm{H} & -6.26261551 & 3.36857230 & -2.13841282\end{array}$

4AdPh_pi_1Nap_pi_60
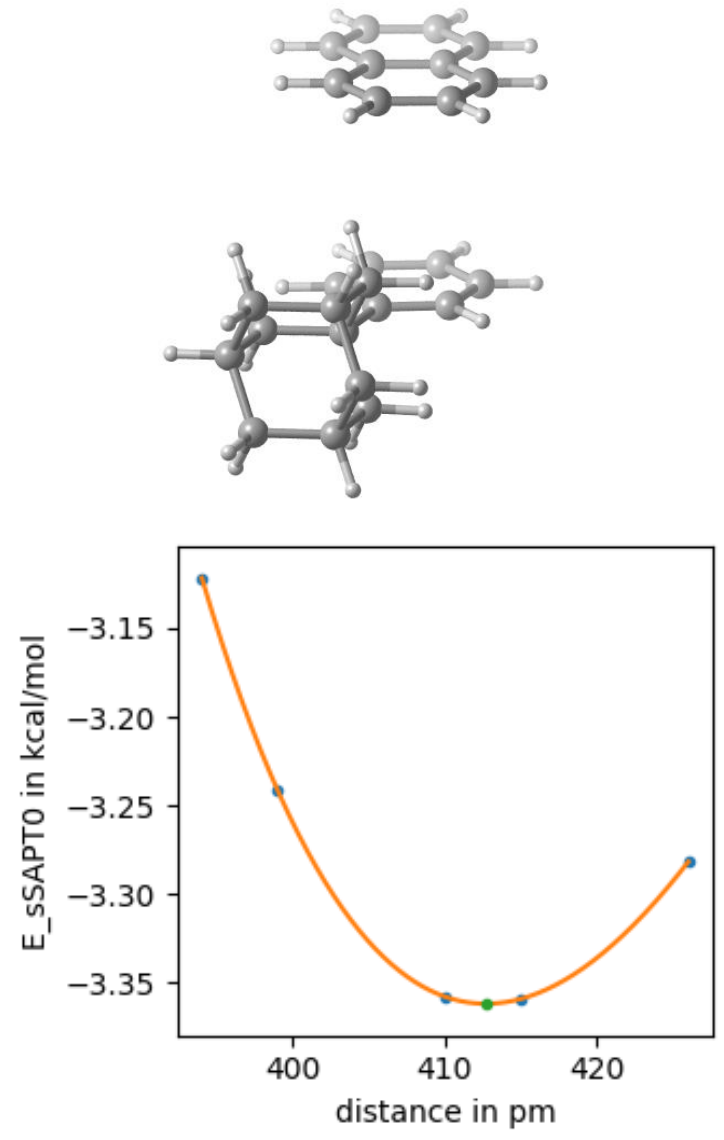

$\begin{array}{cccc}\mathrm{H} & -4.21489869 & 2.10823779 & 0.99997296 \\ \mathrm{C} & -1.50186069 & 0.04547079 & 1.00003496 \\ \mathrm{H} & -1.63822169 & -2.10721521 & 1.00001196 \\ \mathrm{H} & -1.76866769 & 2.18568279 & 0.99999896 \\ \mathrm{C} & 0.02432131 & 0.04597979 & 1.00001696 \\ \mathrm{C} & 0.55299131 & -0.68839721 & -0.25574504 \\ \mathrm{C} & 0.61562531 & 1.46952779 & 0.99962896 \\ \mathrm{C} & 0.55304431 & -0.68773121 & 2.25614696 \\ \mathrm{H} & 0.18630831 & -0.17327021 & -1.15295804 \\ \mathrm{H} & 0.15106831 & -1.70832321 & -0.28631404 \\ \mathrm{C} & 2.08801531 & -0.73763521 & -0.25537904 \\ \mathrm{H} & 0.26267531 & 2.01628379 & 1.88396396 \\ \mathrm{H} & 0.26264731 & 2.01581579 & 0.11501596 \\ \mathrm{C} & 2.15102431 & 1.42884979 & 0.99960696 \\ \mathrm{H} & 0.15113631 & -1.70764521 & 2.28727396 \\ \mathrm{H} & 0.18639031 & -0.17213221 & 3.15310096 \\ \mathrm{C} & 2.08806731 & -0.73696321 & 2.25574796 \\ \mathrm{H} & 2.43134431 & -1.26741721 & -1.15157504 \\ \mathrm{C} & 2.64484831 & 0.69282179 & -0.25412404 \\ \mathrm{C} & 2.57474631 & -1.47711121 & 1.00037196 \\ \mathrm{H} & 2.53497331 & 2.45557179 & 0.99932396 \\ \mathrm{C} & 2.64489931 & 0.69349279 & 2.25371096 \\ \mathrm{H} & 2.43143031 & -1.26627021 & 3.15221196 \\ \mathrm{H} & 2.31642131 & 1.22449379 & -1.15601004 \\ \mathrm{H} & 3.74172831 & 0.67110979 & -0.26941004 \\ \mathrm{H} & 2.19408231 & -2.50641621 & 1.00065496 \\ \mathrm{H} & 3.67039831 & -1.53542421 & 1.00036396 \\ \mathrm{H} & 3.74178031 & 0.67178779 & 2.26896296 \\ \mathrm{H} & 2.31650931 & 1.22564379 & 3.15532696 \\ \mathrm{H} & -1.5589969 & -2.08650301 & -3.12744855 \\ \mathrm{C} & -2.15540908 & -1.17993418 & -3.12712400 \\ \mathrm{C} & -1.51055358 & 0.08137299 & -3.13493184 \\ \mathrm{C} & -3.52374899 & -1.25604287 & -3.11919831 \\ \mathrm{H} & -0.42658571 & 0.12839908 & -3.14116848 \\ \mathrm{C} & -2.24938756 & 1.23560920 & -3.13462402 \\ \mathrm{C} & -4.31657711 & -0.07779374 & -3.11866534 \\ \mathrm{H} & -4.02393335 & -2.22064783 & -3.11319075 \\ \mathrm{C} & -3.66867276 & 1.18896161 & -3.12650738 \\ \mathrm{H} & -1.75988860 & 2.20572113 & -3.14059792 \\ \mathrm{C} & -5.73594138 & -0.12443344 & -3.11054529 \\ \mathrm{C} & -4.46151016 & 2.36717282 & -3.12597123 \\ \mathrm{C} & -6.47501053 & 1.02970971 & -3.11022984 \\ \mathrm{H} & -6.22531106 & -1.09458906 & -3.10457496 \\ \mathrm{C} & -5.82986375 & 2.29098243 & -3.11803920 \\ \mathrm{H} & -3.96131761 & 3.33179379 & -3.13198189 \\ \mathrm{H} & -7.55911633 & 0.98271039 & -3.10398951 \\ \mathrm{H} & -6.42619068 & 3.19758264 & -3.11770627\end{array}$

54

4AdPh_pi_1Nap_pi_60-412

H $\quad-5.40699069-0.070204210 .99997496$

$\begin{array}{llll}\text { C } & -4.32228069 & -0.03862821 & 0.99999096\end{array}$

$\begin{array}{llll}\text { C } & -3.58278169 & -1.21943021 & 0.99999896\end{array}$

$\begin{array}{llll}\text { C } & -3.65363169 & 1.17895579 & 0.99998996\end{array}$

$\begin{array}{llll}\text { C } & -2.19429369 & -1.17367121 & 1.00001796\end{array}$

H $\quad-4.08912469-2.17970721 \quad 0.99998896$

$\begin{array}{llll}\text { C } & -2.25971469 & 1.21924079 & 1.00000596\end{array}$ 
4AdPh_pi_1Nap_pi_60_2
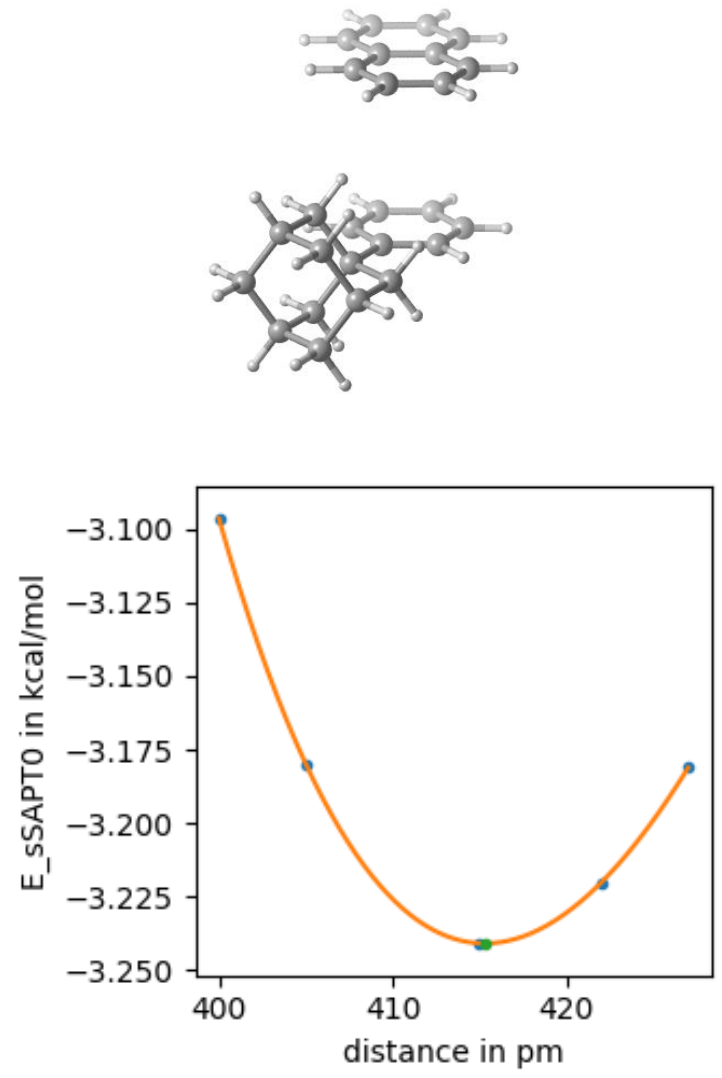

54

4AdPh_pi_1Nap_pi_60_2-415

H $\quad-5.40699838-0.07046942 \quad-1.99997493$

C $-4.32228838-0.03889342 \quad-1.99999093$

C $\quad-3.58278938-1.21969542 \quad-1.99999893$

C $\quad-3.65363938 \quad 1.17869058 \quad-1.99998993$

C $-2.19430138-1.17393642 \quad-2.00001793$

H $\quad-4.08913238-2.17997242 \quad-1.99998893$

C $\quad-2.25972238 \quad 1.21897558 \quad-2.00000593$

H $\quad-4.21490638 \quad 2.10797258 \quad-1.99997293$

C $\quad-1.50186838 \quad 0.04520558-2.00003493$

H $-1.63822938-2.10748042 \quad-2.00001193$

H $\quad-1.76867538 \quad 2.18541758 \quad-1.99999893$

C $\quad 0.02431362 \quad 0.04571458 \quad-2.00001693$

C $\quad 0.55298362 \quad-0.68866242 \quad-0.74425493$

C $\quad 0.61561762 \quad 1.46926258 \quad-1.99962893$

C $\quad 0.55303662 \quad-0.68799642 \quad-3.25614693$

H $\quad 0.18630062 \quad-0.17353542 \quad 0.15295807$

H $\quad 0.15106062 \quad-1.70858842 \quad-0.71368593$

C $2.08800762-0.73790042 \quad-0.74462093$

H $\quad 0.26266762 \quad 2.01601858 \quad-2.88396393$

H $\quad 0.26263962 \quad 2.01555058-1.11501593$

C $2.15101662 \quad 1.42858458 \quad-1.99960693$

H $\quad 0.15112862 \quad-1.70791042 \quad-3.28727393$

H $\quad 0.18638262-0.17239742 \quad-4.15310093$

C $\quad 2.08805962 \quad-0.73722842 \quad-3.25574793$

$\begin{array}{lrrr}\mathrm{H} & 2.43133662 & -1.26768242 & 0.15157507 \\ \mathrm{C} & 2.64484062 & 0.69255658 & -0.74587593 \\ \mathrm{C} & 2.57473862 & -1.47737642 & -2.00037193 \\ \mathrm{H} & 2.53496562 & 2.45530658 & -1.99932393 \\ \mathrm{C} & 2.64489162 & 0.69322758 & -3.25371093 \\ \mathrm{H} & 2.43142262 & -1.26653542 & -4.15221193 \\ \mathrm{H} & 2.31641362 & 1.22422858 & 0.15601007 \\ \mathrm{H} & 3.74172062 & 0.67084458 & -0.73058993 \\ \mathrm{H} & 2.19407462 & -2.50668142 & -2.00065493 \\ \mathrm{H} & 3.67039062 & -1.53568942 & -2.00036393 \\ \mathrm{H} & 3.74177262 & 0.67152258 & -3.26896293 \\ \mathrm{H} & 2.31650162 & 1.22537858 & -4.15532693 \\ \mathrm{H} & -0.42594034 & 0.15382691 & 2.15300964 \\ \mathrm{C} & -1.50862173 & 0.08044920 & 2.15294872 \\ \mathrm{C} & -2.28962419 & 1.26229903 & 2.16078860 \\ \mathrm{C} & -2.11539433 & -1.14836255 & 2.14532289 \\ \mathrm{H} & -1.79740280 & 2.22921104 & 2.16678583 \\ \mathrm{C} & -3.65799122 & 1.18674343 & 2.16081177 \\ \mathrm{C} & -3.53122655 & -1.25911829 & 2.14513656 \\ \mathrm{H} & -1.52156337 & -2.05831400 & 2.13929242 \\ \mathrm{C} & -4.31547297 & -0.07193415 & 2.15301072 \\ \mathrm{H} & -4.26187272 & 2.09010008 & 2.16681179 \\ \mathrm{C} & -4.18875406 & -2.51786084 & 2.13733212 \\ \mathrm{C} & -5.73127672 & -0.18271662 & 2.15282122 \\ \mathrm{C} & -5.55715573 & -2.59366697 & 2.13734770 \\ \mathrm{H} & -3.58477094 & -3.42112640 & 2.13133564 \\ \mathrm{C} & -6.33798493 & -1.41158046 & 2.14518903 \\ \mathrm{H} & -6.32511761 & 0.72724984 & 2.15885478 \\ \mathrm{H} & -6.04946827 & -3.56068589 & 2.13134682 \\ \mathrm{H} & -7.42064816 & -1.48486224 & 2.14511970\end{array}$

4AdPh_pi_2Furane_pi_300_N1
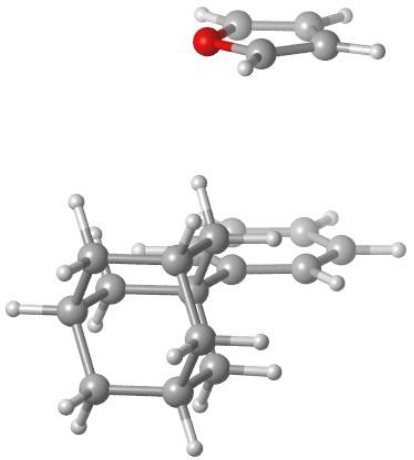


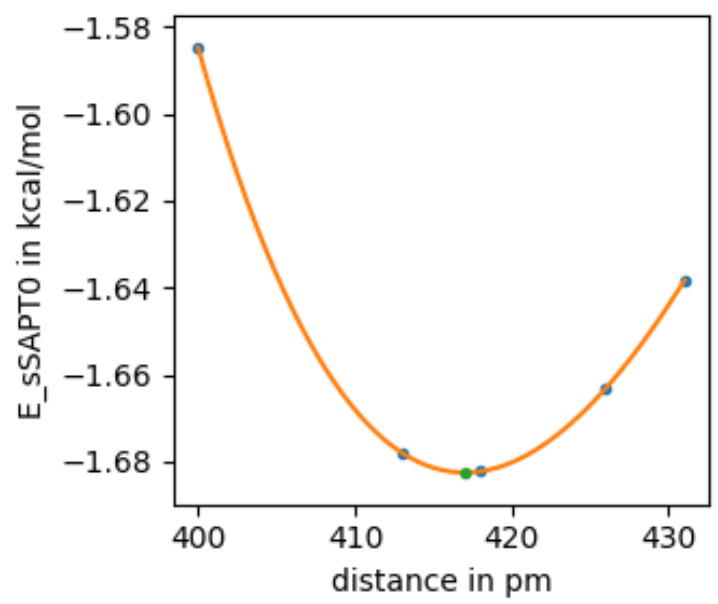

45

4AdPh_pi_2Furane_pi_300_N1-417

$\begin{array}{llll}\mathrm{H} & -5.40699838 & -0.07046942 & 1.99997493 \\ \mathrm{C} & -4.32228838 & -0.03889342 & 1.99999093\end{array}$

$\begin{array}{llll}\text { C } & -4.32228838 & -0.03889342 & 1.99999093\end{array}$

$\begin{array}{llll}\text { C } & -3.58278938 & -1.21969542 & 1.99999893\end{array}$

$\begin{array}{llll}\text { C } & -3.65363938 & 1.17869058 & 1.99998993\end{array}$

$\begin{array}{llll}\text { C } & -2.19430138 & -1.17393642 & 2.00001793\end{array}$

H $-4.08913238-2.17997242 \quad 1.99998893$

$\begin{array}{llll}\text { C } & -2.25972238 & 1.21897558 & 2.00000593\end{array}$

$\begin{array}{llll}\mathrm{H} & -4.21490638 & 2.10797258 & 1.99997293\end{array}$

C $-1.50186838 \quad 0.04520558 \quad 2.00003493$

H $-1.63822938-2.10748042 \quad 2.00001193$

$\begin{array}{llll}\mathrm{H} & -1.76867538 & 2.18541758 & 1.99999893\end{array}$

$\begin{array}{llll}\text { C } & 0.02431362 & 0.04571458 & 2.00001693\end{array}$

$\begin{array}{llll}\text { C } & 0.55298362 & -0.68866242 & 0.74425493\end{array}$

$\begin{array}{llll}\text { C } & 0.61561762 & 1.46926258 & 1.99962893\end{array}$

$\begin{array}{llll}\text { C } & 0.55303662 & -0.68799642 & 3.25614693\end{array}$

$\begin{array}{llll}\mathrm{H} & 0.18630062 & -0.17353542 & -0.15295807\end{array}$

H $\quad 0.15106062 \quad-1.70858842 \quad 0.71368593$

$\begin{array}{llll}\text { C } & 2.08800762 & -0.73790042 & 0.74462093\end{array}$

$\begin{array}{llll}\mathrm{H} & 0.26266762 & 2.01601858 & 2.88396393\end{array}$

$\begin{array}{llll}\mathrm{H} & 0.26263962 & 2.01555058 & 1.11501593\end{array}$

C $\quad 2.15101662 \quad 1.42858458 \quad 1.99960693$

$\mathrm{H} \quad 0.15112862 \quad-1.70791042 \quad 3.28727393$

$\begin{array}{llll}\mathrm{H} & 0.18638262 & -0.17239742 & 4.15310093\end{array}$

C $\quad 2.08805962 \quad-0.73722842 \quad 3.25574793$

$\mathrm{H} \quad 2.43133662 \quad-1.26768242 \quad-0.15157507$

$\begin{array}{llll}\text { C } & 2.64484062 & 0.69255658 & 0.74587593\end{array}$

$\begin{array}{llll}\text { C } & 2.57473862 & -1.47737642 & 2.00037193\end{array}$

$\begin{array}{llll}\mathrm{H} & 2.53496562 & 2.45530658 & 1.99932393\end{array}$

C $\quad 2.64489162 \quad 0.69322758 \quad 3.25371093$

$\mathrm{H} \quad 2.43142262-1.26653542 \quad 4.15221193$

H $\quad 2.31641362 \quad 1.22422858 \quad-0.15601007$

$\begin{array}{llll}\mathrm{H} & 3.74172062 & 0.67084458 & 0.73058993\end{array}$

$\mathrm{H} \quad 2.19407462 \quad-2.50668142 \quad 2.00065493$

H $\quad 3.67039062 \quad-1.53568942 \quad 2.00036393$

H $3.74177262 \quad 0.67152258 \quad 3.26896293$

$\begin{array}{llll}\mathrm{H} & 2.31650162 & 1.22537858 & 4.15532693\end{array}$

C $-1.84190990 \quad-0.40872971-2.17017930$

$\begin{array}{llll}\text { C } & -3.52417111 & 0.97461115 & -2.16971047\end{array}$
$\begin{array}{rrrr}\mathrm{C} & -2.95748412 & -1.18016006 & -2.17021604 \\ \mathrm{O} & -2.17151143 & 0.90468628 & -2.16974642 \\ \mathrm{C} & -4.06516958 & -0.26909908 & -2.17010070 \\ \mathrm{H} & -0.78654676 & -0.62979128 & -2.17030834 \\ \mathrm{H} & -3.94415227 & 1.96773507 & -2.16942375 \\ \mathrm{H} & -2.99315540 & -2.25869055 & -2.17041379 \\ \mathrm{H} & -5.11652407 & -0.51227063 & -2.17020104\end{array}$

4AdPh_pi_2Furane_pi_300_N2
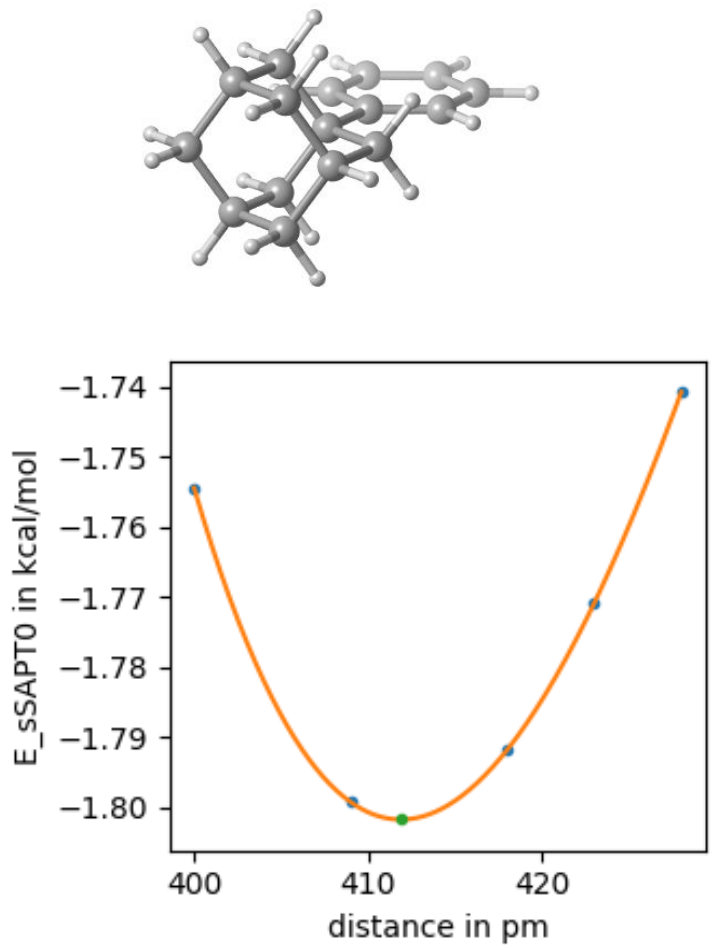

45

4AdPh_pi_2Furane_pi_300_N2-411

H $\quad-5.40699838-0.07046942 \quad 1.99997493$

$\begin{array}{llll}\text { C } & -4.32228838 & -0.03889342 & 1.99999093\end{array}$

$\begin{array}{llll}\text { C } & -3.58278938 & -1.21969542 & 1.99999893\end{array}$

$\begin{array}{llll}\text { C } & -3.65363938 & 1.17869058 & 1.99998993\end{array}$

$\begin{array}{llll}\text { C } & -2.19430138 & -1.17393642 & 2.00001793\end{array}$

H $\quad-4.08913238-2.17997242 \quad 1.99998893$

C $\quad-2.25972238 \quad 1.21897558 \quad 2.00000593$

$\begin{array}{llll}\mathrm{H} & -4.21490638 & 2.10797258 & 1.99997293\end{array}$

$\begin{array}{llll}\text { C } & -1.50186838 & 0.04520558 & 2.00003493\end{array}$

$\mathrm{H}-1.63822938-2.10748042 \quad 2.00001193$

$\begin{array}{llll}\mathrm{H} & -1.76867538 & 2.18541758 & 1.99999893\end{array}$

$\begin{array}{llll}\text { C } & 0.02431362 & 0.04571458 & 2.00001693\end{array}$

$\begin{array}{llll}\text { C } & 0.55298362 & -0.68866242 & 0.74425493\end{array}$

$\begin{array}{llll}\text { C } & 0.61561762 & 1.46926258 & 1.99962893\end{array}$

C $\quad 0.55303662 \quad-0.68799642 \quad 3.25614693$

H $\quad 0.18630062 \quad-0.17353542 \quad-0.15295807$ 


$\begin{array}{lrrr}\mathrm{H} & 0.15106062 & -1.70858842 & 0.71368593 \\ \mathrm{C} & 2.08800762 & -0.73790042 & 0.74462093 \\ \mathrm{H} & 0.26266762 & 2.01601858 & 2.88396393 \\ \mathrm{H} & 0.26263962 & 2.01555058 & 1.11501593 \\ \mathrm{C} & 2.15101662 & 1.42858458 & 1.99960693 \\ \mathrm{H} & 0.15112862 & -1.70791042 & 3.28727393 \\ \mathrm{H} & 0.18638262 & -0.17239742 & 4.15310093 \\ \mathrm{C} & 2.08805962 & -0.73722842 & 3.25574793 \\ \mathrm{H} & 2.43133662 & -1.26768242 & -0.15157507 \\ \mathrm{C} & 2.64484062 & 0.69255658 & 0.74587593 \\ \mathrm{C} & 2.57473862 & -1.47737642 & 2.00037193 \\ \mathrm{H} & 2.53496562 & 2.45530658 & 1.99932393 \\ \mathrm{C} & 2.64489162 & 0.69322758 & 3.25371093 \\ \mathrm{H} & 2.43142262 & -1.26653542 & 4.15221193 \\ \mathrm{H} & 2.31641362 & 1.22422858 & -0.15601007 \\ \mathrm{H} & 3.74172062 & 0.67084458 & 0.73058993 \\ \mathrm{H} & 2.19407462 & -2.50668142 & 2.00065493 \\ \mathrm{H} & 3.67039062 & -1.53568942 & 2.00036393 \\ \mathrm{H} & 3.74177262 & 0.67152258 & 3.26896293 \\ \mathrm{H} & 2.31650162 & 1.22537858 & 4.15532693 \\ \mathrm{C} & -3.52405299 & 0.97440975 & -2.11868703 \\ \mathrm{C} & -3.00022158 & -1.13964687 & -2.11912877 \\ \mathrm{C} & -2.16854707 & 0.92735350 & -2.11851265 \\ \mathrm{O} & -4.04367936 & -0.27606508 & -2.11893558 \\ \mathrm{C} & -1.82374928 & -0.46480793 & -2.11899697 \\ \mathrm{H} & -4.23877578 & 1.78177022 & -2.11855692 \\ \mathrm{H} & -3.25613755 & -2.18711349 & -2.11938198 \\ \mathrm{H} & -1.49608994 & 1.77133325 & -2.11819507 \\ \mathrm{H} & -0.83511412 & -0.89733442 & -2.11913185\end{array}$

4AdPh_pi_2Furane_pi_60_N1

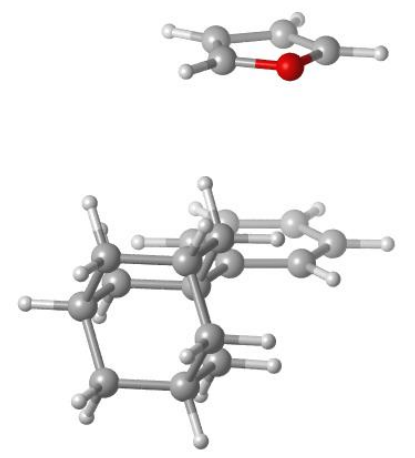

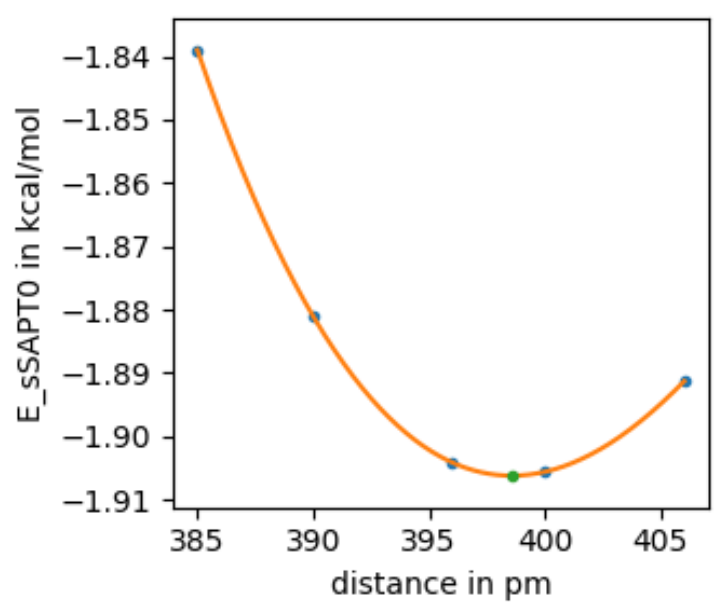

45

4AdPh_pi_2Furane_pi_60_N1-398

H $\quad-5.40699838-0.07046942 \quad 1.99997493$

$\begin{array}{llll}\text { C } & -4.32228838 & -0.03889342 & 1.99999093\end{array}$

$\begin{array}{llll}\text { C } & -3.58278938 & -1.21969542 & 1.99999893\end{array}$

$\begin{array}{llll}\text { C } & -3.65363938 & 1.17869058 & 1.99998993\end{array}$

$\begin{array}{llll}\text { C } & -2.19430138 & -1.17393642 & 2.00001793\end{array}$

$\begin{array}{llll}\mathrm{H} & -4.08913238 & -2.17997242 & 1.99998893\end{array}$

$\begin{array}{llll}\text { C } & -2.25972238 & 1.21897558 & 2.00000593\end{array}$

$\begin{array}{llll}\mathrm{H} & -4.21490638 & 2.10797258 & 1.99997293\end{array}$

$\begin{array}{llll}\text { C } & -1.50186838 & 0.04520558 & 2.00003493\end{array}$

$\begin{array}{llll}\mathrm{H} & -1.63822938 & -2.10748042 & 2.00001193\end{array}$

$\begin{array}{llll}\mathrm{H} & -1.76867538 & 2.18541758 & 1.99999893\end{array}$

$\begin{array}{llll}\text { C } & 0.02431362 & 0.04571458 & 2.00001693\end{array}$

$\begin{array}{llll}\text { C } & 0.55298362 & -0.68866242 & 0.74425493\end{array}$

$\begin{array}{llll}\text { C } & 0.61561762 & 1.46926258 & 1.99962893\end{array}$

C $\quad 0.55303662 \quad-0.68799642 \quad 3.25614693$

H $\quad 0.18630062-0.17353542 \quad-0.15295807$

$\begin{array}{llll}\mathrm{H} & 0.15106062 & -1.70858842 & 0.71368593\end{array}$

$\begin{array}{llll}\text { C } & 2.08800762 & -0.73790042 & 0.74462093\end{array}$

$\begin{array}{llll}\mathrm{H} & 0.26266762 & 2.01601858 & 2.88396393\end{array}$

$\begin{array}{llll}\mathrm{H} & 0.26263962 & 2.01555058 & 1.11501593\end{array}$

C $\quad 2.15101662 \quad 1.42858458 \quad 1.99960693$

$\begin{array}{llll}\mathrm{H} & 0.15112862 & -1.70791042 & 3.28727393\end{array}$

H $\quad 0.18638262 \quad-0.17239742 \quad 4.15310093$

C $\quad 2.08805962 \quad-0.73722842 \quad 3.25574793$

$\begin{array}{llll}\mathrm{H} & 2.43133662 & -1.26768242 & -0.15157507\end{array}$

$\begin{array}{llll}\text { C } & 2.64484062 & 0.69255658 & 0.74587593\end{array}$

C $\quad 2.57473862 \quad-1.47737642 \quad 2.00037193$

$\begin{array}{llll}\mathrm{H} & 2.53496562 & 2.45530658 & 1.99932393\end{array}$

C $\quad 2.64489162 \quad 0.69322758 \quad 3.25371093$

H $\quad 2.43142262-1.26653542 \quad 4.15221193$

$\mathrm{H} \quad 2.31641362 \quad 1.22422858 \quad-0.15601007$

$\begin{array}{llll}\mathrm{H} & 3.74172062 & 0.67084458 & 0.73058993\end{array}$

$\mathrm{H} \quad 2.19407462 \quad-2.50668142 \quad 2.00065493$

$\begin{array}{llll}\mathrm{H} & 3.67039062 & -1.53568942 & 2.00036393\end{array}$

$\begin{array}{llll}\mathrm{H} & 3.74177262 & 0.67152258 & 3.26896293\end{array}$

$\begin{array}{llll}\mathrm{H} & 2.31650162 & 1.22537858 & 4.15532693\end{array}$

C $\quad-3.46366853-1.00151005 \quad-1.98592938$

$\begin{array}{llll}\text { C } & -1.86892602 & 0.48187492 & -1.98540882\end{array}$ 


$\begin{array}{cccc}\mathrm{C} & -4.07991455 & 0.20673298 & -1.98543725 \\ \mathrm{O} & -2.11808890 & -0.84947605 & -1.98579163 \\ \mathrm{C} & -3.02965791 & 1.18344182 & -1.98528791 \\ \mathrm{H} & -3.82309552 & -2.01810816 & -1.98628028 \\ \mathrm{H} & -0.82877695 & 0.76607427 & -1.98529888 \\ \mathrm{H} & -5.14412513 & 0.38549630 & -1.98530946 \\ \mathrm{H} & -3.13087493 & 2.25779452 & -1.98503152\end{array}$

4AdPh_pi_2Furane_pi_60_N2
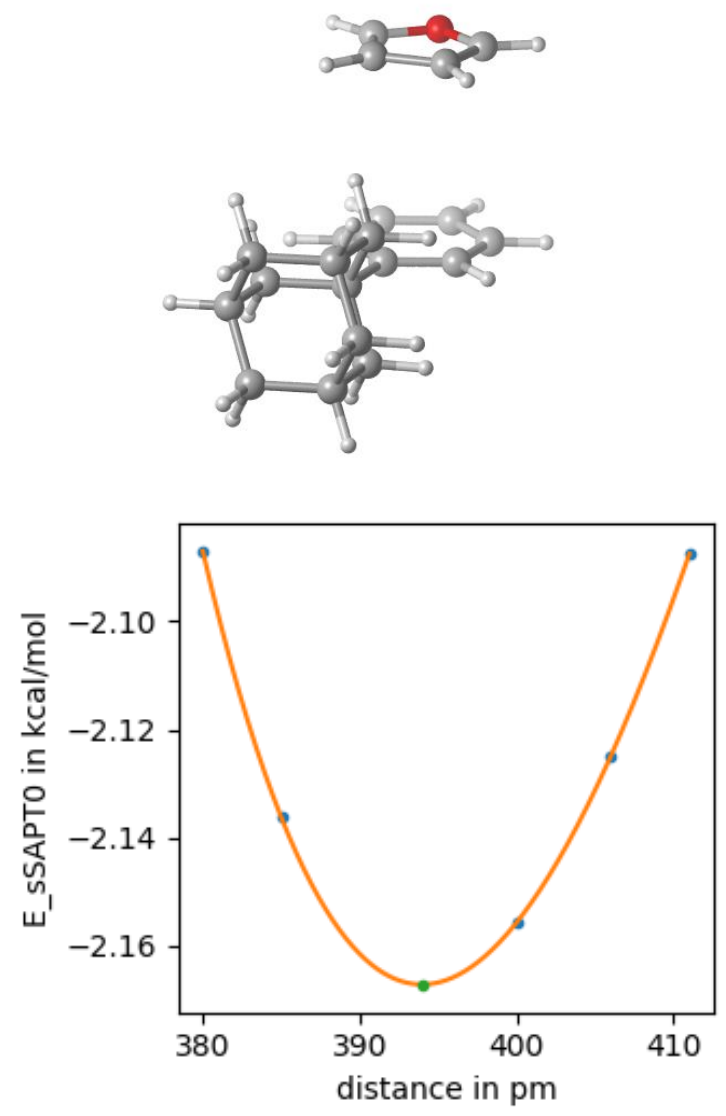

45

4AdPh_pi_2Furane_pi_60_N2-393
H $\quad-5.40699838-0.07046942$
C $-4.32228838-0.03889342$
C $\quad-3.58278938-1.21969542$
C $-3.65363938 \quad 1.17869058$
C $-2.19430138-1.17393642$
H $\quad-4.08913238-2.17997242$
C $\quad-2.25972238 \quad 1.21897558$
H -4.21490638
2.10797258
0.04520558
C -1.50186838
H -1.63822938
$-2.10748042$
H -1.76867538
2.18541758
C 0.02431362
0.04571458
C $\quad 0.55298362 \quad-0.68866242$
C $\quad 0.61561762 \quad 1.46926258$
C $0.55303662-0.68799642$
H $\quad 0.18630062-0.17353542$
1.99997493
1.99999093
1.99999893
1.99998993
2.00001793
1.99998893
2.00000593
1.99997293
2.00003493
2.00001193
1.99999893
2.00001693
0.74425493
1.99962893
3.25614693
$-0.15295807$

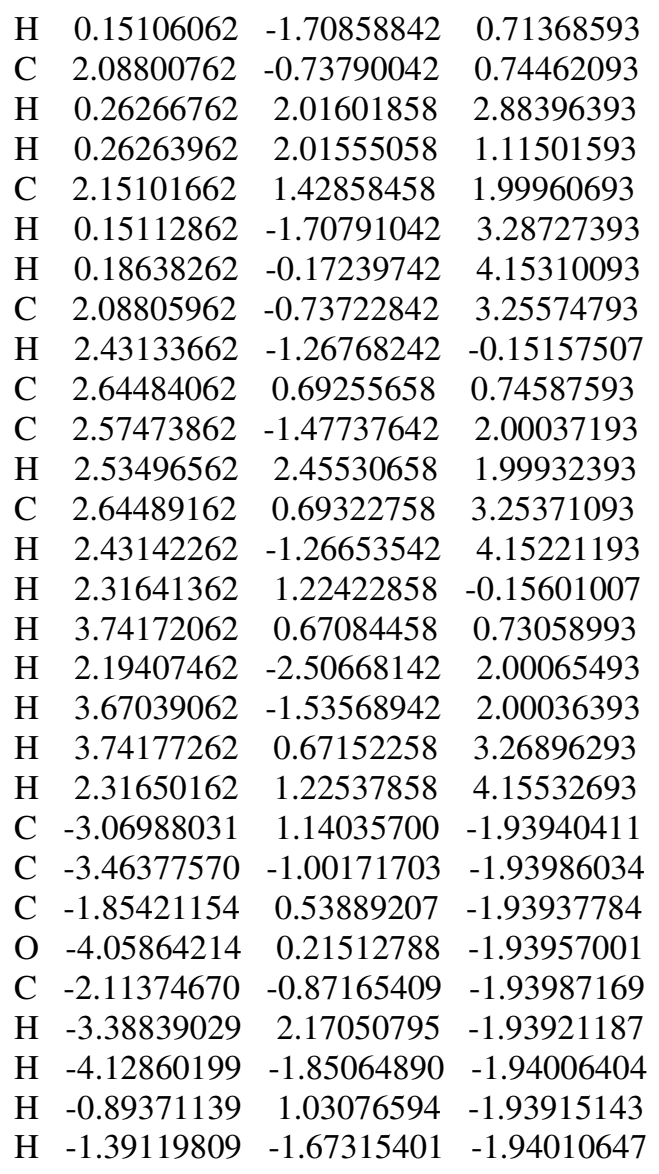

4AdPh_pi_2MePh_pi_60_2
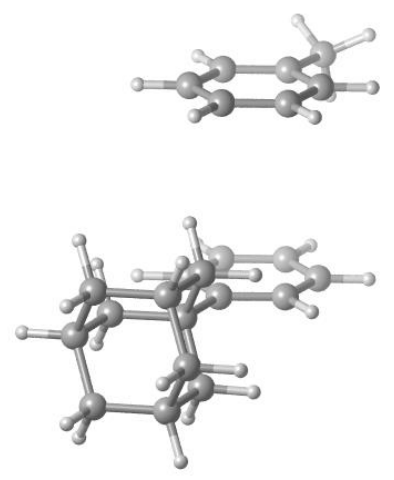


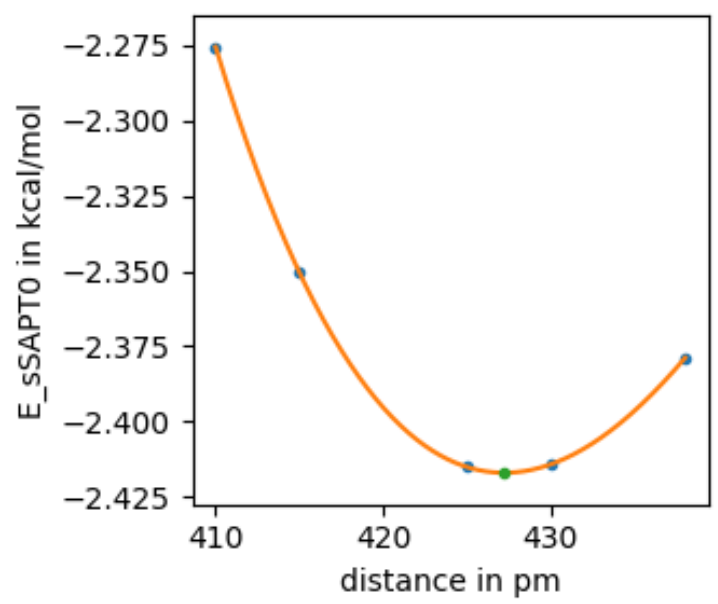

51

4AdPh_pi_2MePh_pi_60_2-427

H $\quad-5.40698300 \quad-0.06993900 \quad-0.00002500$

$\begin{array}{llll}\text { C } & -4.32227300 & -0.03836300 & -0.00000900\end{array}$

$\begin{array}{llll}\text { C } & -3.58277400 & -1.21916500 & -0.00000100\end{array}$

C $\quad-3.65362400 \quad 1.17922100 \quad-0.00001000$

$\begin{array}{llll}\text { C } & -2.19428600 & -1.17340600 & 0.00001800\end{array}$

$\begin{array}{llll}\mathrm{H} & -4.08911700 & -2.17944200 & -0.00001100\end{array}$

$\begin{array}{llll}\text { C } & -2.25970700 & 1.21950600 & 0.00000600\end{array}$

$\mathrm{H} \quad-4.21489100 \quad 2.10850300 \quad-0.00002700$

$\begin{array}{llll}\text { C } & -1.50185300 & 0.04573600 & 0.00003500\end{array}$

$\begin{array}{llll}\mathrm{H} & -1.63821400 & -2.10695000 & 0.00001200\end{array}$

$\begin{array}{llll}\mathrm{H} & -1.76866000 & 2.18594800 & -0.00000100\end{array}$

$\begin{array}{llll}\text { C } & 0.02432900 & 0.04624500 & 0.00001700\end{array}$

$\begin{array}{llll}\text { C } & 0.55299900 & -0.68813200 & -1.25574500\end{array}$

$\begin{array}{llll}\text { C } & 0.61563300 & 1.46979300 & -0.00037100\end{array}$

$\begin{array}{llll}\text { C } & 0.55305200 & -0.68746600 & 1.25614700\end{array}$

$\begin{array}{llll}\mathrm{H} & 0.18631600 & -0.17300500 & -2.15295800\end{array}$

$\begin{array}{llll}\mathrm{H} & 0.15107600 & -1.70805800 & -1.28631400\end{array}$

C $\quad 2.08802300 \quad-0.73737000 \quad-1.25537900$

$\begin{array}{llll}\mathrm{H} & 0.26268300 & 2.01654900 & 0.88396400\end{array}$

$\mathrm{H} \quad 0.26265500 \quad 2.01608100 \quad-0.88498400$

C $\quad 2.15103200 \quad 1.42911500 \quad-0.00039300$

$\mathrm{H} \quad 0.15114400 \quad-1.70738000 \quad 1.28727400$

H $\quad 0.18639800 \quad-0.17186700 \quad 2.15310100$

C $\quad 2.08807500 \quad-0.73669800 \quad 1.25574800$

H $\quad 2.43135200 \quad-1.26715200 \quad-2.15157500$

$\begin{array}{llll}\text { C } & 2.64485600 & 0.69308700 & -1.25412400\end{array}$

$\begin{array}{llll}\text { C } & 2.57475400 & -1.47684600 & 0.00037200\end{array}$

$\begin{array}{llll}\mathrm{H} & 2.53498100 & 2.45583700 & -0.00067600\end{array}$

C $\quad 2.64490700 \quad 0.69375800 \quad 1.25371100$

$\mathrm{H} \quad 2.43143800 \quad-1.26600500 \quad 2.15221200$

H $\quad 2.31642900 \quad 1.22475900 \quad-2.15601000$

$\mathrm{H} \quad 3.74173600 \quad 0.67137500 \quad-1.26941000$

$\mathrm{H} \quad 2.19409000 \quad-2.50615100 \quad 0.00065500$

H $\quad 3.67040600 \quad-1.53515900 \quad 0.00036400$

H $\quad 3.74178800 \quad 0.67205300 \quad 1.26896300$

$\begin{array}{llll}\mathrm{H} & 2.31651700 & 1.22590900 & 2.15532700\end{array}$

C $\quad-1.50825451 \quad 0.04365143 \quad-4.27205376$

$\begin{array}{llll}\text { C } & -2.24284944 & 1.22660335 & -4.27264815\end{array}$
C $-2.17623276-1.17789612-4.27322676$

$\begin{array}{llll}\text { C } & -3.63396089 & 1.18513451 & -4.27374723\end{array}$

C $-3.56775202-1.21338588 \quad-4.27423381$

$\begin{array}{llll}\text { C } & -4.31589634 & -0.03401495 & -4.27209595\end{array}$

$\mathrm{H} \quad-0.42310186 \quad 0.07371184 \quad-4.27489541$

$\mathrm{H}-1.73173344 \quad 2.18441354-4.27645552$

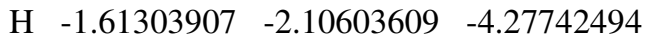

H $\quad-4.20229816 \quad 2.11185623 \quad-4.27769711$

H $\quad-4.08392591 \quad-2.17009907 \quad-4.27852106$

C $\quad-5.82295162-0.07482217 \quad-4.24042181$

$\mathrm{H} \quad-6.19271300 \quad-0.05971389-3.20995864$

$\begin{array}{llll}\mathrm{H} & -6.25308314 & 0.78660855 & -4.75686889\end{array}$

H $\quad-6.20441196 \quad-0.98240737 \quad-4.71407784$

4AdPh_pi_2Nap_pi1_300
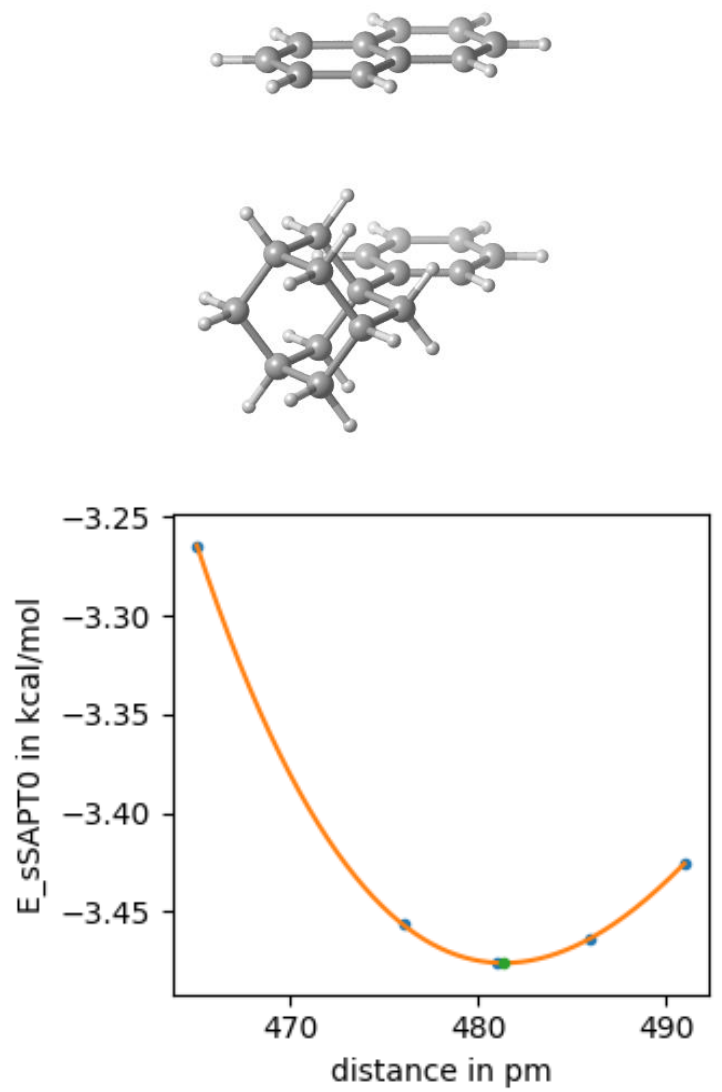

54

4AdPh_pi_2Nap_pi1_300-481

$\begin{array}{llll}\text { H } & -5.40699838 & -0.07046942 & 1.99997493\end{array}$

$\begin{array}{llll}\text { C } & -4.32228838 & -0.03889342 & 1.99999093\end{array}$

$\begin{array}{llll}\text { C } & -3.58278938 & -1.21969542 & 1.99999893\end{array}$

$\begin{array}{llll}\text { C } & -3.65363938 & 1.17869058 & 1.99998993\end{array}$

$\begin{array}{llll}\text { C } & -2.19430138 & -1.17393642 & 2.00001793\end{array}$

$\begin{array}{llll}\mathrm{H} & -4.08913238 & -2.17997242 & 1.99998893\end{array}$

$\begin{array}{llll}\text { C } & -2.25972238 & 1.21897558 & 2.00000593\end{array}$

$\begin{array}{llll}\mathrm{H} & -4.21490638 & 2.10797258 & 1.99997293\end{array}$

$\begin{array}{llll}\text { C } & -1.50186838 & 0.04520558 & 2.00003493\end{array}$

$\begin{array}{llll}\mathrm{H} & -1.63822938 & -2.10748042 & 2.00001193\end{array}$ 


$\begin{array}{lrrr}\mathrm{H} & -1.76867538 & 2.18541758 & 1.99999893 \\ \mathrm{C} & 0.02431362 & 0.04571458 & 2.00001693 \\ \mathrm{C} & 0.55298362 & -0.68866242 & 0.74425493 \\ \mathrm{C} & 0.61561762 & 1.46926258 & 1.99962893 \\ \mathrm{C} & 0.55303662 & -0.68799642 & 3.25614693 \\ \mathrm{H} & 0.18630062 & -0.17353542 & -0.15295807 \\ \mathrm{H} & 0.15106062 & -1.70858842 & 0.71368593 \\ \mathrm{C} & 2.08800762 & -0.73790042 & 0.74462093 \\ \mathrm{H} & 0.26266762 & 2.01601858 & 2.88396393 \\ \mathrm{H} & 0.26263962 & 2.01555058 & 1.11501593 \\ \mathrm{C} & 2.15101662 & 1.42858458 & 1.99960693 \\ \mathrm{H} & 0.15112862 & -1.70791042 & 3.28727393 \\ \mathrm{H} & 0.18638262 & -0.17239742 & 4.15310093 \\ \mathrm{C} & 2.08805962 & -0.73722842 & 3.25574793 \\ \mathrm{H} & 2.43133662 & -1.26768242 & -0.15157507 \\ \mathrm{C} & 2.64484062 & 0.69255658 & 0.74587593 \\ \mathrm{C} & 2.57473862 & -1.47737642 & 2.00037193 \\ \mathrm{H} & 2.53496562 & 2.45530658 & 1.99932393 \\ \mathrm{C} & 2.64489162 & 0.69322758 & 3.25371093 \\ \mathrm{H} & 2.43142262 & -1.26653542 & 4.15221193 \\ \mathrm{H} & 2.31641362 & 1.22422858 & -0.15601007 \\ \mathrm{H} & 3.74172062 & 0.67084458 & 0.73058993 \\ \mathrm{H} & 2.19407462 & -2.50668142 & 2.00065493 \\ \mathrm{H} & 3.67039062 & -1.53568942 & 2.00036393 \\ \mathrm{H} & 3.74177262 & 0.67152258 & 3.26896293 \\ \mathrm{H} & 2.31650162 & 1.22537858 & 4.15532693 \\ \mathrm{H} & -4.23345941 & 2.11558708 & -2.81320107 \\ \mathrm{C} & -3.65075775 & 1.20014073 & -2.81335066 \\ \mathrm{C} & -4.31453137 & -0.05130960 & -2.82183498 \\ \mathrm{C} & -2.28142715 & 1.25563362 & -2.80536903 \\ \mathrm{H} & -5.39908431 & -0.08200585 & -2.82810892 \\ \mathrm{C} & -3.59316037 & -1.21653952 & -2.82213108 \\ \mathrm{C} & -1.50642970 & 0.06558040 & -2.80545209 \\ \mathrm{H} & -1.76677564 & 2.21259468 & -2.79884405 \\ \mathrm{C} & -2.17333384 & -1.19127158 & -2.81397354 \\ \mathrm{H} & -4.09721059 & -2.17916783 & -2.82862520 \\ \mathrm{C} & -0.08652423 & 0.09083927 & -2.79729114 \\ \mathrm{C} & -1.39832655 & -2.38128701 & -2.81405341 \\ \mathrm{C} & 0.63508332 & -1.07430114 & -2.79757956 \\ \mathrm{H} & 0.41739739 & 1.05351322 & -2.79080057 \\ \mathrm{C} & -0.02898103 & -2.32571265 & -2.80606538 \\ \mathrm{H} & -1.91298647 & -3.33826397 & -2.82058149 \\ \mathrm{H} & 1.71977376 & -1.04363375 & -2.79130195 \\ \mathrm{H} & 0.55362770 & -3.24118898 & -2.80620660\end{array}$

4AdPh_pi_2Nap_pi1_60
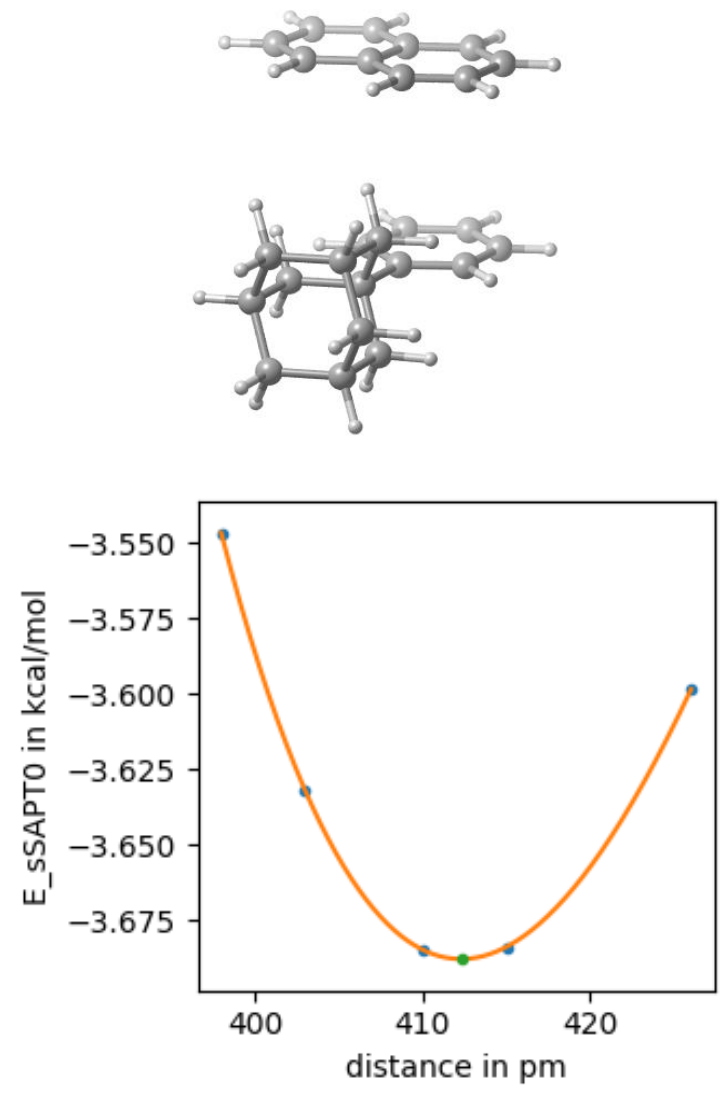

54

4AdPh_pi_2Nap_pi1_60-412

H $\quad-5.40699838-0.07046942 \quad 1.99997493$

$\begin{array}{llll}\text { C } & -4.32228838 & -0.03889342 & 1.99999093\end{array}$

$\begin{array}{llll}\text { C } & -3.58278938 & -1.21969542 & 1.99999893\end{array}$

$\begin{array}{llll}\text { C } & -3.65363938 & 1.17869058 & 1.99998993\end{array}$

$\begin{array}{llll}\text { C } & -2.19430138 & -1.17393642 & 2.00001793\end{array}$

H $\quad-4.08913238-2.17997242 \quad 1.99998893$

$\begin{array}{llll}\text { C } & -2.25972238 & 1.21897558 & 2.00000593\end{array}$

$\begin{array}{llll}\mathrm{H} & -4.21490638 & 2.10797258 & 1.99997293\end{array}$

$\begin{array}{llll}\text { C } & -1.50186838 & 0.04520558 & 2.00003493\end{array}$

$\begin{array}{llll}\mathrm{H} & -1.63822938 & -2.10748042 & 2.00001193\end{array}$

$\begin{array}{llll}\mathrm{H} & -1.76867538 & 2.18541758 & 1.99999893\end{array}$

$\begin{array}{llll}\text { C } & 0.02431362 & 0.04571458 & 2.00001693\end{array}$

$\begin{array}{llll}\text { C } & 0.55298362 & -0.68866242 & 0.74425493\end{array}$

$\begin{array}{llll}\text { C } & 0.61561762 & 1.46926258 & 1.99962893\end{array}$

C $\quad 0.55303662 \quad-0.68799642 \quad 3.25614693$

H $\quad 0.18630062-0.17353542-0.15295807$

$\begin{array}{llll}\mathrm{H} & 0.15106062 & -1.70858842 & 0.71368593\end{array}$

$\begin{array}{llll}\text { C } & 2.08800762 & -0.73790042 & 0.74462093\end{array}$

$\begin{array}{llll}\mathrm{H} & 0.26266762 & 2.01601858 & 2.88396393\end{array}$

$\begin{array}{llll}\mathrm{H} & 0.26263962 & 2.01555058 & 1.11501593\end{array}$

C $\quad 2.15101662 \quad 1.42858458 \quad 1.99960693$

H $\quad 0.15112862 \quad-1.70791042 \quad 3.28727393$

H $\quad 0.18638262 \quad-0.17239742 \quad 4.15310093$

C $\quad 2.08805962 \quad-0.73722842 \quad 3.25574793$ 


$\begin{array}{lrrr}\mathrm{H} & 2.43133662 & -1.26768242 & -0.15157507 \\ \mathrm{C} & 2.64484062 & 0.69255658 & 0.74587593 \\ \mathrm{C} & 2.57473862 & -1.47737642 & 2.00037193 \\ \mathrm{H} & 2.53496562 & 2.45530658 & 1.99932393 \\ \mathrm{C} & 2.64489162 & 0.69322758 & 3.25371093 \\ \mathrm{H} & 2.43142262 & -1.26653542 & 4.15221193 \\ \mathrm{H} & 2.31641362 & 1.22422858 & -0.15601007 \\ \mathrm{H} & 3.74172062 & 0.67084458 & 0.73058993 \\ \mathrm{H} & 2.19407462 & -2.50668142 & 2.00065493 \\ \mathrm{H} & 3.67039062 & -1.53568942 & 2.00036393 \\ \mathrm{H} & 3.74177262 & 0.67152258 & 3.26896293 \\ \mathrm{H} & 2.31650162 & 1.22537858 & 4.15532693 \\ \mathrm{H} & -4.07965591 & -2.19570655 & -2.12359685 \\ \mathrm{C} & -3.57820695 & -1.23334899 & -2.12324906 \\ \mathrm{C} & -2.16253036 & -1.18246544 & -2.13137200 \\ \mathrm{C} & -4.31093242 & -0.07522350 & -2.11499116 \\ \mathrm{H} & -1.59366864 & -2.10636522 & -2.13787010 \\ \mathrm{C} & -1.51409711 & 0.02487511 & -2.13103942 \\ \mathrm{C} & -3.66781485 & 1.19097048 & -2.11442382 \\ \mathrm{H} & -5.39701231 & -0.10800522 & -2.10874098 \\ \mathrm{C} & -2.24589502 & 1.24184375 & -2.12258238 \\ \mathrm{H} & -0.42840989 & 0.06967142 & -2.13725415 \\ \mathrm{C} & -4.39964437 & 2.40801202 & -2.10596334 \\ \mathrm{C} & -1.60281509 & 2.50802736 & -2.12201186 \\ \mathrm{C} & -3.75140693 & 3.61551266 & -2.10562308 \\ \mathrm{H} & -5.48530681 & 2.36308152 & -2.09975222 \\ \mathrm{C} & -2.33561859 & 3.66612503 & -2.11374759 \\ \mathrm{H} & -0.51671723 & 2.54080974 & -2.12826513 \\ \mathrm{H} & -4.32031240 & 4.53954596 & -2.09912125 \\ \mathrm{H} & -1.83409719 & 4.62841710 & -2.11339144\end{array}$

4AdPh_pi_2Nap_pi2_300

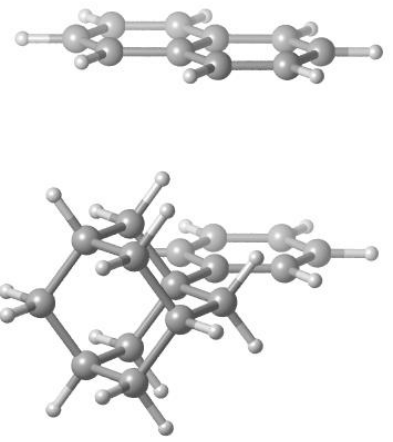

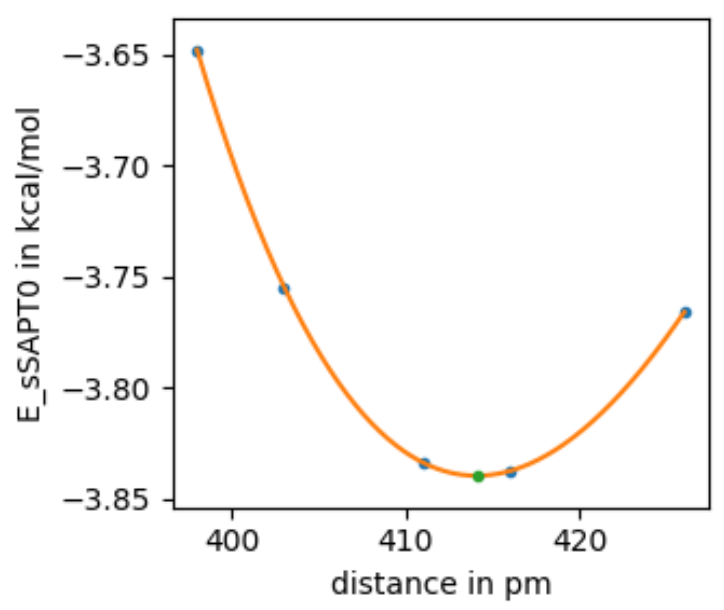

54

4AdPh_pi_2Nap_pi2_300-414

$\begin{array}{llll}\mathrm{H} & -5.40699838 & -0.07046942 & 1.99997493\end{array}$

$\begin{array}{llll}\text { C } & -4.32228838 & -0.03889342 & 1.99999093\end{array}$

$\begin{array}{llll}\text { C } & -3.58278938 & -1.21969542 & 1.99999893\end{array}$

$\begin{array}{llll}\text { C } & -3.65363938 & 1.17869058 & 1.99998993\end{array}$

$\begin{array}{llll}\text { C } & -2.19430138 & -1.17393642 & 2.00001793\end{array}$

$\begin{array}{llll}\mathrm{H} & -4.08913238 & -2.17997242 & 1.99998893\end{array}$

$\begin{array}{llll}\text { C } & -2.25972238 & 1.21897558 & 2.00000593\end{array}$

$\begin{array}{llll}\mathrm{H} & -4.21490638 & 2.10797258 & 1.99997293\end{array}$

$\begin{array}{llll}\text { C } & -1.50186838 & 0.04520558 & 2.00003493\end{array}$

$\begin{array}{llll}\mathrm{H} & -1.63822938 & -2.10748042 & 2.00001193\end{array}$

$\begin{array}{llll}\mathrm{H} & -1.76867538 & 2.18541758 & 1.99999893\end{array}$

$\begin{array}{llll}\text { C } & 0.02431362 & 0.04571458 & 2.00001693\end{array}$

$\begin{array}{llll}\text { C } & 0.55298362 & -0.68866242 & 0.74425493\end{array}$

$\begin{array}{llll}\text { C } & 0.61561762 & 1.46926258 & 1.99962893\end{array}$

C $\quad 0.55303662 \quad-0.68799642 \quad 3.25614693$

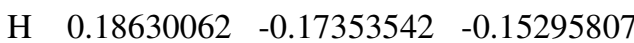

$\begin{array}{llll}\mathrm{H} & 0.15106062 & -1.70858842 & 0.71368593\end{array}$

$\begin{array}{llll}\text { C } & 2.08800762 & -0.73790042 & 0.74462093\end{array}$

$\begin{array}{llll}\mathrm{H} & 0.26266762 & 2.01601858 & 2.88396393\end{array}$

$\begin{array}{llll}\mathrm{H} & 0.26263962 & 2.01555058 & 1.11501593\end{array}$

C $\quad 2.15101662 \quad 1.42858458 \quad 1.99960693$

$\begin{array}{llll}\mathrm{H} & 0.15112862 & -1.70791042 & 3.28727393\end{array}$

H $\quad 0.18638262 \quad-0.17239742 \quad 4.15310093$

C $\quad 2.08805962 \quad-0.73722842 \quad 3.25574793$

$\begin{array}{llll}\mathrm{H} & 2.43133662 & -1.26768242 & -0.15157507\end{array}$

$\begin{array}{llll}\text { C } & 2.64484062 & 0.69255658 & 0.74587593\end{array}$

$\begin{array}{llll}\text { C } & 2.57473862 & -1.47737642 & 2.00037193\end{array}$

$\begin{array}{llll}\mathrm{H} & 2.53496562 & 2.45530658 & 1.99932393\end{array}$

$\begin{array}{llll}\text { C } & 2.64489162 & 0.69322758 & 3.25371093\end{array}$

H $\quad 2.43142262 \quad-1.26653542 \quad 4.15221193$

$\mathrm{H} \quad 2.31641362 \quad 1.22422858 \quad-0.15601007$

$\begin{array}{llll}\mathrm{H} & 3.74172062 & 0.67084458 & 0.73058993\end{array}$

$\mathrm{H} \quad 2.19407462 \quad-2.50668142 \quad 2.00065493$

H $\quad 3.67039062 \quad-1.53568942 \quad 2.00036393$

$\begin{array}{llll}\mathrm{H} & 3.74177262 & 0.67152258 & 3.26896293\end{array}$

$\begin{array}{llll}\mathrm{H} & 2.31650162 & 1.22537858 & 4.15532693\end{array}$

H $\quad-1.71448564 \quad 2.18804680 \quad-2.14011765$

C $\quad-2.22904047 \quad 1.23263246-2.14028628$ 


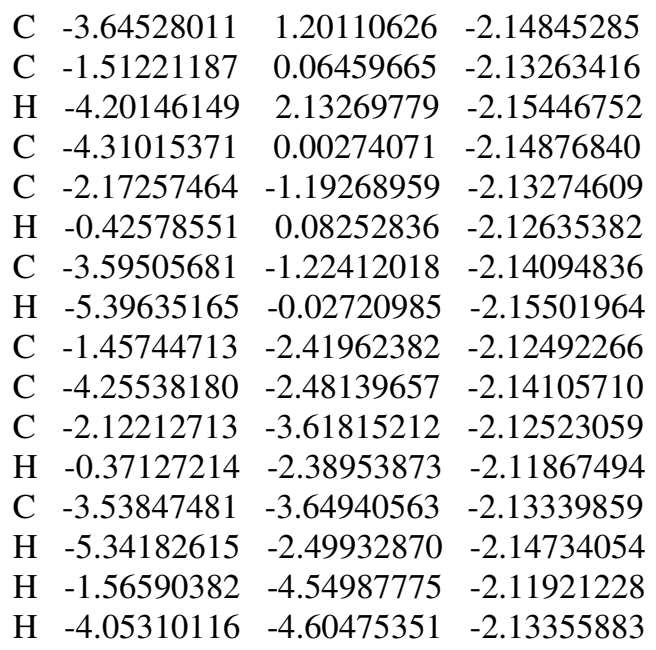

4AdPh_pi_2Nap_pi2_60
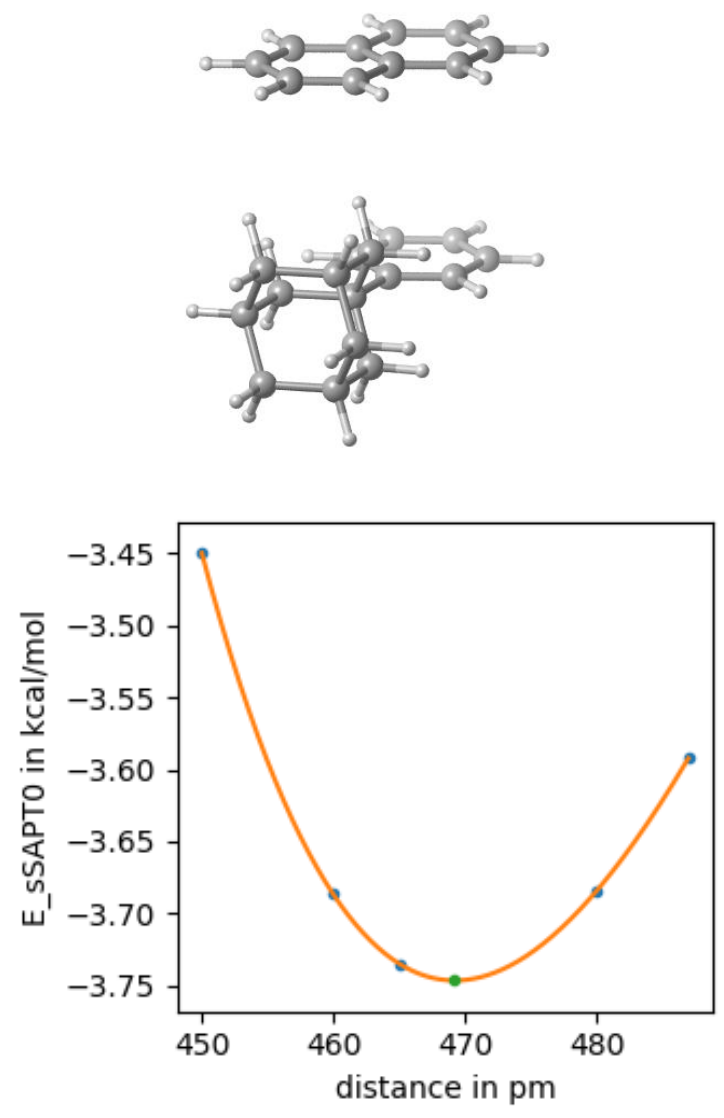

$\begin{array}{cccc}\mathrm{H} & -4.21490638 & 2.10797258 & 1.99997293 \\ \mathrm{C} & -1.50186838 & 0.04520558 & 2.00003493 \\ \mathrm{H} & -1.63822938 & -2.10748042 & 2.00001193 \\ \mathrm{H} & -1.76867538 & 2.18541758 & 1.99999893 \\ \mathrm{C} & 0.02431362 & 0.04571458 & 2.00001693 \\ \mathrm{C} & 0.55298362 & -0.68866242 & 0.74425493 \\ \mathrm{C} & 0.61561762 & 1.46926258 & 1.99962893 \\ \mathrm{C} & 0.55303662 & -0.68799642 & 3.25614693 \\ \mathrm{H} & 0.18630062 & -0.17353542 & -0.15295807 \\ \mathrm{H} & 0.15106062 & -1.70858842 & 0.71368593 \\ \mathrm{C} & 2.08800762 & -0.73790042 & 0.74462093 \\ \mathrm{H} & 0.26266762 & 2.01601858 & 2.88396393 \\ \mathrm{H} & 0.26263962 & 2.01555058 & 1.11501593 \\ \mathrm{C} & 2.15101662 & 1.42858458 & 1.99960693 \\ \mathrm{H} & 0.15112862 & -1.70791042 & 3.28727393 \\ \mathrm{H} & 0.18638262 & -0.17239742 & 4.15310093 \\ \mathrm{C} & 2.08805962 & -0.73722842 & 3.25574793 \\ \mathrm{H} & 2.43133662 & -1.26768242 & -0.15157507 \\ \mathrm{C} & 2.64484062 & 0.69255658 & 0.74587593 \\ \mathrm{C} & 2.57473862 & -1.47737642 & 2.00037193 \\ \mathrm{H} & 2.53496562 & 2.45530658 & 1.99932393 \\ \mathrm{C} & 2.64489162 & 0.69322758 & 3.25371093 \\ \mathrm{H} & 2.43142262 & -1.26653542 & 4.15221193 \\ \mathrm{H} & 2.31641362 & 1.22422858 & -0.15601007 \\ \mathrm{H} & 3.74172062 & 0.67084458 & 0.73058993 \\ \mathrm{H} & 2.19407462 & -2.50668142 & 2.00065493 \\ \mathrm{H} & 3.67039062 & -1.53568942 & 2.00036393 \\ \mathrm{H} & 3.74177262 & 0.67152258 & 3.26896293 \\ \mathrm{H} & 2.31650162 & 1.22537858 & 4.15532693 \\ \mathrm{H} & -5.40204757 & -0.05037494 & -2.69250188 \\ \mathrm{C} & -4.31735705 & -0.01828534 & -2.69239632 \\ \mathrm{C} & -3.58193277 & -1.22901852 & -2.70085908 \\ \mathrm{C} & -3.66422456 & 1.18652121 & -2.68411538 \\ \mathrm{H} & -4.11062253 & -2.17647903 & -2.70737187 \\ \mathrm{C} & -2.21168093 & -1.20563315 & -2.70083495 \\ \mathrm{C} & -2.24520135 & 1.24327332 & -2.68386283 \\ \mathrm{H} & -4.22296857 & 2.11842569 & -2.67760530 \\ \mathrm{C} & -1.50673861 & 0.02708617 & -2.69236268 \\ \mathrm{H} & -1.64264250 & -2.13133041 & -2.70731103 \\ \mathrm{C} & -1.54021083 & 2.47605581 & -2.67538713 \\ \mathrm{C} & -0.08774283 & 0.08386607 & -2.69210694 \\ \mathrm{C} & -0.16991484 & 2.49969021 & -2.67535528 \\ \mathrm{H} & -2.10935428 & 3.40166593 & -2.66891464 \\ \mathrm{C} & 0.56532730 & 1.28872713 & -2.68381961 \\ \mathrm{H} & 0.47101053 & -0.84805379 & -2.69862012 \\ \mathrm{H} & 0.35887009 & 3.44725408 & -2.66883878 \\ \mathrm{H} & 1.64999602 & 1.32072155 & -2.68370570\end{array}$

54

4AdPh_pi_2Nap_pi2_60-469

H $\quad-5.40699838-0.07046942$

C $-4.32228838-0.03889342$

C $-3.58278938-1.21969542$

C $-3.65363938 \quad 1.17869058$

C $-2.19430138-1.17393642$

H $\quad-4.08913238-2.17997242$

C $\quad-2.25972238 \quad 1.21897558$
1.99997493

1.99999093

1.99999893

1.99998993

2.00001793

1.99998893

2.00000593 
4AdPh_pi_2OMePh_pi_60
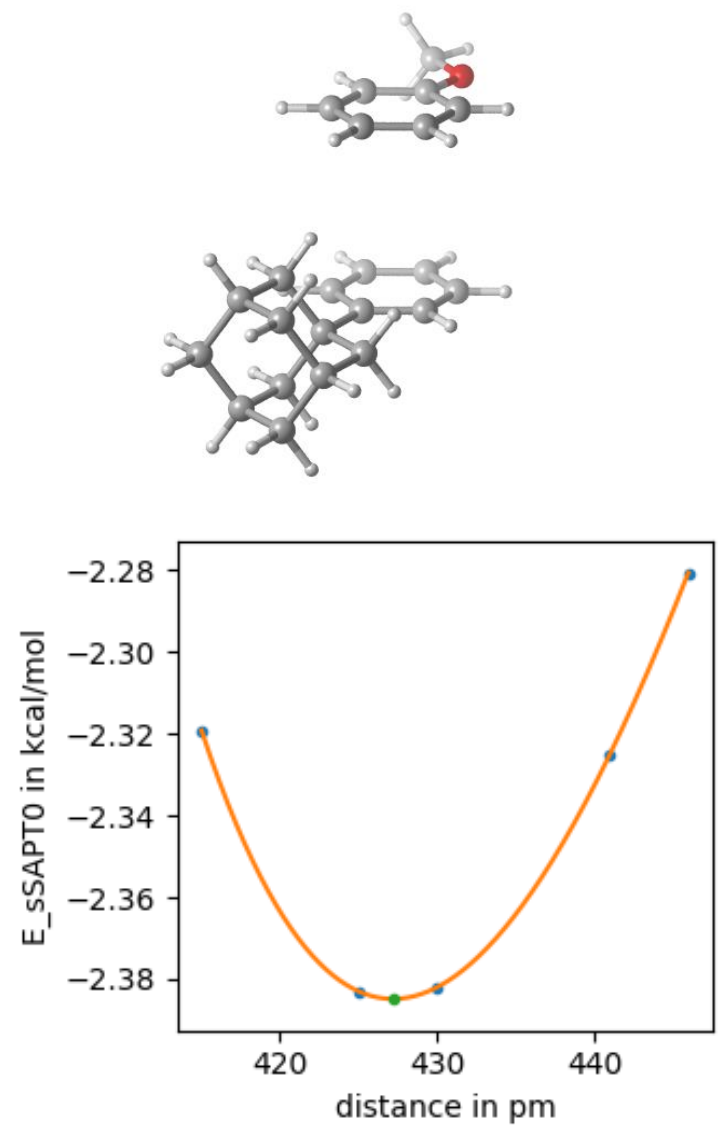

52

4AdPh_pi_2OMePh_pi_60-427

$\begin{array}{llll}\mathrm{H} & -5.40699069 & -0.07020421 & 0.99997496 \\ \mathrm{C} & -4.32228069 & -0.03862821 & 0.99999096\end{array}$

$\begin{array}{llll}\text { C } & -3.58278169 & -1.21943021 & 0.99999896\end{array}$

$\begin{array}{llll}\text { C } & -3.65363169 & 1.17895579 & 0.99998996\end{array}$

$\begin{array}{llll}\text { C } & -2.19429369 & -1.17367121 & 1.00001796\end{array}$

$\begin{array}{llll}\text { H } & -4.08912469 & -2.17970721 & 0.99998896\end{array}$

$\begin{array}{llll}\text { C } & -2.25971469 & 1.21924079 & 1.00000596\end{array}$

$\begin{array}{llll}\mathrm{H} & -4.21489869 & 2.10823779 & 0.99997296\end{array}$

$\begin{array}{llll}\text { C } & -1.50186069 & 0.04547079 & 1.00003496\end{array}$

$\begin{array}{llll}\mathrm{H} & -1.63822169 & -2.10721521 & 1.00001196\end{array}$

$\begin{array}{llll}\mathrm{H} & -1.76866769 & 2.18568279 & 0.99999896\end{array}$

$\begin{array}{llll}\text { C } & 0.02432131 & 0.04597979 & 1.00001696\end{array}$

$\begin{array}{llll}\text { C } & 0.55299131 & -0.68839721 & -0.25574504\end{array}$

$\begin{array}{llll}\text { C } & 0.61562531 & 1.46952779 & 0.99962896\end{array}$

$\begin{array}{llll}\text { C } & 0.55304431 & -0.68773121 & 2.25614696\end{array}$

H $\quad 0.18630831 \quad-0.17327021 \quad-1.15295804$

$\begin{array}{lllll}\mathrm{H} & 0.15106831 & -1.70832321 & -0.28631404\end{array}$

$\begin{array}{llll}\text { C } & 2.08801531 & -0.73763521 & -0.25537904\end{array}$

$\begin{array}{llll}\mathrm{H} & 0.26267531 & 2.01628379 & 1.88396396\end{array}$

$\begin{array}{llll}\mathrm{H} & 0.26264731 & 2.01581579 & 0.11501596\end{array}$

$\begin{array}{llll}\text { C } & 2.15102431 & 1.42884979 & 0.99960696\end{array}$

H $\quad 0.15113631 \quad-1.70764521 \quad 2.28727396$

H $\quad 0.18639031 \quad-0.17213221 \quad 3.15310096$

$\begin{array}{llll}\text { C } & 2.08806731 & -0.73696321 & 2.25574796\end{array}$

$\begin{array}{lrrr}\mathrm{H} & 2.43134431 & -1.26741721 & -1.15157504 \\ \mathrm{C} & 2.64484831 & 0.69282179 & -0.25412404 \\ \mathrm{C} & 2.57474631 & -1.47711121 & 1.00037196 \\ \mathrm{H} & 2.53497331 & 2.45557179 & 0.99932396 \\ \mathrm{C} & 2.64489931 & 0.69349279 & 2.25371096 \\ \mathrm{H} & 2.43143031 & -1.26627021 & 3.15221196 \\ \mathrm{H} & 2.31642131 & 1.22449379 & -1.15601004 \\ \mathrm{H} & 3.74172831 & 0.67110979 & -0.26941004 \\ \mathrm{H} & 2.19408231 & -2.50641621 & 1.00065496 \\ \mathrm{H} & 3.67039831 & -1.53542421 & 1.00036396 \\ \mathrm{H} & 3.74178031 & 0.67178779 & 2.26896296 \\ \mathrm{H} & 2.31650931 & 1.22564379 & 3.15532696 \\ \mathrm{C} & -4.30690008 & -0.02851290 & -3.27132747 \\ \mathrm{C} & -3.58499717 & -1.22239653 & -3.27170146 \\ \mathrm{C} & -3.63129858 & 1.19685450 & -3.27150510 \\ \mathrm{C} & -2.18996484 & -1.17720412 & -3.27225107 \\ \mathrm{C} & -2.24578662 & 1.22363135 & -3.27205418 \\ \mathrm{C} & -1.51329858 & 0.03495472 & -3.27243409 \\ \mathrm{O} & -5.66499026 & 0.04766870 & -3.27077698 \\ \mathrm{H} & -4.08914470 & -2.18105614 & -3.27157495 \\ \mathrm{H} & -4.22047856 & 2.10729041 & -3.27120184 \\ \mathrm{H} & -1.63423031 & -2.10962825 & -3.27254135 \\ \mathrm{H} & -1.73179047 & 2.17961715 & -3.27218889 \\ \mathrm{H} & -0.42920164 & 0.05822861 & -3.27286494 \\ \mathrm{C} & -6.38399345 & -1.16523958 & -3.27047781 \\ \mathrm{H} & -7.43922018 & -0.89446813 & -3.26999910 \\ \mathrm{H} & -6.16214938 & -1.76173198 & -2.37756066 \\ \mathrm{H} & -6.16295002 & -1.76169370 & -4.16361949\end{array}$

4AdPh_pi_2OMePh_pi_60_2
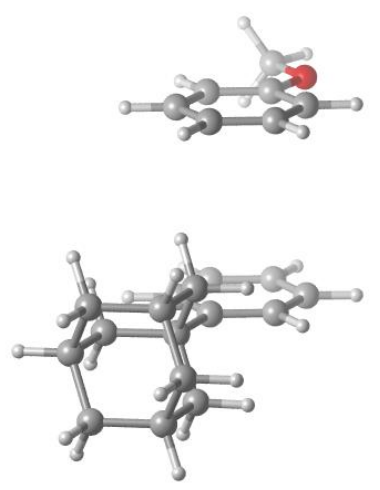


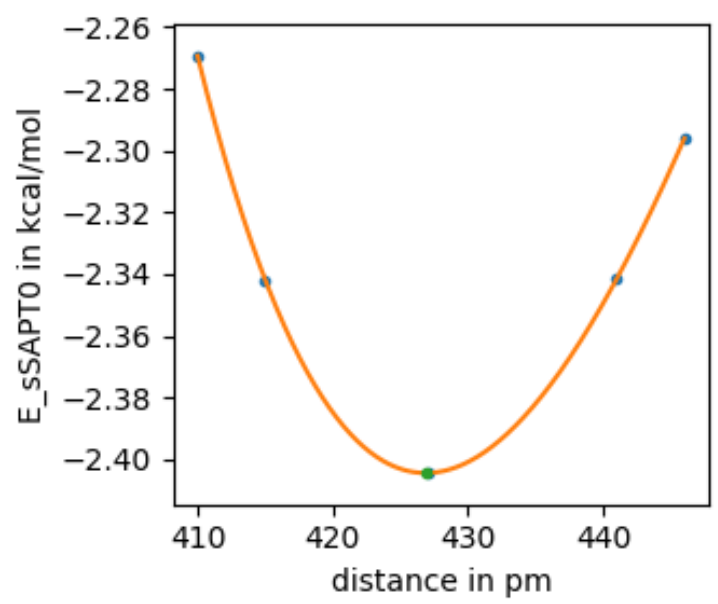

52

4AdPh_pi_2OMePh_pi_60_2-426

$\begin{array}{llll}\mathrm{H} & -5.40699069 & -0.07020421 & 0.99997496\end{array}$

$\begin{array}{llll}\text { C } & -4.32228069 & -0.03862821 & 0.99999096\end{array}$

$\begin{array}{llll}\text { C } & -3.58278169 & -1.21943021 & 0.99999896\end{array}$

$\begin{array}{llll}\text { C } & -3.65363169 & 1.17895579 & 0.99998996\end{array}$

$\begin{array}{llll}\text { C } & -2.19429369 & -1.17367121 & 1.00001796\end{array}$

$\begin{array}{llll}\mathrm{H} & -4.08912469 & -2.17970721 & 0.99998896\end{array}$

$\begin{array}{llll}\text { C } & -2.25971469 & 1.21924079 & 1.00000596\end{array}$

$\begin{array}{llll}\mathrm{H} & -4.21489869 & 2.10823779 & 0.99997296\end{array}$

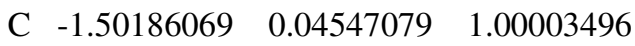

$\begin{array}{llll}\mathrm{H} & -1.63822169 & -2.10721521 & 1.00001196\end{array}$

$\begin{array}{llll}\mathrm{H} & -1.76866769 & 2.18568279 & 0.99999896\end{array}$

$\begin{array}{llll}\text { C } & 0.02432131 & 0.04597979 & 1.00001696\end{array}$

$\begin{array}{llll}\text { C } & 0.55299131 & -0.68839721 & -0.25574504\end{array}$

$\begin{array}{llll}\text { C } & 0.61562531 & 1.46952779 & 0.99962896\end{array}$

$\begin{array}{llll}\text { C } & 0.55304431 & -0.68773121 & 2.25614696\end{array}$

H $\quad 0.18630831 \quad-0.17327021 \quad-1.15295804$

H $\quad 0.15106831 \quad-1.70832321 \quad-0.28631404$

C $\quad 2.08801531 \quad-0.73763521 \quad-0.25537904$

$\begin{array}{llll}\mathrm{H} & 0.26267531 & 2.01628379 & 1.88396396\end{array}$

$\begin{array}{llll}\mathrm{H} & 0.26264731 & 2.01581579 & 0.11501596\end{array}$

C $\quad 2.15102431 \quad 1.42884979 \quad 0.99960696$

H $\quad 0.15113631 \quad-1.70764521 \quad 2.28727396$

H $\quad 0.18639031 \quad-0.172132213 .15310096$

$\begin{array}{llll}\text { C } & 2.08806731 & -0.73696321 & 2.25574796\end{array}$

H $\quad 2.43134431 \quad-1.26741721 \quad-1.15157504$

$\begin{array}{llll}\text { C } & 2.64484831 & 0.69282179 & -0.25412404\end{array}$

$\begin{array}{llll}\text { C } & 2.57474631 & -1.47711121 & 1.00037196\end{array}$

$\begin{array}{llll}\mathrm{H} & 2.53497331 & 2.45557179 & 0.99932396\end{array}$

$\begin{array}{llll}\text { C } & 2.64489931 & 0.69349279 & 2.25371096\end{array}$

$\mathrm{H} \quad 2.43143031 \quad-1.26627021 \quad 3.15221196$

H $\quad 2.31642131 \quad 1.22449379 \quad-1.15601004$

H $\quad 3.74172831 \quad 0.67110979 \quad-0.26941004$

$\begin{array}{llll}\mathrm{H} & 2.19408231 & -2.50641621 & 1.00065496\end{array}$

H $\quad 3.67039831 \quad-1.53542421 \quad 1.00036396$

$\begin{array}{llll}\mathrm{H} & 3.74178031 & 0.67178779 & 2.26896296\end{array}$

$\begin{array}{llll}\mathrm{H} & 2.31650931 & 1.22564379 & 3.15532696\end{array}$

C $\quad-4.30708003 \quad-0.01976622-3.26815795$

$\begin{array}{llll}\text { C } & -3.63488168 & 1.20279314 & -3.26789147\end{array}$
C $\quad-3.58166140 \quad-1.21631534 \quad-3.26897750$

$\begin{array}{llll}\text { C } & -2.23917095 & 1.21500467 & -3.26844981\end{array}$

$\begin{array}{llll}\text { C } & -2.19622025 & -1.18609532 & -3.26952568\end{array}$

$\begin{array}{llll}\text { C } & -1.51323160 & 0.03169671 & -3.26926775\end{array}$

$\begin{array}{llll}\mathrm{O} & -5.66088880 & -0.15172997 & -3.26766263\end{array}$

$\mathrm{H}-4.17802402 \quad 2.13991052-3.26726247$

H $\quad-4.13290479-2.15020900 \quad-3.26916310$

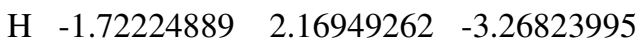

H $\quad-1.64334757 \quad-2.12013617-3.27016135$

$\begin{array}{lllll}\mathrm{H} & -0.42909458 & 0.05302212 & -3.26969912\end{array}$

C $-6.42916009 \quad 1.03058578-3.26672860$

$\mathrm{H} \quad-7.472359820 .71665094 \quad-3.26640488$

$\mathrm{H} \quad-6.23202243 \quad 1.63522292 \quad-2.37349310$

$\begin{array}{llll}\mathrm{H} & -6.23284027 & 1.63609815 & -4.15955169\end{array}$

4AdPh_pi_3MePh_pi2_60_2_300_1
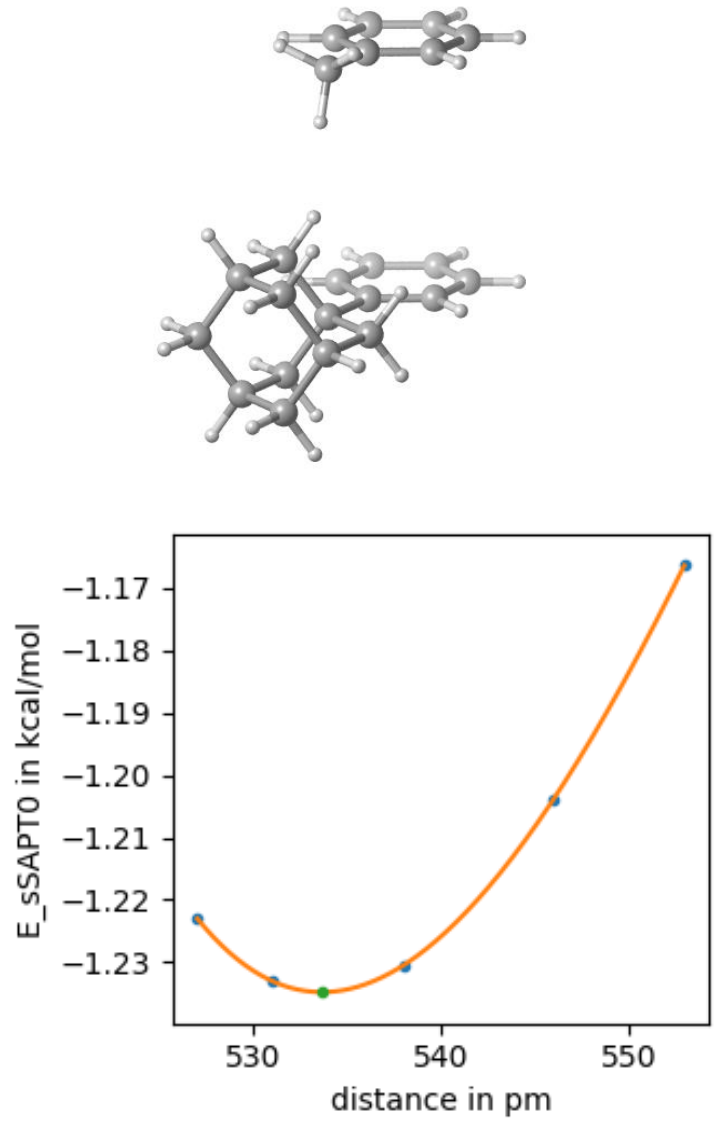

51

4AdPh_pi_3MePh_pi2_60_2_300_1-533

H $\quad-5.40699838 \quad-0.07046942 \quad 1.99997493$

$\begin{array}{llll}\text { C } & -4.32228838 & -0.03889342 & 1.99999093\end{array}$

$\begin{array}{llll}\text { C } & -3.58278938 & -1.21969542 & 1.99999893\end{array}$

$\begin{array}{llll}\text { C } & -3.65363938 & 1.17869058 & 1.99998993\end{array}$

$\begin{array}{llll}\text { C } & -2.19430138 & -1.17393642 & 2.00001793\end{array}$

$\begin{array}{llll}\mathrm{H} & -4.08913238 & -2.17997242 & 1.99998893\end{array}$

$\begin{array}{llll}\text { C } & -2.25972238 & 1.21897558 & 2.00000593\end{array}$

$\begin{array}{llll}\mathrm{H} & -4.21490638 & 2.10797258 & 1.99997293\end{array}$

$\begin{array}{llll}\text { C } & -1.50186838 & 0.04520558 & 2.00003493\end{array}$ 


$\begin{array}{cccc}\mathrm{H} & -1.63822938 & -2.10748042 & 2.00001193 \\ \mathrm{H} & -1.76867538 & 2.18541758 & 1.99999893 \\ \mathrm{C} & 0.02431362 & 0.04571458 & 2.00001693 \\ \mathrm{C} & 0.55298362 & -0.68866242 & 0.74425493 \\ \mathrm{C} & 0.61561762 & 1.46926258 & 1.99962893 \\ \mathrm{C} & 0.55303662 & -0.68799642 & 3.25614693 \\ \mathrm{H} & 0.18630062 & -0.17353542 & -0.15295807 \\ \mathrm{H} & 0.15106062 & -1.70858842 & 0.71368593 \\ \mathrm{C} & 2.08800762 & -0.73790042 & 0.74462093 \\ \mathrm{H} & 0.26266762 & 2.01601858 & 2.88396393 \\ \mathrm{H} & 0.26263962 & 2.01555058 & 1.11501593 \\ \mathrm{C} & 2.15101662 & 1.42858458 & 1.99960693 \\ \mathrm{H} & 0.15112862 & -1.70791042 & 3.28727393 \\ \mathrm{H} & 0.18638262 & -0.17239742 & 4.15310093 \\ \mathrm{C} & 2.08805962 & -0.73722842 & 3.25574793 \\ \mathrm{H} & 2.43133662 & -1.26768242 & -0.15157507 \\ \mathrm{C} & 2.64484062 & 0.69255658 & 0.74587593 \\ \mathrm{C} & 2.57473862 & -1.47737642 & 2.00037193 \\ \mathrm{H} & 2.53496562 & 2.45530658 & 1.99932393 \\ \mathrm{C} & 2.64489162 & 0.69322758 & 3.25371093 \\ \mathrm{H} & 2.43142262 & -1.26653542 & 4.15221193 \\ \mathrm{H} & 2.31641362 & 1.22422858 & -0.15601007 \\ \mathrm{H} & 3.74172062 & 0.67084458 & 0.73058993 \\ \mathrm{H} & 2.19407462 & -2.50668142 & 2.00065493 \\ \mathrm{H} & 3.67039062 & -1.53568942 & 2.00036393 \\ \mathrm{H} & 3.74177262 & 0.67152258 & 3.26896293 \\ \mathrm{H} & 2.31650162 & 1.22537858 & 4.15532693 \\ \mathrm{C} & -4.31566878 & -0.04458473 & 7.33652509 \\ \mathrm{C} & -3.65540614 & 1.18140782 & 7.33742636 \\ \mathrm{C} & -3.57364697 & -1.22262448 & 7.33739202 \\ \mathrm{C} & -2.26438448 & 1.22578640 & 7.33852541 \\ \mathrm{C} & -2.18258689 & -1.17225303 & 7.33840059 \\ \mathrm{C} & -1.50857990 & 0.05100145 & 7.33656878 \\ \mathrm{H} & -5.40061030 & -0.08148720 & 7.33936617 \\ \mathrm{H} & -4.22460340 & 2.10588201 & 7.34147380 \\ \mathrm{H} & -4.07854480 & -2.18372335 & 7.34134953 \\ \mathrm{H} & -1.75426534 & 2.18578478 & 7.34271564 \\ \mathrm{H} & -1.60840896 & -2.09532251 & 7.34244806 \\ \mathrm{C} & -0.00187605 & 0.10319725 & 7.30489568 \\ \mathrm{H} & 0.36624198 & 0.14133682 & 6.27443918 \\ \mathrm{H} & 0.37432996 & 0.98937725 & 7.82156540 \\ \mathrm{H} & 0.43481949 & -0.77926302 & 7.77832342 \\ & & & \end{array}$

4AdPh_pi_3MePh_pi_300_2
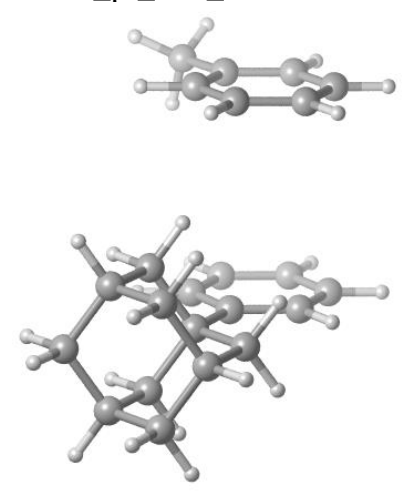

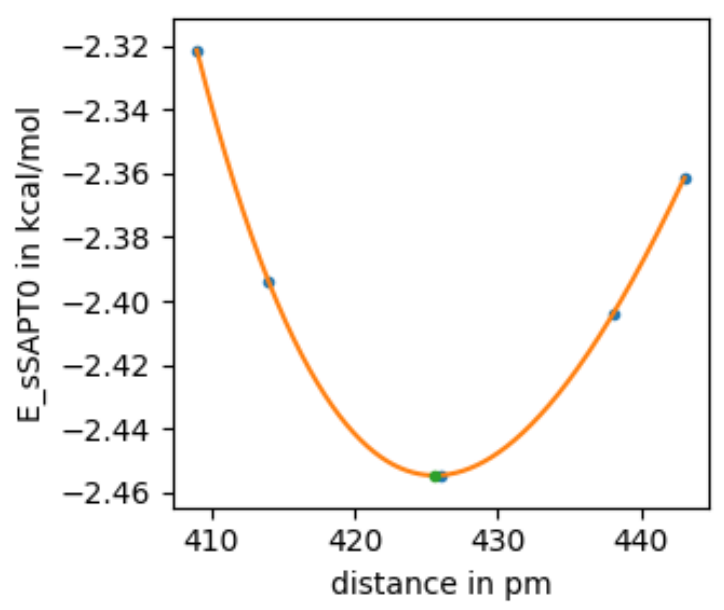

51

4AdPh_pi_3MePh_pi_300_2-425

H $\quad-5.40699838-0.07046942 \quad 1.99997493$

$\begin{array}{llll}\text { C } & -4.32228838 & -0.03889342 & 1.99999093\end{array}$

$\begin{array}{llll}\text { C } & -3.58278938 & -1.21969542 & 1.99999893\end{array}$

$\begin{array}{llll}\text { C } & -3.65363938 & 1.17869058 & 1.99998993\end{array}$

$\begin{array}{llll}\text { C } & -2.19430138 & -1.17393642 & 2.00001793\end{array}$

$\begin{array}{llll}\mathrm{H} & -4.08913238 & -2.17997242 & 1.99998893\end{array}$

$\begin{array}{llll}\text { C } & -2.25972238 & 1.21897558 & 2.00000593\end{array}$

$\begin{array}{llll}\mathrm{H} & -4.21490638 & 2.10797258 & 1.99997293\end{array}$

$\begin{array}{llll}\text { C } & -1.50186838 & 0.04520558 & 2.00003493\end{array}$

$\begin{array}{llll}\mathrm{H} & -1.63822938 & -2.10748042 & 2.00001193\end{array}$

$\begin{array}{llll}\mathrm{H} & -1.76867538 & 2.18541758 & 1.99999893\end{array}$

$\begin{array}{llll}\text { C } & 0.02431362 & 0.04571458 & 2.00001693\end{array}$

$\begin{array}{llll}\text { C } & 0.55298362 & -0.68866242 & 0.74425493\end{array}$

$\begin{array}{llll}\text { C } & 0.61561762 & 1.46926258 & 1.99962893\end{array}$

C $\quad 0.55303662 \quad-0.68799642 \quad 3.25614693$

$\mathrm{H} \quad 0.18630062-0.17353542 \quad-0.15295807$

$\begin{array}{llll}\mathrm{H} & 0.15106062 & -1.70858842 & 0.71368593\end{array}$

$\begin{array}{llll}\text { C } & 2.08800762 & -0.73790042 & 0.74462093\end{array}$

$\begin{array}{llll}\mathrm{H} & 0.26266762 & 2.01601858 & 2.88396393\end{array}$

$\begin{array}{llll}\mathrm{H} & 0.26263962 & 2.01555058 & 1.11501593\end{array}$

C $\quad 2.15101662 \quad 1.42858458 \quad 1.99960693$

$\begin{array}{llll}\mathrm{H} & 0.15112862 & -1.70791042 & 3.28727393\end{array}$

H $\quad 0.18638262 \quad-0.17239742 \quad 4.15310093$

C $\quad 2.08805962 \quad-0.73722842 \quad 3.25574793$

$\begin{array}{llll}\mathrm{H} & 2.43133662 & -1.26768242 & -0.15157507\end{array}$

$\begin{array}{llll}\text { C } & 2.64484062 & 0.69255658 & 0.74587593\end{array}$

C $\quad 2.57473862 \quad-1.47737642 \quad 2.00037193$

$\begin{array}{llll}\mathrm{H} & 2.53496562 & 2.45530658 & 1.99932393\end{array}$

C $\quad 2.64489162 \quad 0.69322758 \quad 3.25371093$

$\mathrm{H} \quad 2.43142262 \quad-1.26653542 \quad 4.15221193$

$\begin{array}{llll}\mathrm{H} & 2.31641362 & 1.22422858 & -0.15601007\end{array}$

$\begin{array}{llll}\mathrm{H} & 3.74172062 & 0.67084458 & 0.73058993\end{array}$

$\mathrm{H} \quad 2.19407462-2.50668142 \quad 2.00065493$

H $\quad 3.67039062 \quad-1.53568942 \quad 2.00036393$

$\begin{array}{llll}\mathrm{H} & 3.74177262 & 0.67152258 & 3.26896293\end{array}$

$\begin{array}{llll}\mathrm{H} & 2.31650162 & 1.22537858 & 4.15532693\end{array}$

$\begin{array}{llll}\text { C } & -2.24932099 & 1.24238596 & -2.25567547\end{array}$

$\begin{array}{llll}\text { C } & -3.64087079 & 1.19145856 & -2.25660216\end{array}$ 


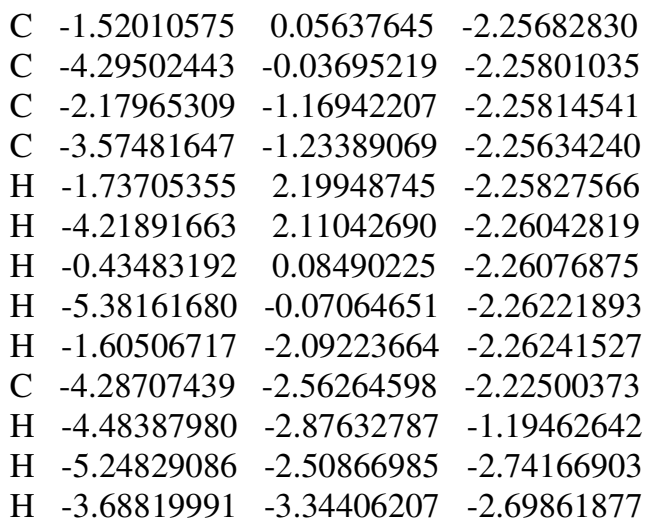

4AdPh_pi_3MePh_pi_60_1
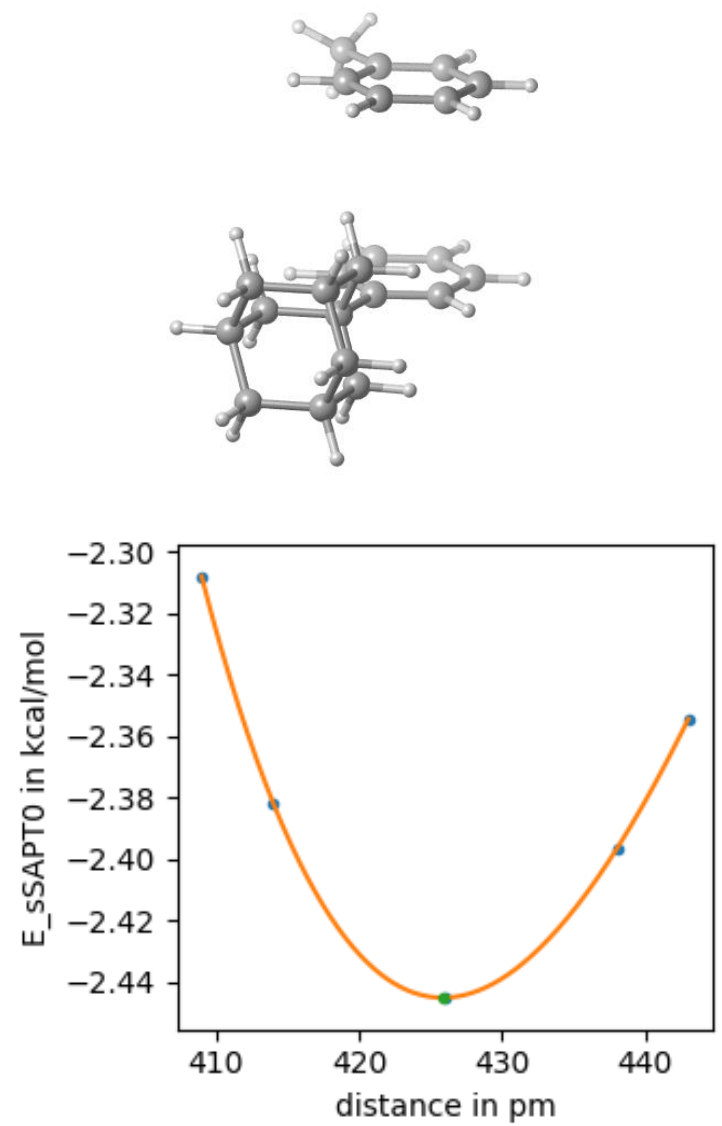

51

4AdPh_pi_3MePh_pi_60_1-425

H $\quad-5.40699069-0.07020421$

C $-4.32228069-0.03862821$

C $\quad-3.58278169-1.21943021$

C $-3.65363169 \quad 1.17895579$

C $-2.19429369-1.17367121$

H $-4.08912469-2.17970721$

C $\quad-2.25971469 \quad 1.21924079$

H $\quad-4.21489869 \quad 2.10823779$

C -1.501860690 .04547079$

H $-1.63822169-2.10721521$
0.99997496 0.99999096 0.99999896 0.99998996 1.00001796 0.99998896 1.00000596 0.99997296 1.00003496 1.00001196

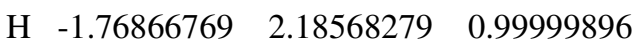

$\begin{array}{llll}\text { C } & 0.02432131 & 0.04597979 & 1.00001696\end{array}$

$\begin{array}{llll}\text { C } & 0.55299131 & -0.68839721 & -0.25574504\end{array}$

$\begin{array}{llll}\text { C } & 0.61562531 & 1.46952779 & 0.99962896\end{array}$

$\begin{array}{llll}\text { C } & 0.55304431 & -0.68773121 & 2.25614696\end{array}$

H $\quad 0.18630831 \quad-0.17327021 \quad-1.15295804$

H $\quad 0.15106831 \quad-1.70832321 \quad-0.28631404$

C $\quad 2.08801531 \quad-0.73763521 \quad-0.25537904$

$\begin{array}{llll}\mathrm{H} & 0.26267531 & 2.01628379 & 1.88396396\end{array}$

$\begin{array}{llll}\mathrm{H} & 0.26264731 & 2.01581579 & 0.11501596\end{array}$

$\begin{array}{llll}\text { C } & 2.15102431 & 1.42884979 & 0.99960696\end{array}$

$\begin{array}{llll}\mathrm{H} & 0.15113631 & -1.70764521 & 2.28727396\end{array}$

H $\quad 0.18639031 \quad-0.17213221 \quad 3.15310096$

$\begin{array}{llll}\text { C } & 2.08806731 & -0.73696321 & 2.25574796\end{array}$

H $\quad 2.43134431 \quad-1.26741721 \quad-1.15157504$

$\begin{array}{llll}\text { C } & 2.64484831 & 0.69282179 & -0.25412404\end{array}$

$\begin{array}{llll}\text { C } & 2.57474631 & -1.47711121 & 1.00037196\end{array}$

$\begin{array}{llll}\mathrm{H} & 2.53497331 & 2.45557179 & 0.99932396\end{array}$

$\begin{array}{llll}\text { C } & 2.64489931 & 0.69349279 & 2.25371096\end{array}$

H $\quad 2.43143031 \quad-1.266270213 .15221196$

H $\quad 2.31642131 \quad 1.22449379 \quad-1.15601004$

H $\quad 3.74172831 \quad 0.67110979 \quad-0.26941004$

H $\quad 2.19408231 \quad-2.50641621 \quad 1.00065496$

H $\quad 3.67039831 \quad-1.53542421 \quad 1.00036396$

$\begin{array}{llll}\mathrm{H} & 3.74178031 & 0.67178779 & 2.26896296\end{array}$

$\begin{array}{llll}\mathrm{H} & 2.31650931 & 1.22564379 & 3.15532696\end{array}$

C $\quad-2.17117794-1.18843921 \quad-3.25874719$

$\begin{array}{llll}\text { C } & -1.51950765 & 0.04214229 & -3.25931830\end{array}$

$\begin{array}{llll}\text { C } & -3.56290015 & -1.22695301 & -3.25961201\end{array}$

C $-2.25626602 \quad 1.22286176-3.26008820$

$\begin{array}{llll}\text { C } & -4.29469938 & -0.04286862 & -3.26029055\end{array}$

C $\quad-3.65294876 \quad 1.19761183 \quad-3.25812579$

$\mathrm{H}-1.59843805-2.11062540 \quad-3.26184533$

$\begin{array}{lllll}\mathrm{H} & -0.43463567 & 0.08326212 & -3.26336436\end{array}$

$\mathrm{H} \quad-4.08083385-2.18108899-3.26382540$

$\mathrm{H} \quad-1.74215090 \quad 2.18072718 \quad-3.26402150$

H $\quad-5.38117410 \quad-0.07906569-3.26433805$

$\begin{array}{llll}\text { C } & -4.44754877 & 2.47881009 & -3.22609557\end{array}$

H $\quad-4.62057776 \quad 2.80567272 \quad-2.19554819$

$\begin{array}{llll}\mathrm{H} & -3.92030854 & 3.284468887 & -3.74255006\end{array}$

H $\quad-5.42381548 \quad 2.35106913 \quad-3.69954936$

4AdPh_pi_3MePh_pi_60_2_300_1
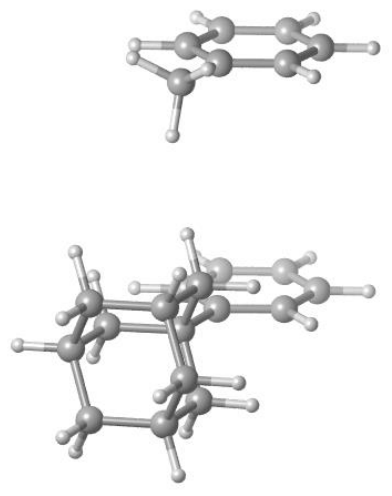


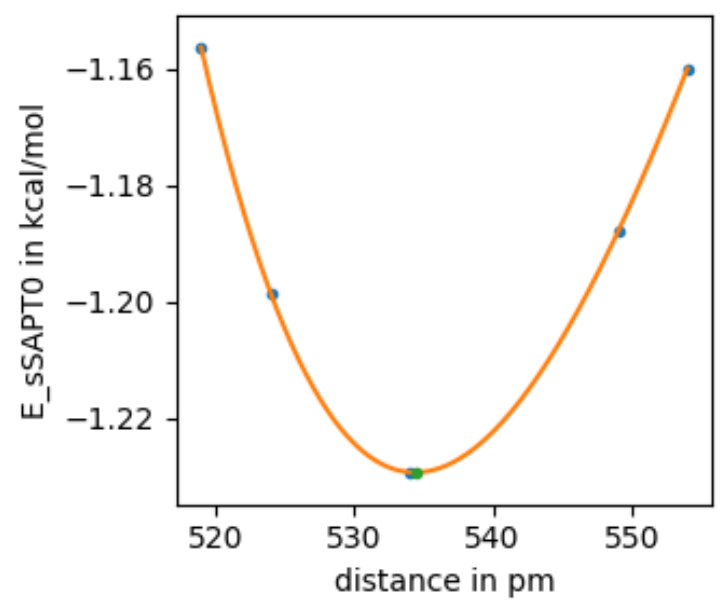

51

4AdPh_pi_3MePh_pi_60_2_300_1-534

H $\quad-5.40699838-0.07046942 \quad 1.99997493$

$\begin{array}{llll}\text { C } & -4.32228838 & -0.03889342 & 1.99999093\end{array}$

$\begin{array}{llll}\text { C } & -3.58278938 & -1.21969542 & 1.99999893\end{array}$

$\begin{array}{llll}\text { C } & -3.65363938 & 1.17869058 & 1.99998993\end{array}$

$\begin{array}{llll}\text { C } & -2.19430138 & -1.17393642 & 2.00001793\end{array}$

$\begin{array}{llll}\mathrm{H} & -4.08913238 & -2.17997242 & 1.99998893\end{array}$

C $\quad-2.25972238 \quad 1.21897558 \quad 2.00000593$

$\begin{array}{llll}\mathrm{H} & -4.21490638 & 2.10797258 & 1.99997293\end{array}$

C $-1.50186838 \quad 0.04520558 \quad 2.00003493$

$\begin{array}{llll}\mathrm{H} & -1.63822938 & -2.10748042 & 2.00001193\end{array}$

$\begin{array}{llll}\mathrm{H} & -1.76867538 & 2.18541758 & 1.99999893\end{array}$

$\begin{array}{llll}\text { C } & 0.02431362 & 0.04571458 & 2.00001693\end{array}$

$\begin{array}{llll}\text { C } & 0.55298362 & -0.68866242 & 0.74425493\end{array}$

$\begin{array}{llll}\text { C } & 0.61561762 & 1.46926258 & 1.99962893\end{array}$

$\begin{array}{llll}\text { C } & 0.55303662 & -0.68799642 & 3.25614693\end{array}$

H $\quad 0.18630062 \quad-0.17353542 \quad-0.15295807$

$\begin{array}{llll}\mathrm{H} & 0.15106062 & -1.70858842 & 0.71368593\end{array}$

C $\quad 2.08800762-0.73790042 \quad 0.74462093$

$\begin{array}{llll}\mathrm{H} & 0.26266762 & 2.01601858 & 2.88396393\end{array}$

$\begin{array}{llll}\mathrm{H} & 0.26263962 & 2.01555058 & 1.11501593\end{array}$

C $\quad 2.15101662 \quad 1.42858458 \quad 1.99960693$

H $\quad 0.15112862-1.70791042 \quad 3.28727393$

H $\quad 0.18638262 \quad-0.17239742 \quad 4.15310093$

C $\quad 2.08805962 \quad-0.73722842 \quad 3.25574793$

$\begin{array}{llll}\mathrm{H} & 2.43133662 & -1.26768242 & -0.15157507\end{array}$

$\begin{array}{llll}\text { C } & 2.64484062 & 0.69255658 & 0.74587593\end{array}$

$\begin{array}{llll}\text { C } & 2.57473862 & -1.47737642 & 2.00037193\end{array}$

$\begin{array}{llll}\mathrm{H} & 2.53496562 & 2.45530658 & 1.99932393\end{array}$

C $\quad 2.64489162 \quad 0.69322758 \quad 3.25371093$

H $\quad 2.43142262 \quad-1.26653542 \quad 4.15221193$

H $\quad 2.31641362 \quad 1.22422858 \quad-0.15601007$

$\begin{array}{llll}\mathrm{H} & 3.74172062 & 0.67084458 & 0.73058993\end{array}$

$\begin{array}{llll}\mathrm{H} & 2.19407462 & -2.50668142 & 2.00065493\end{array}$

H $\quad 3.67039062-1.53568942 \quad 2.00036393$

H $\quad 3.741772620 .67152258 \quad 3.26896293$

H $\quad 2.31650162 \quad 1.22537858 \quad 4.15532693$

C $\quad-4.31563160-0.04053819-3.34403064$

$\begin{array}{llll}\text { C } & -3.57575206 & -1.22019157 & -3.34524029\end{array}$ $\begin{array}{llll}\text { C } & -3.65312462 & 1.18398580 & -3.34454469\end{array}$

C $-2.18484003-1.17249943-3.34629432$

$\begin{array}{llll}\text { C } & -2.26177802 & 1.22570071 & -3.34550987\end{array}$

$\begin{array}{llll}\text { C } & -1.50836530 & 0.04968742 & -3.34398513\end{array}$

$\begin{array}{llll}\mathrm{H} & -5.40063887 & -0.07545121 & -3.34690621\end{array}$

$\mathrm{H} \quad-4.08257812 \quad-2.18027651-3.34956412$

$\mathrm{H} \quad-4.22046465 \quad 2.10959912-3.34825993$

H $\quad-1.61236269-2.09666719-3.35072630$

H $\quad-1.74988912 \quad 2.18471555 \quad-3.34928113$

$\begin{array}{llll}\text { C } & -0.00150811 & 0.09721923 & -3.31226439\end{array}$

$\mathrm{H} \quad 0.36830253 \quad 0.08321862-2.28180324$

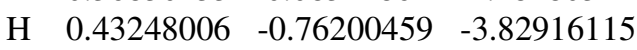

H $\quad 0.37589505 \quad 1.00675294 \quad-3.78543267$

4AdPh_pi_4CF3Ph_pi2_300
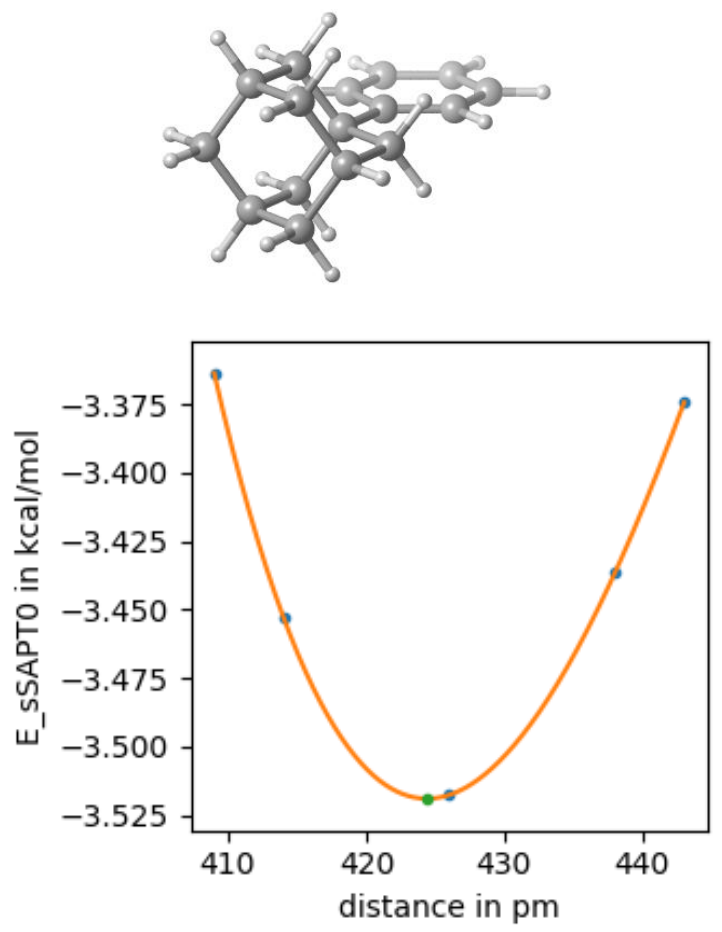

51

4AdPh_pi_4CF3Ph_pi2_300-424

H $\quad-5.40699838-0.07046942 \quad 1.99997493$

$\begin{array}{llll}\text { C } & -4.32228838 & -0.03889342 & 1.99999093\end{array}$

$\begin{array}{llll}\text { C } & -3.58278938 & -1.21969542 & 1.99999893\end{array}$

$\begin{array}{llll}\text { C } & -3.65363938 & 1.17869058 & 1.99998993\end{array}$

$\begin{array}{llll}\text { C } & -2.19430138 & -1.17393642 & 2.00001793\end{array}$

$\begin{array}{llll}\mathrm{H} & -4.08913238 & -2.17997242 & 1.99998893\end{array}$

$\begin{array}{llll}\text { C } & -2.25972238 & 1.21897558 & 2.00000593\end{array}$

$\begin{array}{llll}\mathrm{H} & -4.21490638 & 2.10797258 & 1.99997293\end{array}$

$\begin{array}{llll}\text { C } & -1.50186838 & 0.04520558 & 2.00003493\end{array}$

$\begin{array}{llll}\mathrm{H} & -1.63822938 & -2.10748042 & 2.00001193\end{array}$ 


$\begin{array}{crrr}\text { H } & -1.76867538 & 2.18541758 & 1.99999893 \\ \mathrm{C} & 0.02431362 & 0.04571458 & 2.00001693 \\ \mathrm{C} & 0.55298362 & -0.68866242 & 0.74425493 \\ \mathrm{C} & 0.61561762 & 1.46926258 & 1.99962893 \\ \mathrm{C} & 0.55303662 & -0.68799642 & 3.25614693 \\ \mathrm{H} & 0.18630062 & -0.17353542 & -0.15295807 \\ \mathrm{H} & 0.15106062 & -1.70858842 & 0.71368593 \\ \mathrm{C} & 2.08800762 & -0.73790042 & 0.74462093 \\ \mathrm{H} & 0.26266762 & 2.01601858 & 2.88396393 \\ \mathrm{H} & 0.26263962 & 2.01555058 & 1.11501593 \\ \mathrm{C} & 2.15101662 & 1.42858458 & 1.99960693 \\ \mathrm{H} & 0.15112862 & -1.70791042 & 3.28727393 \\ \mathrm{H} & 0.18638262 & -0.17239742 & 4.15310093 \\ \mathrm{C} & 2.08805962 & -0.73722842 & 3.25574793 \\ \mathrm{H} & 2.43133662 & -1.26768242 & -0.15157507 \\ \mathrm{C} & 2.64484062 & 0.69255658 & 0.74587593 \\ \mathrm{C} & 2.57473862 & -1.47737642 & 2.00037193 \\ \mathrm{H} & 2.53496562 & 2.45530658 & 1.99932393 \\ \mathrm{C} & 2.64489162 & 0.69322758 & 3.25371093 \\ \mathrm{H} & 2.43142262 & -1.26653542 & 4.15221193 \\ \mathrm{H} & 2.31641362 & 1.22422858 & -0.15601007 \\ \mathrm{H} & 3.74172062 & 0.67084458 & 0.73058993 \\ \mathrm{H} & 2.19407462 & -2.50668142 & 2.00065493 \\ \mathrm{H} & 3.67039062 & -1.53568942 & 2.00036393 \\ \mathrm{H} & 3.74177262 & 0.67152258 & 3.26896293 \\ \mathrm{H} & 2.31650162 & 1.22537858 & 4.15532693 \\ \mathrm{C} & -3.64262818 & 1.18229858 & -2.24473191 \\ \mathrm{C} & -4.30575984 & -0.04447074 & -2.24560031 \\ \mathrm{C} & -2.25215470 & 1.22643813 & -2.24364315 \\ \mathrm{C} & -3.57676671 & -1.22656105 & -2.24537360 \\ \mathrm{C} & -1.51597887 & 0.04404506 & -2.24342868 \\ \mathrm{C} & -2.18349262 & -1.17498639 & -2.24429368 \\ \mathrm{H} & -4.21349836 & 2.10513751 & -2.24487571 \\ \mathrm{H} & -5.39000518 & -0.07741685 & -2.24640273 \\ \mathrm{H} & -1.73775901 & 2.18147386 & -2.24292916 \\ \mathrm{H} & -4.07941740 & -2.18871384 & -2.24593572 \\ \mathrm{H} & -0.43225347 & 0.06399969 & -2.24253832 \\ \mathrm{C} & -1.42528145 & -2.47108330 & -2.24466308 \\ \mathrm{~F} & -1.72347763 & -3.21265795 & -3.32330965 \\ \mathrm{~F} & -1.73149004 & -3.21789982 & -1.17188769 \\ \mathrm{~F} & -0.09872838 & -2.28463995 & -2.23933941\end{array}$

4AdPh_pi_4CF3Ph_pi2_60
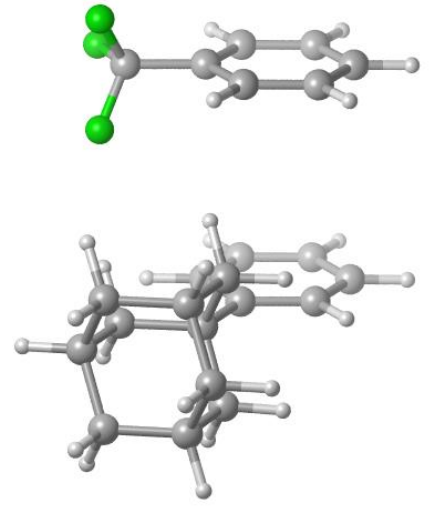

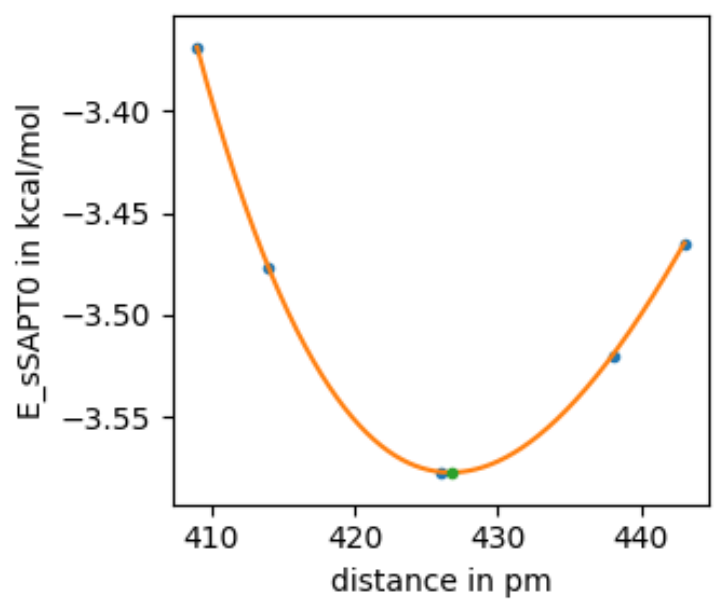

51

4AdPh_pi_4CF3Ph_pi2_60-426

H $\quad-5.40699838-0.07046942 \quad 1.99997493$

$\begin{array}{llll}\text { C } & -4.32228838 & -0.03889342 & 1.99999093\end{array}$

$\begin{array}{llll}\text { C } & -3.58278938 & -1.21969542 & 1.99999893\end{array}$

$\begin{array}{llll}\text { C } & -3.65363938 & 1.17869058 & 1.99998993\end{array}$

$\begin{array}{llll}\text { C } & -2.19430138 & -1.17393642 & 2.00001793\end{array}$

$\begin{array}{llll}\mathrm{H} & -4.08913238 & -2.17997242 & 1.99998893\end{array}$

$\begin{array}{llll}\text { C } & -2.25972238 & 1.21897558 & 2.00000593\end{array}$

$\begin{array}{llll}\mathrm{H} & -4.21490638 & 2.10797258 & 1.99997293\end{array}$

$\begin{array}{llll}\text { C } & -1.50186838 & 0.04520558 & 2.00003493\end{array}$

$\begin{array}{llll}\mathrm{H} & -1.63822938 & -2.10748042 & 2.00001193\end{array}$

$\begin{array}{llll}\mathrm{H} & -1.76867538 & 2.18541758 & 1.99999893\end{array}$

$\begin{array}{llll}\text { C } & 0.02431362 & 0.04571458 & 2.00001693\end{array}$

$\begin{array}{llll}\text { C } & 0.55298362 & -0.68866242 & 0.74425493\end{array}$

$\begin{array}{llll}\text { C } & 0.61561762 & 1.46926258 & 1.99962893\end{array}$

C $\quad 0.55303662 \quad-0.68799642 \quad 3.25614693$

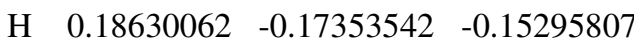

$\begin{array}{llll}\mathrm{H} & 0.15106062 & -1.70858842 & 0.71368593\end{array}$

$\begin{array}{llll}\text { C } & 2.08800762 & -0.73790042 & 0.74462093\end{array}$

$\begin{array}{llll}\mathrm{H} & 0.26266762 & 2.01601858 & 2.88396393\end{array}$

$\begin{array}{llll}\mathrm{H} & 0.26263962 & 2.01555058 & 1.11501593\end{array}$

C $\quad 2.15101662 \quad 1.42858458 \quad 1.99960693$

$\begin{array}{llll}\mathrm{H} & 0.15112862 & -1.70791042 & 3.28727393\end{array}$

H $\quad 0.18638262 \quad-0.17239742 \quad 4.15310093$

C $\quad 2.08805962 \quad-0.73722842 \quad 3.25574793$

H $\quad 2.43133662 \quad-1.26768242 \quad-0.15157507$

$\begin{array}{llll}\text { C } & 2.64484062 & 0.69255658 & 0.74587593\end{array}$

C $\quad 2.57473862 \quad-1.47737642 \quad 2.00037193$

$\begin{array}{llll}\mathrm{H} & 2.53496562 & 2.45530658 & 1.99932393\end{array}$

$\begin{array}{llll}\text { C } & 2.64489162 & 0.69322758 & 3.25371093\end{array}$

$\mathrm{H} \quad 2.43142262 \quad-1.26653542 \quad 4.15221193$

H $\quad 2.31641362 \quad 1.22422858-0.15601007$

$\begin{array}{llll}\mathrm{H} & 3.74172062 & 0.67084458 & 0.73058993\end{array}$

$\mathrm{H} \quad 2.19407462 \quad-2.50668142 \quad 2.00065493$

H $\quad 3.67039062 \quad-1.53568942 \quad 2.00036393$

H $\quad 3.74177262 \quad 0.67152258 \quad 3.26896293$

$\mathrm{H} \quad 2.31650162 \quad 1.22537858 \quad 4.15532693$

C $\quad-3.56695165-1.21742065 \quad-2.26827696$

C $-2.17297226-1.17832462 \quad-2.26879382$ 


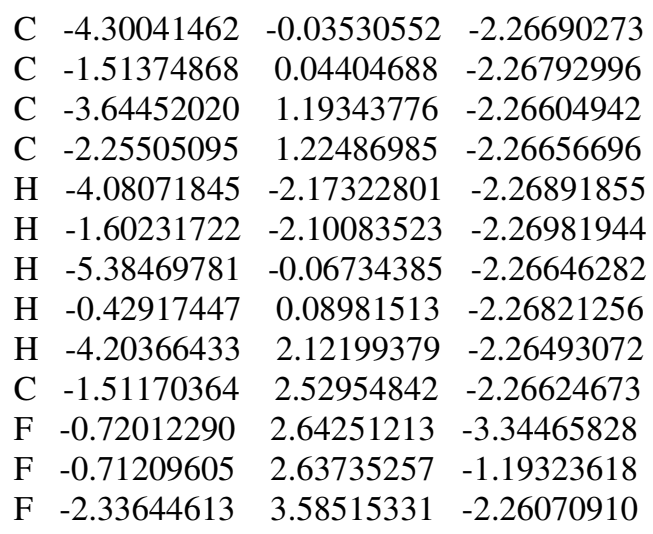

4AdPh_pi_4CF3Ph_pi_300
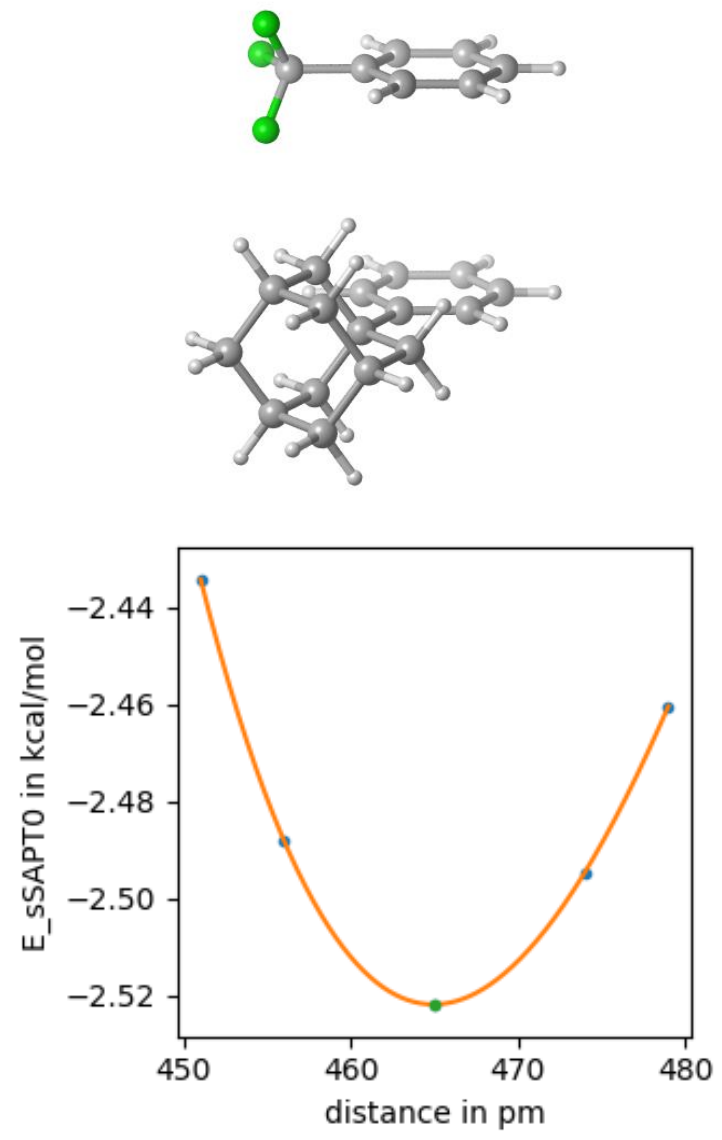

51

4AdPh_pi_4CF3Ph_pi_300-464

H $\quad-5.40699838 \quad-0.07046942 \quad 1.99997493$

$\begin{array}{llll}\text { C } & -4.32228838 & -0.03889342 & 1.99999093\end{array}$

$\begin{array}{llll}\text { C } & -3.58278938 & -1.21969542 & 1.99999893\end{array}$

$\begin{array}{llll}\text { C } & -3.65363938 & 1.17869058 & 1.99998993\end{array}$

$\begin{array}{llll}\text { C } & -2.19430138 & -1.17393642 & 2.00001793\end{array}$

$\begin{array}{llll}\mathrm{H} & -4.08913238 & -2.17997242 & 1.99998893\end{array}$

C $\quad-2.25972238 \quad 1.21897558 \quad 2.00000593$

$\begin{array}{llll}\mathrm{H} & -4.21490638 & 2.10797258 & 1.99997293\end{array}$

$\begin{array}{llll}\text { C } & -1.50186838 & 0.04520558 & 2.00003493\end{array}$

H $\quad-1.63822938-2.10748042 \quad 2.00001193$

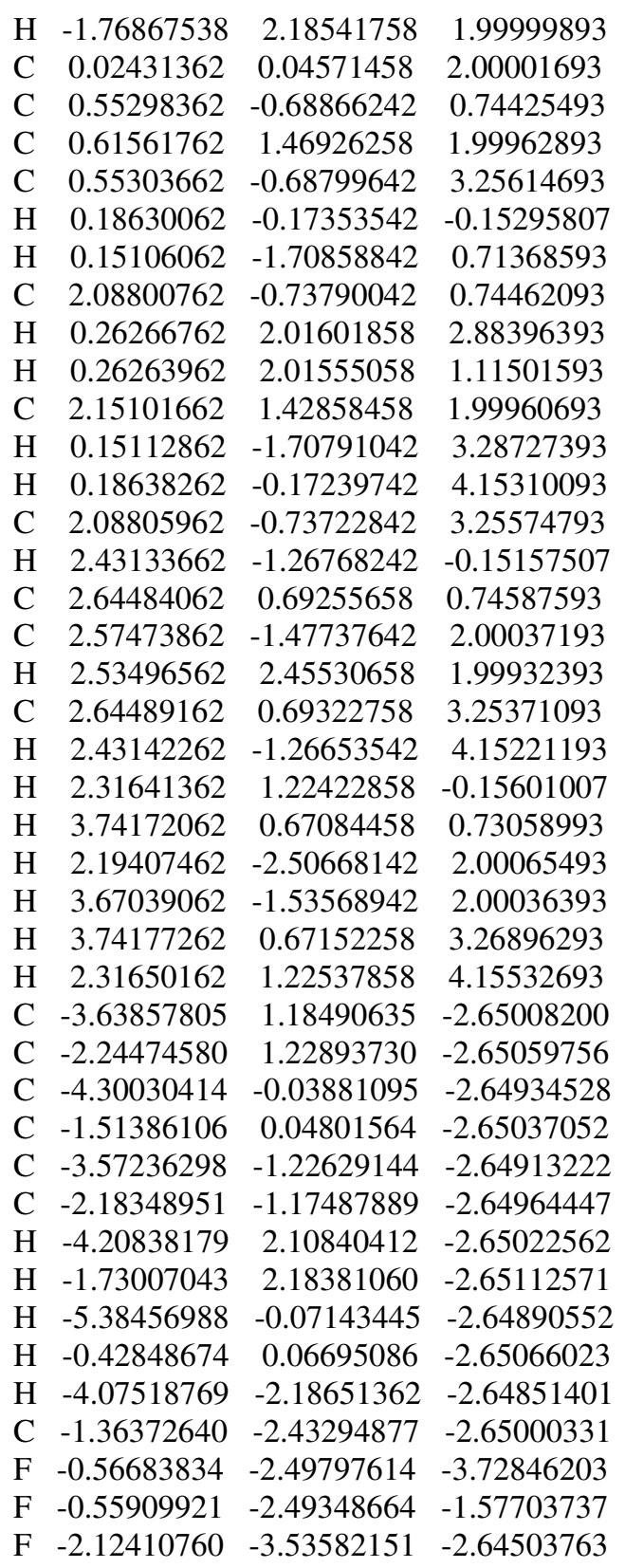

4AdPh_pi_4CF3Ph_pi_60
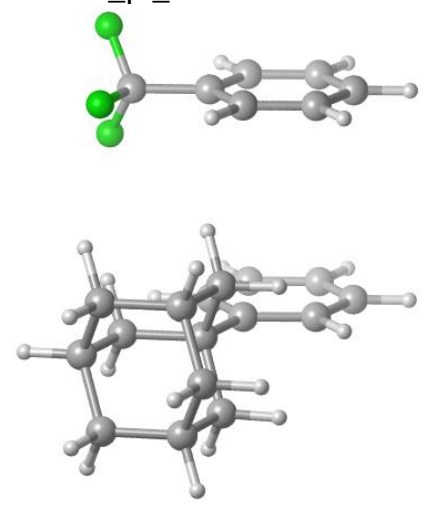


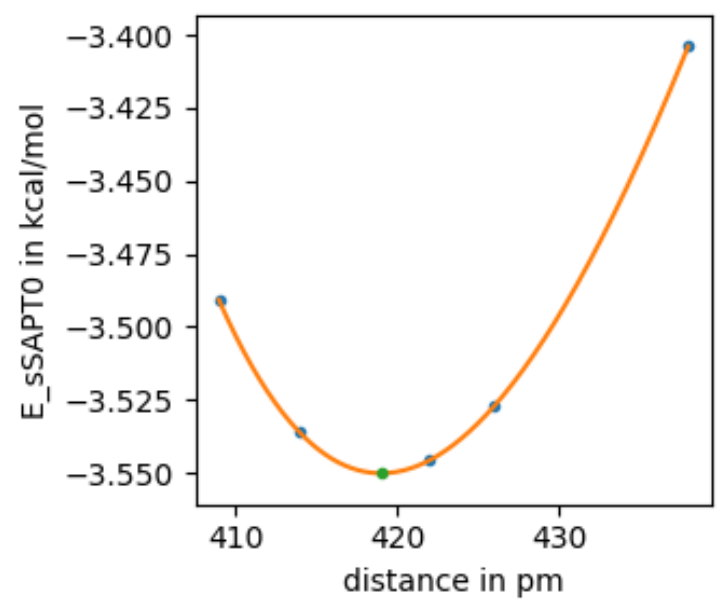

51

4AdPh_pi_4CF3Ph_pi_60-419

H $\quad-5.40699838-0.07046942$

C $-4.32228838-0.03889342$

$\begin{array}{llll}\text { C } & -3.58278938 & -1.21969542 & 1.99999893\end{array}$

$\begin{array}{llll}\text { C } & -3.65363938 & 1.17869058 & 1.99998993\end{array}$

$\begin{array}{llll}\text { C } & -2.19430138 & -1.17393642 & 2.00001793\end{array}$

$\begin{array}{llll}\mathrm{H} & -4.08913238 & -2.17997242 & 1.99998893\end{array}$

$\begin{array}{llll}\text { C } & -2.25972238 & 1.21897558 & 2.00000593\end{array}$

$\begin{array}{llll}\mathrm{H} & -4.21490638 & 2.10797258 & 1.99997293\end{array}$

$\begin{array}{llll}\text { C } & -1.50186838 & 0.04520558 & 2.00003493\end{array}$

$\begin{array}{llll}\mathrm{H} & -1.63822938 & -2.10748042 & 2.00001193\end{array}$

$\begin{array}{llll}\mathrm{H} & -1.76867538 & 2.18541758 & 1.99999893\end{array}$

$\begin{array}{llll}\text { C } & 0.02431362 & 0.04571458 & 2.00001693\end{array}$

$\begin{array}{llll}\text { C } & 0.55298362 & -0.68866242 & 0.74425493\end{array}$

$\begin{array}{llll}\text { C } & 0.61561762 & 1.46926258 & 1.99962893\end{array}$

C $\quad 0.55303662 \quad-0.68799642 \quad 3.25614693$

H $\quad 0.18630062 \quad-0.17353542 \quad-0.15295807$

$\begin{array}{llll}\mathrm{H} & 0.15106062 & -1.70858842 & 0.71368593\end{array}$

C $\quad 2.08800762-0.73790042 \quad 0.74462093$

$\mathrm{H} \quad 0.26266762 \quad 2.01601858 \quad 2.88396393$

$\begin{array}{llll}\mathrm{H} & 0.26263962 & 2.01555058 & 1.11501593\end{array}$

C $\quad 2.15101662 \quad 1.42858458 \quad 1.99960693$

H $\quad 0.15112862 \quad-1.70791042 \quad 3.28727393$

H $\quad 0.18638262-0.17239742 \quad 4.15310093$

C $\quad 2.08805962 \quad-0.73722842 \quad 3.25574793$

$\begin{array}{llll}\mathrm{H} & 2.43133662 & -1.26768242 & -0.15157507\end{array}$

$\begin{array}{llll}\text { C } & 2.64484062 & 0.69255658 & 0.74587593\end{array}$

$\begin{array}{llll}\text { C } & 2.57473862 & -1.47737642 & 2.00037193\end{array}$

$\begin{array}{llll}\mathrm{H} & 2.53496562 & 2.45530658 & 1.99932393\end{array}$

C $\quad 2.64489162 \quad 0.69322758 \quad 3.25371093$

H $\quad 2.43142262-1.26653542 \quad 4.15221193$

H $\quad 2.31641362 \quad 1.22422858 \quad-0.15601007$

H $\quad 3.741720620 .67084458 \quad 0.73058993$

$\begin{array}{llll}\mathrm{H} & 2.19407462 & -2.50668142 & 2.00065493\end{array}$

H $\quad 3.67039062-1.53568942 \quad 2.00036393$

H $\quad 3.74177262 \quad 0.67152258 \quad 3.26896293$

H $\quad 2.31650162 \quad 1.22537858 \quad 4.15532693$

C $\quad-3.57114105-1.21518629-2.19162639$

$\begin{array}{llll}\text { C } & -4.30618897 & -0.03010746 & -2.19185570\end{array}$
C $-2.18050796-1.17639964-2.19053905$

$\begin{array}{llll}\text { C } & -3.64892327 & 1.19331790 & -2.19099157\end{array}$

$\begin{array}{llll}\text { C } & -1.51609036 & 0.04775591 & -2.18968690\end{array}$

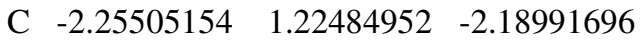

$\mathrm{H} \quad-4.08601164-2.17039952-2.19226784$

H $\quad-5.39047103 \quad-0.06182187-2.19265780$

H $\quad-1.61012241-2.09908971-2.19032263$

$\begin{array}{llll}\mathrm{H} & -4.20800874 & 2.12381210 & -2.19105218\end{array}$

$\begin{array}{lllll}\mathrm{H} & -0.43310138 & 0.09240770 & -2.18878998\end{array}$

C $\quad-1.57541258 \quad 2.56382012-2.18958812$

F $\quad-1.91728112 \quad 3.28688092 \quad-3.26784659$

F $-1.92555765 \quad 3.29049692-1.11642229$

$\begin{array}{llll}\text { F } & -0.24010740 & 2.45674501 & -2.18434223\end{array}$

4AdPh_pi_4ClPh_pi_300
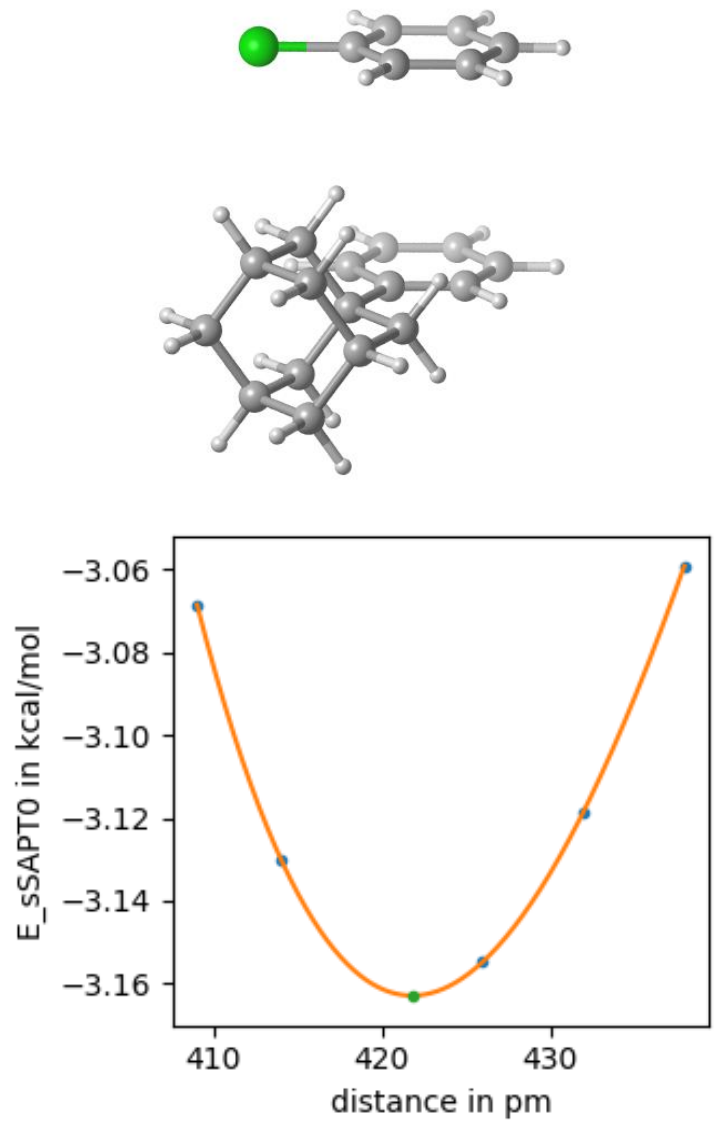

48

4AdPh_pi_4ClPh_pi_300-421

H $\quad-5.40700607 \quad-0.07073463 \quad 2.99997489$

$\begin{array}{llll}\text { C } & -4.32229607 & -0.03915863 & 2.99999089\end{array}$

$\begin{array}{llll}\text { C } & -3.58279707 & -1.21996063 & 2.99999889\end{array}$

$\begin{array}{llll}\text { C } & -3.65364707 & 1.17842537 & 2.99998989\end{array}$

$\begin{array}{llll}\text { C } & -2.19430907 & -1.17420163 & 3.00001789\end{array}$

$\begin{array}{llll}\mathrm{H} & -4.08914007 & -2.18023763 & 2.99998889\end{array}$

$\begin{array}{llll}\text { C } & -2.25973007 & 1.21871037 & 3.00000589\end{array}$

$\begin{array}{llll}\mathrm{H} & -4.21491407 & 2.10770737 & 2.99997289\end{array}$

$\begin{array}{llll}\text { C } & -1.50187607 & 0.04494037 & 3.00003489\end{array}$

H $\quad-1.63823707 \quad-2.10774563 \quad 3.00001189$ 


$\begin{array}{lrrr}\mathrm{H} & -1.76868307 & 2.18515237 & 2.99999889 \\ \mathrm{C} & 0.02430593 & 0.04544937 & 3.00001689 \\ \mathrm{C} & 0.55297593 & -0.68892763 & 1.74425489 \\ \mathrm{C} & 0.61560993 & 1.46899737 & 2.99962889 \\ \mathrm{C} & 0.55302893 & -0.68826163 & 4.25614689 \\ \mathrm{H} & 0.18629293 & -0.17380063 & 0.84704189 \\ \mathrm{H} & 0.15105293 & -1.70885363 & 1.71368589 \\ \mathrm{C} & 2.08799993 & -0.73816563 & 1.74462089 \\ \mathrm{H} & 0.26265993 & 2.01575337 & 3.88396389 \\ \mathrm{H} & 0.26263193 & 2.01528537 & 2.11501589 \\ \mathrm{C} & 2.15100893 & 1.42831937 & 2.99960689 \\ \mathrm{H} & 0.15112093 & -1.70817563 & 4.28727389 \\ \mathrm{H} & 0.18637493 & -0.17266263 & 5.15310089 \\ \mathrm{C} & 2.08805193 & -0.73749363 & 4.25574789 \\ \mathrm{H} & 2.43132893 & -1.26794763 & 0.84842489 \\ \mathrm{C} & 2.64483293 & 0.69229137 & 1.74587589 \\ \mathrm{C} & 2.57473093 & -1.47764163 & 3.00037189 \\ \mathrm{H} & 2.53495793 & 2.45504137 & 2.99932389 \\ \mathrm{C} & 2.64488393 & 0.69296237 & 4.25371089 \\ \mathrm{H} & 2.43141493 & -1.26680063 & 5.15221189 \\ \mathrm{H} & 2.31640593 & 1.22396337 & 0.84398989 \\ \mathrm{H} & 3.74171293 & 0.67057937 & 1.73058989 \\ \mathrm{H} & 2.19406693 & -2.50694663 & 3.00065489 \\ \mathrm{H} & 3.67038293 & -1.53595463 & 3.00036389 \\ \mathrm{H} & 3.74176493 & 0.67125737 & 4.26896289 \\ \mathrm{H} & 2.31649393 & 1.22511337 & 5.15532689 \\ \mathrm{C} & -3.64300761 & 1.18715303 & -1.21767906 \\ \mathrm{C} & -2.25142282 & 1.22671902 & -1.21765771 \\ \mathrm{C} & -4.30114124 & -0.03960381 & -1.21800854 \\ \mathrm{C} & -1.51266693 & 0.04685723 & -1.21796679 \\ \mathrm{C} & -3.57661769 & -1.22825956 & -1.21831824 \\ \mathrm{C} & -2.18748482 & -1.16880933 & -1.21829264 \\ \mathrm{H} & -4.21313658 & 2.10998460 & -1.21743821 \\ \mathrm{H} & -1.73310124 & 2.17990203 & -1.21740181 \\ \mathrm{H} & -5.38550690 & -0.07656718 & -1.21802628 \\ \mathrm{H} & -0.42885003 & 0.06256799 & -1.21795573 \\ \mathrm{H} & -4.07568793 & -2.19046263 & -1.21857617 \\ \mathrm{Cl} & -1.26987710 & -2.65408270 & -1.21868163\end{array}$

4AdPh_pi_4ClPh_pi_60
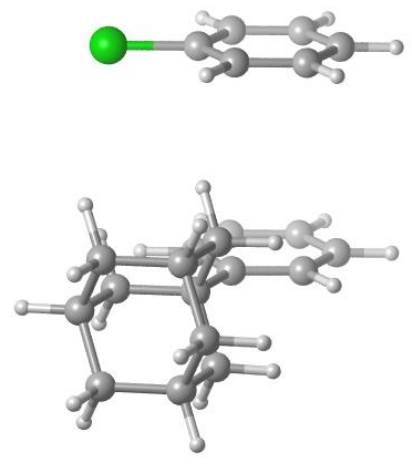

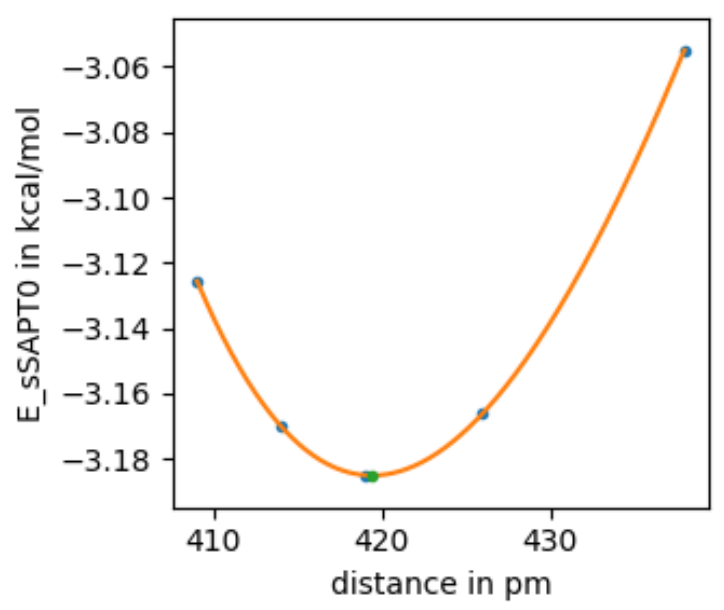

48

4AdPh_pi_4CIPh_pi_60-419

H $\quad-5.40700607 \quad-0.07073463 \quad 2.99997489$

$\begin{array}{llll}\text { C } & -4.32229607 & -0.03915863 & 2.99999089\end{array}$

$\begin{array}{llll}\text { C } & -3.58279707 & -1.21996063 & 2.99999889\end{array}$

$\begin{array}{llll}\text { C } & -3.65364707 & 1.17842537 & 2.99998989\end{array}$

$\begin{array}{llll}\text { C } & -2.19430907 & -1.17420163 & 3.00001789\end{array}$

$\begin{array}{llll}\mathrm{H} & -4.08914007 & -2.18023763 & 2.99998889\end{array}$

$\begin{array}{llll}\text { C } & -2.25973007 & 1.21871037 & 3.00000589\end{array}$

$\begin{array}{llll}\mathrm{H} & -4.21491407 & 2.10770737 & 2.99997289\end{array}$

$\begin{array}{llll}\text { C } & -1.50187607 & 0.04494037 & 3.00003489\end{array}$

$\begin{array}{llll}\mathrm{H} & -1.63823707 & -2.10774563 & 3.00001189\end{array}$

$\begin{array}{llll}\mathrm{H} & -1.76868307 & 2.18515237 & 2.99999889\end{array}$

$\begin{array}{llll}\text { C } & 0.02430593 & 0.04544937 & 3.00001689\end{array}$

$\begin{array}{llll}\text { C } & 0.55297593 & -0.68892763 & 1.74425489\end{array}$

$\begin{array}{llll}\text { C } & 0.61560993 & 1.46899737 & 2.99962889\end{array}$

$\begin{array}{llll}\text { C } & 0.55302893 & -0.68826163 & 4.25614689\end{array}$

$\begin{array}{llll}\mathrm{H} & 0.18629293 & -0.17380063 & 0.84704189\end{array}$

$\begin{array}{llll}\mathrm{H} & 0.15105293 & -1.70885363 & 1.71368589\end{array}$

$\begin{array}{llll}\text { C } & 2.08799993 & -0.73816563 & 1.74462089\end{array}$

$\begin{array}{llll}\mathrm{H} & 0.26265993 & 2.01575337 & 3.88396389\end{array}$

$\begin{array}{llll}\mathrm{H} & 0.26263193 & 2.01528537 & 2.11501589\end{array}$

$\begin{array}{llll}\text { C } & 2.15100893 & 1.42831937 & 2.99960689\end{array}$

$\begin{array}{llll}\mathrm{H} & 0.15112093 & -1.70817563 & 4.28727389\end{array}$

$\begin{array}{llll}\mathrm{H} & 0.18637493 & -0.17266263 & 5.15310089\end{array}$

$\begin{array}{llll}\text { C } & 2.08805193 & -0.73749363 & 4.25574789\end{array}$

$\begin{array}{llll}\mathrm{H} & 2.43132893 & -1.26794763 & 0.84842489\end{array}$

$\begin{array}{llll}\text { C } & 2.64483293 & 0.69229137 & 1.74587589\end{array}$

$\begin{array}{llll}\text { C } & 2.57473093 & -1.47764163 & 3.00037189\end{array}$

$\begin{array}{llll}\mathrm{H} & 2.53495793 & 2.45504137 & 2.99932389\end{array}$

$\begin{array}{llll}\text { C } & 2.64488393 & 0.69296237 & 4.25371089\end{array}$

$\mathrm{H} \quad 2.43141493 \quad-1.26680063 \quad 5.15221189$

$\begin{array}{llll}\mathrm{H} & 2.31640593 & 1.22396337 & 0.84398989\end{array}$

$\begin{array}{llll}\mathrm{H} & 3.74171293 & 0.67057937 & 1.73058989\end{array}$

H $\quad 2.19406693 \quad-2.50694663 \quad 3.00065489$

H $3.67038293 \quad-1.53595463 \quad 3.00036389$

$\begin{array}{llll}\mathrm{H} & 3.74176493 & 0.67125737 & 4.26896289\end{array}$

H $\quad 2.31649393 \quad 1.22511337 \quad 5.15532689$

C $-3.57121411 \quad-1.22059061 \quad-1.19446166$

C $-4.30127166-0.03522586-1.19415275$ 


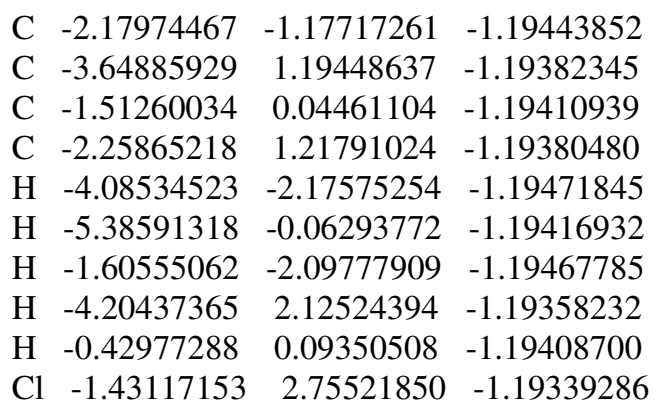

4AdPh_pi_4FPh_pi_300
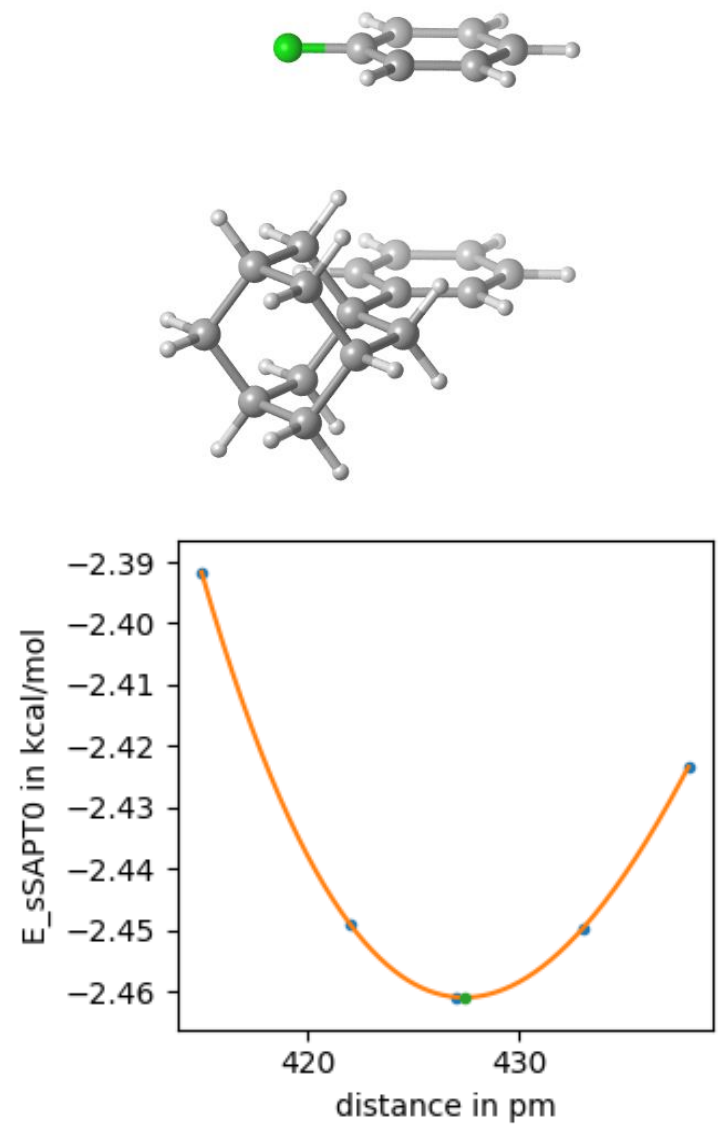

48

4AdPh_pi_4FPh_pi_300-427

H $\quad-5.40699069-0.07020421$

C $-4.32228069-0.03862821$

C $-3.58278169-1.21943021$

$\begin{array}{lll}\text { C } & -3.65363169 & 1.17895579\end{array}$

C $-2.19429369-1.17367121$

H $\quad-4.08912469-2.17970721$

C $\quad-2.25971469 \quad 1.21924079$

H $\quad-4.214898692 .10823779$

C -1.501860690 .04547079$

H $\quad-1.63822169-2.10721521$

H $\quad-1.768667692 .18568279$

$\begin{array}{lll}\text { C } & 0.02432131 & 0.04597979\end{array}$

C $\quad 0.55299131 \quad-0.68839721$

0.99997496

0.99999096

0.99999896

0.99998996

1.00001796

0.99998896

1.00000596

0.99997296

1.00003496

1.00001196

0.99999896

1.00001696

$-0.25574504$

$\begin{array}{lrrr}\mathrm{C} & 0.61562531 & 1.46952779 & 0.99962896 \\ \mathrm{C} & 0.55304431 & -0.68773121 & 2.25614696 \\ \mathrm{H} & 0.18630831 & -0.17327021 & -1.15295804 \\ \mathrm{H} & 0.15106831 & -1.70832321 & -0.28631404 \\ \mathrm{C} & 2.08801531 & -0.73763521 & -0.25537904 \\ \mathrm{H} & 0.26267531 & 2.01628379 & 1.88396396 \\ \mathrm{H} & 0.26264731 & 2.01581579 & 0.11501596 \\ \mathrm{C} & 2.15102431 & 1.42884979 & 0.99960696 \\ \mathrm{H} & 0.15113631 & -1.70764521 & 2.28727396 \\ \mathrm{H} & 0.18639031 & -0.17213221 & 3.15310096 \\ \mathrm{C} & 2.08806731 & -0.73696321 & 2.25574796 \\ \mathrm{H} & 2.43134431 & -1.26741721 & -1.15157504 \\ \mathrm{C} & 2.64484831 & 0.69282179 & -0.25412404 \\ \mathrm{C} & 2.57474631 & -1.47711121 & 1.00037196 \\ \mathrm{H} & 2.53497331 & 2.45557179 & 0.99932396 \\ \mathrm{C} & 2.64489931 & 0.69349279 & 2.25371096 \\ \mathrm{H} & 2.43143031 & -1.26627021 & 3.15221196 \\ \mathrm{H} & 2.31642131 & 1.22449379 & -1.15601004 \\ \mathrm{H} & 3.74172831 & 0.67110979 & -0.26941004 \\ \mathrm{H} & 2.19408231 & -2.50641621 & 1.00065496 \\ \mathrm{H} & 3.67039831 & -1.53542421 & 1.00036396 \\ \mathrm{H} & 3.74178031 & 0.67178779 & 2.26896296 \\ \mathrm{H} & 2.31650931 & 1.22564379 & 3.15532696 \\ \mathrm{C} & -3.64213592 & 1.18631450 & -3.27289140 \\ \mathrm{C} & -2.24963143 & 1.22600915 & -3.27326613 \\ \mathrm{C} & -4.30079779 & -0.04120644 & -3.27362359 \\ \mathrm{C} & -1.51029813 & 0.04666224 & -3.27437721 \\ \mathrm{C} & -3.57699427 & -1.23014871 & -3.27472552 \\ \mathrm{C} & -2.19238785 & -1.16030202 & -3.27508032 \\ \mathrm{H} & -4.21212102 & 2.10891587 & -3.27203131 \\ \mathrm{H} & -1.73238312 & 2.17978492 & -3.27270810 \\ \mathrm{H} & -5.38521326 & -0.07694216 & -3.27333275 \\ \mathrm{H} & -0.42645366 & 0.04564111 & -3.27470255 \\ \mathrm{H} & -4.06111830 & -2.19986189 & -3.27531088 \\ \mathrm{~F} & -1.48716866 & -2.30179944 & -3.27614729\end{array}$

4AdPh_pi_4FPh_pi_60
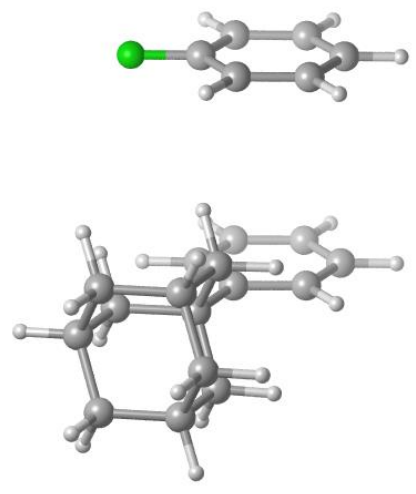


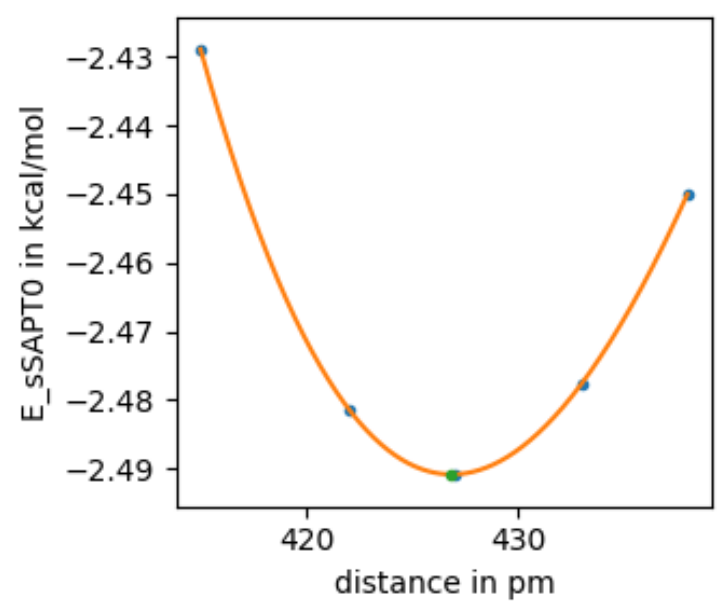

$\begin{array}{rrrr}\text { C } & -2.17803249 & -1.17526489 & -3.26851683 \\ \text { C } & -3.64937864 & 1.19744458 & -3.26869726 \\ \text { C } & -1.51027981 & 0.04603876 & -3.26897996 \\ \text { C } & -2.26307194 & 1.21021986 & -3.26906098 \\ \text { H } & -4.08443173 & -2.17353012 & -3.26777487 \\ \text { H } & -5.38567491 & -0.06144841 & -3.26793939 \\ \text { H } & -1.60487678 & -2.09652847 & -3.26844810 \\ \text { H } & -4.19041648 & 2.13659209 & -3.26878576 \\ \text { H } & -0.42842144 & 0.11163178 & -3.26927871 \\ \text { F } & -1.62711555 & 2.39170658 & -3.26951240\end{array}$

4AdPh_pi_4MePh_pi_300

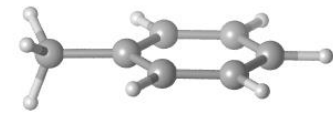

48

4AdPh_pi_4FPh_pi_60-426

H $\quad-5.40699069-0.070204210 .99997496$

$\begin{array}{llll}\text { C } & -4.32228069 & -0.03862821 & 0.99999096\end{array}$

$\begin{array}{llll}\text { C } & -3.58278169 & -1.21943021 & 0.99999896\end{array}$

$\begin{array}{llll}\text { C } & -3.65363169 & 1.17895579 & 0.99998996\end{array}$

$\begin{array}{llll}\text { C } & -2.19429369 & -1.17367121 & 1.00001796\end{array}$

H $-4.08912469-2.179707210 .99998896$

$\begin{array}{llll}\text { C } & -2.25971469 & 1.21924079 & 1.00000596\end{array}$

$\begin{array}{cccc}\mathrm{H} & -4.21489869 & 2.10823779 & 0.99997296\end{array}$

$\begin{array}{llll}\text { C } & -1.50186069 & 0.04547079 & 1.00003496\end{array}$

$\begin{array}{llll}\mathrm{H} & -1.63822169 & -2.10721521 & 1.00001196\end{array}$

$\begin{array}{llll}\mathrm{H} & -1.76866769 & 2.18568279 & 0.99999896\end{array}$

$\begin{array}{llll}\text { C } & 0.02432131 & 0.04597979 & 1.00001696\end{array}$

$\begin{array}{llll}\text { C } & 0.55299131 & -0.68839721 & -0.25574504\end{array}$

$\begin{array}{llll}\text { C } & 0.61562531 & 1.46952779 & 0.99962896\end{array}$

$\begin{array}{llll}\text { C } & 0.55304431 & -0.68773121 & 2.25614696\end{array}$

H $\quad 0.18630831 \quad-0.17327021 \quad-1.15295804$

$\begin{array}{lllll}\mathrm{H} & 0.15106831 & -1.70832321 & -0.28631404\end{array}$

C $\quad 2.08801531 \quad-0.73763521 \quad-0.25537904$

$\begin{array}{llll}\mathrm{H} & 0.26267531 & 2.01628379 & 1.88396396\end{array}$

$\begin{array}{llll}\mathrm{H} & 0.26264731 & 2.01581579 & 0.11501596\end{array}$

$\begin{array}{llll}\text { C } & 2.15102431 & 1.42884979 & 0.99960696\end{array}$

H $\quad 0.15113631 \quad-1.70764521 \quad 2.28727396$

H $\quad 0.18639031 \quad-0.17213221 \quad 3.15310096$

$\begin{array}{llll}\text { C } & 2.08806731 & -0.73696321 & 2.25574796\end{array}$

H $\quad 2.43134431 \quad-1.26741721 \quad-1.15157504$

$\begin{array}{llll}\text { C } & 2.64484831 & 0.69282179 & -0.25412404\end{array}$

C $\quad 2.57474631 \quad-1.47711121 \quad 1.00037196$

$\begin{array}{llll}\mathrm{H} & 2.53497331 & 2.45557179 & 0.99932396\end{array}$

$\begin{array}{llll}\text { C } & 2.64489931 & 0.69349279 & 2.25371096\end{array}$

H $\quad 2.43143031 \quad-1.26627021 \quad 3.15221196$

H $\quad 2.31642131 \quad 1.22449379 \quad-1.15601004$

H $\quad 3.74172831 \quad 0.67110979-0.26941004$

$\mathrm{H} \quad 2.19408231 \quad-2.50641621 \quad 1.00065496$

H $\quad 3.67039831 \quad-1.53542421 \quad 1.00036396$

H $\quad 3.74178031 \quad 0.67178779 \quad 2.26896296$

$\begin{array}{llll}\mathrm{H} & 2.31650931 & 1.22564379 & 3.15532696\end{array}$

C $\quad-3.57042788-1.21860763-3.26813746$

$\begin{array}{llll}\text { C } & -4.30105660 & -0.03251058 & -3.26822447\end{array}$
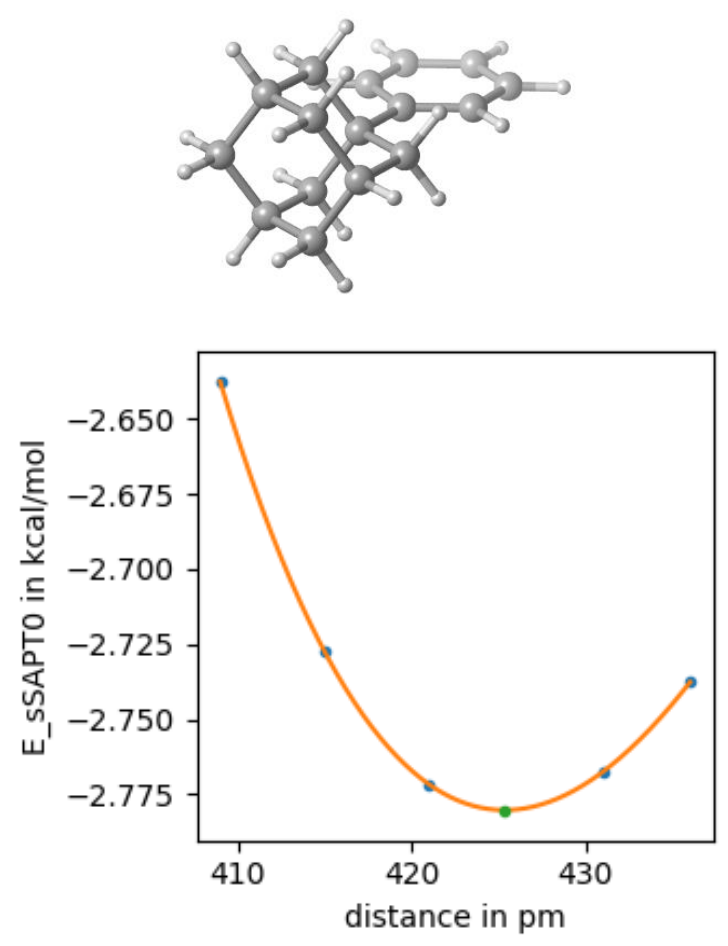

51

4AdPh_pi_4MePh_pi_300-425

$\mathrm{H} \quad-5.40698300 \quad-0.06993900 \quad-0.00002500$

C $-4.32227300 \quad-0.03836300 \quad-0.00000900$

$\begin{array}{llll}\text { C } & -3.58277400 & -1.21916500 & -0.00000100\end{array}$

C $\quad-3.65362400 \quad 1.17922100 \quad-0.00001000$

$\begin{array}{llll}\text { C } & -2.19428600 & -1.17340600 & 0.00001800\end{array}$

$\mathrm{H} \quad-4.08911700 \quad-2.17944200 \quad-0.00001100$

$\begin{array}{llll}\text { C } & -2.25970700 & 1.21950600 & 0.00000600\end{array}$

$\begin{array}{llll}\mathrm{H} & -4.21489100 & 2.10850300 & -0.00002700\end{array}$

C $-1.50185300 \quad 0.04573600 \quad 0.00003500$

$\mathrm{H} \quad-1.63821400 \quad-2.10695000 \quad 0.00001200$

$\begin{array}{llll}\mathrm{H} & -1.76866000 & 2.18594800 & -0.00000100\end{array}$

$\begin{array}{llll}\text { C } & 0.02432900 & 0.04624500 & 0.00001700\end{array}$

$\begin{array}{llll}\text { C } & 0.55299900 & -0.68813200 & -1.25574500\end{array}$ 


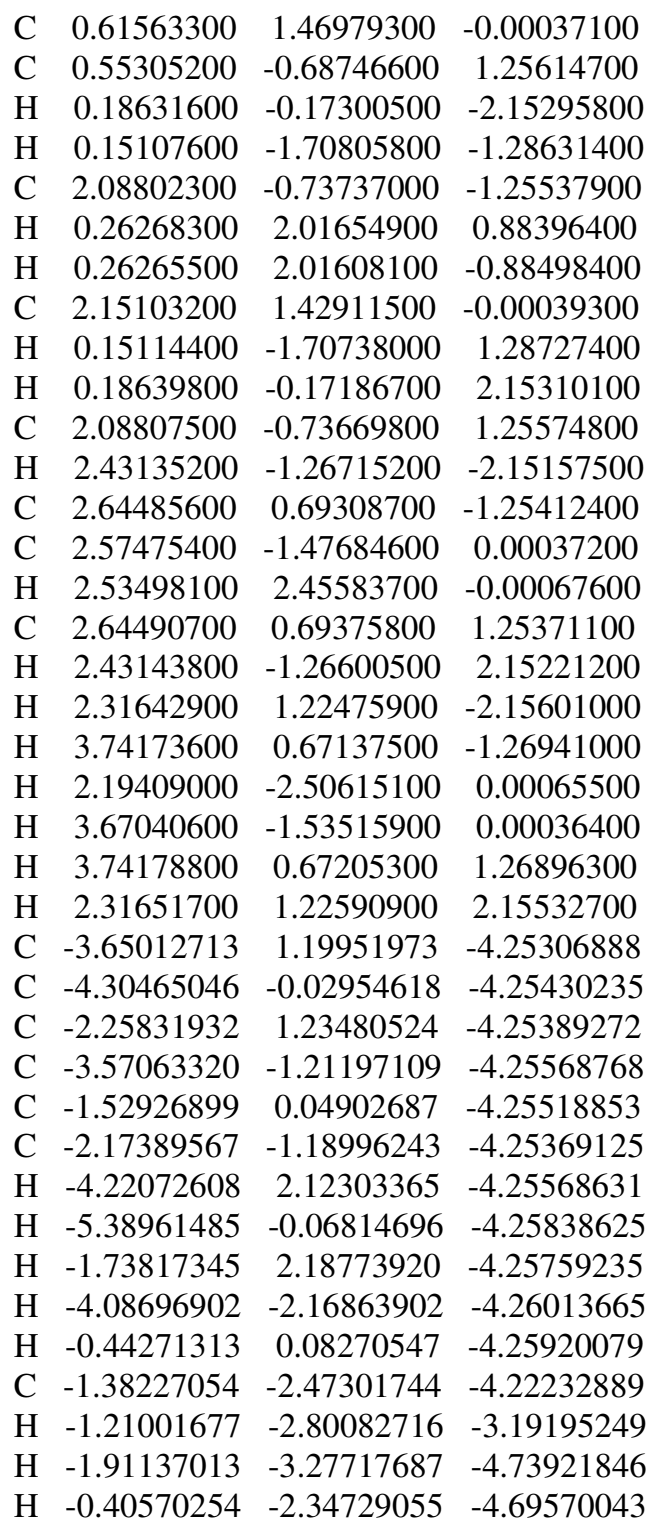

4AdPh_pi_4MePh_pi_60
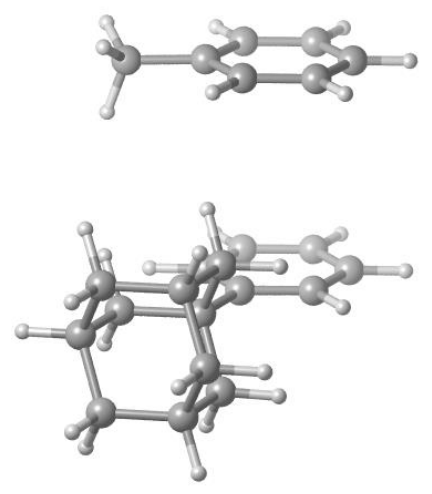

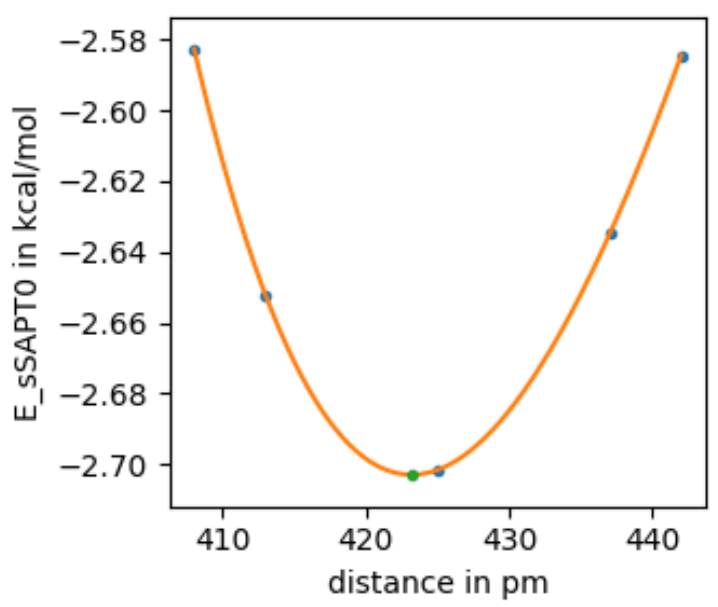

51

4AdPh_pi_4MePh_pi_60-423

$\mathrm{H} \quad-5.40698300 \quad-0.06993900-0.00002500$

$\begin{array}{llll}\text { C } & -4.32227300 & -0.03836300 & -0.00000900\end{array}$

C $\quad-3.58277400 \quad-1.21916500 \quad-0.00000100$

$\begin{array}{llll}\text { C } & -3.65362400 & 1.17922100 & -0.00001000\end{array}$

$\begin{array}{llll}\text { C } & -2.19428600 & -1.17340600 & 0.00001800\end{array}$

$\mathrm{H} \quad-4.08911700 \quad-2.17944200 \quad-0.00001100$

C $\quad-2.25970700 \quad 1.21950600 \quad 0.00000600$

$\begin{array}{llll}\mathrm{H} & -4.21489100 & 2.10850300 & -0.00002700\end{array}$

$\begin{array}{llll}\text { C } & -1.50185300 & 0.04573600 & 0.00003500\end{array}$

$\mathrm{H} \quad-1.63821400 \quad-2.10695000 \quad 0.00001200$

$\begin{array}{llll}\mathrm{H} & -1.76866000 & 2.18594800 & -0.00000100\end{array}$

$\begin{array}{llll}\text { C } & 0.02432900 & 0.04624500 & 0.00001700\end{array}$

C $\quad 0.55299900 \quad-0.68813200 \quad-1.25574500$

$\begin{array}{llll}\text { C } & 0.61563300 & 1.46979300 & -0.00037100\end{array}$

$\begin{array}{llll}\text { C } & 0.55305200 & -0.68746600 & 1.25614700\end{array}$

H $\quad 0.18631600 \quad-0.17300500 \quad-2.15295800$

H $\quad 0.15107600 \quad-1.70805800 \quad-1.28631400$

C $\quad 2.08802300 \quad-0.73737000 \quad-1.25537900$

$\begin{array}{llll}\mathrm{H} & 0.26268300 & 2.01654900 & 0.88396400\end{array}$

$\begin{array}{llll}\mathrm{H} & 0.26265500 & 2.01608100 & -0.88498400\end{array}$

C $\quad 2.15103200 \quad 1.42911500 \quad-0.00039300$

$\mathrm{H} \quad 0.15114400 \quad-1.70738000 \quad 1.28727400$

$\begin{array}{llll}\mathrm{H} & 0.18639800 & -0.17186700 & 2.15310100\end{array}$

$\begin{array}{llll}\text { C } & 2.08807500 & -0.73669800 & 1.25574800\end{array}$

$\mathrm{H} \quad 2.43135200 \quad-1.26715200 \quad-2.15157500$

$\begin{array}{llll}\text { C } & 2.64485600 & 0.69308700 & -1.25412400\end{array}$

$\begin{array}{llll}\text { C } & 2.57475400 & -1.47684600 & 0.00037200\end{array}$

$\begin{array}{llll}\mathrm{H} & 2.53498100 & 2.45583700 & -0.00067600\end{array}$

$\begin{array}{llll}\text { C } & 2.64490700 & 0.69375800 & 1.25371100\end{array}$

$\mathrm{H} \quad 2.43143800 \quad-1.26600500 \quad 2.15221200$

$\mathrm{H} \quad 2.31642900 \quad 1.22475900 \quad-2.15601000$

$\mathrm{H} \quad 3.74173600 \quad 0.67137500 \quad-1.26941000$

$\mathrm{H} \quad 2.19409000 \quad-2.50615100 \quad 0.00065500$

H $\quad 3.67040600 \quad-1.53515900 \quad 0.00036400$

$\begin{array}{llll}\mathrm{H} & 3.74178800 & 0.67205300 & 1.26896300\end{array}$

$\begin{array}{llll}\mathrm{H} & 2.31651700 & 1.22590900 & 2.15532700\end{array}$

C $\quad-3.57763204-1.23175174 \quad-4.23209592$

C $-2.18596782-1.18405224 \quad-4.23297504$ 


$\begin{array}{rrrr}\text { C } & -4.30409389 & -0.04405327 & -4.23263041 \\ \text { C } & -1.52896614 & 0.04283821 & -4.23372199 \\ \text { C } & -3.64170459 & 1.18021238 & -4.23328757 \\ \text { C } & -2.24639544 & 1.24144324 & -4.23142977 \\ \text { H } & -4.09211842 & -2.18766074 & -4.23521136 \\ \text { H } & -1.61005538 & -2.10435696 & -4.23728001 \\ \text { H } & -5.38943092 & -0.07005919 & -4.23660202 \\ \text { H } & -0.44229846 & 0.07401389 & -4.23789665 \\ \text { H } & -4.21414807 & 2.10435959 & -4.23707643 \\ \text { C } & -1.53105738 & 2.56852579 & -4.19937577 \\ \text { H } & -1.33354127 & 2.88120371 & -3.16882931 \\ \text { H } & -0.56996044 & 2.51259393 & -4.71605543 \\ \text { H } & -2.12810987 & 3.35158019 & -4.67258508\end{array}$

4AdPh_pi_4OMePh_pi1_300
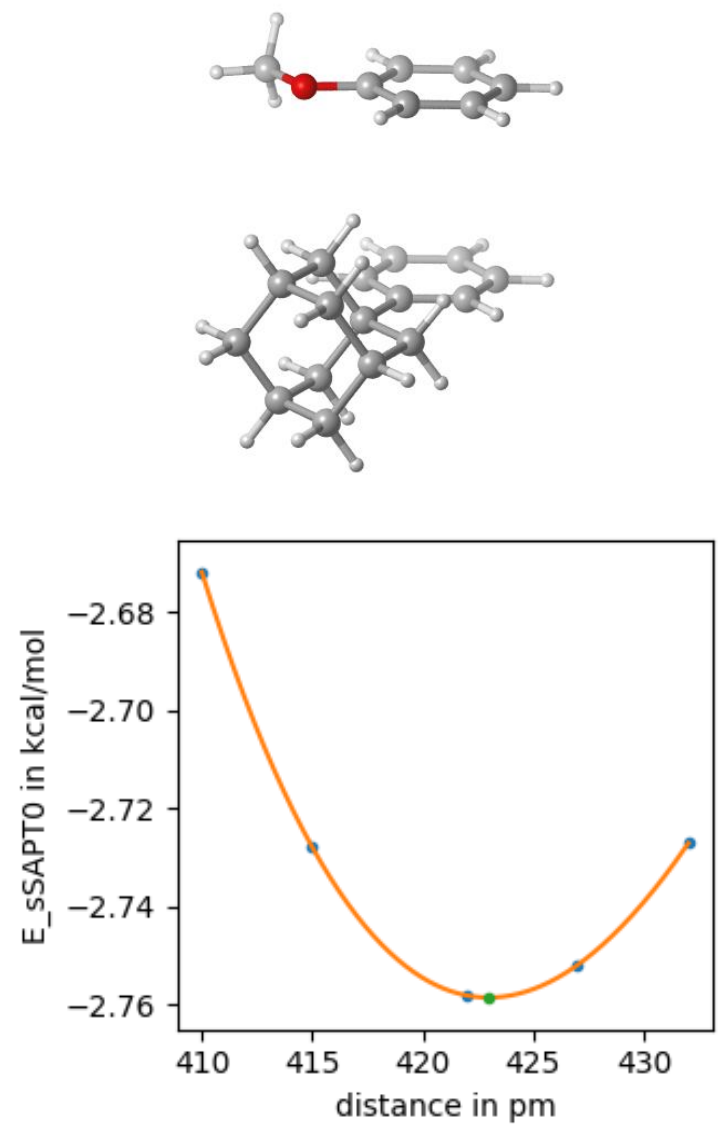

52

4AdPh_pi_4OMePh_pi1_300-422

H $\quad-5.40699069-0.070204210 .99997496$

$\begin{array}{llll}\text { C } & -4.32228069 & -0.03862821 & 0.99999096\end{array}$

$\begin{array}{llll}\text { C } & -3.58278169 & -1.21943021 & 0.99999896\end{array}$

$\begin{array}{llll}\text { C } & -3.65363169 & 1.17895579 & 0.99998996\end{array}$

$\begin{array}{llll}\text { C } & -2.19429369 & -1.17367121 & 1.00001796\end{array}$

H $\quad-4.08912469-2.17970721 \quad 0.99998896$

$\begin{array}{llll}\text { C } & -2.25971469 & 1.21924079 & 1.00000596\end{array}$

$\begin{array}{llll}\text { H } & -4.21489869 & 2.10823779 & 0.99997296\end{array}$

$\begin{array}{llll}\text { C } & -1.50186069 & 0.04547079 & 1.00003496\end{array}$

H $-1.63822169-2.10721521 \quad 1.00001196$

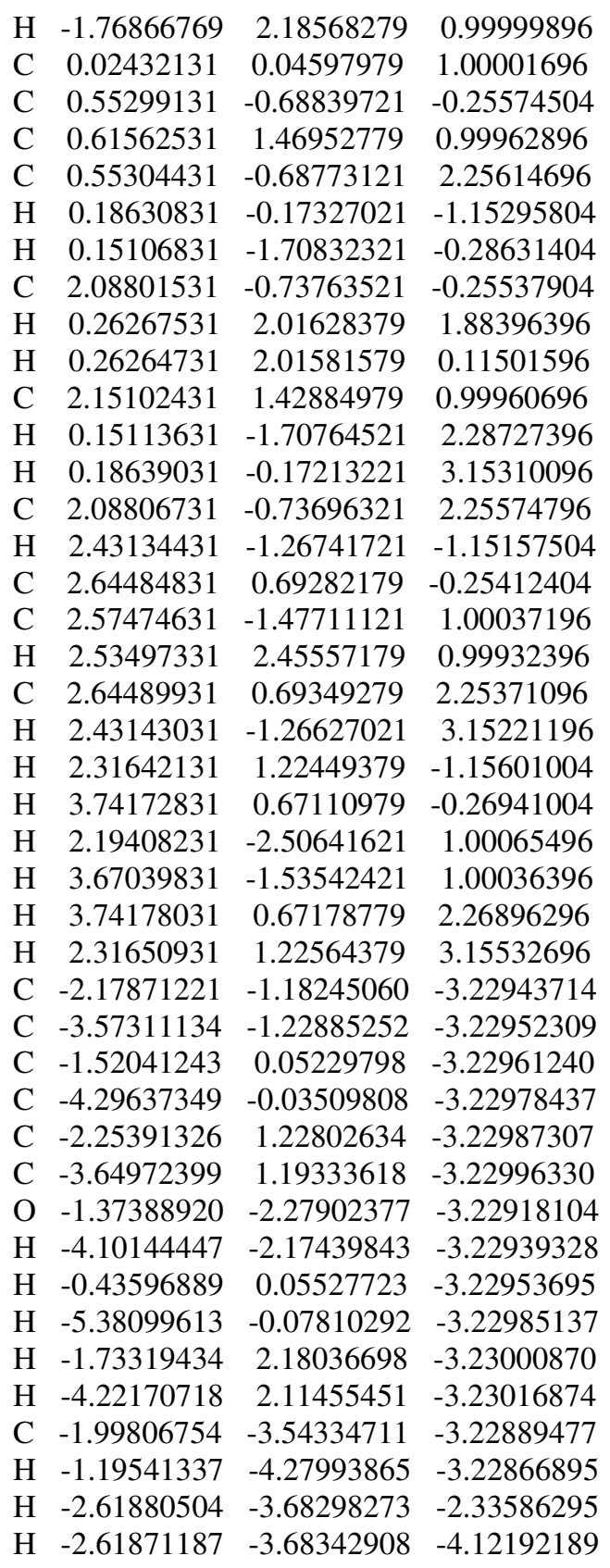

4AdPh_pi_4OMePh_pi1_60
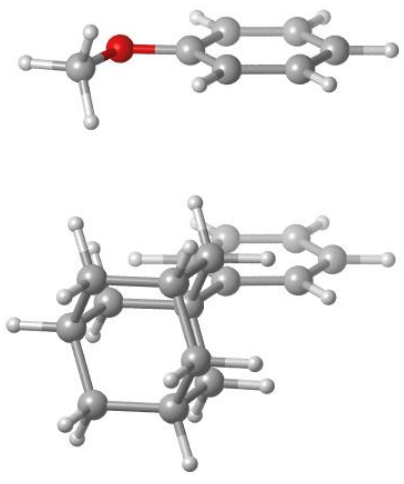


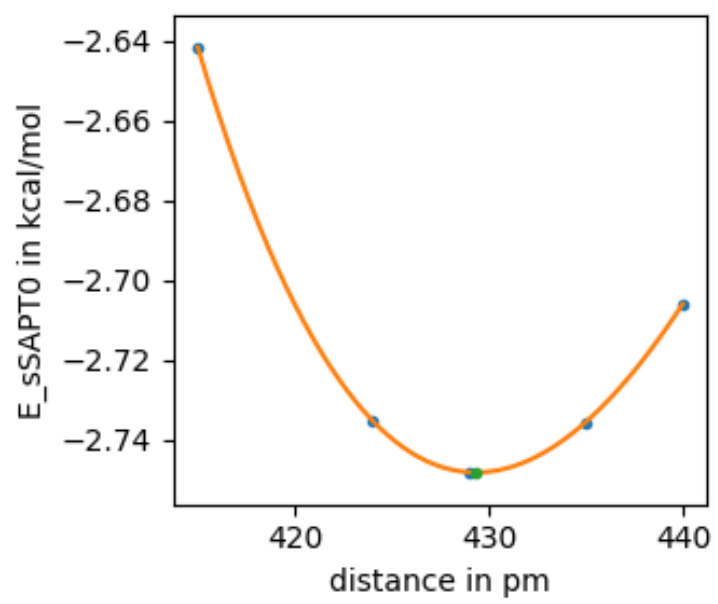

52

4AdPh_pi_4OMePh_pi1_60-429

H $\quad-5.40699069 \quad-0.07020421 \quad 0.99997496$

$\begin{array}{llll}\text { C } & -4.32228069 & -0.03862821 & 0.99999096\end{array}$

$\begin{array}{llll}\text { C } & -3.58278169 & -1.21943021 & 0.99999896\end{array}$

$\begin{array}{llll}\text { C } & -3.65363169 & 1.17895579 & 0.99998996\end{array}$

$\begin{array}{llll}\text { C } & -2.19429369 & -1.17367121 & 1.00001796\end{array}$

H $\quad-4.08912469-2.17970721 \quad 0.99998896$

$\begin{array}{llll}\text { C } & -2.25971469 & 1.21924079 & 1.00000596\end{array}$

H $\quad-4.214898692 .108237790 .99997296$

$\begin{array}{llll}\text { C } & -1.50186069 & 0.04547079 & 1.00003496\end{array}$

H $-1.63822169-2.10721521 \quad 1.00001196$

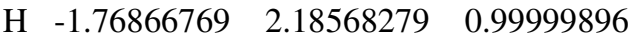

$\begin{array}{llll}\text { C } & 0.02432131 & 0.04597979 & 1.00001696\end{array}$

$\begin{array}{llll}\text { C } & 0.55299131 & -0.68839721 & -0.25574504\end{array}$

$\begin{array}{llll}\text { C } & 0.61562531 & 1.46952779 & 0.99962896\end{array}$

$\begin{array}{llll}\text { C } & 0.55304431 & -0.68773121 & 2.25614696\end{array}$

$\mathrm{H} \quad 0.18630831 \quad-0.17327021 \quad-1.15295804$

H $\quad 0.15106831 \quad-1.70832321 \quad-0.28631404$

$\begin{array}{llll}\text { C } & 2.08801531 & -0.73763521 & -0.25537904\end{array}$

$\begin{array}{llll}\mathrm{H} & 0.26267531 & 2.01628379 & 1.88396396\end{array}$

$\begin{array}{llll}\mathrm{H} & 0.26264731 & 2.01581579 & 0.11501596\end{array}$

$\begin{array}{llll}\text { C } & 2.15102431 & 1.42884979 & 0.99960696\end{array}$

H $\quad 0.15113631 \quad-1.70764521 \quad 2.28727396$

H $\quad 0.18639031 \quad-0.17213221 \quad 3.15310096$

$\begin{array}{llll}\text { C } & 2.08806731 & -0.73696321 & 2.25574796\end{array}$

H $\quad 2.43134431 \quad-1.26741721 \quad-1.15157504$

$\begin{array}{llll}\text { C } & 2.64484831 & 0.69282179 & -0.25412404\end{array}$

C $\quad 2.57474631 \quad-1.47711121 \quad 1.00037196$

$\begin{array}{llll}\mathrm{H} & 2.53497331 & 2.45557179 & 0.99932396\end{array}$

$\begin{array}{llll}\text { C } & 2.64489931 & 0.69349279 & 2.25371096\end{array}$

H $\quad 2.43143031 \quad-1.266270213 .15221196$

H $\quad 2.31642131 \quad 1.22449379-1.15601004$

$\mathrm{H} \quad 3.74172831 \quad 0.67110979 \quad-0.26941004$

H $\quad 2.19408231 \quad-2.50641621 \quad 1.00065496$

H $\quad 3.67039831 \quad-1.53542421 \quad 1.00036396$

$\begin{array}{llll}\mathrm{H} & 3.74178031 & 0.67178779 & 2.26896296\end{array}$

$\begin{array}{llll}\mathrm{H} & 2.31650931 & 1.22564379 & 3.15532696\end{array}$

$\begin{array}{llll}\text { C } & -2.25073901 & 1.23313808 & -3.29256006\end{array}$

$\begin{array}{llll}\text { C } & -1.51335418 & 0.04875404 & -3.29293142\end{array}$ $\begin{array}{llll}\text { C } & -3.64921245 & 1.18586830 & -3.29309114\end{array}$

C $-2.18554468-1.17448633-3.29383332$

$\begin{array}{llll}\text { C } & -4.30067257 & -0.03722598 & -3.29398737\end{array}$

$\begin{array}{llll}\text { C } & -3.57272462 & -1.22868839 & -3.29436806\end{array}$

$\begin{array}{llll}\text { O } & -1.70349039 & 2.47842160 & -3.29168485\end{array}$

H $\quad-0.43032090 \quad 0.06397694 \quad-3.29253441$

H $\quad-4.19401435 \quad 2.12353427 \quad-3.29278033$

H $\quad-1.60599006-2.09229462-3.29411953$

$\begin{array}{llll}\mathrm{H} & -5.38578315 & -0.06244035 & -3.29439461\end{array}$

H $\quad-4.08453141 \quad-2.18464933-3.29507088$

C $-0.29646520 \quad 2.57002874 \quad-3.29102336$

$\mathrm{H} \quad-0.05988538 \quad 3.63344323 \quad-3.29032451$

$\mathrm{H} \quad 0.13461613 \quad 2.10192279 \quad-2.39807056$

$\begin{array}{llll}\mathrm{H} & 0.13538691 & 2.10292529 & -4.18412912\end{array}$

4AdPh_pi_4OMePh_pi2_300
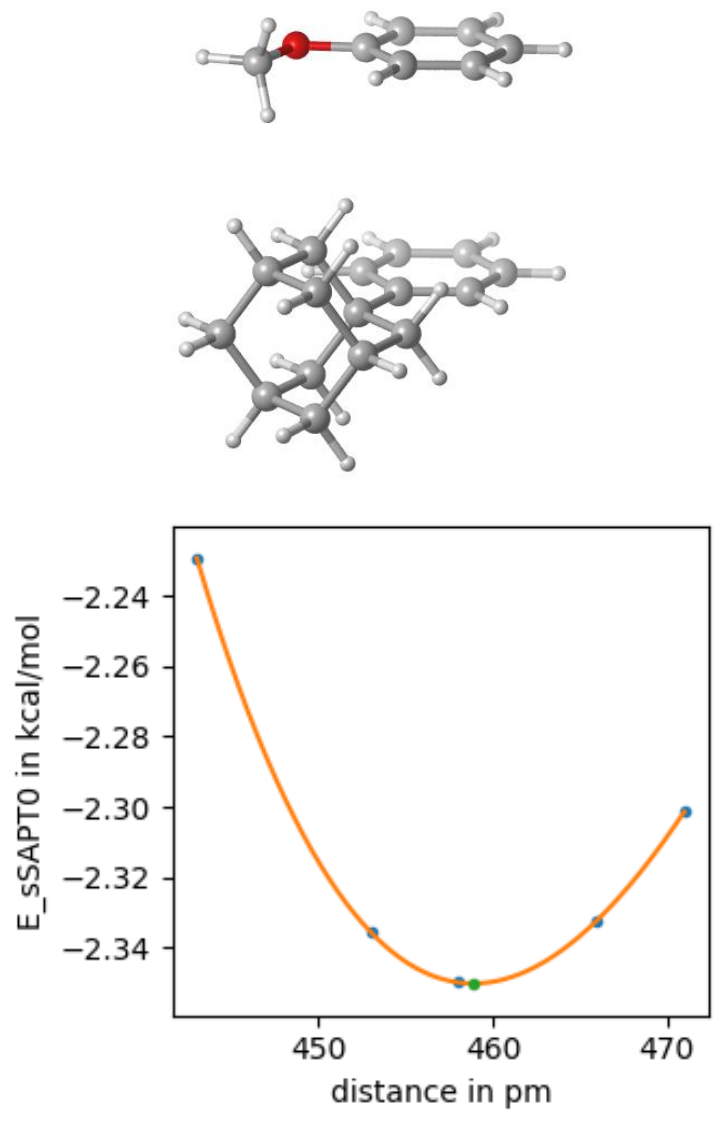

52

4AdPh_pi_4OMePh_pi2_300-458

H $\quad-5.40699069-0.07020421 \quad 0.99997496$

$\begin{array}{llll}\text { C } & -4.32228069 & -0.03862821 & 0.99999096\end{array}$

$\begin{array}{llll}\text { C } & -3.58278169 & -1.21943021 & 0.99999896\end{array}$

$\begin{array}{llll}\text { C } & -3.65363169 & 1.17895579 & 0.99998996\end{array}$

$\begin{array}{llll}\text { C } & -2.19429369 & -1.17367121 & 1.00001796\end{array}$

H $\quad-4.08912469-2.17970721 \quad 0.99998896$

$\begin{array}{llll}\text { C } & -2.25971469 & 1.21924079 & 1.00000596\end{array}$

H $\quad-4.21489869 \quad 2.10823779 \quad 0.99997296$

$\begin{array}{llll}\text { C } & -1.50186069 & 0.04547079 & 1.00003496\end{array}$ 


$\begin{array}{crrc}\mathrm{H} & -1.63822169 & -2.10721521 & 1.00001196 \\ \mathrm{H} & -1.76866769 & 2.18568279 & 0.99999896 \\ \mathrm{C} & 0.02432131 & 0.04597979 & 1.00001696 \\ \mathrm{C} & 0.55299131 & -0.68839721 & -0.25574504 \\ \mathrm{C} & 0.61562531 & 1.46952779 & 0.99962896 \\ \mathrm{C} & 0.55304411 & -0.68773121 & 2.25614696 \\ \mathrm{H} & 0.18630831 & -0.17327021 & -1.15295804 \\ \mathrm{H} & 0.15106831 & -1.70832321 & -0.28631404 \\ \mathrm{C} & 2.08801531 & -0.73763521 & -0.25537904 \\ \mathrm{H} & 0.26267531 & 2.01628379 & 1.88396396 \\ \mathrm{H} & 0.26264731 & 2.01581579 & 0.11501596 \\ \mathrm{C} & 2.15102431 & 1.42884979 & 0.99960696 \\ \mathrm{H} & 0.15113631 & -1.70764521 & 2.28727396 \\ \mathrm{H} & 0.18639031 & -0.17213221 & 3.15310096 \\ \mathrm{C} & 2.08806731 & -0.73696321 & 2.25574796 \\ \mathrm{H} & 2.43134431 & -1.26741721 & -1.15157504 \\ \mathrm{C} & 2.64484831 & 0.69282179 & -0.25412404 \\ \mathrm{C} & 2.57474631 & -1.47711121 & 1.00037196 \\ \mathrm{H} & 2.53497331 & 2.45557179 & 0.99932396 \\ \mathrm{C} & 2.64489931 & 0.69349279 & 2.25371096 \\ \mathrm{H} & 2.43143031 & -1.26627021 & 3.15221196 \\ \mathrm{H} & 2.31642131 & 1.22449379 & -1.15601004 \\ \mathrm{H} & 3.74172831 & 0.67110979 & -0.26941004 \\ \mathrm{H} & 2.19408231 & -2.50641621 & 1.00065496 \\ \mathrm{H} & 3.67039831 & -1.53542421 & 1.00036396 \\ \mathrm{H} & 3.74178031 & 0.67178779 & 2.26896296 \\ \mathrm{H} & 2.31650931 & 1.22564379 & 3.15532696 \\ \mathrm{C} & -2.17870945 & -1.18235537 & -3.58851873 \\ \mathrm{C} & -1.51320365 & 0.04385996 & -3.58825132 \\ \mathrm{C} & -3.57751479 & -1.21849430 & -3.58904687 \\ \mathrm{C} & -2.25708405 & 1.22487637 & -3.58851614 \\ \mathrm{C} & -4.30069293 & -0.03638855 & -3.58930576 \\ \mathrm{C} & -3.64502899 & 1.19633029 & -3.58904409 \\ \mathrm{O} & -1.55823555 & -2.39282014 & -3.58829425 \\ \mathrm{H} & -0.43118749 & 0.09319409 & -3.58784528 \\ \mathrm{H} & -4.06547997 & -2.18695526 & -3.58924114 \\ \mathrm{H} & -1.73324471 & 2.17558558 & -3.58830724 \\ \mathrm{H} & -5.38537802 & -0.07587273 & -3.58971678 \\ \mathrm{H} & -4.21288537 & 2.12009818 & -3.58924882 \\ \mathrm{C} & -0.14825191 & -2.40043032 & -3.58765905 \\ \mathrm{H} & 0.15126885 & -3.44785963 & -3.58751953 \\ \mathrm{H} & 0.25418656 & -1.90794385 & -2.69445171 \\ \mathrm{H} & 0.25498751 & -1.90795294 & -4.48051054\end{array}$

4AdPh_pi_4OMePh_pi2 60
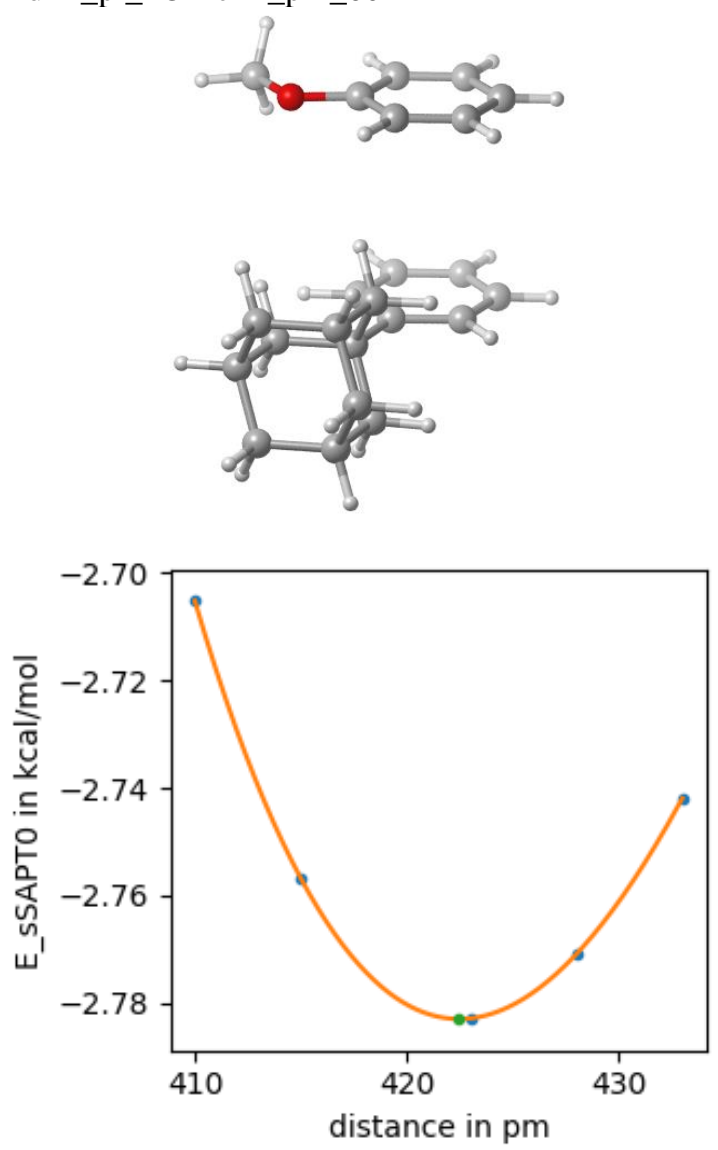

52

4AdPh_pi_4OMePh_pi2_60-422

H $\quad-5.40699069-0.07020421 \quad 0.99997496$

$\begin{array}{llll}\text { C } & -4.32228069 & -0.03862821 & 0.99999096\end{array}$

$\begin{array}{llll}\text { C } & -3.58278169 & -1.21943021 & 0.99999896\end{array}$

$\begin{array}{llll}\text { C } & -3.65363169 & 1.17895579 & 0.99998996\end{array}$

$\begin{array}{llll}\text { C } & -2.19429369 & -1.17367121 & 1.00001796\end{array}$

H $\quad-4.08912469-2.17970721 \quad 0.99998896$

$\begin{array}{llll}\text { C } & -2.25971469 & 1.21924079 & 1.00000596\end{array}$

$\begin{array}{llll}\mathrm{H} & -4.21489869 & 2.10823779 & 0.99997296\end{array}$

$\begin{array}{llll}\text { C } & -1.50186069 & 0.04547079 & 1.00003496\end{array}$

$\begin{array}{llll}\mathrm{H} & -1.63822169 & -2.10721521 & 1.00001196\end{array}$

$\begin{array}{llll}\mathrm{H} & -1.76866769 & 2.18568279 & 0.99999896\end{array}$

$\begin{array}{llll}\text { C } & 0.02432131 & 0.04597979 & 1.00001696\end{array}$

$\begin{array}{llll}\text { C } & 0.55299131 & -0.68839721 & -0.25574504\end{array}$

$\begin{array}{llll}\text { C } & 0.61562531 & 1.46952779 & 0.99962896\end{array}$

$\begin{array}{llll}\text { C } & 0.55304431 & -0.68773121 & 2.25614696\end{array}$

H $\quad 0.18630831 \quad-0.17327021 \quad-1.15295804$

H $\quad 0.15106831 \quad-1.70832321 \quad-0.28631404$

C $\quad 2.08801531 \quad-0.73763521 \quad-0.25537904$

$\begin{array}{llll}\mathrm{H} & 0.26267531 & 2.01628379 & 1.88396396\end{array}$

$\begin{array}{llll}\mathrm{H} & 0.26264731 & 2.01581579 & 0.11501596\end{array}$

$\begin{array}{llll}\text { C } & 2.15102431 & 1.42884979 & 0.99960696\end{array}$

$\begin{array}{llll}\mathrm{H} & 0.15113631 & -1.70764521 & 2.28727396\end{array}$

H $\quad 0.18639031 \quad-0.17213221 \quad 3.15310096$

$\begin{array}{llll}\text { C } & 2.08806731 & -0.73696321 & 2.25574796\end{array}$ 


$\begin{array}{lrrr}\mathrm{H} & 2.43134431 & -1.26741721 & -1.15157504 \\ \mathrm{C} & 2.64484831 & 0.69282179 & -0.25412404 \\ \mathrm{C} & 2.57474631 & -1.47711121 & 1.00037196 \\ \mathrm{H} & 2.53497331 & 2.45557179 & 0.99932396 \\ \mathrm{C} & 2.64489931 & 0.69349279 & 2.25371096 \\ \mathrm{H} & 2.43143031 & -1.26627021 & 3.15221196 \\ \mathrm{H} & 2.31642131 & 1.22449379 & -1.15601004 \\ \mathrm{H} & 3.74172831 & 0.67110979 & -0.26941004 \\ \mathrm{H} & 2.19408231 & -2.50641621 & 1.00065496 \\ \mathrm{H} & 3.67039831 & -1.53542421 & 1.00036396 \\ \mathrm{H} & 3.74178031 & 0.67178779 & 2.26896296 \\ \mathrm{H} & 2.31650931 & 1.22564379 & 3.15532696 \\ \mathrm{C} & -2.25073954 & 1.23311986 & -3.22385546 \\ \mathrm{C} & -3.64542609 & 1.19635713 & -3.22393885 \\ \mathrm{C} & -1.52003951 & 0.03978861 & -3.22467412 \\ \mathrm{C} & -4.29627600 & -0.03837015 & -3.22484363 \\ \mathrm{C} & -2.18218395 & -1.17755467 & -3.22556890 \\ \mathrm{C} & -3.57758177 & -1.22609245 & -3.22566280 \\ \mathrm{O} & -1.51268335 & 2.37569817 & -3.22300603 \\ \mathrm{H} & -4.22915887 & 2.10874337 & -3.22331673 \\ \mathrm{H} & -0.43734512 & 0.10142883 & -3.22458312 \\ \mathrm{H} & -5.38153401 & -0.06006658 & -3.22490499 \\ \mathrm{H} & -1.60564700 & -2.09717723 & -3.22620057 \\ \mathrm{H} & -4.09365996 & -2.17975425 & -3.22636505 \\ \mathrm{C} & -2.21108471 & 3.60058462 & -3.22206017 \\ \mathrm{H} & -1.45374477 & 4.38369169 & -3.22143165 \\ \mathrm{H} & -2.83902517 & 3.70251399 & -2.32896433 \\ \mathrm{H} & -2.83898695 & 3.70391080 & -4.11502279\end{array}$

4AdPh_pi_4PhPh_pi1_300
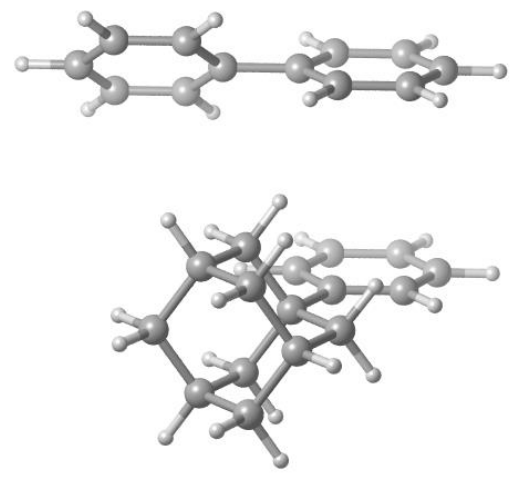

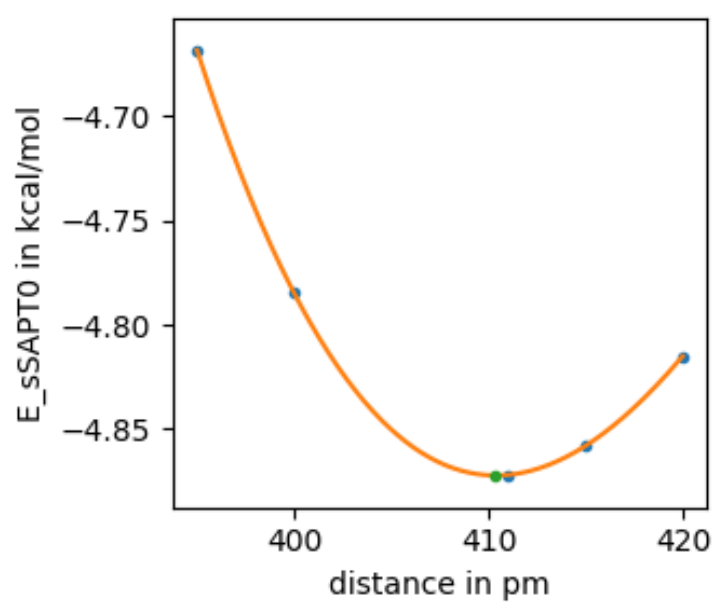

58

4AdPh_pi_4PhPh_pi1_300-410

$\begin{array}{llll}\mathrm{H} & -5.40699838 & -0.07046942 & 1.99997493\end{array}$

$\begin{array}{llll}\text { C } & -4.32228838 & -0.03889342 & 1.99999093\end{array}$

$\begin{array}{llll}\text { C } & -3.58278938 & -1.21969542 & 1.99999893\end{array}$

$\begin{array}{llll}\text { C } & -3.65363938 & 1.17869058 & 1.99998993\end{array}$

$\begin{array}{llll}\text { C } & -2.19430138 & -1.17393642 & 2.00001793\end{array}$

$\begin{array}{llll}\mathrm{H} & -4.08913238 & -2.17997242 & 1.99998893\end{array}$

$\begin{array}{llll}\text { C } & -2.25972238 & 1.21897558 & 2.00000593\end{array}$

$\begin{array}{llll}\mathrm{H} & -4.21490638 & 2.10797258 & 1.99997293\end{array}$

$\begin{array}{llll}\text { C } & -1.50186838 & 0.04520558 & 2.00003493\end{array}$

$\begin{array}{llll}\mathrm{H} & -1.63822938 & -2.10748042 & 2.00001193\end{array}$

$\begin{array}{llll}\mathrm{H} & -1.76867538 & 2.18541758 & 1.99999893\end{array}$

$\begin{array}{llll}\text { C } & 0.02431362 & 0.04571458 & 2.00001693\end{array}$

$\begin{array}{llll}\text { C } & 0.55298362 & -0.68866242 & 0.74425493\end{array}$

$\begin{array}{llll}\text { C } & 0.61561762 & 1.46926258 & 1.99962893\end{array}$

C $\quad 0.55303662 \quad-0.68799642 \quad 3.25614693$

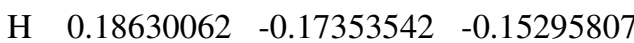

$\begin{array}{llll}\mathrm{H} & 0.15106062 & -1.70858842 & 0.71368593\end{array}$

$\begin{array}{llll}\text { C } & 2.08800762 & -0.73790042 & 0.74462093\end{array}$

$\begin{array}{llll}\mathrm{H} & 0.26266762 & 2.01601858 & 2.88396393\end{array}$

$\begin{array}{llll}\mathrm{H} & 0.26263962 & 2.01555058 & 1.11501593\end{array}$

C $\quad 2.15101662 \quad 1.42858458 \quad 1.99960693$

$\begin{array}{llll}\mathrm{H} & 0.15112862 & -1.70791042 & 3.28727393\end{array}$

H $\quad 0.18638262 \quad-0.17239742 \quad 4.15310093$

C $\quad 2.08805962 \quad-0.73722842 \quad 3.25574793$

$\begin{array}{llll}\mathrm{H} & 2.43133662 & -1.26768242 & -0.15157507\end{array}$

$\begin{array}{llll}\text { C } & 2.64484062 & 0.69255658 & 0.74587593\end{array}$

$\begin{array}{llll}\text { C } & 2.57473862 & -1.47737642 & 2.00037193\end{array}$

$\begin{array}{llll}\mathrm{H} & 2.53496562 & 2.45530658 & 1.99932393\end{array}$

$\begin{array}{llll}\text { C } & 2.64489162 & 0.69322758 & 3.25371093\end{array}$

$\mathrm{H} \quad 2.43142262 \quad-1.26653542 \quad 4.15221193$

$\mathrm{H} \quad 2.31641362 \quad 1.22422858 \quad-0.15601007$

$\begin{array}{llll}\mathrm{H} & 3.74172062 & 0.67084458 & 0.73058993\end{array}$

$\mathrm{H} \quad 2.19407462 \quad-2.50668142 \quad 2.00065493$

H $\quad 3.67039062 \quad-1.53568942 \quad 2.00036393$

H $\quad 3.74177262 \quad 0.67152258 \quad 3.26896293$

$\begin{array}{llll}\mathrm{H} & 2.31650162 & 1.22537858 & 4.15532693\end{array}$

$\begin{array}{llll}\text { C } & -3.64961814 & 1.19809903 & -2.10346597\end{array}$

C $\quad-4.30514771-0.03042950 \quad-2.10379665$ 


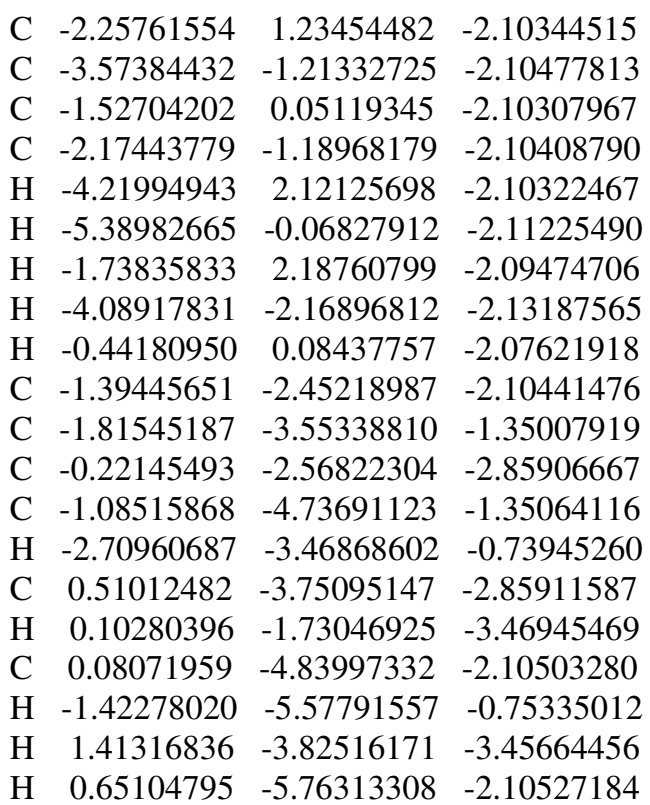

4AdPh_pi_4PhPh_pi1_60
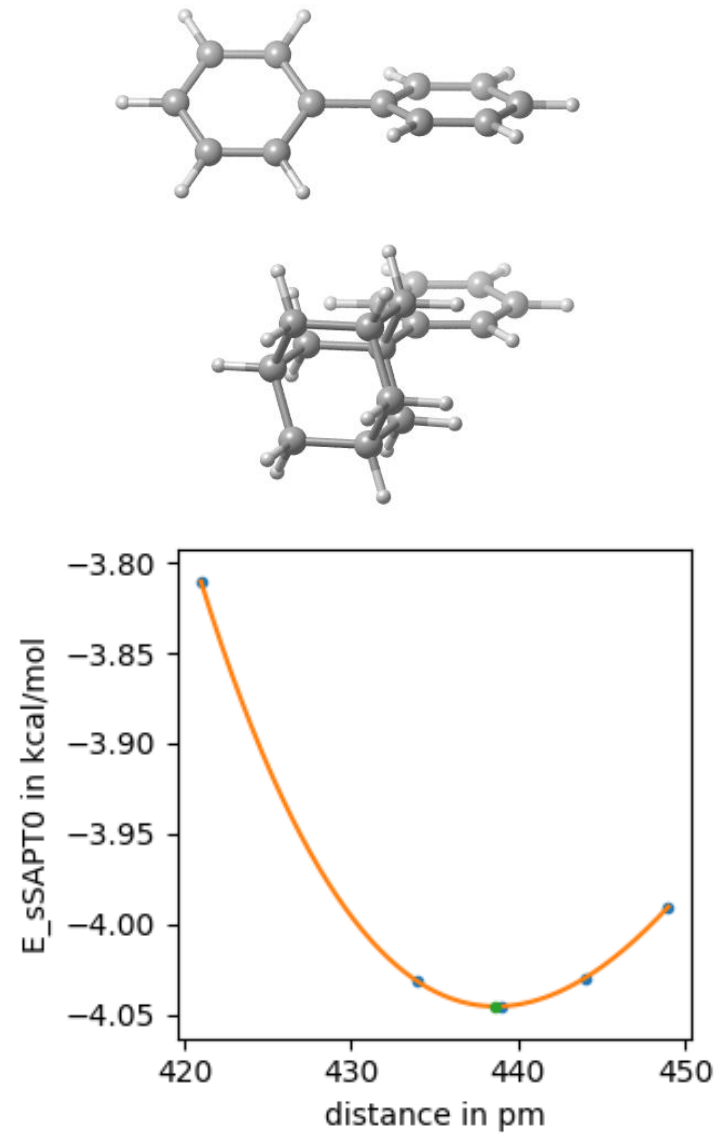

58

4AdPh_pi_4PhPh_pi1_60-438

H $\quad-5.40699838-0.07046942$

C $-4.32228838-0.03889342$

C $-3.58278938-1.21969542$

1.99997493

1.99999093

1.99999893

$\begin{array}{cccc}\mathrm{C} & -3.65363938 & 1.17869058 & 1.99998993 \\ \mathrm{C} & -2.19430138 & -1.17393642 & 2.00001793 \\ \mathrm{H} & -4.08913238 & -2.17997242 & 1.99998893 \\ \mathrm{C} & -2.25972238 & 1.21897558 & 2.00000593 \\ \mathrm{H} & -4.21490638 & 2.10797258 & 1.99997293 \\ \mathrm{C} & -1.50186838 & 0.04520558 & 2.00003493 \\ \mathrm{H} & -1.63822938 & -2.10748042 & 2.00001193 \\ \mathrm{H} & -1.76867538 & 2.18541758 & 1.99999893 \\ \mathrm{C} & 0.02431362 & 0.04571458 & 2.00001693 \\ \mathrm{C} & 0.55298362 & -0.68866242 & 0.74425493 \\ \mathrm{C} & 0.61561762 & 1.46926258 & 1.99962893 \\ \mathrm{C} & 0.55303662 & -0.68799642 & 3.25614693 \\ \mathrm{H} & 0.18630062 & -0.17353542 & -0.15295807 \\ \mathrm{H} & 0.15106062 & -1.70858842 & 0.71368593 \\ \mathrm{C} & 2.08800762 & -0.73790042 & 0.74462093 \\ \mathrm{H} & 0.26266762 & 2.01601858 & 2.88396393 \\ \mathrm{H} & 0.26263962 & 2.01555058 & 1.11501593 \\ \mathrm{C} & 2.15101662 & 1.42858458 & 1.99960693 \\ \mathrm{H} & 0.15112862 & -1.70791042 & 3.28727393 \\ \mathrm{H} & 0.18638262 & -0.17239742 & 4.15310093 \\ \mathrm{C} & 2.08805962 & -0.73722842 & 3.25574793 \\ \mathrm{H} & 2.43133662 & -1.26768242 & -0.15157507 \\ \mathrm{C} & 2.64484062 & 0.69255658 & 0.74587593 \\ \mathrm{C} & 2.57473862 & -1.47737642 & 2.00037193 \\ \mathrm{H} & 2.53496562 & 2.45530658 & 1.99932393 \\ \mathrm{C} & 2.64489162 & 0.69322758 & 3.25371093 \\ \mathrm{H} & 2.43142262 & -1.26653542 & 4.15221193 \\ \mathrm{H} & 2.31641362 & 1.22422858 & -0.15601007 \\ \mathrm{H} & 3.74172062 & 0.67084458 & 0.73058993 \\ \mathrm{H} & 2.19407462 & -2.50668142 & 2.00065493 \\ \mathrm{H} & -1.17728358 & 4.08461597 & -1.03356793 \\ \mathrm{H} & 3.67039062 & -1.53568942 & 2.00036393 \\ \mathrm{H} & 3.74177262 & 0.67152258 & 3.26896293 \\ \mathrm{H} & 2.31650162 & 1.22537858 & 4.15532693 \\ \mathrm{C} & -3.57717233 & -1.23136068 & -2.38691015 \\ \mathrm{C} & -2.18547059 & -1.18480165 & -2.38688692 \\ \mathrm{C} & -4.30473662 & -0.04407399 & -2.38660041 \\ \mathrm{C} & -1.52670259 & 0.03997478 & -2.38723041 \\ \mathrm{C} & -3.64521115 & 1.18029662 & -2.38559692 \\ \mathrm{C} & -2.24688359 & 1.24007334 & -2.38624447 \\ \mathrm{H} & -4.09148495 & -2.18686102 & -2.38716665 \\ \mathrm{H} & -1.61035035 & -2.10523303 & -2.39556647 \\ \mathrm{H} & -5.38974421 & -0.07091897 & -2.37817453 \\ \mathrm{H} & -0.44141983 & 0.07151340 & -2.41405380 \\ \mathrm{H} & -4.21657219 & 2.10353296 & -2.35851312 \\ \mathrm{C} & -1.54351013 & 2.54681096 & -2.38589054 \\ \mathrm{C} & -0.37952880 & 2.73252223 & -1.63120147 \\ \mathrm{H} & -2.02934122 & 3.62097186 & -3.14023955 \\ \mathrm{H} & -2.97374133 & -3.73695239 \\ \mathrm{H} & -2.38496220\end{array}$


4AdPh_pi_4PhPh_pi2_300
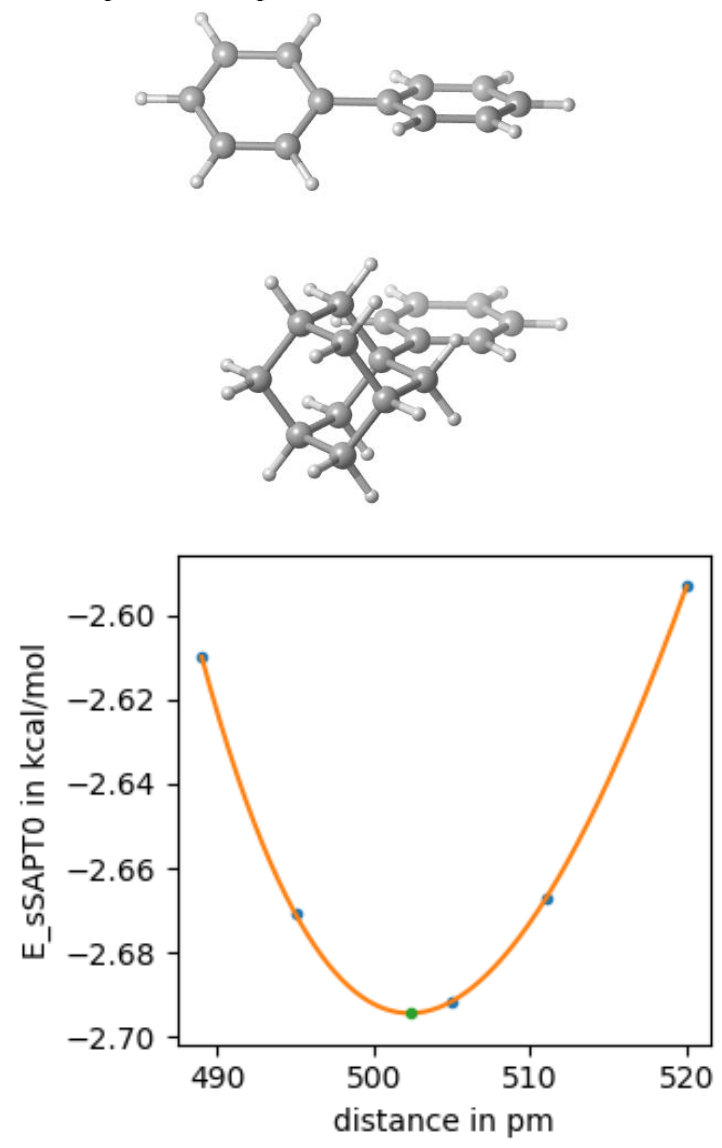

58

4AdPh_pi_4PhPh_pi2_300-502

H $-5.40699838 \quad-0.07046942 \quad 1.99997493$

$\begin{array}{llll}\text { C } & -4.32228838 & -0.03889342 & 1.99999093\end{array}$

$\begin{array}{llll}\text { C } & -3.58278938 & -1.21969542 & 1.99999893\end{array}$

$\begin{array}{llll}\text { C } & -3.65363938 & 1.17869058 & 1.99998993\end{array}$

$\begin{array}{llll}\text { C } & -2.19430138 & -1.17393642 & 2.00001793\end{array}$

$\begin{array}{llll}\mathrm{H} & -4.08913238 & -2.17997242 & 1.99998893\end{array}$

C $\quad-2.25972238 \quad 1.21897558 \quad 2.00000593$

$\begin{array}{llll}\mathrm{H} & -4.21490638 & 2.10797258 & 1.99997293\end{array}$

C $\quad-1.50186838 \quad 0.04520558 \quad 2.00003493$

$\begin{array}{llll}\mathrm{H} & -1.63822938 & -2.10748042 & 2.00001193\end{array}$

$\begin{array}{llll}\mathrm{H} & -1.76867538 & 2.18541758 & 1.99999893\end{array}$

$\begin{array}{llll}\text { C } & 0.02431362 & 0.04571458 & 2.00001693\end{array}$

$\begin{array}{llll}\text { C } & 0.55298362 & -0.68866242 & 0.74425493\end{array}$

$\begin{array}{llll}\text { C } & 0.61561762 & 1.46926258 & 1.99962893\end{array}$

C $\quad 0.55303662 \quad-0.68799642 \quad 3.25614693$

$\mathrm{H} \quad 0.18630062-0.17353542 \quad-0.15295807$

H $\quad 0.15106062 \quad-1.70858842 \quad 0.71368593$

C $\quad 2.08800762 \quad-0.73790042 \quad 0.74462093$

$\begin{array}{llll}\mathrm{H} & 0.26266762 & 2.01601858 & 2.88396393\end{array}$

$\begin{array}{llll}\mathrm{H} & 0.26263962 & 2.01555058 & 1.11501593\end{array}$

C $\quad 2.15101662 \quad 1.42858458 \quad 1.99960693$

H $\quad 0.15112862 \quad-1.70791042 \quad 3.28727393$

$\begin{array}{llll}\mathrm{H} & 0.18638262 & -0.17239742 & 4.15310093\end{array}$

C $\quad 2.08805962 \quad-0.73722842 \quad 3.25574793$

$\begin{array}{lrrr}\mathrm{H} & 2.43133662 & -1.26768242 & -0.15157507 \\ \mathrm{C} & 2.64484062 & 0.69255658 & 0.74587593 \\ \mathrm{C} & 2.57473862 & -1.47737642 & 2.00037193 \\ \mathrm{H} & 2.53496562 & 2.45530658 & 1.99932393 \\ \mathrm{C} & 2.64489162 & 0.69322758 & 3.25371093 \\ \mathrm{H} & 2.43142262 & -1.26653542 & 4.15221193 \\ \mathrm{H} & 2.31641362 & 1.22422858 & -0.15601007 \\ \mathrm{H} & 3.74172062 & 0.67084458 & 0.73058993 \\ \mathrm{H} & 2.19407462 & -2.50668142 & 2.00065493 \\ \mathrm{H} & 3.67039062 & -1.53568942 & 2.00036393 \\ \mathrm{H} & 3.74177262 & 0.67152258 & 3.26896293 \\ \mathrm{H} & 2.31650162 & 1.22537858 & 4.15532693 \\ \mathrm{C} & -3.64961107 & 1.19834279 & -3.02260394 \\ \mathrm{C} & -2.25760776 & 1.23478800 & -3.02258339 \\ \mathrm{C} & -4.30514083 & -0.03018486 & -3.02293434 \\ \mathrm{C} & -1.52703450 & 0.05143918 & -3.02356499 \\ \mathrm{C} & -3.57383562 & -1.21308418 & -3.02256874 \\ \mathrm{C} & -2.17443072 & -1.18943803 & -3.02322586 \\ \mathrm{H} & -4.21994136 & 2.12150135 & -3.02236263 \\ \mathrm{H} & -1.73835135 & 2.18785587 & -3.03076649 \\ \mathrm{H} & -5.38982013 & -0.06803987 & -3.01451138 \\ \mathrm{H} & -0.44180115 & 0.08463502 & -3.05038794 \\ \mathrm{H} & -4.08917209 & -2.16873778 & -2.99598281 \\ \mathrm{C} & -1.39444776 & -2.45194507 & -3.02355272 \\ \mathrm{C} & -0.22145728 & -2.56837267 & -2.26894372 \\ \mathrm{C} & -1.81542606 & -3.55274520 & -3.77847806 \\ \mathrm{C} & 0.51012722 & -3.75109803 & -2.26950548 \\ \mathrm{H} & 0.10278846 & -1.73093788 & -1.65810813 \\ \mathrm{C} & -1.08512813 & -4.73626554 & -3.77852748 \\ \mathrm{H} & -2.70956900 & -3.46772477 & -4.38907508 \\ \mathrm{C} & 0.08073685 & -4.83972327 & -3.02417077 \\ \mathrm{H} & 1.41316022 & -3.82562119 & -1.67200160 \\ \mathrm{H} & -1.42273748 & -5.57695594 & -4.37626898 \\ \mathrm{H} & 0.65107006 & -5.76288002 & -3.02440980\end{array}$

4AdPh_pi_4PhPh_pi2_60
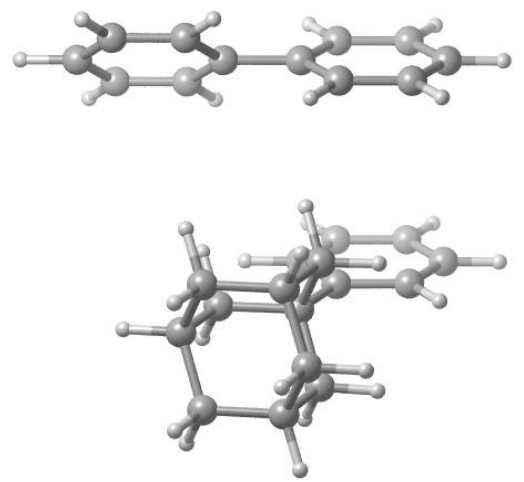


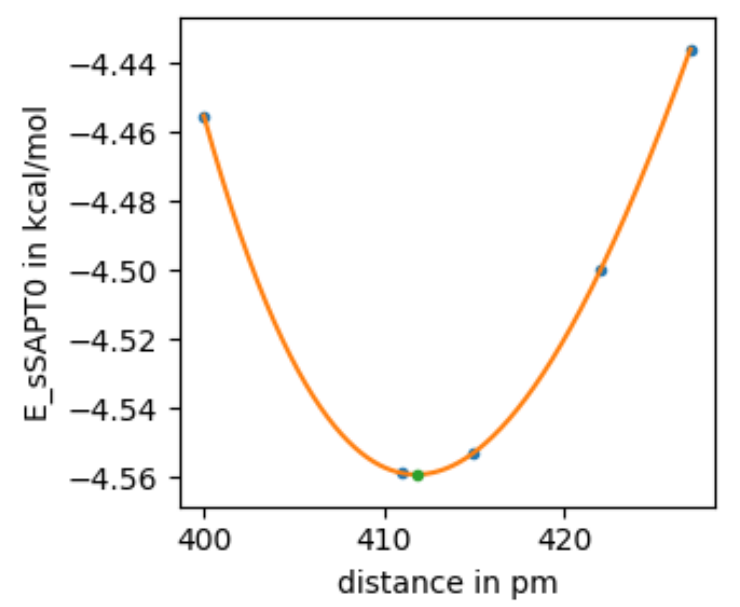

58

4AdPh_pi_4PhPh_pi2_60-411

H $-5.40699069-0.0702042$

C $-4.32228069-0.03862821$

C $\quad-3.58278169-1.21943021$

C $\quad-3.65363169 \quad 1.17895579$

C $-2.19429369-1.17367121$

H $\quad-4.08912469-2.17970721$

C $-2.25971469 \quad 1.21924079$

H $\quad-4.214898692 .10823779$

C -1.501860690 .04547079$

H $-1.63822169-2.10721521$

H $\quad-1.768667692 .18568279$

$\begin{array}{lll}\text { C } & 0.02432131 & 0.04597979\end{array}$

C $\quad 0.55299131 \quad-0.68839721$

C $\quad 0.61562531 \quad 1.46952779$

C $\quad 0.55304431 \quad-0.68773121$

H $\quad 0.18630831 \quad-0.17327021$

H $\quad 0.15106831-1.70832321$

C $2.08801531-0.73763521$

$\mathrm{H} \quad 0.26267531 \quad 2.01628379$

H $\quad 0.26264731 \quad 2.01581579$

C $\quad 2.15102431 \quad 1.42884979$

H $\quad 0.15113631-1.70764521$

$\begin{array}{lll}\mathrm{H} & 0.18639031 & -0.17213221\end{array}$

C $2.08806731 \quad-0.73696321$

H $\quad 2.43134431-1.26741721$

C $2.64484831 \quad 0.69282179$

C $2.57474631-1.47711121$

$\begin{array}{lll}\mathrm{H} & 2.53497331 & 2.45557179\end{array}$

C 2.644899310 .69349279

H $\quad 2.43143031 \quad-1.26627021$

H $\quad 2.31642131 \quad 1.22449379$

H 3.741728310 .67110979

H $2.19408231-2.50641621$

H $\quad 3.67039831 \quad-1.53542421$

H $\quad 3.74178031 \quad 0.67178779$

H $\quad 2.31650931 \quad 1.22564379$

C $\quad-3.57716670-1.23116674$

C $-4.30473085-0.04387915$
0.99997496

0.99999096

0.99999896

0.99998996

1.00001796

0.99998896

1.00000596

0.99997296

1.00003496

1.00001196

0.99999896

1.00001696

$-0.25574504$

0.99962896

2.25614696

$-1.15295804$

$-0.28631404$

$-0.25537904$

1.88396396

0.11501596

0.99960696

2.28727396

3.15310096

2.25574796

$-1.15157504$

$-0.25412404$

1.00037196

0.99932396

3.15221196

$-1.15601004$

$-0.26941004$

1.00065496

1.00036396

2.26896296

3.15532696

$-3.11819093$

$-3.11788147$
2.25371096
C $-2.18546563-1.18460832-3.11816743$

$\begin{array}{llll}\text { C } & -3.64520713 & 1.18049050 & -3.11822507\end{array}$

$\begin{array}{llll}\text { C } & -1.52669751 & 0.04016994 & -3.11716384\end{array}$

C $-2.24687796 \quad 1.24026728-3.11752526$

$\mathrm{H}-4.09148036-2.18666652-3.11844744$

H $\quad-5.38973803 \quad-0.07072056-3.12633679$

H $-1.61034462-2.10504391 \quad-3.10996577$

$\begin{array}{llll}\mathrm{H} & -4.21656578 & 2.10374269 & -3.14482470\end{array}$

$\begin{array}{llll}\mathrm{H} & -0.44141542 & 0.07169178 & -3.09030379\end{array}$

C $\quad-1.54350624 \quad 2.54700584-3.11717132$

C $\quad-2.02935424 \quad 3.62076397 \quad-2.36225942$

C $-0.37951415 \quad 2.73312318-3.87174316$

$\begin{array}{llll}\text { C } & -1.37087619 & 4.84569749 & -2.36218321\end{array}$

H $\quad-2.91686423 \quad 3.48261278 \quad-1.75169189$

C $\quad 0.28029554 \quad 3.95733979 \quad-3.87115457$

H $\quad-0.00592524 \quad 1.91650134-4.48256956$

C $-0.21322190 \quad 5.01844222-3.11650176$

$\mathrm{H} \quad-1.75799787 \quad 5.66477484 \quad-1.76445226$

H $\quad 1.17730361 \quad 4.08554045 \quad-4.46862962$

$\mathrm{H} \quad 0.30108873 \quad 5.97394364 \quad-3.11624299$

4AdPh_pi_Ph_pi_60
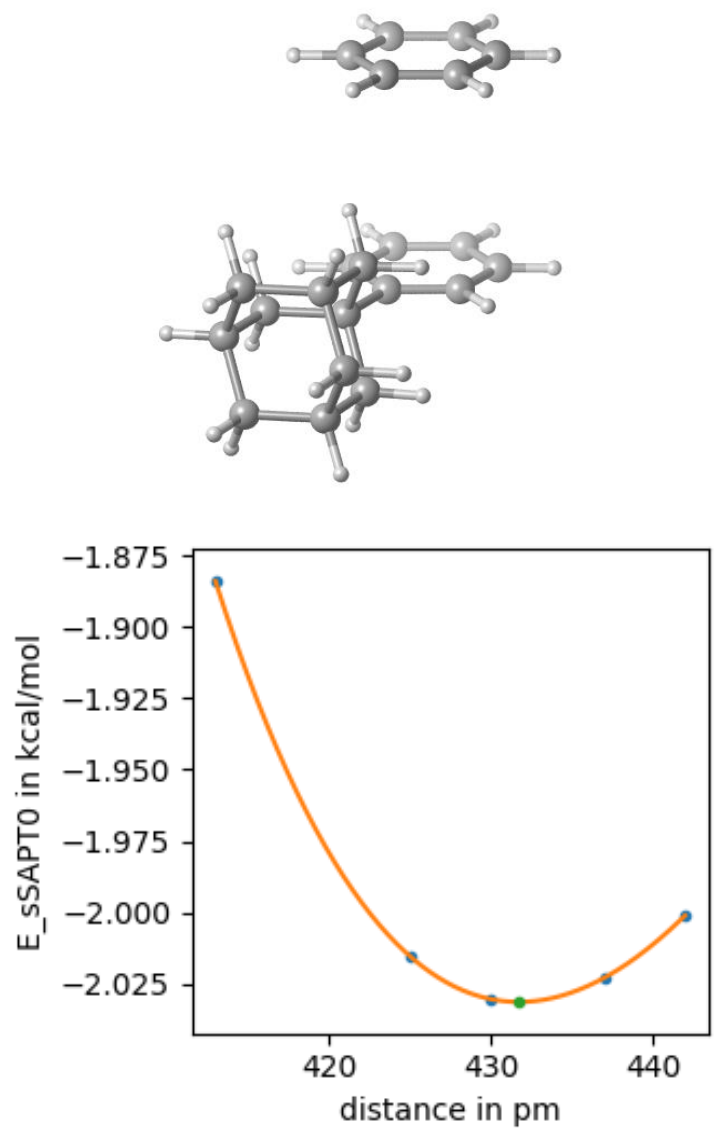

48

4AdPh_pi_Ph_pi_60-431

H $\quad-5.40699069-0.07020421 \quad 0.99997496$

$\begin{array}{llll}\text { C } & -4.32228069 & -0.03862821 & 0.99999096\end{array}$

$\begin{array}{llll}\text { C } & -3.58278169 & -1.21943021 & 0.99999896\end{array}$ 


$\begin{array}{lrrr}\mathrm{C} & -3.65363169 & 1.17895579 & 0.99998996 \\ \mathrm{C} & -2.19429369 & -1.17367121 & 1.00001796 \\ \mathrm{H} & -4.08912469 & -2.17970721 & 0.99998896 \\ \mathrm{C} & -2.25971469 & 1.21924079 & 1.00000596 \\ \mathrm{H} & -4.21489869 & 2.10823779 & 0.99997296 \\ \mathrm{C} & -1.50186069 & 0.04547079 & 1.00003496 \\ \mathrm{H} & -1.63822169 & -2.10721521 & 1.00001196 \\ \mathrm{H} & -1.76866769 & 2.18568279 & 0.99999896 \\ \mathrm{C} & 0.02432131 & 0.04597979 & 1.00001696 \\ \mathrm{C} & 0.55299131 & -0.68839721 & -0.25574504 \\ \mathrm{C} & 0.61562531 & 1.46952779 & 0.99962896 \\ \mathrm{C} & 0.55304431 & -0.68773121 & 2.25614696 \\ \mathrm{H} & 0.18630831 & -0.17327021 & -1.15295804 \\ \mathrm{H} & 0.15106831 & -1.70832321 & -0.28631404 \\ \mathrm{C} & 2.08801531 & -0.73763521 & -0.25537904 \\ \mathrm{H} & 0.26267531 & 2.01628379 & 1.88396396 \\ \mathrm{H} & 0.26264731 & 2.01581579 & 0.11501596 \\ \mathrm{C} & 2.15102431 & 1.42884979 & 0.99960696 \\ \mathrm{H} & 0.15113631 & -1.70764521 & 2.28727396 \\ \mathrm{H} & 0.18639031 & -0.17213221 & 3.15310096 \\ \mathrm{C} & 2.08806731 & -0.73696321 & 2.25574796 \\ \mathrm{H} & 2.43134431 & -1.26741721 & -1.15157504 \\ \mathrm{C} & 2.64484831 & 0.69282179 & -0.25412404 \\ \mathrm{C} & 2.57474631 & -1.47711121 & 1.00037196 \\ \mathrm{H} & 2.53497331 & 2.45557179 & 0.99932396 \\ \mathrm{C} & 2.64489931 & 0.69349279 & 2.25371096 \\ \mathrm{H} & 2.43143031 & -1.26627021 & 3.15221196 \\ \mathrm{H} & 2.31642131 & 1.22449379 & -1.15601004 \\ \mathrm{H} & 3.74172831 & 0.67110979 & -0.26941004 \\ \mathrm{H} & 2.19408231 & -2.50641621 & 1.00065496 \\ \mathrm{H} & 3.67039831 & -1.53542421 & 1.00036396 \\ \mathrm{H} & 3.74178031 & 0.67178779 & 2.26896296 \\ \mathrm{H} & 2.31650931 & 1.22564379 & 3.15532696 \\ \mathrm{C} & -4.30443514 & -0.03710191 & -3.31731020 \\ \mathrm{C} & -3.64398646 & 1.18942393 & -3.31700089 \\ \mathrm{C} & -3.57175656 & -1.22187557 & -3.31774062 \\ \mathrm{C} & -2.25179638 & 1.23117991 & -3.31732390 \\ \mathrm{C} & -2.17953688 & -1.18022222 & -3.31776266 \\ \mathrm{C} & -1.51964643 & 0.04631316 & -3.31757529 \\ \mathrm{H} & -5.38938365 & -0.06958807 & -3.31721405 \\ \mathrm{H} & -4.21474104 & 2.11296202 & -3.31682932 \\ \mathrm{H} & -4.08619512 & -2.17792652 & -3.31792003 \\ \mathrm{H} & -1.73705395 & 2.18710949 & -3.31724957 \\ \mathrm{H} & -1.60855778 & -2.10366188 & -3.31801724 \\ \mathrm{H} & -0.43441968 & 0.07885057 & -3.31778247\end{array}$

4CF3Ph_pi_4CF3Ph_pi2_300
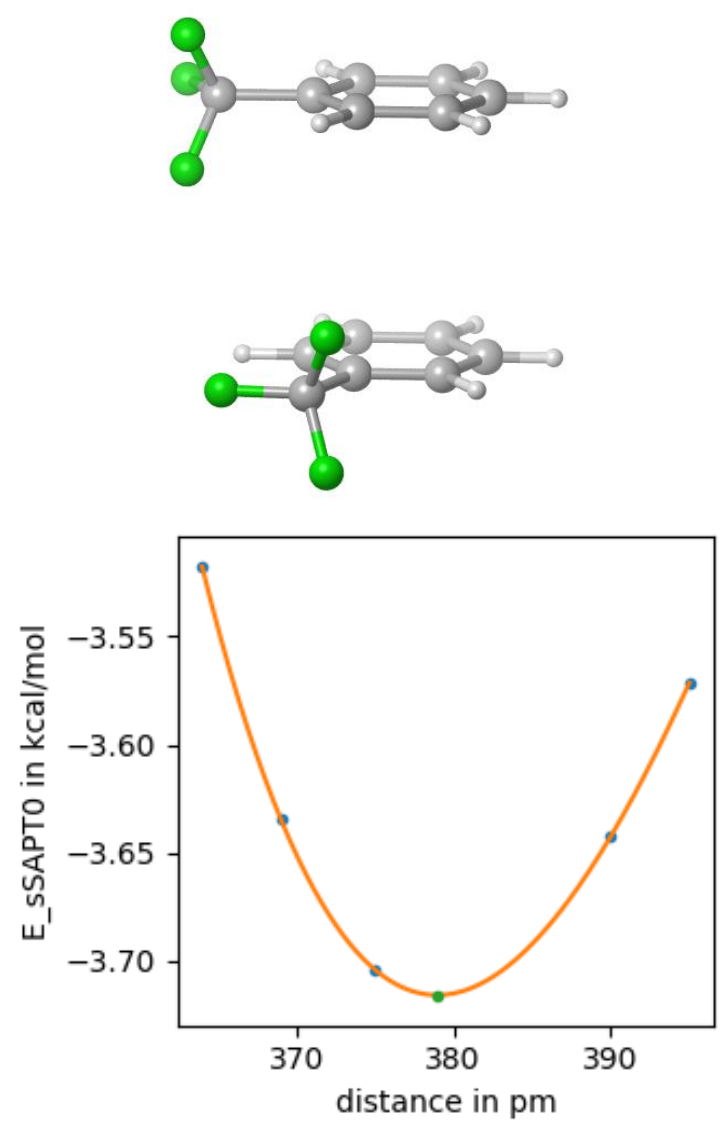

30

4CF3Ph_pi_4CF3Ph_pi2_300-378

$\begin{array}{llll}\mathrm{H} & 0.69232956 & 1.25809605 & 2.00207273\end{array}$

$\begin{array}{llll}\text { C } & -0.24312665 & 0.70814581 & 2.00113799\end{array}$

$\begin{array}{llll}\text { C } & -1.45465706 & 1.39872587 & 2.00189782\end{array}$

$\begin{array}{llll}\text { C } & -0.23029987 & -0.68296795 & 1.99914700\end{array}$

$\begin{array}{llll}\text { C } & -2.65285853 & 0.69652913 & 2.00065945\end{array}$

$\begin{array}{llll}\mathrm{H} & -1.46318687 & 2.48343736 & 2.00340442\end{array}$

$\begin{array}{llll}\text { C } & -1.42896571 & -1.39233876 & 1.99791598\end{array}$

H $\quad 0.71291409-1.21873256 \quad 1.99851876$

$\begin{array}{llll}\text { C } & -2.63266156 & -0.69755192 & 1.99867876\end{array}$

$\begin{array}{llll}\mathrm{H} & -3.60345207 & 1.22071205 & 2.00112484\end{array}$

$\begin{array}{llll}\mathrm{H} & -1.43341235 & -2.47623792 & 1.99631597\end{array}$

$\begin{array}{llll}\text { C } & -3.94549875 & -1.42639313 & 1.99796619\end{array}$

$\begin{array}{llll}\text { F } & -4.68067050 & -1.11228235 & 3.07647871\end{array}$

$\begin{array}{llll}\text { F } & -4.68473729 & -1.10274946 & 0.92506041\end{array}$

$\begin{array}{llll}\text { F } & -3.78896332 & -2.75680349 & 1.99184628\end{array}$

$\begin{array}{llll}\text { C } & -2.64223082 & 0.690884444 & -2.21083620\end{array}$

$\begin{array}{llll}\text { C } & -1.44531667 & 1.40591506 & -2.20960686\end{array}$

$\begin{array}{llll}\text { C } & -2.64150694 & -0.69893989 & -2.21176234\end{array}$

$\begin{array}{llll}\text { C } & -3.93016663 & 1.46287716 & -2.21055967\end{array}$

$\begin{array}{llll}\text { C } & -0.23780896 & 0.71984395 & -2.20929141\end{array}$

H $\quad-1.46710995 \quad 2.49123563 \quad-2.20899908$

C $-1.42755545-1.38182344 \quad-2.21145836$

H $\quad-3.58219089-1.23743075 \quad-2.21276911$

F $\quad-4.02530301 \quad 2.25644367 \quad-1.13188752$ 


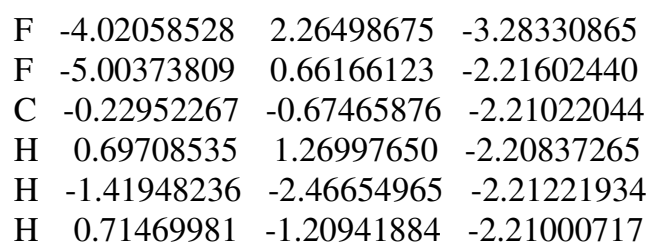

4CF3Ph_pi_4CF3Ph_pi2_60
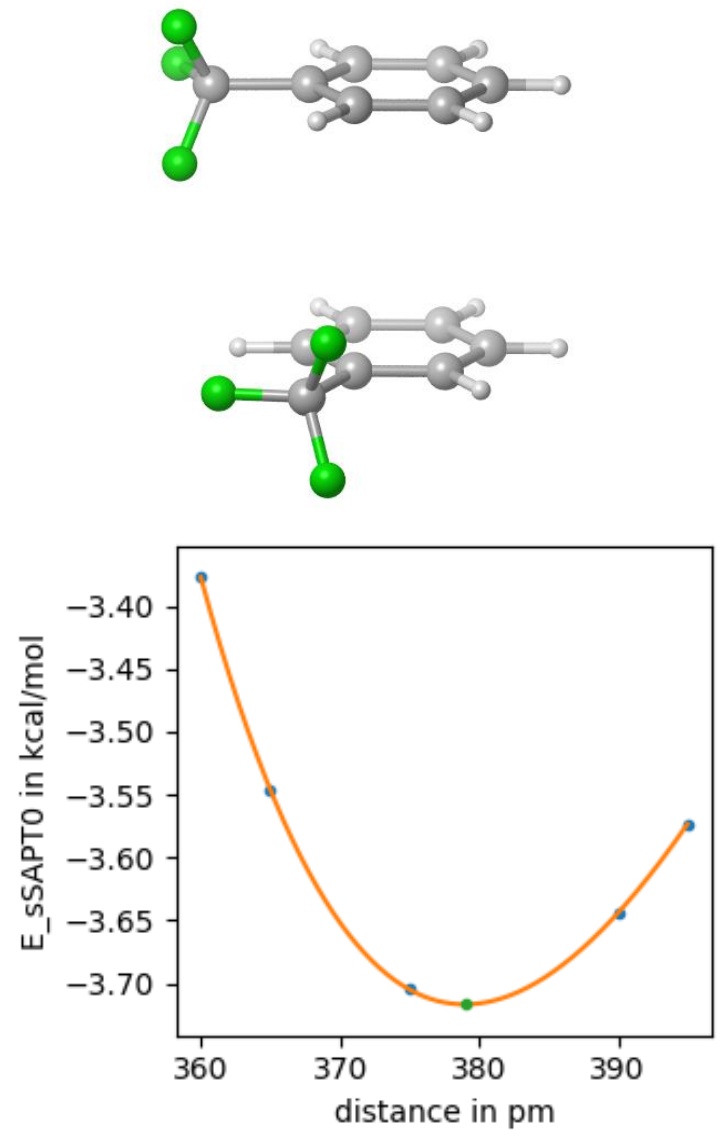

30

4CF3Ph_pi_4CF3Ph_pi2_60-378
$\begin{array}{llll}\mathrm{H} & 0.69232956 & 1.25809605 & 2.00207273\end{array}$
$\begin{array}{llll}\text { C } & -0.24312665 & 0.70814581 & 2.00113799\end{array}$
$\begin{array}{llll}\text { C } & -1.45465706 & 1.39872587 & 2.00189782\end{array}$
$\begin{array}{llll}\text { C } & -0.23029987 & -0.68296795 & 1.99914700\end{array}$
$\begin{array}{llll}\text { C } & -2.65285853 & 0.69652913 & 2.00065945\end{array}$
$\begin{array}{llll}\mathrm{H} & -1.46318687 & 2.48343736 & 2.00340442\end{array}$
$\begin{array}{llll}\text { C } & -1.42896571 & -1.39233876 & 1.99791598\end{array}$
$\mathrm{H} \quad 0.71291409-1.21873256 \quad 1.99851876$
$\begin{array}{llll}\text { C } & -2.63266156 & -0.69755192 & 1.99867876\end{array}$
$\begin{array}{llll}\mathrm{H} & -3.60345207 & 1.22071205 & 2.00112484\end{array}$
H $-1.43341235-2.47623792 \quad 1.99631597$
$\begin{array}{llll}\text { C } & -3.94549875 & -1.42639313 & 1.99796619\end{array}$
$\begin{array}{llll}\text { F } & -4.68067050 & -1.11228235 & 3.07647871\end{array}$
$\begin{array}{llll}\text { F } & -4.68473729 & -1.10274946 & 0.92506041\end{array}$
$\begin{array}{llll}\text { F } & -3.78896332 & -2.75680349 & 1.99184628\end{array}$
$\begin{array}{llll}\text { C } & -1.42158093 & -1.37901177 & -2.21172617\end{array}$
$\begin{array}{llll}\text { C } & -2.64132127 & -0.70365500 & -2.21099877\end{array}$
$\begin{array}{llll}\text { C } & -0.22042828 & -0.67983736 & -2.21102384\end{array}$
$\begin{array}{llll}\text { C } & -1.44163802 & -2.88046047 & -2.21271538\end{array}$
$\begin{array}{llll}\text { C } & -2.65512022 & 0.68507679 & -2.20954081\end{array}$
H $\quad-3.56863757 \quad-1.26799540 \quad-2.21166863$
$\begin{array}{llll}\text { C } & -0.24022164 & 0.71286304 & -2.20957927\end{array}$
$\mathrm{H} \quad 0.71790612 \quad-1.22241258 \quad-2.21166361$
$\begin{array}{llll}\text { F } & -2.08080674 & -3.36246270 & -1.13500041\end{array}$
F $-2.08868783 \quad-3.36088946-3.28642866$
$\begin{array}{llll}\text { F } & -0.20938008 & -3.40586469 & -2.21754259\end{array}$
$\begin{array}{llll}\text { C } & -1.45372405 & 1.39314041 & -2.20883377\end{array}$
$\mathrm{H} \quad-3.60060891 \quad 1.21679559-2.20900431$
$\mathrm{H} \quad 0.69347696 \quad 1.26504360 \quad-2.20906716$
H $\quad-1.46600115 \quad 2.47820839 \quad-2.20772911$

4CF3Ph_pi_4CF3Ph_pi_300
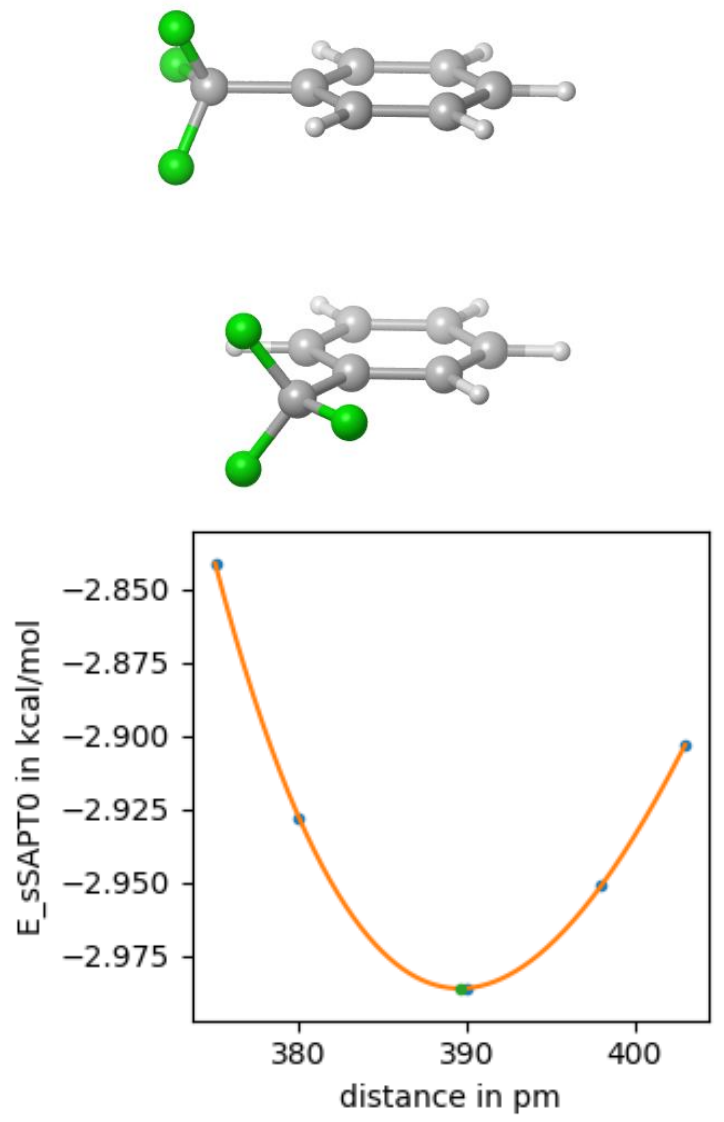

30

4CF3Ph_pi_4CF3Ph_pi_300-389
H 0.69232956
C -0.24312665
\begin{tabular}{ll}
$1.25809605 \quad 2.00207273$ \\
\hline
\end{tabular}
C -1.45465706
0.70814581
2.00113799
C -0.23029987
1.39872587
2.00189782
C -2.65285853
$-0.68296795$
1.99914700
H $\quad-1.46318687 \quad 2.48343736$
2.00065945
C $-1.42896571-1.39233876$
H $\quad 0.71291409-1.21873256$
C $-2.63266156-0.69755192$
H $\quad-3.60345207 \quad 1.22071205$
2.00340442
1.99791598
1.99851876
1.99867876
2.00112484 


$\begin{array}{lrrr}\text { H } & -1.43341235 & -2.47623792 & 1.99631597 \\ \text { C } & -3.94549875 & -1.42639313 & 1.99796619 \\ \text { F } & -4.68067050 & -1.11228235 & 3.07647871 \\ \text { F } & -4.68473729 & -1.10274946 & 0.92506041 \\ \text { F } & -3.78896332 & -2.75680349 & 1.99184628 \\ \text { C } & -2.64225169 & 0.69081376 & -2.10477642 \\ \text { C } & -2.64152489 & -0.70341338 & -2.10676103 \\ \text { C } & -1.44911831 & 1.40358597 & -2.10353005 \\ \text { C } & -3.96587878 & 1.39987143 & -2.10459578 \\ \text { C } & -1.43292024 & -1.38754989 & -2.10751380 \\ \text { H } & -3.58414482 & -1.24180264 & -2.10760841 \\ \text { C } & -0.23994167 & 0.71228317 & -2.10427526 \\ \text { H } & -1.46983094 & 2.48729634 & -2.10193511 \\ \text { F } & -4.69581872 & 1.07476671 & -3.18340390 \\ F & -4.70061009 & 1.06516736 & -1.03198739 \\ \text { F } & -3.82932866 & 2.73248105 & -2.09841674 \\ \text { C } & -0.23189005 & -0.67886641 & -2.10626720 \\ \text { H } & -1.42517043 & -2.47226723 & -2.10902056 \\ \text { H } & 0.69512564 & 1.26214207 & -2.10326806 \\ \text { H } & 0.71171412 & -1.21471652 & -2.10682283\end{array}$

4CF3Ph_pi_4CF3Ph_pi_60
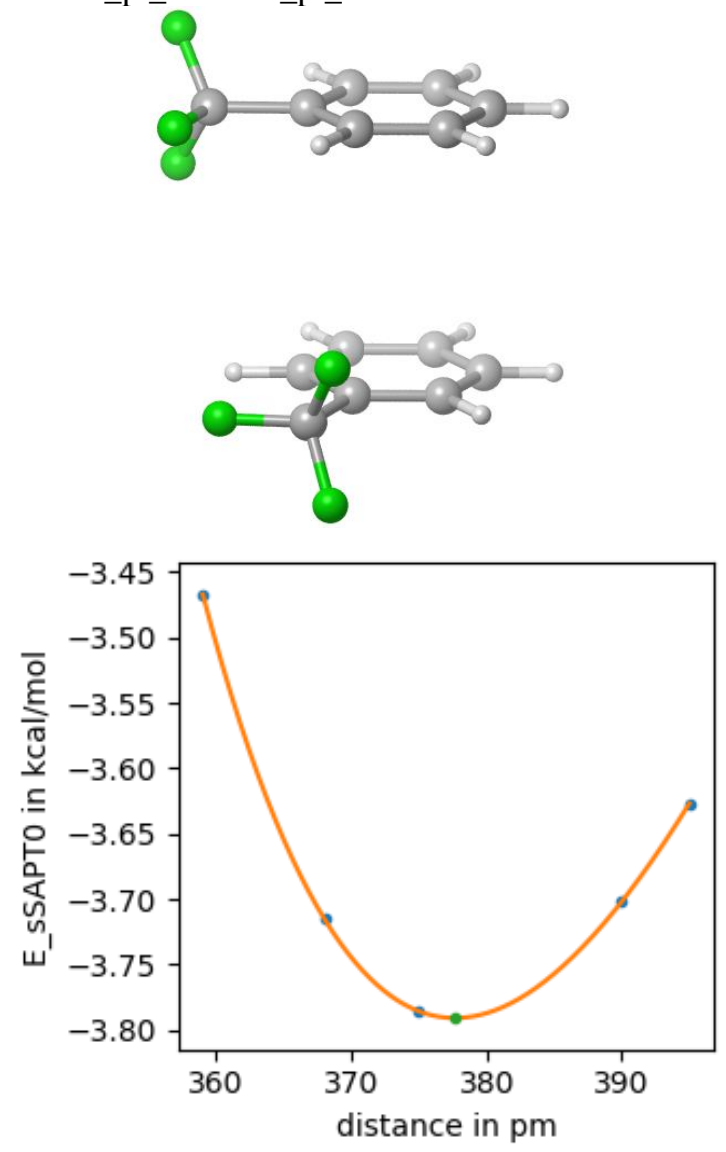

$\begin{array}{llll}\text { C } & -0.23029987 & -0.68296795 & 1.99914700\end{array}$

$\begin{array}{llll}\text { C } & -2.65285853 & 0.69652913 & 2.00065945\end{array}$

$\begin{array}{llll}\mathrm{H} & -1.46318687 & 2.48343736 & 2.00340442\end{array}$

C $-1.42896571-1.39233876 \quad 1.99791598$

$\mathrm{H} \quad 0.71291409 \quad-1.21873256 \quad 1.99851876$

C $-2.63266156-0.69755192 \quad 1.99867876$

$\begin{array}{llll}\mathrm{H} & -3.60345207 & 1.22071205 & 2.00112484\end{array}$

H $\quad-1.43341235 \quad-2.47623792 \quad 1.99631597$

C $-3.94549875 \quad-1.42639313 \quad 1.99796619$

$\begin{array}{llll}\text { F } & -4.68067050 & -1.11228235 & 3.07647871\end{array}$

$\begin{array}{llll}\text { F } & -4.68473729 & -1.10274946 & 0.92506041\end{array}$

$\begin{array}{llll}\text { F } & -3.78896332 & -2.75680349 & 1.99184628\end{array}$

C $-1.41682115-1.37894368 \quad-2.22499958$

$\begin{array}{llll}\text { C } & -0.21427714 & -0.67342149 & -2.22534828\end{array}$

$\begin{array}{llll}\text { C } & -2.63501225 & -0.70989740 & -2.22425681\end{array}$

$\begin{array}{llll}\text { C } & -1.35938099 & -2.87942730 & -2.22601982\end{array}$

C $\quad-0.23506513 \quad 0.71522398 \quad-2.22496148$

H $\quad 0.72680492 \quad-1.21449484 \quad-2.22582681$

$\begin{array}{lllll}\text { C } & -2.64990651 & 0.68286470 & -2.22385388\end{array}$

$\mathrm{H} \quad-3.55954416-1.27567154-2.22393905$

$\begin{array}{llll}\text { F } & -0.70890404 & -3.34393850 & -3.30464924\end{array}$

$\begin{array}{llll}\text { F } & -0.70007395 & -3.34501275 & -1.15322437\end{array}$

F $\quad-2.57817092 \quad-3.43536072-2.22136613$

C $-1.45372103 \quad 1.39315065-2.22421148$

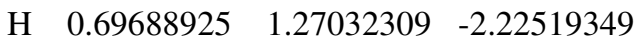

$\mathrm{H} \quad-3.59706601 \quad 1.21162285 \quad-2.22323109$

$\begin{array}{llll}\mathrm{H} & -1.46846843 & 2.47818838 & -2.22387538\end{array}$

4CF3Ph_pi_4OMePh_pi1_300
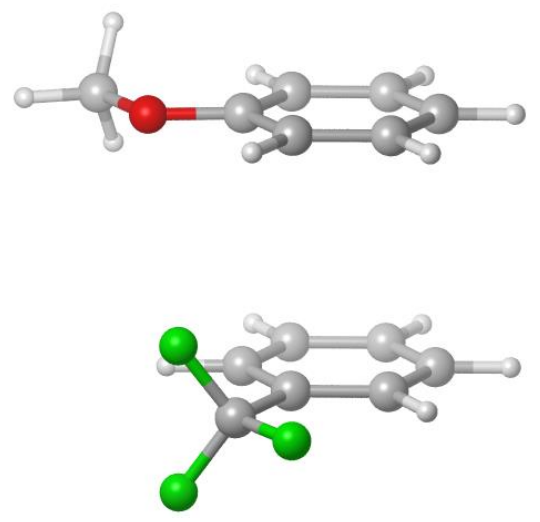

30

4CF3Ph_pi_4CF3Ph_pi_60-377

$\begin{array}{rrrr}\mathrm{H} & 0.69232956 & 1.25809605 & 2.00207273 \\ \mathrm{C} & -0.24312665 & 0.70814581 & 2.00113799 \\ \mathrm{C} & -1.45465706 & 1.39872587 & 2.00189782\end{array}$ 


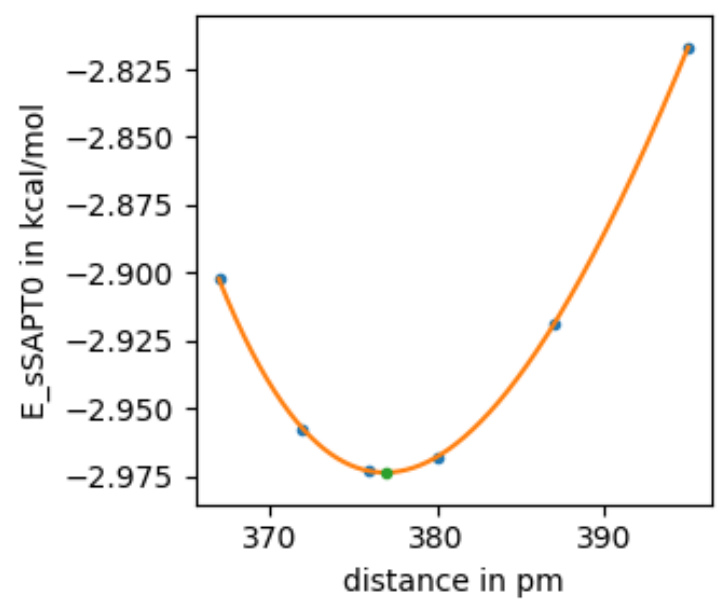

31

4CF3Ph_pi_4OMePh_pi1_300-376

$\mathrm{H} \quad-3.90811076-0.03308048 \quad 1.00030676$

$\begin{array}{llll}\text { C } & -2.82309076 & -0.01707948 & 1.00014676\end{array}$

$\begin{array}{llll}\text { C } & -2.11090776 & -1.21603848 & 1.00002576\end{array}$

$\begin{array}{llll}\text { C } & -2.14698176 & 1.19875052 & 1.00002376\end{array}$

$\begin{array}{llll}\text { C } & -0.72223076 & -1.19747348 & 0.99977676\end{array}$

H $-2.63937876-2.16334648 \quad 1.00007576$

$\begin{array}{llll}\text { C } & -0.75435676 & 1.22332352 & 0.99979176\end{array}$

$\begin{array}{llll}\mathrm{H} & -2.70236376 & 2.13054852 & 1.00007876\end{array}$

$\begin{array}{llll}\text { C } & -0.05106376 & 0.02457752 & 0.99966776\end{array}$

$\begin{array}{llll}\mathrm{H} & -0.15471076 & -2.12284748 & 0.99957476\end{array}$

$\begin{array}{llll}\mathrm{H} & -0.21500476 & 2.16351452 & 0.99964776\end{array}$

$\begin{array}{llll}\text { C } & 1.45044524 & 0.00967352 & 0.99998476\end{array}$

$\begin{array}{llll}\text { F } & 1.93370024 & -0.62786748 & 2.07810276\end{array}$

$\begin{array}{llll}\text { F } & 1.93402624 & -0.63569348 & -0.07332624\end{array}$

$\begin{array}{llll}\text { F } & 1.97162124 & 1.24372752 & 0.99564576\end{array}$

C $\quad-0.72167823-1.19086724-2.76974025$

C $-2.11663249-1.21545486-2.77009403$

$\begin{array}{llll}\text { C } & -0.04414742 & 0.03343440 & -2.76929876\end{array}$

C $-2.82113573-0.01053451-2.77000191$

$\begin{array}{llll}\text { C } & -0.75917001 & 1.22049101 & -2.76921514\end{array}$

$\begin{array}{llll}\text { C } & -2.15535253 & 1.20763578 & -2.76956872\end{array}$

$\begin{array}{llll}\text { O } & 0.06589581 & -2.29989383 & -2.76978370\end{array}$

$\mathrm{H} \quad-2.65968941-2.15262190 \quad-2.77044084$

H $\quad 1.04021005 \quad 0.01945242 \quad-2.76902840$

$\mathrm{H} \quad-3.90629828 \quad-0.03657042 \quad-2.77028004$

$\mathrm{H} \quad-0.22362010 \quad 2.16457103 \quad-2.76887276$

$\mathrm{H} \quad-2.71285781 \quad 2.13768733 \quad-2.76950391$

C $\quad-0.57798031 \quad-3.55430030-2.77012005$

$\begin{array}{llll}\mathrm{H} & 0.21305530 & -4.30335540 & -2.77004863\end{array}$

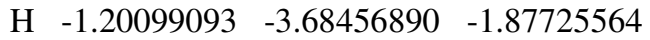

H $\quad-1.20057446 \quad-3.68429969-3.66331457$
4CF3Ph_pi_4OMePh_pi1_60
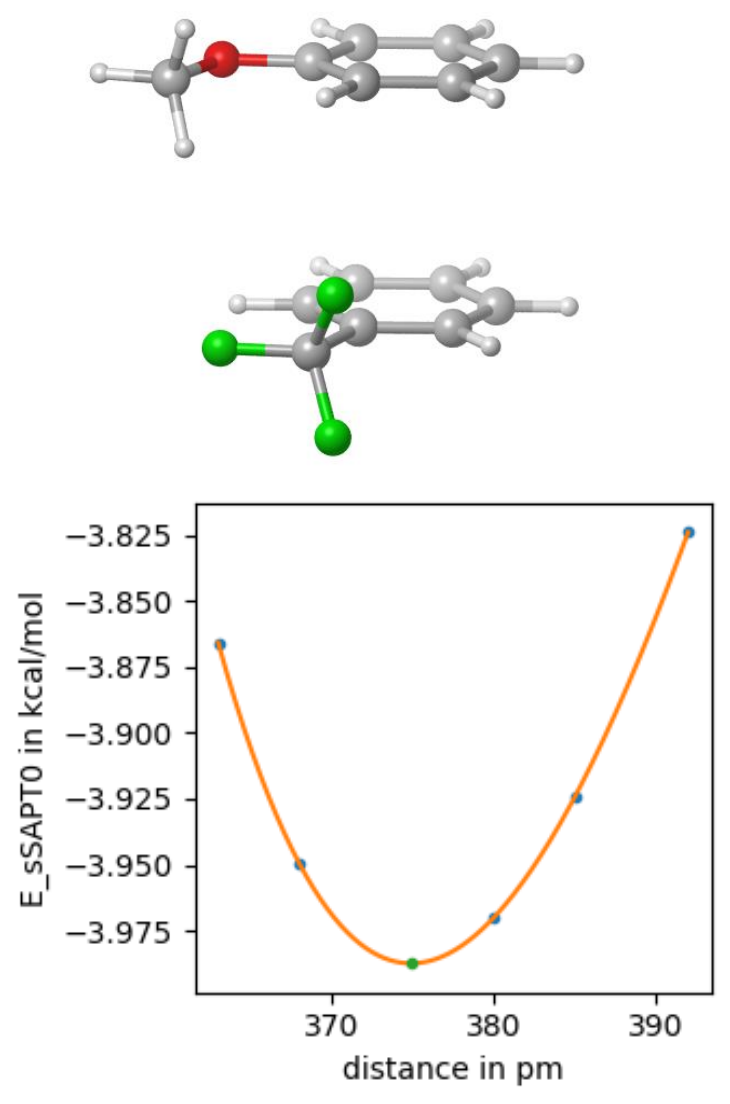

31

4CF3Ph_pi_4OMePh_pi1_60-374

$\mathrm{H} \quad-3.90811076-0.03308048 \quad 1.00030676$

$\begin{array}{llll}\text { C } & -2.82309076 & -0.01707948 & 1.00014676\end{array}$

$\begin{array}{llll}\text { C } & -2.11090776 & -1.21603848 & 1.00002576\end{array}$

$\begin{array}{llll}\text { C } & -2.14698176 & 1.19875052 & 1.00002376\end{array}$

$\begin{array}{llll}\text { C } & -0.72223076 & -1.19747348 & 0.99977676\end{array}$

$\mathrm{H} \quad-2.63937876 \quad-2.16334648 \quad 1.00007576$

$\begin{array}{llll}\text { C } & -0.75435676 & 1.22332352 & 0.99979176\end{array}$

$\begin{array}{llll}\mathrm{H} & -2.70236376 & 2.13054852 & 1.00007876\end{array}$

$\begin{array}{llll}\text { C } & -0.05106376 & 0.02457752 & 0.99966776\end{array}$

$\mathrm{H} \quad-0.15471076-2.12284748 \quad 0.99957476$

$\begin{array}{llll}\mathrm{H} & -0.21500476 & 2.16351452 & 0.99964776\end{array}$

C $\quad 1.45044524 \quad 0.00967352 \quad 0.99998476$

$\begin{array}{llll}\text { F } & 1.93370024 & -0.62786748 & 2.07810276\end{array}$

F $1.93402624 \quad-0.63569348 \quad-0.07332624$

F $\quad 1.97162124 \quad 1.24372752 \quad 0.99564576$

C $\quad-0.75592109 \quad 1.22552181 \quad-2.74813391$

$\begin{array}{llll}\text { C } & -0.03715023 & 0.02975009 & -2.74885235\end{array}$

$\begin{array}{llll}\text { C } & -2.15496267 & 1.20013033 & -2.74893390\end{array}$

C $\quad-0.72838988-1.18282706 \quad-2.75036884$

$\begin{array}{llll}\text { C } & -2.82547218 & -0.01262497 & -2.75044095\end{array}$

$\begin{array}{llll}\text { C } & -2.11624775 & -1.21532659 & -2.75117327\end{array}$

$\begin{array}{llll}\text { O } & -0.18926328 & 2.46209352 & -2.74665752\end{array}$

$\mathrm{H} \quad 1.04598858 \quad 0.02803219 \quad-2.74825574$

H $\quad-2.68503282 \quad 2.14620218 \quad-2.74834133$ 


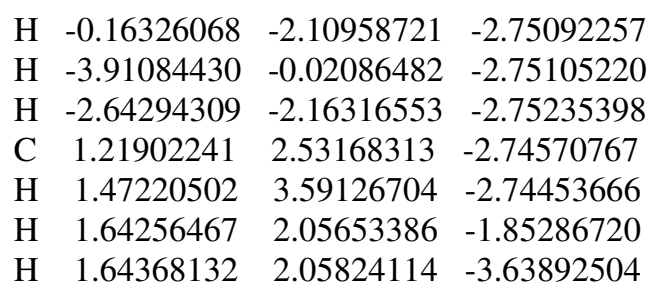

4CF3Ph_pi_4OMePh_pi2_300
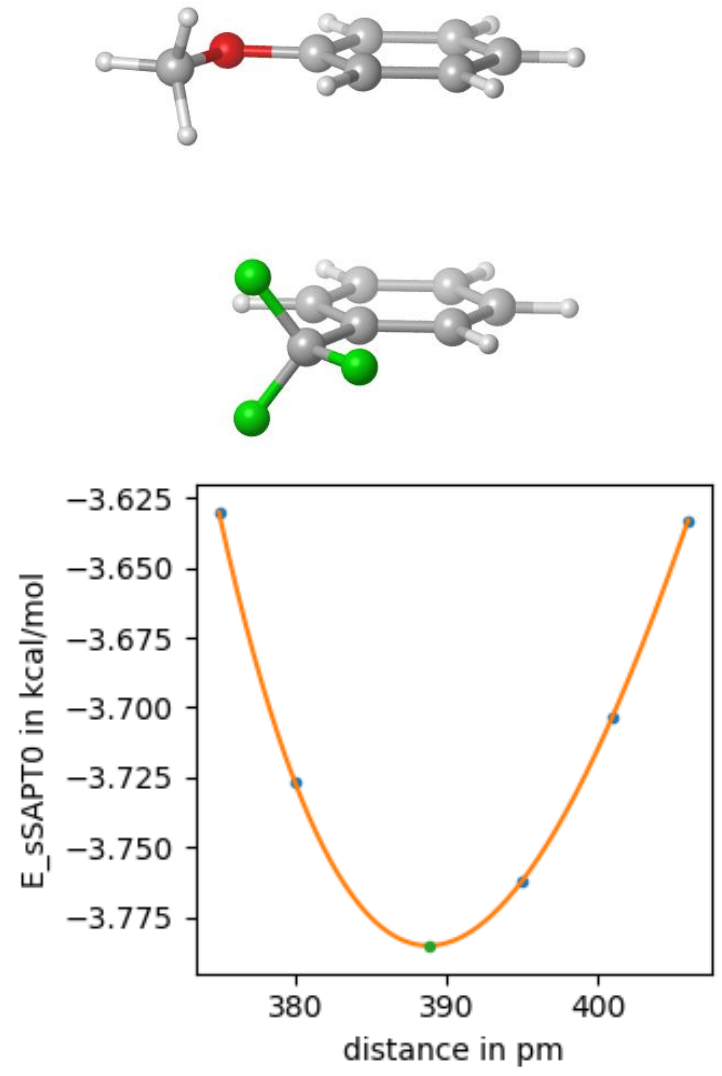

31

4CF3Ph_pi_4OMePh_pi2_300-388

$\begin{array}{lrrc}\mathrm{H} & -3.90811076 & -0.03308048 & 1.00030676 \\ \mathrm{C} & -2.82309076 & -0.01707948 & 1.00014676 \\ \mathrm{C} & -2.11090776 & -1.21603848 & 1.00002576 \\ \mathrm{C} & -2.14698176 & 1.19875052 & 1.00002376 \\ \mathrm{C} & -0.72223076 & -1.19747348 & 0.99977676 \\ \mathrm{H} & -2.63937876 & -2.16334648 & 1.00007576 \\ \mathrm{C} & -0.75435676 & 1.22332352 & 0.99979176 \\ \mathrm{H} & -2.70236376 & 2.13054852 & 1.00007876 \\ \mathrm{C} & -0.05106376 & 0.02457752 & 0.99966776 \\ \mathrm{H} & -0.15471076 & -2.12284748 & 0.99957476 \\ \mathrm{H} & -0.21500476 & 2.16351452 & 0.99964776 \\ \mathrm{C} & 1.45044524 & 0.00967352 & 0.99998476 \\ \mathrm{~F} & 1.93370024 & -0.62786748 & 2.07810276 \\ \mathrm{~F} & 1.93402624 & -0.63569348 & -0.07332624 \\ \mathrm{~F} & 1.97162124 & 1.24372752 & 0.99564576 \\ \mathrm{C} & -0.72165475 & -1.19078771 & -2.88907582\end{array}$

$\begin{array}{llll}\text { C } & -0.03705233 & 0.02486884 & -2.88819383\end{array}$

$\begin{array}{llll}\text { C } & -2.12085409 & -1.20504451 & -2.88986843\end{array}$

$\begin{array}{llll}\text { C } & -0.76237052 & 1.21737519 & -2.88811407\end{array}$

$\begin{array}{llll}\text { C } & -2.82545552 & -0.01177274 & -2.88977861\end{array}$

$\begin{array}{llll}\text { C } & -2.15059205 & 1.21054045 & -2.88890148\end{array}$

$\begin{array}{llll}\mathrm{O} & -0.12018853 & -2.41080870 & -2.88922986\end{array}$

H $\quad 1.04560298 \quad 0.05727390 \quad-2.88757457$

H $\quad-2.62390632-2.16575501-2.89054137$

$\mathrm{H} \quad-0.22372616 \quad 2.15977504 \quad-2.88742726$

$\begin{array}{llll}\mathrm{H} & -3.91062537 & -0.03428731 & -2.89039934\end{array}$

$\mathrm{H} \quad-2.70393114 \quad 2.14307671 \quad-2.88883419$

C $\quad 1.28950348 \quad-2.44047052 \quad-2.88834589$

H $\quad 1.57260573 \quad-3.49245624 \quad-2.88857631$

H $\quad 1.69943007 \quad-1.95469708 \quad-1.99486765$

H $1.70056106-1.95400173-3.78092616$

4CF3Ph_pi_4OMePh_pi2_60
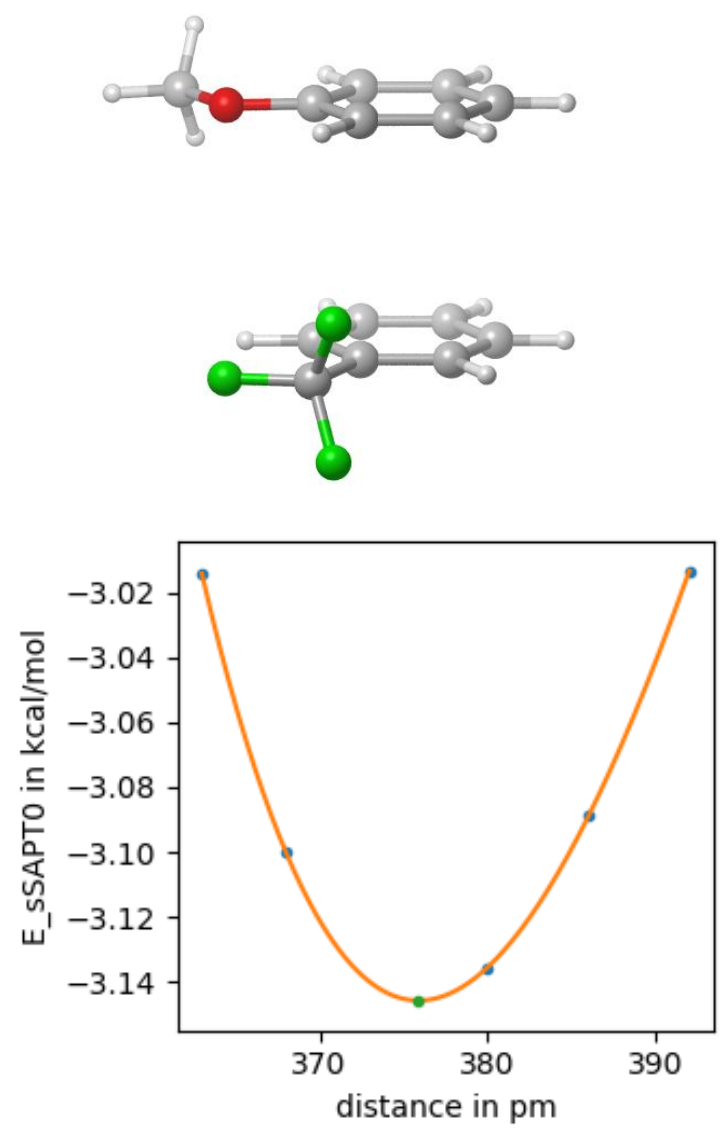

31

4CF3Ph_pi_4OMePh_pi2_60-375

$\mathrm{H} \quad-3.90811076-0.03308048 \quad 1.00030676$

$\begin{array}{llll}\text { C } & -2.82309076 & -0.01707948 & 1.00014676\end{array}$

$\begin{array}{llll}\text { C } & -2.11090776 & -1.21603848 & 1.00002576\end{array}$

$\begin{array}{llll}\text { C } & -2.14698176 & 1.19875052 & 1.00002376\end{array}$

$\begin{array}{llll}\text { C } & -0.72223076 & -1.19747348 & 0.99977676\end{array}$

$\begin{array}{llll}\mathrm{H} & -2.63937876 & -2.16334648 & 1.00007576\end{array}$

$\begin{array}{llll}\text { C } & -0.75435676 & 1.22332352 & 0.99979176\end{array}$

H $\quad-2.70236376 \quad 2.13054852 \quad 1.00007876$ 


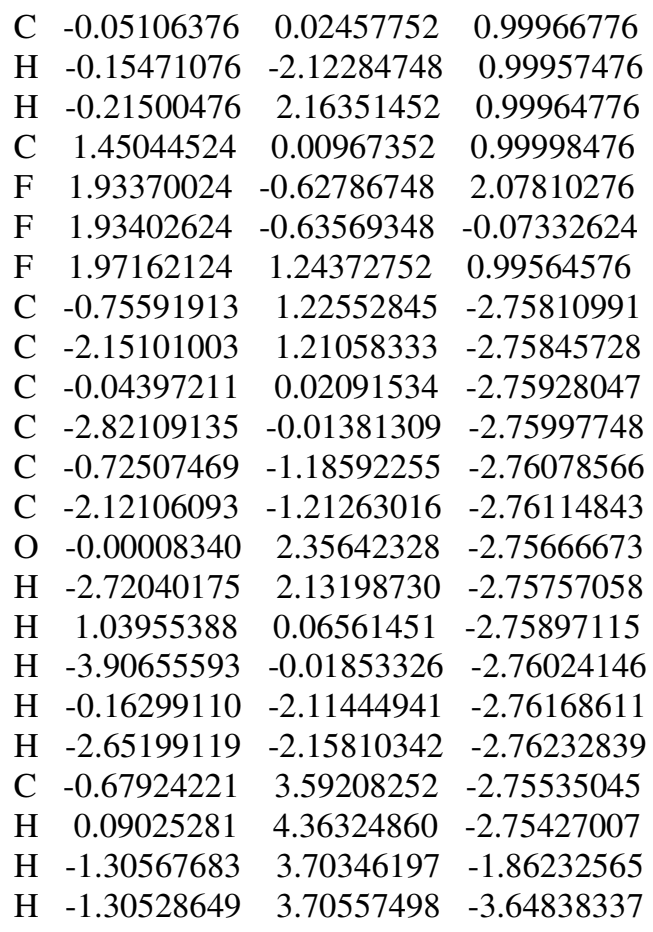

4CF3Ph_pi_4PhPh_pi1_300
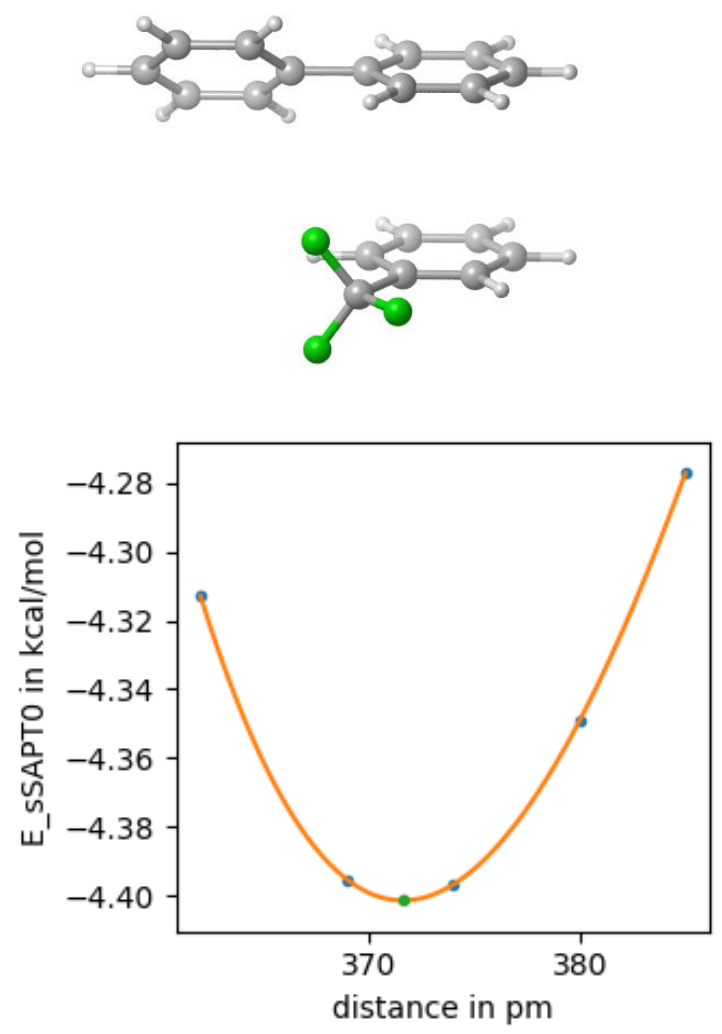

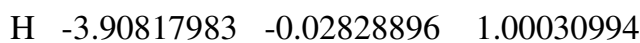

$\begin{array}{llll}\text { C } & -2.82313085 & -0.01439191 & 1.00014854\end{array}$

$\begin{array}{llll}\text { C } & -2.11327404 & -1.21472962 & 1.00002617\end{array}$

$\begin{array}{llll}\text { C } & -2.14466555 & 1.20012479 & 1.00002513\end{array}$

$\begin{array}{llll}\text { C } & -0.72456365 & -1.19885738 & 0.99977538\end{array}$

$\begin{array}{llll}\mathrm{H} & -2.64358093 & -2.16101110 & 1.00007649\end{array}$

$\begin{array}{llll}\text { C } & -0.75199552 & 1.22199736 & 0.99979134\end{array}$

$\begin{array}{llll}\mathrm{H} & -2.69823970 & 2.13299796 & 1.00008120\end{array}$

$\begin{array}{llll}\text { C } & -0.05102828 & 0.02188989 & 0.99966597\end{array}$

$\begin{array}{llll}\mathrm{H} & -0.15883907 & -2.12533010 & 0.99957229\end{array}$

$\begin{array}{llll}\mathrm{H} & -0.21082145 & 2.16114076 & 0.99964700\end{array}$

C $\quad 1.45044899 \quad 0.00407440 \quad 0.99998103$

$\begin{array}{llll}\text { F } & 1.93246825 & -0.63440287 & 2.07809816\end{array}$

F $1.93277629 \quad-0.64222867 \quad-0.07333084$

$\begin{array}{llll}\text { F } & 1.97401691 & 1.23711549 & 0.99564182\end{array}$

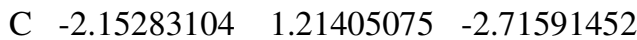

$\begin{array}{llll}\text { C } & -2.82985536 & -0.00276422 & -2.71685853\end{array}$

$\begin{array}{llll}\text { C } & -0.76040288 & 1.22602099 & -2.71563211\end{array}$

$\begin{array}{llll}\text { C } & -2.11945878 & -1.19833422 & -2.71818896\end{array}$

$\begin{array}{llll}\text { C } & -0.05074429 & 0.03000973 & -2.71561590\end{array}$

$\begin{array}{llll}\text { C } & -0.71985297 & -1.19929274 & -2.71724101\end{array}$

$\begin{array}{llll}\mathrm{H} & -2.70684619 & 2.14709169 & -2.71540081\end{array}$

$\begin{array}{llll}\mathrm{H} & -3.91503047 & -0.02153710 & -2.72552443\end{array}$

H $\quad-0.22447377 \quad 2.16980536 \quad-2.70645631$

$\begin{array}{llll}\mathrm{H} & -2.65150719 & -2.14475744 & -2.74576453\end{array}$

$\begin{array}{llll}\mathrm{H} & 1.03489891 & 0.04410073 & -2.68854955\end{array}$

C $\quad 0.03781439 \quad-2.47531673 \quad-2.71794041$

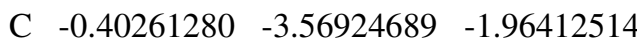

C $\quad 1.20873414 \quad-2.61164911 \quad-3.47243119$

C $0.30676266 \quad-4.76542459-1.96503650$

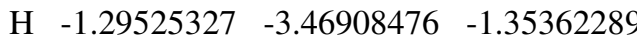

C $\quad 1.91940984 \quad-3.80705505-3.47282923$

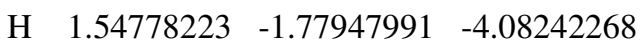

C $\quad 1.47078816 \quad-4.88866277 \quad-2.71926303$

H $\quad-0.04570072-5.60060366-1.36814566$

$\mathrm{H} \quad 2.82111949 \quad-3.89688847 \quad-4.07022775$

H $\quad 2.02480035 \quad-5.82170547 \quad-2.71977446$

4CF3Ph_pi_4PhPh_pi1_60
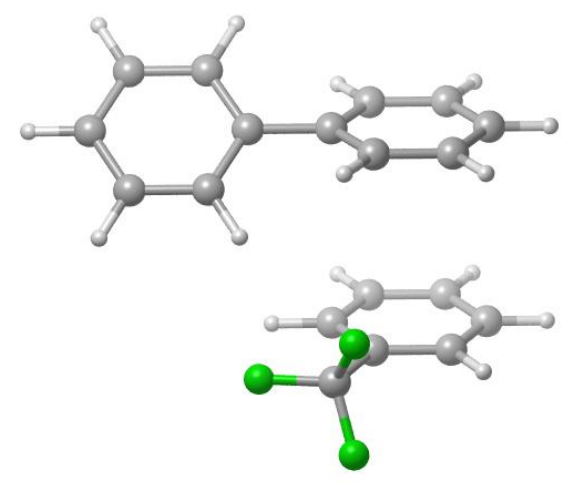

37

4CF3Ph_pi_4PhPh_pi1_300-371 


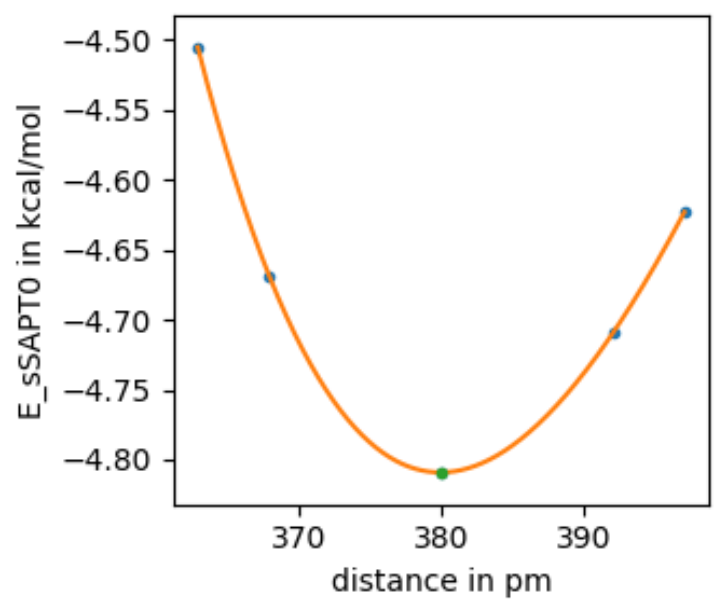

37

4CF3Ph_pi_4PhPh_pi1_60-379

$\begin{array}{llll}\mathrm{H} & -3.90817983 & -0.02828896 & 1.00030994\end{array}$

$\begin{array}{llll}\text { C } & -2.82313085 & -0.01439191 & 1.00014854\end{array}$

C $\quad-2.11327404-1.21472962 \quad 1.00002617$

$\begin{array}{llll}\text { C } & -2.14466555 & 1.20012479 & 1.00002513\end{array}$

C $-0.72456365-1.19885738 \quad 0.99977538$

$\begin{array}{llll}\mathrm{H} & -2.64358093 & -2.16101110 & 1.00007649\end{array}$

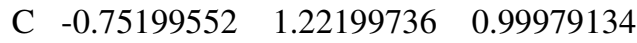

$\begin{array}{llll}\mathrm{H} & -2.69823970 & 2.13299796 & 1.00008120\end{array}$

$\begin{array}{llll}\text { C } & -0.05102828 & 0.02188989 & 0.99966597\end{array}$

$\begin{array}{llll}\mathrm{H} & -0.15883907 & -2.12533010 & 0.99957229\end{array}$

$\begin{array}{llll}\mathrm{H} & -0.21082145 & 2.16114076 & 0.99964700\end{array}$

$\begin{array}{llll}\text { C } & 1.45044899 & 0.00407440 & 0.99998103\end{array}$

$\begin{array}{llll}\text { F } & 1.93246825 & -0.63440287 & 2.07809816\end{array}$

$\begin{array}{llll}\text { F } & 1.93277629 & -0.64222867 & -0.07333084\end{array}$

$\begin{array}{llll}\text { F } & 1.97401691 & 1.23711549 & 0.99564182\end{array}$

$\begin{array}{llll}\text { C } & -2.12309045 & -1.21632587 & -2.80109868\end{array}$

C $-0.73078532-1.19423866-2.80080983$

$\begin{array}{llll}\text { C } & -2.82967122 & -0.01643307 & -2.80043755\end{array}$

$\begin{array}{llll}\text { C } & -0.05058890 & 0.01876812 & -2.80054094\end{array}$

$\begin{array}{llll}\text { C } & -2.14872484 & 1.19615411 & -2.79882171\end{array}$

$\begin{array}{llll}\text { C } & -0.74956245 & 1.23134076 & -2.79919711\end{array}$

H $\quad-2.65412013-2.16263732-2.80183301$

H $-0.17193249-2.12463424-2.80975983$

$\begin{array}{llll}\mathrm{H} & -3.91498460 & -0.02420401 & -2.79221493\end{array}$

$\mathrm{H} \quad 1.03508550 \quad 0.03123454 \quad-2.82715912$

$\mathrm{H} \quad-2.70377314 \quad 2.12928075 \quad-2.77146566$

C $\quad-0.02332680 \quad 2.52551166 \quad-2.79818971$

$\begin{array}{llll}\text { C } & 1.14360006 & 2.69042973 & -2.04321917\end{array}$

$\begin{array}{llll}\text { C } & -0.49006120 & 3.60834971 & -3.55219016\end{array}$

C $\quad 1.82483296 \quad 3.90285570 \quad-2.04253079$

$\begin{array}{llll}\mathrm{H} & 1.50264477 & 1.86679312 & -1.43308634\end{array}$

$\begin{array}{llll}\text { C } & 0.18985277 & 4.82151542 & -3.55098899\end{array}$

$\begin{array}{lllll}\mathrm{H} & -1.37973243 & 3.48639580 & -4.16305931\end{array}$

C $\quad 1.35020555 \quad 4.97317585-2.79628426$

H $\quad 2.72382990 \quad 4.01470743 \quad-1.44476254$

H $\quad-0.18268289 \quad 5.64783095 \quad-4.14802764$

H $\quad 1.88123823 \quad 5.91948562 \quad-2.79554766$
4CF3Ph_pi_4PhPh_pi2_300
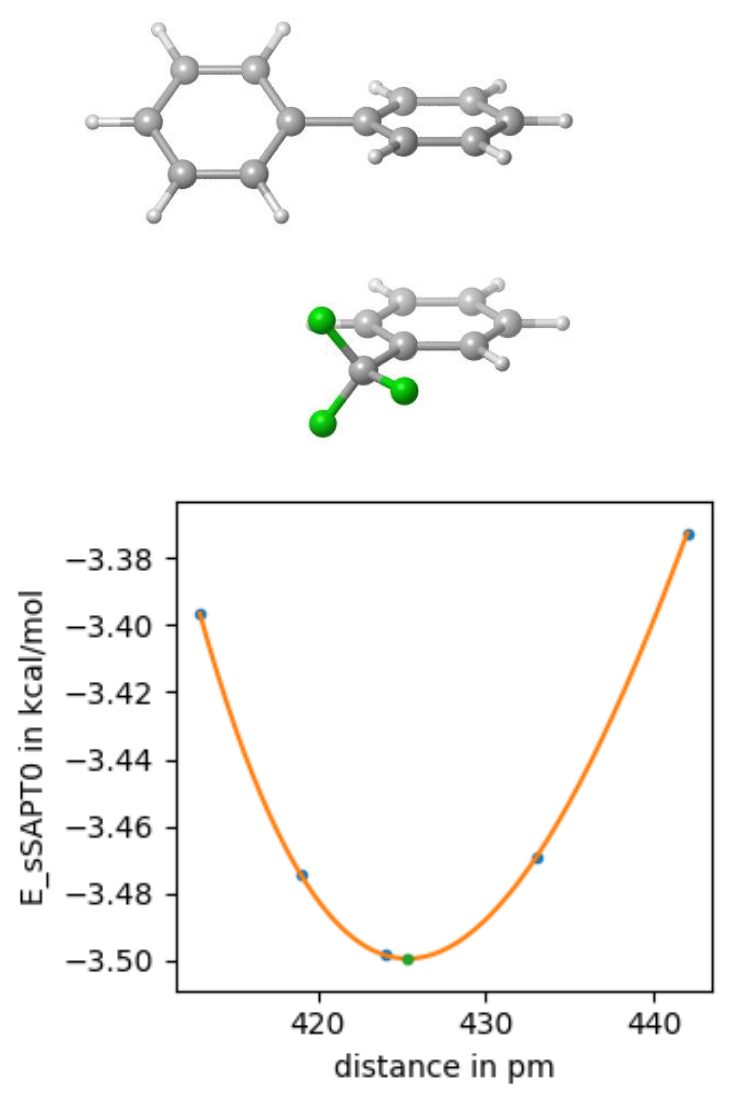

37

4CF3Ph_pi_4PhPh_pi2_300-425

H $\quad-3.90817983-0.02828896 \quad 1.00030994$

$\begin{array}{llll}\text { C } & -2.82313085 & -0.01439191 & 1.00014854\end{array}$

$\begin{array}{llll}\text { C } & -2.11327404 & -1.21472962 & 1.00002617\end{array}$

$\begin{array}{llll}\text { C } & -2.14466555 & 1.20012479 & 1.00002513\end{array}$

$\begin{array}{llll}\text { C } & -0.72456365 & -1.19885738 & 0.99977538\end{array}$

$\begin{array}{llll}\mathrm{H} & -2.64358093 & -2.16101110 & 1.00007649\end{array}$

$\begin{array}{llll}\text { C } & -0.75199552 & 1.22199736 & 0.99979134\end{array}$

$\begin{array}{llll}\mathrm{H} & -2.69823970 & 2.13299796 & 1.00008120\end{array}$

$\begin{array}{llll}\text { C } & -0.05102828 & 0.02188989 & 0.99966597\end{array}$

$\begin{array}{llll}\mathrm{H} & -0.15883907 & -2.12533010 & 0.99957229\end{array}$

$\begin{array}{llll}\mathrm{H} & -0.21082145 & 2.16114076 & 0.99964700\end{array}$

$\begin{array}{llll}\text { C } & 1.45044899 & 0.00407440 & 0.99998103\end{array}$

$\begin{array}{llll}\text { F } & 1.93246825 & -0.63440287 & 2.07809816\end{array}$

F $1.93277629 \quad-0.64222867 \quad-0.07333084$

$\begin{array}{llll}\text { F } & 1.97401691 & 1.23711549 & 0.99564182\end{array}$

$\begin{array}{llll}\text { C } & -2.15272527 & 1.21440902 & -3.25346479\end{array}$

$\begin{array}{llll}\text { C } & -0.76029641 & 1.22637868 & -3.25318266\end{array}$

$\begin{array}{llll}\text { C } & -2.82974976 & -0.00240505 & -3.25440853\end{array}$

$\begin{array}{llll}\text { C } & -0.05063778 & 0.03037051 & -3.25451352\end{array}$

C $\quad-2.11935164-1.19797720 \quad-3.25439188$

$\begin{array}{llll}\text { C } & -0.71974720 & -1.19893447 & -3.25479128\end{array}$

H $\quad-2.70673940 \quad 2.14745056 \quad-3.25295108$

$\begin{array}{llll}\mathrm{H} & -0.22436490 & 2.17017452 & -3.26088805\end{array}$

H $\quad-3.91492844 \quad-0.02119011-3.24619322$ 


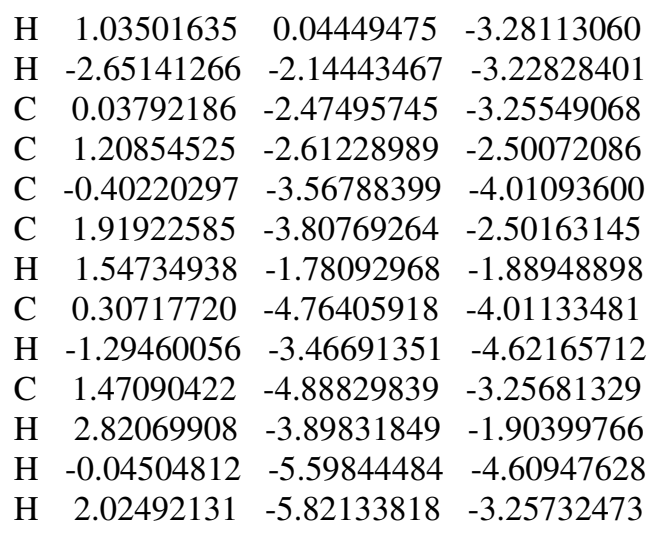

4CF3Ph_pi_4PhPh_pi2_60
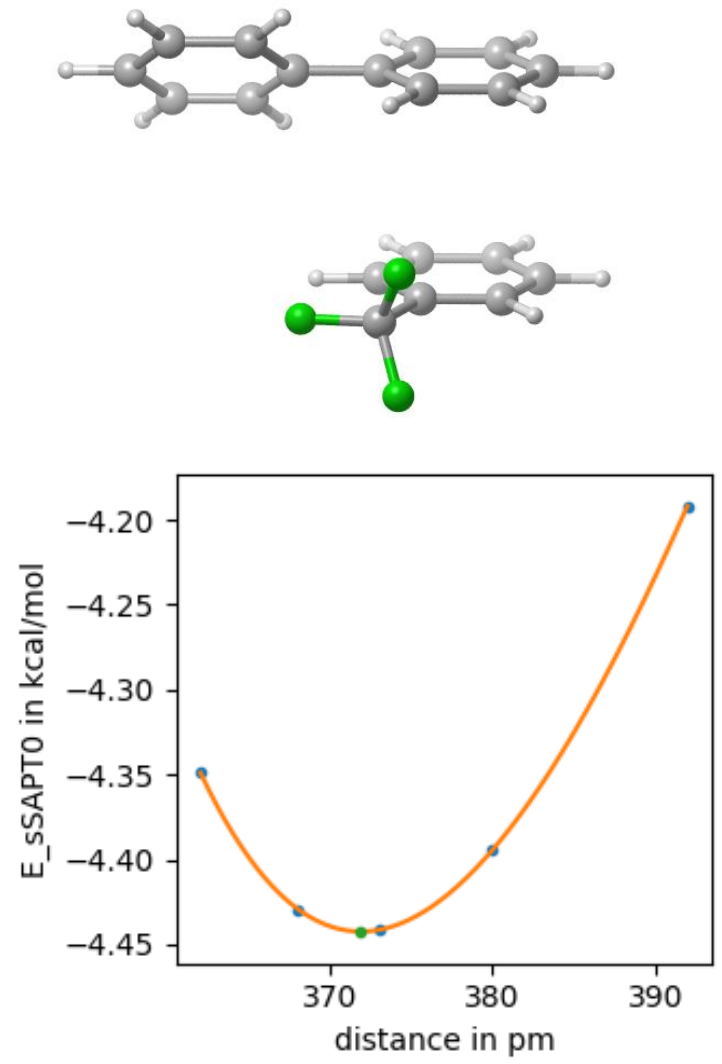

37

4CF3Ph_pi_4PhPh_pi2_60-371

$\mathrm{H} \quad-3.90817983 \quad-0.02828896 \quad 1.00030994$

$\begin{array}{llll}\text { C } & -2.82313085 & -0.01439191 & 1.00014854\end{array}$

$\begin{array}{llll}\text { C } & -2.11327404 & -1.21472962 & 1.00002617\end{array}$

$\begin{array}{llll}\text { C } & -2.14466555 & 1.20012479 & 1.00002513\end{array}$

$\begin{array}{llll}\text { C } & -0.72456365 & -1.19885738 & 0.99977538\end{array}$

$\begin{array}{llll}\mathrm{H} & -2.64358093 & -2.16101110 & 1.00007649\end{array}$

C $\quad-0.75199552 \quad 1.22199736 \quad 0.99979134$

$\begin{array}{llll}\mathrm{H} & -2.69823970 & 2.13299796 & 1.00008120\end{array}$

$\begin{array}{llll}\text { C } & -0.05102828 & 0.02188989 & 0.99966597\end{array}$

$\begin{array}{llll}\mathrm{H} & -0.15883907 & -2.12533010 & 0.99957229\end{array}$
H $\quad-0.21082145 \quad 2.16114076 \quad 0.99964700$

$\begin{array}{llll}\text { C } & 1.45044899 & 0.00407440 & 0.99998103\end{array}$

$\begin{array}{llll}\text { F } & 1.93246825 & -0.63440287 & 2.07809816\end{array}$

$\begin{array}{llll}\text { F } & 1.93277629 & -0.64222867 & -0.07333084\end{array}$

$\begin{array}{llll}\text { F } & 1.97401691 & 1.23711549 & 0.99564182\end{array}$

C $-2.12310632 \quad-1.21637961-2.72045990$

$\begin{array}{llll}\text { C } & -2.82968694 & -0.01648591 & -2.71979905\end{array}$

$\begin{array}{llll}\text { C } & -0.73080188 & -1.19429300 & -2.72017078\end{array}$

$\begin{array}{llll}\text { C } & -2.14874207 & 1.19610087 & -2.71953028\end{array}$

$\begin{array}{llll}\text { C } & -0.05060555 & 0.01871508 & -2.71855480\end{array}$

C $-0.74957832 \quad 1.23128702-2.71855833$

$\mathrm{H}-2.65413702-2.16269049-2.72119423$

H $\quad-3.91499673-0.02424652 \quad-2.72845763$

$\mathrm{H} \quad-0.17195144 \quad-2.12469959 \quad-2.71223957$

$\begin{array}{llll}\mathrm{H} & -2.70377781 & 2.12926488 & -2.74585766\end{array}$

H $\quad 1.03505799 \quad 0.03114317 \quad-2.69148955$

C $\quad-0.02334440 \quad 2.52545889-2.71755093$

$\begin{array}{llll}\text { C } & -0.49038110 & 3.60728865 & -1.96229079\end{array}$

C $\quad 1.14387869 \quad 2.69138866 \quad-3.47184099$

$\begin{array}{llll}\text { C } & 0.18952810 & 4.82045743 & -1.96160217\end{array}$

$\begin{array}{llll}\mathrm{H} & -1.38029507 & 3.48451517 & -1.35193659\end{array}$

C $\quad 1.82510662 \quad 3.90381702 \quad-3.47064004$

$\mathrm{H} \quad 1.50316723 \quad 1.86857100 \quad-4.08293150$

C $\quad 1.35017925 \quad 4.97312796-2.71564548$

$\mathrm{H} \quad-0.18324555 \quad 5.64597337 \quad-1.36360851$

$\begin{array}{llll}\mathrm{H} & 2.72433987 & 4.01646928 & -4.06790410\end{array}$

H $\quad 1.88120696 \quad 5.91944052 \quad-2.71490888$

4CF3Ph_pi_Ph_pi_60
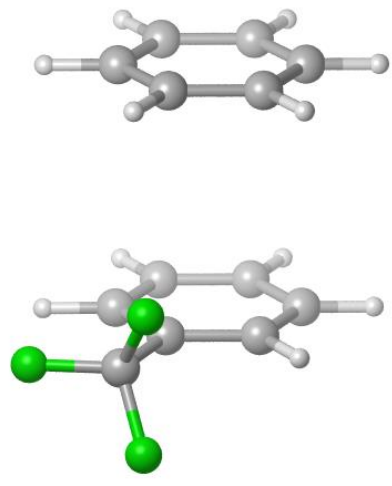


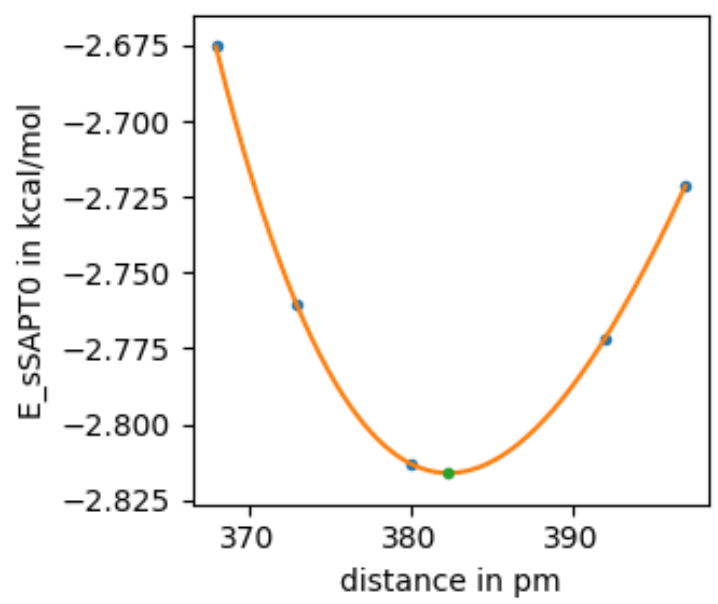

27

4CF3Ph_pi_Ph_pi_60-382

$\begin{array}{lrrl}\mathrm{H} & -3.84245403 & -0.56217066 & 0.99996705 \\ \mathrm{C} & -2.78611520 & -0.31383071 & 0.99995626 \\ \mathrm{C} & -1.83335676 & -1.33214238 & 1.00000299 \\ \mathrm{C} & -2.38650680 & 1.01871539 & 0.99985664 \\ \mathrm{C} & -0.48097719 & -1.01617081 & 0.99994506 \\ \mathrm{H} & -2.14635447 & -2.37075034 & 1.00003454 \\ \mathrm{C} & -1.03155969 & 1.34140190 & 0.99981591 \\ \mathrm{H} & -3.12881337 & 1.80971325 & 0.99978102 \\ \mathrm{C} & -0.08753004 & 0.32139158 & 0.99985840 \\ \mathrm{H} & 0.27180715 & -1.79829086 & 0.99987499 \\ \mathrm{H} & -0.70640774 & 2.37539219 & 0.99969228 \\ \mathrm{C} & 1.38223405 & 0.62887284 & 1.00038402 \\ \mathrm{~F} & 1.99082182 & 0.10984358 & 2.07860567 \\ \mathrm{~F} & 1.99313611 & 0.10221220 & -0.07282281 \\ \mathrm{~F} & 1.62660688 & 1.94598924 & 0.99604592 \\ \mathrm{C} & -0.48303989 & -1.00824577 & -2.82475726 \\ \mathrm{C} & -1.83944693 & -1.32560657 & -2.82525673 \\ \mathrm{C} & -0.08048346 & 0.32534124 & -2.82391109 \\ \mathrm{C} & -2.79267968 & -0.31008494 & -2.82511226 \\ \mathrm{C} & -1.03365903 & 1.34095277 & -2.82346555 \\ \mathrm{C} & -2.38970350 & 1.02316747 & -2.82408716 \\ \mathrm{H} & 0.25978358 & -1.79968867 & -2.82502548 \\ \mathrm{H} & -2.15348881 & -2.36486614 & -2.82608013 \\ \mathrm{H} & 0.97656394 & 0.57299239 & -2.82345995 \\ \mathrm{H} & -3.84983732 & -0.55742792 & -2.82566825 \\ \mathrm{H} & -0.71983956 & 2.38031545 & -2.82272515 \\ \mathrm{H} & -3.13274959 & 1.81478500 & -2.82392990\end{array}$

4MePh_pi_Ph_pi_60
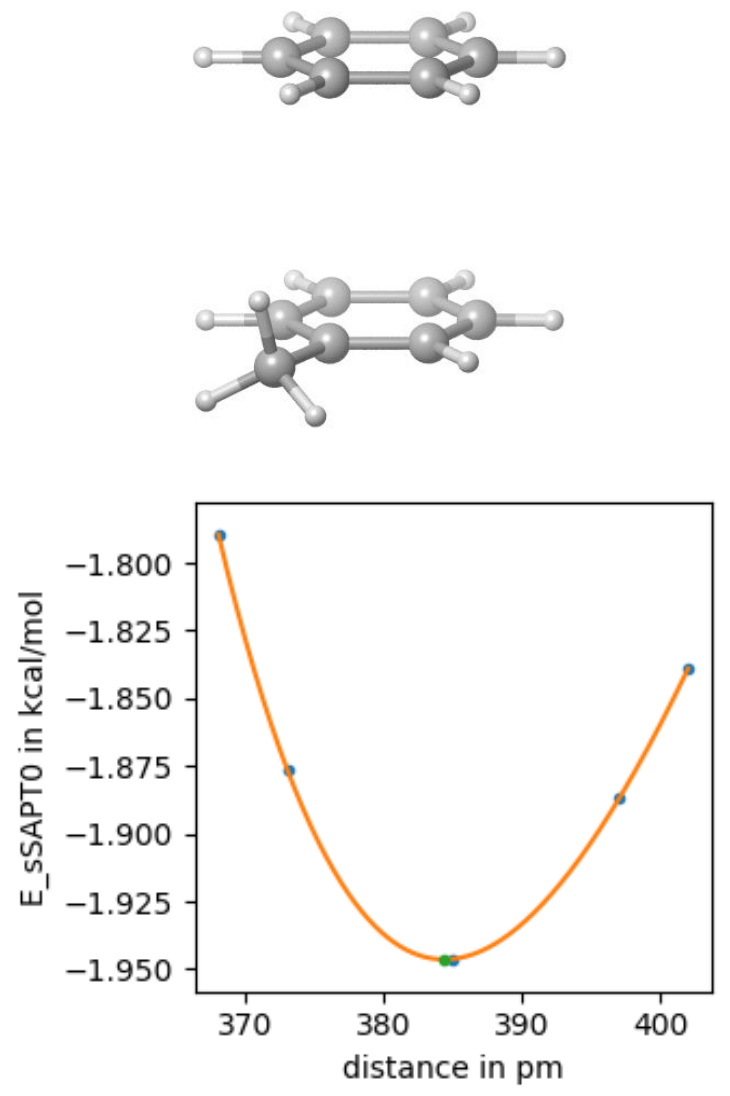

27

4MePh_pi_Ph_pi_60-384

H $\quad 2.12550555-1.27494126 \quad 0.76876672$

$\begin{array}{llll}\text { C } & 1.19469567 & -0.71658509 & 0.76874815\end{array}$

$\begin{array}{llll}\text { C } & 1.21758873 & 0.67626597 & 0.76872723\end{array}$

$\begin{array}{llll}\text { C } & -0.02338433 & -1.39244591 & 0.76874293\end{array}$

$\begin{array}{llll}\text { C } & 0.02322135 & 1.39280167 & 0.76890307\end{array}$

$\begin{array}{llll}\mathrm{H} & 2.16676406 & 1.20328553 & 0.76889520\end{array}$

$\begin{array}{llll}\text { C } & -1.21782760 & -0.67598525 & 0.76861777\end{array}$

$\begin{array}{llll}\mathrm{H} & -0.04154939 & -2.47796445 & 0.76869751\end{array}$

$\begin{array}{llll}\text { C } & -1.19444282 & 0.71660143 & 0.76871884\end{array}$

$\begin{array}{llll}\mathrm{H} & 0.04106195 & 2.47836275 & 0.76905348\end{array}$

H $\quad-2.16715076-1.20280924 \quad 0.76853280$

$\begin{array}{llll}\mathrm{H} & -2.12547047 & 1.27513823 & 0.76881127\end{array}$

$\begin{array}{llll}\text { C } & 1.20874732 & 0.67388027 & -3.07652906\end{array}$

$\begin{array}{llll}\text { C } & 0.01672335 & 1.39267412 & -3.07571085\end{array}$

C $\quad 1.20480457 \quad-0.72276616-3.07465380$

$\begin{array}{llll}\mathrm{H} & 2.15859841 & 1.20257810 & -3.08045956\end{array}$

$\begin{array}{llll}\text { C } & -1.20357912 & 0.72242332 & -3.07499448\end{array}$

$\mathrm{H} \quad 0.04138862 \quad 2.47804238-3.07969910$

C $\quad-0.02597152-1.38348536 \quad-3.07676276$

$\begin{array}{llll}\text { C } & 2.49706742 & -1.49924828 & -3.04277442\end{array}$

$\begin{array}{llll}\mathrm{H} & -2.13444064 & 1.28095339 & -3.07798413\end{array}$

C $-1.22086881 \quad-0.66995078 \quad-3.07585430$

$\mathrm{H} \quad-0.04553590-2.47042415 \quad-3.08092149$

H $\quad 2.80033511 \quad-1.71112680 \quad-2.01226856$ 
$\begin{array}{rrrr}\mathrm{H} & 2.39627673 & -2.45669474 & -3.55942814 \\ \mathrm{H} & 3.30706085 & -0.93939033 & -3.51610585 \\ \mathrm{H} & -2.16704462 & -1.20229319 & -3.08001809\end{array}$

4nPrPh_Ph_pi_1_pi_0
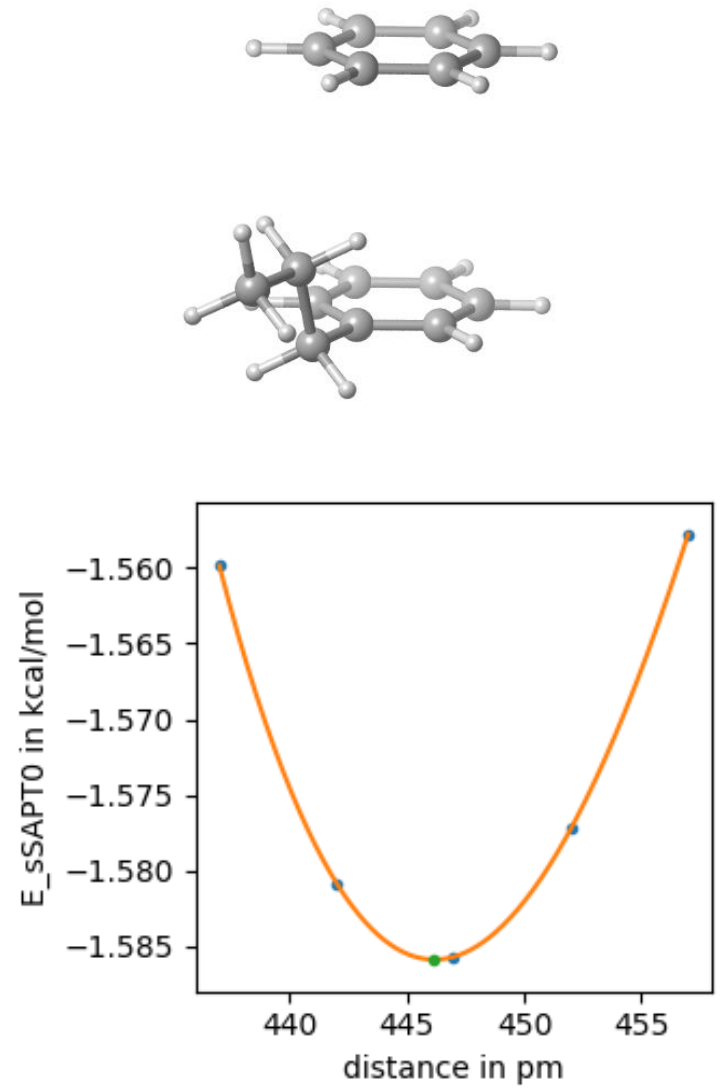

33

4nPrPh_Ph_pi_1_pi_0-446

$\begin{array}{llll}\mathrm{H} & 2.38305942 & -0.68129007 & 1.00002909\end{array}$

$\begin{array}{llll}\text { C } & 1.33943933 & -0.38291826 & 1.00000511\end{array}$

$\begin{array}{llll}\text { C } & 1.00099442 & 0.96838231 & 0.99994138\end{array}$

$\begin{array}{llll}\text { C } & 0.33783412 & -1.35105758 & 1.00003572\end{array}$

$\begin{array}{llll}\text { C } & -0.33814639 & 1.35131666 & 1.00011026\end{array}$

$\begin{array}{llll}\mathrm{H} & 1.78139033 & 1.72314429 & 1.00008141\end{array}$

$\begin{array}{llll}\text { C } & -1.00136057 & -0.96821536 & 0.99990361\end{array}$

$\begin{array}{llll}\mathrm{H} & 0.60128884 & -2.40427745 & 1.00002367\end{array}$

$\begin{array}{llll}\text { C } & -1.33926208 & 0.38295712 & 0.99996188\end{array}$

H $-0.60192552 \quad 2.40449362 \quad 1.00022731$

$\begin{array}{llll}\mathrm{H} & -1.78194990 & -1.72282671 & 0.99984657\end{array}$

$\begin{array}{llll}\mathrm{H} & -2.38313927 & 0.68144704 & 1.00004889\end{array}$

$\begin{array}{llll}\text { C } & -1.34868579 & 0.38558163 & -3.46451231\end{array}$

$\begin{array}{llll}\text { C } & -1.00593604 & -0.96401713 & -3.46252577\end{array}$

$\begin{array}{llll}\text { C } & -0.34438768 & 1.35007065 & -3.46251533\end{array}$

$\begin{array}{llll}\text { C } & 0.33309994 & -1.34358227 & -3.45919424\end{array}$

$\begin{array}{llll}\text { C } & 0.99294877 & 0.96452115 & -3.45920839\end{array}$

C $\quad 1.34993553 \quad-0.38590659-3.45925914$

$\begin{array}{llll}\mathrm{H} & -2.39206158 & 0.68386579 & -3.47041245\end{array}$

H $\quad-1.78253012 \quad-1.72249550 \quad-3.46728248$

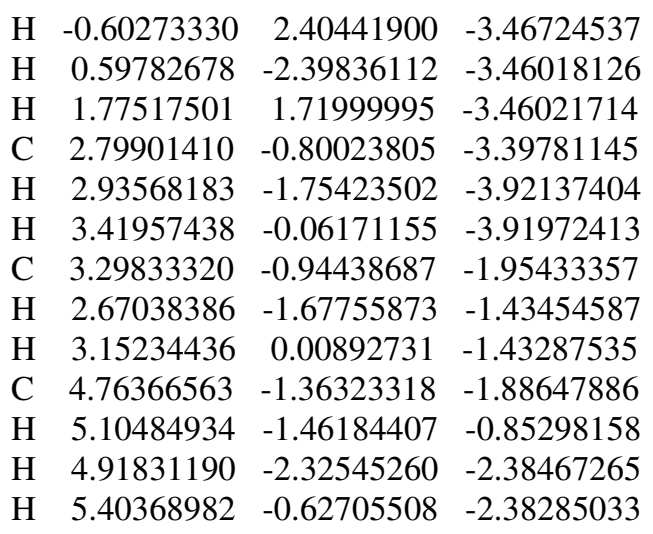

4nPrPh_Ph_pi_2_pi_0
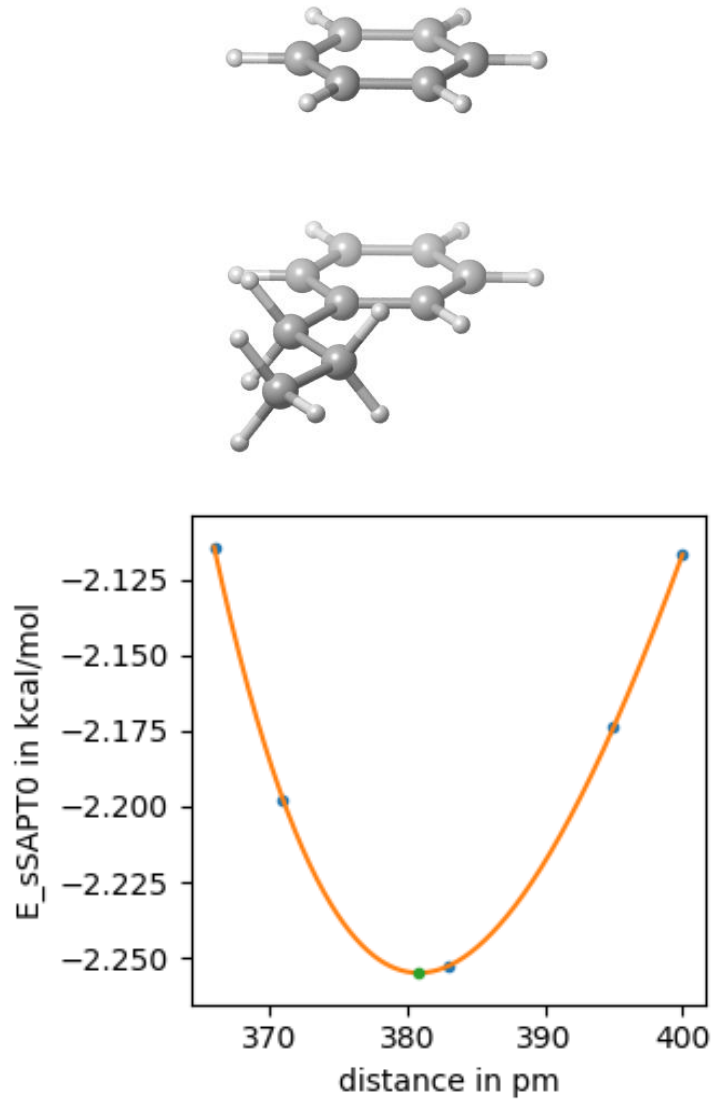

33

4nPrPh_Ph_pi_2_pi_0-380

$\begin{array}{llll}\mathrm{H} & 2.47818743 & -0.04130912 & 1.00002014\end{array}$

$\begin{array}{llll}\text { C } & 1.39290347 & -0.02321566 & 1.00000007\end{array}$

$\begin{array}{llll}\text { C } & 0.71624531 & 1.19444271 & 0.99989932\end{array}$

$\begin{array}{llll}\text { C } & 0.67600314 & -1.21760279 & 1.00007275\end{array}$

$\begin{array}{llll}\text { C } & -0.67637615 & 1.21773013 & 1.00007324\end{array}$

$\begin{array}{llll}\mathrm{H} & 1.27470068 & 2.12546958 & 1.00000658\end{array}$

C $\quad-0.71664652-1.194418320 .99994566$

$\begin{array}{llll}\mathrm{H} & 1.20307668 & -2.16674660 & 1.00008955\end{array}$

$\begin{array}{llll}\text { C } & -1.39274665 & 0.02325697 & 0.99996691\end{array}$

H $\quad-1.20375194 \quad 2.16674853 \quad 1.00016143$ 


$\begin{array}{lrrl}\mathrm{H} & -1.27532771 & -2.12534975 & 0.99992141 \\ \mathrm{H} & -2.47830951 & 0.04139798 & 1.00005785 \\ \mathrm{C} & -0.68192366 & 1.22923057 & -2.81125235 \\ \mathrm{C} & -1.39433691 & 0.03068329 & -2.81046275 \\ \mathrm{C} & 0.70709110 & 1.19653425 & -2.80946938 \\ \mathrm{C} & -0.71600098 & -1.18123752 & -2.80791236 \\ \mathrm{C} & 1.38371453 & -0.02328913 & -2.80691629 \\ \mathrm{C} & 0.68301079 & -1.22951090 & -2.80609832 \\ \mathrm{H} & -1.20783203 & 2.17844057 & -2.81323951 \\ \mathrm{H} & -2.47986104 & 0.04223762 & -2.81183672 \\ \mathrm{H} & 1.27289879 & 2.12302273 & -2.81006103 \\ \mathrm{H} & -1.27588351 & -2.11374386 & -2.80730247 \\ \mathrm{H} & 2.46888554 & -0.02537018 & -2.80556460 \\ \mathrm{C} & 1.37059819 & -2.57979617 & -2.80335701 \\ \mathrm{H} & 1.02352227 & -3.14625331 & -1.92865248 \\ \mathrm{H} & 1.02543906 & -3.14882492 & -3.67714855 \\ \mathrm{C} & 2.89626545 & -2.55725125 & -2.80169337 \\ \mathrm{H} & 3.25370808 & -2.00705903 & -1.92316861 \\ \mathrm{H} & 3.25565352 & -2.00915242 & -3.68073832 \\ \mathrm{C} & 3.48400327 & -3.96567870 & -2.79934696 \\ \mathrm{H} & 4.57639663 & -3.94296770 & -2.79809635 \\ \mathrm{H} & 3.15849451 & -4.52335071 & -1.91576867 \\ \mathrm{H} & 3.16055576 & -4.52544064 & -3.68235826\end{array}$

4nPrPh_Ph_pi_3_pi1_0
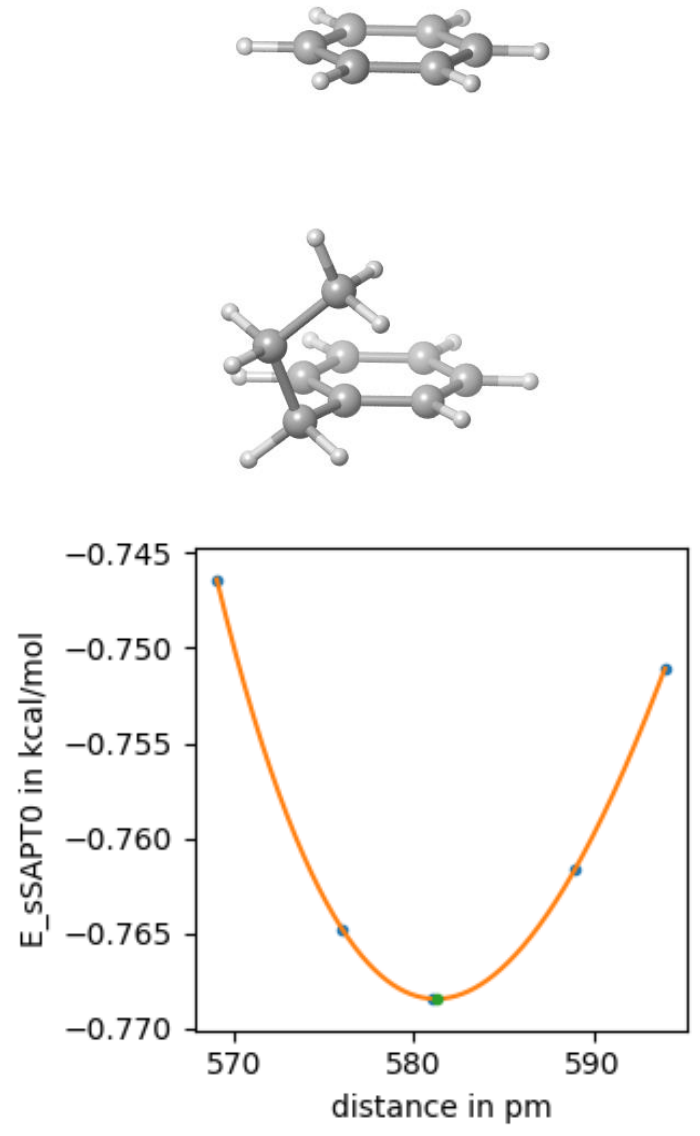

$\begin{array}{llll}\text { H } & 2.47830959 & -0.04134128 & 0.00002015\end{array}$

$\begin{array}{llll}\text { C } & 1.39302564 & -0.02324781 & 0.00000008\end{array}$

$\begin{array}{llll}\text { C } & 0.71636748 & 1.19441056 & -0.00010067\end{array}$

$\begin{array}{llll}\text { C } & 0.67612530 & -1.21763495 & 0.00007275\end{array}$

$\begin{array}{llll}\text { C } & -0.67625398 & 1.21769798 & 0.00007325\end{array}$

$\begin{array}{llll}\mathrm{H} & 1.27482284 & 2.12543743 & 0.00000658\end{array}$

$\begin{array}{llll}\text { C } & -0.71652436 & -1.19445047 & -0.00005433\end{array}$

$\begin{array}{llll}\mathrm{H} & 1.20319885 & -2.16677876 & 0.00008956\end{array}$

$\begin{array}{llll}\text { C } & -1.39262449 & 0.02322482 & -0.00003308\end{array}$

$\begin{array}{llll}\mathrm{H} & -1.20362977 & 2.16671637 & 0.00016144\end{array}$

$\mathrm{H}-1.27520554 \quad-2.12538191 \quad-0.00007858$

$\begin{array}{llll}\mathrm{H} & -2.47818735 & 0.04136582 & 0.00005785\end{array}$

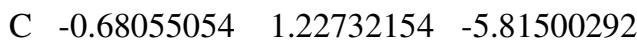

$\begin{array}{llll}\text { C } & -1.39290951 & 0.02998811 & -5.81376457\end{array}$

$\begin{array}{llll}\text { C } & 0.71071687 & 1.19864973 & -5.81311586\end{array}$

$\begin{array}{llll}\text { C } & -0.71499030 & -1.18459868 & -5.81025868\end{array}$

$\begin{array}{llll}\text { C } & 1.38403437 & -0.02067523 & -5.81075592\end{array}$

$\begin{array}{llll}\text { C } & 0.68237305 & -1.22779765 & -5.80985649\end{array}$

$\begin{array}{llll}\mathrm{H} & -1.20755508 & 2.17593261 & -5.81978781\end{array}$

$\begin{array}{llll}\mathrm{H} & -2.47836915 & 0.04314819 & -5.81936059\end{array}$

H $\quad 1.27425851 \quad 2.12642396 \quad-5.81529438$

$\mathrm{H} \quad-1.27459321 \quad-2.11733151-5.81327440$

H $\quad 2.47115300 \quad-0.03974594 \quad-5.80955431$

C $\quad 1.40264886 \quad-2.55383672 \quad-5.76149621$

H $\quad 0.92913631 \quad-3.25160001 \quad-6.46124057$

H $\quad 2.43824851 \quad-2.42030144 \quad-6.09616937$

C $\quad 1.40531592 \quad-3.17372222-4.35495521$

H $\quad 1.86484322-4.16694753 \quad-4.40881569$

H $\quad 0.36830365-3.32420865 \quad-4.03143668$

C $\quad 2.14361211 \quad-2.31706195 \quad-3.33057983$

$\mathrm{H} \quad 2.15490160 \quad-2.79591652-2.34828066$

H $3.18250963 \quad-2.15405356-3.63699334$

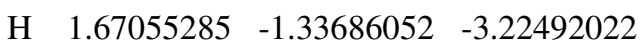

4nPrPh_Ph_pi_3_pi2_0
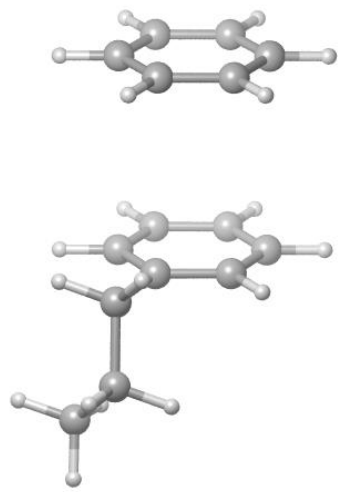

33

4nPrPh_Ph_pi_3_pi1_0-581 


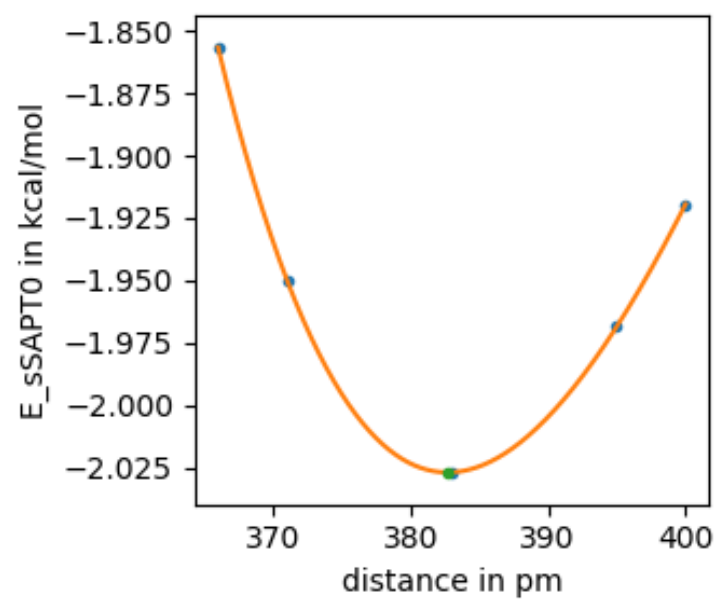

33

4nPrPh_Ph_pi_3_pi2_0-382

H $\quad-2.16089594 \quad-1.22405477-11.99764044$

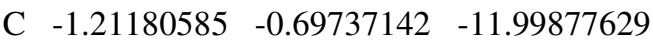

$\begin{array}{llll}\text { C } & -1.18776638 & 0.69545162 & -12.00370130\end{array}$

$\begin{array}{llll}\text { C } & -0.01718454 & -1.41387309 & -11.99530759\end{array}$

$\begin{array}{llll}\mathrm{C} & 0.03006013 & 1.37134561 & -12.00535875\end{array}$

$\mathrm{H} \quad-2.11864945 \quad 1.25413930 \quad-12.00656943$

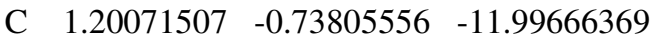

$\begin{array}{llll}\mathrm{H} & -0.03559658 & -2.49938046 & -11.99140727\end{array}$

$\begin{array}{llll}\text { C } & 1.22425429 & 0.65451937 & -12.00171021\end{array}$

H $\quad 0.04879796 \quad 2.45688417 \quad-12.00936392$

$\mathrm{H} \quad 2.13175254 \quad-1.29655308 \quad-11.99387913$

$\begin{array}{llll}\mathrm{H} & 2.17356820 & 1.18137549 & -12.00295751\end{array}$

$\begin{array}{llll}\text { C } & 0.02647319 & 1.39590055 & -8.17567441\end{array}$

$\begin{array}{llll}\text { C } & -1.18911453 & 0.71515880 & -8.17451415\end{array}$

$\begin{array}{llll}\text { C } & 1.21701014 & 0.67543622 & -8.17378963\end{array}$

$\begin{array}{llll}\text { C } & -1.20931278 & -0.67566402 & -8.17108788\end{array}$

$\begin{array}{llll}\text { C } & 1.19045764 & -0.71718905 & -8.17150962\end{array}$

$\begin{array}{llll}\text { C } & -0.02076012 & -1.41175712 & -8.17068892\end{array}$

$\mathrm{H} \quad 0.04437956 \quad 2.48092437 \quad-8.18039712$

H $\quad-2.12256993 \quad 1.26928590 \quad-8.18010900$

H $\quad 2.16893870 \quad 1.19714157 \quad-8.17590765$

H $-2.16030944-1.20363272 \quad-8.17416443$

H $\quad 2.12239460 \quad-1.27726416 \quad-8.17030957$

$\begin{array}{llll}\text { C } & -0.06000408 & -2.92028129 & -8.12241556\end{array}$

$\mathrm{H} \quad-0.81893692 \quad-3.28776602 \quad-8.82220541$

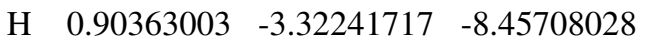

C $-0.36768272-3.45853127-6.71591510$

H $-0.46633131-4.54845021 \quad-6.76984066$

H $\quad-1.34101540 \quad-3.07036857 \quad-6.39240611$

$\begin{array}{llll}\text { C } & 0.69999743 & -3.08584797 & -5.69148391\end{array}$

$\mathrm{H} \quad 0.47031527 \quad-3.50624870 \quad-4.70921606$

$\mathrm{H} \quad 1.68122322 \quad-3.46410992 \quad-5.99788706$

H $\quad 0.78041339-2.00044500 \quad-5.58576007$
4PhPh_pi_ph_pi_60
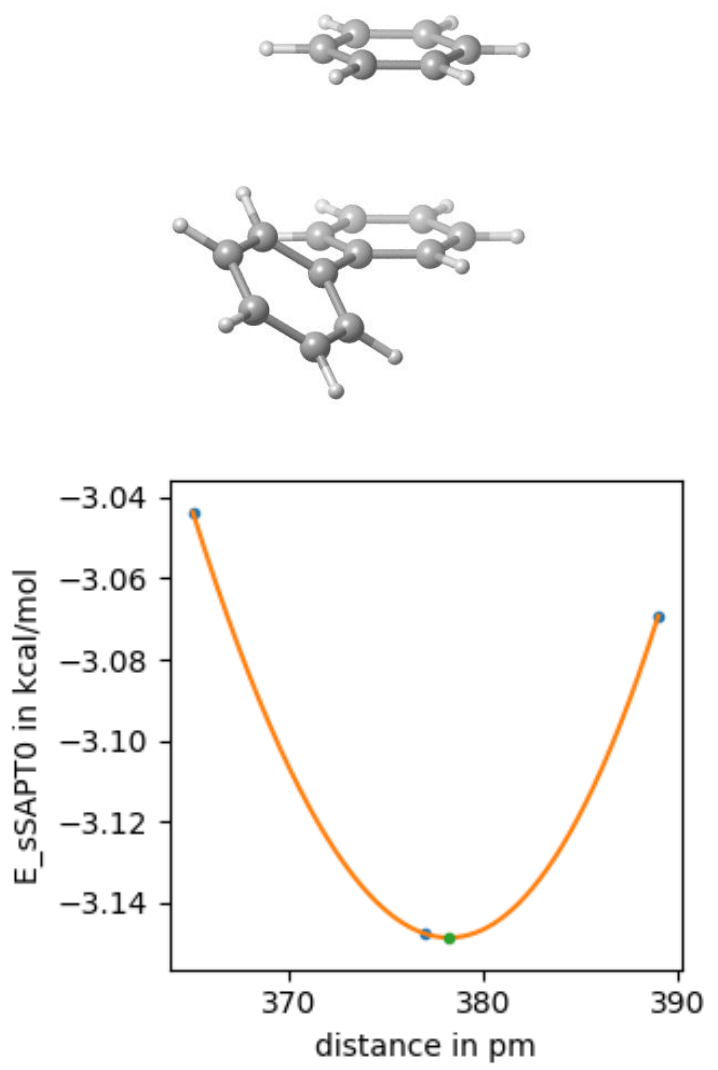

34

4PhPh_pi_ph_pi_60-376

$\begin{array}{llll}\mathrm{H} & 2.07331734 & 1.35807154 & 0.99992568\end{array}$

$\begin{array}{llll}\text { C } & 1.16533979 & 0.76330653 & 0.99994698\end{array}$

$\begin{array}{llll}\text { C } & -0.07853608 & 1.39046759 & 0.99979592\end{array}$

$\begin{array}{lrrr}\text { C } & 1.24330586 & -0.62753061 & 1.00012307\end{array}$

$\begin{array}{llll}\text { C } & -1.24367980 & 0.62733133 & 1.00002292\end{array}$

$\begin{array}{llll}\mathrm{H} & -0.13961661 & 2.47441990 & 0.99982257\end{array}$

$\begin{array}{llll}\text { C } & 0.07819666 & -1.39076790 & 1.00004908\end{array}$

$\begin{array}{llll}\mathrm{H} & 2.21257978 & -1.11659971 & 1.00017911\end{array}$

$\begin{array}{llll}\text { C } & -1.16522707 & -0.76327933 & 1.00002000\end{array}$

$\begin{array}{llll}\mathrm{H} & -2.21313328 & 1.11612688 & 1.00007190\end{array}$

$\begin{array}{llll}\mathrm{H} & 0.13903676 & -2.47476812 & 1.00010543\end{array}$

$\begin{array}{llll}\mathrm{H} & -2.07346206 & -1.35816170 & 1.00015231\end{array}$

$\begin{array}{llll}\text { C } & -0.08491919 & 1.39076656 & -2.76772244\end{array}$

$\begin{array}{llll}\text { C } & -1.25241263 & 0.63184718 & -2.76784112\end{array}$

$\begin{array}{lllll}\text { C } & 1.15657818 & 0.76407540 & -2.76687693\end{array}$

$\begin{array}{llll}\mathrm{H} & -0.14064317 & 2.47467496 & -2.75932276\end{array}$

$\begin{array}{llll}\mathrm{H} & -2.22126847 & 1.12053601 & -2.76797434\end{array}$

C $-1.16881424-0.75812145 \quad-2.76778604$

$\begin{array}{lrrr}\text { C } & 1.25356590 & -0.63216478 & -2.76749434\end{array}$

$\begin{array}{llll}\mathrm{H} & 2.06427783 & 1.35981708 & -2.73994185\end{array}$

C $\quad 0.07306844 \quad-1.38404465-2.76828758$

H $\quad-2.07360720-1.35754573-2.77631716$

C $\quad 2.57856848 \quad-1.30049529 \quad-2.76730901$

H $\quad 0.13348909-2.46810372 \quad-2.79508544$ 


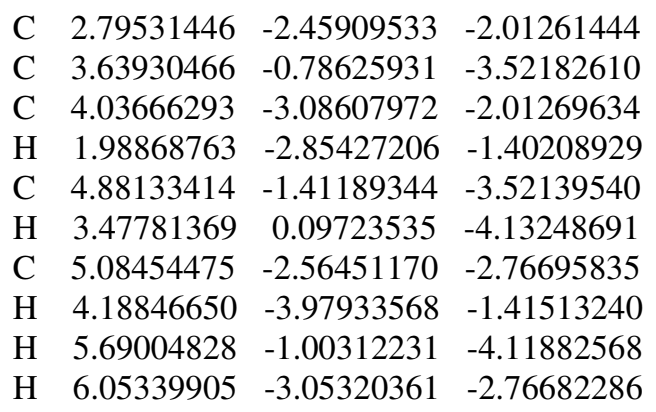

4tBuPh_pi_Ph_pi0
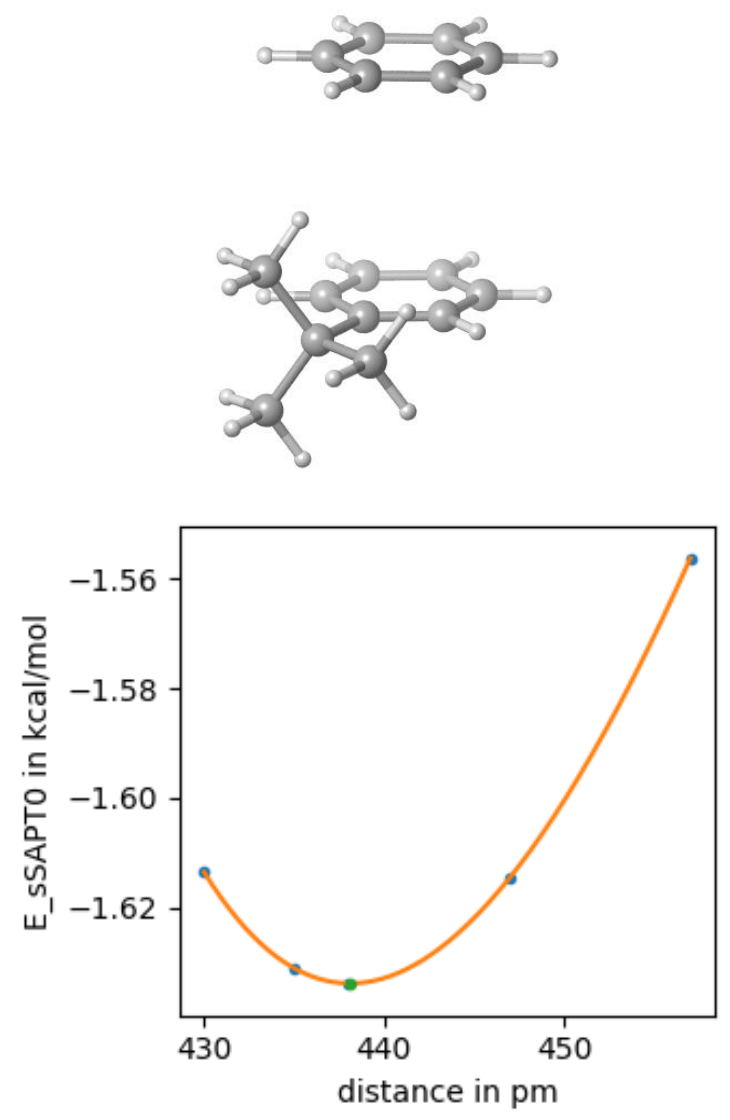

36

4tBuPh_pi_Ph_pi0-438
H $\quad-3.99020222 \quad-0.02114875 \quad 0.00001071$
$\begin{array}{llll}\text { C } & -2.90514422 & -0.00688675 & -0.00000229\end{array}$
$\begin{array}{llll}\text { C } & -2.18469622 & -1.19952675 & -0.00001429\end{array}$
$\begin{array}{llll}\text { C } & -2.21712322 & 1.19969625 & 0.00000571\end{array}$
C $-0.79567222-1.17597275-0.00003429$
$\mathrm{H} \quad-2.70652522-2.15145475-0.00000629$
C $-0.82251122 \quad 1.21733125 \quad-0.00001029$
$\begin{array}{llll}\mathrm{H} & -2.76322822 & 2.13795125 & 0.00002371\end{array}$
$\begin{array}{llll}\text { C } & -0.08421822 & 0.03180925 & -0.00004829\end{array}$
H $\quad-0.25447922-2.11833575-0.00001929$
$\begin{array}{llll}\mathrm{H} & -0.31527522 & 2.17555325 & -0.00000629\end{array}$
$\begin{array}{llll}\text { C } & 1.44717978 & 0.00652025 & -0.00000029\end{array}$
$\begin{array}{llll}\text { C } & 1.94443278 & -0.73085975 & 1.25485971\end{array}$

$\begin{array}{lrrc}\text { C } & 1.94459178 & -0.72995875 & -1.25532729 \\ \mathrm{C} & 2.04833178 & 1.41565725 & 0.00052871 \\ \mathrm{H} & 1.60028378 & -0.22559475 & 2.16223371 \\ \mathrm{H} & 1.58290578 & -1.76238475 & 1.28564271 \\ \mathrm{H} & 3.03919278 & -0.75726375 & 1.26854671 \\ \mathrm{H} & 1.60053578 & -0.22405775 & -2.16238229 \\ \mathrm{H} & 3.03935378 & -0.75631375 & -1.26890329 \\ \mathrm{H} & 1.58312378 & -1.76147675 & -1.28689929 \\ \mathrm{H} & 3.14025378 & 1.34721625 & 0.00047671 \\ \mathrm{H} & 1.74864078 & 1.98131725 & -0.88719029 \\ \mathrm{H} & 1.74869678 & 1.98063225 & 0.88870171 \\ \mathrm{C} & -0.07540878 & 0.10570352 & 4.37225775 \\ \mathrm{C} & -0.75222793 & 1.32316207 & 4.35586523 \\ \mathrm{C} & -0.79212524 & -1.08854170 & 4.39680576 \\ \mathrm{C} & -2.14482527 & 1.34639500 & 4.36421665 \\ \mathrm{C} & -2.18475262 & -1.06541675 & 4.40485813 \\ \mathrm{C} & -2.86101299 & 0.15206079 & 4.38858387 \\ \mathrm{H} & 1.00985732 & 0.08765512 & 4.36590406 \\ \mathrm{H} & -0.19391488 & 2.25408106 & 4.33689617 \\ \mathrm{H} & -0.26492659 & -2.03753080 & 4.40952401 \\ \mathrm{H} & -2.67232550 & 2.29526047 & 4.35160735 \\ \mathrm{H} & -2.74329098 & -1.99623892 & 4.42390996 \\ \mathrm{H} & -3.94655733 & 0.17015858 & 4.39504945\end{array}$

Nap_pi_Ph_pi0
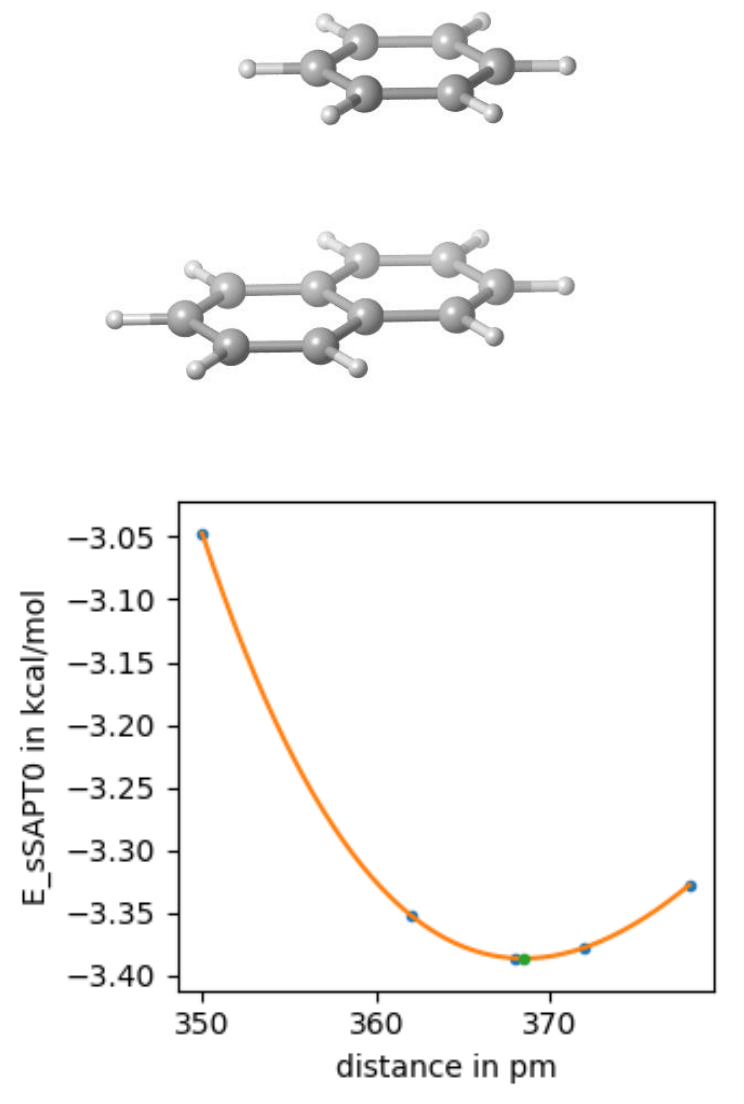

30

Nap_pi_Ph_pi0-368 


$\begin{array}{lrrr}\text { H } & 3.36933800 & 1.24391000 & 0.00000300 \\ \text { C } & 2.42572600 & 0.70834200 & 0.00000200 \\ \text { C } & 1.24227900 & 1.39942100 & -0.00000100 \\ \text { C } & 2.42607300 & -0.70827200 & 0.00000200 \\ \text { C } & -0.00003000 & 0.71146700 & -0.00000200 \\ \text { H } & 1.23624300 & 2.48603300 & -0.00000600 \\ \text { C } & 1.24253700 & -1.39925100 & -0.00000200 \\ \text { H } & 3.36993500 & -1.24372300 & 0.00001200 \\ \text { C } & -1.24247400 & 1.39929400 & 0.00000000 \\ \text { C } & 0.00008400 & -0.71138600 & -0.00000300 \\ \text { H } & 1.23655200 & -2.48582700 & -0.00000500 \\ \text { C } & -2.42598500 & 0.70823600 & 0.00000200 \\ \text { H } & -1.23648900 & 2.48588800 & 0.00000000 \\ \text { C } & -1.24229900 & -1.39936900 & -0.00000100 \\ \text { C } & -2.42591300 & -0.70848000 & 0.00000200 \\ \text { H } & -3.36977900 & 1.24375700 & 0.00000100 \\ \text { H } & -1.23612800 & -2.48596100 & 0.00000100 \\ \text { H } & -3.36966000 & -1.24408700 & 0.00000400 \\ \text { C } & -0.00167454 & 0.68397294 & 3.68462231 \\ \text { C } & -0.00945605 & -0.70902144 & 3.67660438 \\ \text { C } & 1.20899447 & 1.37297268 & 3.69278472 \\ \text { C } & 1.19260631 & -1.41257159 & 3.67695052 \\ \text { C } & 2.41113295 & 0.66950010 & 3.69283056 \\ \text { C } & 2.40285647 & -0.72323593 & 3.68493437 \\ \text { H } & -0.93848123 & 1.23220817 & 3.68450779 \\ \text { H } & -0.95285310 & -1.24628025 & 3.67040574 \\ \text { H } & 1.21538254 & 2.45860677 & 3.69897292 \\ \text { H } & 1.18654278 & -2.49824529 & 3.67086822 \\ \text { H } & 3.35467966 & 1.20656449 & 3.69911160 \\ \text { H } & 3.33988248 & -1.27165005 & 3.68515965\end{array}$

Ph_pi_4OMePh_pi_0

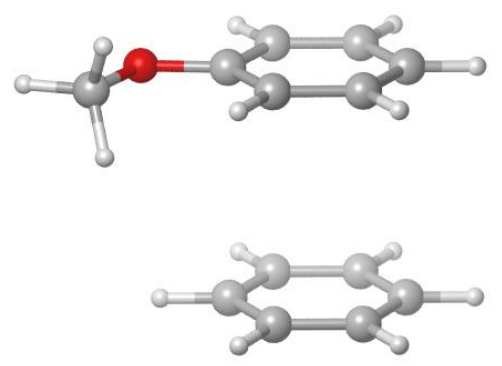

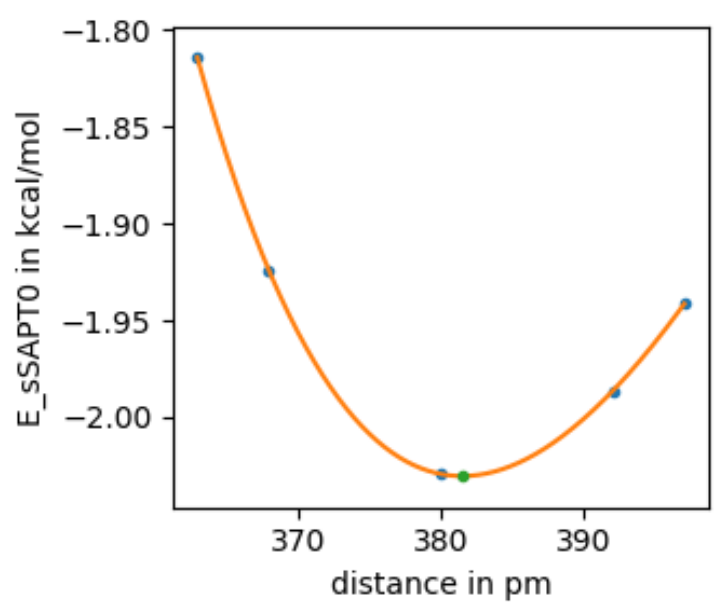

28

Ph_pi_4OMePh_pi_0-381

$\begin{array}{llll}\mathrm{H} & 2.39213446 & 0.64825143 & 1.99998746\end{array}$

$\begin{array}{llll}\text { C } & 1.34448088 & 0.36436655 & 1.99998170\end{array}$

$\begin{array}{llll}\text { C } & 0.35640276 & 1.34633386 & 1.99985048\end{array}$

$\begin{array}{llll}\text { C } & 0.98730915 & -0.98208621 & 2.00010321\end{array}$

$\begin{array}{llll}\text { C } & -0.98795138 & 0.98212407 & 2.00004276\end{array}$

$\begin{array}{llll}\mathrm{H} & 0.63446358 & 2.39579354 & 1.99991968\end{array}$

C $\quad-0.35704351-1.34640275 \quad 1.99999449$

H $\quad 1.75714374 \quad-1.74761556 \quad 2.00014377$

C $-1.34459023-0.36426428 \quad 1.99998526$

$\begin{array}{llll}\mathrm{H} & -1.75804153 & 1.74744903 & 2.00010721\end{array}$

$\begin{array}{llll}\mathrm{H} & -0.63534777 & -2.39583342 & 2.00000830\end{array}$

$\begin{array}{llll}\mathrm{H} & -2.39252495 & -0.64818090 & 2.00009051\end{array}$

C $\quad 0.98964192 \quad-0.98406573-1.81427755$

$\begin{array}{llll}\text { C } & -0.35575344 & -1.35340752 & -1.81449295\end{array}$

$\begin{array}{llll}\text { C } & 1.34280923 & 0.36990446 & -1.81478574\end{array}$

C $-1.33675346-0.36053476 \quad-1.81521703$

$\begin{array}{llll}\text { C } & 0.35604238 & 1.34286456 & -1.81550441\end{array}$

$\begin{array}{llll}\text { C } & -0.99344899 & 0.98458535 & -1.81572805\end{array}$

$\begin{array}{llll}\text { O } & 2.02737194 & -1.86345687 & -1.81358176\end{array}$

$\mathrm{H} \quad-0.64975842-2.39588242 \quad-1.81411071$

H $\quad 2.39683984 \quad 0.62494588-1.81459884$

$\mathrm{H} \quad-2.38165191 \quad-0.65454647 \quad-1.81538249$

$\begin{array}{llll}\mathrm{H} & 0.64106199 & 2.39017759 & -1.81589547\end{array}$

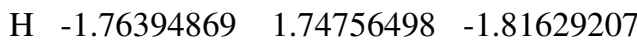

C $\quad 1.71426734 \quad-3.23825768-1.81294943$

$\mathrm{H} \quad 2.66618872 \quad-3.76803713-1.81240110$

$\mathrm{H} \quad 1.14285091 \quad-3.51813337 \quad-0.91993663$

H $\quad 1.14336029 \quad-3.51906715 \quad-2.70599532$ 
Ph_pi_Ph_piO
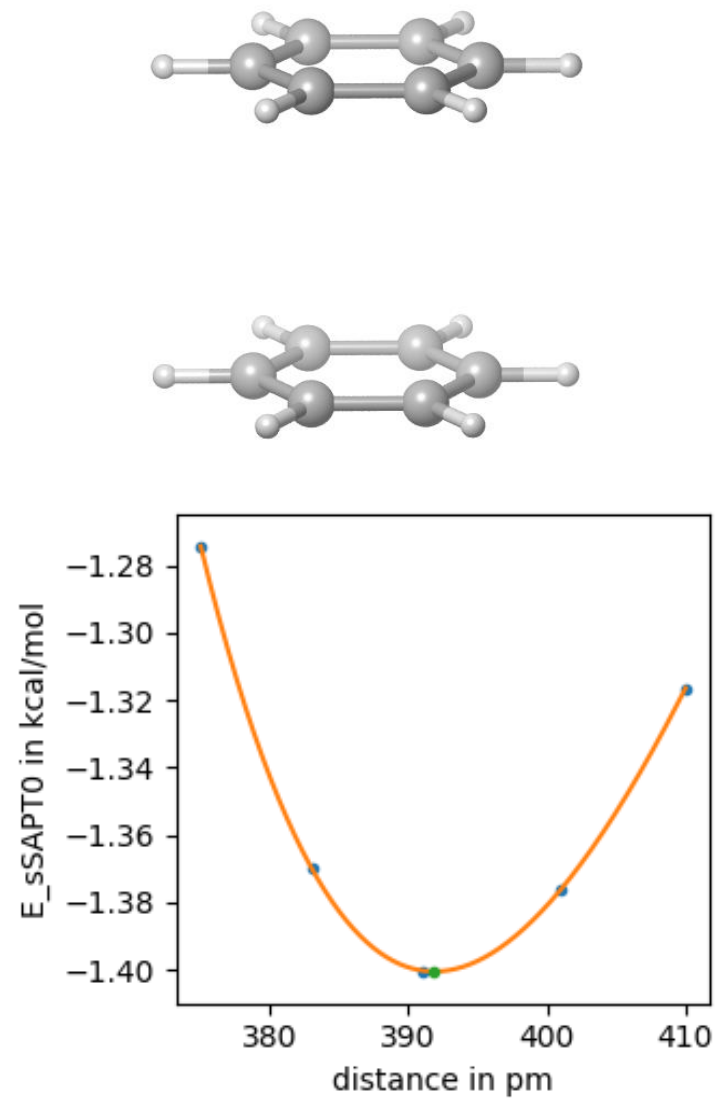

24

Ph_pi_Ph_pi0-391

H $\quad 2.12766703 \quad-1.27139451 \quad 0.50001687$

C $\quad 1.19592490 \quad-0.71459541 \quad 0.49999824$

$\begin{array}{llll}\text { C } & 1.21648911 & 0.67829199 & 0.49997703\end{array}$

$\begin{array}{llll}\text { C } & -0.02102337 & -1.39249188 & 0.49999322\end{array}$

C $\quad 0.02092537 \quad 1.39282973 \quad 0.50015279$

H $2.16478195 \quad 1.20689780 \quad 0.50014485$

C $\quad-1.21666287 \quad-0.67802930 \quad 0.49986798$

$\mathrm{H} \quad-0.03737345 \quad-2.47803928 \quad 0.49994803$

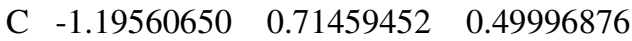

H $\quad 0.03695092 \quad 2.47841911 \quad 0.50030298$

H $-2.16510387 \quad-1.20643980 \quad 0.49978316$

H $\quad-2.12756670 \quad 1.27157389 \quad 0.50006114$

C $\quad-1.19534779 \quad 0.71409590 \quad-3.41897675$

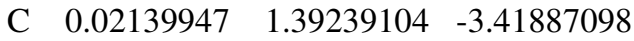

C $\quad-1.21546568-0.67877954 \quad-3.41905626$

C $\quad 1.21724098 \quad 0.67831828 \quad-3.41904672$

C $\quad-0.01965444-1.39295465 \quad-3.41893101$

C $\quad 1.19662746 \quad-0.71435107 \quad-3.41894724$

$\mathrm{H} \quad-2.12725034 \quad 1.27062646 \quad-3.41899539$

H $\quad 0.03739561 \quad 2.47794507 \quad-3.41897290$

H $-2.16358493 \quad-1.20769388-3.41907698$

H $\quad 2.16555102 \quad 1.20696676 \quad-3.41913101$

H $\quad-0.03540837 \quad-2.47854657 \quad-3.41891209$

H $2.12879211 \quad-1.27098820 \quad-3.41903960$ 


\section{Crystallographic data}

\section{Crystal data and structure refinement for compound 6}

Identification code

Formula

Formula weight

Temperature

Diffractometer, wavelength

Crystal system, space group

Unit cell dimensions

Volume, Z

Density (calculated)

Absorption coefficient

$\mathrm{F}(000)$

Crystal colour / morphology

Crystal size

$\theta$ range for data collection

Index ranges

Reflns collected / unique

Reflns observed [F>4 $\sigma(\mathrm{F})]$

Absorption correction

Max. and min. transmission

Refinement method

Data / restraints / parameters

Goodness-of-fit on $\mathrm{F}^{2}$

Final $R$ indices $[F>4 \sigma(F)]$

$\mathrm{R}$ indices (all data)

Largest diff. peak, hole

Mean and maximum shift/error

Bond Lengths $[\AA ̊]$ and angles.

$\mathrm{C} 1 \mathrm{C} 2$

$\mathrm{C} 1 \mathrm{C} 6$

$1.388(4)$

$\mathrm{C} 2 \mathrm{C} 3$

$1.395(4)$

$\mathrm{C} 3 \mathrm{C} 4$

$1.386(4)$

$1.377(4)$

$\mathrm{C} 4 \mathrm{C} 5$

$1.391(4)$

C5 C6

$1.382(3)$

C6 C7

$1.530(3)$

$\mathrm{C} 7 \mathrm{C} 8$

$1.504(3)$

C7 $\mathrm{C} 10$

$1.549(3)$

C8 C9

$1.318(4)$

C10 C11

$1.537(4)$

$\mathrm{C} 10 \mathrm{C} 12$

$1.504(3)$
ME001

C17 H24 N1 B1 F4

329.18

$100.15 \mathrm{~K}$

Pilatus 200K, $1.54184 \AA$

Orthorhombic, P $2 \mathrm{ac} 2 \mathrm{ab}$

$\mathrm{a}=8.73380(10) \AA$

$\alpha=90^{\circ}$

$\mathrm{b}=13.7551(2) \AA$

$\beta=90^{\circ}$

$\mathrm{c}=14.4231(2) \AA$

$\gamma=90^{\circ}$

1732.71(4) $\AA^{3}, 4$

$1.262 \mathrm{Mg} / \mathrm{m}^{3}$

$0.866 \mathrm{~mm}^{-1}$

696

Colourless plates

$0.12 \times 0.10 \times 0.04 \mathrm{~mm}^{3}$

4.442 to $74.491^{\circ}$

$-10<=\mathrm{h}<=8,-17<=\mathrm{k}<=17,-17<=\mathrm{l}<=18$

19080 / 3532

3377

multi-scan

1.00000 and 0.72516

Full-matrix least-squares on $\mathrm{F}^{2}$

3532 / 0 / 237

1.042

$\mathrm{R} 1=0.0401, \mathrm{wR} 2=0.1056$

$\mathrm{R} 1=0.0419, \mathrm{wR} 2=0.1069$

$0.366,-0.354 \mathrm{e}^{-3}$

0.000 and 0.000 


\begin{tabular}{|c|c|}
\hline $\mathrm{C} 12 \mathrm{C} 13$ & $1.489(3)$ \\
\hline C12 N1 & $1.287(3)$ \\
\hline C14 C15 & $1.505(4)$ \\
\hline C14 N1 & $1.488(4)$ \\
\hline C15 C16 & $1.346(7)$ \\
\hline C15 C16A & $1.708(10)$ \\
\hline C16 C17 & $1.625(6)$ \\
\hline C16A C17 & $1.325(8)$ \\
\hline C17 N1 & $1.486(3)$ \\
\hline B1 F1 & $1.393(3)$ \\
\hline B1 F2 & $1.394(3)$ \\
\hline B1 F3 & $1.394(3)$ \\
\hline B1 F4 & $1.390(3)$ \\
\hline C2 C1 H1 & 119.8 \\
\hline C2 C1 C6 & $120.4(3)$ \\
\hline C3 C2 C1 & $120.5(3)$ \\
\hline C4 C3 C2 & $119.2(2)$ \\
\hline C3 C4 C5 & $120.5(3)$ \\
\hline C6 C5 C4 & $120.8(2)$ \\
\hline C1 C6 C7 & $121.7(2)$ \\
\hline C5 C6 C1 & $118.6(2)$ \\
\hline C5 C6 C7 & 119.7(2) \\
\hline C6 C7 C10 & $110.9(2)$ \\
\hline C8 C7 C6 & $111.10(19)$ \\
\hline C8 C7 C10 & $111.0(2)$ \\
\hline C9 C8 C7 & $124.8(3)$ \\
\hline $\mathrm{C} 11 \mathrm{C} 10 \mathrm{C} 7$ & $112.2(2)$ \\
\hline $\mathrm{C} 12 \mathrm{C} 10 \mathrm{C} 7$ & $112.3(2)$ \\
\hline C12 C10 C11 & $107.0(2)$ \\
\hline C13 C12 C10 & 119.7(2) \\
\hline N1 C12 C10 & $120.0(2)$ \\
\hline N1 C12 C13 & $120.2(2)$ \\
\hline N1 C14 C15 & $105.4(3)$ \\
\hline C14 C15 C16A & $103.5(3)$ \\
\hline C16 C15 C14 & $106.7(4)$ \\
\hline C15 C16 C17 & $107.8(3)$ \\
\hline C17 C16A C15 & $104.3(5)$ \\
\hline C16A C17 N1 & 111.3(4) \\
\hline N1 C17 C16 & $98.6(3)$ \\
\hline F1 B1 F2 & $109.6(2)$ \\
\hline F1 B1 F3 & $109.0(2)$ \\
\hline F2 B1 F3 & $109.52(19)$ \\
\hline F4 B1 F1 & $109.4(2)$ \\
\hline F4 B1 F2 & 109.2(2) \\
\hline F4 B1 F3 & $110.2(2)$ \\
\hline C12 N1 C14 & $125.0(2)$ \\
\hline C12 N1 C17 & $124.6(2)$ \\
\hline C17 N1 C14 & $110.3(2)$ \\
\hline
\end{tabular}



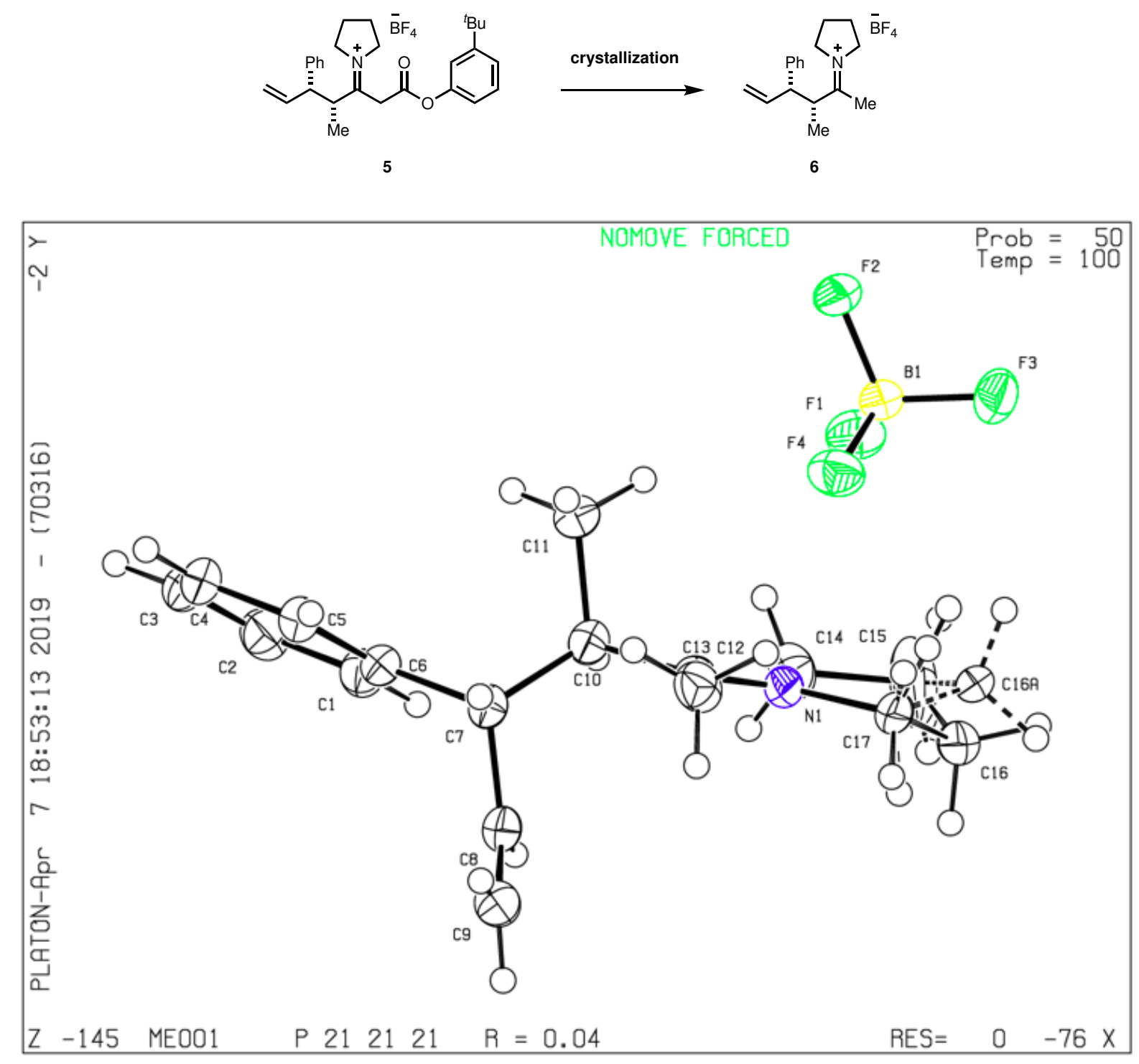


\section{Crystal data and structure refinement for compound 34}

A colourless block $0.12 \times 0.11 \times 0.06 \mathrm{~mm}$ in size was mounted on a Cryoloop with Paratone oil. Data were collected in a nitrogen gas stream at $100(2) \mathrm{K}$ using omega scans. Crystal-to-detector distance was $30.23 \mathrm{~mm}$ and exposure time was 0.50 seconds per frame at low angles and 1.88 seconds per frame at high angles using a scan width of $0.5^{\circ}$. Data collection was $100 \%$ complete to $74.000^{\circ}$ in $\theta$. A total of 35517 reflections were collected covering the indices $12<=\mathrm{h}<=14,-33<=\mathrm{k}<=32,-11<=\mathrm{l}<=11.5714$ reflections were founded to be symmetry independent, with an $\mathrm{R}_{\text {int }}$ of 0.0391. Indexing and unit cell refinement indicated a primitive, orthorhombic lattice. The space group was found to be P 21212 (No. 18). The data were integrated using the CrysAlis ${ }^{\text {Pro }}$ 1.171.39.46e software program and scaled using the SCALE3 ABSPACK scaling algorithm. Solution by intrinsic phasing (SHELXT-2015) produced a heavy-atom phasing model consistent with the proposed structure. All non-hydrogen atoms were refined anisotropically by fullmatrix least-squares (SHELXL-2014). All hydrogen atoms were placed using a riding model. Their positions were constrained relative to their parent atom using the appropriate HFIX command in SHELXL-2014.

\begin{tabular}{|c|c|c|}
\hline Identification code & \multicolumn{2}{|l|}{ JM_IV_380_4 } \\
\hline Empirical formula & \multicolumn{2}{|l|}{$\mathrm{C} 65.50 \mathrm{H} 61 \mathrm{Cl} 3 \mathrm{O} 2$} \\
\hline Formula weight & \multicolumn{2}{|l|}{986.49} \\
\hline Temperature & \multicolumn{2}{|l|}{$100(2) \mathrm{K}$} \\
\hline Wavelength & \multicolumn{2}{|l|}{$1.54184 \AA$} \\
\hline Crystal system & \multicolumn{2}{|l|}{ Orthorhombic } \\
\hline Space group & \multicolumn{2}{|l|}{ P 21212} \\
\hline \multirow[t]{3}{*}{ Unit cell dimensions } & $\mathrm{a}=11.60670(10) \AA$ & $\alpha=90^{\circ}$. \\
\hline & $\mathrm{b}=26.8486(3) \AA$ & $\beta=90^{\circ}$. \\
\hline & $c=8.97260(10) \AA$ & $\gamma=90^{\circ}$. \\
\hline Volume & \multicolumn{2}{|l|}{$2796.07(5) \AA^{3}$} \\
\hline $\mathrm{Z}$ & \multicolumn{2}{|l|}{2} \\
\hline Density (calculated) & \multicolumn{2}{|l|}{$1.172 \mathrm{Mg} / \mathrm{m}^{3}$} \\
\hline Absorption coefficient & \multicolumn{2}{|l|}{$1.807 \mathrm{~mm}^{-1}$} \\
\hline $\mathrm{F}(000)$ & \multicolumn{2}{|l|}{1042} \\
\hline Crystal size & \multicolumn{2}{|c|}{$0.120 \times 0.110 \times 0.060 \mathrm{~mm}^{3}$} \\
\hline Theta range for data collection & \multicolumn{2}{|c|}{4.150 to $74.473^{\circ}}$. \\
\hline Index ranges & \multicolumn{2}{|c|}{$-12<=\mathrm{h}<=14,-33<=\mathrm{k}<=32,-11<=\mathrm{l}<=11$} \\
\hline Reflections collected & \multicolumn{2}{|c|}{35517} \\
\hline Independent reflections & \multicolumn{2}{|c|}{$5714[\mathrm{R}($ int $)=0.0391]$} \\
\hline Completeness to theta $=74.000^{\circ}$ & \multicolumn{2}{|c|}{$99.8 \%$} \\
\hline Absorption correction & \multicolumn{2}{|c|}{ Semi-empirical from equivalents } \\
\hline Max. and min. transmission & \multicolumn{2}{|c|}{1.00000 and 0.69992} \\
\hline Refinement method & \multicolumn{2}{|c|}{ Full-matrix least-squares on $\mathrm{F}^{2}$} \\
\hline Data / restraints / parameters & \multicolumn{2}{|c|}{$5714 / 0 / 348$} \\
\hline Goodness-of-fit on $\mathrm{F}^{2}$ & \multicolumn{2}{|l|}{1.067} \\
\hline Final $\mathrm{R}$ indices $[\mathrm{I}>2 \operatorname{sigma}(\mathrm{I})]$ & \multicolumn{2}{|c|}{$\mathrm{R} 1=0.0537, \mathrm{wR} 2=0.1504$} \\
\hline $\mathrm{R}$ indices (all data) & \multicolumn{2}{|c|}{$\mathrm{R} 1=0.0556, \mathrm{wR} 2=0.1522$} \\
\hline Absolute structure parameter & \multicolumn{2}{|l|}{$0.039(6)$} \\
\hline Extinction coefficient & \multicolumn{2}{|l|}{$\mathrm{n} / \mathrm{a}$} \\
\hline Largest diff. peak and hole & \multicolumn{2}{|c|}{1.028 and $-0.378 \mathrm{e} . \AA^{-3}$} \\
\hline
\end{tabular}


Atomic coordinates $\left(\mathrm{x} \mathrm{10}^{4}\right)$ and equivalent isotropic displacement parameters $\left(\AA^{2} \times 10^{3}\right)$ for jm_iv_380_4. U(eq) is defined as one third of the trace of the orthogonalized $\mathrm{U}^{\mathrm{ij}}$ tensor.

\begin{tabular}{|c|c|c|c|c|}
\hline & $\mathrm{x}$ & $\mathrm{y}$ & $\mathrm{z}$ & $\mathrm{U}(\mathrm{eq})$ \\
\hline $\mathrm{C}(1)$ & $5389(2)$ & $5633(1)$ & 1973(3) & $24(1)$ \\
\hline$C(2)$ & $5493(2)$ & $5179(1)$ & 2786(3) & $25(1)$ \\
\hline$C(3)$ & $6487(3)$ & $5086(1)$ & $3594(3)$ & $27(1)$ \\
\hline $\mathrm{C}(4)$ & $6644(3)$ & $4606(1)$ & $4425(5)$ & $39(1)$ \\
\hline$C(5)$ & $7378(2)$ & $5453(1)$ & $3625(3)$ & $26(1)$ \\
\hline$C(6)$ & $8417(3)$ & $5374(1)$ & $4439(4)$ & $32(1)$ \\
\hline$C(7)$ & $9262(3)$ & $5728(1)$ & $4488(4)$ & $35(1)$ \\
\hline $\mathrm{C}(8)$ & $9118(3)$ & $6182(1)$ & $3735(4)$ & $31(1)$ \\
\hline $\mathrm{C}(9)$ & $8131(2)$ & $6274(1)$ & $2927(4)$ & $27(1)$ \\
\hline$C(10)$ & $7241(2)$ & 5911(1) & 2837(3) & $24(1)$ \\
\hline $\mathrm{C}(11)$ & $6221(2)$ & $5996(1)$ & 1991(3) & $23(1)$ \\
\hline $\mathrm{C}(12)$ & $6033(2)$ & $6469(1)$ & $1150(3)$ & $23(1)$ \\
\hline $\mathrm{C}(13)$ & $6654(2)$ & $6565(1)$ & $-203(3)$ & $25(1)$ \\
\hline$C(14)$ & $7497(3)$ & $6230(1)$ & $-750(4)$ & $32(1)$ \\
\hline$C(15)$ & $8098(3)$ & $6325(1)$ & $-2039(4)$ & $38(1)$ \\
\hline$C(16)$ & $7879(3)$ & 6761(1) & $-2852(4)$ & $36(1)$ \\
\hline $\mathrm{C}(17)$ & 7071(3) & 7093(1) & $-2371(4)$ & $33(1)$ \\
\hline $\mathrm{C}(18)$ & $6432(3)$ & 7010(1) & $-1037(3)$ & $26(1)$ \\
\hline C(19) & $5571(3)$ & $7350(1)$ & $-519(3)$ & $27(1)$ \\
\hline$C(20)$ & $5343(3)$ & $7828(1)$ & $-1333(4)$ & $37(1)$ \\
\hline $\mathrm{C}(21)$ & $4983(2)$ & $7242(1)$ & 759(3) & $26(1)$ \\
\hline $\mathrm{C}(22)$ & $5210(2)$ & 6806(1) & $1620(3)$ & $24(1)$ \\
\hline $\mathrm{C}(23)$ & $4548(2)$ & $6740(1)$ & $3020(3)$ & $24(1)$ \\
\hline$C(24)$ & $3347(2)$ & $6729(1)$ & $3002(3)$ & $27(1)$ \\
\hline$C(25)$ & $2715(2)$ & 6696(1) & 4311(3) & $28(1)$ \\
\hline$C(26)$ & $3253(3)$ & $6676(1)$ & $5699(3)$ & $27(1)$ \\
\hline $\mathrm{C}(27)$ & $2572(3)$ & 6679(1) & $7160(4)$ & $35(1)$ \\
\hline $\mathrm{C}(28)$ & 1331(3) & $6506(2)$ & $6944(5)$ & $64(1)$ \\
\hline C(29) & $2572(4)$ & $7212(2)$ & $7760(5)$ & $50(1)$ \\
\hline $\mathrm{C}(30)$ & $3152(4)$ & $6342(2)$ & $8332(4)$ & $46(1)$ \\
\hline $\mathrm{C}(31)$ & $4458(3)$ & $6683(1)$ & $5710(3)$ & $31(1)$ \\
\hline $\mathrm{C}(32)$ & $5089(2)$ & 6712(1) & $4405(3)$ & $28(1)$ \\
\hline $\mathrm{C}(33)$ & $8271(8)$ & 4798(3) & $358(11)$ & $53(2)$ \\
\hline $\mathrm{C}(34)$ & 5000 & 5000 & $8294(12)$ & $79(6)$ \\
\hline $\mathrm{Cl}(1)$ & $8377(2)$ & 4920(1) & $-1506(2)$ & $58(1)$ \\
\hline $\mathrm{Cl}(2)$ & $9158(3)$ & $4313(1)$ & 989(3) & $76(1)$ \\
\hline $\mathrm{Cl}(3)$ & $5624(2)$ & $5476(1)$ & $7260(3)$ & $56(1)$ \\
\hline $\mathrm{O}(1)$ & $4395(2)$ & $5689(1)$ & $1162(2)$ & $29(1)$ \\
\hline
\end{tabular}




\section{Bond lengths $[\AA]$ and angles $\left[{ }^{\circ}\right]$ for $j \mathrm{jm} \_i v \_380 \_4$.}

\begin{tabular}{|c|c|}
\hline $\mathrm{C}(1)-\mathrm{O}(1)$ & $1.372(3)$ \\
\hline$C(1)-C(11)$ & $1.372(4)$ \\
\hline $\mathrm{C}(1)-\mathrm{C}(2)$ & $1.425(4)$ \\
\hline $\mathrm{C}(2)-\mathrm{C}(3)$ & $1.385(4)$ \\
\hline $\mathrm{C}(2)-\mathrm{C}(2) \# 1$ & $1.496(5)$ \\
\hline $\mathrm{C}(3)-\mathrm{C}(5)$ & $1.430(4)$ \\
\hline $\mathrm{C}(3)-\mathrm{C}(4)$ & $1.499(4)$ \\
\hline $\mathrm{C}(4)-\mathrm{H}(4 \mathrm{~A})$ & 0.9800 \\
\hline $\mathrm{C}(4)-\mathrm{H}(4 \mathrm{~B})$ & 0.9800 \\
\hline $\mathrm{C}(4)-\mathrm{H}(4 \mathrm{C})$ & 0.9800 \\
\hline$C(5)-C(6)$ & $1.426(4)$ \\
\hline$C(5)-C(10)$ & $1.427(4)$ \\
\hline$C(6)-C(7)$ & $1.366(4)$ \\
\hline $\mathrm{C}(6)-\mathrm{H}(6)$ & 0.9500 \\
\hline$C(7)-C(8)$ & $1.405(5)$ \\
\hline $\mathrm{C}(7)-\mathrm{H}(7)$ & 0.9500 \\
\hline $\mathrm{C}(8)-\mathrm{C}(9)$ & $1.377(4)$ \\
\hline $\mathrm{C}(8)-\mathrm{H}(8)$ & 0.9500 \\
\hline C(9)-C(10) & $1.422(4)$ \\
\hline $\mathrm{C}(9)-\mathrm{H}(9)$ & 0.9500 \\
\hline $\mathrm{C}(10)-\mathrm{C}(11)$ & $1.425(4)$ \\
\hline $\mathrm{C}(11)-\mathrm{C}(12)$ & $1.492(4)$ \\
\hline$C(12)-C(22)$ & $1.381(4)$ \\
\hline$C(12)-C(13)$ & $1.436(4)$ \\
\hline$C(13)-C(14)$ & $1.417(4)$ \\
\hline$C(13)-C(18)$ & $1.432(4)$ \\
\hline$C(14)-C(15)$ & $1.374(5)$ \\
\hline $\mathrm{C}(14)-\mathrm{H}(14)$ & 0.9500 \\
\hline$C(15)-C(16)$ & $1.402(5)$ \\
\hline $\mathrm{C}(15)-\mathrm{H}(15)$ & 0.9500 \\
\hline$C(16)-C(17)$ & $1.365(5)$ \\
\hline $\mathrm{C}(16)-\mathrm{H}(16)$ & 0.9500 \\
\hline $\mathrm{C}(17)-\mathrm{C}(18)$ & $1.425(4)$ \\
\hline $\mathrm{C}(17)-\mathrm{H}(17)$ & 0.9500 \\
\hline $\mathrm{C}(18)-\mathrm{C}(19)$ & $1.432(4)$ \\
\hline$C(19)-C(21)$ & $1.366(4)$ \\
\hline $\mathrm{C}(19)-\mathrm{C}(20)$ & $1.499(4)$ \\
\hline $\mathrm{C}(20)-\mathrm{H}(20 \mathrm{~A})$ & 0.9800 \\
\hline $\mathrm{C}(20)-\mathrm{H}(20 \mathrm{~B})$ & 0.9800 \\
\hline $\mathrm{C}(20)-\mathrm{H}(20 \mathrm{C})$ & 0.9800 \\
\hline $\mathrm{C}(21)-\mathrm{C}(22)$ & $1.427(4)$ \\
\hline $\mathrm{C}(21)-\mathrm{H}(21)$ & 0.9500 \\
\hline$C(22)-C(23)$ & $1.483(4)$ \\
\hline$C(23)-C(32)$ & $1.394(4)$ \\
\hline$C(23)-C(24)$ & $1.394(4)$ \\
\hline$C(24)-C(25)$ & $1.388(4)$ \\
\hline $\mathrm{C}(24)-\mathrm{H}(24)$ & 0.9500 \\
\hline $\mathrm{C}(25)-\mathrm{C}(26)$ & $1.394(4)$ \\
\hline
\end{tabular}




\begin{tabular}{|c|c|}
\hline $\mathrm{C}(25)-\mathrm{H}(25)$ & 0.9500 \\
\hline$C(26)-C(31)$ & $1.398(4)$ \\
\hline$C(26)-C(27)$ & $1.531(4)$ \\
\hline $\mathrm{C}(27)-\mathrm{C}(28)$ & $1.525(5)$ \\
\hline$C(27)-C(29)$ & $1.529(5)$ \\
\hline $\mathrm{C}(27)-\mathrm{C}(30)$ & $1.543(5)$ \\
\hline $\mathrm{C}(28)-\mathrm{H}(28 \mathrm{~A})$ & 0.9800 \\
\hline $\mathrm{C}(28)-\mathrm{H}(28 \mathrm{~B})$ & 0.9800 \\
\hline $\mathrm{C}(28)-\mathrm{H}(28 \mathrm{C})$ & 0.9800 \\
\hline $\mathrm{C}(29)-\mathrm{H}(29 \mathrm{~A})$ & 0.9800 \\
\hline $\mathrm{C}(29)-\mathrm{H}(29 \mathrm{~B})$ & 0.9800 \\
\hline C(29)-H(29C) & 0.9800 \\
\hline $\mathrm{C}(30)-\mathrm{H}(30 \mathrm{~A})$ & 0.9800 \\
\hline $\mathrm{C}(30)-\mathrm{H}(30 \mathrm{~B})$ & 0.9800 \\
\hline $\mathrm{C}(30)-\mathrm{H}(30 \mathrm{C})$ & 0.9800 \\
\hline $\mathrm{C}(31)-\mathrm{C}(32)$ & $1.384(4)$ \\
\hline $\mathrm{C}(31)-\mathrm{H}(31)$ & 0.9500 \\
\hline $\mathrm{C}(32)-\mathrm{H}(32)$ & 0.9500 \\
\hline $\mathrm{C}(33)-\mathrm{Cl}(1)$ & $1.709(10)$ \\
\hline $\mathrm{C}(33)-\mathrm{Cl}(2)$ & $1.753(10)$ \\
\hline $\mathrm{C}(33)-\mathrm{H}(33 \mathrm{~A})$ & 0.9900 \\
\hline $\mathrm{C}(33)-\mathrm{H}(33 \mathrm{~B})$ & 0.9900 \\
\hline $\mathrm{C}(34)-\mathrm{Cl}(3)$ & $1.737(6)$ \\
\hline $\mathrm{C}(34)-\mathrm{Cl}(3) \# 1$ & $1.738(6)$ \\
\hline $\mathrm{C}(34)-\mathrm{H}(34 \mathrm{~A})$ & 0.9900 \\
\hline $\mathrm{C}(34)-\mathrm{H}(34 \mathrm{~B})$ & 0.9900 \\
\hline $\mathrm{O}(1)-\mathrm{H}(1)$ & $0.74(8)$ \\
\hline $\mathrm{O}(1)-\mathrm{C}(1)-\mathrm{C}(11)$ & $121.3(3)$ \\
\hline $\mathrm{O}(1)-\mathrm{C}(1)-\mathrm{C}(2)$ & $115.9(2)$ \\
\hline $\mathrm{C}(11)-\mathrm{C}(1)-\mathrm{C}(2)$ & $122.8(3)$ \\
\hline $\mathrm{C}(3)-\mathrm{C}(2)-\mathrm{C}(1)$ & $119.6(3)$ \\
\hline $\mathrm{C}(3)-\mathrm{C}(2)-\mathrm{C}(2) \# 1$ & $121.3(3)$ \\
\hline $\mathrm{C}(1)-\mathrm{C}(2)-\mathrm{C}(2) \# 1$ & $119.0(3)$ \\
\hline $\mathrm{C}(2)-\mathrm{C}(3)-\mathrm{C}(5)$ & 119.2(3) \\
\hline$C(2)-C(3)-C(4)$ & $121.2(3)$ \\
\hline $\mathrm{C}(5)-\mathrm{C}(3)-\mathrm{C}(4)$ & $119.6(3)$ \\
\hline $\mathrm{C}(3)-\mathrm{C}(4)-\mathrm{H}(4 \mathrm{~A})$ & 109.5 \\
\hline $\mathrm{C}(3)-\mathrm{C}(4)-\mathrm{H}(4 \mathrm{~B})$ & 109.5 \\
\hline $\mathrm{H}(4 \mathrm{~A})-\mathrm{C}(4)-\mathrm{H}(4 \mathrm{~B})$ & 109.5 \\
\hline $\mathrm{C}(3)-\mathrm{C}(4)-\mathrm{H}(4 \mathrm{C})$ & 109.5 \\
\hline $\mathrm{H}(4 \mathrm{~A})-\mathrm{C}(4)-\mathrm{H}(4 \mathrm{C})$ & 109.5 \\
\hline $\mathrm{H}(4 \mathrm{~B})-\mathrm{C}(4)-\mathrm{H}(4 \mathrm{C})$ & 109.5 \\
\hline$C(6)-C(5)-C(10)$ & $118.4(3)$ \\
\hline$C(6)-C(5)-C(3)$ & $121.3(3)$ \\
\hline$C(10)-C(5)-C(3)$ & $120.3(3)$ \\
\hline$C(7)-C(6)-C(5)$ & $121.3(3)$ \\
\hline $\mathrm{C}(7)-\mathrm{C}(6)-\mathrm{H}(6)$ & 119.3 \\
\hline $\mathrm{C}(5)-\mathrm{C}(6)-\mathrm{H}(6)$ & 119.3 \\
\hline $\mathrm{C}(6)-\mathrm{C}(7)-\mathrm{C}(8)$ & $120.2(3)$ \\
\hline
\end{tabular}




$\begin{array}{ll}\mathrm{C}(6)-\mathrm{C}(7)-\mathrm{H}(7) & 119.9 \\ \mathrm{C}(8)-\mathrm{C}(7)-\mathrm{H}(7) & 119.9 \\ \mathrm{C}(9)-\mathrm{C}(8)-\mathrm{C}(7) & 120.5(3) \\ \mathrm{C}(9)-\mathrm{C}(8)-\mathrm{H}(8) & 119.8 \\ \mathrm{C}(7)-\mathrm{C}(8)-\mathrm{H}(8) & 119.8 \\ \mathrm{C}(8)-\mathrm{C}(9)-\mathrm{C}(10) & 120.7(3) \\ \mathrm{C}(8)-\mathrm{C}(9)-\mathrm{H}(9) & 119.6 \\ \mathrm{C}(10)-\mathrm{C}(9)-\mathrm{H}(9) & 119.6 \\ \mathrm{C}(9)-\mathrm{C}(10)-\mathrm{C}(11) & 121.6(3) \\ \mathrm{C}(9)-\mathrm{C}(10)-\mathrm{C}(5) & 118.8(3) \\ \mathrm{C}(11)-\mathrm{C}(10)-\mathrm{C}(5) & 119.7(2) \\ \mathrm{C}(1)-\mathrm{C}(11)-\mathrm{C}(10) & 118.5(2) \\ \mathrm{C}(1)-\mathrm{C}(11)-\mathrm{C}(12) & 119.7(2) \\ \mathrm{C}(10)-\mathrm{C}(11)-\mathrm{C}(12) & 121.8(2) \\ \mathrm{C}(22)-\mathrm{C}(12)-\mathrm{C}(13) & 119.2(2) \\ \mathrm{C}(22)-\mathrm{C}(12)-\mathrm{C}(11) & 120.3(3) \\ \mathrm{C}(13)-\mathrm{C}(12)-\mathrm{C}(11) & 120.5(2) \\ \mathrm{C}(14)-\mathrm{C}(13)-\mathrm{C}(18) & 118.2(3) \\ \mathrm{C}(14)-\mathrm{C}(13)-\mathrm{C}(12) & 121.7(3) \\ \mathrm{C}(18)-\mathrm{C}(13)-\mathrm{C}(12) & 120.1(3) \\ \mathrm{C}(15)-\mathrm{C}(14)-\mathrm{C}(13) & 121.6(3) \\ \mathrm{C}(15)-\mathrm{C}(14)-\mathrm{H}(14) & 119.2 \\ \mathrm{C}(13)-\mathrm{C}(14)-\mathrm{H}(14) & 119.2 \\ \mathrm{C}(14)-\mathrm{C}(15)-\mathrm{C}(16) & 120.0(3) \\ \mathrm{C}(14)-\mathrm{C}(15)-\mathrm{H}(15) & 120.0 \\ \mathrm{C}(16)-\mathrm{C}(15)-\mathrm{H}(15) & 120.0 \\ \mathrm{C}(17)-\mathrm{C}(16)-\mathrm{C}(15) & 120.4(3) \\ \mathrm{C}(17)-\mathrm{C}(16)-\mathrm{H}(16) & 119.8 \\ \mathrm{C}(15)-\mathrm{C}(16)-\mathrm{H}(16) & 119.8 \\ \mathrm{C}(16)-\mathrm{C}(17)-\mathrm{C}(18) & 121.3(3) \\ \mathrm{C}(16)-\mathrm{C}(17)-\mathrm{H}(17) & 119.4 \\ \mathrm{C}(18)-\mathrm{C}(17)-\mathrm{H}(17) & 119.4 \\ \mathrm{C}(17)-\mathrm{C}(18)-\mathrm{C}(13) & 118.5(3) \\ \mathrm{C}(17)-\mathrm{C}(18)-\mathrm{C}(19) & 122.3(3) \\ \mathrm{C}(13)-\mathrm{C}(18)-\mathrm{C}(19) & 119.2(3) \\ \mathrm{C}(21)-\mathrm{C}(19)-\mathrm{C}(18) & 119.0(3) \\ \mathrm{C}(21)-\mathrm{C}(19)-\mathrm{C}(20) & 120.2(3) \\ \mathrm{C}(18)-\mathrm{C}(19)-\mathrm{C}(20) & 120.8(3) \\ \mathrm{C}(19)-\mathrm{C}(20)-\mathrm{H}(20 \mathrm{~A}) & 109.5 \\ \mathrm{C}(19)-\mathrm{C}(20)-\mathrm{H}(20 \mathrm{~B}) & 109.5 \\ \mathrm{H}(20 \mathrm{~A})-\mathrm{C}(20)-\mathrm{H}(20 \mathrm{~B}) & 109.5 \\ \mathrm{C}(19)-\mathrm{C}(20)-\mathrm{H}(20 \mathrm{C}) & 109.5 \\ \mathrm{H}(20 \mathrm{~A})-\mathrm{C}(20)-\mathrm{H}(20 \mathrm{C}) & 109.5 \\ \mathrm{H}(20 \mathrm{~B})-\mathrm{C}(20)-\mathrm{H}(20 \mathrm{C}) & 109.5 \\ \mathrm{C}(19)-\mathrm{C}(21)-\mathrm{C}(22) & 122.5(3) \\ \mathrm{C}(19)-\mathrm{C}(21)-\mathrm{H}(21) & 118.7 \\ \mathrm{C}(22)-\mathrm{C}(21)-\mathrm{H}(21) & 118.7 \\ \mathrm{C}(12)-\mathrm{C}(22)-\mathrm{C}(21) & 120.0(3) \\ \mathrm{C}(12)-\mathrm{C}(22)-\mathrm{C}(23) & 12.5(2) \\ \mathrm{C}(21)-\mathrm{C}(22)-\mathrm{C}(23) & \\ & \end{array}$




\begin{tabular}{|c|c|}
\hline$C(32)-C(23)-C(24)$ & $117.4(3)$ \\
\hline $\mathrm{C}(32)-\mathrm{C}(23)-\mathrm{C}(22)$ & $121.9(2)$ \\
\hline $\mathrm{C}(24)-\mathrm{C}(23)-\mathrm{C}(22)$ & $120.7(3)$ \\
\hline $\mathrm{C}(25)-\mathrm{C}(24)-\mathrm{C}(23)$ & 121.3(3) \\
\hline $\mathrm{C}(25)-\mathrm{C}(24)-\mathrm{H}(24)$ & 119.3 \\
\hline $\mathrm{C}(23)-\mathrm{C}(24)-\mathrm{H}(24)$ & 119.3 \\
\hline $\mathrm{C}(24)-\mathrm{C}(25)-\mathrm{C}(26)$ & $121.4(3)$ \\
\hline $\mathrm{C}(24)-\mathrm{C}(25)-\mathrm{H}(25)$ & 119.3 \\
\hline $\mathrm{C}(26)-\mathrm{C}(25)-\mathrm{H}(25)$ & 119.3 \\
\hline $\mathrm{C}(25)-\mathrm{C}(26)-\mathrm{C}(31)$ & $117.0(3)$ \\
\hline $\mathrm{C}(25)-\mathrm{C}(26)-\mathrm{C}(27)$ & $122.2(3)$ \\
\hline $\mathrm{C}(31)-\mathrm{C}(26)-\mathrm{C}(27)$ & $120.7(3)$ \\
\hline $\mathrm{C}(28)-\mathrm{C}(27)-\mathrm{C}(29)$ & 109.2(4) \\
\hline $\mathrm{C}(28)-\mathrm{C}(27)-\mathrm{C}(26)$ & $112.2(3)$ \\
\hline $\mathrm{C}(29)-\mathrm{C}(27)-\mathrm{C}(26)$ & 107.9(3) \\
\hline $\mathrm{C}(28)-\mathrm{C}(27)-\mathrm{C}(30)$ & 108.7(3) \\
\hline C(29)-C(27)-C(30) & $108.0(3)$ \\
\hline $\mathrm{C}(26)-\mathrm{C}(27)-\mathrm{C}(30)$ & $110.8(3)$ \\
\hline $\mathrm{C}(27)-\mathrm{C}(28)-\mathrm{H}(28 \mathrm{~A})$ & 109.5 \\
\hline $\mathrm{C}(27)-\mathrm{C}(28)-\mathrm{H}(28 \mathrm{~B})$ & 109.5 \\
\hline $\mathrm{H}(28 \mathrm{~A})-\mathrm{C}(28)-\mathrm{H}(28 \mathrm{~B})$ & 109.5 \\
\hline C(27)-C(28)-H(28C) & 109.5 \\
\hline $\mathrm{H}(28 \mathrm{~A})-\mathrm{C}(28)-\mathrm{H}(28 \mathrm{C})$ & 109.5 \\
\hline $\mathrm{H}(28 \mathrm{~B})-\mathrm{C}(28)-\mathrm{H}(28 \mathrm{C})$ & 109.5 \\
\hline C(27)-C(29)-H(29A) & 109.5 \\
\hline C(27)-C(29)-H(29B) & 109.5 \\
\hline $\mathrm{H}(29 \mathrm{~A})-\mathrm{C}(29)-\mathrm{H}(29 \mathrm{~B})$ & 109.5 \\
\hline C(27)-C(29)-H(29C) & 109.5 \\
\hline $\mathrm{H}(29 \mathrm{~A})-\mathrm{C}(29)-\mathrm{H}(29 \mathrm{C})$ & 109.5 \\
\hline $\mathrm{H}(29 \mathrm{~B})-\mathrm{C}(29)-\mathrm{H}(29 \mathrm{C})$ & 109.5 \\
\hline $\mathrm{C}(27)-\mathrm{C}(30)-\mathrm{H}(30 \mathrm{~A})$ & 109.5 \\
\hline $\mathrm{C}(27)-\mathrm{C}(30)-\mathrm{H}(30 \mathrm{~B})$ & 109.5 \\
\hline $\mathrm{H}(30 \mathrm{~A})-\mathrm{C}(30)-\mathrm{H}(30 \mathrm{~B})$ & 109.5 \\
\hline C(27)-C(30)-H(30C) & 109.5 \\
\hline $\mathrm{H}(30 \mathrm{~A})-\mathrm{C}(30)-\mathrm{H}(30 \mathrm{C})$ & 109.5 \\
\hline $\mathrm{H}(30 \mathrm{~B})-\mathrm{C}(30)-\mathrm{H}(30 \mathrm{C})$ & 109.5 \\
\hline $\mathrm{C}(32)-\mathrm{C}(31)-\mathrm{C}(26)$ & $121.6(3)$ \\
\hline $\mathrm{C}(32)-\mathrm{C}(31)-\mathrm{H}(31)$ & 119.2 \\
\hline $\mathrm{C}(26)-\mathrm{C}(31)-\mathrm{H}(31)$ & 119.2 \\
\hline $\mathrm{C}(31)-\mathrm{C}(32)-\mathrm{C}(23)$ & 121.3(3) \\
\hline $\mathrm{C}(31)-\mathrm{C}(32)-\mathrm{H}(32)$ & 119.4 \\
\hline $\mathrm{C}(23)-\mathrm{C}(32)-\mathrm{H}(32)$ & 119.4 \\
\hline $\mathrm{Cl}(1)-\mathrm{C}(33)-\mathrm{Cl}(2)$ & $114.7(5)$ \\
\hline $\mathrm{Cl}(1)-\mathrm{C}(33)-\mathrm{H}(33 \mathrm{~A})$ & 108.6 \\
\hline $\mathrm{Cl}(2)-\mathrm{C}(33)-\mathrm{H}(33 \mathrm{~A})$ & 108.6 \\
\hline $\mathrm{Cl}(1)-\mathrm{C}(33)-\mathrm{H}(33 \mathrm{~B})$ & 108.6 \\
\hline $\mathrm{Cl}(2)-\mathrm{C}(33)-\mathrm{H}(33 \mathrm{~B})$ & 108.6 \\
\hline $\mathrm{H}(33 \mathrm{~A})-\mathrm{C}(33)-\mathrm{H}(33 \mathrm{~B})$ & 107.6 \\
\hline $\mathrm{Cl}(3)-\mathrm{C}(34)-\mathrm{Cl}(3) \# 1$ & $115.4(6)$ \\
\hline $\mathrm{Cl}(3)-\mathrm{C}(34)-\mathrm{H}(34 \mathrm{~A})$ & 108.4 \\
\hline
\end{tabular}




$\begin{array}{ll}\mathrm{Cl}(3) \# 1-\mathrm{C}(34)-\mathrm{H}(34 \mathrm{~A}) & 108.4 \\ \mathrm{Cl}(3)-\mathrm{C}(34)-\mathrm{H}(34 \mathrm{~B}) & 108.4 \\ \mathrm{Cl}(3) \# 1-\mathrm{C}(34)-\mathrm{H}(34 \mathrm{~B}) & 108.4 \\ \mathrm{H}(34 \mathrm{~A})-\mathrm{C}(34)-\mathrm{H}(34 \mathrm{~B}) & 107.5 \\ \mathrm{C}(1)-\mathrm{O}(1)-\mathrm{H}(1) & 100(6)\end{array}$

Symmetry transformations used to generate equivalent atoms:

$\# 1-\mathrm{x}+1,-\mathrm{y}+1, \mathrm{z}$

Anisotropic displacement parameters $\left(\AA^{2} \times 1^{3}\right)$ for jm_iv_380_4.

The anisotropic displacement factor exponent takes the form: $-2 \pi^{2}\left[h^{2} a^{* 2} U^{11}+\ldots+2 h k a^{*} b^{*} U^{12}\right]$

\begin{tabular}{lcccccc}
\hline & $\mathrm{U}^{11}$ & $\mathrm{U}^{22}$ & $\mathrm{U}^{33}$ & $\mathrm{U}^{23}$ & $\mathrm{U}^{13}$ & $\mathrm{U}^{12}$ \\
\hline $\mathrm{C}(1)$ & $21(1)$ & $25(1)$ & $25(1)$ & $-1(1)$ & $-1(1)$ & $2(1)$ \\
$\mathrm{C}(2)$ & $23(1)$ & $22(1)$ & $30(1)$ & $-2(1)$ & $2(1)$ & $-1(1)$ \\
$\mathrm{C}(3)$ & $27(1)$ & $22(1)$ & $34(2)$ & $1(1)$ & $-3(1)$ & $0(1)$ \\
$\mathrm{C}(4)$ & $38(2)$ & $26(2)$ & $53(2)$ & $10(1)$ & $-13(2)$ & $-3(1)$ \\
$\mathrm{C}(5)$ & $23(1)$ & $23(1)$ & $31(1)$ & $2(1)$ & $-2(1)$ & $0(1)$ \\
$\mathrm{C}(6)$ & $28(2)$ & $27(1)$ & $40(2)$ & $3(1)$ & $-7(1)$ & $2(1)$ \\
$\mathrm{C}(7)$ & $25(1)$ & $34(2)$ & $45(2)$ & $2(1)$ & $-9(1)$ & $0(1)$ \\
$\mathrm{C}(8)$ & $24(1)$ & $29(1)$ & $41(2)$ & $0(1)$ & $-4(1)$ & $-5(1)$ \\
$\mathrm{C}(9)$ & $25(1)$ & $25(1)$ & $32(1)$ & $2(1)$ & $0(1)$ & $-2(1)$ \\
$\mathrm{C}(10)$ & $22(1)$ & $23(1)$ & $27(1)$ & $-2(1)$ & $1(1)$ & $1(1)$ \\
$\mathrm{C}(11)$ & $24(1)$ & $20(1)$ & $23(1)$ & $-1(1)$ & $2(1)$ & $1(1)$ \\
$\mathrm{C}(12)$ & $23(1)$ & $23(1)$ & $24(1)$ & $0(1)$ & $-3(1)$ & $-4(1)$ \\
$\mathrm{C}(13)$ & $24(1)$ & $25(1)$ & $26(1)$ & $-1(1)$ & $-2(1)$ & $-4(1)$ \\
$\mathrm{C}(14)$ & $33(2)$ & $30(1)$ & $32(2)$ & $-1(1)$ & $5(1)$ & $-2(1)$ \\
$\mathrm{C}(15)$ & $37(2)$ & $39(2)$ & $36(2)$ & $-6(1)$ & $10(1)$ & $-3(1)$ \\
$\mathrm{C}(16)$ & $36(2)$ & $46(2)$ & $26(2)$ & $2(1)$ & $5(1)$ & $-11(1)$ \\
$\mathrm{C}(17)$ & $35(2)$ & $37(2)$ & $27(2)$ & $4(1)$ & $-4(1)$ & $-10(1)$ \\
$\mathrm{C}(18)$ & $25(1)$ & $29(1)$ & $24(1)$ & $1(1)$ & $-5(1)$ & $-8(1)$ \\
$\mathrm{C}(19)$ & $25(1)$ & $28(1)$ & $29(1)$ & $5(1)$ & $-7(1)$ & $-7(1)$ \\
$\mathrm{C}(20)$ & $32(2)$ & $36(2)$ & $42(2)$ & $14(2)$ & $-4(1)$ & $-2(1)$ \\
$\mathrm{C}(21)$ & $25(1)$ & $25(1)$ & $29(1)$ & $2(1)$ & $-6(1)$ & $-1(1)$ \\
$\mathrm{C}(22)$ & $23(1)$ & $22(1)$ & $25(1)$ & $1(1)$ & $-3(1)$ & $-2(1)$ \\
$\mathrm{C}(23)$ & $23(1)$ & $22(1)$ & $27(1)$ & $0(1)$ & $-2(1)$ & $1(1)$ \\
$\mathrm{C}(24)$ & $23(1)$ & $33(1)$ & $26(1)$ & $3(1)$ & $-4(1)$ & $2(1)$ \\
$\mathrm{C}(25)$ & $21(1)$ & $31(1)$ & $31(2)$ & $-1(1)$ & $-2(1)$ & $4(1)$ \\
$\mathrm{C}(26)$ & $25(1)$ & $27(1)$ & $28(1)$ & $-4(1)$ & $1(1)$ & $-1(1)$ \\
$\mathrm{C}(27)$ & $28(2)$ & $50(2)$ & $28(2)$ & $-5(1)$ & $3(1)$ & $-4(1)$ \\
$\mathrm{C}(28)$ & $34(2)$ & $122(4)$ & $34(2)$ & $-3(2)$ & $6(2)$ & $-30(2)$ \\
$\mathrm{C}(29)$ & $41(2)$ & $58(2)$ & $52(2)$ & $-19(2)$ & $6(2)$ & $12(2)$ \\
$\mathrm{C}(30)$ & $48(2)$ & $58(2)$ & $32(2)$ & $5(2)$ & $8(2)$ & $-2(2)$ \\
$\mathrm{C}(31)$ & $25(1)$ & $43(2)$ & $25(1)$ & $-2(1)$ & $-4(1)$ & $-4(1)$ \\
$\mathrm{C}(32)$ & $21(1)$ & $35(2)$ & $28(1)$ & $-1(1)$ & $-4(1)$ & $-1(1)$ \\
$\mathrm{C}(33)$ & $52(4)$ & $43(4)$ & $63(5)$ & $-16(4)$ & $-4(4)$ & $-5(3)$ \\
$\mathrm{C}(34)$ & $142(15)$ & $73(9)$ & $23(5)$ & 0 & 0 & $63(10)$ \\
$\mathrm{Cl}(1)$ & $81(2)$ & $43(1)$ & $48(1)$ & $2(1)$ & $-4(1)$ & $1(1)$ \\
& & & & & &
\end{tabular}




\begin{tabular}{llllccc}
$\mathrm{Cl}(2)$ & $80(2)$ & $65(1)$ & $82(2)$ & $17(1)$ & $-32(1)$ & $-9(1)$ \\
$\mathrm{Cl}(3)$ & $48(1)$ & $52(1)$ & $68(1)$ & $-25(1)$ & $1(1)$ & $-15(1)$ \\
$\mathrm{O}(1)$ & $24(1)$ & $29(1)$ & $33(1)$ & $5(1)$ & $-6(1)$ & $-3(1)$ \\
\hline
\end{tabular}

Hydrogen coordinates $\left(\times 10^{4}\right)$ and isotropic displacement parameters $\left(\AA^{2} \times 10^{3}\right)$ for jm_iv_380_4.

\begin{tabular}{|c|c|c|c|c|}
\hline & $\mathrm{x}$ & $\mathrm{y}$ & $\mathrm{z}$ & $\mathrm{U}(\mathrm{eq})$ \\
\hline $\mathrm{H}(4 \mathrm{~A})$ & 5945 & 4404 & 4334 & 59 \\
\hline $\mathrm{H}(4 \mathrm{~B})$ & 6792 & 4677 & 5479 & 59 \\
\hline $\mathrm{H}(4 \mathrm{C})$ & 7299 & 4423 & 4005 & 59 \\
\hline $\mathrm{H}(6)$ & 8524 & 5069 & 4957 & 38 \\
\hline $\mathrm{H}(7)$ & 9948 & 5667 & 5034 & 41 \\
\hline $\mathrm{H}(8)$ & 9705 & 6428 & 3783 & 38 \\
\hline $\mathrm{H}(9)$ & 8044 & 6583 & 2425 & 32 \\
\hline $\mathrm{H}(14)$ & 7651 & 5933 & -212 & 38 \\
\hline $\mathrm{H}(15)$ & 8662 & 6095 & -2379 & 45 \\
\hline $\mathrm{H}(16)$ & 8295 & 6825 & -3744 & 44 \\
\hline $\mathrm{H}(17)$ & 6933 & 7387 & -2935 & 39 \\
\hline $\mathrm{H}(20 \mathrm{~A})$ & 6040 & 8034 & -1326 & 55 \\
\hline $\mathrm{H}(20 \mathrm{~B})$ & 4714 & 8008 & -840 & 55 \\
\hline $\mathrm{H}(20 \mathrm{C})$ & 5124 & 7754 & -2365 & 55 \\
\hline $\mathrm{H}(21)$ & 4401 & 7465 & 1087 & 32 \\
\hline $\mathrm{H}(24)$ & 2954 & 6744 & 2074 & 33 \\
\hline $\mathrm{H}(25)$ & 1897 & 6687 & 4260 & 33 \\
\hline $\mathrm{H}(28 \mathrm{~A})$ & 918 & 6529 & 7894 & 95 \\
\hline $\mathrm{H}(28 \mathrm{~B})$ & 1327 & 6160 & 6596 & 95 \\
\hline $\mathrm{H}(28 \mathrm{C})$ & 952 & 6719 & 6204 & 95 \\
\hline $\mathrm{H}(29 \mathrm{~A})$ & 2185 & 7220 & 8731 & 75 \\
\hline $\mathrm{H}(29 \mathrm{~B})$ & 2163 & 7430 & 7062 & 75 \\
\hline $\mathrm{H}(29 \mathrm{C})$ & 3368 & 7328 & 7873 & 75 \\
\hline $\mathrm{H}(30 \mathrm{~A})$ & 3242 & 6005 & 7924 & 69 \\
\hline $\mathrm{H}(30 \mathrm{~B})$ & 2672 & 6328 & 9229 & 69 \\
\hline $\mathrm{H}(30 \mathrm{C})$ & 3911 & 6477 & 8587 & 69 \\
\hline $\mathrm{H}(31)$ & 4852 & 6668 & 6637 & 37 \\
\hline $\mathrm{H}(32)$ & 5907 & 6714 & 4453 & 33 \\
\hline $\mathrm{H}(33 \mathrm{~A})$ & 7461 & 4713 & 590 & 63 \\
\hline $\mathrm{H}(33 \mathrm{~B})$ & 8466 & 5104 & 916 & 63 \\
\hline $\mathrm{H}(34 \mathrm{~A})$ & 4402 & 5146 & 8947 & 95 \\
\hline $\mathrm{H}(34 \mathrm{~B})$ & 5598 & 4854 & 8947 & 95 \\
\hline $\mathrm{H}(1)$ & $4500(60)$ & $5940(30)$ & $810(90)$ & $110(30)$ \\
\hline
\end{tabular}




\section{Torsion angles $\left[{ }^{\circ}\right]$ for jm_iv_380_4.}

\begin{tabular}{|c|c|}
\hline $\mathrm{O}(1)-\mathrm{C}(1)-\mathrm{C}(2)-\mathrm{C}(3)$ & $177.8(3)$ \\
\hline $\mathrm{C}(11)-\mathrm{C}(1)-\mathrm{C}(2)-\mathrm{C}(3)$ & $-2.4(4)$ \\
\hline $\mathrm{O}(1)-\mathrm{C}(1)-\mathrm{C}(2)-\mathrm{C}(2) \# 1$ & $-4.1(4)$ \\
\hline$C(11)-C(1)-C(2)-C(2) \# 1$ & $175.7(2)$ \\
\hline $\mathrm{C}(1)-\mathrm{C}(2)-\mathrm{C}(3)-\mathrm{C}(5)$ & $1.6(4)$ \\
\hline $\mathrm{C}(2) \# 1-\mathrm{C}(2)-\mathrm{C}(3)-\mathrm{C}(5)$ & $-176.5(2)$ \\
\hline $\mathrm{C}(1)-\mathrm{C}(2)-\mathrm{C}(3)-\mathrm{C}(4)$ & $-178.1(3)$ \\
\hline $\mathrm{C}(2) \# 1-\mathrm{C}(2)-\mathrm{C}(3)-\mathrm{C}(4)$ & $3.9(4)$ \\
\hline $\mathrm{C}(2)-\mathrm{C}(3)-\mathrm{C}(5)-\mathrm{C}(6)$ & $-179.9(3)$ \\
\hline$C(4)-C(3)-C(5)-C(6)$ & $-0.3(5)$ \\
\hline$C(2)-C(3)-C(5)-C(10)$ & $0.3(4)$ \\
\hline $\mathrm{C}(4)-\mathrm{C}(3)-\mathrm{C}(5)-\mathrm{C}(10)$ & $179.9(3)$ \\
\hline $\mathrm{C}(10)-\mathrm{C}(5)-\mathrm{C}(6)-\mathrm{C}(7)$ & $0.9(5)$ \\
\hline$C(3)-C(5)-C(6)-C(7)$ & $-179.0(3)$ \\
\hline$C(5)-C(6)-C(7)-C(8)$ & $0.3(5)$ \\
\hline$C(6)-C(7)-C(8)-C(9)$ & $-0.6(5)$ \\
\hline $\mathrm{C}(7)-\mathrm{C}(8)-\mathrm{C}(9)-\mathrm{C}(10)$ & $-0.2(5)$ \\
\hline $\mathrm{C}(8)-\mathrm{C}(9)-\mathrm{C}(10)-\mathrm{C}(11)$ & $-179.2(3)$ \\
\hline C(8)-C(9)-C(10)-C(5) & $1.3(4)$ \\
\hline $\mathrm{C}(6)-\mathrm{C}(5)-\mathrm{C}(10)-\mathrm{C}(9)$ & $-1.6(4)$ \\
\hline C(3)-C(5)-C(10)-C(9) & $178.2(3)$ \\
\hline$C(6)-C(5)-C(10)-C(11)$ & $178.8(3)$ \\
\hline $\mathrm{C}(3)-\mathrm{C}(5)-\mathrm{C}(10)-\mathrm{C}(11)$ & $-1.4(4)$ \\
\hline $\mathrm{O}(1)-\mathrm{C}(1)-\mathrm{C}(11)-\mathrm{C}(10)$ & $-179.0(3)$ \\
\hline $\mathrm{C}(2)-\mathrm{C}(1)-\mathrm{C}(11)-\mathrm{C}(10)$ & $1.2(4)$ \\
\hline $\mathrm{O}(1)-\mathrm{C}(1)-\mathrm{C}(11)-\mathrm{C}(12)$ & $1.8(4)$ \\
\hline $\mathrm{C}(2)-\mathrm{C}(1)-\mathrm{C}(11)-\mathrm{C}(12)$ & $-178.0(3)$ \\
\hline $\mathrm{C}(9)-\mathrm{C}(10)-\mathrm{C}(11)-\mathrm{C}(1)$ & $-178.9(3)$ \\
\hline $\mathrm{C}(5)-\mathrm{C}(10)-\mathrm{C}(11)-\mathrm{C}(1)$ & $0.6(4)$ \\
\hline $\mathrm{C}(9)-\mathrm{C}(10)-\mathrm{C}(11)-\mathrm{C}(12)$ & $0.3(4)$ \\
\hline $\mathrm{C}(5)-\mathrm{C}(10)-\mathrm{C}(11)-\mathrm{C}(12)$ & $179.8(3)$ \\
\hline $\mathrm{C}(1)-\mathrm{C}(11)-\mathrm{C}(12)-\mathrm{C}(22)$ & $69.2(4)$ \\
\hline $\mathrm{C}(10)-\mathrm{C}(11)-\mathrm{C}(12)-\mathrm{C}(22)$ & $-110.0(3)$ \\
\hline $\mathrm{C}(1)-\mathrm{C}(11)-\mathrm{C}(12)-\mathrm{C}(13)$ & $-107.1(3)$ \\
\hline $\mathrm{C}(10)-\mathrm{C}(11)-\mathrm{C}(12)-\mathrm{C}(13)$ & 73.7(4) \\
\hline $\mathrm{C}(22)-\mathrm{C}(12)-\mathrm{C}(13)-\mathrm{C}(14)$ & $-179.2(3)$ \\
\hline $\mathrm{C}(11)-\mathrm{C}(12)-\mathrm{C}(13)-\mathrm{C}(14)$ & $-2.9(4)$ \\
\hline $\mathrm{C}(22)-\mathrm{C}(12)-\mathrm{C}(13)-\mathrm{C}(18)$ & $1.0(4)$ \\
\hline $\mathrm{C}(11)-\mathrm{C}(12)-\mathrm{C}(13)-\mathrm{C}(18)$ & $177.3(2)$ \\
\hline $\mathrm{C}(18)-\mathrm{C}(13)-\mathrm{C}(14)-\mathrm{C}(15)$ & $0.3(4)$ \\
\hline $\mathrm{C}(12)-\mathrm{C}(13)-\mathrm{C}(14)-\mathrm{C}(15)$ & $-179.4(3)$ \\
\hline $\mathrm{C}(13)-\mathrm{C}(14)-\mathrm{C}(15)-\mathrm{C}(16)$ & $-0.3(5)$ \\
\hline $\mathrm{C}(14)-\mathrm{C}(15)-\mathrm{C}(16)-\mathrm{C}(17)$ & $0.1(5)$ \\
\hline $\mathrm{C}(15)-\mathrm{C}(16)-\mathrm{C}(17)-\mathrm{C}(18)$ & $0.1(5)$ \\
\hline $\mathrm{C}(16)-\mathrm{C}(17)-\mathrm{C}(18)-\mathrm{C}(13)$ & $0.0(4)$ \\
\hline $\mathrm{C}(16)-\mathrm{C}(17)-\mathrm{C}(18)-\mathrm{C}(19)$ & $-179.2(3)$ \\
\hline $\mathrm{C}(14)-\mathrm{C}(13)-\mathrm{C}(18)-\mathrm{C}(17)$ & $-0.2(4)$ \\
\hline $\mathrm{C}(12)-\mathrm{C}(13)-\mathrm{C}(18)-\mathrm{C}(17)$ & $179.6(3)$ \\
\hline
\end{tabular}


C(14)-C(13)-C(18)-C(19)

C(12)-C(13)-C(18)-C(19)

C(17)-C(18)-C(19)-C(21)

$\mathrm{C}(13)-\mathrm{C}(18)-\mathrm{C}(19)-\mathrm{C}(21)$

$\mathrm{C}(17)-\mathrm{C}(18)-\mathrm{C}(19)-\mathrm{C}(20)$

C(13)-C(18)-C(19)-C(20)

$\mathrm{C}(18)-\mathrm{C}(19)-\mathrm{C}(21)-\mathrm{C}(22)$

$\mathrm{C}(20)-\mathrm{C}(19)-\mathrm{C}(21)-\mathrm{C}(22)$

C(13)-C(12)-C(22)-C(21)

$\mathrm{C}(11)-\mathrm{C}(12)-\mathrm{C}(22)-\mathrm{C}(21)$

$\mathrm{C}(13)-\mathrm{C}(12)-\mathrm{C}(22)-\mathrm{C}(23)$

$\mathrm{C}(11)-\mathrm{C}(12)-\mathrm{C}(22)-\mathrm{C}(23)$

$\mathrm{C}(19)-\mathrm{C}(21)-\mathrm{C}(22)-\mathrm{C}(12)$

$\mathrm{C}(19)-\mathrm{C}(21)-\mathrm{C}(22)-\mathrm{C}(23)$

$\mathrm{C}(12)-\mathrm{C}(22)-\mathrm{C}(23)-\mathrm{C}(32)$

$\mathrm{C}(21)-\mathrm{C}(22)-\mathrm{C}(23)-\mathrm{C}(32)$

$\mathrm{C}(12)-\mathrm{C}(22)-\mathrm{C}(23)-\mathrm{C}(24)$

$\mathrm{C}(21)-\mathrm{C}(22)-\mathrm{C}(23)-\mathrm{C}(24)$

$\mathrm{C}(32)-\mathrm{C}(23)-\mathrm{C}(24)-\mathrm{C}(25)$

$\mathrm{C}(22)-\mathrm{C}(23)-\mathrm{C}(24)-\mathrm{C}(25)$

$\mathrm{C}(23)-\mathrm{C}(24)-\mathrm{C}(25)-\mathrm{C}(26)$

$\mathrm{C}(24)-\mathrm{C}(25)-\mathrm{C}(26)-\mathrm{C}(31)$

$\mathrm{C}(24)-\mathrm{C}(25)-\mathrm{C}(26)-\mathrm{C}(27)$

$\mathrm{C}(25)-\mathrm{C}(26)-\mathrm{C}(27)-\mathrm{C}(28)$

$\mathrm{C}(31)-\mathrm{C}(26)-\mathrm{C}(27)-\mathrm{C}(28)$

$\mathrm{C}(25)-\mathrm{C}(26)-\mathrm{C}(27)-\mathrm{C}(29)$

$\mathrm{C}(31)-\mathrm{C}(26)-\mathrm{C}(27)-\mathrm{C}(29)$

$\mathrm{C}(25)-\mathrm{C}(26)-\mathrm{C}(27)-\mathrm{C}(30)$

$\mathrm{C}(31)-\mathrm{C}(26)-\mathrm{C}(27)-\mathrm{C}(30)$

$\mathrm{C}(25)-\mathrm{C}(26)-\mathrm{C}(31)-\mathrm{C}(32)$

$\mathrm{C}(27)-\mathrm{C}(26)-\mathrm{C}(31)-\mathrm{C}(32)$

$\mathrm{C}(26)-\mathrm{C}(31)-\mathrm{C}(32)-\mathrm{C}(23)$

$\mathrm{C}(24)-\mathrm{C}(23)-\mathrm{C}(32)-\mathrm{C}(31)$

$\mathrm{C}(22)-\mathrm{C}(23)-\mathrm{C}(32)-\mathrm{C}(31)$
179.1(3)

$-1.2(4)$

179.2(3)

$0.0(4)$

$-2.9(4)$

177.9(3)

$1.4(4)$

$-176.5(3)$

$0.3(4)$

$-176.0(2)$

$-178.7(2)$

5.0(4)

$-1.6(4)$

$177.5(3)$

60.0(4)

$-119.0(3)$

$-123.4(3)$

$57.6(4)$

$0.5(4)$

$-176.2(3)$

$0.4(5)$

$-0.8(4)$

175.3(3)

22.2(5)

$-161.9(4)$

$-98.2(3)$

77.8(4)

143.8(3)

$-40.3(4)$

$0.3(5)$

$-175.8(3)$

$0.6(5)$

$-1.0(4)$

175.7(3)

Symmetry transformations used to generate equivalent atoms:

\#1-x+1,-y+1,z 


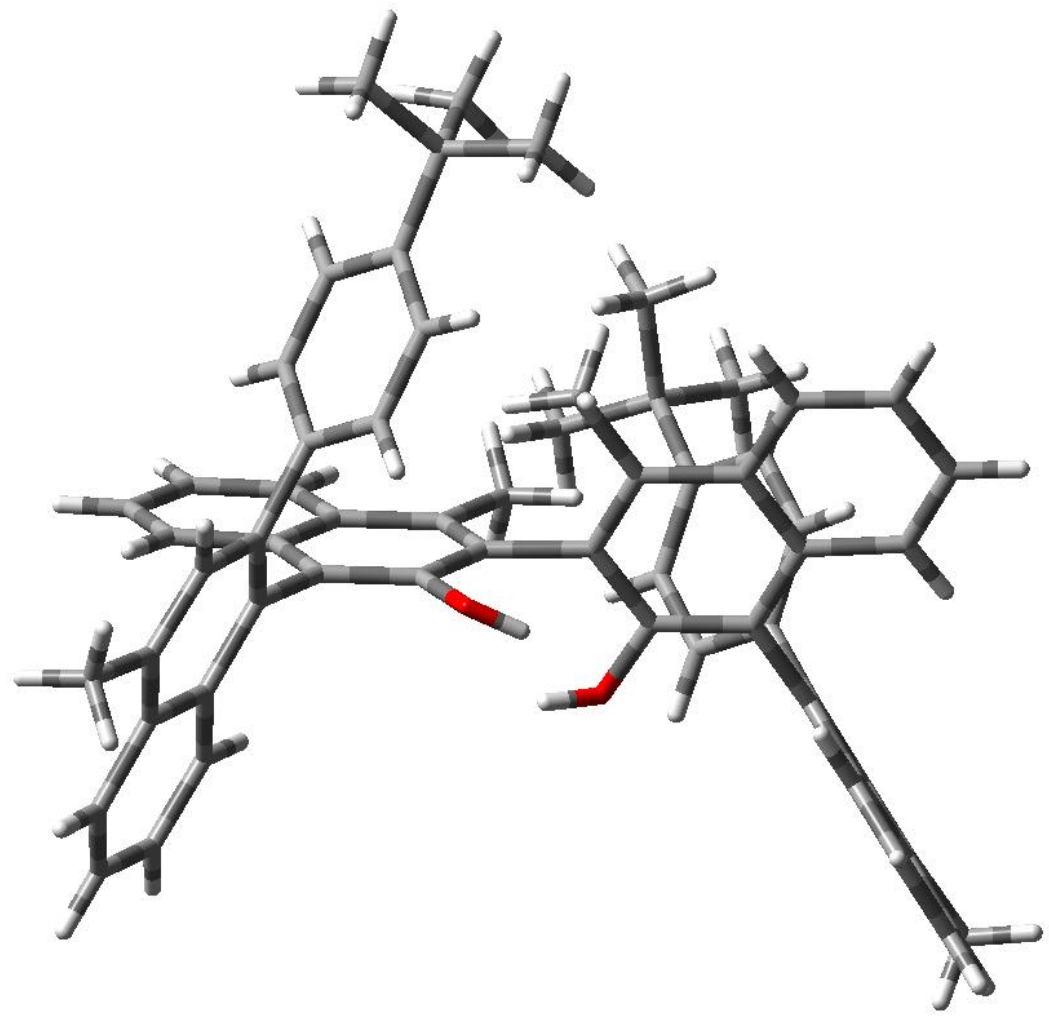




\section{References}

1. Lambert, T. H.; MacMillan, D. W. C. Development of a New Lewis Acid-catalyzed [3,3]-Sigmatropic Rearrangement: the Allenoate-Claisen Rearrangement. J. Am. Chem. Soc. 2002, 124, 13646-13647.

2. Registered ${ }^{19} \mathrm{~F}$ NMR signals of the tetrafluoroborate anions show two components corresponding to the ${ }^{11} \mathrm{~B}$ and ${ }^{10} \mathrm{~B}$ isotopomers. Just signals corresponding to the most abundant ${ }^{10} \mathrm{~B}$ isotopomer are indicated.

3. (a) Li, Y.-L.; Li, J.; Deng, J. Practical Access to Difluoromethyl Ketones via Straightforward Decarboxylative Difluorination of $\beta$-Ketoacids. Adv. Synth. Catal. 2017, 359, 1407-1412. (b) Peng, W.; Shreeve, J. M. Reactions of Enamines with Selectfluor: A Straightforward Route to Difluorinated Carbonyl Compounds. J. Org. Chem. 2005, 70, 5760-5763.

4. Morphy, J. R.; Rankovic, Z.; York, M. Reagent Concentration Effects in the REM Resin Solid Phase Synthesis of Tertiary Amines. Tetrahedron, 2003, 59, 2137-2145.

5. Non-commercially available allylic alcohols were prepared according to reported procedures in the literature.

6. Hirata, G.; Satomura, H.; Kumagae, H.; Shimizu, A.; Onodera, G.; Kimura, M. Direct Allylic Amination of Allylic Alcohol Catalyzed by Palladium Complex Bearing Phosphine-Borane Ligand. Org. Lett. 2017, 19, 61486151.

7. (a) Honjo, T.; Phipps, R. J.; Rauniyar, V.; Toste, F. D. A Doubly Axially Chiral Phosphoric Acid Catalyst for the Asymmetric Tandem Oxyfluorination of Enamides. Angew. Chem. Int. Ed. 2012, 51, 9684-9688. (b) Hiramatsu, K.; Honjo, T.; Rauniyar, V.; Toste, F. D. Enantioselective Synthesis of Fluoro-Dihydroquinazolones and Benzooxazinones by Fluorination-Initiated Asymmetric Cyclization Reactions. ACS Catal. 2016, 6, 151-154.

8. Kim, H. Y., Oh, K. Org. Lett. 2014, 16, 5934-5936.

9. $\quad$ Dai, Y., Feng, X.; Liu, H., Jiang, H., Bao, M. J. Org. Chem. 2011, 76, 10068-10077.

10. In this case, hydrolysis of intermediate phosphoryl chloride was completed after 24 hours at $95{ }^{\circ} \mathrm{C}$.

11. M. J. Frisch, G. W. Trucks, H. B. Schlegel, G. E. Scuseria, M. A. Robb, J. R. Cheeseman, G. Scalmani, V. Barone, G. A. Petersson, H. Nakatsuji, X. Li, M. Caricato, A. V. Marenich, J. Bloino, B. G. Janesko, R. Gomperts, B. Mennucci, H. P. Hratchian, J. V. Ortiz, A. F. Izmaylov, J. L. Sonnenberg, D. Williams-Young, F. Ding, F. Lipparini, F. Egidi, J. Goings, B. Peng, A. Petrone, T. Henderson, D. Ranasinghe, V. G. Zakrzewski, J. Gao, N. Rega, G. Zheng, W. Liang, M. Hada, M. Ehara, K. Toyota, R. Fukuda, J. Hasegawa, M. Ishida, T. Nakajima, Y. Honda, O. Kitao, H. Nakai, T. Vreven, K. Throssell, J. A. Montgomery, Jr., J. E. Peralta, F. Ogliaro, M. J. Bearpark, J. J. Heyd, E. N. Brothers, K. N. Kudin, V. N. Staroverov, T. A. Keith, R. Kobayashi, J. Normand, K. Raghavachari, A. P. Rendell, J. C. Burant, S. S. Iyengar, J. Tomasi, M. Cossi, J. M. Millam, M. Klene, C. Adamo, R. Cammi, J. W. Ochterski, R. L. Martin, K. Morokuma, O. Farkas, J. B. Foresman, and D. J. Fox, Gaussian 16, Revision A.03 (2016) Gaussian, Inc., Wallingford CT.

12. Legault, C. Y. CYLview, 1.0b; Université de Sherbrooke, 2009.

13. (a) Johnson, E. R.; Keinan, S.; Mori-Sánchez, P.; Contreras-García, J.; Cohen, A. J.; Yang, W. Revealing noncovalent interactions, J. Am. Chem. Soc. 2010, 132, 6498-6506. (b) Contreras-García, J.; Johnson, E. R.; Keinan, S.; Chaudret, R.; Piquemal, J.-P.; Beratan, D. N.; Yang, W. NCIPLOT: A Program for Plotting Noncovalent Interaction Regions, J. Chem. Theory Comput. 2011, 7, 625-632.

14. Grimme, S.; Ehrlich, S.; Goerigk, L. Effect of the damping function in dispersion corrected density functional theory, J. Comput. Chem. 2011, 32, 1456-1465.

15. Goerigk, L.; Grimme, S. A thorough benchmark of density functional methods for general main group thermochemistry, kinetics, and noncovalent interactions, Phys. Chem. Chem. Phys. 2011, 13, 6670-6688.

16. (a) Dunning, T. H. Gaussian basis sets for use in correlated molecular calculations I The atoms boron through neon and hydrogen, J. Chem. Phys. 1989, 90, 1007. (b) Kendall, R. A.; Dunning, T. H.; Harrison, R. J. Electron affinities of the first-row atoms revisited Systematic basis sets and wave functions, J. Chem. Phys. 1992, 96, 6796-6806. (c) Papajak, E.; Zheng, J.; Xu, X.; Leverentz, H. R.; Truhlar, D. G. Perspectives on Basis Sets Beautiful: Seasonal Plantings of Diffuse Basis Functions, J. Chem. Theory Comput. 2011, 7, 3027-3034.

17. Chai, J.-D.; Head-Gordon, M. Long-range corrected hybrid density functionals with damped atom-atom dispersion corrections, Phys. Chem. Chem. Phys. 2008, 10, 6615-6620.

18. Marenich, A. V.; Cramer, C. J.; Truhlar, D. G. Universal Solvation Model Based on Solute Electron Density and on a Continuum Model of the Solvent Defined by the Bulk Dielectric Constant and Atomic Surface Tensions, $J$. Phys. Chem. B 2009, 113, 6378-6396.

19. Ribeiro, R. F.; Marenich, A. V.; Cramer, C. J.; Truhlar, D. G. Use of solution-phase vibrational frequencies in continuum models for the free energy of solvation, J. Phys. Chem. B 2011, 115, 14556-14562.

20. Funes-Ardoiz, I.; Paton, R. S. GoodVibes: version2.0.3. 2018. DOI: 10.5281/zenodo.595246.

21. Glendening, E. D.; Reed, A. E.; Carpenter, J. E.; Weinhold, F. NBO Version 3.1 (1990). 
22. Verloop, A. in Drug Design Ed. Ariens, E. J. (Academic Press, Vol. III., 1976).

23. Jackson, K.; Paton, R. S. Sterimol: version1.0. 2018. DOI: 10.5281/zenodo. 1320767

24. (a) Pollice, R.; Chen, P. A Universal Quantitative Descriptor of the Dispersion Interaction Potential, Angew. Chem. Int. Ed. 2019, 58, 9758-9769. (b) https://gitlab.com/robpollice/ldp.

25. (a) Lu, T.; Chen, F. Multiwfn: A multifunctional wavefunction analyzer, J. Comput. Chem. 2012, 33, $580-592$. (b) Lu, T.; Chen, F. Quantitative analysis of molecular surface based on improved Marching Tetrahedra algorithm, J. Mol. Graph. Model. 2012, 38, 314-323.

26. (a) Caldeweyher, E.; Bannwarth, C.; Grimme, S. Extension of the D3 dispersion coefficient model, J. Chem. Phys. 2017, 147, 034112. (b) Caldeweyher, E.; Ehlert, S.; Hansen, A.; Neugebauer, H.; Spicher, S.; Bannwarth, C.; Grimme, S. A generally applicable atomic-charge dependent London dispersion correction, J. Chem. Phys. 2019, $150,154122$.

27. Zhao, Y.; Truhlar, D. G. The M06 suite of density functionals for main group thermochemistry, thermochemical kinetics, noncovalent interactions, excited states, and transition elements: two new functionals and systematic testing of four M06-class functionals and 12 other functionals, Theor. Chem. Account. 2008, 120, $215-241$.

28. (a) Hehre, W. J.; Ditchfield, R.; Pople, J. A. Self-Consistent Molecular Orbital Methods XII Further Extensions of Gaussian-Type Basis Sets for Use in Molecular Orbital Studies of Organic Molecules, J. Chem. Phys. 1972, 56, 2257-2261. (b) Hariharan, P. C.; Pople, J. A. The influence of polarization functions on molecular orbital hydrogenation energies, Theor. Chim. Acta 1973, 28, 213-222.

29. Santiago, C. B.; Milo, A.; Sigman, M. S. Developing a Modern Approach to Account for Steric Effects in Hammett-Type Correlations, J. Am. Chem. Soc. 2016, 138, 13424-13430.

30. Weigend, F.; Ahlrichs, R. Balanced basis sets of split valence, triple zeta valence and quadruple zeta valence quality for H to Rn: Design and assessment of accuracy, Phys. Chem. Chem. Phys. 2005, 7, 3297.

31. Schrödinger Release 2018-1: MacroModel (Schrödinger, LLC, New York, NY, 2018).

32. Ermanis, K.; Goodman, J. M. PyDP4. https://github.com/KristapsE/PyDP4/

33. Orlandi, M.; Coelho, J. A. S.; Hilton, M. J.; Toste, F. D.; Sigman, M. S. Parametrization of Non-covalent Interactions for Transition State Interrogation Applied to Asymmetric Catalysis, J. Am. Chem. Soc. 2017, 139, 6803-6806.

34. (a) Hohenstein, E. G.; Sherrill, C. D. Density fitting and Cholesky decomposition approximations in symmetryadapted perturbation theory: Implementation and application to probe the nature of $\pi-\pi$ interactions in linear acenes. J. Chem. Phys. 2010, 132, 184111. (b) Parker, T. M.; Burns, L. A.; Parrish, R. M.; Ryno, A. G.; Sherrill, C. D. Levels of symmetry adapted perturbation theory (SAPT) I Efficiency and performance for interaction energies. J. Chem. Phys. 2014, 140, 094106. (c) Gonthier, J. F.; Sherrill, C. D. Density-fitted open-shell symmetry-adapted perturbation theory and application to $\pi$-stacking in benzene dimer cation and ionized DNA base pair steps. J. Chem. Phys. 2016, 145, 134106.

35. Parrish, R. M.; Burns, L. A.; Smith, D. G. A.; Simmonett, A. C.; DePrince, A. E.; Hohenstein, E. G.; Bozkaya, U.; Sokolov, A. Y.; Di Remigio, R.; Richard, R. M.; Gonthier, J. F.; James, A. M.; McAlexander, H. R.; Kumar, A.; Saitow, M.; Wang, X.; Pritchard, B. P.; Verma, P.; Schaefer, H. F.; Patkowski, K.; King, R. A.; Valeev, E. F.; Evangelista, F. A.; Turney, J. M.; Crawford, T. D.; Sherrill, C. D. Psi4 1.1: An Open-Source Electronic Structure Program Emphasizing Automation, Advanced Libraries, and Interoperability. J. Chem. Theory Comput. 2017, 13, 3185-3197.

36. Guo, J.-Y.; Minko, Y.; Santiago, C. B.; Sigman, M. S. Developing Comprehensive Computational Parameter Sets To Describe the Performance of Pyridine-Oxazoline and Related Ligands, ACS Catal. 2017, 7, 4144-4151. 

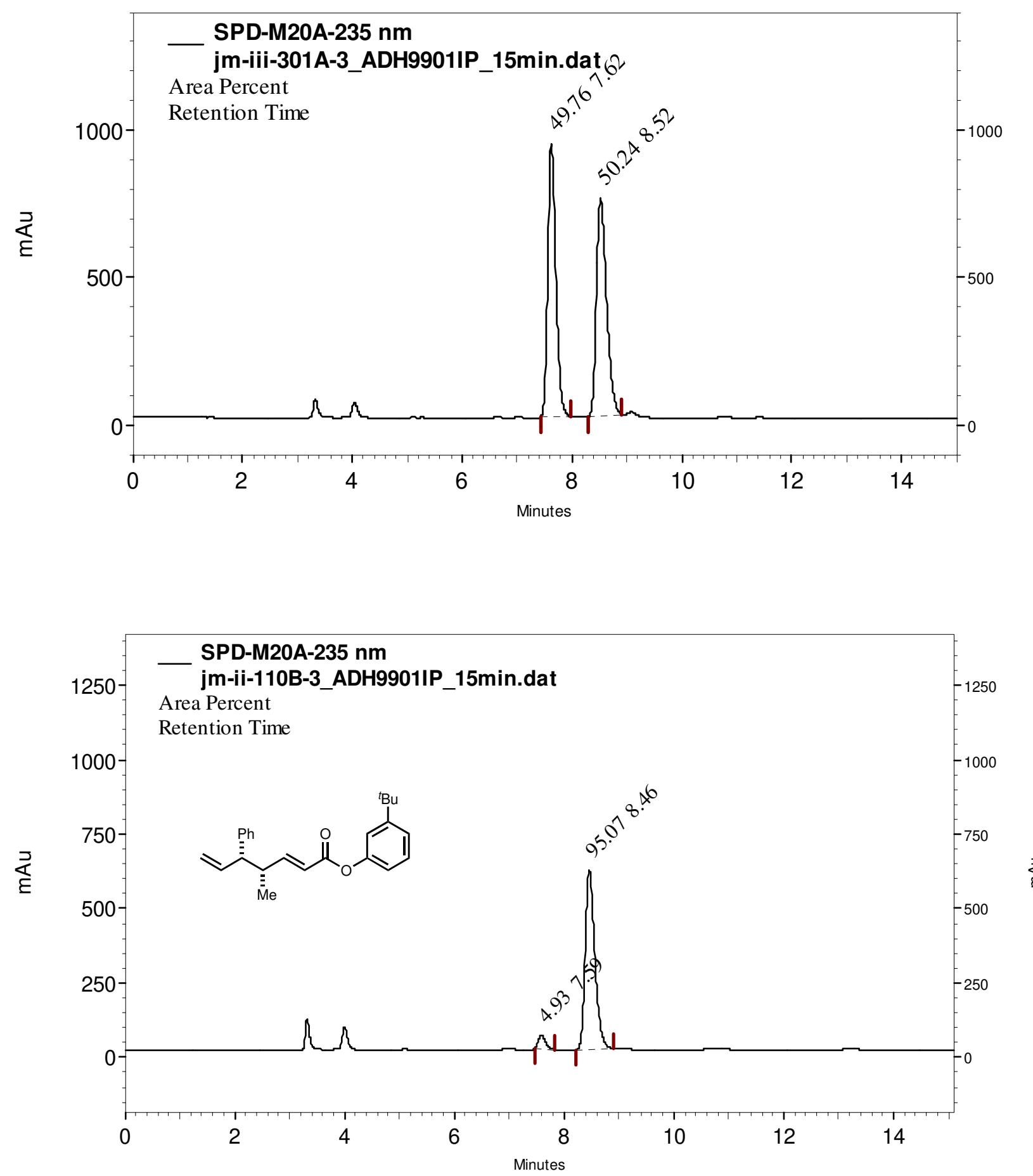

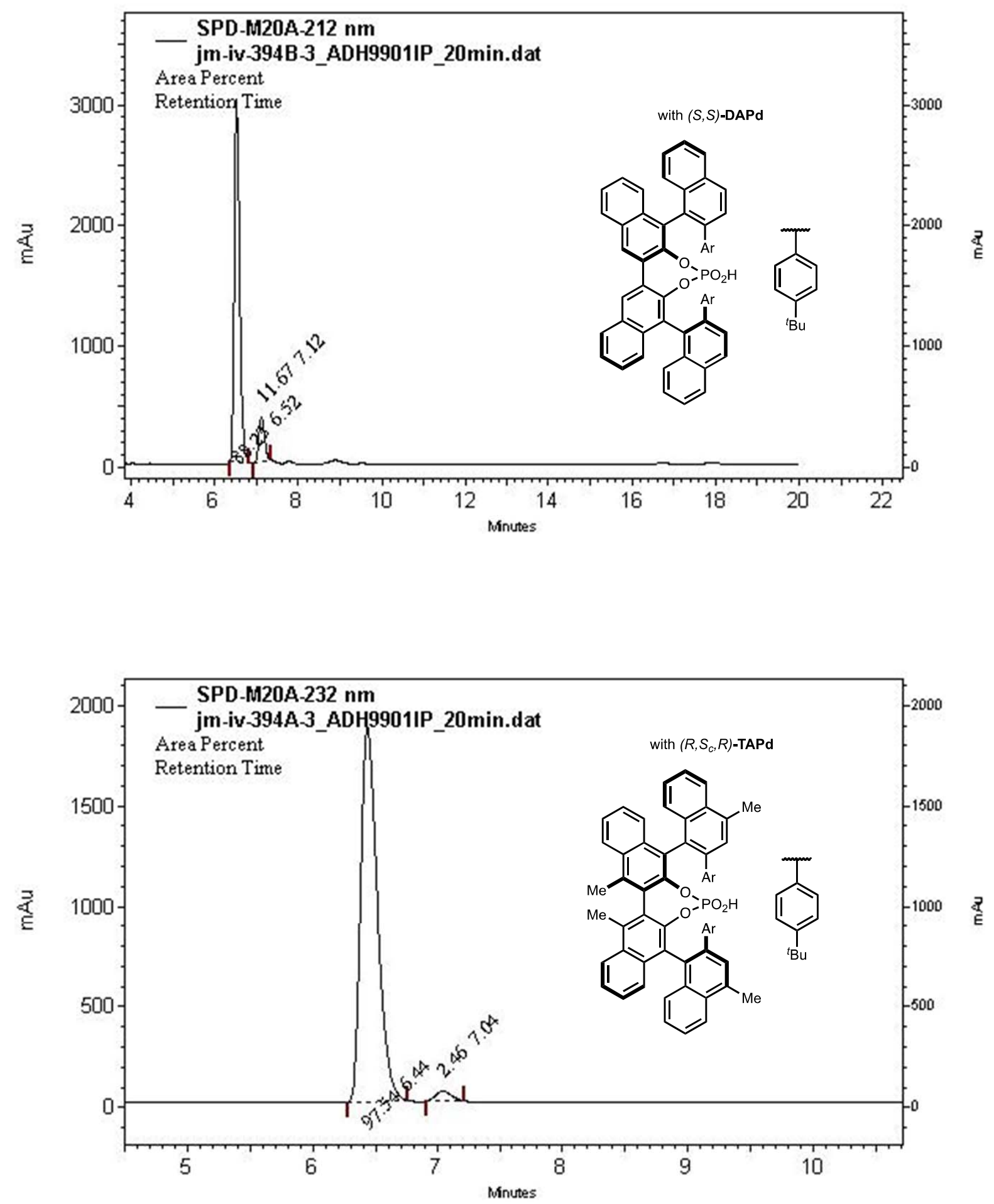

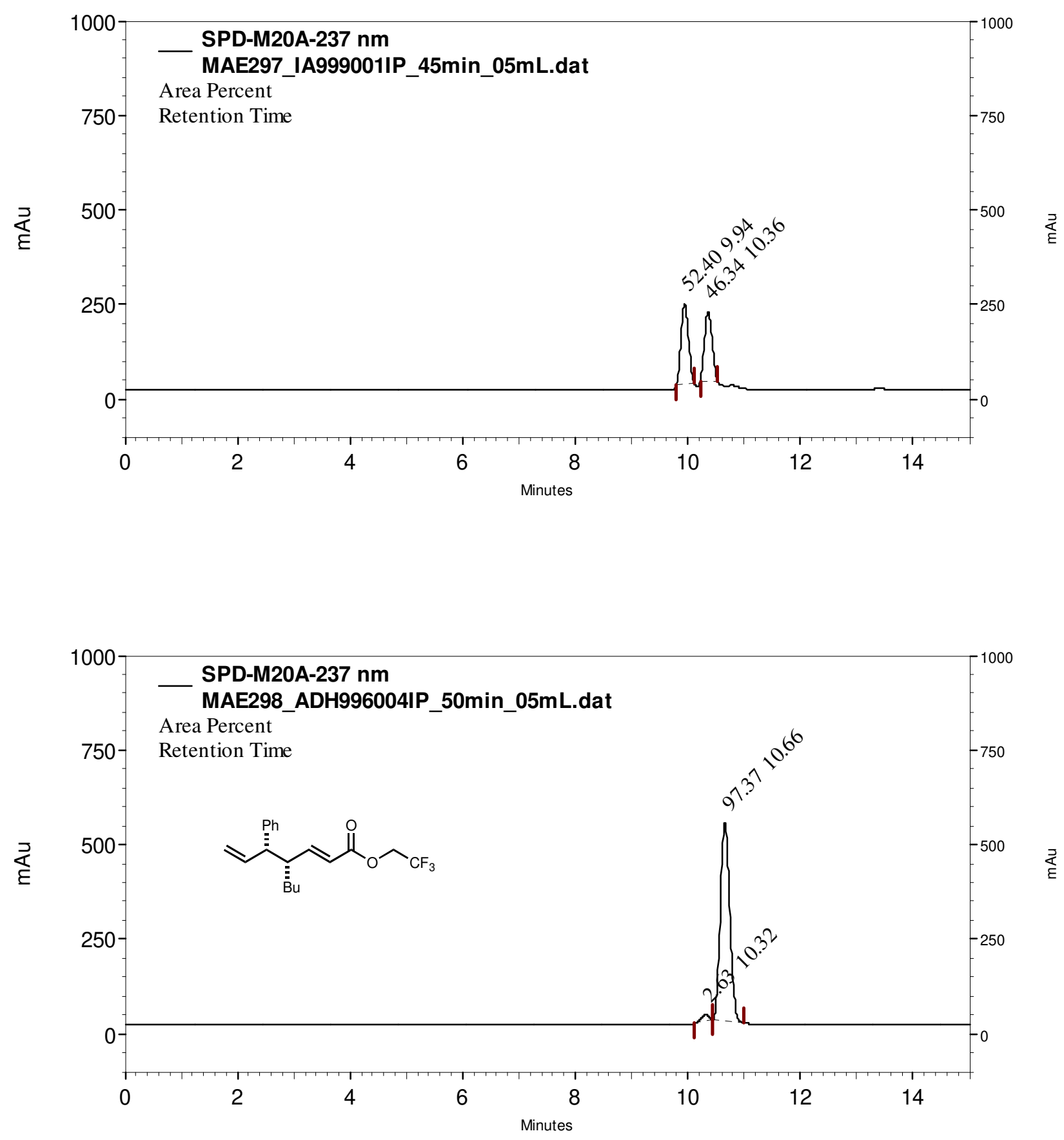

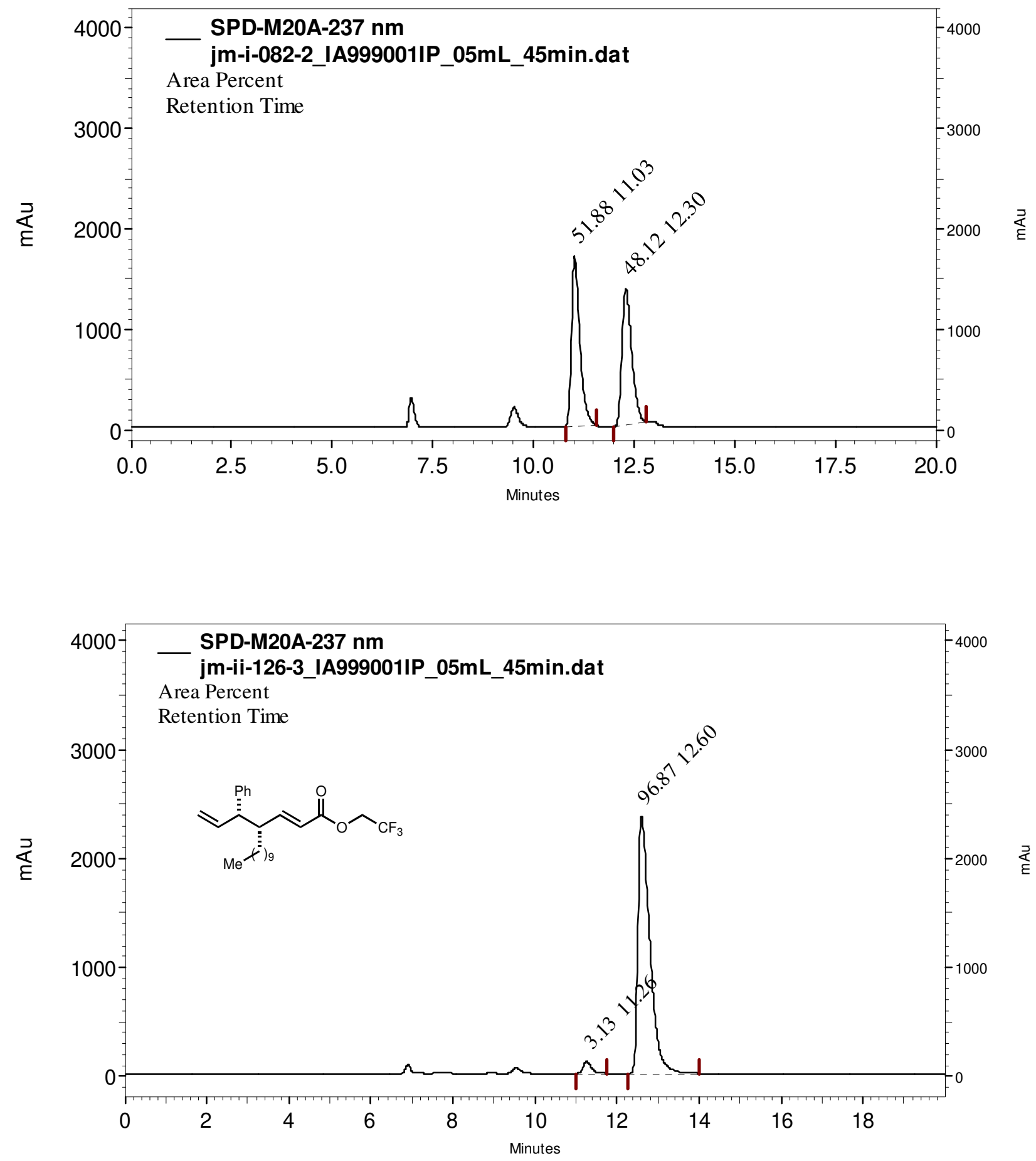

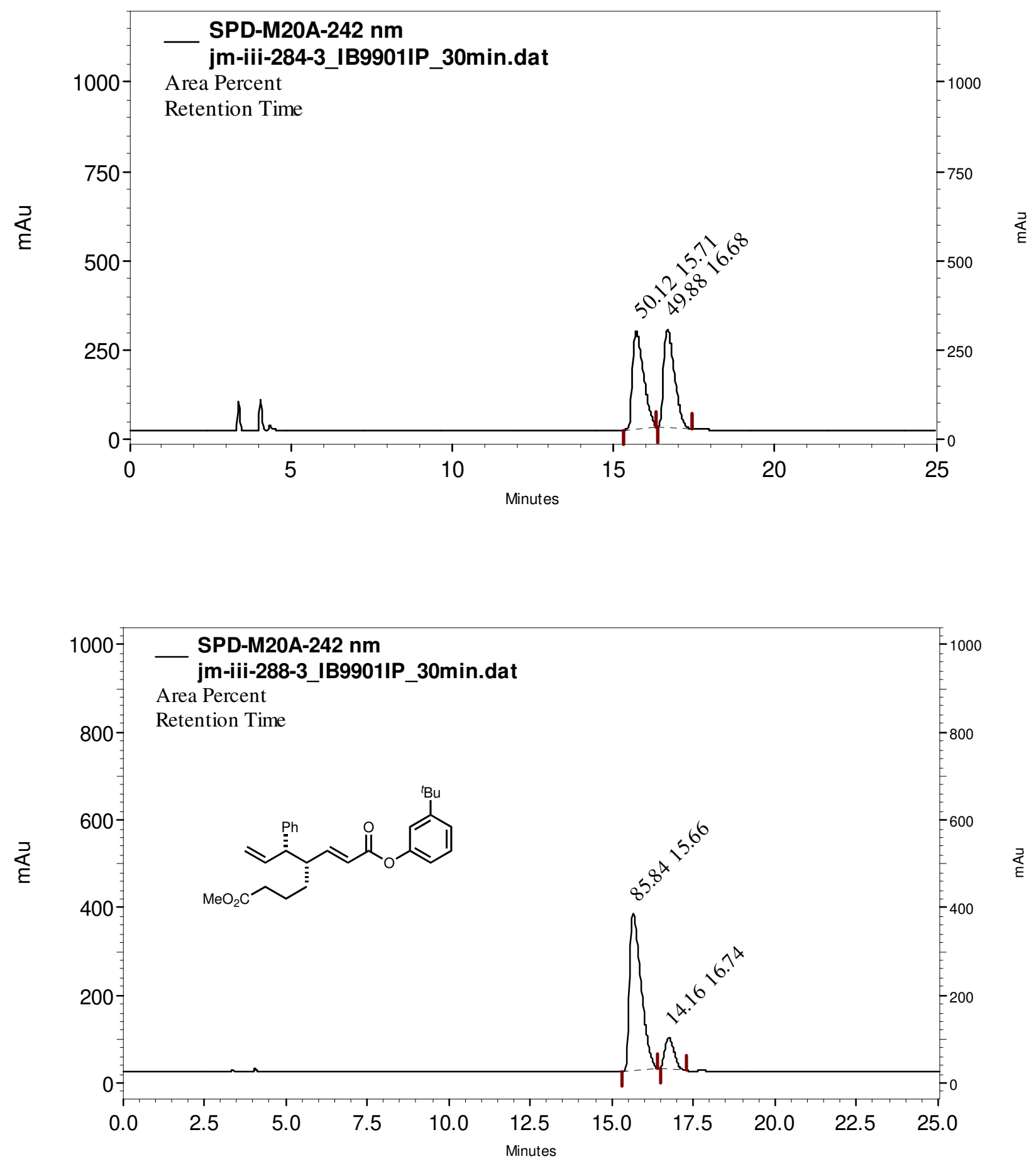

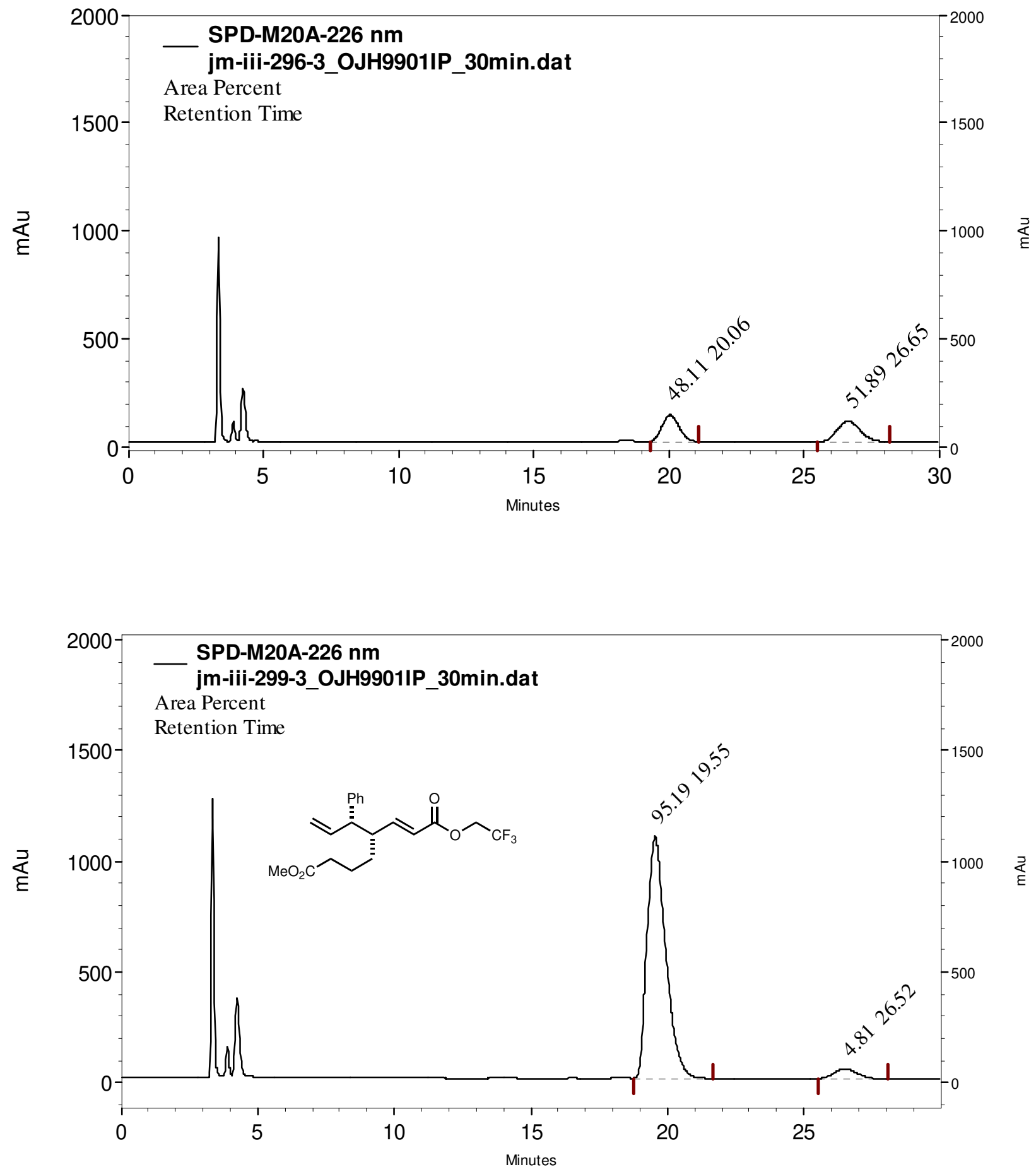

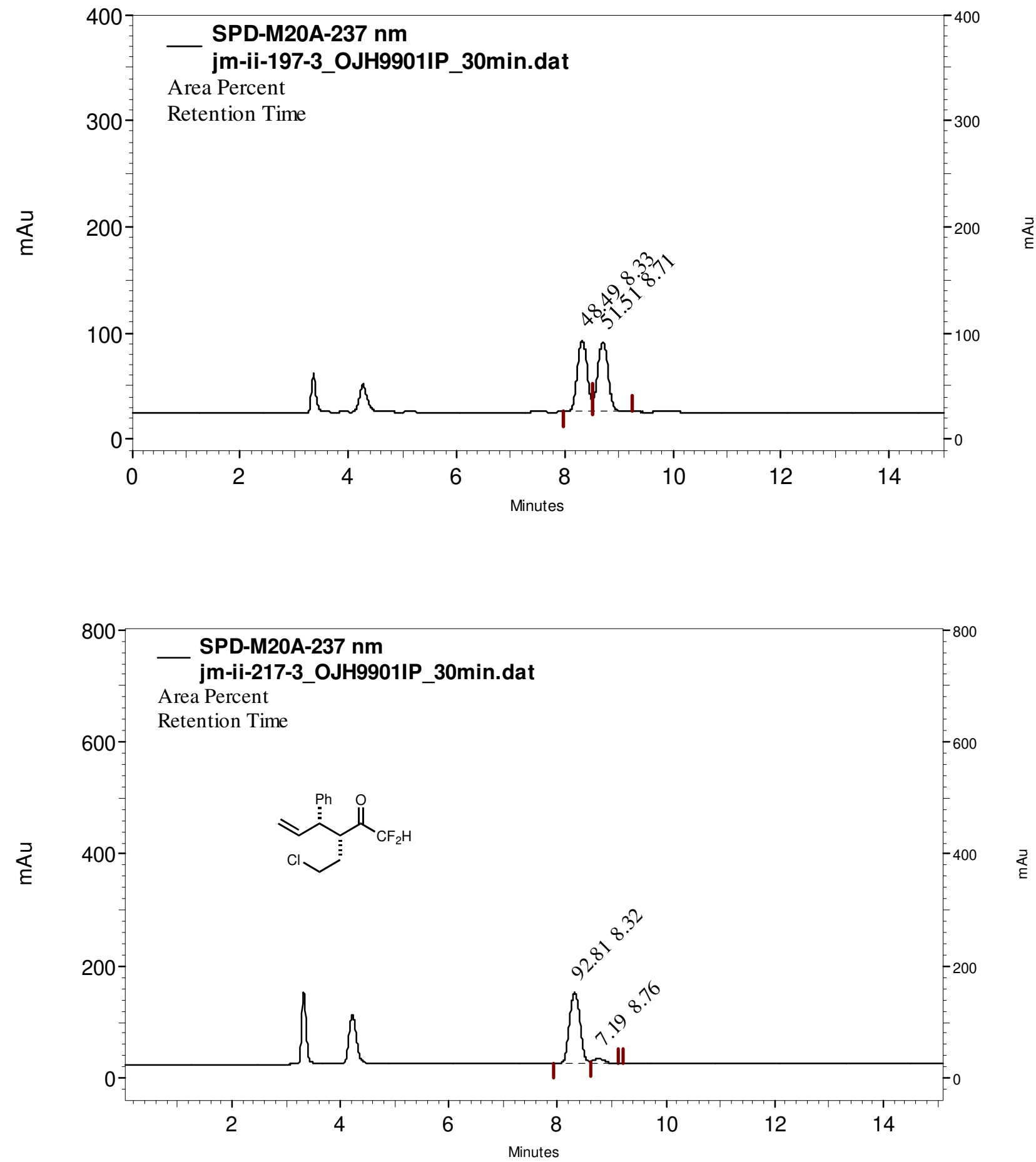

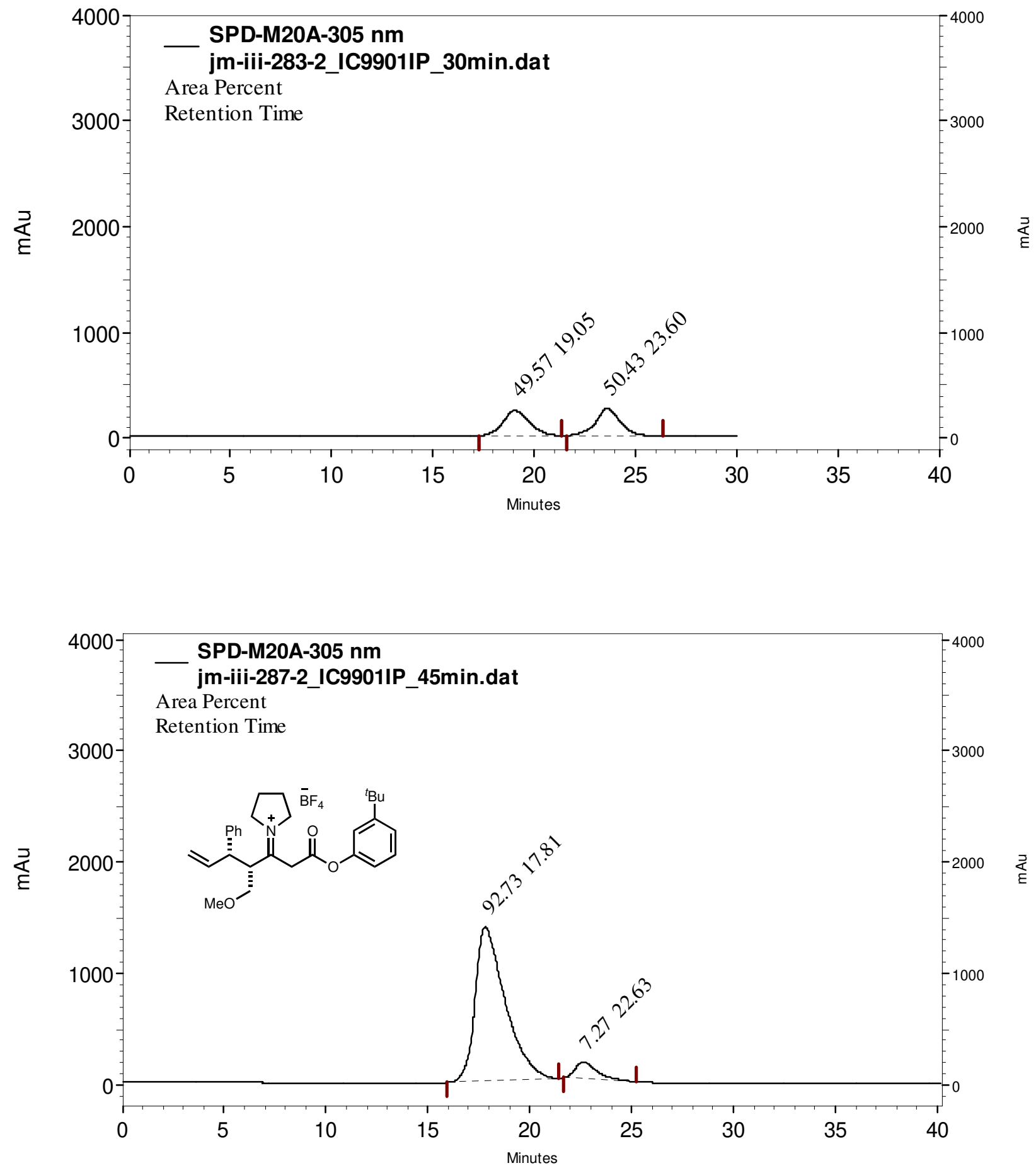

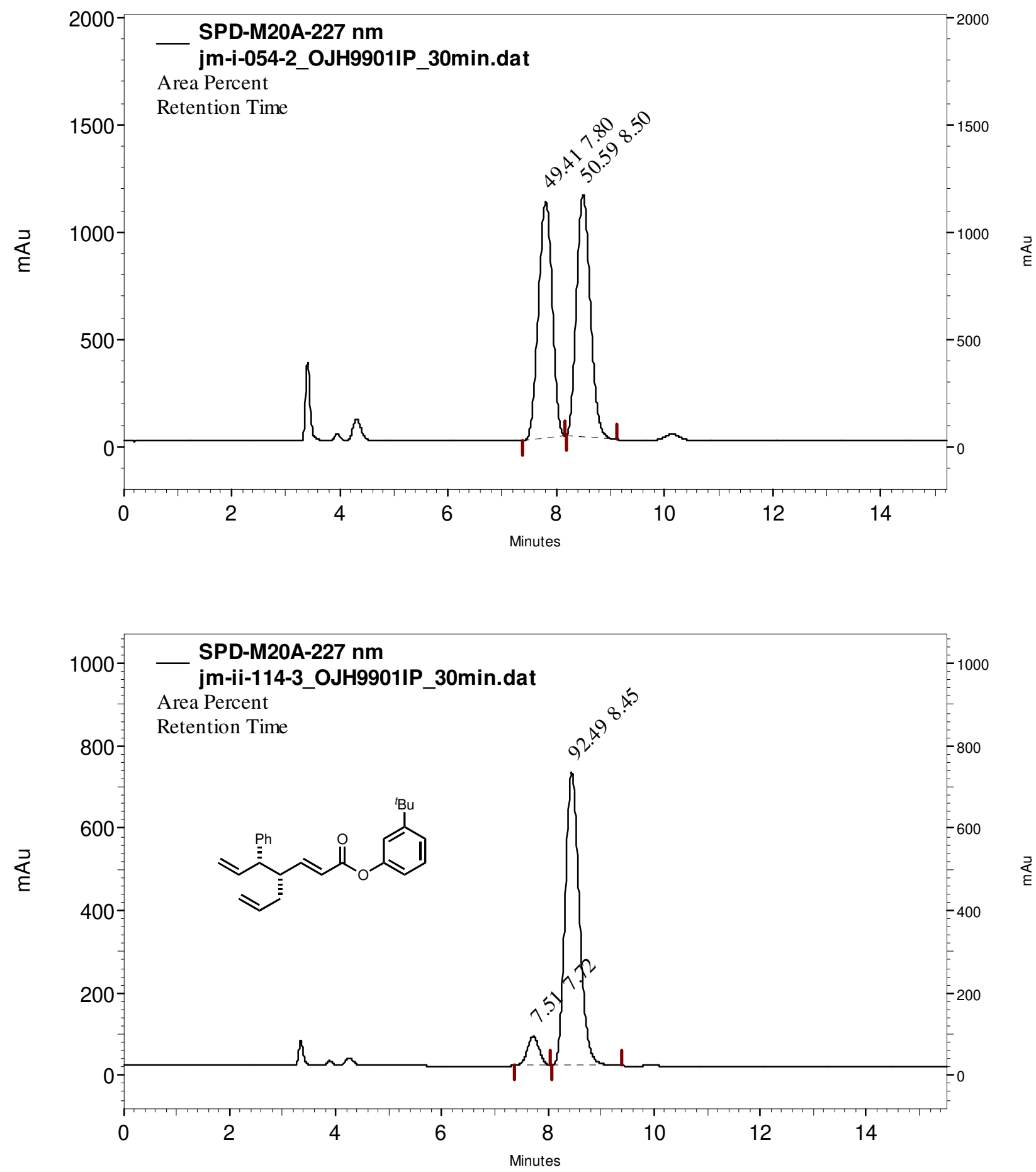

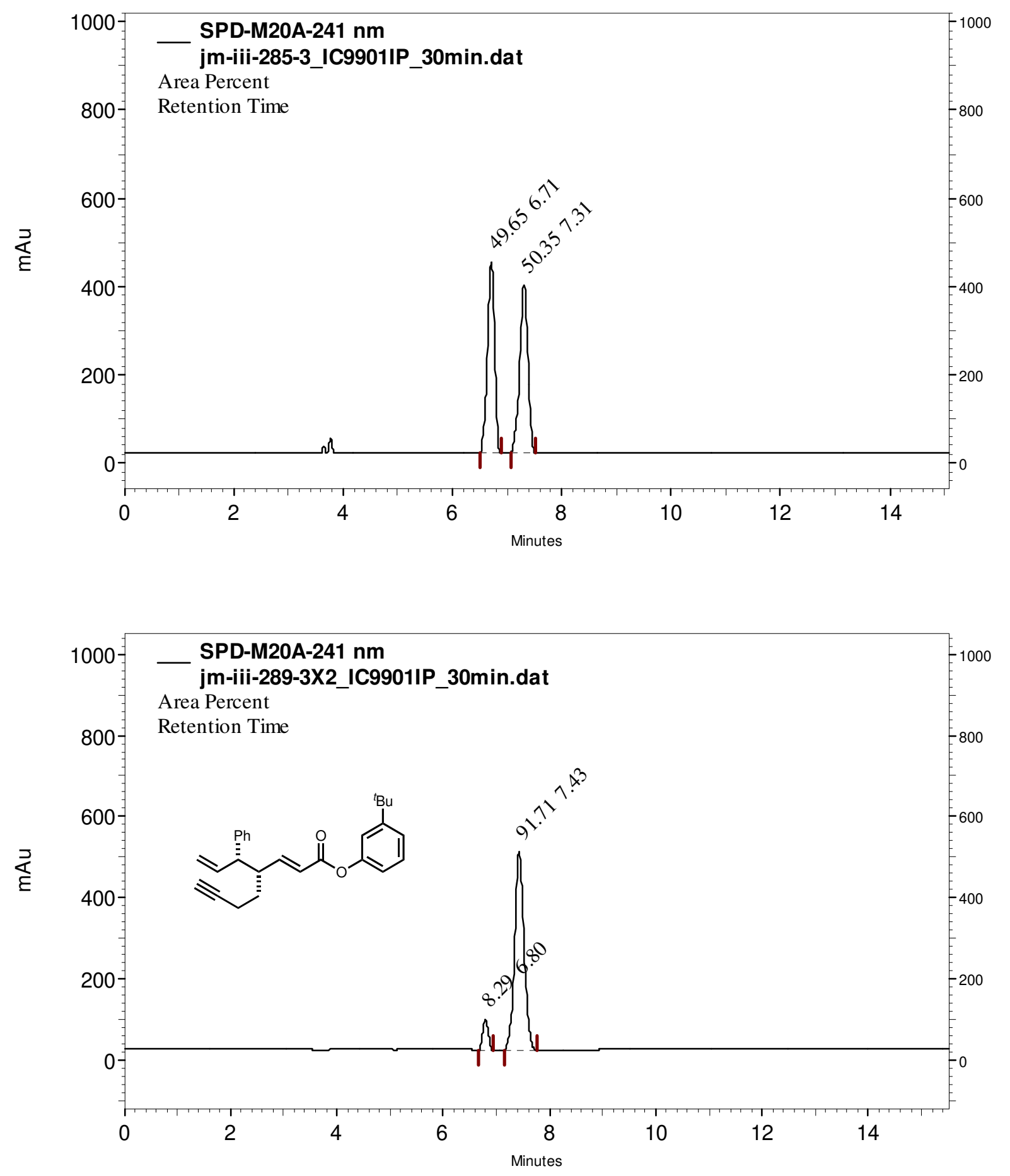

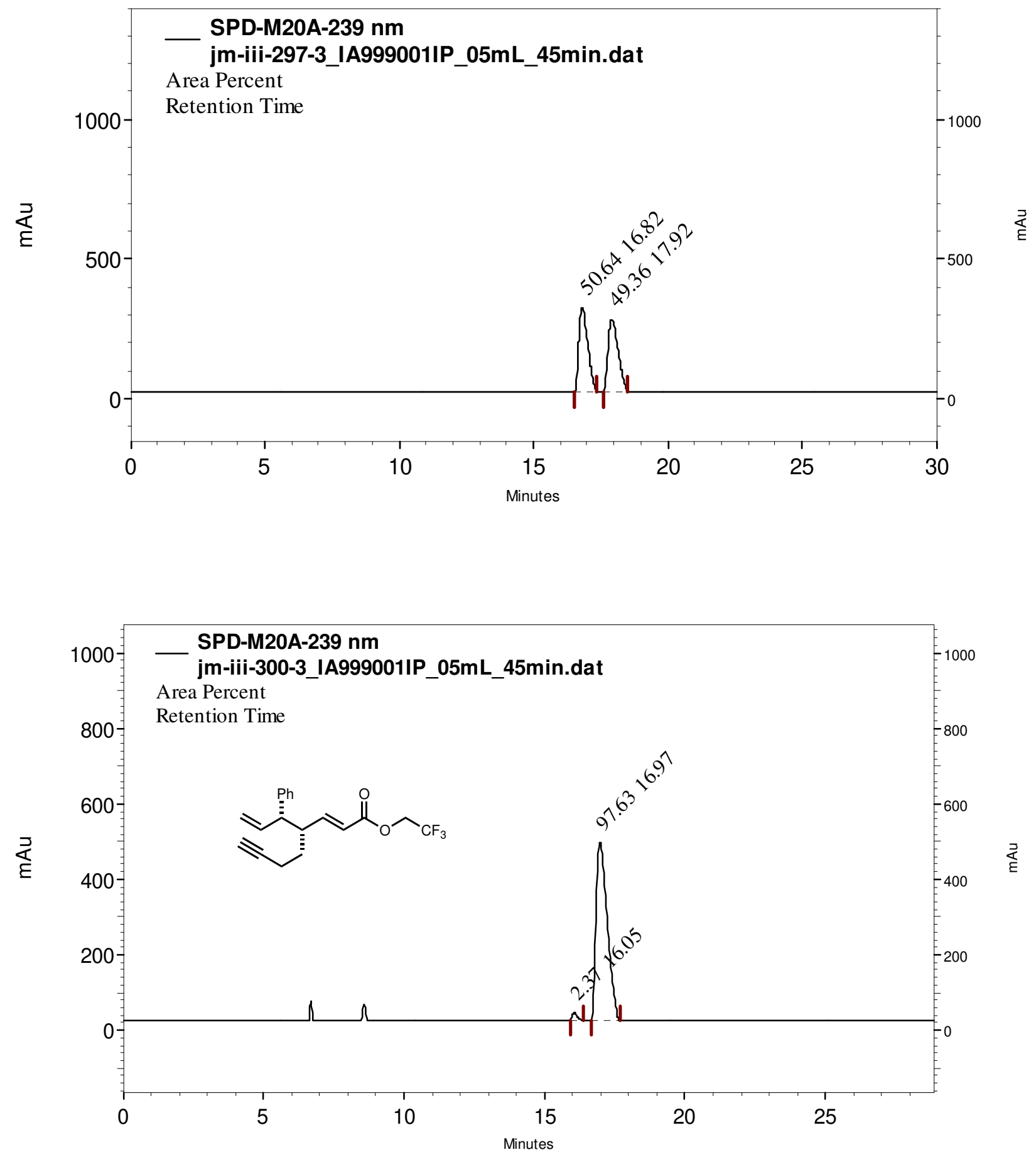

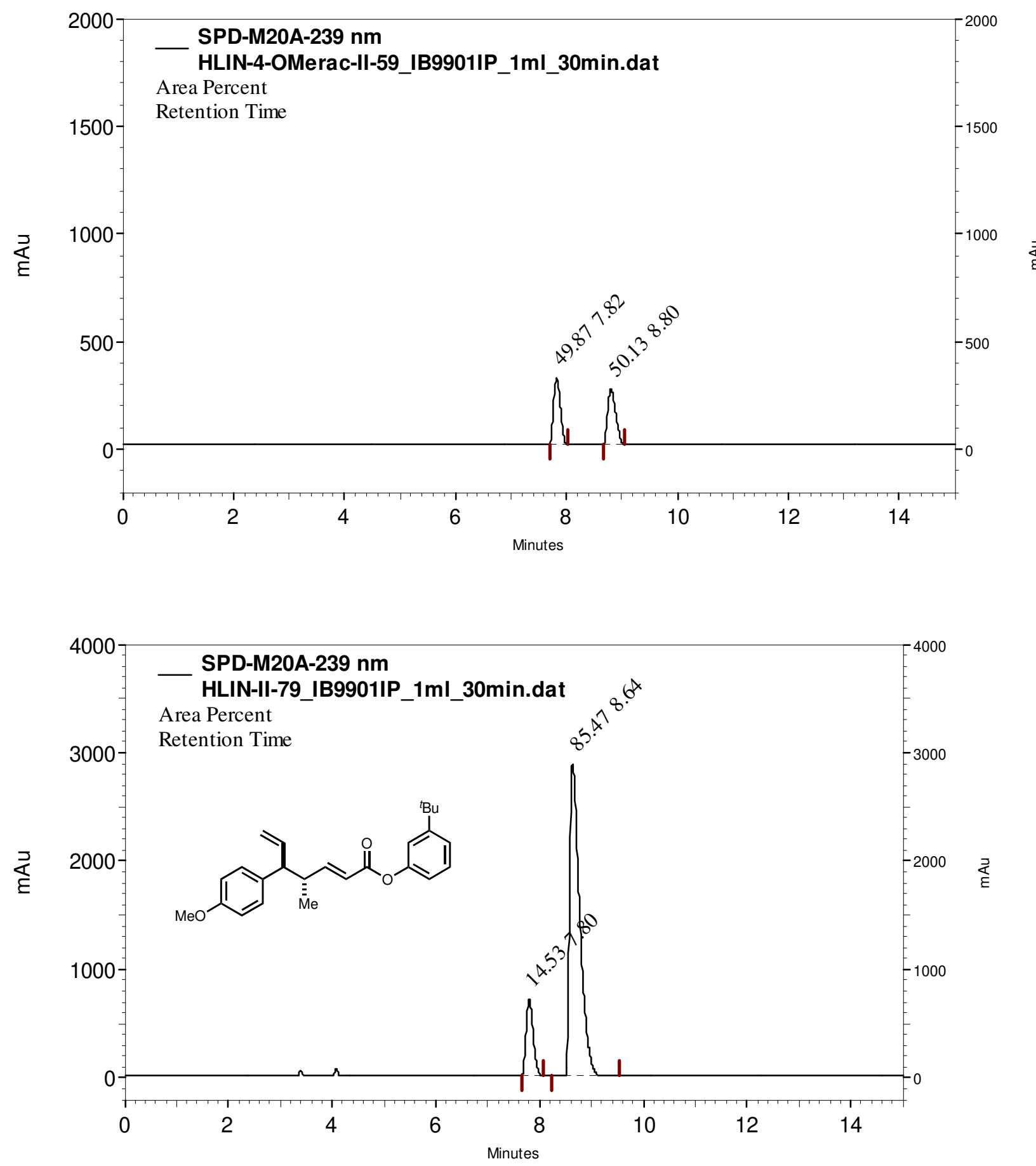


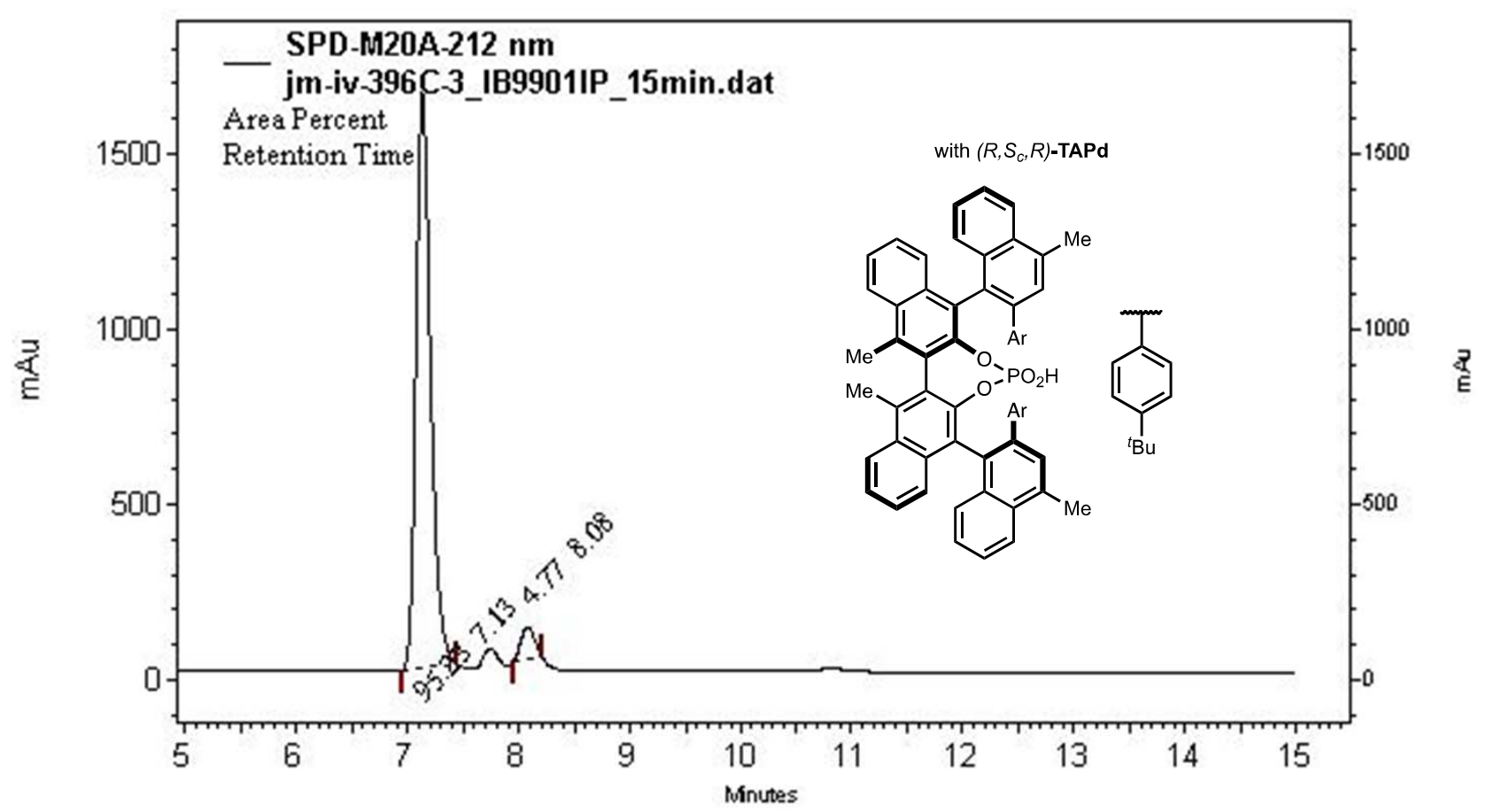



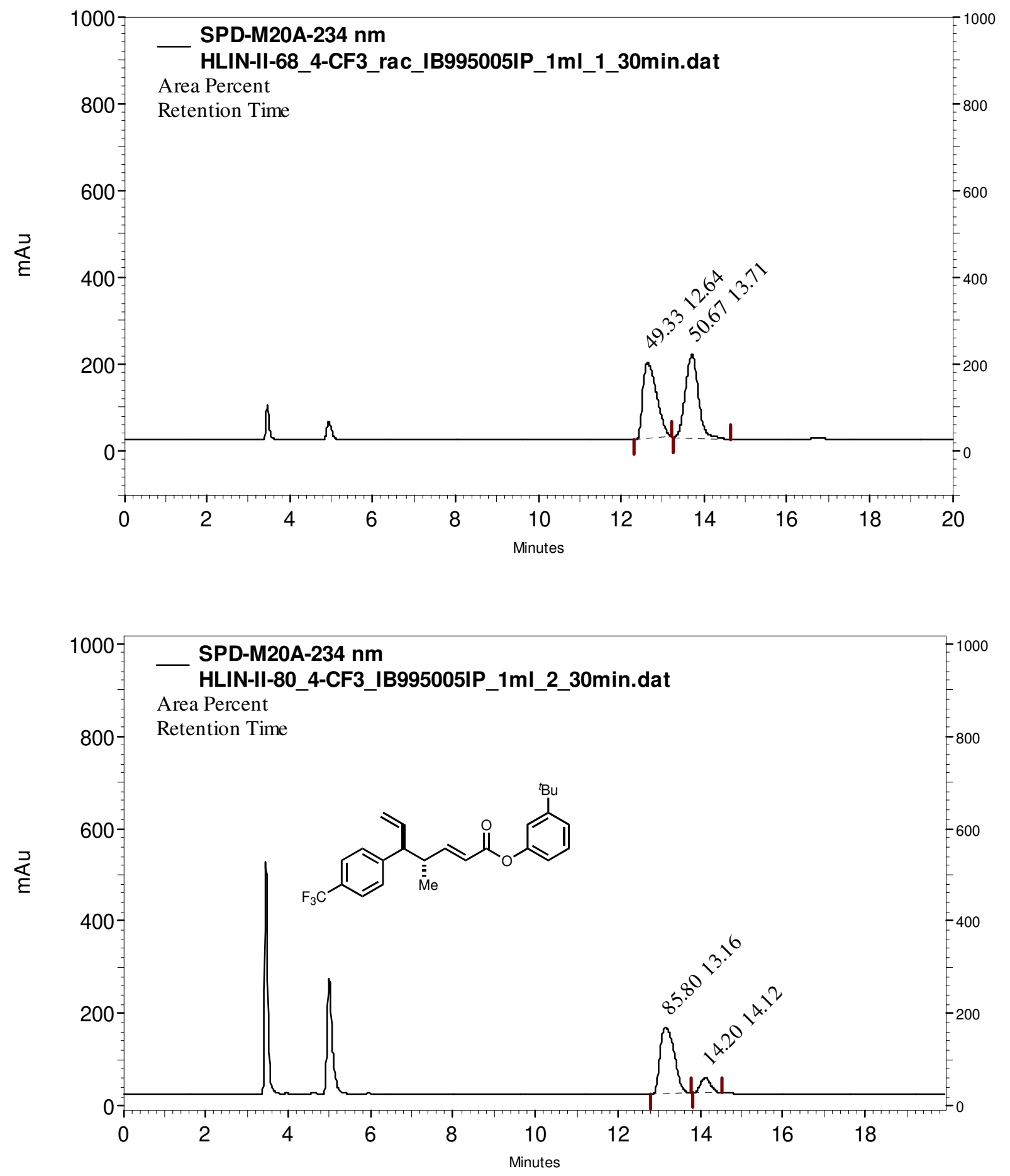

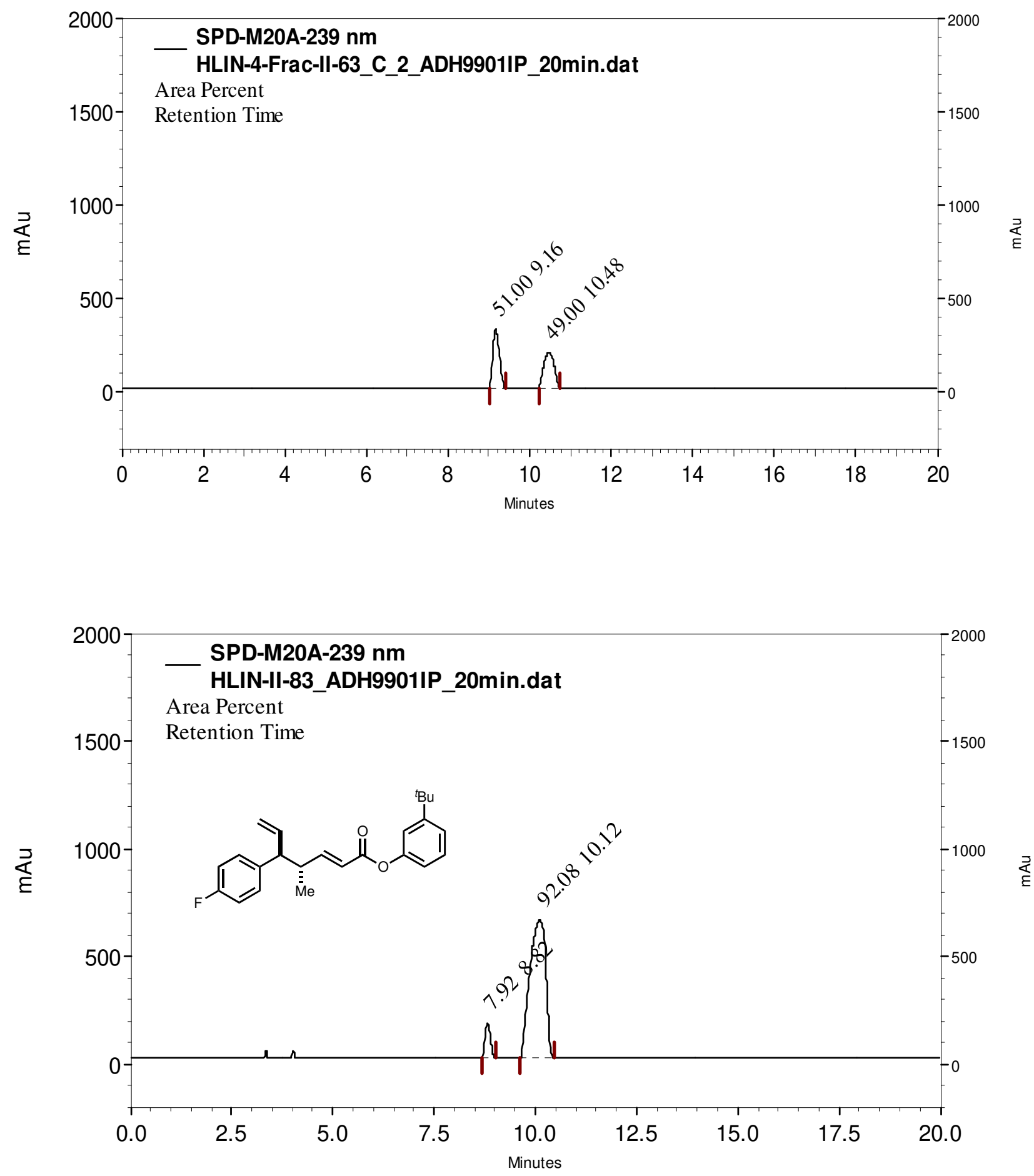

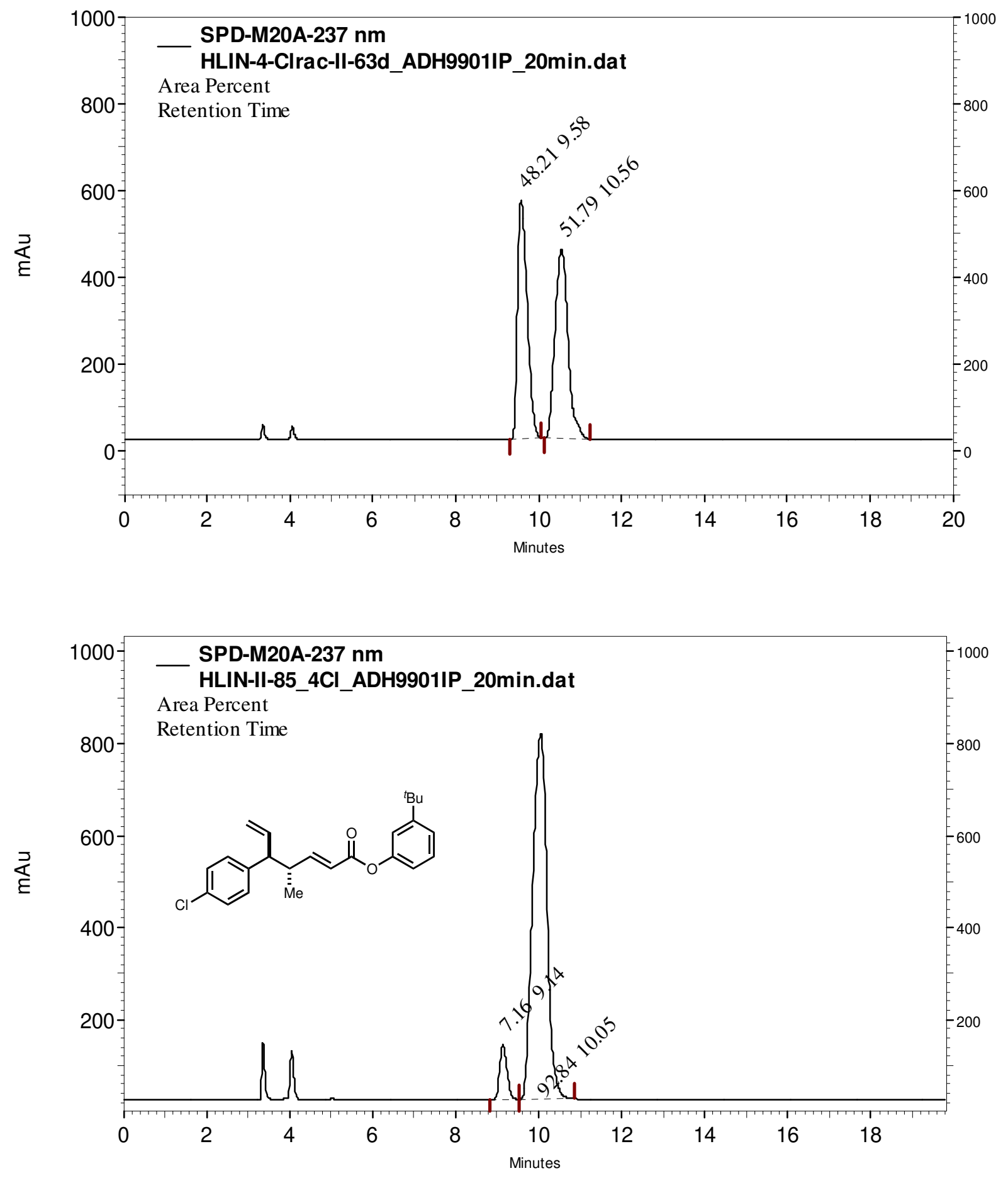

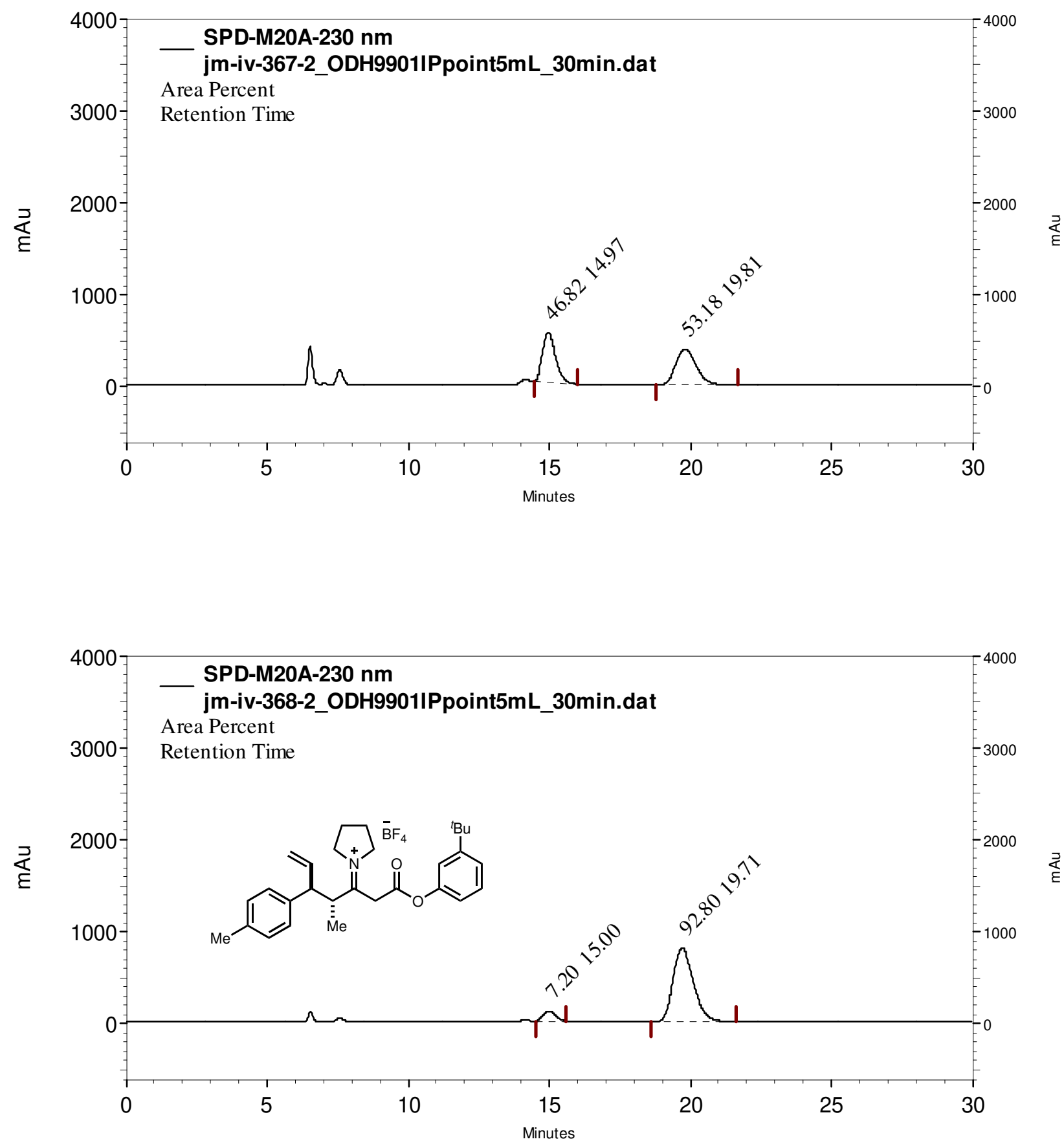

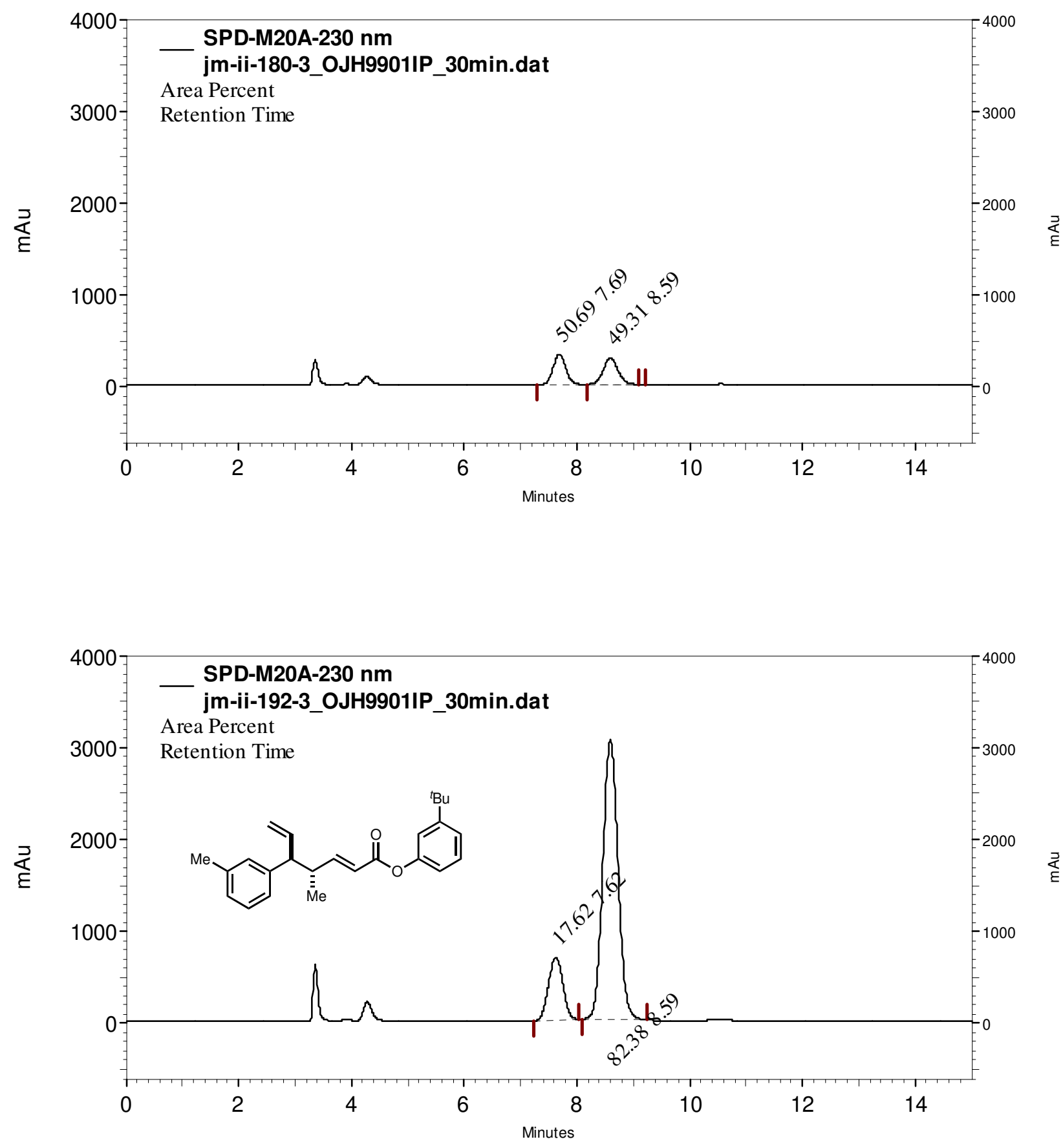

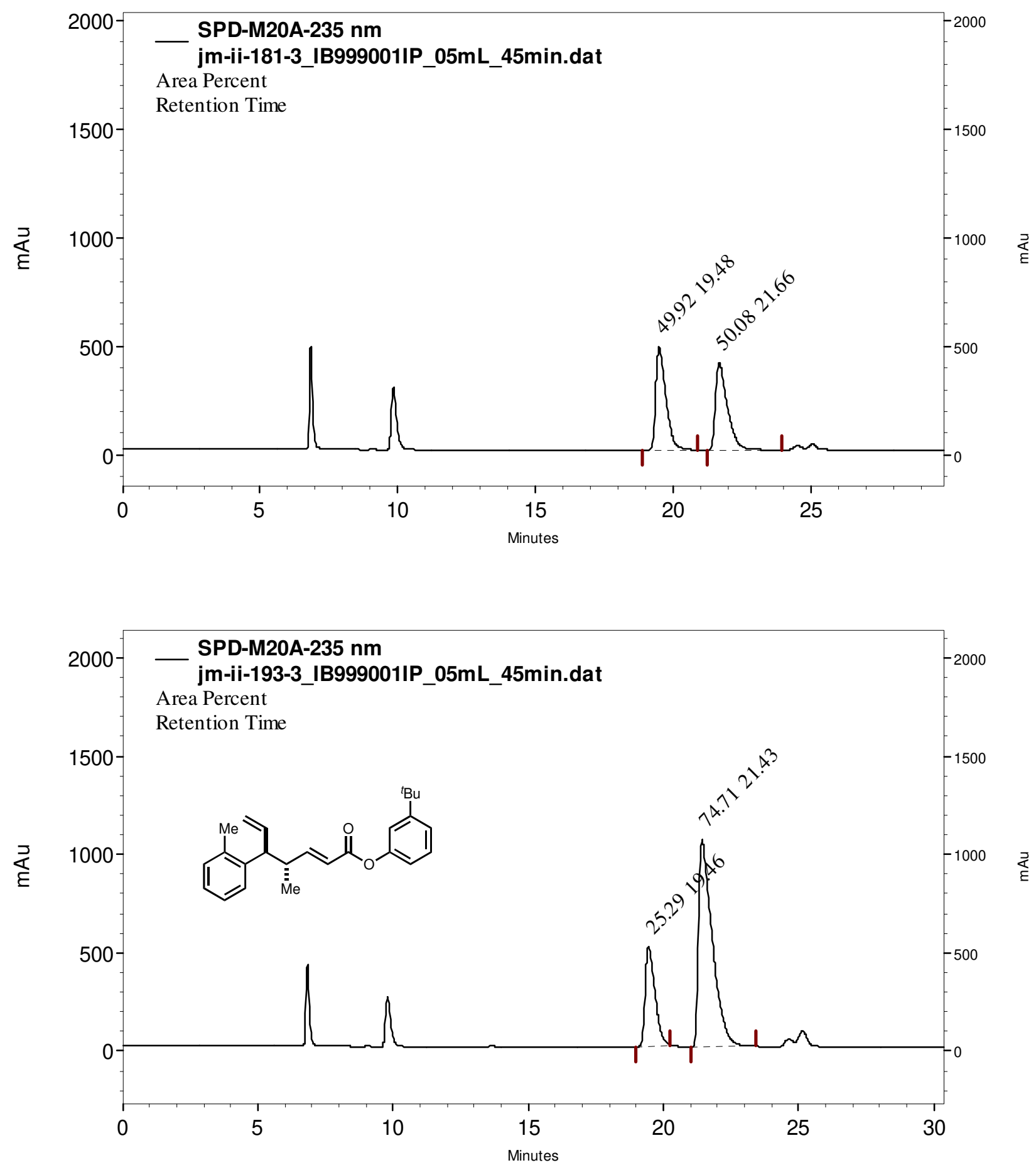

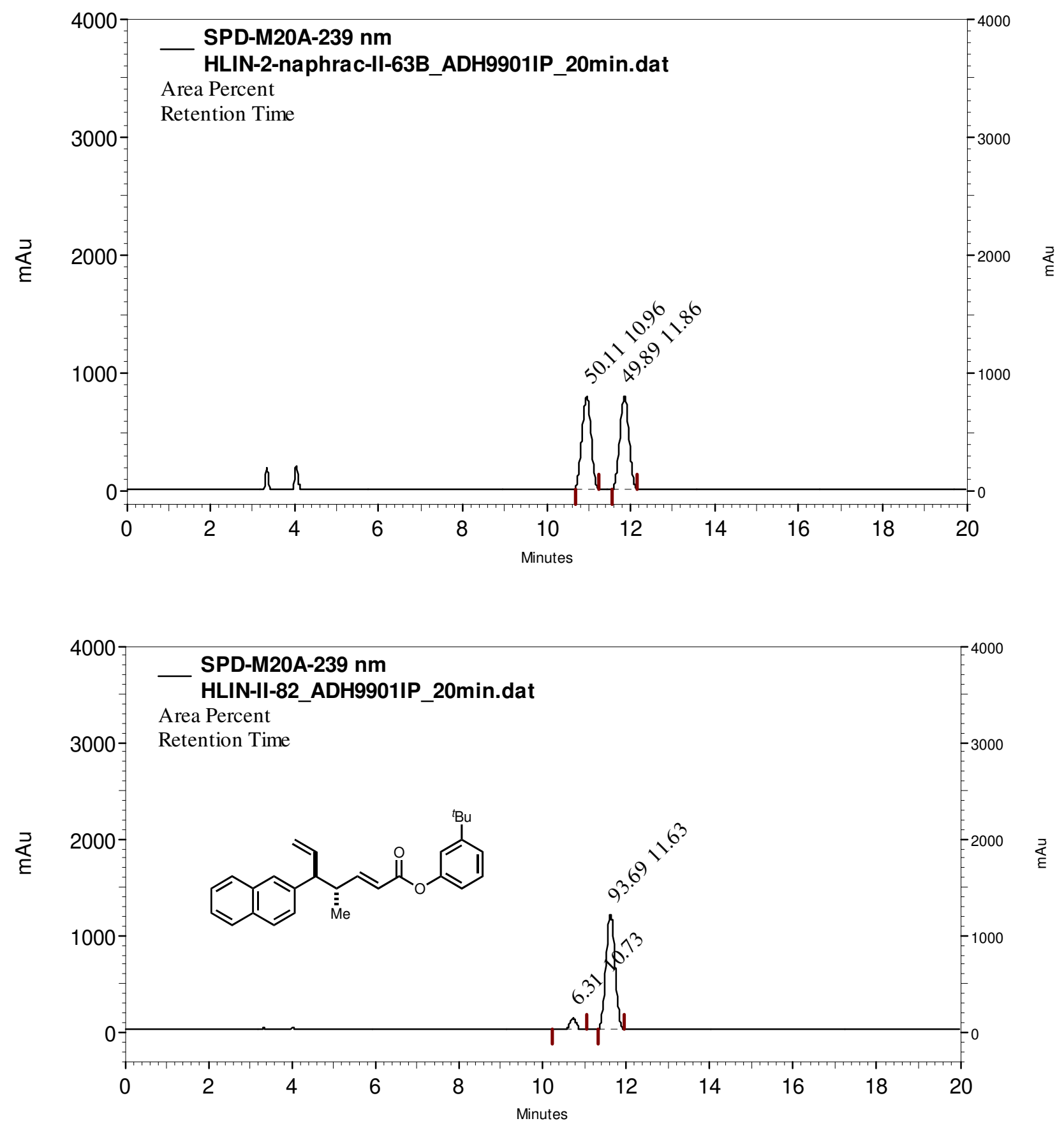

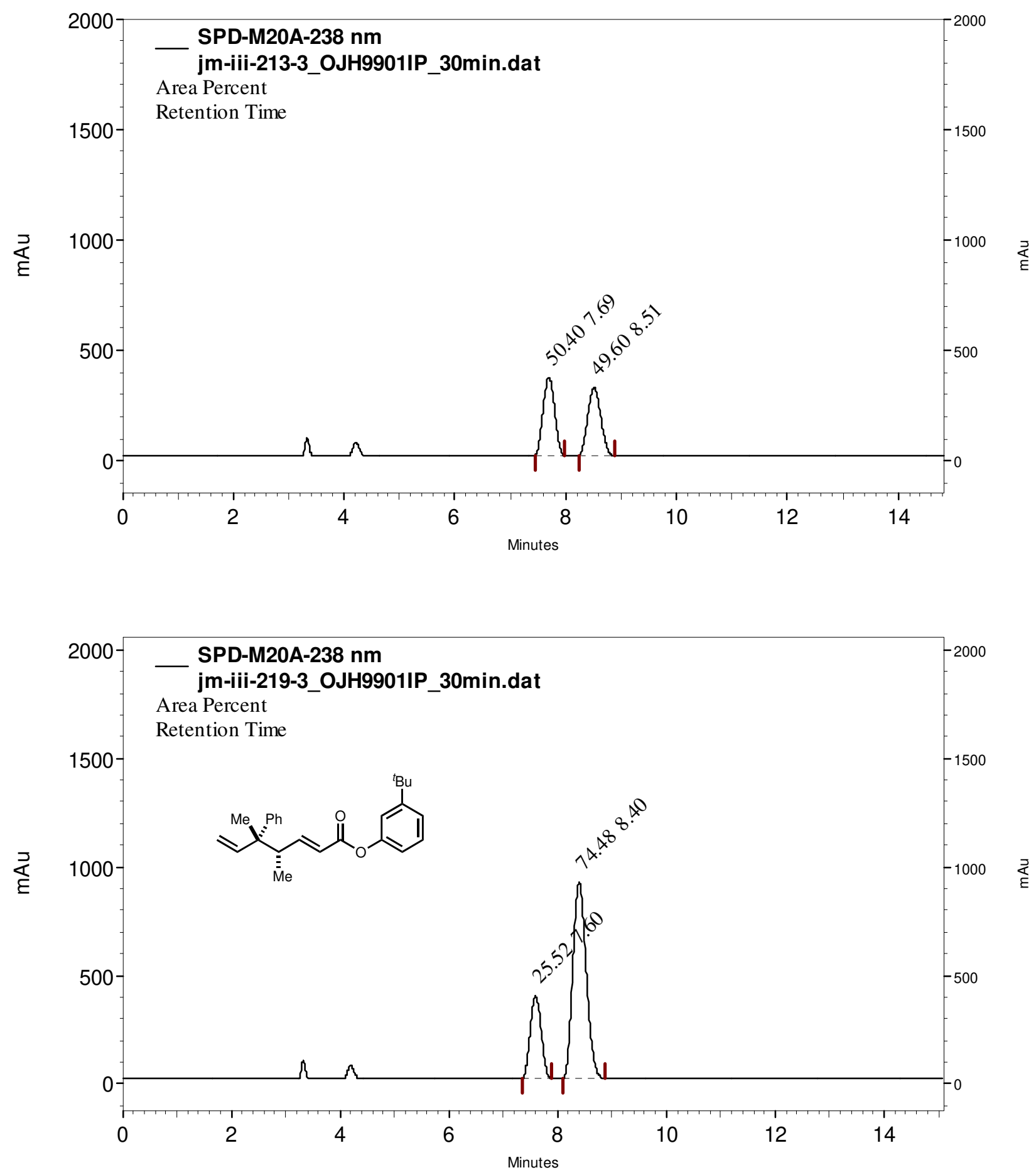


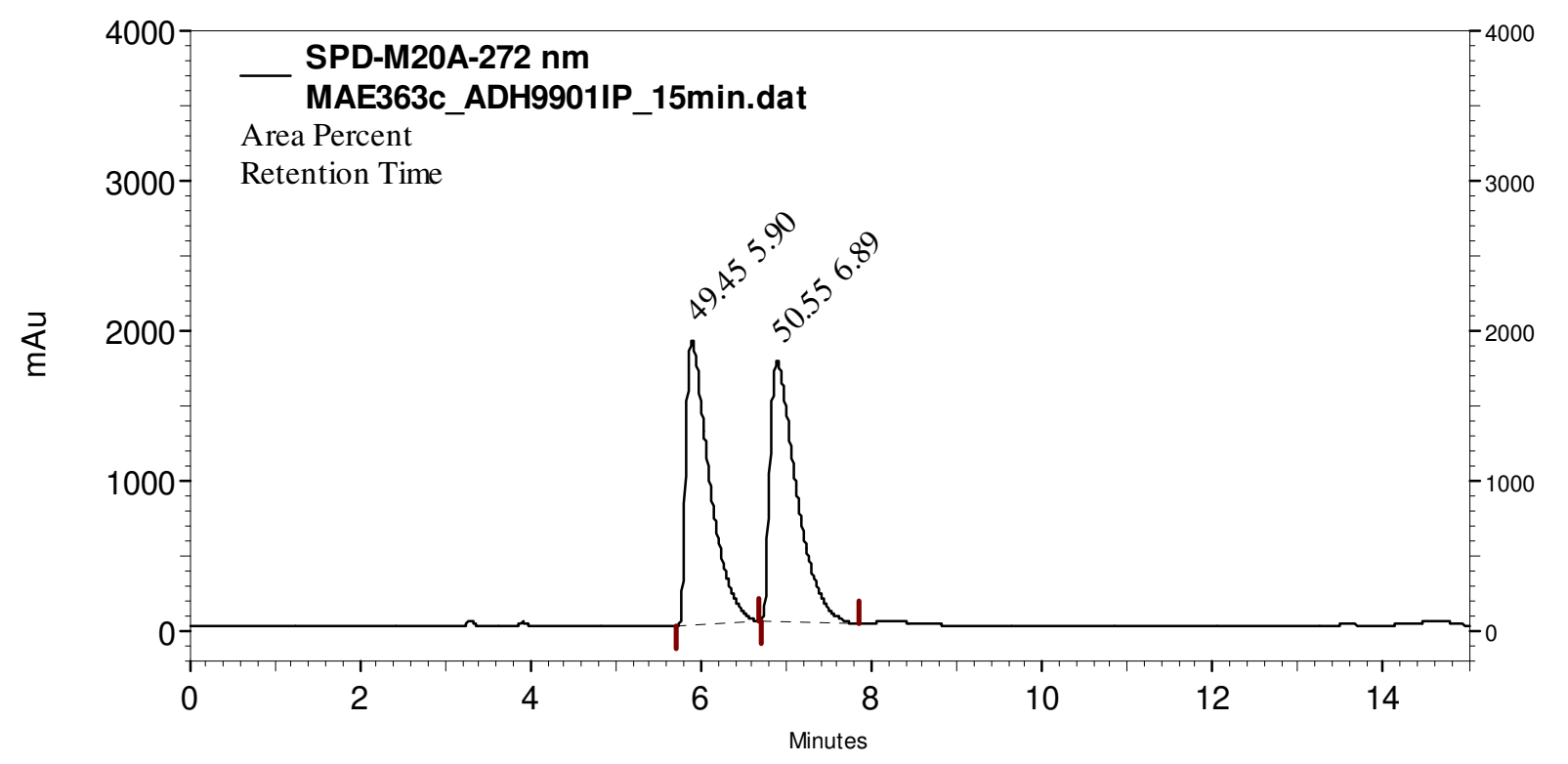

ङ

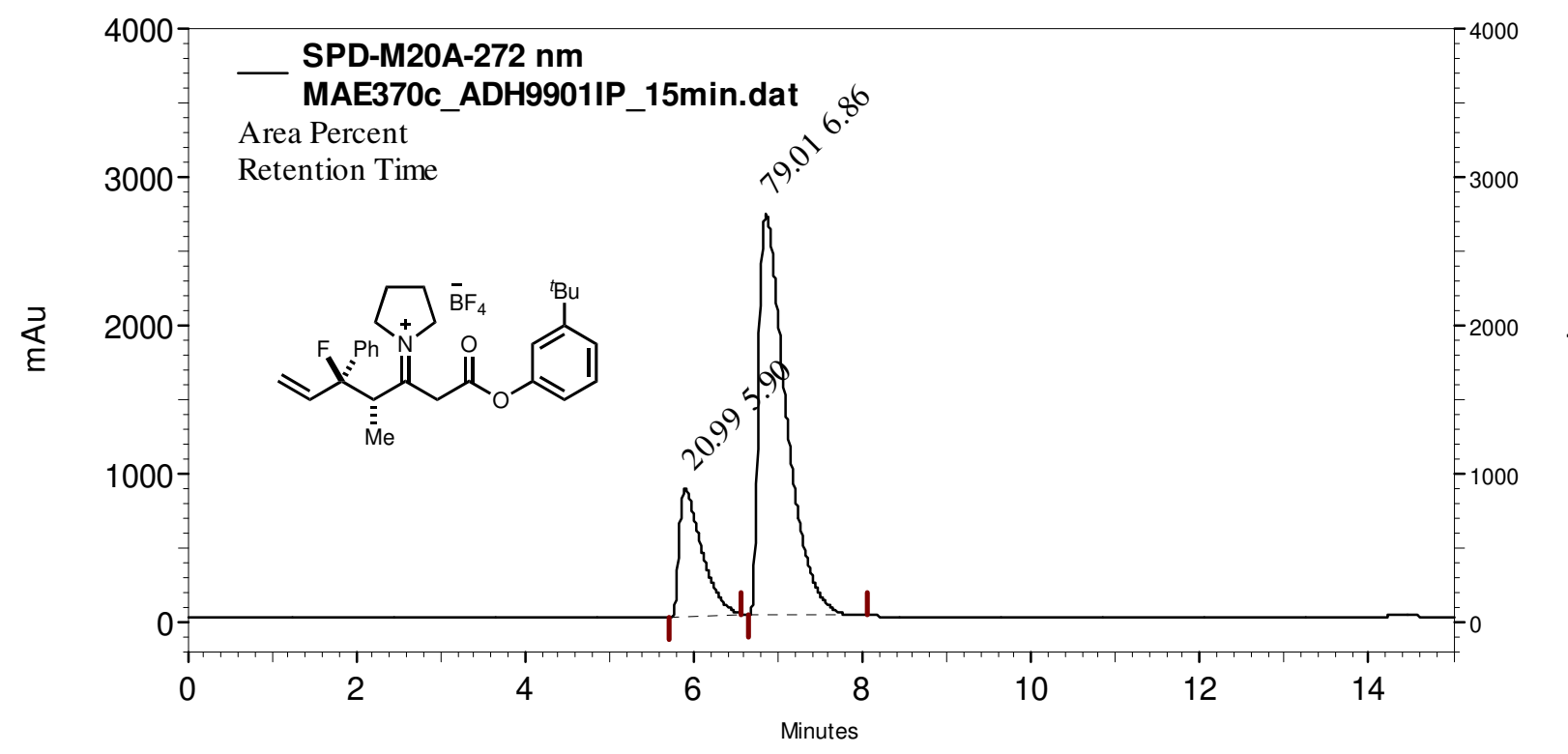



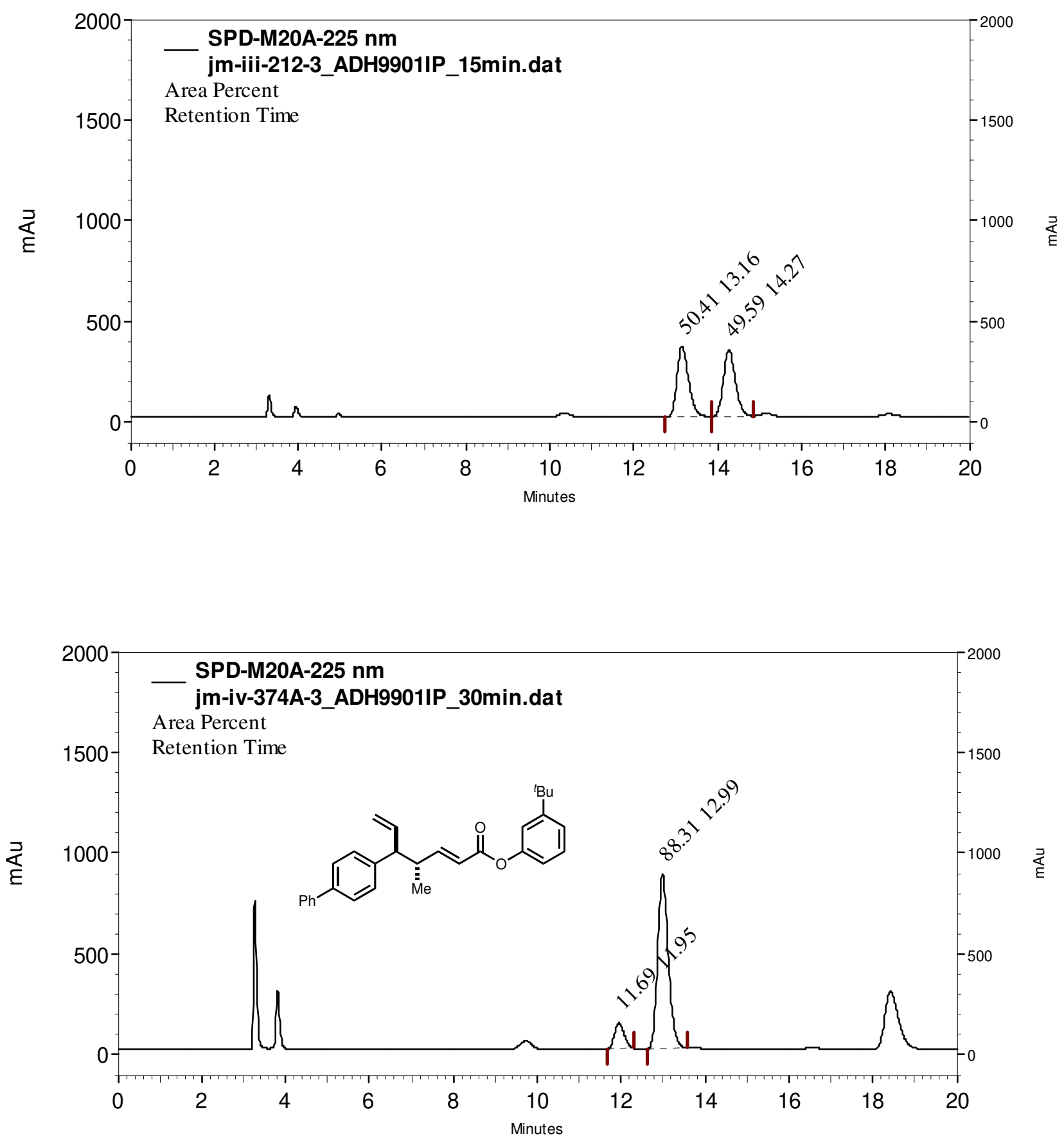

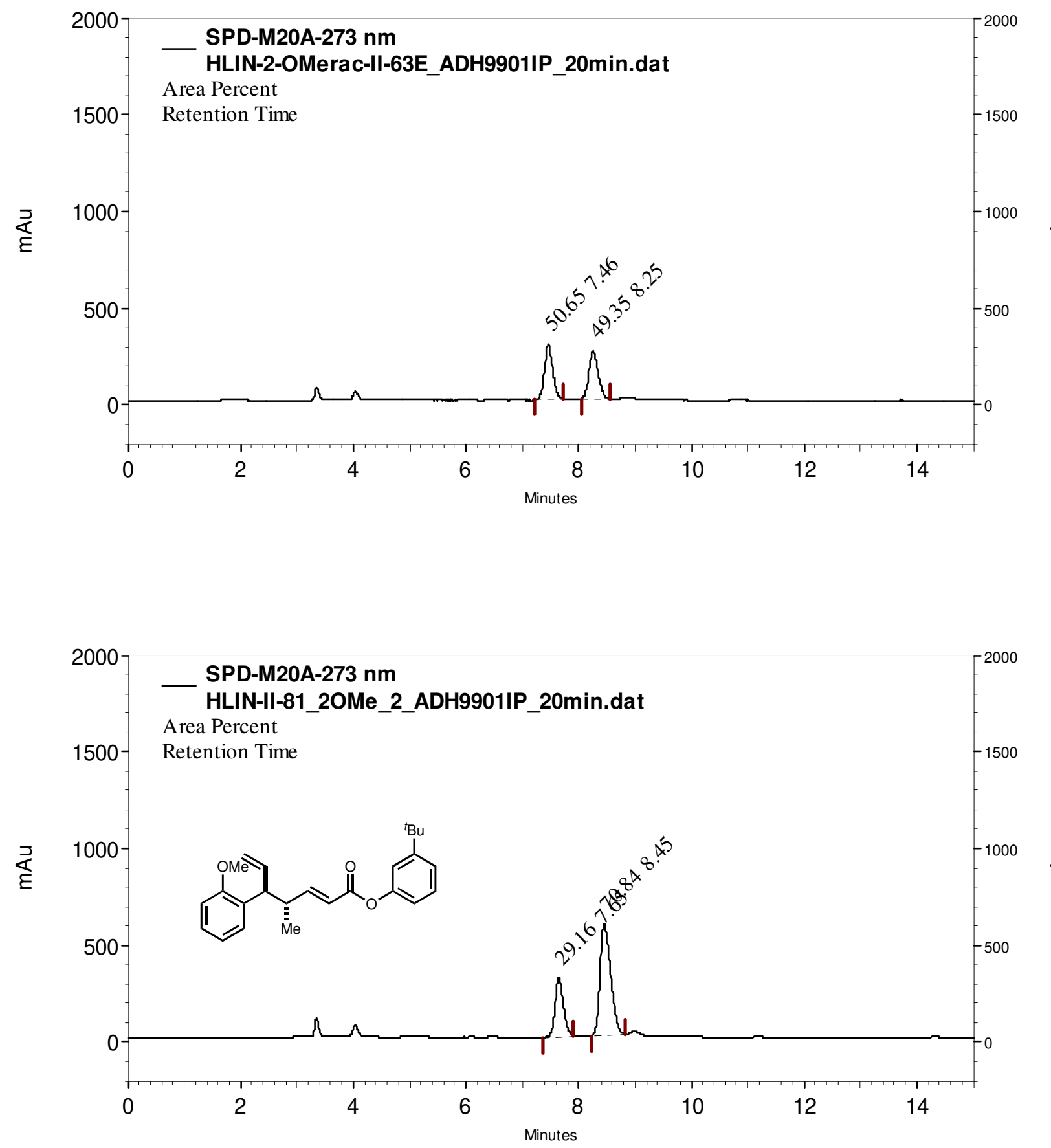

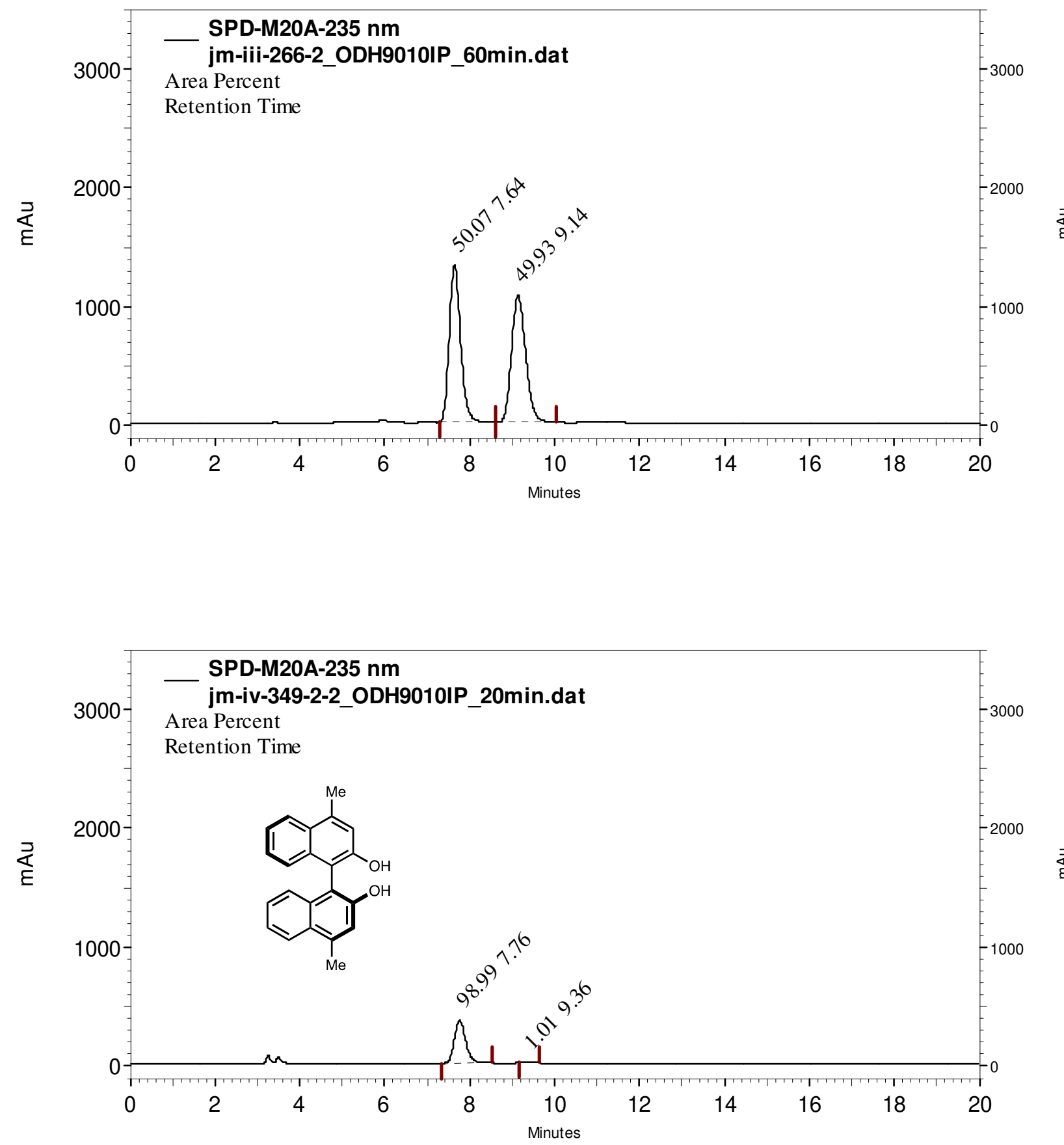


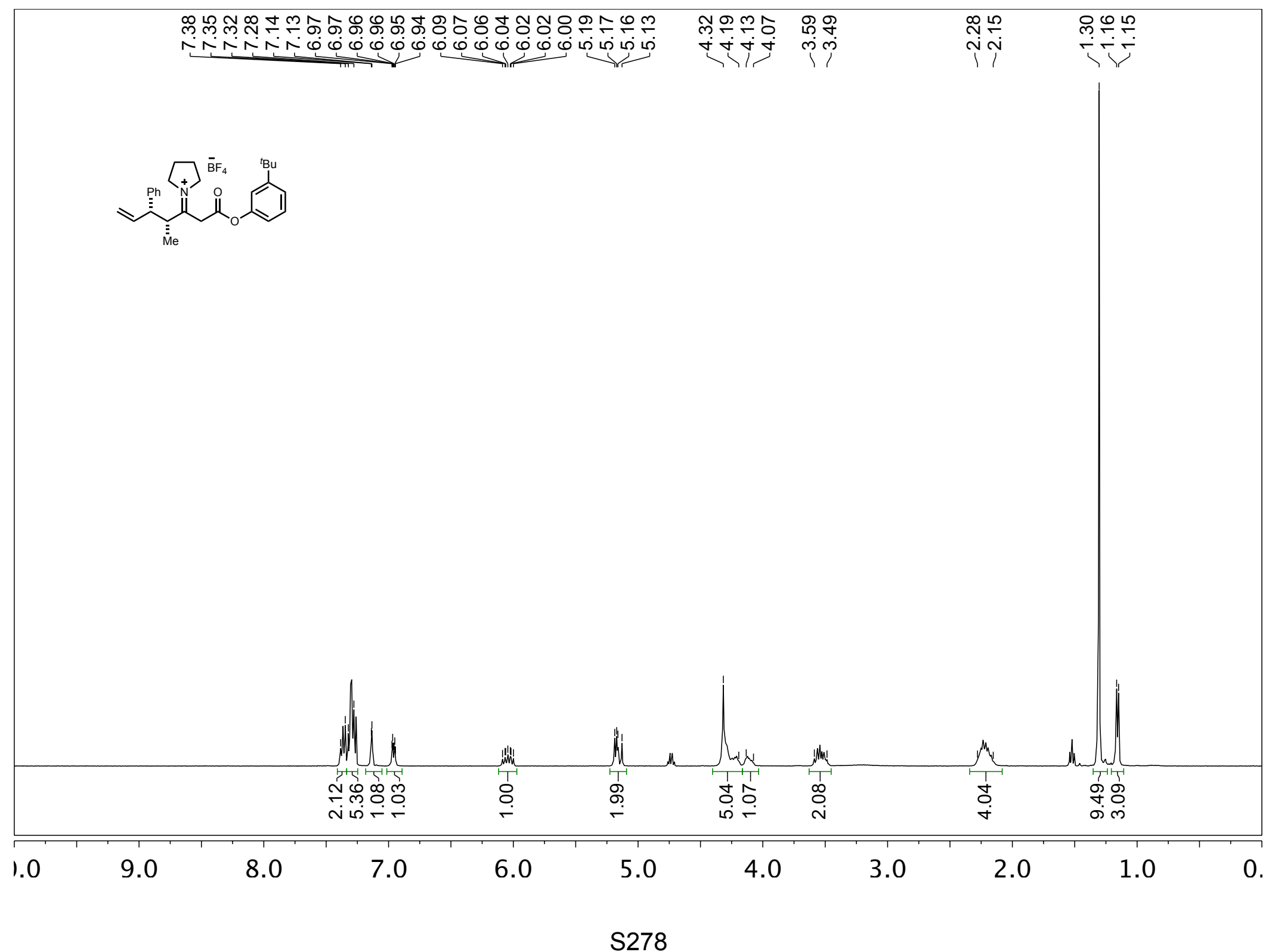




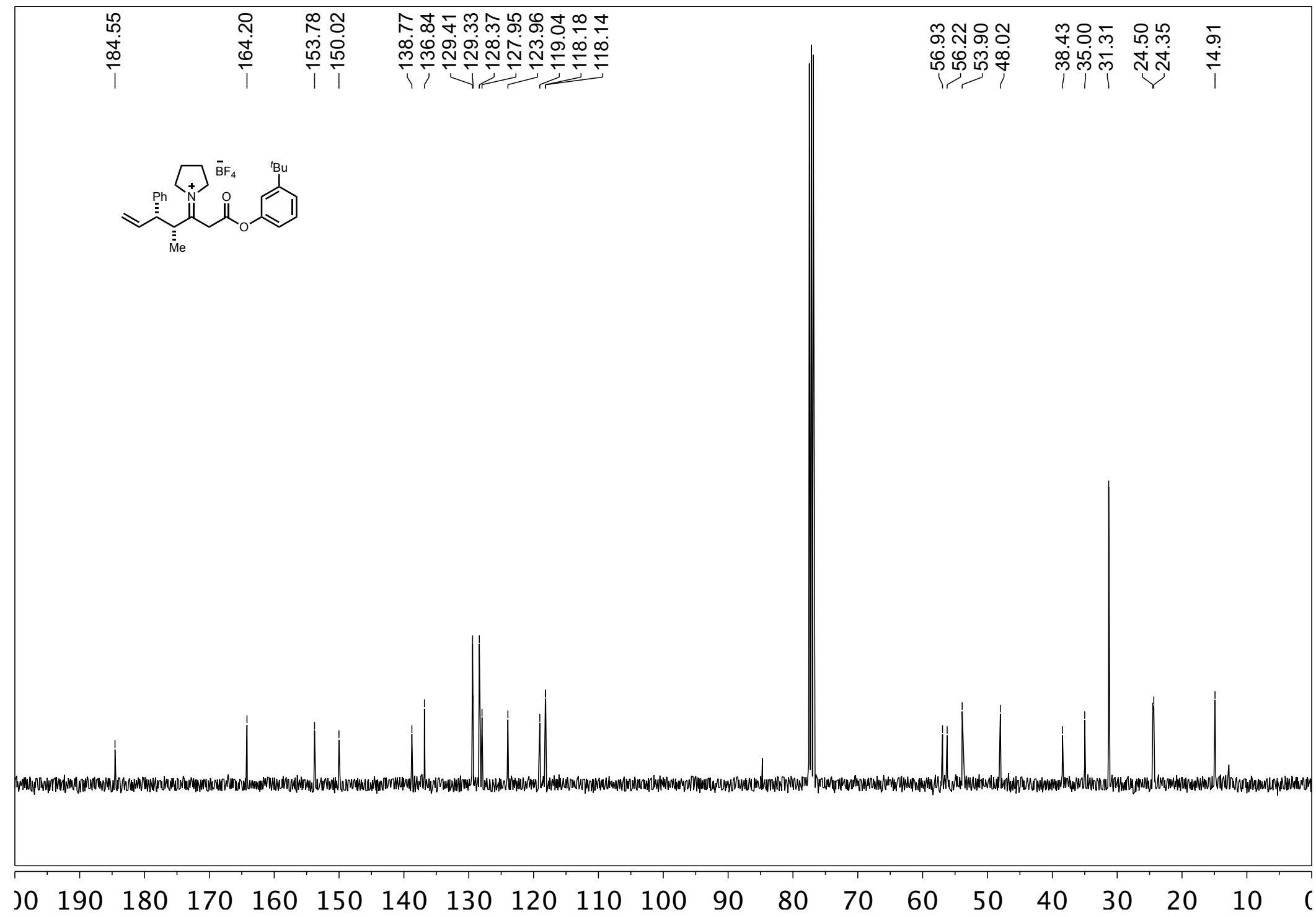




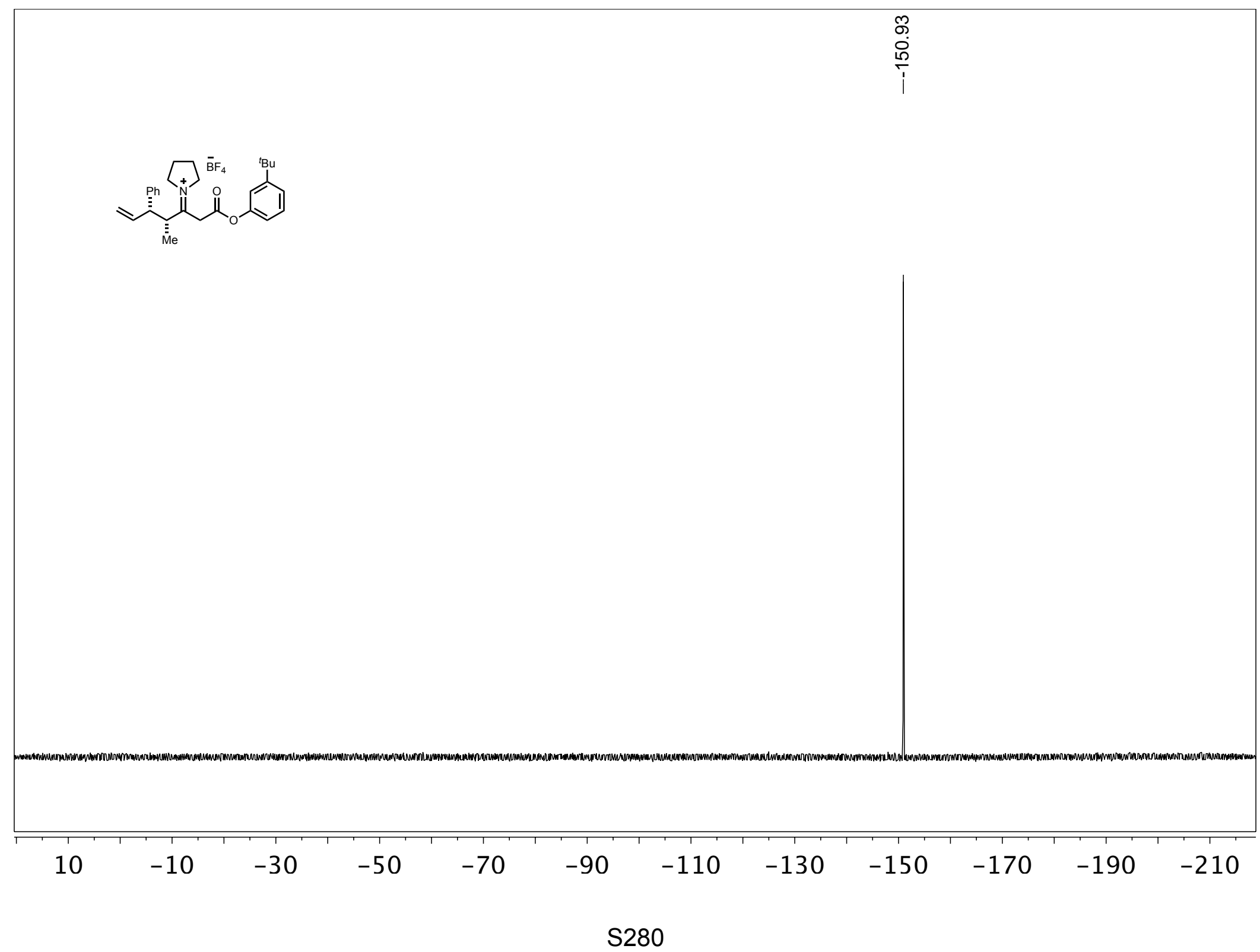




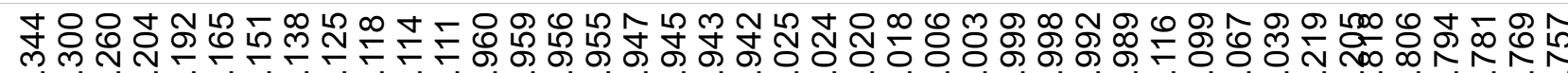

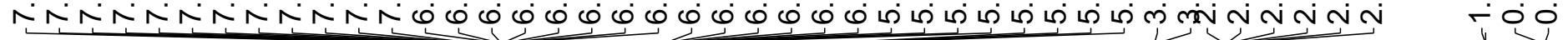

ఇ్ల
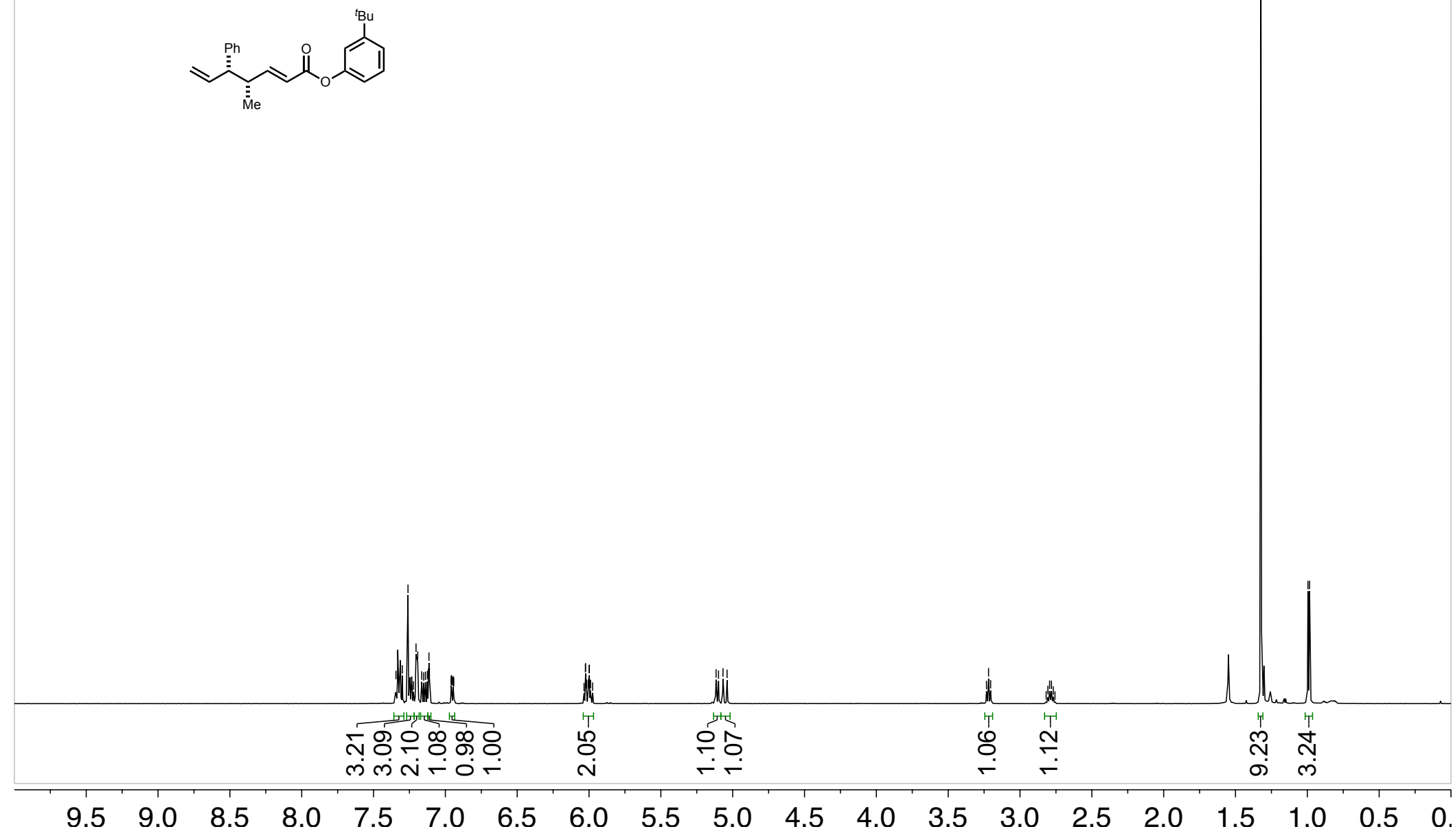


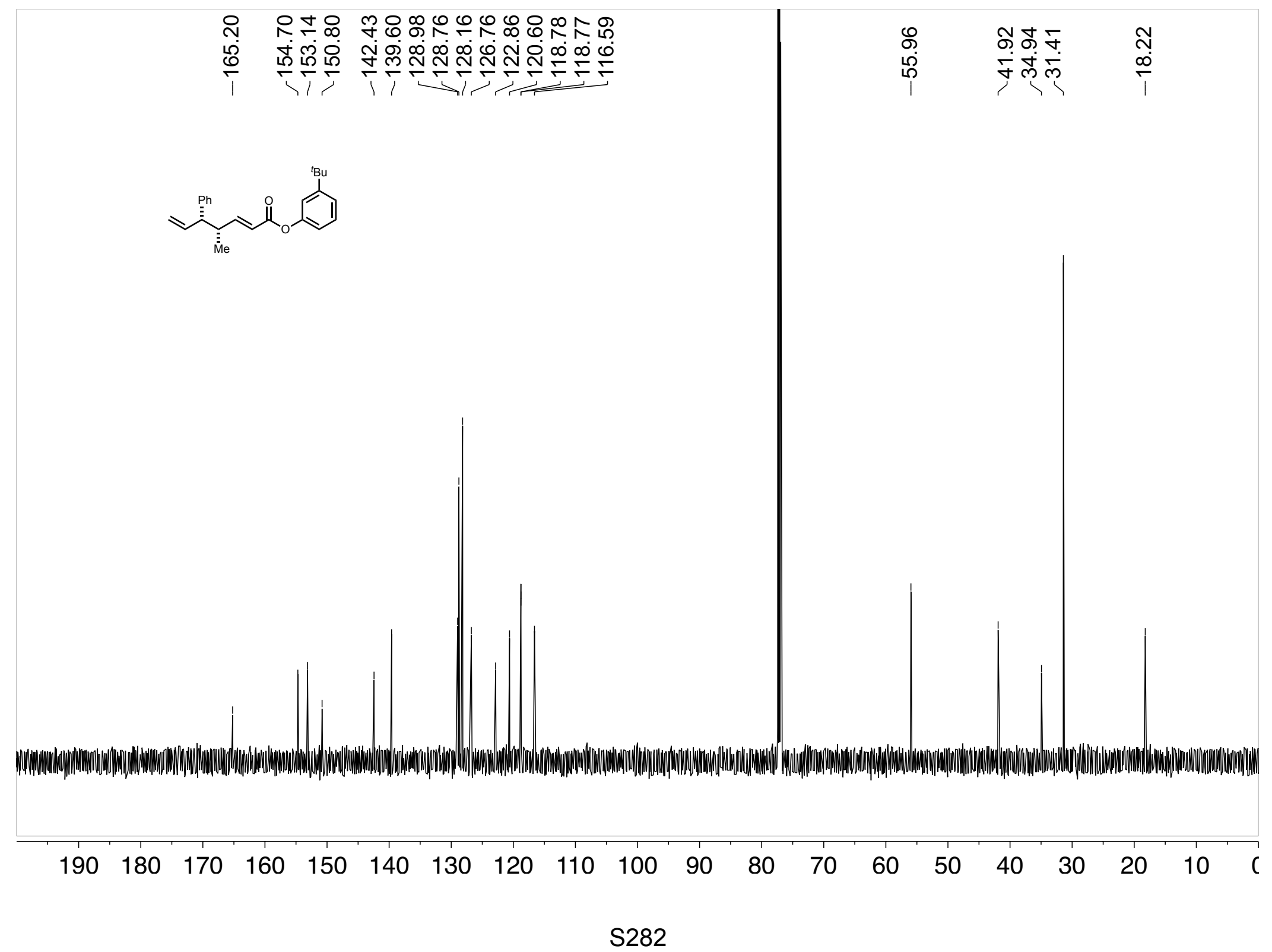




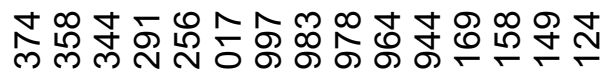

N N N

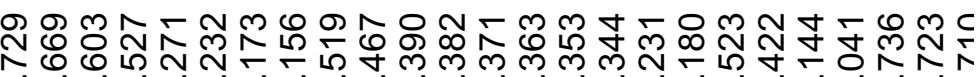

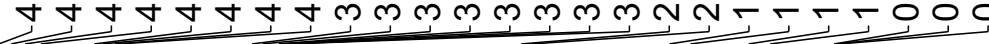
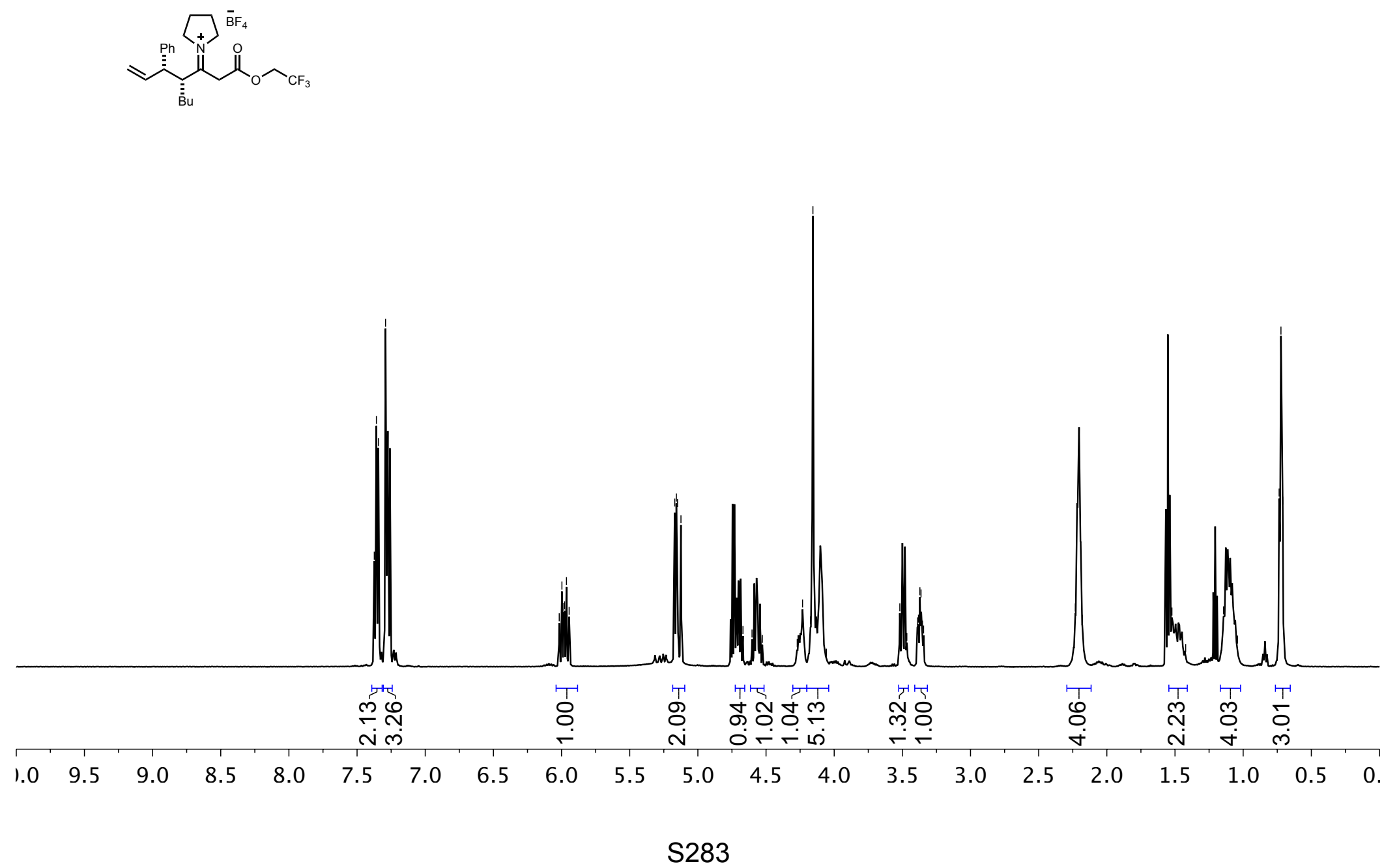


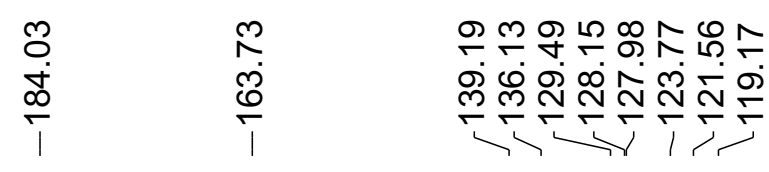

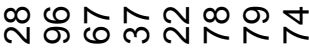

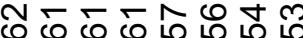

r

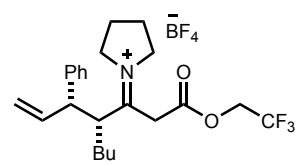

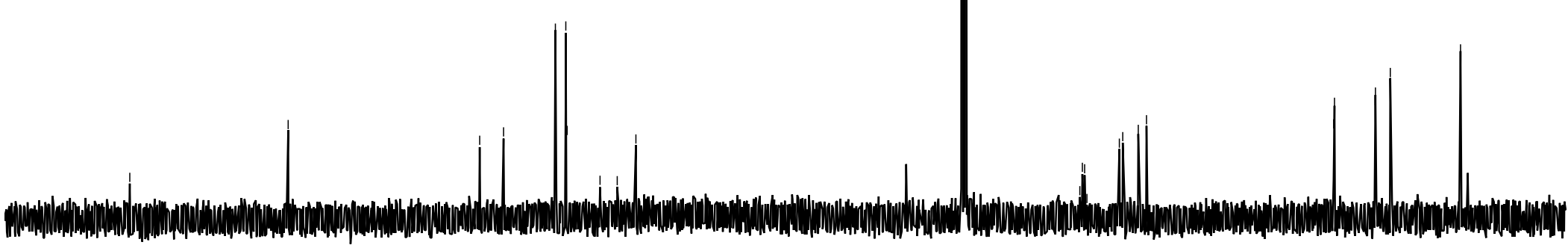



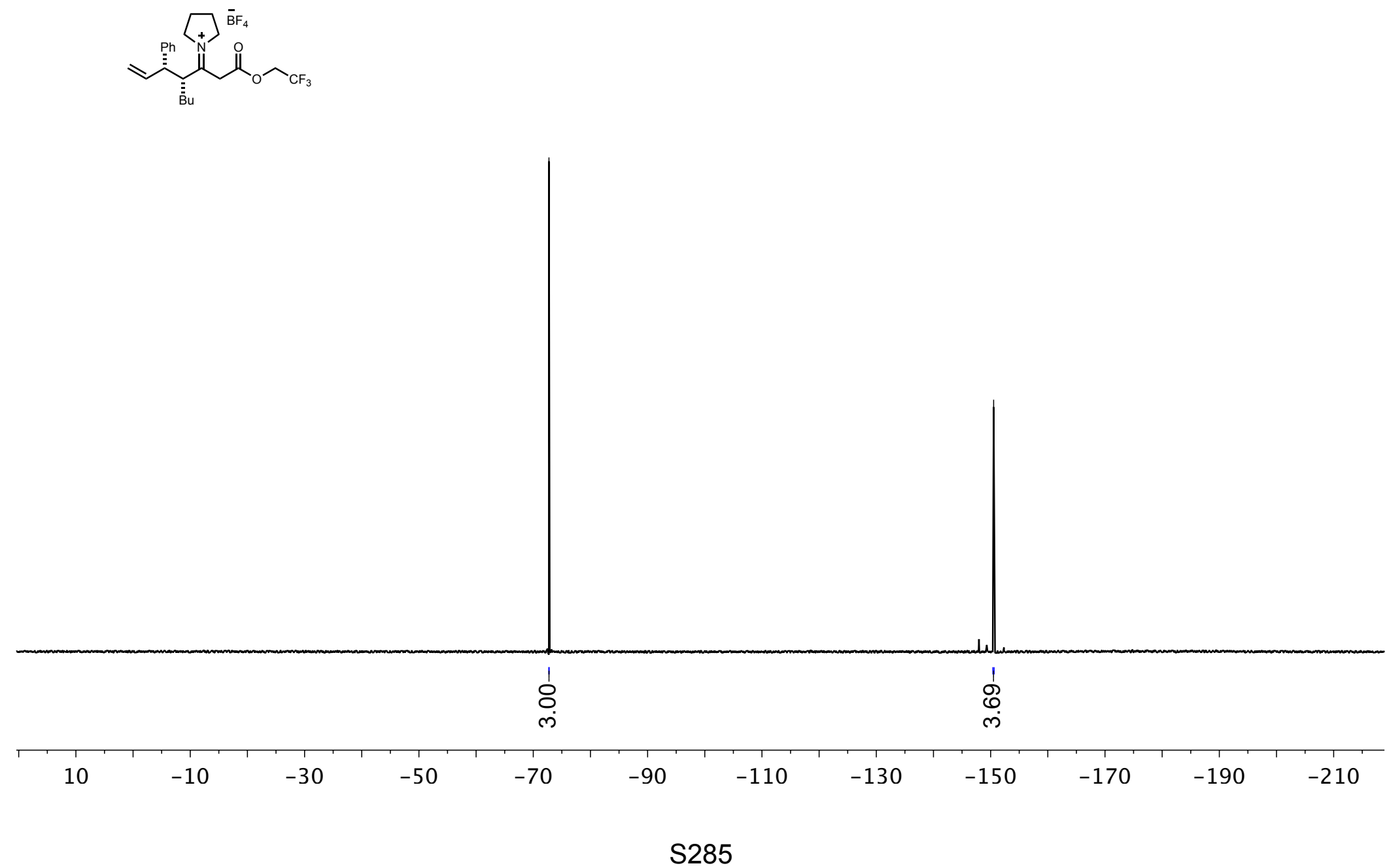


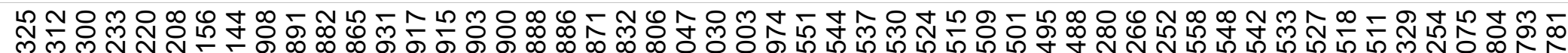
-<smiles>C=C[C@H](C/C=C/C(=O)OCC(F)F)[C@H](C)COC(F)(F)c1ccccc1</smiles>

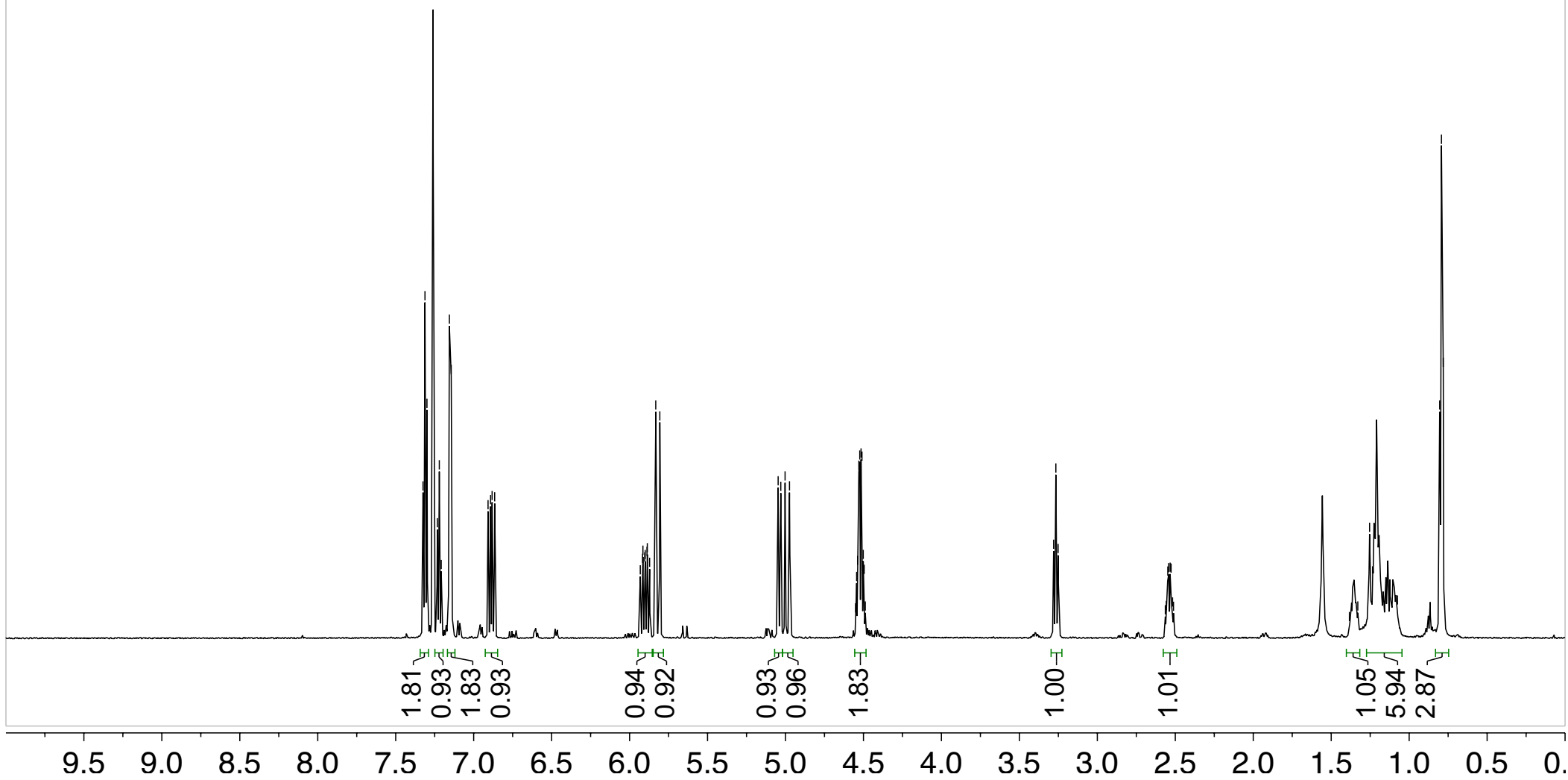




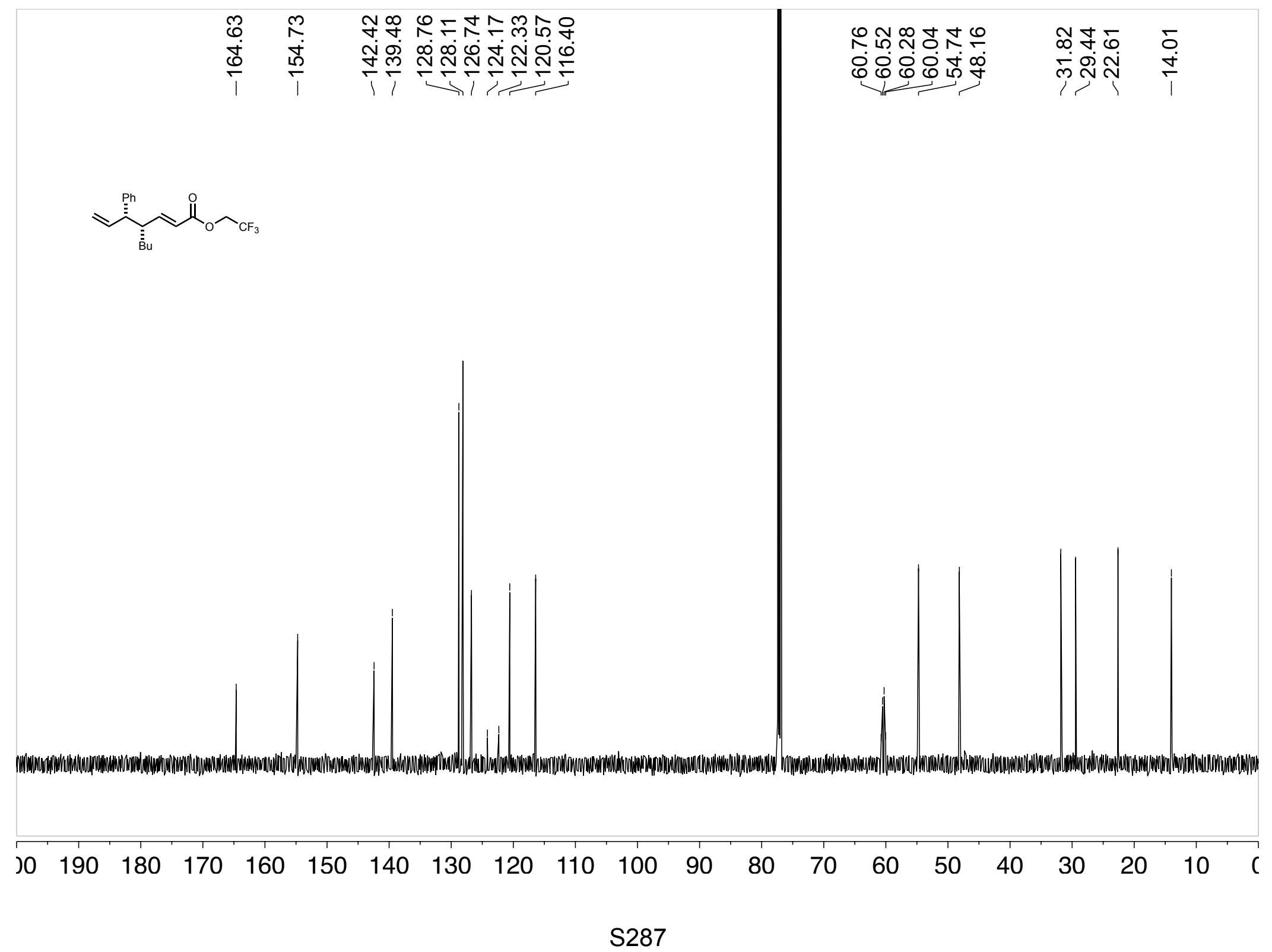




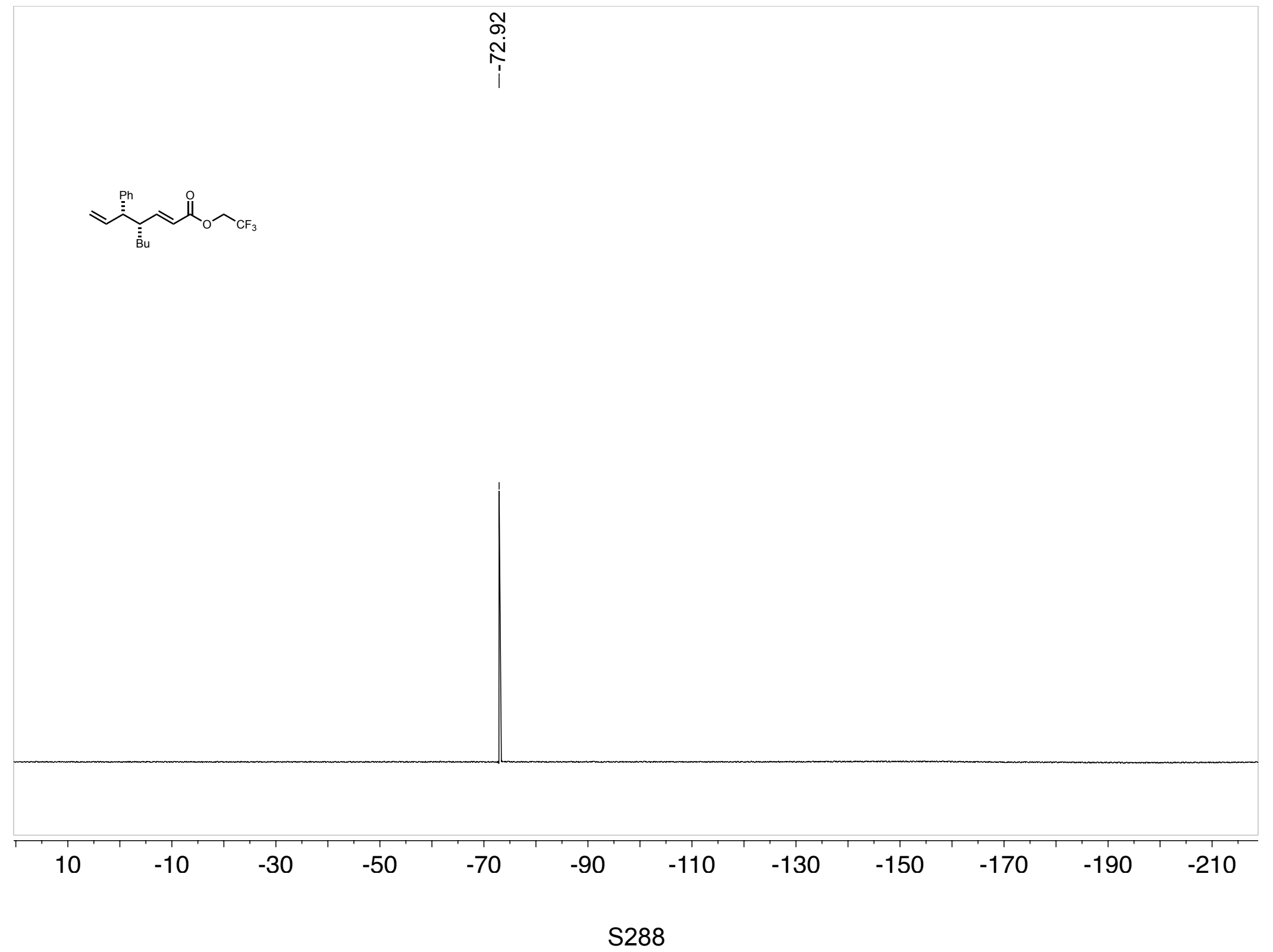




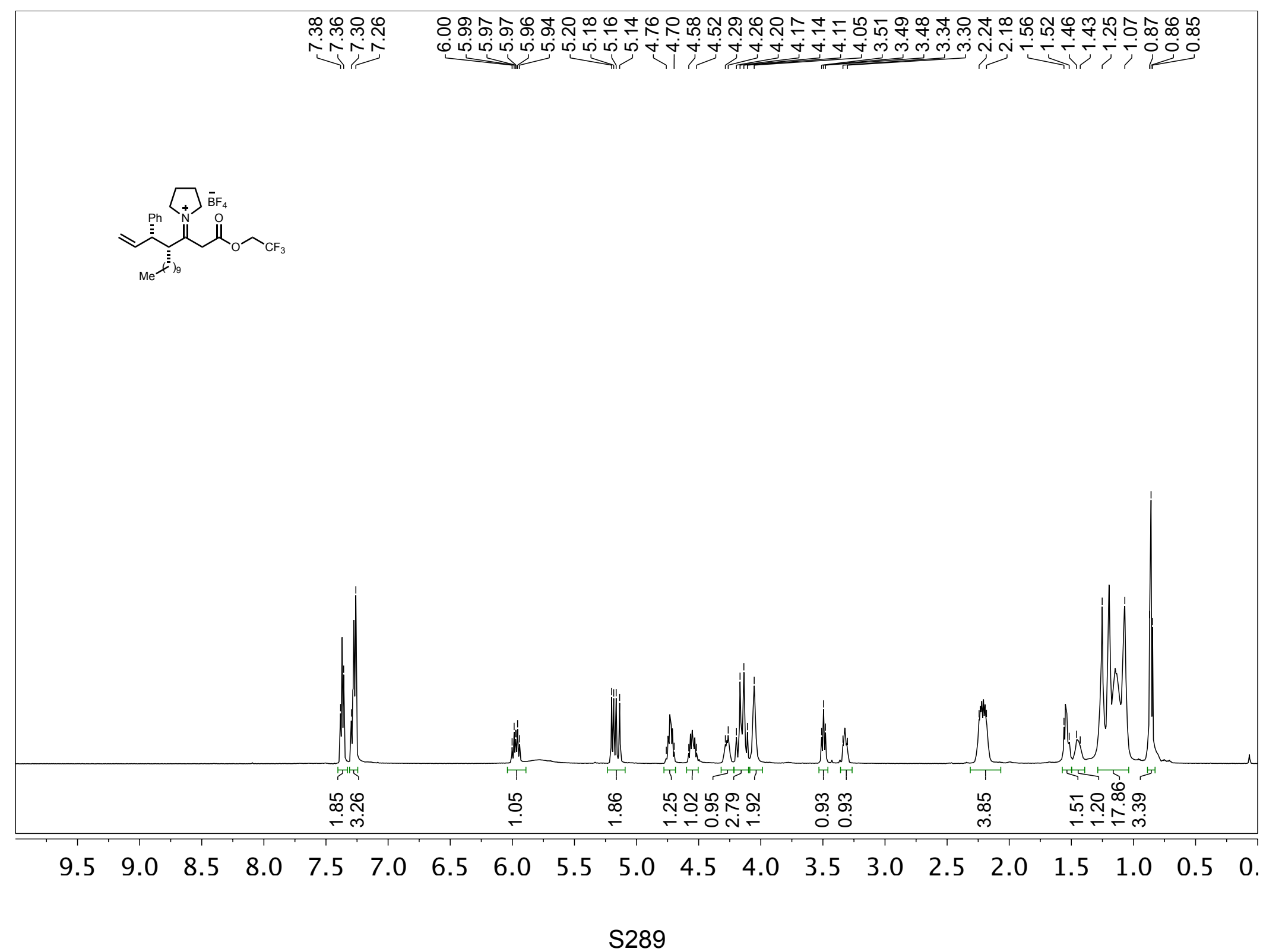




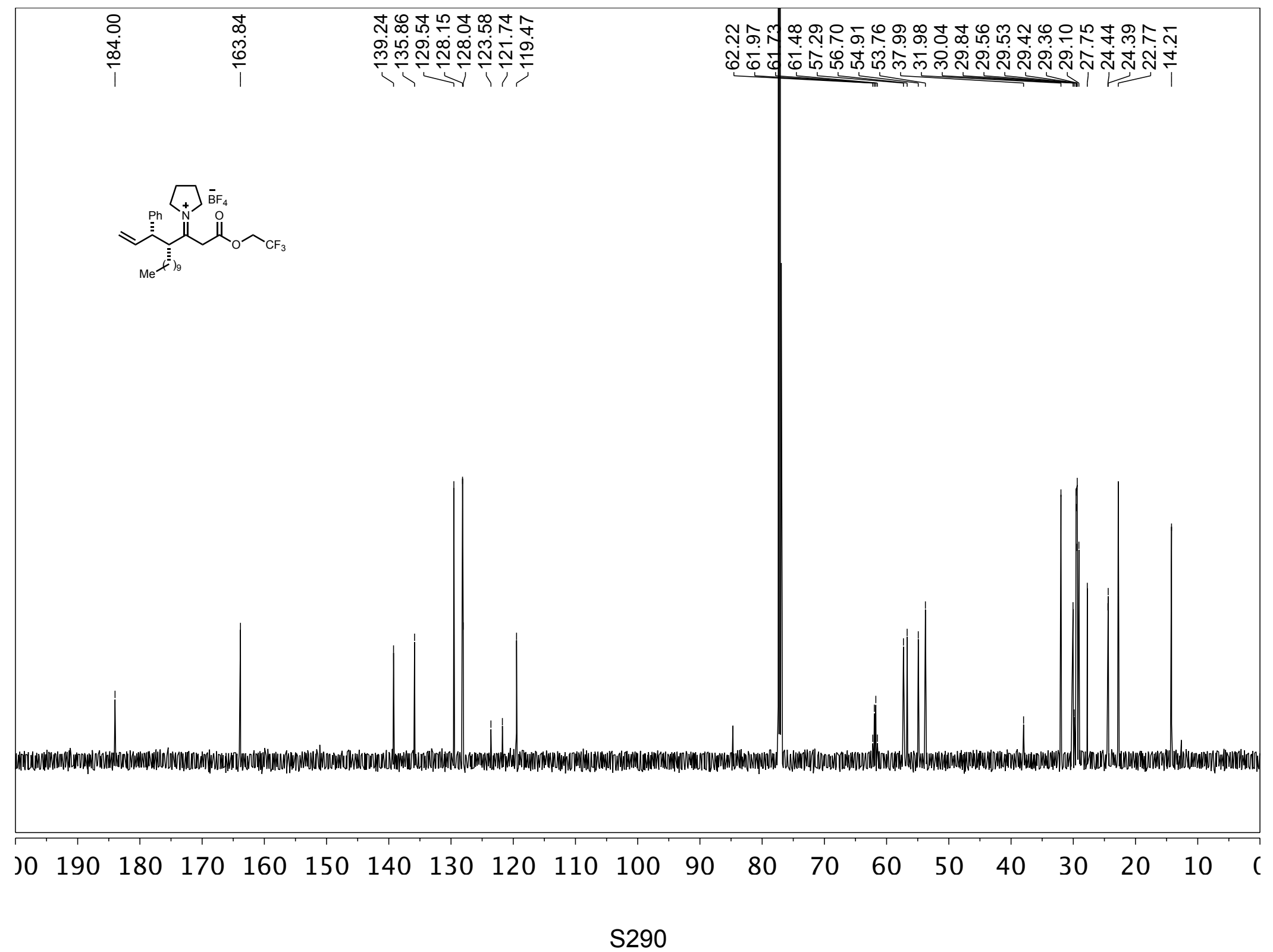




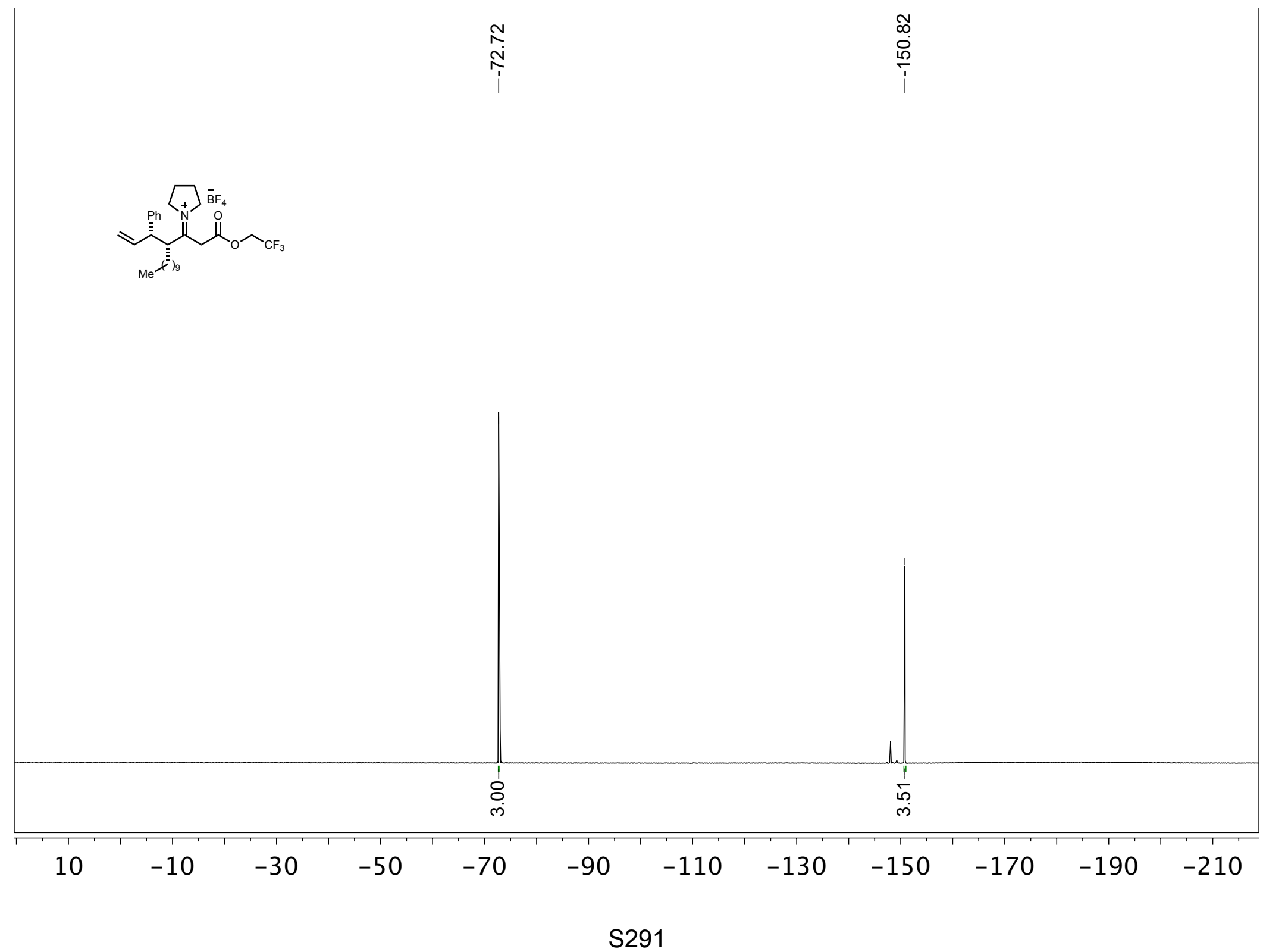




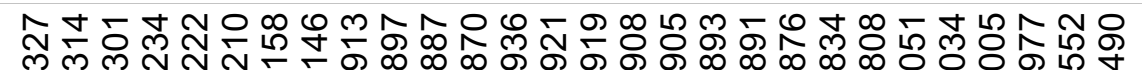

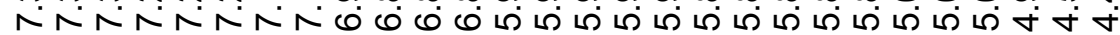

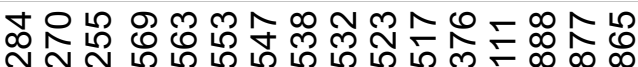

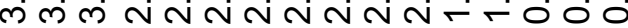
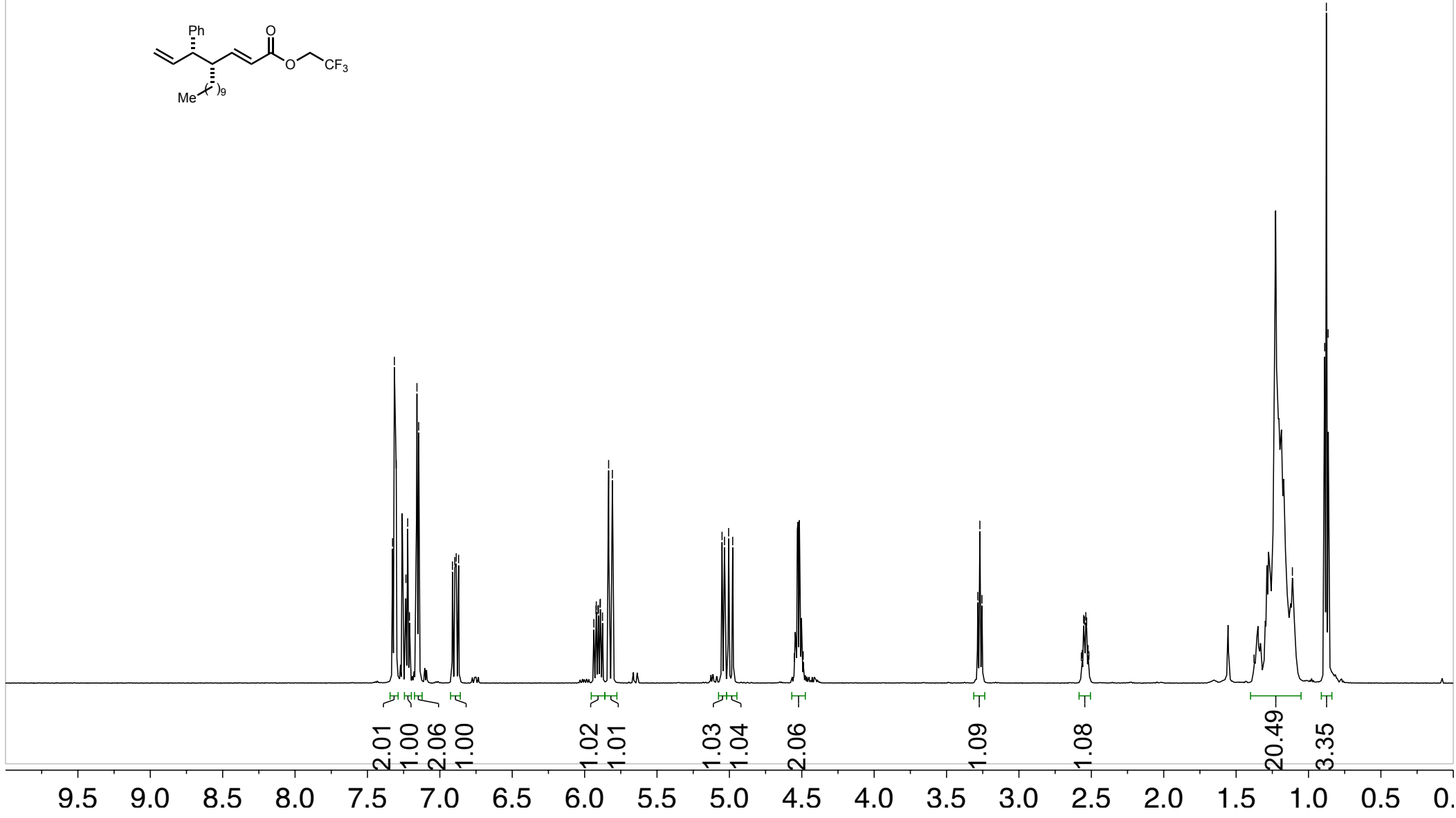


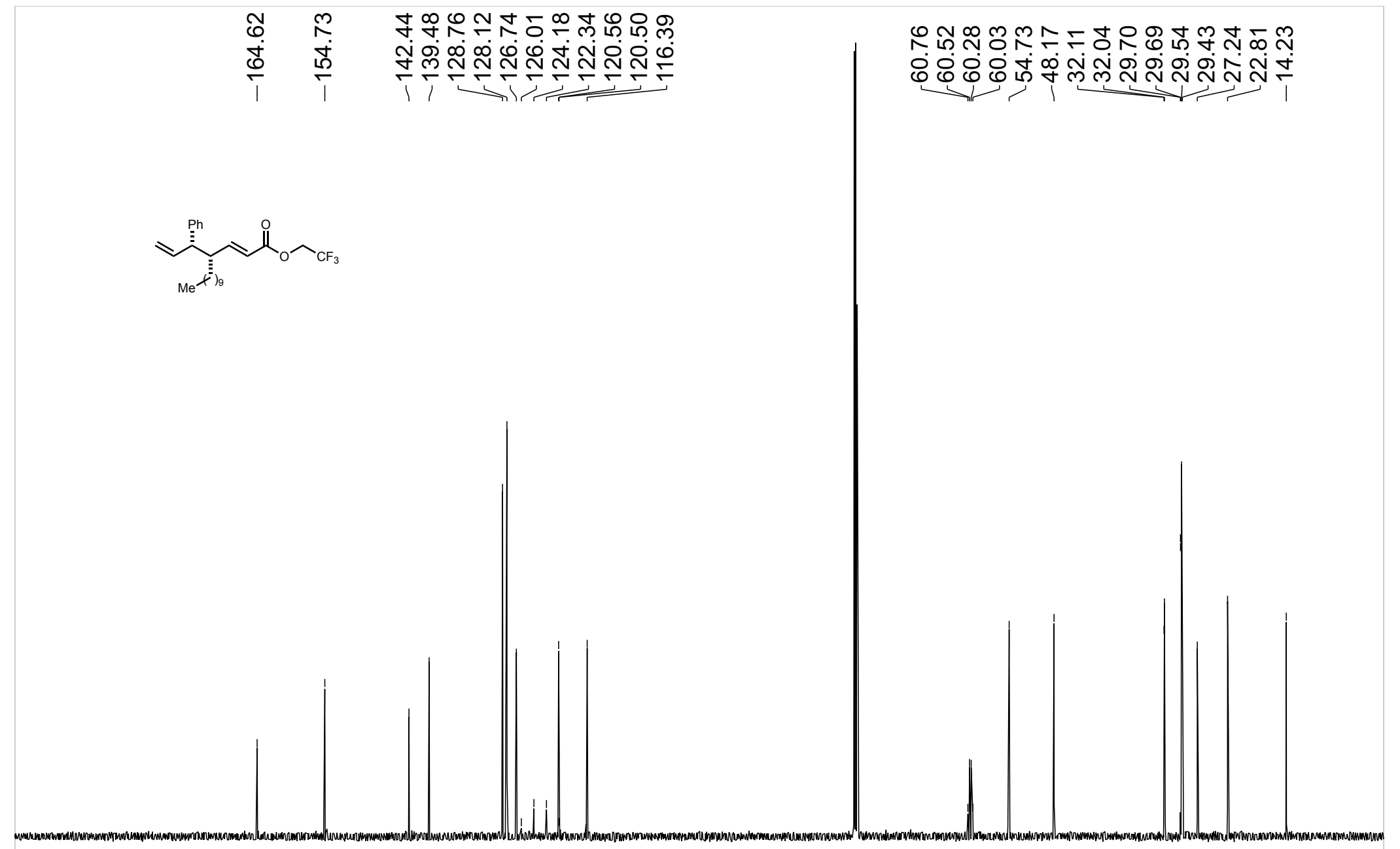

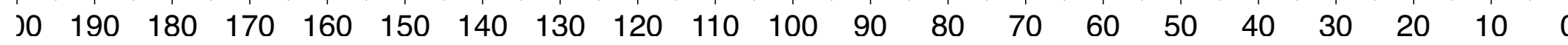




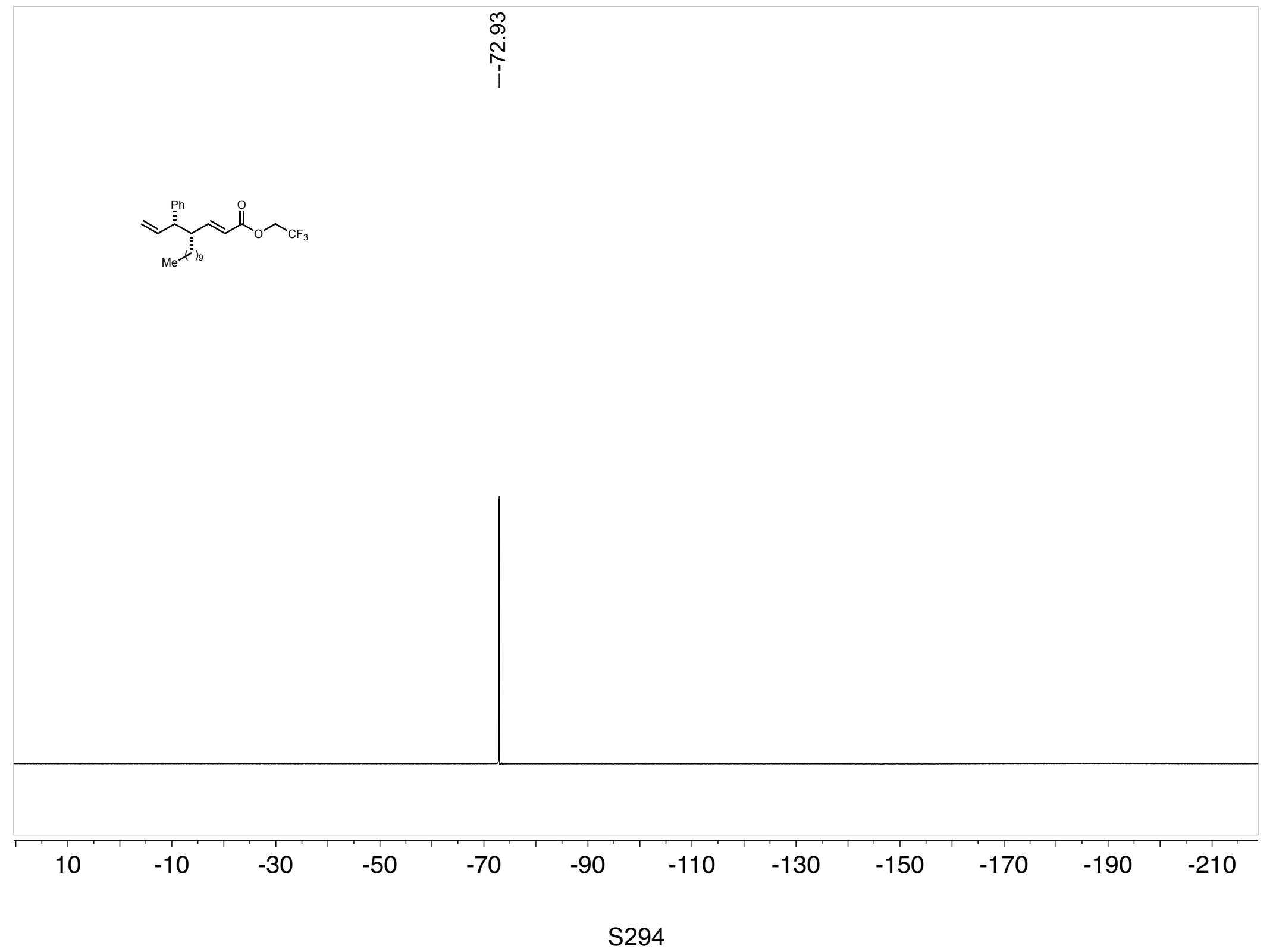




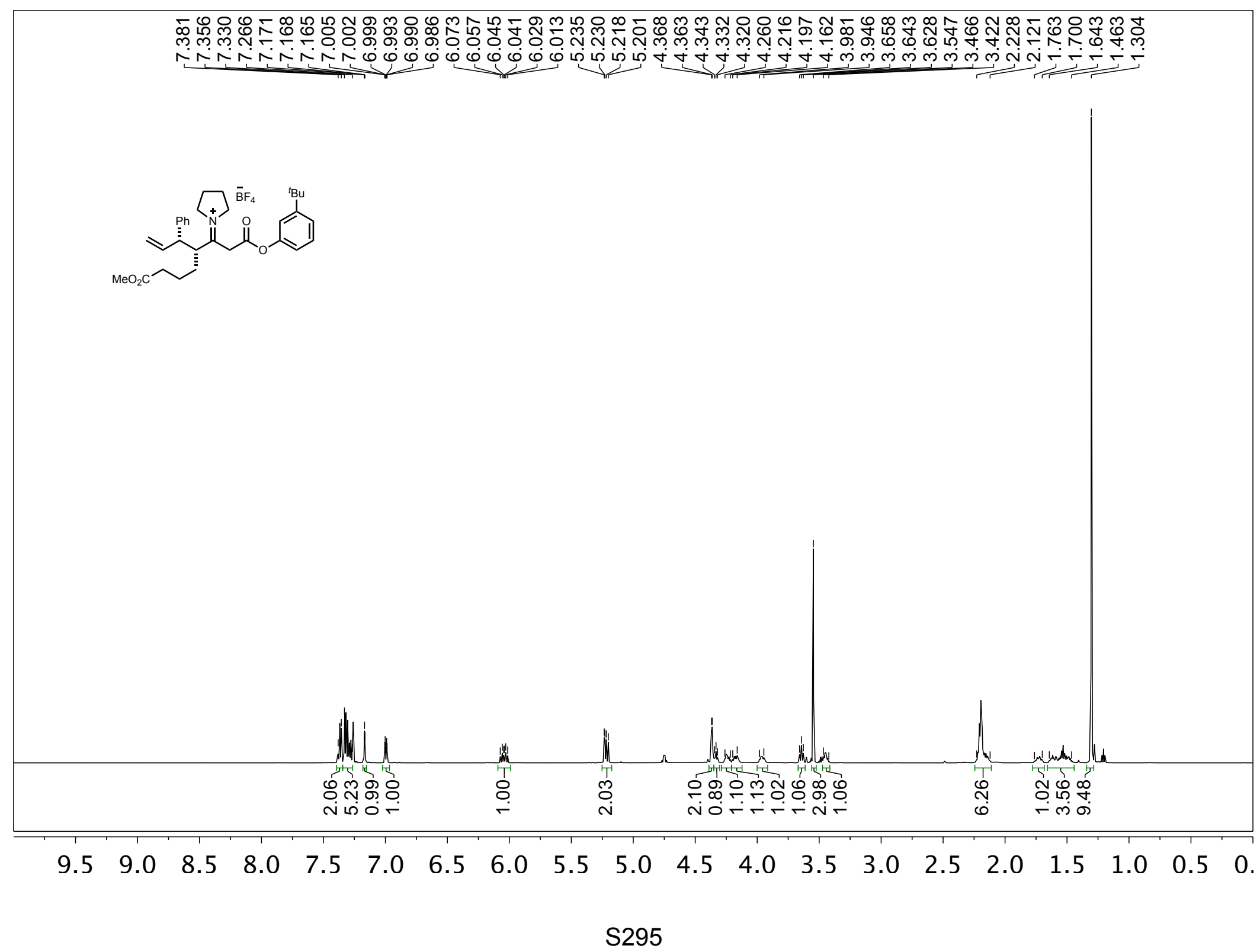




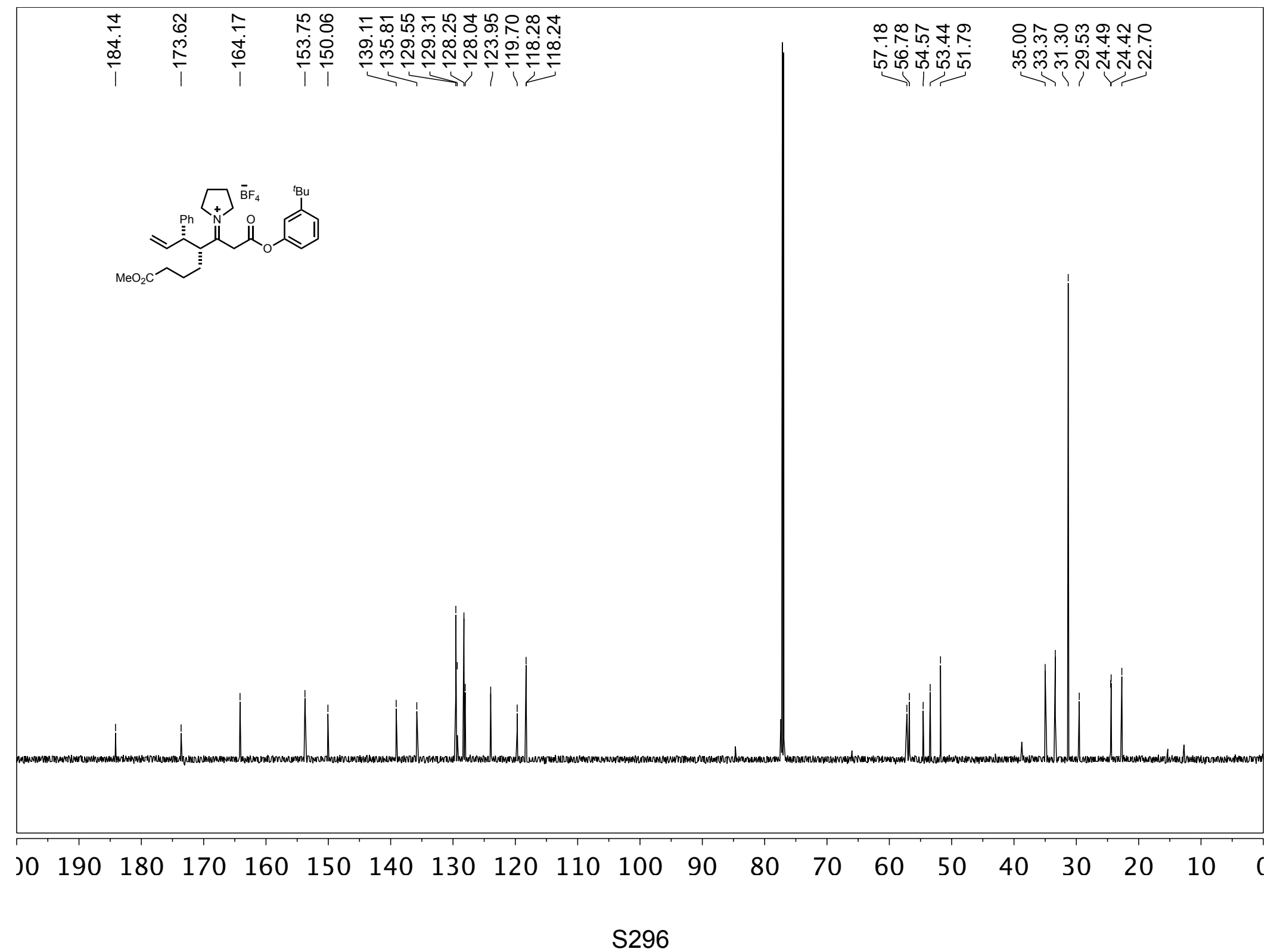




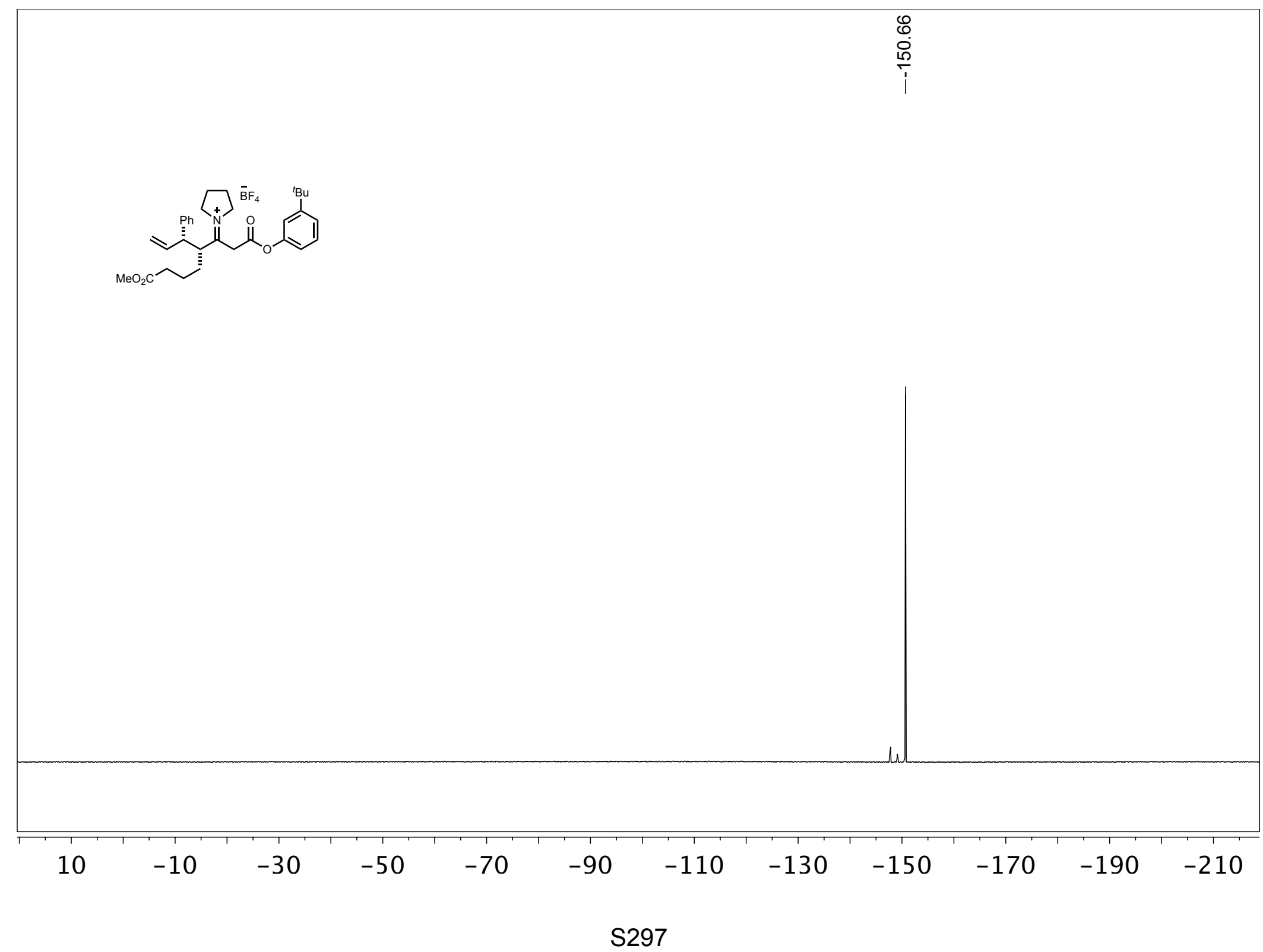




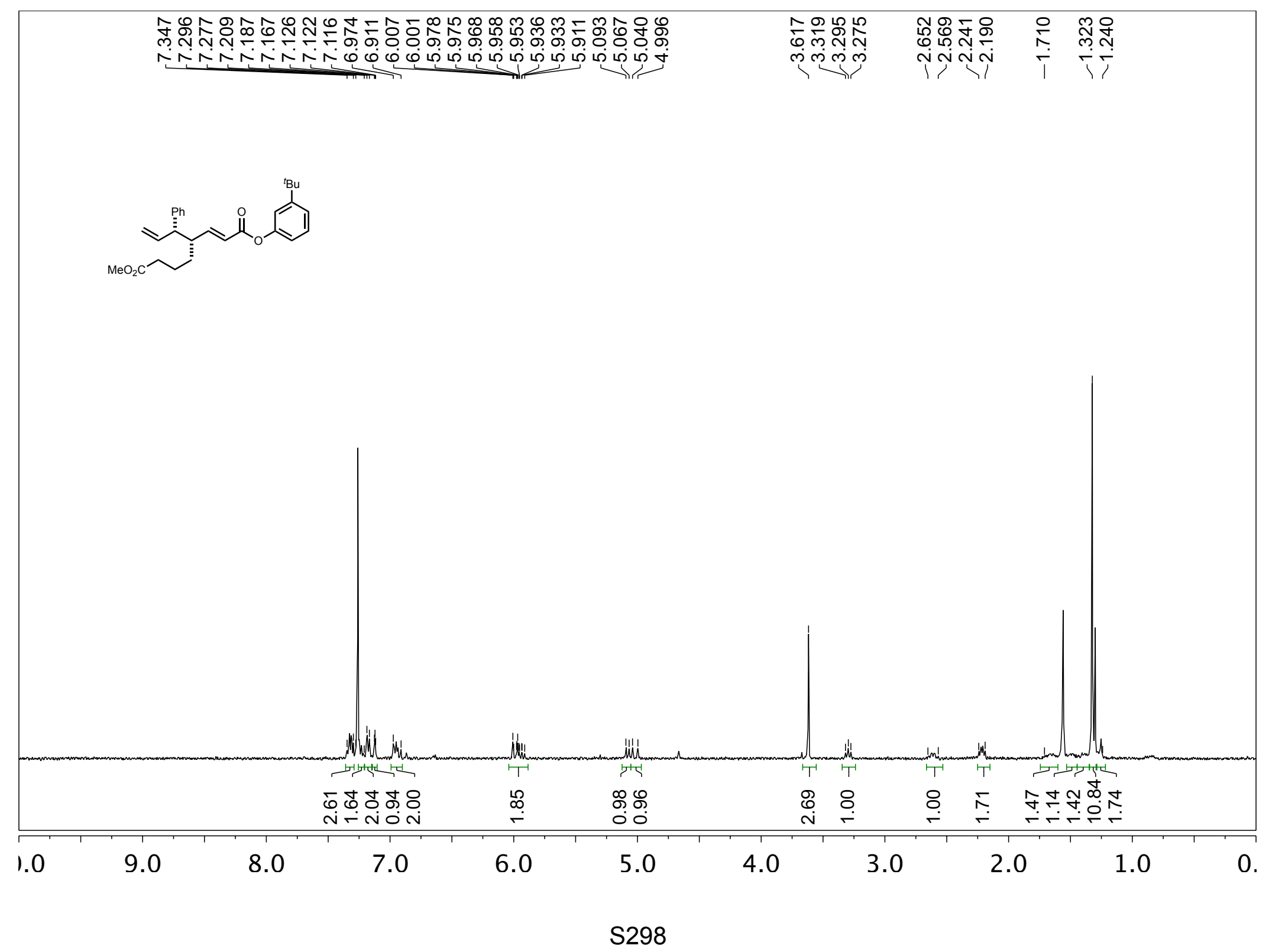




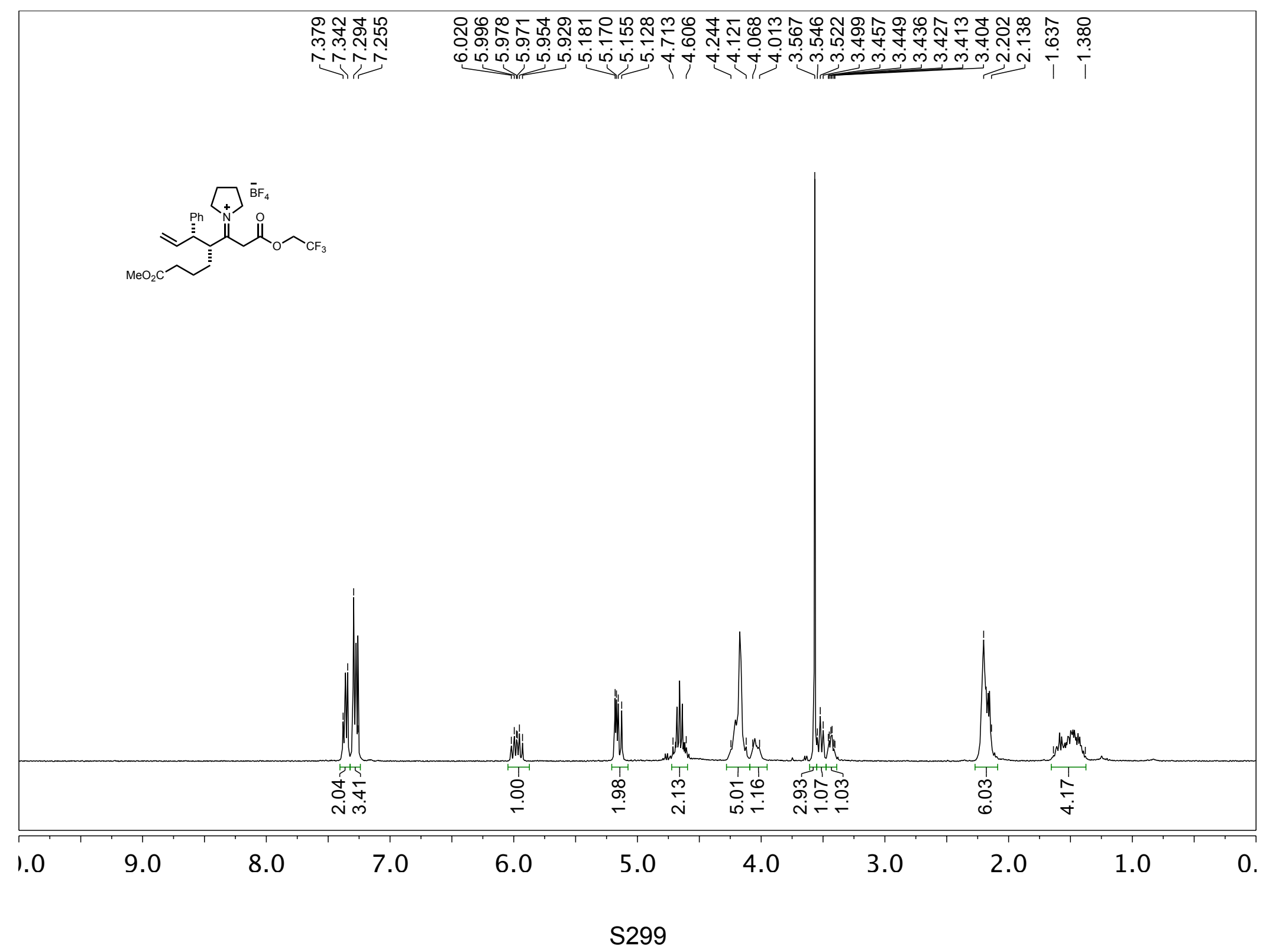




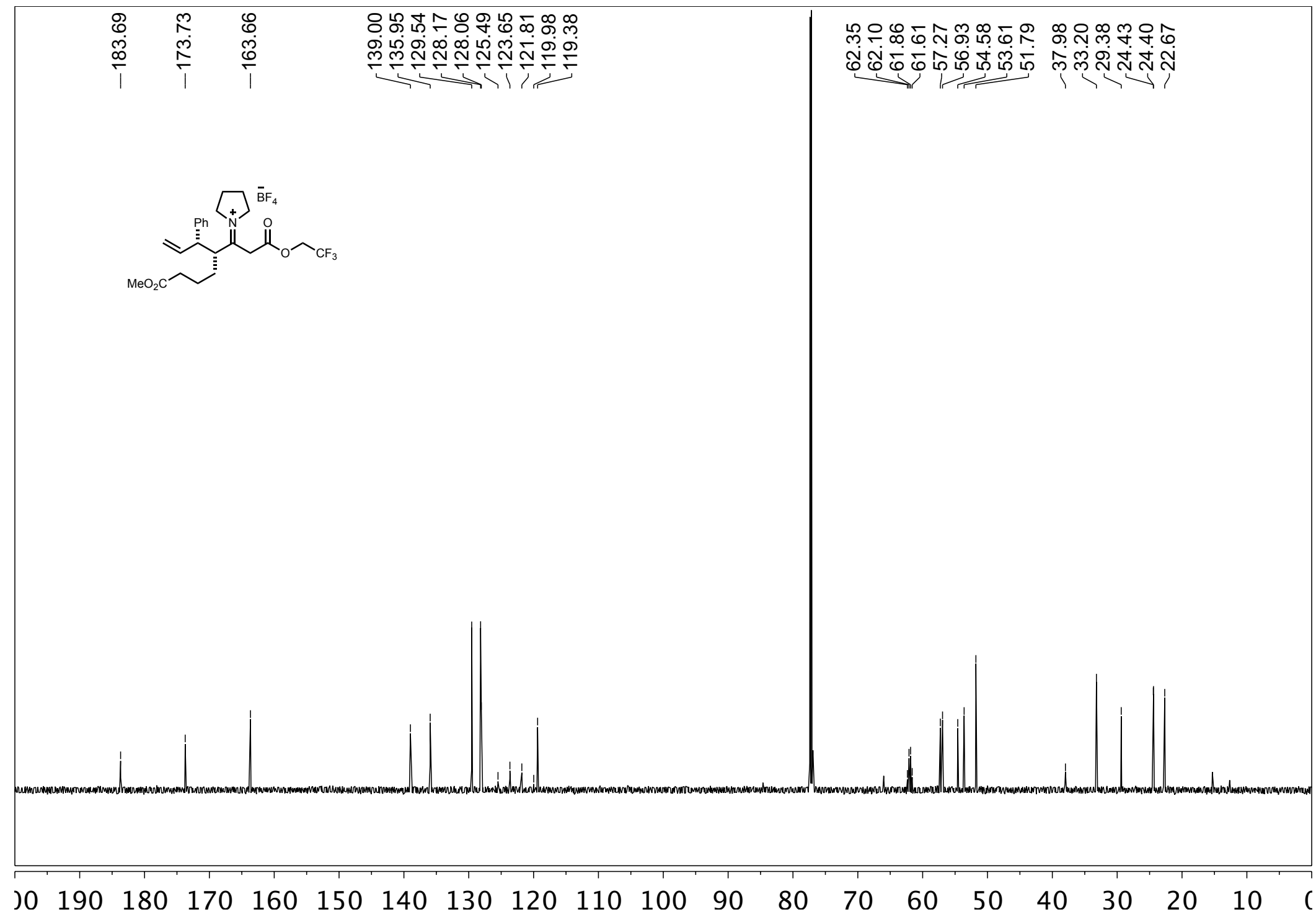




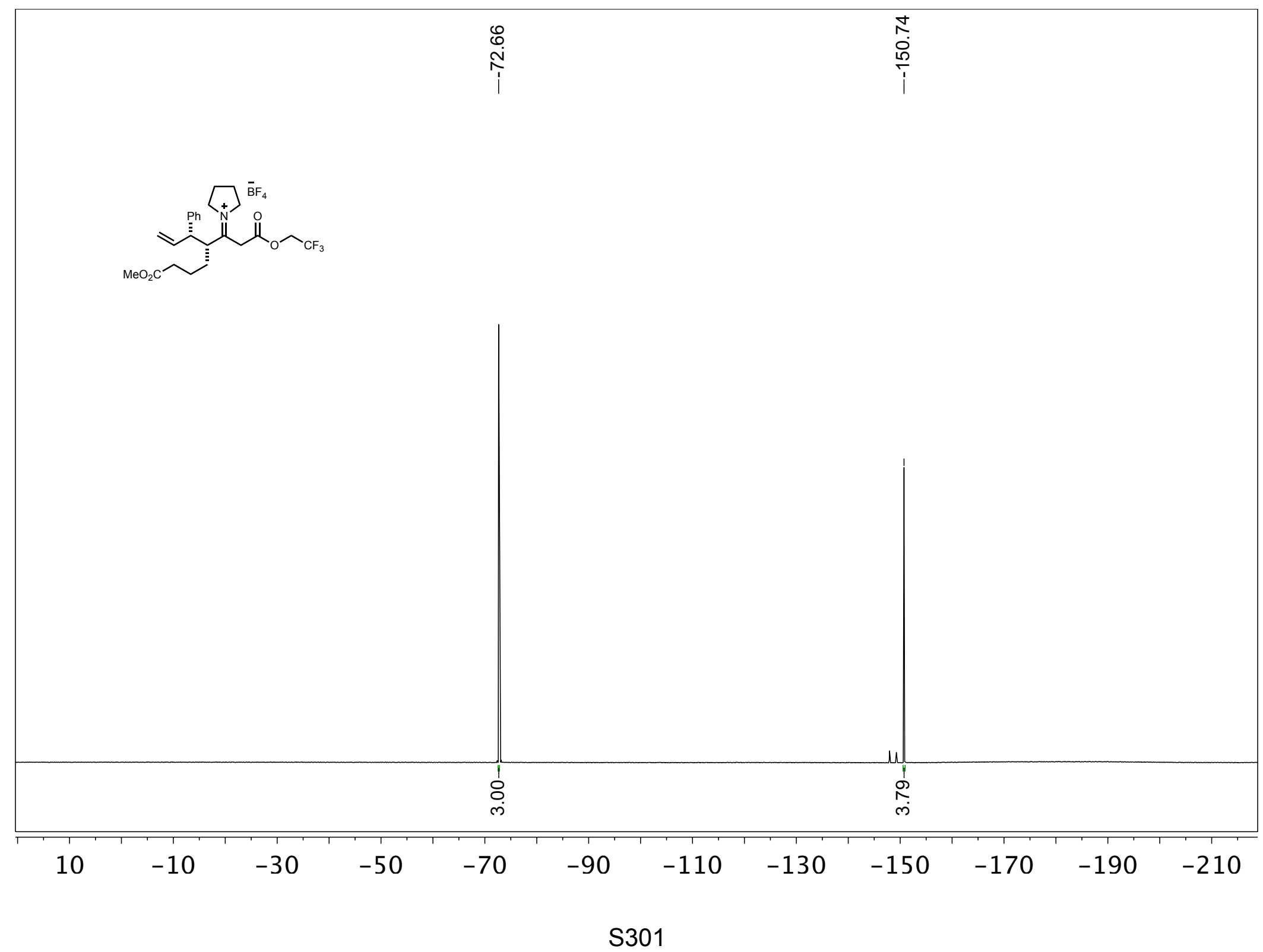




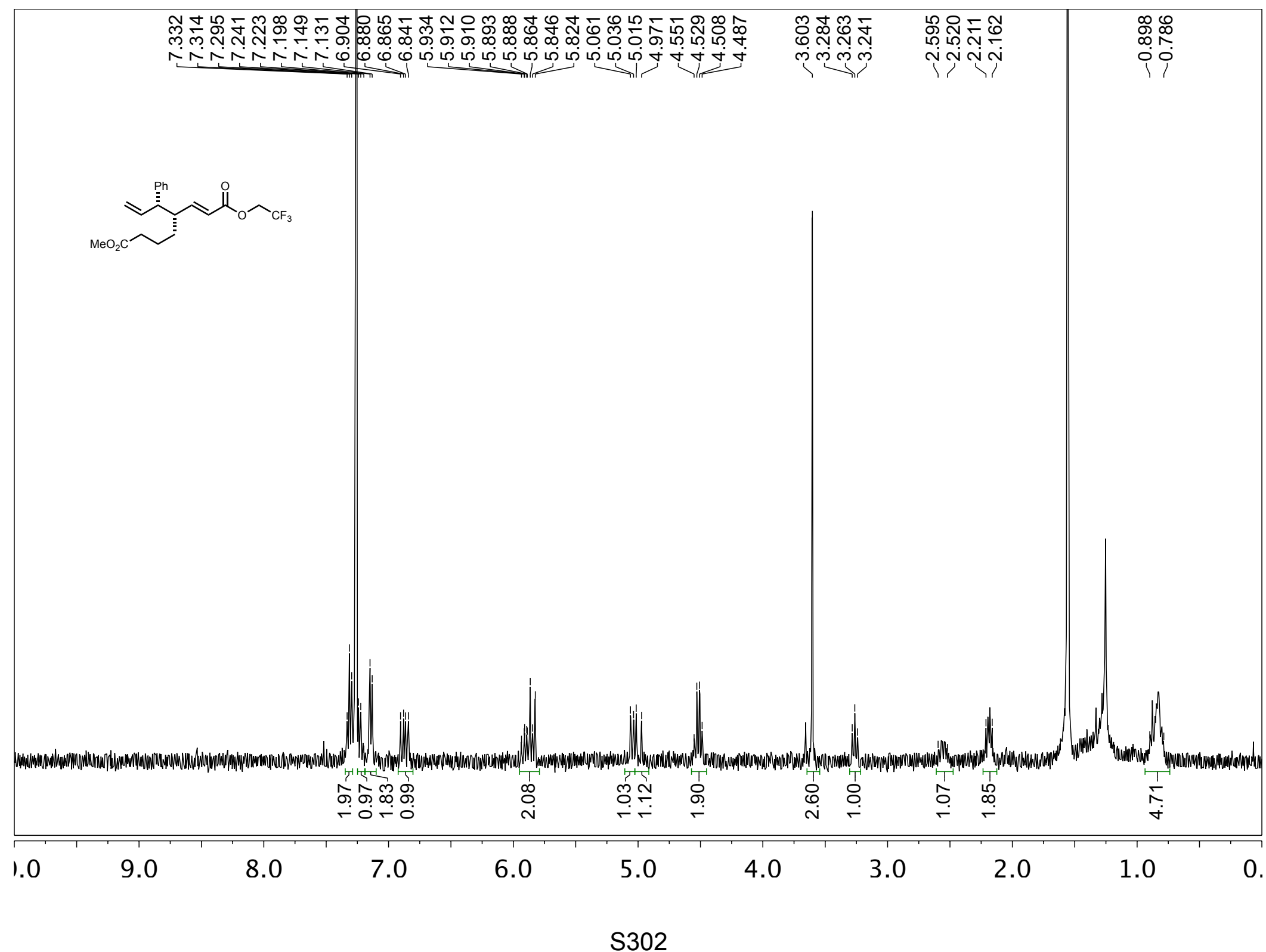




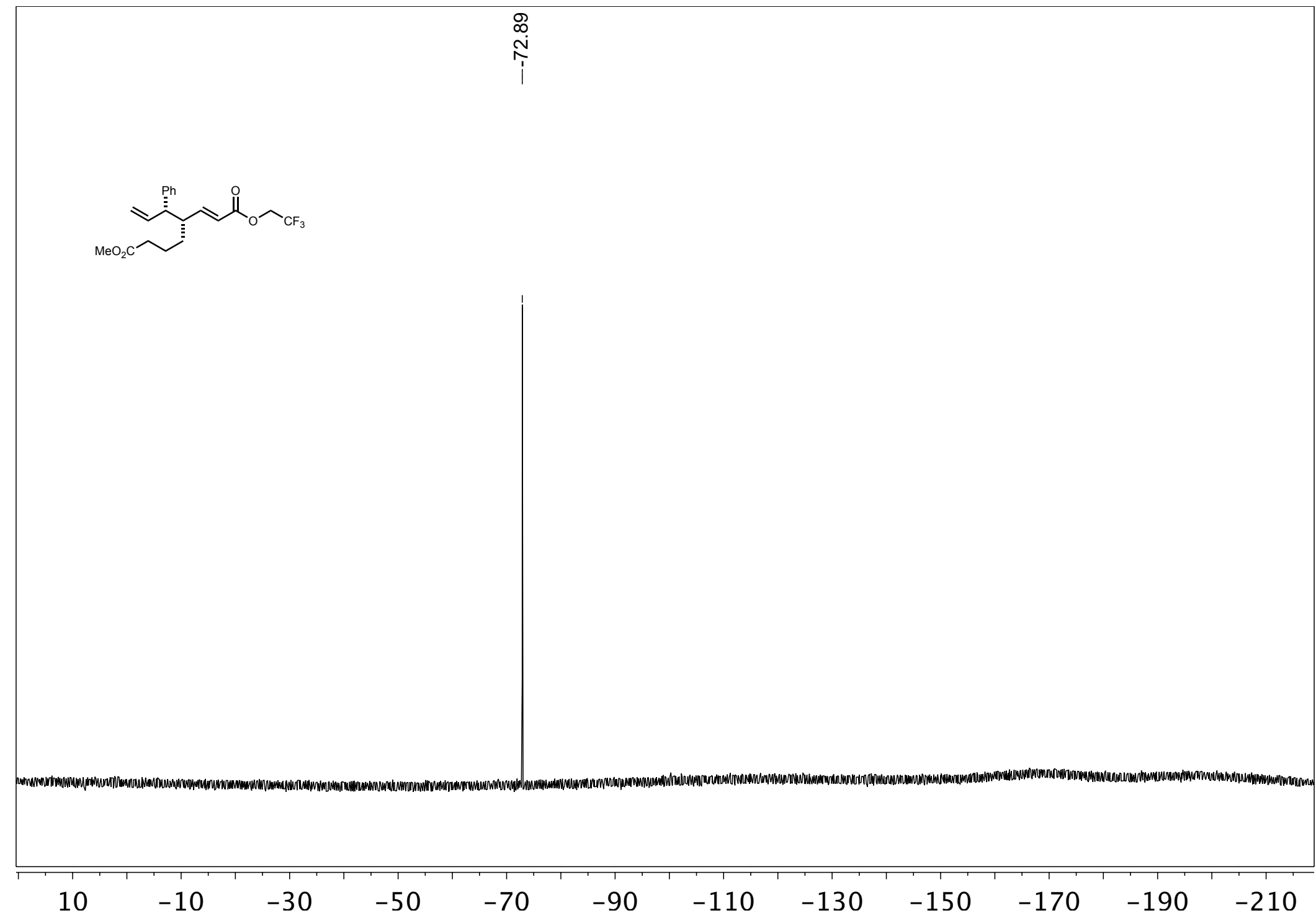




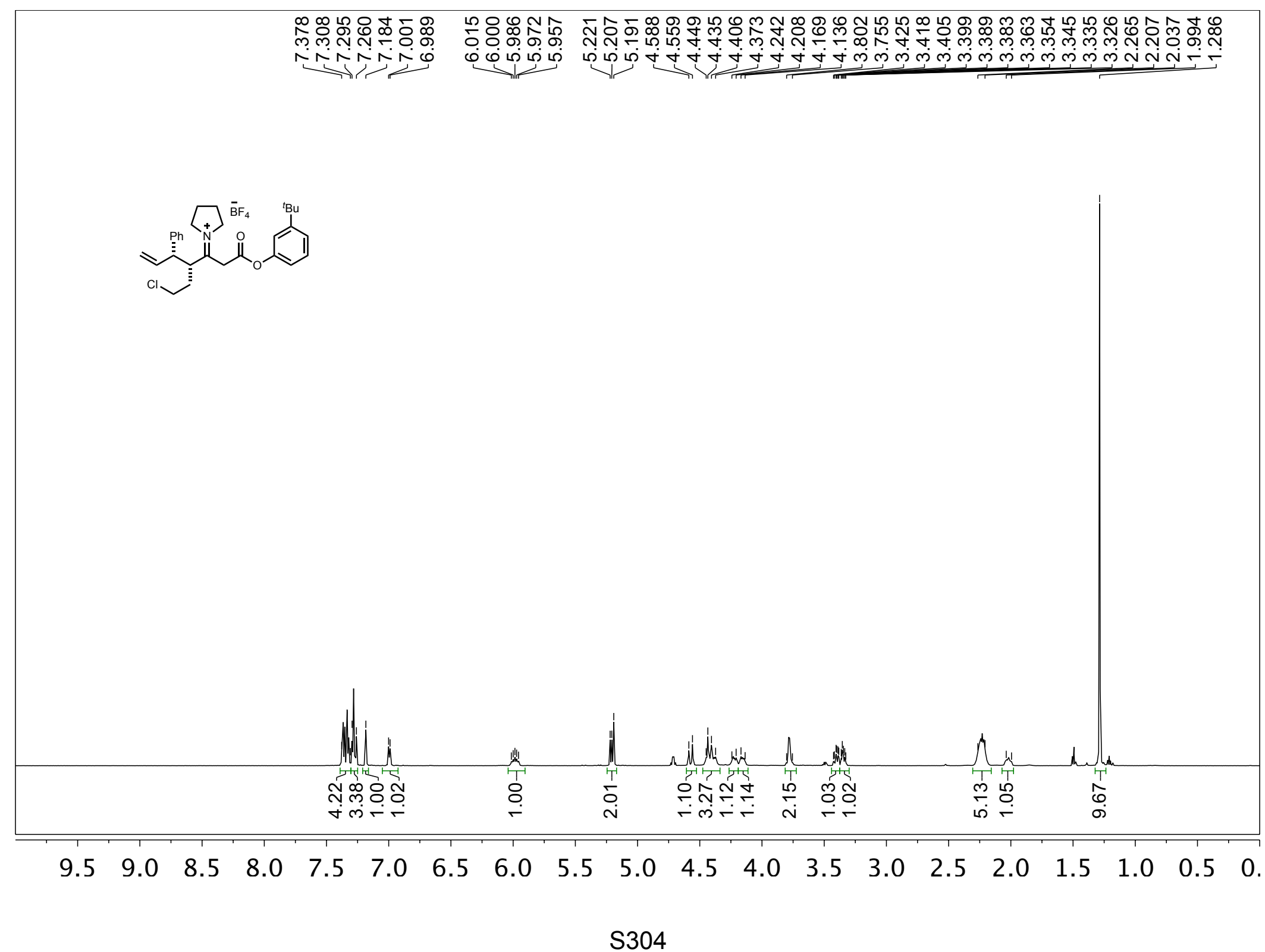




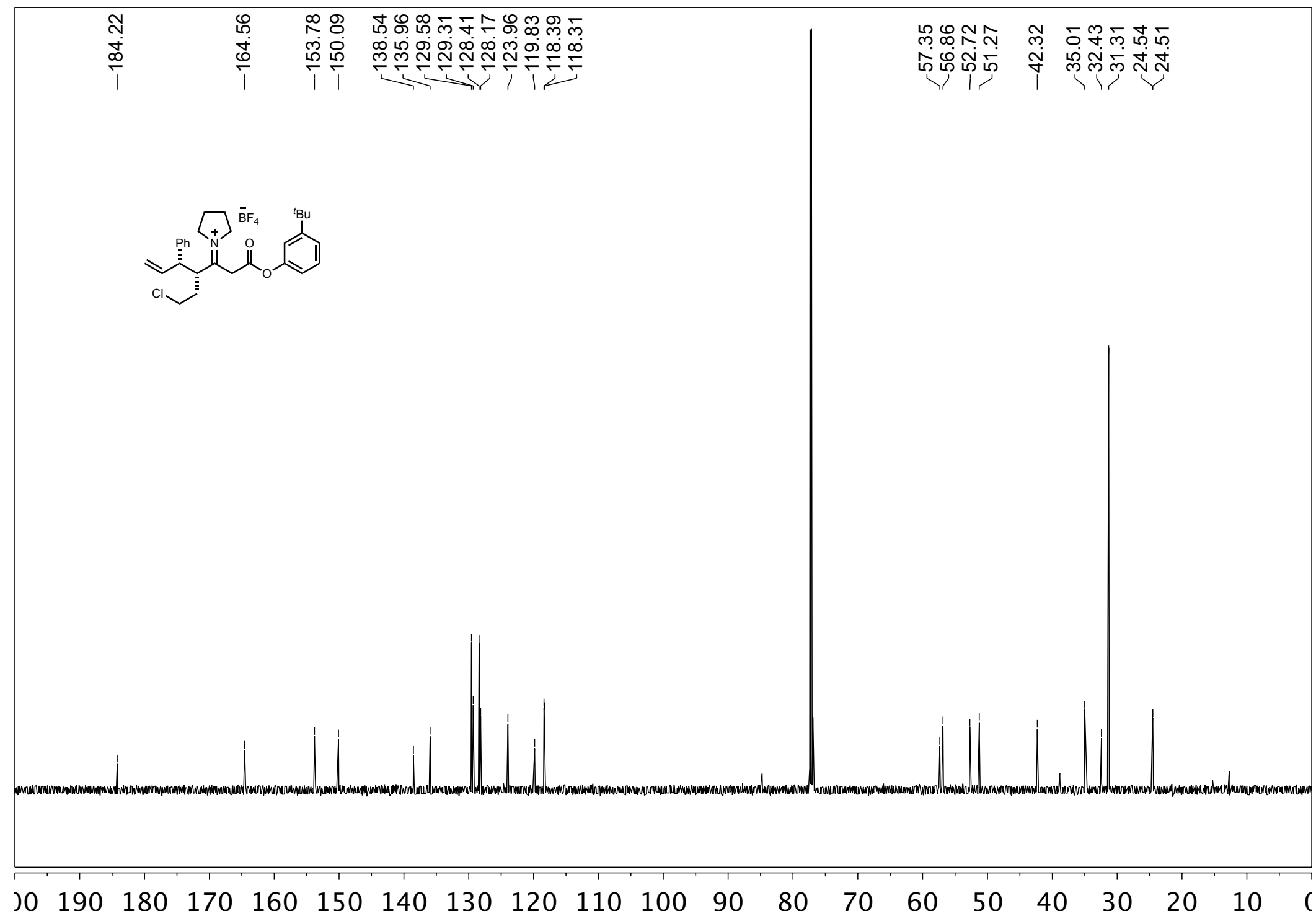




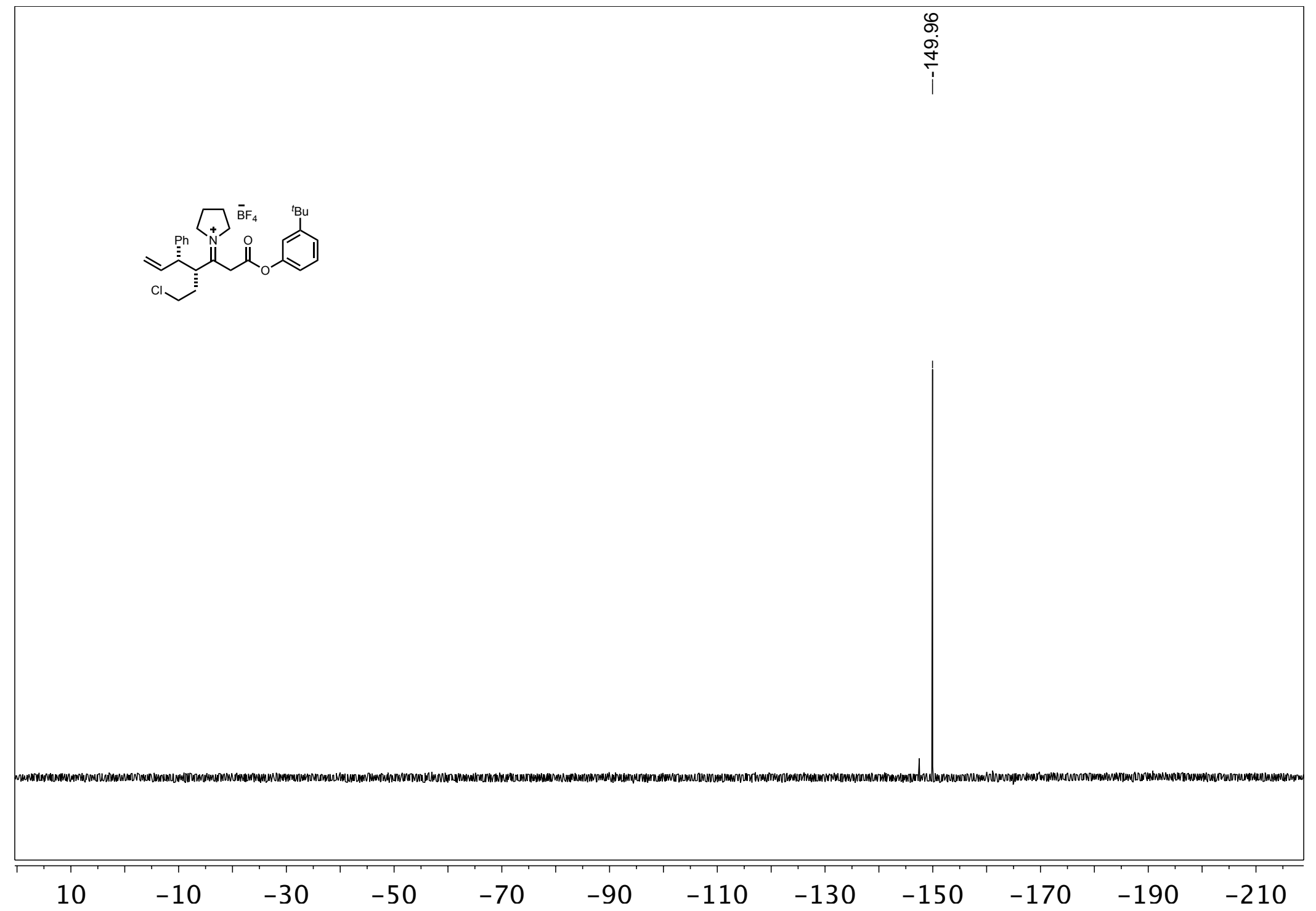




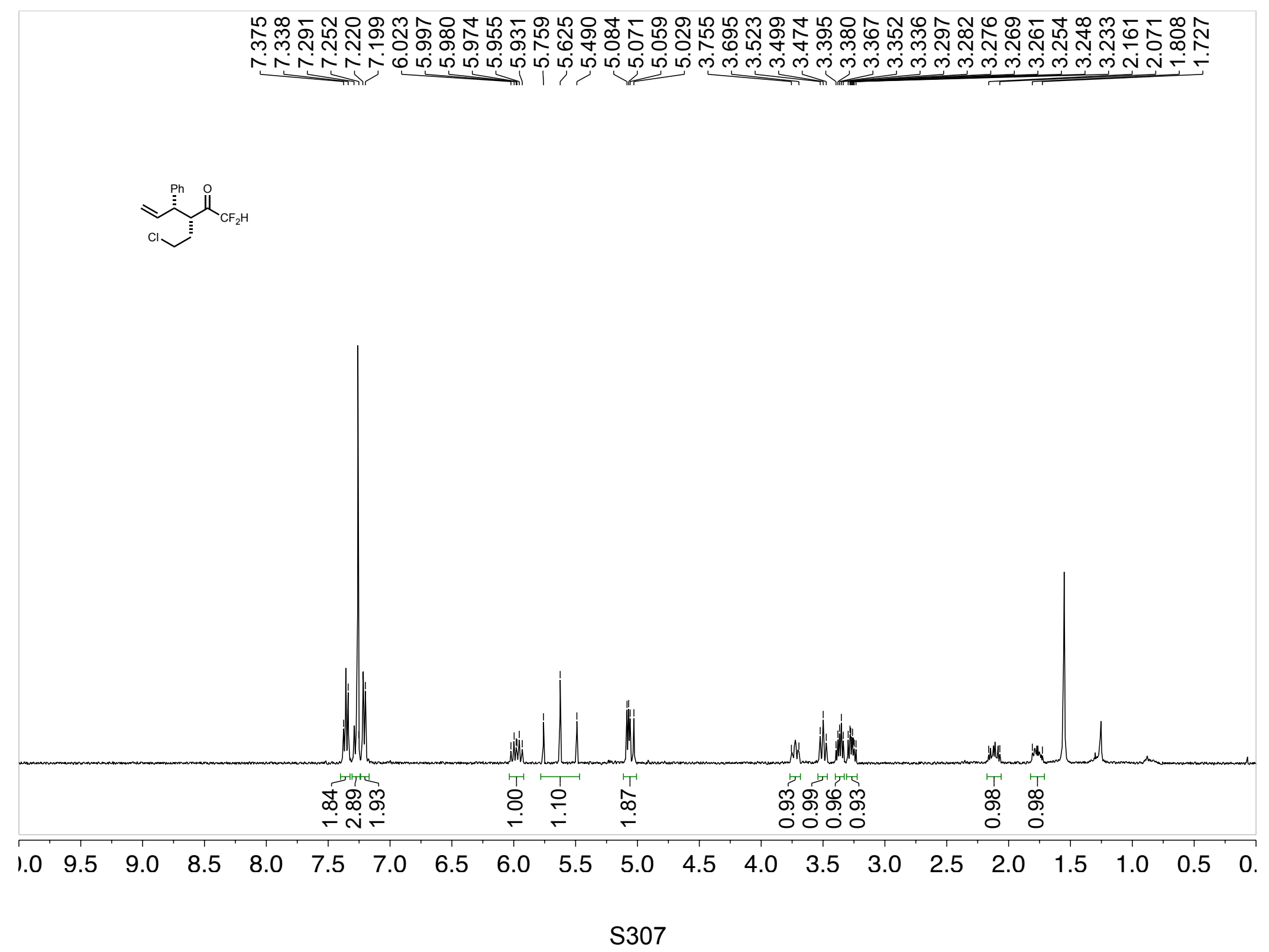




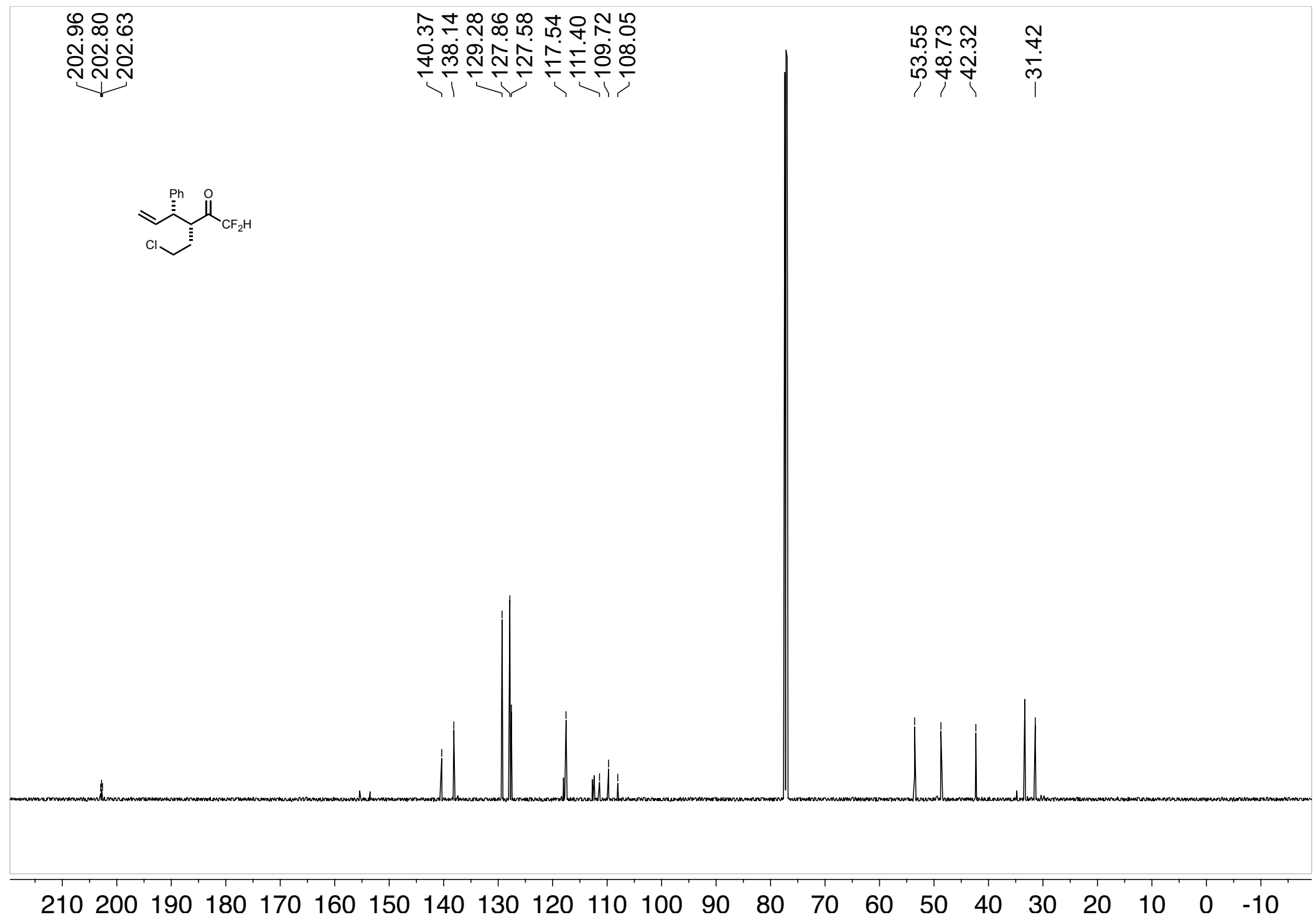

S308 


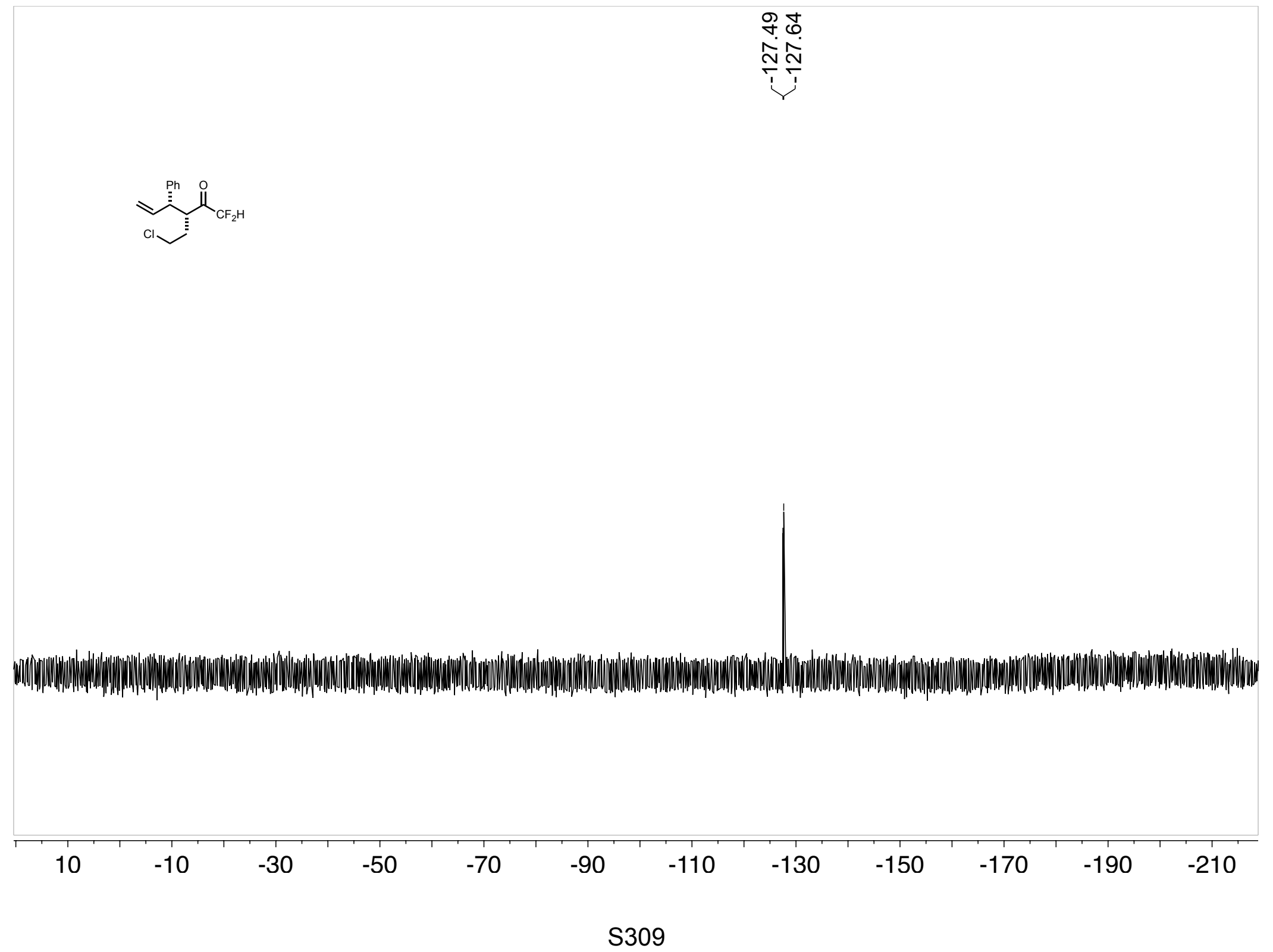




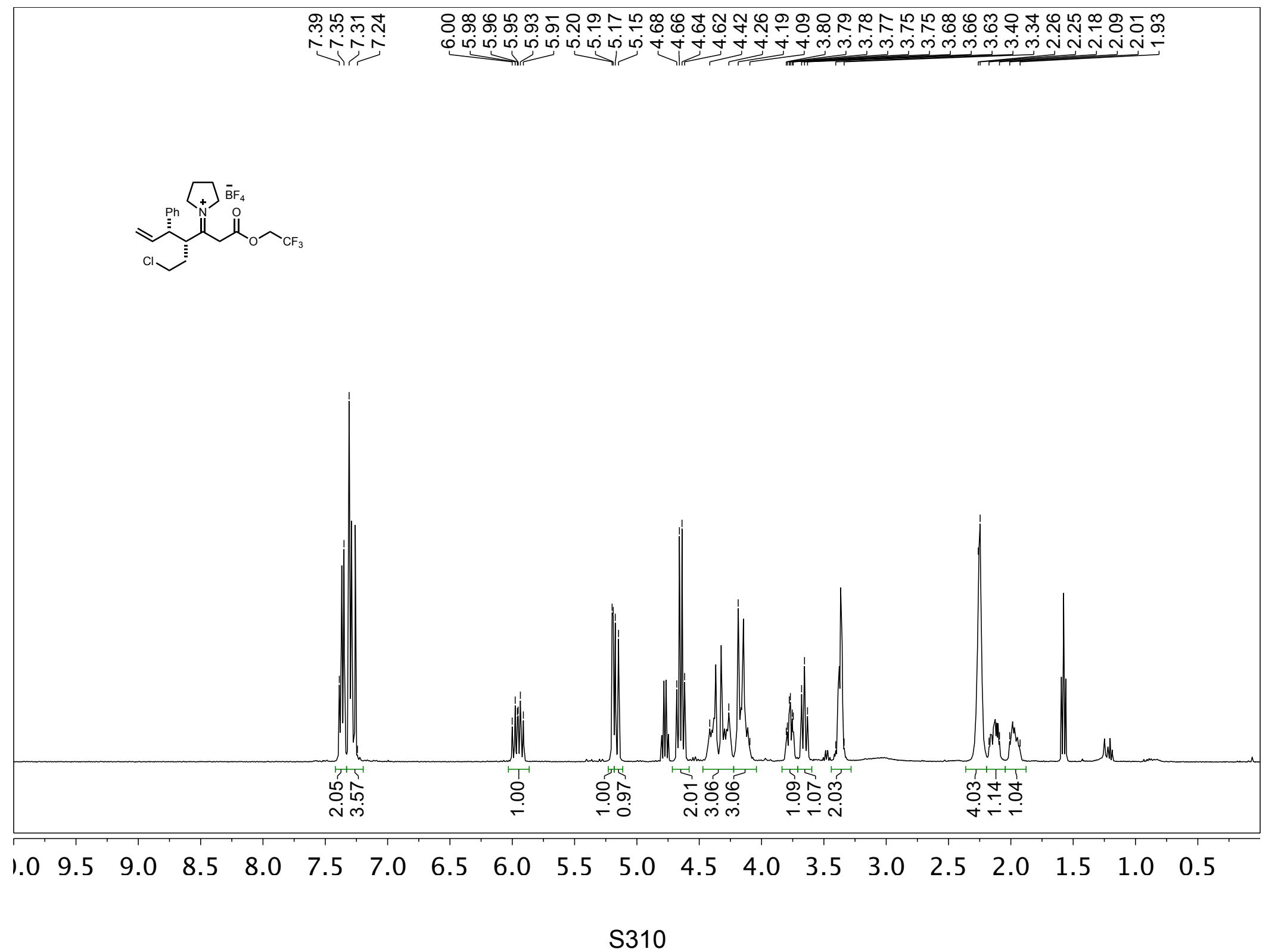




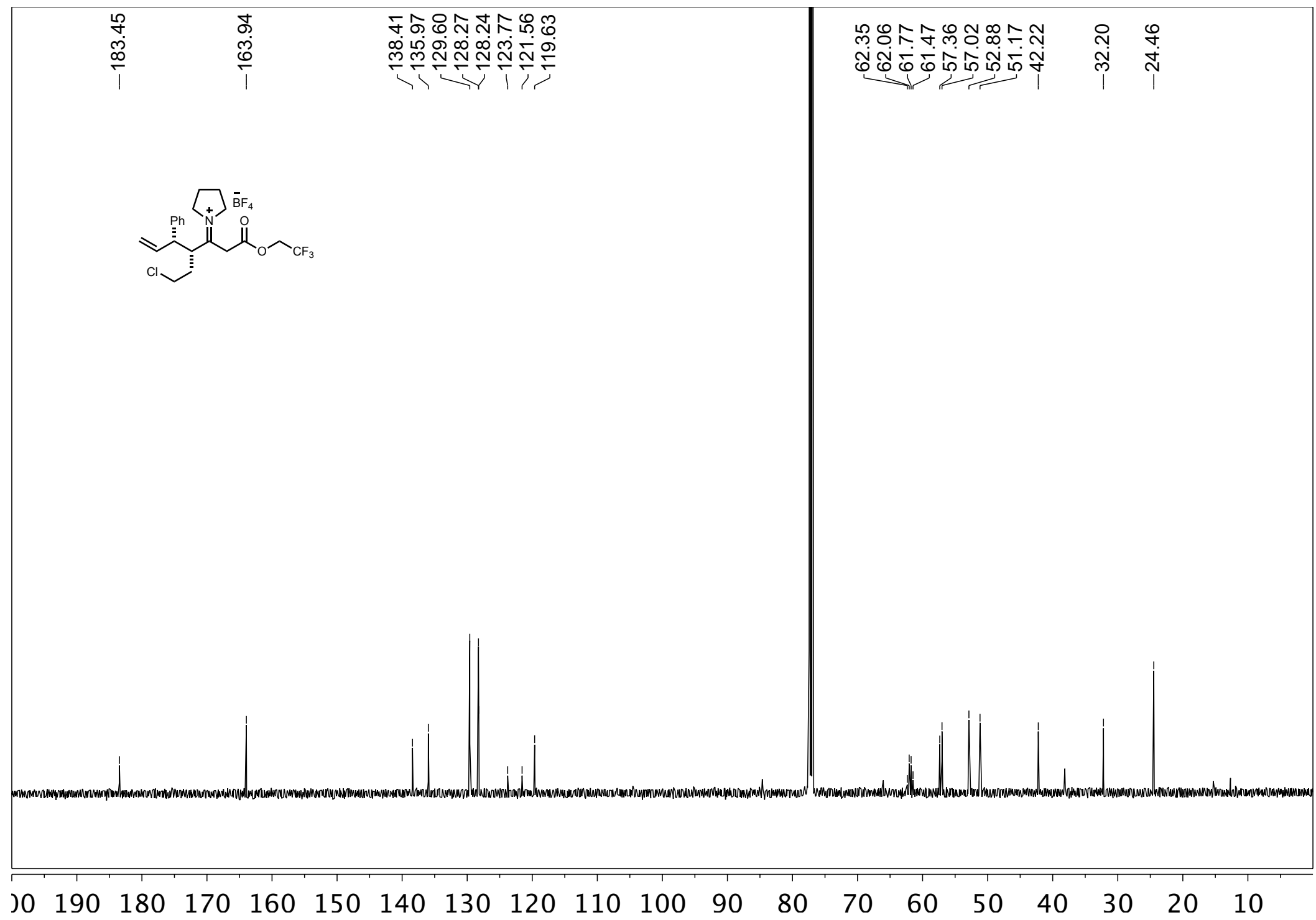

S311 


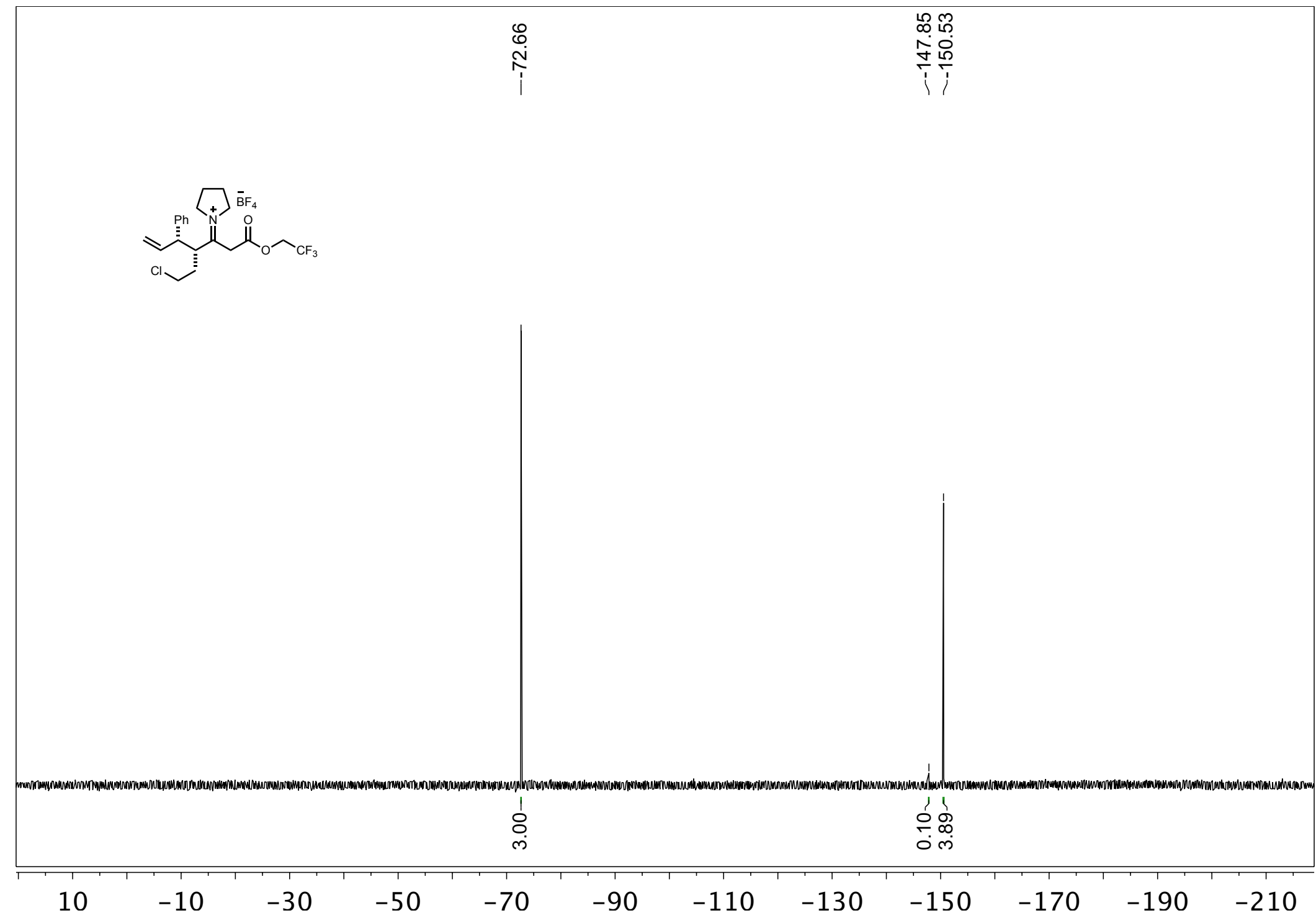




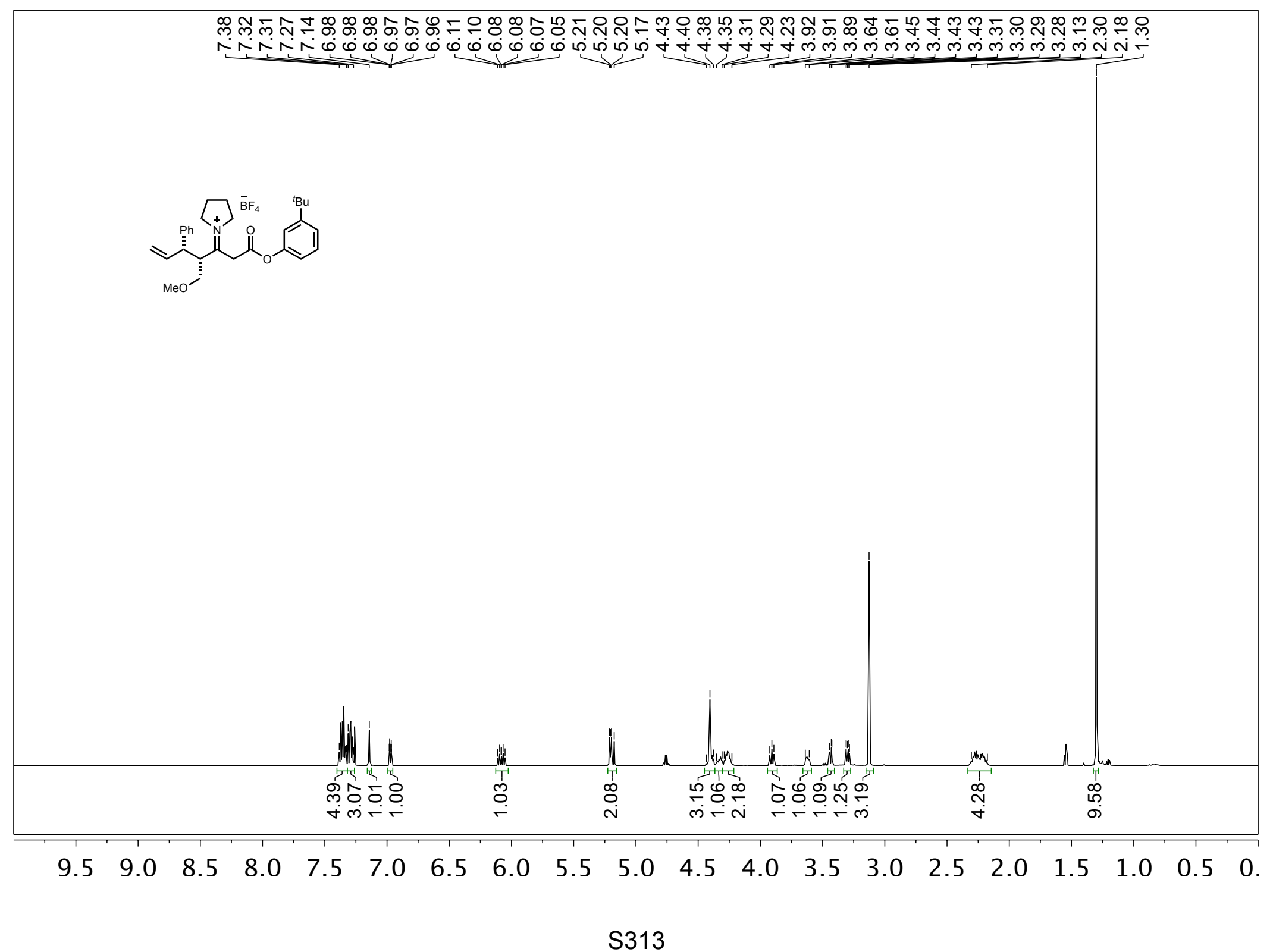




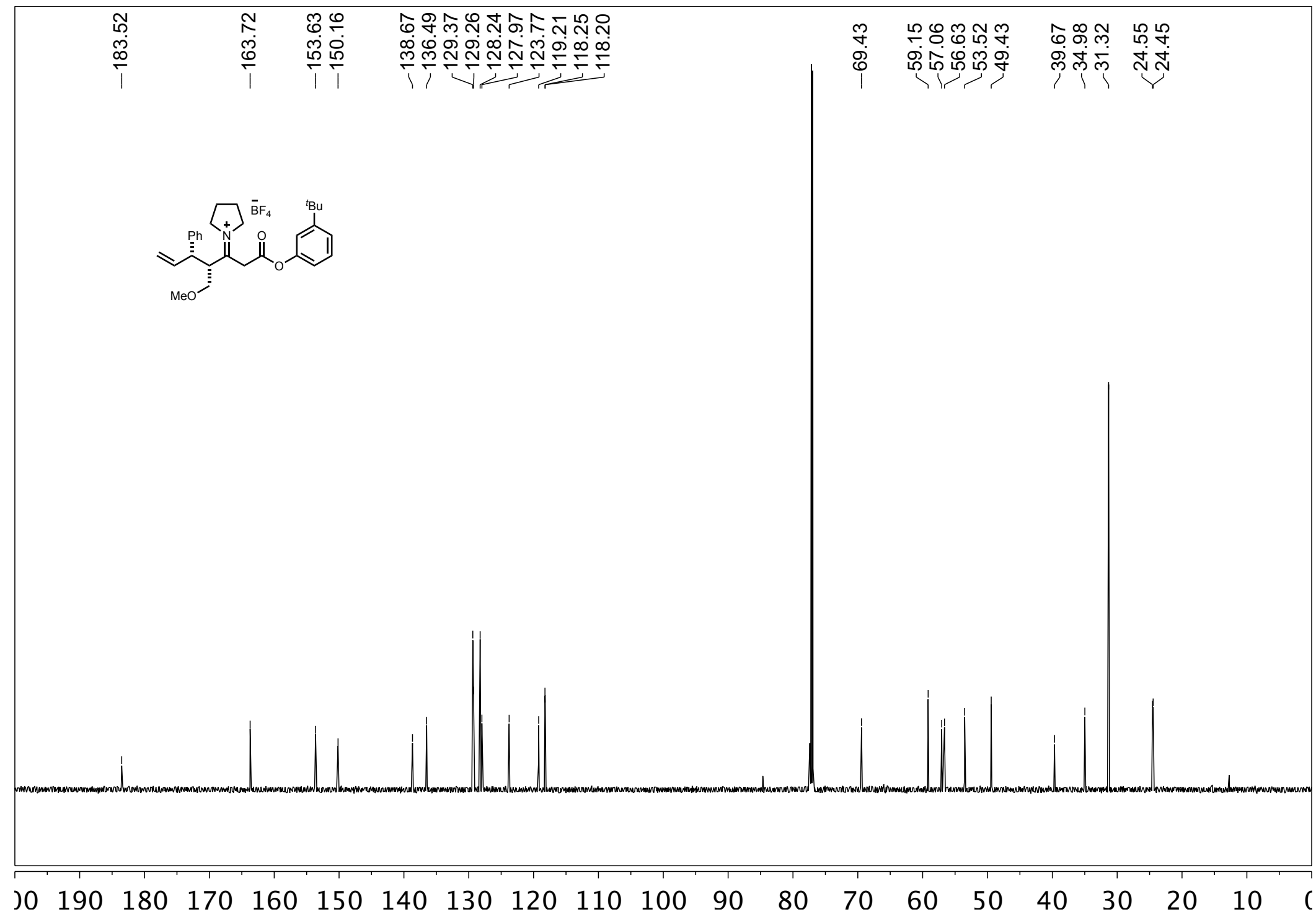

S314 


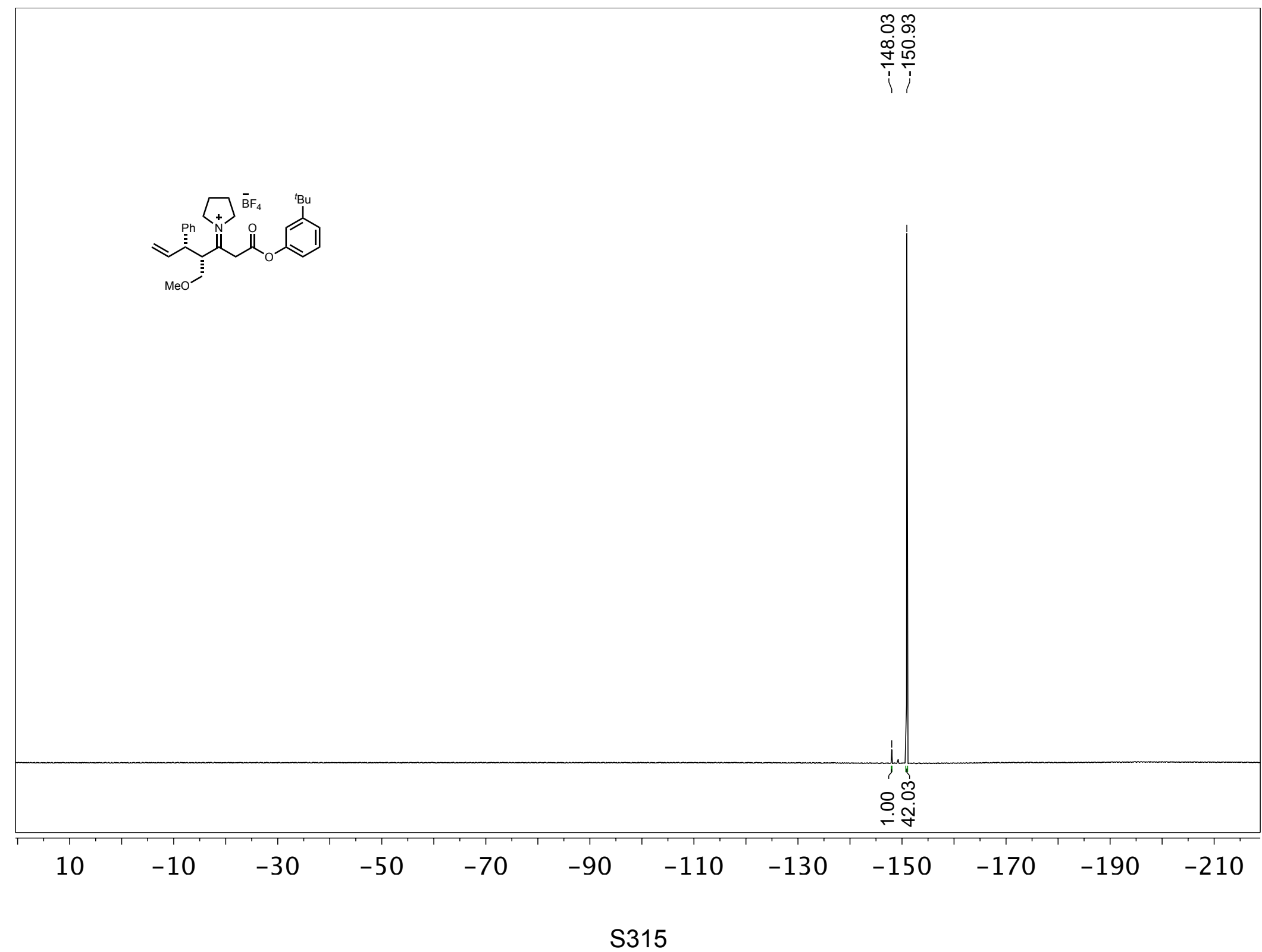




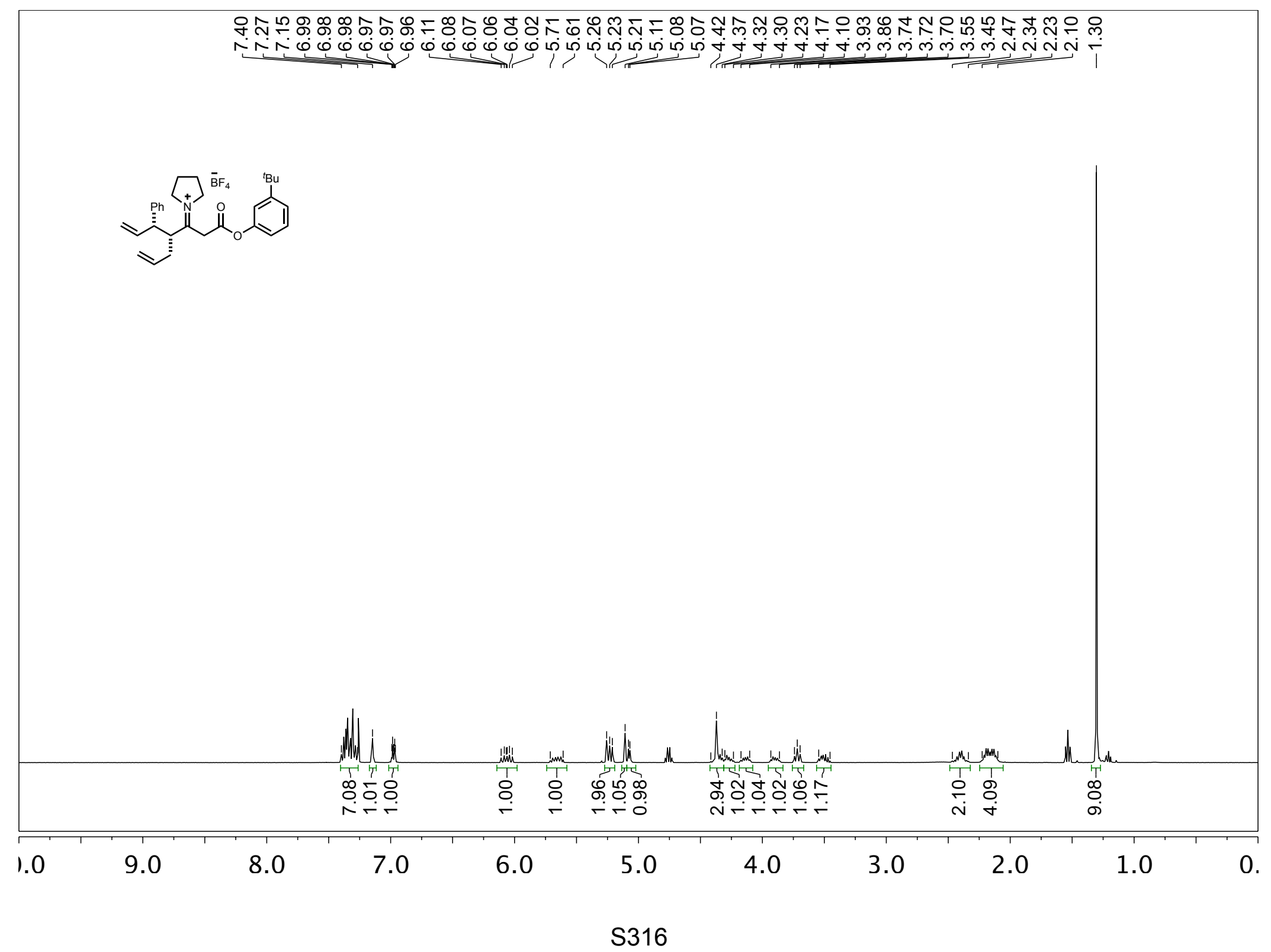




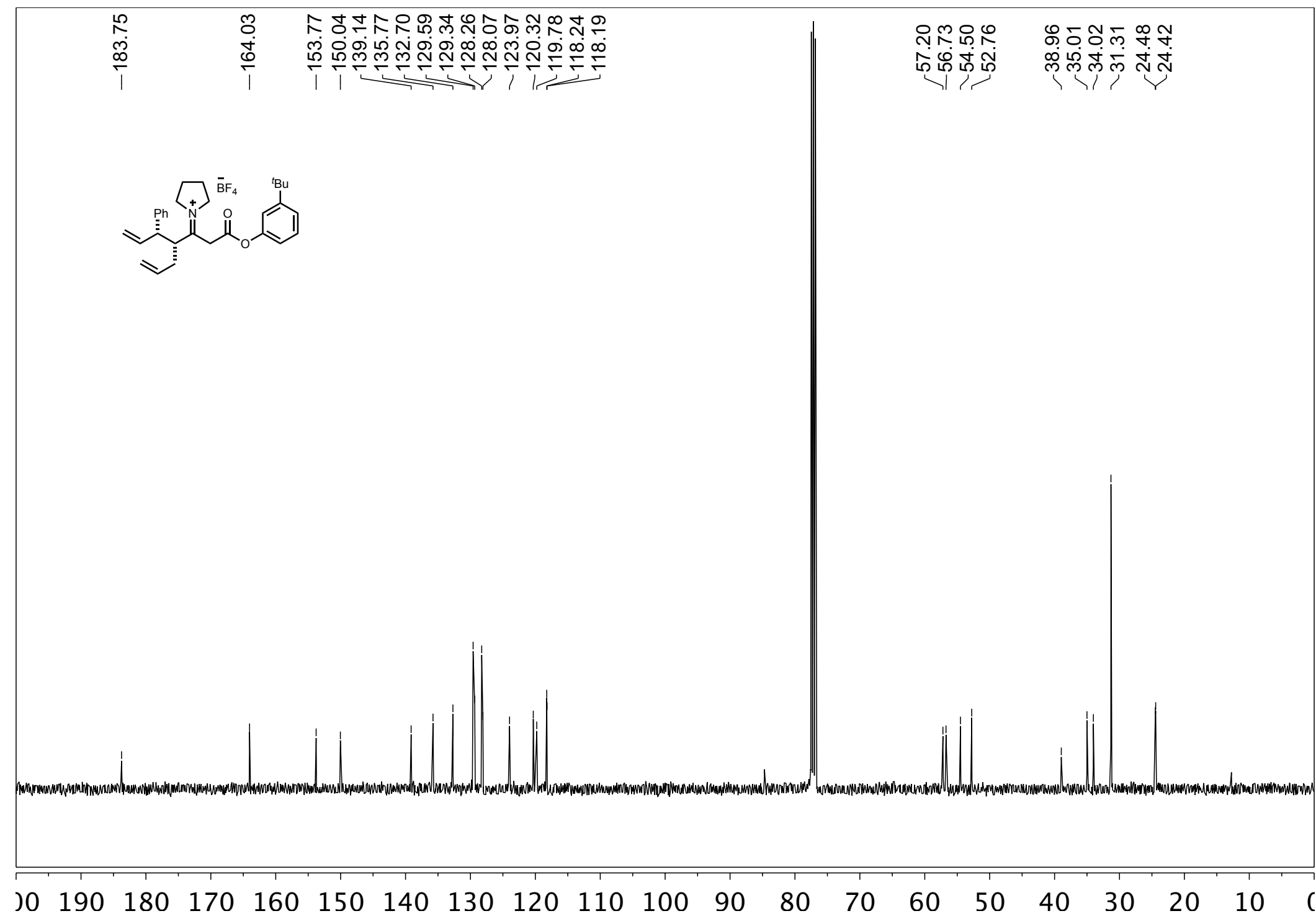




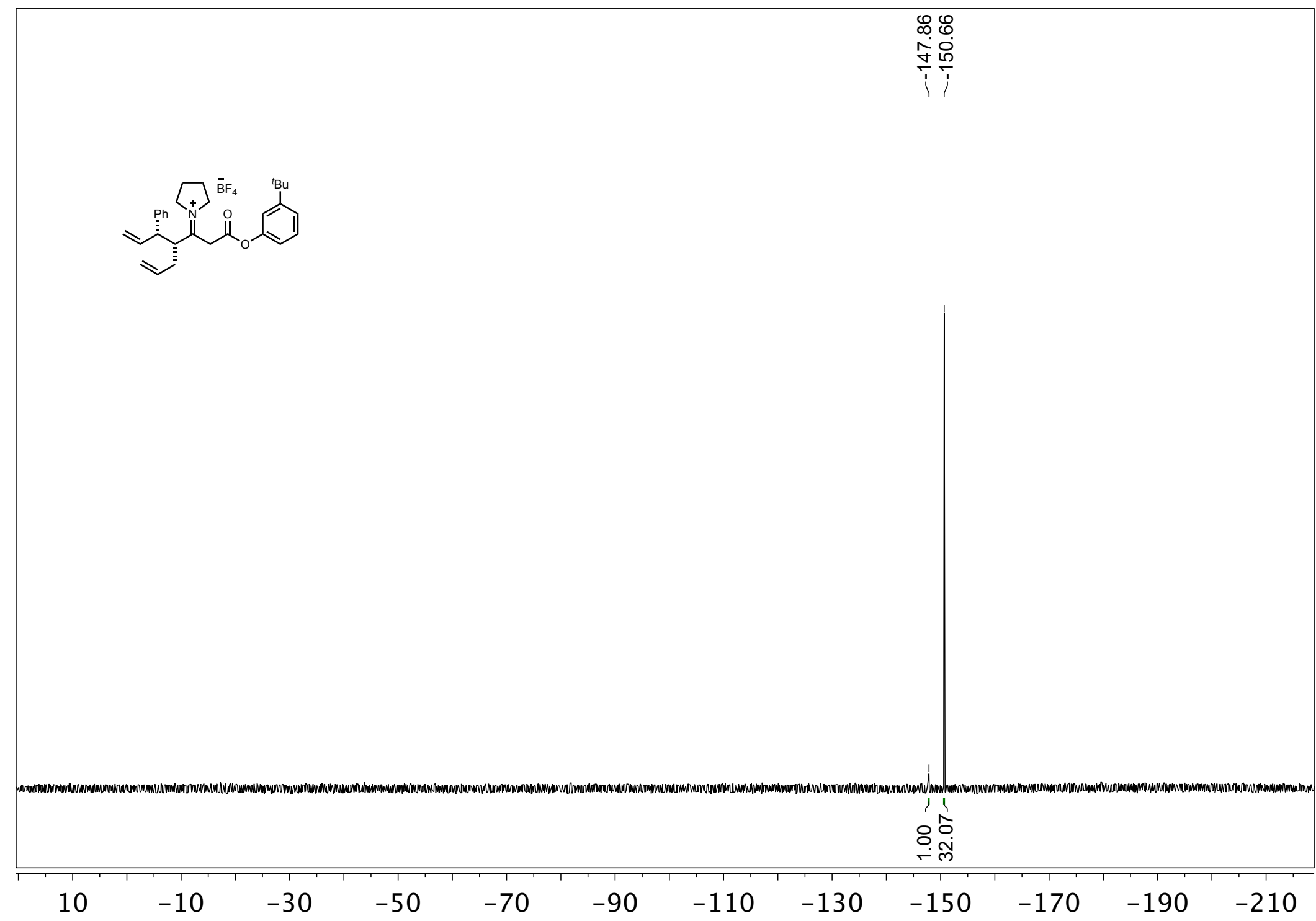




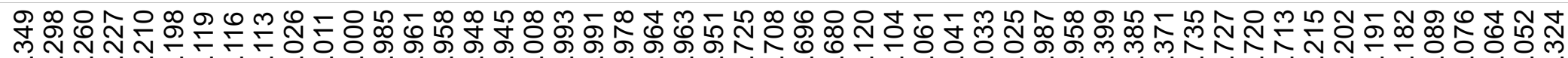

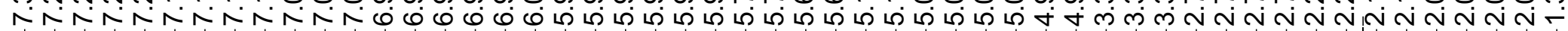
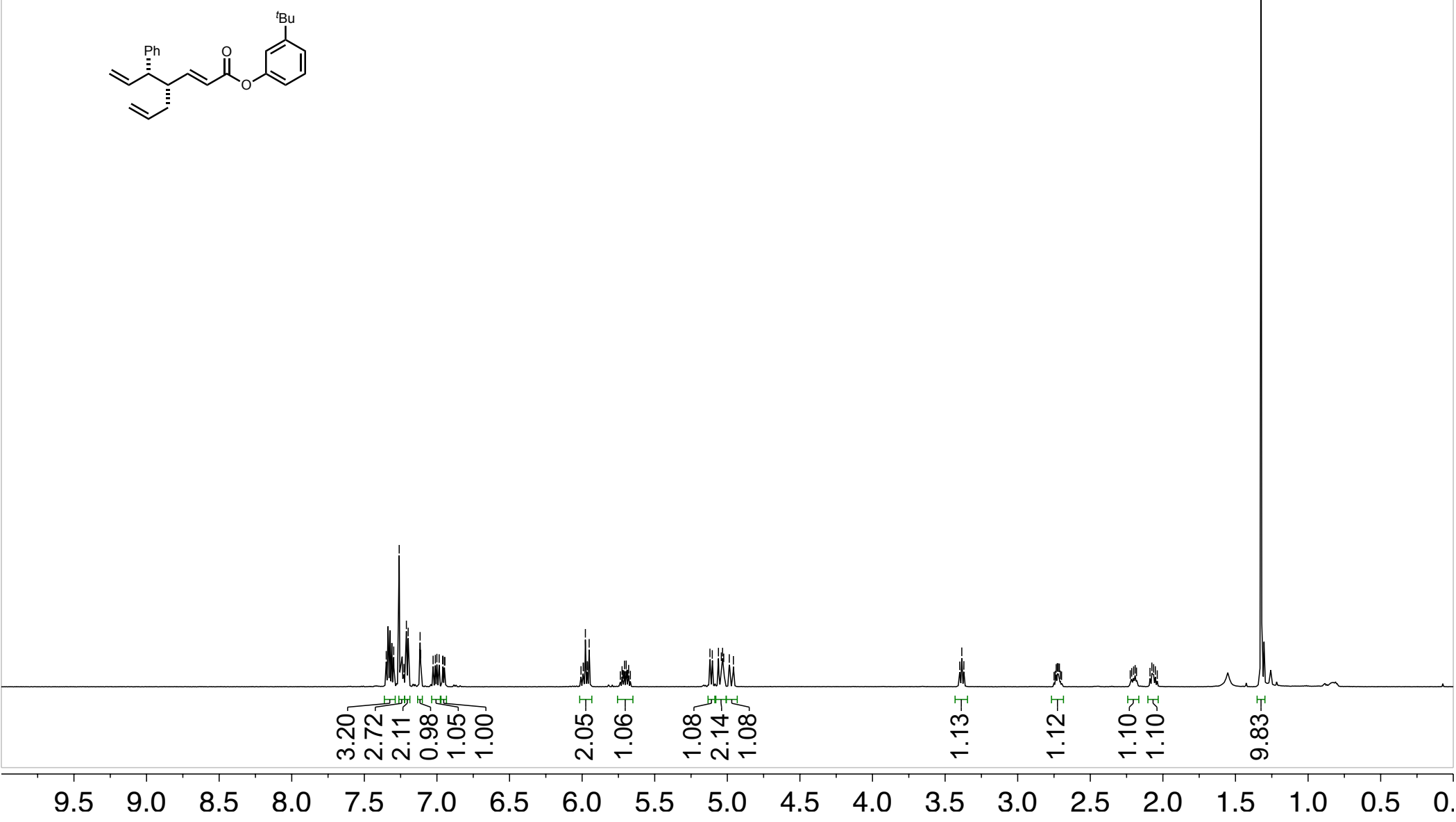


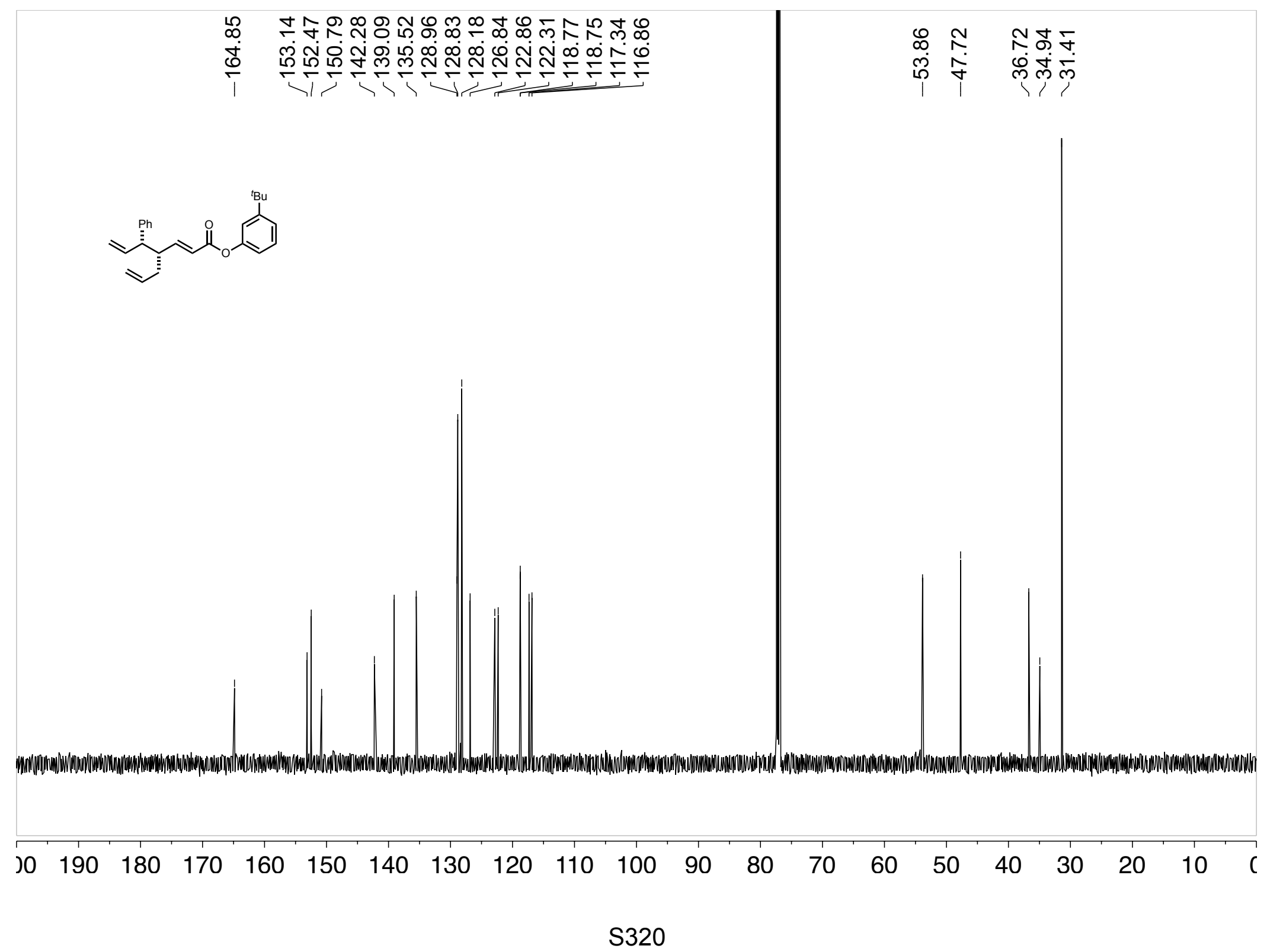




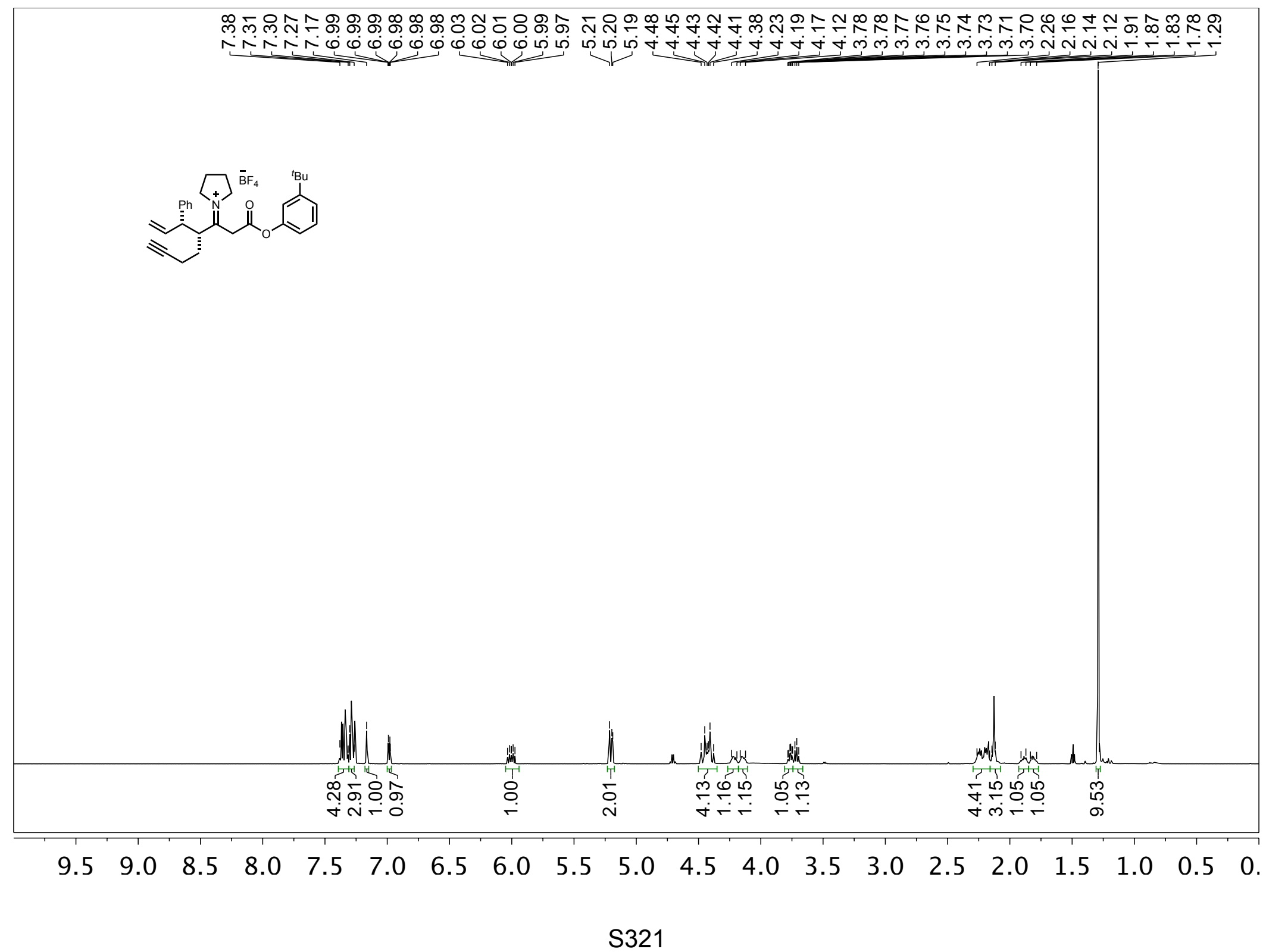




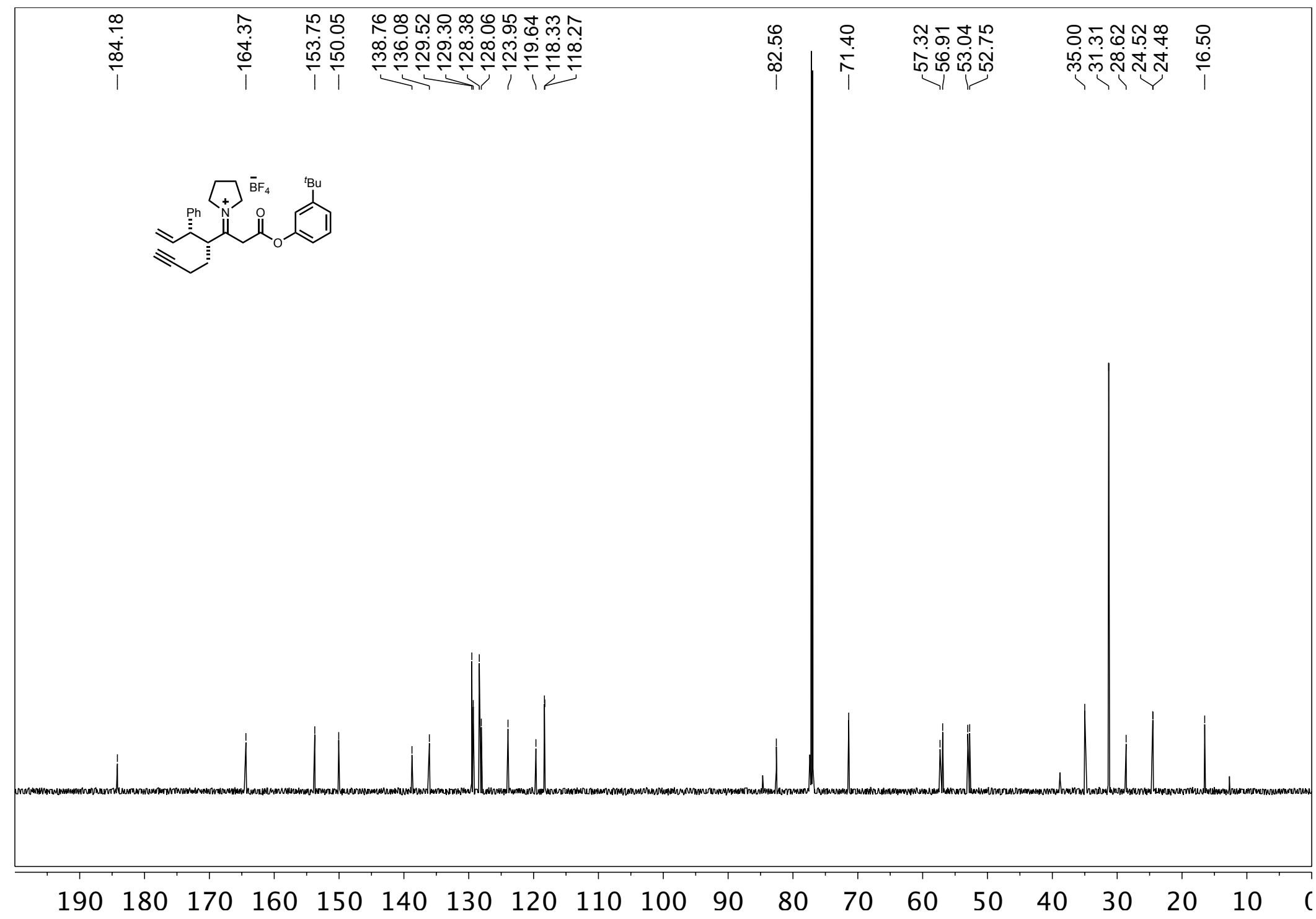




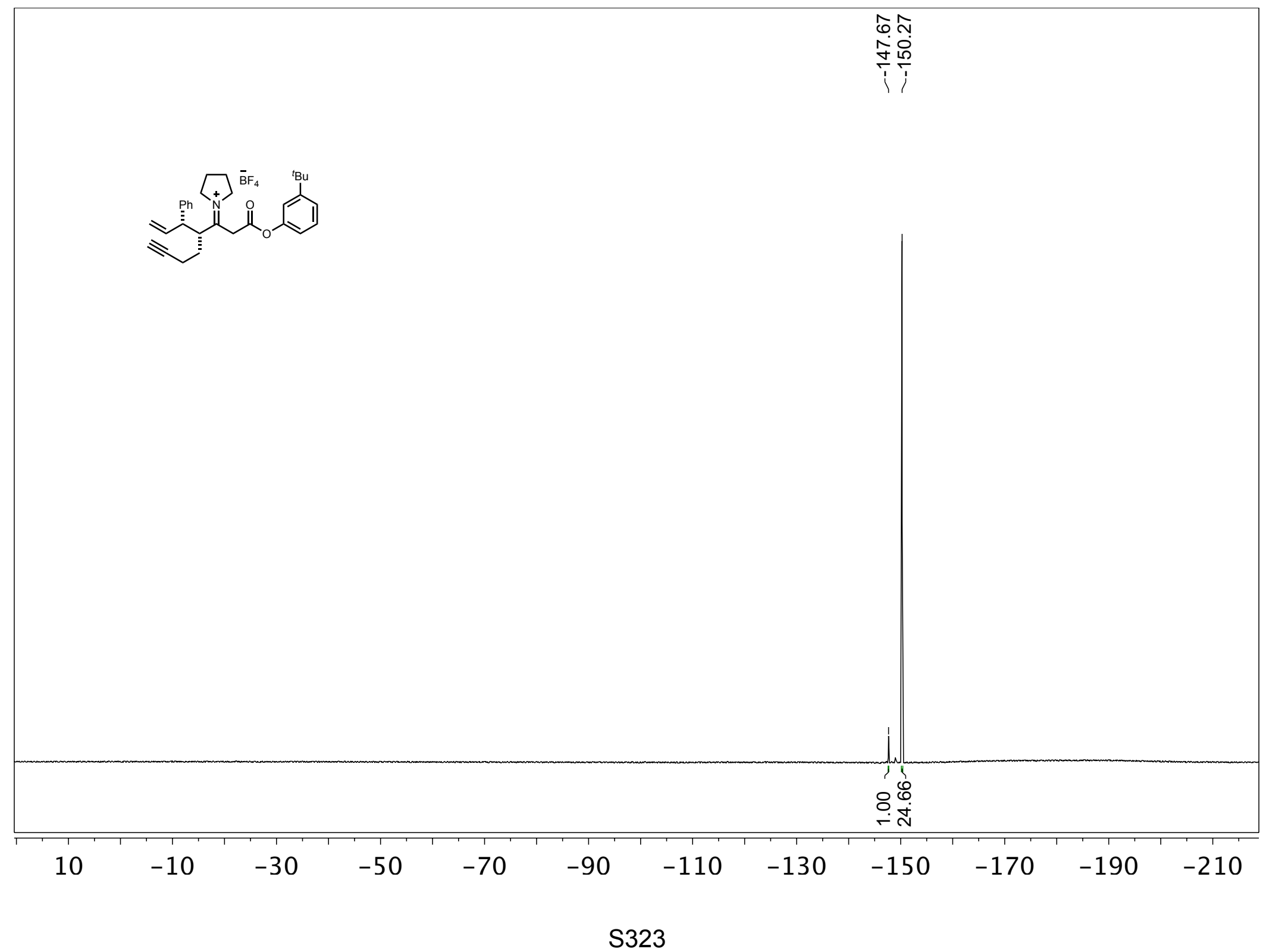




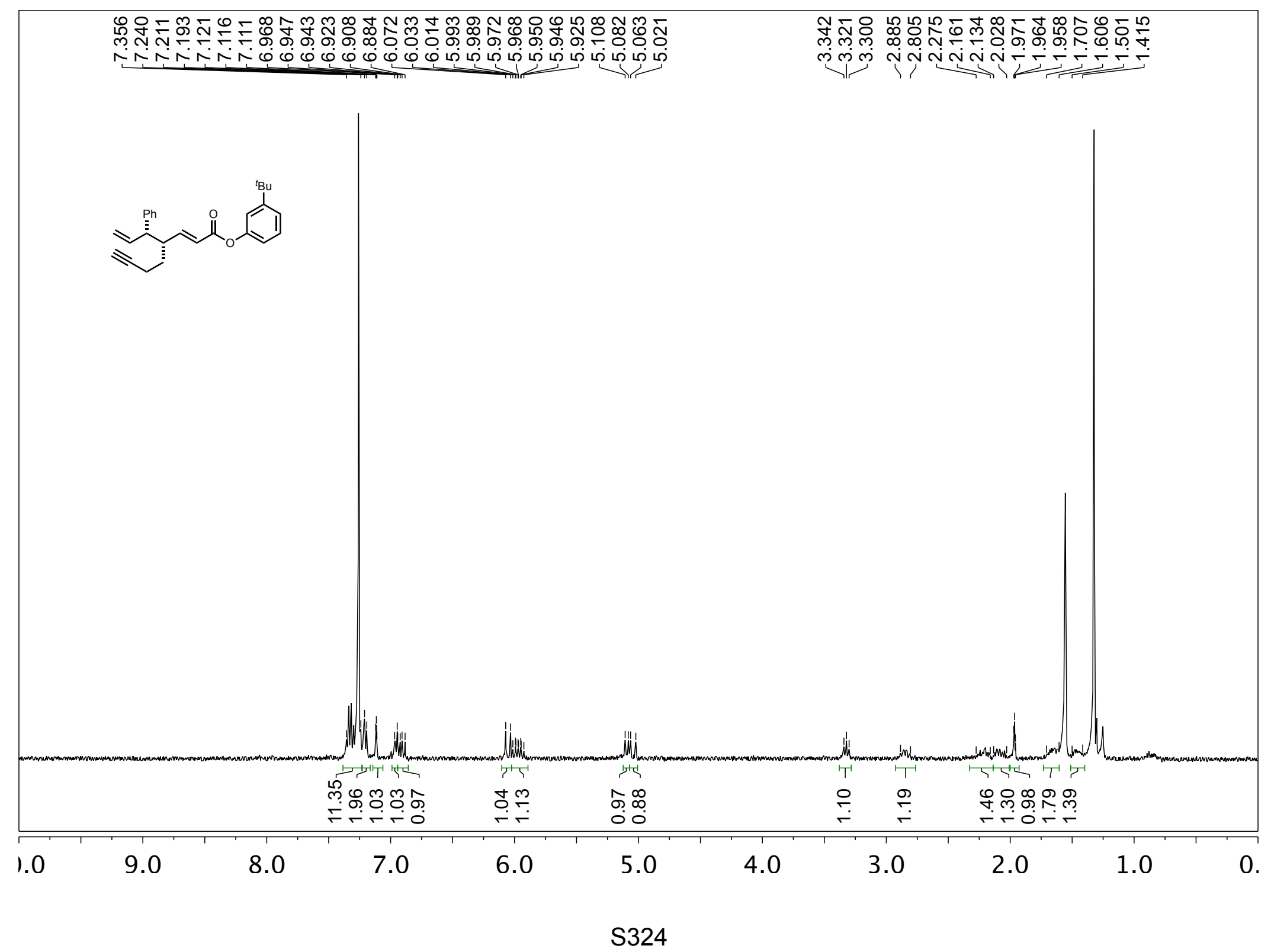




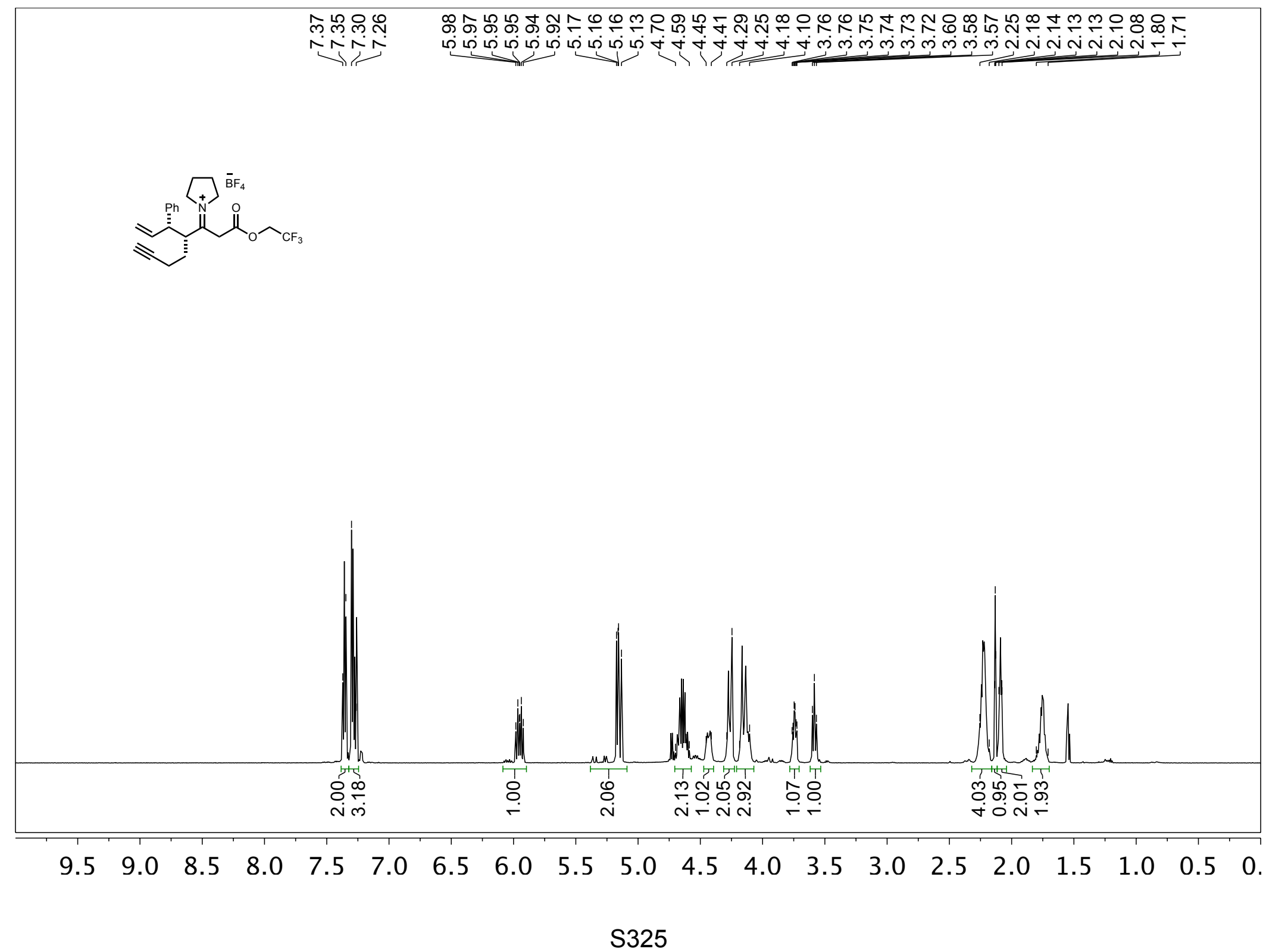




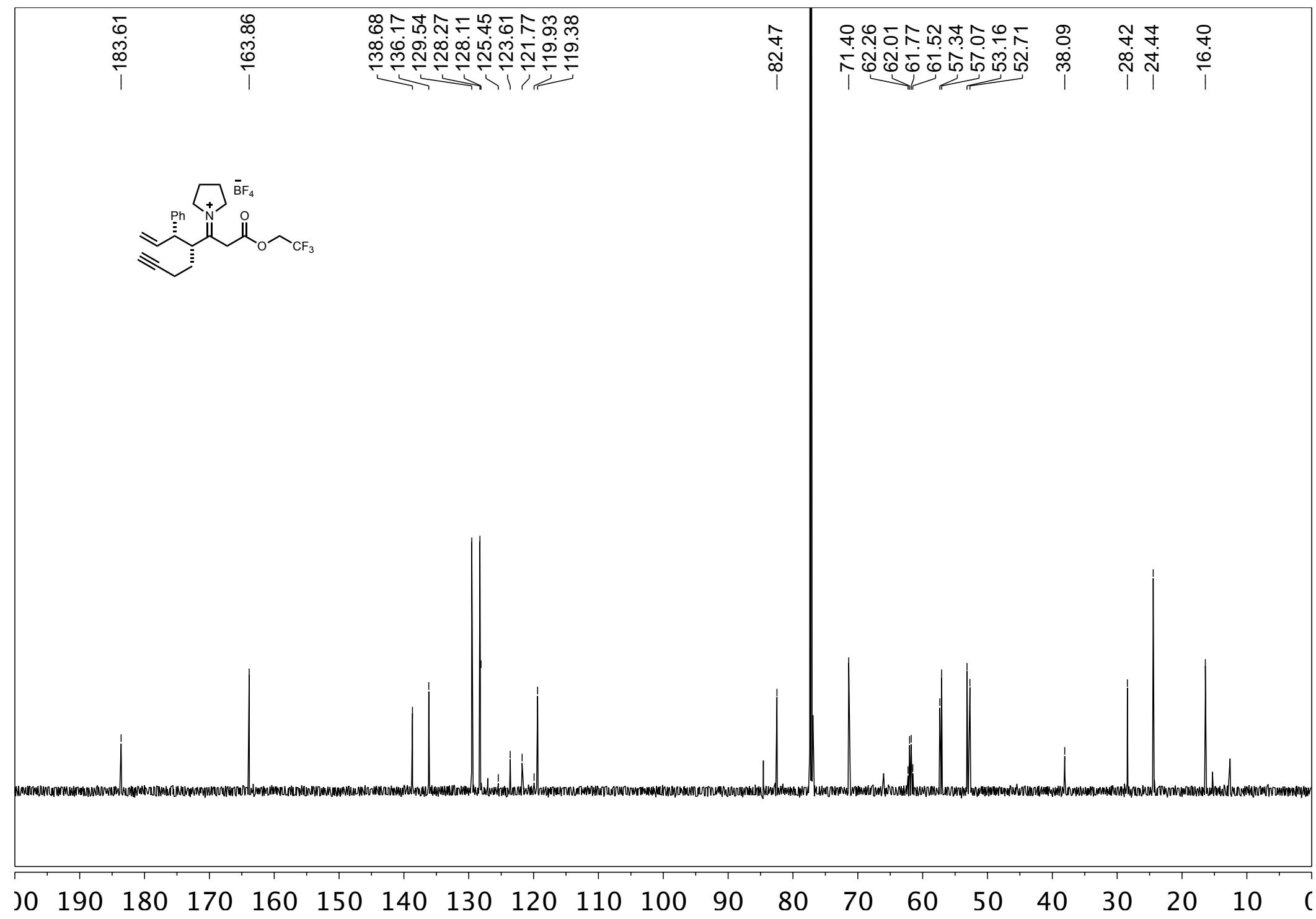




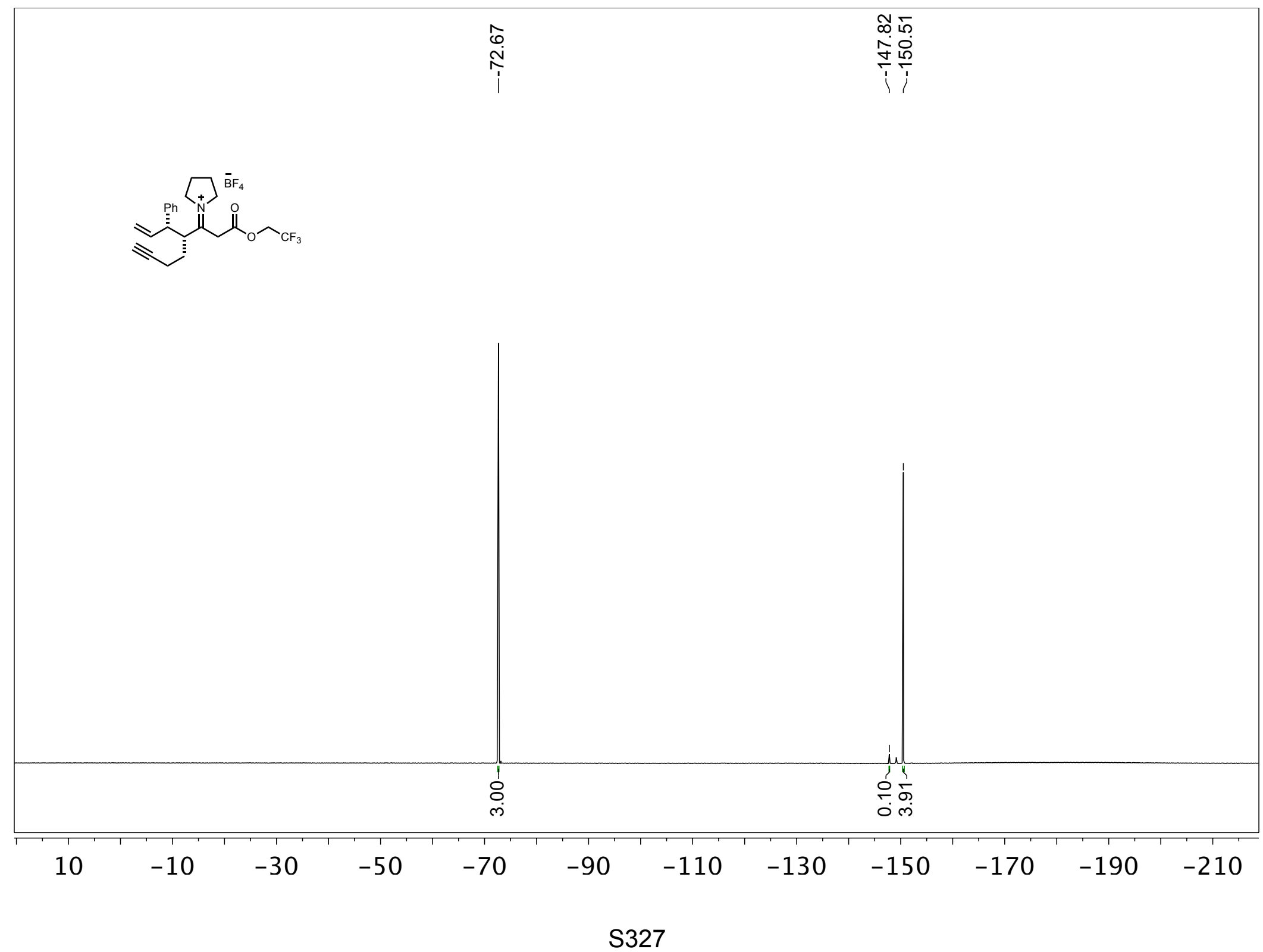




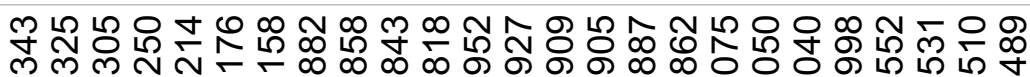

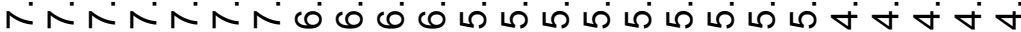

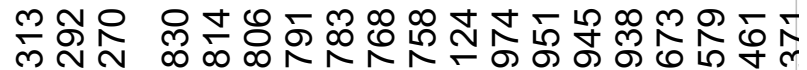
लं लें

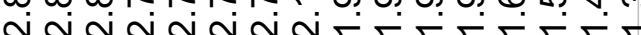

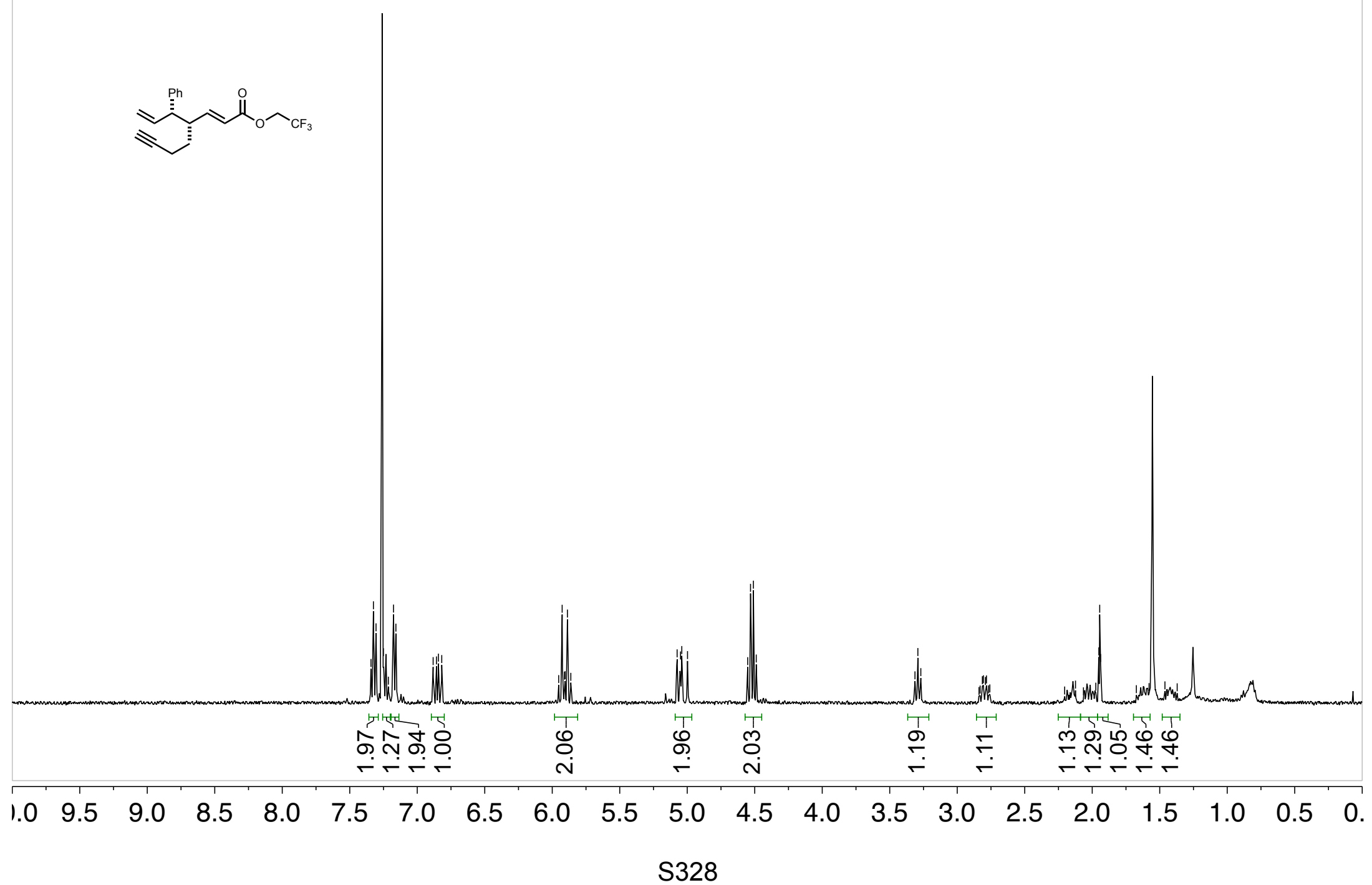




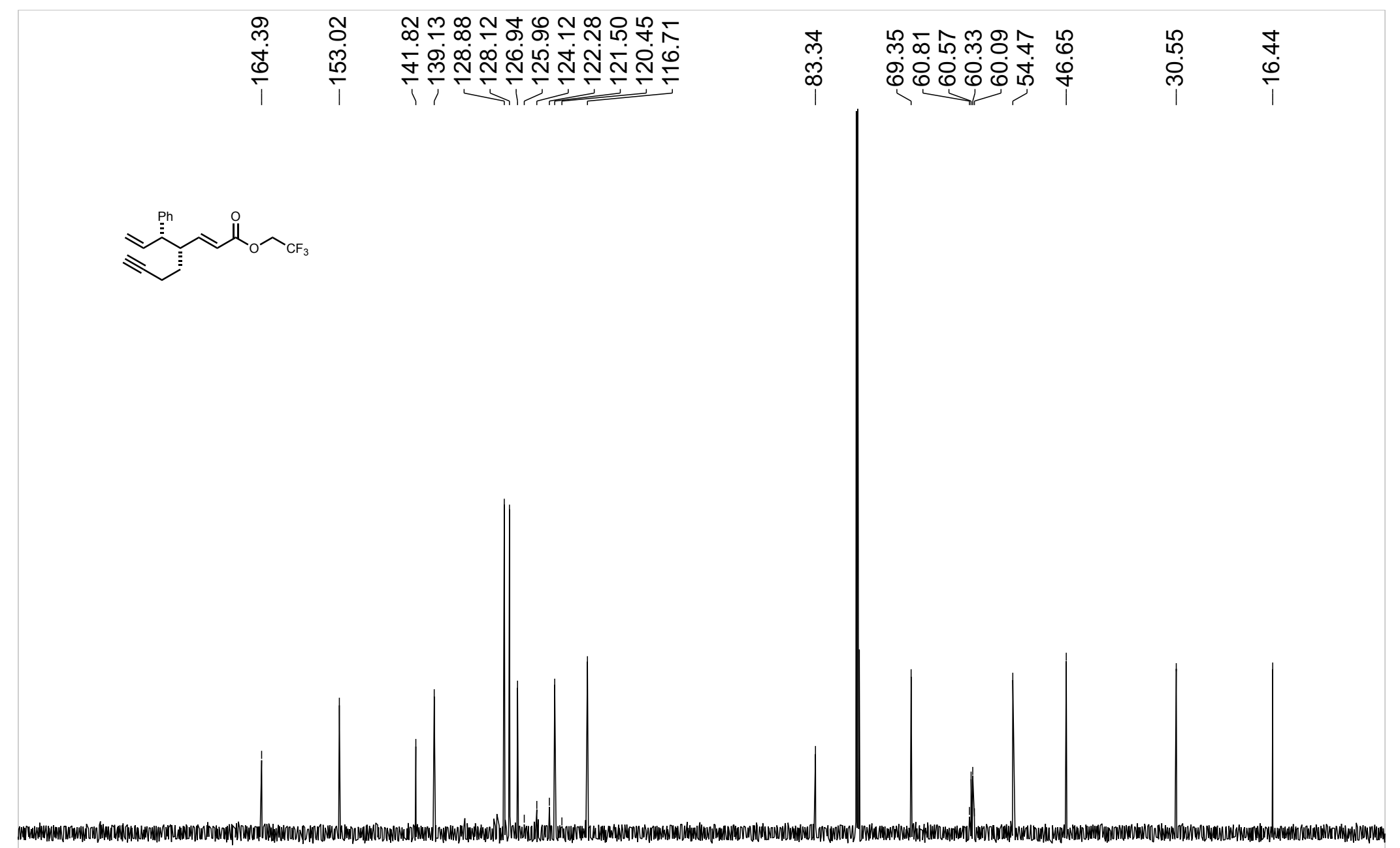

60

50




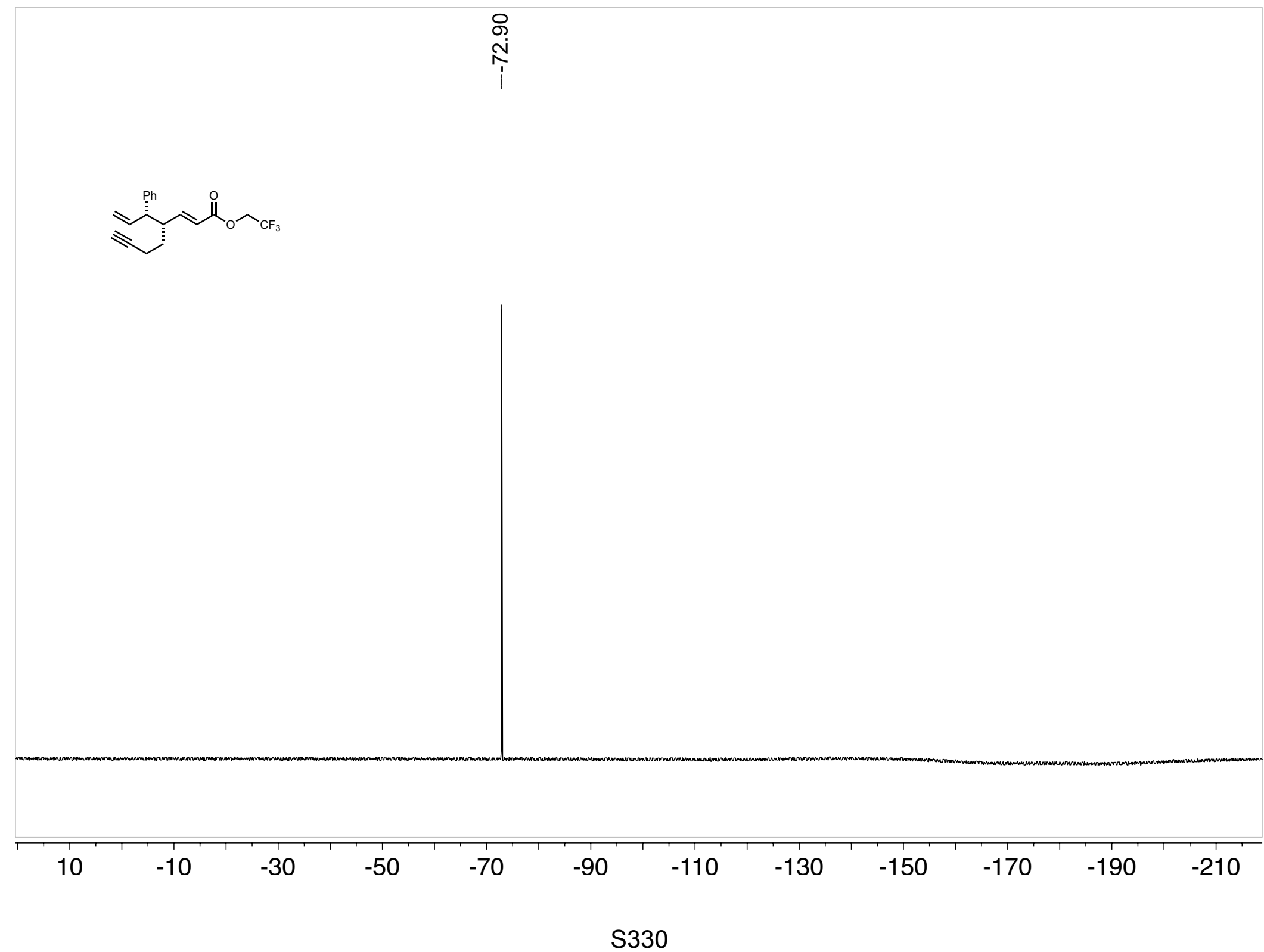




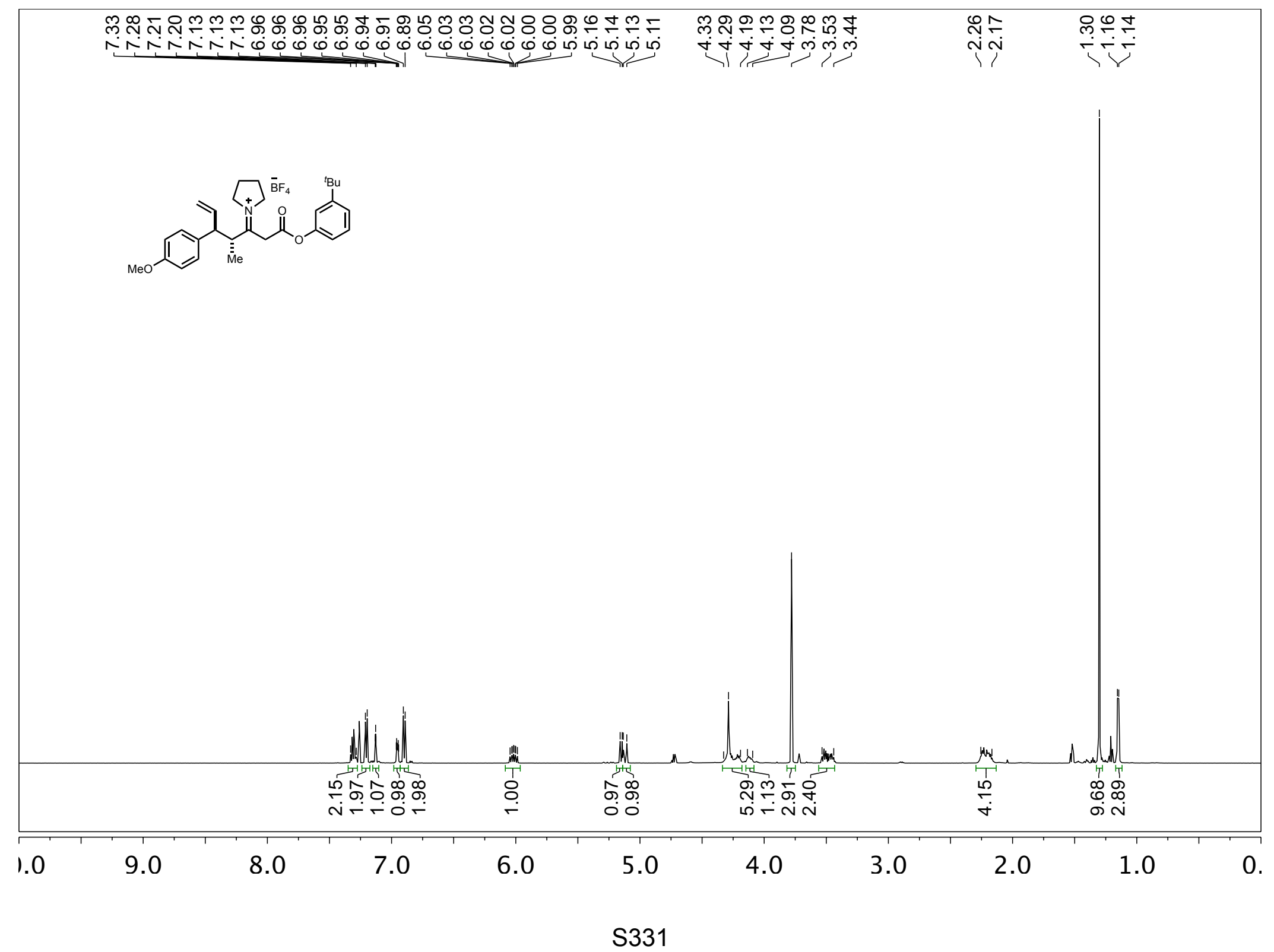




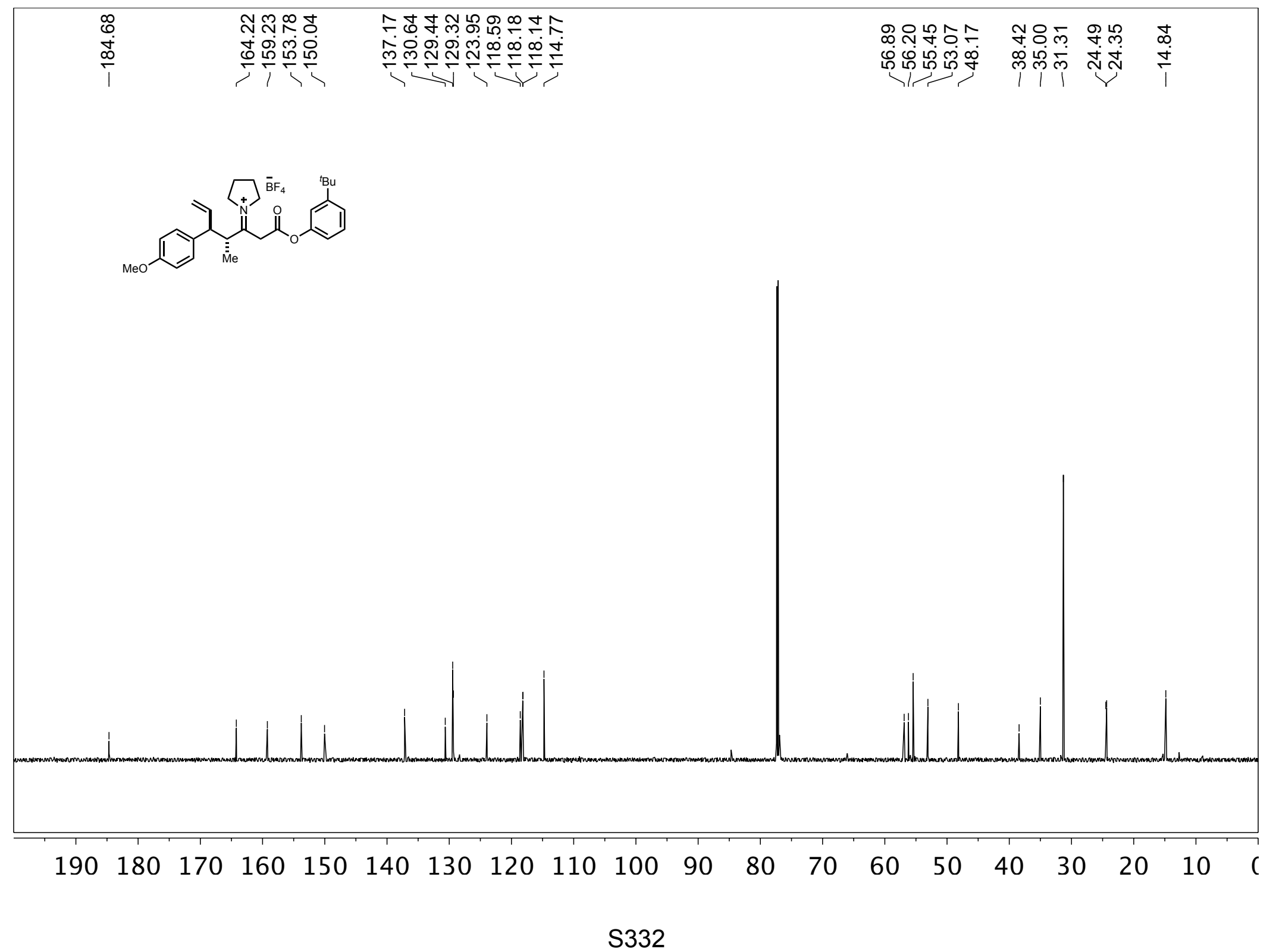




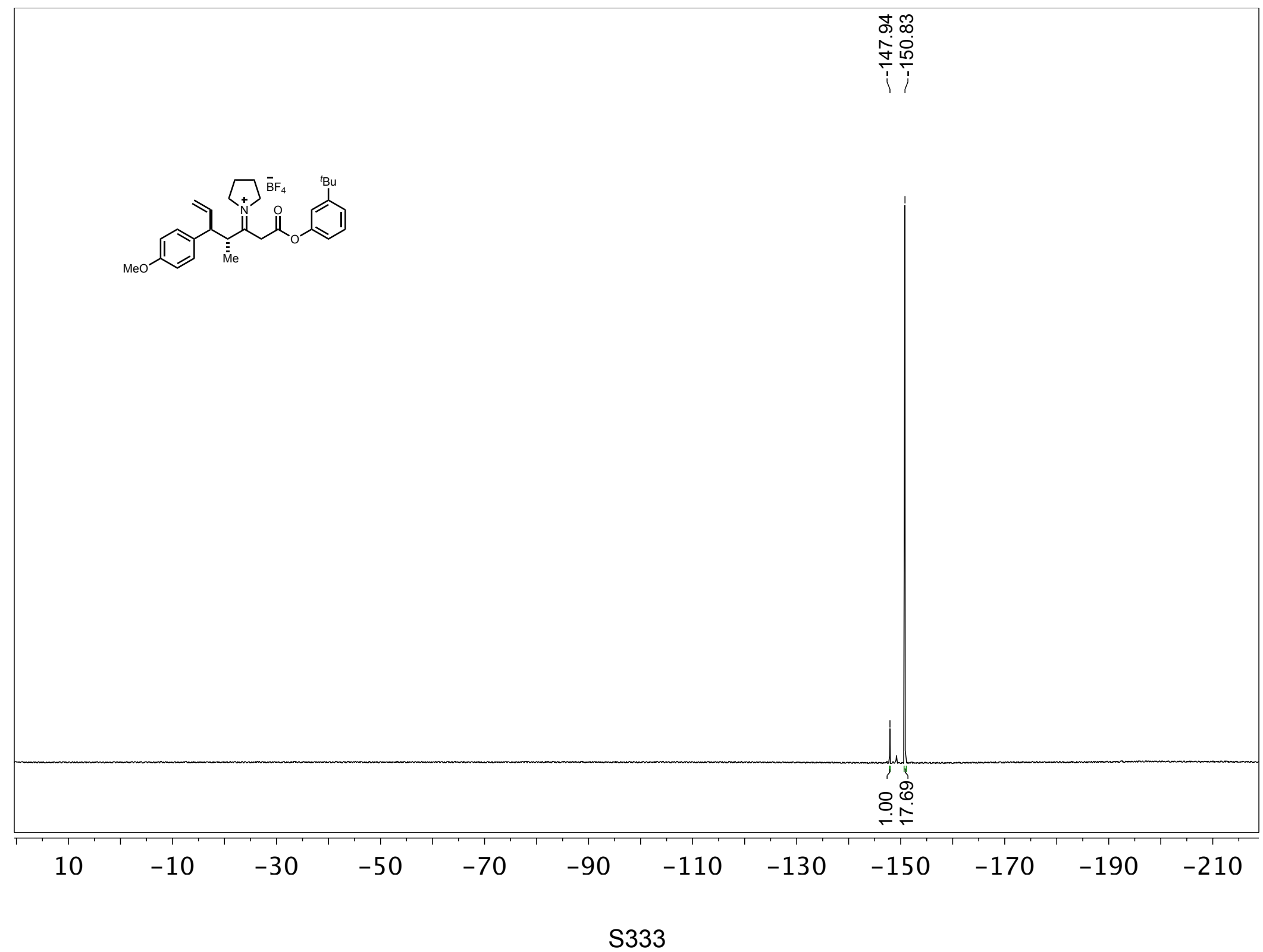




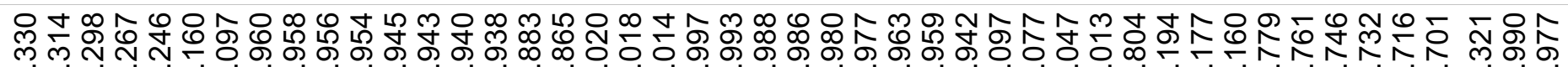
N-N N N

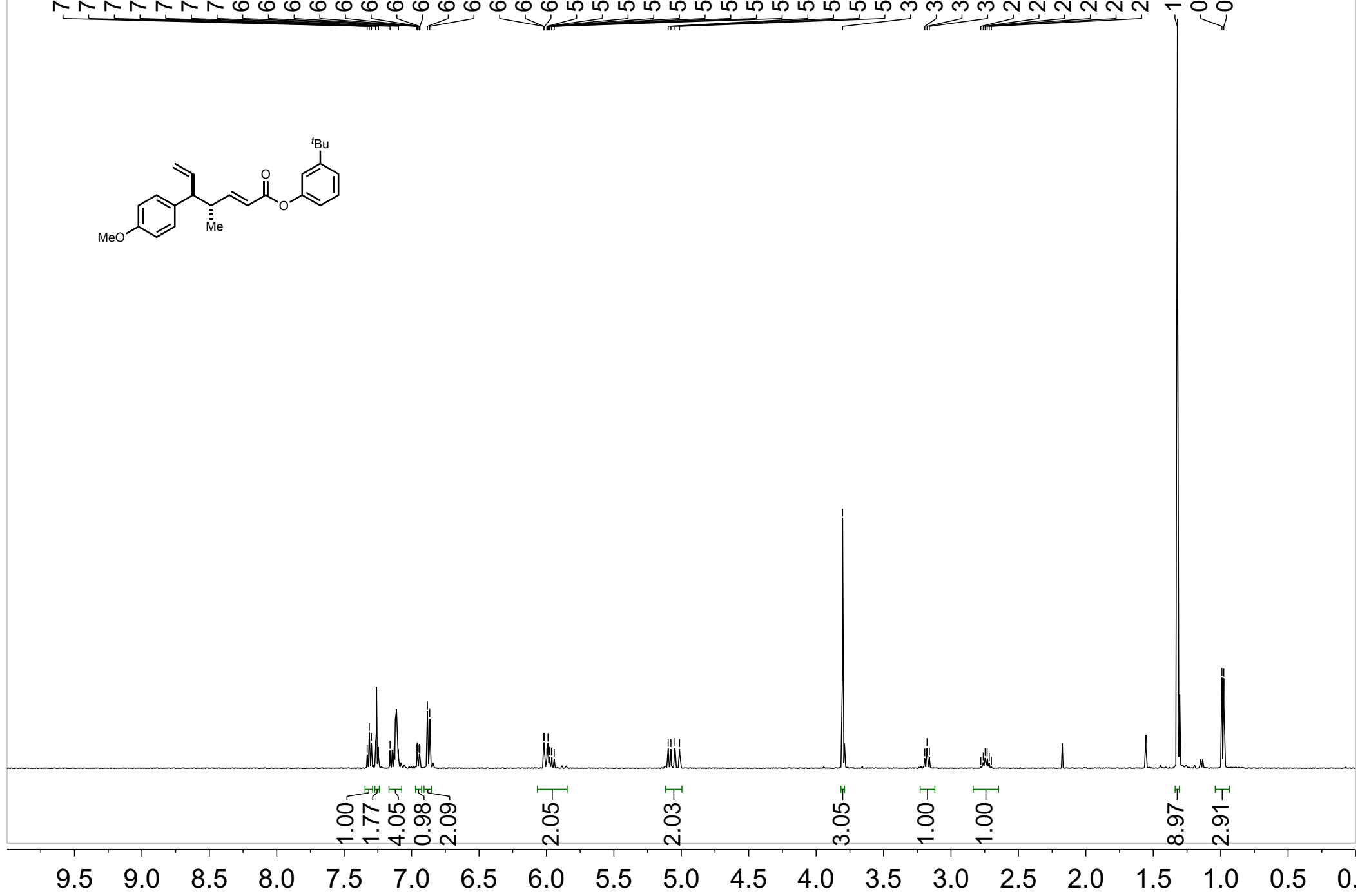




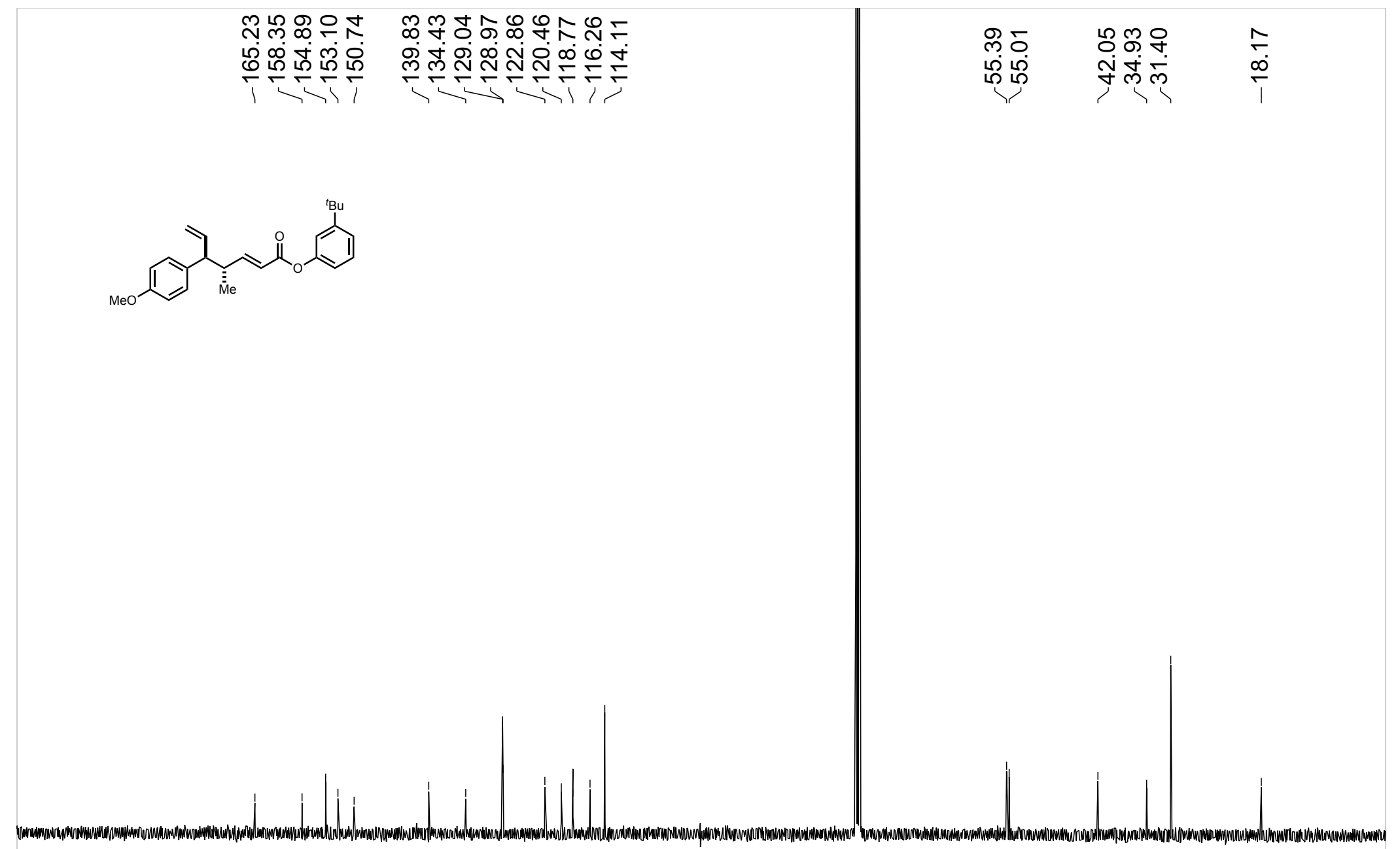

$\begin{array}{llllllllll}190 & 180 & 170 & 160 & 150 & 140 & 130 & 120 & 110 & 100\end{array}$

$90 \quad 80$

80

70

$60 \quad 50$




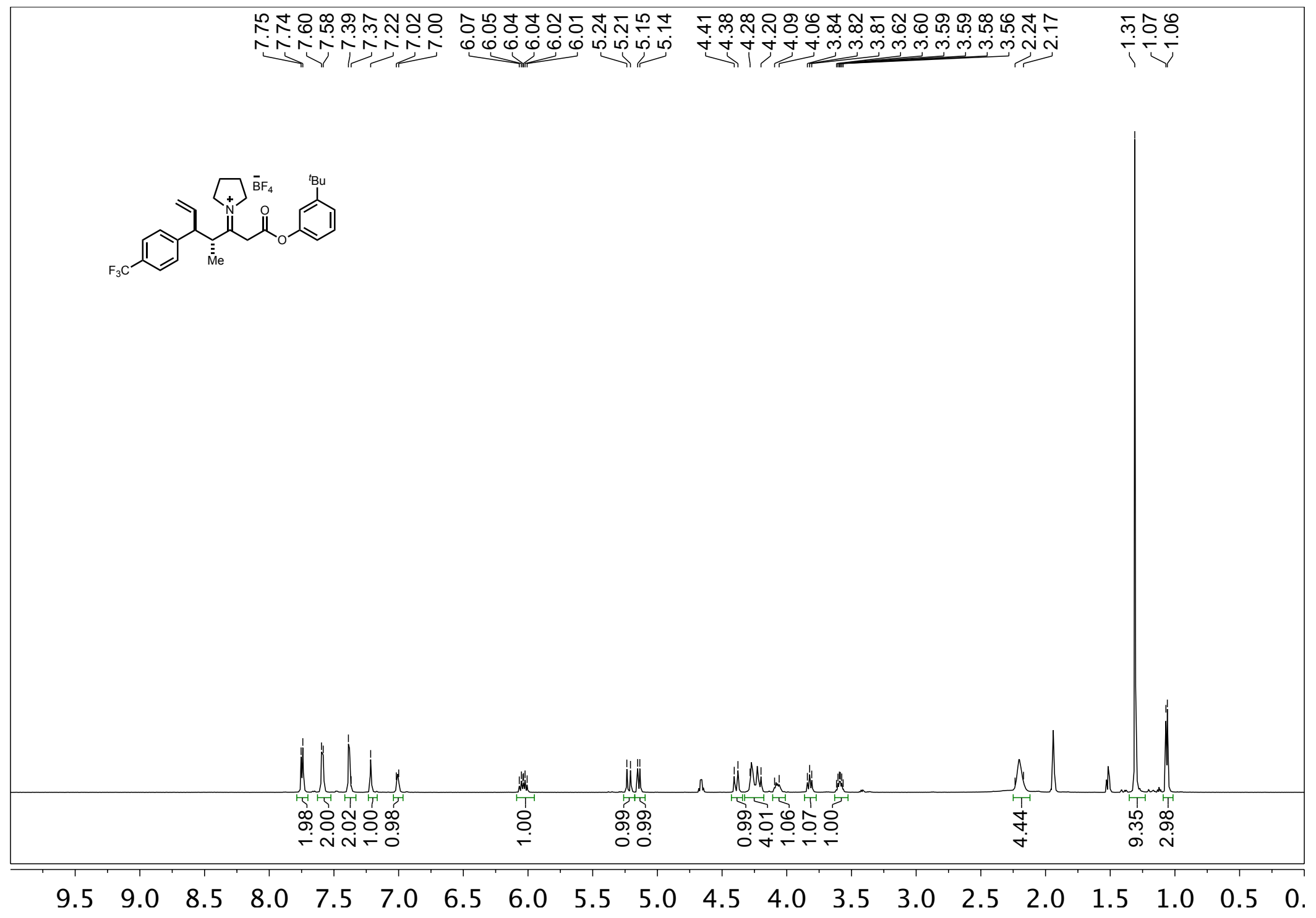

S336 


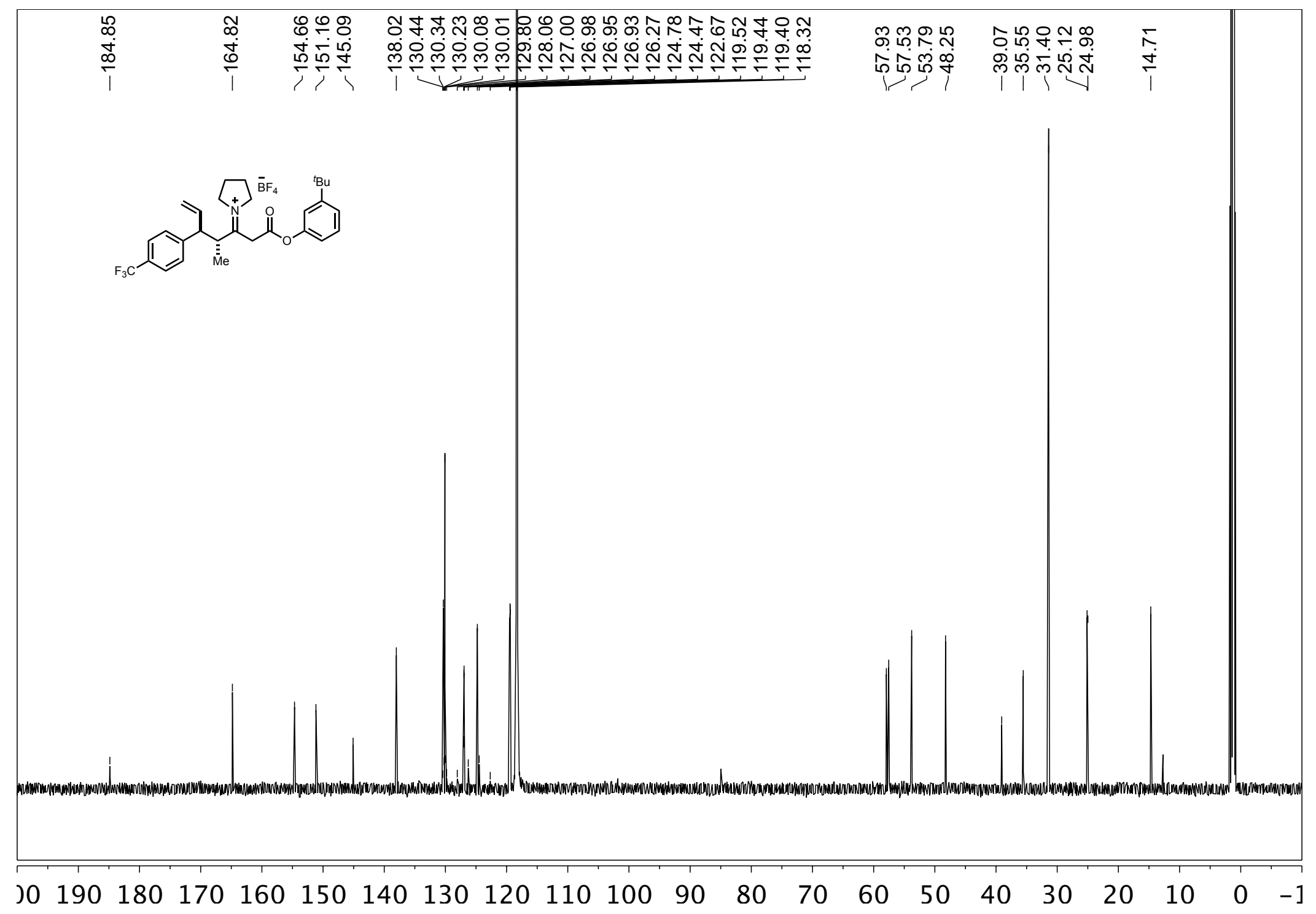




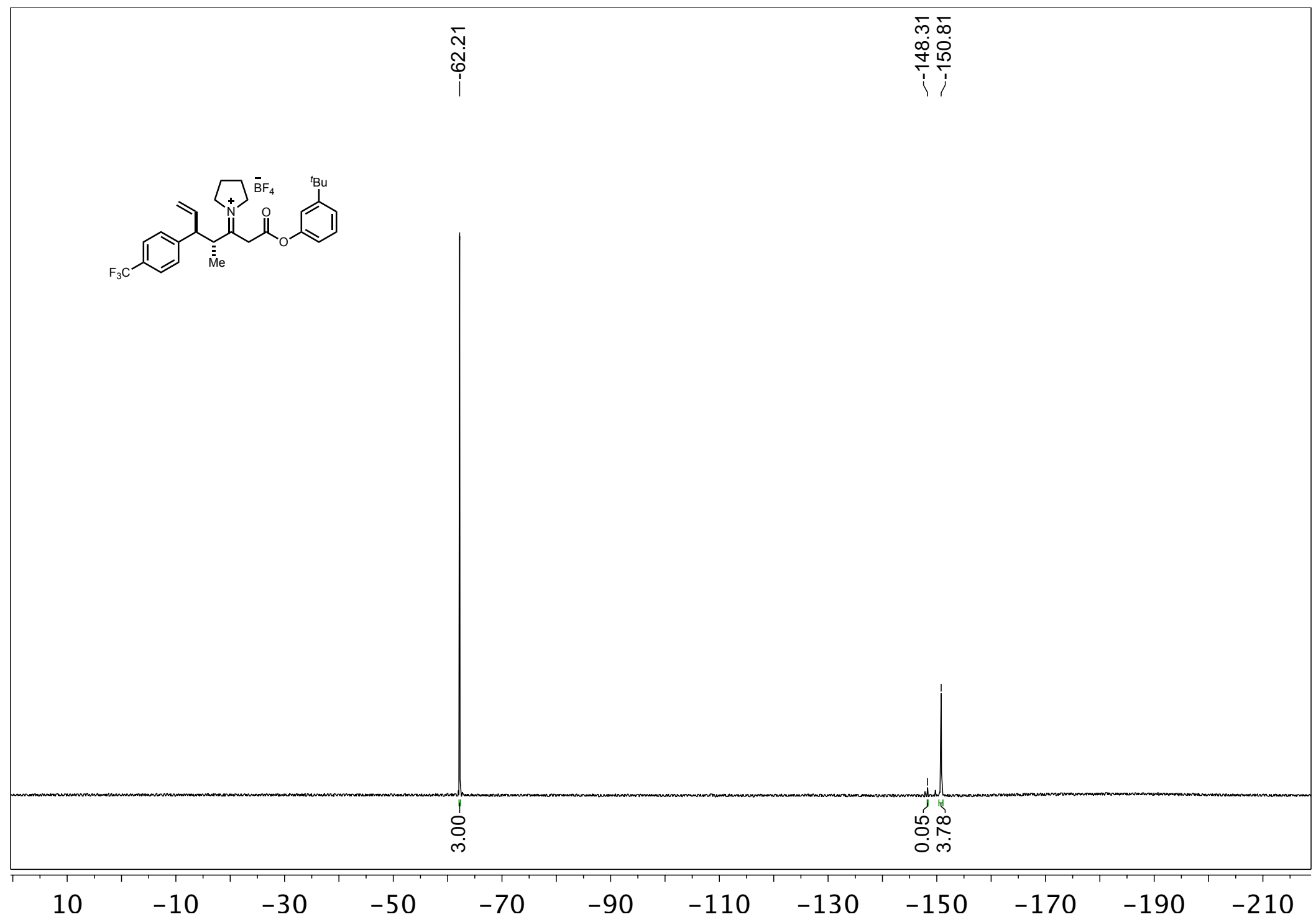

S338 


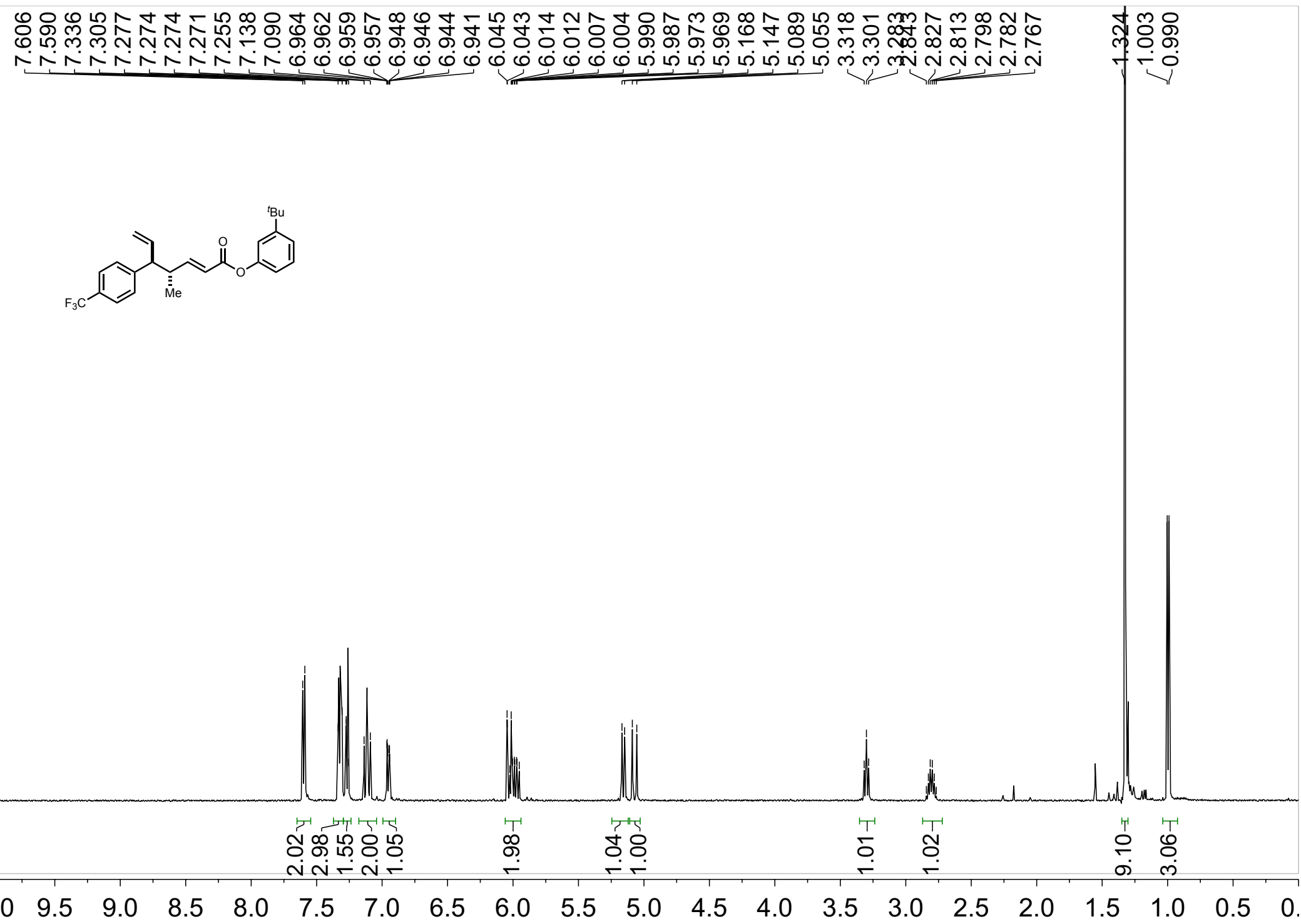




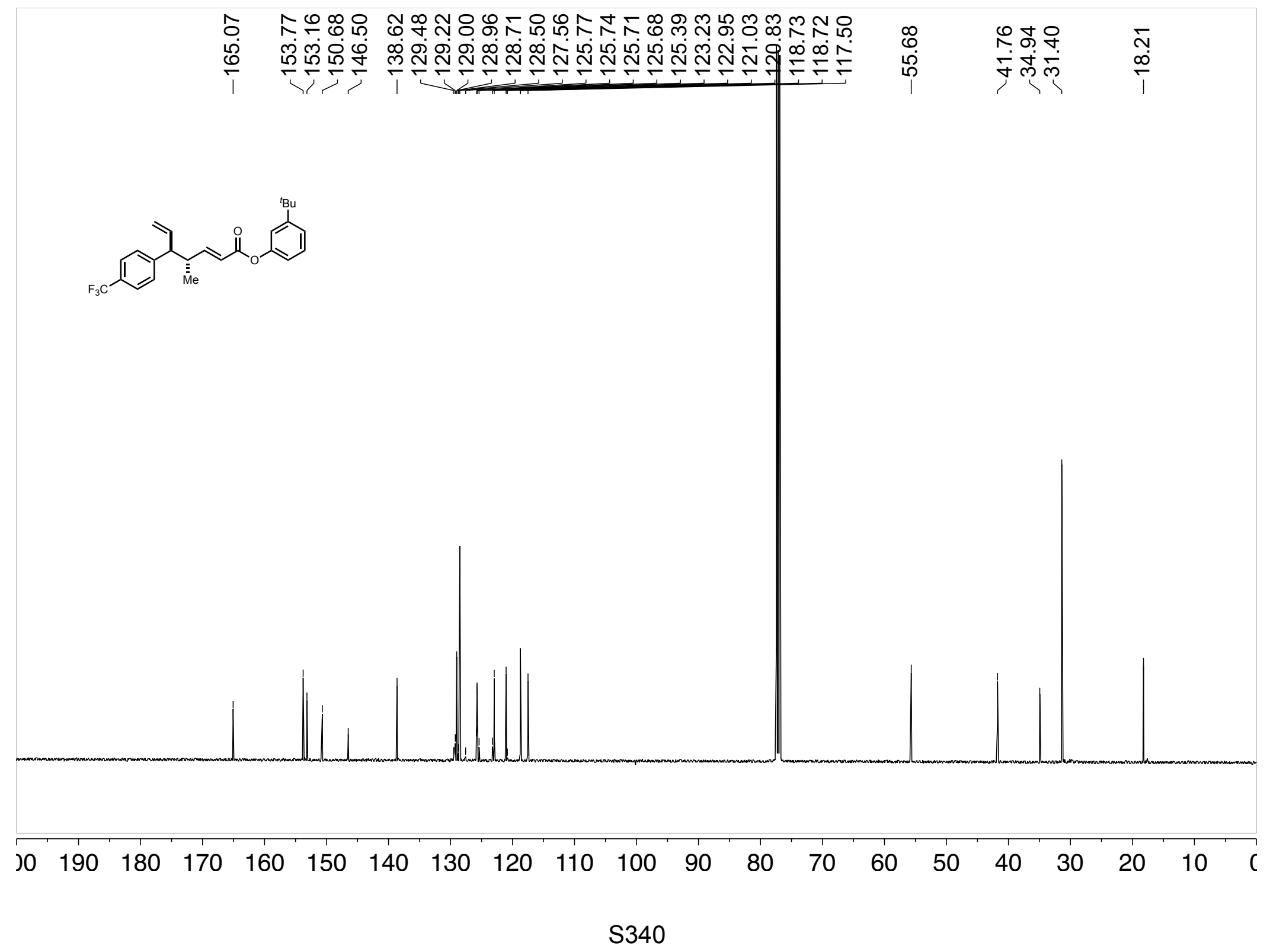




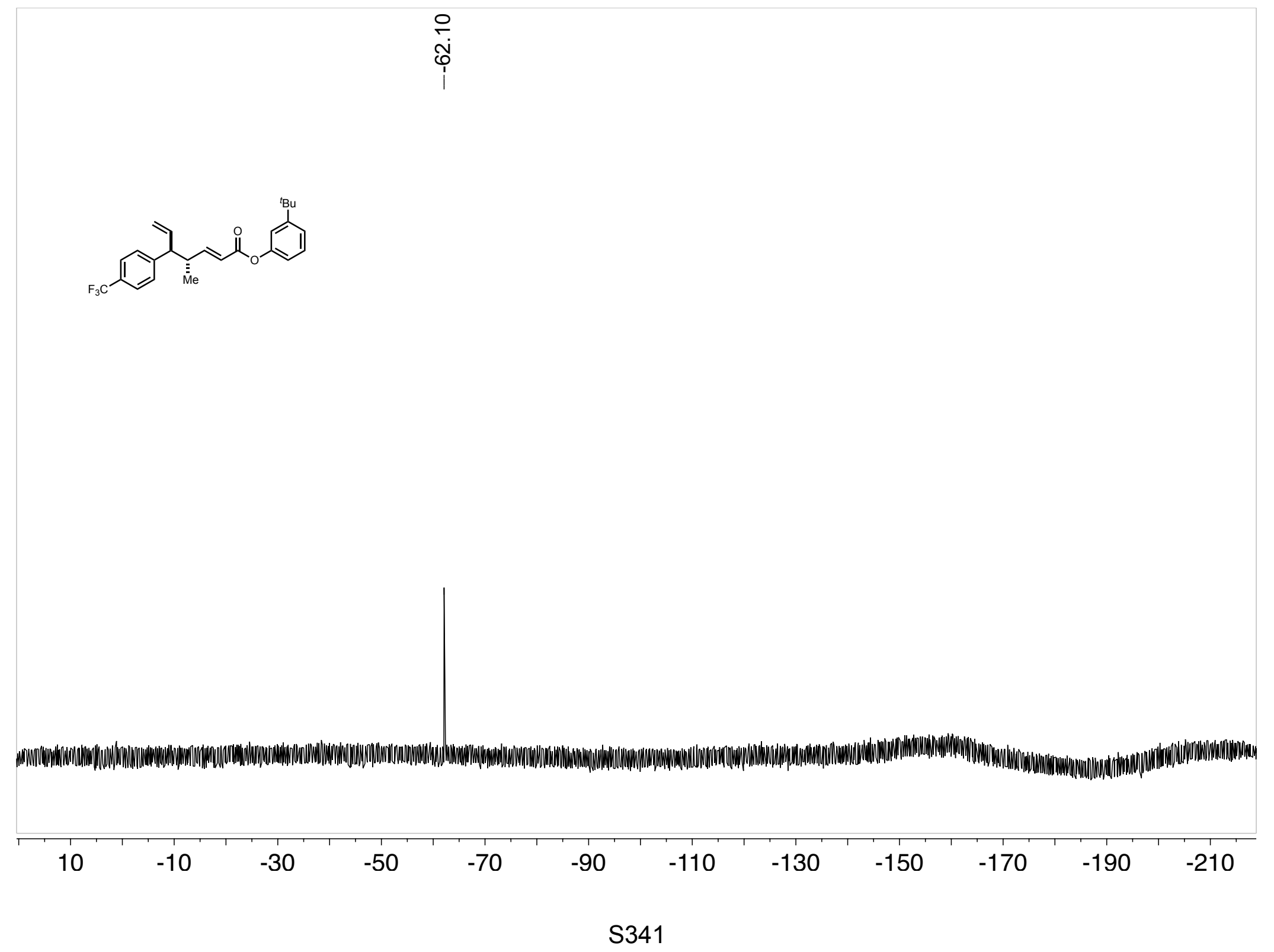




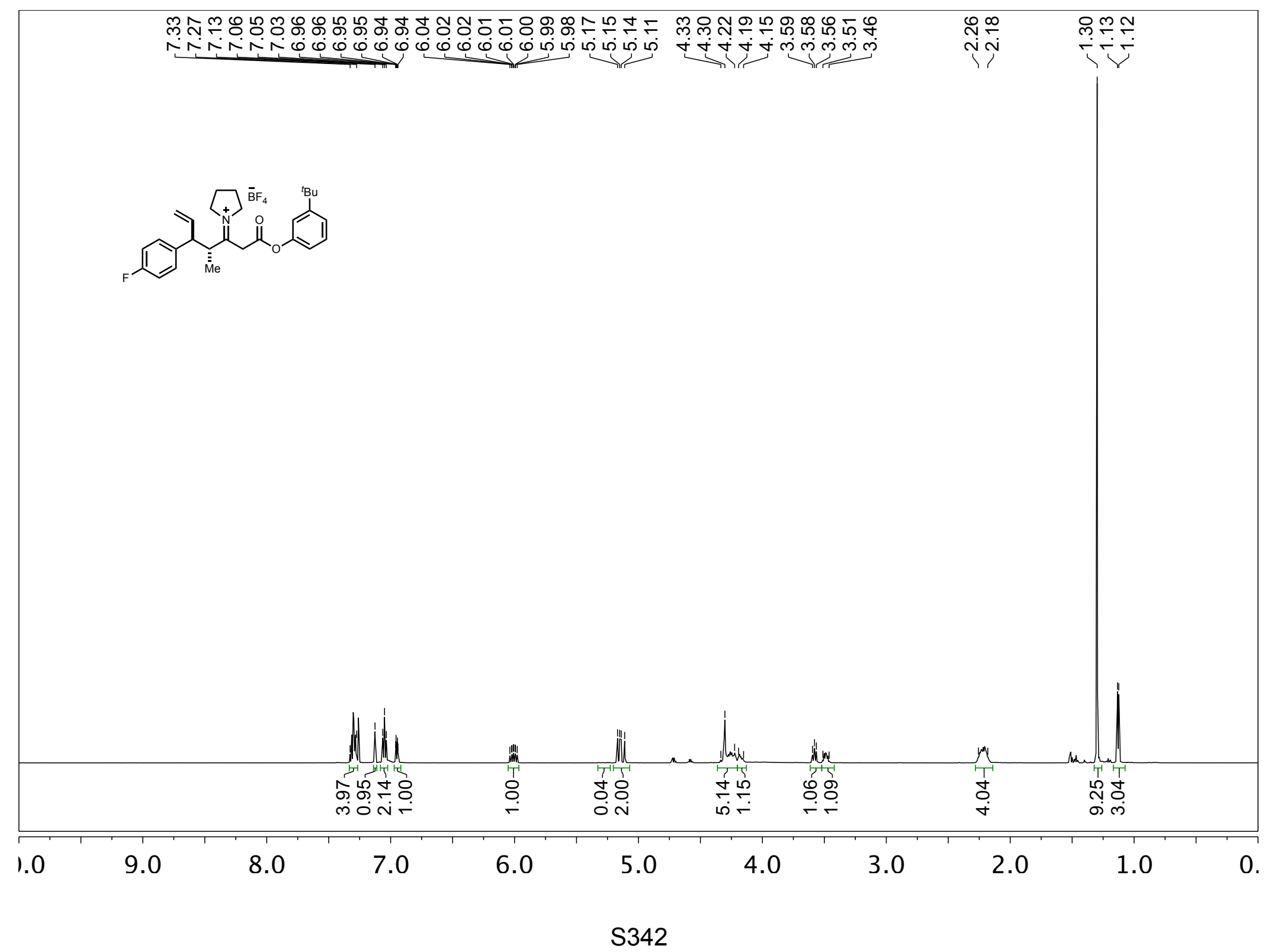




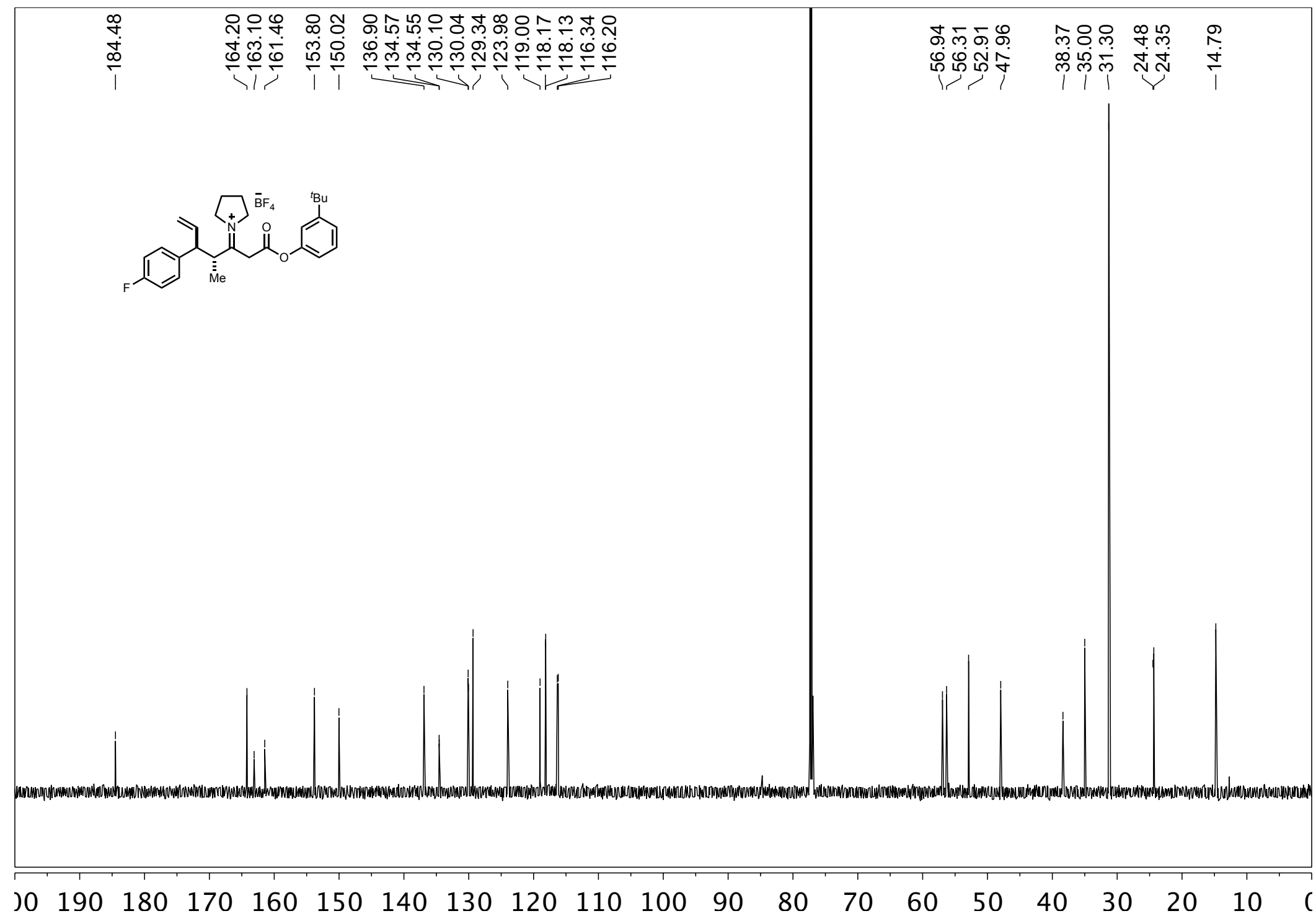




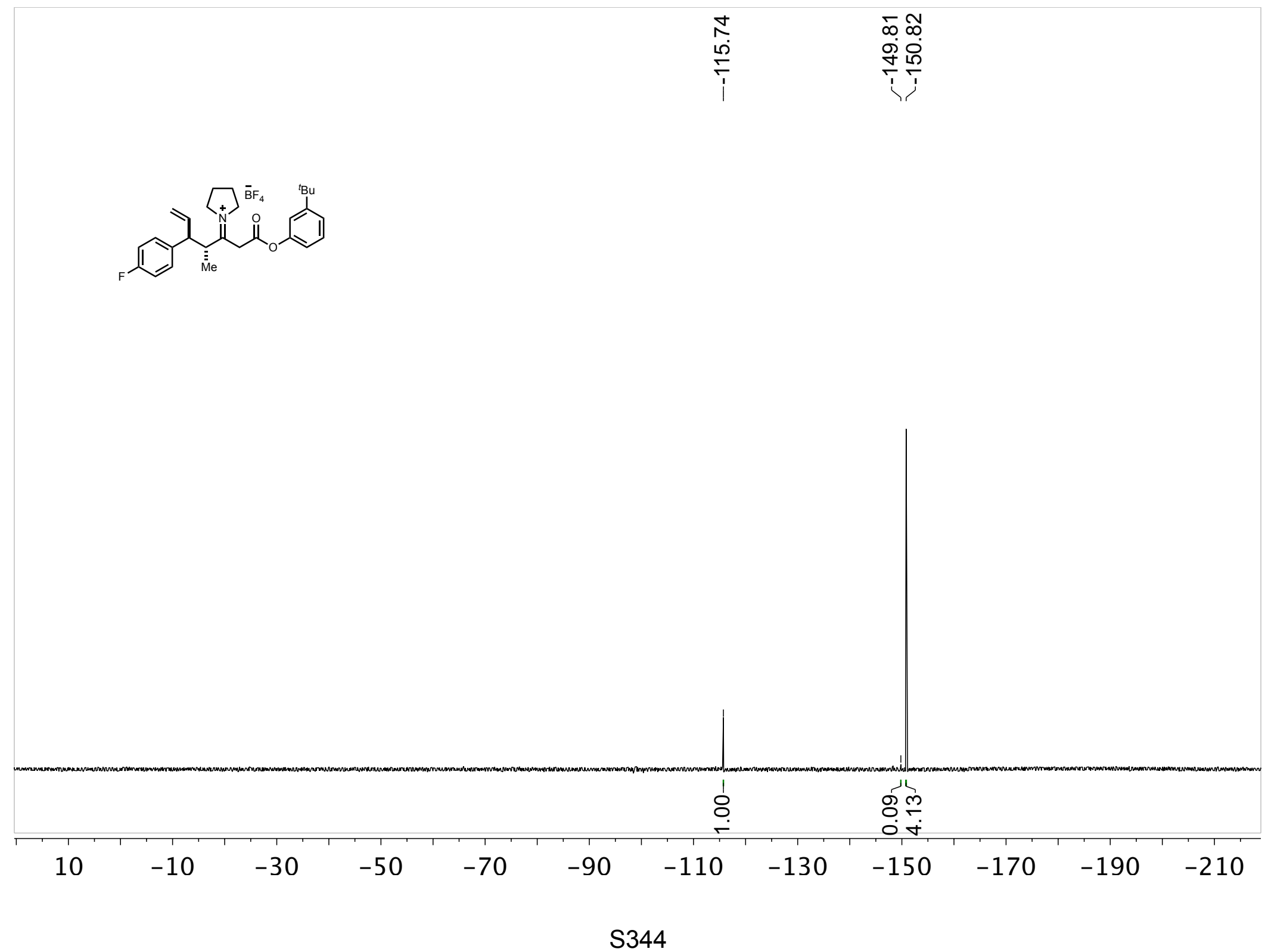




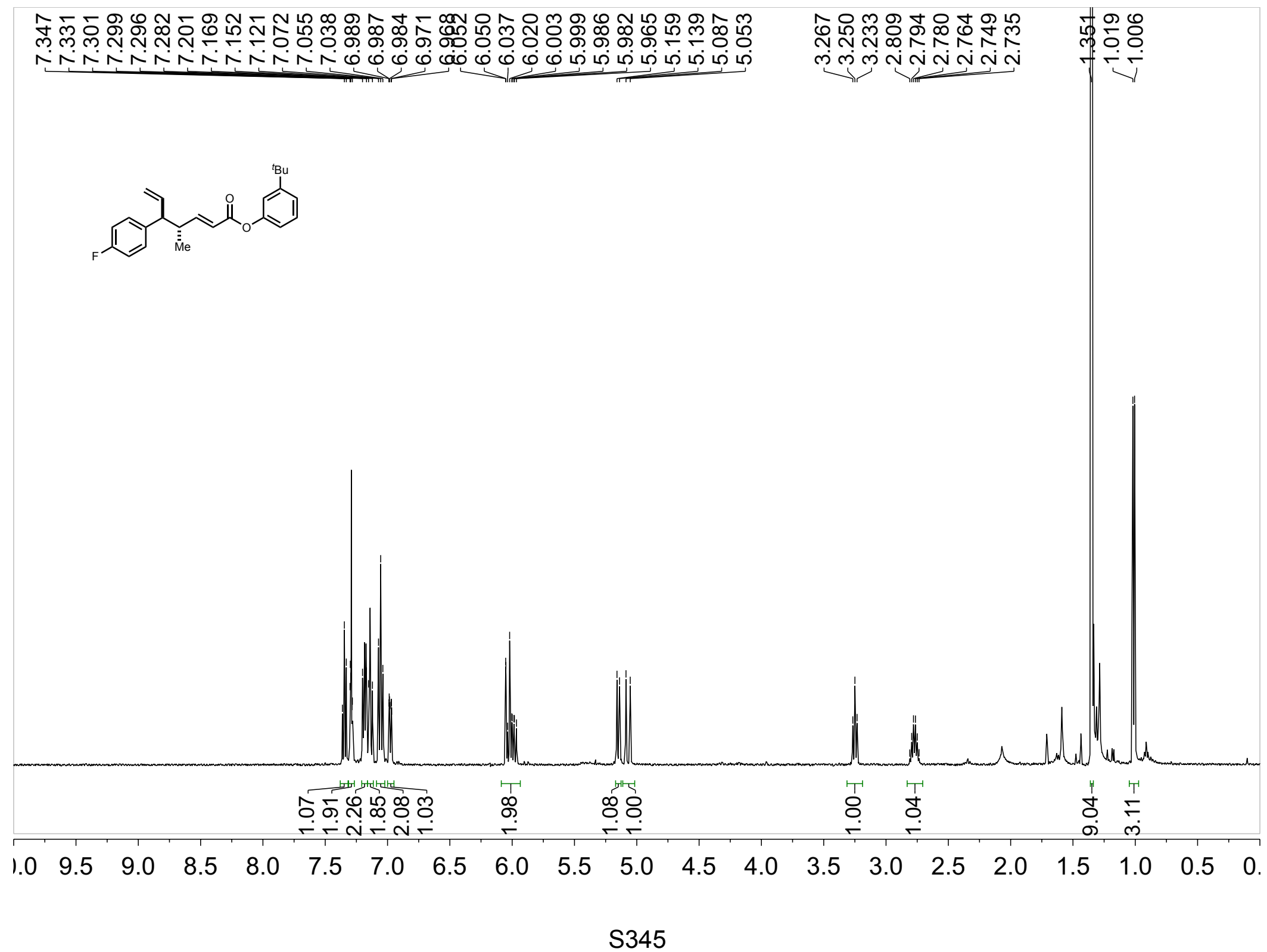




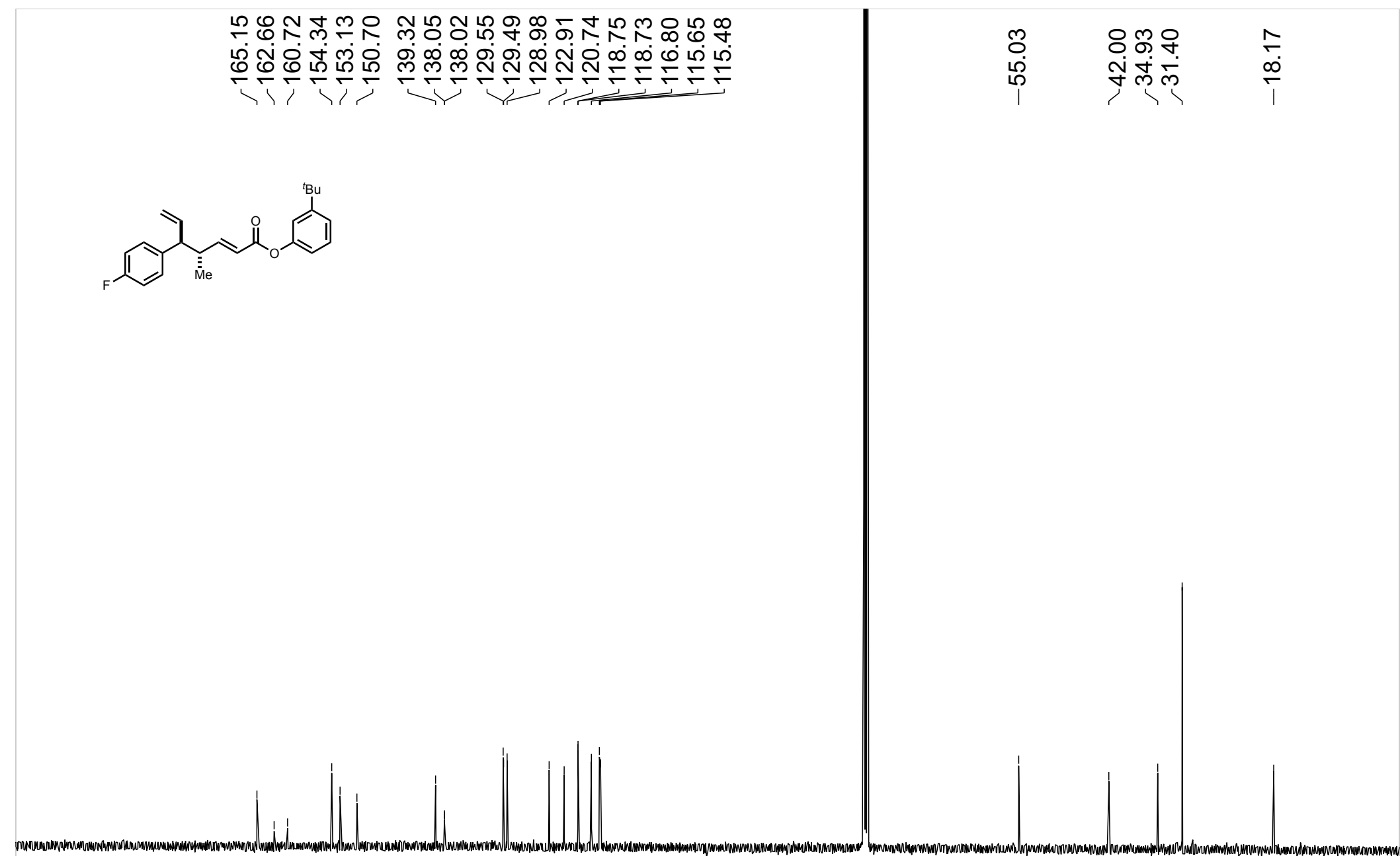

$\begin{array}{llllllllllllllllllll}190 & 180 & 170 & 160 & 150 & 140 & 130 & 120 & 110 & 100 & 90 & 80 & 70 & 60 & 50 & 40 & 30 & 20 & 10 & (\end{array}$




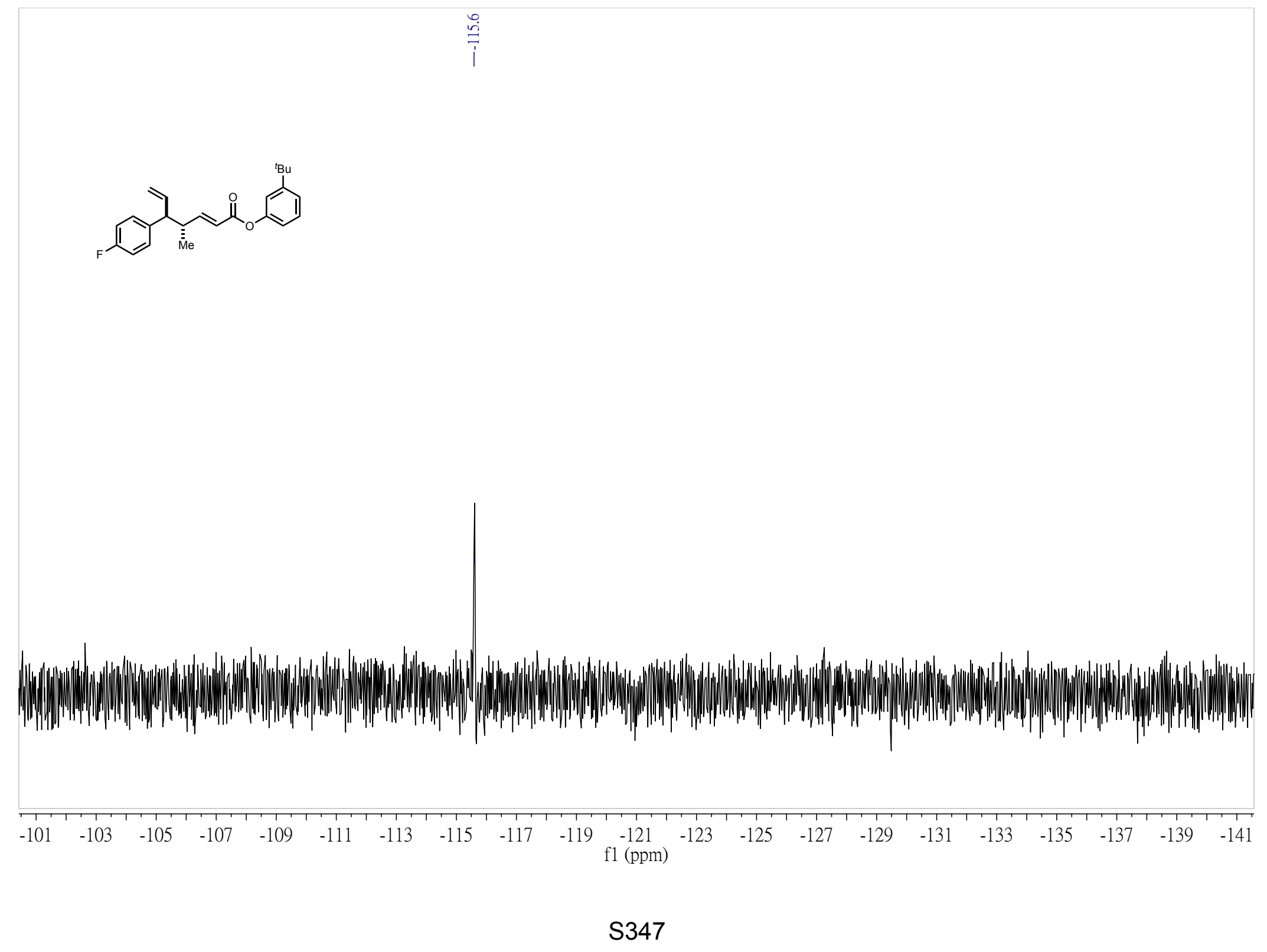




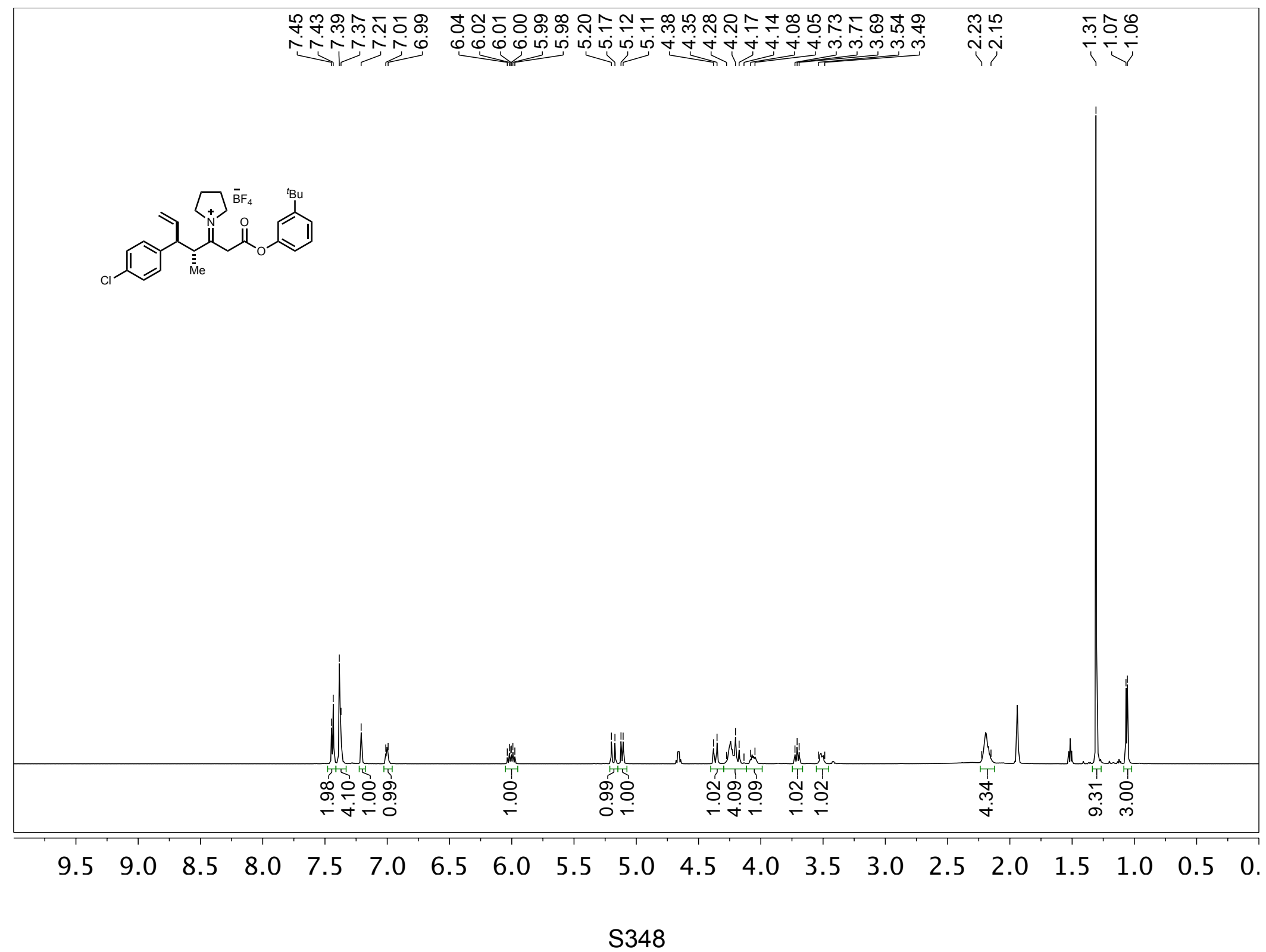




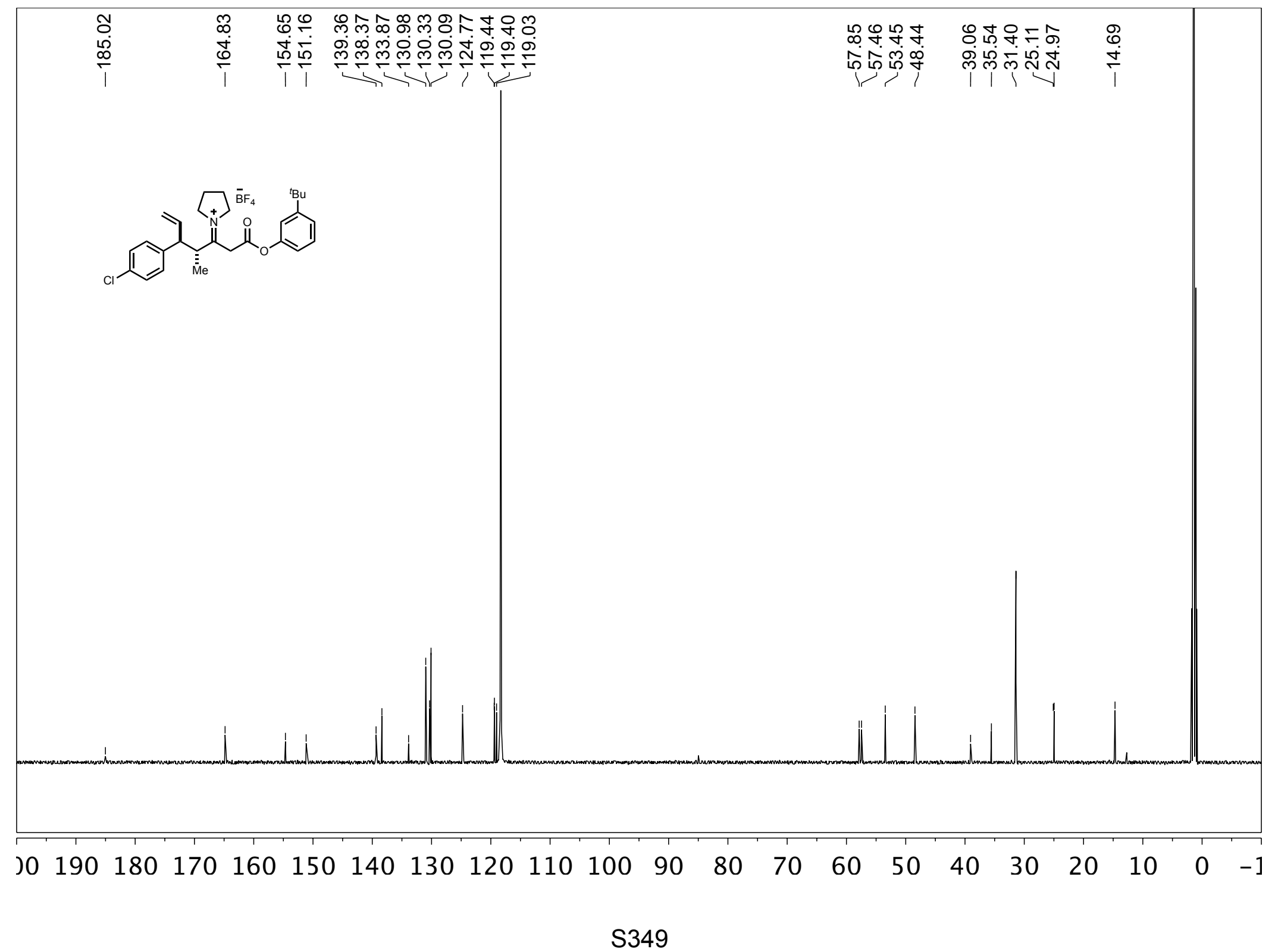




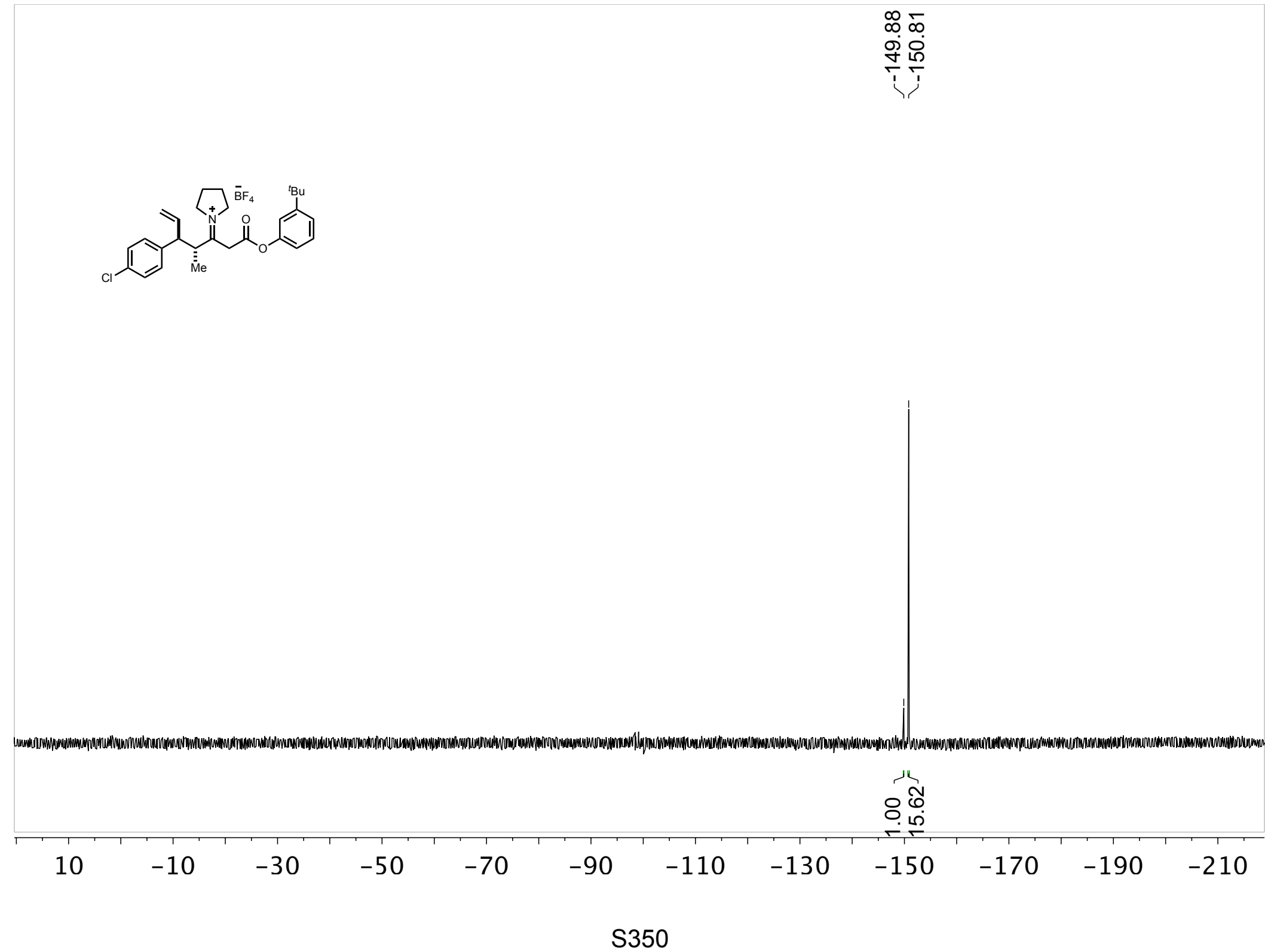




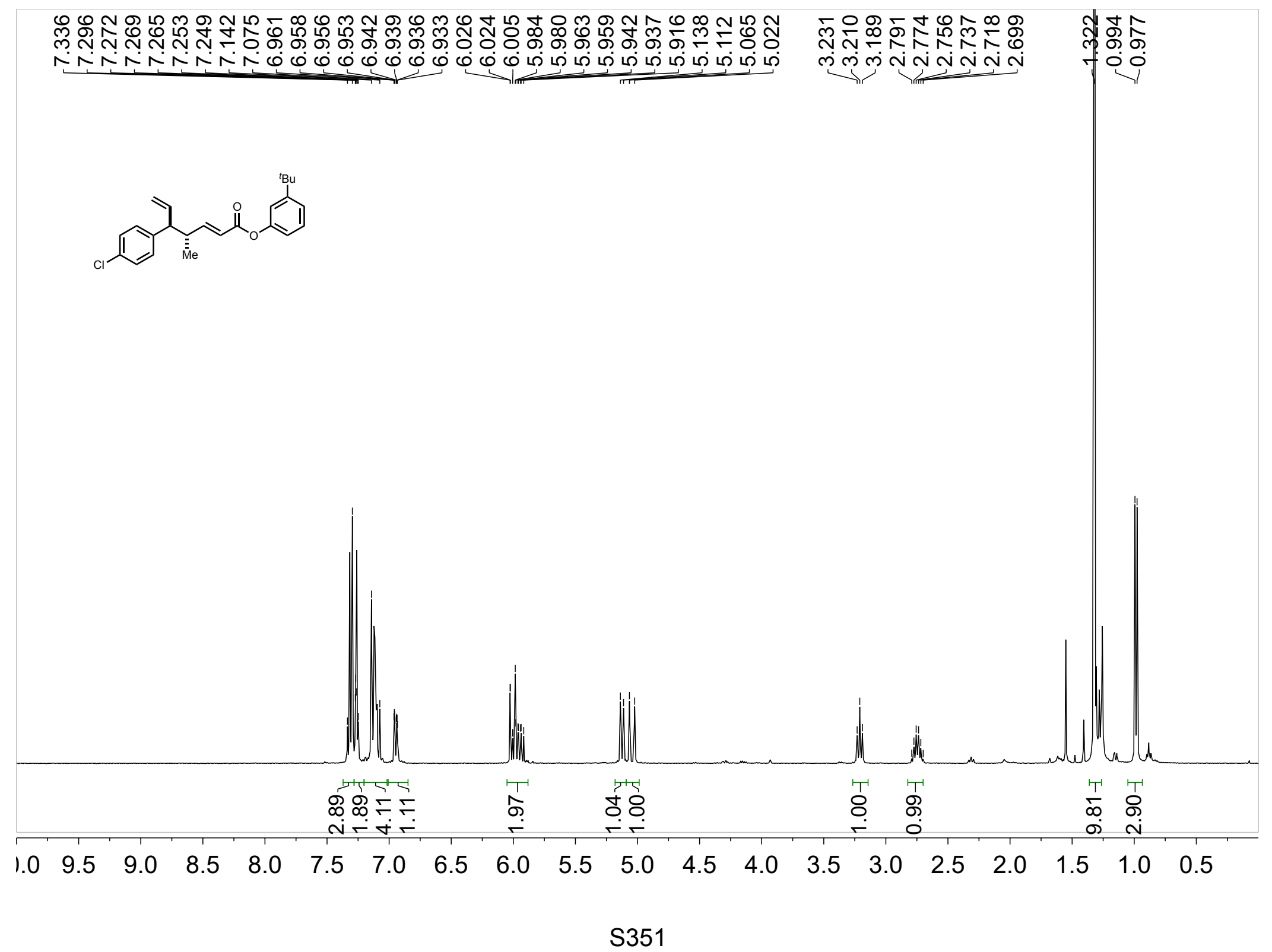




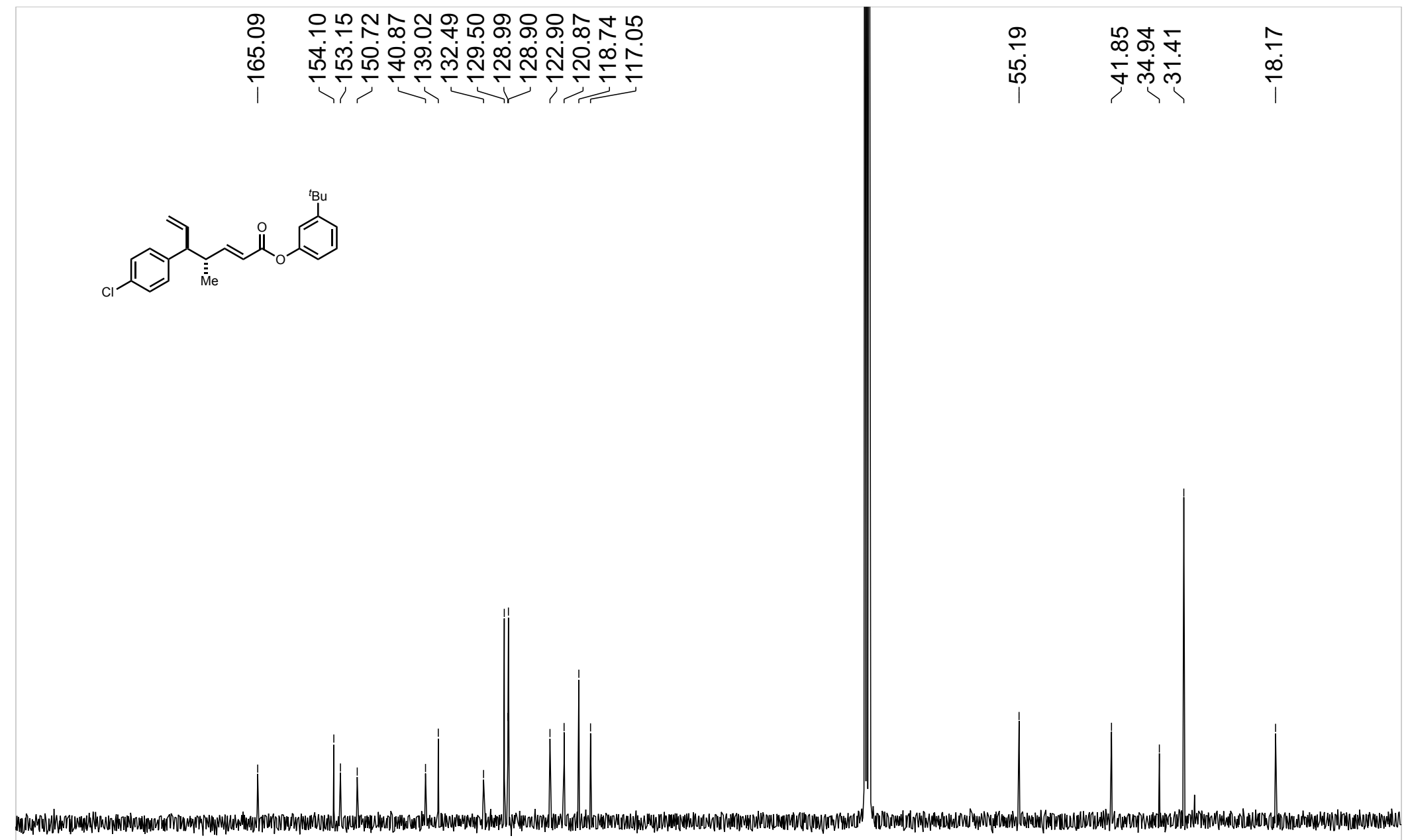

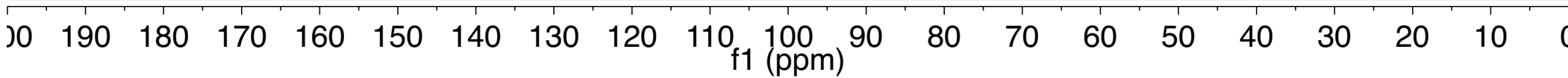




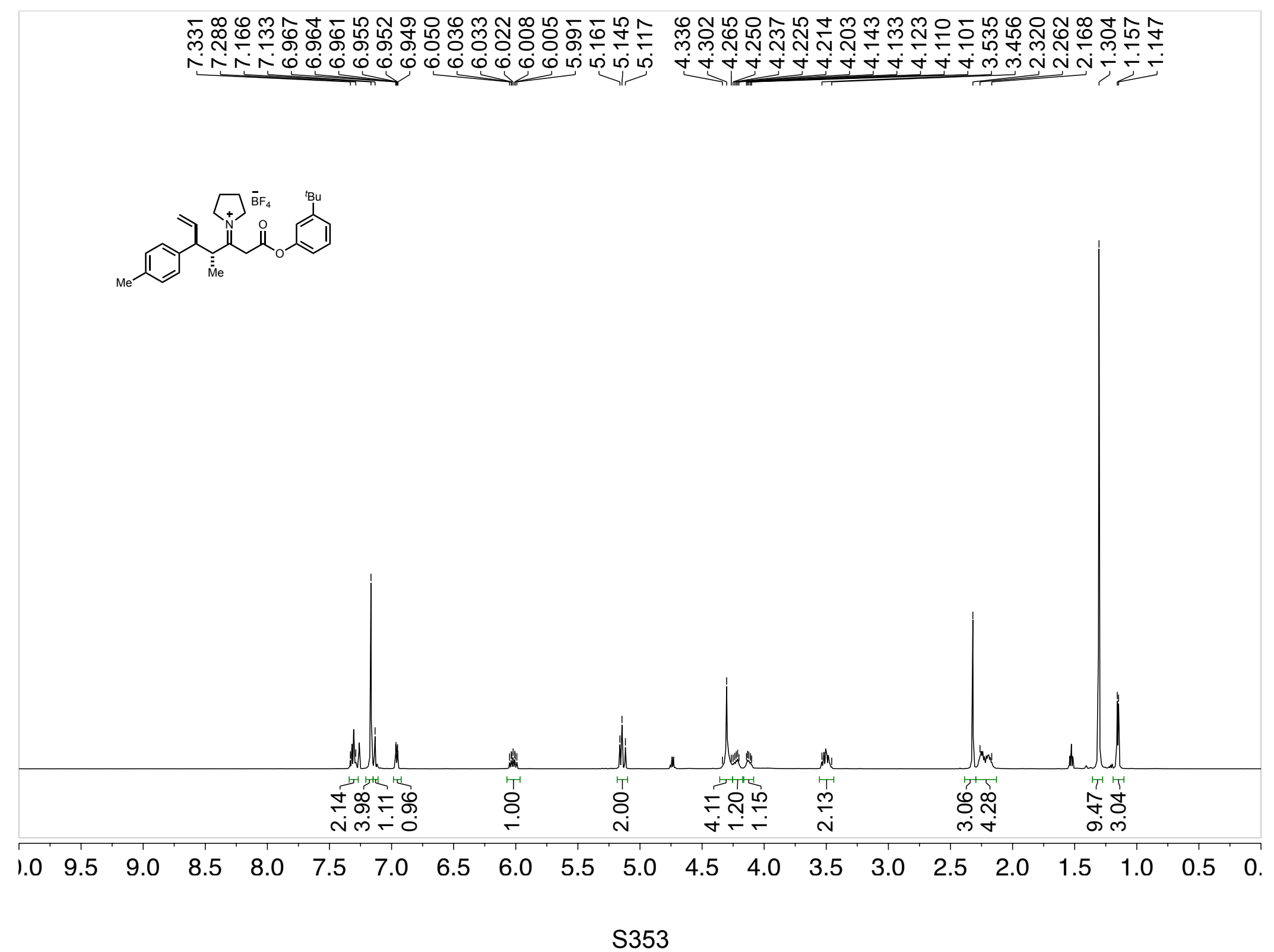



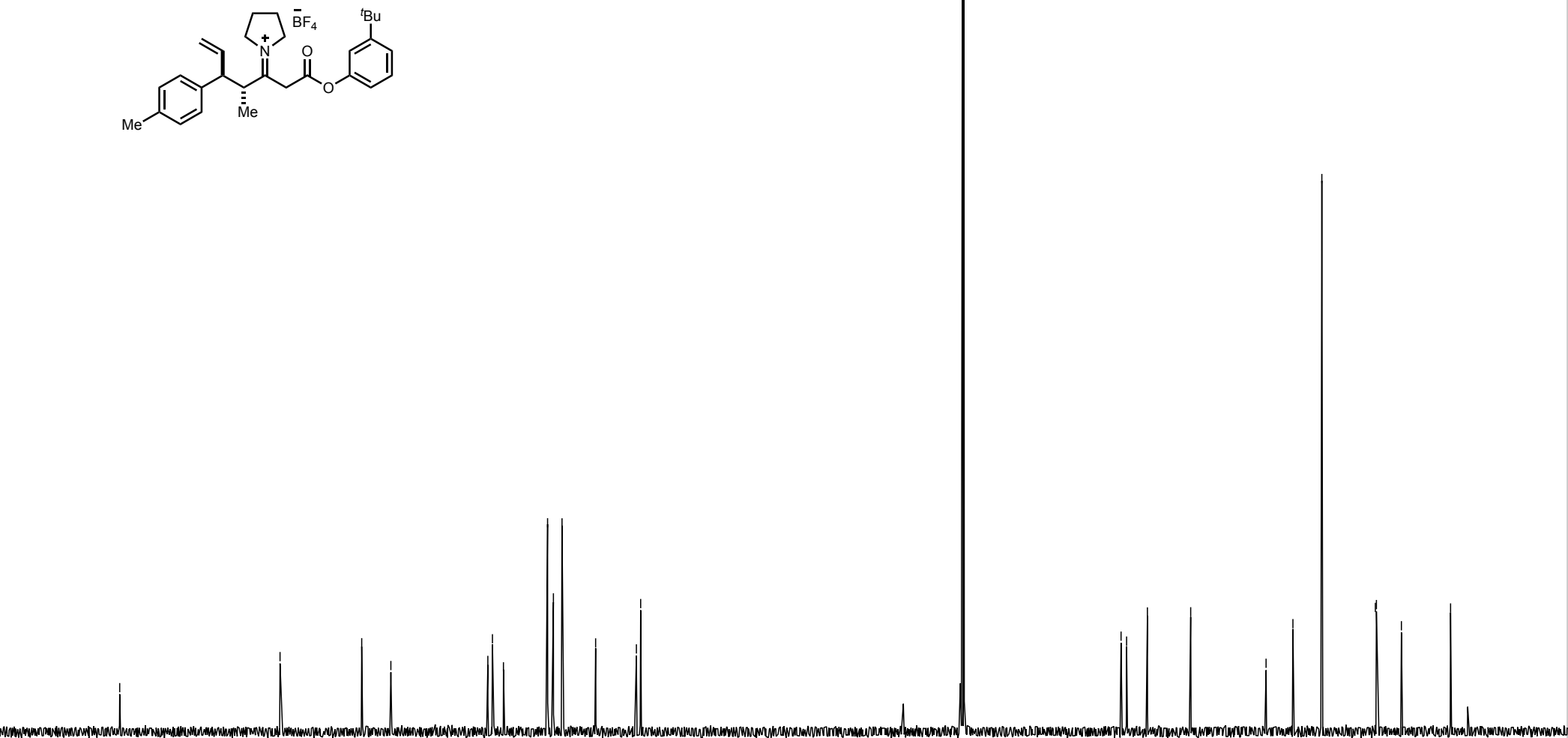


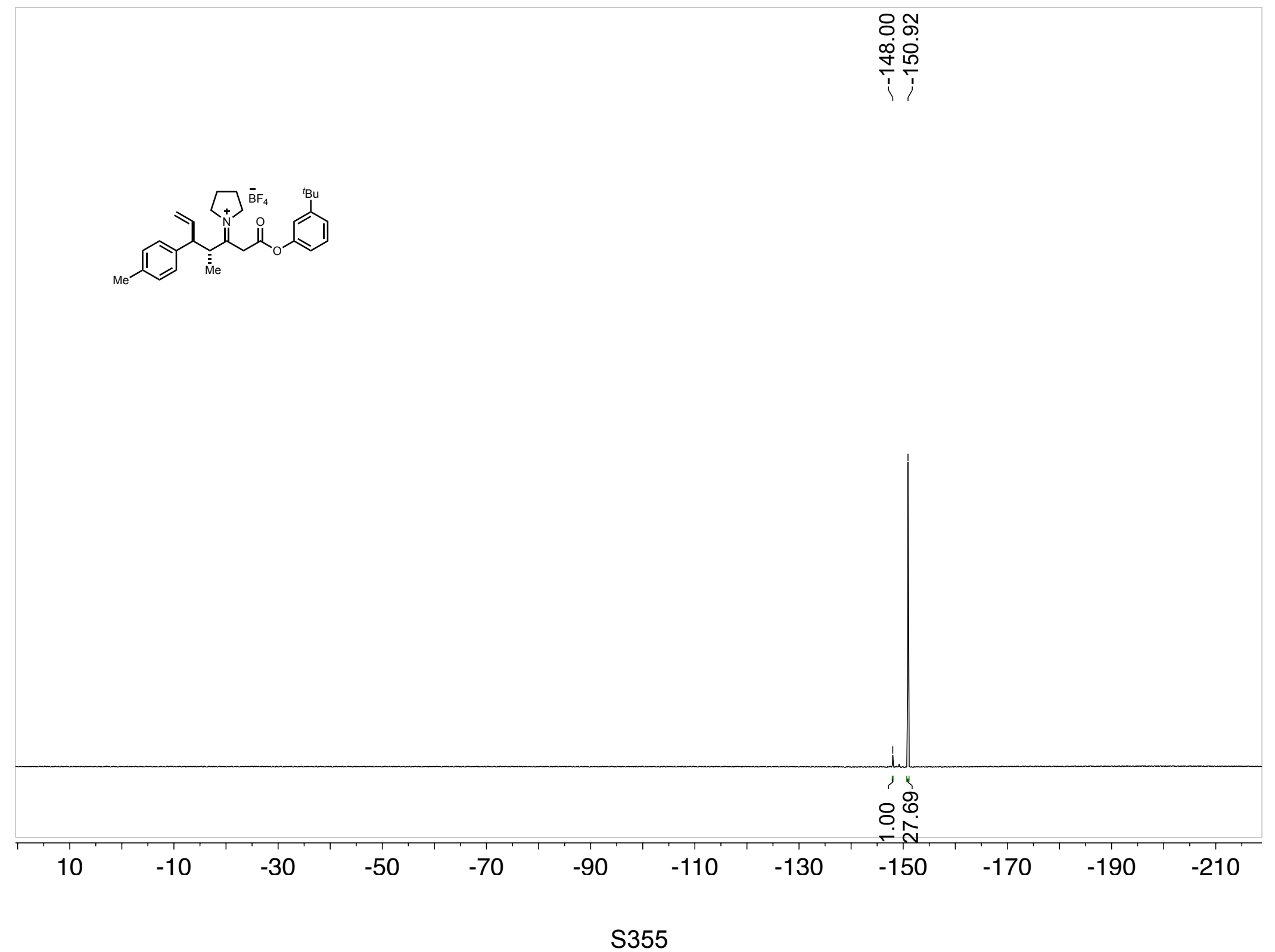




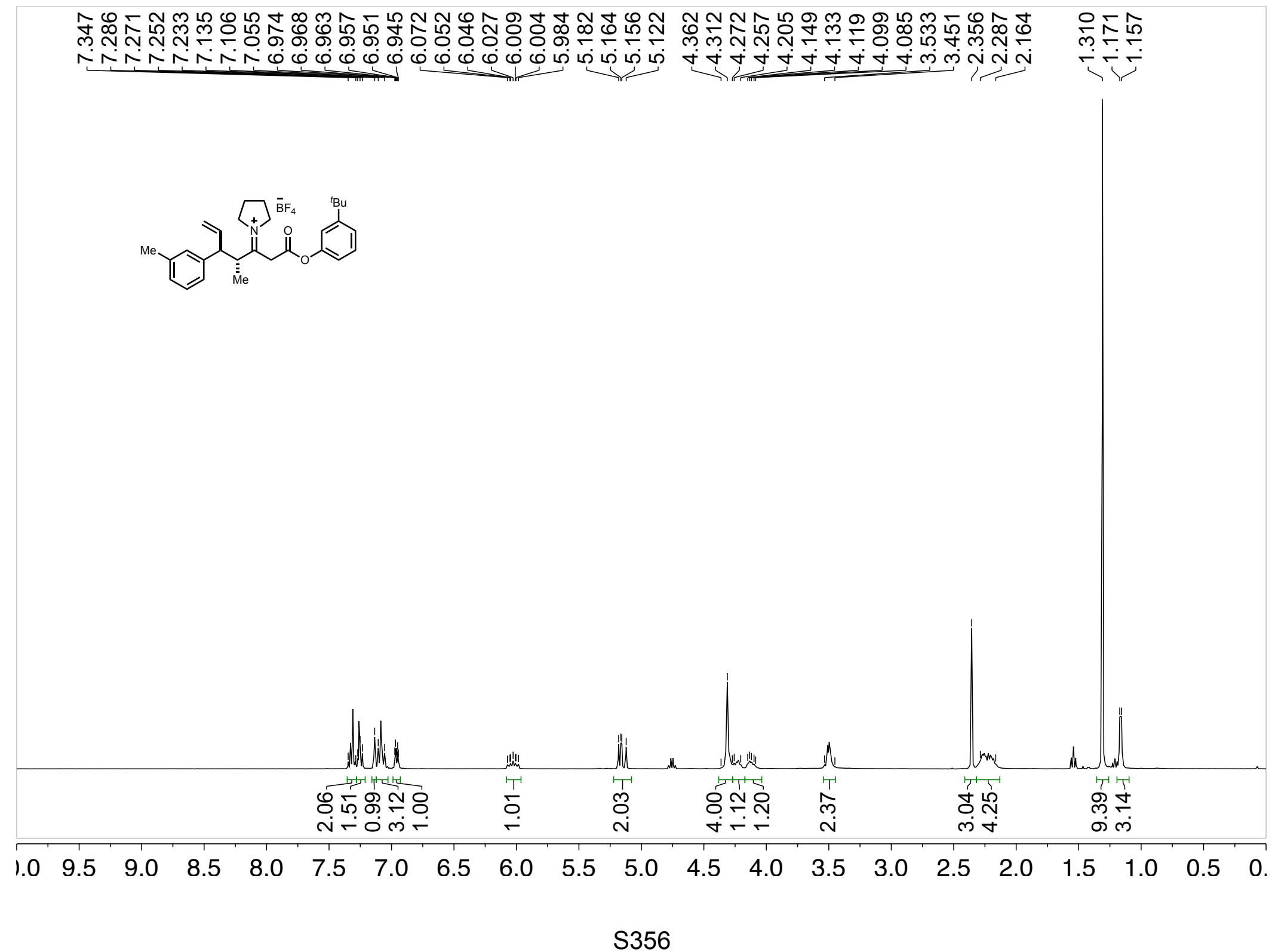



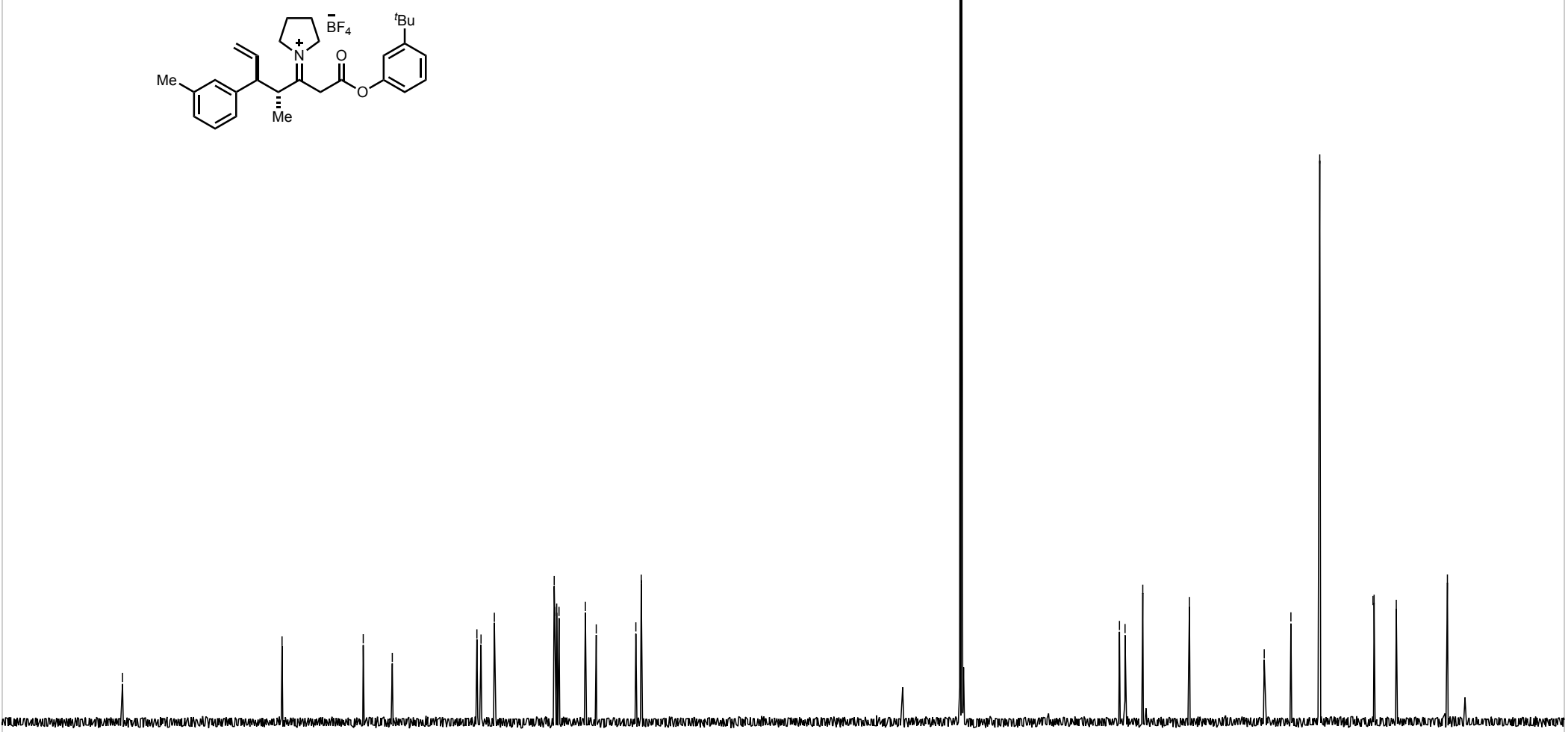
可

于

i

(1)

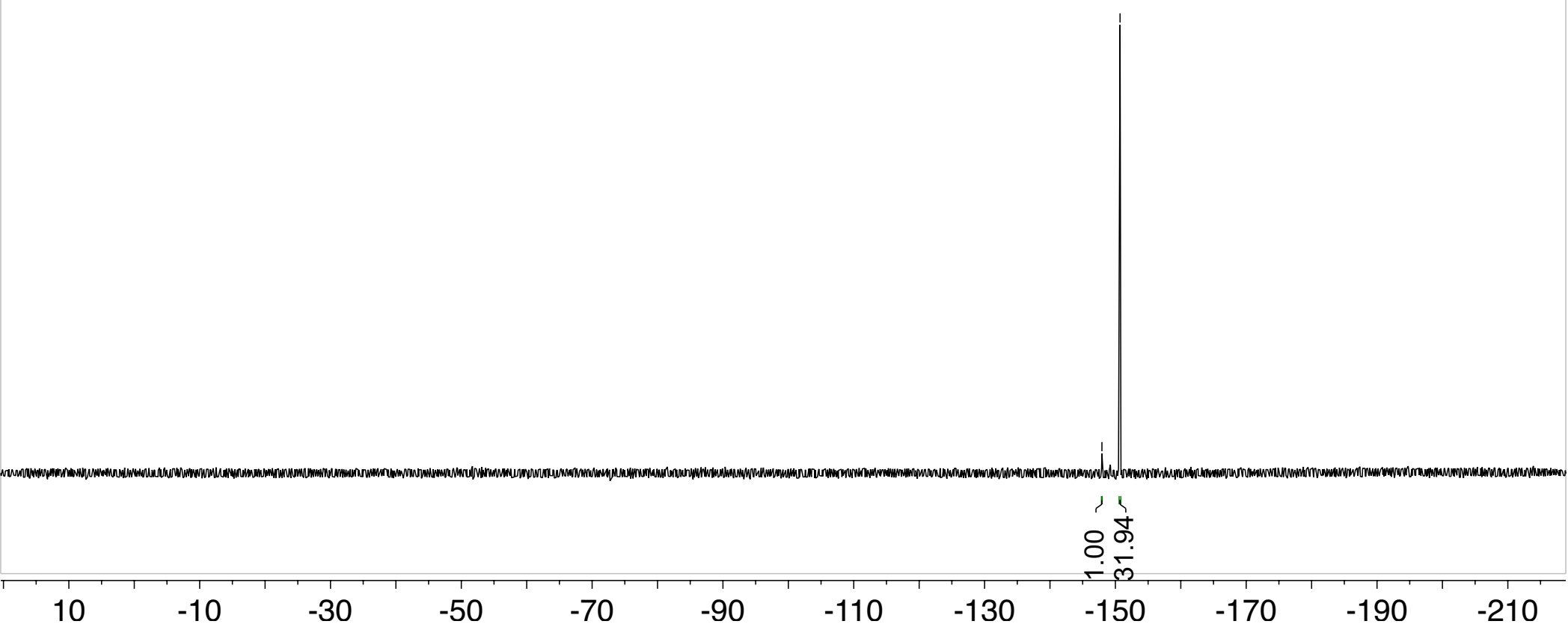




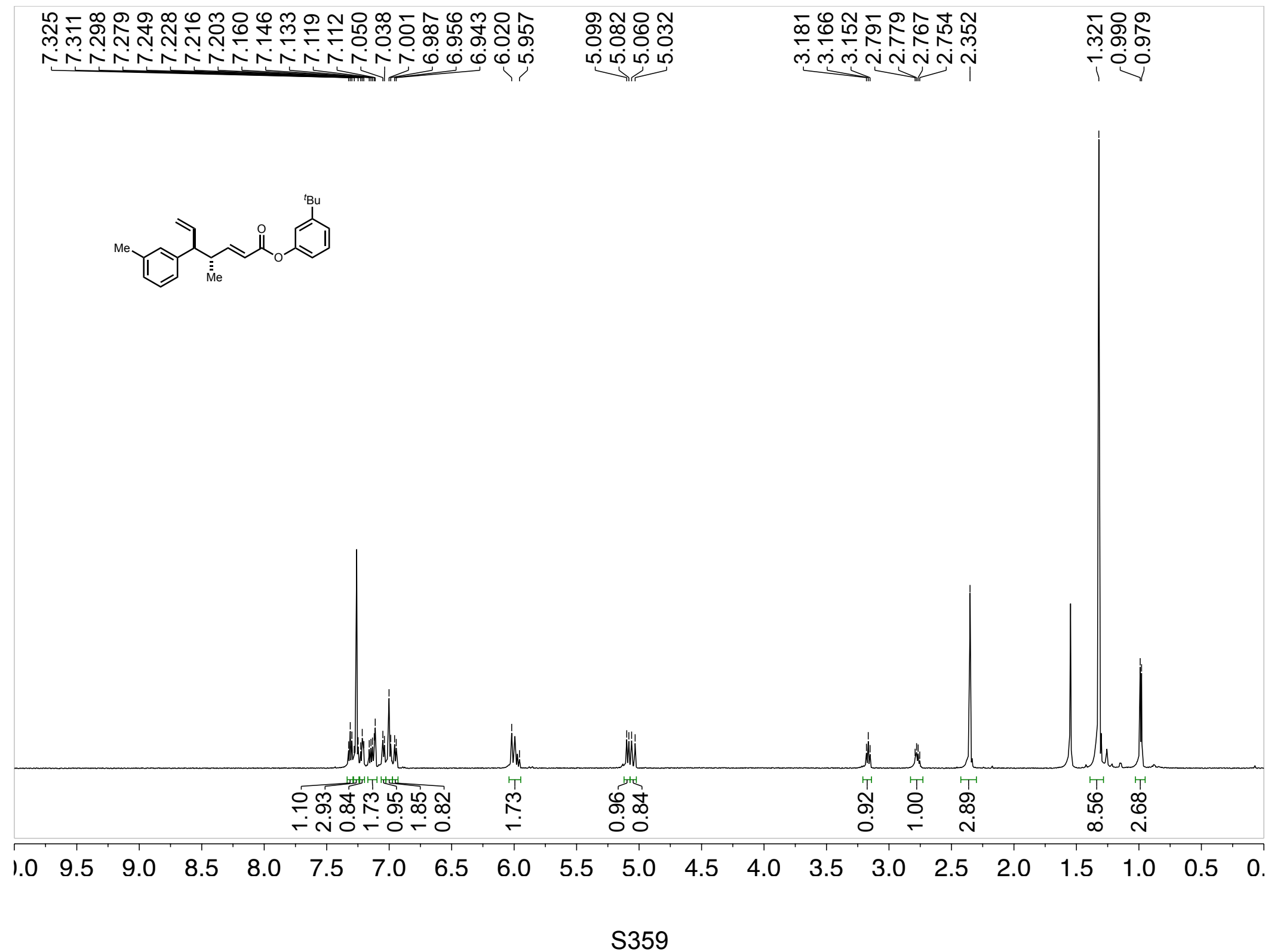




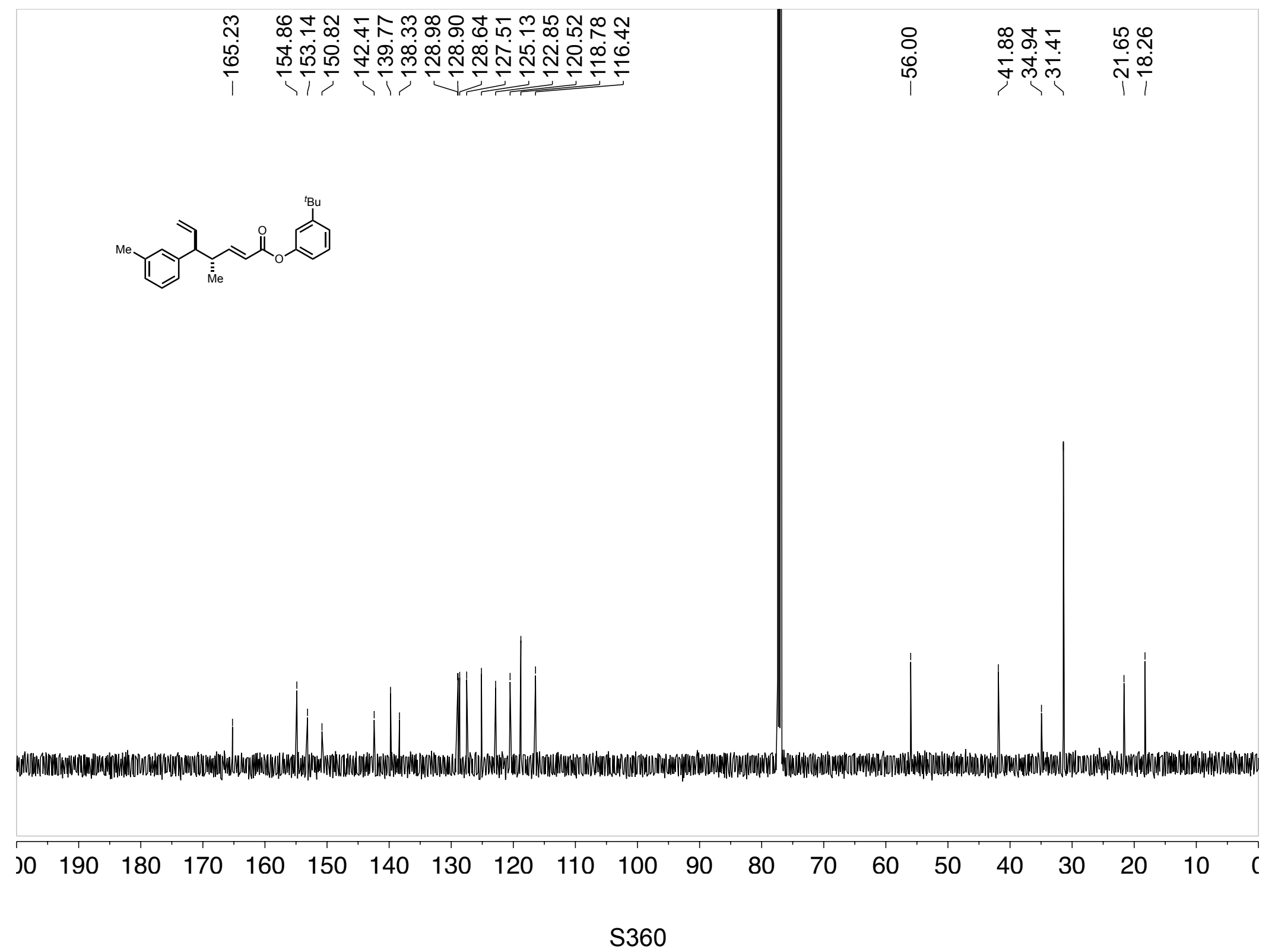




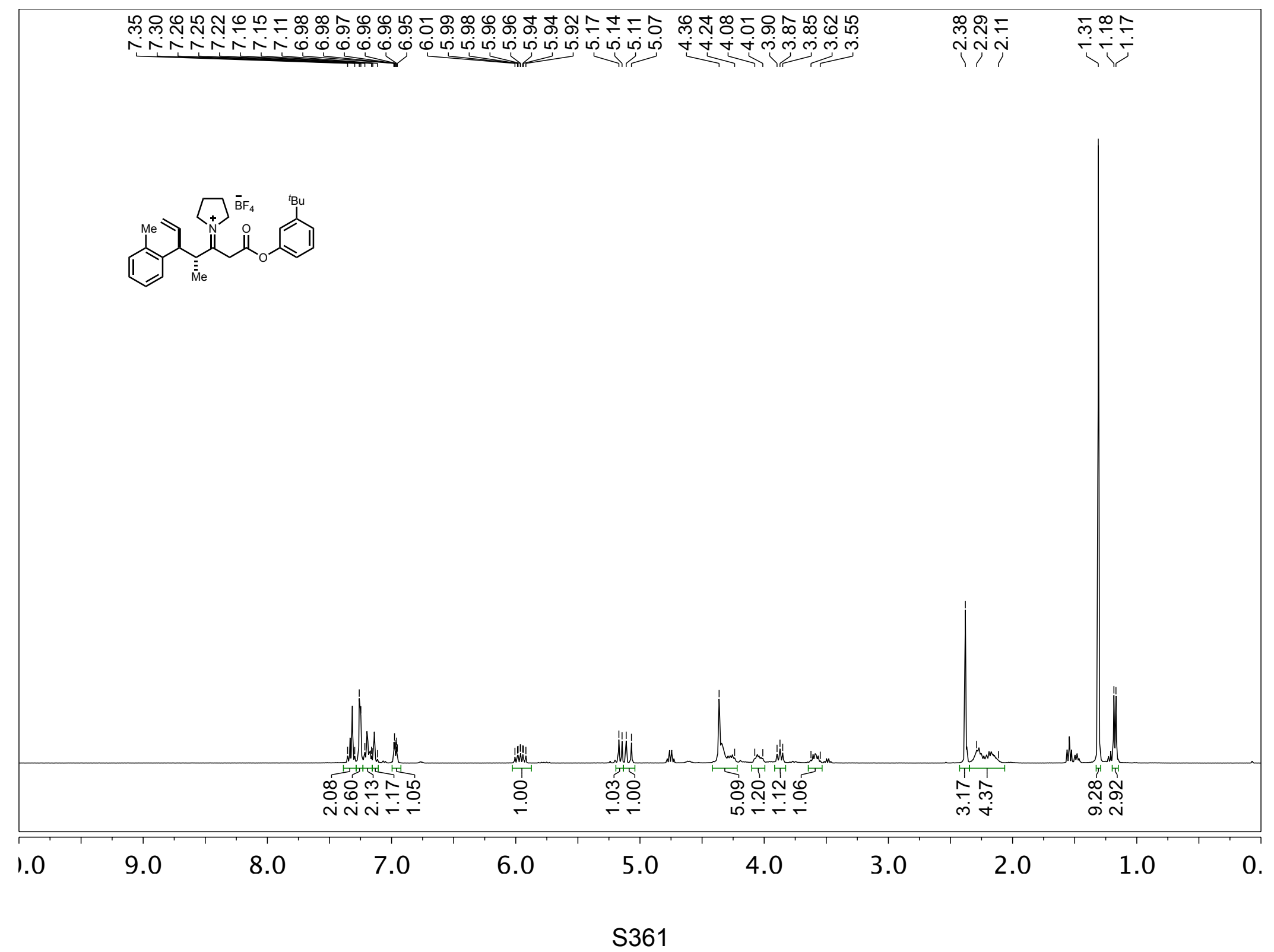




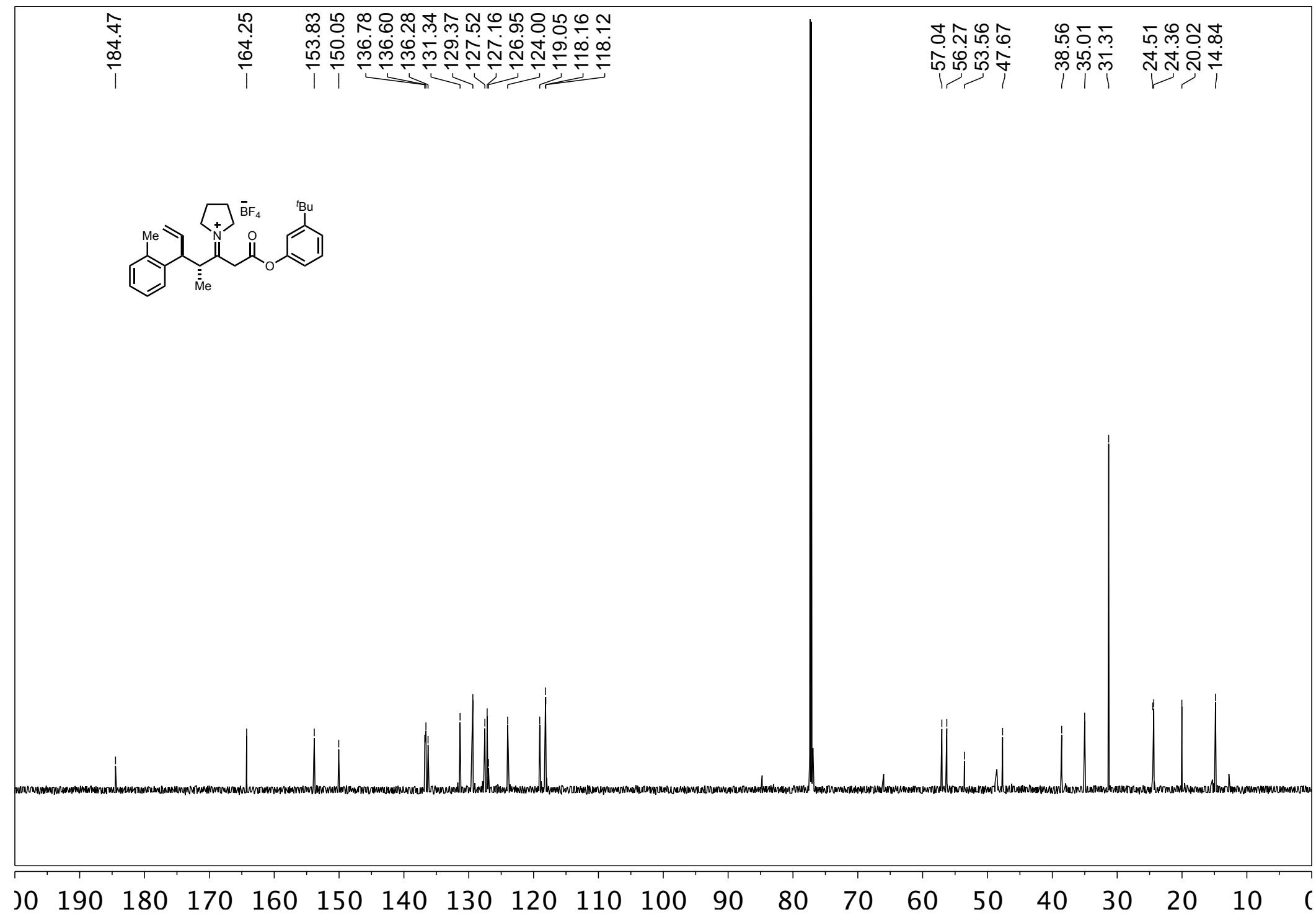




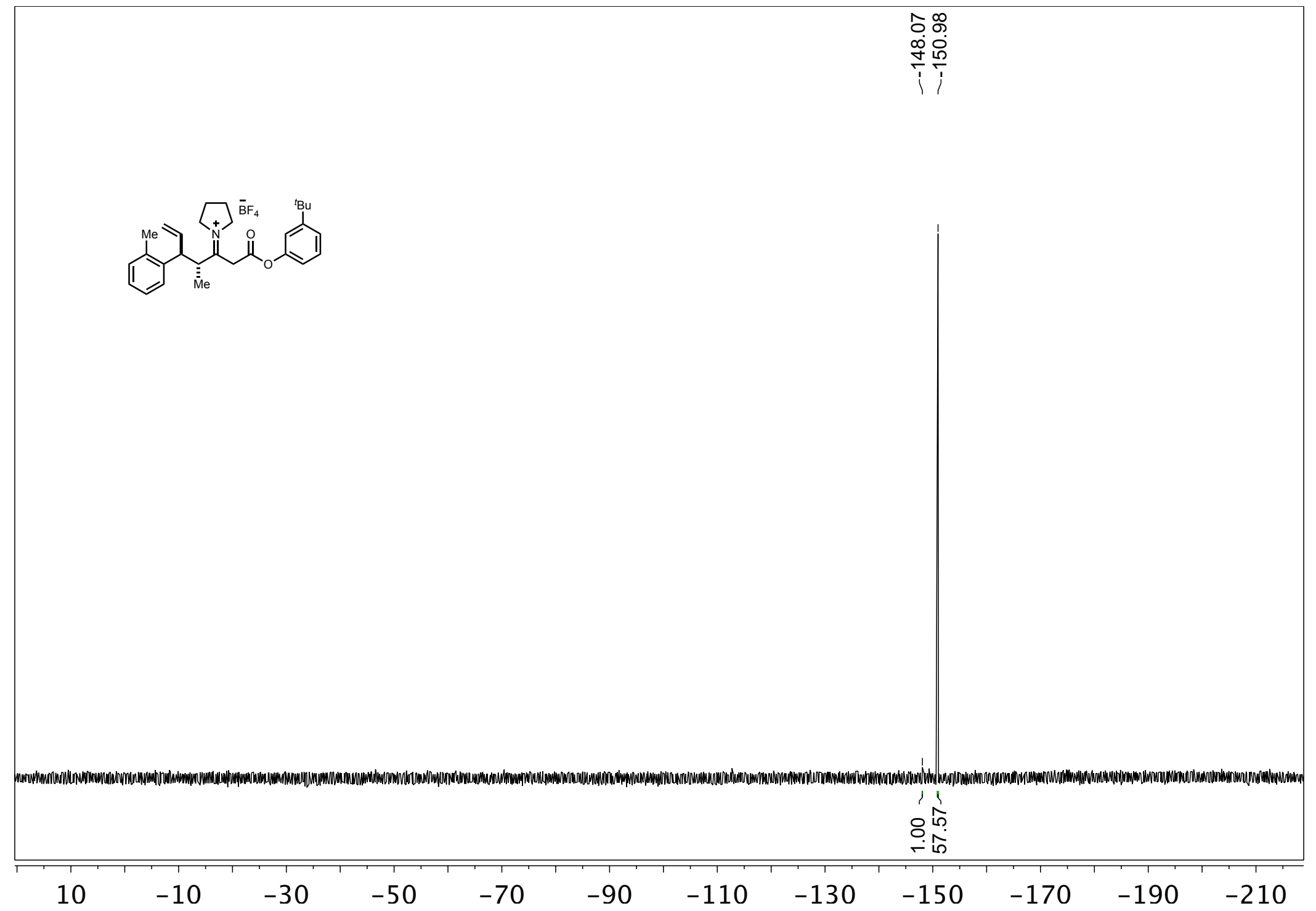




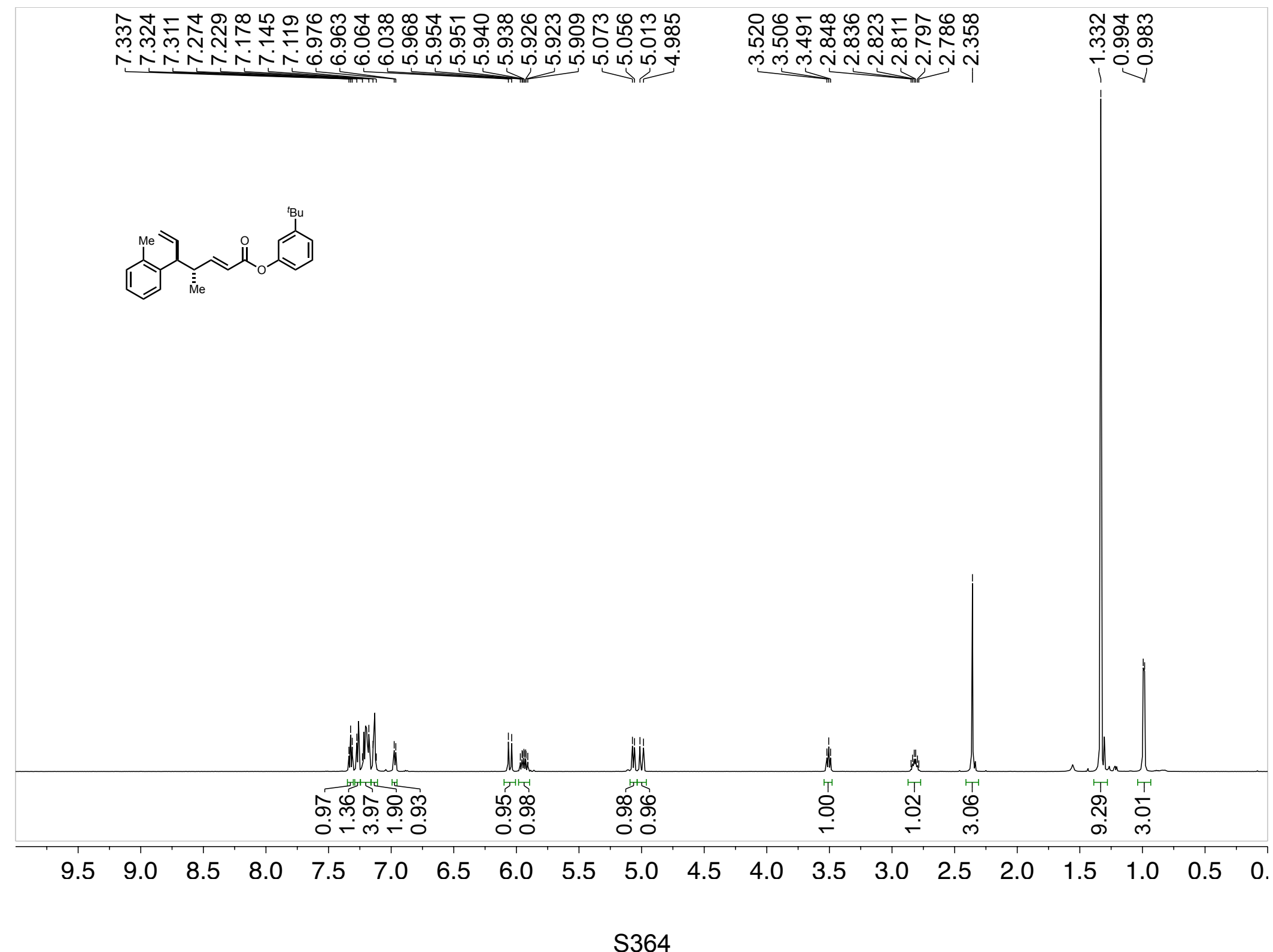




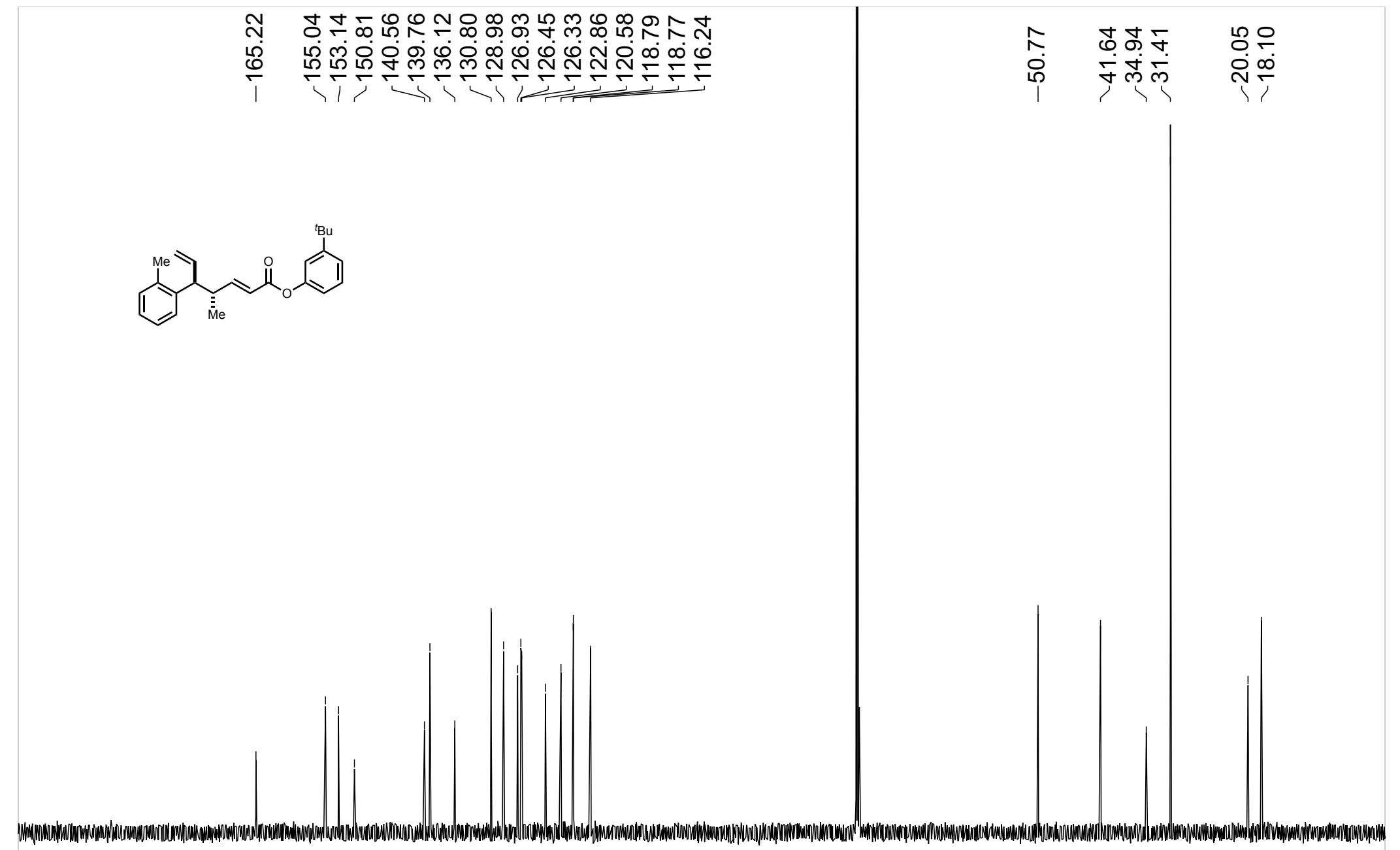

$\begin{array}{lllllllllllllllllllll}30 & 190 & 180 & 170 & 160 & 150 & 140 & 130 & 120 & 110 & 100 & 90 & 80 & 70 & 60 & 50 & 40 & 30 & 20 & 10 & (\end{array}$




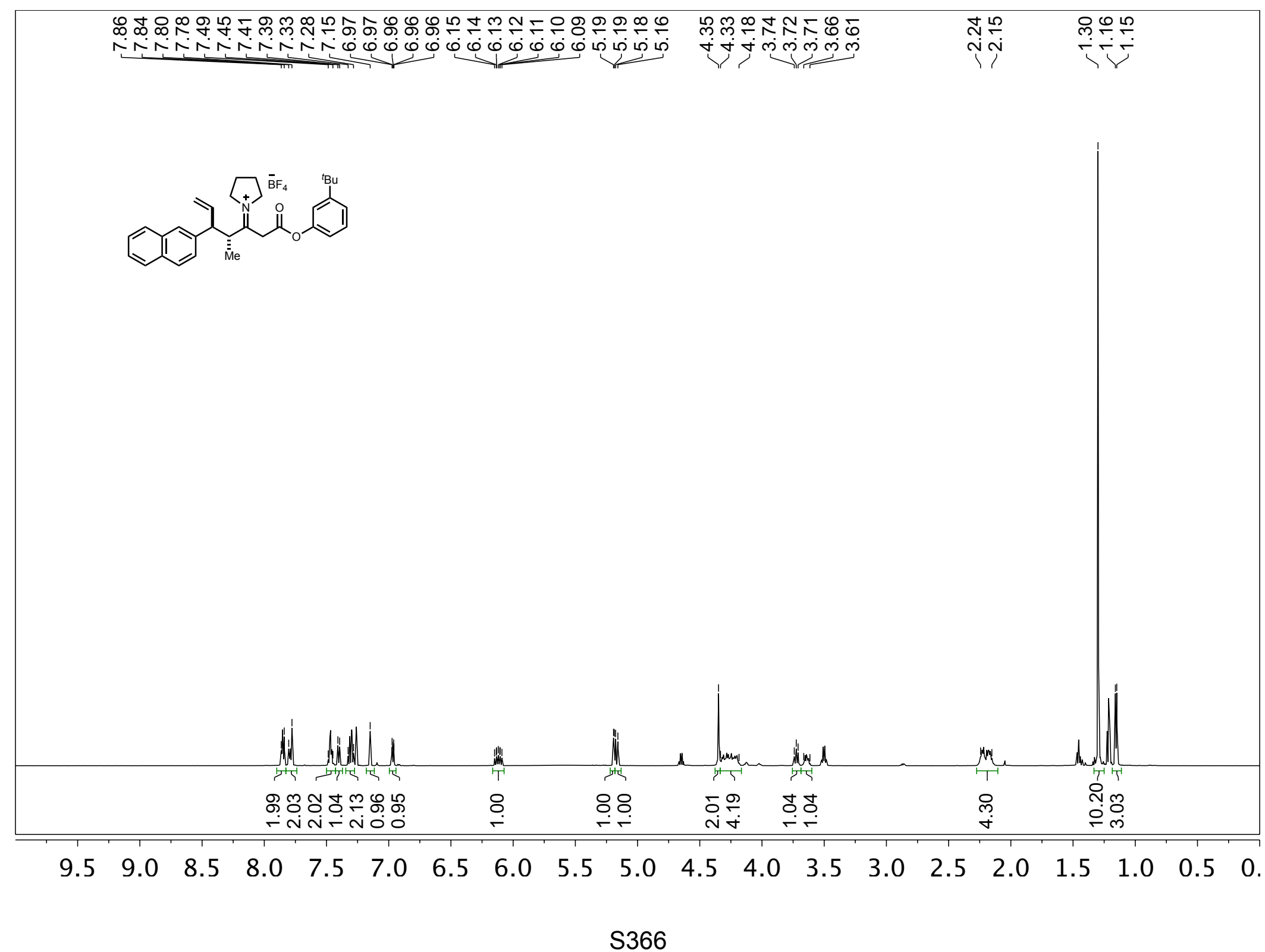




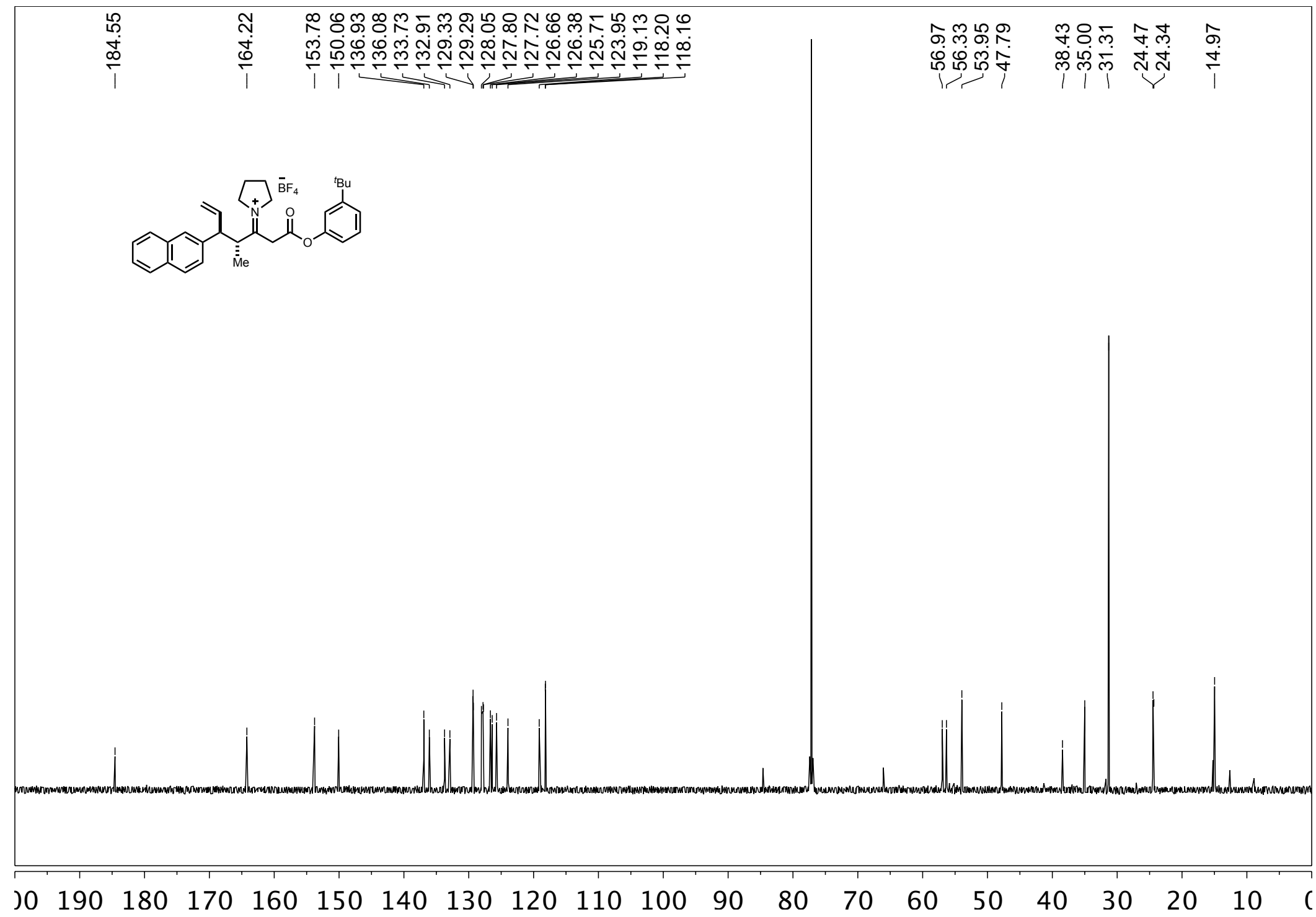


న

守通
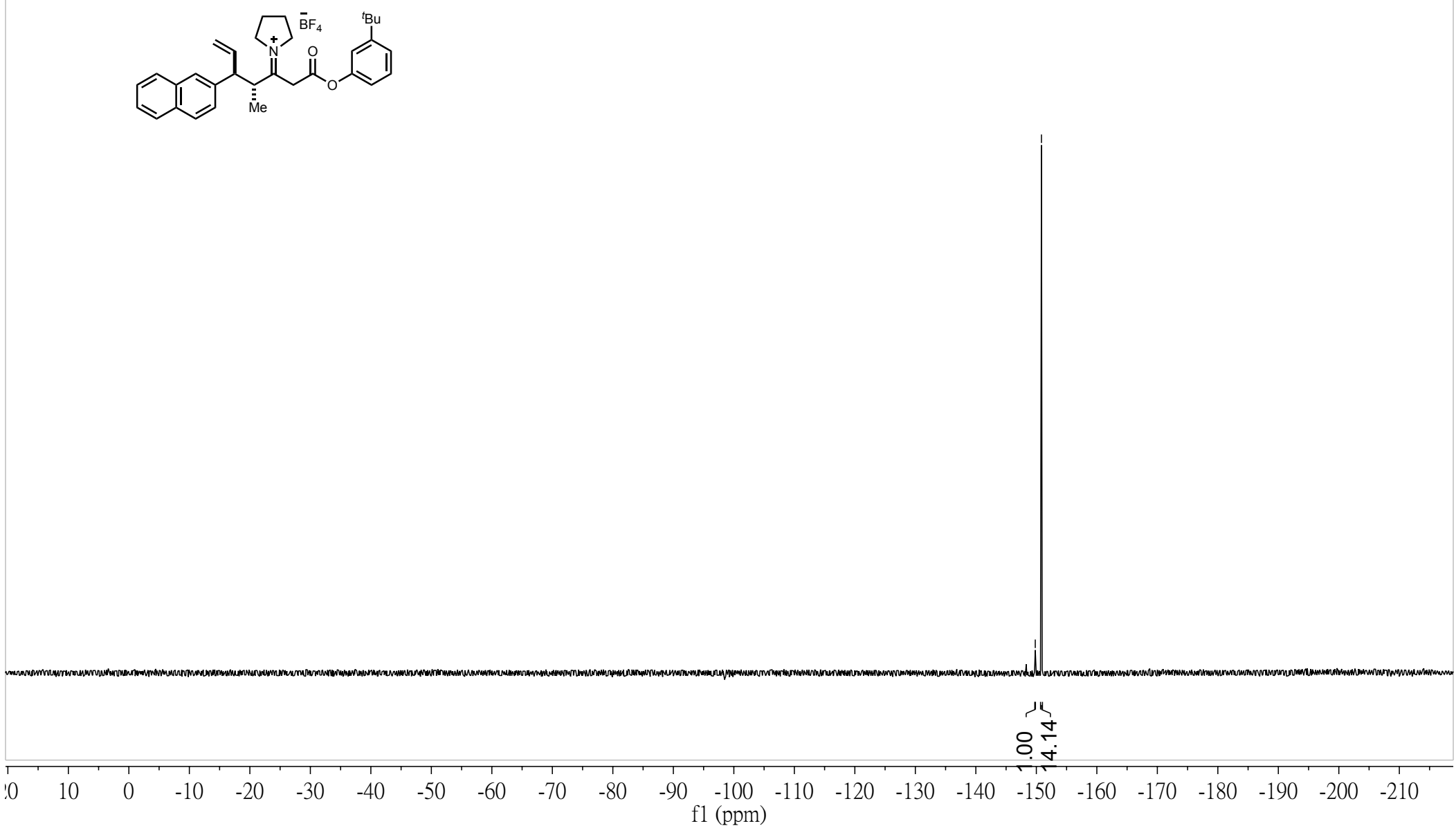

S368 


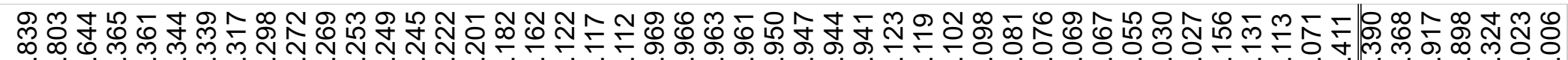

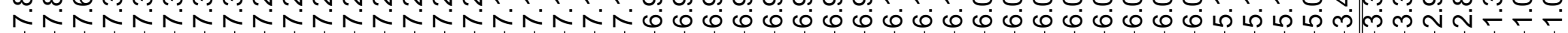

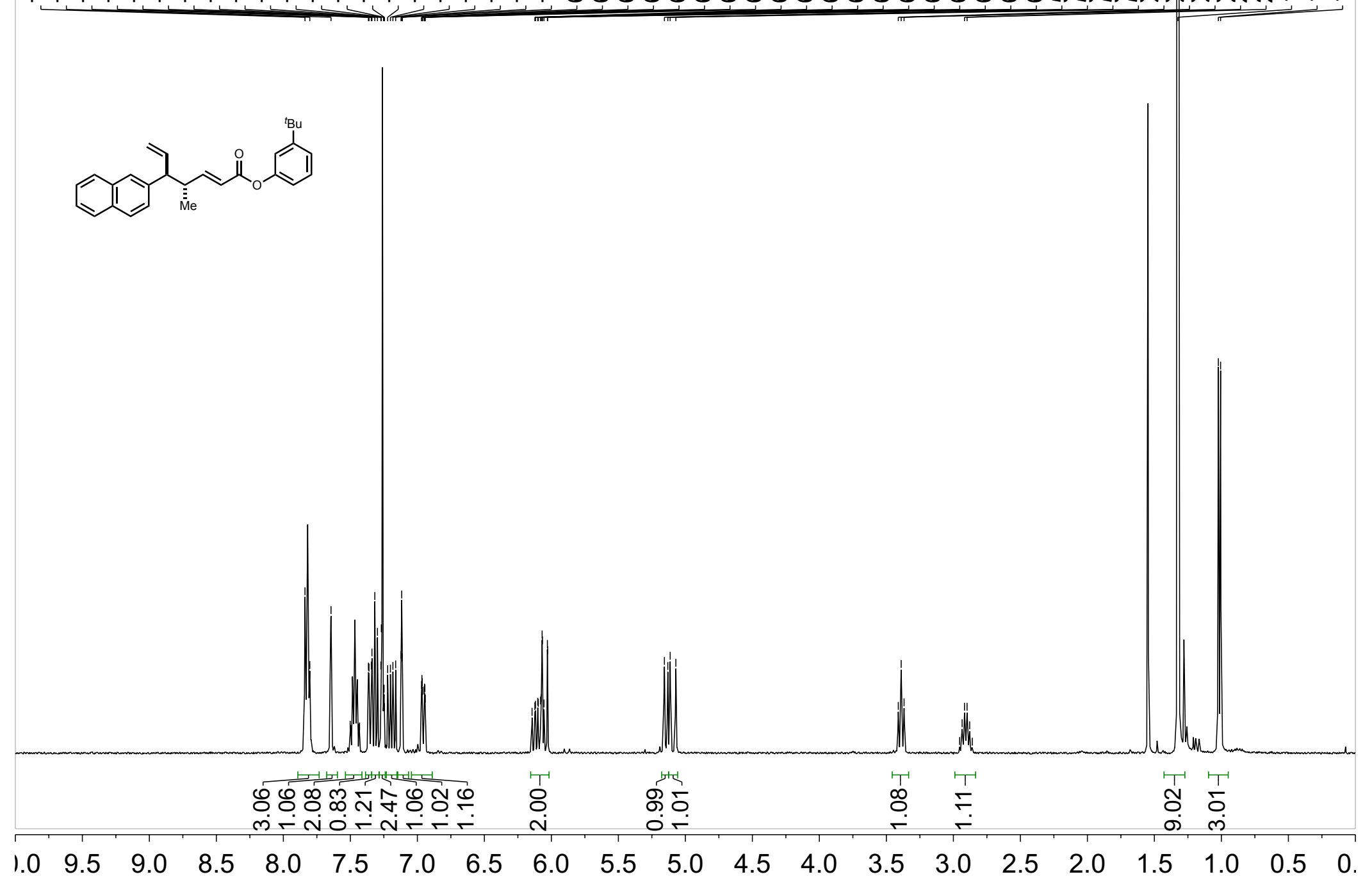




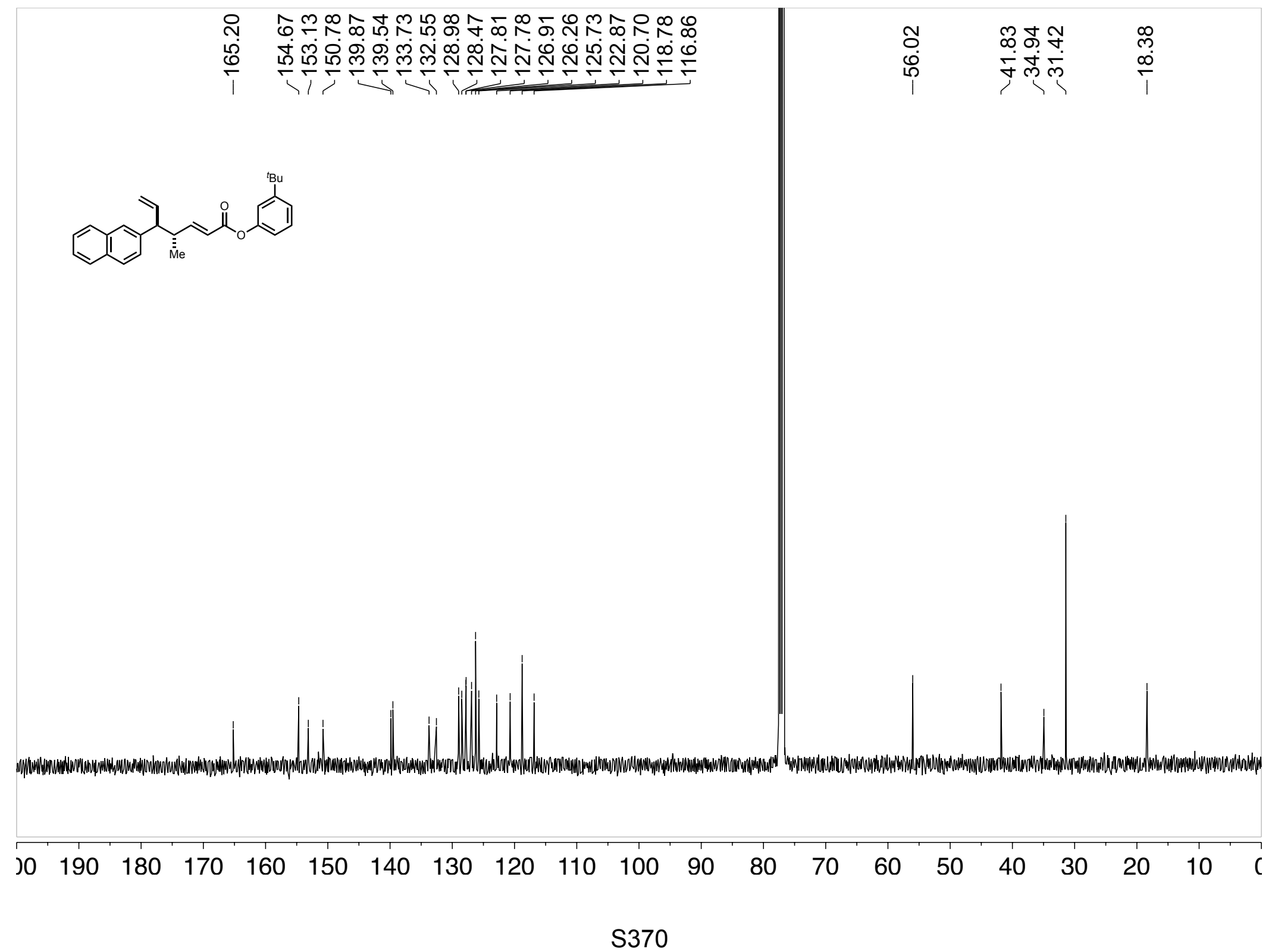




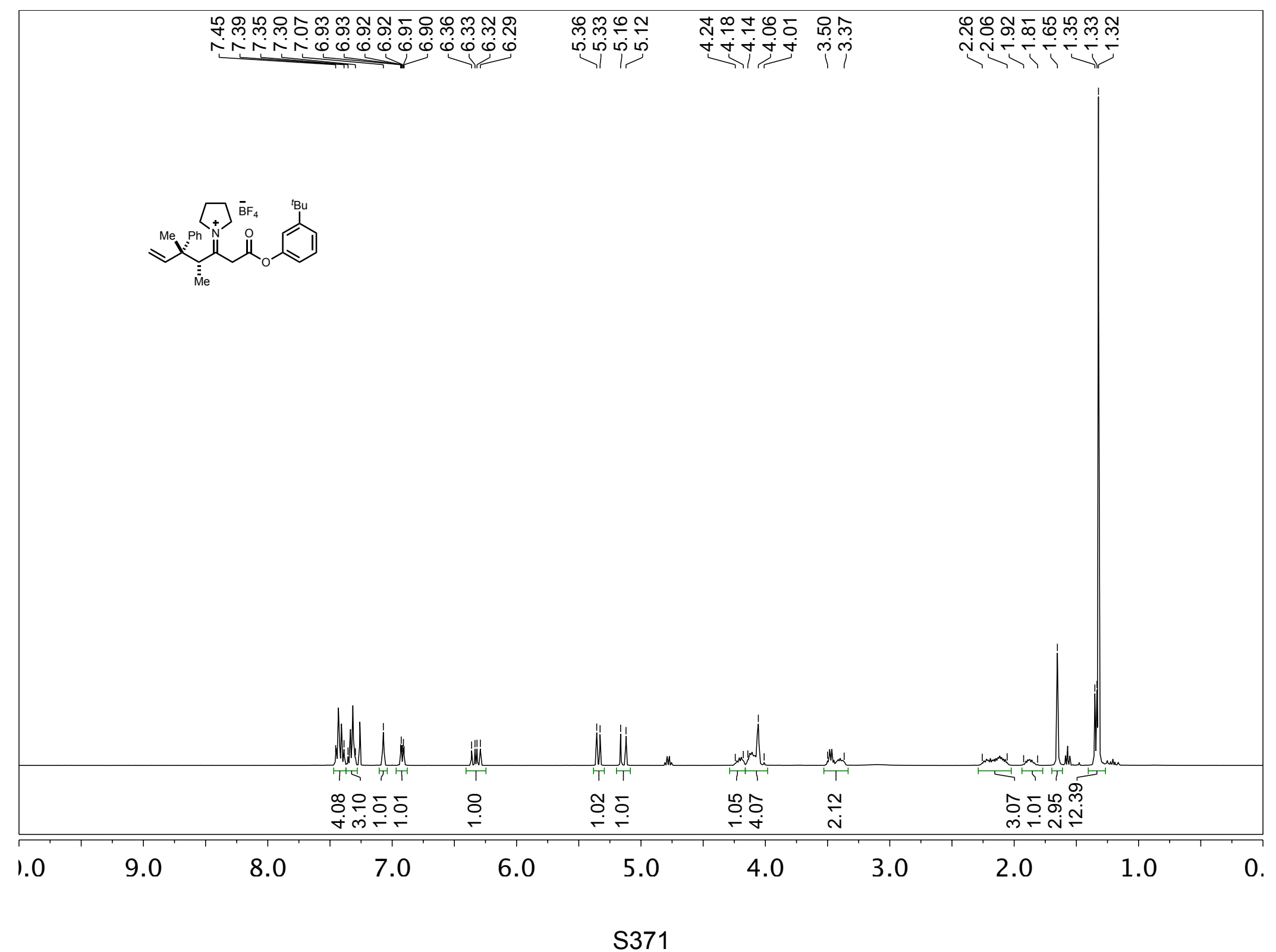




$$
=
$$




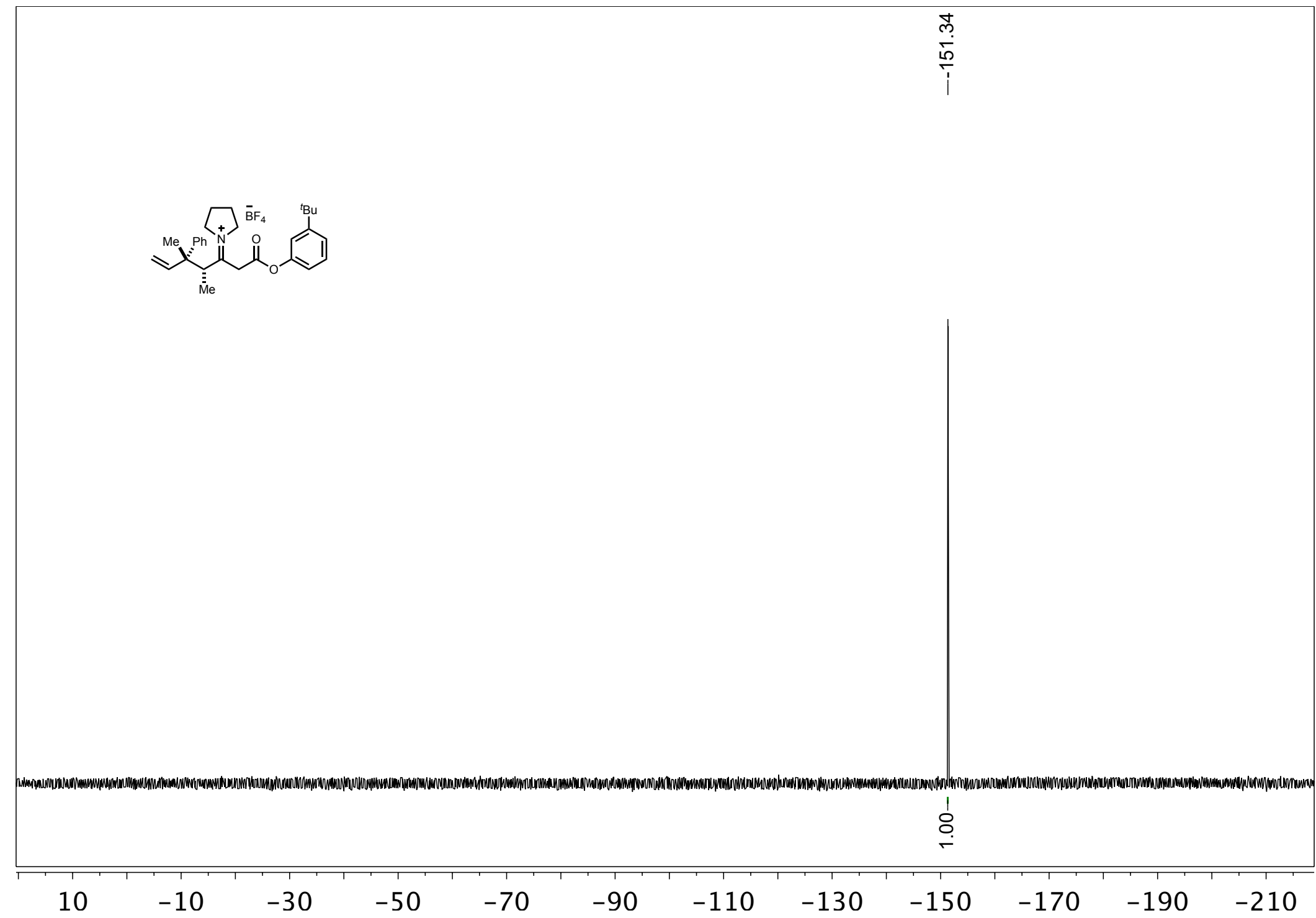




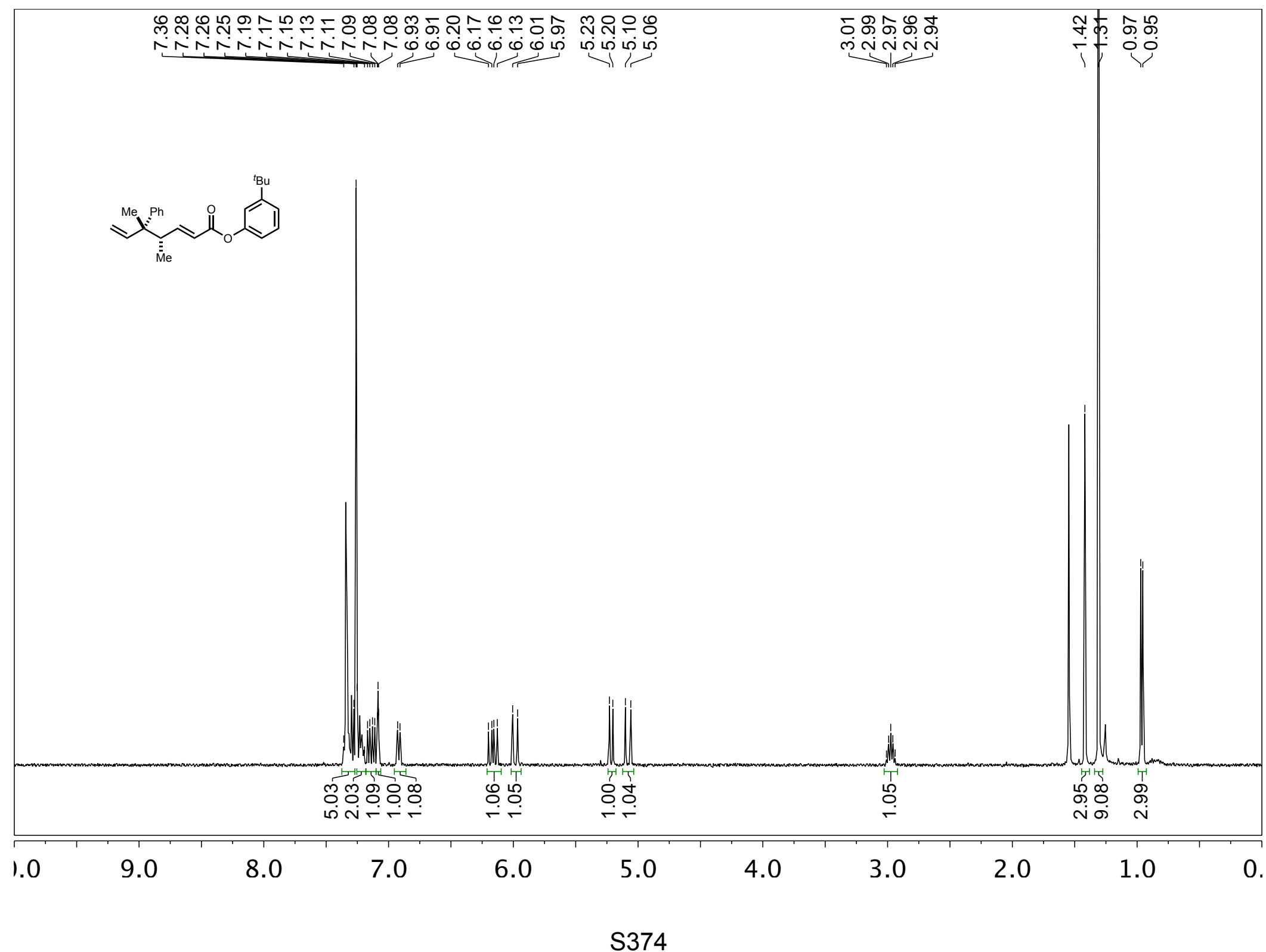




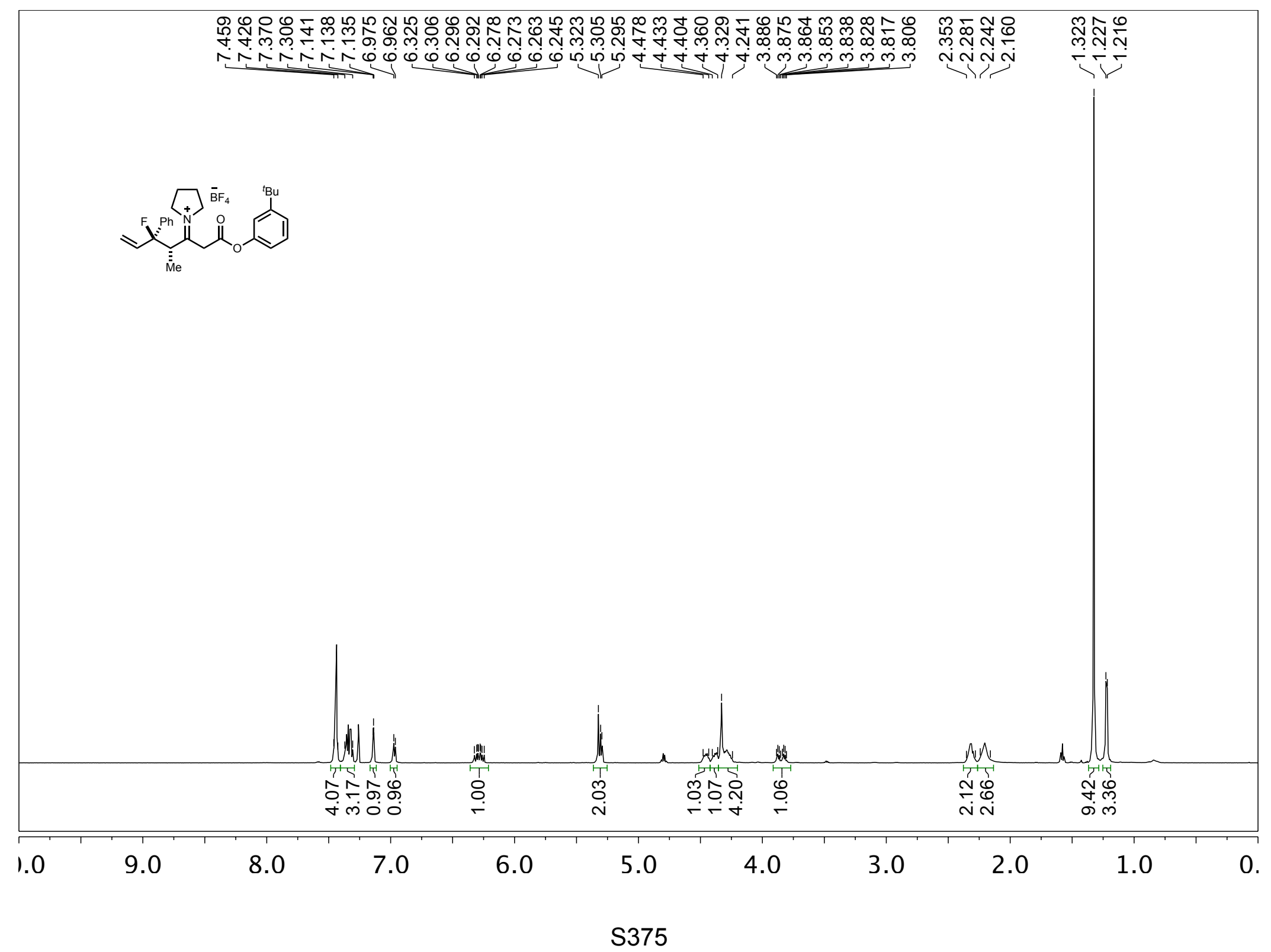




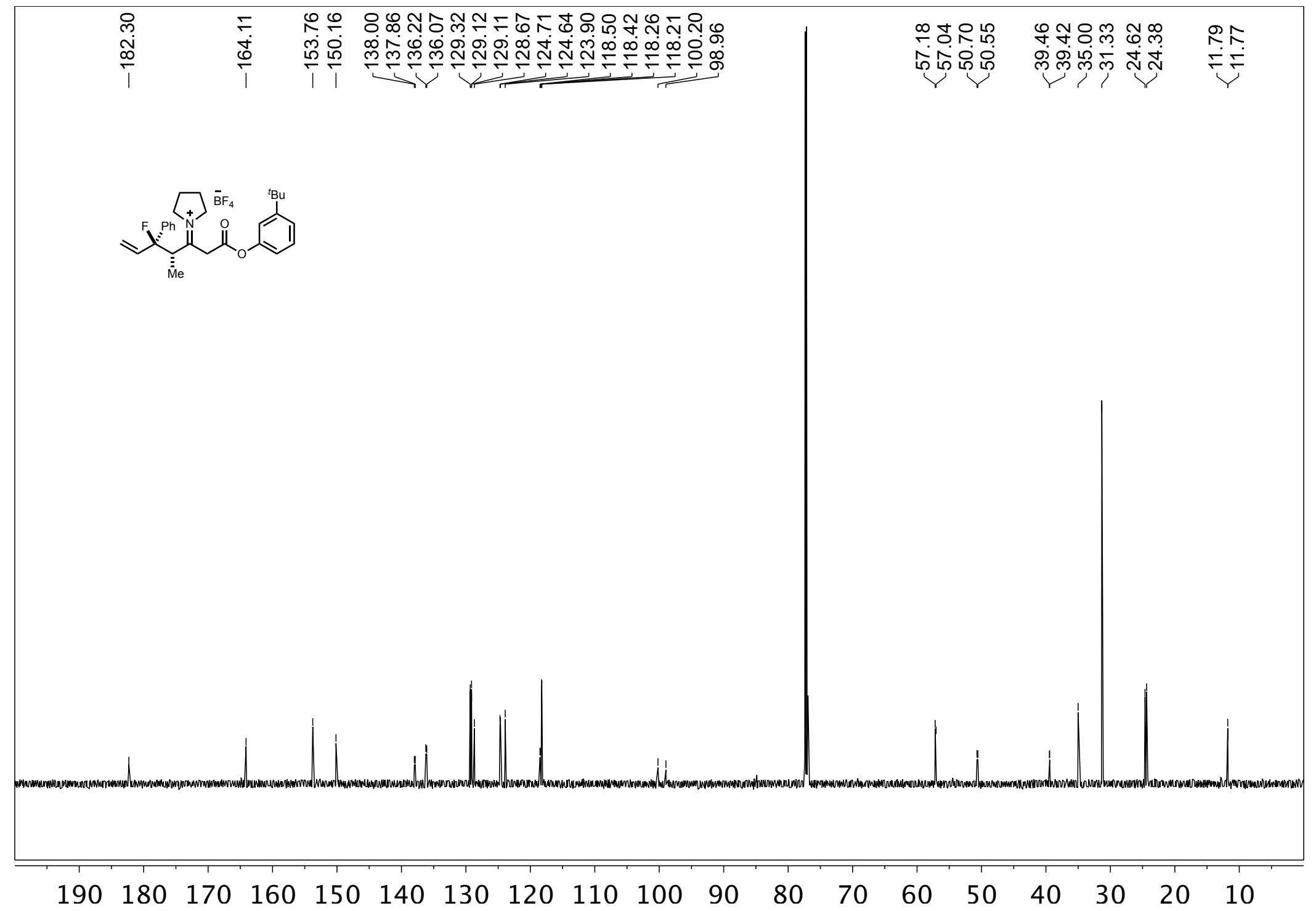




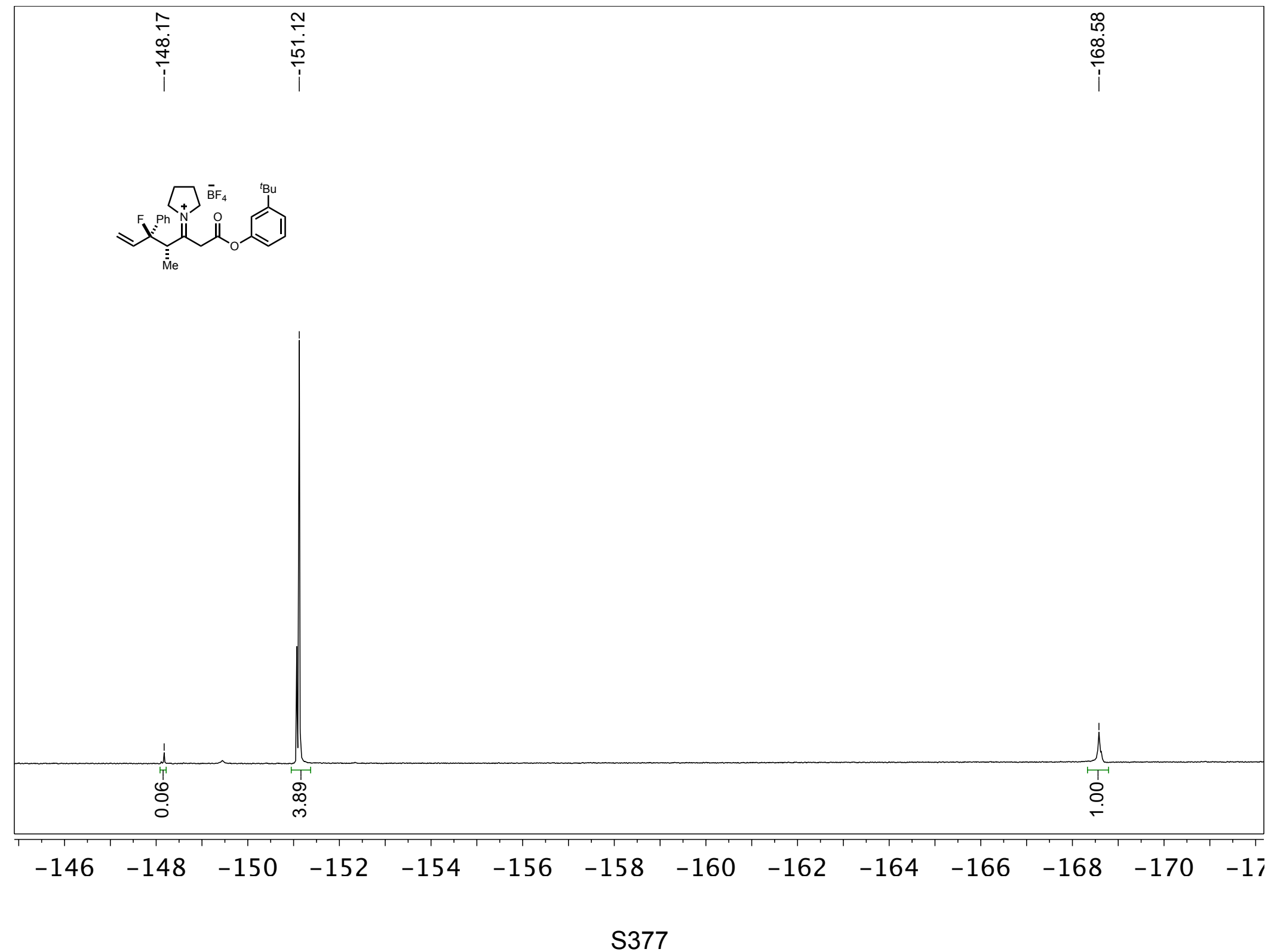




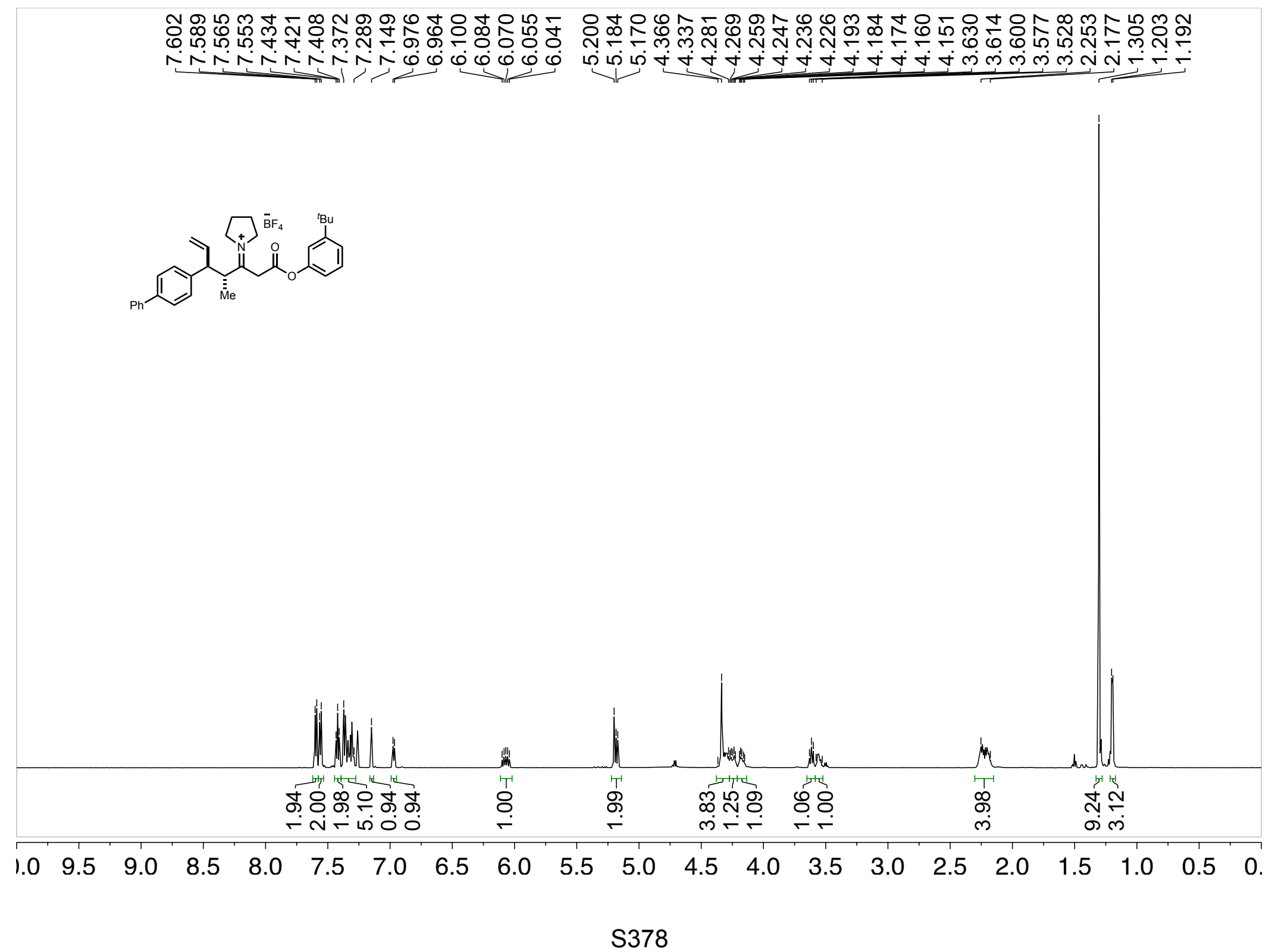




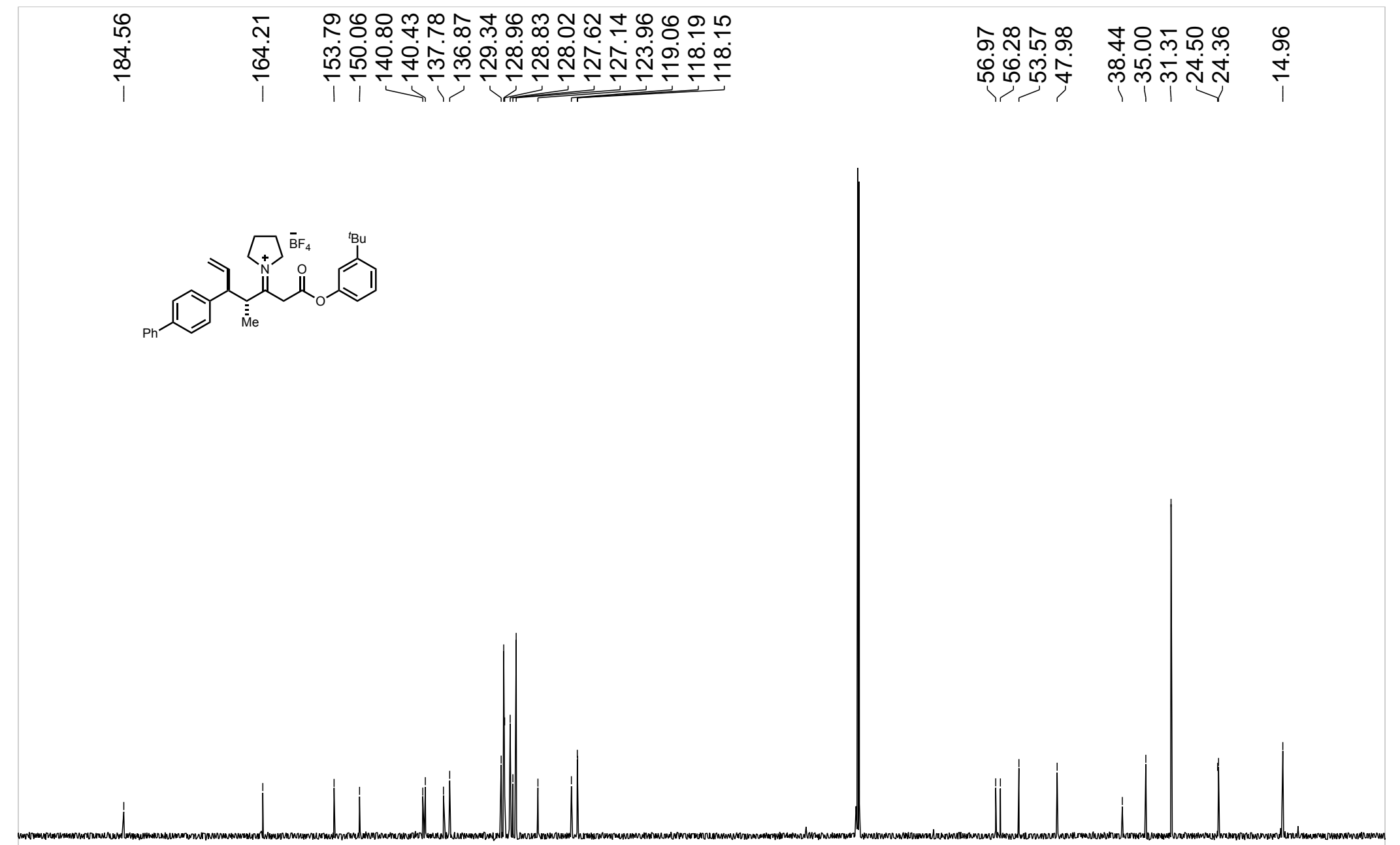

J0 $190 \begin{array}{lllllllllllllllllll}180 & 170 & 160 & 150 & 140 & 130 & 120 & 110 & 100 & 90 & 80 & 70 & 60 & 50 & 40 & 30 & 20 & 10 & (\end{array}$ 


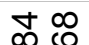

安官

i

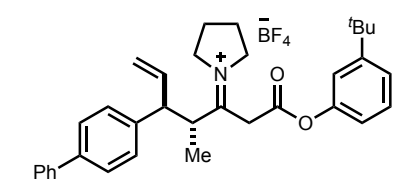

mowW

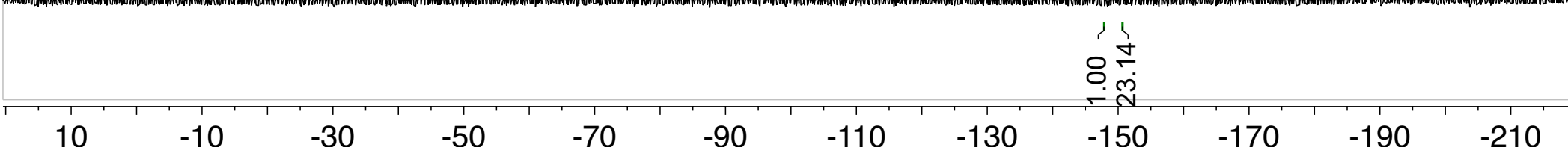




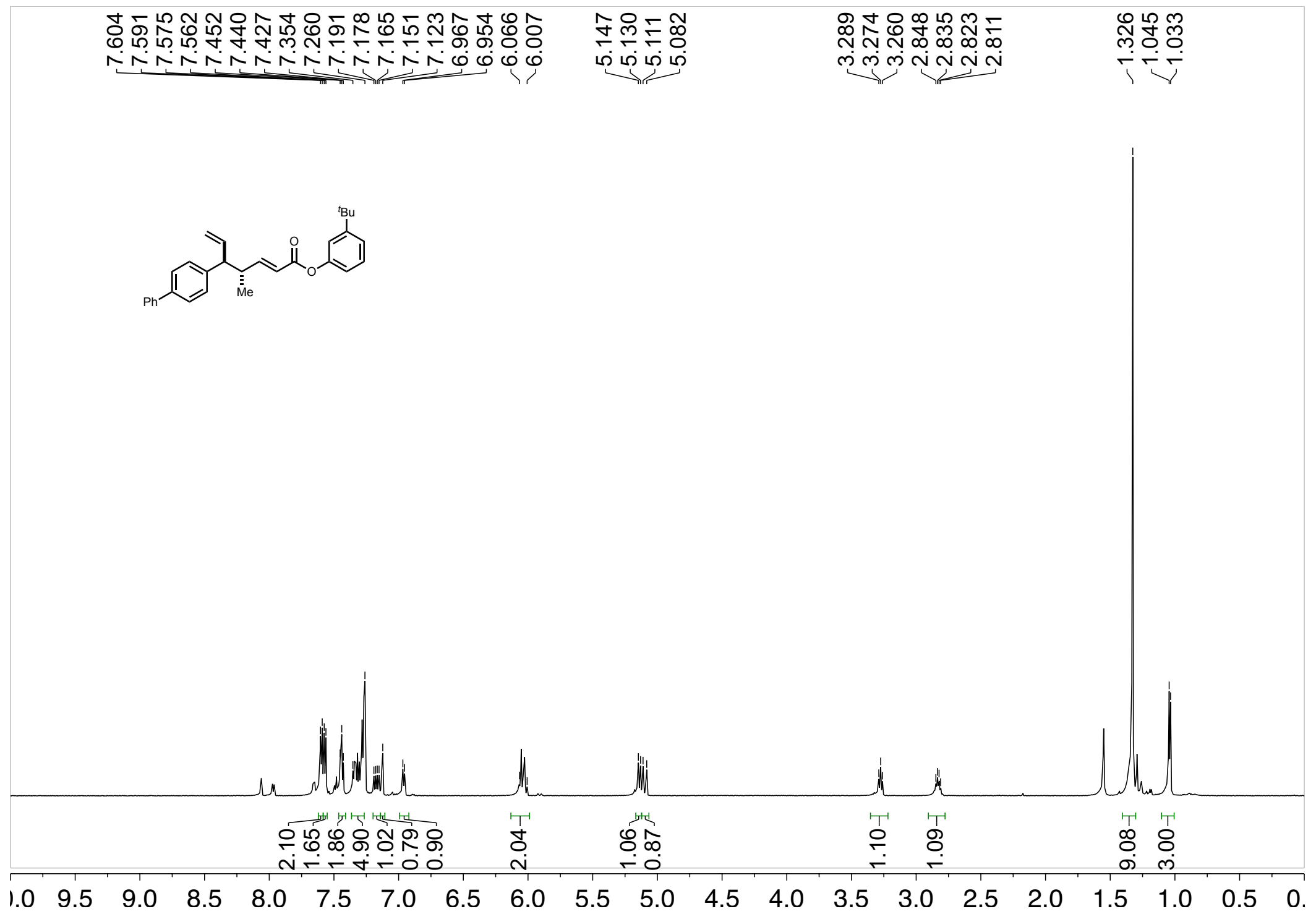

S381 


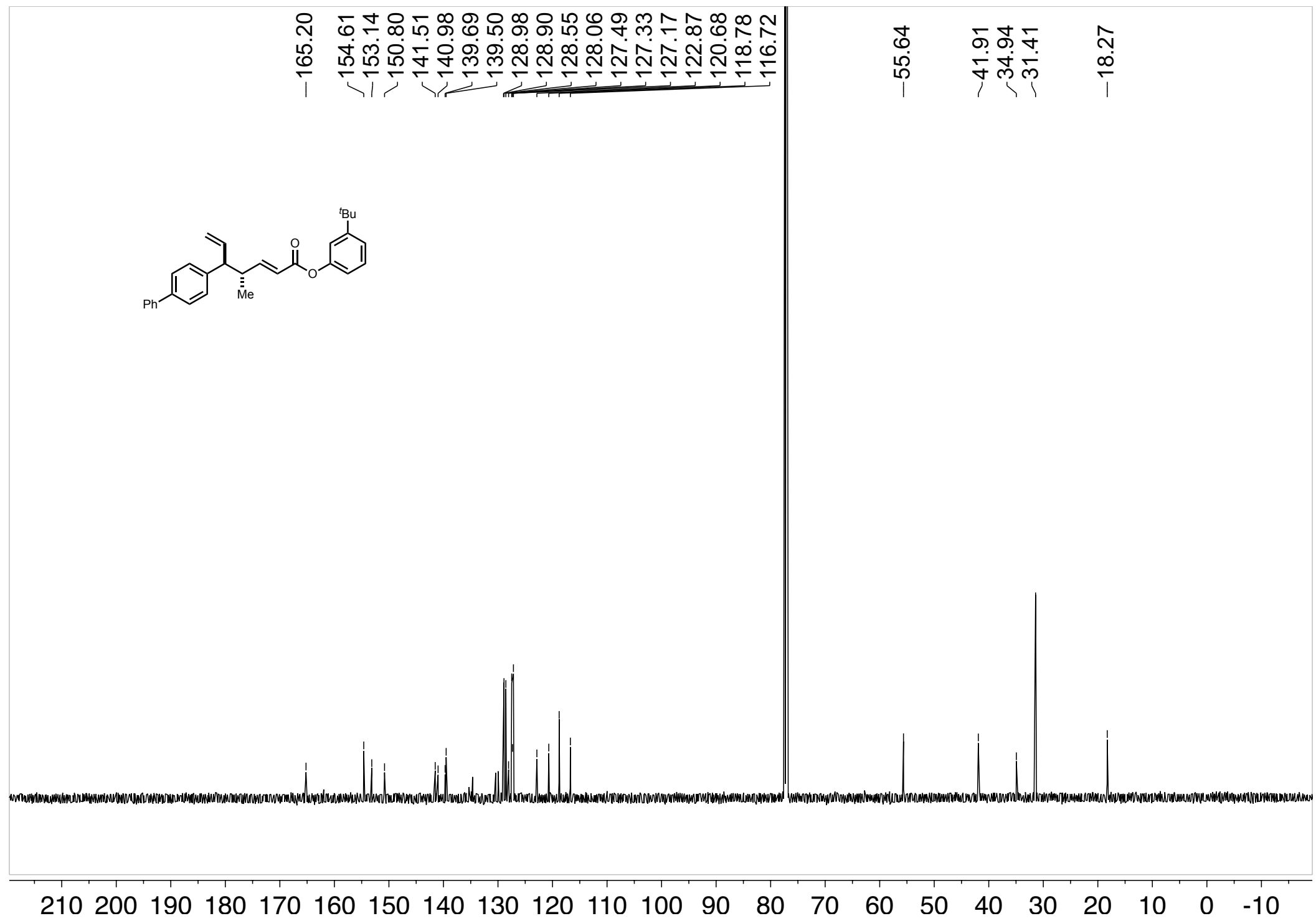

S382 


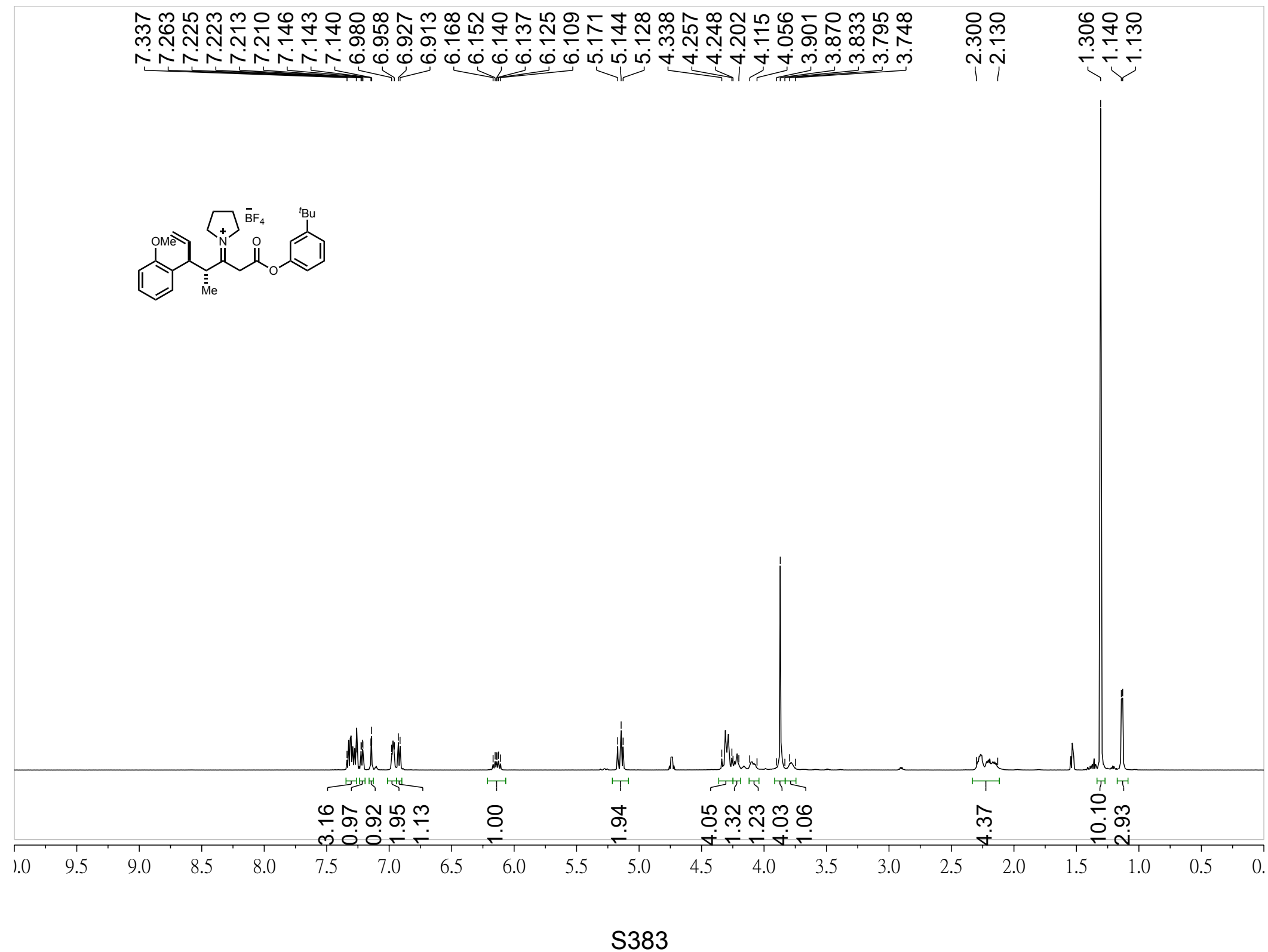



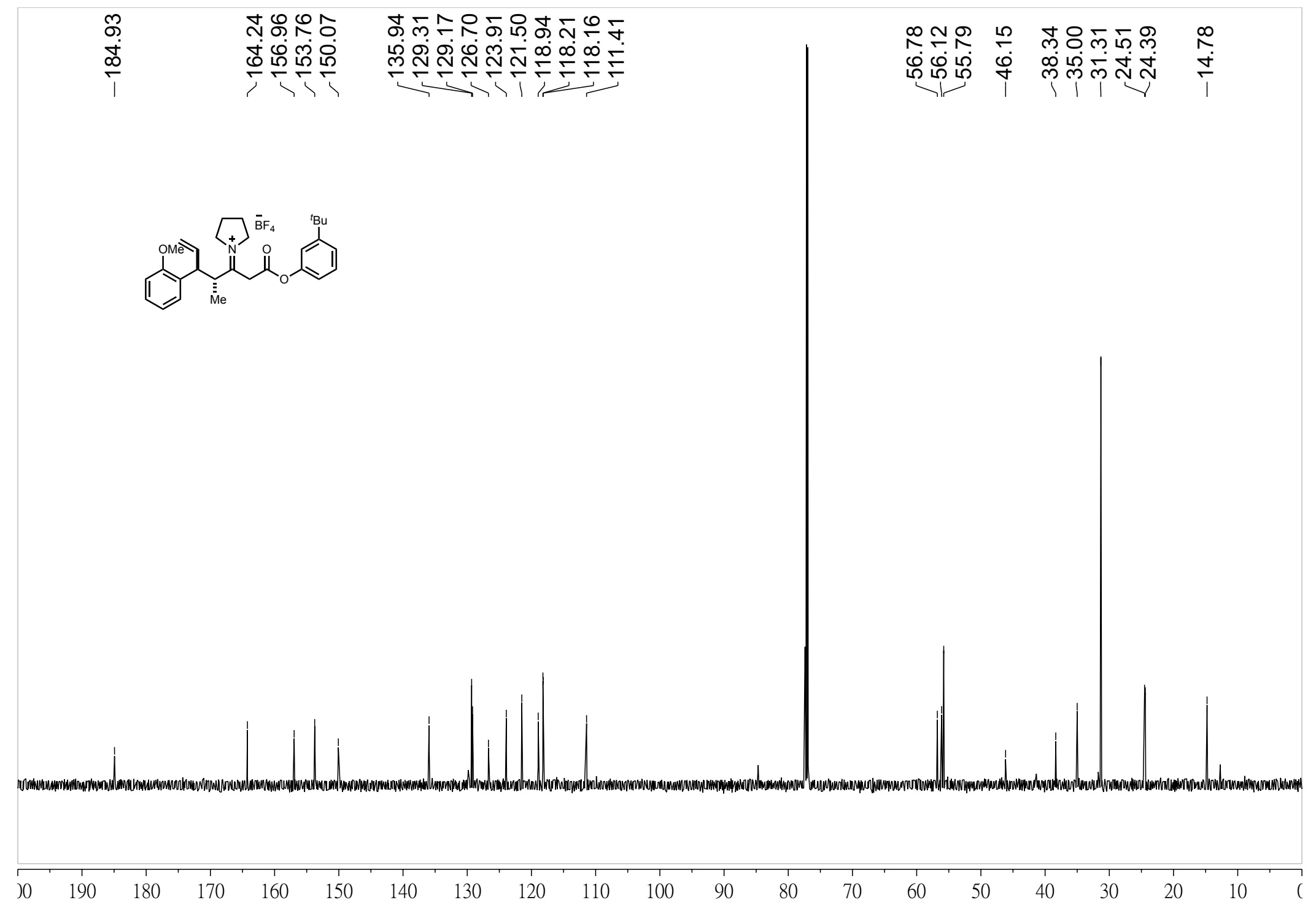


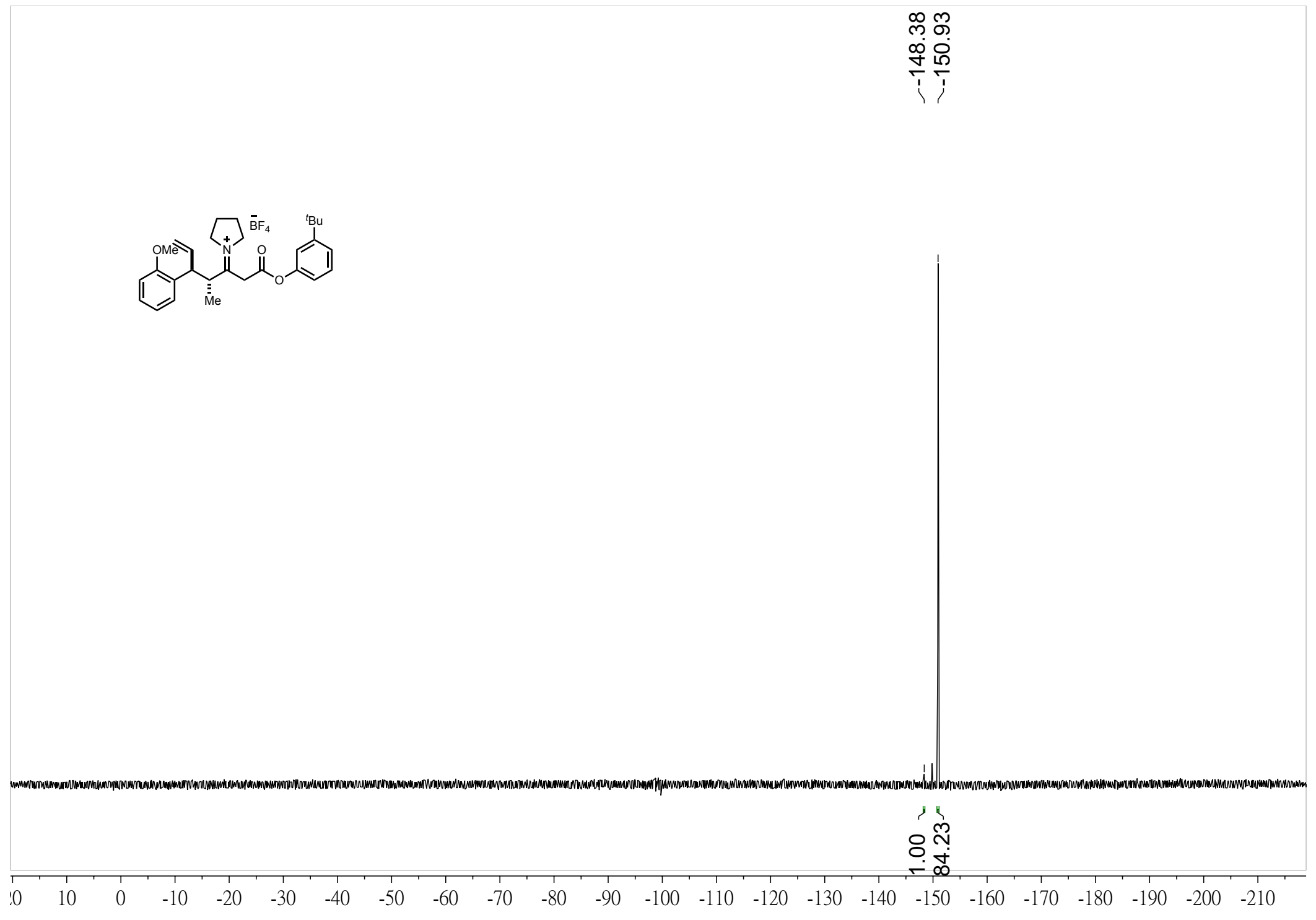

S385 


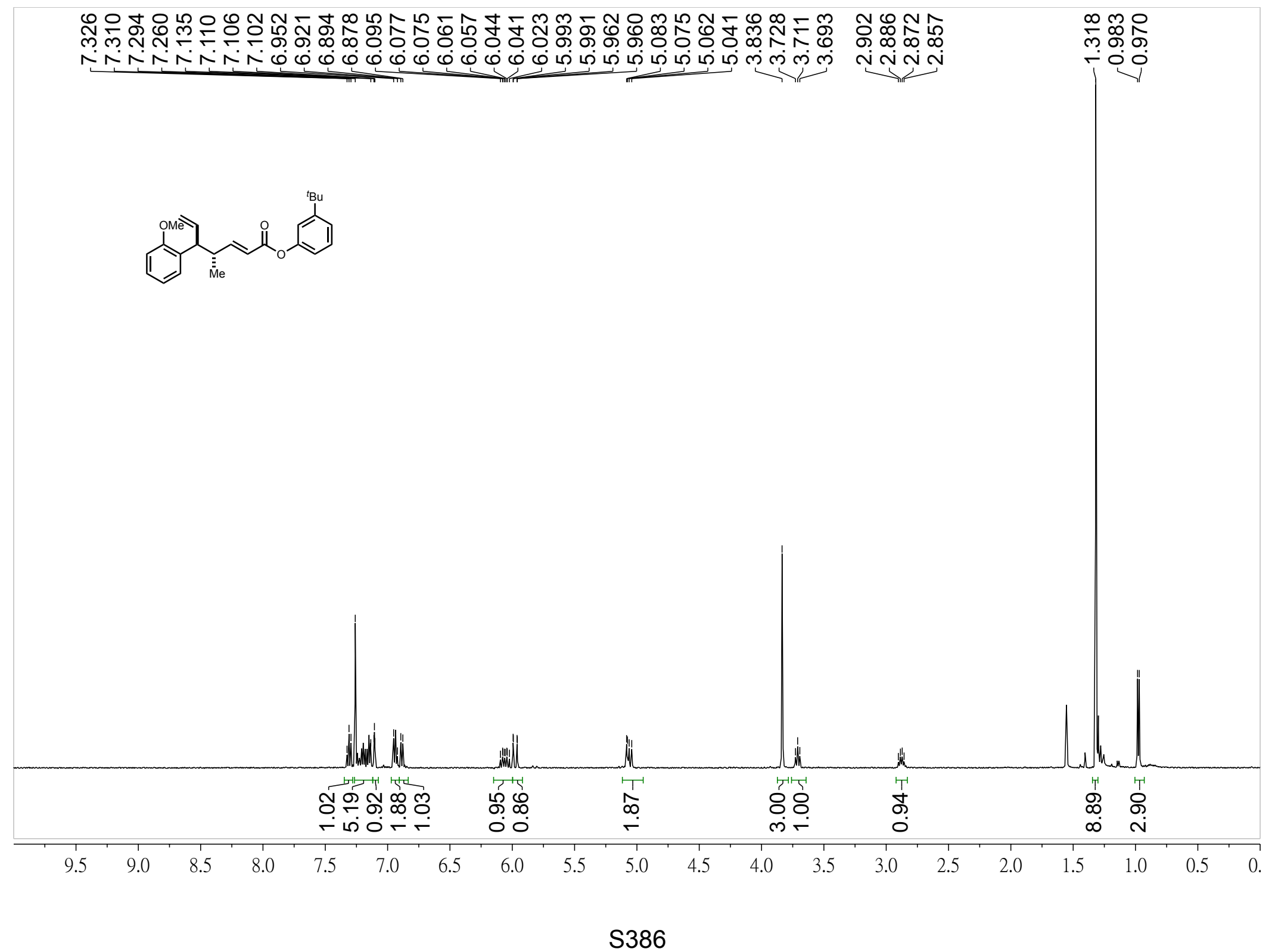



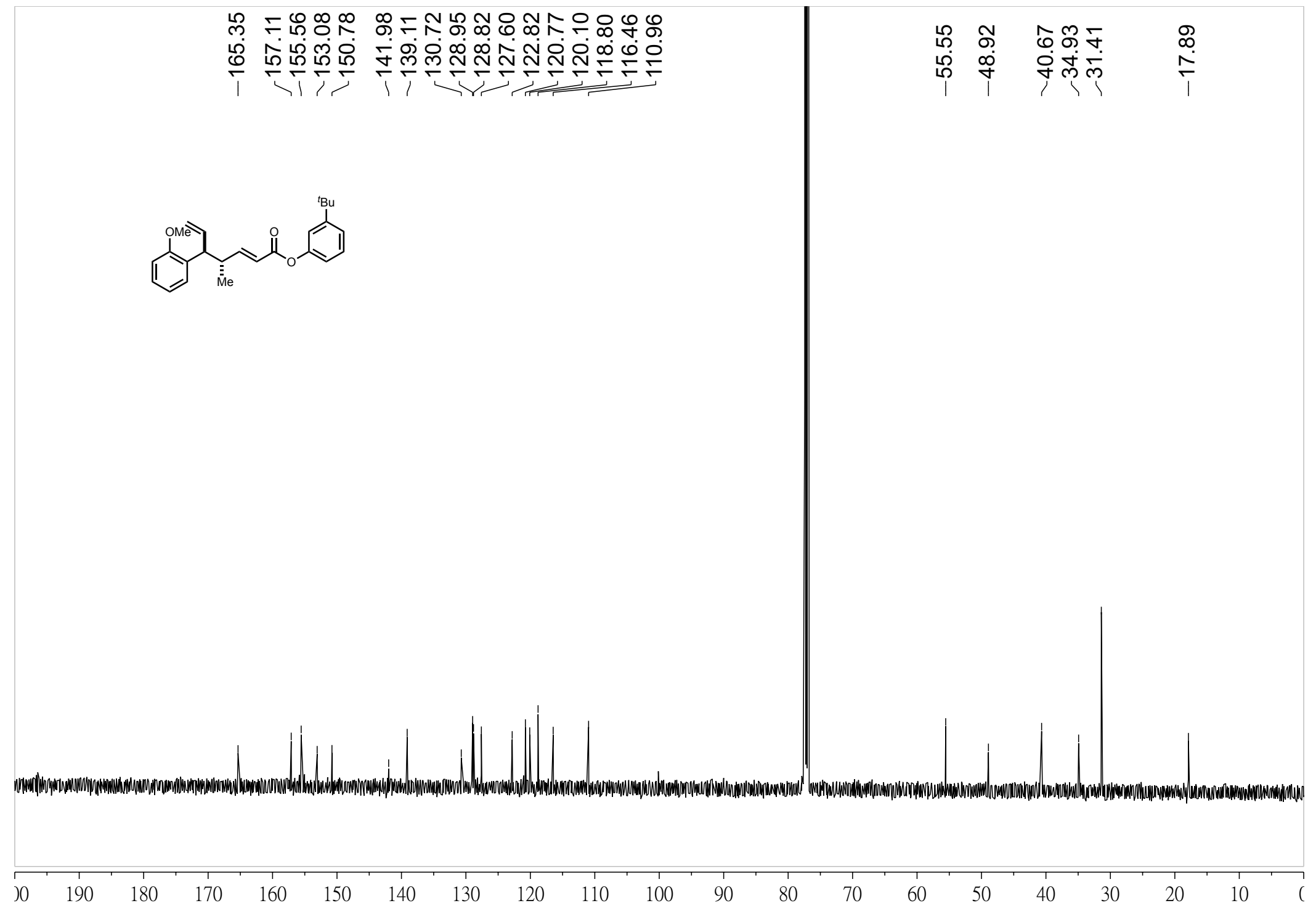


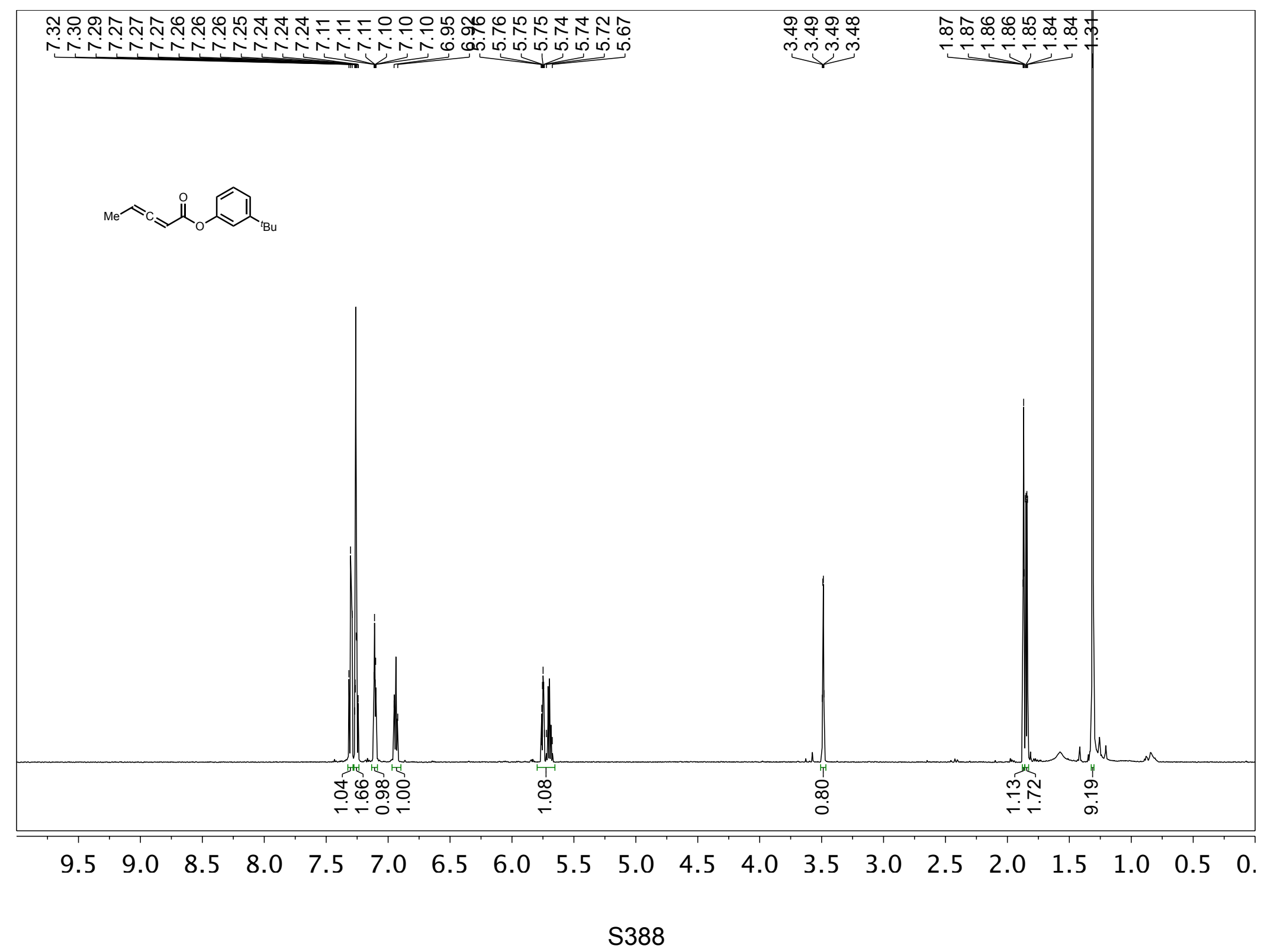




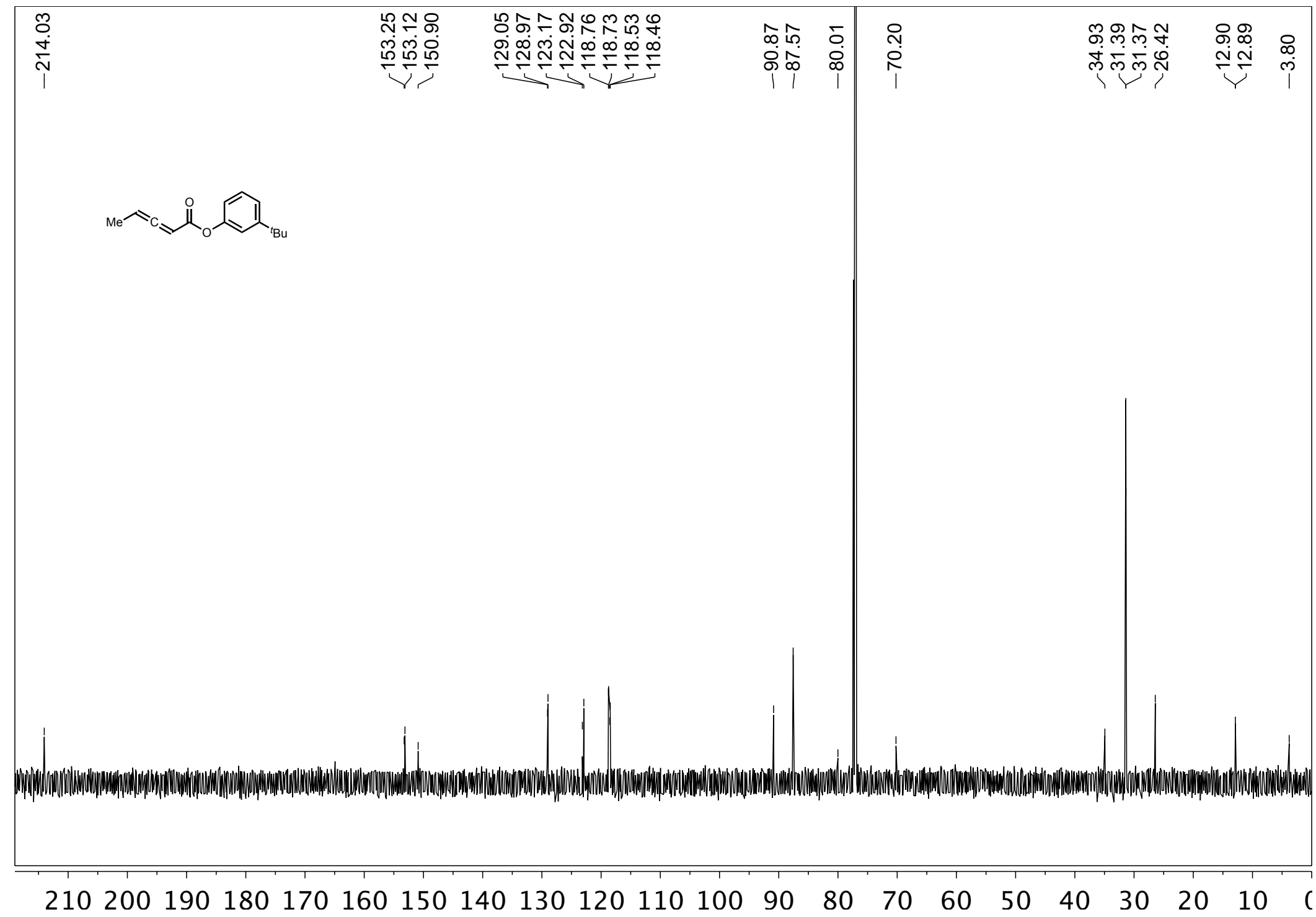




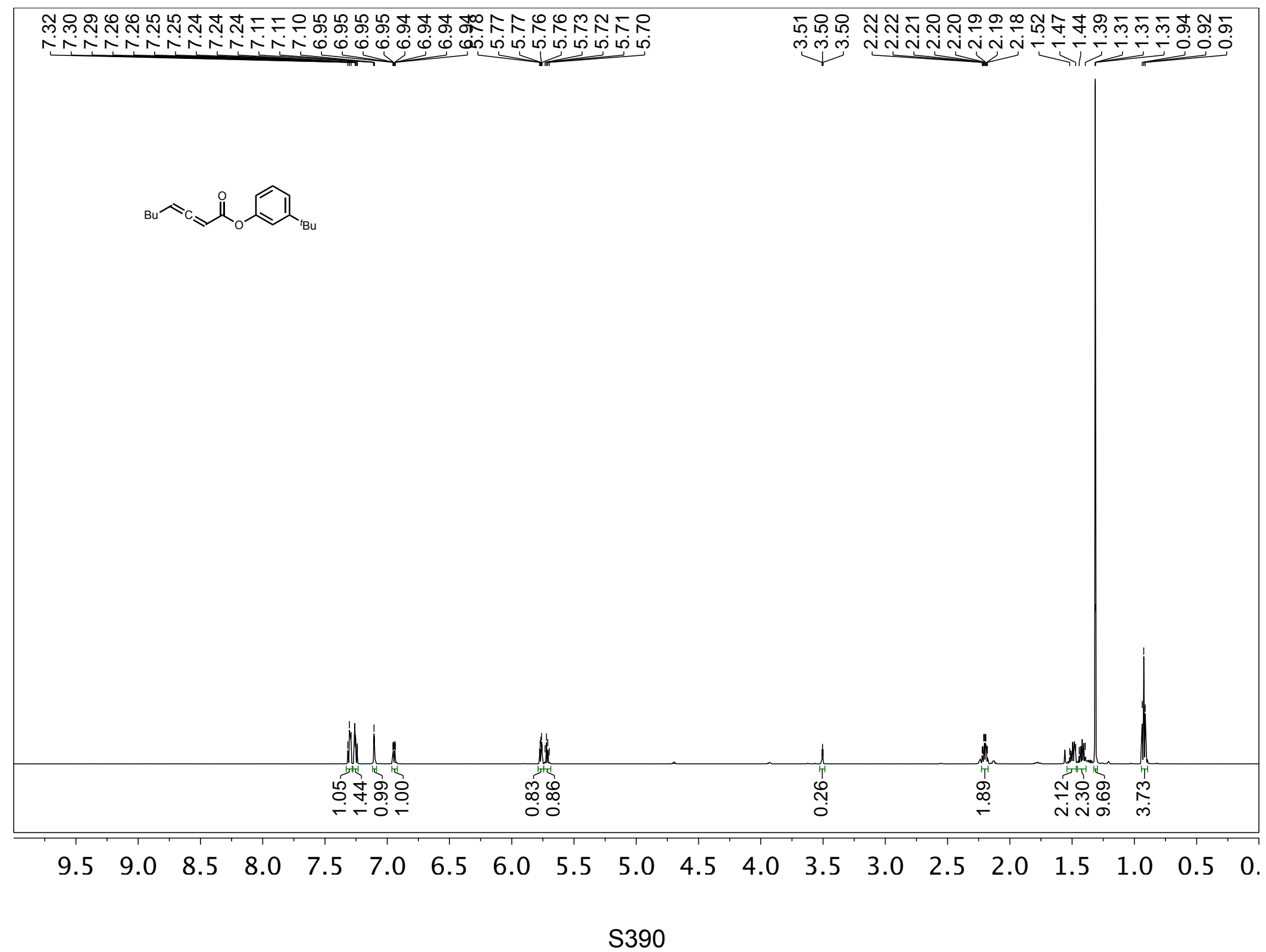




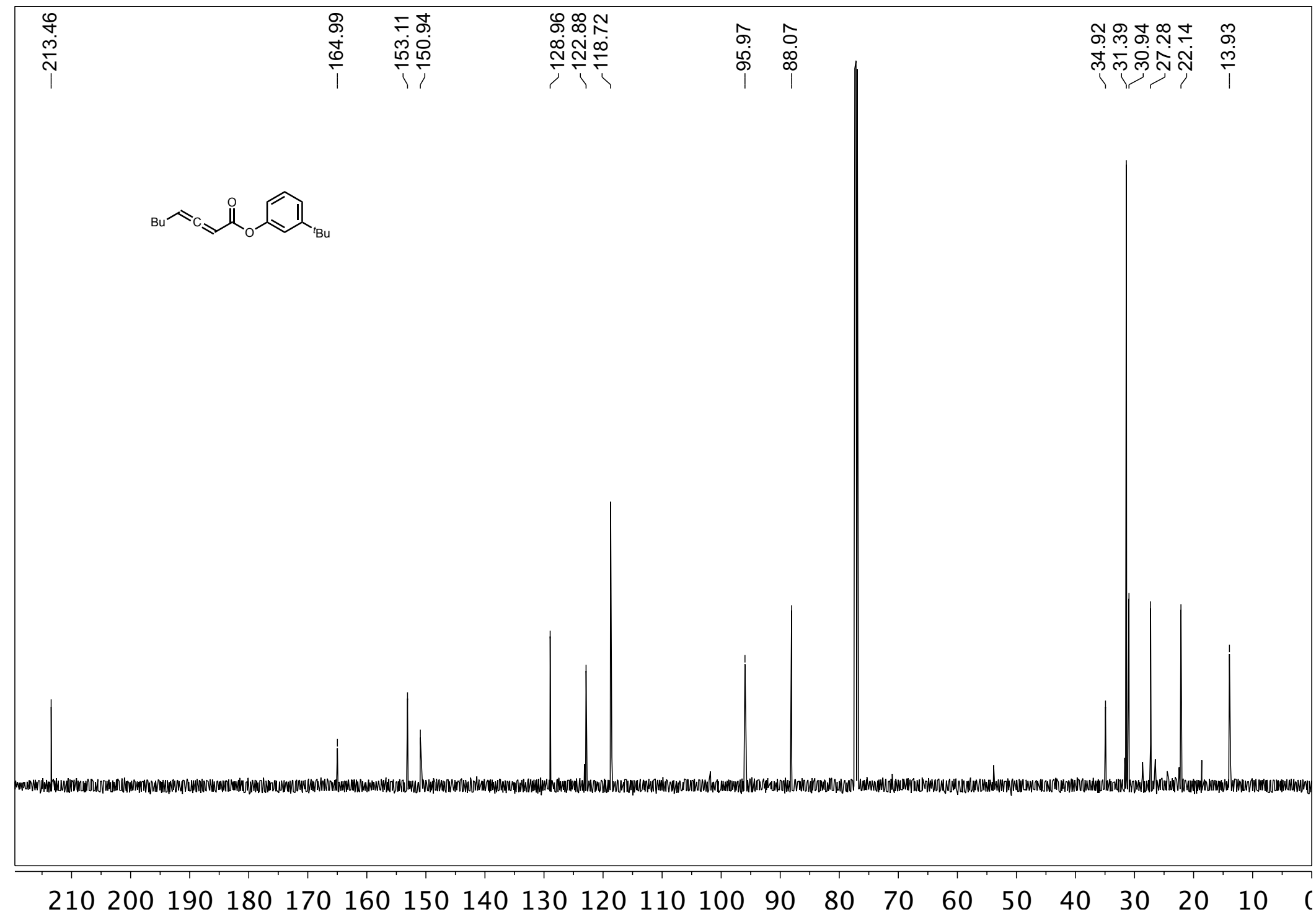




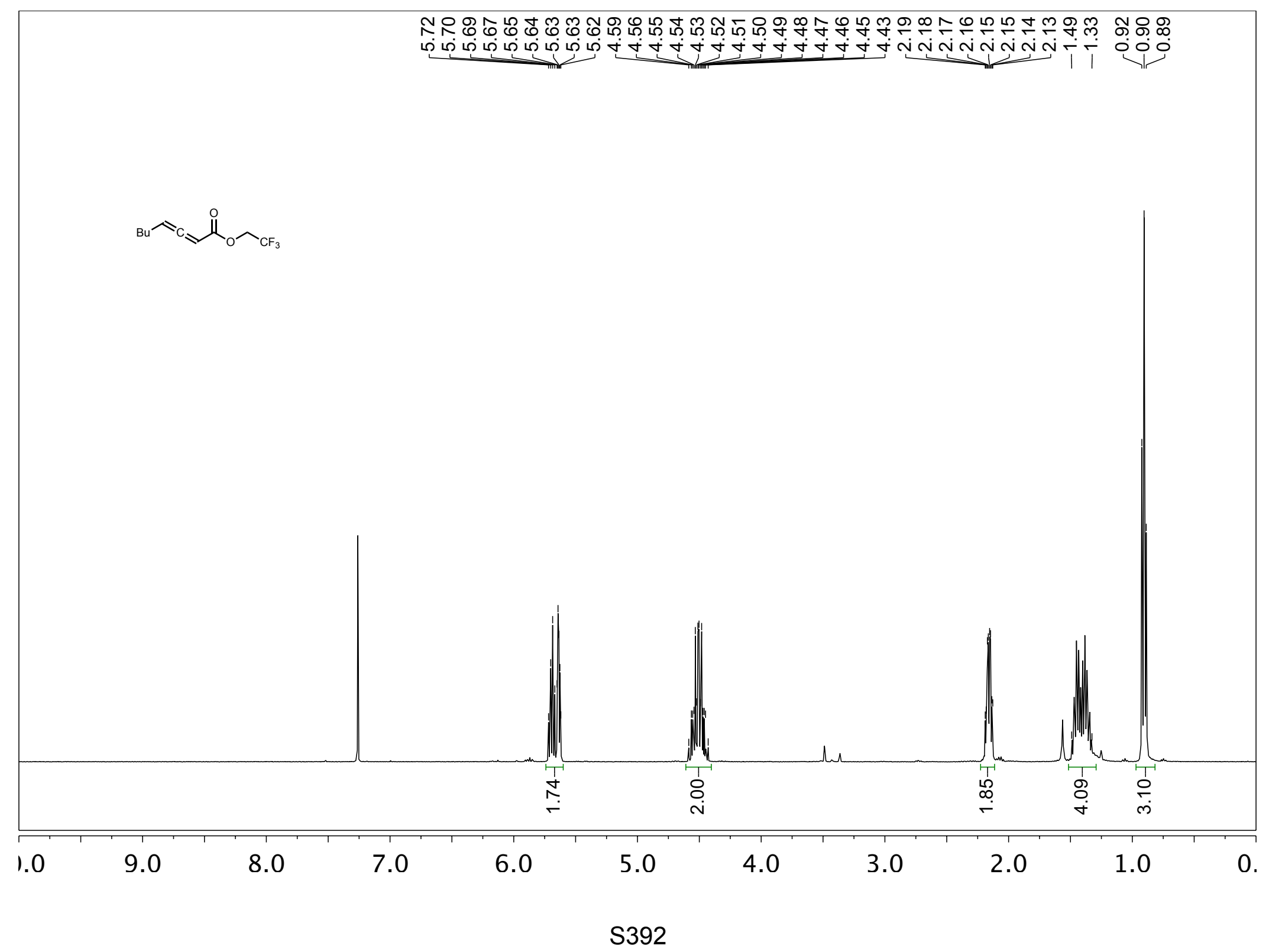




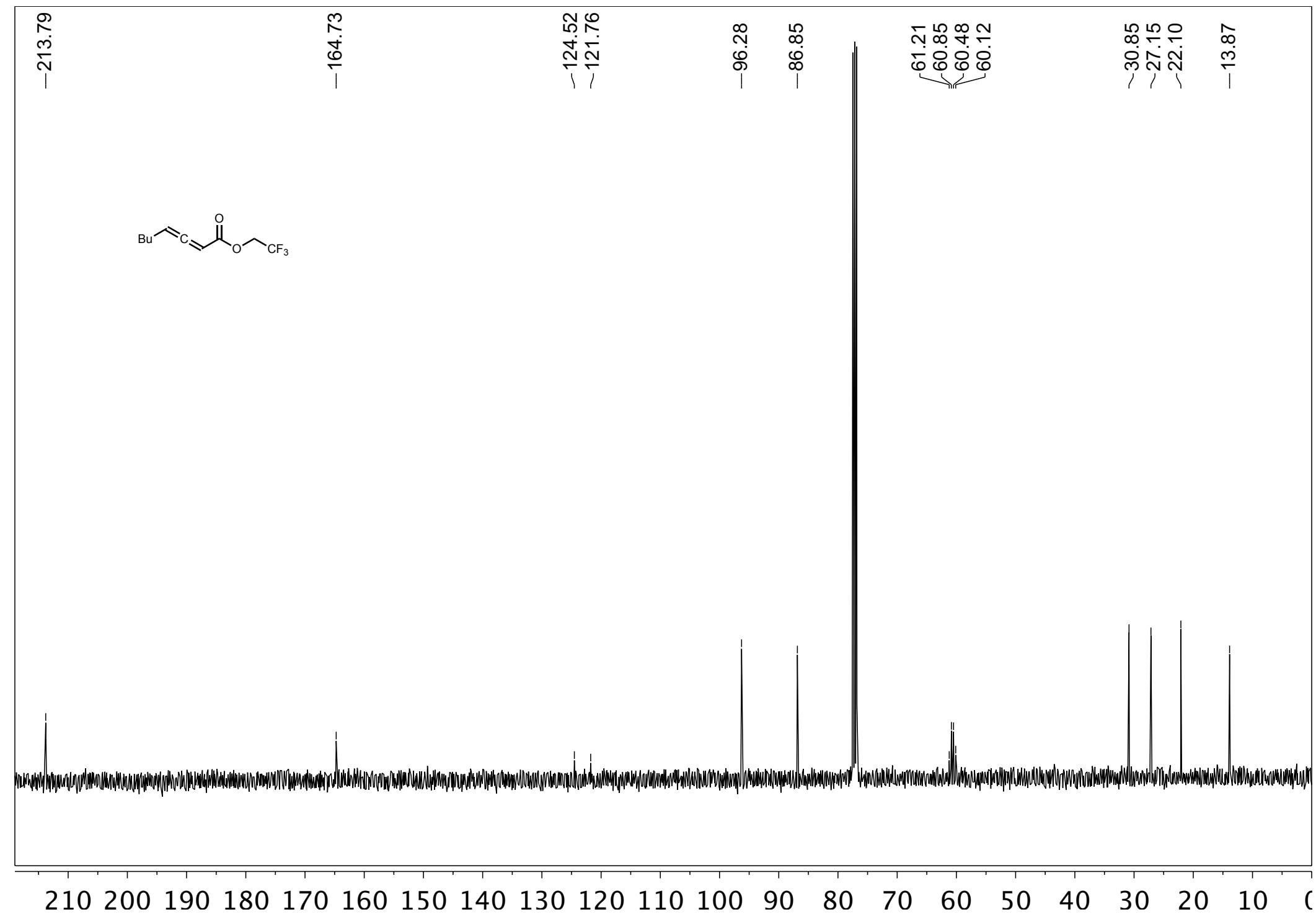




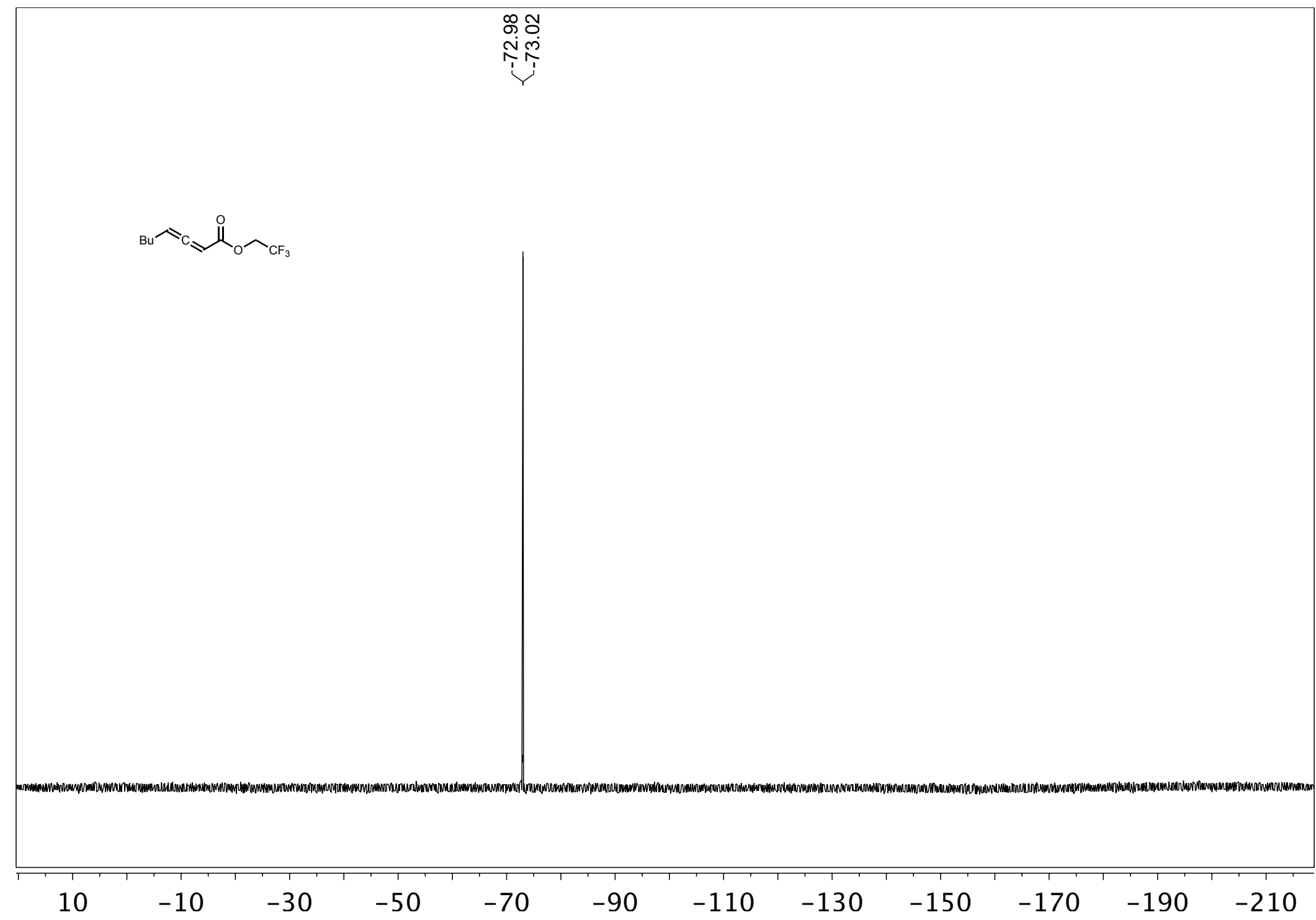




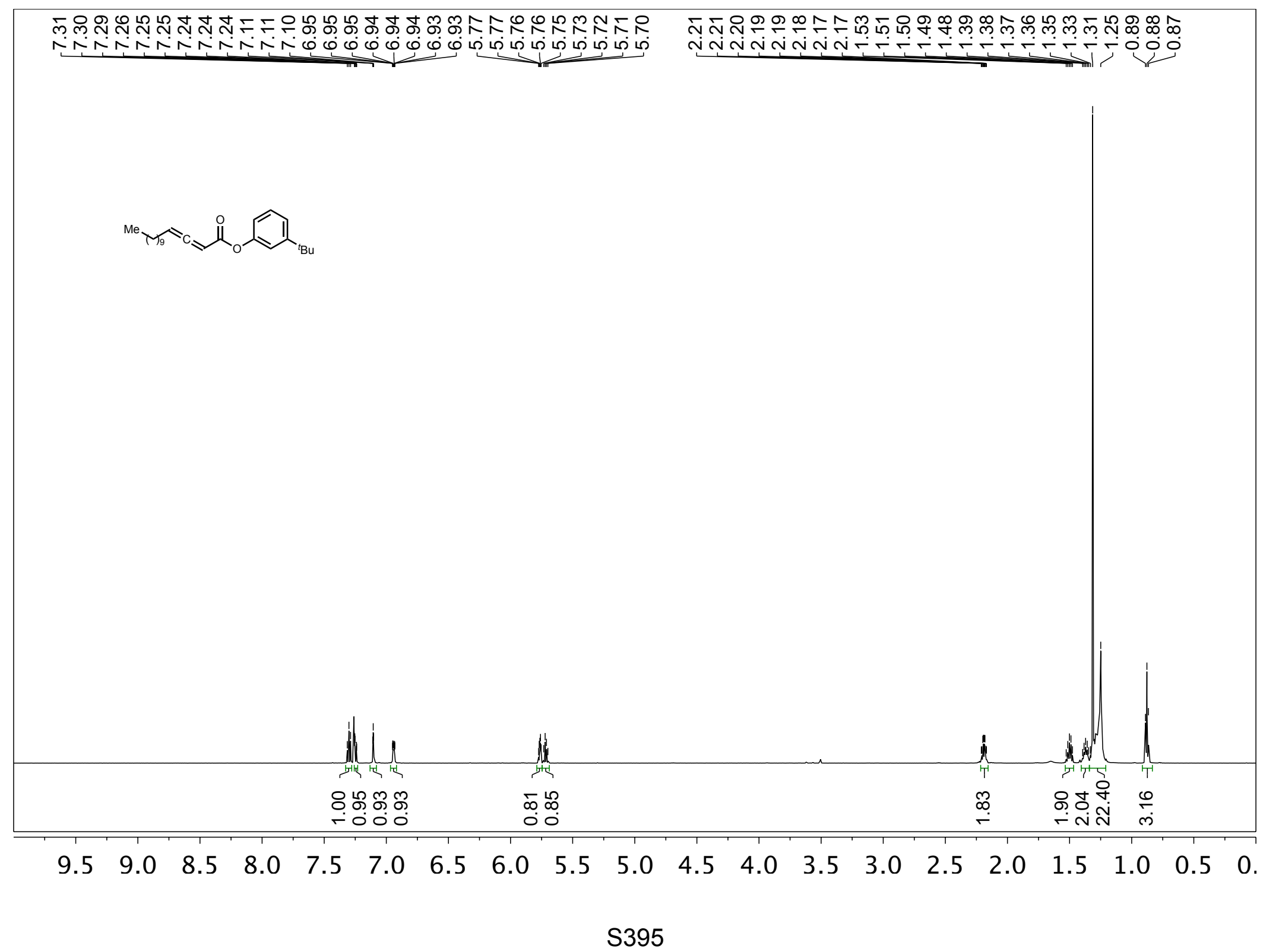




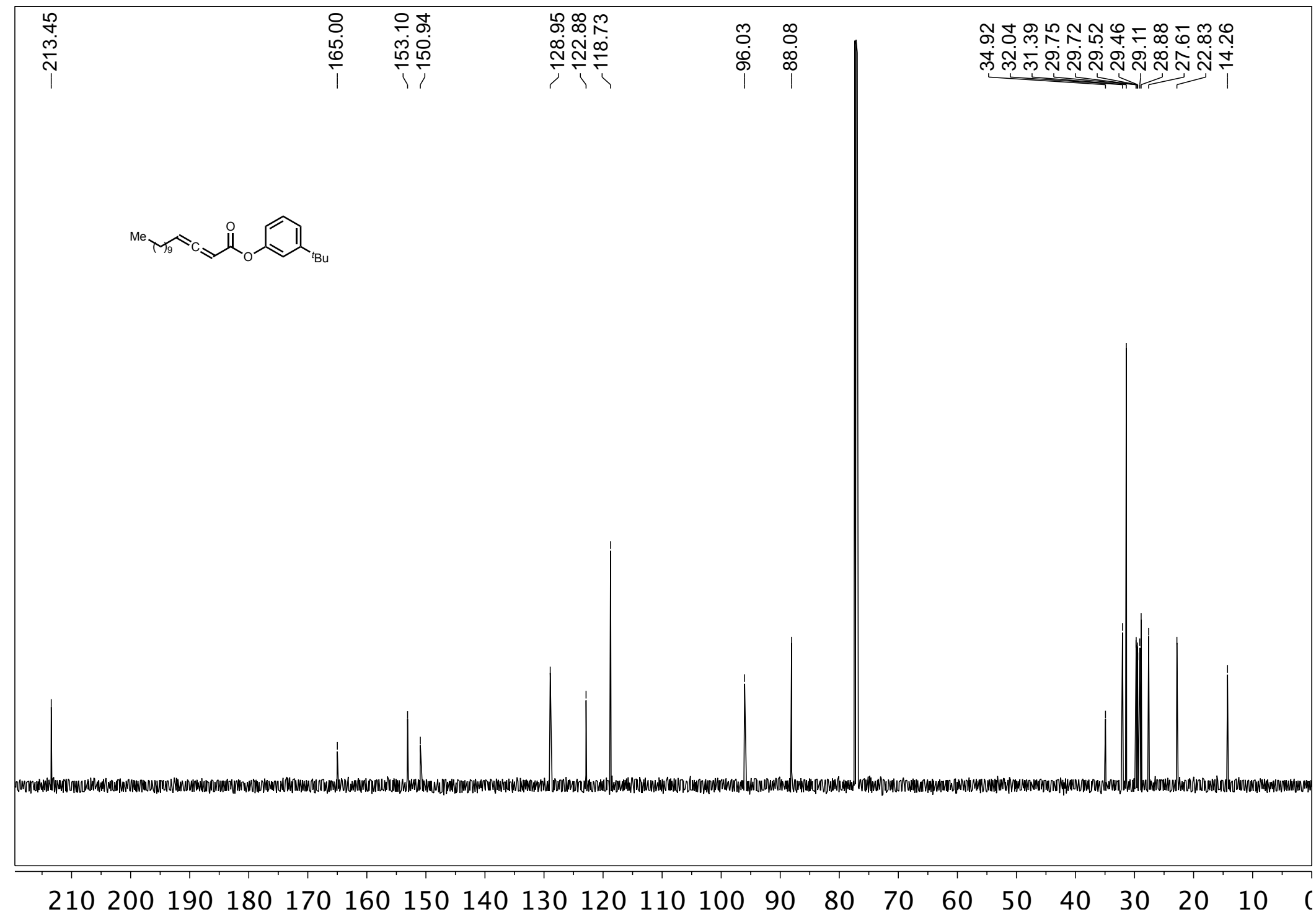




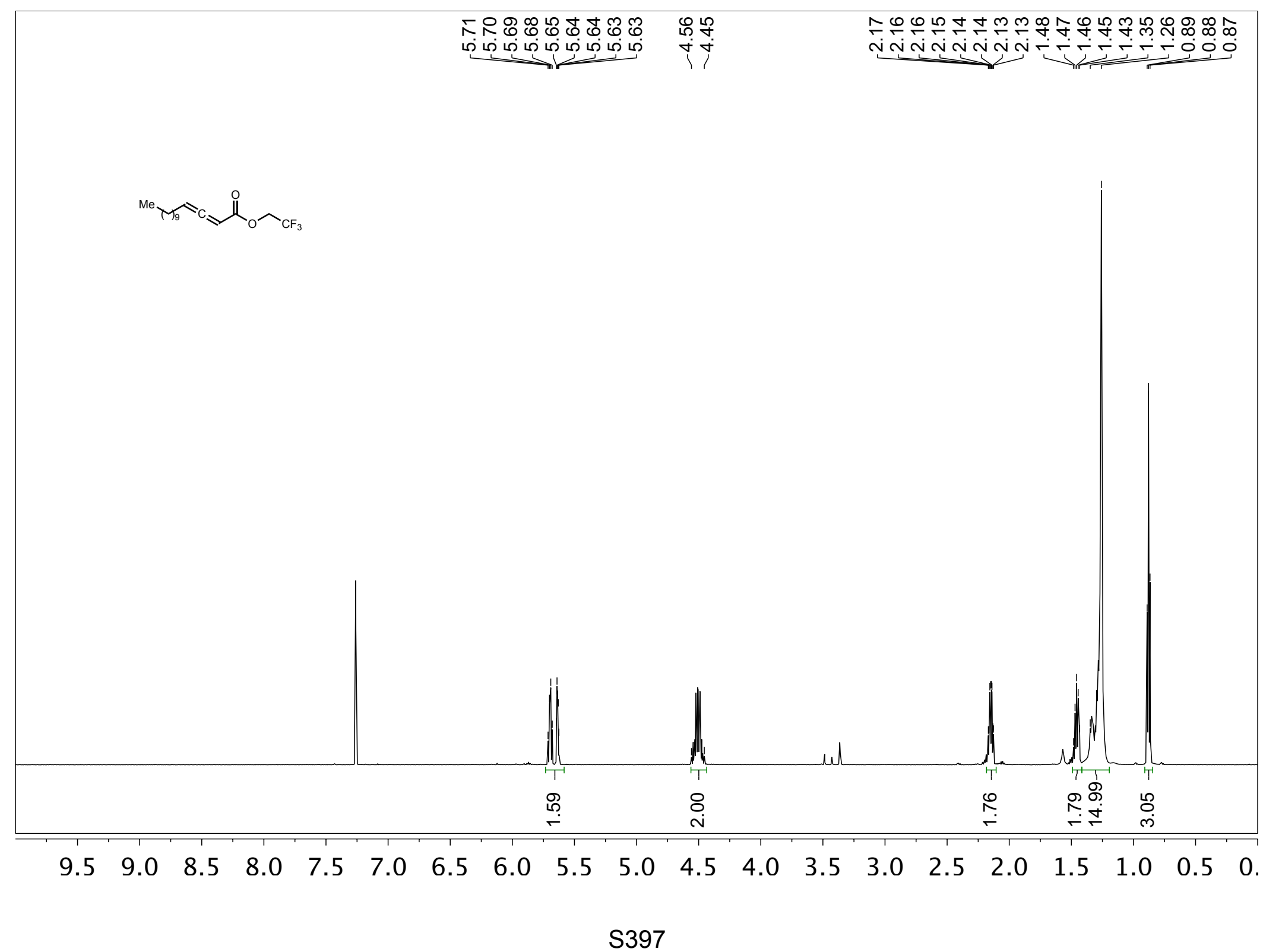




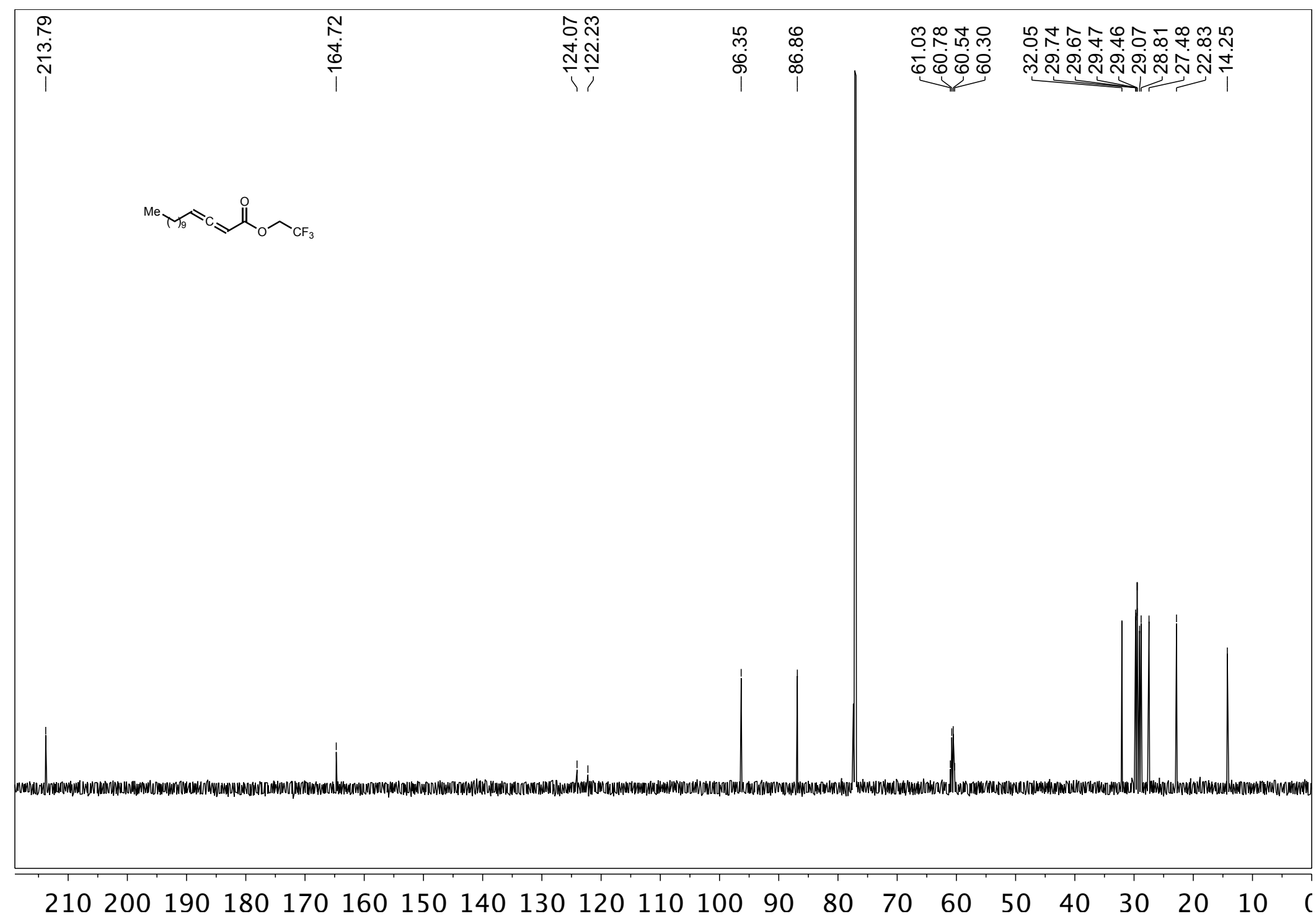




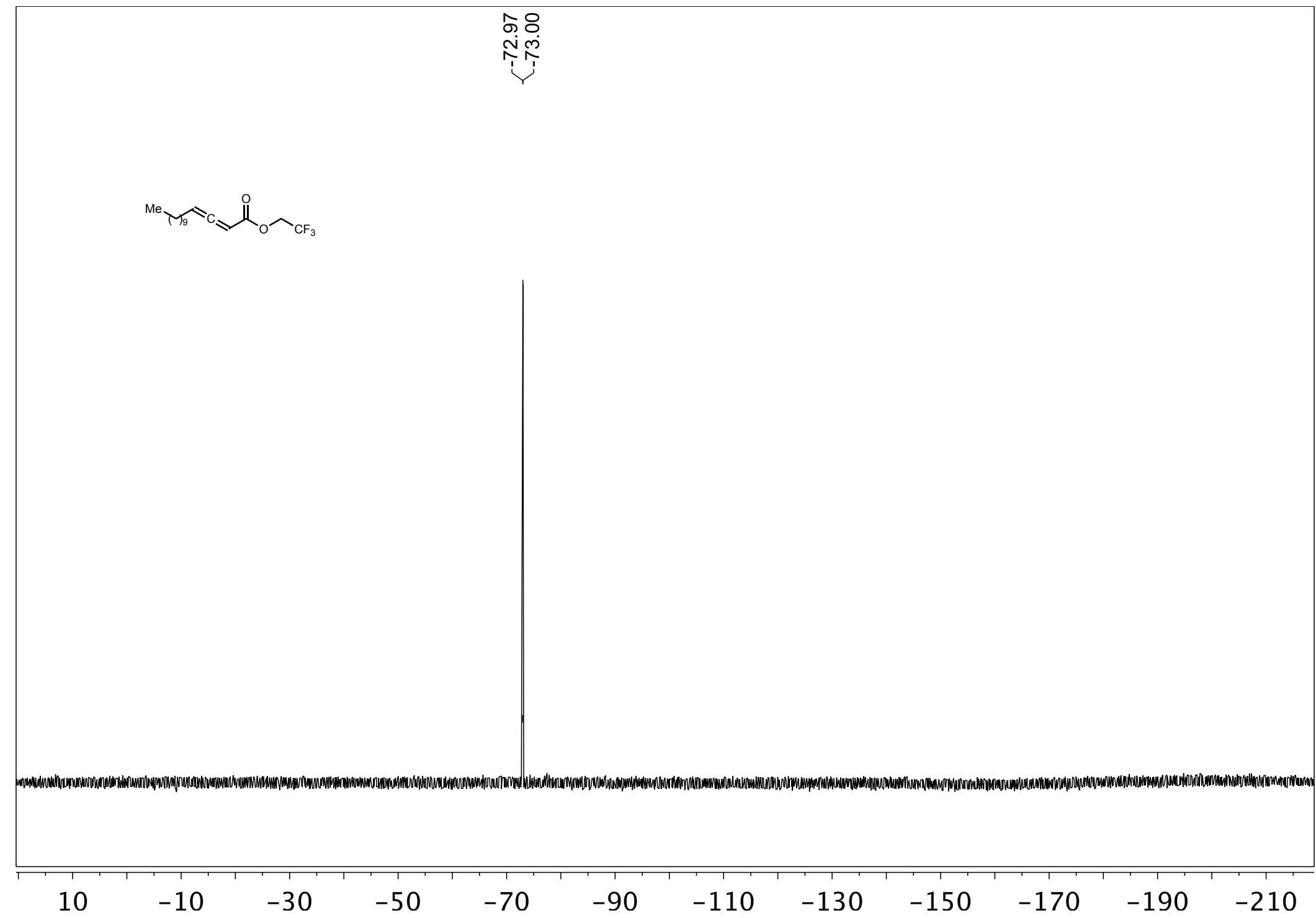




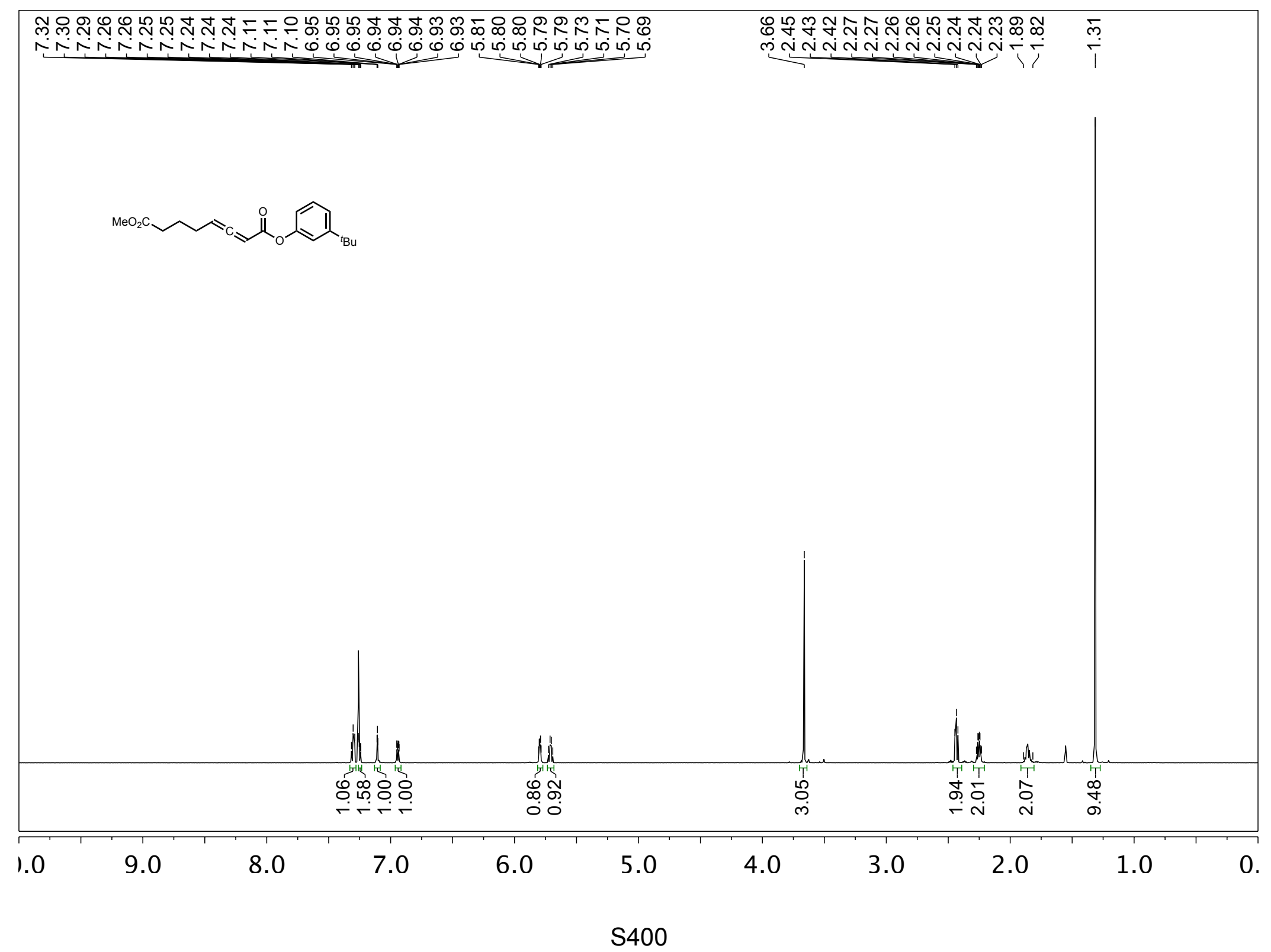




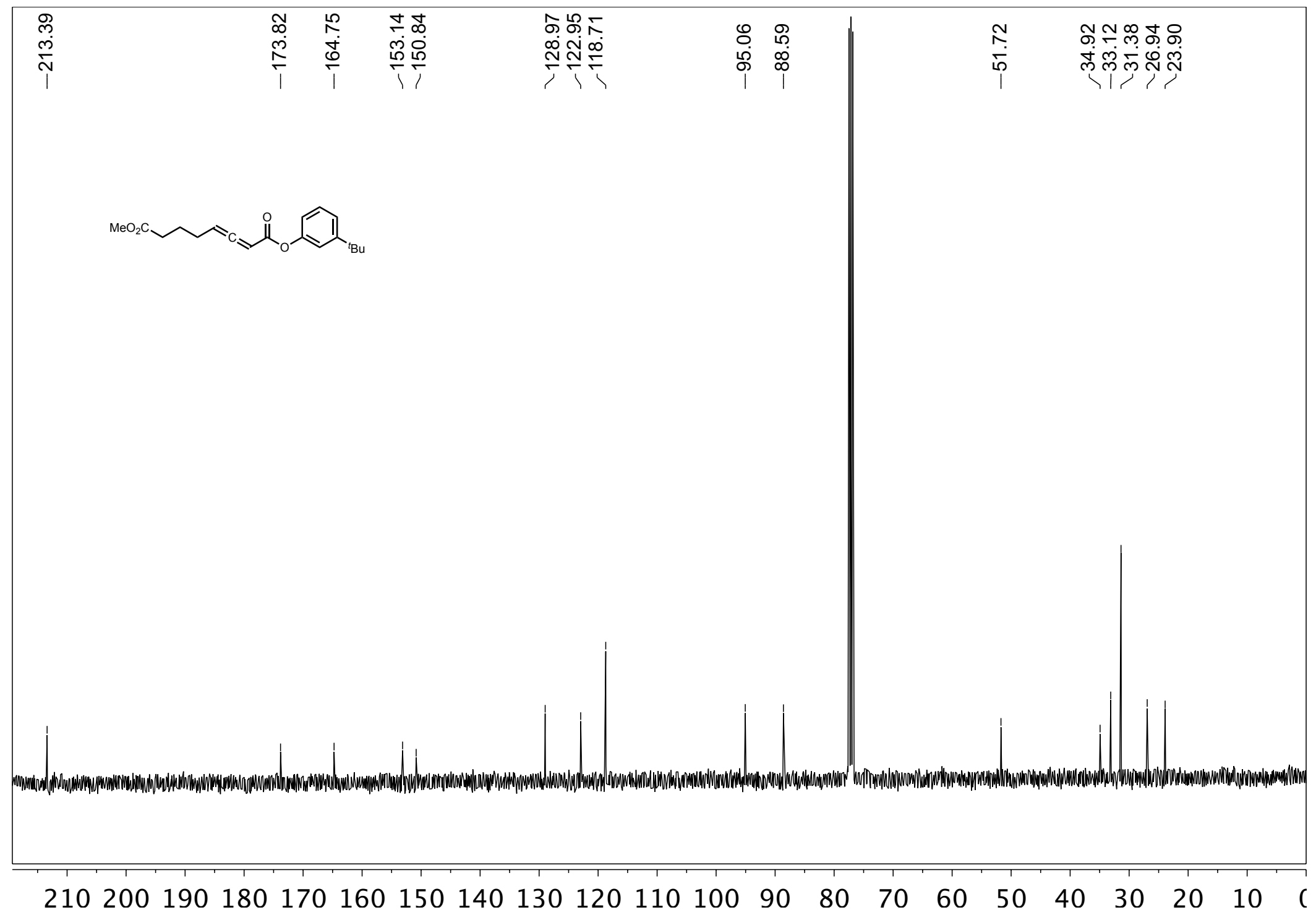

S401 


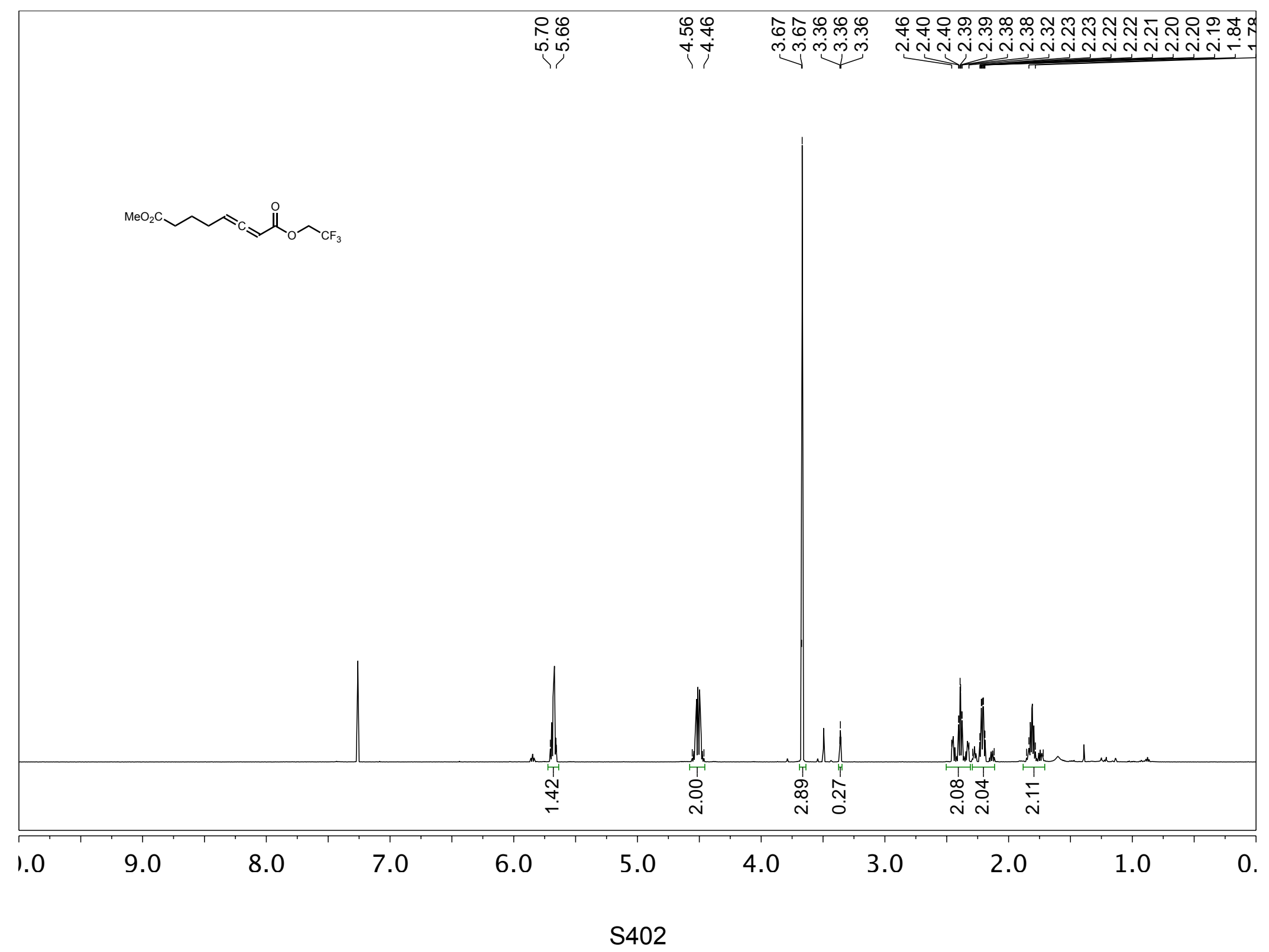




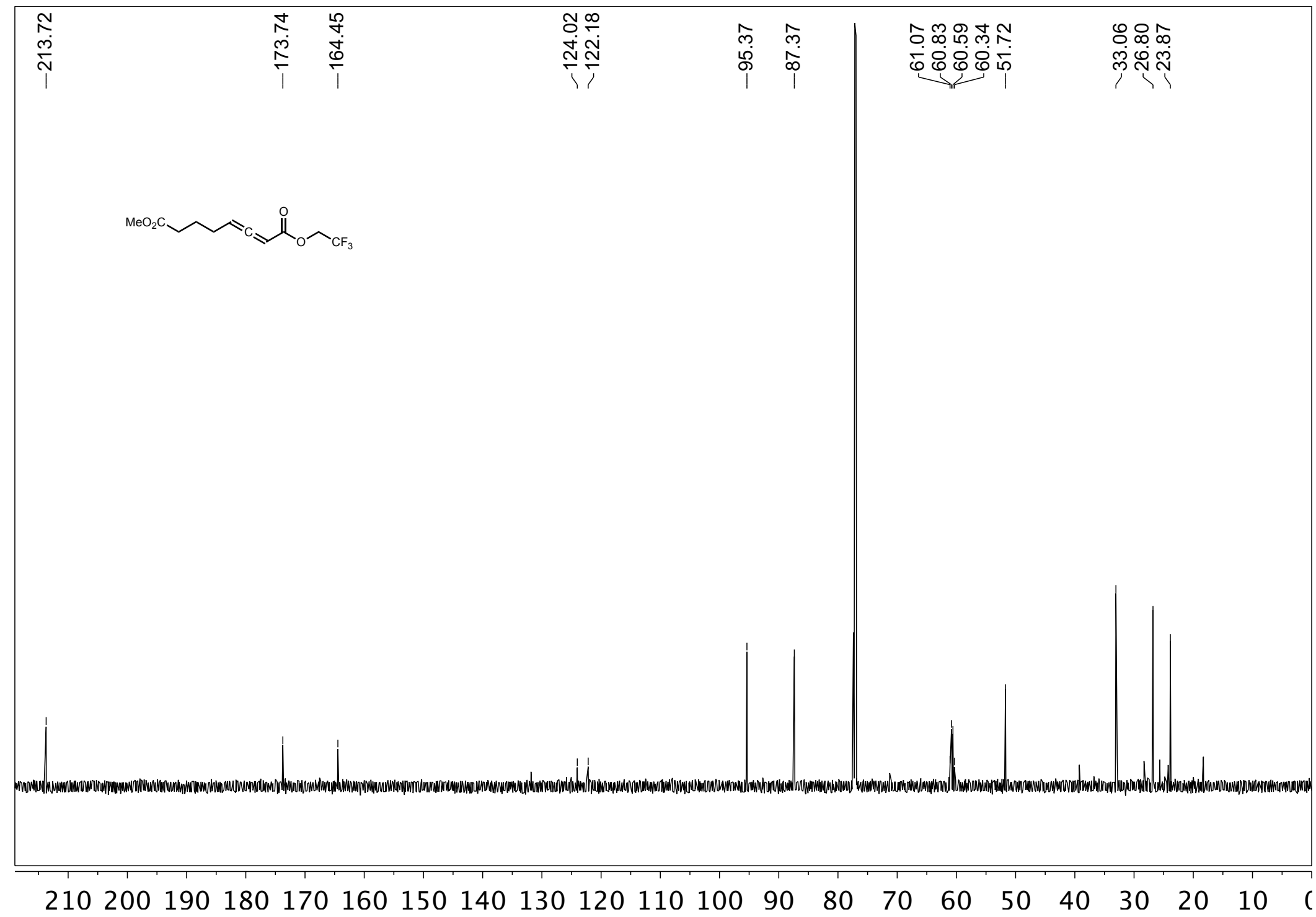




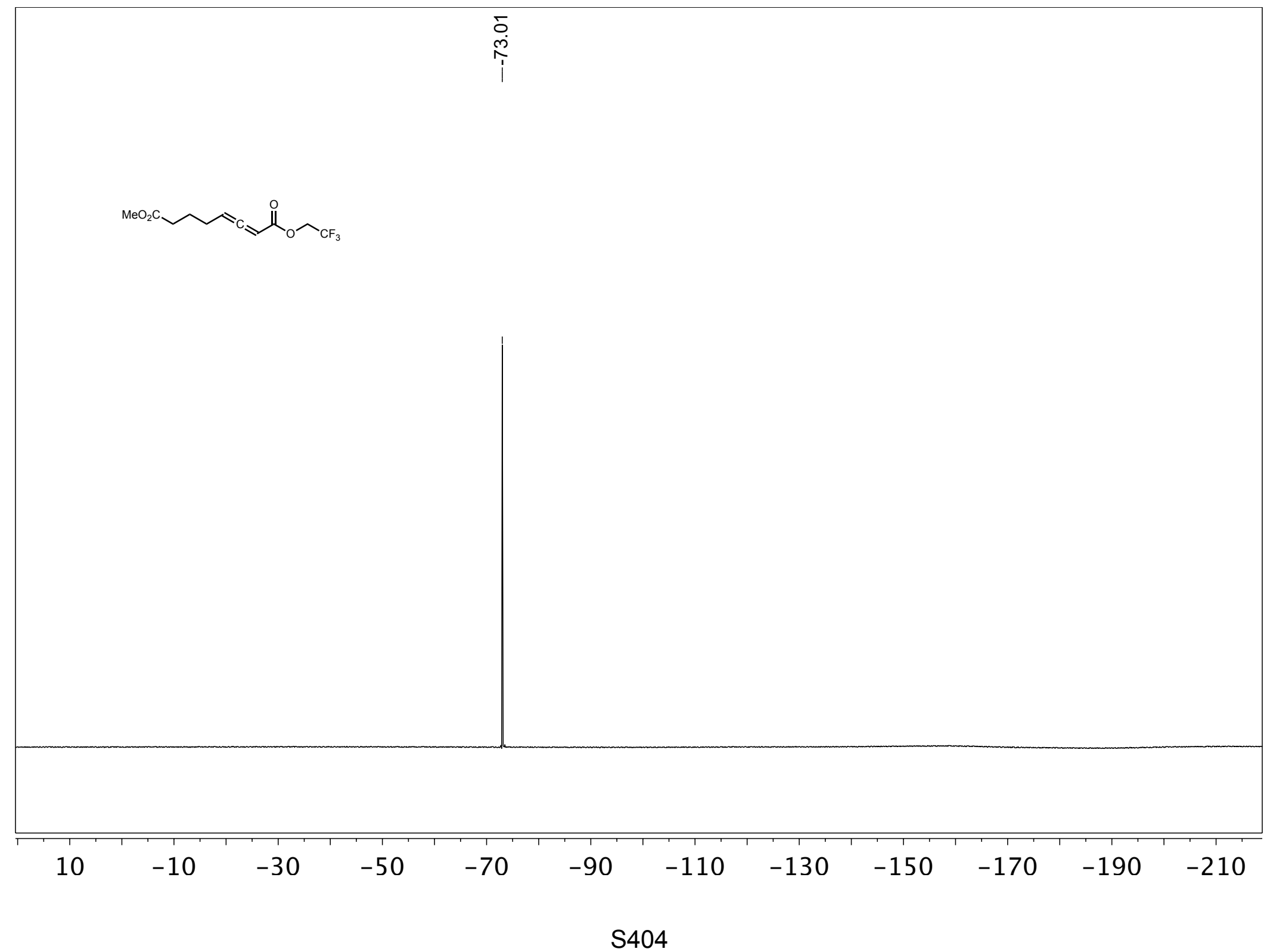




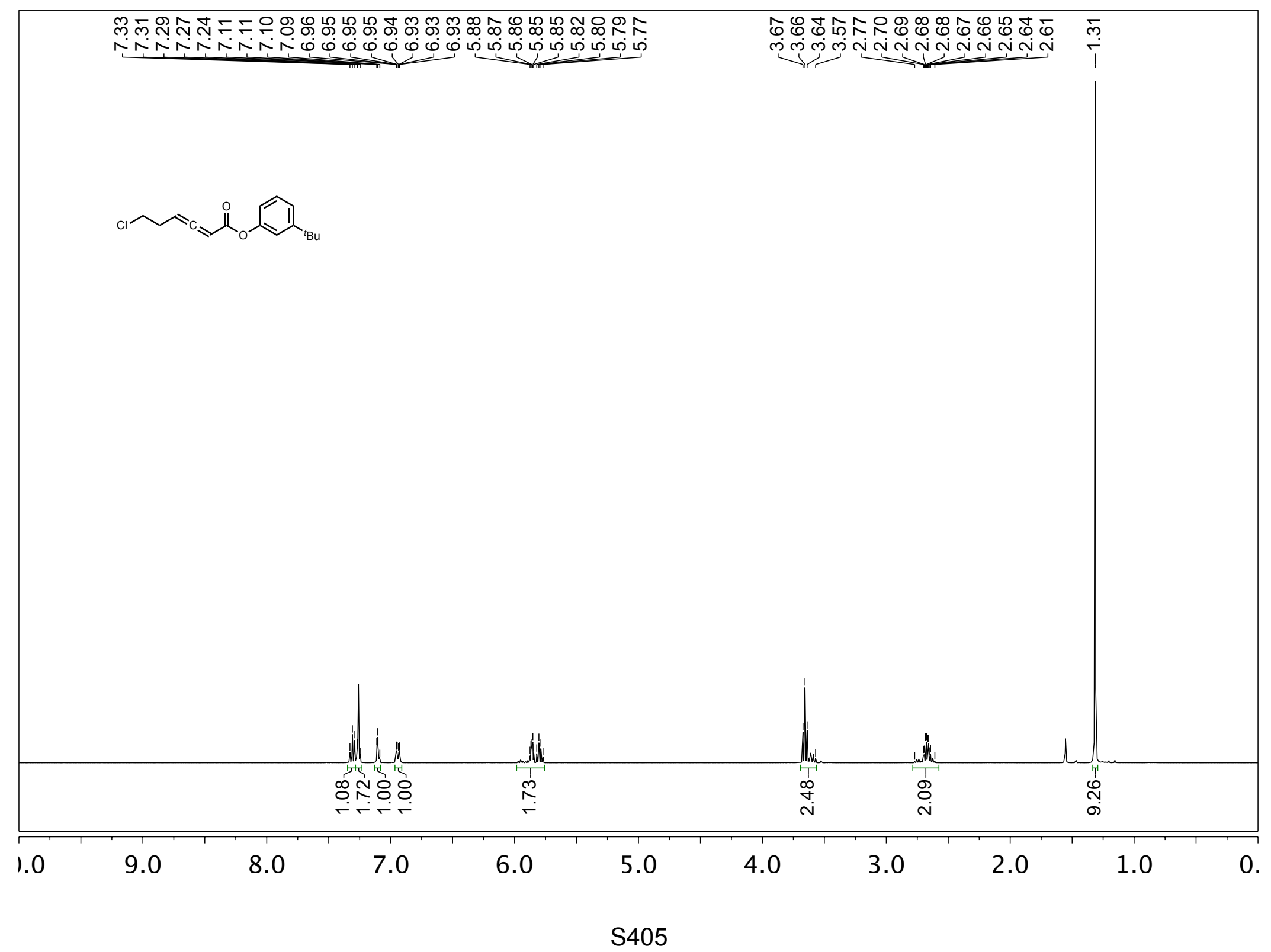




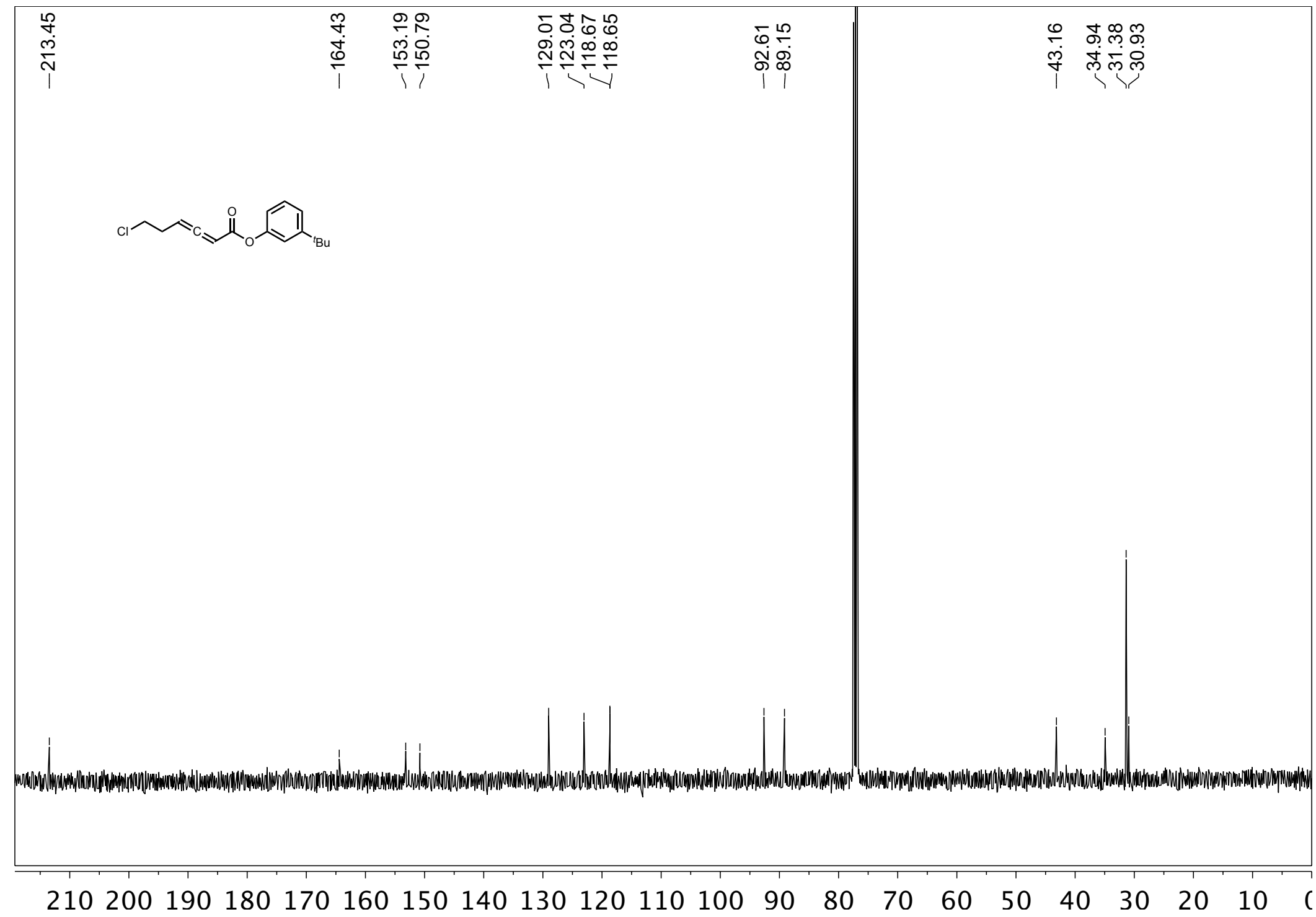




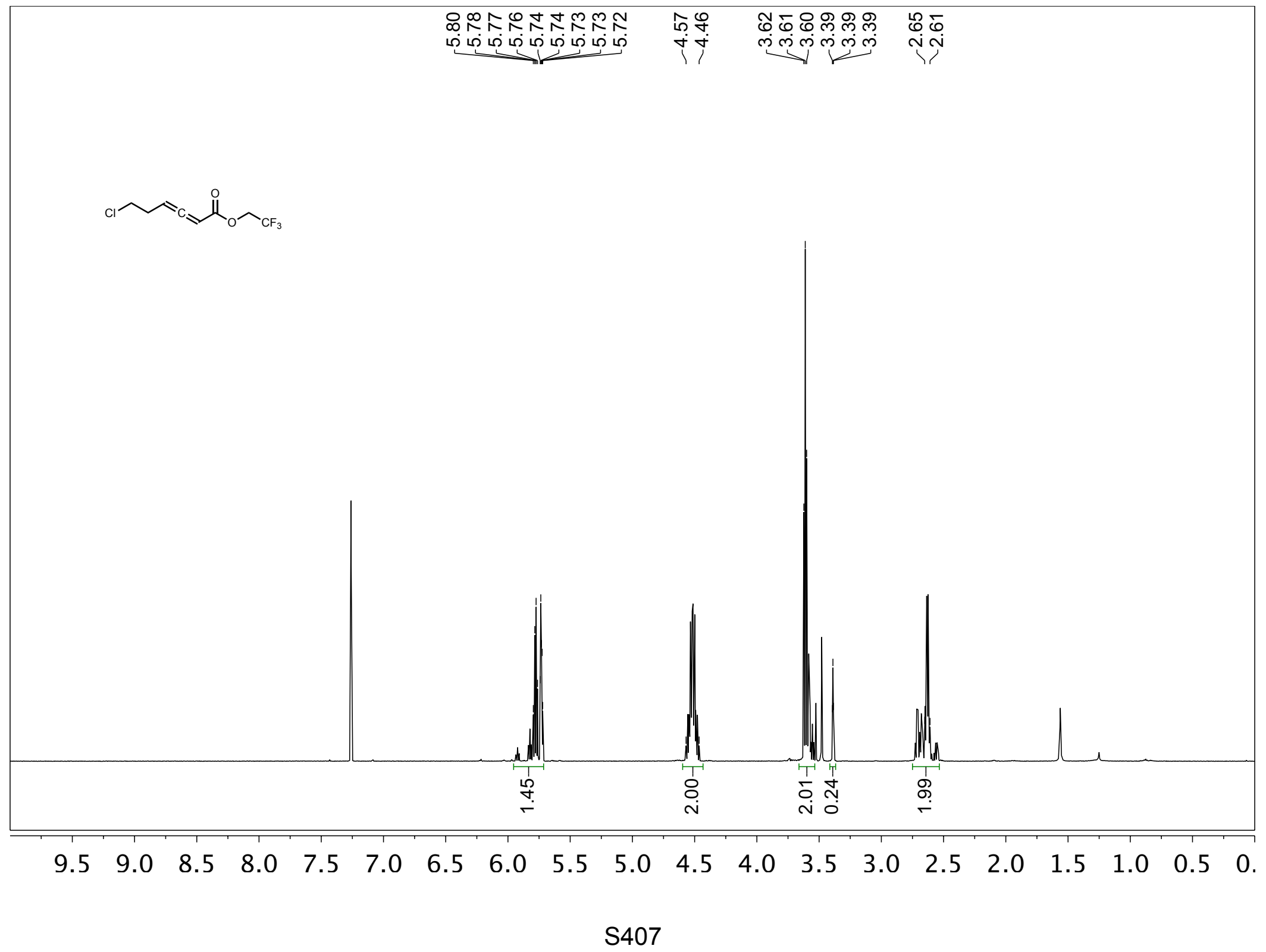




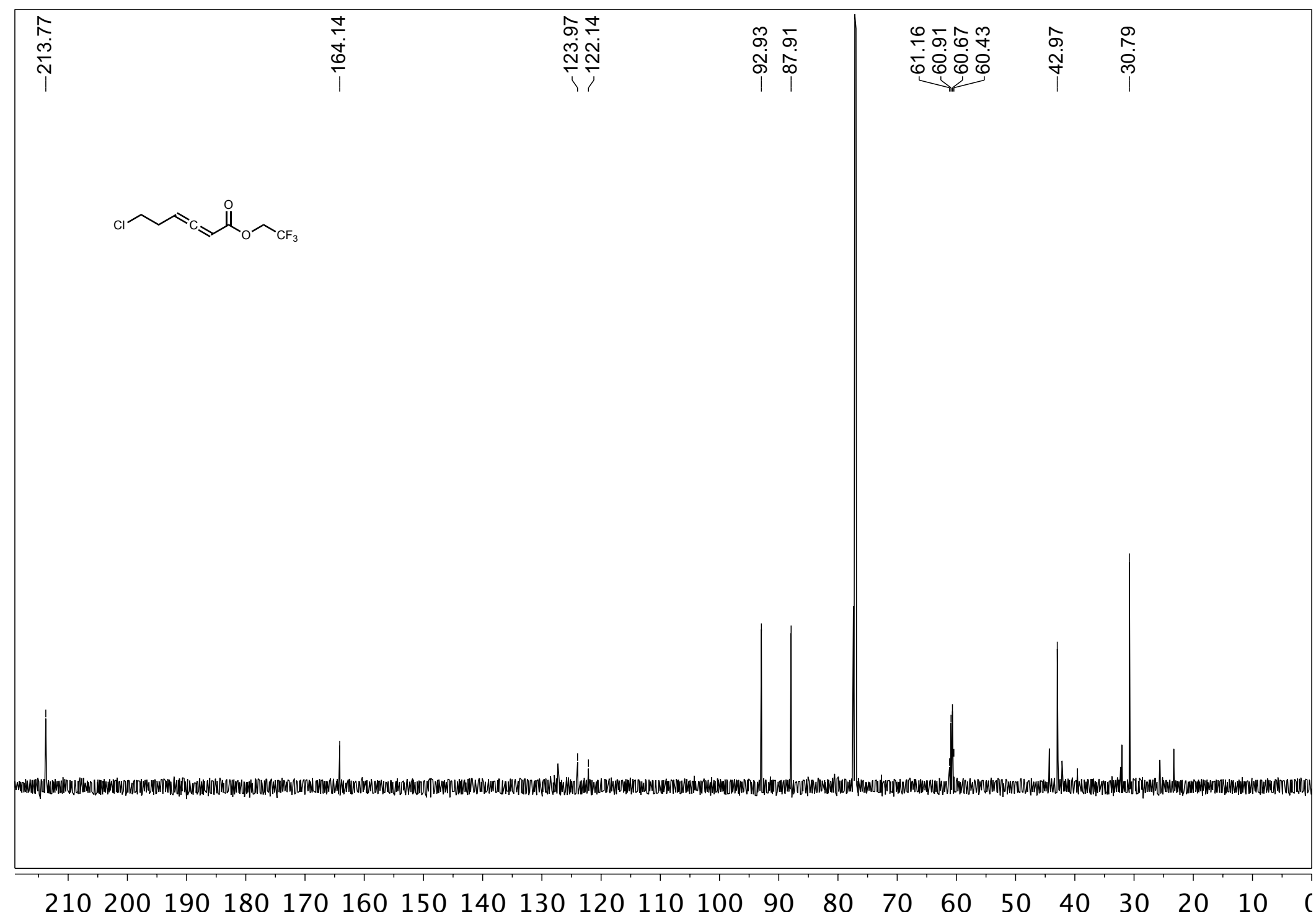




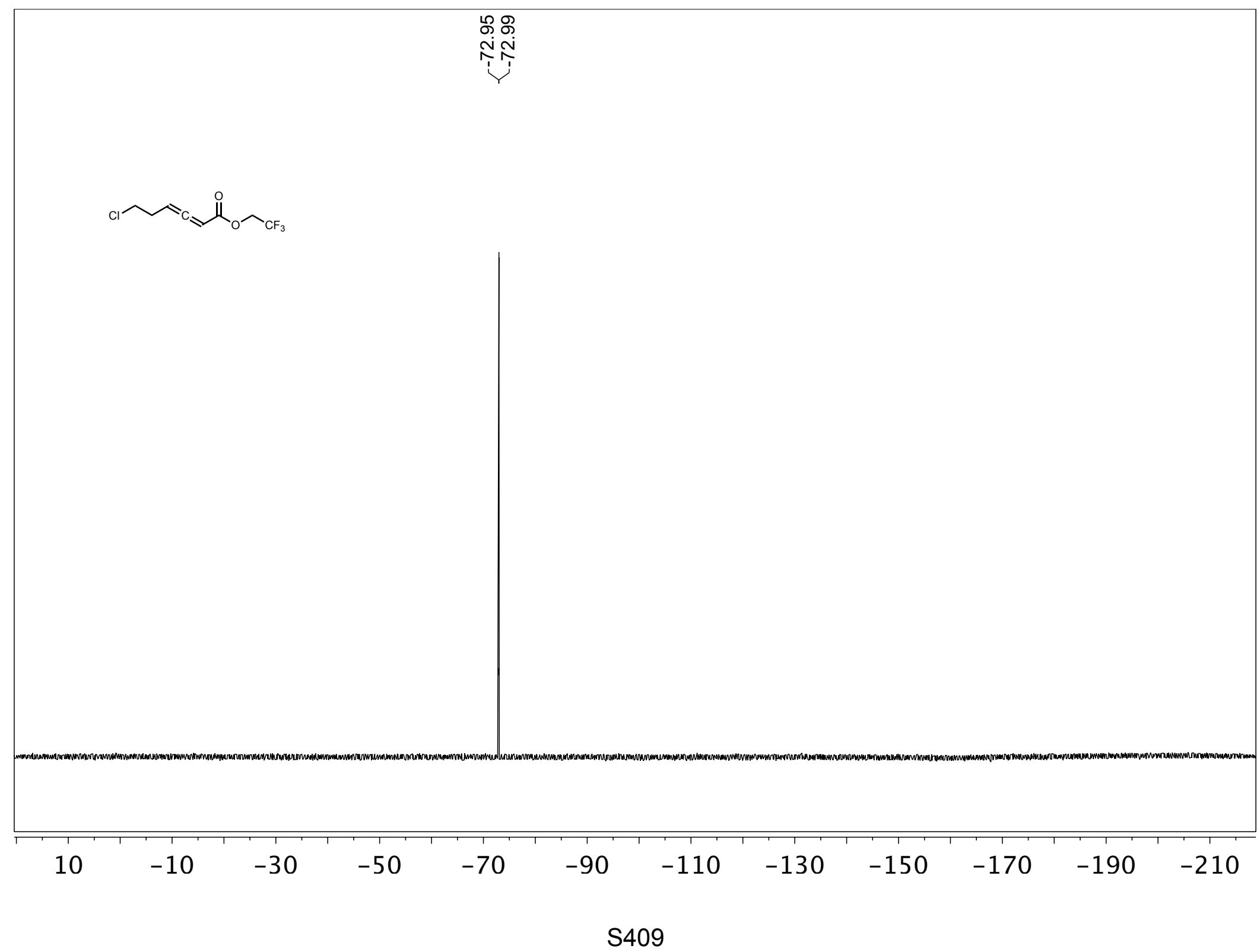




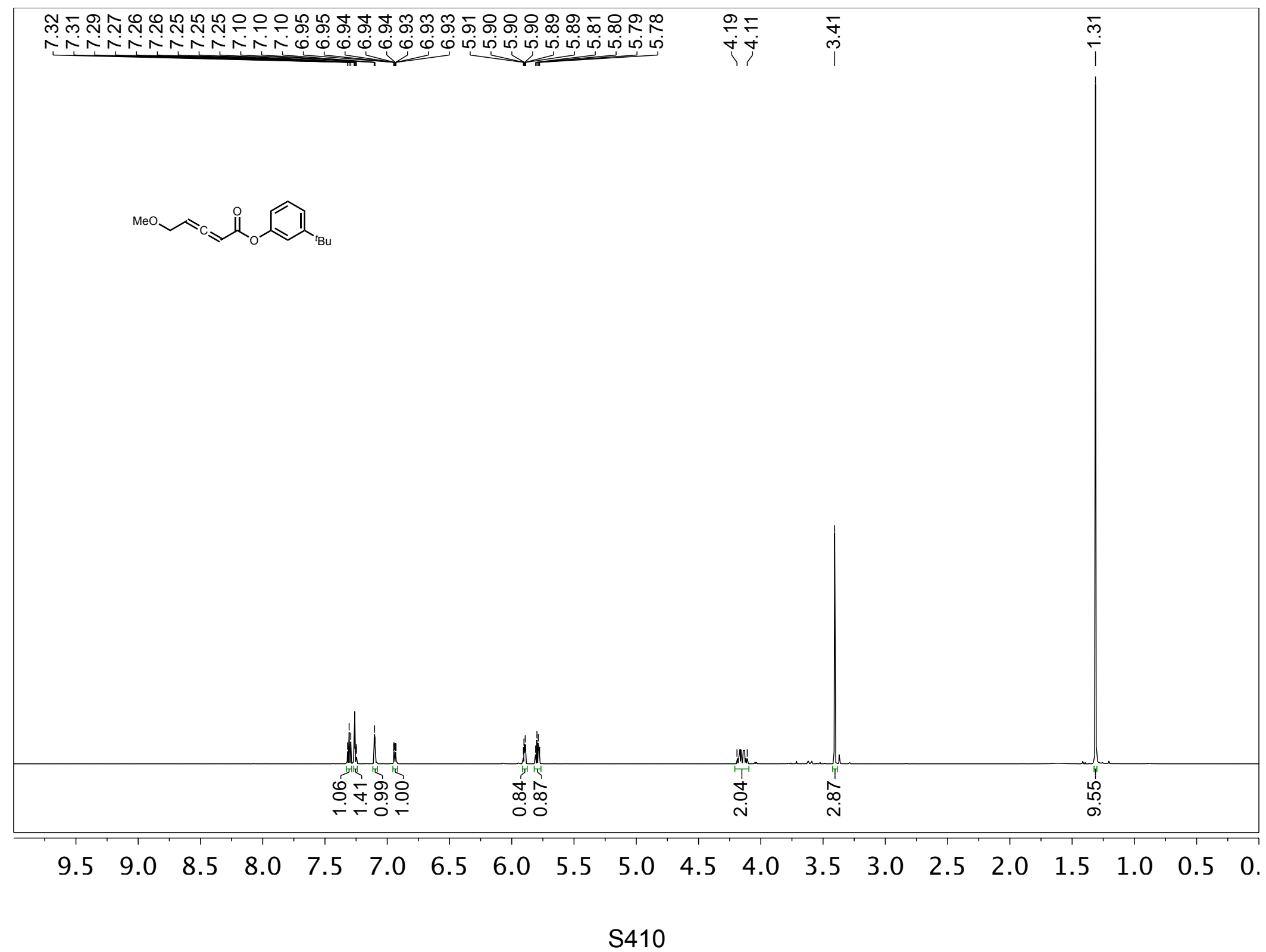




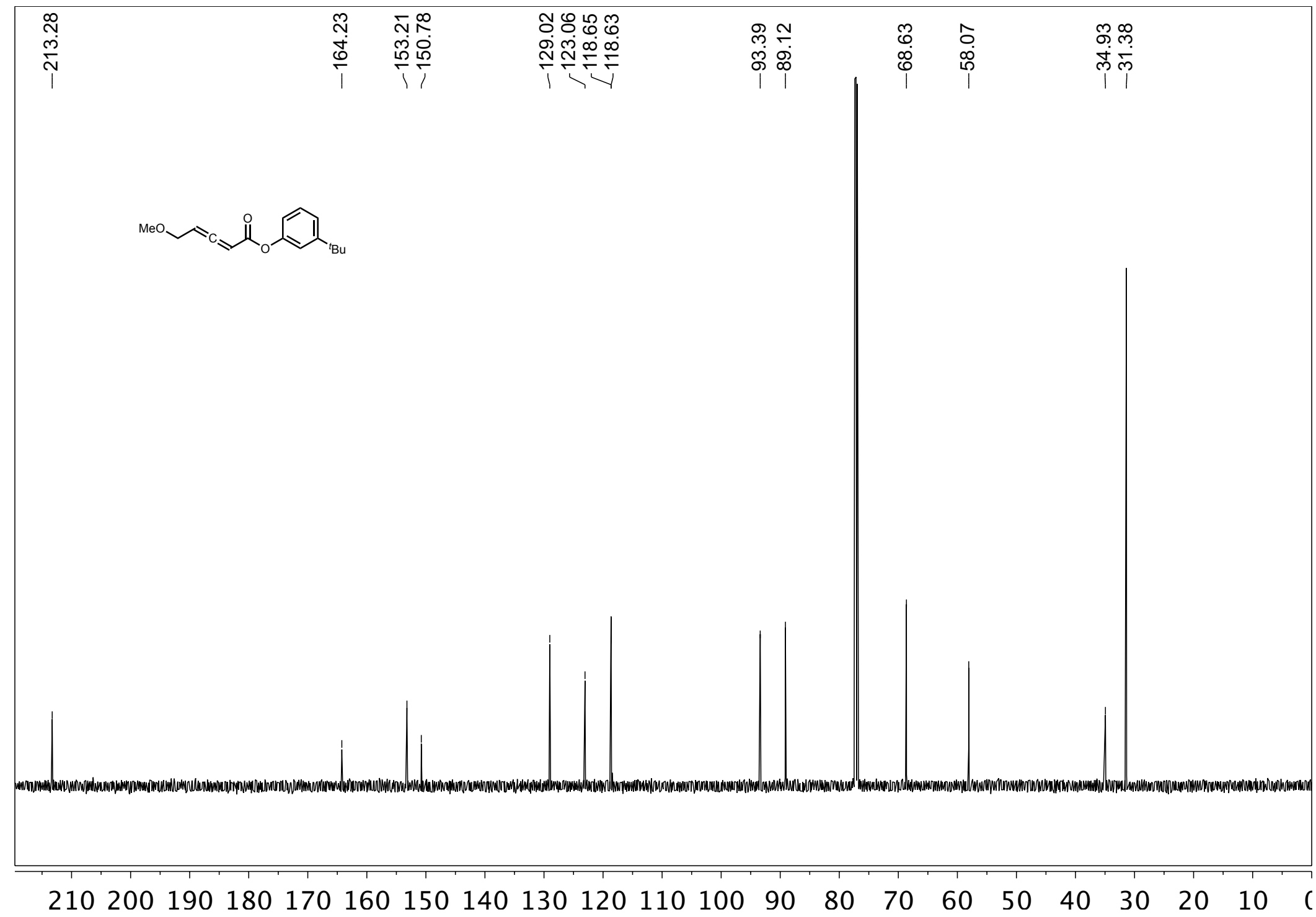




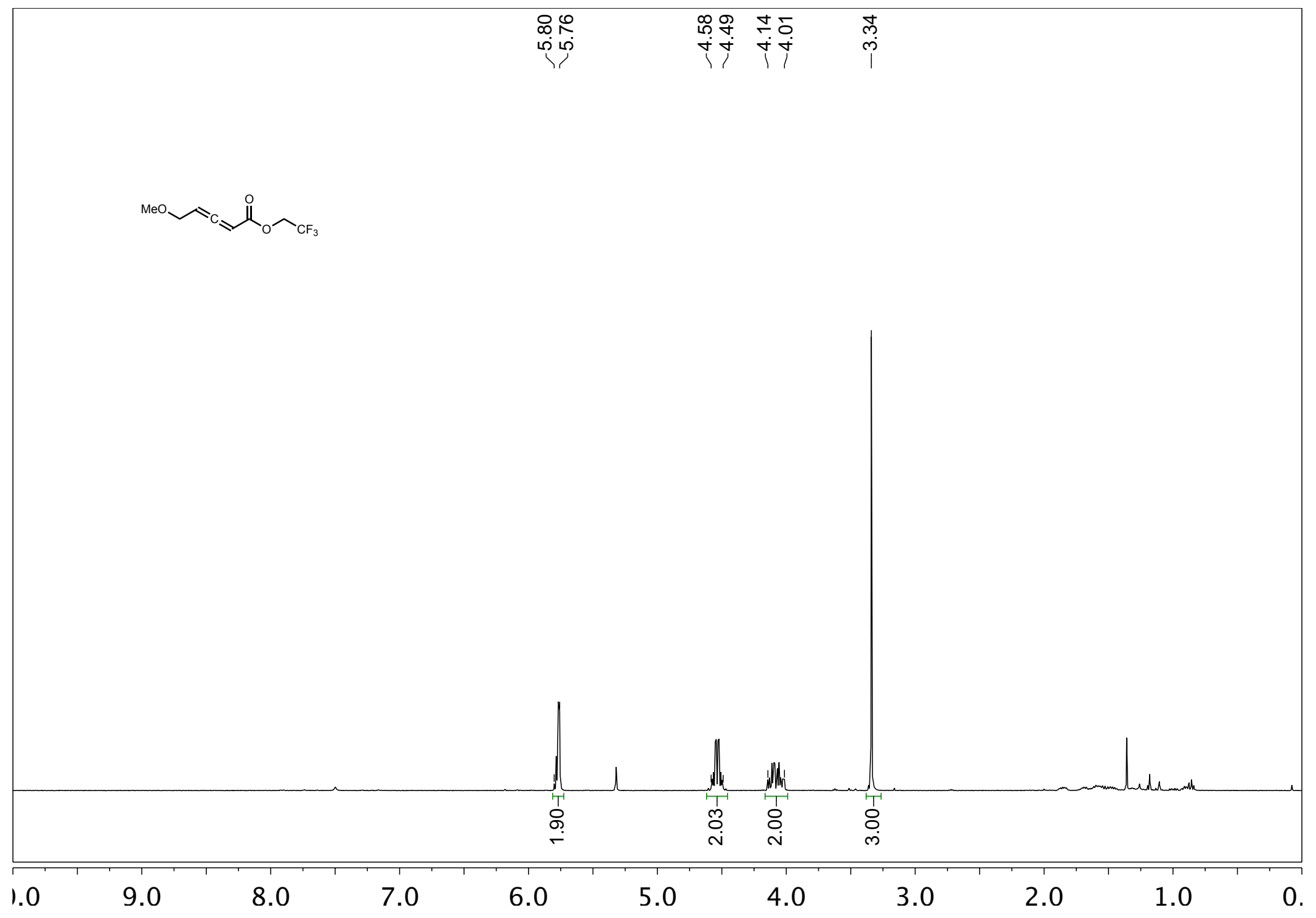

S412 


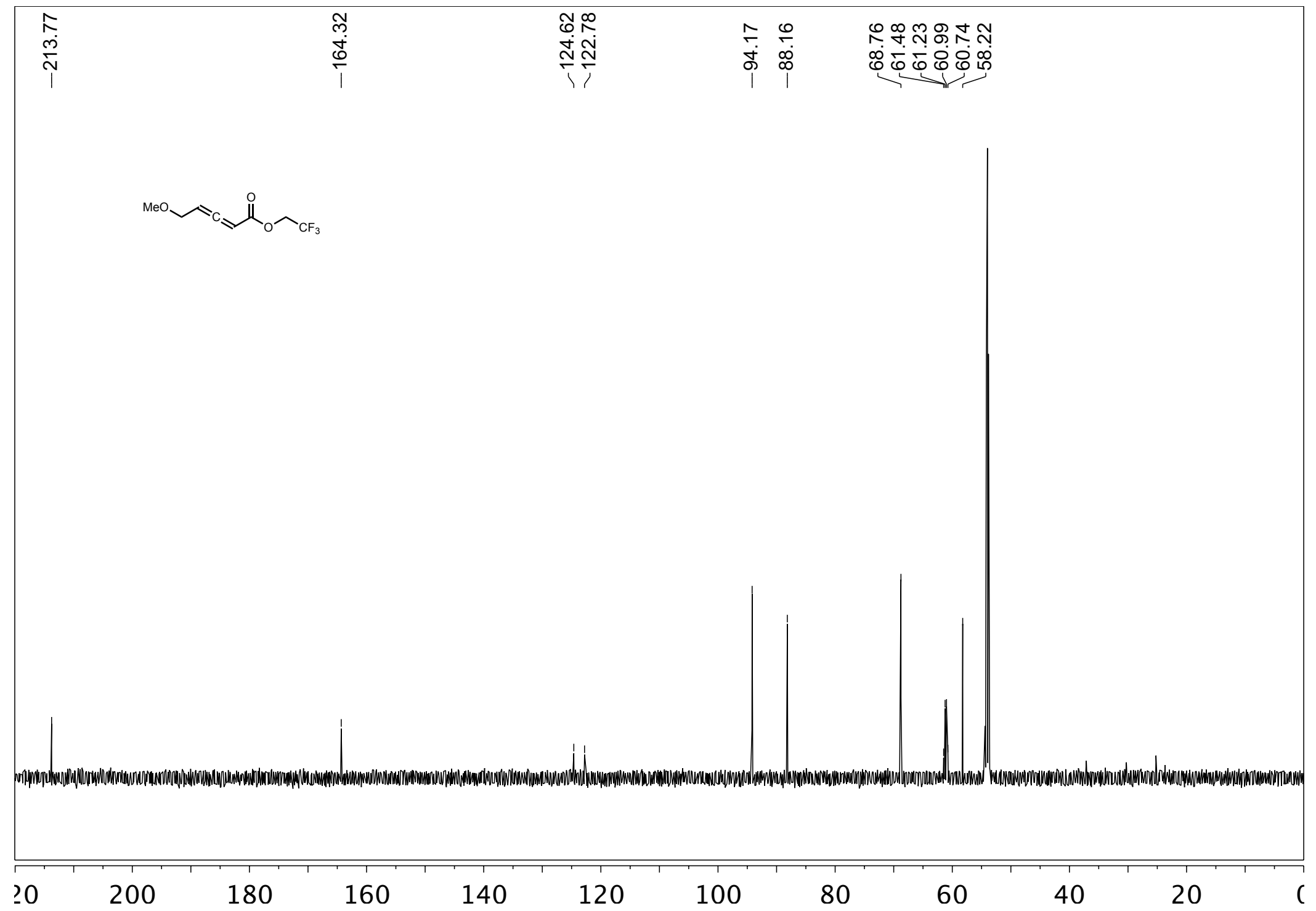




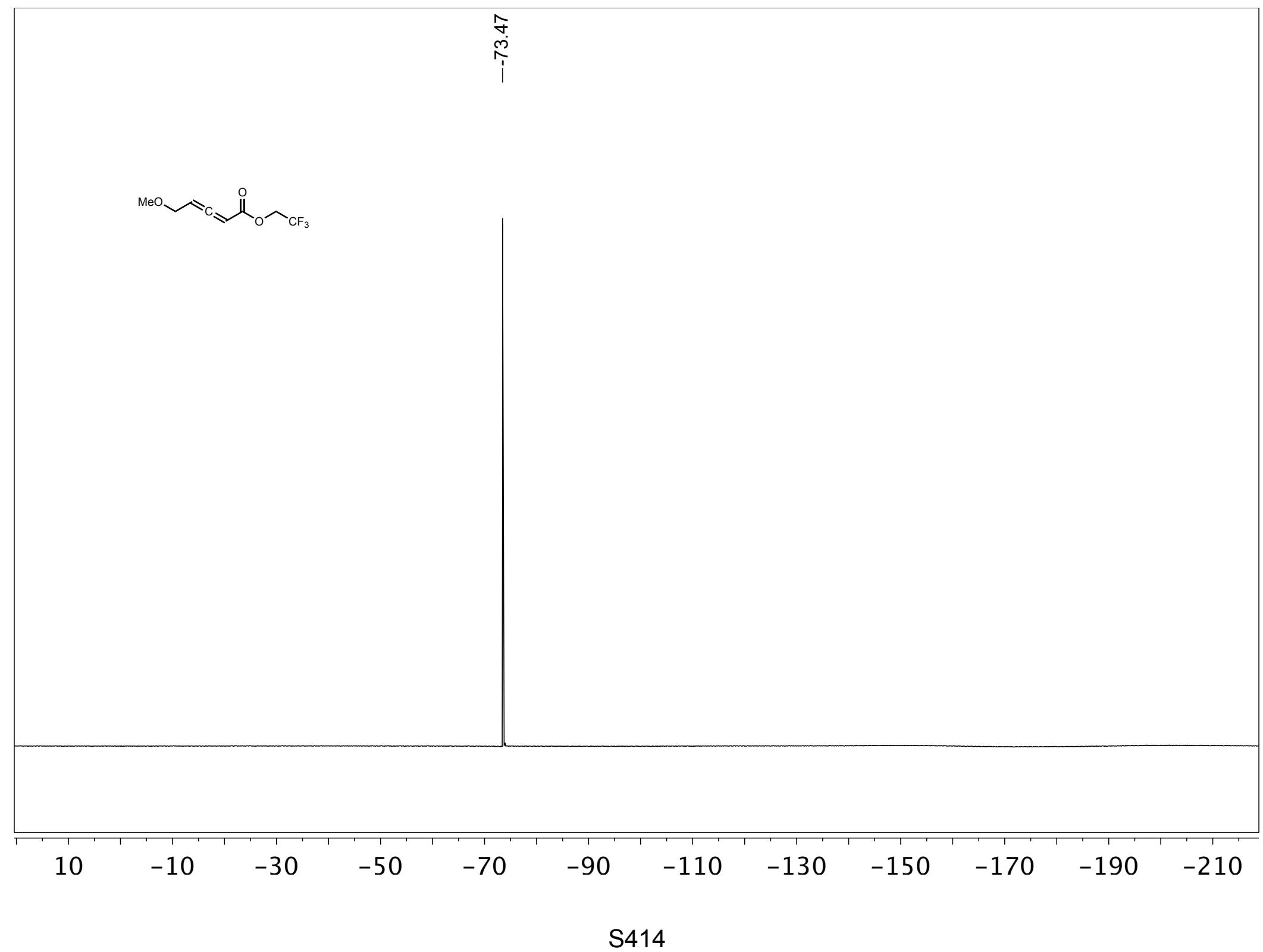




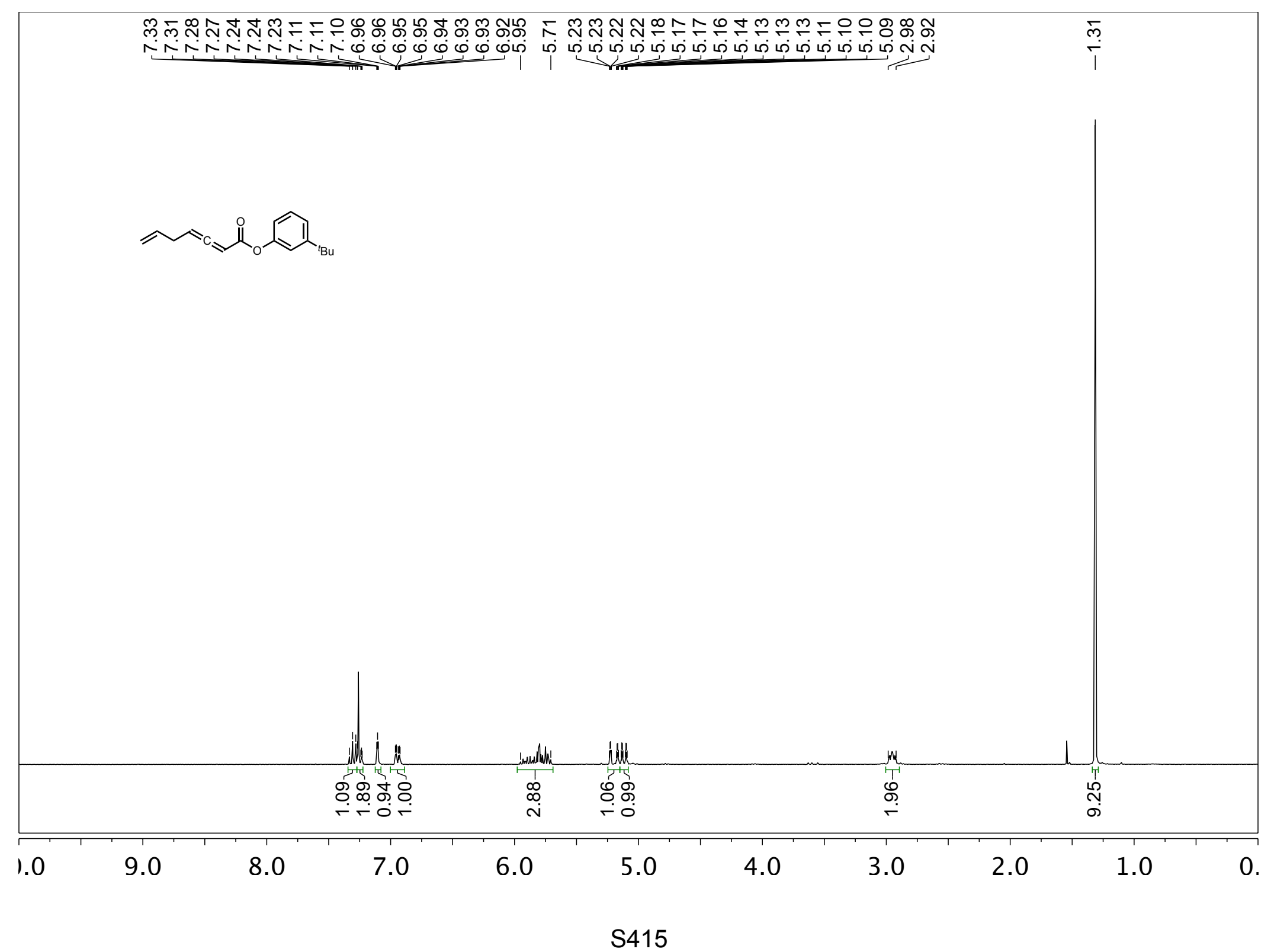




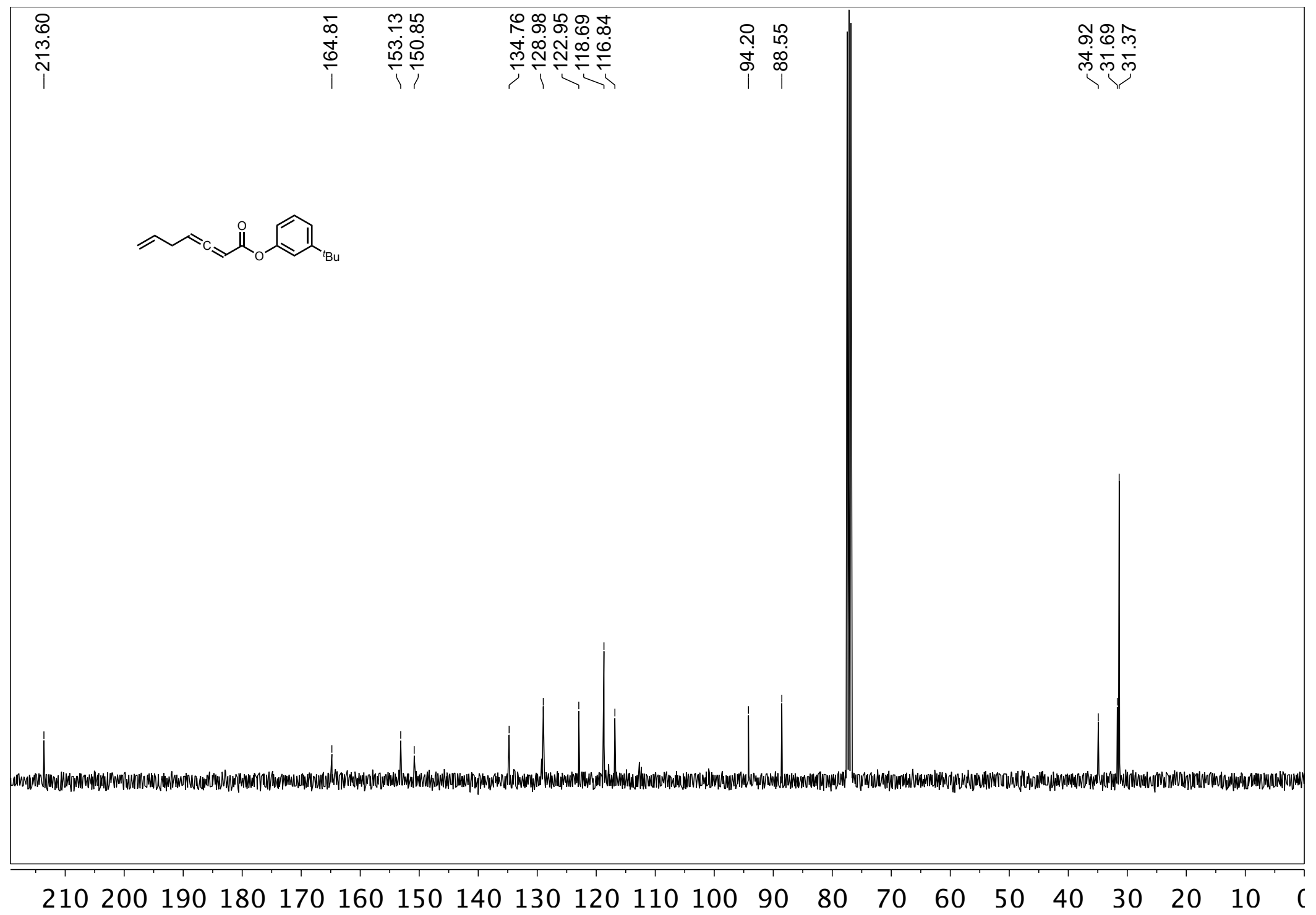

S416 


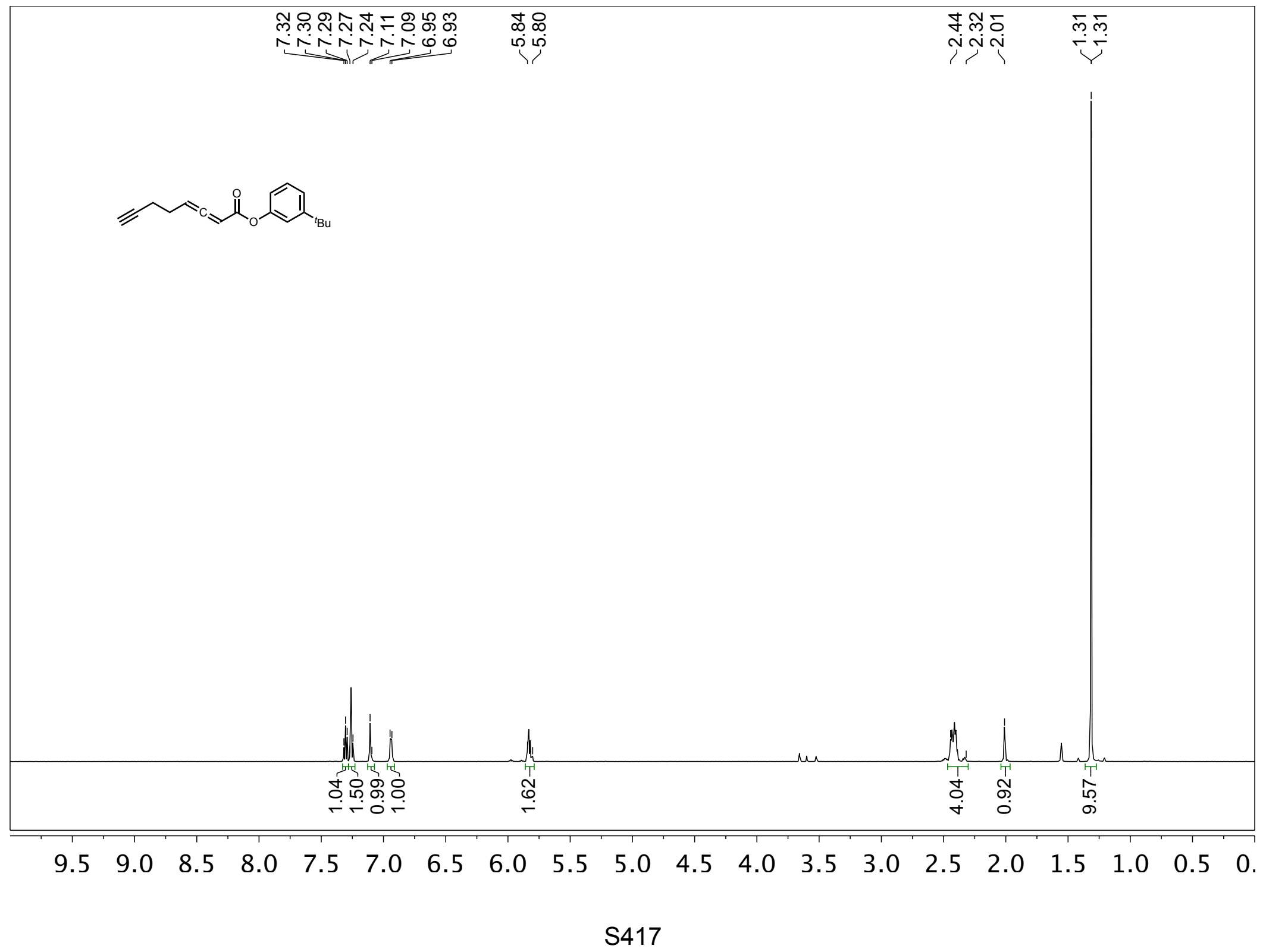




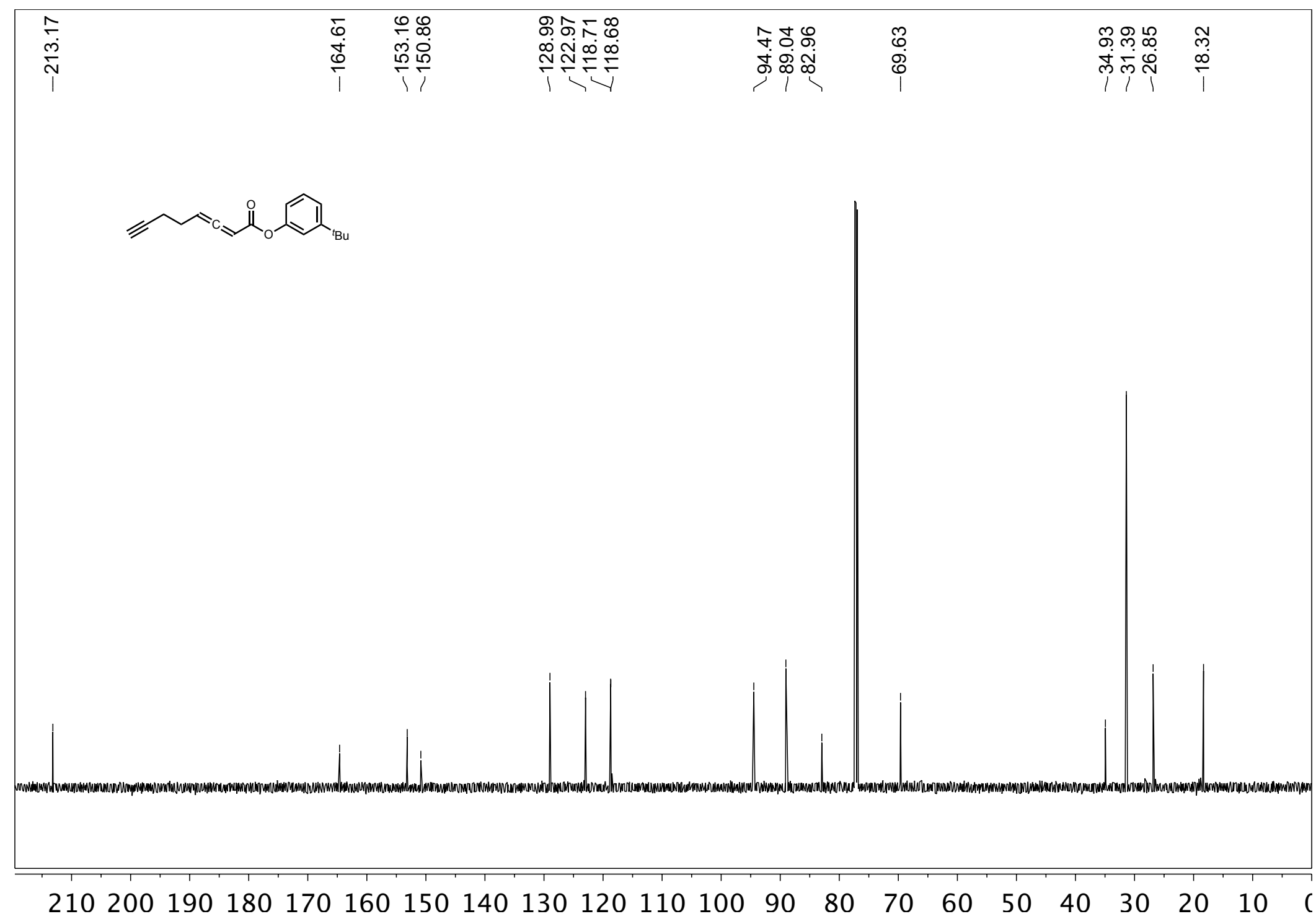




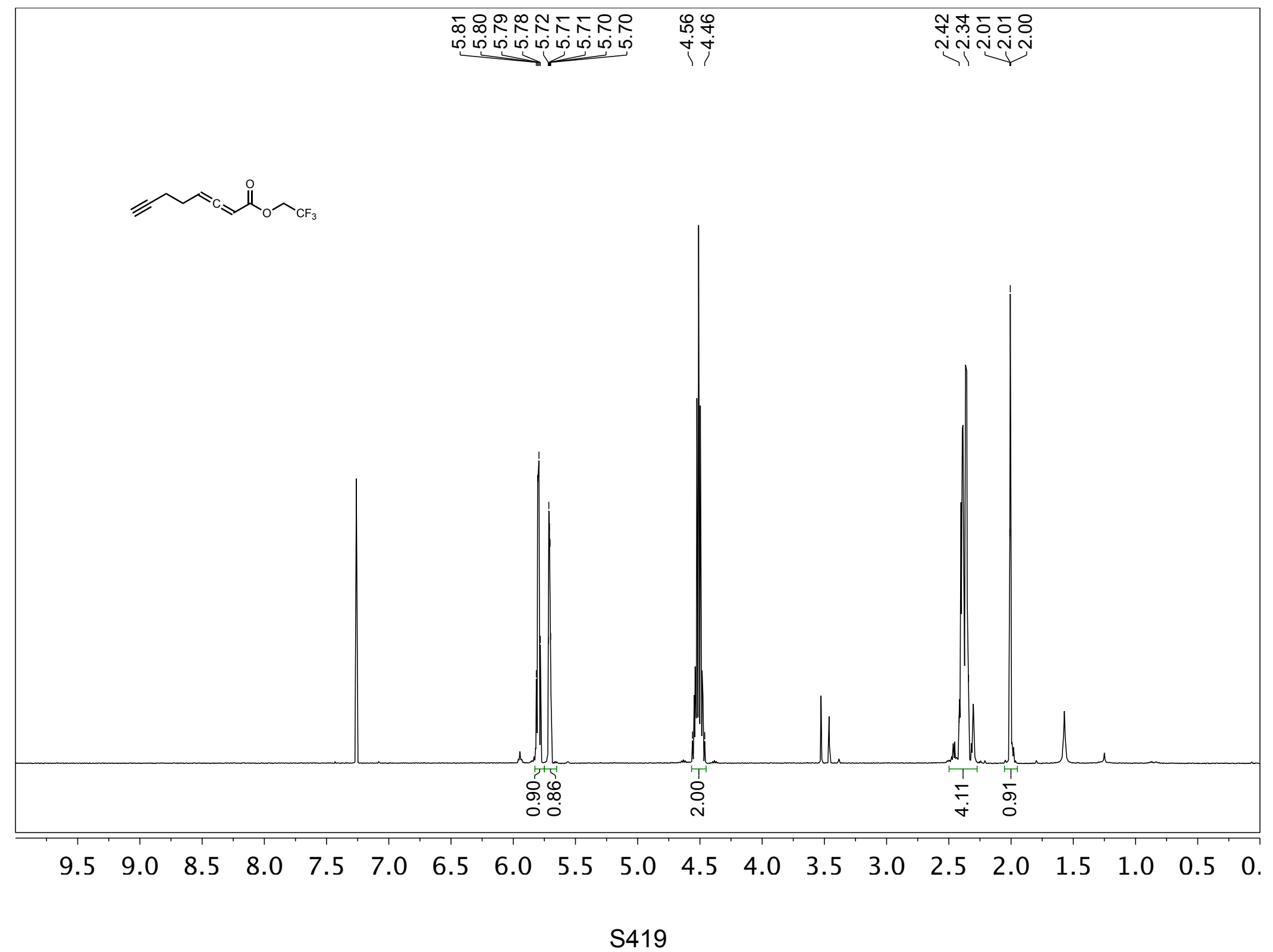




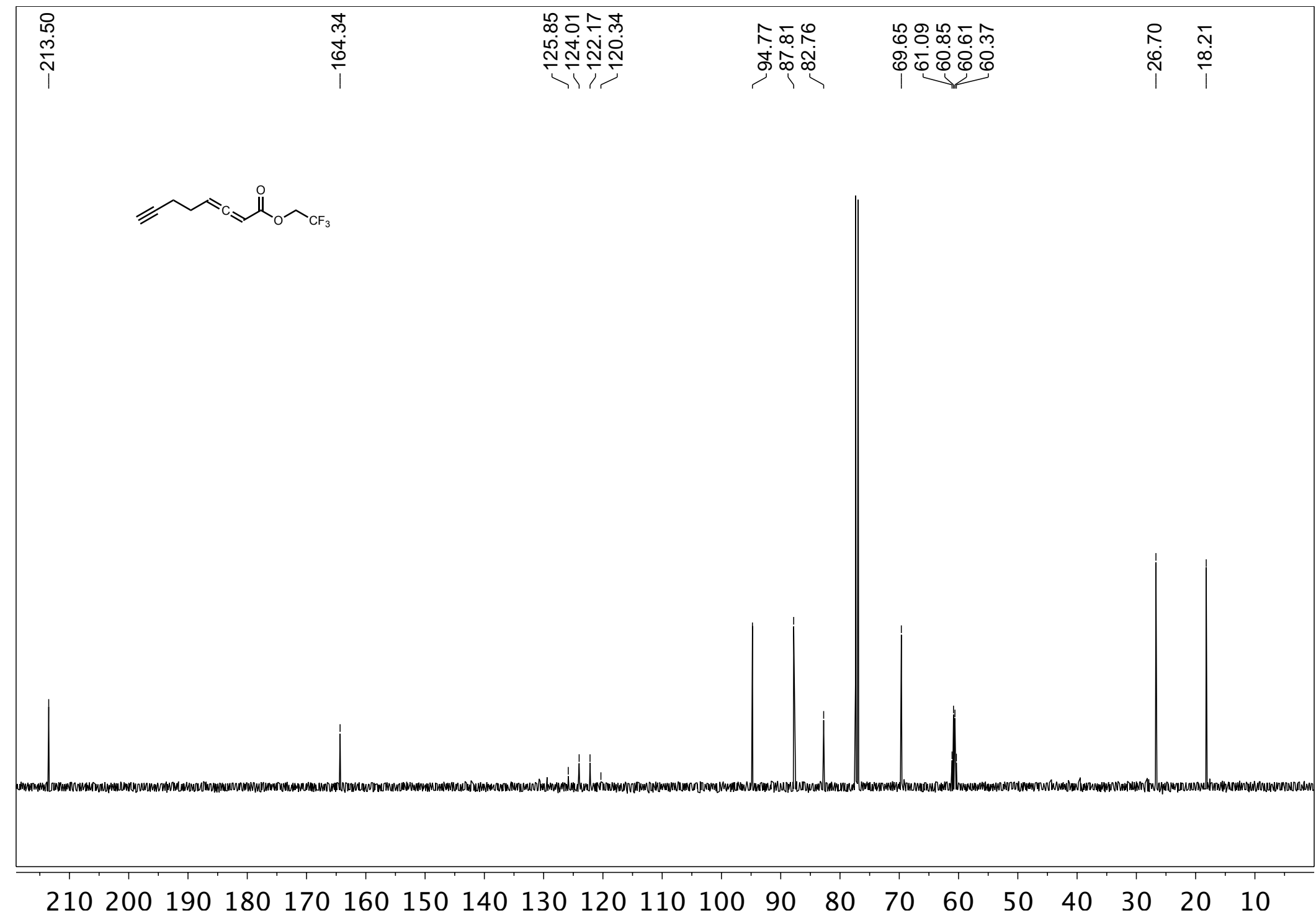




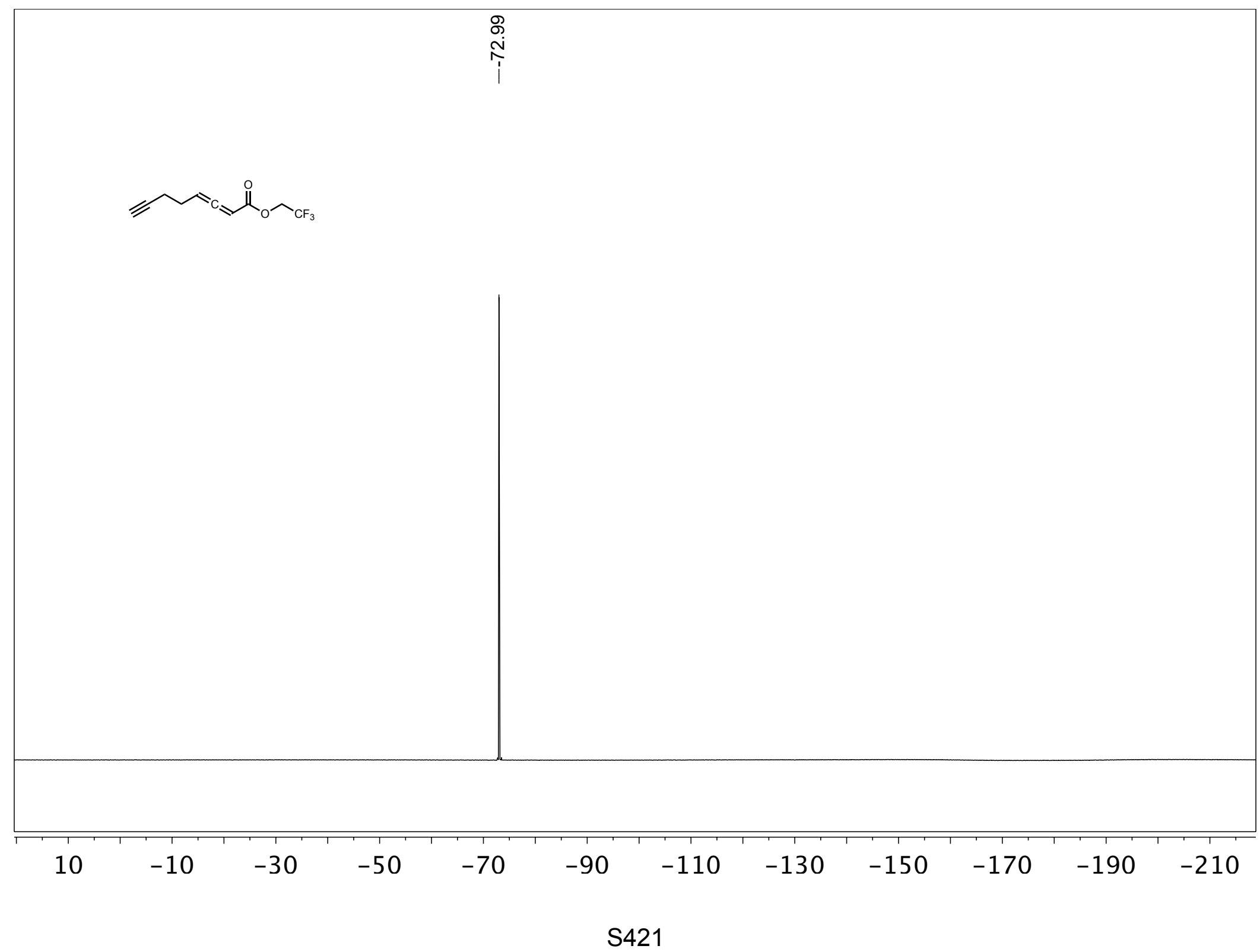




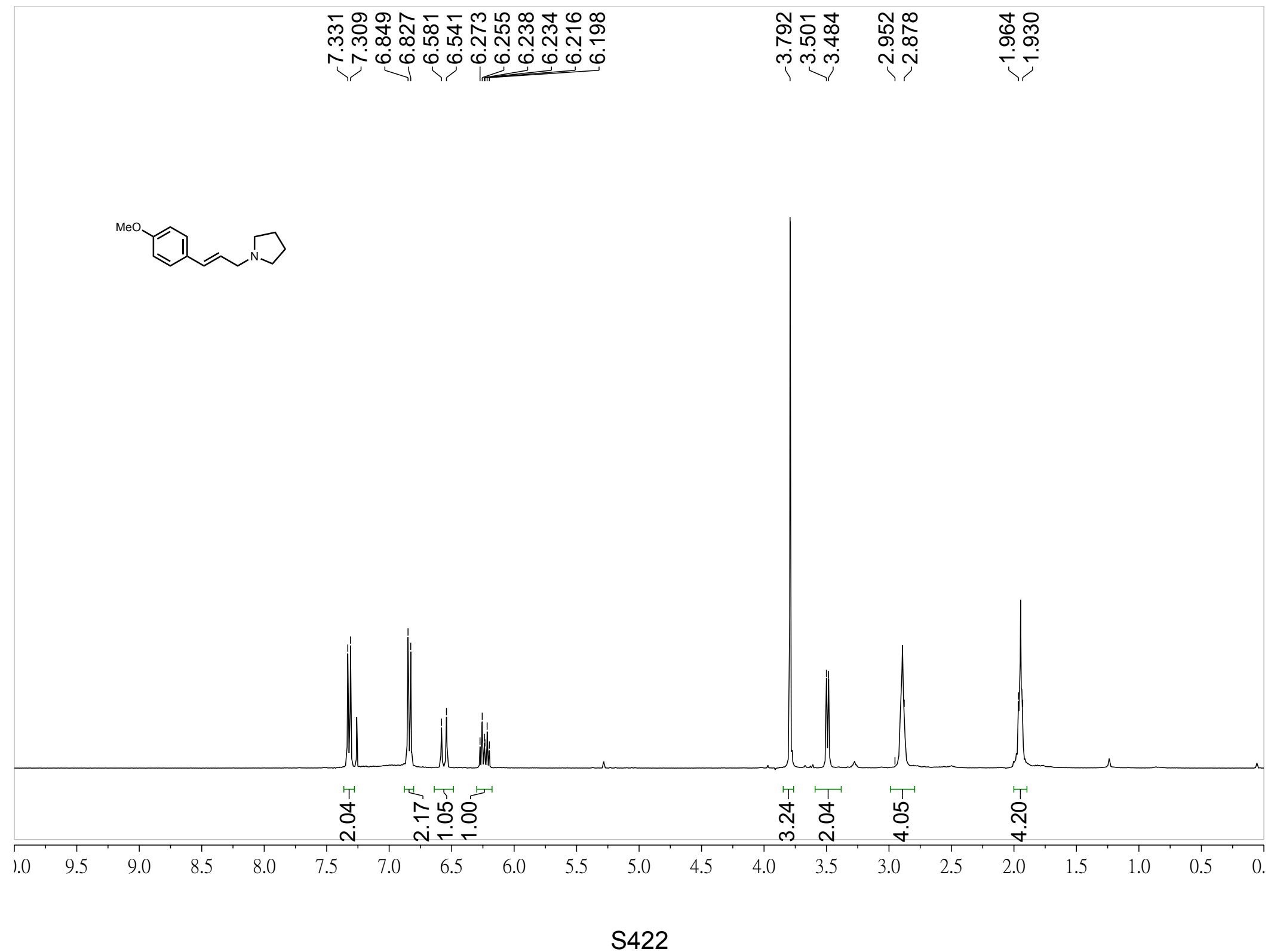




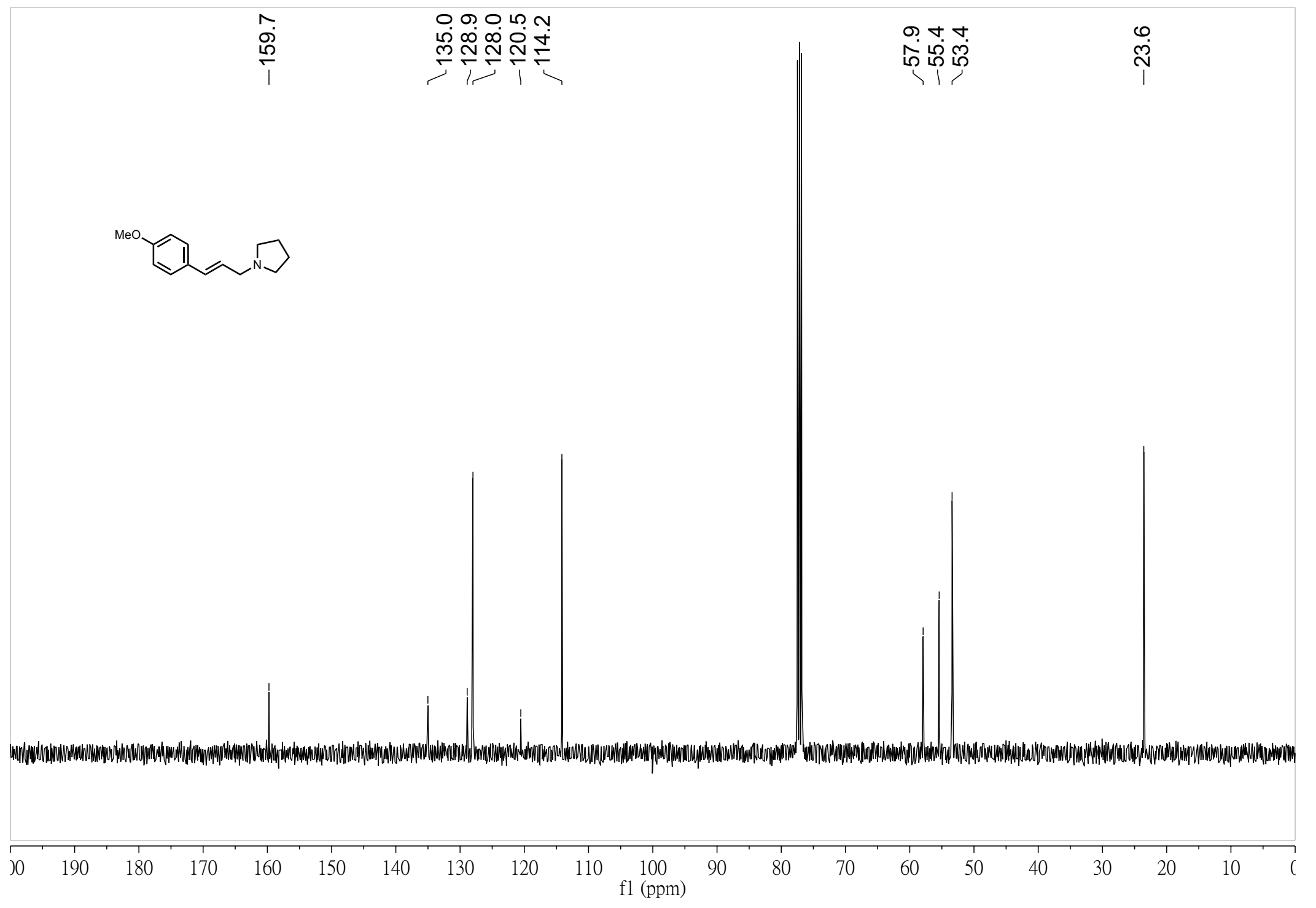

S423 


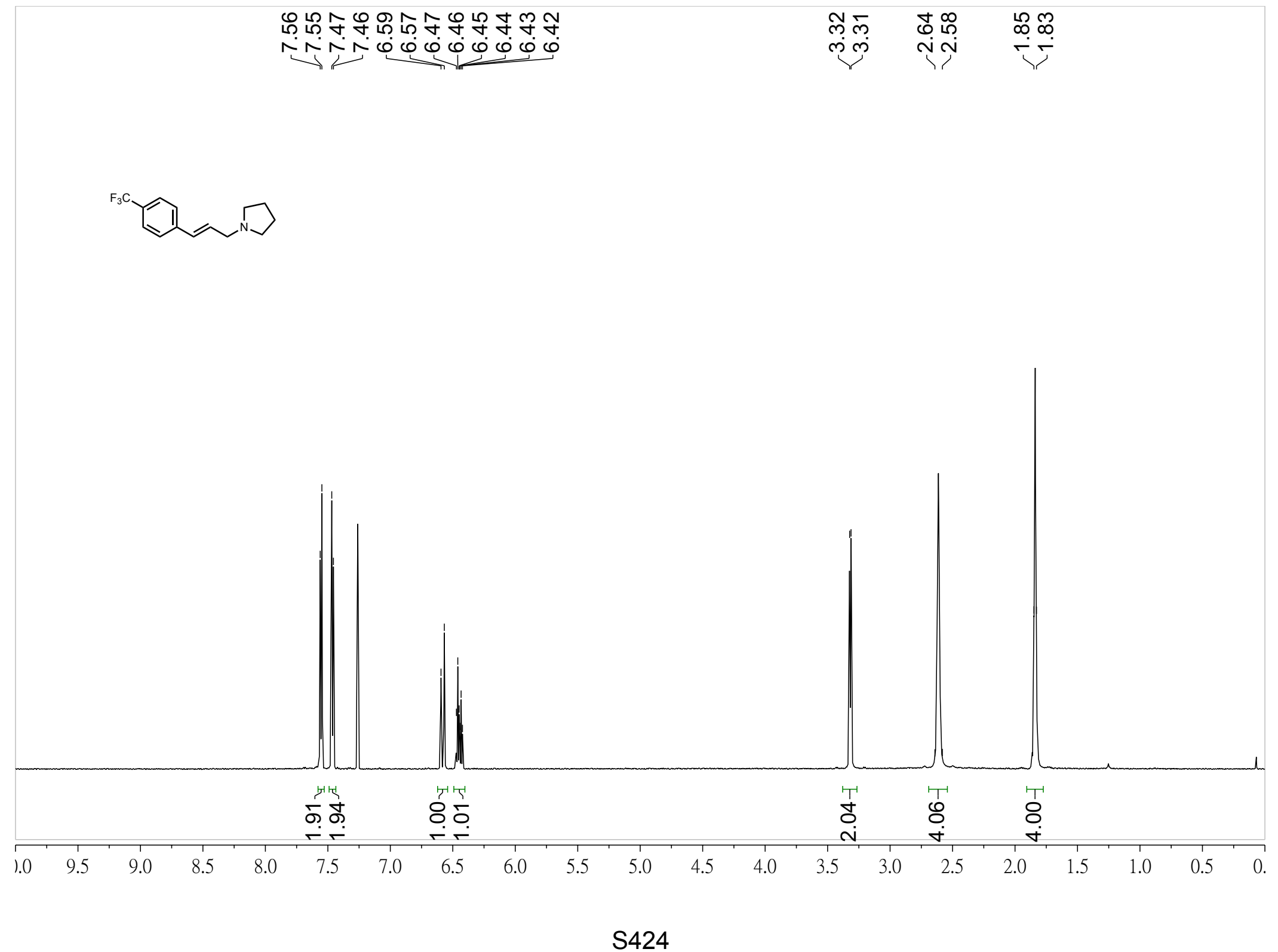




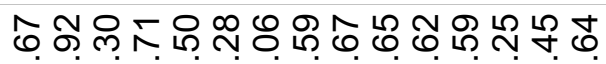

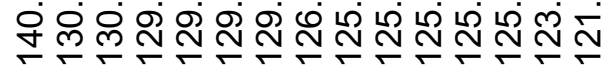

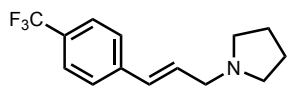

$\stackrel{\text { N }}{\stackrel{2}{*}}$

$\stackrel{1}{\infty} \dot{0}$

๔0

ஸ

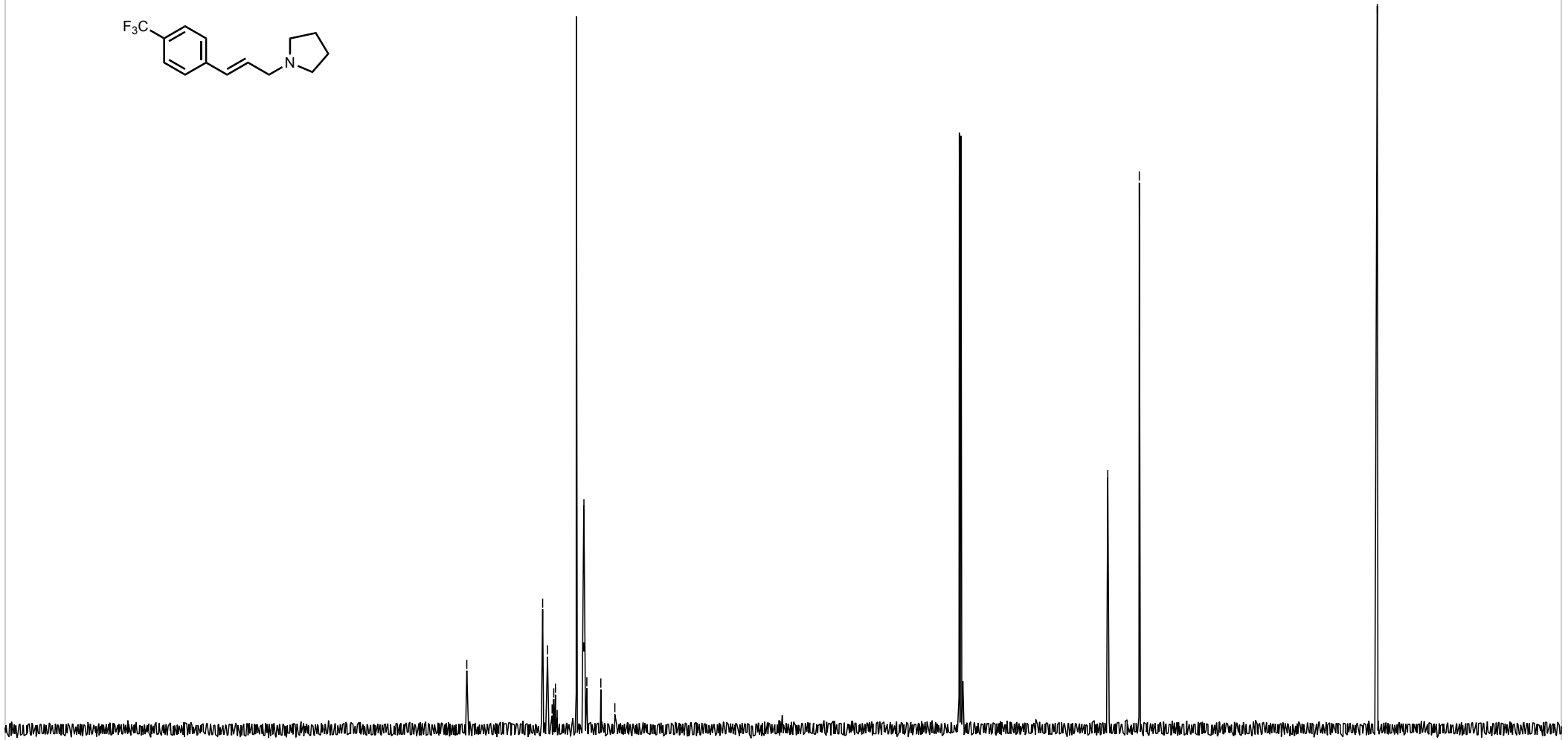

(n)

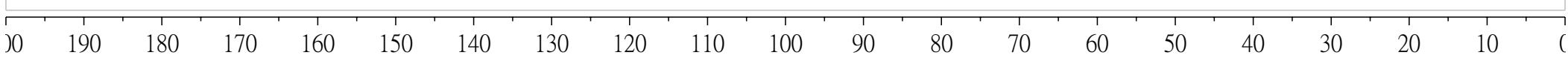




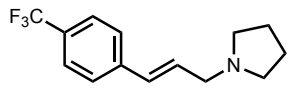

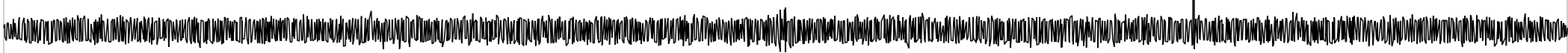

\begin{tabular}{|llllllllllllllllllllllll}
\hline 20 & 110 & 100 & 90 & 80 & 70 & 60 & 50 & 40 & 30 & 20 & 10 & 0 & -10 & -20 & -30 & -40 & -50 & -60 & -70 & -80 & -90 & -100 & -110
\end{tabular}




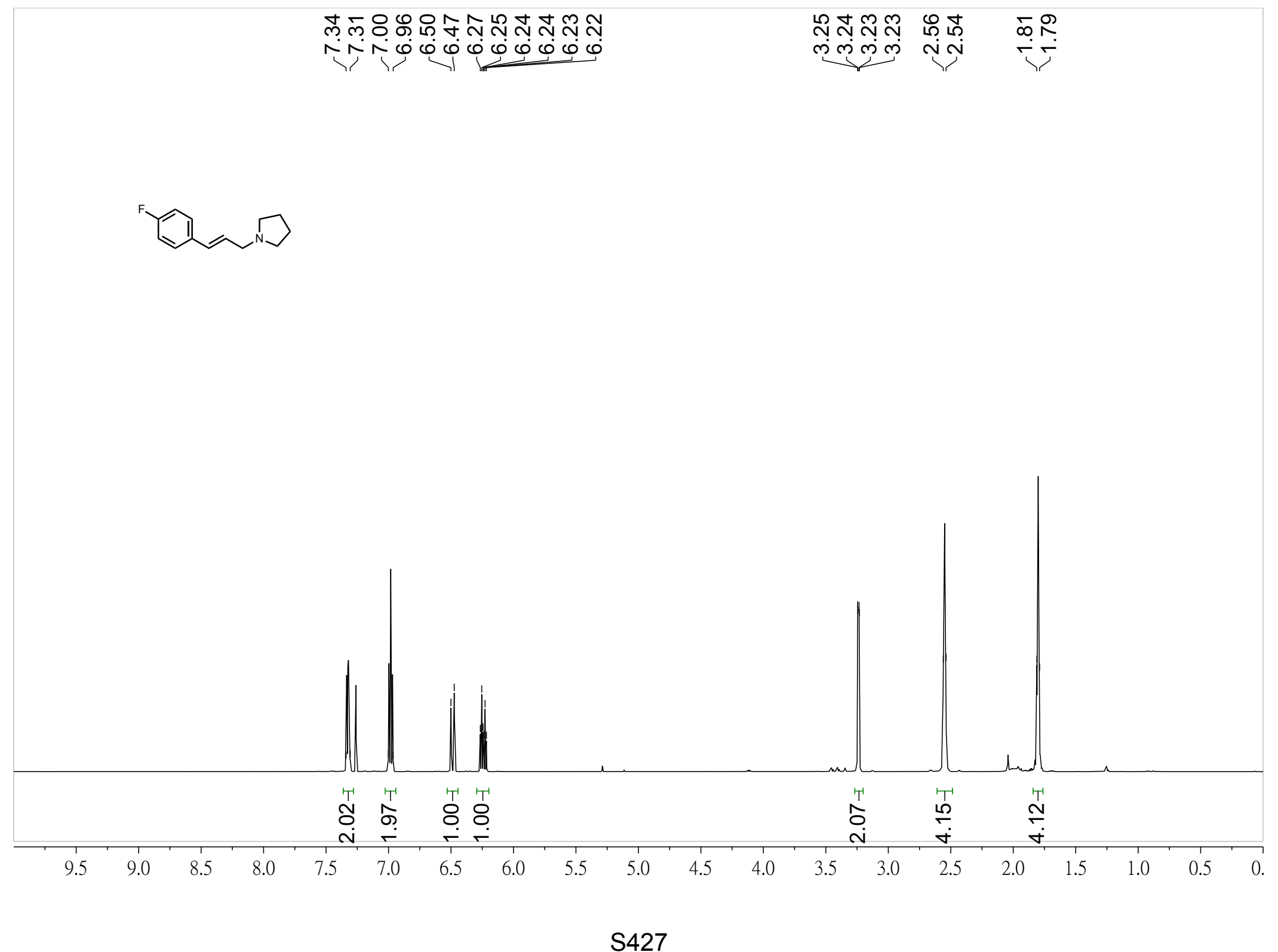




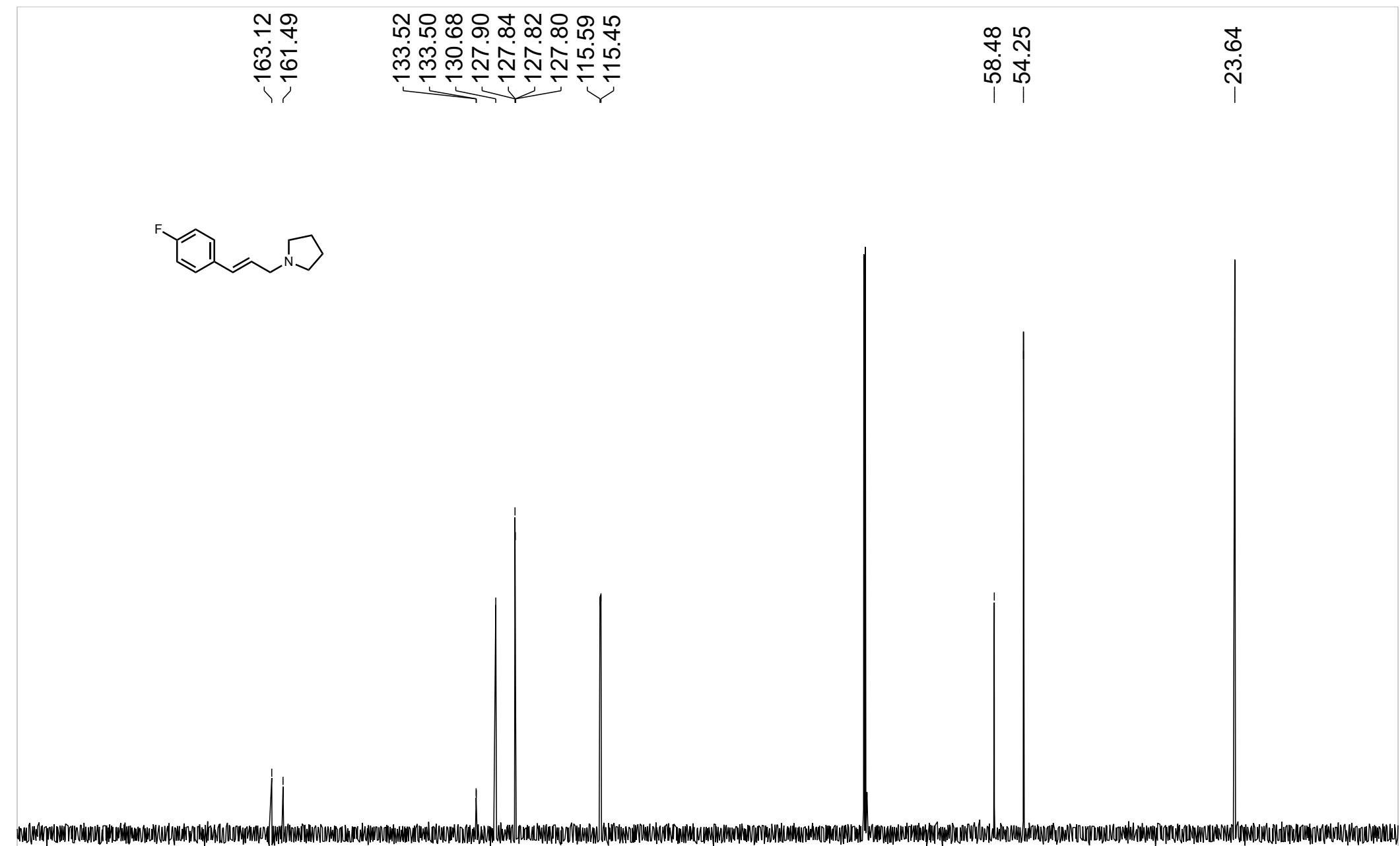

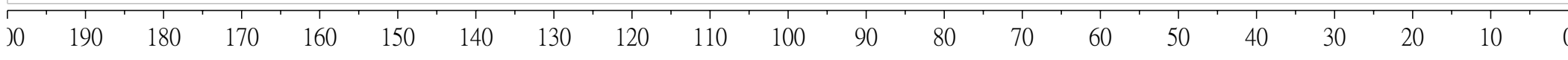




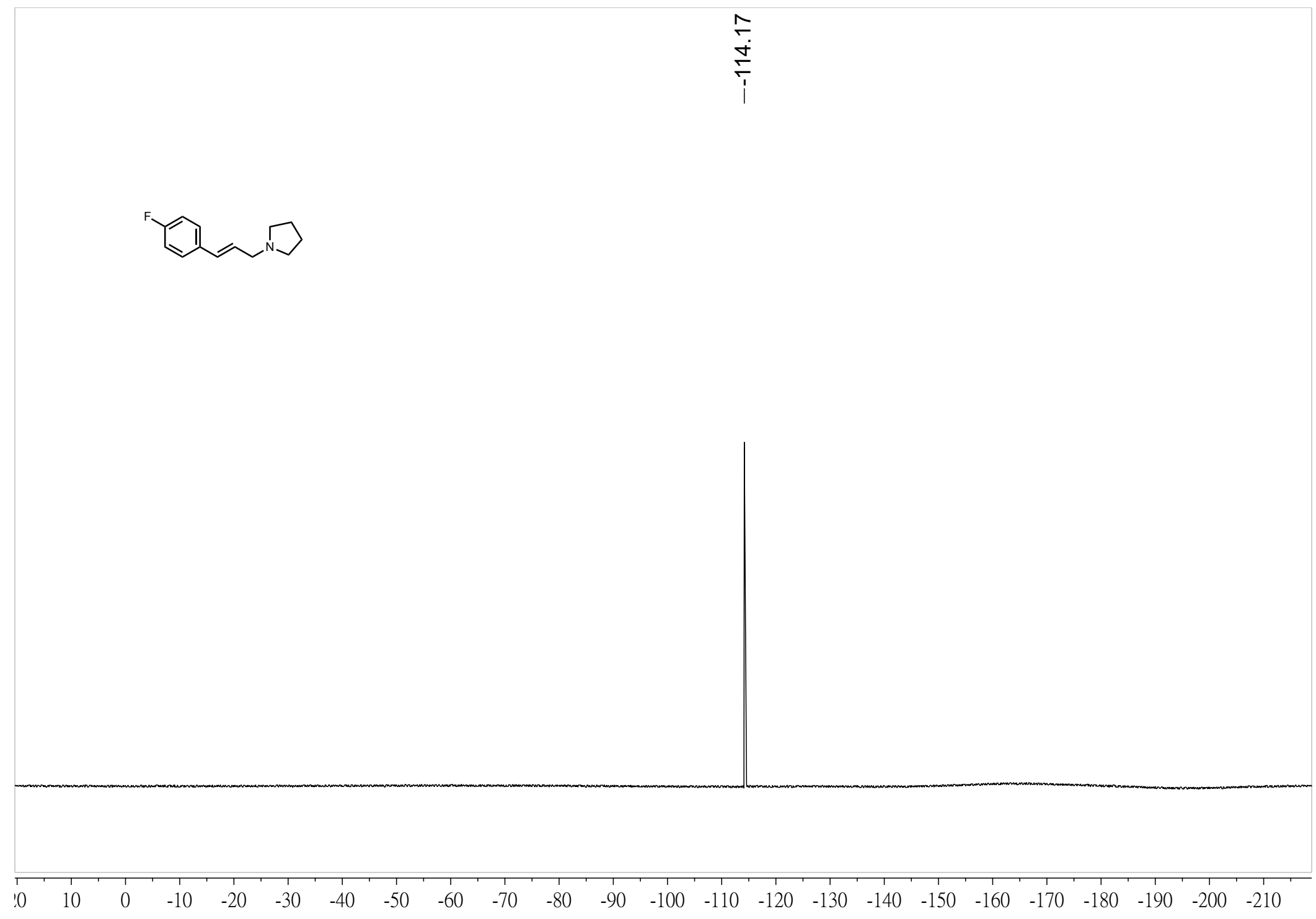

S429 


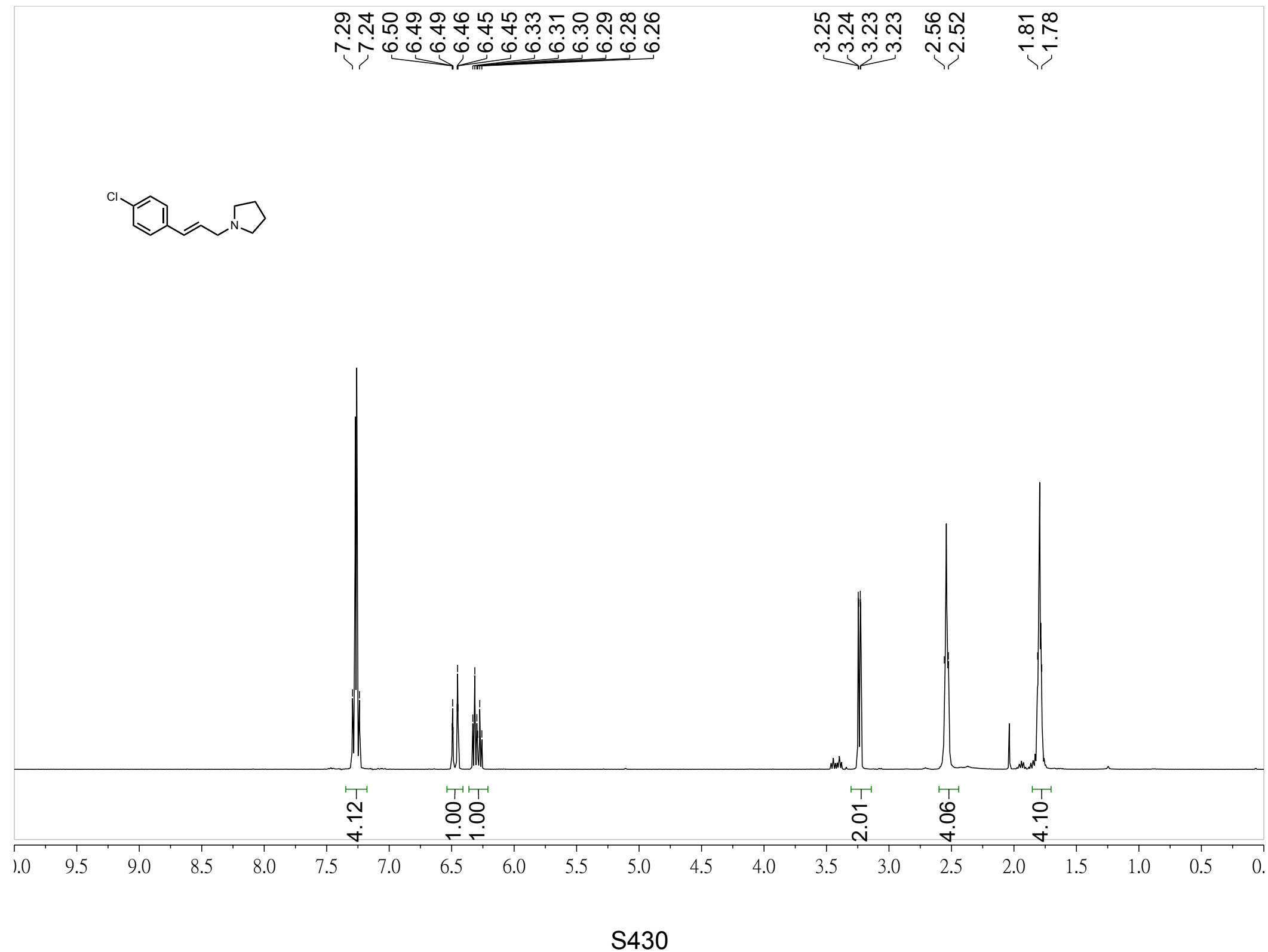




$$
\text { تلت }
$$




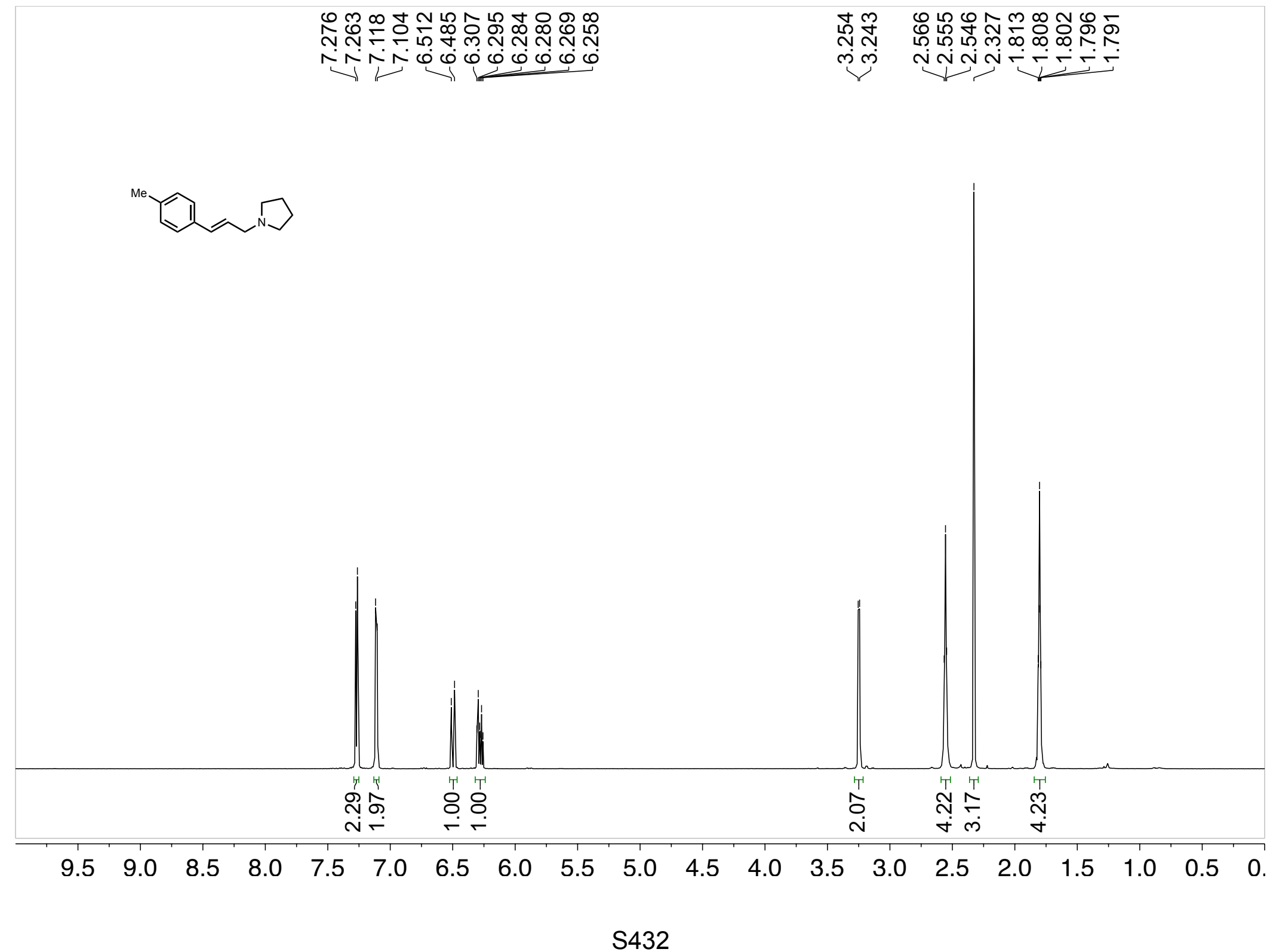




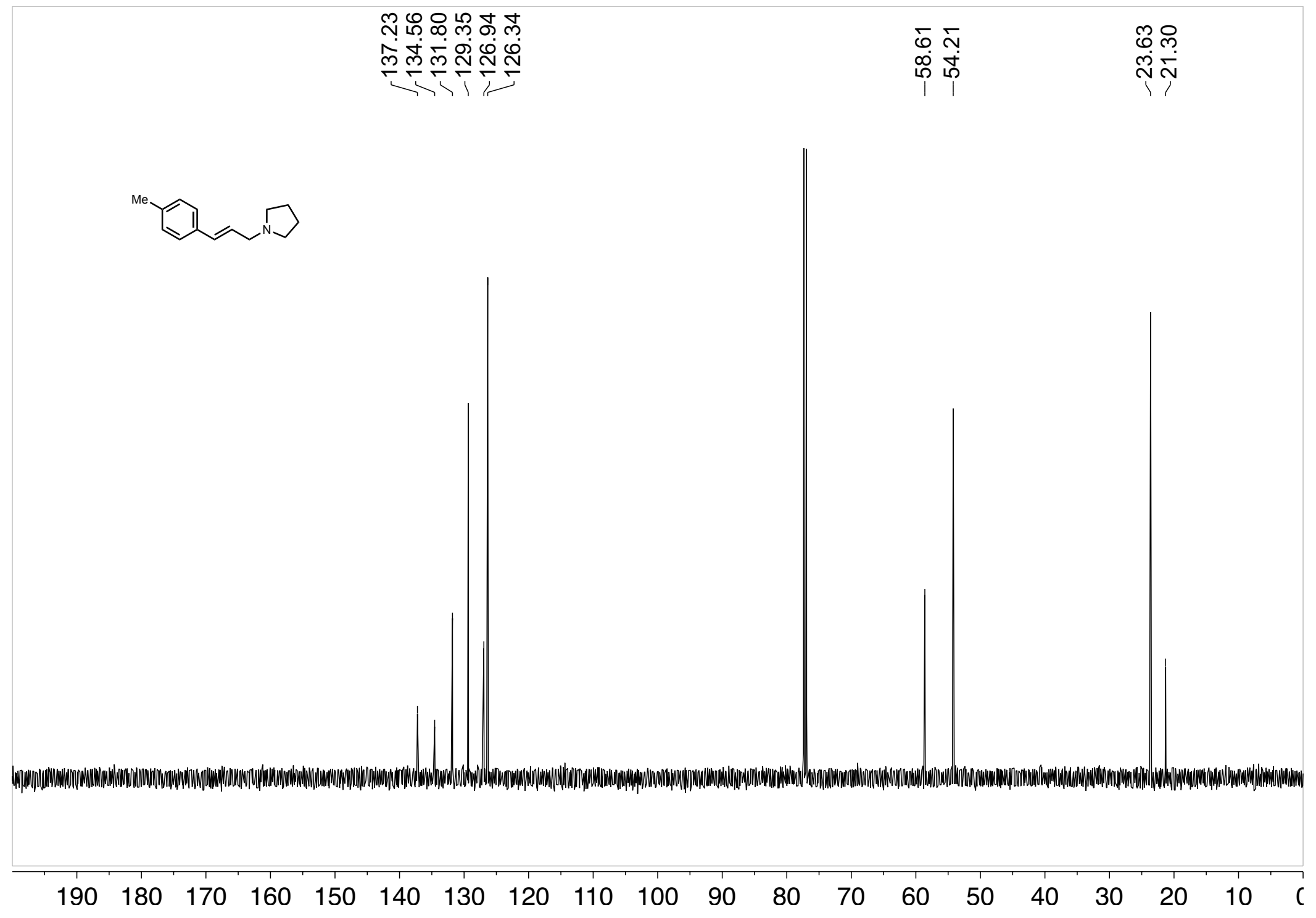

S433 


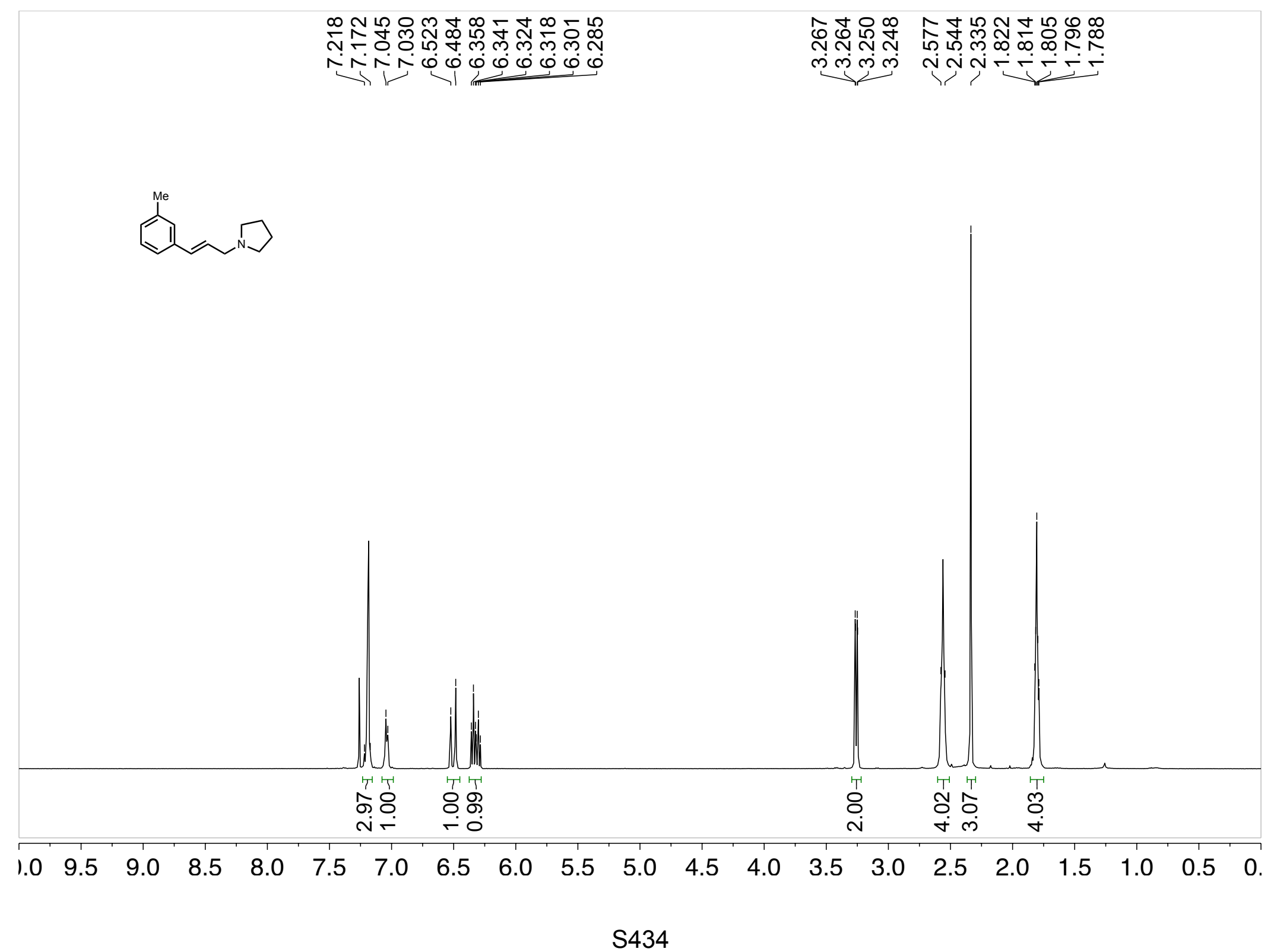




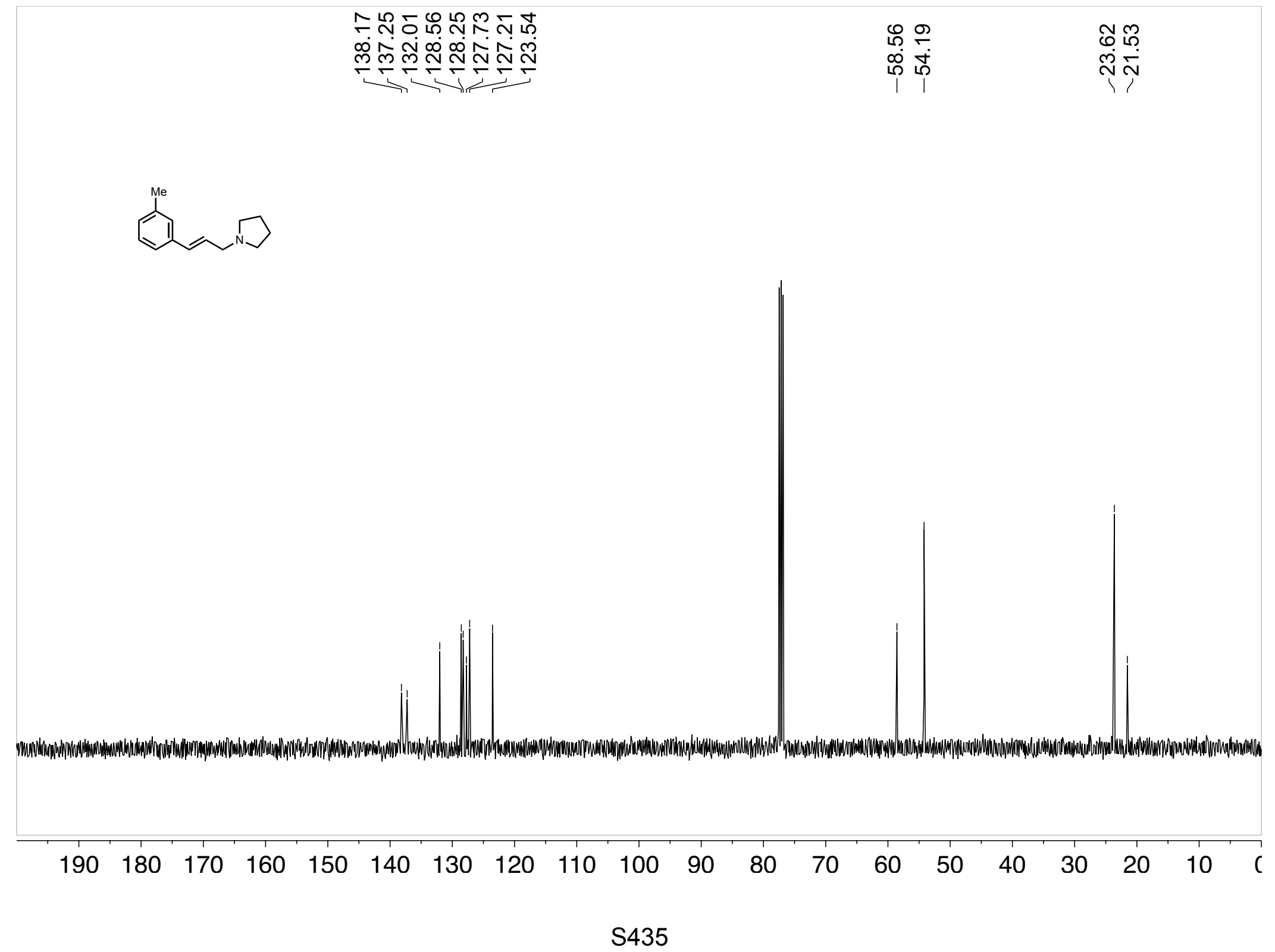




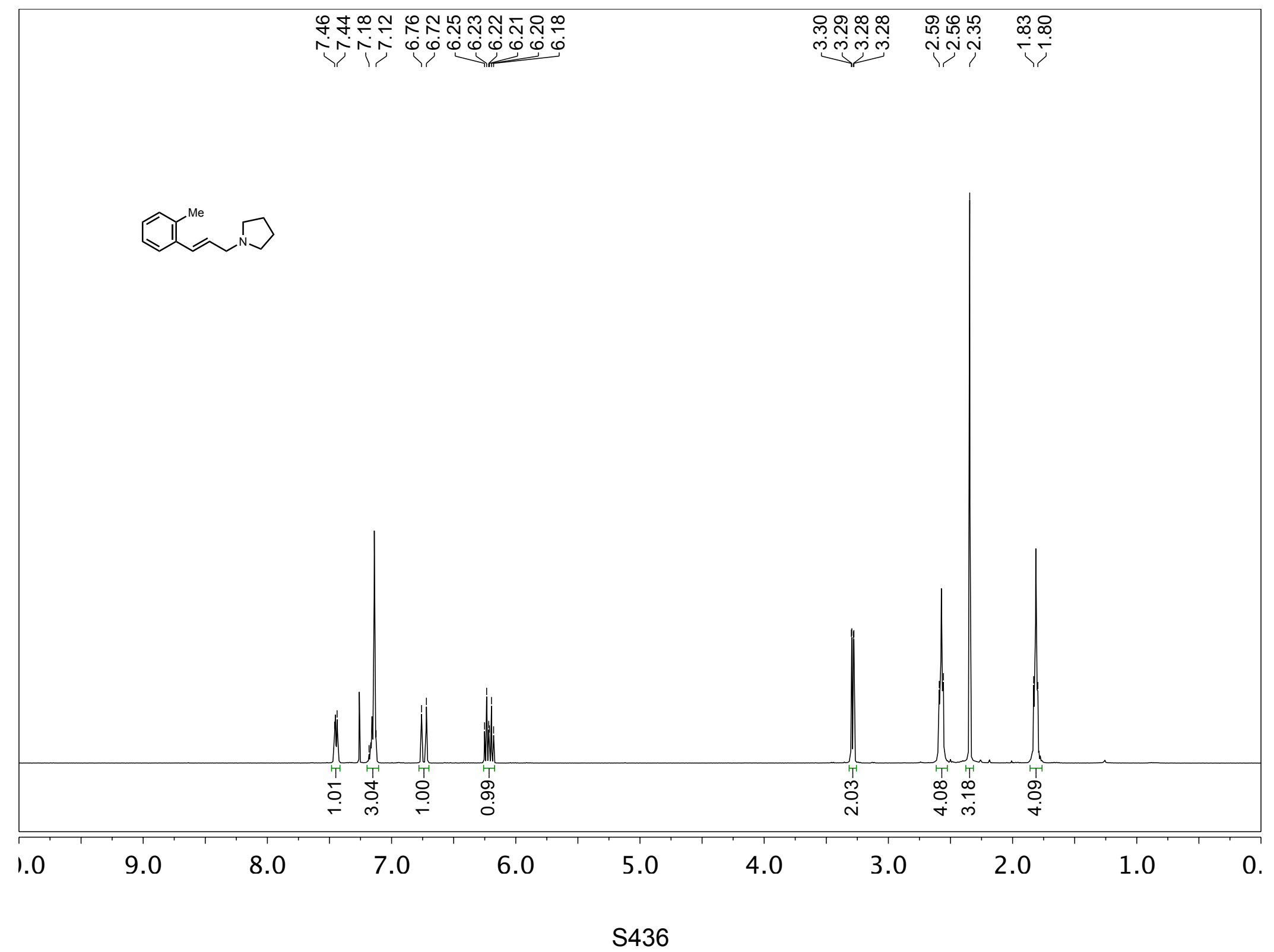




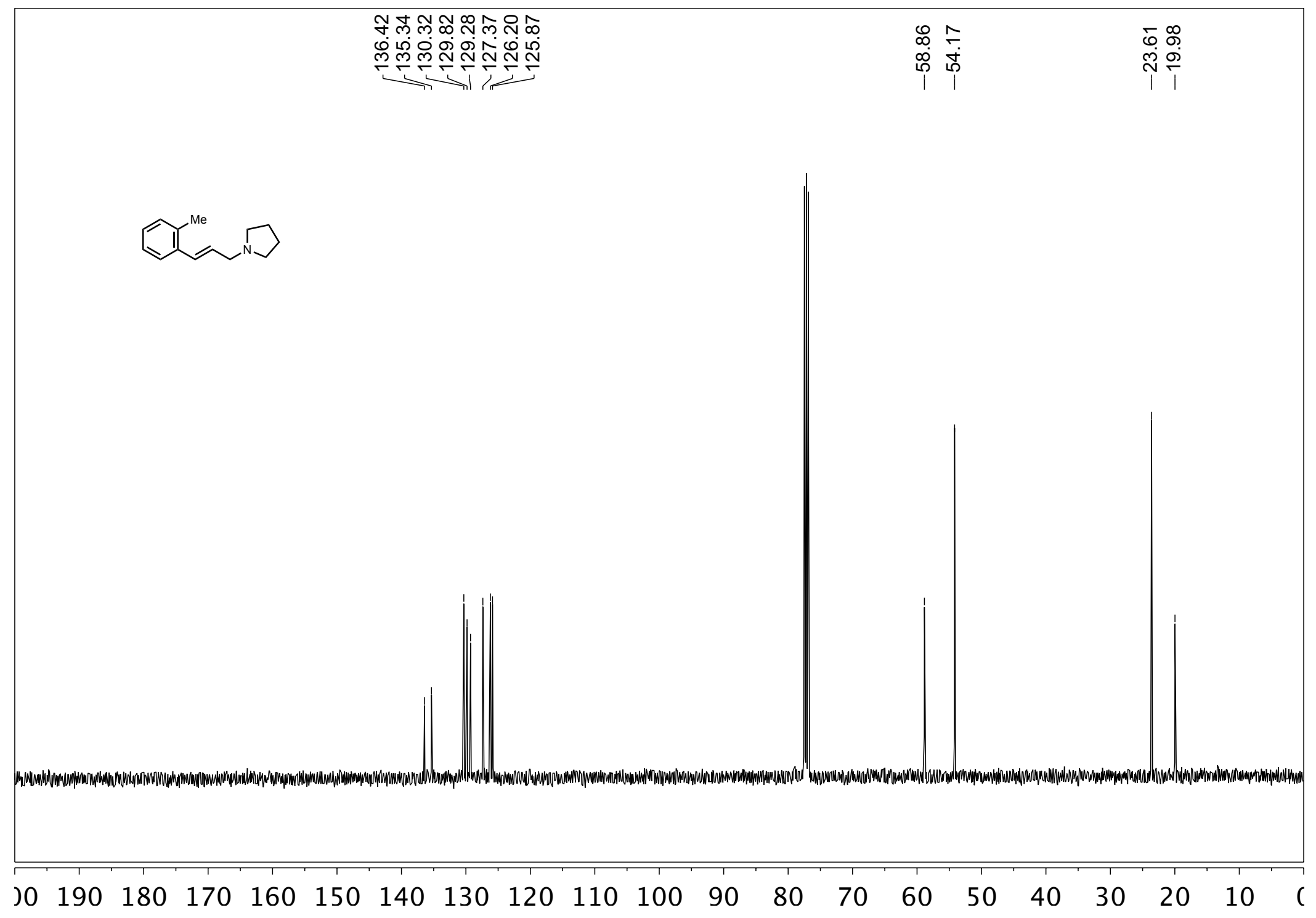

S437 


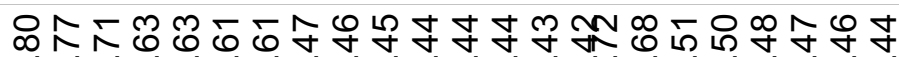

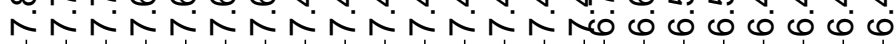

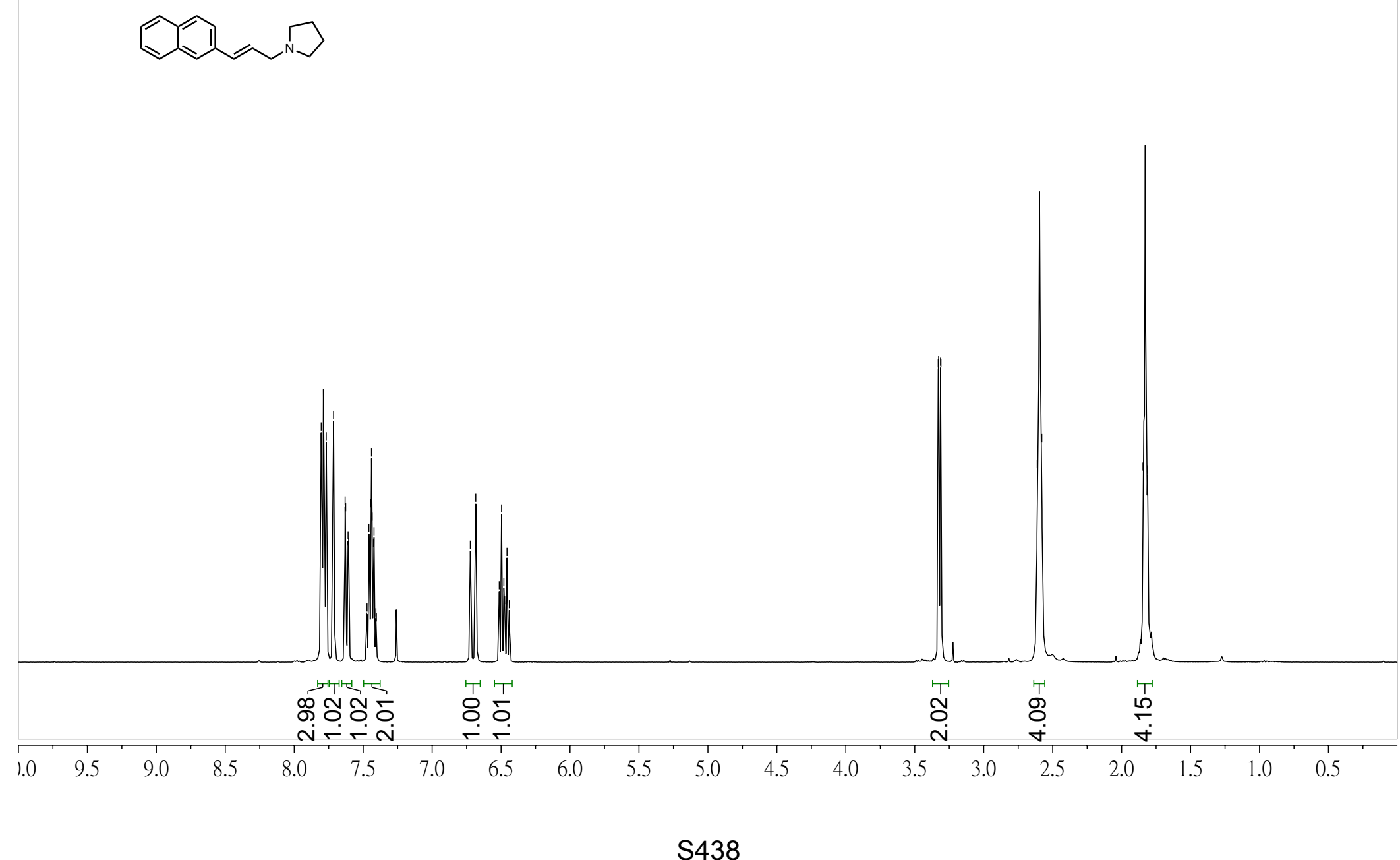

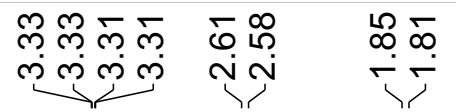




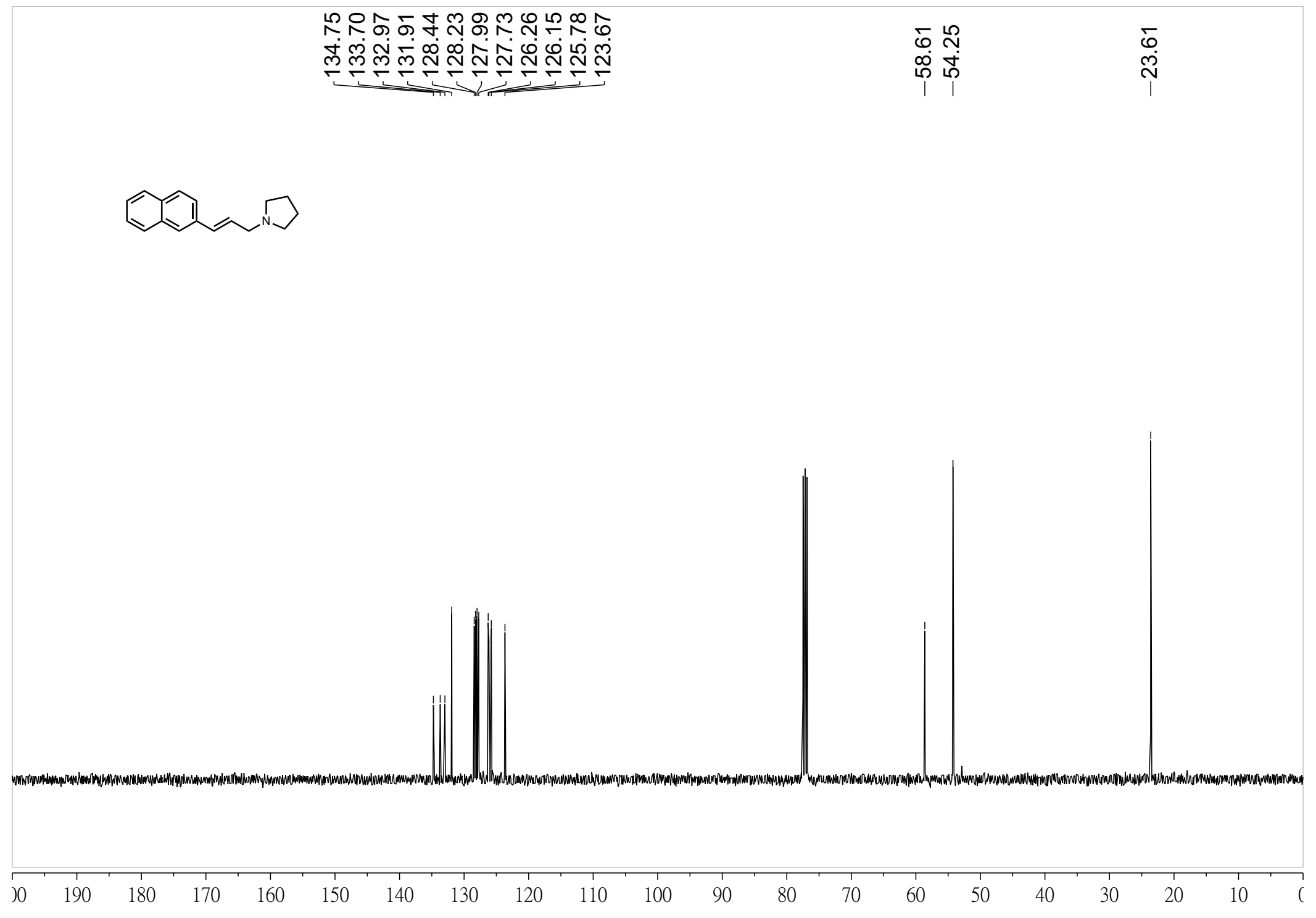

S439 


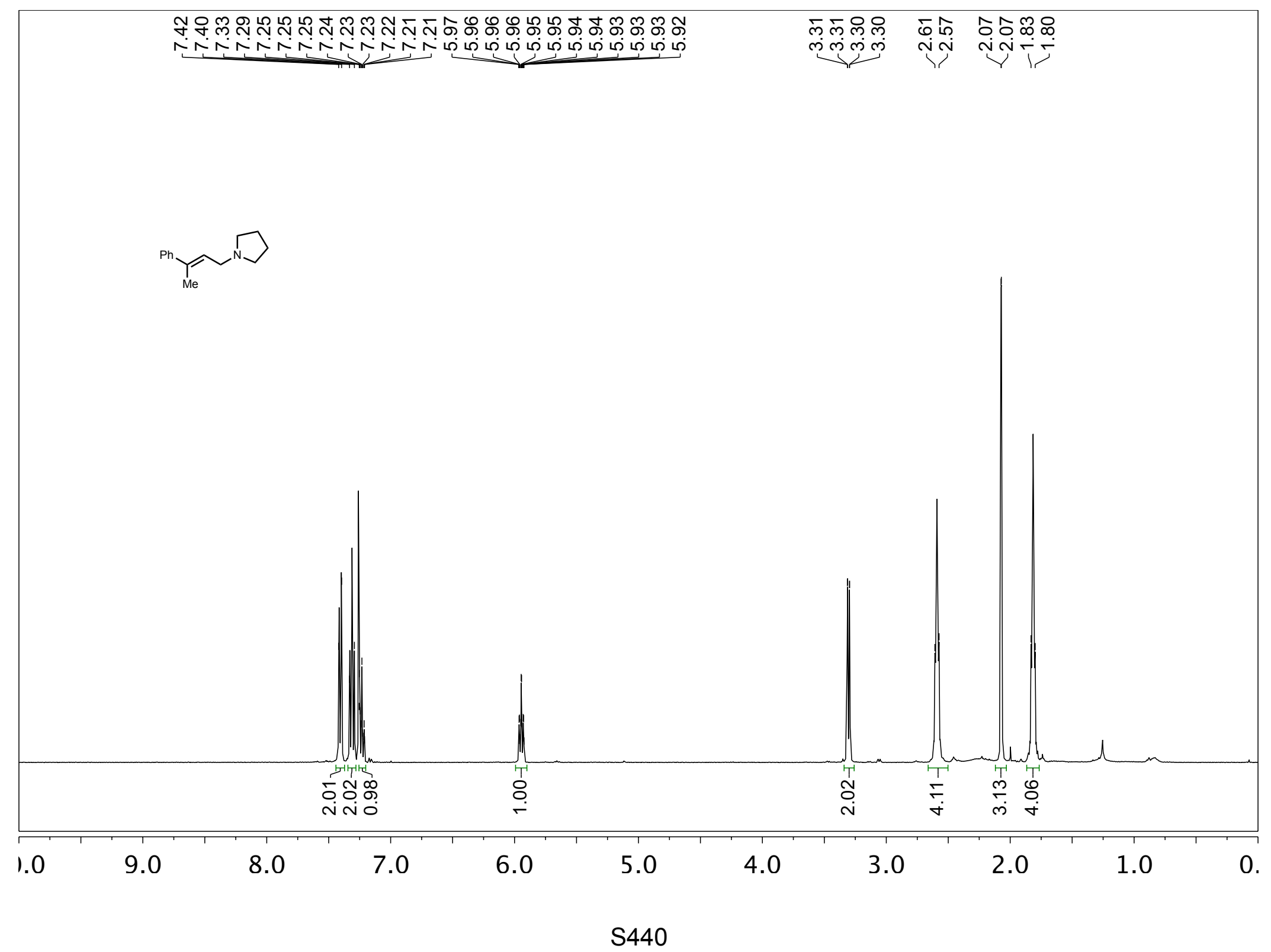




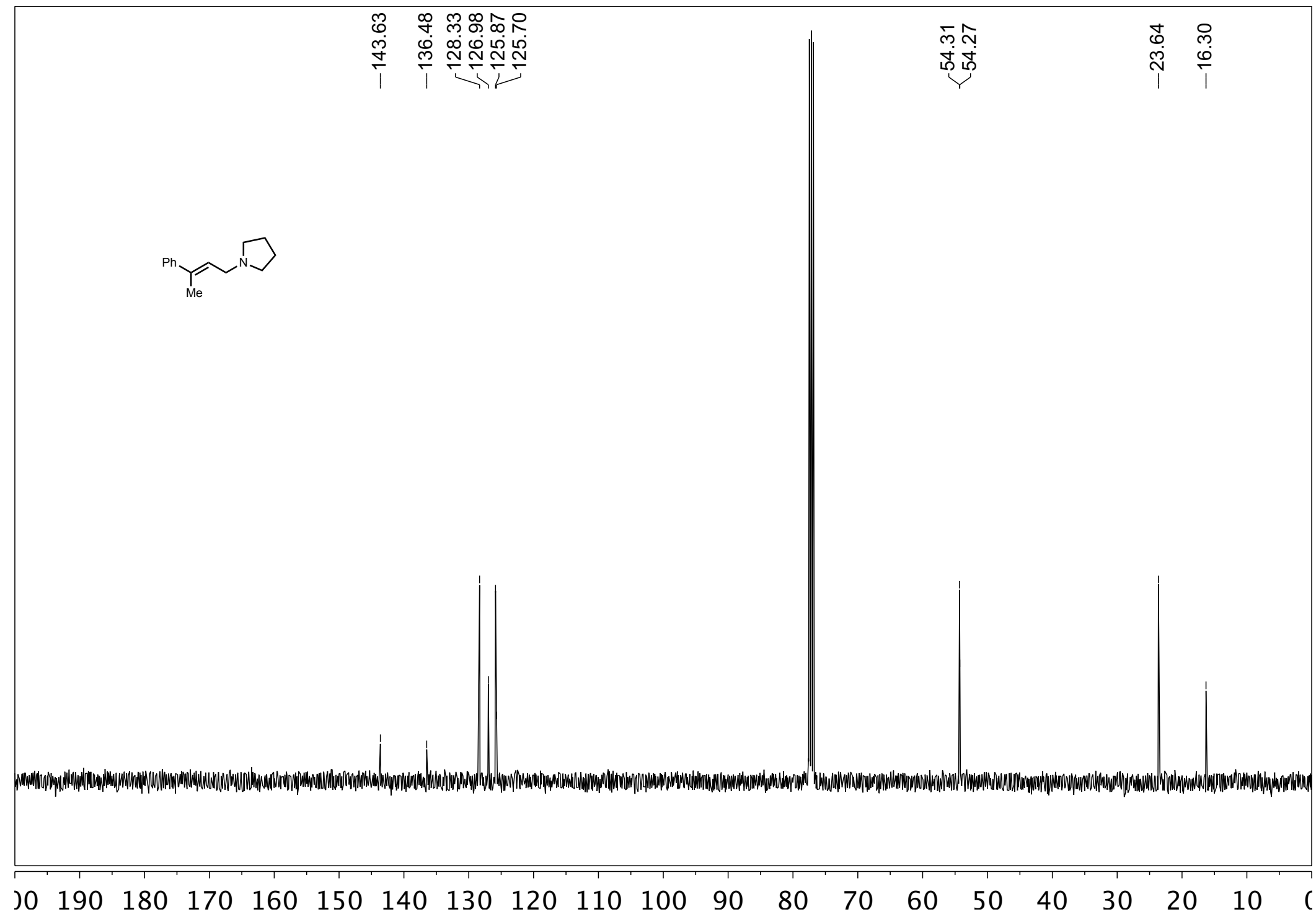




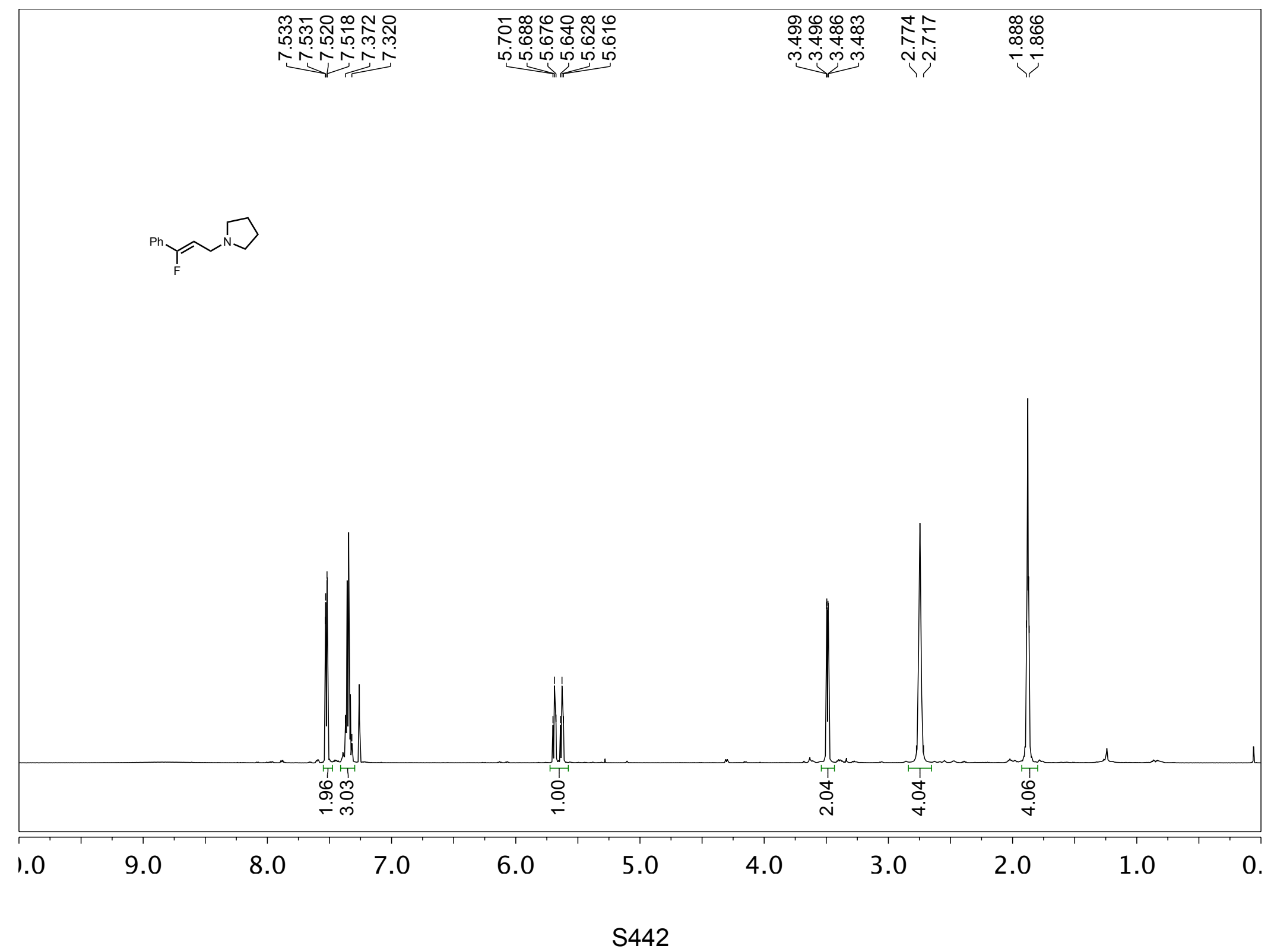




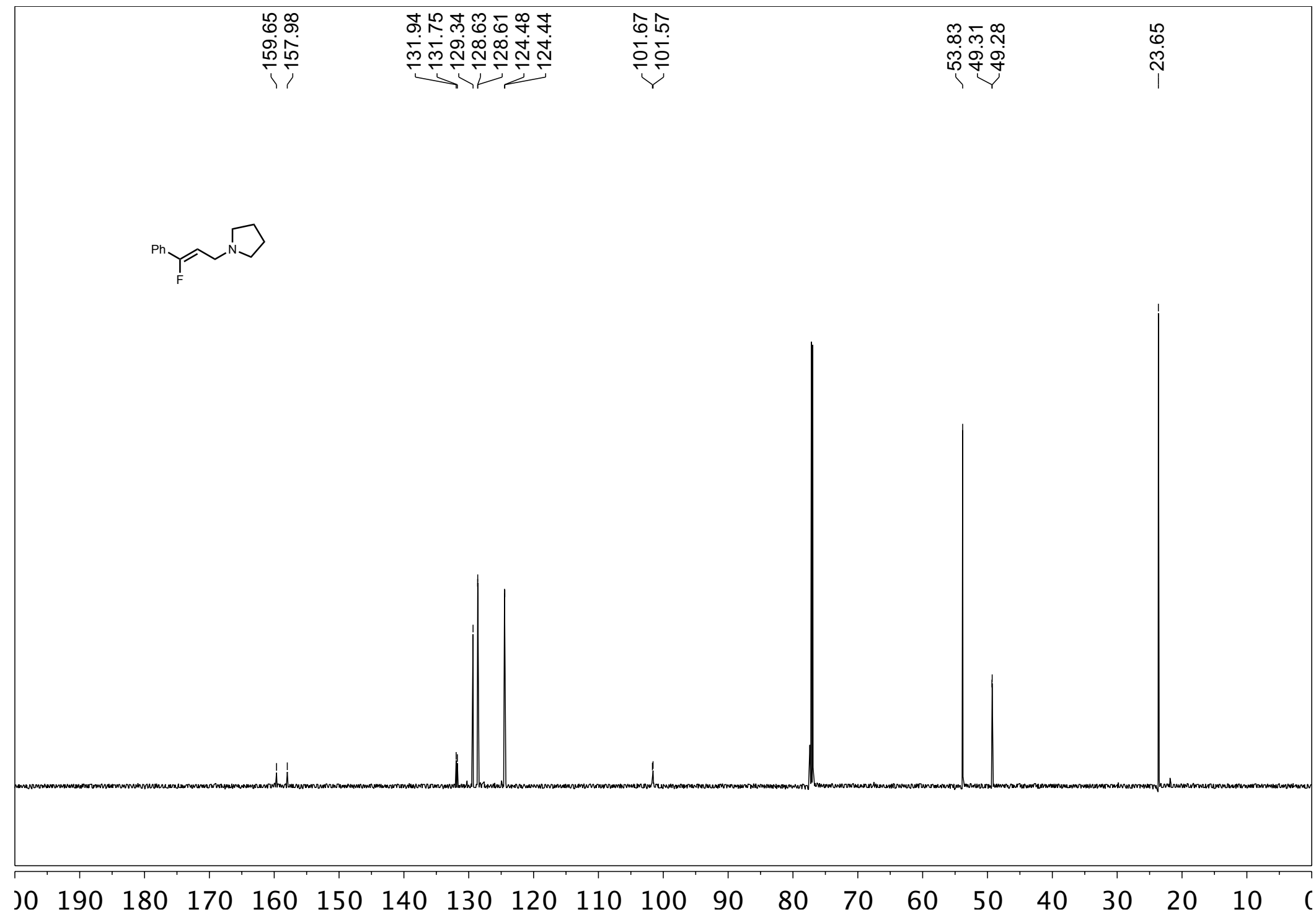

S443 


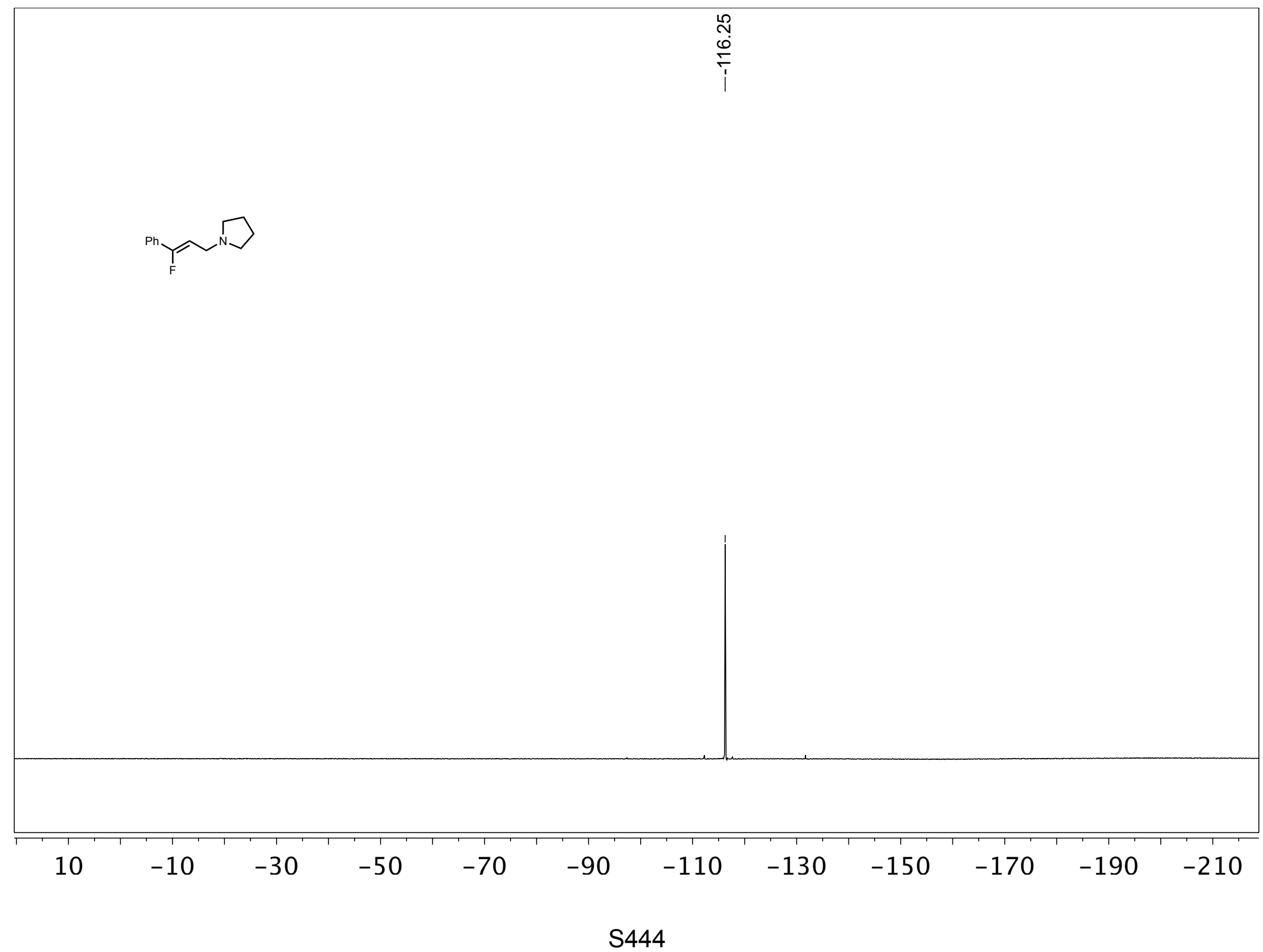




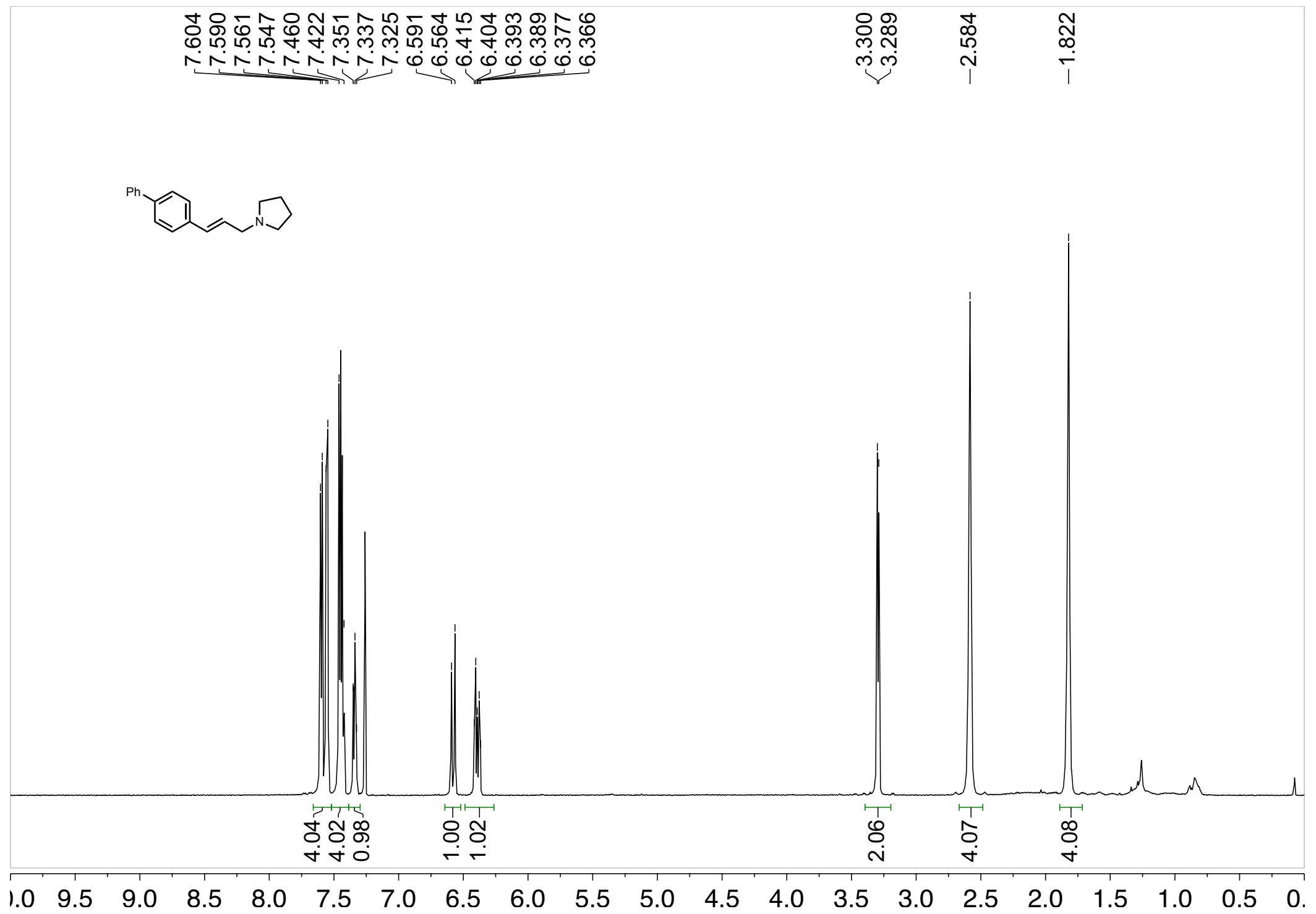

S445 


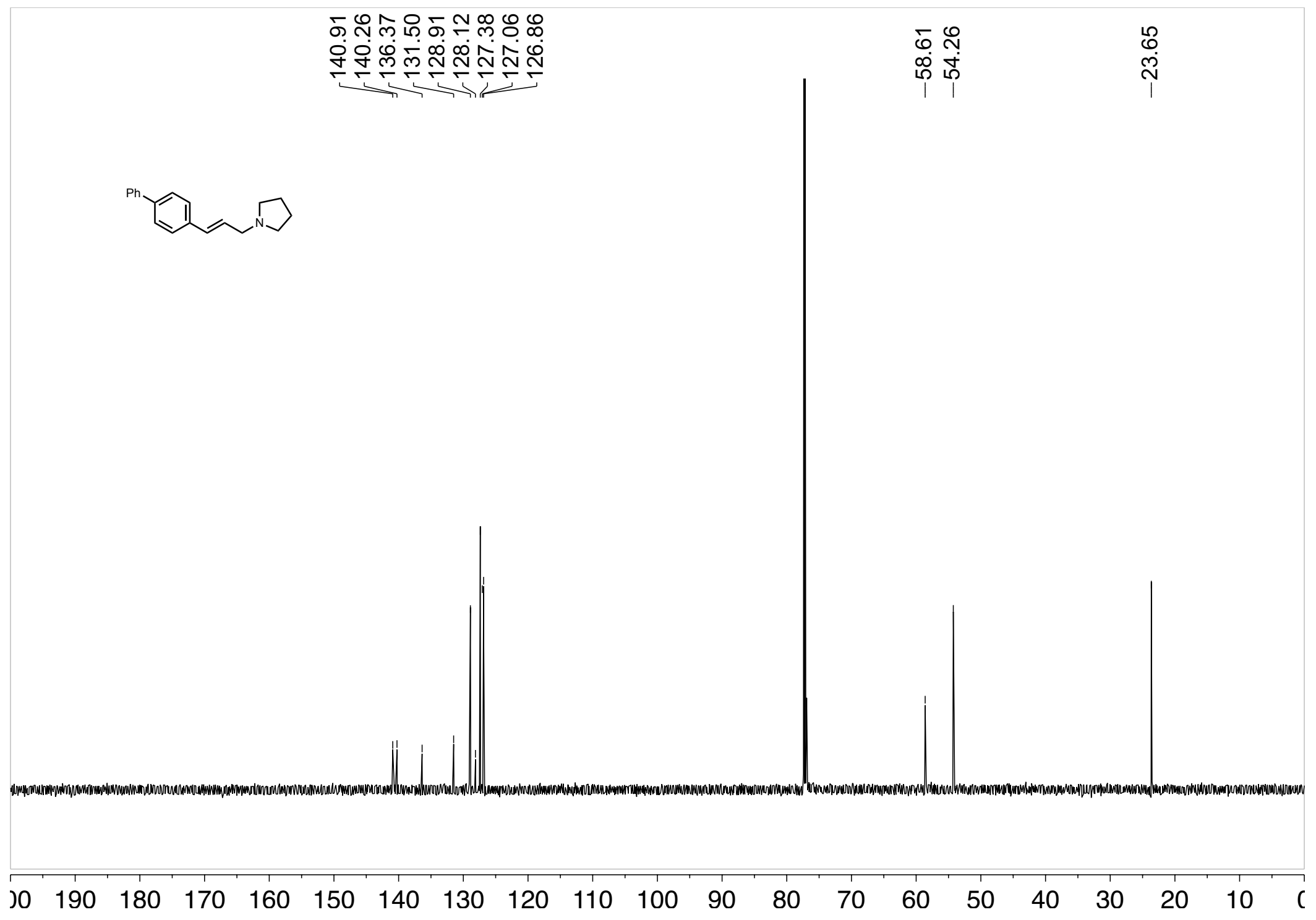

S446 


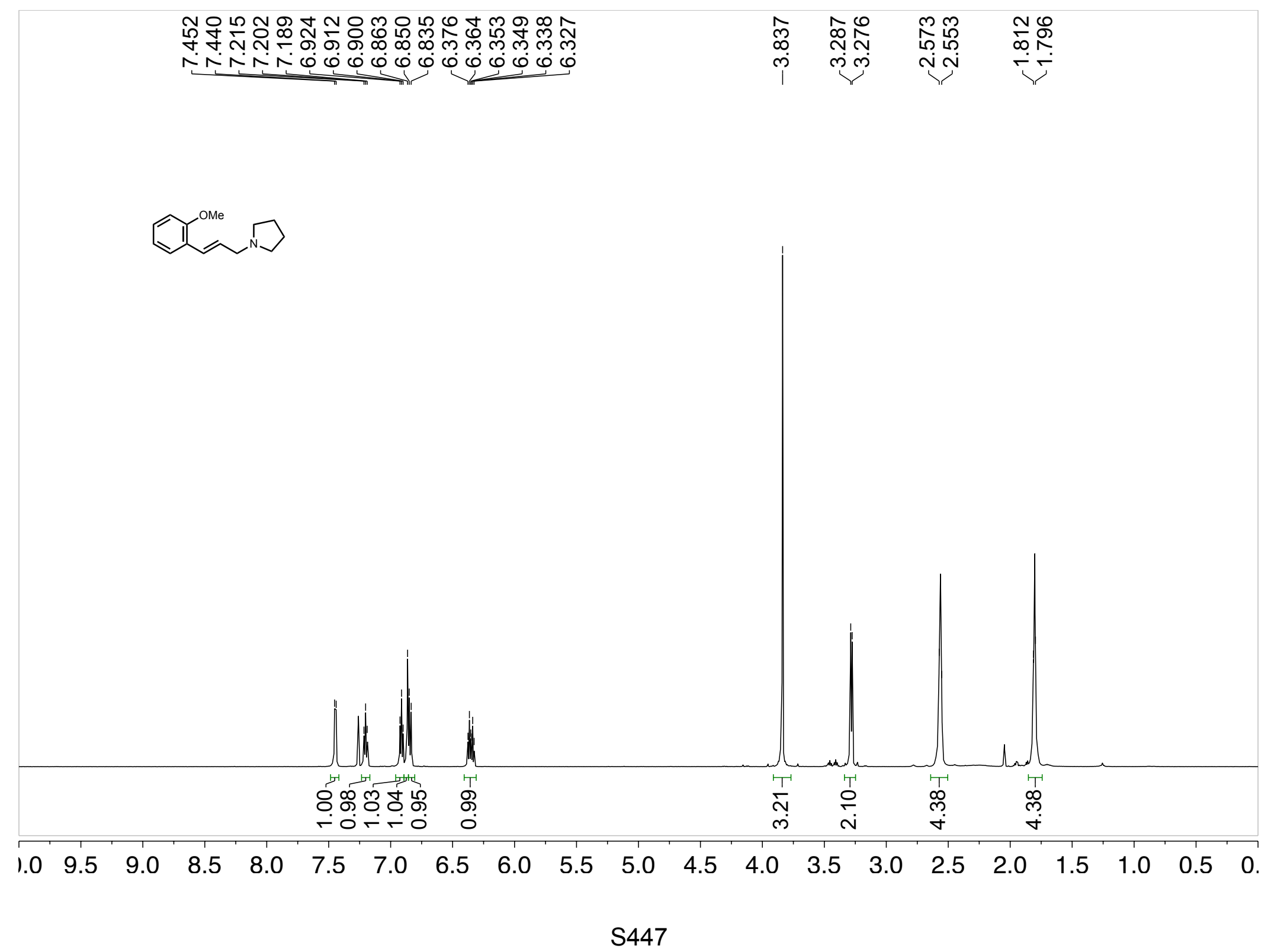




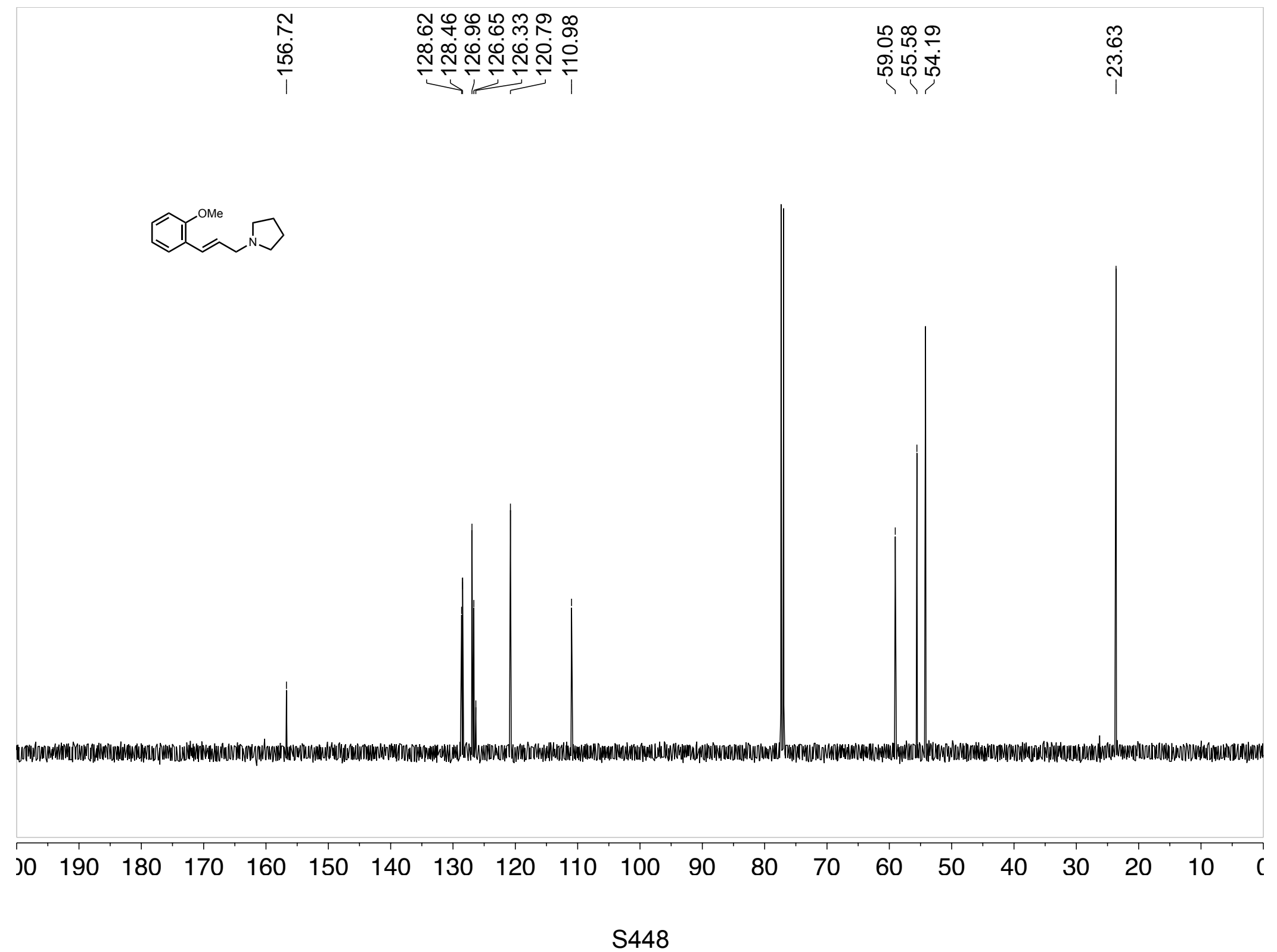



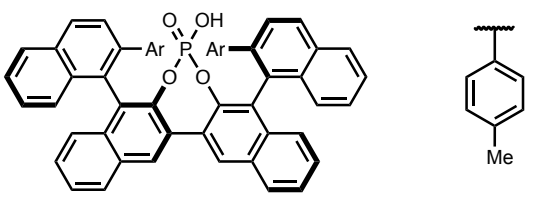

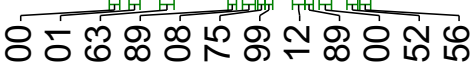
N 


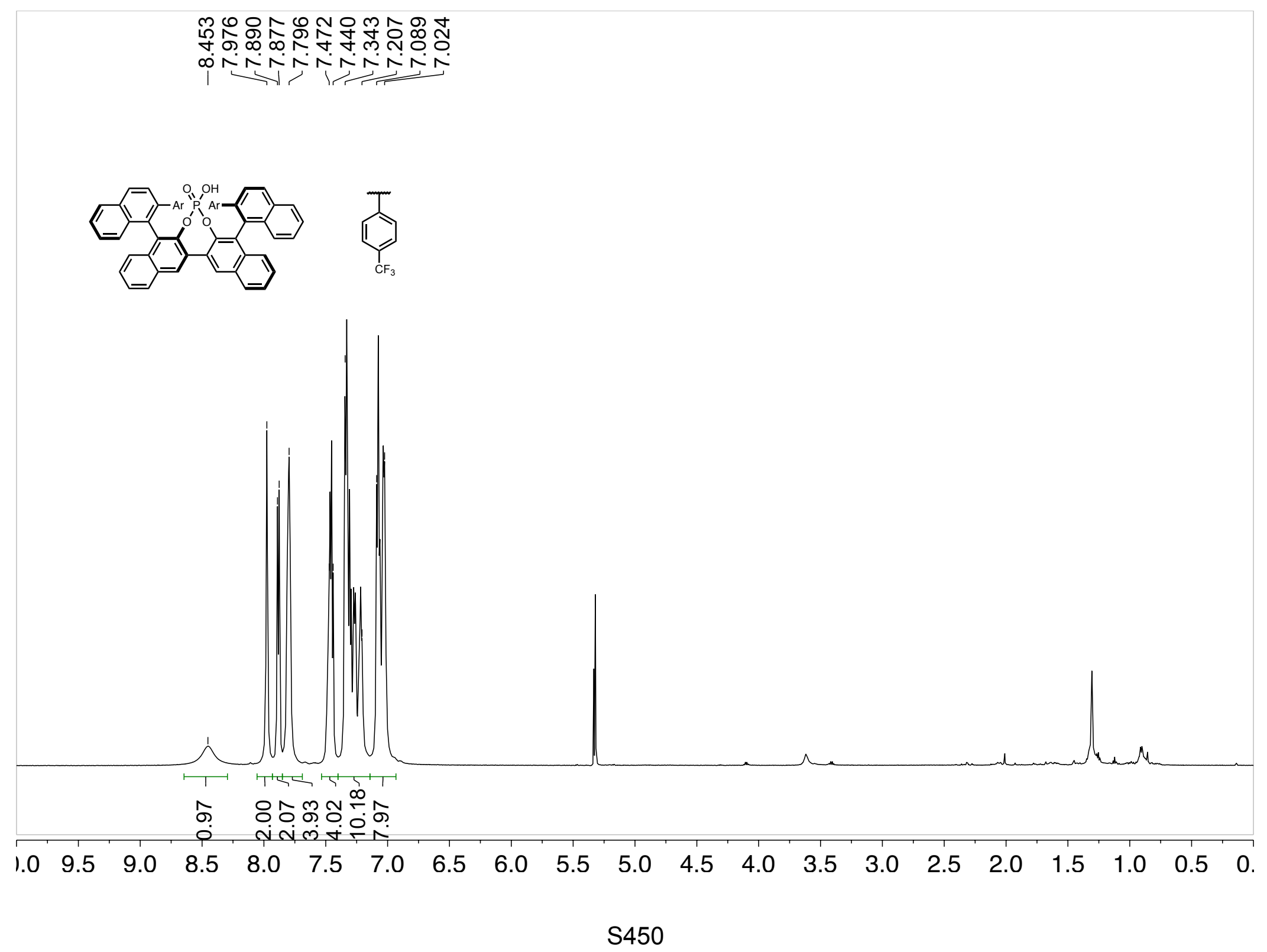


Мุર

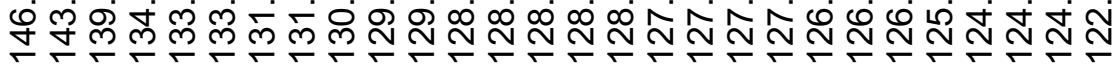
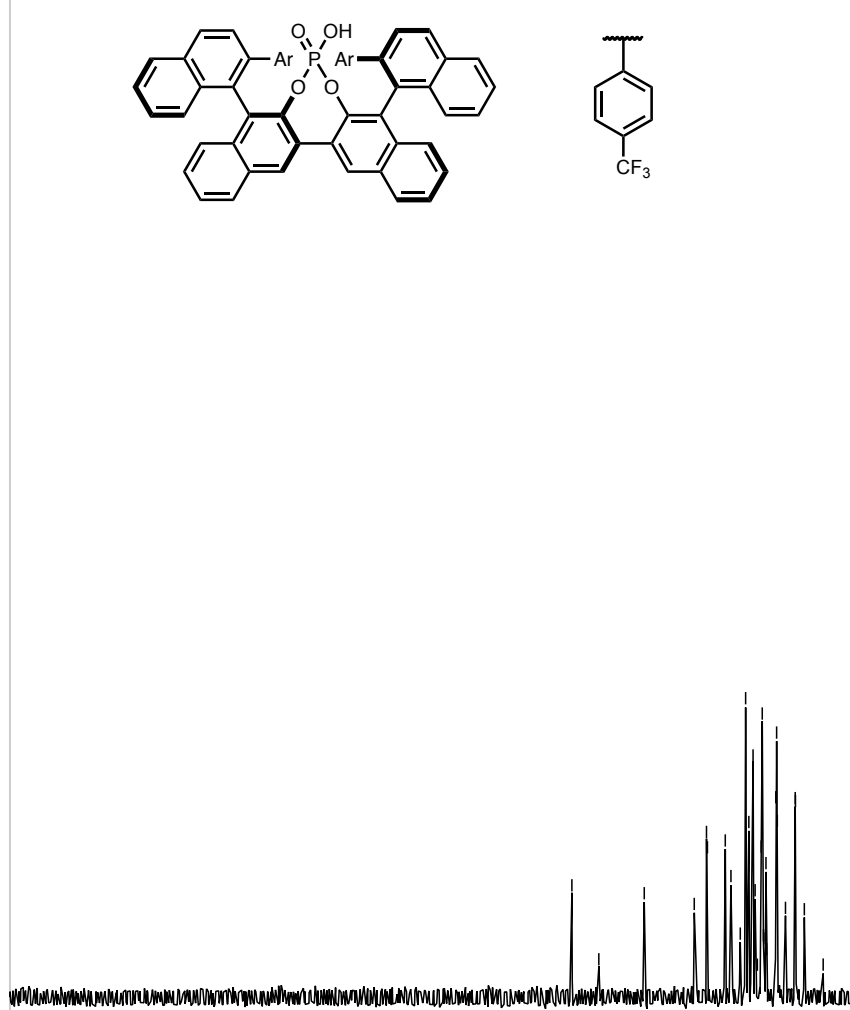

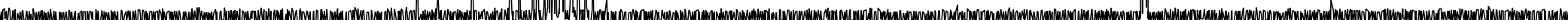

J0 $190 \begin{array}{lllllllllllllllllll}180 & 170 & 160 & 150 & 140 & 130 & 120 & 110 & 100 & 90 & 80 & 70 & 60 & 50 & 40 & 30 & 20 & 10 & (\end{array}$




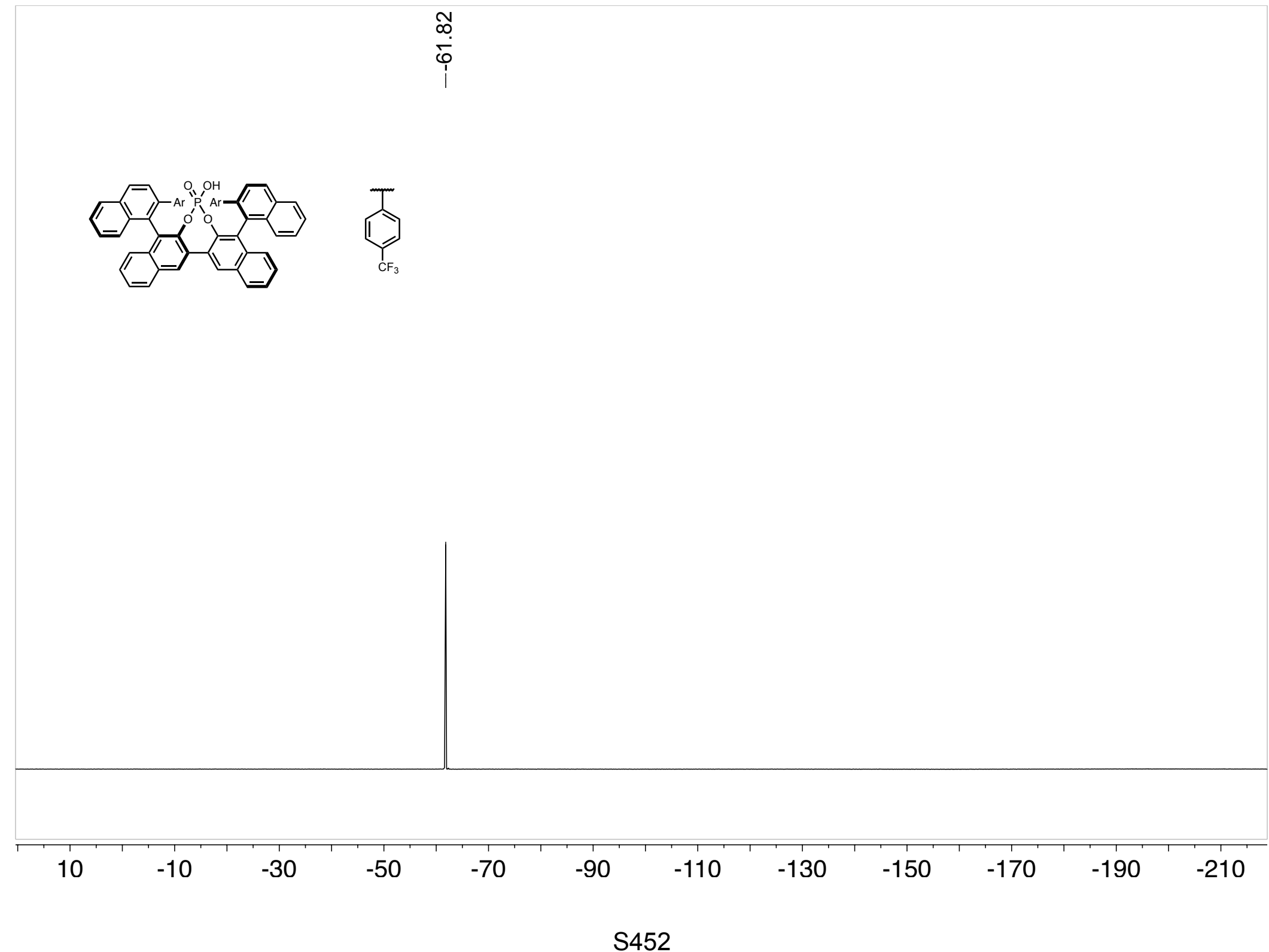




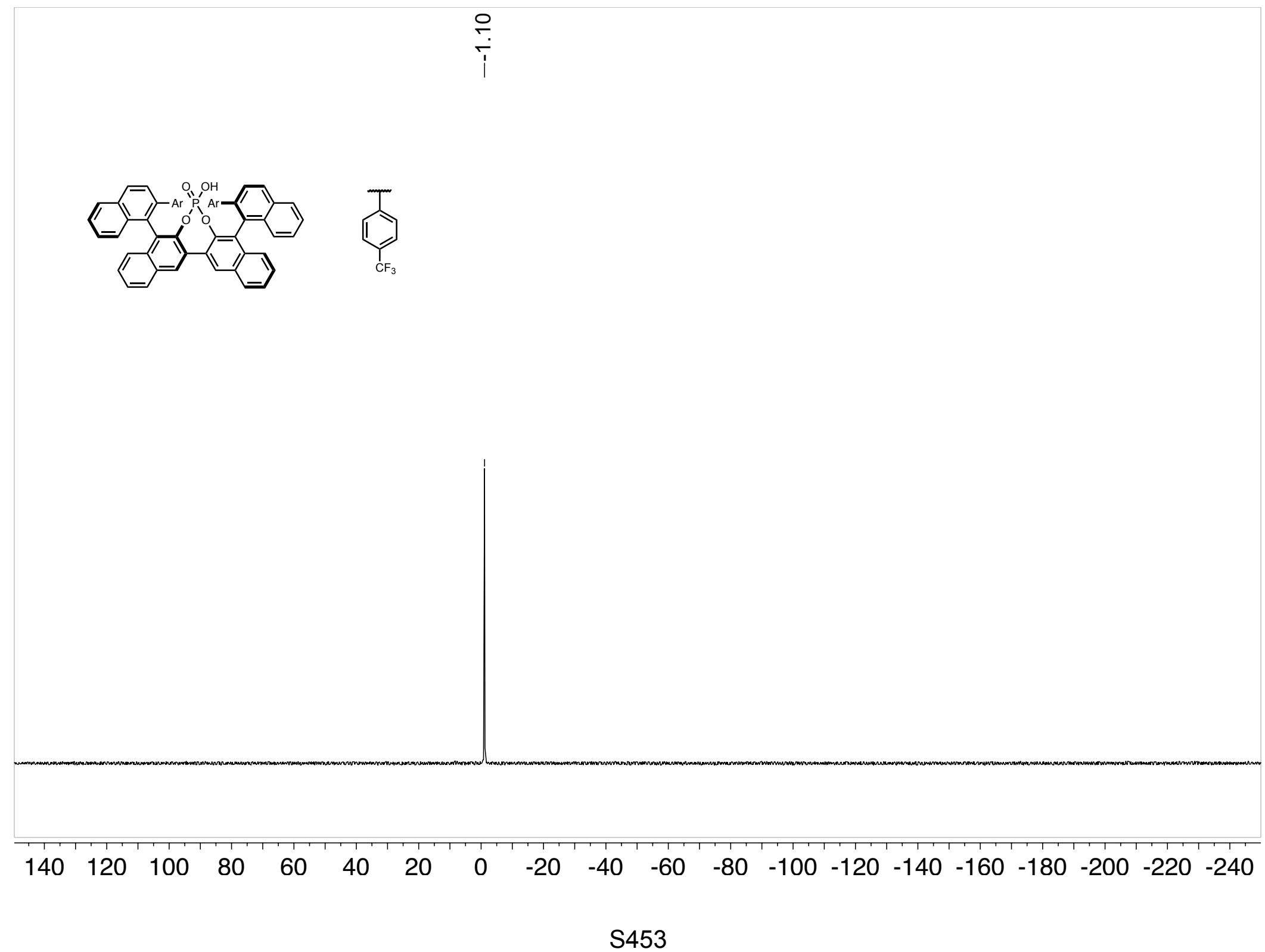




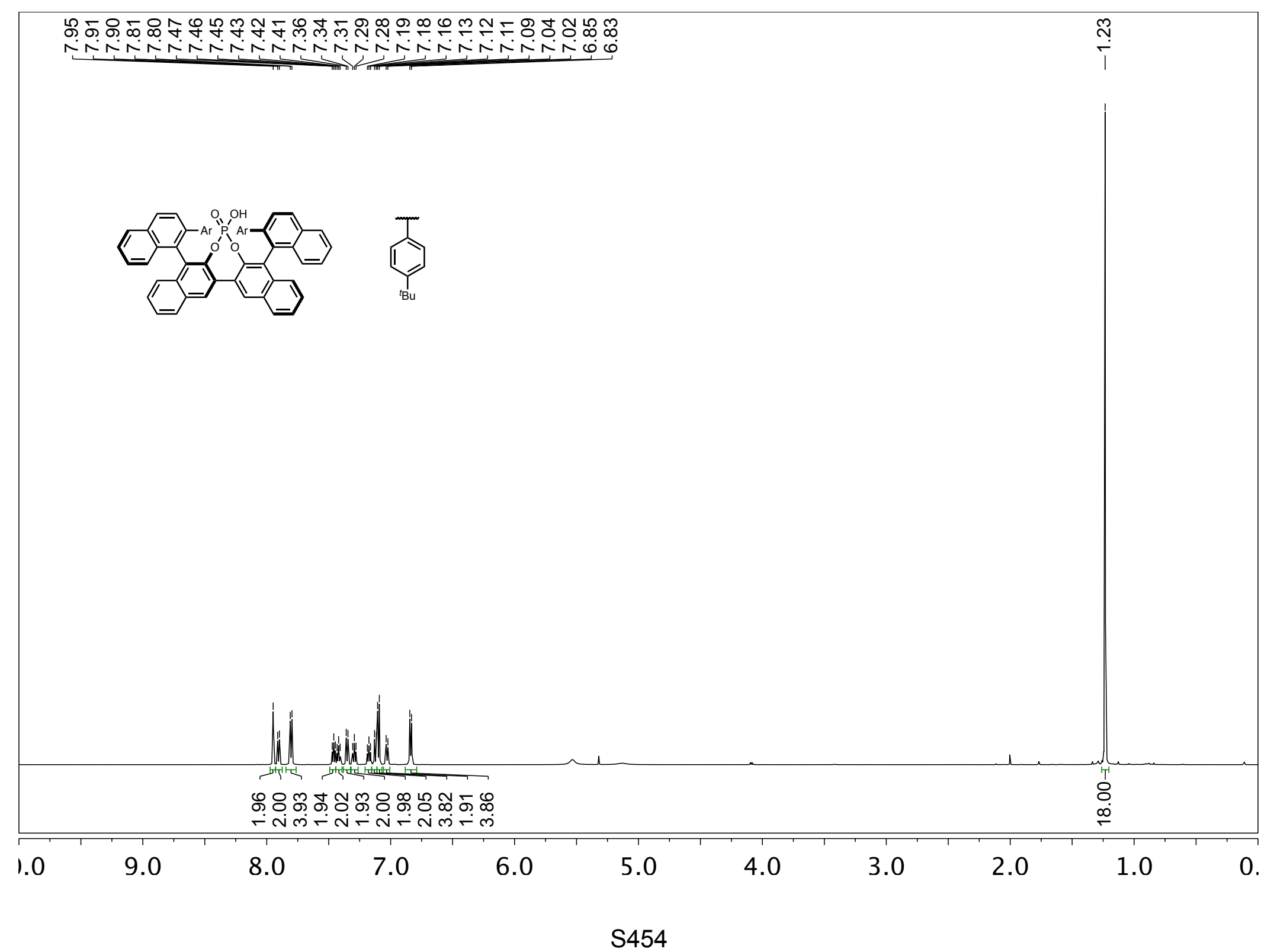




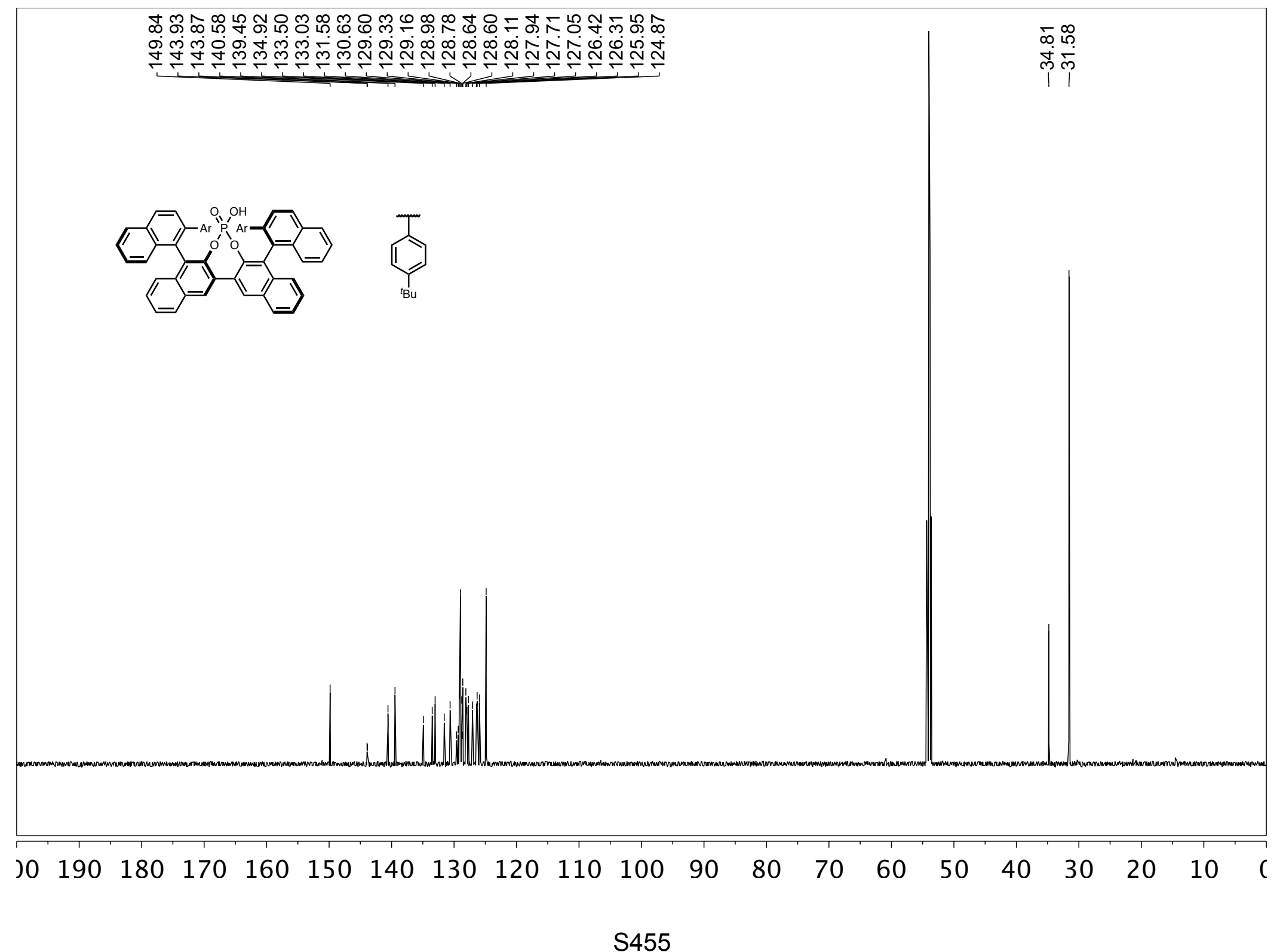




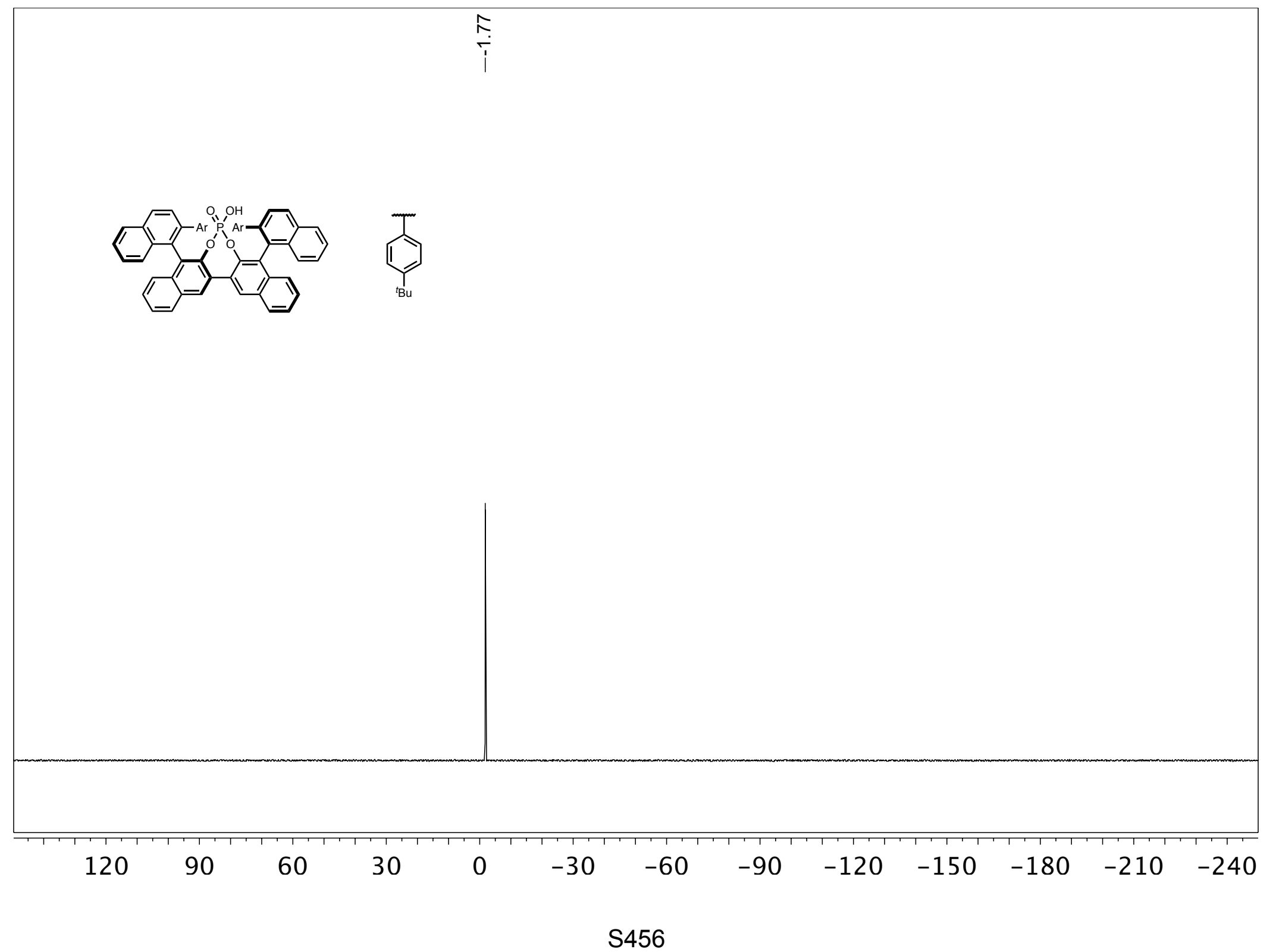



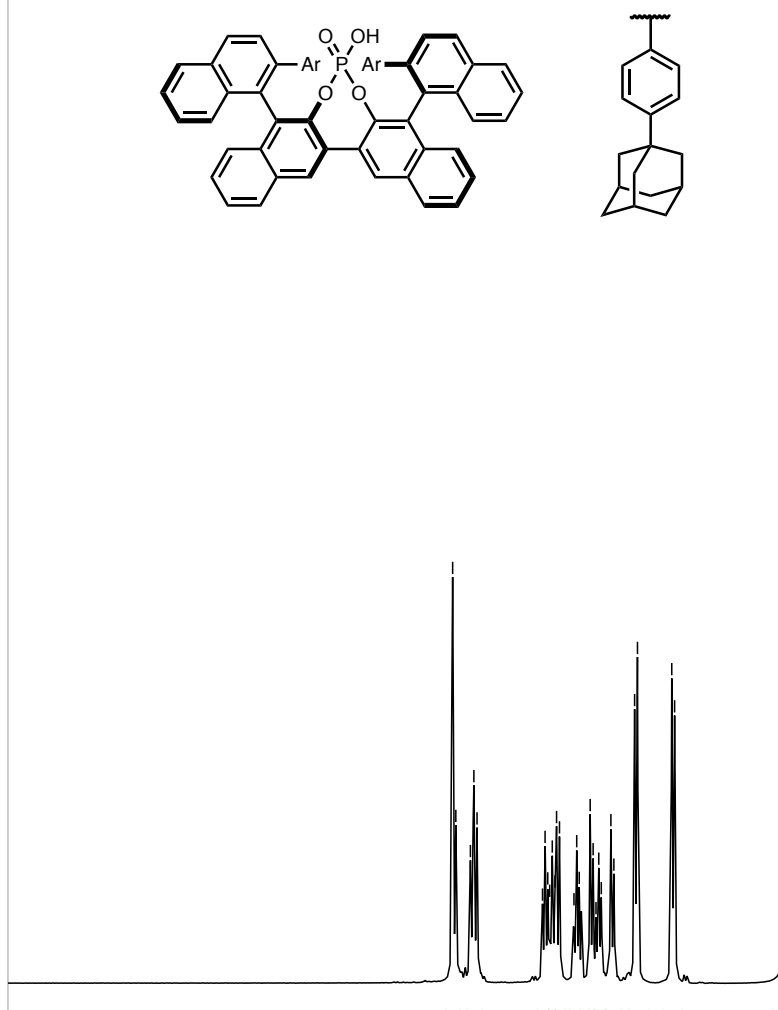

D年

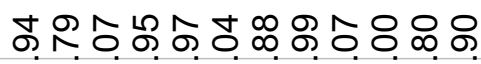

$\begin{array}{lllllllllllll}1.0 & 9.5 & 9.0 & 8.5 & 8.0 & 7.5 & 7.0 & 6.5\end{array}$

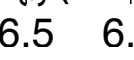



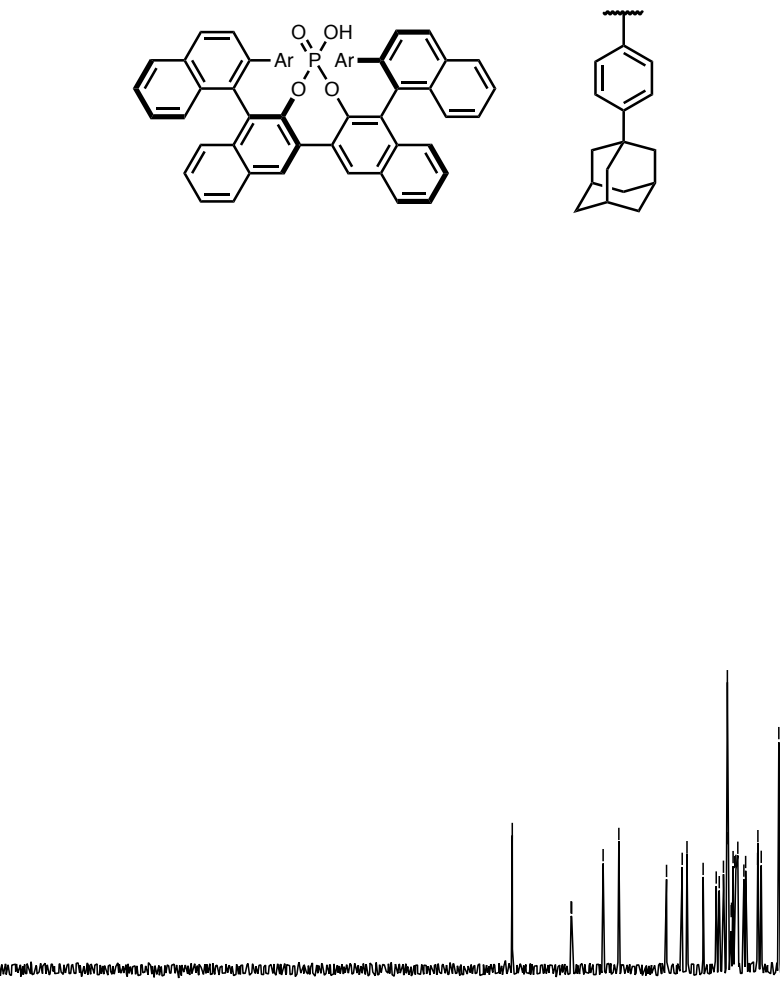


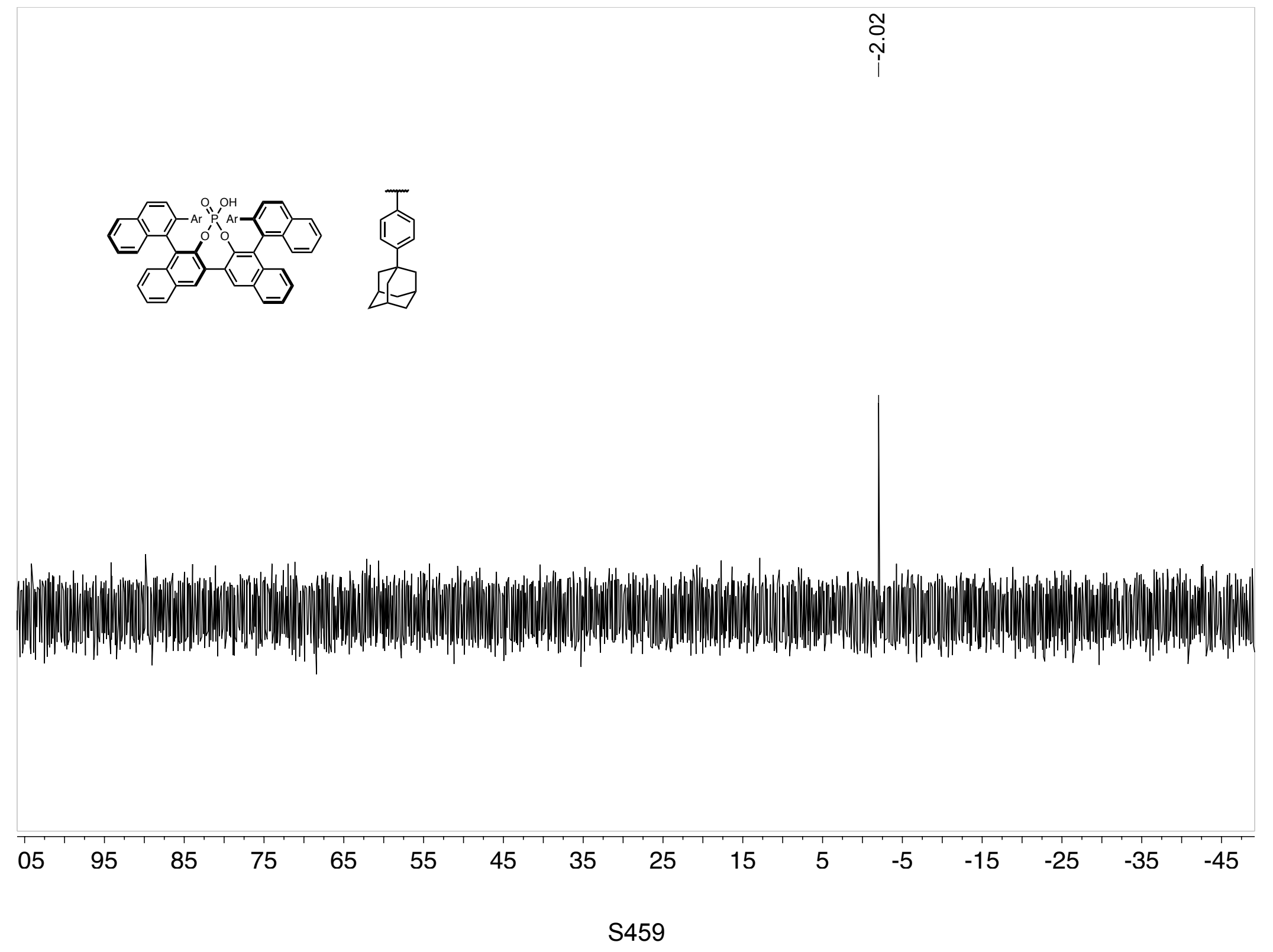




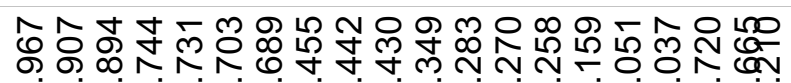

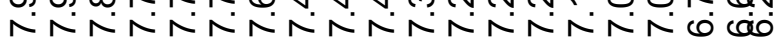
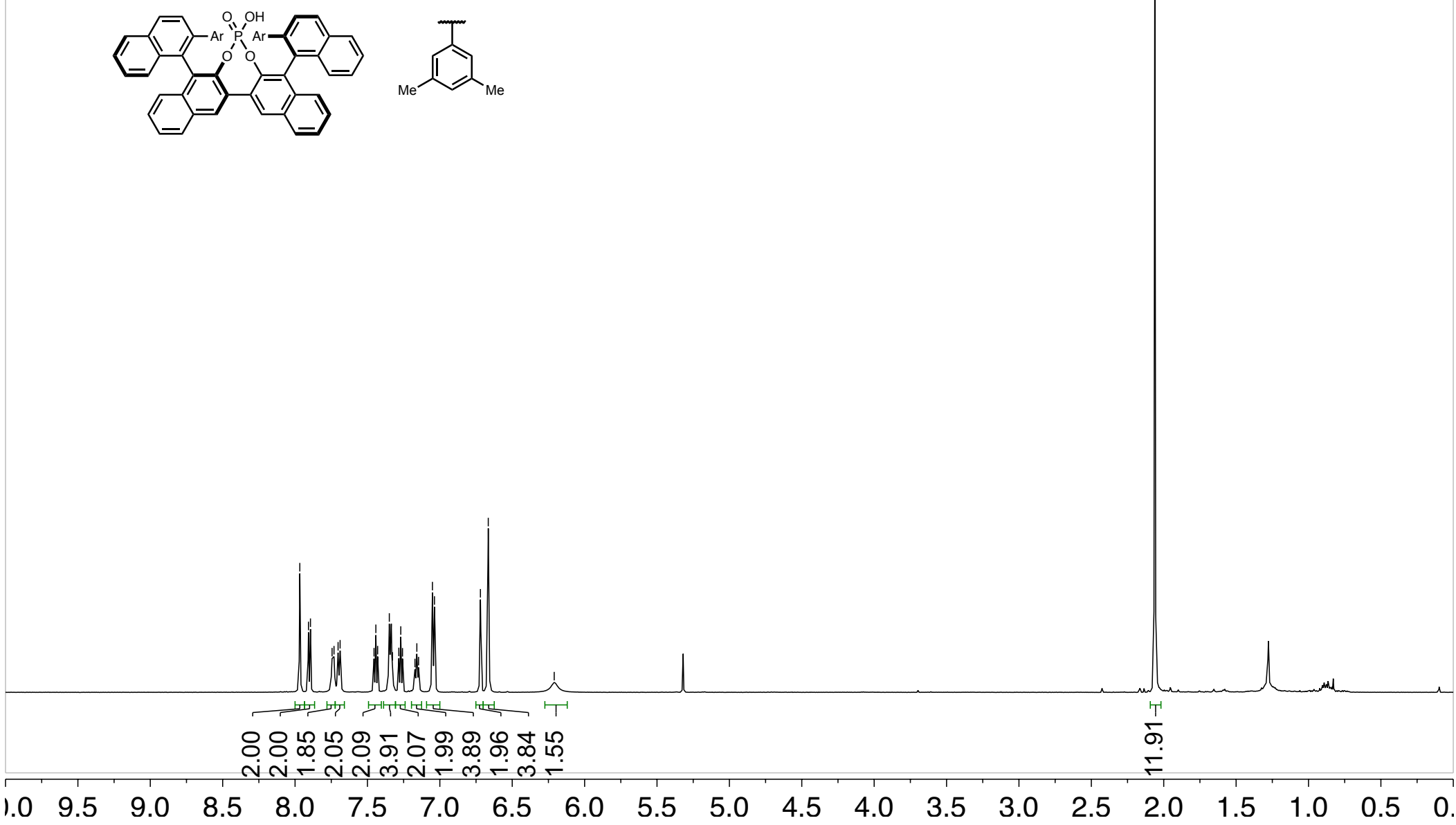
ๆ

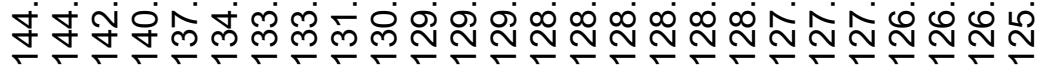
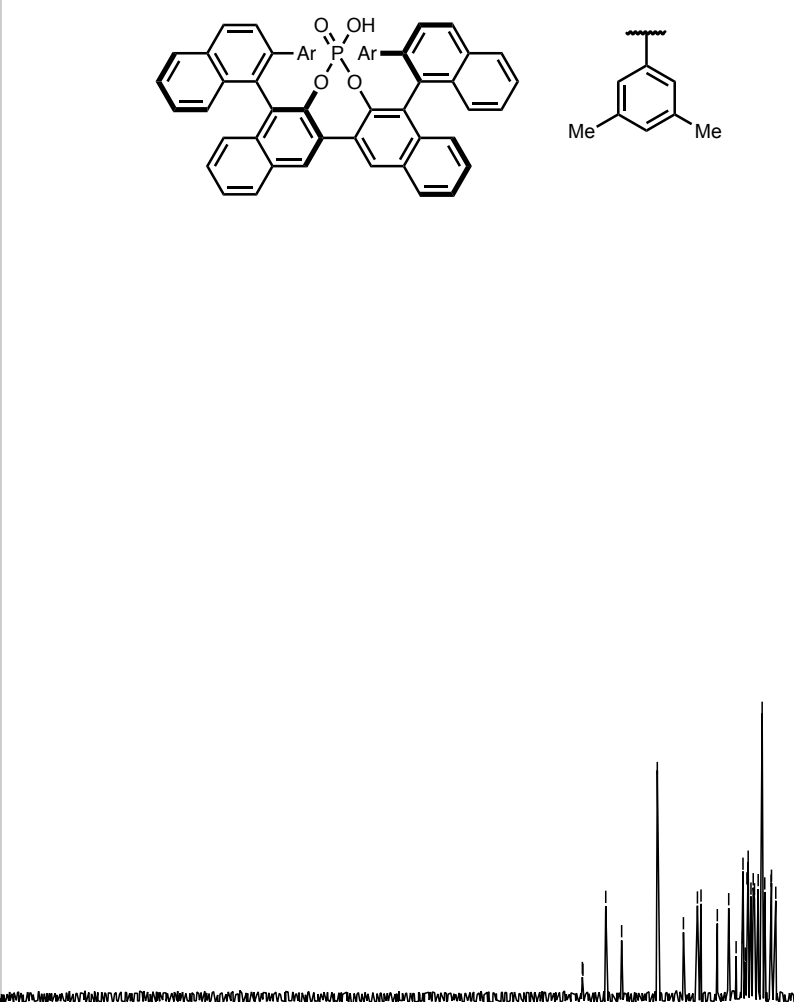


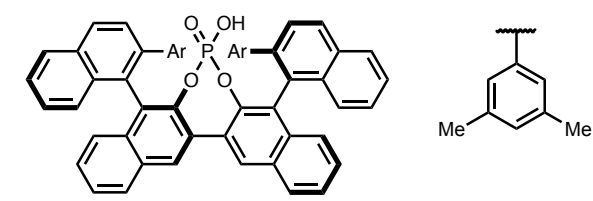

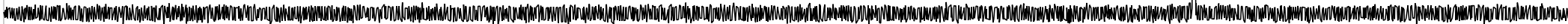

$\begin{array}{lllllllllllllllllllll}50 & 140 & 130 & 120 & 110 & 100 & 90 & 80 & 70 & 60 & 50 & 40 & 30 & 20 & 10 & 0 & -10 & -20 & -30 & -40 & -5\end{array}$




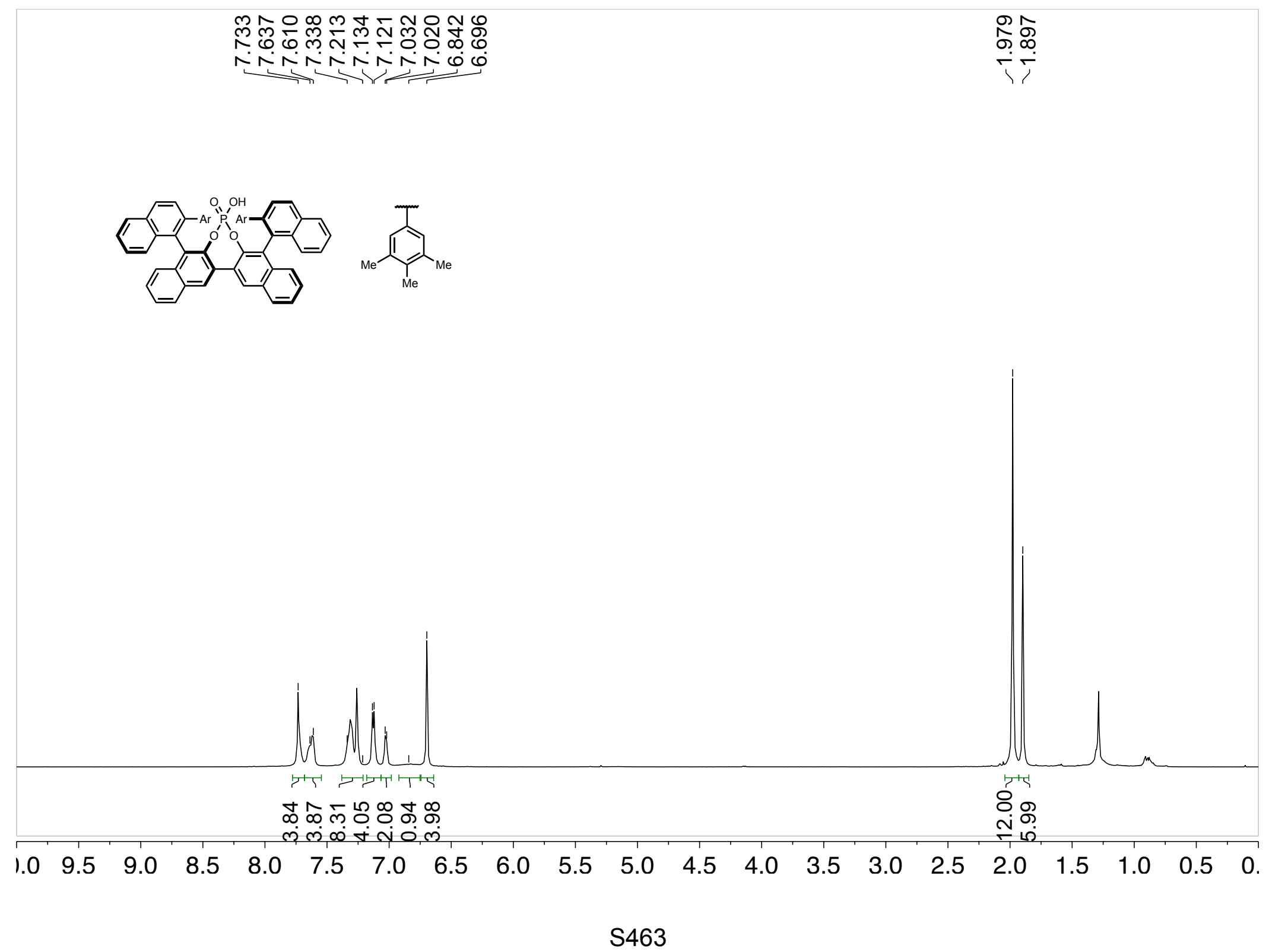




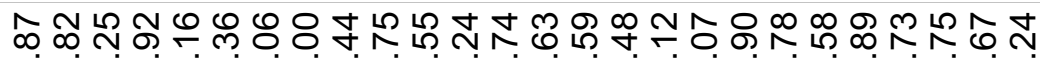

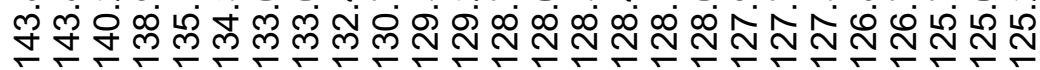
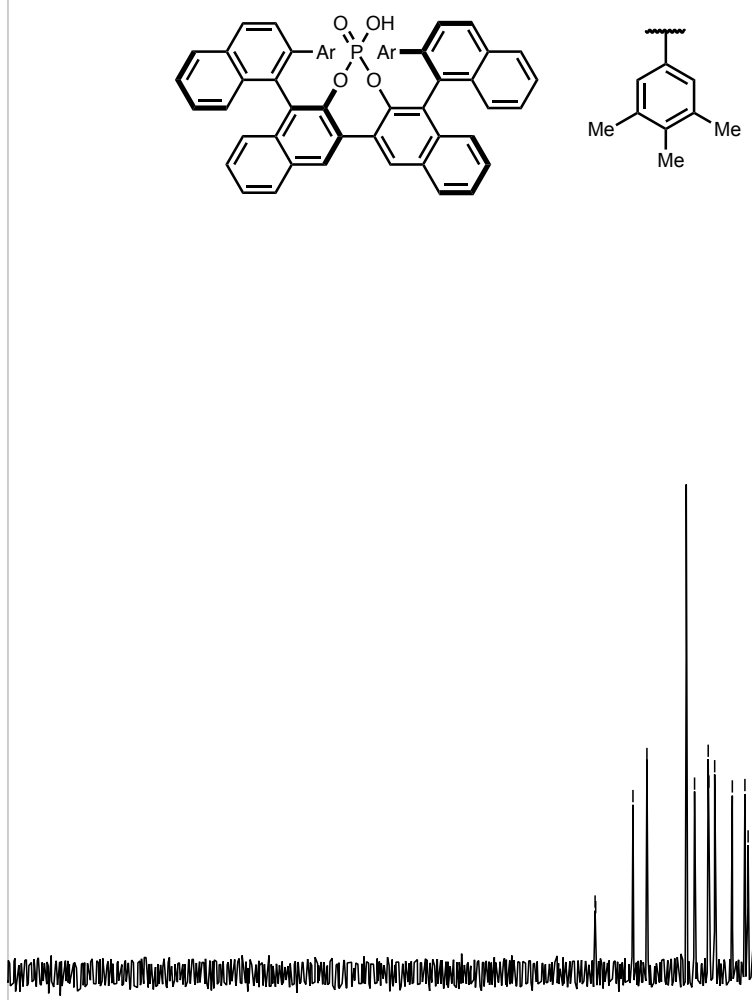

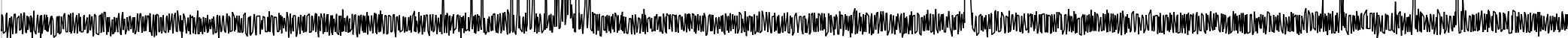

60

50




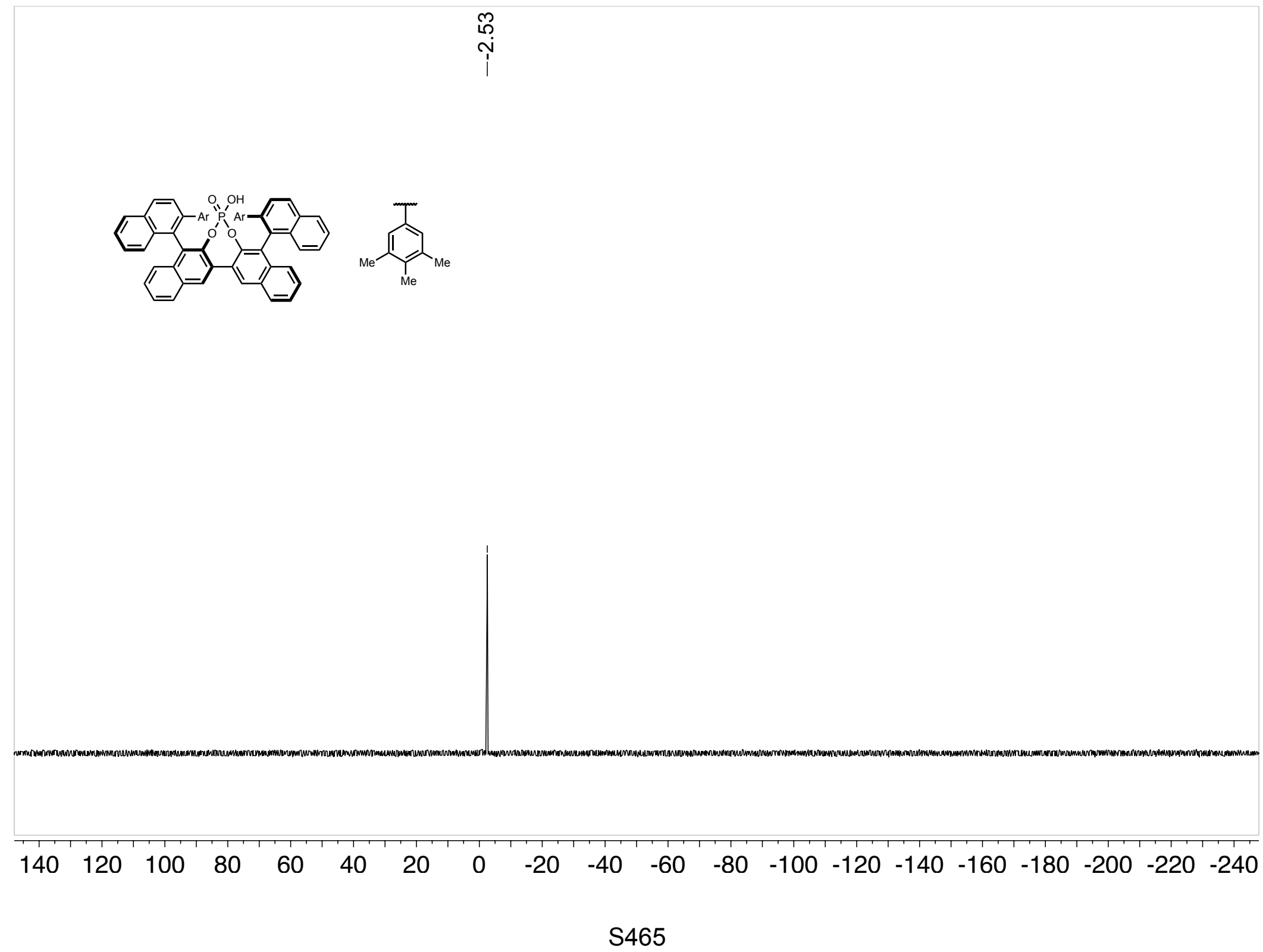




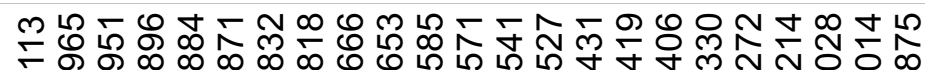

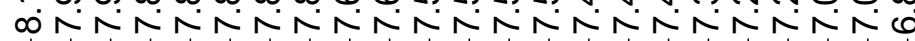
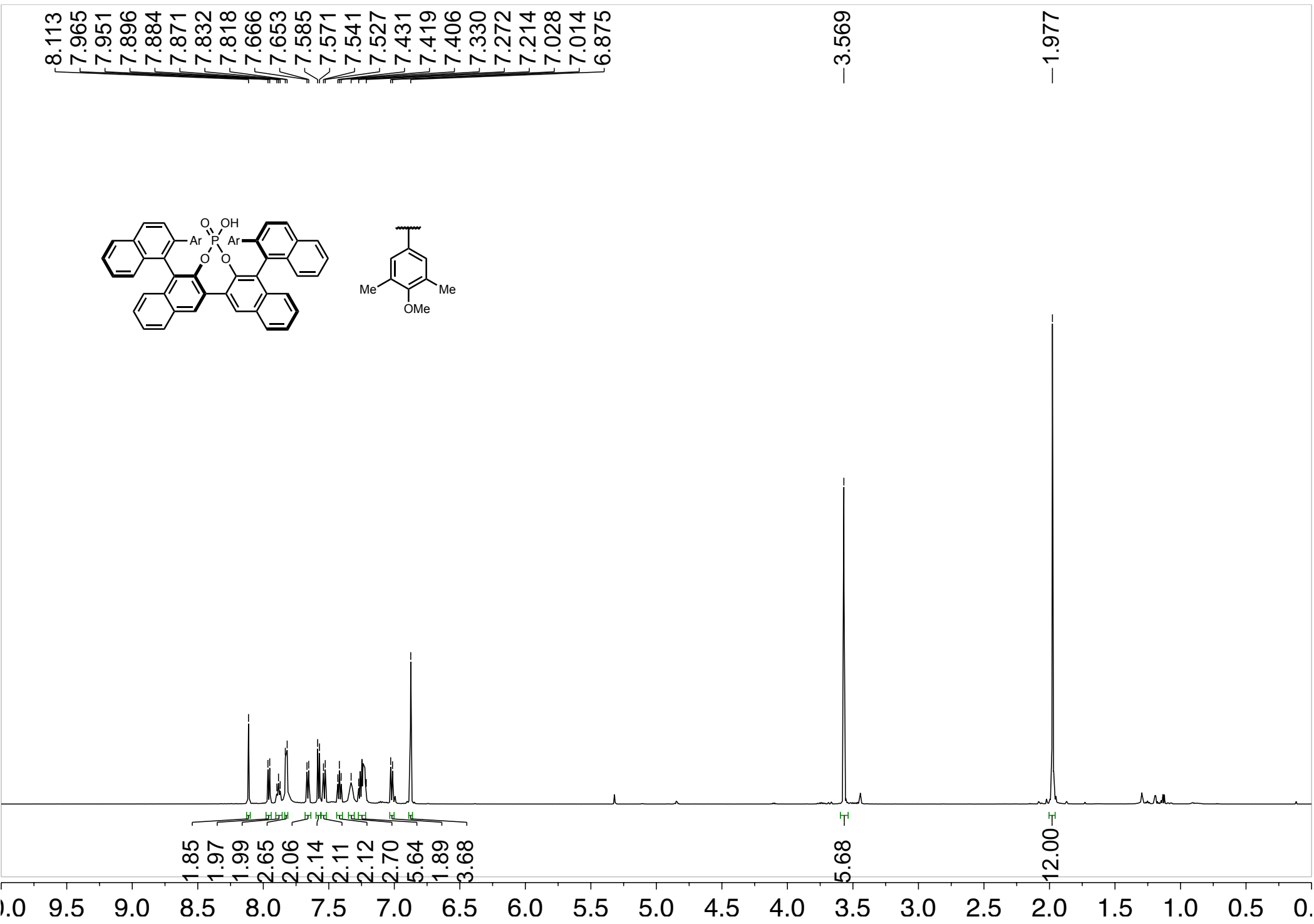


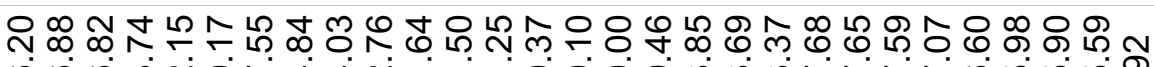

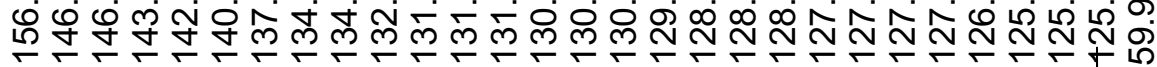

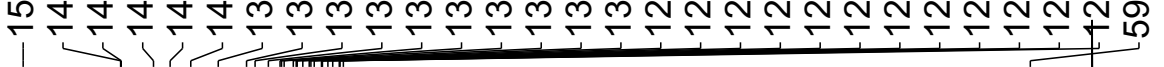
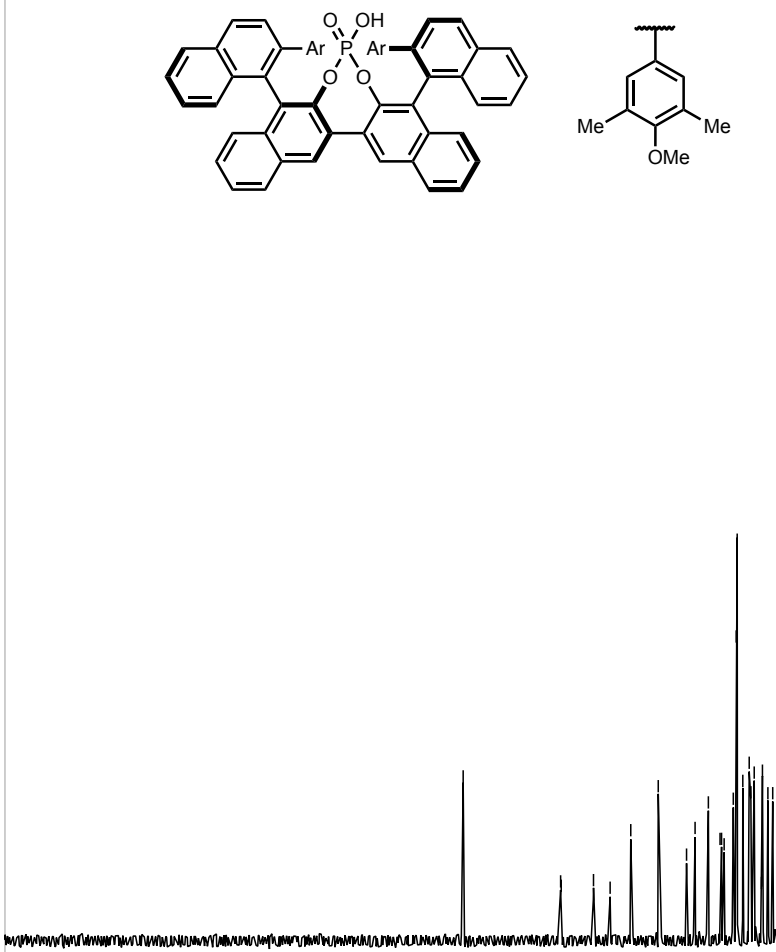


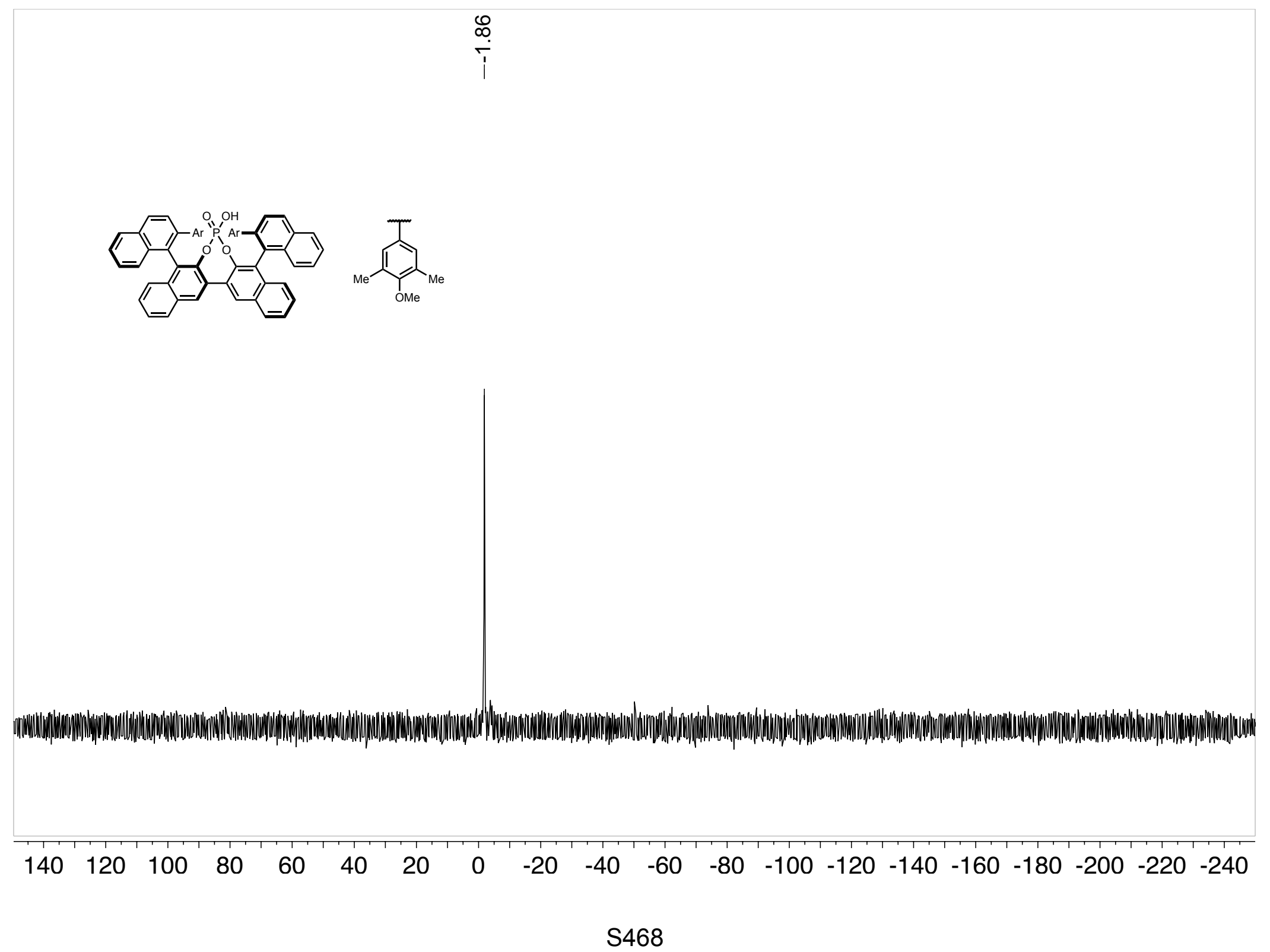




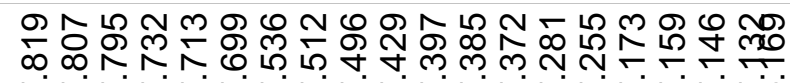

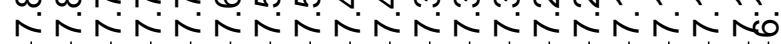
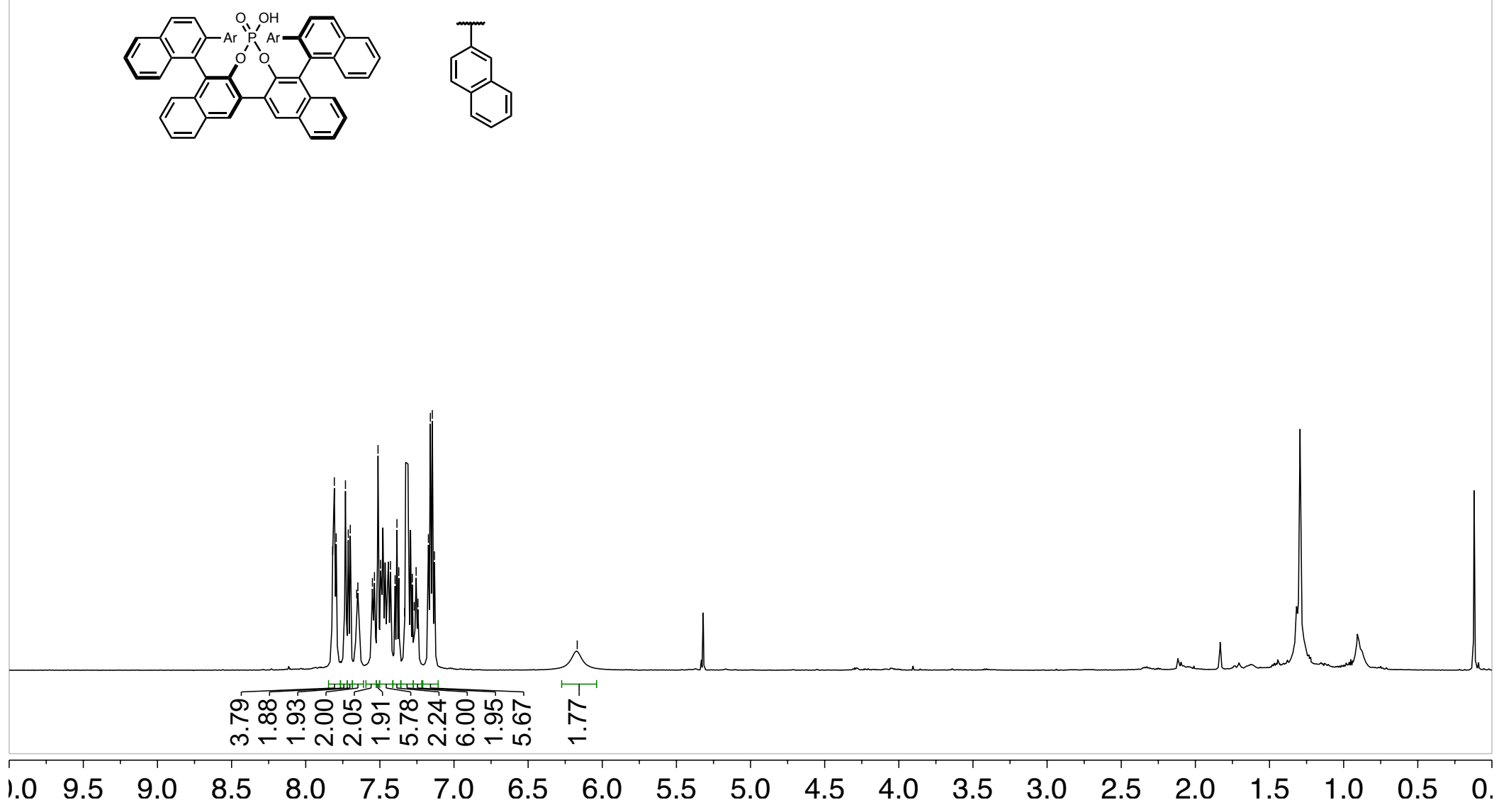


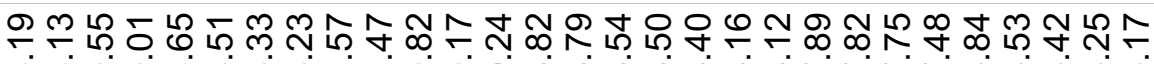

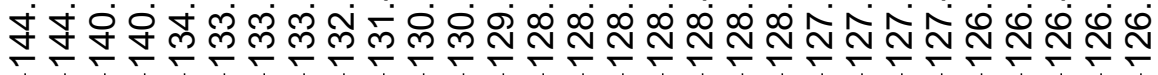
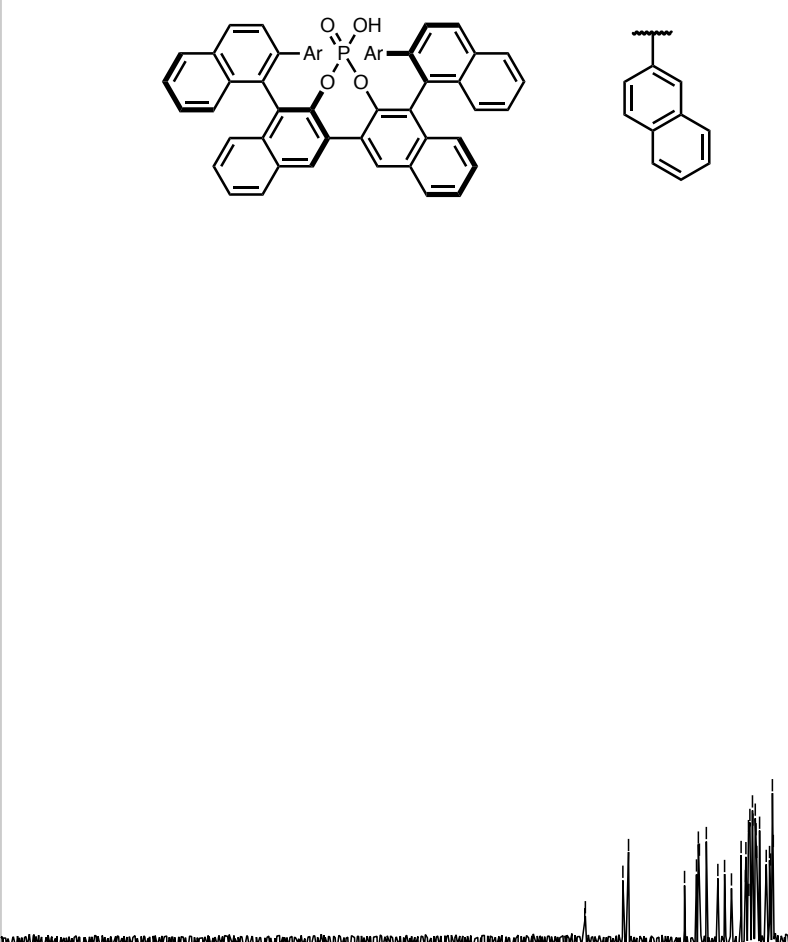


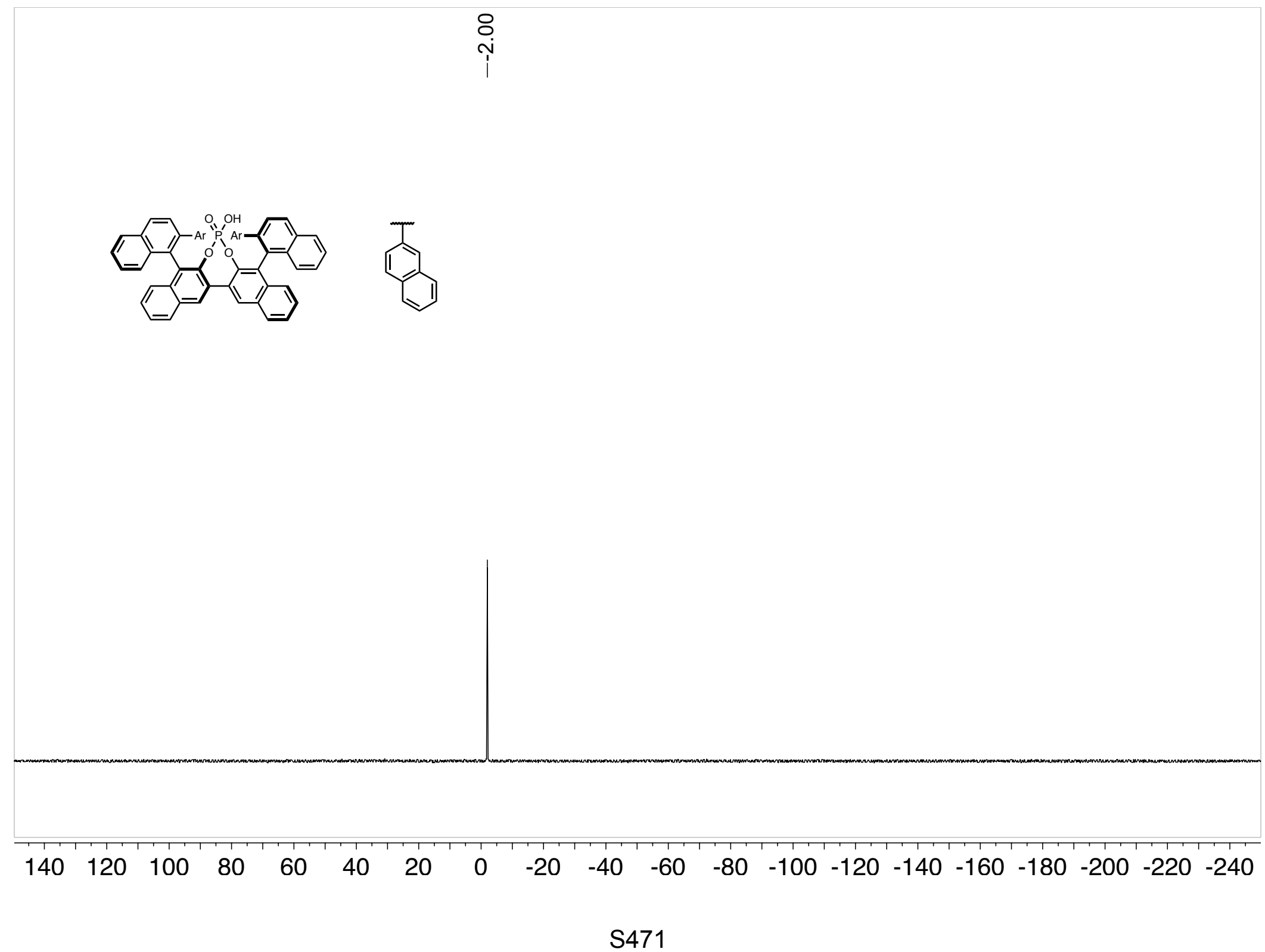




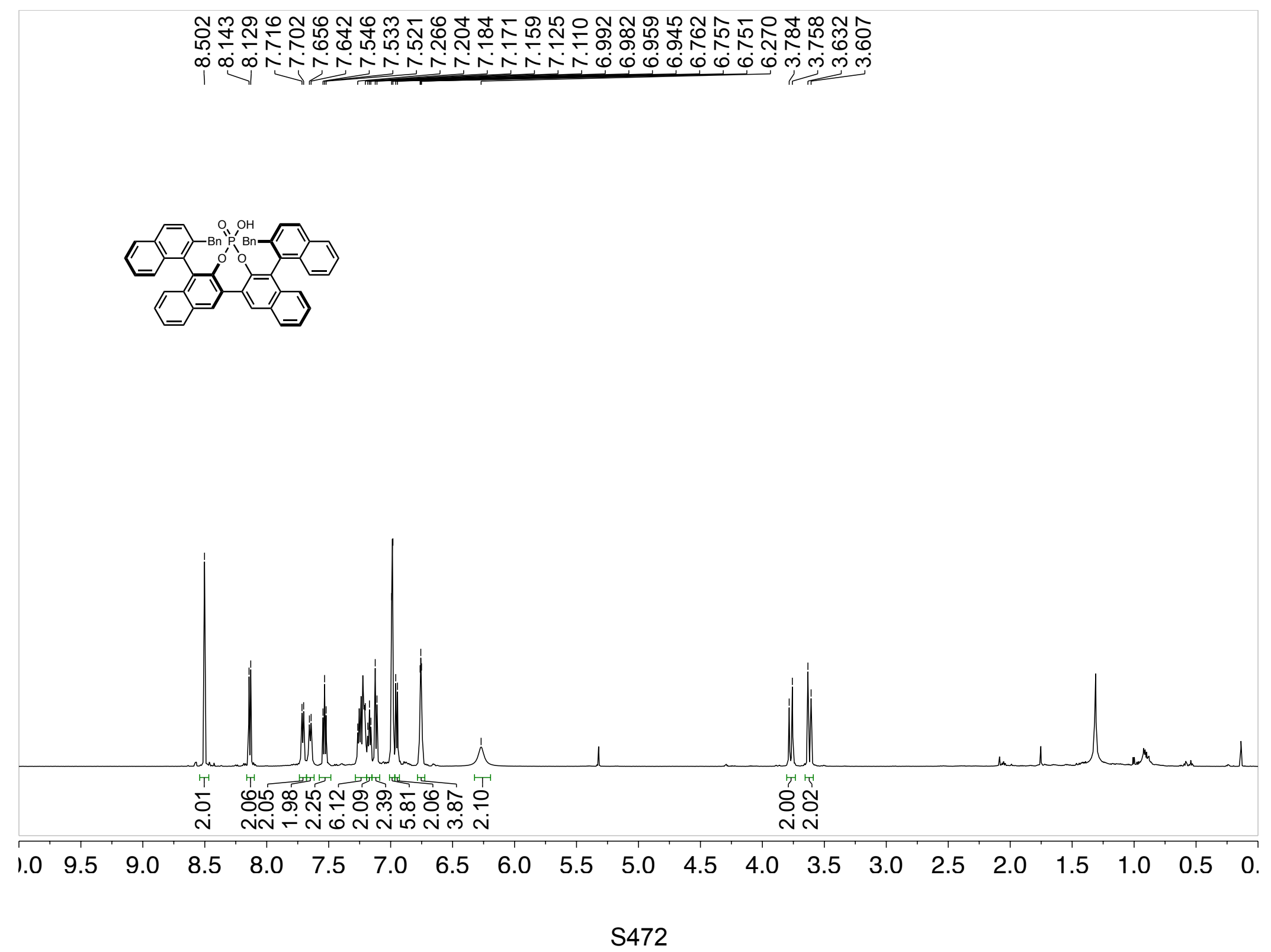




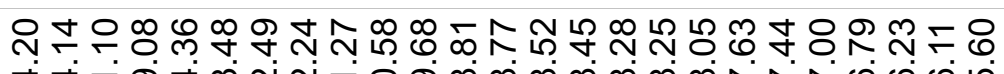

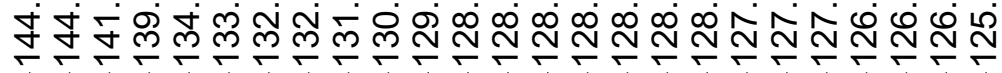
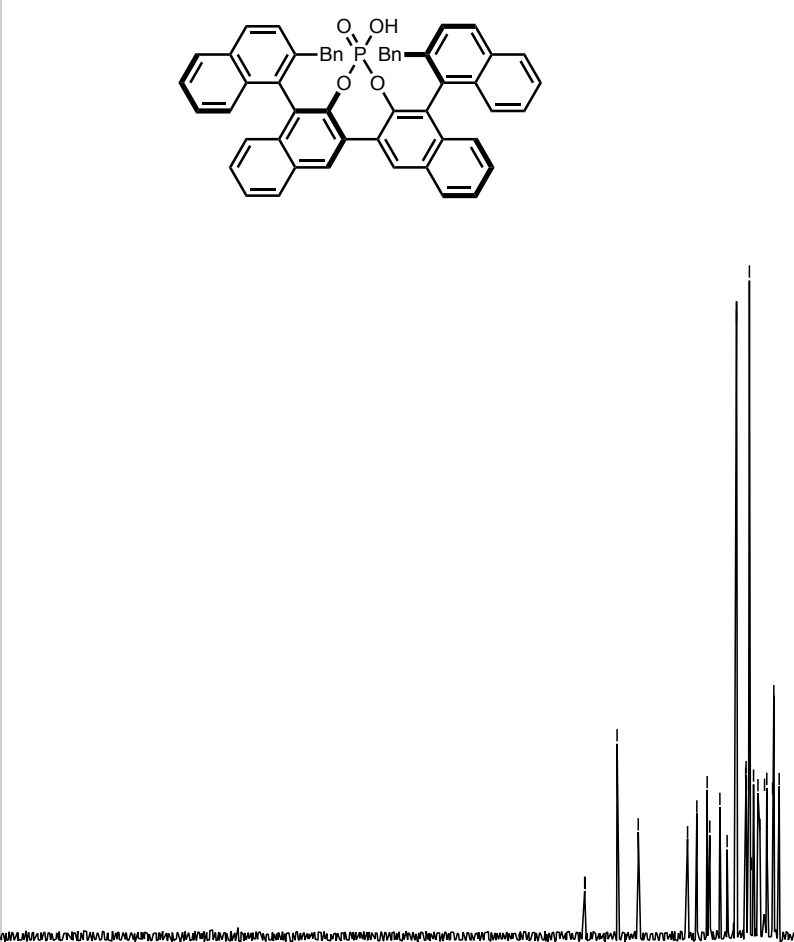


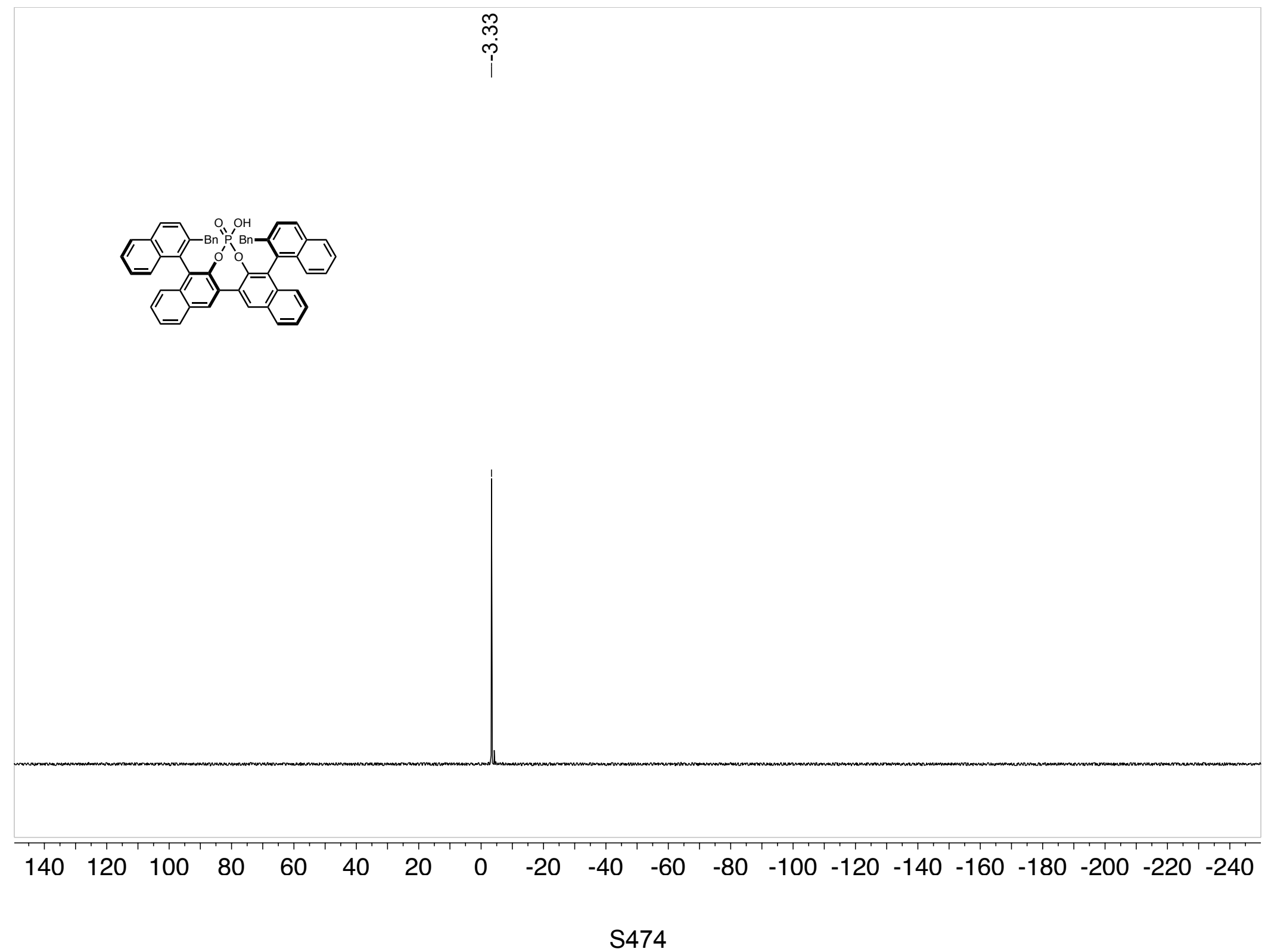




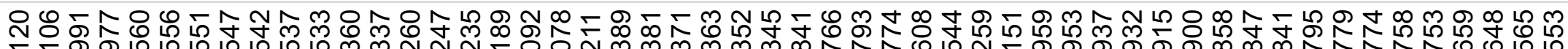

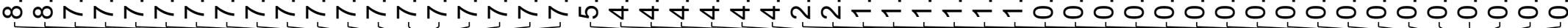
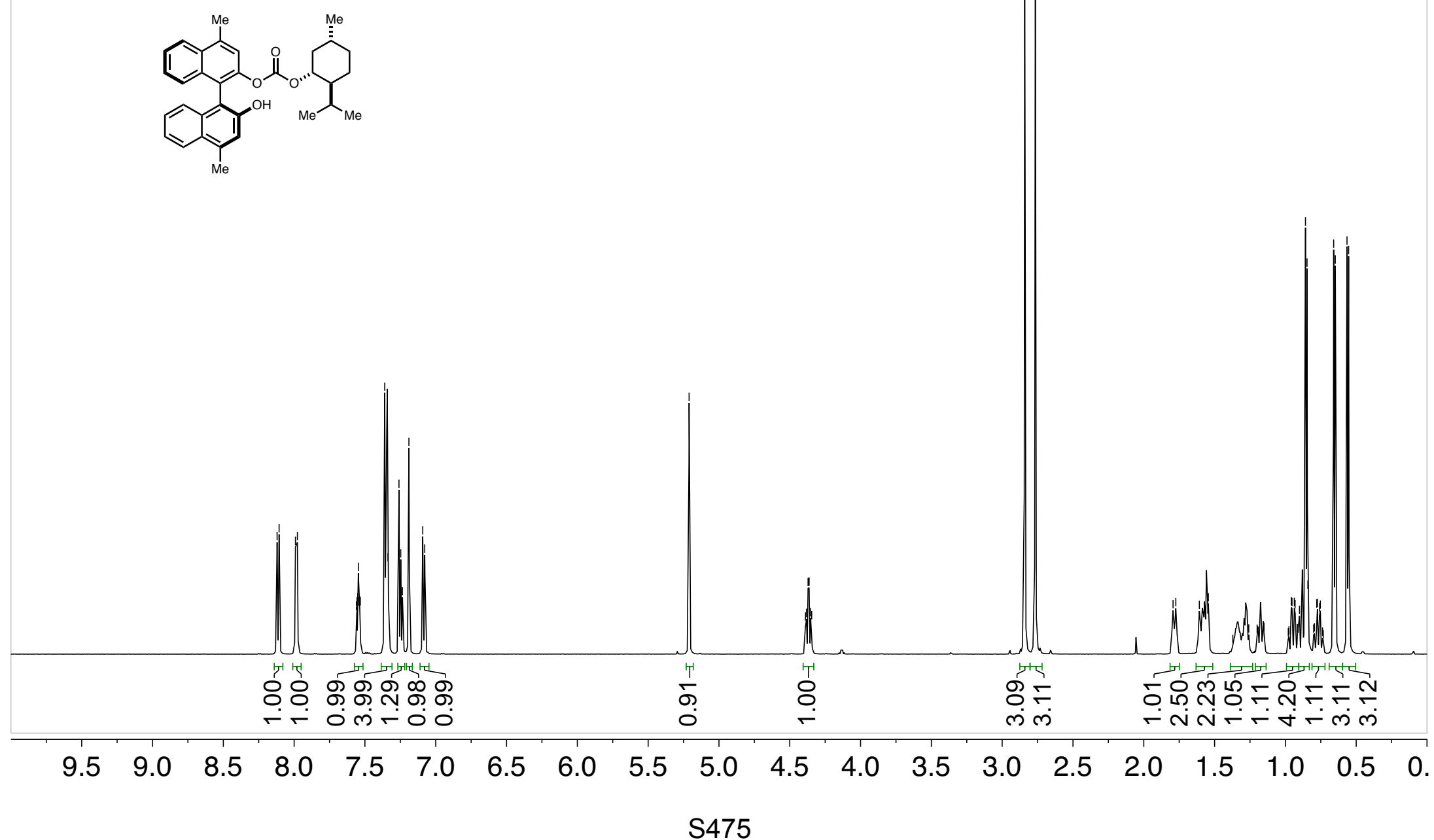

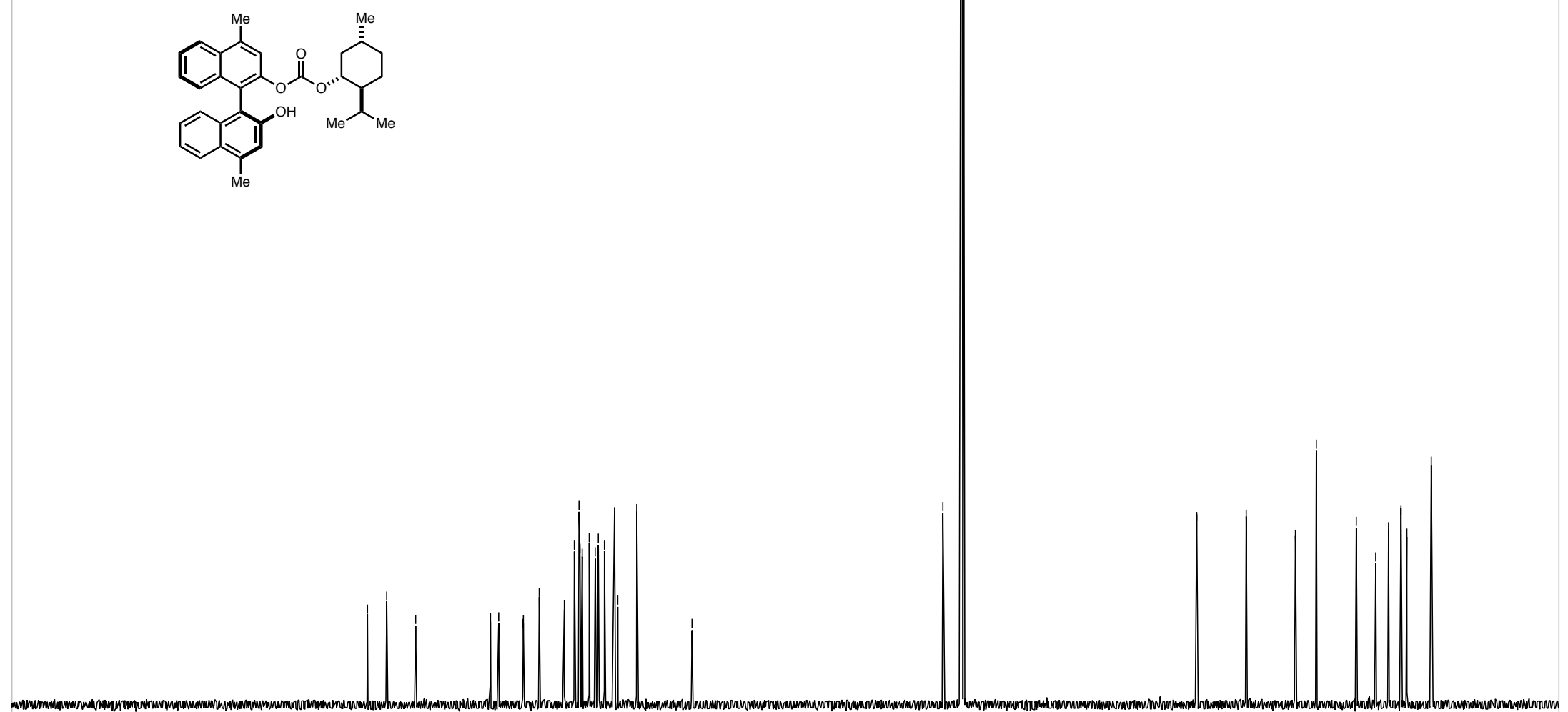

6050

40




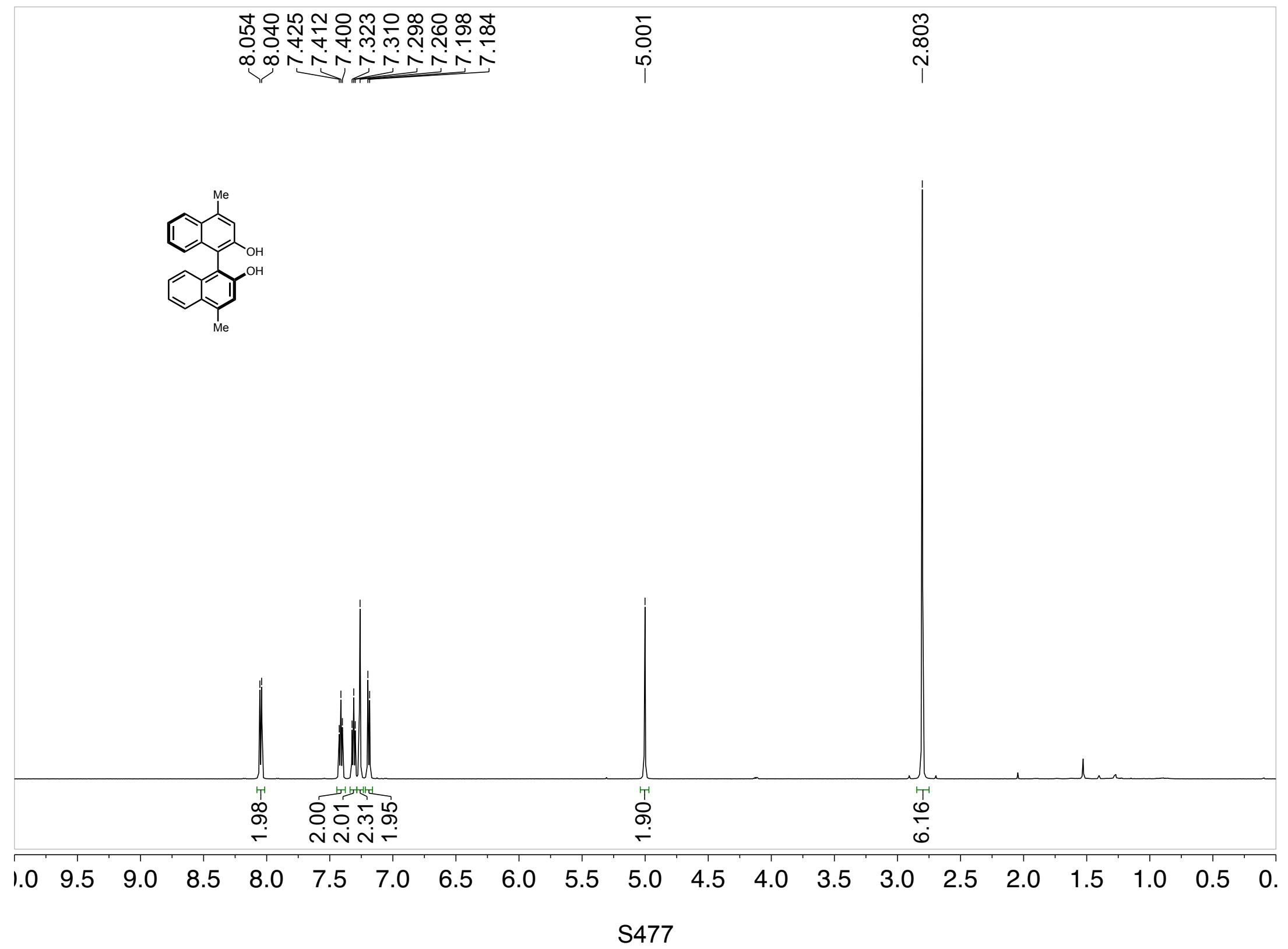




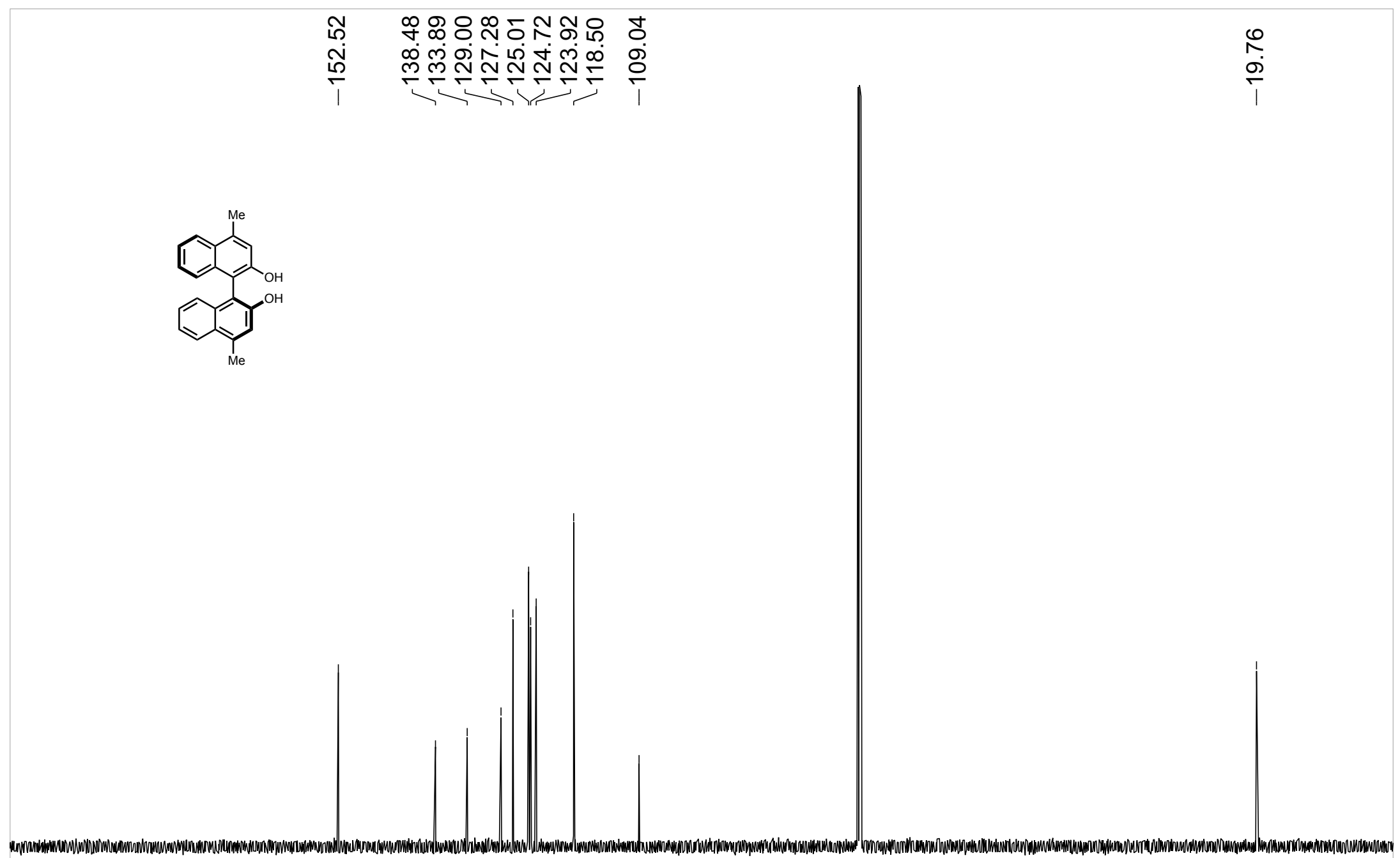

$\begin{array}{lllllllllllllllllllll}190 & 180 & 170 & 160 & 150 & 140 & 130 & 120 & 110 & 100 & 90 & 80 & 70 & 60 & 50 & 40 & 30 & 20 & 10 & C\end{array}$




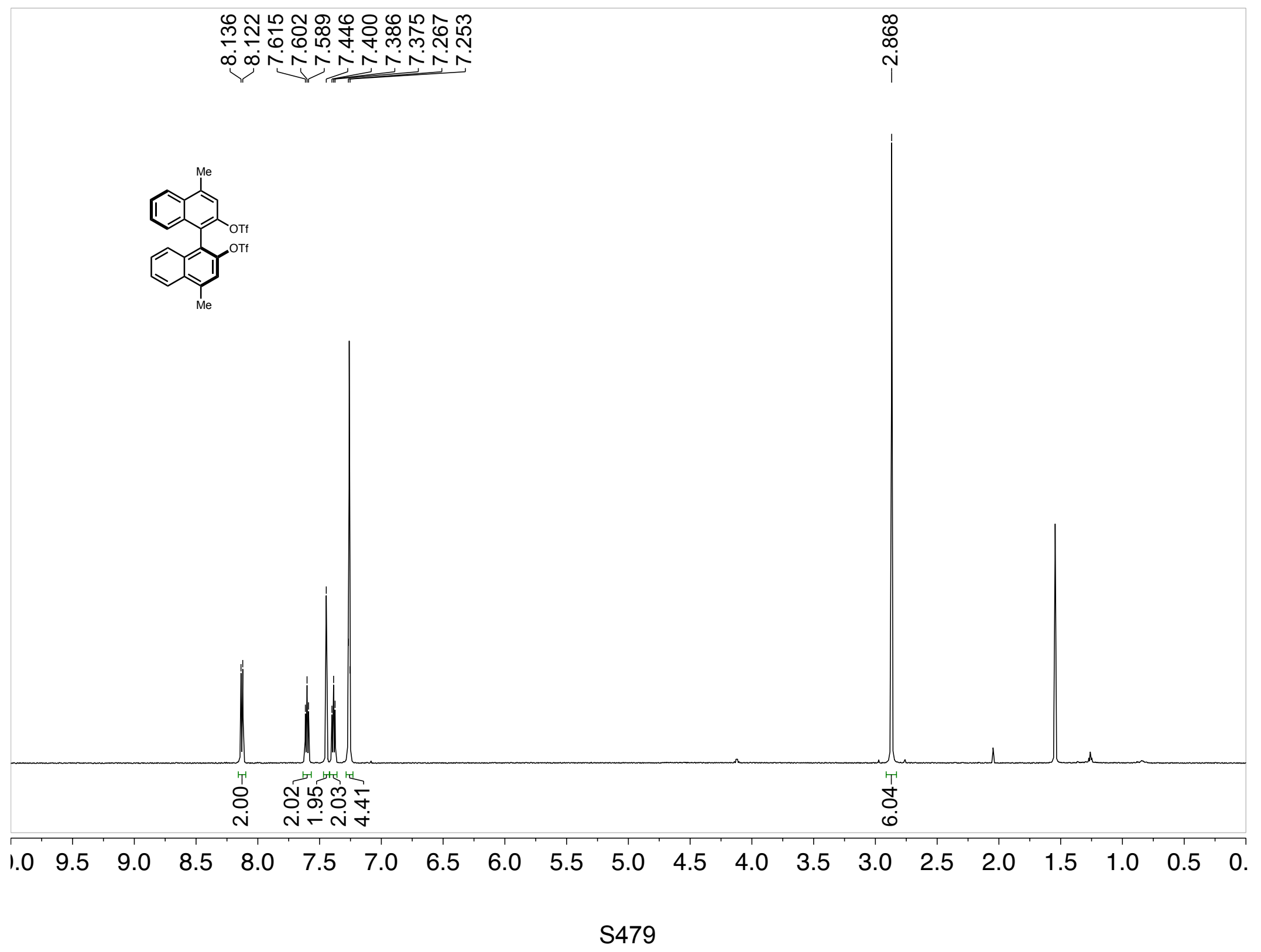




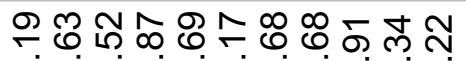

宁

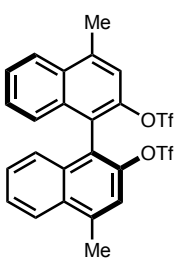

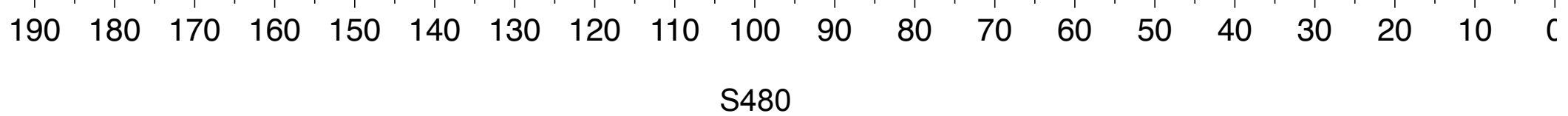




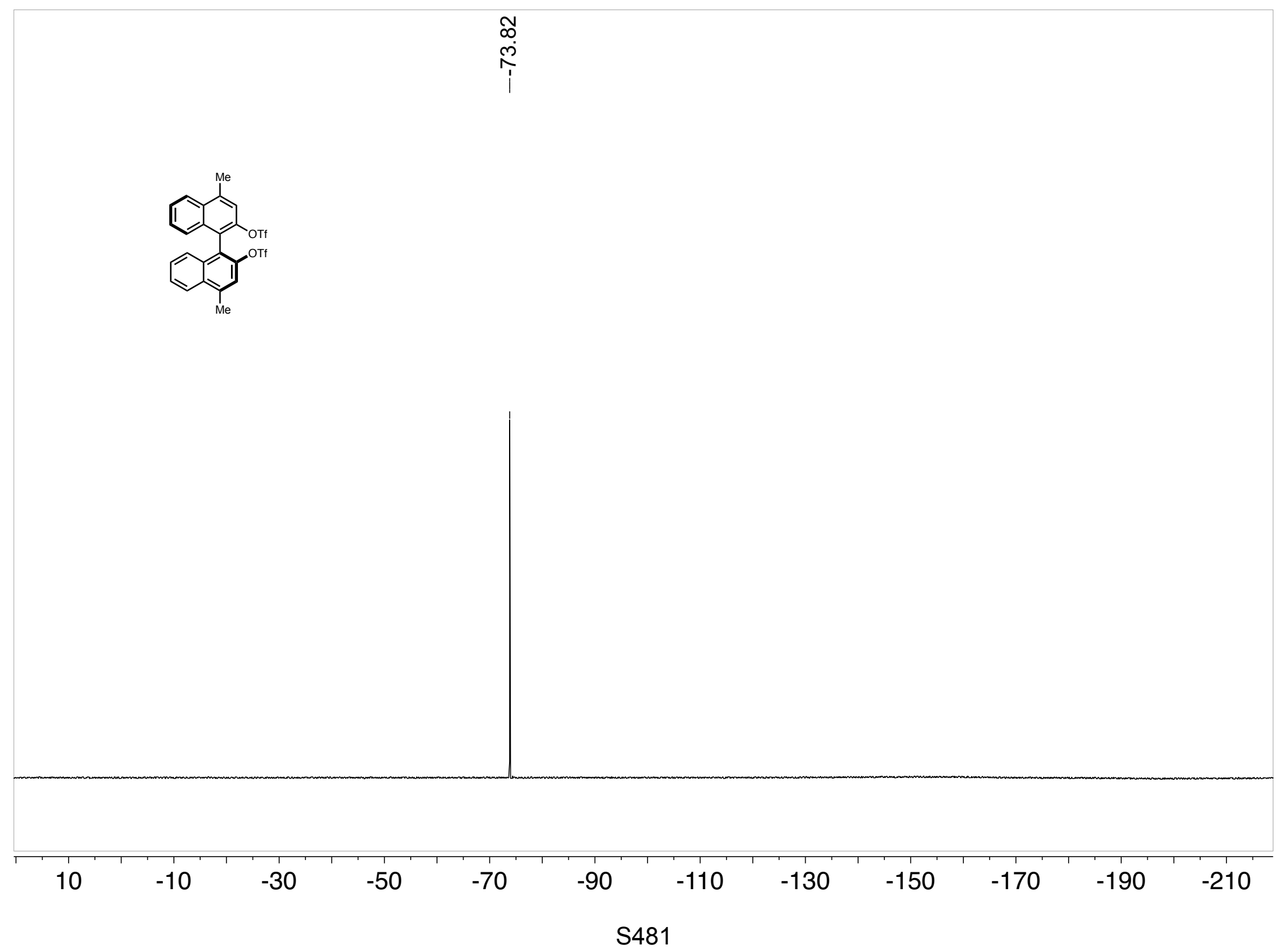


స్

$\infty \infty \infty \infty N N N$ N $\infty$ NNNNN

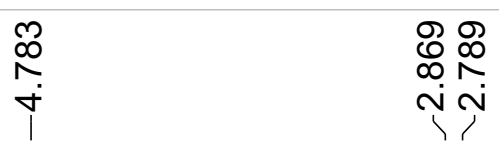

๖

N

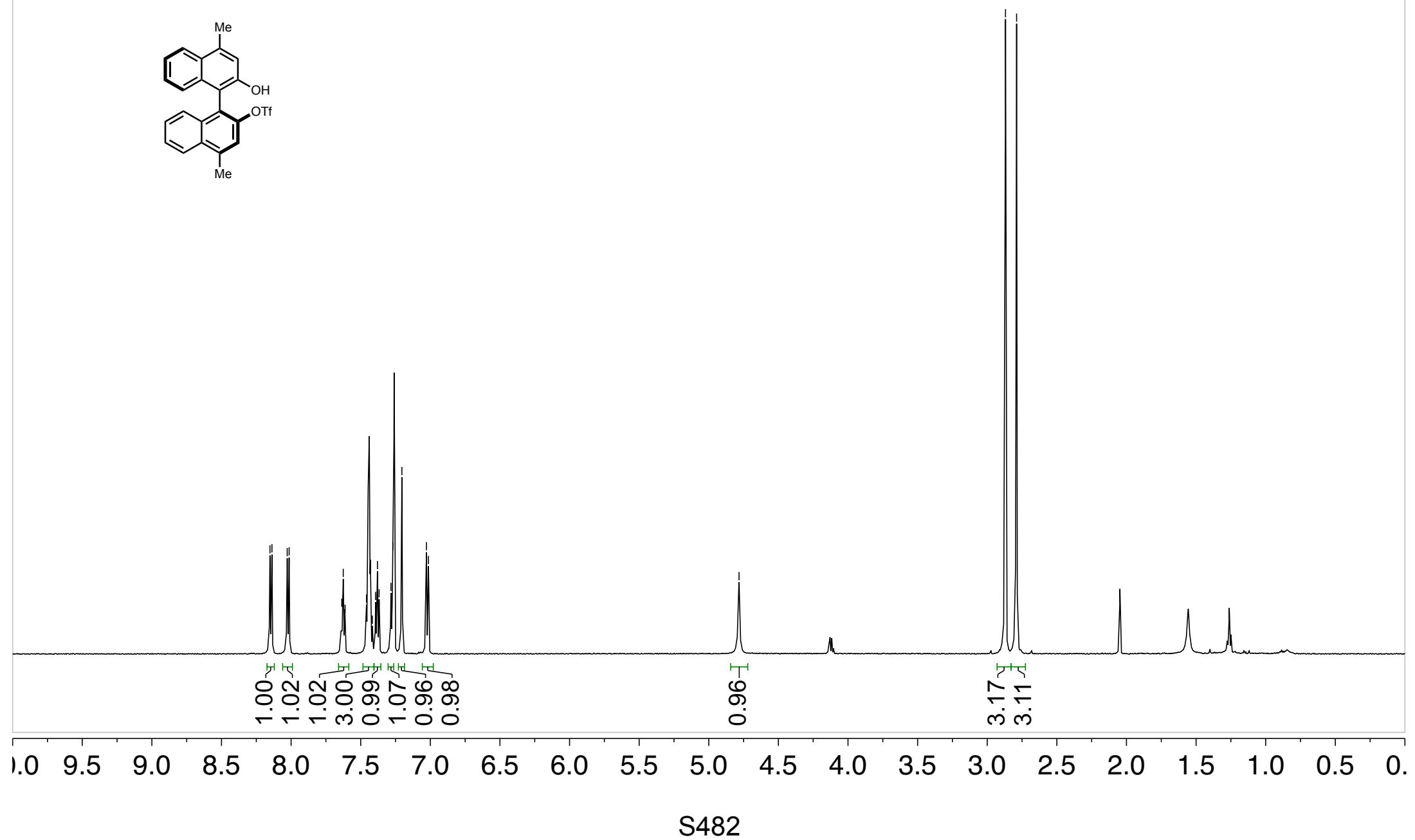



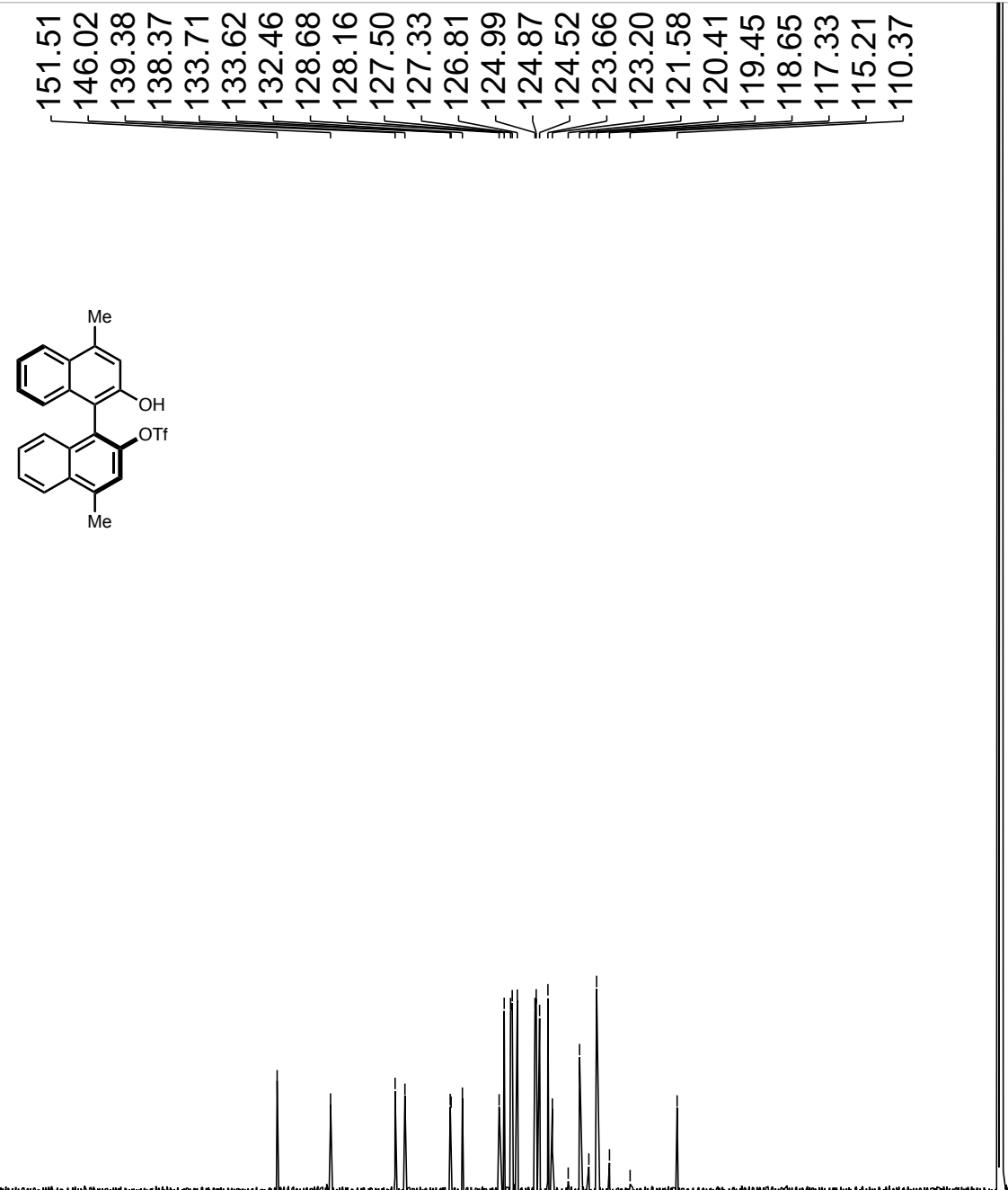

50



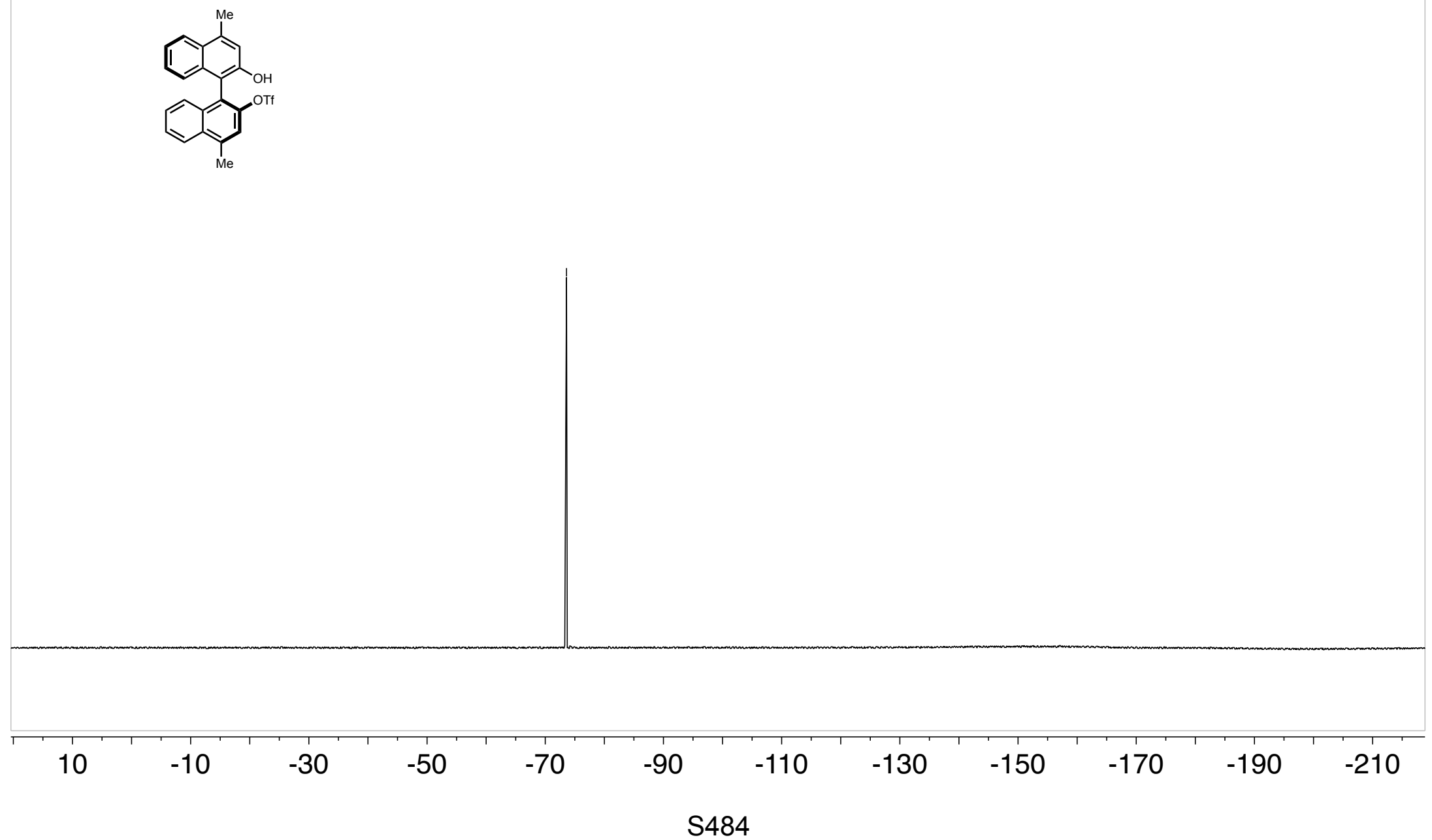

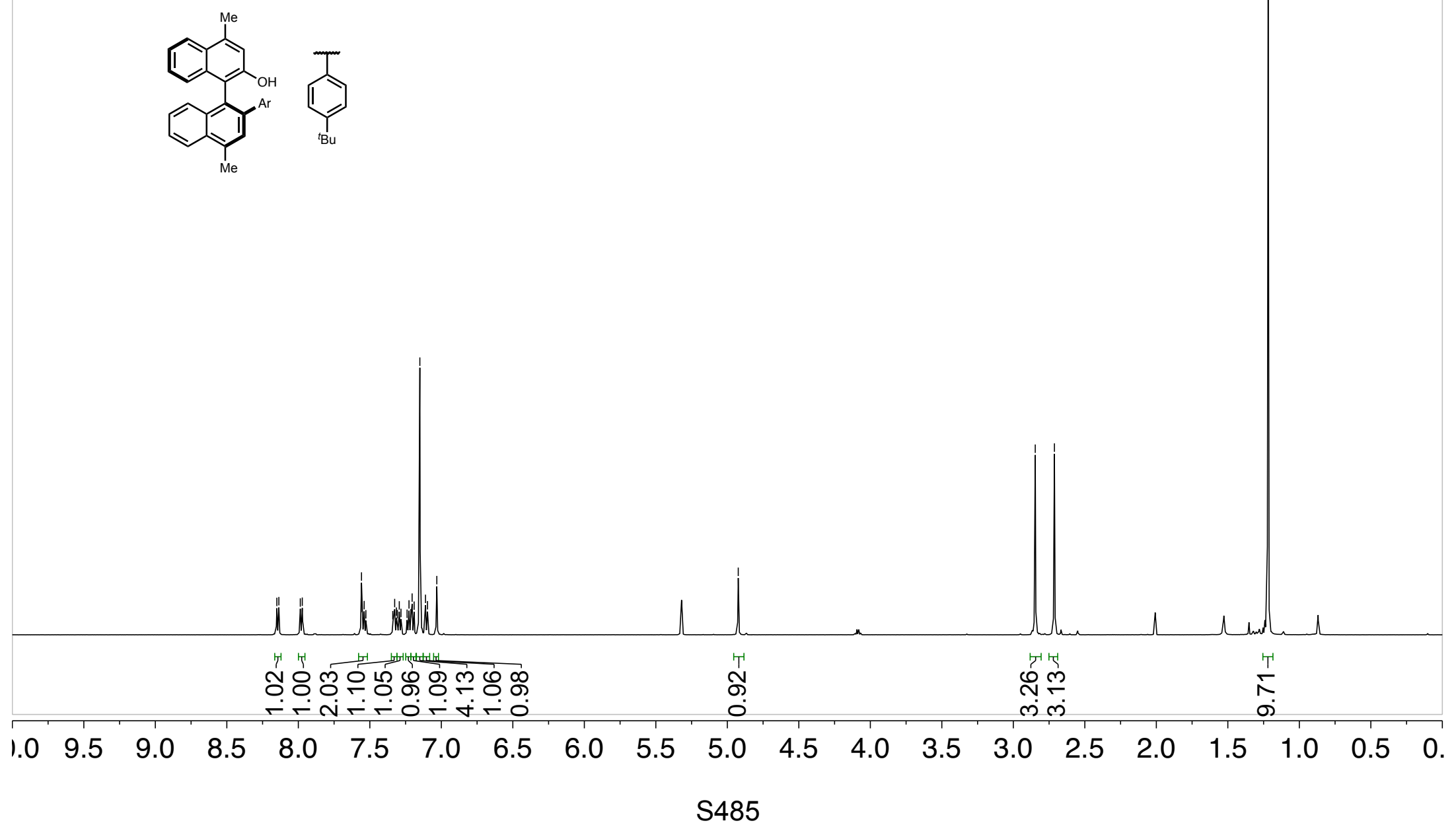


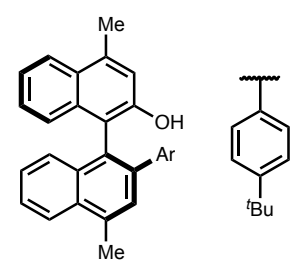

$$
\begin{array}{llllllllll}
190 & 180 & 170 & 160 & 150 & 140 & 130 & 120 & 110 & 100
\end{array}
$$



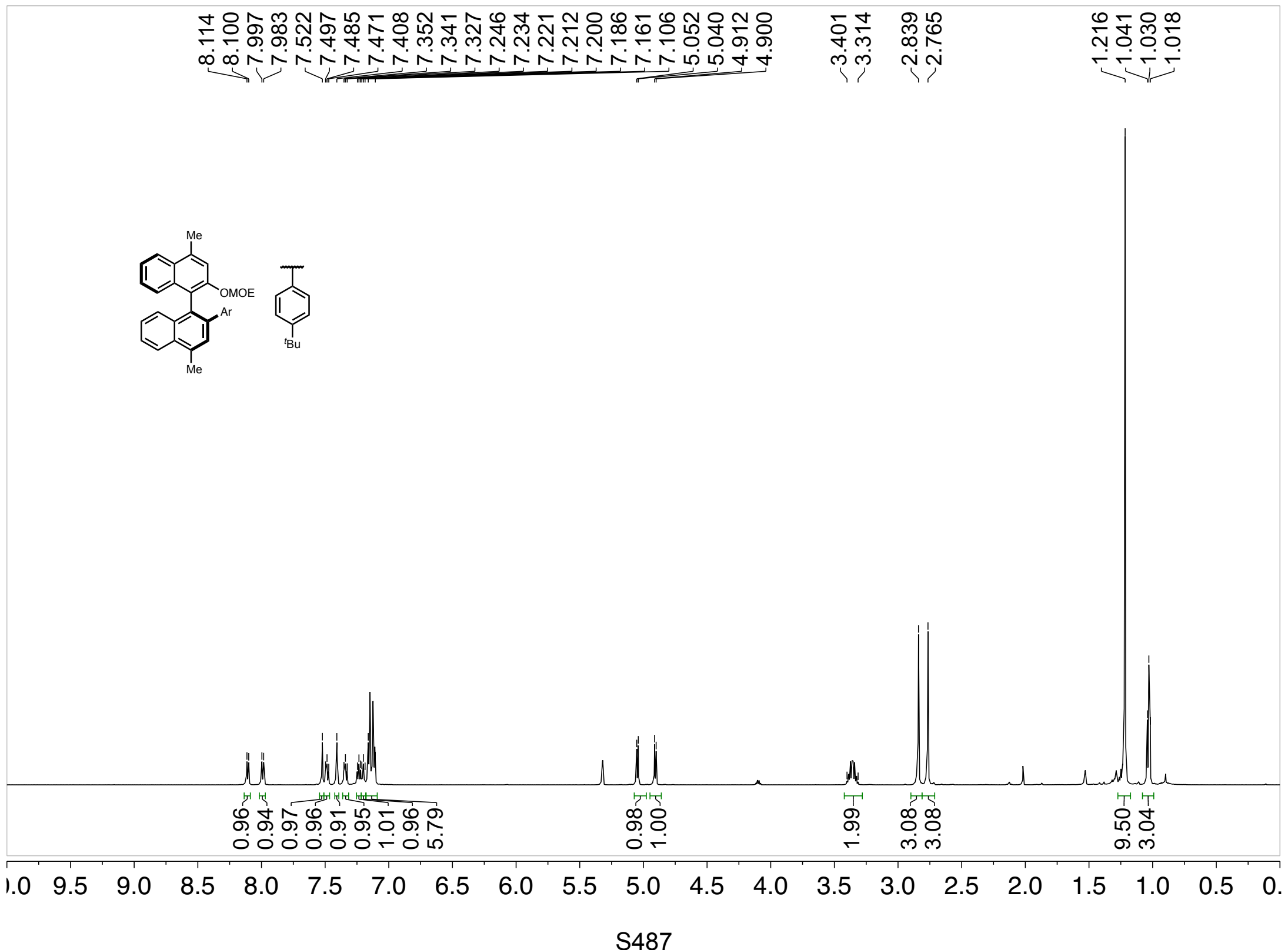


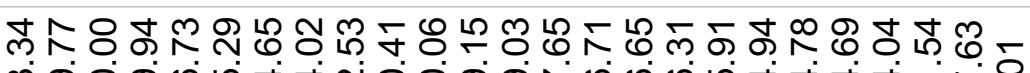

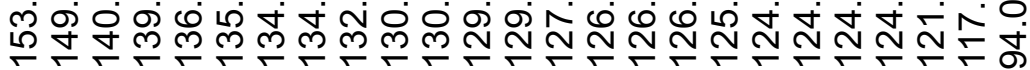

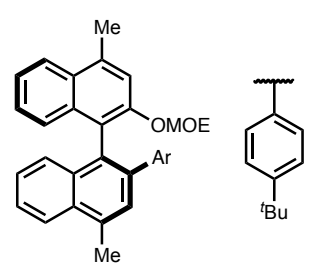

$\stackrel{\infty}{+}$

छ்

$\infty \infty$

लें

సิธ

กิ

1 . 1 Nं 


\begin{tabular}{|c|c|c|c|}
\hline 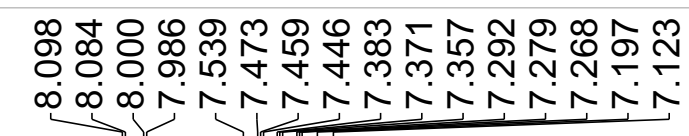 & $\begin{array}{l}\stackrel{0}{+} \\
\infty \\
\dot{+} \\
j\end{array}$ & $\underset{\substack{\infty \\
\infty}}{\substack{N \\
j}}$ & 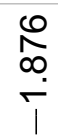 \\
\hline
\end{tabular}
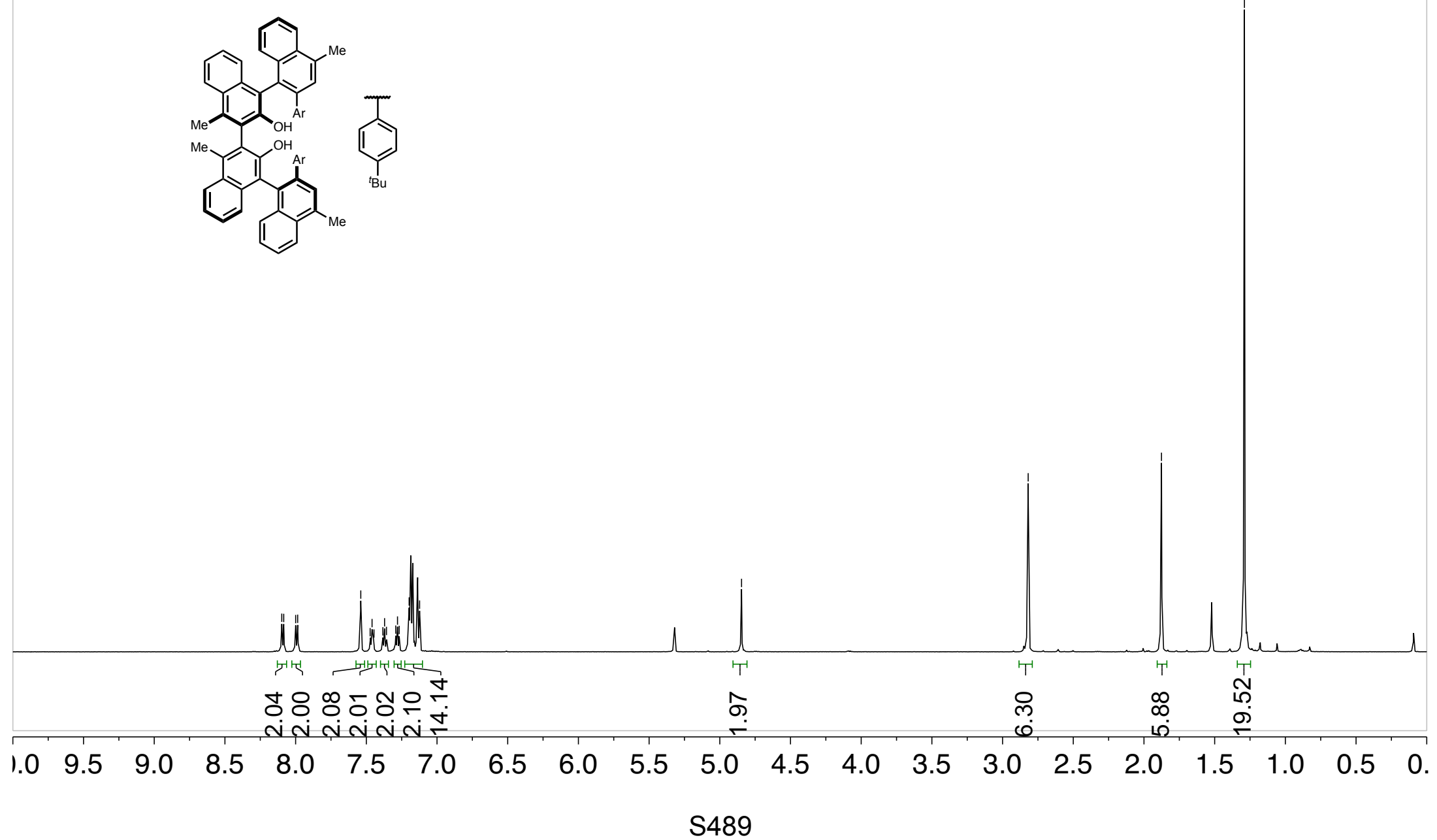


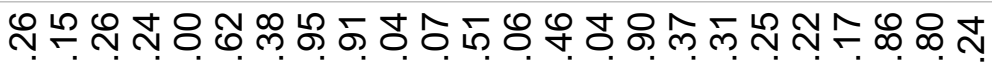

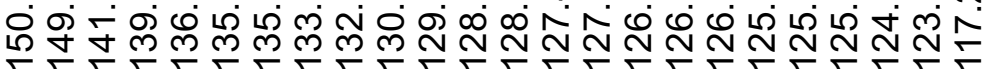

৪)

8 N

㐫穴

i,

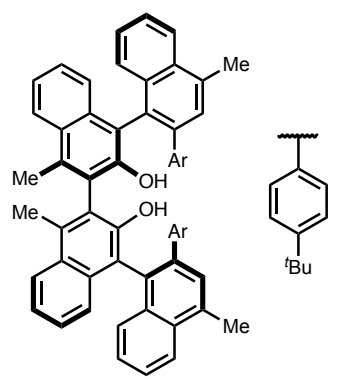

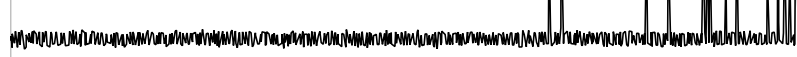




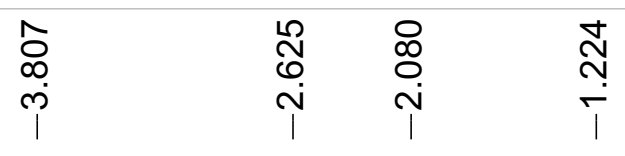
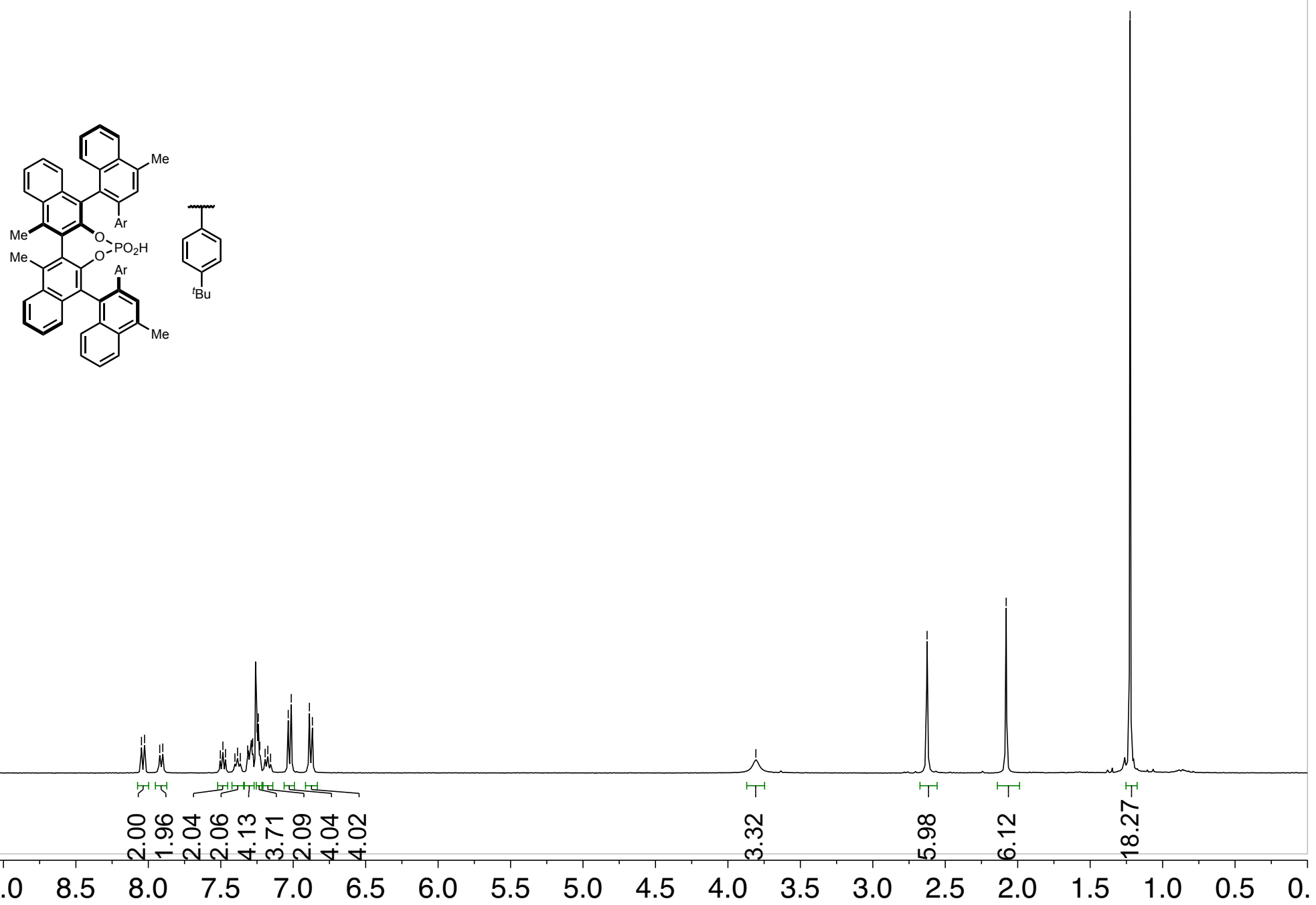

$\begin{array}{lllllllllll}1.0 & 9.5 & 9.0 & 8.5 & 8.0 & 7.5 & 7.0 & 6.5 & 6.0 & 5.5 & 5.0\end{array}$ 


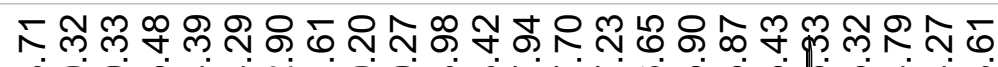

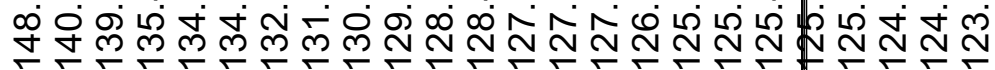
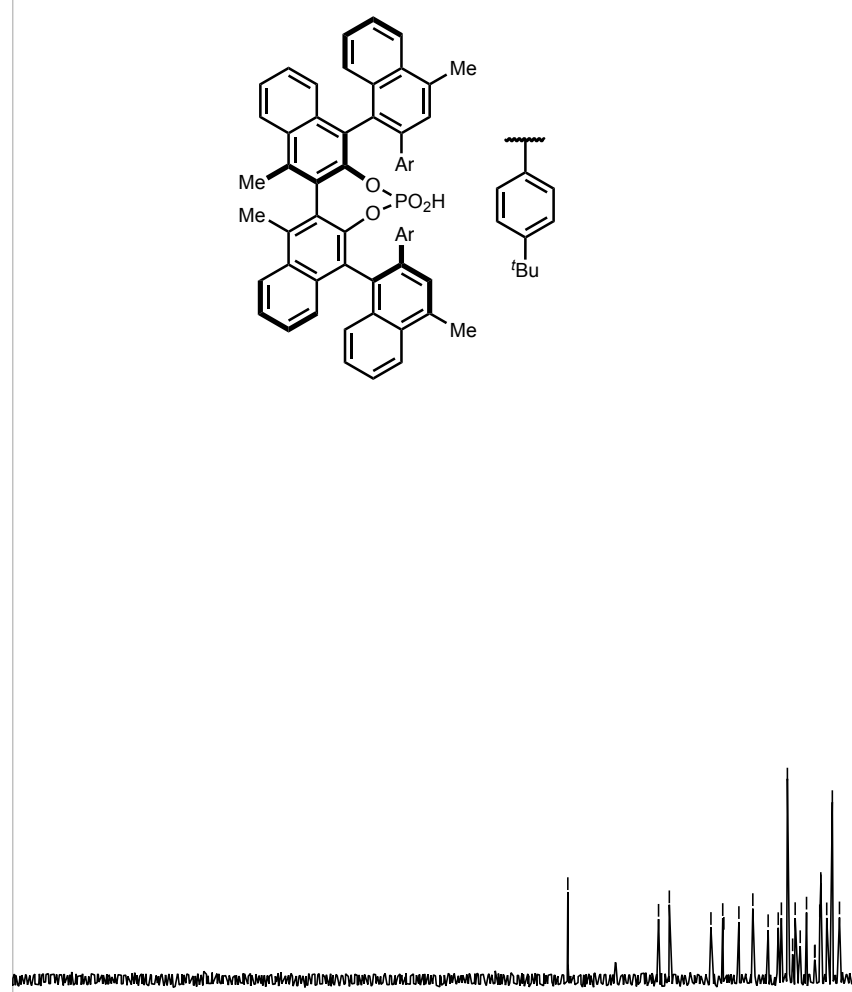

50




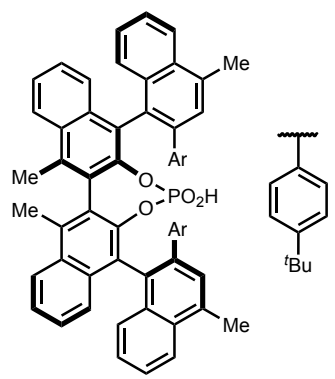

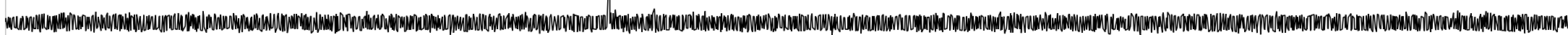

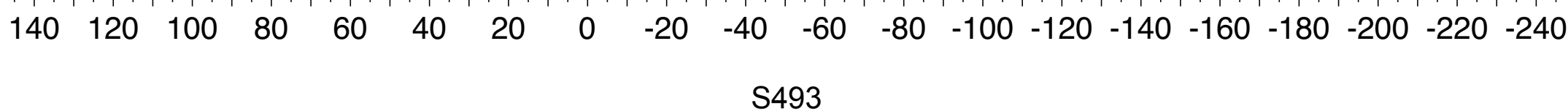

